\title{
TRAC-PF1/MOD1: An Advanced Best-Estimate Computer Program for Pressurized Water Reactor Thermal-Hydraulic Analysis
}

Safety Code Development Group

Energy Division

Manuscript submitted: May 1986 Date published: July 1986

Prepared for

Civision of Accident Evaluation

Office or Nuclear Regulatory Research

US Nuclear hiegulatory Commission

Washington, DC 20555

NRC FIN No. A7016

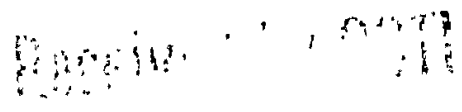

Nub i i 1980 
An Affirmative Action/Equal Opportunity Employer

Edited by Janet Holmes Stanford

Prepared by Vera B. Metzger, Group Q-9 Seates Govermment nor any asency thereor, of any of their employers, maker any warrenty, expressed of implied, or asumes any kgal bibility or responsibitity for any third pany's uxe, of the results of such use, of any information, apparatus, product or process disctosed in this report, or mopresents that its use by such third party would nox infrinze pnvately owned rights. 
If you wish to receive quarterly updates, to this docunent, please complete this form and return it to Rick Jenks at the address shown below.

Rick Jenks

Code User's Support, Safety Code Development, Q-9

Los Alamos National Laboratory

P.O. Box 1663

MS-K 555

Los Alamos, NM 87545

Name:

Organization:

Address :

Telephone: 
Many people contributed to the TRAC-PF1/MODI code development and to this report. Because it was a team effort, there wes, considerable overlap in responsibilities and contributions. The participants are listed according to their primary activity. "Those with the prime responilility for each area are listed first.

PRINCIPAL INVESTIGAT:JRS :

FLUID DYNAMICS :

HEAT TRANSFER:

CODE DEVELOPMENT ANE PROGRAMMING:

TRIPS AND CONTROLS:

GRAPHICS :

SAMPLE PROBLEM:

REPORT COMPILATION :

REPORT TYPING:
Dennis:R. JAlies and John H. Mahaffy

John 'H. Mahatfy, Susan B. Woodruff, Dennis K. Ljles, and Manjit S. Sahota

Ralụh A. Neison and Frank L. Addessio Paul. T. Giguere, Thad D. Knight, Richard, P. Jeiks, and Jennie R. Netuschil is:

Robert G Steinke

Victor Martinez and Michael R. Turner is

Paul T. Giguere and Manjit S. Sahota i.t.

Vera B. Metzger, Thad D. Knight, Dennis R. Liles, and John H. Mahaffy ",

Yyera B. Metzger and Gloria E. Mirabal

In addition to those 1isted above, we acknowledge all others who contributed to earlier, versions of TRAC. In particular, major contributions to the code architecture by Richard J. Pryor, Savannah River Laboratory, and James Sicilian, Flow' Science Inc., still are u'sed in the code". "We also acknowledge useful tiiscussions and technical exchanges with Louis M. Shotkin and Novak Zuber, US. Nuclear Regulatory Commission;: Terrence F. Boti, Francis H. Harlow, David A.c Mándell, and Burton Wenágoff, Los Alamos National Laboratory; John E. Meyer and Peter Griffith, Massachusetts Institute of Technology; S. George Bankoff, Northwestern Uaiversity; Garrett Birkhoff, Harvard University; and Ronald $P$. Harper, Flow Sirience Inc. We also thank all Los Alamos users and external users, both domesilic and foreign, who supplied comments and corrections to this document.

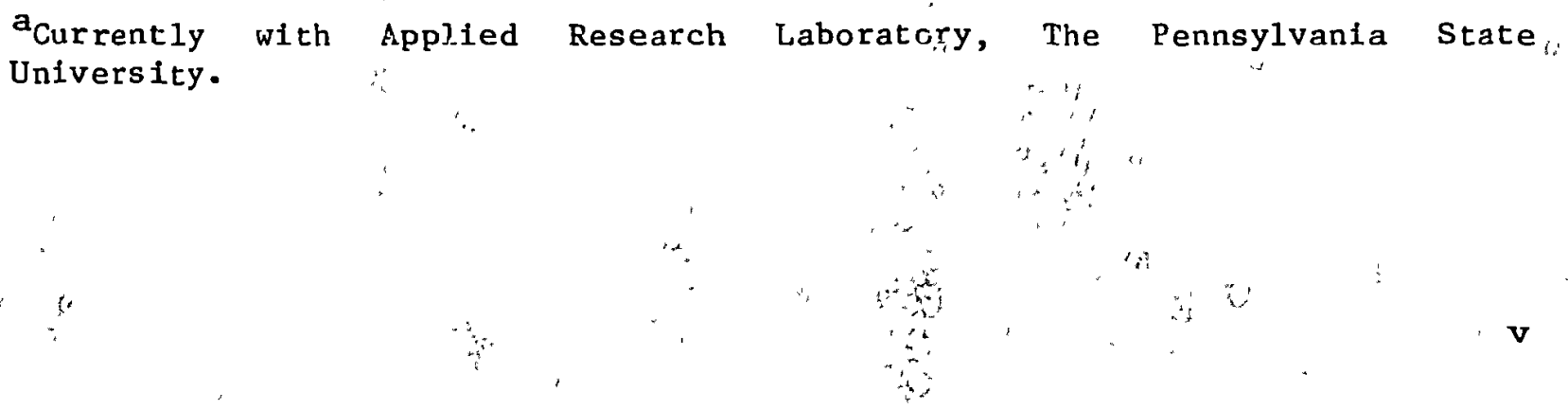


CONTENTS

AUTHORS AND ACKNOWLEDGMENTS . . . . . . . . . . . . . . . • • . v v

LIST OF FIGURES . . . . . . . . . . . . . . . . . . . xiv

LIST OF TABLES . . . . . . . . . . . . . . . . . . . . xvii

STANDARD NOMENCLATURE . . . . . . . . . . . . . . . . . . Xix

CONVERSION TABLE $\cdot$. . . . . . . . . . . . . . . . . . .

ABSTRACT . . . . . . . . . . . . . . . . . . . . . . . . . . . 1

I. COMPUTER PROGRAM OUTLINE ....................... 3

A. Program Name ..................... 3

B. Computers...................... 3

C. Problem or Function Description . . . . . . . . . . . 3

D. Solution Method . . . . . . . . . . . . . . . . 3

E. Problem Complexity Restrictions . . . . . . ....... 3

F. Typical Running Time . . . . . . . . . . . . . . 3

G. Unusual Progran Features . . . . . . . . . . . . . . . 4

H. Related and Auxiliary Programs . . . . . . . . . . . 4

I. Status ................... . . . . . 4

J. References ...................... 4

K. Machine Requirements .................. 4

L. Programming Languages... . . . . . . . . . . . . . 4

M. Operating Systems or Monitors . . . . . . . . . . . . . . 4

N. Other Programming, Operating Information, or Restrictions . . . 5

0. Avallable Materials . . . . . . . . . . . . . . 5

II. InTRODUCTION ...."................... . . . . . . 7

A. TRAC Characteristics ................ . 8

1. Variable-Dimensional Fluid Dynamics . . . . . . . . 8

2. Nonhomogeneous, Nonequilibrium Modeling . . . . . . 9

3. Flow-Regime-Dependent Constitutive Equation Package . •. 9

4. Comprehensive Heat-Transfer Capability . . . . . . . . 9

5. Consistent Analysis of Entire Accident Sequences . . . . 9

6. Component and Functional Modularity . . . . . . . . . 9

B. Physical Phenomena Treated .............. 10

C. Significant Changes from Previous TRAC Versions . . . . . . 11

D. Planned Improvements .................. . 11

E. Scope of TRAC Manual . . . . . . . . . . . . . 12

III. BASIC METHODS . . . . . . . . . . . . . . . . . . . . 15

A. Hydrodynamics . . . . . . . . . . . . . . . . 15

1. Field Equations . . . . . . . . . . . . . . . 15

2. Three-Dimensional Finite-Difference Equations . . . . . 19

a. Solution of Difference Equations . . . . . . . . 25

b. Pressure Matrix Solution ........... 27

c. Water Packing . . . . . . . . . . . . . 28

3. One-Dimensional Finite-Difference Methods . . . . . . 29

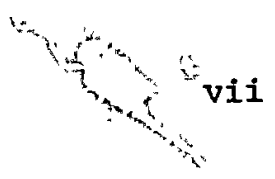


4. Constitutive Equations . . . . . . . . . . . 38

a. One-Dimensional Wall Shear and Form Losses . . . . 38

i. Homogeneous Model ............ 39

ii. Annular-Flow Model ........... 40

iii. Form Losses... . . . . . . . . . . . 41

b. Three-Dimensional Wall Shear Coefficients... . . . 43

c. Interfacial Heat Transfer and Shear . . . . . . . . 44

d. Interface Sharpener . . . . . . . . . . . . 51

e. Horizontal Stratified Flow ........... 51

f. Subcooled Boiling Model ............. 53

B. Structural Heat t'ransfer ................ . 53

1. Heat-Conduction Models .............. . 53

a. Cylindrical Wall Heat Conduction . . . . . . . 54

b. Slab Heat Conduction . . . . . . . . . . 57

c. Rod Heat Conduction .............. 60

i. Numerical Model ............. 60

ii. Reflood Algorithm ............ 65

iii. Fuel-Cladding Gap Conductance. . . . . . . 68

iv. Metal-Water Reaction .......... 72

2. Wall-to-Fluid Heat Transfer ........... 74

a. Wall-to-Fluid HTC Selection Logic......... 75

b. HTC Correlations ............... 77

i. Single-Phase Liquid (Heat-Transfer

Regimes 1 and 12) ............ . 77

ii. Nucleate-Boiling HTCs (Heat-Transfer Regime 2) 80

iii. Transition-Boiling HTCs (Heat-Transfer

Regime 3) . . . . . . . . . . . 83

iv. Film-Boiling HTCs (Heat-Transfer Regime 4) . . 84

v. Single-Phase Vapor HTCs (Heat-Transfer

Regime 6)............... 87

vi. Condensation HTCs (Heat-Transfer Regime 11) . . 87

vii. Two-Phase Mixture HTCs (Heat-Transfer

Regime 7) . . . . . . . . . . 88

c. Critical Heat Flux (CHF) . . . . . . . . . 90

d. Minimum Stable Film-Boiling Temperature, $\mathrm{T}_{\text {min }}$. . . 92

e. Steady-State Calculations ....... . . . . . 94

C. Reactor Kinetics . . . . . . . . . . . . . . . 94

D. Overall Solution Strategy ............... 110

1. Transient Solutions............... 110

a. Outer Iteration Strategy . . . . . . . . . . 110

b. Details of the Solution Method .......... . 111

2. Steady-State Solutions . . . . . . . . . . . . 114

E. Control Procedure .................... 116

1. Component-Action Table .............. 119

2. Rate-Factor Table . . . . . . . . . . . . 122

3. Signal Variables . . . . . . . . . . . . . . 124

4. Control Blocks ................. . . 132

5. Trips ....................... 148

6. Control-Parameter Evaluation ............ 155 
IV. COMPONENT MODELS . . . . . . . . . . . . . . . . . . . 159

A. Pipe ........................ 159

B. Accumulator ..................... 161

C. Break and Fill .................. . . 163

D. Core ........................ 165

E. Plenum ....................... 166

F. Pressurizer .................... 167

G. Pump ....................... 169

H. Steam Generator ................... 182

I. Tec........................ 190

J. Turbine ....................... 191

K. Valve........................ 204

L. Vessel ...................... 206

V. USER INFORMATION . . . . . . . . . . . . . . . 215

A. Input Organization and Format ............ 215

B. Dump/Restart Features . . . . . . . . . . . . 217

C. TRAC-PF1 Input Specifications . . . . . . . . . . 218

1. Maln Data................... . . . 219

2. Material-Properties Data . . . . . . . . . . 228

3. Interactive Control-Panel-Vector Data . . . . . . . 229

4. Control-Parameter Data . . . . . . . . . . . 233

a. Multipass Control-Parameter Evaluation . . . . . 233

b. Signal-Variable Data . . . . . . . . . . 234

c. Control-Block Data ............. . . 236

d. Trip Data... . . . . . . . . . . . 239

5. Component Data . . . . . . . . . . . . . . . 249

a. Accumulator Cc ponent (ACCUM) . . . . . . . . 251

b. Break Component (BREAK) . . . . . . . . . . 255

c. One-Dimensiona1 Core Component (CORE) . . . . . . 263

d. Fill Component (FILL) . . . . . . . . . . . 285

e. Pipe Component (PIPE) . . . . . . . . . . . . 293

f. Plenum Component (PLENUM) . . . . . . . . . . 303

g. Pressurizer Component (PRIZER) .......... 305

h. Pump Component (PUMP) .............. 311

i. Steam-Generator Component (STGEN) . . . . . . . . 327

j. Tee Component (TEE) . . . . . . . . . . . . 339

k. Turbine Component (TURB) . . . . . . . . . . 357

1. Valve Component (VALVE) . . . . . . . . . . 369

m. Vessel Component (VESSEL) . . . . . . . . . . 381

6. Time-Step Data ................. 406

D. LOAD Subroutine . . . . . . . . . . . . . . 407

E. Free Format . . . . . . . . . . . . . . . 408

1. Free-Format Comments, Problem Title Cards,

and Hollerith Component Descriptions ......... 410

2. Free-Format Input-Error Handling . . . . . . . . 411

F. NAMELIST Format . . . . . . . . . . . . . . . 411

G. Output Files ..................... 412 
VI. PROGRAMMING DETAILS . . . . . . . . . . . . . . .

A. Overall Code Organization . . . . . . . . . . . . . .

B. Input Processing . . . . . . . . . . . . . . . 423

C. Component Initialization . . . . . . . . . . . . 432

D. Transient Calculation . . . . . . . . . . . . 434

1. General ................... . . 434

2. Time-Step Selection and Output Control . . . . . . 436

3. Prepass Calculations . . . . . . . . . . . 437

4. Outer Iterations . . . . . . . . . . . . . 439

5. Postpass Calculations and Backup ... . . . . . . . 442

6. Vesscl Data Structure................ 442

E. Steady-State Calculations . . . . . . . . . . . . 443

F. Output Processing . . . . . . . . . . . . . . . 447

G. Storage Requirements .. . . . . . ......... 453

H. TRAC for Various Machine Configurations........... 462

APPENDIX A. THERMODYNAMIC AND TRANSPORT FLUID PROPERTIES . . . . . . 465

I. THERMODYNAMIC PROPERTIES . . . . . . . . . . . . 465

A. Saturation Properties . . . . . . . . . . . . 466

1. Relationship Between Saturation Temperature and Pressure ................ . . 466

2. Internal Energy of Steam . . . . . . . . . . 467

3. Heat Capacity of Steam at Constant Pressure . . . . 469

4. Enthalpy of Steam . . . . . . . . . . . . 470

5. Density of Steam ............... 471

6. Enthalpy of Liquid .............. 471

B. Liquid Properties... . . . . . . . . . 472

1. Internal Energy .. . . . . . . . . . . . 472

2. Density .................. . . 474

C. Vapor Properties ... . . . . . . . . . . 477

1. Superheated Steam ................ 477

2. Subcooled Vapor ................ 482

3. Noncondensable Gas (Air) ........... . 4 482

II. TRANSPORT PROPERTIES .................... . . 483

A. Latent Heat of Vaporization . . . . . . . . . . 484

B. Constant Pressure Specific Heats . .. . . . . . . . 484

C. Fluid Viscosities ................ 485

1. Liquid ................... . . 485

2. Steam . . . . . . . . . . . . . . . 486

3. Noncondensable Gas .............. 486

D. Fluid Thermal Conductivities ........... 487

E. Surface Tension.................. 488

III. VERIFICATION ...................... 488 
ÁPENDIX B. MATERIAL PROPERTIES . . . . . . . . . . . . . 497

I. INTRODUCTION . . . . . . . . . . . . .... 497

II. NUCLEAR FUEL MIXED-OXIDE PROPERTIES . . . . . . . . . . 499

A. Density .................... 499

B. Specific Heat .................. 500

C. Thermal Conductivity . . . . . . . . . . . 501

D. Spectral Emissivity ................ 502

III. ZIRCALOY CLADDING PROPERTIES . . . . . . . . . . . 502

A. Density . . . . . . . . . . . . . . . 503

B. Specific Heat . . . . . . . . . . . . . 504

C: Thermal Conductivity . . . . . . . . . . 504

D. Spectral Emissivity ................ 504

IV. FUEL-CLADDING GAP-GAS PROPERTIES . . . . . . . . . . 505

V. ELECTRICAL FUEL-ROD INSULATOR (BN) PROPERTIES . . . . . . . 507

A. Density . . . . . . . . . . . . . . . . 507

B. Specific Heat ................. 507

C. Thermal Conductivity . . . . . . . . . . 507

D. Spectral Emissivity ................. 507

4 VI. ELECTRICAL FUEL-ROD HEATER-COIL (CONSTANTAN) PROPERTIES • • 508

A. Density . . . . . . . . . . . . . . . 50u

B. Specific Heat . . . . . . . . . . . . 508

C. Thermal Conductivity ............... 508

D. Spectral Emissivity . . . . . . . . . . . . 508

VII. STRUCTURAL MATERIAL PROPERTIES . . . . . . . . . . 508

APPENDIX C. SETS NUMERICAL METHOD . • • • • . • • • • . • • . 517

I'. BASIC EqUATIONS ..................... 517

II. SOLUTION OF THE BASIC EQUATION SET . . . . . . . . . . 522

APPENDIX D. CHOKED-FLOW MODEL . . . . . . . . . . . . . . 527

I. INTRODUCTION ....................... 527

II. FEATURES . . . . . . . . . . . . . . . . . . 528

A. Two-Phase Flow Choking Criterion ........... 528

B. Subcooled-Flow Choking Criterion .......... 530

C. Single-Phase Vapor Choking Criterion . . . . . . . 533

D. Transition Regimes . . . . . . . . . . . 533

III. RESULTS . . . . . . . . . . . . . . . . . . 534

A. Comparison of the TRAC-PF1/MOD1 Choked-Flow Model
with Other Models . . . . . . . . . . . 534

B. Comparison of the TRAC-PF1/MOD1 Choked-Flow Mode1

Calculations with the Fine-Mesh Calculations and

the Test Data................ 536

1. Marviken Test Facility . . . . . . . . . . 536

2. Edwards ${ }^{-}$Blowdown Experiment . . . . . . . . \$541

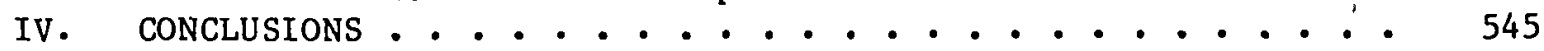


APPENDIX E. PWR SAMPLE PROBLEM • . . . . . . . . . . . . . . . 547

I. LOFT SYSTEM DESCRIPTION . . . . . . . . . . . . . . 547

II. TEST DESCRIPTION . . . . . . . . . . . . . . . . . 550

III. TRAC ONE-DIMENSIONAL MODEL . . . . . . . . . . . . . 550

A. Pressurizer Heaters . . . . . . . . . . . . . 557

B. Pressurizer Liquid Level . . . . . . . . . . . 559

C. Pump-Speed Controller . . . . . . . . . . . . 561

D. Steam-Generator Secondary-Side Feedwater Controller . . . 561

E. Steam-Generator Secondary-Side Steam-Flow Valve Controller . . . . . . . . . . . . 565

IV. STEADY-STATE CALCULATION . . . . . . . . . . . . . 567

V. TRANSIENT CALCUlation . . . . . . . . . . . . . . 570

VI. INPUT LISTINGS . . . . . . . . . . . . . . . . . . . 579

A. Steady-State Ont-Dimensional Input for LOFT L6-1 . . . 581

B. Restart Input Deck for a Transient Calculation

C. LOFT Three-Dimensional VESSEL Input . . . . . . . 623

APPENDIX F. TRAC ERROR MESSAGES • . . . • . . . . . . • . 637

APPENDIX G. TRAC SUBPROGRAMS . . . . . . . . . . . . . . . 673

APPENDIX H. DESCRIPTION OF COMMON-BLOCK VARIABLES . . . . . . . . 695

APPENDIX I. COMPONENT DATA TABLES . . . . . . . . . . . . . 743

I. GENERAL POINTER TABLES" . . . . . . . . . . ... 743

A. DUALPT . . . . . . . . . . ....... 743

B. HYDROPT ..................... 747

C. $\operatorname{INTPT} \cdot . \cdot . \cdot . \cdot . \cdot . \cdot . \cdot . \cdot . \cdot . \cdot . \cdot 750$

D. HÉATPT . . . . . . . . . . . . . . . . . . 750

II. ACCUMULATOR MODULE . . . . . . . . . . . . . . . 751

A. ACCUMVLT--ACCUM Variable-Length Table . . . . . . . 751

B. ACCUMPT-ACCUM Pointer Table ... . . . . . . 752

C. ACCDATA-ACCUM Data Table . . . . . . . . . . 753

III. BREAK MODULE .................... 753

A. BREAKVLT--BREAK Variable-Length Table . . . . . . . 753

B. BREAKPT--BREAK Pointer Table ... . . . . . . 755

C. BRKDATA-BREAK Data Table . . . . . . . . . . 755

IV. CORE MODULE . . . . . . . . . . . . . . . . 755

A. COREVLT-Core Variable-Length Table . . . . . . . 755

B. COREPT--Core Pointer Table . . . . . . . . . . 762

C. COREDATA-Core Data Table,.............. 767

V. FILL MODULE . . . . . . . . . . . . . . .. 767

A. FILLVLT--FILL Variable-Length Table . . . . . . . 767

B. FILLPT-FILl Pointer Table ... .......... 769

C. FILLDATA-FILL Data Table . . . . . . ..... 770

VI. PIPE MODULE ....................... . . 770

A. PIPEVLT-PIPE Variable-Length Table ......... . 770

B. PIPEPT-PIPE Pointer Table ............. 773

C. PIPEDATA--PIPE Data Table ........... 774

xii 
VII. PLENUM MODULE . . . . . . . . . . . . . . . . . 774

A. VLTAB-PLENUM Variable-Length Table . . . . . . . . 774

B. PTAB--PLENUM Pointer Table . . . . . . . . . . . . 776

C. Equivalences Defined for BLANKCOM . . . . . . . . . 777

VIII. PRESSURIZER MODULE . . . . . . . . . . . . . . . . . 782

A. PRIZEVLT--PRIZER Variable-Length Table . . . . . . . 782

B. PRIZEPT--PRIZER Pointer Table . . . . . . . . . . 784

C. PRZDATA--PRIZER Data Table . . . . . . . . . . 784

IX. PUMP MODULE . . . . . . . . . . . . . . . . . . . 784

A. PUMPVLT--PUMP Variable-Length Table . . . . . . . . . 784

B. PUMPPT--PUMP Pointer Table ........... . 789

C. PUMPDATA-PUMP Data Table .............. i90

X. STEAM-GENERATOR MODULE . . . . . . . . . . . . . . 790

A. STGENVLT-STGEN Variable-Length Table . . . . . . . . 790

B. STGENPT-STGEN Pointer Table ............ 794

C. STGNDATA-STGEN Data Table ............. 799

XI. TEE MODULE ....................... . . 799

A. TEEVLT-TEE Variable-Length Table .......... 799

B. TEEPT-TEE Pointer Table . . . . . . . . . . 806

C. TEEDATA-TEE Data Table ............. 807

XII. TURBINE MODULE . . . . . . . . . . . . . . . . . 807

A. TURBNVLT-TURB Variable-Length Table .......... 897

B. TURBPT-TURB Pointer Table .............. . 811

C. TURBDATA-TURB Data Table . . . . . . ....... 811

XIII. VAlVE MODULE ...................... 812

A. VALVEVLT--VALVE Variable-Length Table . . . . . . 812

B. VLVEPT-VVALVE Pointer Table .............". 815

C. VLVEDATA--VALVE Data Table . . . . . . . . . . . 816

XIV. VESSEL MODULE ....................... 816

A. VSSELVLT-VESSEL Variable-Length Table . . . . . . 816

B. VSSLPT-VESSEL Pointer Table . . . . . . . . . . 825

C. VSSLDATA-VESSEL Data Table . . . . . . . . . 840

REFERENCES . . . . . . . . . . . . . . . . . . . 841 
FIGURES

1. Three-dimensional mesh-cell velocities . . . . . . . . 20

2. Flow-regime map for three-dimensional hydrodynamics . . . . . . 44

3. Semi-implicit coupling between hydrodynamics and structural

heat transfer . . . . . . . . . . . . . 54

4. Cylindrical wall geometry . . . . . . . . . . . . 56

5. Rod geometry . . . . . . . . . . . . . . . . 61

6. Node located at the interface between two dissimilar materials . 64

7. Fine-mesh rezoning . . . . . . . . . . . . . . . . 66

8. Insertion of conduclion nodes during reflood . . . . . . . . 66

9. Fuel-rod geometry . . . . . . . . . . . . . . . . 69

10. TRAC-PFl/MODl boiling curve .................... 74

11. HTC correlation selection logic . . . . . . . . . . . 76

12. Comrionent network with one three-dimensional vessel.... .112

13. TRAC simulation model of a PWR power plant ........... 117

14. Proportional plus integral controller diagram . . . . . . . . . 144

15. Proportional plus integral plus derivative controller diagram . 145

16. Control-block diagram . . . . . . . . . . . . . . . 146

17. Implicit control-block loop example . . . . . . . . . . 157

1.8. Plpe noding diagram . . . . . . . . . . . . . . . . 160

19. Accumulator noding diagram . . . . . . . . . . . 162

20. Break noding diagram . . . . ........... . . 163

21. Fill noding diagram ................. . . . 164

22. Core noding diagram ................. . . . 165

23. Pressurizer noding diagram . . . . . . . . . . . 168

24. Pump noding diagram . . . . . . . . . . . . . . . 170

25. Semiscale single-phase homologous head curves . . . . . . . . 179

26. Semiscale fully degraded homologous head curves . . . . . . . ' 179

27. Semiscale head degradation multiplier curve . . . . . . . 179

28. Semiscale single-phase homologous torque curves . . . . . . ' 179

29. Semiscale torque degradation multiplier curve . . . . . . . 180

30. LOFT single-phase homologous head curves . . . . . . . . 180

31. LOFT fully degraded homologous head curves . . . . . . . . . 180

32. LOFT head degradation multiplier curve . . . . . . . . . 180

33. LOFT single-phase homologous torque curves . . . . . . . . 181

34. LOFT torque degradation multiplier curve . . . . . . . . . . 181

35. TRAC steam-generator noding diagrams . . . . . . . . . . 189

36. Tee noding diagram . . . . . . . . . . . . . . . 191

37. Steam expansion through a turbine stage . . . . . . . . . 193

38. Turbine stage noding diagram depicting momentum and energy . . 194

39. Velocity diagram for two rows of moving blades . . . . . . . 197

40. A typical noding diagram showing the use of special valve options for modeling turbines . . . . . . . . . . 202

41. Valve noding diagram . . . . . . . . . . . . . . . . 204

42. Cell noding diagram for a typical PWR vessel . . . . . . . . 207

43. Vessel geometry, three-dimensional mesh construction 3 . . . 207

44. Boundaries of a three-dimensional mesh cell . . . . . . . 208

45. Flow restrictions and downcomer modeling . . . . . . . . . 210

46. Pipe connections to the vesse1 . . . . . . . . . . . 210

47. Core region inside the vessel . . . . . . . . . . . . 211 
48. TRAC input deck organization . . . . . . . . . . . Flow diagram for steady-state calculation . . . . . . . . . Overall graphics file structure . . . . . . . . . . . .

58. Structure of graphics file catalog. . . . . . . . . . .

59. Structure of graphics file time-edit data section . . . . . . Graphics file catalog and time-edit data correspondence . . . Dump-file overall structure . . . . . . . . . . . . LCM data organization . . . . . . . . . . . . . . . . .

D-3. Comparison of the subcooled critical mass fluxes. The TRAC-PF1/MODl calculations, the test data from the Burnell model, and the HEM tables in Ref. 67 were used . . . .

1-4. Comparison of the TRAC-calculated two-phase critical mass flux with the test data from the HEM tables in Ref. 67 . . . .

D-5. Marviken pressure vessel . . . . . . . . . . . . .

D-6. Marviken discharge pipe, test nozzle, and rupture-disk assembly . . . . . . . . . . . . . . . . .

D-7. Comparison of the nozzle mass flows for Marviken Test 4 between the test data and the TRAC-PF1/MOD1 models. The fine-mesh and choking criteria were used . . . .

D-8. Comparison of the system pressure histories for Marviken Test 4 between the test data and the TRAC-PFl/MOD1 models. The fine-mesh and choking criteria were used . . . . . . . .

D-9. Comparison of the nozzle mass flows for Marviken Test 24 between the test data and the TRAC-PFl/MOD1 models. The fine-mesh and choking criteria were used . . . . . . . .

D-10. Comparison of the system pressure histories for Marviken Test 24 between the test data and the TRAC-PFI/MODI models. The fine-mesh and choking criteria were used . . . . . . . . .

D-11. Comparison of the nozzle mass flows for Marviken Test 24 between the test data and the TRAC-PF1/MOD1 models. The fine-mesh and choking criteria obtained from the seven-equation frozen model were used ..............

D-12. Comparison of the system pressure histories for Marviken Test 24 between the test data and the TRAC-PF1/MOD1 models. The fine-mesh and choking criteria obtained from the seven-equation frozen model were used ............. 
D-13. Edwards horizontal-pipe blowdown experiment (adapted from Ref. 71) ...................

D-14. Comparison of the system pressure histories for the Edwards blowdown experiment between the test data and the TRAC-PF1/MOD1 calculations. The fine-mesh and choking criteria were used . . . . . . . . . . . . . .

E-6. Three-dimensional vessel noding combining all the components shown in Fig. E-5 . . . . . . . . . . . . 552

E-7. Pressurizer liquid-level calculation using control blocks . . . 560

E-8. Pump-speed control system . . . . . . . . . . . . . . 562

E-9. Steam-generator secondary-side feedwater control system . . . . 563

E-10. Steam-generator secondary-side steam-flow valve control system . 566

E-11. Steady-state upper-plenum pressure for Test L6-1 . . . . . . . 567

E-12. Steady-state pump number 1 speed for Test L6-1 . . . . . . . 568

E-13. Steady-state primary-coolant mass flow for Test L6-1 . . . . . 568

E-14. Steady-state steam-generator primary-side inlet temperature for Test L6-1 . . . . . . . . . . . . 569

E-15. Steady-state steam-generator primary-side outlet cemperature for Test L6-1 . . . . . . . . . . . 569

E-16. Steady-state steam-generator secondary-side pressure

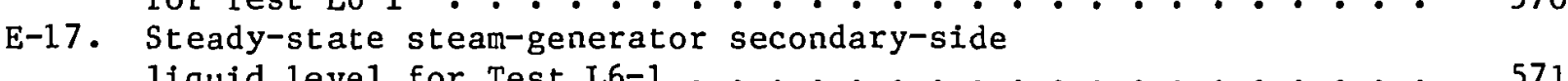
liquid level for Test L6-1 . . . . . . . . . . . . . 571

E-18. Steady-state steam-generator secondary-side
feedwater mass flow for Test L6-1 . . . . . . . . . . 571

E-19. Steady-state steam-generator secondary-side

E-20. Comparison of the TRAC-calculated with the measured core powers for Test L6-1 . . . . . . . . . . . . 575

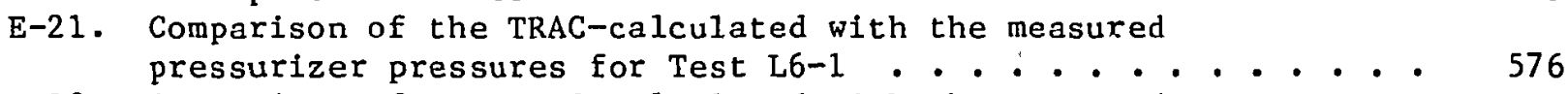

E-22. Comparison of the TRAC-calculated with the measured

E-23. Comparison of the TRAC-calculated with the measured steam-generator secondary-side feedwater mass flows for Test L6-1 . . . . . . . . . . . 577

E-24. Comparison of the TRAC-calculated with the measured steam-generator secondary-side steam mass flows for Test L6-1 . . . . . . . . . . . 578

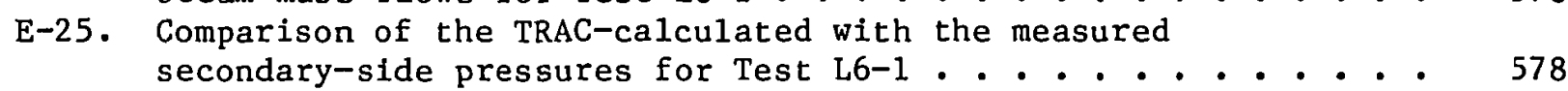

E-26. Comparison of the TRAC-calculated with the measured
pressurizer liquid-level histories for Test L6-1 . . . . . 579 
TABLES

I. TRAC-PF1/MOD1 HEAT-TRANSFER REGIMES . . . . . . . . 78

II. DELAYED-NEUTRON CONSTANTS . . . . . . . . . . . . . 97

III. DECAY-HEAT CONSTANTS . . . . . . . . . . . . . . . 97

IV. REACTIVITY-COEFFICIENT FORMS . . . . . . . . . . . . . 99

V. ADJUSTABLE COMPONENT HARDWARE ACTIONS

BY THE CONTROL PROCEDURE . . . . . . . . . . . . . . . 118

VI. DEFINED FORMS OF THE COMPONENT-ACTION TABLE'S

VII. SIGNAL-VARIABLE PARAMETERS . . . . . . . . . . . . . . . 126

VIII. CONTROL-BLOCK FUNCTION OPERATIONS . . . . . . . . . . . . 134

IX. TRIP SIGNAL-RANGE TYPES . . . . . . . . . . . . . . . . . . 149

$X$. ID NUMBERS OF CONTROL PARAMETERS EVALUATED DURING

EACH OF FIVE CONTROL-PARAMETER EVALUATION PASSES . . . . . . 158

XI. DEFINITIONS OF THE FOUR CURVE SEGMENTS THAT

XII. SPECIFICATION OF THE FLUID CELLS ASSOCIATED

WITH THE CONDUCTION PATHS FOR AN ASPIRATED

ONCE-THROUGH STEAM GENERATOR . . . . . . . . . . . . . . .

XIII. SPECIFICATION OF THE FLUID CELLS ASSOCIATED

WITH THE CONDUCTION PATHS FOR A U-TUBE

STEAM GENERATOR WITH A COUNTERFLOW PREHEATER . . . . . . . .

XIV. ARITHMETIC-OPERATOR ID NUMBERS OF THE Jth

ARITHMETIC SUBEXPRESSIONS . . . . . . . . . . . . . . 243

XV. IMPORTANT LOW-LEVEL SUBPROGRAMS . . . . . . . . . . . . 416

XVI. TRAC DVERLAYS . . . . . . . . . . . . . . . . . . . . . 419

XVII. FIXED SEGMENT ALLOCATIONS FOR THE BLANK COMMON AREA . . . • . 424

XVIII. COMPONENT INPUT SUBROUTINES . . . . . . . . . . . . . . 428

XIX. FIRST INDEX FOR THE JUNCTION-COMPONENT PAIR ARRAY • • • • • . 429

XX. BOUNDARY ARRAY DATA . . . . . . . . . . . . . . . 429

XXI. COMPONENT INITIALIZATION SUBROUTINES . . . . . . . . . . 433

XXII. ITERATION SUBROUTINES . . . . . . . . . . . . . . . 439

XXIII. STEADY-STATE CALCULATION EFFECTS . . . . . . . . . . . . 444

XXIV. EXAMPLE OF A STEADY-STATE CONVERGENCE EDIT . . . . . . . . . . 445

XXV. COMPONENT EDIT SUBROUTINES . . . . . . . . . . . . . . . 448

XXVI. COMPONENT DUMP SUBROUTINES . . . . . . . . . . . . . . 458

XXVII. TRAC STORAGE ALLOCATIONS . . . . . . . . . . . . . 458

XXVIII. TRAC DEFINITIONS . . . . . . . . . . . . . . . . . 463

A-I. POLYNOMIAL CONSTANTS FOR THERMO . . . . . . . . . . . . 489

A-II. DERIVED CONSTANTS FOR THERMODYNAMIC PROPERTIES OF WATER

AND NONCONDENSABLE GAS . . . . . . . . . . . . . . . . 491

A-III. BASIC CONSTANTS FOR TRANSPORT PROPERTIES OF WATER AND AIR • • 492

A-IV. LIQUID VISCOSITY CONSTANTS . . . . . . . . . . . . . . . . 493

A-V. VAPOR VISCOSITY CONSTANTS . . . . . . . . . . . . . . . 494

A-VI. THERMAL CONDUCTIVITY CONSTANTS . . . . . . . . . . . 495 


\section{TABLES (cont.)}

B-I. SPEClFiC HEAT VS TEMPERATURE ................ 509

B-II. STRUCTURAL MATERIAL PROPERTIES

STAINLESS STEEL, TYPE 304 . . . . . . . . . . . . 510

B-III. STRUCTURAL MATERIAL PROPERTIES

STAINLESS STEEL, TYPE 316 . . . . . . . . . . . . 511

B-IV. STRUCTURAL MATERIAL PROPERTIES

STAINLESS STEEL, TYYE 347 . . . . . . . . . . . . . . 512

B-V. GTRUCTURAL MATERIAL PROPERTIES

Carbon S'TELL, TYPE 508 . . . . . . . . . . . . . . 513

B-VI. STRUCTIRAL MATERIAL PROPERTIES

INCONIL, TYPE 718 . . . . . . . . . . . . . . 514

B-VII. STRUCTURAL MATERIAL PROPERTIES

INCONEL, TYPE 600 .................... 515

E-I. TRAC MODEL COMPONENTS . . . . . . . . . . . . . 553

E-II. MAJOR TRIP SET POINTS FOR TEST L6-1 . . . . . . . . . . . 558

E-III. TEST L6-1 INITIAL CONDITIONS . . . . . . . . . . . . . 573

E-IV. TEST L6-1 SEQUENCE OF EVENTS . . . . . . . . . . . . . . 574 
STANDARD NOMENCLATURE

\section{Independent Variables}

$r$

$t$

$\theta$

$\mathrm{x}$

$\mathbf{Z}$
Radial coordinate in cylindrical geometry.

Time.

Azinuthal coordinate in cylindrical geometry. Coordinate for one-dinensional geometry. Axial coordinate in cylindrical geometry.

\section{Other Variables}

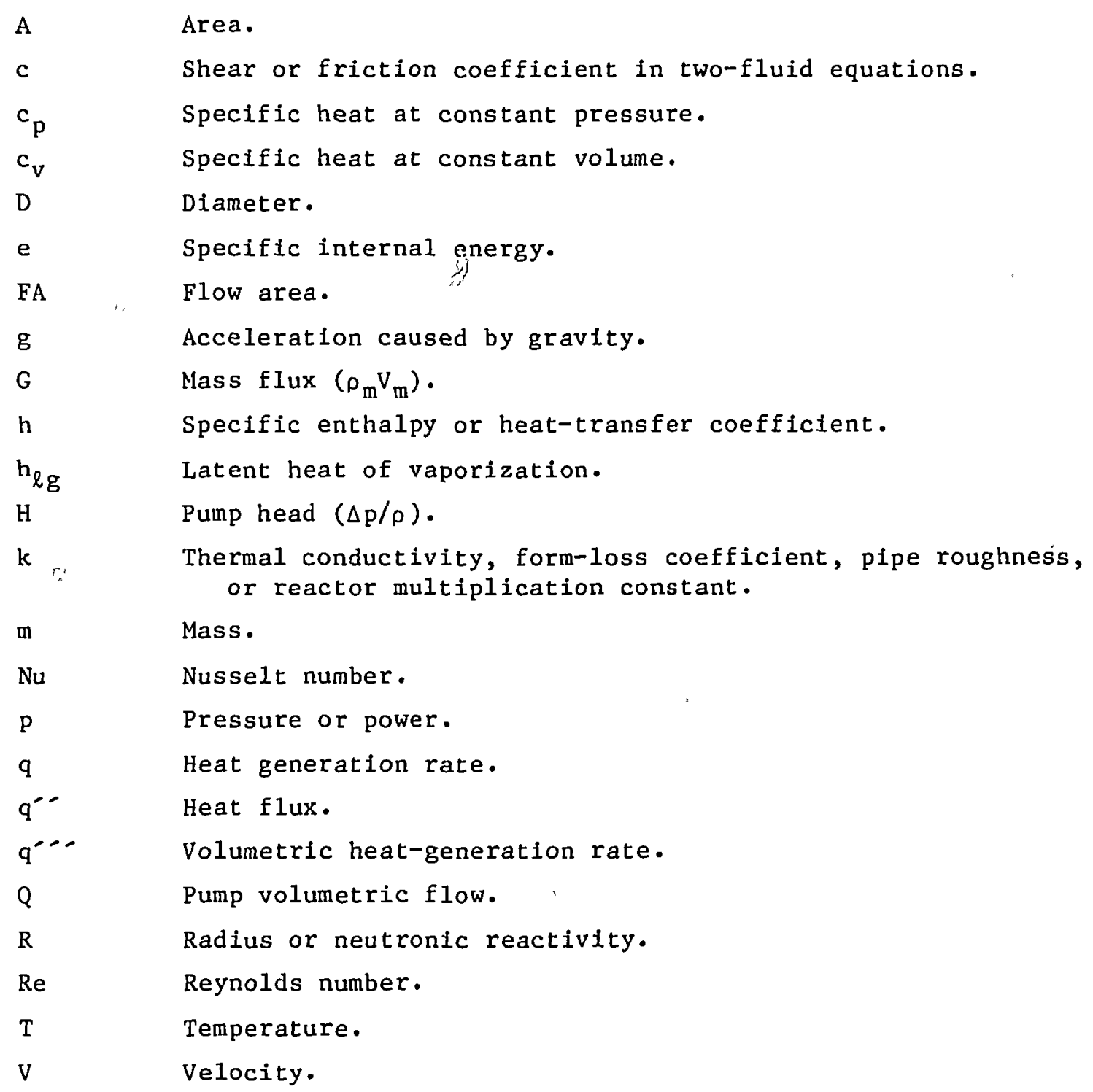

A

c

$c_{p}$

$c_{v}$

D

e

FA

g

G

h

$\mathrm{h}_{\ell \mathrm{g}}$

$\mathrm{H}$

k

m

Nu

p

q

$q^{-e}$

R

$\mathrm{Re}$

T

V

Area,

Shear or friction coefficient in two-fluid equations.

Specific heat at constant pressure.

Specific heat at constant volume.

Diameter.

Specific internal genergy.

Flow area.

Acceleration caused by gravity.

Mass flux $\left(\rho_{\mathrm{m}} \mathrm{V}_{\mathrm{m}}\right)$.

Specific enthalpy or heat-transfer coeffictent.

Latent heat of vaporization.

Pump head $(\Delta \mathrm{p} / \mathrm{\rho})$.

Thermal conductivity, form-loss coefficient, pipe roughness, or reactor multiplication constant.

Mass.

Nusselt number.

Pressure or power.

Heat generation rate.

Heat flux.

Volumetric heat-generation rate.

Pump volumetric flow.

Radius or neutronic reactivity.

Reynolds number.

Temperature.

Velocity. 
$\therefore i$

STANDARD NOMENG:ATURE (cont.)

\section{Other Variables}

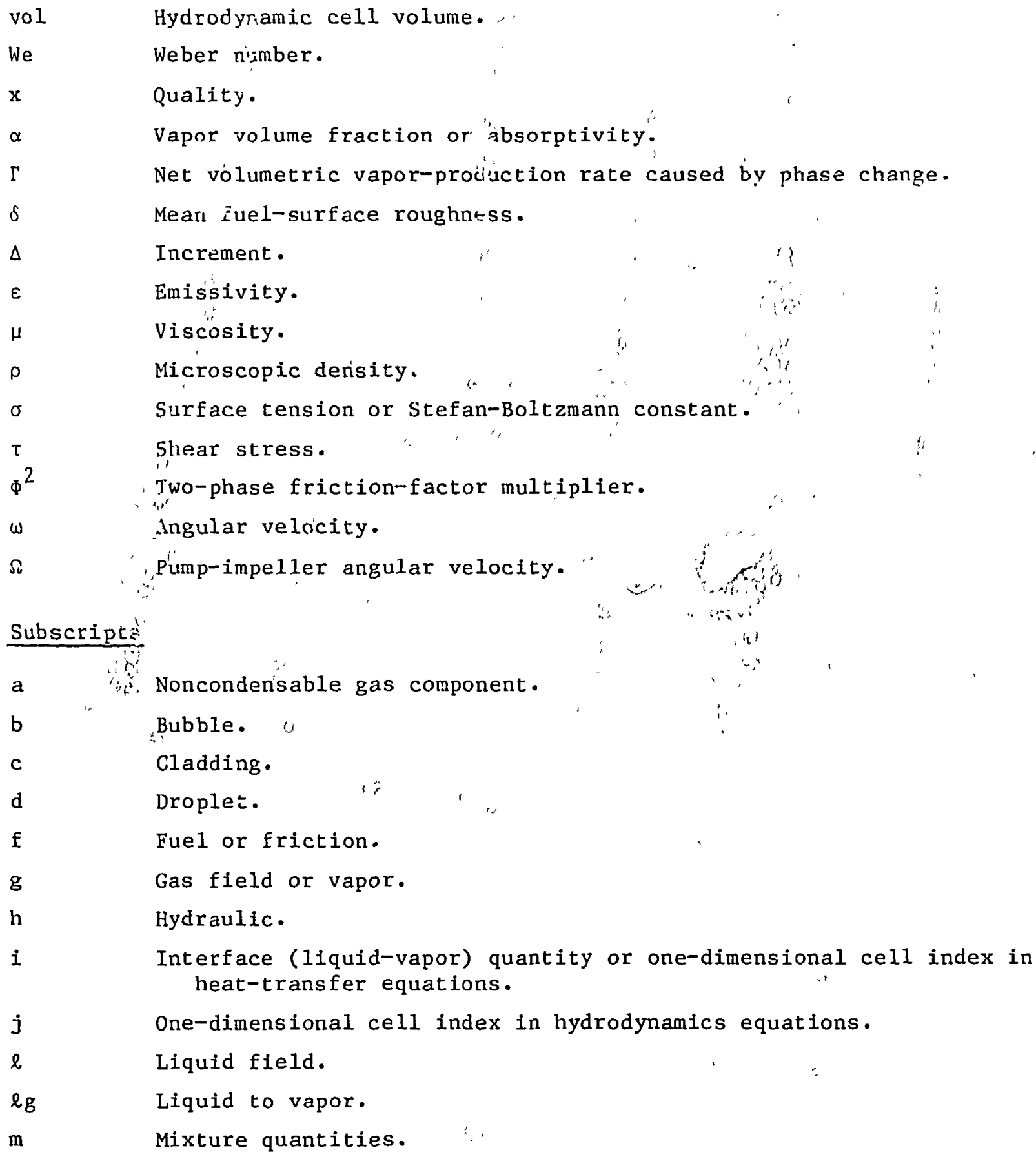


TRAC-PF1/MOD1:

AN ADVANCED BEST-ESTIMATE COMPU'TER PROGRAM

FOR PRESSURIZED WATER REACTOR THERMAL-HYDRAULIC ANALYSIS

by

Safety Code Development Group

Energy Division

\begin{abstract}
The Los Alamos National Laboratory is developing the Transient Reactor Analysis Code (TRAC) to provide advanced best-estimate predictions of postulated accidents in light-water reactors. The TRAC-PF1/MOD1 program provides this capability for pressurized water reactors and for many thermal-hydraulic test facilities. The code features either a one- or a three-dimensional treatment of the pressure vessel and its associated internals, a two-fluid nonequilibrium hydrodynamics model with a noncondensable gas field and solute tracking, flow-regime-dependent constitutive equation treatinent, optional reflood tracking capability for bottom-flood and falling-film quench fronts, and consistent treatment of entire accident sequences including the generation of consistent initial conditions. The stability-enhancing twostep (SETS) numerical algorithm is used in the onedimensional hydrodynamics and permits this portion of the fluid dynamics to violate the material Courant condition. This technique permits large time steps and, hence, reduced running time for slow transients.

In addition to the components contained, in previous TRAC versions, TRAC-PF1/MOD 1 includes an improved steam-generator model, a turbine component, and a control system together with modifled constitutive relations to model the balance of plant on the secondary side and to extend the applications to transients without loss of coolant. This report describes the thermal-hydraulic models and the numerical solution methods used in the code. Detailed programming and user information are provided.
\end{abstract}


I. COMPUTER PROGRAM OUTLINE

A. Program Name

The program natne is TRAC-PF1/MOD1, version 12.1

B. Computers

The code is designed for the Control Data Corporation CDC 7600 and for the Cray Research, Inc., Cray-l computers. Efforts have been made to make the programming as machine independent as possible.

c. Problem or Function Description

TRAC-PF1/MODI performs best-estimate analyses of loss-of-coolant accidents (LOCAS) and other transients in pressurized light-water reactors (LWRs). The code also models a wide range of thermal-hydraulic experiments in reduced-scale facilities. Models used include reflood, raultidimensional twophase flow, nonequilibrium thermodynamics, generalized heat transfer, and reactor kinetics. Automatic steady-state and dump/restart capabilities also are provided.

\section{Solution Method}

The partial differential equations that describe the two-phase flow and the heat transfer are solved by finite differences. The heat-transfer equations are treated using a semi-implicit differencing technique. The fluid-dynamics equations in the one-dimensional components use a multistep procedure that allows the material Courant condition to be violated. The three-dimensional vessel option uses semi-impiicit differencing. The finitedifference equations for hydrodynamic phenomena form a system of coupled, nonlinear equations that are sulved by a Newton iteration procedure.

E. Problem Complexity Restrictions

All storage arrays in the code are allocated dynarically so the only limit on the problem size is the amount of core memory. The number of reactor components in the problem and the manner in which they are coupled are arbitrary. Reactor components in TRAC-PF1/MOD1 are accumulators, breaks and filits; cores, pipes, pressurizers, pumps, steam generators, tees, turbines, valves, and vessels with associated internals.

F. Typical Running Time

Running time is highly problem dependent and is a function of the total number of mesh cells, the maximum allowable time-step size, and the selection of a one- or three-dimensional vessel. For a one-dimensional model, very 
large time steps can be used for slow transients. For a three-dimensional model, a material Courant limit in the vessel may reduce the maximum time-step size allowed and may increase the running time. Typical times for, a CDC 7600 computer average 2-3 ms per time step per mesh cell.

G. Unusual Program Features

The highly versatile TRAC-PFl/MODl describes most thermal-hydraulic experiments in addition to a wide variety of LWR system designs. The code's modularity allows better geometric problem descriptions, more detailed models of physical processes, and reduced maintenance costs.

H. Related and Auxiliary Programs

One output file, TRCGRF, contains graphics information that can be used to produce plots and movies. Two auxiliary programs, TRAP and EXCON, also are available for this purpose and are documented separately. These programs may require changes to be compatible with graphics software and hardware at various installations.

I. Status

The code is used at the Los Alamos National Laboratory and at many other installations.

J._._References

References are provided in the manual.

K. Machine Requirements

For most applications, a CDC 7600 gomputer with 60000 words of smallcore memory (SCM) and 220000 words of flarge-core memory (LCM) is required. For very large system simulations, a ${ }^{\prime}$ Cray-l or a similar computer is recommended.

L. Programining Languages

The programming language is FORTRAN-IV. (Although COMPASS matrix inversion subroutines are available, they are not mandatory.)

M. Operating Systems or Monitors

The code compiles on a standard Fortran Extended (FTN) compiler. The Cray-1 version compiles on a Cray Fortran (CFT) compiler. Los Alamos operates its CDC 7600 computers on the Livermore Time-Sharing System (LTSS) and $\mathrm{Cray}^{-1}$ computers on a similar Cray "Time-Sharing System (CTSS). Compatibility with the CDC SCOPE operating systeln has been maximized. 
N. Other Programining, Operating Information, or Restrictions

The code is modified through CDC Update files.

0. Available Materials

A source listing, a TRAC-PFl/MODl manual, and sample problems are available. 
The Transient Reactor Analysis Code (TRAC), an advanced best-estimate systems code for analyzing LWR accidents, is being developed at the Los Alamos National Laboratory under the sponsorship of the Reactor Safety Research Division of the US Nuclear Regulatory Commission (NRC). A preliminary TRAC version consisting of only one-dimensional components was completed in December 1976. Although this version was not released publicly nor dúcumented formally, it was used in TRAC-PI development and formed the basis for the onedimensional loop-component modules. The first publicly released version, TRAC-P1, was completed in December 1977. It is described in Los Alamos report LA-7279-MS (June 1978).

The TRAC-P1 program was designed primarily for the analysis of largebreak LOCAs in pressurized water reactors (PWRs). However, because of its versatility, it could be applied directly to many analyses ranging from blowdowns in simple pipes to integral LOCA tests in multiloop facilities. A refined version, TRAC-P1A, was released to the National Energy Software Center (NESC) in March 1979. It is described in Los Alamos report LA-7777-MS (May 1979). Although it still treats the same class of pioblems, TRAC-PlA is more efficient than TRAC-P1 and incorporates improved hydrodynamic and heattransfer models. It also is easier to implement on various computers. TRAC-PD2 contains improvements in reflood, heat-transfer models, and numerical solution methods. Although a large-break LOCA code, it has been applied successfully to sinall-break problems and to the Three Mile Island incident.

TRAC-PF1 was designed to improve the ability of TRAC-PD2 to handle small-break LOCAs and other transients. TRAC-PFi has all of the major improvements of TRAC-PD2; in addition, it uses a full two-fluid model with two-step numerics in the one-dimensional components. The two-fluid model, in conjunction with a stratifled-flow regime, handles countercurrent flow better than the drift-flux model used previously. The two-step numerics allow large time steps for slow transients. A one-dimensional core component permits calculations with.reduced dimensionality although the three-dimensional vessel option has been retained. A noncondensable gas field has been added to the one- and the three-dimensional hydrodynamics. Significant improvements also have been made to the trip logic and the input. TRAC-PF1 was released publicly in July 1981. 
TRAC-PF1/MOD1 provides full balance-of-plant modeling through the addition of a general capability to model plant control systems. The steamgenerator model was replaced to allow a wider variety of feedwater connections and better modeling of steam-tube ruptures. New components were not required to model condensers, heaters, and pumps in the secondary system; however, a special turbine component was added. The TRAC-PF1/MOD1 physical models also have been modified; the condensation model contains the most significant changes. During condensation the liquid-side interfacidi heat-transfer coefficient (HTC), which is sensitive to the flow regime, includes a special model for thermally stratified configurations. Wall heat transfer in the condensation and film-boiling regimes has been improved. The motion equations include momentum transport caused by phase change, and their momentum flux terms in the three-dimensional flow equations have been modified. This last modification can change substantially, the computed pressure drop across a vessel from that calculated by previous codes. These model changes make TRAC-PF1/MOD1 a superior code not only for small-break and operational transients but also for large-break analyses.

A. TRAC Characteristics

Some distinguishing characteristics of f́RAC-PFl/MODl are summarized below. Withín restrictions imposed by computer running times, we are incorporating state-of-the-art technology in two-phase thermal hydraulics into the code.

1. Variable-Dimensional Fluid Dynamics. A three-dimensional $(r, \theta, z)$ flow calculation can be used within the reactor vessel; the flow within the loop components is treated one dimensionally to allow an accurate calculation of the complex multidinensional flow patterns inside the reactor vessel that are important in determining accident behavior. For example", phenomena such as emérgency core-coolant (ECC) downcomer penetration during blowdown, multidimensional plenum and core flow effects, and upper-plenum pool formation and core penetration during reflootican be treated directly. However, a onedimensional vessel model may be constructed that allows fast transient calculations because the usual time-step restrictions are removed by the special stabilizing numerical treatment. 
2. Nonhomogeneous, Nonequilibrium Modeling. A tull two-fluid (sixequation) hydrodynamics model describes the steam-water flow, thereby allowing important phenomena such as countercurrent flow to be treated explicitly. A stratified-flow regime has been added to the one-dimensional hydrodynamics; a seventh field equation (mass balance) describes a noncondensable gas field; and an eighth field equation tracks the solutes in the liquid.

3. Flow-Regime-Dependent Constitutive Equation Package. The thermal-hydraulic equations describe the transfer of mass, energy, and momentum between the steam-water phases and the interaction of these phases with the heat flow from the system structures. Because these interactions are dependent on the flow topology, a flow-regime-dependent constitutive equation package has, been incorporated into the code. Although this package undoubtedly will be improved in future code versions, assessment calculations performed to date indicate that many flow conditions can be handled adequately with the current package.

4. Comprehensive Heat-'Transfer Capability. TRAC-PF1/MOD1 incorporates detailed heat-transfer analyses of the vessel and the loop components. Included is a two-dimensional $(r, z)$ treatment of fuel-rod heat conduction with dynamic fine-mesh rezoning to resolve both bottom-flood and fallirg-film quench fronts. The heat transfer from the fuel rods and other system structures is calculated using flow-regime-dependent HTCs obtained from a generalized boiling curve based on local conditions.

5. Consistent Analysis of Entire Accident Sequences. An important TRAC feature is its ability to address entire accident sequences, including computation of initial conditions, with a consistent and continuous calculation. For example, the code models the blowdown, refill, and reflood phases of a LOCA. This modeling eliminates the need to perform calculations using different codes to analyze a given accident. In addition, a steadystate solution capability provides self-consistent initial conditions for subsequent transient calculations. Both a steady-state and a transient calculation can be performed in the same run, if desiréd.

6. Component and Functional Modularity. The TRAC program is completely modular by component. The components in a calculation are specified through input data; available components allow the user to model virtually any PWR design or experimental configuration. Thus, TRAC has great 
versatility in its range of applications. This feature also allows component modules to be improved, modified, or added without disturbing the remainder of the code. TRAC component modules currently include accumulators, breaks and fills, cores, pipes, pressurizers, pumps, steam generators, tees, turbines, valves, and vessels with associated internals (downcomer, lower plenum, core, upper plenum, etc.).

The TRAC program also is modular by function; that is, the major aspects of the calculations are performed in separate modules. For example, the basic one-dimensional hydrodynamics solution algorithm, the wall-temperature field solution algorithm, HTC selection, and other functions are performed in separate sets of routines that are accessed by all component modules. This modularity allows the code to be upgraded readily as improved correlations and test information become available.

B. Physical Phenomena Treated

Because of the detailed modeling in TRAC, the code can simulate physical phenomena important in large- and small-break LOCA analysis, such as

1. ECC downcomer penetration and bypass, including the effects of countercurrent flow and hot walls;

2. lower-plenum refill with entrainment and phase separation effects;

3. bottom-flood and falling-film reflood quench fronts;

4. multidimensional flow patterns in the core and plenum regions;

5. pool formation and countercurrent flow at the upper-core support-plate (UCSP) región;

6. pool formation in the upper plenum;

7. steam biading;

8. average-rod and hot-rod cladding-temperature histories;

9. alternate ECC injection systems, including hot-leg and upper-head injection;

10. direct injection of subcooled ECC water, without artificial mixing zones;

11. critical flow (choking); 


\section{2. liquid carryover during reflood;}

13. metal-water reaction;

14. water-hammer effects;

15. wall friction losses; and

16. horizontally stratified flow, including reflux cooling. C. Significant Changes from Previous TRAC Versions

Because our funding has been insufficient to provide the automatic inputdeck conversions that were released in the past, we wish to alert the user to the many significant changes that have been made to the TRAC-PF1/MOD1 input. Substantial changes have been made to all input sections that cover the use of trips and signal variables to provide a more general control capability. The initial definition cards for almost all components were modified. The user is cautioned to be especially careful when setting the parameter that regulates the maximum rate of change for quantities controlled by tables. The definitions of the wall heat sources and the direct power to the fluid were changed. The capabilities of the input loss coefficients and hydraulic diameters were expanded.

Four significant model changes should be considered when the TRAC-PF1/MOD1 results are compared with those from previous TRAC versions. Many details that affect the core reflood were changed; thus, the TRAC-PF1/MOD1 reflood calculations are significantly better than those from TRAC-PFl. The condensation model improvements include a more detailed flow-regime dependence and a special model for condensation on a stagnant interface in a vertical onedimensional component. Also, the choked-flow model was improved. Finally, the axial-momentum transport terms in the vessel were modified so that internally generated losses at orifices were eliminated. Loss coefficients probably will be required at core support plates to match results from previous TRAC versions.

D. Planned Improvements

TRAC-PFI/MOD1 combines all of the PWR accident analysis capabilities thus far requested by the NRC into a single code. This code represents such an important milestone in the TRAC series that future versions will be designed to accept any TRAC-PF1/MOD1 input deck. 
Most of our short-term plans involve increasing the speed of the code and making it more flexible and convenient for the user. Recent studies indicate that a change in the data base combined with recoding some key subroutines will cut run times by at least 30\%. Most user-convenience features are being developed under the Nuclear Plant Analyzer (NPA) project. However, we expect to simplify the standard TRAC input by adding an option that allows input of the trips and controllers in Fortran-like code.

E. Scope of TRAC Manual

We wrote this manual to describe TRAC-PF1/MOD1, version 12.1 (the frozen version). Relative to the initial release of TRAC-PF1/MOD1, the frozen code includes error corrections and new features such as a PLENUM component and super-critical water properties. Earlier versions of TRAC-PF1/MOD1 do not necessarily provide all features and capabilities described in this manual.

This manual describes TRAC basic methods and models and provides user information and programing details. Section III describes the basic hydrodynamics and heat-transfer methods and discusses the overall strategies for transient and steady-state solutions. Section III is supplemented by Apps. A and B that supply, respectively, the fluid and material properties for the thermal-hydraulic analyses. Appendix $C$ describes the stability-enhancing two-step (SETS) method. Appendix D describes the TRAC-PF1/MOD1 choking model.

A standard nomenclature guide is included. Quintities that are not included in the standard nomenclature list are defined 'in' the text. All units are metric as given in National Bureau of Standards Special Publication 330, "The International System of Units (SI)," unless otherwise specified.

Section IV describes the component models. The user should study these descriptions if questions arise when preparing detailed input specifications for a TRAC problem.

Section $V$ provides input specifications and other user information. Appendix E provides three sample input decks for LOFT experiments to provide additional guidance for input preparation. Appendix F explains the error messages that might occur during a calculation.

Section VI discusses the overall code organization, input and output processing, storage requirements, and other programming details associated with both transient and steady-state solutions. Appendix G provides a list of TRAC 
subprograms; App. H, a compilation of COMMON arrays; and App. I, component data tables.

An important aspect of the TRAC program involves the developmental assessment of the code through comparisons with measurements obtained from test facilities. Developmental assessment calculations already have been performed with TRAC-PF1/MOD1. A Los Alamos report entitled "TRAC-PF1/MOD1 Developmental Assessment," which summarizes the key developmental assessment results, will be published. 
III. BASIC METHODS

A. Hydrodynamics

1. Field Equations. Unlike previous TRAC versions, TRAC-PF1/MOD1 uses the same two-phase two-fluid model for fluid flow in both the one- and the three-dimensional components. We added a noncondensable gas component in the vapor field and a solute field for boron tracking that require two extra mass-continuity equations. We assumed that homogeneity and thermal equilibrium exist in the combined gas field.

Eight differential equations describe the four-component, two-fluid mode1.1,2

Liquid Mass Equation

$\frac{\partial(1-\alpha) \rho_{\ell}}{\partial t}+\nabla \cdot\left[(1-\alpha) \rho_{\ell} \vec{V}_{\ell}\right]=-\Gamma$

Combined Vapor Mass Equatiori

$\frac{\partial\left(\alpha \rho_{g}\right)}{\partial t}+\nabla \cdot\left(\alpha \rho_{g} \vec{V}_{g}\right)=\Gamma \quad$.

Noncondensable Gas Mass Equation

$\frac{\partial\left(\alpha \rho_{a}\right)}{\partial t}+\nabla \cdot\left(\alpha \rho_{a} \vec{v}_{g}\right)=0$.

Solute Concentration Equation

$\frac{\partial(1-\alpha) \mathrm{mo}_{\ell}}{\partial t}+\nabla \cdot\left[(1-\alpha) \mathrm{mo}_{\ell} \overrightarrow{\mathrm{v}}_{\ell}\right]=\mathrm{s}_{\mathrm{c}}$. 


$$
\begin{aligned}
\frac{\partial \vec{v}_{g}}{\partial t}+\vec{v}_{g} \cdot \nabla \vec{v}_{g}=-\frac{1}{\rho_{g}} \nabla p & -\frac{c_{i}}{\alpha \rho_{g}}\left(\vec{v}_{g}-\vec{v}_{l}\right)\left|\vec{v}_{g}-\vec{v}_{l}\right| \\
& -\frac{\Gamma^{+}}{\alpha \rho_{g}}\left(\vec{v}_{g}-\vec{v}_{l}\right)-\frac{c_{w g}}{\alpha \rho_{g}} \vec{v}_{g}\left|\vec{v}_{g}\right|+\vec{g} .
\end{aligned}
$$

\section{Liquid Equation of Motion}

$$
\begin{aligned}
& \frac{\partial \vec{v}_{\ell}}{\partial t}+\vec{v}_{\ell} \cdot \nabla \vec{v}_{\ell}=-\frac{1}{\rho_{\ell}} \nabla p+\frac{c_{1}}{(1-\alpha) p_{\ell}}\left(\vec{v}_{g}-\vec{v}_{\ell}\right)\left|\vec{v}_{g}-\vec{v}_{\ell}\right| \\
& \left.-\frac{\Gamma^{-}}{(1-\alpha) \rho_{\ell}} \stackrel{+}{v}_{g}-\vec{v}_{\ell}\right)-\frac{c_{w \ell}}{(1-\alpha) p_{l}}-\vec{v}_{\ell}\left|\vec{v}_{\ell}\right|++\vec{g} .
\end{aligned}
$$

Combined Vapor Energy Equation

$$
\begin{aligned}
\frac{\partial}{\partial t}\left(\alpha p_{g} e_{g}\right)+\nabla \cdot\left(\alpha p_{g} e_{g}^{+} \vec{V}_{g}\right)=-p \frac{\partial \alpha}{\partial t}-p \nabla \cdot\left(\alpha v_{g}\right) \\
+q_{w g}+q_{1 g}+\Gamma h_{s g}
\end{aligned}
$$

Total Energy Equation

$$
\begin{array}{r}
\frac{\partial\left[(1-\alpha) \rho_{\ell} e_{\ell}+\alpha \rho_{g} e_{g}\right]}{\partial t}+\nabla \cdot\left[(1-\alpha) \rho_{\ell} e_{\ell} \vec{v}_{\ell}+\alpha \rho g e_{g} \vec{V}_{g}\right] \\
=-p \nabla \cdot\left[(1-\alpha) \vec{V}_{\ell}+\alpha \vec{v}_{g}\right]+q_{w \ell}+q_{w g}
\end{array}
$$


In these equations the vapor densities and energies are sums of the steam and the noncondensable components,

$\rho_{\mathrm{g}}=\rho_{\mathrm{s}}+\rho_{\mathrm{a}}$

and

$\rho_{g} e_{g}=\rho_{s} e_{s}+\rho_{a} e_{a}$

We assume Dalton's law applies; therefore,

$\mathrm{p}=\mathrm{p}_{\mathrm{s}}+\mathrm{p}_{\mathrm{a}}$

A subscript, a, is used for the noncondensable gas because the internal thermodynamic properties model air. It would be easy to replace these properties with others describing//ifferent noncondensable gases.

In addition to the thermodynamic relations that are required for closure [Eqs. (28)-(29)], specifications/for the interfacial-drag coefficients ( $\left.c_{i}\right)$, the interfacial heat transfer $\left(q_{i g}\right)$, the phase-change rate $(\Gamma)$, the solute source term $\left(S_{c}\right)$, the wall-shear coefficients ( $c_{w g}$ and $c_{w \ell}$ ), and the wall heat transfers ( $q_{w g}$ and $q_{w \ell}$ ) are required. Gamma is evaluated from a simple thermal-energy jump relation,

$\Gamma=\frac{-\left(q_{i g}+q_{i \ell}\right)}{h_{g}^{\prime}-h_{\ell}^{\prime}}$,

where

$q_{i g}=h_{i g} A_{i} \frac{\left(T_{s s}-T_{g}\right)}{\text { vol }}$ 
and

$q_{1 \ell}=h_{i \ell} A_{i} \frac{\left(T_{s s}-T_{\ell}\right)}{\text { vol }}$

Here $A_{i}$ and the $h_{i}$ terms are the interfacial area and HTCs, and $T_{S S}$ is the saturation temperature corresponding to the partial steam pressure. The term $\Gamma^{+}$is equal to $\Gamma$ for positive $\Gamma$ and zero for negative $\Gamma$; $\Gamma^{-}$is equal to $\Gamma$ for negative. $\Gamma$ and zero for all other values of $\Gamma$. The quantities $h_{g}^{\prime}$ and $h_{l}^{\prime}$ are the appropriate enthalpies of the vapor and liquid, respectively. These enthalpies are the bulk fluid enthalpy for the phase moving to the interface and the saturation enthalpy for the product of the phase change.

Wall heat-transfer terms assume the form

$q_{w g}=h_{w g} A_{w g} \frac{\left(T_{w}-T_{g}\right)}{v_{01}}$

and

$q_{w \ell}=h_{w \ell} A_{w \ell} \frac{\left(T_{w}-T_{\ell}\right)}{v_{01}}$,

where $A_{w g}$ and $A_{w l}$ are the actual heated surface areas of the ce1l, except during reflood when the average HTC values reflect the fraction of the heated surface area that is quenched.

TRAC-PF1/MOD1 includes a mass-continuity equation for a solute moving with the liquid field instead of a physical diffusion model. The solute loes not affect the hydrodynamics directly. If we assume that the solute represents boron, the amount of the dissolved and the plated-out boron in the core may affect the hydrodynamics indirectly through reactivity feedback corresponding to user-specified input values obtained from a boron-mass reactivity-coefficient table (Sec. III.C). If the solute concentration exceeds the orthoboric-acid solubility at the fluid temperature in a specific hydrodynamic cell, we assume that the solute in that cell plates out. Plating also occurs if the cell fluid flashes or boils. We also assume that any 
plated-out solute instantaneously redissolves to the maximum allowable concentration if more liquid enters the cell. Because the solute does not affect the hydrodynamics directly, the solute variable may be used as a tag to track the movement of fluid from a specific source through the system.

2. Three-Dimensional Finite-Difference Equations. The momentum equations are separated into three coordinate components. Only the vapor equation is discussed with the understanding that the liquid-momentum equation is treated analogously. The vapor-momentum differential equation has three components.

Axia1 (z) Component

$$
\begin{aligned}
\frac{\partial v_{g z}}{\partial t}= & -\left(v_{g r} \frac{\partial v_{g z}}{\partial r}+\frac{v_{g \theta}}{r} \frac{\partial v_{g z}}{\partial \theta}+v_{g z} \frac{\partial v_{g z}}{\partial z}\right)-\frac{1}{\rho_{g}} \frac{\partial p}{\partial z}-\frac{\Gamma^{+}}{\alpha \rho_{g}}\left(v_{g z}-v_{\ell z}\right) \\
& -\frac{c_{i z}}{\alpha \rho g}\left(v_{g z}-v_{\ell z}\right)\left|\vec{v}_{g}-\vec{v}_{\ell}\right|-\frac{\Gamma}{\alpha \rho_{g}}\left(v_{g z}-v_{i g z}\right)-\frac{c_{w g z}}{\alpha \rho g} v_{g z}\left|\vec{v}_{g}\right|+g .
\end{aligned}
$$

$\underline{\text { Radial }(r) \text { Component }}$

$$
\begin{aligned}
\frac{\partial v_{g r}}{\partial t}= & -\left(v_{g r} \frac{\partial v_{g r}}{\partial r}+\frac{v_{g \theta}}{r} \frac{\partial v_{g r}}{\partial \theta}-\frac{v_{g \theta}^{2}}{r}+v_{g z} \frac{\partial v_{g r}}{\partial z}\right)-\frac{1}{\rho_{g}} \frac{\partial p}{\partial r}-\frac{\Gamma}{\alpha \rho_{g}}\left(v_{g r}-v_{\ell r}\right) \\
& -\frac{c_{i r}}{\alpha \rho_{g}}\left(v_{g r}-v_{\ell r}\right)\left|\vec{v}_{g}-\vec{v}_{\ell}\right|-\frac{\Gamma}{\alpha \rho_{g}}\left(v_{g r}-v_{i g r}\right)-\frac{c_{w g r}}{\alpha \rho_{g}} v_{g r}\left|\vec{v}_{g}\right|
\end{aligned}
$$




$$
\begin{aligned}
\frac{\partial v_{g \theta}}{\partial t}= & -\left(v_{g r} \frac{\partial v_{g \theta}}{\partial r}+\frac{v_{g \theta}}{r} \frac{\partial v_{g \theta}}{\partial \theta}+\frac{v_{g r} v_{g \theta}}{r}+v_{g z} \frac{\partial v_{g \theta}}{\partial z}\right)-\frac{1}{\rho_{g} r} \frac{\partial p}{\partial \theta}-\frac{\Gamma}{\alpha \rho_{g}}\left(v_{g \theta}-v_{l \theta}\right) \\
& -\frac{c_{i \theta}}{\alpha \rho_{g}}\left(v_{g \theta}-v_{\ell \theta}\right)\left|\vec{v}_{g}-\vec{v}_{\ell}\right|-\frac{\Gamma}{\alpha \rho_{g}}\left(v_{g \theta}-v_{i g \theta}\right)-\frac{c_{w g}}{\alpha \rho_{g}} v_{g \theta}\left|\stackrel{v}{g}_{g}\right|
\end{aligned}
$$

In the TRAC-staggered scheme 3,4 the velocities are defined on the meshcell surfaces at the locations shown in Fig. 1 , where the subscript a stands for either $\ell$ or $g$. However, the volume properties $p, \alpha, T, e$, and $\rho$ are located at the mesh-cell centers. The scalar field equations are written over a given mesh cell, whereas the momentum equations are staggered between mesh cells in the three component directions.

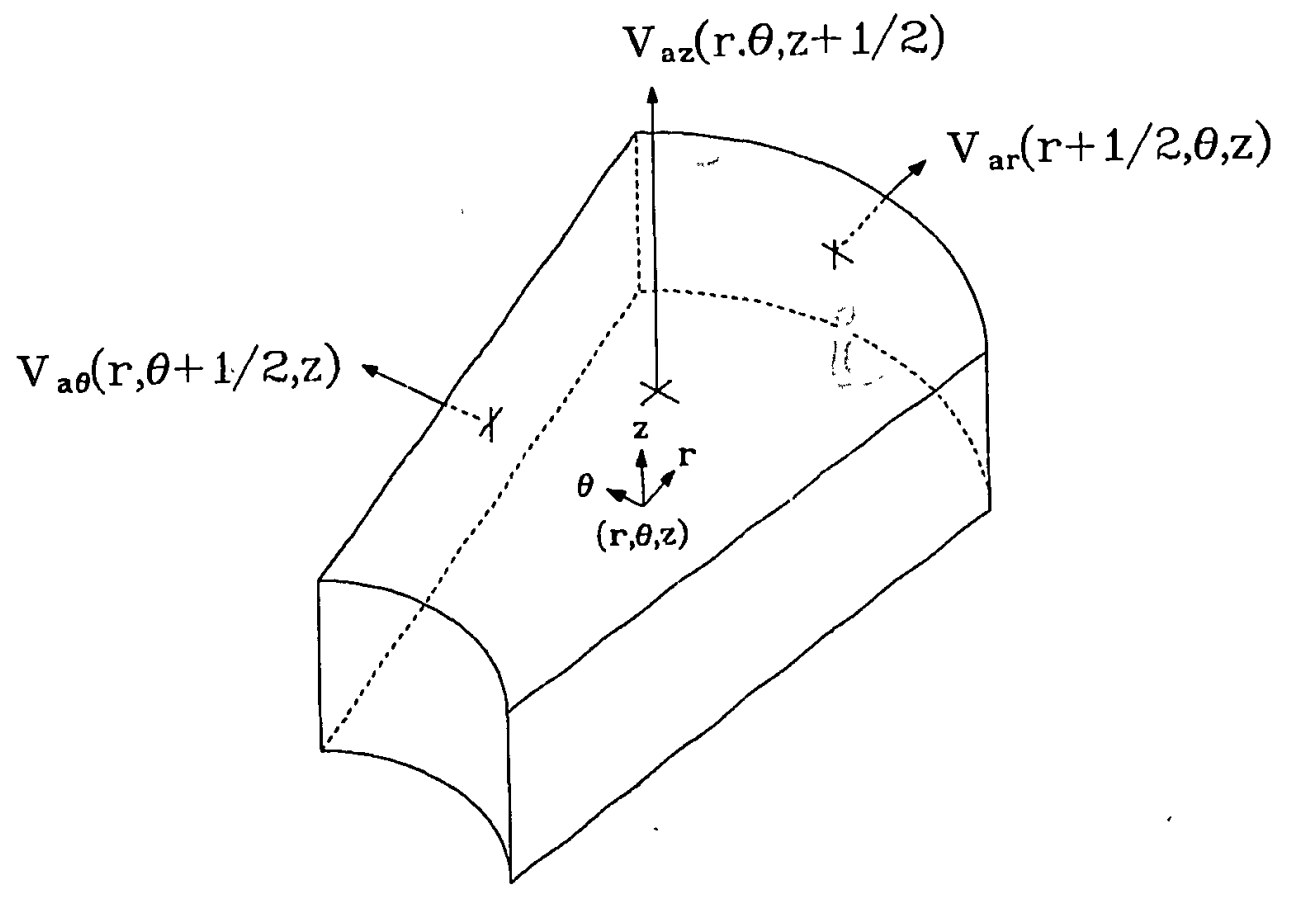

Fig. 1 .

Three-dimensional mesh-cell velocities. 
The written difference scheme for each of the momentum equations is lengthy because of the cross-derivative terms. Therefore, only the vapor zdirection finite-difference equations for a typical mesh cell are given to illustrate the procedure used. The time levels are indicated by the superscript $n$ (old time) or $n+1$ (new time). The subscript $g$ (for vapor) is dropped unless it is needed for clarity.

Using these conventions, the finite-difference vapor-momentum equation in the $z$-direction is

$$
\begin{aligned}
& v_{z}^{n+1}(r, \theta, z+1 / 2)=v_{z}^{n}(r, \theta, z+1 / 2) \\
& -\Delta t\left\{\frac{v_{r}^{n}(r, \theta, z+1 / 2)}{\Delta r}\left[v_{z}(r+1 / 2, \theta, z+1 / 2)-v_{z}(r-1 / 2, \theta, z+1 / 2)\right]^{n}\right. \\
& +\frac{\mathrm{v}_{\theta}^{\mathrm{n}}(\mathrm{r}, \theta, \mathrm{z}+1 / 2)}{\mathrm{r} \Delta \theta}\left[\mathrm{v}_{\mathrm{z}}(\mathrm{r}, \theta+1 / 2, \mathrm{z}+1 / 2)-\mathrm{v}_{\mathrm{z}}(\mathrm{r}, \theta-1 / 2, \mathrm{z}+1 / 2)\right]^{\mathrm{n}} \\
& +\frac{v_{z}^{n}(r, \theta, z+1 / 2)}{\Delta z}\left[v_{z}(r, \theta, z+1)-v_{z}(r, \theta, z)\right]^{n} \\
& -\frac{[p(r, \theta, z+1)-p(r, \theta, z)]^{n+1}}{\rho^{n}(r, \theta, z+1 / 2) \Delta z} \\
& -\frac{c_{i z}^{n}(r, \theta, z+1 / 2)\left[v_{z g}(r, \theta, z+1 / 2)-v_{z \ell}(r, \theta, z+1 / 2)\right]^{n+}{ }_{1}}{\alpha^{n}(r, \theta, z+1 / 2) \rho^{n}(r, \theta, z+1 / 2)} \\
& \times\left|\overrightarrow{\mathrm{v}}_{g}(r, \theta, z+1 / 2)-\overrightarrow{\mathrm{v}}_{\ell}(r, \theta, z+1 / 2)\right|^{n} \\
& -\frac{\Gamma^{+n}(r, \theta, z+1 / 2)\left[v_{g z}(r, \theta, z+1 / 2)-v_{\ell z}(r, \theta, z+1 / 2)\right]^{n+1}}{\alpha^{n}(r, \theta, z+1 / 2) \rho^{n}(r, \theta, z+1 / 2)} \\
& \left.-\frac{c_{w z}^{n}(r, \theta, z+1 / 2) v_{z}^{n+1}(r, \theta, z+1 / 2)\left|\vec{v}^{n}(r, \theta, z+1 / 2)\right|}{\alpha^{n}(r, \theta, z+1 / 2) \rho^{n}(r, \theta, z+1 / 2)}\right\},
\end{aligned}
$$

where $\Delta t$ is the time-step size.

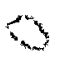


In any finite-difference scheme, certain quantities are required at locations where they are not defined formally; therefore, additional relations are needed. The volume properties $\Gamma, \alpha$, and $\rho_{\mathrm{g}}$ are donor-celled depending on the direction of $\mathrm{V}_{z}(x, \theta, z+1 / 2)$.

For example,

$$
\begin{aligned}
\alpha(r, \theta, z+1 / 2) & =\alpha(r, \theta, z), \quad \text { if } v_{z}(r, \theta, z+1 / 2)<0 ; \\
& =\alpha(r, \theta, z+1), \text { if } v_{z}(r, \theta, z+1 / 2)<0 .
\end{aligned}
$$

The radial component of the velocity at the axial location $(z+1 / 2)$ is obtained from

$$
\begin{aligned}
v_{r}(r, \theta, z+1 / 2)= & \frac{1}{4}\left[v_{r}(r+1 / 2, \theta, z)+v_{r}(r-1 / 2, \theta, z)+v_{r}(r+1 / 2, \theta, z+1)\right. \\
& \left.+v_{r}(r-1 / 2, \theta, z+1)\right] ;
\end{aligned}
$$

a similar expression applies to $V_{\theta}(r, \theta, z+1 / 2)$. The spatial differences for $V_{z}$ are, in the r-direction,

$$
\begin{aligned}
& v_{z}(r+1 / 2, \theta, z+1 / 2)-v_{z}(r-1 / 2, \theta, z+1 / 2)=v_{z}(r, \theta, z+1 / 2)-v_{z}(r-1, \theta, z+1 / 2), \\
& \quad \text { if } v_{r}(r, \theta, z+1 / 2) \geq 0 \text {, or } \\
& x_{z}(r+1 / 2, \theta, z+1 / 2)-v_{z}(r-1 / 2, \theta, z+1 / 2)=v_{z}(r+1, \theta, z+1 / 2)-v_{z}(r, \theta, z+1 / 2), \\
& \quad \text { if } v_{r}(r, \theta, z+1 / 2)<0 .
\end{aligned}
$$


In the $\theta$-direction,

$$
\begin{aligned}
& v_{,}(r, \theta+1 / 2, z+1 / 2)-v_{z}(r, \theta-1 / 2, z+1 / 2)=v_{z}(r, \theta, z+1 / 2)-v_{z}(r, \theta-1, z+1 / 2), \\
& \quad \text { if } v_{\theta}(r, \theta, z+1 / 2) 20 \text {, or } \\
& v_{z}(r, \theta+1 / 2, z+1 / 2)-v_{z}(r, \theta-1 / 2, z+1 / 2)=v_{z}(r, \theta+1, z+1 / 2)-v_{z}(r, \theta, z+1 / 2), \\
& \quad \text { if } v_{\theta}(r, \theta, z+1 / 2)<0 .
\end{aligned}
$$

In the $z$-direction,

$$
V_{z}(r, \theta, z+1)-V_{z}(r, \theta, z)=\frac{V_{z}(r, \theta, z+1 / 2) \cdot F A_{z+1 / 2}}{F A_{z+1}}-\frac{V_{z}(r, \theta, z-1 / 2) \cdot F A_{z-1 / 2}}{F A_{z}},
$$

if $v_{z}(r, \theta, z+1 / 2) \geq 0$, or

$$
V_{z}(r, \theta, z+1)-V_{z}(r, \theta, z)=\frac{V_{z}(r, \theta, z+3 / 2) \cdot F A_{z+3 / 2}}{F_{z+1}}-\frac{V_{z}(r, \theta, z+1 / 2) \cdot F_{z+1 / 2}}{F_{z}},
$$

$$
\text { if } v_{z}(r, \theta, z+1 / 2)<0
$$

The convective terms in the finite-difference relations for the scalar field equations are written in conservative form. The finite-difference form of the overall mixture mass equation is

$$
\begin{aligned}
& \rho_{m}^{n+1}=\rho_{m}^{n}+\left[\frac{\Delta t}{v o 1}\right]\left(F A_{z-1 / 2}\left\{\left[(1-\alpha) \rho_{\ell}\right]^{n_{v} n+1}+\left(\alpha \rho_{g}\right)^{n_{v^{n}}^{n+1}}\right\}_{z-1 / 2}^{\prime}\right. \\
& -F A_{z+1 / 2}\left\{\left[(1-\alpha) \rho_{\ell}\right]^{n_{V_{\ell}^{n}}^{n+1}}+\left(\alpha \rho_{g}\right)^{n_{V_{g}}^{n+1}}\right\}_{z+1 / 2} \\
& +F A_{r-1 / 2}\left\{\left[(1-\alpha) \rho_{\ell}\right]^{n_{V_{\ell}^{n+1}}^{n+1}}+\left(\alpha \rho_{g}\right)^{n_{V}}{ }_{g}^{n+1}\right\}_{r-1 / 2} \\
& -{ }^{F A_{r+1 / 2}}\left\{\left[(1-\alpha) \rho_{\ell}\right]^{n_{V_{\ell}^{n+1}}}+\left(\alpha \rho_{g}\right)^{n_{V_{g}^{n+1}}^{n+1}}{ }_{r+1 / 2}\right. \\
& +F A_{\theta-1 / 2}\left\{\left[(1-\alpha) \rho_{\ell}\right]^{n_{V_{\ell}}^{n+1}}+\left(\alpha \rho_{g}\right)^{n_{V}}{ }_{g}^{n+1}\right\}_{\theta-1 / 2} \\
& \left.-F A_{\theta+1 / 2}\left\{\left[(1-\alpha) \rho_{\ell}\right]^{n_{V}}{ }_{\ell}^{n+1}-\left(\alpha \rho_{g}\right)^{n_{V}^{n+1}}\right\}_{\theta+1 / 2}\right) \text {, }
\end{aligned}
$$


where vol is the hydrodynamic cell volume and FA is the flow area at the meshcell edge. The other scalar equations are differenced similarly.

All of the field equations [Eqs. (1)-(8)] have additional source terms to allow piping to be connected anywhere in the mesh. The source terms in the mass and energy equations are given below. The subscripts $p$ and $v$ refer to pipe and vessel quantities, respectively.

Overall Mass-Continuity Source Term

$\left[\alpha \rho_{g}^{n} V_{g}^{n+1} F A+\left((1-\alpha) \rho_{\ell}\right)^{n} V_{\ell}^{n+1} F A\right]_{p}$.

Vapor Mass-Continuity Source Term

$\left[\left(\alpha \rho_{g}\right)^{n} F A v_{g}^{n+1}\right]_{p}$

Overall Energy Source Term

$$
\begin{aligned}
& \left.\left[(1-\alpha) \rho_{\ell} e_{\ell}\right)^{n} F A v_{\ell}^{n+1}\right]_{p}+\left[\left(\alpha \rho_{g} e_{g}\right)^{n} F v_{g}^{n+1}\right]_{p} \\
& +P_{v}\left[\alpha^{n} F A v_{g}^{n+1}+(1-\alpha)^{n} F A v_{\ell}^{n+1}\right]_{p}
\end{aligned}
$$

Vapor Energy Source Term

$\left[\left(\alpha \rho_{g} e_{g}\right)^{n} F A V_{g}^{n+1}\right]_{p}+p_{v}\left(\alpha^{n} F A V_{g}^{n+1}\right)_{p}$

The momentum source terms are complicated because of the staggered differencing and the fact that pipes may enter at an arbitrary angle. For TRAC-PF1/MOD1, we have assumed that the pipe enters normal to the vessel meshcell face. The basic forms for the liquid- and vapor-momentum source terms follow.

\section{Liquid-Momentum Source Term}

$$
\left(\frac{{ }^{v_{\ell}}{ }_{p}^{v_{\ell}}{ }^{n}}{\Delta x_{v}}\right)^{n}
$$




$$
\left(\frac{\mathrm{v}_{\mathrm{g}} \mathrm{v}_{\mathrm{g}_{\mathrm{v}}}}{\Delta \mathrm{x}_{\mathrm{v}}}\right)^{\mathrm{n}}
$$

The existence of the momentum source terms is dependent on the sign of the velocities in the vessel to keep the vessel donor-cell momentum equations consistent. For example, if the nearest vessel liquid velocity indicates lhat the flow is into the pipe from the vessel, then the source term is set equal to zero.

If structure exists in the mesh cell, the hydrodynamic FA and vol are reduced from their geometric mesh-cell values. Thus, FA may be less than or equal to the geometric mesh-cell area and vol may be less than or equal to the geometric mesh-cell volume. When the FA is zero, all fluxes across that plane, as well as the individual velocities of each phase, are suppressed. This procedure allows large obstacles such as the downcomer walls to be modeled properly. The user specifies the flow and volume restrictions.

The finite-difference equations thus formed are semi-implicit, because the pressure gradient terms in the vapor- and liquid-momentum equations are treated at the new time. A Courant stability criterion,

$\Delta t<\frac{L}{|V|}=\min \left(\frac{\Delta x}{V_{g z}}, \frac{r \Delta \theta}{V_{g \theta}}, \frac{\Delta r}{V_{g r}}, \frac{\Delta z}{V_{\ell z}}, \frac{r \Delta \theta}{V_{\ell \theta}}, \frac{\Delta r}{V_{\ell r}}\right)$

is necessary.

a. Solution of Difference Equations. "A linearization procedure is needed to solve the finite-difference equations. All the scalar equations are reduced to a linear system in $\mathrm{V}_{\ell}, \mathrm{V}_{\mathrm{g}}, \mathrm{T}_{\ell}, \mathrm{T}_{\mathrm{g}}, \alpha$, and $\mathrm{p}$ by using the thermal equations of state,

$\rho_{\ell}=\rho_{\ell}\left(p, T_{\ell}\right)$ 
and

$\rho_{g}=\rho_{g}\left(p, T_{g}\right) ;$

the caloric equations of state,

$e_{\ell}=e_{\ell}\left(p, T_{\ell}\right)$

and

$e_{g}=e_{g}\left(p, T_{g}\right)$

and the definitions for $\rho_{\mathrm{m}}$ and $e_{\mathrm{m}}$.

A further system reduction is accomplished by observing that the finitedifference vapor- and liquid-momentum equations yield equations of the form

$$
\mathrm{V}^{\mathrm{n}+1}=\mathrm{V}^{\mathrm{n}}+\left[\operatorname{conv}^{\mathrm{n}}+\frac{1}{\rho_{\ell}} \nabla \mathrm{p}^{\mathrm{n}+1}+\text { FRIC }\right] \Delta t
$$

where conv designates the explicit convection terms and FRIC includes both the wall and the interfacial shears. Equation (32) indicates that changes in $\mathrm{V}$ are linearly dependent (after an explicit pass on the explicit parts of the momentum equations) on changes in pressure. Therefore, the system of variables may be reduced further to $\mathrm{T}_{\ell}, \mathrm{T}_{g}, \mathrm{p}$, and $\alpha$ and solved by a block Gauss-Seidel method. Reference 5 provides a much more detailed description of the basic block Gauss-Seidel numerical technique.

An improvement to the method proposed in Ref. 5 has been implemented to reduce the computing cost. The linear system that results from this method is a seven-stripe block matrix. In performing the Gauss-Seidel operation, if the nonlinear terms are not updated, the matrix coefficients remain constant for the time step. In this case a Gauss elimination technique can be applied once 
at each time step to the seven-stripe block array that allows its reduction to a seven-stripe single-element array. This results in a much faster iteration (after the first iteration) for the pressure. The actual iteration is performed in subroutine ITRl. When the vessel pressures are obtained for a specifled convergence criterion, a backward substitution in subroutine FF3D is performed to unfold $\mathrm{T}_{\ell}, \mathrm{T}_{g}, \alpha$, and the velocities for each phase. A call to THERMO in FF3D then updates all of the thermodynamic properties and their derivatives in preparation for the next time step.

\section{b. Pressure Matrix Solution.}

Two options are avallable for solving the vessel pressure matrix. The first is a direct inversion of the pressure solution matrix when the number of vessel cells is sufficiently small $(580)$. The second feature is a coarse-mesh rebalance method for other cases where Gauss-Seidel iteration is required. During the iteration the pressure solution is scaled nonuniformly to reduce the overall iteration error. Such scaling can be represented by

$P^{-(i)}=S^{(i)}{ }_{P}^{(i)}$,

where $\mathrm{P}^{(i)}$ is the pressure solution vector after $i$ iterations and $s^{(i)}$ is its scaling matrix that is diagonal with scalar elements $s_{j}$. We define coarsemesh regions as those vessel regions having the same scale factor. The scaled pressure solution vector is then

$\mathrm{P}^{-}=s_{1} \mathrm{P}_{1}+s_{2} \mathrm{P}_{2}+\ldots$,

where $P_{i}$ is a vector of pressures belonging to coarse-mesh region $i$. With this equation in the vessel pressure equation,

$A \cdot P=B$, 
and with the requirement that the least-squares error in $P^{-}$be a minimum yield an equation for the scale factors,

$\mathrm{U} \cdot \mathrm{S}=\mathrm{V}$,

where $U_{i j}=\left(P_{i}, A P_{j}\right), V_{i}=\left(P_{i}, B\right)$, and the notation $(X, Y)$ means the inner product of the vectors $X$ and $Y$. The matrix size of $U$ is equal to the number of coarse-mesh regions and normally is small enough to be solved by direct inversion.

The choice of coarse-mesh regions is extremely important. We have selected a scheme that follows the flow path in the vessel so that coarse-mesh regions are coupled in the flow direction. We use the facts that the vessel matrix $A$ is a seven-stripe matrix for a three-dimensional vessel and that coupling occurs only between nearest neighbors (there is no coupling if neighbors are separated by a wall such as a downcomer boundary). Based on these facts we select coarse-mesh regions as follows. All mesh cells on a level in the downcomer form a single coarse-mesh region, and all other mesh cells on a level form another coarse-mesh region. Hence, the total number of coarse-mesh regions is equal to the number of downcomer levels plus the total number of vessel levels. Although this choice of coarse-mesh regions is not unique, we have found it to be very effective in reducing the number of vessel iterations (typically a factor of 10 ).

c. Water Packing. The three-dimensional hydrodynamics package has a water-packing algorithm that is similar in function to the one-dimensional algorithm but somewhat different in construction. In subroutine TF3DI, after the initial block matrix inversion, the center diagonal $\delta P$ is calculated. If the resulting predicted pressure is either very low or very high and the mesh cell has a large liquid fraction, then the surrounding region is scanned for additional information. If an adjoining celj. has a liquid fraction less than 0.9 , the momentum derivative $\delta \mathrm{V}_{\ell} / \delta \mathrm{P}_{i}$ (derived from the momentum equation) is multiplied by a large constant wherever it appears in terms within the offending mesh cell. This step reduces the liquid inertia and consequently allows the resultant velocity to adjust during the time step to ameliorate the 
effects of the sudden pressure change. The matrix is then re-evaluated and the calculation continues.

3. One-Dimensional Finite-Difference Methods. The stabilityenhancing two-step (SETS) method 6,7 used for one-dimensional flow eliminates the material Courant stability limit from all one-dimensional components. As a result, this upper time-step bound applies only in vessel cells and at junctions to the vessel. We recommend that users unfamiliar with the SETS method read the simplified description provided in App. C before completing this section.

The SETS method consists of a basic step (which is almost identical to the standard semi-implicit method used in the vessel) and a stabilizing step. For homogeneous flow, the order of these steps does not matter; however, for two-fluid flow with noticeable relative velocity, the stabilizing step for the equations of motion must be performed before the basic step. When this stabilizing step precedes the basic step, an initial explicit prediction of velocities gives strong coupling through the interfacial-drag terms without requiring direct communication between the stabilizing equations for liquid and vapor motion. To provide improved conservation and to minimize machine storage required by TRAC, the stabilizing steps for mass and energy equations are done as the final portion of the calculation.

The spatial mesh used for the finite-difference equations is staggered with thermodynamic properties evaluated at the cell centers and velocities evaluated at the cell edges. For stability, flux terms at cell edges require donor-cell averages of the form,

$$
\begin{array}{rlrl}
\langle Y V\rangle_{j+1 / 2} & =Y_{j} V_{j+1 / 2}, & & \text { if } V_{j+1 / 2}<0 ; \\
& =Y_{j+1} V_{j+1 / 2}, \quad \text { if } V_{j+1 / 2}<0,
\end{array}
$$


where $Y$ can be any cell-center state variable or a combination of such variables and $V$ may be either liquid or vapor velocity. With this notation the finite-difference divergence operator for one-dimensional calculations is

$\nabla_{j} \cdot(Y V)=\frac{\left(A_{j+1 / 2}\langle Y V\rangle_{j+1 / 2}-A_{j-1 / 2}\langle Y V\rangle_{j-1 / 2}\right)}{\operatorname{Vol}_{j}}$,

where $A$ is the local cross-sectional area and $v^{\prime} j$ is the volume of the $j t h$ cell. For the equations of motion, the donor-cell form of any $V \cdot \nabla V$ term is

$$
\begin{aligned}
v_{j+1 / 2} \nabla_{j+1 / 2} v & =\frac{v_{j+1 / 2}\left(v_{j+1 / 2}-v_{j-1 / 2}\right)}{\Delta x_{j+1 / 2}}, \text { if } v_{j+1 / 2} \geq 0 ; \\
& =\frac{v_{j+1 / 2}\left(v_{j+3 / 2}-v_{j+1 / 2}\right)}{\Delta x_{j+1 / 2}}, \text { if } v_{j+1 / 2}<0 ;
\end{aligned}
$$

where $\Delta x_{j+1 / 2}$ is half the sum of $\Delta x_{j}$ and $\Delta x_{j+1}$.

The following finite-difference equations (roughly in order of their calculation) currently are used.

\section{Predictors for Equations of Motion}

Vapor

$$
\begin{aligned}
\frac{\left(\hat{V}_{g}^{n+1}-v_{g}^{n}\right)}{\Delta t}+v_{g}^{n} \nabla_{j+1 / 2} \tilde{V}_{g}^{n}+\beta\left(\hat{v}_{g}^{n+1}-v_{g}^{n}\right) \nabla_{j+1 / 2} \tilde{V}_{g}^{n} \\
+\frac{\left(c_{i}^{n}\left|v_{g}^{n}-v_{l}^{n}\right|+\Gamma_{j+l / 2}^{+n}\right)}{(\overline{\alpha \rho g})_{j+1 / 2}^{n}}\left[2\left(\hat{v}_{g}^{n+1}-\hat{v}_{l}^{n+1}\right)-\left(v_{g}^{n}-v_{l}^{n}\right)\right]
\end{aligned}
$$




$$
\begin{aligned}
& +\frac{1}{\left(\overline{\rho_{g}}\right)_{j+1 / 2}^{n}} \frac{\left(p_{j+1}^{n_{j}}-p_{j}^{n}\right)}{\Delta x_{j+1 / 2}} \\
& +\frac{c_{w g}}{\left(\overline{\alpha \rho_{g}}\right)_{j+1 / 2}^{n}}\left(2 \hat{v}_{g}^{n+1}-v_{g}^{n}\right)\left|v_{g}^{n}\right|+g \cos \theta=0,
\end{aligned}
$$

where

$$
\begin{aligned}
B & =0, \text { if } \nabla_{j+1 / 2} \mathrm{~V}^{\mathrm{n}} \leq 0 \text {; } \\
& =1, \text { if } \nabla_{j+1 / 2} \mathrm{~V}^{\mathrm{n}}>0 \text {. }
\end{aligned}
$$

\section{Liquid}

$$
\begin{aligned}
& \frac{\left(\hat{V}_{\ell}^{n+1}-v_{\ell}^{n}\right)}{\Delta t}+v_{\ell}^{n} \nabla_{j+1 / 2} \tilde{V}_{\ell}^{n}+\beta\left(\hat{V}_{\ell}^{n+1}-v_{\ell}^{n}\right) \nabla_{j+1 / 2} \tilde{V}_{\ell}^{n}
\end{aligned}
$$

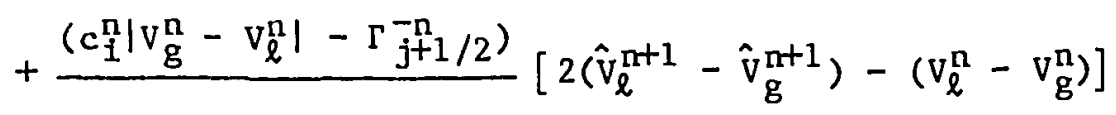

$$
\begin{aligned}
& {\left[(1-\alpha) p_{\ell}\right]_{j+1 / 2}^{n}} \\
& +\frac{1}{\left(\overline{p_{\ell}}\right)_{j+1 / 2}^{n}} \frac{\left(p_{j+1}^{n}-p_{j}^{n}\right)}{\Delta X_{j+1 / 2}}
\end{aligned}
$$

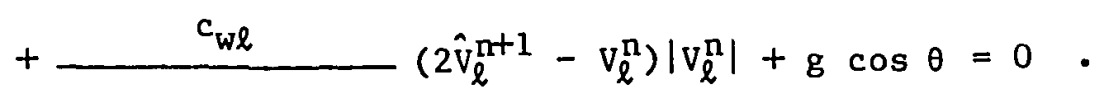

$$
\begin{aligned}
& {\left[\overline{(1-\alpha) p_{\ell}}\right]_{j+1 / 2}^{n}}
\end{aligned}
$$


Stabilizer Equations of Motion

Vapor

$$
\begin{aligned}
\frac{\left(\tilde{v}_{g}^{n+1}-v_{g}^{n}\right)}{\Delta t}+ & v_{g}^{n} \nabla_{j+1 / 2} \tilde{v}_{g}^{n+1}+\beta\left(\tilde{v}_{g}^{n+1}-v_{g}^{n}\right) \nabla_{j+1 / 2} \tilde{v}_{g}^{n} \\
& +\frac{\left(c_{i}^{n}\left|v_{g}^{n}-v_{l}^{n}\right|+\Gamma_{j+1 / 2}^{+n}\right)}{\left(\overline{\alpha \rho_{g}}\right)_{j+1 / 2}^{n}}\left[2\left(\hat{v}_{g}^{n+1}-\hat{v}_{l}^{n+1}\right)-\left(v_{g}^{n}-v_{l}^{n}\right)\right] \\
& +\frac{1}{\left(\overline{\rho_{g}}\right)_{j+1 / 2}^{n}} \frac{\left(p_{j+1}^{n}-p_{j}^{n}\right)}{\Delta x_{j+1 / 2}} \\
& +\frac{c_{w g}}{(\overline{\alpha \rho})_{j+1 / 2}^{n}}\left(2 \tilde{v}_{g}^{n+1}-v_{g}^{n}\right)\left|v_{g}^{n}\right|+g \cos \theta=0
\end{aligned}
$$

\section{Liquid}

$$
\begin{aligned}
\frac{\left(\tilde{v}_{\ell}^{n+1}-v_{\ell}^{n}\right)}{\Delta t} & +v_{\ell}^{n} \nabla_{j+1 / 2} \tilde{v}_{l}^{n+1}+\beta\left(\tilde{v}_{\ell}^{n+1}-v_{\ell}^{n}\right) \nabla_{j+1 / 2} \tilde{v}_{\ell}^{n} \\
& +\frac{\left.c_{i}^{n}\left|v_{g}^{n}-v_{\ell}^{n}\right|-\Gamma_{j+1 / 2}^{-n}\right)}{\left[\overline{(1-\alpha) \rho_{\ell}}\right]_{j+1 / 2}^{n}}\left[2\left(\hat{v}_{\ell}^{n+1}-\hat{v}_{g}^{n+1}\right)-\left(v_{\ell}^{n}-v_{g}^{n}\right)\right] \\
& +\frac{1}{\left(\overline{\rho_{\ell}}\right)_{j+1 / 2}^{n} \frac{\left(p_{j+1}^{n}-p_{j}^{n}\right)}{\Delta x_{j+1 / 2}}} \quad ; \\
& \left.+\frac{c_{w \ell}}{\left[(1-\alpha) \rho_{\ell}\right.}\right]_{j+1 / 2}^{n}
\end{aligned}
$$




\section{Basic Equations of Motion}

Vapor

$$
\begin{aligned}
\frac{\left(v_{g}^{n+1}-v_{g}^{n}\right)}{\Delta t} & +v_{g}^{n} \nabla_{j+1 / 2} \tilde{v}_{g}^{n+1}+\beta\left(v_{g}^{n+1}-v_{g}^{n}\right) \nabla_{j+1 / 2} \tilde{v}_{g}^{n} \\
& +\frac{\left(c_{i}^{n}\left|v_{g}^{n}-v_{l}^{n}\right|+\Gamma_{j+1 / 2}^{+n}\right)}{\left(\overline{\alpha \rho_{g}}\right)_{j+1 / 2}^{n}}\left[2\left(v_{g}^{n+1}-v_{\ell}^{n+1}\right)-\left(v_{g}^{n}-v_{\ell}^{n}\right)\right] \\
& +\frac{1}{\left(\overline{\rho_{g}}\right)_{j+1 / 2}^{n}} \frac{\left(\tilde{p}_{j+1}^{n+1}-\tilde{p}_{j}^{n+1}\right)}{\Delta X_{j+1 / 2}} \\
& +\frac{c_{w g}}{(\overline{\alpha \rho g})_{j+1 / 2}^{n}}\left(2 v_{g}^{n+1}-v_{g}^{n}\right)\left|v_{g}^{n}\right|+g \cos \theta=0 .
\end{aligned}
$$

Liquid

$$
\begin{aligned}
& \frac{\left(v_{\ell}^{n+1}-v_{\ell}^{n}\right)}{\Delta t}+v_{\ell}^{n} \nabla_{j+1 / 2} \tilde{v}_{\ell}^{n+1}+B\left(v_{\ell}^{n+1}-v_{\ell}^{n}\right) \nabla_{j+1 / 2} \tilde{v}_{\ell}^{n} \\
& +\frac{\left(c_{i}^{n}\left|v_{\ell}^{n}-v_{g}^{n}\right|-\Gamma_{j+1 / 2}^{-n}\right)}{\left[(1-\alpha) \rho_{\ell}\right]_{j+1 / 2}^{n}}\left[2\left(v_{\ell}^{n+1}-v_{g}^{n+1}\right)-\left(v_{\ell}^{n}-v_{g}^{n}\right)\right] \\
& +\frac{1}{\left(\overline{\rho_{\ell}}\right)_{j+1 / 2}^{n}} \frac{\left(\tilde{p}_{j+1}^{n+1}-\tilde{p}_{j+1}^{n+1}\right)}{\Delta X_{j+1 / 2}}
\end{aligned}
$$

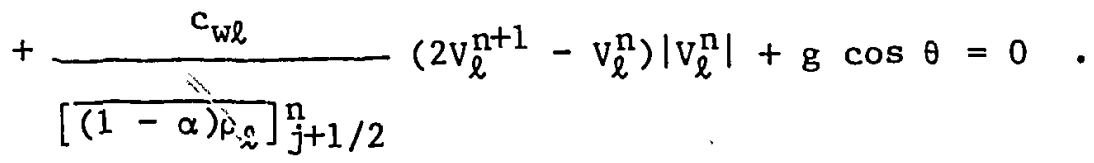




\section{Vapor}

$\frac{\left[\left(\tilde{\alpha}_{g}\right)^{n+1}-\left(\alpha \rho_{g}\right)^{n}\right]}{\Delta t}+\nabla_{j} \cdot\left(\alpha \rho_{g} v_{g}^{n+1}\right)=\tilde{\Gamma}^{n+1} \cdot$

Noncondensable Gas

$$
\frac{\left[\left(\tilde{\alpha}_{a}\right)^{n+1}-\left(\alpha \rho_{a}\right)^{n}\right]}{\Delta t}+\nabla_{j} \cdot\left(\alpha \rho_{a} v_{g}^{n+1}\right)=0 .
$$

\section{$\underline{\text { Liquid }}$}

$$
\begin{aligned}
& \frac{\left[(1-\tilde{\alpha})^{n+1} \tilde{\rho}_{\ell}^{n+1}-(1-\alpha)^{n} \rho_{\ell}^{n}\right]}{\Delta t}+\nabla_{j} \cdot\left[(1-\alpha) \rho_{\ell} v_{\ell}^{n+1}\right] \\
& =-\tilde{\Gamma}^{n+1} .
\end{aligned}
$$

\section{Basic Energy Equations}

Vapor

$$
\begin{aligned}
& \frac{\left[\left(\tilde{\alpha}_{\rho}^{g} \tilde{e}_{g}\right)^{n+1}-\left(\alpha \rho_{g} e_{g}\right)^{n}\right]}{\Delta t}+\nabla_{j} \cdot\left(\alpha \rho_{g} e_{g} v_{g}^{n+1}\right) \\
& +\tilde{p}^{n+1}\left[\frac{\left(\tilde{\alpha}^{n+1}-\alpha^{n}\right)}{\Delta t}+\nabla_{j} \cdot\left(\alpha^{n} v_{g}^{n+1}\right)\right]
\end{aligned}
$$

$$
=\tilde{q}_{w g}^{n+1}+\tilde{q}_{i g}^{n+1}+\tilde{\Gamma}^{n+1} \tilde{h}_{s g}^{n+1} .
$$




$$
\begin{aligned}
& \frac{\left\{\left[\tilde{\alpha \tilde{\rho}_{g}} \tilde{\mathrm{e}}_{g}+(1-\tilde{\alpha}) \tilde{\rho}_{\ell} \tilde{\mathrm{e}}_{\ell}\right]^{\mathrm{n}+1}-\left[\alpha \rho_{g} \mathrm{e}_{g}+(1-\alpha) \rho_{\ell} e_{l}\right]^{\mathrm{n}}\right\}}{\Delta t} \\
& \quad+\nabla_{j} \cdot\left[\left(\alpha \rho_{g} \mathrm{e}_{g}\right) v_{g}^{\mathrm{n}+1}+(1-\alpha) \rho_{\ell} e_{\ell} v_{\ell}^{\mathrm{n}+1}\right] \\
& \quad+\tilde{\mathrm{p}}^{\mathrm{n}+1} \nabla_{j} \cdot\left[(1-\alpha)^{\mathrm{n}} v_{\ell}^{\mathrm{n}+1}+\alpha^{\mathrm{n}} v_{\mathrm{v}}^{\mathrm{n}+1}\right]=\tilde{\mathrm{q}}_{\mathrm{wg}}^{\mathrm{n}+1}+\tilde{\mathrm{q}}_{\mathrm{wl}}^{\mathrm{n}+1} \cdot
\end{aligned}
$$

\section{Stabilizing Mass Equations}

\section{Vapor}

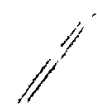

$\frac{\left[\left(\alpha \rho_{g}\right)^{n+1}-\left(\alpha \rho_{g}\right)^{n}\right]}{\Delta t}+\nabla_{j} \cdot\left[\left(\alpha \rho_{g}\right)^{n+1} v_{g}^{n+1}\right]=\tilde{\Gamma}^{n+1}$.

Noncondensable Gas

$\frac{\left[\left(\alpha \rho_{a}\right)^{n+1}-\left(\alpha \rho_{a}\right)^{n}\right]}{\Delta t}+\nabla_{j} \cdot\left[\left(\alpha \rho_{a}\right)^{n+1} v_{g}^{n+1}\right]=0$

$\underline{\text { Liquid }}$

$$
\begin{gathered}
\frac{\left[(1-\alpha)^{n+1} \rho_{\ell}^{n+1}-(1-\alpha)^{n} \rho_{\ell}^{n}\right]}{\Delta t}+\nabla_{j} \cdot\left[(1-\alpha)^{n+1} \rho_{\ell}^{n+1} v_{\ell}^{n+1}\right] \\
=-\tilde{\Gamma}^{n+1} .
\end{gathered}
$$


Solute

$$
\begin{aligned}
& \frac{\left[(1-\alpha)^{n+1} \tilde{m}^{n+1} \rho_{\ell}^{n+1}-(1-\alpha)^{n} m^{n} \rho_{\ell}^{n}\right]}{\Delta t} \\
& \quad+\nabla_{j} \cdot\left[(1-\alpha)^{n+1} \tilde{m}^{n+1} \rho_{\ell}^{n+1} v_{\ell}^{n+1}\right]=0 ; \\
& m^{n+1}=\min \left(\tilde{m}^{n+1}+s_{c}^{N}, m_{\max }\right) ; \text { and } \\
& s^{n+1}=\tilde{m}^{n+1}+s^{n}-c^{n+1} .
\end{aligned}
$$

\section{Stabilizing Energy Equations}

Vapor

$$
\begin{gathered}
\frac{\left[\left(\alpha \rho_{g} e_{g}\right)^{n+1}-\left(\alpha \rho_{g} e\right)^{n}\right]}{\Delta t}+\nabla_{j} \cdot\left[\left(\alpha \rho_{g} e_{g}\right)^{n+1} v_{g}^{n+1}\right] \\
+\tilde{p}^{n+1}\left[\frac{\left(\tilde{\alpha}^{n+1}-\alpha^{n}\right)}{\Delta t}+\nabla_{j} \cdot\left(\alpha^{n} v_{g}^{n+1}\right)\right] \\
=\tilde{q}_{1 g}^{n+1}+\tilde{q}_{w g}^{n+1}+\tilde{\Gamma}^{n+1} \tilde{h}_{s g}^{n+1} .
\end{gathered}
$$

\section{Liquid}

$$
\begin{gathered}
\frac{\left\{\left[(1-\alpha) \rho_{\ell} e_{\ell}\right]^{n+1}-\left[(1-\alpha) \rho_{\ell} e_{\ell}\right]^{n}\right\}}{\Delta t}+\nabla_{j} \cdot\left\{\left[(1-\alpha) \rho_{\ell} e_{\ell}\right]^{n+1} v_{\ell}^{n+1}\right\} \\
+\tilde{p}^{n+1}\left\{\frac{\left(\alpha^{n}-\tilde{\alpha}^{n+1}\right)}{\Delta t}+\nabla_{j} \cdot\left[(1-\alpha)^{n} v_{\ell}^{n+1}\right]\right\} \\
=\tilde{q}_{w \ell}^{n+1}-\tilde{q}_{\tilde{i} g}^{n+1}-\tilde{\Gamma}^{n+1} \tilde{h}_{s g}^{n+1} .
\end{gathered}
$$


A caret above velocities denotes explicit predictor values. A tilde' above a variable indicates that it is the result of an intermediate step and not a final value for the end of the time step. A horizontal line above a quantity indicates that it is obtained with a $50 \%$ average between values at adjacent cells. If there are no subscripts denoting cell location, we assume subscript $j$ for mass and energy equations and subscript $j+1 / 2$ for equations of motion. Finally, theta is the angle between a vector from the center of cell $j$ to the center of cell $j+1$ and a vector directed against gravity.

Timc levels were omitted from some flux terms in Eqs. (42)-(46) because these terms contain both old and new time quantities. If $X$ is a combination of state variables without a time superscript, then the correct definition for the divergence term in which it appears is

$$
\begin{aligned}
\nabla_{j}\left(X v_{j}^{n+1}\right)= & \left\{A_{j+1 / 2} v_{j+1 / 2}^{n+1}\left[f_{j+1 / 2} x_{j}^{m}+\left(1-f_{j+1 / 2}\right) x_{j+1}^{n}\right]\right. \\
& \left.-A_{j-1 / 2} v_{j-1 / 2}^{n+1}\left[f_{j-1 / 2} x_{j-1}^{n}+\left(1-f_{j-1 / 2}\right) x_{j}^{m}\right]\right\} / v o 1_{j},
\end{aligned}
$$

where

$x_{j}^{m}=g^{-} x^{n}+\left(1-g^{-}\right) x_{j}^{n+1}$

The weighting function used to obtain donor-cell averaging [Eq. (33)] is f, and $g^{-}$is a weighting factor that depends on the rate of phase change, which goes to unity as the phase change disappears and to zero as the phase change approaches the total outflow of the phase created in the cell. For nonzero $g^{-}$, this form of the divergence operator is nonconservative, but total conservation is maintained by the stabilizer step.

Equations (36) and (37) do not involve any implicit coupling between cells and can be solved rapidly for each cell.: Because Eqs. (38) and (39) do not couple, each one requires only the solution of a tridiagonal linear system. Equations (40)-(46), combined with the necessary thermodynamic and constitutive equations, form a coupled system of nonlinear equations. Equations (40) and (41) are solved directly to obtain $v_{g}^{n+1}$ and $v_{\ell}^{n+1}$ as dependent variables. After substituting these equations for velocity into 
Eqs. (42)-(46), the resulting system is solved for the independent variables $\tilde{\mathrm{p}}^{\mathrm{n}+1}, \tilde{\mathrm{p}}_{\mathrm{a}}^{\mathrm{n}+1}, \tilde{\mathrm{T}}_{\mathrm{g}}^{\mathrm{n}+1}, \tilde{\mathrm{T}}_{\ell}^{\mathrm{n}+1}$, and $\tilde{\alpha}^{\mathrm{n}+1}$ with a standard Newton iteration, including all coupling between cells. In practice, the linearized equations solved during this iteration can be reduced easily to a tridiagonal system involving only cotal pressures. The final eight stabilizing equations [Eqs. (47)-(54)] alsu are simple tridiagonal linear systems because $v_{g}^{n+1}$ and $v_{\ell}^{n+1}$ are known after solving Eqs. (40)-(46). Because the pressure and fluid temperatures are used mainly as the starting point for the next iteration, the start of timestep values for these quantities are the tilde values from the end of the previous basic step. This approximation prevents an extra evaluation of the thermodynamic and transport properties. A void fraction consistent with the stabilizer mass and energy quantities is calculated by a simple linearization. 4. Constitutive Equations. The field equations [Eqs. (1)-(8)] require certain auxiliary or constitutive equations to effect closure. Thermal and caloric equations of state for each phase are required, and these are discussed in App. A. In addition; the liquid and vapor wall shear, interfacial drag, wall heat transfer, interfacial heat transfer, and the net vaporization rate are necessary.

The wall heat transfers, $q_{w g}$ and $q_{w l}$, are accounted for in the standard way [see Eqs. (15) and (16)]. The surface areas represent an actual estimate of the total wall surface area wetted by each phase, whereas “ $h_{w \ell}$ and $h_{w g}$ are based on heat-transfer correlations from the literature. In many two-phase flow situations the walls are wetted totally by the liquid phase, in which case wall heat transfer to the vapor is zero.

a. One-Dimensional Wall Shear and Form Losses. The total pressure gradient calculated in the momentum equations is expressed as the sum of the frictional dissipation, acceleration head, and potential head terms. Subroutine FWALL calculates coefficients for the frictional dissipation terms and for losses associated with abrupt area changes. Under single-phase flow conditions, pressure drops associated with frictional losses are correlated as functions of fluid velocity, fluid density, fluid viscosity, channel hydraulic diameter, and surface roughness of the channel wall. When a two-phase mixture is flowing in a channel, a correction to the single-phase frictional loss is necessary to account for added dissipation between phases and interactions 
with the channel walls. This correction factor is the two-phase flow multiplier.

The wall shear coefficients, $c_{w g}$ and $c_{w \ell}$, are defined as

$c_{w g}=\alpha \rho_{g} \frac{c_{f g}}{D_{h}}$

and

$c_{w \ell}=(1-\alpha) p_{\ell} \frac{c_{f \ell}}{D_{h}}$,

where $c_{f g}$ and $c_{f l}$ are the vapor and liquid friction factors. The options available to calculate the wall friction are

$\mathrm{NFF}=0$, constant value (user input);

$\mathrm{NFF}=1$, homogeneous model; and

$\mathrm{NFF}=2$, annular model;

where NFF is the user-spectfied index. Use of a negative index value results in an automatic calculation of an appropriate form-loss coefficient in addition to the selected two-phase flow friction factor if there are abrupt area changes. This option is not avallable for the accumulator component where a constant value for the friction factor is used.

i. Homogeneous Model. The homogeneous friction-factor model alters the single-phase value by using a two-phase viscosity $(\bar{\mu})$ defined in terms of the flow quality $(x), 8$

$\frac{1}{\bar{\mu}}=\frac{x}{\mu_{g}}+\frac{(1-x)}{\mu_{\ell}}$ 
The homogeneous friction factor ${ }^{9}$ then is given by

$$
\begin{array}{ll}
f=0.046(\operatorname{Re})^{-0.2,} & \text { if } \operatorname{Re}>5000 ; \\
f=0.032, & \text { if } \operatorname{Re} \leq 500 ; \\
f=0.032-5.25 \times 10^{-6}(\operatorname{Re}-500), & \text { if } 500<\operatorname{Re}<5000 ;
\end{array}
$$

where $\operatorname{Re}=\mathrm{GD}_{\mathrm{h}} / \bar{\mu}$ and $\mathrm{G}=\rho_{\mathrm{m}} \mathrm{V}_{\mathrm{m}}$. The development of $\mathrm{Eq} \cdot$ (58) assumes sinooth pipe walls.

Equations (58)-(60) represent a constant friction factor for $\operatorname{Re} S 500$ and a linear interpolation between $500<\operatorname{Re}<5000$, where the friction factor is given by Eq. (58). The coefficient of friction for the liquid is

$c_{f_{\ell}}=2.0 \mathrm{f}$,

and, if the void fraction is greater than 0.90 , the vapor coefficient of friction is

$c_{f_{g}}=(10.0 \alpha-9.0)^{2}(21.0-20.0 \alpha) c_{f_{\ell}}$

The purpose of this function is to ensure a smooth transition from zero-vapor wall friction at $\alpha=0.9$ to the single-phase vapor value at $\alpha=1.0$.

ii. Annular-Flow Model. The annular-flow friction-factor method from Ref. 10 is adopted with a modification at high vapor fractions. The single-phase friction factor $\left(f_{s p}\right)$ from Ref. 11 is

$f_{s p}=a+b e^{-c}$, 
where

$$
\begin{aligned}
& a=0.026\left(\frac{k}{D}\right)^{0.225}+0.133\left(\frac{k}{D}\right), \\
& b=22.0\left(\frac{k}{D}\right)^{0.44,}, \\
& c=1.62\left(\frac{k}{D}\right)^{0.134,},
\end{aligned}
$$

and $k / D$ is the relative pipe wall roughness. A value of $k=5.0 \times 10^{-6} \mathrm{~m}$, which is $\sim 23.3$ times the roughness of of drawn tubing and $\sim 20.11$ times the roughness of commercial steel pipe, is used for the absolute pipe roughness. The annular-flow friction factor is then

$$
\mathrm{f}=\mathrm{f}_{\mathrm{sp}} \Phi^{2}
$$

where

$$
\Phi^{2}=\frac{\rho_{\ell}}{\rho_{\text {m }}}
$$

At vapor fractions above 0.90 , the annular-flow friction factor is merged linearly into the homogeneous model with full transition occurring at $\alpha=0.9995$. As discussed in Sec. III.A.1, the wall friction is partitioned between the liquid and vapor phases for a void fraction greater than 0.90 .

iii. Form Losses. The finite-difference equations yield the correct pressure loss for an abrupt expansion. However, this is not true for an abrupt contraction or for an orifice. Form-loss corrections can be included in a TRAC calculation in two ways. For one-dimensional components, the simplest method is to specify a negative value for the input friction-option variable NFF (see any one-dimensional component in Sec.V.C.5) at the location of any abrupt area change. This triggers logic in the code that examines the local pipe geometry, the flow direction, and the implicitness level of the 
difference equations to determine an appropriate loss correction. An extra term in the Bernoulli equation of the form,

$\Delta \mathrm{p}=\frac{\mathrm{kp} \mathrm{V}^{2}}{2}$

where $k$ is a form-loss coefficient, accounts for these corrections. The values used for $k$ are

$k=\left(1-\frac{A_{1}}{A_{2}}\right)^{2}$

for an abrupt expansion or zero-length orifice, and

$k=0.5-0.7\left(\frac{\mathrm{A}_{1}}{\mathrm{~A}_{2}}\right)+0.2\left(\frac{\mathrm{A}_{1}}{\mathrm{~A}_{2}}\right)^{2}$

for an abrupt contraction where $A_{1}$ and $A_{2}$ are the smaller and the larger FAs, respectively. Equation (71) is a curve that was fitted to the values reported in Ref. 12. Experience has shown that these classic form losses often do not match pressure drops in real systems. This option should be used with caution.

The other way to account for form losses is through the use of the FRIC input array. Losses computed using this array are added to those specified with the NFF option. The pressure loss that results from FRIC is

$\Delta p_{j}=\frac{\left(\Delta x_{j}+\Delta x_{j-1}\right)}{2 D_{h, j}} \operatorname{FRIC}_{j} \rho_{m}^{\prime \prime} v_{m}\left|v_{m}\right|$, 
where $j$ is the mesh-cell index.

An input option allows standard $k$ factors 13,14 to be input.

b. Three-Dimensional Wall Shear Coefficients. The wall shear coefficients, $c_{w g}$ and $c_{w \ell}$, are defined similarly to the one-dimensional wall friction. The standard homogeneous correlation is used to provide the wall friction factors for two-phase flow. These factors approach the appropriate single-phase values for $\alpha=0$ and $\alpha=1$.

The velocity used by the correlations in the $z$ direction is determined from the average mesh-cell porosity; therefore, at locations where orifice plates exist and velocities are high, the wall friction is calculated using

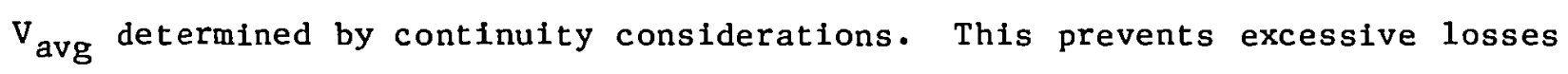
at major flow restrictions. A total friction factor is calculated from the information above and is ascribed completely to the liquid-momentum equation until a vapor fraction of 0.75 is reached. If $0.75<\alpha<0.95$, the shear is assigned with linear weighting (in $\alpha$ ) to both the liquid and vapor. If $\alpha>0.95$, the entire skin friction is assigned to the vapor.

A single friction coefficient is generated from this procedure for both the outer radial and upper axial cell face. However, the hydraulic diameter used in the radial and axial directions, in general, will vary depending on the geometry. If nonzero hydraulic diameters are specified in the problem input, these are used. If the hydraulic diameters are zero in the input, then TF3DE calculates where $i=(\theta, z, r)$ and the wetted perimeter $\left(P_{i}\right)$ normal to direction $i$ includes the surface area of any rods, wall heat slabs, or flow boundaries. If there is no solid material in a mesh cell, the wall shear is " zero. A similar procedure is used to calculate a wall shear in the theta direction. However, in this case vector velocities and properties on the appropriate theta face (rather than the cell-centered averages) are used to achleve theta symmetry where such symmetry should exist.

The basic finite-difference scheme properly calculates classical Bourda form losses at an expansion but overpredicts the losses at a contraction (see discussion in Sec. III.A.4.a.iii). The user can specify an additional constant hydraulic loss factor in any of the coordinate directions to account for geometric details whose scale is smaller than the mesh-cell size. The relation between this input friction term and the pressure drop that it 
produces is the same as the one ir 7 . (72) except that the hydraulic diameter $\left(D_{h}\right)$ is omitted from the expressici:

c. Interfacial Heat Trans ar and Shear. The interfacial constitutive equations basically are identical for the one- and the three-dimensional portions of TRAC-PFI/MODl. A generic description of these relations will be given and any differences between the one- and the three-dimensional sections will be noted. The interfacial heat transfer during boiling and the interfacial shear are calculated in conjunction with a sinple flow-regime map. 15 This flow-regitne map, although originally developed for vertical pipe flow, is the simplest prescription that provides a rational means for defining the constitutive equations. Figure 2 illustrates how the flow map is implemented in the code.

If the void fraction is less than or equal to 0.3 (or $\alpha \leq 0.5$ if G $22700 \mathrm{~kg} / \mathrm{m}^{2} \mathrm{~s}$ ), a bubbly flow is assumed. The interfacial surface area in this regime is calculated in conjunction with a critical bubble Weber number, $W_{b}$. A value of $W_{b}=7.5$ is used in TRAC-PF1/MODI. This choice was based on comparisons between the TRAC predictions and the experimental results for low

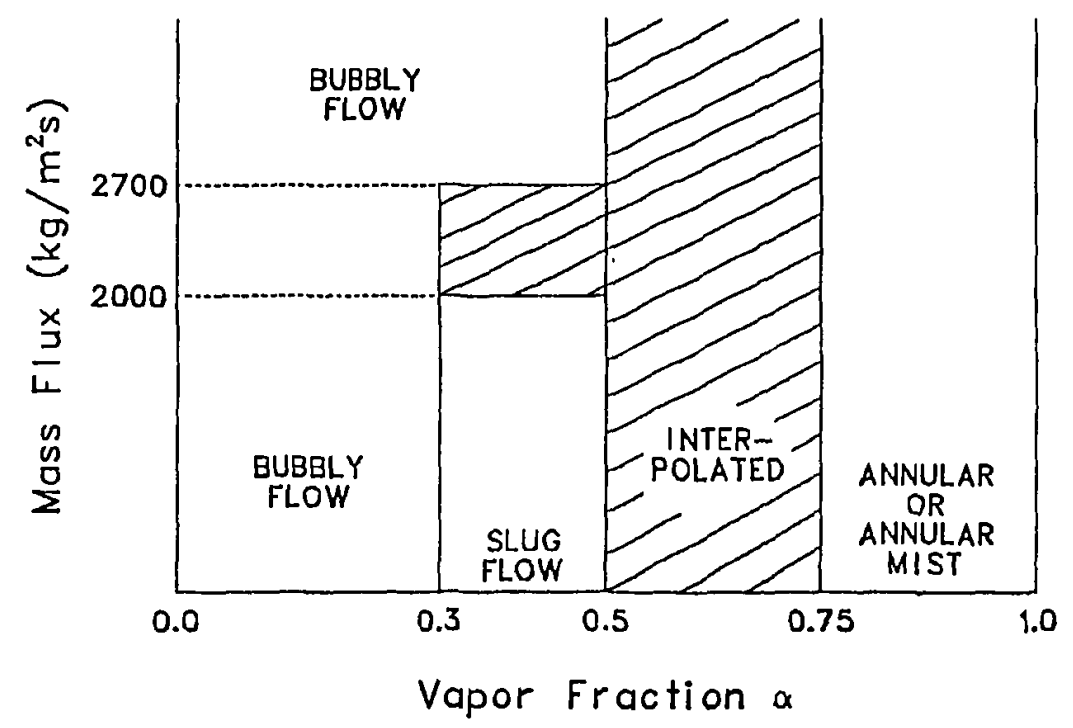

Fig. 2.

Flow-regime map for three-dimensional hydrodynamics. (Cross-hatched regions are transition zones.) 
subcooling (that is, shear-dominated) downcomer tests performed by Creare, Inc. ${ }^{16}$ The expression relating interfacial surface area to $W_{b}$ is

$$
\frac{\rho_{\ell} V_{r}^{2} D_{b}}{\sigma}=W e_{b}
$$

or

$D_{b}=\frac{W e_{b} \sigma}{\rho_{\ell} V_{r}^{2}}$,

where $D_{b}$ is the bubble diameter. The bubble diameter must lie between the mesh-cell hydraulic diameter and $10^{-4} \mathrm{~m}$. For this diameter, with the assumption of a uniform bubble distribution within the mesh-cell vol, the number of bubbles is

$\mathrm{CNB}=\frac{6 \alpha \text { vol }}{\pi D_{b}^{3}}$,

and the interfacial area is

$A_{1}=6 \alpha \operatorname{vol} \rho_{\ell} \frac{v_{r}^{2}}{W_{b^{\sigma}}}$.

If the relative velocity is very small, this area can become small enough to allow significant nonequilibrium. Another surface area,

$A=4.83 \alpha^{2 / 3}\left(N_{B}\right)^{1 / 3}$ vol,

based on a minimum number density $\left(\mathrm{N}_{\mathrm{B}}=10^{10}\right.$ bubbles $\left./ \mathrm{m}^{3}\right)$, is computed, and the actual surface area used is the larger of the two. 
For boiling, the liquid-side interfacial HTC is the larger of an approximate formulation of the Plesset-Zwick bubble growth model, 17

$\mathrm{Nu}=\frac{12}{\pi}\left(\mathrm{T}_{\ell}-\mathrm{T}_{\mathrm{s}}\right) \rho_{\ell} \frac{\frac{\partial \mathrm{e}_{\ell}}{\partial \mathrm{T}_{\ell}}}{\left[\rho_{g}\left(\mathrm{~h}_{\mathrm{sg}}-\mathrm{h}_{\mathrm{s \ell}}\right)\right]}$

and a sphere convection coefficient, ${ }^{18}$

$\mathrm{Nu}=2.0+0.74 \mathrm{Re}_{\mathrm{b}}^{0.5}$

where

$\operatorname{Re}_{\mathrm{b}}=\rho_{\ell} \mathrm{V}_{\mathrm{r}_{\mathrm{t}}} \frac{\mathrm{D}_{\mathrm{b}}}{\mu_{\ell}}$.

For subcooled liquid in a dispersed bubbly regime, the liquid side interfacial HTC, obtained from turbulent convection, is

$h_{i \ell}=0.02 \rho_{\ell} c_{p \ell} v_{r}$

In the bubbly regime, the vapor-to-interface HTC is one of two constants. If the vapor is superheated, $h_{i g}$ is 1000 ; if it is subcooled, $h_{i g}$ is 10000 . The interfacial shear coefficient is provided by a standard set of formulas for a sphere, 11

$c_{i}=\frac{3 c_{b} \alpha \rho_{\ell}}{4 D_{b}}$, 
where

$$
\begin{aligned}
c_{b} & =180, \quad \text { if } \operatorname{Re}_{b}<0.1 ; \\
& =\frac{18}{\operatorname{Re}_{b}}\left(1 .+.15 \mathrm{R}^{0.687}\right), \quad \text { if } 0.1 \leq \mathrm{Re}_{\mathrm{b}} \leq 989 ;
\end{aligned}
$$

or

$$
=0.33 \text {, if } \operatorname{Re}_{b}>989 \text {. }
$$

If the cell-average mass flux is less than $2000 \mathrm{~kg} / \mathrm{m}^{2} \mathrm{~s}$ and the vapor fraction is between 0.3 and 0.5 , the flow enters the slug regime. At the maximum alpha of $0.5,40 \%$ of the vapor is assumed to exist in the form of trailing bubbles with the remainder contained in the slug. These bubbles probably contribute to the majority of the interfacial heat transfer and the liquid-side coefficient is calculated from the heat-transfer relations for the entrained bubbles. If the mass flux is greater than $2700 \mathrm{~kg} / \mathrm{m}^{2} \mathrm{~s}$, all of the vapor is assumed to exist in bubbly form. Linear interpolation in mass flux is used in the range of 2000 to $2700 \mathrm{~kg} / \mathrm{m}^{2} \mathrm{~s}$. In the slug regime the bubble diameter is determined by a linear weighting in $\alpha$ between the Weber number criterion and the channel hydraulic diame ex.s. hydraulic diameter at a void fraction of 0.5 and the value determined by the Weber number size at an $\alpha$ of 0.3 .

In the vapor-fraction range of 0.75 to 1.0 , an annular or annular-mist regime is assumed. The liquid fraction that exists as mist is obtained from an entrainment, correlation, which combines a simple s-shaped entrainment correlation based on the critical Weber number and a correlation by Kataoka and Ishii. 19 Thus,

$$
E=\max \left\{1-\exp \left[\frac{\left(V_{E}-V_{g}\right)}{V_{E}}\right] 0.5,7.75 \times 10^{-7} W e_{e}\left(R e_{\ell} W e_{e}\right)^{1 / 4}\right\},
$$


where

$V_{E}=2.33\left[\frac{\left(\rho_{\ell}-\rho_{g}\right) \sigma W e_{d}}{\rho_{g}^{2}}\right]^{1 / 4}$

and

$W e_{e}=\frac{\rho_{g}^{2 / 3}\left(\alpha V_{g}\right)^{2} D_{h}\left(\rho_{\ell}-\rho_{g}\right)^{1 / 3}}{\sigma}$

This method appears to provide reasonable results for the Dartmouth flooding tests.

The remainder of the liquid is in a film or sheet. The interfacial , shear and heat transfer are volume averages of the film and droplet relations in the annular-mist regime. The wetted surface area of the mesh cell is determined from the rod or slab heat-transfer area in the cell and the portion of the geometric FA that is blocked. If the cell is in a region devoid of any structure, the geometric surface area is used as a scaling factor. This, of course, is artifjctal but in a realistic PWR simulation very few, if any, of the mesh cells are completely free of metal structure. The total interfacial surface area is determined by the sum of the areas contained in the wetted film and the droplets. A critical Weber number equal to four for the drops is used with a calculation procedure similar to that for bubbly flow. This value of the Weber number is appropriate for accelerating drops. For those cases where sensitivity to $\mathrm{We}_{\mathrm{d}}$ was tested, the results were not influenced strongly by $W_{\mathrm{d}}$ in the range of $2 \leq \mathrm{We}_{\mathrm{d}} \leq 12$.

For superheated drops, the liquid-side HTC is sinply

$h_{i \ell}=\frac{a k_{\ell}}{D_{d}}$,

where $a$, a constant, has been adjusted to drive the drops to equilibrium under a variety of flow conditions. In TRAC-PF1/MOD1, $a=15000$, which implies a 
droplet thermal boundary layer that is approximately one ten-thousandth of the drop diameter.

For subcooled drops, $h_{i l}$ is obtained by assuming circulation in the drop giving

$$
h_{i \ell}=0.02 \rho_{\ell} c_{p \ell} v_{c i r c},
$$

where

$v_{\text {circ }}=0.5\left(\frac{\mu_{g}}{\mu_{g}+\mu_{l}}\right) v_{r}$

At some point, this expression for $V_{c i r c}$ produces centripetal source terms large enough compared to the surface tension to produce distortion and eventually to break up the drop. Hence, $v_{\text {circ }}$ can not exceed $1.4 \sqrt{\sigma /\left(D_{\mathrm{drop}} \rho_{\ell}\right)}$. The heat transfer between the vapor and the droplet interface is obtained from

$\mathrm{Nu}=2.0+0.74 \operatorname{Re}_{\mathrm{g}}^{1 / 2} \operatorname{Pr}_{\mathrm{g}}{ }^{1 / 3}$

For any wall film that inay exist along with the drops, the liquid-side interfacial HTC is

$h_{i \ell}=0.02 \rho_{\ell} c_{p \ell} V_{\ell} ;$

for the vapor side,

$h_{i g}=0.02 \rho_{g} c_{p g} V_{g}$. 
The interfacial-drag coefficient for the annular-drop regime combines the droplet drag [analogous to Eq. (80)] and Wallis' correlation for annular flow, 20

$c_{i}=\frac{0.01 \rho g}{D_{h}}(1.0+75.0(1-\alpha)(1-E))$,

where $E$ is given by Eq. (88). To avoid a singularity in the liquid acceleration, a void fraction, $\alpha_{\text {cur }}$, is calculated that corresponds to the minimum allowed film thickness. Above this void-fraction value, Eq. ( 88 ) is multiplied by $(1-\alpha) /\left(1-\alpha_{c u t}\right)$. To obtain the total interfacial-drag coefficient, droplet drag is weighted by the liquid fraction that is entrained, and Wallis annular flow is weighted by the fraction remaining as film.

For the regime between the droplet and the bubbly slug flow, a cubic spline interpolation in the vapor fraction is made between the conditions that would exist if the vapor fraction were 0.75 in the annular or annular-mist topology and the conditions that would exist if the void fraction were 0.5 in the bubbly slug regime. This interpolation assures that the correlation for the interfacial shear, interfacial heat transfer, and surface area is a continuous function of the vapor fraction, the relative velocity, the "mass flux, and the various fluid thermodynamic and transport properties.

If the liquid is subcooled, several other considerations are used to determine the liquid-side interfacial heat transfer. Local conditions are tested for horizontally stratified flow (Sec. III.A.4.e). If it occurs, Eq. (86) is used for $h_{i l}$, and the interfacial area is taken as the area of the stratified interface. If flow is not stratified, adjacent void fractions are tested for a plug interface. If this is detected, Eq. (86) again is used, and the surface area is obtained from an estimate of the flow cross section. If a pipe enters a given three-d sisensional mesh cell and the liquid flows into that cell, then a jet is assumed with an $\alpha$-weighted diameter, a surface area for condensation based on a right circular cylinder is provided, and $h_{i l}$ is given by Eq. (86) multiplied by $\left(\Delta x / D_{j e t}\right)^{2}$. Local conditions also are tested for vertically stratified flow (sharp mixture-density discontinuity with low 
cell-average vapor velocity). If the flow is stratified, $h_{i \ell}$ is reduced by a factor of $10^{3}$, and the interfacial area is the sum of the stratified interface area and the wall area exposed to the gas phase. However, if the liquid velocity into the cell from the top cell edge exceeds a threshold value (for example, a criterion that would be satisfied by a pressurizer spray), $h_{j \ell}$ instead is increased by a factor of $10^{4}$.

If a noncondensable gas is present, the condensation rate is reduced according to the formula,

$\frac{h_{n c}}{h_{i \ell}}=0.168\left(\frac{\alpha \rho_{s}^{2}}{\rho_{a}(1-\alpha) \rho_{\ell}}\right)^{0.1}$,

where $h_{n c}$ is the liquid interfacial HTC when a noncondensable gas is present. Small cutoffs on $(1-\alpha)$ and $\rho_{a}$ prevent the denominator from ever coming so close to zero as to cause difficulties. This model is based on Russian jet data $^{21}$ and can reduce dramatically condensation when a vapor other than steam is in a region.

d. Interface Sharpener. An interface sharpener is used in the lower plenum and core of the three-dimensional vessel to improve the liquid distribution during reflood. Simple void-fraction tests are used to detect any sharp mixture-density discontinuity. The entrainment model then is used to predict the void fraction for convection from the positive face of the mesh cell. This vold fraction always will be greater than or equal to the actual void fraction in the cell. The interfacial shear constitutive relations are calculated based on this void fraction, and the scalar field equations use this new value in the $z$-direction convective terms. A cubic spline is used to merge the sharpened alpha as the mesh cell fills.

e. Horizontal Stratified Flow. In TRAC-PF1/MOD1 the one-dimensional components have an additional flow regime if the angle from the horizontal is less than $30^{\circ}$. A stratification criterion based on the modified Froude number analysis developed by $Y$. Taitel and A. E. Dukler ${ }^{22}$ is used to determine if the flow is stratified. 


$$
U_{\text {crit }}=C_{2}\left[\frac{\left(\rho_{\ell}-\rho_{g}\right) g \cos \beta A_{g}}{\rho_{g} \frac{d A_{\ell}}{d h_{\ell}}}\right]^{1 / 2},
$$

where

$$
\begin{aligned}
C_{2} & =1-\frac{h_{\ell}}{D}, \\
\frac{d A_{\ell}}{d h_{\ell}} & =\left[D^{2}-\left(2 h_{\ell}-D\right)^{2}\right]^{1 / 2},
\end{aligned}
$$

$h_{\ell}$ is the collapsed liquid height (determined by a standard mensuration formula), and $D$ is the pipe inside diameter. If the absolute value of the vapor velocity is more than double the $U_{c r i t}$ value, the standard flow map is used. Below the $U_{\text {crit }}$ value, the wall shear coefficients are calculated by the Blasius relation (but are based on a minimum turbulent Reynolds number); the interfacial shear coefficient is assumed to be a constant $(0.01)$ times the vapor density. A cubic spline employing the independent variable $A B S\left(V_{g}\right)$ connects the two end points. This form of interpolation is necessary to prevent oscillations in the flow pattern with the large time steps often used by the code.

In addition, the hydraulic approximation for the difference in gravitational head caused by collapsed liquid height variations is added explicitly into the liquid equation of motion. Because this calculation is explicit, horlzontal manometer oscillations can occur at larger time-step sizes. To prevent this difficulty, the magnitude of this added term is reduced as the time-step size is increaged beyond the basic stability limit. This reduction eliminates the undesired oscillations. 
f. Subcooled Boiling Model. If hot metal surfaces are present in a region, then the flashing rate 1 is modified to include the effects of subcooled boiling. If rods or slabs exist and $T_{w}>T_{S}$, the $h_{w x}$ is compared to the Dittus-Boelter liquid convective coefficient. If $h_{w x}$ is larger, the difference in wall flux $\left(q_{s b}\right)$ is attributed to subcooled boiling and

$I^{\prime}=\frac{q_{i \ell}+q_{i g}+q_{s b}}{h_{\ell g}}$.

Both in the vapor-continuity equation and in the vapor thermal-energy equation, the potentials, $\left(T_{s}-T_{g}\right)$ and $\left(T_{s}-T_{\ell}\right)$, are evaluated at the new time level, whereas $h_{i g} A_{i}$ and $h_{i \ell} A_{i}$ are evaluated at the old time.

B. Structural Heat Transfer

The TRAC code models three fundamental heat-transfer mechanisms that include the interfacial heat transfer between the vapor and liquid phases, conduction within the reactor structural components, and the heat transfer between the structures and the fluid. Interfacial heat transfer was discussed in Sec. III.A.4.c. The remaining two mechanisms are discussed here.

The thermal history of the structural reactor materials is obtained from a solution of the heat-conduction equation. The energy exchange between the structures and the fluid is modeled using Newton's law. The coupling algorithm (Fig. 3) is semi-implicit. For each new time step, the fluid-dynamics equations are solved based on previous values for the wall HTCs $(h)$ and surface wall temperatures $\left(T_{W}\right)$; that is,

$\mathrm{q}_{\mathrm{w}}^{\mathrm{n}+1}=\mathrm{h}^{\mathrm{n}}\left(\mathrm{T}_{\mathrm{w}}^{\mathrm{n}}-\mathrm{T}_{\mathrm{f}}^{\mathrm{n}+1}\right)$

After the fluid-dynamics equations are solved, the wall temperature distributions are deduced from the conduction equation.

1. Heat-Conduction Models. For simplicity, as well as computing efficiency, the conduction models are separated according to their geometric function. They include conduction within cylindrical walls, slabs, and core rods. The first model analyzes heat conduction within the walls of the 


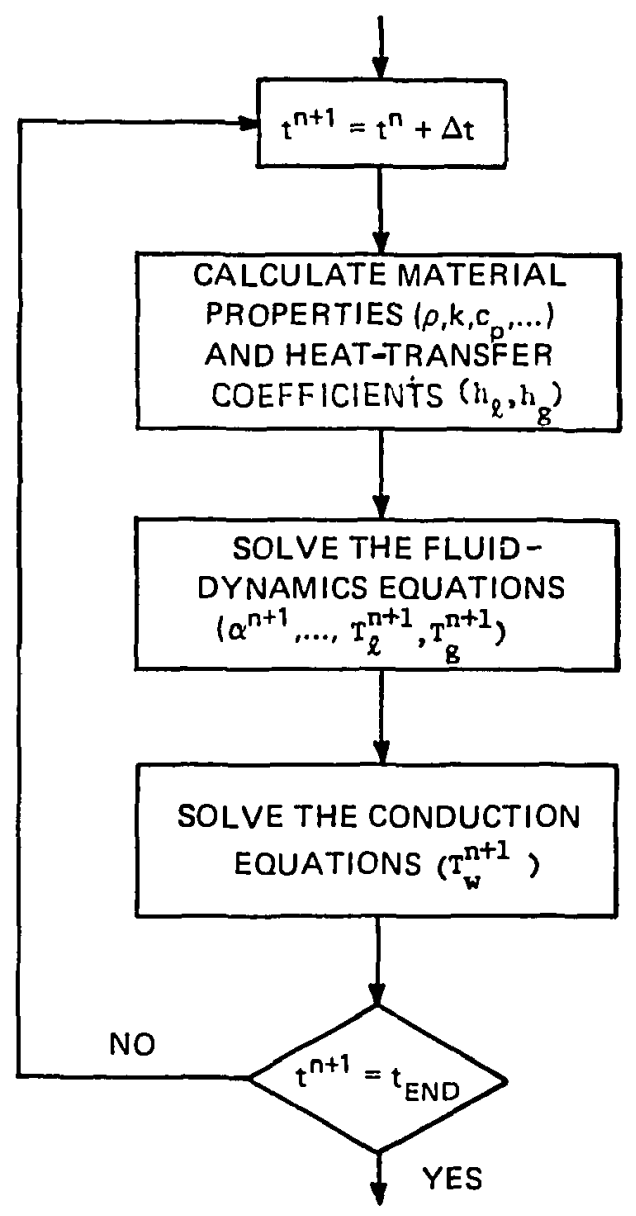

PRE PASS

POST PASS

$\mathrm{F} 1 \mathrm{~g} \cdot 3$.

Semi-implicit coupling between hydrodynamics and structural heat transfer.

one-dimensional loop components, such as the pipe walls. The latter two are associated with heat transfer within structural components of the vessel. Each of these models will be discussed in detall.

a. Cylindrical Wall Heat Conduction. The temperature distribution within the walls of the one-dimensional components is determined by subroutine CYLHT. A solution is obtained from a finite-difference approximation to the one-dimensional conduction equation,

$\rho c_{p} \frac{\partial T}{\partial t}=\frac{1}{r}\left[\frac{\partial}{\partial r}\left(r k \frac{\partial T}{\partial r}\right)\right]+\dot{q} \cdots$ 
The finite-difference equations are derived by applying an integral method 23 to the elemental volumes shown in Fig. 4. The general form for the ith volume $(1<i<N)$ is

$\frac{r_{i-1 / 2} k_{i-1 / 2}}{\Delta r_{i-1}} T_{i-1}^{n+1}-\left\{\frac{r_{i-1 / 2} k_{i-1 / 2}}{\Delta r_{i-1}}+\frac{r_{i+1 / 2} k_{i+1 / 2}}{\Delta r_{i}}\right.$

$\left.+\frac{1}{2 \Delta t}\left[\left(r_{i} \Delta r_{i-1}-\frac{\Delta r_{i-1}^{2}}{4}\right)\left(\rho c_{p}\right)_{i-1 / 2}+\left(r_{i} \Delta r_{i}+\frac{\Delta r_{i}^{2}}{4}\right)\left(\rho c_{p}\right)_{i+1 / 2}\right]\right\} T_{i}^{n+1}$

$+\frac{r_{i+1 / 2} k_{i+1 / 2}}{\Delta r_{i}} T_{i+1}^{n+1}=-\frac{1}{2}\left\{\left(r_{i} \Delta r_{i-1}-\frac{\Delta r_{i-1}^{2}}{4}\right)\left[\frac{\left(\rho c_{p}\right)_{i-1 / 2}}{\Delta t} T_{i}^{n}+\dot{q}^{\cdots}\right]\right.$

$\left.+\left(r_{i} \Delta r_{i}+\frac{\Delta r_{i}^{2}}{4}\right)\left[\frac{\left(\rho c_{p}\right)_{i+1 / 2}}{\Delta t} T_{i, 1}^{n}+\dot{q}^{\cdots-}\right]\right\}$,

where

$f_{i}^{n} \equiv f\left(t^{n}, r_{i}\right)$

The boundary conditions applied to the inner $(i=1)$ and outer ( $i=N)$ surfaces are

$-\left.k \frac{\partial T}{\partial r}\right|_{i=1, N}= \pm\left[h_{\ell}\left(T_{\ell}-T_{i}\right)+h_{g}\left(T_{g}-T_{i}\right)\right]$. 


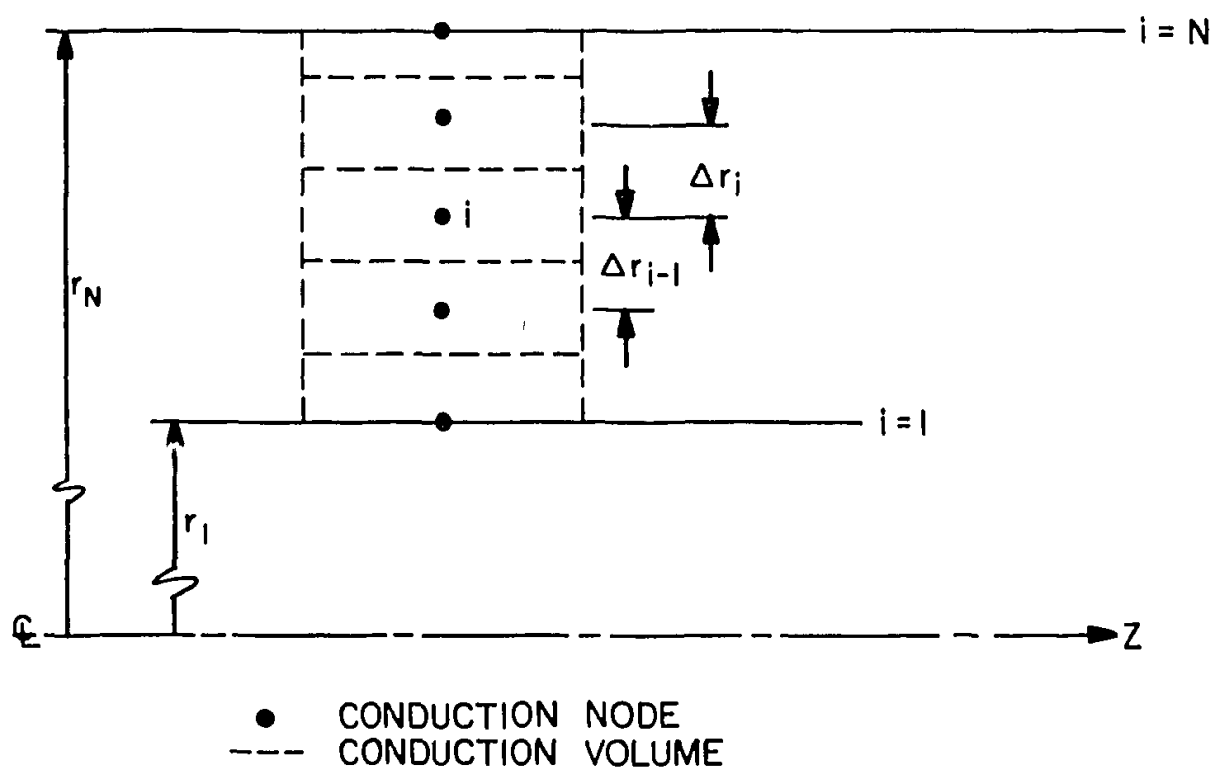

Fig. 4.

Cylindrical wall geometry.

For example, application of this boundary condition to the inner surface ( $i=1$ ) yields

$$
\begin{aligned}
& -\left\{\frac{r_{3 / 2} k_{3 / 2}}{\Delta r_{1}}+\frac{1}{2}\left[r_{1} \Delta r_{1}+\frac{\Delta r_{1}^{2}}{4}\right] \frac{\left(\rho c_{p}\right)_{3 / 2}}{\Delta t}+f_{s s} r_{1}^{\prime}\left(h_{\ell}+h_{g}\right)\right\} T_{1}^{n+1}+\frac{r_{3 / 2} k_{3 / 2}}{\Delta r_{1}} T_{2}^{n+1} \\
& =-\frac{1}{2}\left(r_{1} \Delta r_{1}+\frac{\Delta r_{1}^{2}}{4}\right)\left[\frac{\left(\rho c_{p}\right)_{3 / 2}}{\Delta t} T_{1}^{n}+\dot{q}^{-\cdots}\right] \\
& \quad+r_{1}\left[h_{\ell}\left(f_{t} T_{1}^{n}-T_{\ell}^{n+1}\right)+h_{g}\left(f_{t} T_{1}^{n}-T_{g}^{n+1}\right)\right] .
\end{aligned}
$$

The parameters $f_{t}$ and $f_{s s}$ are 0 and 1 , respectively, to provide maximal stability.

Note that the above formulation conveniently positions nodal points on material interfaces. Material properties are evaluated between nodes. 
The resulting linear equations are solved in a sequential fashion in the axial ( $z$ ) direction. For each axial position a solution is achieved using Gaussian elimination.

A lumped-parameter solution is available to the user if the number of nodes equals one. For this option the wall temperature is obtained from the equation,

$$
\begin{aligned}
T^{n+1} & =\left\{\frac{1}{2}\left(2 \Delta r+\frac{\Delta r^{2}}{R_{i}}\right)\left(\frac{\rho c p}{\Delta t} T^{n}+\dot{q}^{\cdots-}\right)+h_{\ell_{i}}\left(T_{\ell}^{n+1}-f_{t} T^{n}\right)\right. \\
& \left.+h_{g_{i}}\left(T_{g_{i}}^{n+1}-f_{t} T^{n}\right)-\left(1+\frac{\Delta r}{R_{i}}\right)\left[h_{\ell}\left(f_{t} T^{n}-T_{\ell}^{n+1}\right)+h_{g_{o}}\left(f_{t} T^{n}-T_{g_{o}}^{n+1}\right)\right]\right\} \\
& /\left\{\frac{1}{2}\left(2 \Delta r+\frac{\Delta r^{2}}{R_{i}}\right)\left(\frac{\rho c}{\Delta t}\right)+f_{s s}\left[h_{\ell}+h_{g_{i}}+\left(1+\frac{\Delta r}{R_{i}}\right)\left(h_{\ell}+h_{g_{o}}\right)\right]\right\} .
\end{aligned}
$$

The subscripts 1 and o refer to the inner and outer radii, respectively.

b. Slab Heat Conduction. Heat conduction within vessel structures (such as downcomer walls and support plates) is modeled in subroutine SLABHT. Only one $s$ lab is allowed in each fluid-dynamic cell. The number of nodes used to determine the temperature distributions is identical for all slabs within the vessel. The model includes the ability to account for the temperature effects in the slab properties ( $\rho, c_{p}, k$, etc.). The temperature distribution is obtained from the one-dimensional conduction equation,

$\rho c_{p} \frac{\partial T}{\partial t}=\frac{\partial}{\partial x}\left(k \frac{\partial T}{\partial x}\right)$

The appropriate finfte-difference equation is derived by applying an integral method to an elemental volume in a manner similar to that used for the cylindrical wall heat-conduction solution technique (Sec. III.B.1.a). The provision for a variable node spacing allows the location of nodes in regions where large or rapid temperature variations are anticipated. 
The slab conduction model includes a symmetry (adiabatic) condition $\left(\partial \mathrm{T} /\left.\partial \mathrm{x}\right|_{i=1}=0\right)$ at the first node. Newton's law

$\left.-\left.k \frac{\partial T}{\partial x}\right|_{i=N}=\left[h_{\ell}\left(T_{\ell}-T_{i}\right)\right)+h_{g}\left(T_{g}-T_{1}\right)\right]$.

is applied to the outer $(1=N)$ surface.

The ability to model an arbitrary number of interfaces between dissimilar materials also has been included in the slab conduction model. The solution technique is identical to the method used in the rod conduction model (Sec. III.B.1.c.i).

Heat slabs of arbitracy thickness and surface areas can be defined in any mesh cell (including core regions) to model the heat capacity of structures within the vessel. One HTC is computed for each slab using the local fluid conditions.

Although the slab conduction model is simplistic, it effectively analyzes the energy exchange between the fluid and the structures in a computational cell. However, the heat capacity and the effective time constant of the structural materials within a cel1 24 must be modeled carefully. If there is only one structural component associated with a cell, the user must conserve the volume of that structure. If the characteristic thickness (distance to an adiabatic surface) or the surface area is well defined, the remaining geometric component (HSA or HSX in the TRAC input) may be calculated.

If several structural components are associated with one computational cell, an accurate slab model becomes more difficult to define. A useful technique 24 first defines an effective volume,

$\mathrm{V}=\frac{1}{\rho \mathrm{c}_{\mathrm{p}}} \sum_{\mathrm{i}}\left(\rho_{\mathrm{i}} \mathrm{c}_{\mathrm{pi}} \mathrm{v}_{\mathrm{i}}\right), \quad, \quad$,

where the sum includes all material structures within the computational cell, and $\rho$ and $c_{p}$ are the user-specified density and specific heat (typically equal 
to those of one of the cell materials). The rate of energy exchange between the fluid and the structures then may be modeled by calculating a characteristic thickness ( $L$ ). The $L$ value for an important time during the transient under consideration may be obtained from the transcendental equation,

$\rho c_{p} v\left(1-\sum_{n}^{N} D_{n} e^{-\gamma_{n}^{2} F o}\right)=\sum_{i} \rho_{i} c_{p i} v_{i}\left[1-\sum_{n}^{N} D_{n i} e^{-\gamma_{n i}^{2} F o_{i}}\right]$.

Equation (100) represents a series solution (composed of $N$ terms) to the transient one-dimensional conduction equation. In Eq. (100),

Fo $\equiv \frac{\alpha t}{L^{2}}$ (Fourier Number) ;

$\alpha \equiv \frac{k}{\rho c_{p}}$

$\gamma_{n}$ is a constant obtained from the transcendental equation, $\gamma_{n} \tan \gamma_{n}=B 1$;

$B i \equiv \frac{h L}{k}(B i o t$ Number) ;

and

$D_{n}=\frac{\left(2 \sin ^{2} \gamma_{n}\right)}{\left(\gamma_{n}^{2}+\gamma_{n} \sin \gamma_{n} \cos \gamma_{n}\right)}$.

The right side of Eq. (100) is the total energy exchange for the time interval $0 \leq t^{\prime} \leq t$ for each structure (i). The left side is the energy exchange for the effective slab. As in Eq. (99), the properties ( $\left.p, c_{p}, k, h, \ldots\right)$ for the effective slab are specified by the user. With the effective length ( $L=H S X)$ determined from Eq. (100), the vorlume (V) froin Eq. (99), and the userspecified properties, the remaining variable, which is the surface area per 
cell (HSA $=\mathrm{V} / \mathrm{L}$ ), may be computed. The above formulation effectively can model the energy exchange between the vessel structures and the fluid.

c. Rod heat Conduction. Subroutine RODHT analyzes the conduction of the reactor rods on a rod-by-rod basis. The formulation can model diverse rod geometries. Both nuclear and electrically heated rods can be analyzed. The effects of internal heat generation, gap conduction, metal-water reaction, and variable rod properties are included.

The numerical procedures can model the entire LOCA in a consistent and mechanistic fashion. The model also can resolve large axial (z) gradients characteristic of the reflood phase.

One computational rod is associated with each segment [that is, for each $(r, \theta)$ region] within the core. This "average" rod is coupled to the fluid by Newton's law of cooling. Any number of additional user-specified rods may be included in each segment. The rod power factors (that is, relative to the average rod located within each segment) also are specified by the user. The supplemental rods allow the user to include hot rods in the reactor vessel. Such rods do not affect the fluid-dynamics calculations.

i. Numerical Model. The thermal response of the vessel rods is modeled using the two-dimensional $(r, z)$ cylindrical conduction equation. Azimuthal symmetry has been assumed.

$\rho c_{p} \frac{\partial T}{\partial t}=\dot{q} \cdots+\frac{1}{r} \frac{\partial}{\partial r}\left(r k \frac{\partial T}{\partial r}\right)+\frac{\partial}{\partial z}\left(k \frac{\partial T}{\partial z}\right)$.

The effects of internal heat generation resulting from nuclear fission, electrical current, or the metal-water reaction may be included.

Appropriate finite-difference equations are obtained by applying an integral method ${ }^{23}$ to appropriate differential volumes. The noding within the rods (Fig. 5) is staggered with respect to the nodes used in the fluiddynamics calculations. This noding scheme is necessary to sinplify the algorithm that generates the fine mesh required by the reflood calculations. The staggered mesh gives the further advantage of providing axial numerical smoothing • 


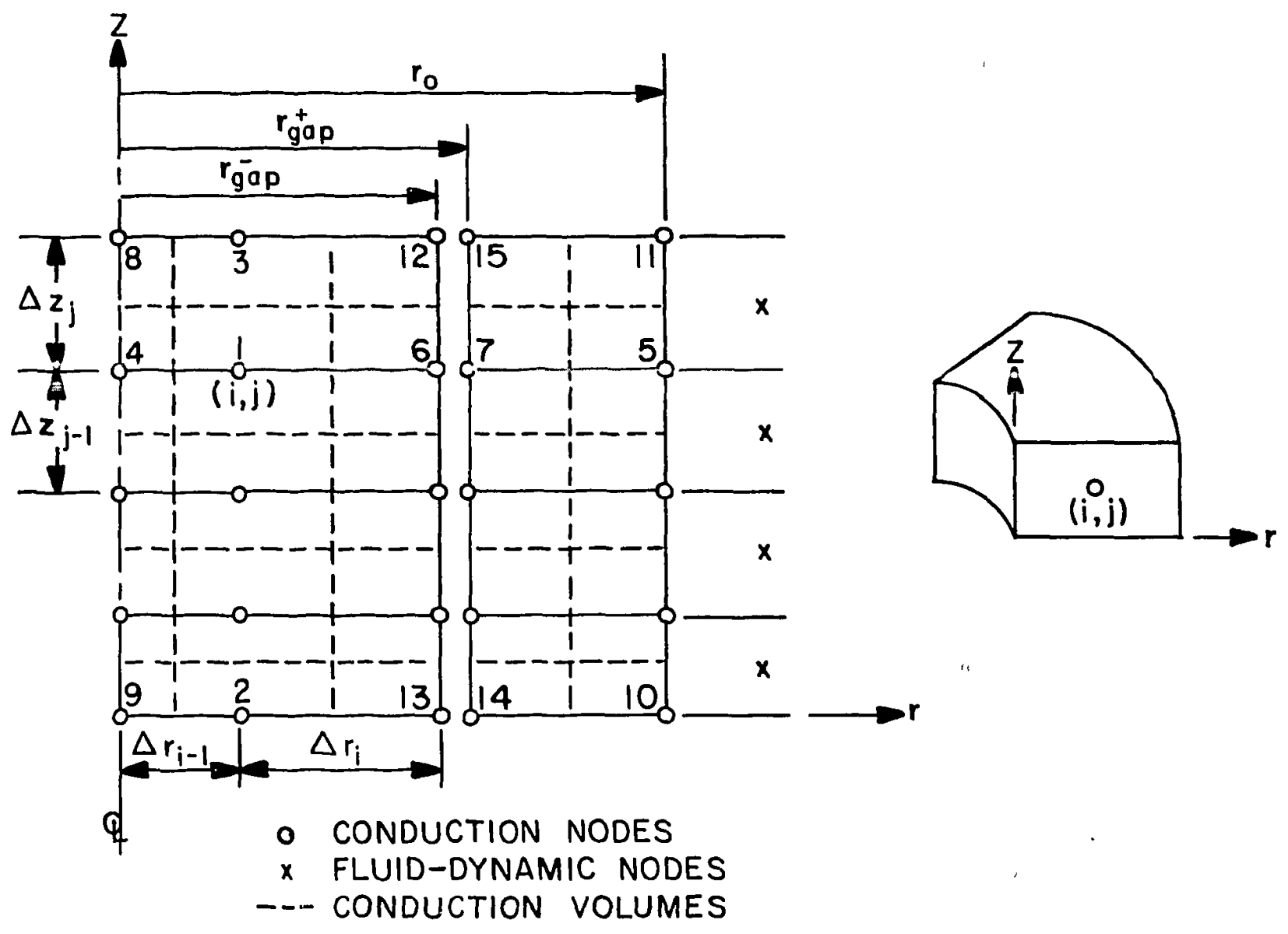

Fig. 5 .

Rod geometry.

Differencing in the radial ( $r$ ) direction is implicit. Therefore, large radial power variations do not create any numerical difficulties. Differencing in the axial ( $z$ ) direction is explicit to simplify the computations and to reduce computer costs. The explicit differencing does limit the minimum axial spacing between nodes for a given time increment. However, this spacing is normally orders of magnitude less than that used by the fluid-dynamics calculations. The resulting finite-difference equations form a tridiagonal matrix for each row ( $z$ fixed) within the rod. The temperature distribution is obtained by using Gaussian elimination on each row in a sequential manner. 
Consider a general differential volume (that is, the volume labeled 1 in Fig. 5). The finite-difference equation for this volume is

$$
\begin{aligned}
& \left.\tilde{i}^{\prime}\left(\rho c_{p}\right)_{i j} \frac{T_{i j}^{n+1}-T_{i j}^{n}}{\Delta t}-\dot{q}_{i j}^{\prime}-\right\} \frac{1}{2}\left[\left(r_{i} \Delta r_{i}+\frac{\Delta r_{i}^{2}}{4}\right)+\left(r_{i} \Delta r_{i-1}-\frac{\Delta r_{i-1}^{2}}{4}\right)\right]\left[\frac{\Delta z_{j}+\Delta z_{j-1}}{2}\right] \\
& =\left\{r_{i+1 / 2} k_{i+1 / 2, j}\left(\frac{T_{i+1, j}^{n+1}-T_{i j}^{n+1}}{\Delta r_{i}}\right)+r_{i-1 / 2} k_{i-1 / 2, j}\left(\frac{T_{i-1, j}^{n+1}-T_{i j}^{n+1}}{\Delta r_{i-1}}\right)\right\} \\
& \times\left[\frac{\Delta z_{j}+\Delta z_{j-1}}{2}\right]+\left\{k_{i, j+1 / 2}\left(\frac{T_{i, j+1}^{n}-T_{i j}^{n}}{\Delta z_{j}}\right)+k_{i, j-1 / 2}\left(\frac{T_{i, j-1}^{n}-T_{i j}^{n}}{\Delta z_{j}-1}\right)\right\} \\
& \times \frac{1}{2}\left[\left(r_{i} \Delta r_{i}+\frac{\Delta r_{i}^{2}}{4}\right)+\left(r_{i} \Delta r_{i-1}-\frac{\Delta r_{i-1}^{2}}{4}\right)\right],
\end{aligned}
$$

where $f_{i j}^{n} \equiv f\left(t^{n}, r_{i}, z_{j}\right)$. In Fig. 5, the locations of nodes within the volumes located at the boundaries differ. This difference should be considered "when values are assigned for the relative power densities at each node.

The boundary conditions applied to the vessel rods are

- the top $\left(z=z_{u}\right)$ and bottom $\left(z=z_{1}\right)$ of the rods are assumed to be insulated,

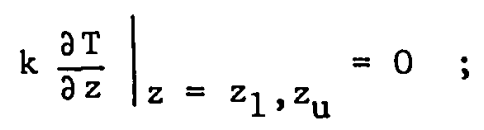

- the rod centerline $(r=0)$ is a line of symmetry,

$$
\left.\frac{\partial T}{\partial r}\right|_{r=0}=0 ; \text { and }
$$


- heat transfer at the inner and outer gap surfaces $\left(r=r_{\text {gap }}^{-}, r_{\text {gap }}^{+}\right)$ and at the cladding surface $\left(r=r_{0}\right)$ is specified using Newton's law,

$$
\begin{aligned}
& \left.k \frac{\partial T}{\partial r}\right|_{r=r_{\text {gap }}^{ \pm}}=-h_{\text {gap }}^{ \pm}\left(T_{r_{\text {gap }}^{-}}-T_{r_{\text {gap }}^{+}}\right) \text {and } \\
& \left.k \frac{\partial T}{\partial r}\right|_{r=r_{o}}=-h_{\text {fluid }}\left(T_{r_{o}}-T_{\text {fluid }}\right),
\end{aligned}
$$

where $h_{\text {gap }}^{+}=h_{\text {gap }}^{-}\left(r_{\text {gap }}^{-} / r_{\text {gap }}^{+}\right)$to conserve energy.

All properties (that is, $\rho, c_{p}$, and $k$ ) required by the difference equations are stored at the node locations. Linear interpolation is used to obtain properties between nodes (that is, at cell surfaces). A node located at the interface between two dissimilar materials requires two sets of properties. Consider the differential volume provided in Fig. 6. Application of an integral technique to this volume results in the differential equation (after dividing through by the volume),

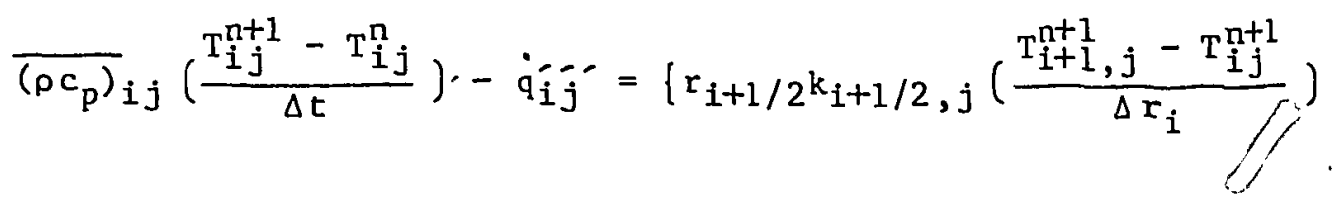

$\left.+r_{i-1 / 2} k_{i-1 / 2, j}\left(\frac{T_{i-1, j}^{n+1}-T_{i j}^{n+1}}{\Delta r_{i-1}}\right)\right\}\left[\frac{\left(r_{i} \Delta r_{i}+\frac{\Delta r_{i}^{2}}{4}\right)+\left(r_{i} \Delta r_{i-1}+\frac{\Delta r_{i}^{2}}{4}\right)}{2}\right]-1$

$+\left\{\bar{k}_{i, j+1 / 2}\left(\frac{T_{i, j+1}^{n}-T_{i j}^{n}}{\Delta z_{j}}\right)+\bar{k}_{i, j-1 / 2}\left(\frac{T_{i, j-1}^{n}-T_{i j}^{n}}{\Delta z_{j-1}}\right)\right\}\left[\frac{\Delta z_{j}+\Delta z_{j-1}-1}{2}\right]^{-}$

where

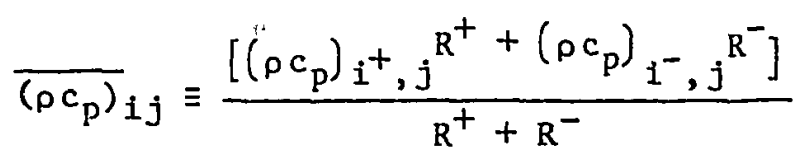




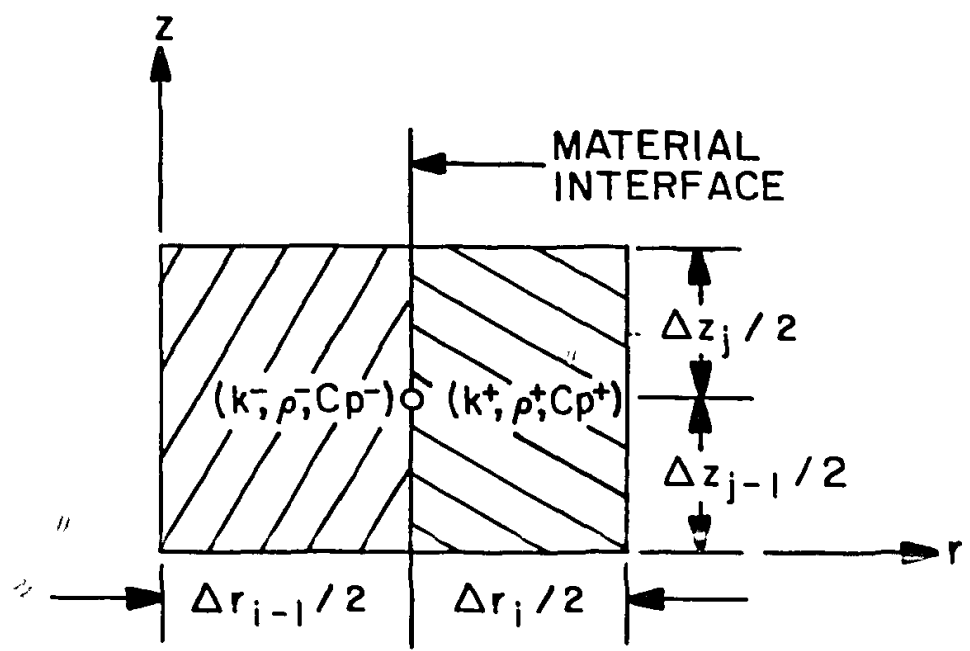

Fig. 6.

Node located at the interface between two dissimilar materials.

and

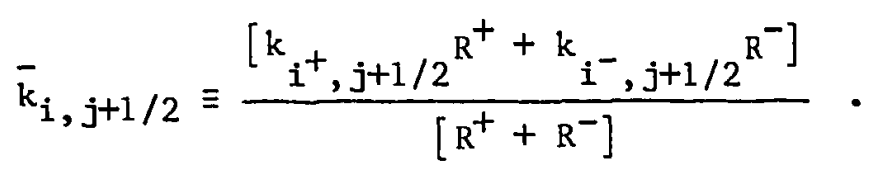

In the above equations,

$R^{+} \equiv\left(r_{i}+\frac{\Delta r_{i}}{4}\right) \frac{\Delta r_{i}}{2}$

and

$R^{-} \equiv\left(r_{i}-\frac{\Delta r_{i-1}}{4}\right) \frac{\Delta r_{i-1}}{2}$

The superscript $+(-)$ refers to the material to the right (left) of the interface.

s. 
ii. Reflood AIgorithm. The reflood phase of a postulated LOCA is characterized by a sequence of heat-transfer and two-phase flow regimes advancing rapidly through the vessel core. A correctly predicted thermal response from the rods during reflood requires a numerical technique that can model the rewetting phenomena associated with the quench-front motion.

The leading edge of the rewetting region is characterized by large variations of temperatures and heat fluxes within sinall axial distances $(\Delta \mathrm{z} \sim 1 \mathrm{~mm}) .25$ The front advancement is controlled by two heat-removal mechanisms, the first being axial conduction from the dry region ahead of the quenched region to the wetted region behind the advancing film. The second is the precursory rod cooling associated with heat transfer to the droplets entrained in the advancing vapor field. The rod' conduction model contains the necessary physics to analyze such phenomena. The need remains to define an algorithm capable of resolving the large gradients.

When reflood begins, supplemental rows of conduction nodes (Fig. 7) are inserted in the rod. The number of rows inserted within each fluid level is specified by the user. The rows are uniformly spaced (that is, $\Delta z$ is constant) within each fluid level. The temperature values at the supplemental nodes are determined from a three-point Lagrangian interpolation technique. The interpolation is normalized to conserve the total rod energy. The nodes added in this fashion remain during the entire reflootd phase.

To model the inherently nonstationary, Lagrangian quench-front motion and to resolve the related thermal gradients, a fine-mesh rezoning technique 26 is used during the reflood conduction calculations. The axial gradients encountered within the rewetting region are resolved by the insertion of rows of transitory nodes (Fig. 7). These nodes are added whenever the temperature difference between adjacent rod surface nodes exceeds a user-spectfied value $\left(T_{\max }\right)$. The temperatures assigned to the, nodes are required to "conserve energy (Fig. 8),

$$
\sum_{j=j_{0}}^{j_{0}+2} \rho_{i j}^{\prime} c_{p_{i j}}^{-} T_{i j}^{\prime} V_{i j}^{\prime}=\sum_{j=j_{0}}^{j_{0}+1} \rho_{i j} c_{p_{i j}} T_{i j} V_{i j} ; \quad i=1, \ldots, \text { nodes . }
$$

The primed quantities denote rod properties after the nodes have been added. The values of $\rho^{-}, c_{p^{\prime}}$, and $T^{-}$at the original node locations are set equal to 

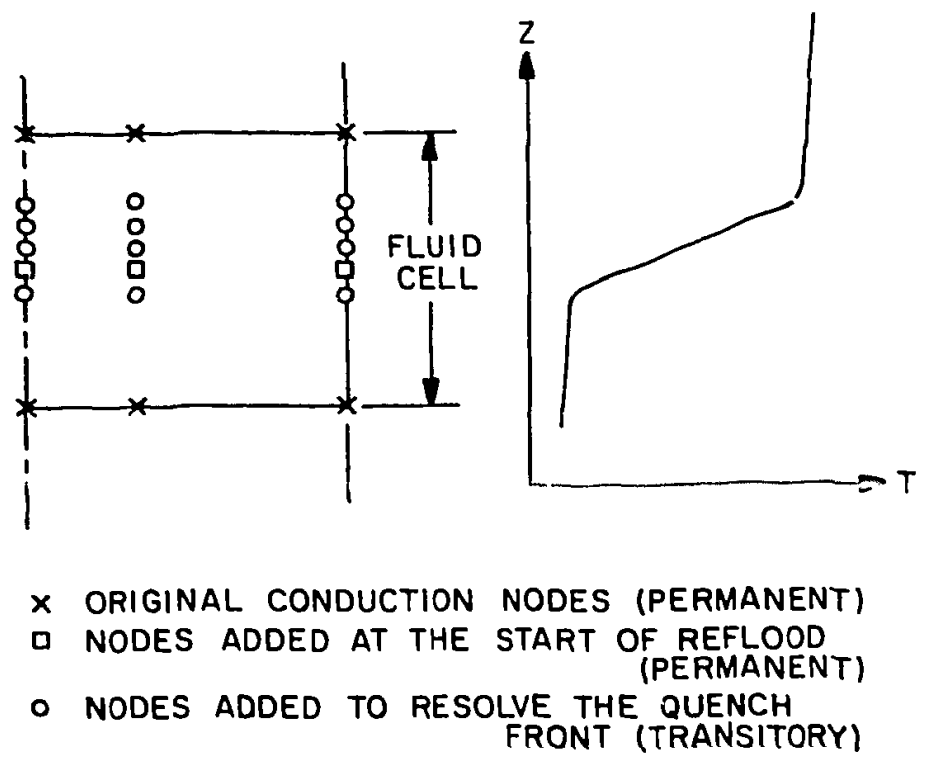

Fig. 7 .

Fine-mesh rezoning.

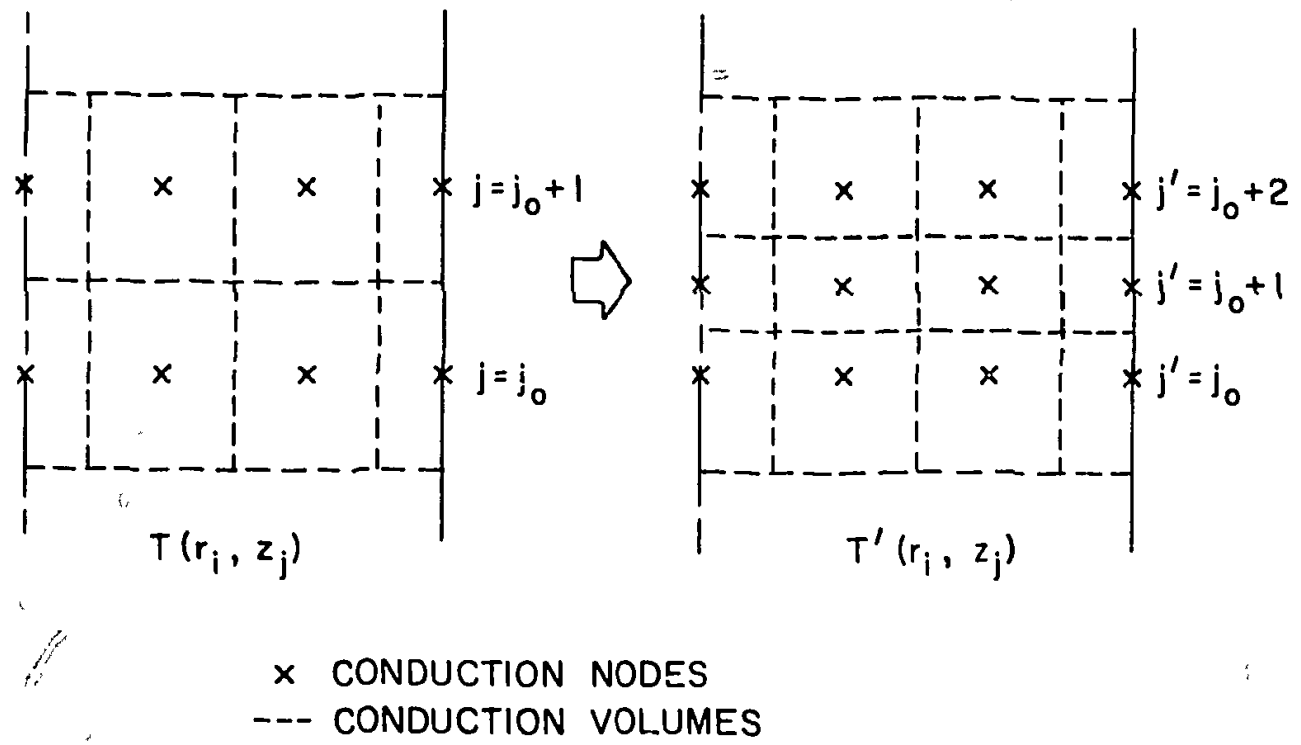

Fig. 8.

Insertion of conduction nodes during reflood. 
their original values. After the quench front has progressed beyond the location of the inserted rows and the surface temperature difference falls below a prescribed value $\left(\Delta \mathrm{T}_{\mathrm{min}}\right)$, the transitory nodes are eliminated. Temperatures at nodes axially adjacent to those deleted retain their original values. For small $\Delta \mathrm{T}_{\mathrm{min}}$, this results in a negligible effect on the total rod energy.

Two values for $\Delta \mathrm{T}_{\max }$ are specified by the user. The first and smaller value is applied to the part of the rewetting region that is in a nucleale- or transitional-boiling regime. The largest wall heat fluxes occur in these heat-transfer regimes. The second $\Delta \mathrm{T}_{\max }$ value is applied to all other heattransfer regimes. The specified values for the $\Delta \mathrm{T}_{\max }$ are the bases of the $\Delta \mathrm{T}_{\text {min }}$ values, which are computed internally.

The above algorithm can analyze multiple quench fronts simultaneously. Both quenching and dryout are modeled automatically.

During the reflood phase, a number of surface conduction nodes are located within each fluid-dynamics cell. Therefore, it is necessary to calculate an effective wall temperature and HTC for the fluid-dynamics computations. These values are obtained. by ensuring the conservation of the total energy transferred to the fluid within each cell. Values applied to the liquid phase that satisfy this criterion are

$\tilde{h}_{\ell} \equiv \frac{\sum_{j} h_{\ell} A_{j}}{\sum_{j} A_{j}}$

and

$\bar{T}_{w_{\ell}} \equiv \frac{\sum h_{\ell}{ }_{j} A_{j} T_{j}}{\sum h_{\ell} A_{j}}$,

where the sum is taken over all surface nodes in each fluid cell. Similar values are used in the vapor phase. 
It already has been noted that, for a given time step $(\Delta t)$, a minimum spacing $(\Delta z)$ between rows of conduction nodes exists because of the explicit axial differencing. For reflood calculations, this axial spacing can be violated, resulting in stability problems. To avoid such problems, the time step is limited internally by a diffusion number. The user also can specify minimum spacing $\left(\Delta z_{\mathrm{min}}\right)$ beyond which supplemental rows of conduction nodes will not be added. This additional advantage can prevent excessively large computer costs.

Computing costs are reduced further by calculating material properties only at those nodes located at the edges of the fluid cells. Linear interpolation is used to obtain the properties at any additional locations required by the reflood calculations. However, the HTCs are obtained directly from the boiling curve for all rod surface nodes.

iii. Fuel-Cladding Gap Conductance. Two options are available in TRAC-PF1/MOD1 for the fuel-cladding gap conductance. If the input variable $\mathrm{NFCI}=0$, a constant input value for the gap conductance is used throughout the entire calculation. If the input variable $\mathrm{NFCI}=1$, the input value for the gap conductance becomes the initial value, and a thermal-expansion model is used to calculate the transient gap conductance.

Subroutine GAPHT calculates the gap HTC ( $\left.\mathrm{h}_{\mathrm{gap}}\right)$ as a function of three components: gap gas conductance, fuel-cladding interfacial contact, and fuelcladding thermal radiation;

$h_{\text {gap }}=h_{\text {gas }}+h_{\text {contact }}+h_{\text {radiation }}$,

where

$$
\begin{aligned}
& \mathrm{h}_{\text {gas }}=\frac{\mathrm{k}_{\text {gas }}}{\Delta \mathrm{r}_{\text {gap }}+\delta}, \\
& \mathrm{h}_{\text {radiation }}=\frac{\sigma \mathrm{F}\left(\mathrm{T}_{\mathrm{f}}^{4}-\mathrm{T}_{\mathrm{c}}^{4}\right)}{\left(\mathrm{T}_{\mathrm{f}}-\mathrm{T}_{\mathrm{c}}\right)},
\end{aligned}
$$


and

$$
F=\frac{1}{\frac{1}{\varepsilon_{f}}+\frac{R_{f}}{R_{c}}\left(\frac{1}{\varepsilon_{c}}-1\right)} \text {. }
$$

Subscripts $f$ and $c$ refer to fuel and cladding, respectively, and $\sigma$ is the Stefan-Boltzmann constant. A value of $4.4 \times 10^{-6} \mathrm{~m}$ is used for $\delta$, which includes the mean surface roughness of the fuel and the cladding plus the temperature jump distances. 27,28 The contact conductance, h contact, is zero in the present code.

The fuel-cladding radial gas gap, $\Delta \mathrm{r}_{\text {gap }}$, is found by using, the uncoupled, quasi-static approximation. 29 In this approximation the mechanical coupling term in the energy equation and the inertial term in the mechanical force balance are omitted. By neglecting these terms we assume that the fuelcladding strains minimally affect the temperature distribution and that displacements are instantaneous. Figure 9 shows the fuel-cladding gap system modeled in three regions: solid fuel, cracked fuel, and cladding. Gap changes

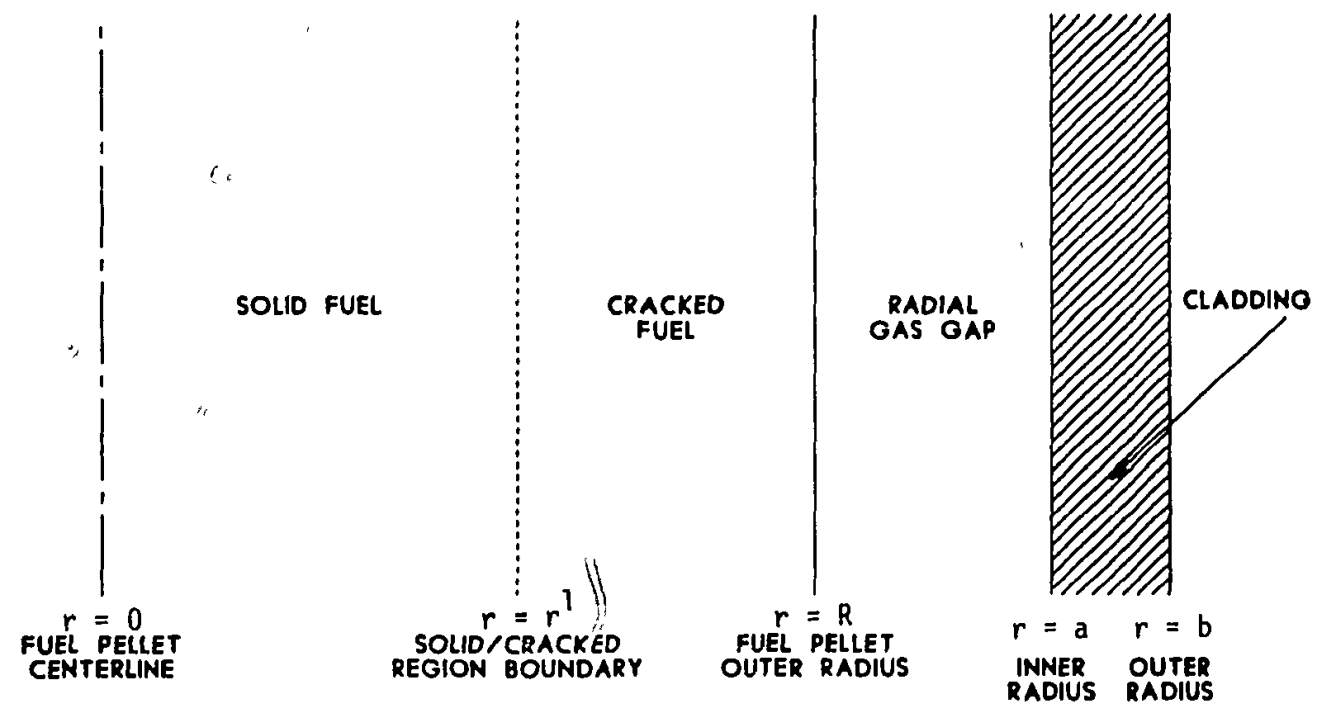

Fig. 9 .

Fuel-rod geometry. 
are found by calculating the radial displacement of each region caused by thermal expansion.

The calculations for the deformation of a hollow or solid circular cylindrical body of outer radius $b$ and of height $h$ are given in Ref. 29 for the case of plane strain where the ratio $\mathrm{h} / \mathrm{b}$ is large compared to unity. other assumptions are made that the cylindrical surfaces are free of forces and that axial displacement is allowed. Because the uncoupled, quasi-static approximation is used, the temperature distributions are assumed to be known from the energy balance. The radial displacement $u$ is given by

$u(r)=\frac{\alpha}{r(1-v)}\left[(1+v) \int_{a}^{r} \operatorname{Tr} d r+\frac{(1-3 v) r^{2}+a^{2}(1+v)}{b^{2}-a^{2}} \int_{a}^{b} \operatorname{Tr} d r\right]$,

where $a$ is the inner radius of a cylindrical shell, $v$ is Poisson's ratio, and $\alpha$ is the linear thermal-expansion coefficient. Equation (111) is used to calculate the radial displacement of the cladding inner radius and the solidfuel radius, $r=a$ and $r=r^{\prime}$, respectively. The results are

$u(a)=\frac{2 \alpha_{c} a}{b^{2}-a^{2}} \int_{a}^{b} T_{c} r d r$

and

$u\left(r^{-}\right)=\frac{2 \alpha_{f}}{r^{\prime}} \int_{0}^{r^{\prime}} T_{f} r d r$

The cladding inner radius and the solid-fuel radius after thermal expansion are

$a_{\text {new }}=a+u(a)$ 
and

$$
r_{\text {new }}^{\prime}=r^{-}+u\left(r^{-}\right)
$$

A parabolic radial temperature distribution is assumed across the fuel pellet,

$T_{f}=T_{c l}+\frac{\left(T_{\text {surf }}-T_{c l}\right) r^{2}}{R^{2}}$,

where $T_{c l}$ is the fuel centerline temperature and $T_{\text {surf }}$ is the fuel surface temperature. A linear temperature profile is assumed across the cladding,

$T=\frac{\left(T_{c o}-T_{c i}\right)(r-a)}{b-a}+T_{c i}$

where $\mathrm{T}_{c o}$ and $\mathrm{T}_{\mathrm{cl}}$ are the cladding outside and inside temperatures, respectively; and $a$ and $b$ are the cladding inside and outside radif.

The following equation is used for the cracked-fuel thickness,

$t=t_{0}\left[1+\frac{\alpha_{f}}{R-r^{-}} \int_{r^{-}}^{R} T_{f} d r\right]$,

where $t_{0}$ is the initial undeformed radial thickness of the cracked fuel, $t_{0}=R-r^{-}$ 
The radial gap width after thermal expansion is

gap width $=a_{\text {new }}-\left(r_{\text {new }}^{-}+t\right)$

or

$$
\begin{aligned}
\text { gap width }= & \left(a-r^{-}\right)+\frac{2 \alpha_{c^{a}}}{b^{2}-a^{2}} \int_{a}^{b} T_{c} r d r-\frac{2 \alpha_{f}}{r^{-}} \int_{0}^{r^{-}} T_{f} r d r \\
& \left.-t_{0} x^{1-} \frac{\alpha_{f}}{R-r^{-}} \int_{r^{-}}^{R} T_{f} d r\right] .
\end{aligned}
$$

Subroutine DELTAR evaluates the fuel-cladding radial spacing.

iv. Metal-Water Reaction. When Zircaloy reaches a súficiently high temperature in a steam environment, an exothermic reaction may occur that influences the peak cladding temperatures. The zirconium-steam reaction equation is

$\mathrm{Zr}+2 \mathrm{H}_{2} \mathrm{O}+\mathrm{ZrO}_{2}+2 \mathrm{H}_{2}+$ heat.

Given sufficient steam, the reaction rate equation 30,31 , is assuned valid,

$\tau \frac{\mathrm{d} \tau}{\mathrm{dt}}=\mathrm{A} \exp \left(-\frac{\mathrm{B}}{\mathrm{T}}\right)$,

where $\tau=$ total oxygen consumed $\left(\mathrm{kg} / \mathrm{m}^{2}\right), \quad A=16.8 \mathrm{~kg}^{2} / \mathrm{m}^{4} \mathrm{~s}, \quad$ and $B=2.007 \times 10^{4} \mathrm{~K}$. 
The kinetic parameter $\tau$ is converted to an effective zirconium-oxide layer thickness according to

$1.5\left(R_{o}-r\right)=\frac{\tau}{0.26 \mathrm{p} \mathrm{ZO}_{2}}$,

where

$r=$ reacting surface radius (in),

$\mathrm{R}_{0}=$ cladding outer radius $(\mathrm{m})$, and

$\rho_{\mathrm{ZrO}_{2}}=$ density of zirconium oxide $\left(\mathrm{kg} / \mathrm{m}^{3}\right)$.

Equation (124) is based on a reacted-material volume expansion of $50 \%$ in the radial direction. This assumption leads to $\rho_{\mathrm{ZrO}_{2}}=0.90 \rho \mathrm{Zr}$. Equation (124) allows Eq. (123) to be rewritten as

$\tau \frac{d \tau}{d t}=-C\left(R_{0}-r\right) \frac{d r}{d t}$,

where $C=\left(0.351 \mathrm{o}_{\mathrm{Zr}}\right)^{2}$.

The method outlined in Ref. 30 is used to calculate the zirconium-oxide penetration depth and associated heat source. The mass per unit length of zirconium ( $m_{Z R}^{-}$) consumed by the reaction in one time step is

$m_{Z r}^{-}=\pi \rho_{Z r}\left[\left(r^{n}\right)^{2}-\left(r^{n+1}\right)^{2}\right]$.

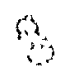

Equation (123) is used to calculate $r^{n+1}$, yielding

$r^{n+1}=R_{o}-\left[\left(R_{o}-r^{n}\right)^{2}+2 \frac{A}{C} \Delta t \exp \left(-\frac{B}{T}\right)\right]^{1 / 2}, \cdot$ 
If a one-region cladding is assumed, the heat source $\left(\dot{q}_{m w}^{-}\right)$added to the conduction equations is

$\dot{q}_{\mathrm{mw}}^{-\cdots}=6.45 \times 10^{6} \mathrm{~m}_{\mathrm{zr}}^{-}\left[\Delta t\left(\mathrm{R}_{0}^{2}-\mathrm{R}_{\mathrm{i}}^{2}\right) \pi\right]^{-1}$,

where $R_{i}$ is the inner cladding radius and $6.45 \times 10^{6} \mathrm{~J} / \mathrm{kg}$ corresponds to the energy released per kilogram of oxidized zirconium.

2. Wall-to-Fluld Heat Transfer. The wall-to-fluid HTCs are obtained from a generalized boiling curve constructed within subroutine HTCOR. The HTC correlations in HTCOR are used by all TRAC components under all"conditions. Figure 10 shows a portion of the boiling curve, which is not dependent on the flow regime. The single-phase vapor and condensation regimes are not shown in this figure.

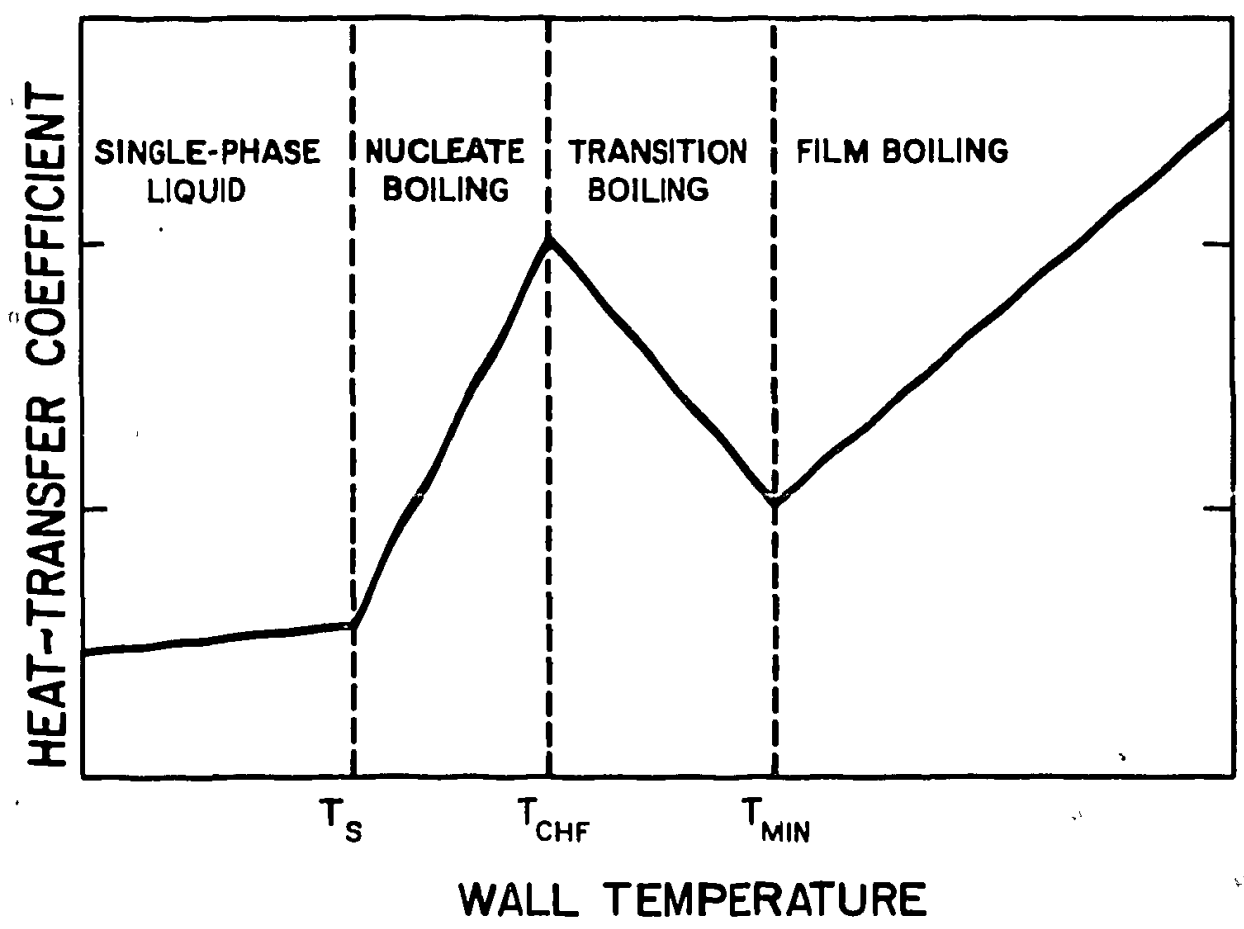

Fig. 10 .

TRAC-PF1/MOD1 bolling curve. 
The individual correlations used for each heat-transfer regime and the method of partitioning the energy between the two phases are discussed in this section. We have tried to make the boiling curve continuous between regimes; thus, we also discuss our methods to smooth the boiling curve.

a. Wall-to-Fluid HTC Selection Logic. Figure 11 outlines the HTC selection logic. The following sequence, corresponding to the numbers on the left side of Fig. 11, is used. If one step is not satisfied, then the next step is examined.

Step 1. Initialize subroutine HTCOR by calculating absolute values, the slip, and the flow and equilibrium qualities. If the slip is less than or equal to zero in HTCOR, it is set equal to 1.0 .

Step 2. If $\mathrm{T}_{\mathrm{w}}<\mathrm{T}_{\mathrm{S}}, \alpha>0.05$ and $\mathrm{T}_{\mathrm{w}}<\mathrm{T}_{\mathrm{g}} ; \mathrm{T}_{\mathrm{w}}<\mathrm{T}_{\mathrm{S}}, \alpha>0.05, \mathrm{~T}_{\mathrm{w}}<\mathrm{T}_{\mathrm{g}}$, and $T_{\dot{w}}<T_{\ell}$; or the liquid-vapor interface is present in the cell, the heat transfer is in the condensation regime. The Nusselt ${ }^{8}$ and $\mathrm{Chen}^{32}$ correlations, discussed in Sec. III.B.2.b.ii, are used in this regime to calculate the vapor and liquid HTCs, respectively. If the equilibrium quality, $x_{\ell}$, is less than or equal to $0.71, h_{g}=0$. For $x>0.71, h_{g}$ is found by linear interpolation between zero and the value obtained from the Nusselt analysis. The suppression factor, $S$, for the Chen correlation is set to zero. If $x_{\ell}>0.71$, the limit of Chen's data base, then the Chen correlation is evaluated at $x=0.71$, which gives $h_{\ell}$. Linear interpolation is used between $h_{\ell}$ calculated corresponding to $x_{\ell}=0.71$ and the single-phase vapor $h_{\ell}$ of zero, for an equilibrium quality exceeding 0.71 .

Step 3. If $0.96<\alpha<1.0$, a single-phase vapor HTC is calculated. When $\alpha>0.98$, linear interpolation is used between the HTCs from the appropriate heat-transfer regime and the single-phase vapor values. The $h_{\ell}$ is interpolated between $0.98 \leq \alpha \leq 0.999$; the $h_{g}$ is interpolated between $0.98 \leq \alpha \leq 1.0$.

Step 4. When the input variable $I C H F=0$, the boiling curve is not used to determine the liquid and vapor HTCs. These values are obtained from the two-phase mixture equations only (heat-transfer regimel7), described in Sec. III.B.2.b.vii.

Step 5. The forced-convection and nucleate-bolling HTCs are calculated by using the Chen correlation. At this point the heat-transfer regime has not been determined. 


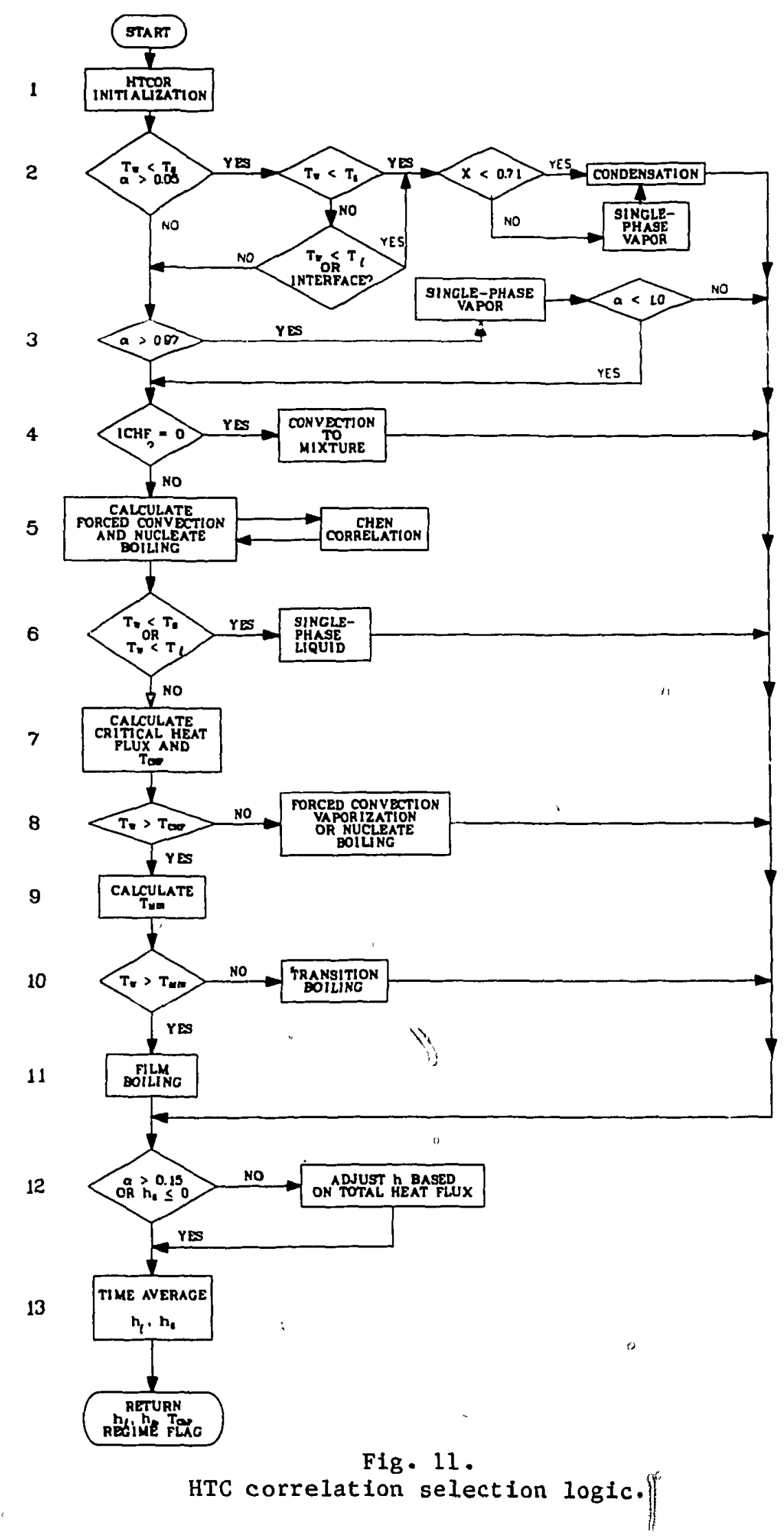


Step 6. If $T_{W}<T_{\ell}$ or $T_{W}<T_{S}$, the heat-transfer regime is singlephase liquid.

Step 7. The critical heat flux (CHF) and the corresponding temperature, $\mathrm{T}_{\mathrm{CHF}}$, are calculated next.

Step 8. If $\mathrm{T}_{\mathrm{w}}<\mathrm{T}_{\mathrm{CHF}}$, nucleate boiling exists.

Step 9. The minimum stable film-boiling temperature, $T_{m i n}$, is calculated by using the homogeneous-nucleation model. This correlation is discussed in Sec. III.B.2.d.

Step 10. If $T_{w}<T_{m i n}$, transition boiling exists. It previously has been determined that $\mathrm{T}_{W}$ is greater than the temperature at CHF conditions (step 8).

Step 11. If $T_{w}>T_{m i n}$, film boiling is occurring.

Step 12. The HTCs are restricted to zero or positive values. (For $\alpha>0.999$, extrapolation occurs and a negative $h_{\ell}$ could result. See step 3.) For $\alpha<0.15$, the vapor heat flux is interpolated linearly from its value at $\alpha=0.15$ to a value of 0.0 at $\alpha=0.01$. The liquid heat flux is adjusted so that the total heat flux remains unchanged. Then, $h_{\ell}$ and $h_{g}$ are recalculated from the adjusted heat fluxes and the known temperature differences to prevent an extremely sinall mass of vapor from becoming superheated to an unrealistic value.

Step 13. The HTCs are averaged between the previous and the current time steps.

b. HTC Correlations. In this section we discuss the HTC correlations used in the construction of the boiling curve. In addition, the correlation for $I C H F=0$ is discussed. Because TRAC-PFI/MODI is a nonequilibrium code, HTCs are needed for the liquid and the vapor phases; this partitioning of energy between the phases also is discussed. Table $I_{i,}$ lists, the TRAC-PF1/MOD1 heat-transfer regimes.

1. Single-Phase Liquid (Heat-Transfer Regimes 1 and 12). Either forced convection (regine 1) or natural convection (regime 12) can occur when single-phase liquid is present. Forced-convection correlations are. used when the ratio of the Grashof number to the Reynolds number squared is less Than or equal to 1.0. Laminar or turbulent forced-convection correlations are 1,40 
TABLE I

TRAC-PF 1/MODI HEAT-TRANSFER REGIMES

IDREG

(IHTF)

1

2

3

4

6

7

11

12
Na11-to-Fluid Heat-Transfer Regime

Forced convection to single-phase liquid

Nucleate boiling

Transition boiling

Film boiling

Convection to single-phase vapor

Convection to two-phase mixture

Condensation

Liquid natùral convection

available. These equations are contained in subroutine CHEN because they constitute one part of the Chen correlation. The laminar equation ${ }^{9}$ is

$\mathrm{h}_{\ell}=4.0 \frac{\mathrm{k}_{\ell}}{\mathrm{D}_{\mathrm{h}}}$

The turbulent HTC is found Erom the Dittus-Boelter equation, 27

$\mathrm{h}_{\ell}=0.023 \frac{\mathrm{k}_{\ell}}{\mathrm{D}_{\mathrm{h}}} \operatorname{Re}_{\ell}^{0.8} \operatorname{Pr}_{\ell}^{0.4}$,

where the liquid Reynolds number is

$\operatorname{Re}=\frac{\rho_{\ell} V_{\ell} D_{h}}{\mu_{\ell}}$, 
and the liquid Prandtl number is

$\operatorname{Pr}_{\ell}=\left(\frac{\mu c_{p}}{k}\right)_{\ell}$

All the properties are evaluated at $\mathrm{T}_{\ell}$. The maximum of Eqs. (118) and (129) is set equal to the single-phase liquid forced-convection HTC, HFORC. In heat-transfer regime 1 , the Chen $F$ factor is set equal to 1.0 .

In heat-transfer regime 12 , natural convection to single-phase liquid, the maximum of the laminar and turbulent correlations for vertical flat plates and cylinders is used. 33 For laminar flow, the equation is

$\mathrm{Nu}=0.59(\mathrm{GrPr})^{0.25} ;$

for turbulent flow,

$\mathrm{Nu}=0.10(\mathrm{GrPr})^{0.3333 ;}$

where the Grashof number is

$G r=\frac{g \beta \mid T_{w}-T_{\ell} \rho_{f}^{2} D_{h}^{3}}{\mu^{2}}$,

the Prandt1 number is

$$
\operatorname{Pr}=\left(\frac{\mu c_{p}}{k}\right)_{\ell},
$$


and the Nusselt number is

$\mathrm{Nu}=\frac{\mathrm{h}_{\ell} \mathrm{D}_{\mathrm{h}}}{\mathrm{k}_{\ell}}$

The maximum of the laminar and turbulent Nusselt numbers is used. To avoid extra calls to the thernodynamic property subroutine THERMU, all the properties are evaluated at $T_{\ell}$ except the density, $\rho_{f}$, and the volume coefficient of expansion, $\beta$; these are evaluated by using a Taylor-series expansion about $\mathrm{T}_{\ell}$,

$\rho_{f}=\rho_{\ell}+\frac{\partial \rho_{\ell}}{\partial T}\left(T_{f}-T_{\ell}\right)$

$\beta=-\frac{\partial \rho_{\ell}}{\partial T} \frac{1}{\rho_{f}}$,

and

$\mathrm{T}_{\mathrm{f}}=\frac{1}{2}\left(\mathrm{~T}_{\mathrm{w}}+\mathrm{T}_{\ell}\right)$

Two points should be noted abuut heat-transfer regimes 1 and 12 . First, because only single-phase liquid is assumed to be present, the vapor HTC, $h_{g}$, $F$ is set equal to zero. Second, in Eq. (132) the hydraulic diameter, $D_{h}$, is used for the characteristic length" even though the axial distance would be more appropriate, because the axial length would approach zero near the bottom of a channel. The characteristic-length term drops out of Eq. (133) and appears only to the -0.25 power in Eq.(132). "Thus, the choice of the characteristic length has a small effect on the HTC. lation $\frac{\text { ii. Nucleate-Boiling HTCs (Heat-Transfer Regime 2). The Chen corre- }}{32}$ is used in heat-transfer regime 2 , nucleate boiling. 
In addition, the nucleate-boiling HTC correlation affects the transition-boiling regime through the Interpolation between the $\mathrm{CHF}$ and the minimum stable film-boiling points. The Chen correlation has two parts, a forced-convection term multiplied by the Reynolds $F$ factor and a nucleateboiling term that contains the $\mathrm{S}$ factor,

$h_{\ell}=h_{\text {forc }}+\min \left(1, \frac{T_{w}-T_{s}}{T_{w}-T_{\ell}}\right) h_{\text {nucb }}$,

where $h_{\text {forc }}$ (HFORC in the code), with the $F$ factor equal to 1.0 , was discussed in the previous section, and the nucleate-botling term is given by

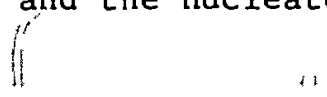

$h_{\text {nucb }}=0.00122 \frac{k_{\ell}^{0.79} c_{p \ell}^{0.45} \rho_{\ell}^{0.49}}{\sigma^{0.5} \mu^{0.29} h_{\ell g}^{0.24} \rho_{g}^{0.24}}\left(T_{w}-T_{s}\right)^{0.24}\left(p_{w}-p\right)^{0.75} s$,

where $\mathrm{p}_{\mathrm{w}}$ is the saturation pressure corresponding to the wall temperature, and $F$ and $S$ are functions that are given in graphical form by Chen. The Reynolds number factor, $F, 34$ can be expressed as

$F=1.0$

, for $x_{T T}^{-1} \leq 0.10$;

and

$\mathrm{F}=2.35\left(x_{\mathrm{TT}}^{-1}+0.213\right)^{0.736} \quad$, for $x_{\mathrm{TT}}^{-1}>0.10 ;$

where $x_{T T}^{-1}$, the Lockhart-Martinelli factor, is

$x_{\mathrm{TT}}^{-1}=\left(\frac{\mathrm{x}}{1-\mathrm{x}}\right)^{0.9}\left(\frac{\rho_{\ell}}{\rho_{\mathrm{g}}}\right)^{0.5}\left(\frac{\mu_{\mathrm{g}}}{\mu_{\ell}}\right)^{0.1}$. 
The value of $x_{\mathrm{TT}}^{-1}$ is restricted to a value less than 100.0 .

The $\mathrm{S}$ factor ${ }^{34}$ can be expressed as

$S_{1}=\left[1+0.12\left(\operatorname{Re}_{t p}\right)^{1.14}\right]^{-1}$, for $\operatorname{Re}_{t p}<32.5$,

or

$S=\left[1+0.42\left(\operatorname{Re}_{t p}\right)^{0.78}\right]^{-1}$, for $32.5 S \operatorname{Re}_{t p} \leq 70.0$,

where

$\operatorname{Re}_{\mathrm{tp}}=\frac{10^{-4}\left|\mathrm{v}_{\ell}\right|_{\rho_{\ell}}(1-\alpha)}{\mu_{\ell}} \mathrm{D}_{\mathrm{h}} \mathrm{F}^{1.25}$

The value of $\operatorname{Re}_{t p}$ is restricted to a value less than 70.0 .

The above correlations for the suppression factor do not approach the correct limit (zero) as the vold fraction approaches one. In the above equations, $S+1.0$ as $\alpha+1.0$. The following procedure is used to ensure that $S$ approaches the correct 1imit. For $\alpha>0.70, S$ is calculated at $\alpha_{s}=0.70$, and at the current value of $\alpha$ and the minimum value of the two suppression factors, $\mathrm{S}_{\mathrm{min}}$, is saved. Linear interpolition is then used between $\mathrm{S}_{\mathrm{min}}$ and $S=0$ at $\alpha=\alpha_{c}$,

$s=\frac{s_{m i n}\left(\alpha_{c}-\alpha\right)}{\alpha_{c}-\alpha_{s}}$

In TRAC-PF1/MOD1, $\alpha_{c}=0.98$ and $\alpha_{s}=0.70$. For $\alpha>\alpha_{c}, S=0.0$.

The properties are evaluated at the liquid and vapor temperatures; $x$ is the equilibrium quality, and $v_{\ell}$ is the liquid velocity parallel to the surface. Because the nucleate-boiling contribution to the Chen correlation was developed for saturated conditions, ${ }^{32} h_{\text {nucb }}$ i.s multiplied by a temperature ratio to adjust the HTC to the actual $\mathrm{T}_{\ell}$, Eq. (140). Because TRAC-PF1/MOD1 
can calculate superheated liquids, the adjustment factor is restricted to a maximum of 1.0 so that the adjustment is made for subcooled liquid only. The vapor HTC goes from zero at $T_{W}=T_{S}$ to the transition-boiling value at $\mathrm{T}_{\mathrm{w}}=\mathrm{T}_{\mathrm{CHF}}$ (Sec. III.B.2.b.iii); thus,

$h_{g}=\left(\frac{T_{w}-T_{s}}{T_{C H F}-T_{s}}\right)^{2} \max \left(h_{n c}, h_{d r}\right)$,

where $h_{n c}$ and $h_{d r}$ are the natural-convection and Dougall-Rohsenow HTCs, respectively. The vapor HTC is calculated in subroutine HVFILM. For a void fraction greater than a cutoff value, $\alpha_{c}$, linear interpolation is used between the current values of $h_{\ell}$ and $h_{g}$ and the values that are calculated for singlephase vapor; that is, $h_{\ell}=0$ and $h_{g}$, calculated as discussed in Sec. III.B.2.b.v. This linear interpolation ensures that the bolling curve is smooth between heat-transfer regimes.

iii. Transition-Boiling HTCs. (Heat-Transfer Regime 3). Transition bolling may be considered as a combination of nucleate and $\mathrm{film}$ boiling. A given spot on the wall surface is wet part of the time and dry during the remainder of the time. Therefore, contributions to the liquid and vapor HTCs exist for all conditions.

The total wall-to-fluid heat flux is obtained from a quadratic interpolation between the $\mathrm{CHF}$ and the minimum stable film-boiling points, ${ }^{34}$

$\mathrm{q}_{\text {trans }}=\delta \mathrm{q}_{\mathrm{CHF}}^{\cdots}+(1.0-\delta) \mathrm{q}_{\mathrm{min}}^{--}$,

where $\mathrm{q}_{\mathrm{CHF}}^{--}$is the heat flux at $\mathrm{CHF}$ conditions, found from the Biasi correlation (Sec. III.B.2.c), and $\mathrm{q}_{\mathrm{min}}$ is the heat flux at the minimum stable film-boiling point, that is, the intersection of the transition- and the film-boiling points. This point is found from the holnogeneous-nucleation 
correlation, as discussed in Sec. III.B.2.d. Delta is a function of the wall temperature and the temperatures corresponding to $\mathrm{q}_{\mathrm{C}}^{-} \overline{\mathrm{H}} \mathrm{F}$ and $\mathrm{q}_{\mathrm{min}}^{\mathrm{i}}$,

$\delta=\left(\frac{\mathrm{T}_{w}-\mathrm{T}_{\mathrm{min}}}{\mathrm{T}_{\mathrm{CHF}}-\mathrm{T}_{\mathrm{min}}}\right)^{2}$.

The vapor HTC is

$h_{g}=\max \left(h_{n c}, h_{D R}\right)$,

where $h_{n c}$ and $h_{D R}$ are the natura1-convection and Douga11-Rohsenow HTCs, respectively. These correlations are discussed in the next section.

As in the nucleate-boiling heat-transfer regime, linear interpolation is used for $\alpha>\alpha_{c}$. The liquid HTC is

$h_{\ell}=\frac{q_{\text {trans }}-h_{g}\left(T_{w}-T_{g}\right)}{T_{w}-T_{\ell}}$.

iv. Film-Boiling HTCs (Heat-Transfer Regime 4). In the film-boiling heat-transfer regime, radiative and dispérsed-flow heat transfers occur between the surface and the liquid; convective heat transfer occurs between the wall and the adjacent vapor. The liquid HTC is given by

$h_{\ell}=\left(h_{r}+h_{f b b}\right)\left[\frac{T_{w}-T_{s}}{T_{w}-T_{\ell}}\right]+h_{d f}$,

where, $h_{d f}$ is the dispersed-flow HTC. 
$h_{r}=(1-\alpha) \sigma \varepsilon\left(\frac{T_{w}^{4}-T_{s}^{4}}{T_{w}-T_{s}}\right)$,

where $\sigma$ is the Stefan-Boltzmann constant and $\varepsilon$ is the wall emissivity. In Eq. (150) the 1iquid absorptivity is 1.0 .

The Bromley film-boiling HTC is $h_{f b b}, 35$

$h_{f b b}=0.62\left[\frac{\rho_{g} k_{g}^{3}\left(\rho_{\ell}-\rho_{g}\right) g h_{\ell g}^{-}}{\mu_{g}\left(T_{w}-T_{s}\right) \lambda}\right]^{1 / 4}$,

where the characteristic length, $\lambda$, is

$\lambda=2 \pi\left[\frac{\sigma}{g\left(\rho_{\ell}-\rho_{g}\right)}\right]^{1 / 2}$.

The latent heat of vaporization is modified as suggested in Ref. 36 ,

$h_{\ell g}^{\prime}=h_{\ell g}+0.5\left(c_{p}\right)_{g}\left(T_{g}-T_{s}\right)$.

The dispersed-flow HTC, $h_{d f}$, uses the Forslund and Rohsenow equation, 37 modified by multiplying $(1-\alpha)$ by the entrained liquid fraction, $E$; thus,

$h_{d f}=0.2 c_{1}[(1-\alpha) E]^{0.6667} B_{B R A C}^{0.25}\left(\frac{T_{w}-T_{s}}{T_{w}-T_{\ell}}\right)$, 
where $c_{1}$ is a constant equal to 1.2760 and

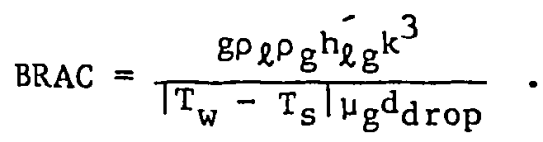

The dispersed-flow HTC is set equal to zero if $(1-\alpha) \mathrm{E}>0.05$.

Equation (152) is multiplied by the temperature ratio to change the base of the HTC from ' $\mathrm{T}_{S}$ to $\mathrm{T}_{\ell}$. The droplet diameter, $\mathrm{d}_{\mathrm{drop}}$, is found from a Weber number criterion of $4: 0$,

$\mathrm{d}_{\mathrm{drop}}=\frac{\text { Weo }}{\rho_{\mathrm{g}}\left(\mathrm{v}_{\mathrm{g}}-\mathrm{v}_{\ell}\right)^{2}}$.

The droplet diameter is restricted to the range,

$1.0 \times 10^{-4} \leq d_{d r o p} \leq 3.0 \times 10^{-3}$

The entrained liquid fraction is found in the following manner,

$\mathrm{E}=0$,

if $\left|v_{g}\right| \leq v_{E}$

or

$E=1.0-\exp 0.23\left[-\left(\left|v_{g}\right|-v_{E}\right)\right]$, if $\left|v_{g}\right|>v_{E}$,

where the entrainment velocity is

$V_{E}=3.65\left[\frac{\left(\rho_{\ell}-\rho_{g}\right)_{\sigma}}{\rho_{g}^{2}}\right]^{1 / 4}$

and $E$ is restricted to values between 0.07 and 1.0 .

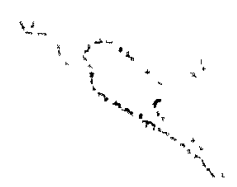


The vapor HTC is the maximum of the Bromley, natural-convection, and Dougal1-Rohsenow values,

$h_{g}=\max \left(h_{n c}, h_{D R}\right)$

The turbulent natural-convection equation ${ }^{38}$ used in this heat-transfer regime is

$h_{n c}=0.13 k_{g}\left(\frac{\rho_{g}^{2} g\left|T_{w}-T_{g}\right|}{\mu^{2} T_{g}}\right)^{0.333} \operatorname{Pr}_{g}^{0.333}$

The forced-convection equation is based on Dougall and Rohsenow's modification 39 to the Dittus-Boelter equation, 38

$h_{f c}=0.023 \frac{k_{g}}{D_{h}}\left\{\frac{p_{g}\left[\alpha\left|v_{g}\right|+(1-\alpha)\left|v_{\ell}\right|\right] D_{h}}{\mu_{g}}\right\}^{0.8}\left[\frac{\mu_{g}\left(c_{p}\right) g}{k_{g}}\right]^{0.4}$,

where the Reynolds number is modified to reflect the volumetric flow rate of the two-phase mixture.

As in the previous heat-transfer regimes, linear interpolation is used for $\alpha>\alpha_{c}$.

v. Single-Puase Vapor HTCs (Heat-Transfer Regime 6). For the single-phase vapor heat-transfer regime, $h_{\ell}=0$ and $h_{g}$ is the maximum of Eq. (154), of turbulent natural convection, and of the Dittus-Boelter Eq. (129) evaluated with the use of vapor properties and flow conditions.

vi. - Condensation HTCs (Heat-Transfer Regime 11). Nusse1t's analysis ${ }^{8}$ is used to calculate the vapor HTC when condensation occurs. For an equilibrium quality $x \leq 0.71, h_{g}$ is set to zero. For $x>0.71, h_{g}$ is found by linear interpolation between zero and the value of $h_{g}$ found from Nusselt's analysis. The liquid HTC is calculated using the chen correlation. ${ }^{32}$ The $\mathrm{S} f$ actor is set equal to zero. The Chen correlation is based upon data taken 
up to $x=0.71$. For $x>0.71$, the Chen correlation is evaluated at $x=0.71$, and $h_{\ell}$ is found by linear interpolation between the value of $h_{\ell}$ at $x=0.71$ and the single-phase vapor $h_{\ell}$ of zero.

vii. Two-Phase Mixture HTCs (Heat-Transfer Regime 7). This heattransfer regime is unique because it is not part of the boiling curve discussed previously. Regime 7 is used only when the input flag ICHF $=0$. When $I C H F=0, h_{\ell}$ and $h_{g}$ are calculated from regime 7 only. Critical heat flux cannot occur in this case.

If the void fraction is less than or equal to the cutoff void fraction, $h_{g}=0$, and $h_{\ell}$ is the maximum of the laminar and turbulent values,

$h_{\ell}=\max \left(h_{\ell 1 a m}, h_{\ell \text { turb }}\right)$,

where

$$
\begin{aligned}
& h_{\ell l a m}=\frac{4 k_{\ell}}{D_{h}} \text {, } \\
& h_{\ell \text { turb }}=\frac{3.023 \mathrm{k}_{\ell} \operatorname{Re}_{\mathrm{m}}^{0.8} \operatorname{Pr}_{\ell}^{0.4}}{D_{h}},
\end{aligned}
$$

and

$$
\operatorname{Re}_{\mathrm{m}}=\frac{G D_{\mathrm{h}}}{\mu_{\mathrm{m}}}
$$


The two-phase viscosity is calculated by using McAdam's equation, 38

$\mu_{m}=\frac{1}{\frac{x_{f}}{\mu_{g}}+\frac{1-x_{f}}{\mu_{\ell}}}$,

where $x_{f}$ is the flow quality.

If the void fraction is greater Lhan the culoff void fraction, $\mathrm{h}_{\mathrm{g}}$ is the maximum of the Dittus-Boelter value for vapor and the turbulent naturalconvection vaiue,

$h_{g}=\max \left(h_{v n c}, h_{v t u r b}\right)$,

where the natural-convection value 38 is

$h_{v n c}=0.13 k_{g}\left(\frac{\rho_{g}^{2} g\left|T_{w}-T_{g}\right|}{\mu_{g}^{2} T_{g}}\right) 0.333 \operatorname{Pr}_{g}^{0.333}$

and the forced-convection HTC is given by the Dittus-Boelter equation,

$\mathrm{h}_{\text {vturb }}=\frac{0.023 \mathrm{~kg}_{\mathrm{g}} \operatorname{Re}_{\mathrm{g}}^{0.8} \mathrm{Pr}_{\mathrm{g}}^{0.333}}{\mathrm{D}_{\mathrm{h}}}$

The Reynolds and Prandtl numbers in Eq. (162) are calculated by using vapor properties evaluated at the vapor temperature.

For $\alpha>\alpha_{c}$, linear interpolation is used between the current values of $h_{\ell}$ and $h_{g}$ and the single-phase values. 
c. Critical heat Flux (CHF). The CHF point has two purposes in relation to the TRAC-PFI/MODl boiling curve. First, the CHF point indicates the change from nucleate boiling (heat-transfer regime 2) to transition boiling (regime 3). Second, the $\mathrm{CHF}$ point is used in the quadratic interpolation that gives the transition-boiling liquid HTC. If the input variable $I C H F=1$, the Biasi forced-flow $C H F$ correlation 40 is used. For ICHF $=0$ and for steady-state calculations (ISTDY = 1), no CHF calculation is performed. In the vessel TCHF $=1$ is used always, and it also is recommended for other components.

The Biasi correlation has a data base that covers the mass-flux range, $G$, between $100 \mathrm{~kg} / \mathrm{m}^{2} \mathrm{~s}$ and $6000 \mathrm{~kg} / \mathrm{m}^{2} \mathrm{~s}$. For mass-flux values between 0 and $200 \mathrm{~kg} / \mathrm{m}^{2} \mathrm{~s}$, the Biasi correlation is evaluated at $200 \mathrm{~kg} / \mathrm{m}^{2} \mathrm{~s}$. For values of $G$, the absolute value of $G$ is used. The Biasi CHF correlation consists of two equations for $\mathrm{q}_{\mathrm{CHF}}^{-}$, and the maximum $\mathrm{CHF}$ value calculated by the two equations is used,

$$
q_{C \bar{C} F}^{-}=\frac{1.883 \times 10^{7}}{D_{h^{n}}^{n_{G}^{1 / 6}}}\left[\frac{f_{p}}{G^{1 / 6}}-x\right]
$$

and

$$
\mathrm{q}_{\mathrm{CH}}^{\prime}=\frac{3.78 \times 10^{7}}{\mathrm{D}_{\mathrm{h}}^{\mathrm{n}_{\mathrm{G}}} 0.6} \mathrm{~h}_{\mathrm{p}}(1-\mathrm{x}) \text {, }
$$

where

$$
\begin{aligned}
n & =0.4, \quad \text { for } D_{h}<1 \mathrm{~cm} ; \\
n & =0.6, \quad \text { for } D_{h}<1 \mathrm{~cm} ; \\
f_{p} & =0.7249+0.099 p \exp (-0.032 p) ;
\end{aligned}
$$




$$
\begin{aligned}
& h_{p}=-1.159+0.149 p \exp (-0.019 p)+\frac{8.99 p}{10+p^{2}} ; \\
& D_{h}=\text { hydraulic diameter }(\mathrm{cm}) ; \\
& G=\text { mass flux }\left(\frac{\mathrm{g}}{\mathrm{cm}^{2} \mathrm{~s}}\right) ; \\
& \mathrm{p}=\text { pressure (bar); and } \\
& \mathrm{x}=\text { equilibrium quality. }
\end{aligned}
$$

Note that the Biasi correlation uses cgs units, but the constants in Eqs. (163) and (164) have been changed so that $\mathrm{q}_{\mathrm{CH}}^{\mathrm{CH}}$ is in $\mathrm{W} / \mathrm{m}^{2}$.

Predictions made with earlier versions of TRAC show that the Biasi correlation sometimes fails to predict $\mathrm{CHF}$ at high vold fraction, even though the data indicate that $\mathrm{CHF}$ has occurred. To correct this problem, "the Biasi correlation is used for a void fraction less than 0.97 , and linear interpolation is used between the HTC at this void fraction and the one at 0.98 , with the assumption that the $\mathrm{T}_{\mathrm{CHF}}$ is one-half degree above $\mathrm{T}_{\boldsymbol{s}}$. For a void fraction greater than 0.98 , the $\mathrm{T}_{\mathrm{CHF}}$ is fixed at one-half degree above $\mathrm{T}_{\mathbf{S}}$.

Once $\mathrm{q}_{\mathrm{C}} \overline{\mathrm{CH}} \mathrm{F}$ is obtained, the temperature corresponding to the CHF point, $\mathrm{T}_{\mathrm{CHF}}$, is calculated by using a Newton-Raphson iteration ${ }^{41}$ to determine the intersection of the heat flux found by using the nucleate-boiling HTC and the CHF. An iteration is required because $T_{W}=T_{C H F}$ must be known to evaluate the Chen correlation; and, in turn, the Chen HTC must be known to calculate the wall temperature,

$$
\mathrm{q}^{C} \overline{C H} \mathrm{~F}=\mathrm{h}\left(\mathrm{T}_{\mathrm{w}}-\mathrm{T}_{\mathrm{S}}\right) \quad:
$$


The equation for $\mathrm{T}_{\mathrm{CHF}}$ is

$T_{C H F}^{n+1}=T_{C H F}^{n}-\frac{\left(T_{C H F}^{n}-T_{s}-\frac{q_{C H F}^{\prime}}{h}\right)}{\left[1+\left(\frac{q_{C}^{\prime} \dot{H} F}{h^{2}} \frac{d h}{d T_{w}}\right)\right]}$,

where $\mathrm{T}_{\mathrm{CHF}}^{\mathrm{n}}$ is the CHF temperature for the $\mathrm{nth}$ iteration, $\mathrm{h}$ is the HTC evaluated by using the Chen correlation, and $d h / \mathrm{dT}_{\mathrm{w}}$ is the derivative of the HTC with respect to the wall temperature.

Convergence occurs if $\left|\mathrm{T}_{\mathrm{CHF}}^{n+1}-\mathrm{T}_{\mathrm{CHF}}^{\mathrm{n}}\right|<1.0$. A maximum of ten iterations is allowed; if convergence does not occur, a message is printed and a fatal error occurs.

The CHF temperature is restricted to the range, $\mathrm{T}_{s}+0.5 \leq \mathrm{T}_{\mathrm{CHF}} \leq \mathrm{T}_{\mathbf{s}}+100$. The $\mathrm{CHF}$ calculations are done in subroutines $\mathrm{CHF}$ and $\mathrm{CHFl}$.

d. Minimum Stable Film-Boiling Temperature, $T_{m i n}$. The minimum stable film-boiling point is the intersection point between the transitionand film-bolling heat-transfer regimes (Fig. 10). In addition, this point is one of the points used in the interpolation scheme for the calculation of the transition-boiling heat flux.

The homogeneous-nucleation minimum stable film-boiling, temperature correlation 42 is,

$T_{\text {min }}=T_{n h}+\left(T_{n h}-T_{\ell}\right) R^{0.5}$,

where

$R=\frac{(k p c)_{\ell}}{(k p c)_{W}}$ 
and $\mathrm{T}_{\mathrm{nh}}$ is the homogeneous-nucleation temperature. In Eq. (168) the subscript $\ell$ indicates liquid properties and the subscript $w$ indicates wall properties. The homogeneous-nucleation temperature is given by Fauske, and a curve fit to these results, from the COBRA-TF code,* is used in TRAC-PF1/MOD1.

$$
\begin{aligned}
\mathrm{T}_{\mathrm{nh}}= & 705.44-\left(4.722 \times 10^{-2}\right) \mathrm{DP} \\
& +\left(2.3907 \times 10^{-5}\right) \mathrm{DP}^{2} \\
& -\left(5.8193 \times 10^{-9}\right) \mathrm{DP}^{3}
\end{aligned}
$$

where $D P=3203.6-P$. The pressure $P$ is in psia units, and $T_{n h}$ is in degrees Fahrenheit. In TRAC-PF1/MOD1, $P$ is converted to a temporary variable in British units, and $T_{n h}$ is converted to Kelvin after the equation is evaluated. After $T_{m i n}$ is evaluated, the corresponding heat flux, $q_{m i n}$, in the transition-boiling HTC interpolation can be calculated,

$\mathrm{q}_{\text {min }}^{-i}=h_{\ell \text { min }}\left(T_{\text {min }}-T_{\ell}\right)+h_{g m i n}\left(T_{m i n}-T_{g}\right)+h_{f b b}\left(T_{m i n}-T_{s a t}\right)$,

where $h_{\ell \min }$ and $h_{g m i n}$ are the liquid and vapor HTCs, respectively, evaluated at the minimum stable film-boiling temperature. Thus,

$h_{\ell \text { min }}=h_{r}\left(\frac{T_{\min }-T_{s}}{T_{\min }-T_{\ell}}\right)+h_{d f}$

and

$h_{\text {gmin }}=\max \left(h_{n c}, h_{D R}\right)$.

\footnotetext{
* This information was provided by M. J. Thurgood and J. M. Kelley, Battelle Pacific Northwest Laboratories (December 1979).
} 
If $\alpha>\alpha_{c}$, linear interpolation again is used between the values of the HTCs at $\alpha_{c}$ and the single-phase vapor values.

e. Steady-State Calculations. The steady-state (ISTDY = 1) and transient (ISTDY $=0$ ) wall-to-fluid HTC code logics differ. The entire bolling curve is not avallable to rods during steady state; only that part of the heat transfer before CHF is calculated (Sec. III.B.2.a).

C. Reactor Kinetics

Subroutine RKIN evaluates power generation in the reactor core by one of two methods. In the first method the user specifies power to be a constant or defined by a power table supplied through input. The table is a function of a system signal-variable parameter or a control-block output parameter. Values between entries in the table are determined by linear interpolation. Power determination can be trip controlled by evaluating the power table when the controlling trip is $O N$ and by holding the power constant when the trip is OFF. In the second method, the user determines power from the solution of the point-reactor kinetics equations. These equations specify the time behavior of the core power level with neutronic reactivity ( $R$ ) [ the sum of progranmed reactivity $\left(R_{\text {prog }}\right)$ and feedback reactivity $\left.\left(R_{f d b k}\right)\right]$ as the driving function. The user inputs programmed reactivity (defining reactivity effects not accounted for by feedback reactivity, such as fuel reactivity and control-rod movement) with the same forms that define power in the first method. Subroutine REDBK evaluates feedback reactivity based on changes in the coreaveraged fuel temperature, coolant temperature, coolant vapor fraction, and boron concentration.

The point-reactor kinetics equations are a coupled set of $(I+1)$ firstorder differential equations defining the total thermal power $P$ and the delayed-neutron precursor concentrations $C_{i}$ as a function of time.

$$
\frac{\mathrm{dP}}{\mathrm{dt}}=\frac{\mathrm{R}-\beta}{\Lambda} \mathrm{P}+\sum_{1}^{\mathrm{I}} \lambda_{1} \mathrm{C}_{i}
$$


and

$\frac{d C_{i}}{d t}=-\lambda_{i} C_{i}+\frac{\beta_{i} P}{\Lambda}$, for $i=1,2, \ldots, I$,

where

$P=$ total thermal power $(W)$ that results from fissions occurring at time $t$,

$t=$ problem time $(s)$,

$R=$ neutronic reactivity $=(k-1) / k=R_{\text {prog }}+R_{f d b k}$,

$k=$ reactor multiplication constant $=k_{\text {prog }}+\Delta k_{f d b k}$,

$R_{\text {prog }}=$ programmed reactivity $=\left(k_{\text {prog }}-1\right) / k_{\text {prog }}$,

$\mathrm{R}_{\mathrm{fdbk}}=$ feedback reactivity $=\left(\Delta \mathrm{k}_{\mathrm{fdbk}} / \mathrm{k}_{\mathrm{prog}}\right) / \mathrm{k}$,

$\beta=$ total fraction of $\left\{\right.$ delayed neutrons $=\sum_{i}^{I} B_{i}$,

$\beta_{i}=$ fraction of delayed neutrons in group $i$,

$\Lambda$ = effective prompt-neutron lifetime (s),

$\lambda_{1}=$ decay constant for the delayed-neutron precursors in group $i\left(s^{-1}\right)$,

$C_{1}=$ concentration of the delayed-neutron precursors in groups $i(W)$, and

$I=$ number of delayed-neutron groups.

The TRAC-PF1/MODI program uses the Kaganove method ${ }^{43}$ to solve these equations. The prompt-fission power solution is applied to the decay-heat equations

$\frac{d H_{j}}{d t}=-\lambda{ }_{j}^{H_{j}}+E_{j} P$, for $j=1,2, \ldots, J$,

where

$H_{j}=$ concentration of decay-heat group $j(W \cdot s)$,

$\lambda_{j}^{\mathrm{H}}=$ decay constant for decay-heat group $\mathrm{j}\left(\mathrm{s}^{-1}\right)$,

$E_{j}=$ effective energy fraction of decay-heat group $j$, and

$J=$ number of decay-heat groups. 
Then, each $j$ th equation is solved for $\mathrm{H}_{j}$. After this is done, the total thermal power generated in the reactor fuel at time $t$ from prompt fission, precursor decay, and delayed fission is

$P_{\text {eff }}=\left(1-\sum_{j}^{J} E_{j}\right) \dot{P}+\sum_{j}^{J} \lambda_{j}^{H_{H}}$

The user inputs the number of delayed-neutron groups, $I$; the delayedneutron parameters, $\lambda_{i}$ and $\beta_{i}$; the number of decay-heat groups, $J$; the decayheat parameters, $\lambda_{j}^{\mathrm{H}}$ and $E_{j}$; and either the prompt-fission power history $P(-t)$ for $-t \leq 0$ or the initial delayed-neutron precursor concentrations $C_{i}(0)$ and decay-heat concentrations, $H_{j}(0)$. If $I \leq 0$ is input, TRAC internally sets I to 6 and defines $\lambda_{1}$ and $\beta_{i}$ with the values in Table II. If $J \leq 0$ is input, TRAC internally sets $J$ to $I 1$ and defines $\lambda_{j}^{H}$ and $E_{j}$ with the values in Table III. The power decay that these parameters evaluate closely approximates the standard American Nuclear Society (ANS) decay-heat curve. 44

If. I $\leq 0$ and/or $J \leq 0$ and no prompt-fission power history is input, we assume that an initial steady-state condition exists to initialize $C_{i}(0)^{\prime \prime}$ and/or $H_{j}(0)$ internally in TRAC. This requires use of the initial power $P(0)$ that is input. If $\mathrm{dC}_{i} / \mathrm{dt}$ and $\mathrm{dH}_{j} / \mathrm{dt}$ are set to zero at the initial time $(t=0)$ in Eqs. (170) and (171), then

$C_{i}(0)=\frac{\beta_{i}}{\lambda_{i}^{\Lambda}} P(0)$, for $i=1,2, \ldots, I$,

and

$H_{j}(0)=\frac{E_{j}}{\lambda_{j}^{H}} P(0)$, for $j=1,2, \ldots, J$. 
TABLE II

DELAYED-NEUTRON CONSTANTS

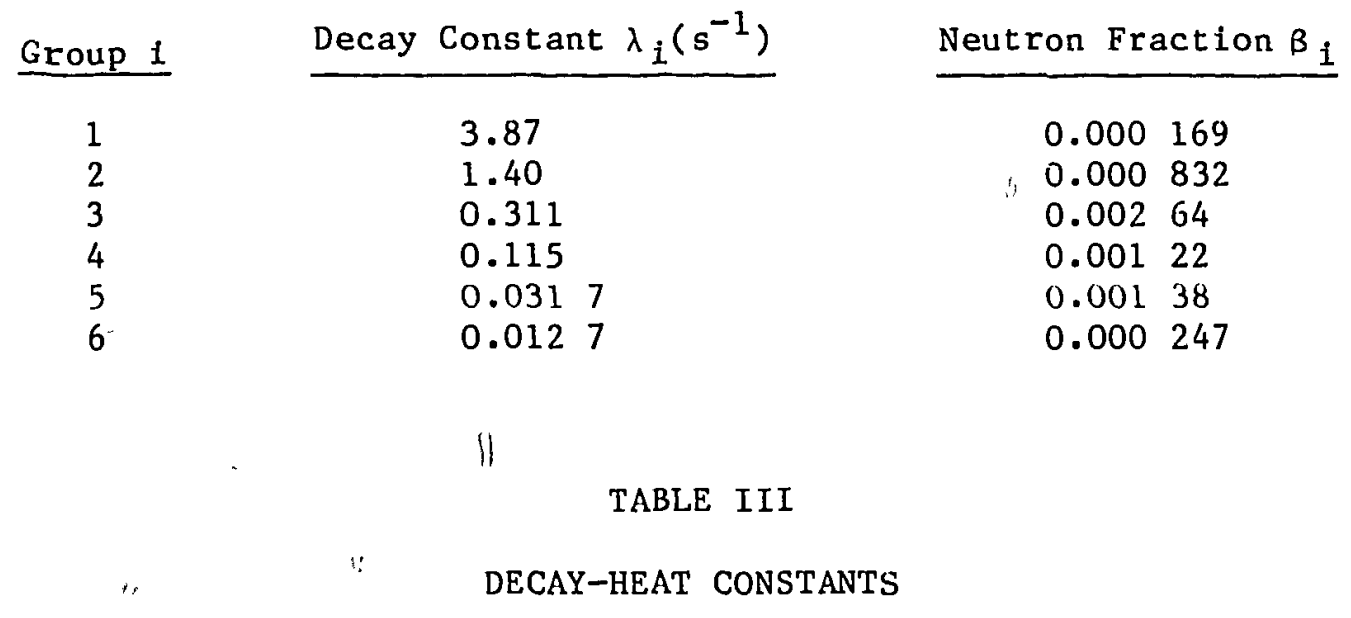

Group $j \quad$ Decay Constant $\lambda_{j}^{H}\left(s^{-1}\right) \quad$ Energy Fraction $E_{j}$

$\begin{array}{rlll}1 & 1.772 & 0.00299 \\ 2 & 0.5774 & 0.00825 \\ 3 & 0.06743 & 0.01550 \\ 4 & 0.006214 & 0.01935 \\ 5 & 4.739 \times 10^{-4} & 0.01165 \\ 6 & 4.810 \times 10^{-5} & 0.00645 \\ 7 & 5.344 \times 10^{-6} & 0.00231 \\ 8 & 5.726 \times 10^{-7} & 0.00164 \\ 9 & 1.036 \times 10^{-7} & 0.00085 \\ 10 & 2.959 \times 10^{-8} & 0.00043 \\ 11 & 7.585 \times 10^{-10} & 0.00057\end{array}$

TRAC evaiuates $C_{i}(0)$ and $H_{j}(0)$ from Eqs. (170) and (171) if the promptfission power history $\mathrm{P}(-t)$ is entered with tabular data $\left(t_{\ell}, \mathrm{P}_{\ell}\right)$ pairs for $\ell=1,2, \ldots, L$ where $-t_{\ell+1}<-t_{\ell} \leq 0$. The prompt-fission power is assumed to vary linearly between data pairs in the power-history table, that is,

$P(t)=a_{\ell}+b_{\ell} t$, over the time interval, $-t_{\ell+1} \leq t \leq-t_{\ell}$. 
Substituting Eq. (175) into Eq. (170) and integrating the resulting equation analytically from $t=-t_{L}\left[\right.$ where $C_{i}\left(-t_{L}\right)=0$ is assumed] to $t=0$ gives

$$
\begin{aligned}
c_{i}(0)= & \frac{\beta_{i}}{\lambda_{i} \Lambda} \sum_{\ell=1}^{L-1}\left\{\left(\frac{a_{\ell}-b_{\ell}}{\lambda_{i}}\right)\left(1-\exp \left[-\lambda_{i}\left(t_{\ell+1}-t_{\ell}\right)\right]\right)\right. \\
& \left.+b_{\ell}\left(t_{\ell+1} \exp \left[-\lambda_{i}\left(t_{\ell+1}-t_{\ell}\right)\right]-t_{\ell}\right)\right\} \exp \left[-\lambda_{i} t_{\ell}\right] .
\end{aligned}
$$

A similar expression for $H_{j}(0)$ is obtained by doing the same for Eq. (171). The expression for $H_{j}(0)$ is $E q .(176)$ with replacement of $C_{i}$ by $H_{j}, \lambda_{i}$ by $\lambda_{j}^{H}$, $\beta_{i}$ by $E_{j}$, and $\Lambda$ by 1 . TRAC-PFI/MODI evaluates these two expressions for $C_{i}(0)$ and $\mathrm{H}_{j}(0)$ when a prompt-fission power-history table is input.

Subroutine RFDBK evaluates reactivity feedback. The reactivity-feedback model is based on the assumption that only changes in the core-averaged fuel temperature $\left(T_{f}\right)$, coolant temperature $\left(T_{C}\right)$, coolant vapor fraction $(\alpha)$, and boron concentration $\left(B_{m}\right.$ or $B_{r}$ ) affect the neutron-multiplication reactivity of the reactor core. The code determines core-averaged values by applying fuelor coolant-mass times power-weighting factors to the temperatures and a powerweighting factor to the coolant vapor fraction and boron concentration. These factors approximate the product of the local adjoint flux, neutron flux, and volume. This weighting-factor product is taken from perturbation theory where it is used to weight spatially the change in reaction-rate cross sections to estimate their reactivity change. It is approximated in TRAC by the product of the coolant-channel volume and the fission-power density distribution raised to a user-specified power POWEXP. For POWEXP $=1.0$, the adjoint-flux distribution is being approximated by the fission reaction-rate cross-section distribution. For POWEXP $=2.0$, when the fission reaction-rate cross section is spatially constant, the adjoint flux is being approximated by the neutron flux (Galerkin approximation). The appropriate value to use for POWEXP will depend on the fuel-loading distribution in the reactor core. However, keep in mind that two levels of approximation are being made: first, that the coolant-channel volume times the fission-power density distribution raised to the POWEXP power is a good approximation for the perturbation-theory product of the adjoint flux, neutron flux, and volume and, second, that the 
theoretical basis for the use of that weighting factor for reaction-rate cross sections can be applied to the reactivity-feedback parameters we wish to average over the reactor core. The user has the option of replacing any of the three spatial shapes (fuel-rod radial, core horizontal plane, and core axial) in the fission-power density distribution with a different shape to weight the spatial averaging of reactivity-feedback parameters over the core. These spatial-shape replacements are specified through input.

The user defines a reactivity coefficient for each of the reactivityfeedback independent variable parameters, $x=T_{f}, T_{c}, \alpha$, and $B_{m}$ or $B_{r}$, by choosing one of the reactivity-coefficient forms in Table IV. Each reactivity coefficient is defined through input by a table of reactivity-coefficient values that are dependent on all four reactivity-feedback parameters. Each parameter has one or more values specified in defining the table. Defining one value for a parameter corresponds to the reactivity coefficient having no dependence on that reactivity-feedback parameter. Defining two or more values for a parameter gives the reactivity-coefficient dependence on it. Linear interpolation is used to evaluate the reactivity coefficient for values of each parameter between its table values. Multidimensional linear-surface interpolation is performed when the reactivity coefficient is dependent on two or more of the reactivity-feedback paraneters.

TABLE IV

REACTIVITY-COEFFICIENT FORMS

Form Number

0

1

2

3
Reactivity-Coefficient Form

$$
\begin{aligned}
& \frac{\partial k}{\partial x} \\
& \frac{1}{k} \frac{\partial k}{\partial x} \simeq \frac{\partial R}{\partial x}
\end{aligned}
$$

$x \frac{\partial k}{\partial x}$

$\frac{x}{k} \frac{\partial k}{\partial x} \simeq x \frac{\partial R}{\partial x}$ 
Boron in the derivative and dependence of a reactivity coefficient are defined in terms of the boron concentration in the coolant channel $B_{m}$ in units of $\mathrm{kg} / \mathrm{m}^{3}$ or the boron mass to liquid-coolant mass ratio $\mathrm{B}_{r}$ in units of boron mass/liquid-coolant mass parts per million (ppm). The boron reactivity coefficient is based on the change in the amount of boron without changing $\mathrm{T}_{\mathrm{c}}$ and $\alpha$ when the coefficient is defined as a derivative of $B_{r}$. The boron dependence of a reactivity coefficient is important when the reactivitycoefficient value is sensitive to a shift in the neutron-energy spectrum. Through the proportionality to the amount of neutron capture in boron, $B_{m}$ or $B_{r}$ dependence characterizes this shift.

All forms of boron in the reactor core are included in the evaluation of $B_{\mathfrak{m}}$ or $B_{r}$ for boron dependence: dissolved boric acid in the liquid coolant, boric acid plated on core structure, borosilicace glass in burnable-poison pins, and boron oxide in control-rod-cluster pins. 'The first two forms are based on boron convection into the reactor core from throughout the modeled system and on plateout of boric acid in the core as a result of coolant dryout. The burnable-poison pin and control-rod-cluster pin forms are to be defined by the user through input with what would be their equivalent boron concentrations in the coolant channel. The equivalent burnable-poison pin boron concentration is input as a first-order polynomial function of the coreaveraged coolant temperature $\mathrm{T}_{C}$,

$\mathrm{BPP}=\mathrm{BPPO}+\mathrm{BPPl} \cdot \mathrm{T}_{\mathrm{C}}\left(\mathrm{kg} / \mathrm{m}^{3}\right.$, boron mass in the coolant-channel volume $) \cdot$

Whereas the physical amount of boron in the burnable-poison pins is constant during the transient, its equivalent concentration for neutron capture Increases with coolant temperature because of reduced spatial self-shielding of boron to neutrons in the burnable-poison pins. The amount of control-rodcluster pin boron in the reactor core depends on the amount of control-rod 
insertion into the core. Its equivalent concentration is input as a firstorder polynomial function of programmed reactivity $R_{p r o g}$,

$B C R=B C R O+B C R 1 \cdot R_{\text {prog }}\left(\mathrm{kg} / \mathrm{m}^{3}\right.$, boron mass in the coolant-channel volume $)$.

Programmed reactivity is assumed to be proportional to the amount of effective boron-mass change caused by control-rod movement in the cora. Initially, $B C R=B C R O$ when the reactor core was at steady-state conditions.

Only the first two forms of boron in the reactor core are included in the evaluation of $B_{m}$ or $B_{r}$ for the boron reactivity-coefficient derivative parameter because the amount of boron in burnable-poison pins is constant during the transient that is being evaluated. Control-rod movement changes the amount of boron, but the reactivity change associated with this change is accounted for in the user-defined programmed reactivity $R_{\text {prog }}$.

After all four reactivity coefficients are evaluated by linear interpolation in their four dinensionally dependent tables, feedback reactivity is evaluated in terms of its change in the neutron multiplication constant over the last time step $\Delta t^{n-1}$

$$
\begin{aligned}
k\left(t^{n}-\Delta t^{n-1}\right) k\left(t^{n}\right) R_{f d b k}\left(t^{n}\right)= & \Delta k_{f d b k}\left(t^{n}\right)=\sum_{i=1}^{4} \frac{1}{2}\left[\frac{\partial k\left(t^{n}\right)}{\partial x_{i}}+\frac{\partial k\left(t^{n}-\Delta t^{n-1}\right)}{\partial x_{i}}\right] \\
& x\left[x_{i}\left(t^{n}\right)-x_{i}\left(t^{n}-\Delta t^{n-1}\right)\right]
\end{aligned}
$$

where $x_{1}=T_{f}, x_{2}=T_{c}, x_{3}=\alpha$, and $x_{4}=B_{m}$ or $B_{r}$. The value of $\partial k\left(t^{n}\right) / \partial x_{i}$ in Eq. (179) for reactivity-coefficient forms 1,2 , and 3 (Table IV) is obtained by multiplying these reactivity coefficients by $k\left(t^{n}-\Delta t^{n-1}\right), 1 / x_{i}\left(t^{n}\right)$, and $k\left(t^{n}-t^{n-1}\right) / x_{i}\left(t^{n}\right)$, respectively. Using $k\left(t^{n}-t^{n-1}\right)$ rather than $k\left(t^{n}\right)$ (which is not known yet) to convert reactivity-coefficient forms 1 and 3 to $k / x_{1}$ is an approximation. The values of $k / x_{i}$ and $x_{i}$ at time $t^{n}$ (the start of the present time step) are evaluated as described above; their values at 
time $t^{n}-\Delta t^{n-1}$ are those saved from their evaluation at the start of the previous time step.

The change in $x_{i}$ over the last time step (with the other $x_{i}$ parameters held constant) is defined in the last factor of $\mathrm{Eq} \cdot(179)$. $\mathrm{B}_{\mathrm{m}}$ or $\mathrm{B}_{r}$ includes only dissolved boric acid in the liquid coolant and boric acid plated on the core structure. There is no change in the $B_{r}$ equivalent boron concentration in burnable-poison pins when $T_{f}, T_{c}$, and $\alpha$ do not vary.

To determine reactivity feedback during the current time step $\Delta t^{n}=L^{n+l}-t^{n}$, TRAC would need to know the end-of-time-step values of $T_{f}$, $\mathrm{T}_{c}, \alpha, \mathrm{B}_{\mathrm{m}}$, and $\mathrm{B}_{\mathrm{r}}$. To evaluate that reactor-core state requires knowing the current core state and the total energy-generation rate, $P_{\text {eff }}(t)$, over the time step $t^{n} \leq t \leq t^{n+1}$. The neutronic reactivity $R(t)$ defined by the reactivity-feedback contribution, $R_{f d b k}(t)$, must be known to determine the value of $P_{\text {eff }}(t)$ froin the solution of the reactor-kinetics equations. Thus, the evaluation of reactivity feedback during the current time step requires first knowing what it is.

This difficulty of needing to know the reactivity feedback to evaluate it is handled in TRAC by assuming its $\Delta \mathrm{k}$ feedback-reactivity rate is zero. The $\Delta k$ programined-reactivity rate, is assumed to be the same as in the previous time step, $\Delta t^{n-1}$,

$\left(\Delta k_{\text {prog }}^{e s t}\right)^{n}=\Delta t^{n}\left\{\frac{\left[k_{p r o g}\left(t^{n}\right)-k_{p r o g}\left(t^{n}-\Delta t^{n-1}\right)\right]}{\Delta t^{n-1}}\right\}$

We estimate $\left(\Delta k_{\text {prog }}\right)^{n}$ because the independent variable needed to evaluate its tabular definition generally is a function of the reactor-core state. A similar approximation for $\left(\Delta k_{f d b k}^{e s t}\right)^{n}$ was tested in TRAC, but near steady state it caused the value sign of $\left(\Delta \mathrm{k}_{\mathrm{fdbk}}^{\text {est }}\right)^{n}$ to change each time step. It is better to assume that $\left(\Delta \mathrm{k}_{\mathrm{fdbk}}^{\text {est }}\right)^{\mathrm{n}}=0$ than to estimate its value based on its value in the 
previous time step. Thus, the reactor multiplication constant and neutronic reactivity are estimated for the end of the present time-step reactor state by

$\left(k^{e s t}\right)^{n}=k\left(t^{n}\right)+\left(\Delta k_{\text {prog }}^{e s t}\right)^{n}$

and

$\left(R^{e s t}\right)^{n}=\frac{\left[\left(k^{e s t}\right)^{n}-1\right]}{\left(k^{e s t}\right)^{n}}$.

After each time-step solution, TRAC compares the actual programmed reactivity with its estimated value. Any discrepancy is corrected by applying

$$
\left(\Delta k^{\operatorname{cor}}\right)^{n+1}=\left[\left(\Delta k_{p r o g}\right)^{n}-\left(\Delta k_{p r o g}^{e s t}\right)^{n}\right] \cdot \min \left(\frac{\Delta t^{n}}{\Delta t^{n+1}}, 2\right)
$$

during the next time step, $\Delta t^{n+1}$. To prevent a time-step reactivity correction from becoming very large by applying it over a much smaller time step $\Delta t^{n+1} \ll \Delta t^{n}, "\left(\Delta k^{c o r}\right)^{n+1}$ in Eq. (183) is constrained to be no more than twice the $\Delta t^{\mathrm{n}}$ time-step value. Including this correction, and the actual reactivity-feedback change in $\Delta k$ during the previous time step in the reactor multiplication constant end-of-time-step estimate gives Eq. (181) a modified form,

$$
\left(k^{e s t}\right)^{n}=k\left(t^{n}\right)+\left(\Delta k_{p r o g}^{e s t}\right)^{n}+\left(\Delta k^{c o r}\right)^{n}+\left(\Delta k_{f d b k}\right)^{n-1} .
$$

In this procedure, we assume $\left(\Delta k^{c o r}\right)^{n}$ and $\left(\Delta k_{f d b k}\right)^{n-1}$ are small enough not to require an iterative evaluation of the present time-step solution but that $\left(\Delta \mathrm{k}^{\mathrm{cor}}\right)^{\mathrm{n}}$ is large enough to be included in the next time-step solution rather 
than be neglected. A similar estimate and correction procedure is applied to the end-of-time-step power in the first method for specifying the total power." The point-reactor kinetics equations [Eqs. (169)-(171)] are solved by the Kaganove method. 43 Ițs derivation in Ref. 43 approxinates the time dependence of $P$ and $k_{e x}=k-1=R /(1-R)$ over each integration time step by second-order polynomials and assumes $\Lambda(t)=\ell /\left[1+k_{e x}(t)\right]$ where $\ell$ is a constant. For TRAC, it is more appropriate to approximate the time dependence of $\mathrm{P}$ by a second-order polynomial, $\mathrm{R}$ by a first-order polynomial, and $\Lambda$ by $a$ constant because TRAC cxtrapolates its sstimate of $R(t)$ over the fluiddynamics time step to be evaluated and because the weak time dependence of $\Lambda$ generally is unknown. These modified assumptions, when applied to the pointreactor kinetics equations, simplify the form that these equations take when analytically integrated over the integration time step $\Delta t$. The derivation of those analytically integrated equations follows. TRAC then evaluates them for $\Delta t^{\mathrm{n}} / \Delta t$ integration time steps during the $\Delta t^{\mathrm{n}}$ fluid-dynamics time step.

We assume that

$P(t)=P(0)+P_{1} t+P_{2} t^{2}$,

$R(t)=R(0)+R_{1} t$,

and $\Lambda, \lambda_{i}, \beta_{i}, \lambda_{j}^{H}$, and $E_{j}$ are constant for $0 \leq t \leq \Delta t$, where $P(0), R(0)$, and $R_{1}=\left[\left(R^{e s t}\right)^{n}-R(0)\right] / \Delta t^{n}$ are known values. Solving $E q \cdot(170)$ for $C_{i}(t)$ in terms of a functional of the prompt-fission power $P(t)$ gives

$C_{1}(t)=C_{i}(0) \exp \left[-\lambda_{i} t\right]+\frac{\beta_{i}}{\Lambda} \int_{0}^{t} \exp \left[-\lambda_{i}(t-\tau)\right] P(\tau) d \tau$ 
Substituting Eq. (187) into Eq. (169) and integrating the resulting equation term-by-term gives

$$
\begin{aligned}
P(t)=P(0)+ & \int_{0}^{t} \frac{R(\tau) P(\tau)}{\Lambda} d \tau-\sum_{i}^{I} \frac{6}{\Lambda} \int_{0}^{t} \exp \left[-\lambda_{i}(t-\tau)\right] P(\tau) d \tau \\
& +\sum_{i}^{I} C_{i}(0)\left(1-\exp \left[-\lambda_{i} t\right]\right) .
\end{aligned}
$$

Substituting Eq. (185) for $P(\tau)$ and Eq. (186) for $R(\tau)$ in Eq. (188), evaluating the integrals, and rearrainging the resulting equation in terms of the $\mathrm{P}_{1}$ and $\mathrm{P}_{2}$ unknowns gives

$a_{1}(t) P_{1}+a_{2}(t) P_{2}=q(t)$,

where

$$
\begin{aligned}
& a_{1}(t)=t \Lambda-t^{2}\left[\frac{1}{2} R(0)+\frac{1}{3} R_{1} t\right]+\sum_{i}^{I} \beta_{i} t^{2} I_{i_{1}, r}(t), \\
& a_{2}(t)=t^{2} \Lambda-t^{3}\left[\frac{1}{3} R(0)+\frac{1}{4} R_{1} t\right]+\sum_{i}^{I} \beta_{i} t^{3} I_{i_{2}}(t), \\
& q(t)=t\left[R(0)+\frac{1}{2} R_{1} t\right] P(0)+\sum_{i}^{I}\left[\Lambda \lambda_{i} C_{i}(0)-\beta_{i} P(0)\right] t I_{i_{0}}(t),
\end{aligned}
$$

and

$$
t^{m+1} I_{i m}(t)=\int_{0}^{t} \exp \left[-\lambda_{i}(t-\tau)\right] \tau^{m} d \tau \quad \text {, for } m=0,1,2
$$

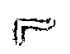


The $P_{1}$ and $P_{2}$ polynomial coefficients are evaluated by requiring Eq. (189) to be satisfied for $t=\Delta t$ (at the end of the integration time step) and $t=\Delta t / 2$ (at the midpoint of the integration time step). Solving the two equations,

$a_{1}(\Delta t) P_{1}+a_{2}(\Delta t) P_{2}=q(\Delta t)$

and

$a_{1}\left(\frac{\Delta t}{2}\right) P_{1}+a_{2}\left(\frac{\Delta t}{2}\right) P_{2}=q\left(\frac{\Delta t}{2}\right)$

for $P_{1}$ and $P_{2}$ gives

$P_{1}=\frac{\left[q(\Delta t) a_{2}\left(\frac{\Delta t}{2}\right)-q\left(\frac{\Delta t}{2}\right) a_{2}(\Delta t)\right]}{D}$

and

$P_{2}=\frac{\left[q\left(\frac{\Delta t}{2}\right) a_{1}(\Delta t)-q(\Delta t) a_{1}\left(\frac{\Delta t}{2}\right)\right]}{D}$

where $D=a_{1}(\Delta t) a_{2}(\Delta t / 2)=a_{1}(\Delta t / 2) a_{2}(\Delta t)$. The prompt-fission power at the end of the integration time step $t=\Delta t$ from Eq. (185) is

$P(\Delta t)=P(0)+P_{1} \Delta t+P_{2} \Delta t^{2}$ 
If we know $P(t)$ for $0 \leq t \leq \Delta t$, we evaluate $C_{i}(\Delta t)$ by substituting Eq. (185) into Eq. (187) and using $\Delta t I_{i 0}(\Delta t)=\left(1-\exp \left[-\lambda_{i} \Delta t\right]\right) / \lambda_{i}$ from analytically integrating its definition in Eq. (189).

$$
\begin{aligned}
C_{i}(\Delta t)= & C_{i}(0)\left[1-\lambda_{i} \Delta t I_{i 0}(\Delta t)\right]+\frac{B_{i}}{\Lambda}\left[P(0) \Delta t I_{i 0}(\Delta t)\right. \\
& \left.+P_{1} \Delta t^{2} I_{i 1}(\Delta t)+P_{2} \Delta t^{3} I_{i 2}(\Delta t)\right] \quad \text {, for } i=1,2, \ldots, I .
\end{aligned}
$$

The decay-heat equations are evaluated in the same way to give

$$
\begin{aligned}
H_{j}(\Delta t)= & H_{j}(0)\left[1-\lambda H_{j}^{H} \Delta t I_{j 0}(\Delta t)\right]+E_{j}\left[P(0) \Delta t I_{j 0}(\Delta t)\right. \\
& \left.+P_{1} \Delta t^{2} I_{j 1}(\Delta t)+P_{2} \Delta t^{3} I_{j 2}(\Delta t)\right] \text {, for } j=1,2, \ldots, J,
\end{aligned}
$$

where $\Delta t^{m+1} I_{j m}(\Delta t)=\int_{0}^{\Delta t} \exp \left[-\lambda{ }_{j}^{H}(\Delta t-\tau)\right] \tau^{m} d \tau$.

Evaluating $\Delta t^{m+1} I_{i m}(\Delta t)$ is done with the following recursion relation,

$$
\Delta t^{m+1} I_{i m}(\Delta t)=\frac{\left[\Delta t^{m}-m \Delta t^{m} I_{i m-1}(\Delta t)\right]}{\lambda_{i}} \text {, for } m=1,2,
$$

once $\Delta t I_{i 0}(\Delta t)=\left(1-\exp \left[-\lambda_{i} \Delta t\right]\right) / \lambda_{i}$ is first evaluated. For $\lambda_{i} \Delta<<1$, use of Eq. (197) results in the loss of several least-significant digits of accuracy with each application of the recursion formula. Thus, for $\lambda_{i} \Delta t<1$, TRAC first evaluates $\Delta t^{3} I_{i 2}(\Delta t)$ using the Maclaurin expansion,

$$
\Delta t^{m+1} I_{i m}(\Delta t)=\Delta t^{m+1} m !\left[\frac{1}{(m+1) !}-\frac{\lambda_{i} \Delta t}{(m+2) !}+\frac{\left(\lambda_{i} \Delta t\right)^{2}}{(m+3) !}-\cdots\right],
$$


for $m=2$ followed by evaluating the reciprocal of the Eq. (197)

$\Delta t^{m_{I-1}}(\Delta t)=\frac{\left[\Delta t^{m}-\lambda_{i} \Delta t^{m+1} I_{i m}(\Delta t)\right]}{m}$, for $m=2,1$.

The accuracy of the second-order polynomial approximation for $P(t)$ can be Increased by decreasing the integration time-step size $\Delta t$. We would like $\Delta t$ to be as large as possible, however, while we maintain a desired level of accuracy in approximating $P(t)$. To achieve this, the following procedure for automatically adjusting the time step in TRAC is used. In the same manner that Eqs. (190) and (191) were defined, Eq. (189) must be satisfied at $t=\Delta t / 4$,

$a_{1}\left(\frac{\Delta t}{4}\right) P_{1}+a_{2}\left(\frac{\Delta t}{4}\right) P_{2}=q\left(\frac{\Delta t}{4}\right)$

The $t=\Delta t / 2$ second equation in Eqs. (190) and (191) and the above equation for $P_{1}=P_{1}^{*}$ and $P_{2}=P_{2}^{*}$ from Eq. (185) are solved over the time range $0 \leq t \leq \Delta t / 2$ giving

$P_{1}^{*}=\frac{\left[q\left(\frac{\Delta t}{2}\right) a_{2}\left(\frac{\Delta t}{4}\right)-q\left(\frac{\Delta t}{4}\right) a_{2}\left(\frac{\Delta t}{2}\right)\right]}{D^{*}}$

and

$P_{2}^{*}=\frac{\left[q\left(\frac{\Delta t}{4}\right) a_{1}\left(\frac{\Delta t}{2}\right)-q\left(\frac{\Delta t}{2}\right) a_{1}\left(\frac{\Delta t}{4}\right)\right]}{D^{*}}$, 
where $D^{*}=a_{1}(\Delta t / 2) a_{2}(\Delta t / 4)-a_{1}(\Delta t / 4) a_{2}(\Delta t / 2)$. This solution over half the integration time step provides a more accurate value for $P(t)$ at $t=\Delta t / 2$,

$\mathrm{P}^{*}\left(\frac{\Delta t}{2}\right)=\mathrm{P}(0)+\mathrm{P}_{1}^{*}\left(\frac{\Delta t}{2}\right)+\mathrm{P}_{2}^{*}\left(\frac{\Delta t}{2}\right)^{2}$

than the solution over the full integration time step at $t=\Delta t / 2$,

$P\left(\frac{\Delta t}{2}\right)=P(0)+P_{1}\left(\frac{\Delta t}{2}\right)+P_{2}\left(\frac{\Delta t}{2}\right)^{2}$

The difference between these two values is used as a measure of the error in the solution over the full integration time step $\Delta t$,

$\varepsilon(\Delta t)=\left|\frac{P\left(\frac{\Delta t}{2}\right)-P^{*}\left(\frac{\Delta t}{2}\right)}{P^{*}\left(\frac{\Delta t}{2}\right)}\right| \cdot$

By defining two error bounds, $\varepsilon_{1}$ and $\varepsilon_{2}, \Delta t$ is doubled if $\varepsilon(\Delta t)$ is too small or halved if $\varepsilon(\Delta t)$ is too large, that is,

1. if $\varepsilon_{1}<\varepsilon(\Delta t)<\varepsilon_{2}$, maintain the present value of $\Delta t$;

2. if $\varepsilon_{1}<\varepsilon_{2} \leq \varepsilon(\Delta t)$, halve the value of $\Delta t$ because the error in $P(\Delta t)$ is too large; and

3. if $\varepsilon(\Delta t) \leq \varepsilon_{1}<\varepsilon_{2}$, double the value of $\Delta t$ because the error in $P(\Delta t)$ is too small.

When $\Delta t$ is halved, the integration time step is reevaluated. When the third criterion is satisfied, the number of remaining integration time steps to be evaluated over the fluid-dynamics time step must be an even number for the integration time step to be doubled (and the number of remaining integration time steps to be halved). TRAC is programmed to use $\varepsilon_{1}=10^{-6}$ and $\varepsilon_{2}=10^{-4}$. A numerical study showed these values to yield a maximum fractional error less than $10^{-4}$ and an average fractional error less than $10^{-5}$ in the prompt-fission 
power solution. The point-reactor kinetics solution required $<1 \%$ of $\mathrm{TRAC}$ execution time.

D. Overall Solution Strategy

Overall solution strategies for both transient and steady-state calculations are described in this section. Each tine step in the transient calrulation consists of several sweeps through all the components in the syslen. The purpose of lhese sweeps is lo converge lo the solulion of the nonlinear finite-difference equations.

1. Transient Solutions.

a. Outer Iteration Strategy. The solution of the thermal-hydraulic flow equations for all components is controlled by subroutines TRANS, PREP, OUTER, and POST. Subroutine TRANS controls the overall strategy whereas the others call each component in turn.

At least six passes are made through each one-dimensional component. Subroutine PREP makes two passes through all components. During the first pass, HTCs are evaluated by calls to subroutine HTCOR and the matrices for the stabilizer motion equations are obtained and reduced by subroutine FEMOM. The second pass in overlay PREP is for a backward substitution on the motion equation done in routine BKSMOM. The next two or more passes call the basic hydrodynamic routines until a solution is found within the convergence criterion or the maximum number of iterations is exceeded. This stage of the calculation is done by a call to subroutine OUTER, which performs both a forward elimination and a backward-substitution pass. The recommended convergence criterion (EPSO) is $10^{-4}$, and the maximum outer iteration count (OUTMAX) generally should range between 6 and 10 . The order in which subroutine OUTER calls the given components is determined by the IORDER array that is set after input by subroutine SRTLP.

If the OUTER iteration process converges, a final pair of passes is made by subroutine POST. The first of these sets up and reduces the stabilizer equations for mass and energy using subroutine STBME. The second calls BKSSTB to compile the solution of these equations, updates the wa11, slab, or rod heat conduction, and generates the information required to begin the next time step. If the OUTER iteration fails to converge, the time-step size is halved (subject to the constraint that $\Delta t$ must be greater than or equal to the minimum time-step size indicated in the input); then, another attempt to 
converge the OUTER iteration cycle begins. After six unsuccessful attempts, the code produces a dump and edit and shuts down.

Programming detalls of the iteration procedure for transient solutions are given in Sec.VI.D. A flow schematic for the TRANS routine is given in that section.

b. Details of the Solution Method. Solution of the fluid flow difference equations is broken into phases. First the stabilizing motion equations are solved, then the basic equation set is solved, and finally the stabilizing mass and energy equations are solved.

Solving the stabilizing motion equations is a relatively simple process because they are linear equations in $\tilde{\mathrm{V}}_{g}$ and $\tilde{\mathrm{V}}_{\ell}$, with no coupling between the $\tilde{v}_{g}$ and $\tilde{v}_{\ell}$ terms. First, the motion equations interior to all components are solved to obtain the interior velocities as linear functions of the junction velocities (done in subroutine FEMOM) in the form,

$\tilde{v}_{j}=a_{j}+b_{j} \tilde{V}_{L}+c_{j} \tilde{v}_{R}+d_{j} \tilde{v}_{T}$

where the subscripts $L, R$, and $T$ indicate the cell faces at the left, right, and tee (where applicable) component junctions. Next, the motion equations at component junctions are applied, and Eq. (206) is substituted into them where necessary to obtain a closed set of equations in the velocities at component junctions. This linear system is solved directly, using lower-upper (LU) decomposition. Finally, a backward substitution through all components is done using the known junction velocities and Eq. (206) to obtain values for the interior velocities (subroutine BKSMOM).

To solve the basic equation set (Secs. III.A.3 and III.A.4), a special junction variable and equation are defined,

$\Delta \mathrm{P}=\delta \mathrm{p}_{+}-\delta \mathrm{p}_{-}$,

where $\delta p_{+}$is the linearized variation in pressure on one side of the junction and $\delta p_{-}$the variation on the other. When the basic equation set is linearized within each one-dimensional component, Eq. (207) is substituted into the 
pressure gradient term of the motion equations at the component junctions. This linearized set of equations is solved in subroutine TEIDS to obtain the variations of independent variables $\left(\delta \mathrm{p}, \delta \mathrm{p}_{\mathrm{a}}, \delta \mathrm{T}_{\mathrm{e}}, \delta \mathrm{T}_{\mathrm{g}}\right.$, and $\left.\delta \alpha\right)$ as linear functions of the $\Delta \mathrm{P}$ junction terms. For example,

$\delta p_{j}=a_{j}^{\prime}+b_{j}^{\prime} \Delta P_{L}+c_{j}^{\prime} \Delta P_{R}+d_{j}^{\prime} \Delta P_{T}$

After this has been done, Eq. (208) is substituted whenever applicable into the definition of Eq. (207) at all junctions, and the solution proceeds with the stabilizing velocities.

When one or more three-dimensional components are present, the situation is slightly more complicated. For the network in Fig. 12, a linear set of

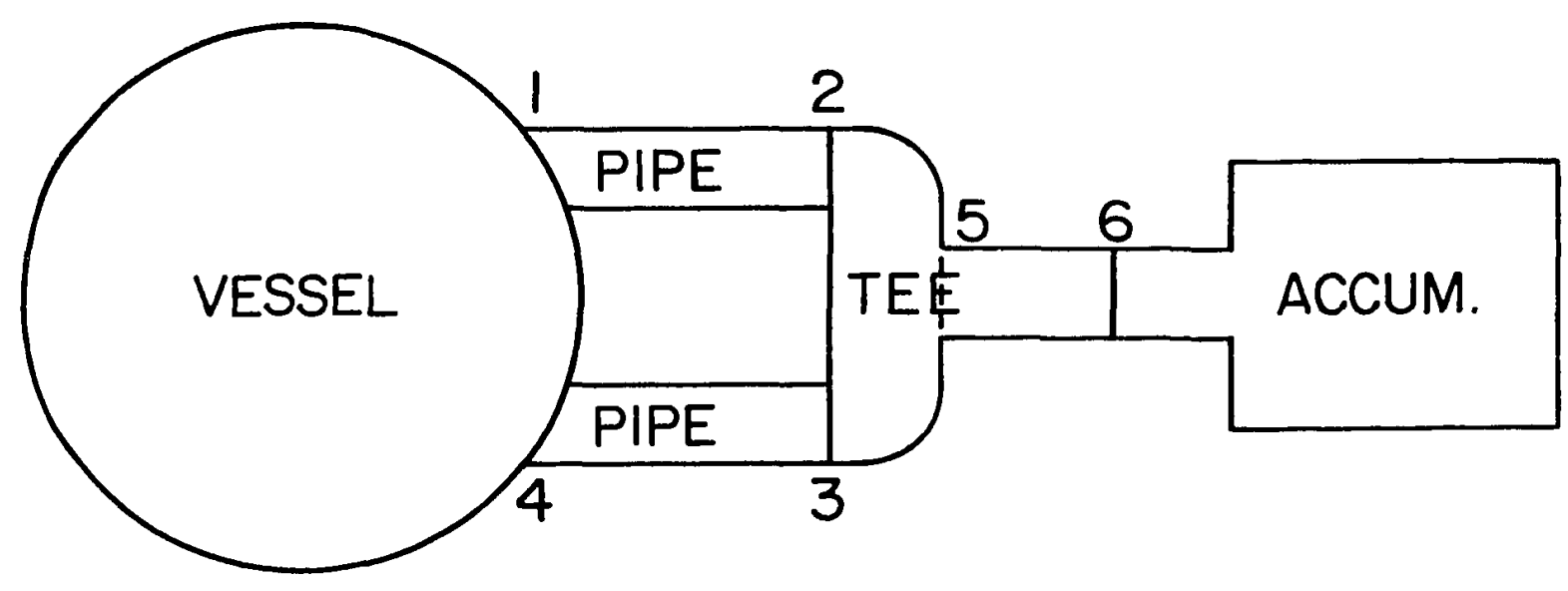

Fig. 12 .

Component network with one three-dimensional vessel. 
equations in $\Delta \mathrm{P}_{i}$ results after all possible substitutions are made. The equations have the form

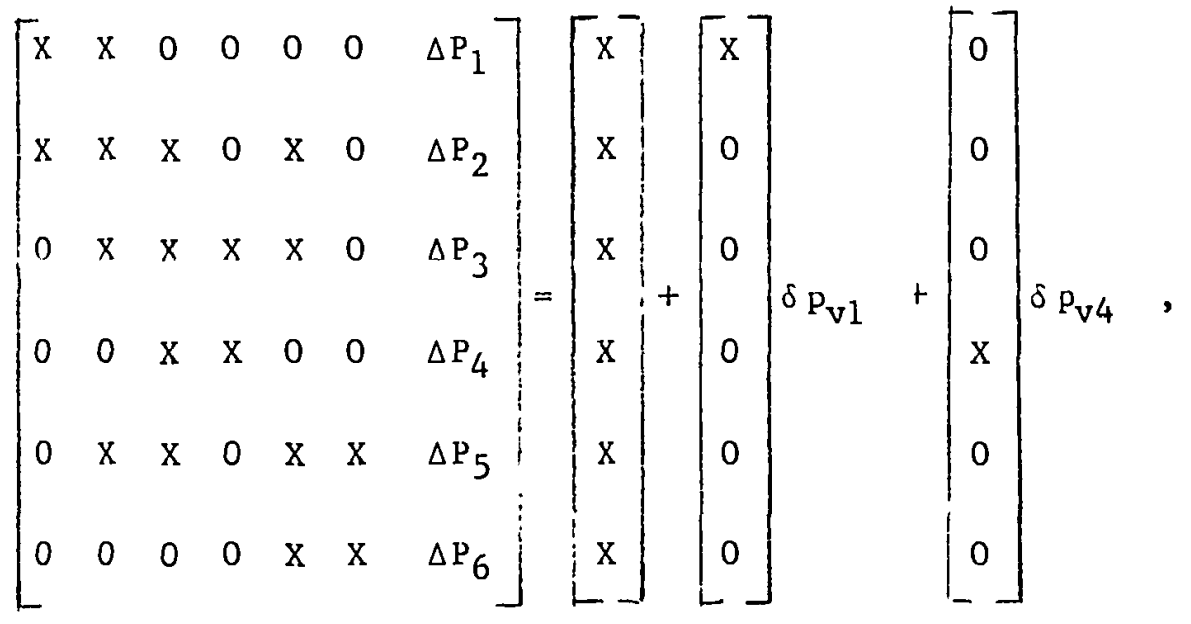

where $X$ indicates nonzero matrix and vector elements and $P_{v 1}$ and $P_{v 4}$ are the linear pressure variations in the vessel cells adjacent to junctions 1 and 4 , respectively. This system is solved directly to obtain,

$\Delta \mathrm{P}_{i}=\mathrm{A}_{i}+\mathrm{B}_{\mathrm{i}} \delta \mathrm{P}_{\mathrm{v} 1}+\mathrm{C}_{i} \delta \mathrm{p}_{\mathrm{v} 4}$

The combination of these equations with the remaining linearized equations in the three-dimensional vessel region provides a closed linear-equation set that is solved in one of two ways. If the input variable IITMAX is set to zero, then the system is solved directly using calls to subroutines STDIR, SOLVE, and BACIT. If this variable is greater than zero, an iterative solution procedure is used. This iteration is a combination of a Gauss-Seidel and a coarse-mesh rebalance, as described in Sec. III.A.2. The reconmended convergence criterion for this iteration (EPSI) is $10^{-5}$, and the maximum allowed number of iterations (IITMAX) is 30-50. Backward substitution through the one-dimensional components again completes the solution of the full linear system.

A single, complete pass through this solution procedure provides the solution for the linearized finite-difference equations. Subsequent passes 
through the procedure for the same time step produce a Newton iteration on the nonlinear difference equations, with quadratic convergence.

Solution of the stabilizing mass and energy equations is quite similar to that for the stabilizing motion equations. Because the unknown quantities are all cell centered, it is necessary to define the junction variables as those in a cell adjacent to the junction that occurs first in the calculational sequence. Special logic is provided to avoid conflicts when this criterion is applied to one cell components.

2. Steady-State Solutions. The TRAC steady-state capability provides time-independent solutions that may be of interest in their own right, or as initial conditions for transient calculations. The generalized steady-state finds the time-independent conditions of an arbitrary system. Desired steady-state parameters such as hot- and cold-leg temperatures may be obtained by using the control system (see Sec. III.E) to adjust pump speeds, steam-generator feed flows, and steam-flow control valves. Appendix $C$ provides a specific example of this procedure.

Steady-state calculations use the transient fluid-dynamics and heattransfer routines to search for time-independent conditions. The search is terminated when the normalized rates of change for the fluid and thermal variables are reduced below a user-specified criterion throughout the system.

Although the same subroutines are used in the transient and the steadystate calculations, there are important ways in which their behavior differs between the two calculations. The most crucial differences are

1. The time-step size used by the heat-transfer and fluid-flow calculations are not required to be equal during a steadystate calculation. The ratio of these time-step sizes is fixed through user-specified input. This permits compensation for the difference between the natural time scales of the two processes. (Caution should be taken not to exceed the diffusion limit in axial rod conduction by using too large a time-step ratio.) 
2. Pressurizers are modeled as pressure boundary conditions during steady-state calculations. Therefore, the energy and mass inventory, as well as the pressure, of each pressurizer remain constant regardless of the flow rate between it and the remainder of the system.

3. Trips with positive identification numbers are not evaluated during steady-state calculations. Thus, even though conditions may exist that would cause the set status of a trip to change, the set status of the trip remains constant at its input value during the steady-state calculation.

4. The reactor power normally is set to zero for a period of time at the beginning of the steady-state calculation. It is increased to its nominal value once the fluid velocity has reached a certain value or its rate of change has fallen below a certain value. Power may be forced on by using the NAMELIST variables IPOWR and TPOWR. POWER may also be forced to stay off by using the variable IPOWR.

5. During the steady-state calculation the pump momentum source is averaged to prevent oscillations.

6. The reactor-kinetics calculation or input power option is not implemented during the steady-state calculation.

7. Control-procedure component actions are evaluated during the steady-state calculation only if they are controlled by the trips and their trip is oN.

numerical first derivatives of the void fraction, pressure, liquid and vapor temperatures, and velocities. The rate of change of variable $x$ for time step $(n+1)$ at cell $i$ is given by

$\delta x_{i}^{n+1}=\frac{x_{i}^{n+1}-x_{1}^{n}}{\sqrt[x_{i}^{n+1}]{\left(t^{n+1} t^{n}\right)}}$. 
Thus, $\delta x_{i}^{n+1}$ is the fractional rate of change for the variable $x_{i}^{n+1}$ in 1 s. This rate of change is checked every 100 time steps during the steadystate calculation. When the maximum absolute value of $\delta x_{i}^{n}$ for all cells in the system is less than a user-specified convergence criterion for all variables, the steady-state calculation is ended. An edit, including the maximum rates of change and the component and cell number where it occurs for each variable, is printed every 100 time steps and at every long edit, dump, or time domain change. Logic is included to limit change rate checking to only those cells where the variable being checked is sufficiently important. For example, liquid temperature change rates are not calculated in cells where the void fraction is above 0.9999 , because the liquid temperature in this cell is not a meaningful value. The variables considered in evaluating the approach to steady state are the two phasic velocities and temperatures, the void fraction, the total pressure, and the partial pressure of a noncondensable gas.

In addition to the normal steady state, a special static-check steady state is available. When this option is selected, all pumps and heat sources are deactivated. If the gravity vectors have been entered correctly, all motion should stop eventually in the system that is being tested. To achieve the best results from this option, we recommend that the user make the initial temperatures as uniform as possible in each group of cells that are coupled hydrodynamically.

More sophisticated steady-state tests can be constructed using the trips and controls to test long-term changes in selected variables or their averages. When these input criteria are met, a trip may be activated that forces a dump and termination of the calculation.

E. Control Procedure

Simulating PWR plant operation involves defining its mode of operation. This requires specifying a control procedure to adjust hardware according to the state of the system and its operating plan. In that procedure one needs to model manual control by operators, automatic control by reguláting hardware, and abnormal hardware behavior. This involves specifying logic for initiating adjustable hardware action when certain conditions occur. For example, when the coolant pressure rises above or falls below a specified value, a valve is to be opened or closed, respectively. Abnormal hardware 
behavior might be opening a valve to model a pipe break occurrence. The control procedure can consist of many such instructions that together direct and limit the mode of operation.

To be able to define a control procedure, one needs to understand how it fits into the TRAC model for the overall PWR plant system. The most basic part of that model is the component description of the physical hardware (pipes, pumps, reactor core, etc.) and the mass, momentum, and energy state of the system (density, velocity, pressure, temperature, etc.). This will be referred to as the PWR physical system model. To apply the control procedure to it, a selected list of system parameters have their values monitored. These values are input to the control procedure along with the control procedure specifications. Evaluating the control procedure results in controlled component hardware actions being adjusted within the PWR physical system model. This process, diagrammed in Fig. 13, is evaluated at the beginning of each time step on the basis of the beginning of the time-step system state. The control procedure determines what adjustments of hardware

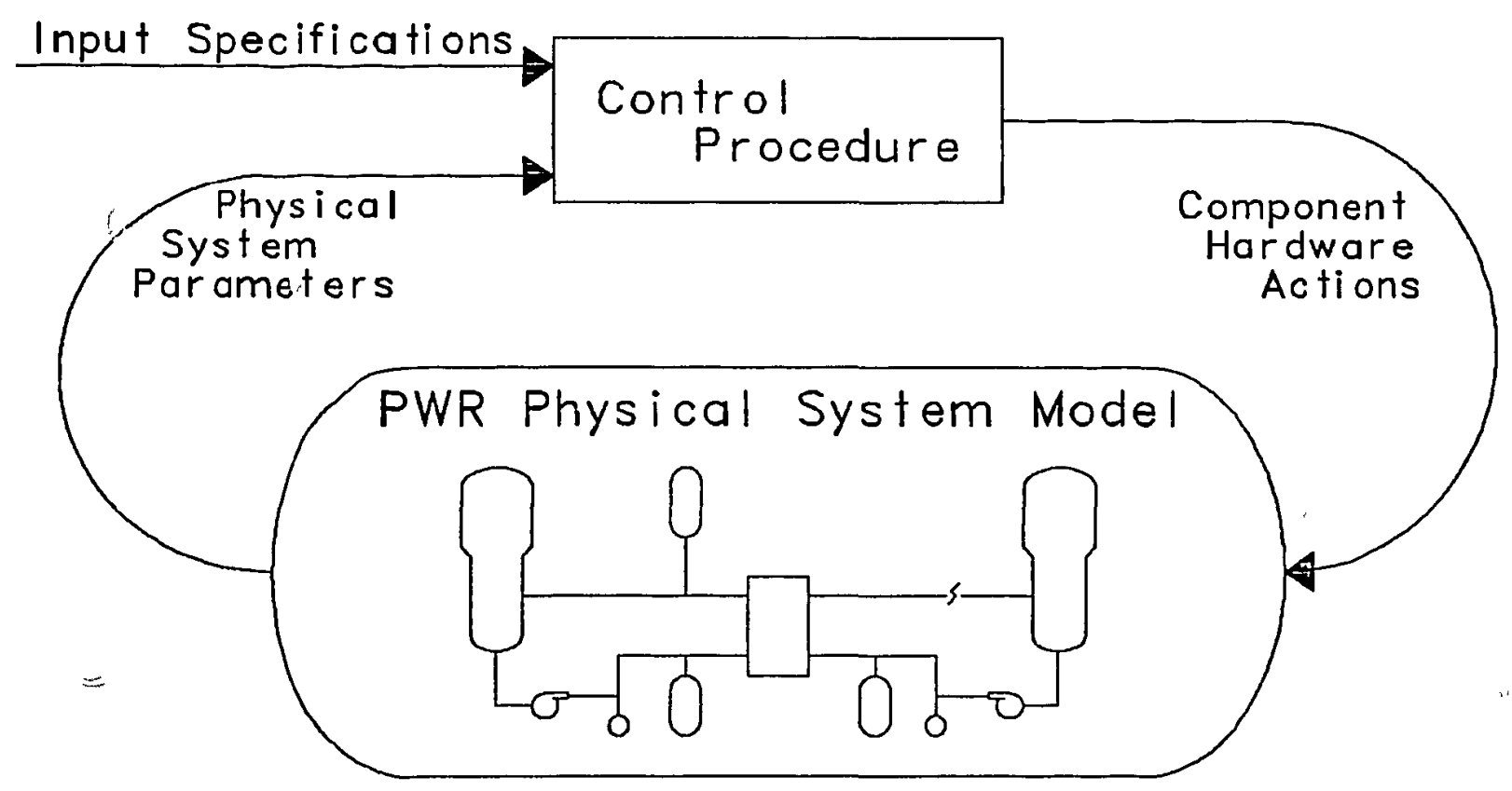

Fig. 13 .

TRAC simulation model of a PWR power plant. 
are needed. The mode of operation of the system is thus directed as well as constrained by the control procedure.

Hardware actions that can be adjusted by the control procedure are listed in Table $V$. The TRAC components to which they are applied are shown along with the FORTRAN variable-name internal letters for defining the action. In addition, the control procedure also implements the use of special timestep data, editing of restart data dumps, and termination of the calculation. These are the adjustable features in the TRAC model over which the user can specify control.

All information for defining the control procedure is specified in the input data for application to the general control model. This fully defines the controlled mode of operation unless TRAC is executed in the interactive mode. In the interactive mode, the control-procedure data can be redefined at any time during the simulation. Preparing the input data involves first defining the PWR physical system model. Each of the hardware components is modeled by its corresponding TRAC component by specifying appropriate values for the component input parameters. For any of the components in Table $V$,

TABLE $V$

ADJUSTABLE COMPONENT HARDWARE ACTIONS BY THE CONTROL PROCEDURE

Actions

Pressure boundary condition
and fluid state
Velocity or mass-flow boundary
condition and fluid state
Reactor-core progranmed
reactivity or power
Reactor-core axial power shape
Energy deposition in the coolant
Energy generation in the wall
Pump rotational speed
Turbine power demand
Valve flow-area fraction or
relative stem position

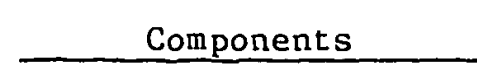

BREAK

EILL

CORE, VESSEL

CORE, VESSEL

PIPE, TEE, TURBINE

PIPE, PUMP, TEE, VALVE

PUMP

TURBINE

VALVE
Variable-Name Letters

B

F

RPW

ZPW

POW or $\mathrm{PW}$

QP3 or QP

PMP or OMG

TRB or POP

V 
modeling one of its hardware actions is done as part of preparing the component input data. Implementing a hardware action involves specifying tabular data and associated control parameters for a component-action table. In the next section the detailed form of a general component-action table is discussed. This includes all of its associated control parameters, which together are part of the component input data.

1. Component-Action Table. Modeling a component adjustable hardware action is done by means of a component-action table, specified through the component input data. The table defines the component hardware action as a tabular function of an independent variable defined by physical system parameters. These parameters monitored from the PWR physical system model are called signal variables in TRAC. They can be applied as the component-action tabla's independent variable directly or after having been operated on by function operators called control blocks. In this section, the independent variable that is discussed is the independent variable of the component-action table.

Determining a hardware action by evaluating its component-action table is based on a component-action type option parameter. Its FORTRAN variable name has the general form IXXXTY where letters XXX are to be replaced by one to three letters characterizing the component action. Those letters for each of the actions in Table $V$ are shown in the rightmost column. Through the option parameter the user specifies if the action is to be held constant, evaluated at all times from the component-action table, or evaluated from the component-action table only when an assigned controlling trip switch is oN but held constant when the trip is OFF. If the component-action table is to be evaluated under trip control, a trip ID number with general form variable name IXXXTR is specified as part of the component inpyt data. The actual definition of the trip with the ID number label referenced here is specified in the trip input data section located elsewhere in the input data file. When IXXXTY defines no trip control for evaluating the conponent-action table, IXXXTR should be defined by zero or a blank field on input.

The independent, variable is defined by physical system parameters in the form of a signal variable or a control-block output variable. The variable to be used is defined by its ID number with the general FORTRAN variable name IXXXSV. Positive ID numbers define signal variables; negative ID numbers 
define control-block output variables. Signal variables and control blocks are described in Secs. III.E.3 and III.E.4.

The number of entry pairs (independent-variable value, component-action value) of tabular input data defining the component-action table is specified by the general FORTRAN variable name NXXXTB. Applied to its value is a plus or minus sign to define the form of the independent variable. When NXXXTB is positive, the independent variable is the IXXXSV variable. When NXXXTB is ncgative, the independent variable is the change in the value of the IXXXsV variable during the time the table is evaluated. For this latter form, the initial value of the component action corresponds to the component-action value in the table with an independent-variable value of zero. Internal to TRAC-PF1, the change in the IXXXSV variable value is incorporated into the table definition for each time step that is evaluated by translation of all the abscissa coordinate (independent variable) values in the table. As a result, the last interpolated component-action value from the table corresponds to the independent variable having a value of zero after translation. The user doesn't need to be concerned with this internal translation of the table's abscissa coordinate values by TRAC. The only time its effect can be seen is when the component-action table is edited on restart. Defining a NXXXTB < 0 table with the initial component-action value corresponding to an abscissa coordinate value of zero is all that need concern the user.

When a NXXXTB < 0 table is to be evaluated under trip control, the change in the IXXXSV variable value over each time step is further multiplied by the value of the controlling trip ON/OFF set status. FORTRAN variable ISET defines the trip set status. OFF has the value 0 , and the two forms of oN,

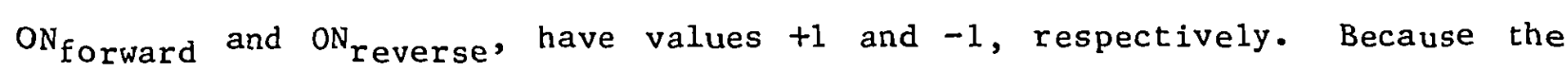
component-action table under trip control is only evaluated when the trip is ON, ISET has the value +1 or -1 when the change in the IXXXSV variable value is multiplied by it to evaluate the independent variable. As a result, the trip set-status value affects the direction of interpolated movement in the table. "The forward and reverse subscripts for $O N$ correspond to the direction of the interpolated movement action. 
The effect the values for IXXXTR, IXXXSV, and NXXXTB have on defining the independent variable is summarized in Table VI. Evaluation of the component-action table is trip controlled when IXXXTR $\neq 0$. No component-action table is defined (the component action is held constant) when IXXXSV $=0$ (or a blank data field) is input. When NXXXTB $=0$ is input with IXXXSV $\neq 0$, a table is defined, but it has no tabular data. This is a special case where the component action is defined directly by ils independentvariable form, that is, a signal variable (IXXXSV >0) or a control-block output variabla (IXXXSV < 0$)$.

In the above definition, the direction of interpolation in the component-action table can be trip controlled when IXXXTR $\neq 0$ and NXXXTB< 0 . The trip set-status value $( \pm 1)$ is applied to the magnitude of the movement defined by the change in the signal variable or control-block output variable. In most situations, this form is sufficient for modeling the quantity that the component action has dependence on. There are a few situations, however, where the user would like further control over the magnitude of the interpolated movement. He can do this with either a connected series of control-block operators defining the independent variable or by applying a rate factor to the independent-variable forms in Table VI. Operating on a parameter signal (signal variable value) with a connected series of control

TABLE VI

DEFINED FORMS OF THE COMPONENT-ACTION TABLE`S INDEPENDENT VARIABLE

\section{Component-Action Table's Defining Variables}

\begin{tabular}{|c|c|c|}
\hline & $x \times \times S V$ & $B$ \\
\hline
\end{tabular}

$\begin{array}{llll}\text { A11 values } & >0 & 20 & A \\ 0 & >0 & <0 & \Delta A \\ \neq 0 & >0 & <0 & \Delta A^{\star} \text { ISET } \\ \text { A11 values } & <0 & 20 & B \\ 0 & <0 & <0 & \Delta B \\ \neq 0 & <0 & <0 & \Delta \mathrm{B}^{\star} \text { ISET }\end{array}$

${ }^{a}$ is a signal variable; $B$ is a control-block output variable. 
blocks and defining the independent-variable form with the last control-block output variable is an extended application of one of the last three forms in Table VI. Applying a rate factor to any one of the six forms is a direct adjustment to the rate of interpolated movement when evaluating the component-action table.

2. Rate-Factor Table. A rate factor can be defined by entering a rate-factor table of tabular data. Specifying a rate-factor table requires that its component-action table be defined (IXXXSV $\neq 0$ ) and that the user decide to apply a rate factor to the independent-variable form in Table.VI. FORTRAN variables NXXXSV and NXXXRF define a rate-factor table in the same way that IXXXSV and NXXXTB, respectively, define the component-action table. In addition to the six independent-variable forms in Table VI, entering IXXXTR $\neq 0, \operatorname{NXXXSV}=0$, and NXXXRF $\neq 0$ defines the independent-variable form to be the difference between the trip signal value and the trip set-point value that turns the trip OFF. This additional form provides a convenient means for adjusting the rate of interpolated movement in the component-action table. The further the trip signal departs from its desired value, specified by its set-point value that turns the trip OFF, the larger the rate factor and the faster the component action can be adjusted in order to return the trip signal to its desired value. Reading Sec. III.E.5 on how trips are defined will help to clarify the explanation for the above form.

When a rate-factor table is defined, the procedure for evaluating the component-action value is as follows. First, TRAC evaluates the independent-variable form (defined by :XXXTR, NXXXSV, and NXXXKF). This value is then used to linearly interpolate in the rate-factor table (when NXXXRF $\neq 0$ ) or define directly (when NXXXRF $=0$ ) the rate-factor value. Next the independent-variable form (defined by IXXXTR, IXXXSV, and NXXXTB) is evaluated and multiplied by the rate factor. This product value is used to interpolate linearly in the component-action table (when NXXXTB $\neq 0$ ) or define directly (when NXXXTB $=0$ ) the component-action value. The component hardware action in the modeled PWR system is then defined with this value. This procedure is evaluated at the beginning of each time step with the current state of the system parameters to evaluate the independent-variable forms for both tables. Not defining a rate-factor table [NXXXSV $=0$ and NXXXRF $=0$ (or blank data fields) are input] reduces the above procedure to evaluating tine 
independent-variable form and then using that value to interpolate linearly in the component-action table or define directly the component-action value.

The component-action table and rate-factor table defining variables IXXXTR, IXXXSV, NXXXTB, NXXXSV, and NXXXRF are specified in that order on one input data card. There is such a card for each type of component action in the component input data unless the IXXXTY option specifies that the component action is to be held constant. Then no component-action table and defining variables are input. There is also a second input data card defining the component action with the following real valued variables: XXXIN, XXXOFF, RXXXMX, and XXXSCL. Variable XXXIN defines the initial value for the component action. This value is used when the component action is to be held constant or when the user is evaluating the component action under trip control and the trip is initially OFF. For component actions that are trip controlled, variable XXXOFF defines the component-action value that is used when the trip is OFF after having been ON. Entering XXXOFF $=-1 \times 10^{19}$ defaults in TRAC to using the last component-action value evaluated when the trip was $O N$. Variable RXXXMX defines the maximum rate of change of the component-action value allowed by the user during the simulation. When the component action evaluated from the table varies faster than this maximum rate, the component action is redefined to vary at the maximum rate. Later when the rate of change of the table-evaluated component action falls below the maxinum rate, the applied component-action value will be able to catch up to the value determined from the table. Variable XXXSCL is a scale factor to be applied during input to the component-action values in the input table.

The component-action table (with $|\operatorname{NXXXTB}| * 2$ values) and its rate-factor table (with $\mid$ NXXXRF|*2 values) are input in the array data section of the component input using the LOAD format described in Sec.V.D. Al1 componentaction tables, with two exceptions, have one component-action value for each independent-variable value. Thus, there are two values for each of the |NXXXTB| entry pairs in their tabular data. The two exceptions are the axial power shape and the energy generation in the wall component-action tables. Corresponding to each independent-variable value is an axial power shape with a relative power value at each axial interface in the reactor-core model or a QPPP factor shape with a QPPP factor value for each mesh cell in the component model. As an example, if the reactor core has five mesh cells axially, each 
axial power shape would have six relative power values defined at the axial interfaces. The axial power shape table would have |NZPWTB| entry "pairs" with each "pair" having one independent-variable value and six axial powershape values. There would be $\mid$ NZPWTB $\mid *(1+6)$ values of tabular data defining the table.

This section has discussed application of the component-action table in its general form. The XXX letters in all the FORTRAN variable names defined will need to be replaced by the component action one to three letters in Table VI to give their FORTRAN variable names. A main point to recognize from this section is that there is one form to the numerical model for applying adjustable control to a component action even though there are nine different component hardware actions that can be modeled. Understanding the general definition of control variables IXXXTY, IXXXTR, IXXXSV, NXXXTB, NXXXSV, NXXXRF, XXXIN, XXXOFF, RXXXMX, and XXXSCL gives the user the ability to model the dynamics of a myriad of possible control procedures encountered in PWR operation. The next four sections on signal variables, control blocks, trips, and ordered evaluation of control parameters go into the details of these modeling tools used to define the control procedure.

3. Signal Variables. Signal variables are PWR modeled-system parameters that the user defines for input to the control-procedure model used to adjust component hardware actions. They have their values determined by TRAC at the beginning of each time step on the basis of the state of the modeled system. Their values are used to determine the magnitudes of all adjustable component actions defined, which are then applied over the time step. This procedure approximates the magnitudes of the signal variables and component actions as being constant over the time step with step changes at the beginning of each time step. In reality, they vary continuously. Using small time steps can approximate this very we11. With the hydrodynamics twostep solution method in TRAC-PFl, very large time steps are possible. When using large time steps, implementing control adjustment can be numerically delayed in time. Both the satisfied trip criteria for starting an action and the evaluated dependence that drives the action encounter fractional time-step lag. It is the accumulation of these small errors over many time steps that can contribute significant error when simulating a control procedure. 
The 57 PWR-modeled system parameters, which are definable as signal variables, are listed in Table VII. Each parameter is defined by a parameter number assigned to FORTRAN variable name ISVN when defining a signal variable. Parameter number 0 is problem time defined by FORTRAN variable name STIME during steady-state calculations and TIMET during transient calculations. Parameter numbers 1 through 15 define control-panel vector (CPV) paraneters that are only evaluated when TRAC is executed in the interactive mode. These parameters are those normally monitored by the plant operator on instrumentation in the control room. Parameter numbers 16 and 17 are dummy parameters, as yet undefined, that the user can define himself. System parameters not defined in Table VII can have their values assigned to either of these parameter numbers by hardwiring their value assignment in subroutine SVSET. The remaining system parameters in the Table VII list, except for the last two, are defined in a specific component. In addition, parameter numbers 20 through 40 require one or two mesh-cell numbers to further delimit the location in the component where the parameter is defined. Specific to the CORE and VESSEL components when fuel rods are present are the parameters with numbers $18,19,25,26$, and 44 through 54. The last two parameters in the list, numbers 55 and 56, are trip parameters that, when defined as signal variables, can be used for other control purposes.

The liquid water level, parameter number 20 , is the geometric distance of collapsed liquid coolant from the bottom interface of mesh cell $\left|\operatorname{cell}_{1}\right|$. It is defined by

liquid water leve1 $=\sum_{i=\left|\operatorname{ce} 11_{1}\right|}^{\mathrm{I} \pm 1} \Delta \mathrm{X}_{1}+\mathrm{f \Delta x} \mathrm{X}_{\mathrm{I}}$

where $\Delta X_{i}$ is the length of mesh cell 1 and the liquid-vapor interface is at a distance $f \Delta X_{I}$ above the lower interface of mesh cell $I$. The values of $f$ and $I$ are defined by requiring that the volume of liquid between mesh cells $\mid$ cell $1_{1} \mid$ and $\left|\operatorname{cell}_{2}\right|$ be collapsed to fill the fluid volume of mesh cells $\left|\operatorname{cell}_{1}\right|$ to \pm 1 ( + when $\left|\operatorname{cell} 1_{1}\right|>\left|\operatorname{cell}_{2}\right|$ and - otherwise) and the fraction $f$ of the volume of mesh cell I, that is, 
TABLE VII

SIGNAL-VARIABLE PARAMETERS

Signal-

Variable

Parameter

Number

\section{0}

1

2
Parameter Description

Problem time (s)

Reactor power (W)

Primary-pressure ( $\mathrm{Pa}$ )

Component pressure ( $\mathrm{Pa})$

Component temperature (K)

Pressurizer water level (m)

Refueling-storage-tank water level (m)

Hot-leg temperature (K)

Cold-leg temperature ( $K$ )

Primary-coolant

$$
\text { mass flow ( } \left.\mathrm{kg} \cdot \mathrm{s}^{-1}\right)
$$

Emergency-core-coolant (ECC) mass flow ( $\left.\mathrm{kg} \cdot \mathrm{s}^{-1}\right)$

Steam-generator-secondary pressure ( $\mathrm{Pa}$ )

Steam-generator-secondary steam outflow ( $\mathrm{kg} \cdot \mathrm{s}^{-1}$ )

Steam-generator-secondary main feedwater flow $\left(\mathrm{kg} \cdot \mathrm{s}^{-1}\right)$

Steam-generator-secondary auxiliary feedwater flow $\left(\mathrm{kg} \cdot \mathrm{s}^{-1}\right)$

Steam-generator-secondary liquid level (m)

Dummy storage variable number 1

Dummy storage variable number 2

Reactor power (W)

Reactor period ( $s$ )

Liquid water level (m)

Cell pressure ( $\mathrm{Pa}$ )

Cell vapor temperature $(K)$

Cell liquid temperature (K)

Cell slab outer-surface temperature $(K)$

Cell fuel-rod outer-surface temperature $(\mathrm{K})$
Additiona1 Location Data

Coolant loop number

Coolant loop number

Coolant loop number

Coolant loop number

Coolant loop number

Coolant loop number

Coolant loop number

Coolant loop number

Coolant loop number

Component number

Component number

Component, cell $1_{1}$, and $c e 11_{2}$ numbers Component, cell $1_{1}$, and cell $1_{2}$ numbers Component, cell $1_{1}$, and $\operatorname{cell}_{2}$ numbers Component, cel1 1 , and ce $11_{2}$ numbers Component, cell 1 , and $\operatorname{cell}_{2}$ numbers

Component, cell 1 , and $\operatorname{cel}_{2} 1_{2}$ numbers 
TABLE VII (cont.)

Signal-

Variable

Parameter Number
Parameter Description

Cell fuel-rod centerline temperature ( $K$ )

Cell vapor volume fraction

$\theta$-interface vapor mass flow $\left(\mathrm{kg} \cdot \mathrm{s}^{-\mathrm{I}}\right)$

$z$-interface vapor mass flow $\left(\mathrm{kg} \cdot \mathrm{s}^{-1}\right)$

$r$-interface vapor mass flow $\left(\mathrm{kg}^{-} \cdot \mathrm{s}^{-1}\right)$

$\theta$-interface liquid mass flow $\left(\mathrm{kg} \cdot \mathrm{s}^{-1}\right)$

z-interface liquid mass flow $\left(\mathrm{kg} \cdot \mathrm{s}^{-1}\right)$ r-interface liquid mass flow $\left(\mathrm{kg} \cdot \mathrm{s}^{-1}\right)$

$\theta$-interface vapor velocity (m $\cdot \mathrm{s}^{-1}$ )

$z$-interface vapor velocity (m $\cdot \mathrm{s}^{-1}$ )

$r$-interface vapor velocity $\left(\mathrm{m} \cdot \mathrm{s}^{-1}\right)$

$\theta$-interface liquid velocity $\left(\mathrm{m} \cdot \mathrm{s}^{-1}\right)$

$z$-interface liquid velocity $\left(\mathrm{m} \cdot \mathrm{s}^{-1}\right)$

r-interface liquid velocity (m $\cdot \mathrm{s}^{-1}$ )

Boron concentration $\left(\mathrm{kg} \cdot \mathrm{m}^{-3}\right)$

Pump rotational speed $\left(\mathrm{rad} \cdot \mathrm{s}^{-1}\right)$

Valve flow-area fraction

Valve stem relative position

Reactor multiplication constant

Programmed reactivity

Total feedback reactivity

Fuel temperature feedback reactivity

Coolant temperature feedback reactivity
Additional Loca ion Data

Component, cell 1 , and cell $1_{2}$ numbers

Component, cell 1 , and $c e 11_{2}$ numbers

Component, cell $1_{1}$, and cell $1_{2}$ numbers

Component, cell 1 , and cell $1_{2}$ numbers

Component, cell $1_{1}$, and $c e l 1_{2}$ numbers

Component, cell 1 , and $\operatorname{cell}_{2}$ numbers

Component, $\operatorname{cell} 1_{1}$, and $\operatorname{cell}_{2}$ numbers

Component, cell 1 , and cell 2 numbers

Component, cell 1 , and cell $l_{2}$ numbers

Component, cell 1 , and $c e l 1_{2}$ numbers

Component, cell 1 , and $\operatorname{cell}_{2}$ numbers

Component, cell 1 , and $\operatorname{cell}_{2}$ numbers

Component, cell 1 , and $\operatorname{cell}_{2}$ numbers

Component, cell 1 , and $\operatorname{cell}_{2}$ numbers

Component, cell 1 , and $\operatorname{cell} 1_{2}$ numbers

Component number

Component number

Component number

Component number

Component number

Component number

Component number

Component number 
TABLE VII (cont.)

Signa1Variable Parameter Number

\section{9}

\section{Parameter Description}

\section{Vapor coolant volume \\ fraction feedback reactivity}

Boron concentration feedback reactivity

Core averaged fuel temperature ( $K$ )

Core averaged coolant temperature (K)

Core averaged vapor coolant volume fraction

Core averaged boron concentration $\left(\mathrm{kg} \cdot \mathrm{m}^{-3}\right)$

$$
\text { Trip signal value }
$$

Trip set-status value

Prompt-fission power (W)

Decay-heat power ( $W$ )

Maximum average fuel-rod temperature (K)

Maximum supplemental fuel-rod temperature (K)

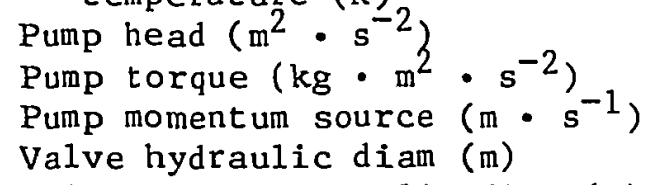

\section{Additional Location Data}

Component number

Component number

Component number

Component number

Component number

Coinponent number

Trip ID number

Trip ID number

Component number

Component number

Component number

Component number

Component number

Component number

Component number

Component number

Component, cell 1 , and cell $1_{2}$ numbers Component, cell 1 , and cel1 2 numbers Component, cell 1 , and $\operatorname{cel} 1_{2}$ numbers Component, cell 1 , and $\operatorname{cell}_{2}$ numbers

Component, cell 1 , and $\operatorname{cell}_{2}$ numbers

Component, cell 1 , and $\operatorname{cell}_{2}$ numbers

Component, $\operatorname{cell} 1$, and $\operatorname{ce}_{1} 1_{2}$ numbers

Component, $\operatorname{cell}_{1}$, and $\operatorname{cell}_{2}$ numbers

Component, cell 1 , and $\operatorname{cell}_{2}$ numbers

Component, $\operatorname{cell} 1_{1}$, and $\operatorname{cell}_{2}$ numbers Component, cell $1_{1}$, and $\operatorname{cell}_{2}$ numbers Component, cell 1 , and cell 2 numbers 
TABLE VII (cont.)

Signal-

Variable

Parameter

Number

77

78

79

80

81

82

83

84

85

86

87

88

89

90

91

92

93

94

95

96

\section{Parameter Description}

Cell air density $\left(\mathrm{kg} \cdot \mathrm{m}^{-3}\right)$

Cell air mass $(\mathrm{kg})$

Cell air pressure $(\mathrm{Pa})$

Cell air internal energy

$\left(W \cdot \mathrm{s} \cdot \mathrm{kg}^{-1}\right)$

Cell vapor internal energy

$\left(\mathrm{W} \cdot \mathrm{s} \cdot \mathrm{kg}^{-1}\right)$

Cell liquid internal energy

$\left(\mathrm{W} \cdot \mathrm{s} \cdot \mathrm{kg}^{-1}\right.$ )

Cell saturation temperature

(K) based on steam pressure

Cell saturation temperature

$(K)$ based on total pressure

Cell vapor specific heat at constant pressure

$\left(\mathrm{W} \cdot \mathrm{s} \cdot \mathrm{kg}^{-1} \cdot \mathrm{K}^{-1}\right)$

Cell liquid specific heat at

constant pressure

$\left(\mathrm{W} \cdot \mathrm{s} \cdot \mathrm{kg}^{-1} \cdot \mathrm{K}^{-1}\right)$

Cell latent heat of vaporization (W.s $\cdot \mathrm{kg}^{-1}$ )

Ce11 average fue1-rod surface heat flux $\left(\mathrm{W} \cdot \mathrm{m}^{-2}\right)$

Cell slab inside surface heat flux $\left(w \cdot \mathrm{m}^{-2}\right)$

Cell coolant interfacial heat flow (W)

Cell vapor heat-transfer coefficient $\left(W \cdot \mathrm{m}^{-2} \cdot \mathrm{K}^{-1}\right)$ at average rod surface

Cell liquid heat-transfer coefficient $\left(W \cdot \mathrm{m}^{-2} \cdot \mathrm{K}^{-1}\right)$ at average rod surface

Cell vapor heat-transfer coefficient $\left(\mathrm{W} \cdot \mathrm{m}^{-2} \cdot \mathrm{K}^{-1}\right)$ at slab inside surface

Cell 1iquid heat-transfer coefficient $\left(W \cdot \mathrm{m}^{-2} \cdot \mathrm{K}^{-1}\right)$ at slab inside surface

Cell coolant interfacial heattransfer coefficient $\left(\mathrm{W} \cdot \mathrm{m}^{-2} \cdot \mathrm{K}^{-1}\right)$

Cell coolant interfacial area $\left(m^{2}\right)$
Additional Location Data

Component, cell 1 , and cell 2 numbers Component, cell $1_{1}$, and cell 2 numbers Component, cell 1 , and cell 2 numbers Component, cell $1_{1}$, and cell 2 numbers

Component, cell $11_{1}$ and cell 2 numbers Component, cell 1 , and cell 2 numbers Component, cell 1 , and cell 2 numbers component, cell $1_{1}$, and $\operatorname{cell}_{2}$ numbers Component, cell 1 , and cell 2 numbers Component, cell 1 , and cell 2 numbers Component, cell 1 , and cell 2 numbers Component, cell 1 , and cell 2 numbers Component, cell 1 , and cell 2 numbers Component, cell 1 , and cell 2 numbers Component, cel1 1 , and cell 2 numbers Component, cell $1_{1}$, and $\mathrm{cell}_{2}$ numbers Component, cell 1 , and $\mathrm{cell}_{2}$ numbers Component, cell 1 , and $\operatorname{cell}_{2}$ numbers Component, cell 1 , and $\mathrm{cell}_{2}$ numbers Component, cell 1 , and $\operatorname{cell}_{2}$ numbers 


\begin{tabular}{|c|c|c|}
\hline $\begin{array}{l}\text { Signal- } \\
\text { Variable } \\
\text { Parameter } \\
\text { Number } \\
\end{array}$ & Parameter Description & Additional Location Data \\
\hline 97 & $\begin{array}{l}\theta \text {-interface coolant inter- } \\
\text { facial Eriction factor } \\
\left(\mathrm{kg} \cdot \mathrm{m}^{4}\right)\end{array}$ & Component, cel1 1 , and cel1 $1_{2}$ numbers \\
\hline 98 & $\begin{array}{l}\text { z-interface coolant inter- } \\
\text { facial fryction factor } \\
\left(\mathrm{kg} \cdot \mathrm{m}^{-4}\right)\end{array}$ & Component, cell 1 , and cell $1_{2}$ numbers \\
\hline 99 & $\begin{array}{l}\text { r-interface coolant inter- } \\
\text { facial friction factor } \\
\quad\left(\mathrm{kg} \cdot \mathrm{m}^{-4}\right)\end{array}$ & Component, cell 1 , and $\operatorname{cell}_{2}$ number \\
\hline 100 & $\begin{array}{l}\text { Celi solute plated on } \\
\text { structure }\left(\mathrm{kg} \cdot \mathrm{m}^{-3}\right)\end{array}$ & Component, cell 1 , and ce $11_{2}$ numbers \\
\hline 101 & $\begin{array}{l}\text { Cell vapor generation rate } \\
\text { per unit volume } \\
\left(\mathrm{kg} \cdot \mathrm{s}^{-1} \cdot \mathrm{m}^{-3}\right)\end{array}$ & Component, cel1 1 , and ce $11_{2}$ numbers \\
\hline 102 & $\begin{array}{l}\text { Component wall heat loss } \\
\text { to the inside (W) }\end{array}$ & One-dimensional component number \\
\hline 103 & $\begin{array}{l}\text { Component wall heat loss } \\
\text { to the outside }(W)\end{array}$ & One-dimensional component number \\
\hline
\end{tabular}

(plus when $\left|c e 11_{1}\right|>\left|c e 11_{2}\right|$ and minus otherwise) and the fraction $f$ of the volume of mesh cell $\mathrm{I}$, that is,

$$
\underset{i=\left|\operatorname{ce} 11_{1}\right|}{\left|\operatorname{ce} 11_{2}\right|}\left(1-\alpha_{i}\right) V_{i} \equiv \underset{i=\left|\sum_{1}^{I \pm 1}\right| V_{1} \mid}{ } V_{i}+f V_{I}
$$

This collapsed liquid column can only be evaluated for mesh cells within a component and only for the primary or secondary side when the component is a TEE. To combine the liquid heights in several connected components requires defining a liquid water-level signal variable for each component (and side) and suming them with control blocks using appropriate weighting factors. The cosine of the angle the component mesh cells are inclined from vertical needs to be multiplied with the liquid water level to give the liquid's vertical height. 
For mesh cells in components on the steam-generator secondary-side (SGS), cell $I_{1}$ and $c e l l_{2}$ are defined with the SGS component ID number in the usual way. For mesh cells on the secondary side of a TEE component, cel1 1 and $\mathrm{cell}_{2}$ are defined as composites of the total number of mesh cells on the primary side, NCELL1, and the desired mesh-cell number on the secondary side, N2, that is,

ce $11_{1}=$ NCFLLI $+\mathrm{N} 2$.

$\operatorname{cell}_{2}$

For the three-dimensional VESSEL component mesh cells, cell 1 and cell $1_{2}$ are defined as composites of the relative cell number in the horizontal $\theta-r$ plane times 1000 plus the axial level number, that is,

$\begin{aligned} & \text { cell } 1 \\ & \text { cell } 11_{2}\end{aligned}=$ (horizontal plane relative cell number)*1000 + (axial level number) .

When the signal-variable definition spans all cells from cell 1 to cell $1_{2}$, a two-dimensional array of mesh cells between the minimum and maximum relative cell numbers and the minimum and maximum axial level numbers (defined by cell 1 and $\mathrm{cell}_{2}$ ) are considered in the VESSEL. Using these composite définitions for TEE secondary and VESSEL mesh cells, enables the TRAC user to define them with the signal-variable definition form for one-dimensional components.

Signal-variable parameter numbers 28 through 39 define mass flow and velocity and have cell 1 and cell $1_{2}$ numbers associated with mesh-cell interfaces. A one-dimensional component mesh cell $i$ has boundary interfaces numbered $i$ and $i+1$. In the three-dimensional VESSEL, the second azimuthal, the outer radial, and the upper axial interfaces are all associated with the mesh-cell number. If mass flow or velocity are specified on $r$ or $\theta$ interfaces for one-dimensional components; their definitions default to the z-interface definition. 
There are five different forms for defining signal-variable parameters when they are location dependent, that is, when they have ISVN parameter numbers 20 through 40 . The signal variable is the

1. parameter value in mesh $\operatorname{cel} 1_{i}\left|\operatorname{cell} 1_{1}\right|$ or $\left|\operatorname{ce} 11_{2}\right|$ when $\operatorname{cell}_{2}=0$ or $\operatorname{cell}_{1}=0$, respectively;

2. maximum value of the parameter in all mesh cells between mesh cells $\left|c e l l_{1}\right|$ and $\left|\operatorname{cell}_{2}\right|$ when $\operatorname{cell}_{1}$ and $\operatorname{cell}_{2}$ are both positive;

3. minimum value of the parameter in all mesh cells between mesh cells $\left|c e l l_{1}\right|$ and $\left|c e 1 l_{2}\right|$ when $\operatorname{cell}_{1}$ and $\operatorname{cell}_{2}$ are both negative;

4. volume-averaged value of the parameter in all mesh cells between mesh cells $\left|c e l l_{1}\right|$ and $\left|c e 1 l_{2}\right|$ when cell $l_{1}$ and $c e l l_{2}$ are of opposite signs; and

5. difference between the parameter values in mesh cells $\left|\operatorname{ce} 11_{1}\right|$ and $\left|c e 11_{2}\right|$ when the ISVN parameter number is prefixed with a minus sign.

The fifth form can only have parameter numbers -40 through -21 . Its signal variable is the parameter value in $\left|c e l 1_{l}\right|$ minus the parameter value in $\left|c e l_{2}\right|$. Mesh-cell numbers are defined above by the positive (absolute) values of cell $l_{1}$ and $\operatorname{cell}_{2}$. Forms 3 and 4 above are the only ones that define cell $l_{1}$ and $c e l l_{2}$ with negative values. For all five forms, it is the absolute value of $\operatorname{cell}_{1}$ and $c e l l_{2}$ that is used to define the mesh-cell number.

A unique, positive identification (ID) number is assigned by the user to each signal variable. This number serves as the signal-variable label for referencing the use of its value within the control procedure.

4. Control Blocks. Control blocks are function operators that operate on input parameter signals to produce an output parameter signal. The output signal can then be used as an input parameter to other control blocks. In this way, a control procedure for a component action can be constructed by coupling control-block operators in series and in parallel. PWR system parameters, defined by signal variables, provide the input data for the initial control block(s) and possible subsequent control blocks in a control procedure. The control procedure ends with a final control block whose output signal value is used to define a component-action table's independent variable or the action directly when the table has no tabular data. 
Table VIII lists the 65 control-block function operations that are available in TRAC-PF1. The user selects the desired function operator for a control block and assigns its operation number (1 through 61, 100, 101, 200, and 201) in the leftmost column to FORTRAN input variable ICBN. The user also specifies for the control block zero to three input parameter signals and zero to two constants based on the required data for each block indicated in the five rightmost columns. Input parameter signals have their ID numbers defined as $X \#$ in the table where $\#=1,2$, or 3 ; input parameters with logical values

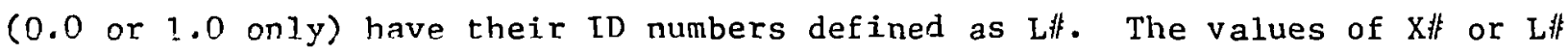
are input through FORTRAN variable name ICB\#. When X\# or L\# in the table is enclosed in parentheses, that is, (X非) or ( $L$ 非), it is not a required input parameter. The parentheses indicate that TRAC internally uses that input variable storage address to save intermediate results from the function evaluation for later use in evaluating the control block. The remaining information in the table is the control-block function name and its defined mathematical operation. XOUT is the control-block output value, which can be referenced with the control-block ID number by other control blocks for their input or by component-action tables for defining their independent variable. The control-block ID number is specified by the user through input with FORTRAN variable name IDCB and is required to be a negative integer greater than -10000. Thus, for a control block, defining ICB\# with a positive ID number inputs a signal-variable value to the control block; a negative ID number inputs a control-block output signal value.

In addition to the five possible X\#, L\#, and C\# control-block input parameters in Table VIII, all control blocks require from input a constant gain factor, G, and constant minimum and maximum limit values, XMIN and XMAX, which constrain the output value of XOUT. XMIN and XMAX enable the user to model the nonlinear saturation of a control signal by electronic hardware. The gain factor can be used to model the amplification or attenuation effect on the signal from such hardware. Variables G, XMIN, XMAX, and C非 are input with FORTRAN variable names CBGAIN, CBXMIN, CBXMAX, and CBCON非, respectively.

Figure 16 presents the general schematic diagram of a control block. The number of input parameters on the left-hand side ranges from zero to three depending on the control-block operation number. On the other hand, there is always one output parameter from a control block. Its value can vary 


\begin{tabular}{|c|c|c|c|c|c|c|c|c|}
\hline \multirow[b]{2}{*}{ No. } & \multirow[b]{2}{*}{ Control-Block Name } & \multirow{2}{*}{\multicolumn{2}{|c|}{$\begin{array}{l}\text { Control-Block } \\
\text { Mathematical Operation a }\end{array}$}} & \multicolumn{3}{|c|}{$\begin{array}{l}\text { Block } b, c \\
\text { Input }\end{array}$} & \multicolumn{2}{|c|}{$\begin{array}{l}\text { Block } \\
\text { Const. }\end{array}$} \\
\hline & & & & 1 & $\underline{2}$ & 3 & 1 & 2 \\
\hline 1 & Absolute value & XOUT & $=G * A B S(X 1)$. & $\mathrm{x} 1$ & & & & \\
\hline 2 & Arccosine & XOUT & $\begin{aligned}= & G^{*} A C O S(X I), \\
& \text { XOUT in radians. }\end{aligned}$ & $\mathrm{X} 1$ & & & & \\
\hline 3 & Add & XouT & $=G^{*}(X 1+X 2)$. & $\mathrm{Xl}$ & $\mathrm{x} 2$ & & & \\
\hline 4 & Integerizer & XouT & $=G^{*} \operatorname{FLOAT}(\operatorname{IFIX}(X 1))$. & $\mathrm{X} 1$ & & & & \\
\hline 5 & Logical "and" & XOUT & $\begin{aligned}= & G \\
& I F((L 1 \cdot E Q \cdot 1 \cdot 0) \\
& . A N D \cdot(L 2 \cdot E Q \cdot 1 \cdot 0)) ; \\
= & 0.0 \text { otherwise. }\end{aligned}$ & L1 & L2 & & & \\
\hline 6 & Arcsine & XOUT & $\begin{aligned}= & G^{*} \operatorname{ASIN}(X 1), \\
& \text { XOUT in radians. }\end{aligned}$ & $\mathrm{X} 1$ & & & & \\
\hline 7 & Arctangent & XOUT & $\begin{aligned}= & G^{*} \operatorname{ATAN}(X 1) \\
& \text { XOUT in radians. }\end{aligned}$ & $\mathrm{X} 1$ & & & & \\
\hline 8 & Arctangent 2 & XOUT & $\begin{aligned}= & G^{*} \operatorname{ATAN} 2(\mathrm{X} 1, \mathrm{X} 2), \\
& \text { XOUT in radians. }\end{aligned}$ & $\mathrm{X} 1$ & $\mathrm{x} 2$ & & & \\
\hline 9 & Constant & XOUT & $=\mathrm{G} * \mathrm{Cl}$. & & & & $\mathrm{Cl}$ & \\
\hline 10 & Cosine & XOUT & $\begin{aligned}= & G * \cos (X 1) \\
& X 1 \text { in radians. }\end{aligned}$ & $\mathrm{X} 1$ & & & & \\
\hline 11 & $\begin{array}{l}\text { Dead band, } \\
\text { dead zone, } \\
\text { or dead space }\end{array}$ & XOUT & $\begin{aligned}= & G^{*}(X 1-C 2) \\
& I F(X 1 \cdot G T \cdot C 2) ; \\
= & G^{*}(X 1-C 1) \\
& I F(X 1 \cdot L T \cdot C 1) ; \text { or } \\
= & 0.0 \text { otherwise. }\end{aligned}$ & $\mathrm{X} 1$ & & & $\mathrm{Cl}$ & $\mathrm{C} 2$ \\
\hline 12 & Derivative & XOUT & $=G^{*}(\mathrm{dXl} / \mathrm{dt})$ & $\mathrm{XI}$ & $(\mathrm{X} 2)$ & $\therefore$ & & \\
\hline
\end{tabular}




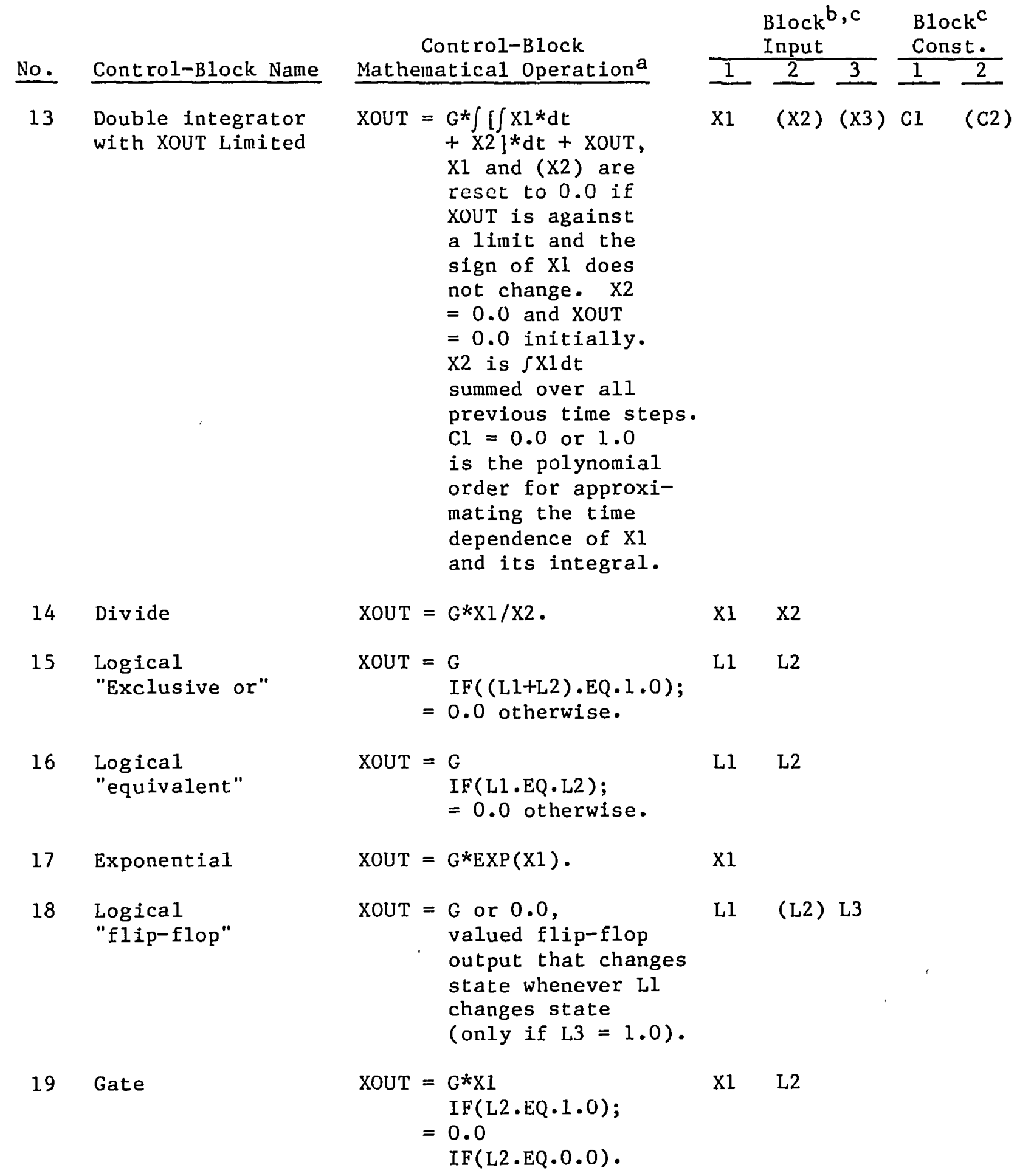




\begin{tabular}{|c|c|c|c|c|c|c|c|c|}
\hline \multirow[b]{2}{*}{ No. } & \multirow[b]{2}{*}{ Control-Block Name } & \multirow{2}{*}{\multicolumn{2}{|c|}{$\begin{array}{l}\text { Control-Block } \\
\text { Mathematical Operation }{ }^{a} \\
\end{array}$}} & \multicolumn{3}{|c|}{$\begin{array}{l}\text { Block }{ }^{b, c} \\
\text { Input }\end{array}$} & \multicolumn{2}{|c|}{$\begin{array}{l}\text { Block } \\
\text { Const. }\end{array}$} \\
\hline & & & & 1 & 2 & 3 & 1 & 2 \\
\hline 20 & $\begin{array}{l}\text { Greater than or } \\
\text { equal to }\end{array}$ & XOUT & $\begin{aligned}= & G \\
& I F(X 1 . G E \cdot X 2) \\
= & 0.0 \text { otherwise. }\end{aligned}$ & $\mathrm{X} 1$ & $\mathrm{x} 2$ & & & \\
\hline 21 & Greater than & XOUT & $\begin{aligned}= & G \\
& I F(X 1, G T \cdot X 2) \\
= & 0.0 \text { otherwise. }\end{aligned}$ & $\mathrm{X} 1$ & $\mathrm{X} 2$; & & & \\
\hline 22 & Input switch & XOUT & $\begin{aligned}= & G^{*} X 1 \\
& I F(L 3 \cdot E Q \cdot 1 \cdot 0) \\
= & G^{*} \times 2 \\
& I F(L 3 \cdot E Q \cdot 0 \cdot 0)\end{aligned}$ & $\mathrm{X} 1$ & $\mathrm{X} 2$ & L3 & & \\
\hline 23 & Integrate & XOUT & $\begin{array}{l}=\mathrm{G} * \int \mathrm{X} 1 * \mathrm{dt} \text { +XOUT, } \\
\mathrm{XOUT}=0.0 \text { initially. } \\
\mathrm{X} 1 \text { is not reset to } \\
0.0 \text { when XOUT is } \\
\text { against limits. } \\
\mathrm{Cl}=0.0 \text { or } 1.0 \\
\text { is the polynomial } \\
\text { order for approxi- } \\
\text { mating the time } \\
\text { dependence of } \mathrm{X} 1 .\end{array}$ & $x 1$ & & & $\mathrm{Cl}$ & (C2) \\
\hline $24^{\circ}$ & $\begin{array}{l}\text { Integrate with } \\
\text { mode control }\end{array}$ & XOUT & $\begin{aligned}= & 0.0 \\
& I F((L 2+L 3) \cdot E Q \cdot 0 \cdot 0), \\
& \text { reset mode; } \\
= & \text { XOUT } \\
& I F((L 2+L 3) \cdot E Q \cdot 1.0), \\
& \text { hold mode; or } \\
= & G * \int X 1 * d t+\text { XOUT } \\
& I F((L 2+L 3) \cdot E Q \cdot 2 \cdot 0), \\
& \text { integrate mode. } \\
& \text { Cl }=0.0 \text { or } 1.0 \\
& \text { is the polynomial } \\
& \text { order for approxi- } \\
& \text { mating the time } \\
& \text { dependence of X1. }\end{aligned}$ & $\mathrm{Xl}$ & $\mathrm{L} 2$ & L3 & $\mathrm{Cl}$ & (C2) \\
\hline 25 & $\begin{array}{l}\text { Logical } \\
\text { "inclusive or" }\end{array}$ & XOUT & $\begin{aligned}= & 0.0 \\
& \operatorname{IF}((L 1+L 2) \cdot E Q \cdot 0 \cdot 0) ; \\
= & G \text { otherwise. }\end{aligned}$ & L1 & L2 & & & \\
\hline
\end{tabular}




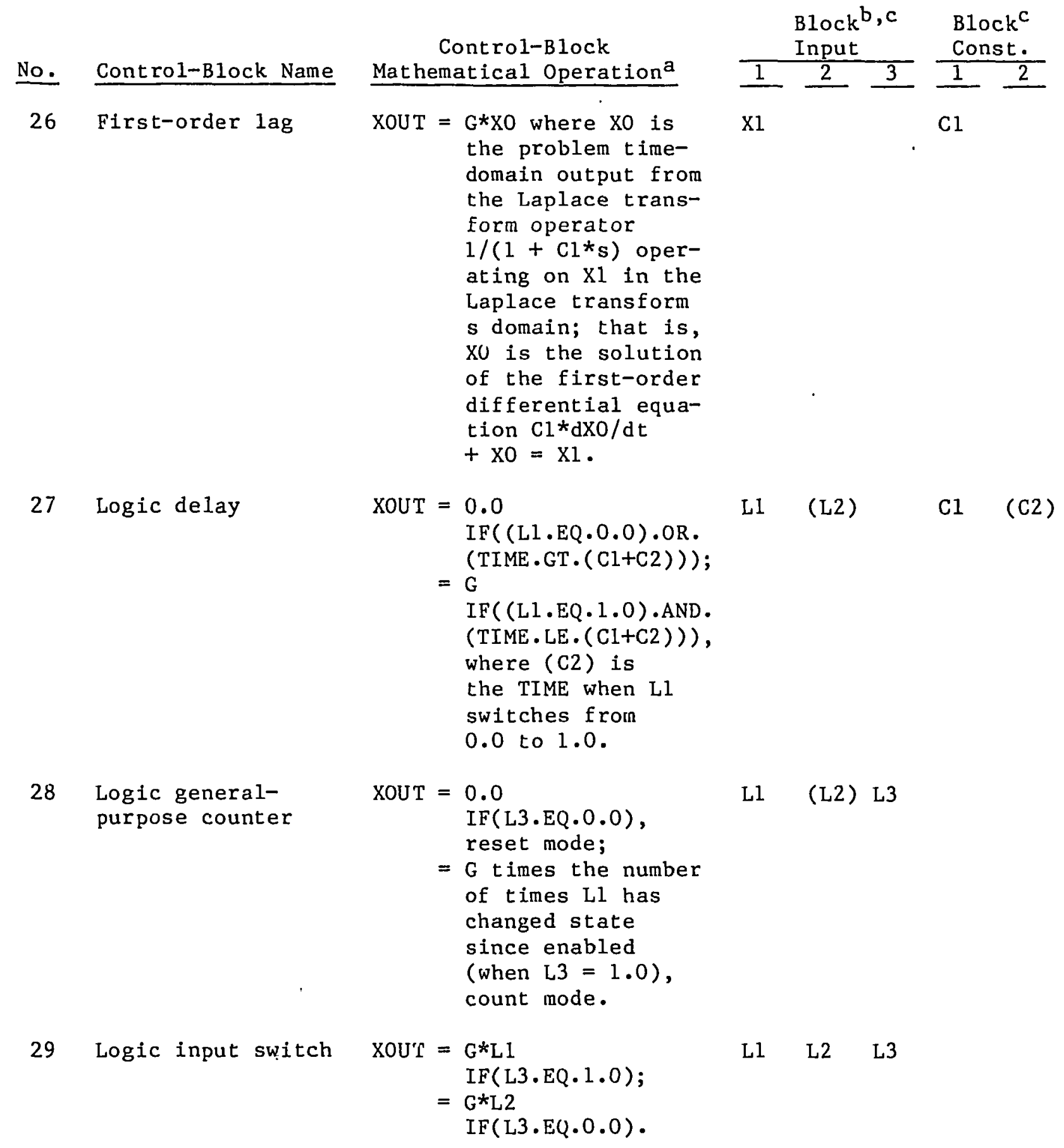




\begin{tabular}{|c|c|c|c|c|c|c|c|c|}
\hline \multirow[b]{2}{*}{ No. } & \multirow[b]{2}{*}{ Control-Block Name } & \multirow{2}{*}{\multicolumn{2}{|c|}{$\begin{array}{l}\text { Control-Block } \\
\text { Mathematical Operationa }\end{array}$}} & \multicolumn{3}{|c|}{$\begin{array}{l}\text { Block }{ }^{b}, c \\
\text { Input }\end{array}$} & \multicolumn{2}{|c|}{$\begin{array}{l}\text { Block } \\
\text { Const. }\end{array}$} \\
\hline & & & & $I$ & 2 & 3 & 1 & 2 \\
\hline 30 & $\begin{array}{l}\text { Lead-lag transfer } \\
\text { function }\end{array}$ & XOUT & 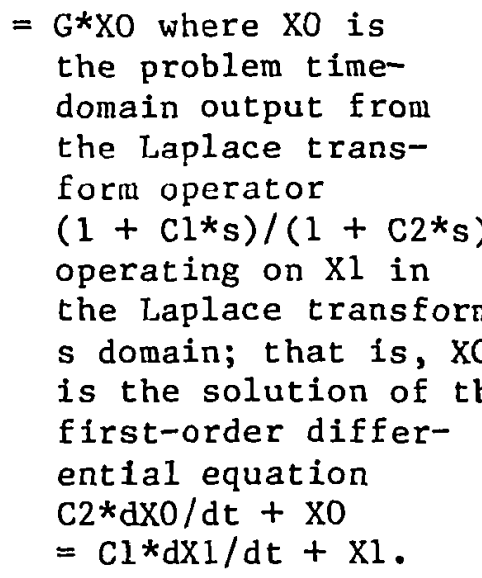 & $\begin{array}{l}n \\
\text { ne } \\
\text { he }\end{array}$ & $(\mathrm{X} 2)$ & (X3) & $\mathrm{Cl}$ & $\mathrm{C} 2$ \\
\hline 31 & Limited integrator & XOUT & $\begin{array}{l}=\mathrm{G} * \mathrm{Xl}{ }^{*} \mathrm{dt}+\mathrm{XOUT} \\
\mathrm{Xl} \text { is set to } 0.0 \text { if } \\
\mathrm{XOUT} \text { is against a } \\
\text { limit and the sign } \\
\text { of } \mathrm{Xl} \text { does not } \\
\text { change; XOUT }=0.0 \\
\text { initially. } \\
\mathrm{C}=0.0 \text { or } 1.0 \\
\text { is the polynomial } \\
\text { order for approxi- } \\
\text { mating the time } \\
\text { dependence of } \mathrm{XI} .\end{array}$ & $\mathrm{X} 1$ & & & $\mathrm{Cl}$ & (C2) \\
\hline 32 & Natural logarithm & XOUT & $=G * A L O G(X 1)$. & $\mathrm{X} 1$ & & & & \\
\hline 33 & $\begin{array}{l}\text { Less than or } \\
\text { equal to }\end{array}$ & XOUT & $\begin{aligned}= & G \\
& I F(X 1 . L E . X 2) ; \\
= & 0.0 \text { otherwise. }\end{aligned}$ & $x 1$ & $\mathrm{X} 2$ & & & \\
\hline 34 & Less than & XOUT & $\begin{aligned}= & G \\
& I F(X 1 . L T . X 2) \\
= & 0.0 \text { otherwise. }\end{aligned}$ & $\mathrm{XI}$ & $\mathrm{x} 2$ & & & \\
\hline 35 & $\begin{array}{l}\text { Maximum of two } \\
\text { signals }\end{array}$ & XOUT & $=G^{*} \operatorname{AMAXI}(X 1, X 2)$ & $\mathrm{X} 1$ & $\mathrm{X} 2$ & & & \\
\hline
\end{tabular}




\begin{tabular}{|c|c|c|c|c|c|c|c|c|}
\hline \multirow[b]{2}{*}{ No. } & \multirow[b]{2}{*}{ Control-Block Name } & \multirow{2}{*}{\multicolumn{2}{|c|}{$\begin{array}{l}\text { Control-Block } \\
\text { Mathematical Operation a }\end{array}$}} & \multicolumn{3}{|c|}{$\begin{array}{l}\text { Block }{ }^{b, c} \\
\text { Input }\end{array}$} & \multicolumn{2}{|c|}{$\begin{array}{l}\text { Block } \\
\text { Const. }\end{array}$} \\
\hline & & & & 1 & 2 & 3 & 1 & 2 \\
\hline 36 & $\begin{array}{l}\text { Maximum during } \\
\text { transient signals }\end{array}$ & XOUT & $=G^{*} \operatorname{AMAX} 1(\mathrm{X} 1, \mathrm{XOUT})$ & $\mathrm{X} 1$ & & & & \\
\hline 37 & Minimum of two & XoUT & $=G^{*} \operatorname{AMTN} 1(X 1, X 2)$. & $\mathrm{x} 1$ & $x 2$ & & & \\
\hline 38 & $\begin{array}{l}\text { Minimum during } \\
\text { transient }\end{array}$ & XOUT & $=G^{*} \operatorname{AMIN} 1(\mathrm{X} 1, \mathrm{XOUT})$ & $\mathrm{X} 1$ & & & & \\
\hline 39 & Multiply & XOUT & $=\mathrm{G} * \mathrm{X} 1 * \mathrm{X} 2$ & $\mathrm{X} 1$ & $\mathrm{x} 2$ & & & \\
\hline 40 & $\begin{array}{l}\text { Logical } \\
\text { "not and" }\end{array}$ & XOUT & $\begin{aligned}= & 0.0 \\
& I F((L 1+L 2) \cdot E Q \cdot 2 \cdot 0) ; \\
= & G \text { otherwise. }\end{aligned}$ & L1 & L2 & & & \\
\hline 41 & $\begin{array}{l}\text { Logical } \\
\text { "not equal" }\end{array}$ & XOUT & $\begin{array}{l}=G \\
=I F(L 1 \cdot N E \cdot L 2) ; \\
=0.0 \text { otherwise. }\end{array}$ & L1 & $\mathrm{L} 2$ & & & \\
\hline 42 & $\begin{array}{l}\text { Logical } \\
\text { "not inclusive or" }\end{array}$ & XOUT & $\begin{array}{l}=G \\
=I F((L 1+L 2) \cdot E Q \cdot 0 \cdot 0) ; \\
=0.0 \text { otherwise. }\end{array}$ & L1 & L2 & & & \\
\hline 43 & $\begin{array}{l}\text { Logical "not" } \\
\text { or negation }\end{array}$ & XOUT & $\begin{aligned}= & G \\
& I F(L 1 \cdot E Q \cdot 0 \cdot 0) ; \\
= & 0.0 \text { IF }(L 1 . E Q \cdot 1.0) .\end{aligned}$ & L1 & & & & \\
\hline 44 & Positive difference & XOUT & $\begin{aligned}= & G^{*}(X 1-X 2) \\
& I F(X 1 \cdot G T \cdot X 2) ; \\
= & 0.0 \text { otherwise. }\end{aligned}$ & $\mathrm{X} 1$ & $\mathrm{x} 2$ & & & \\
\hline 45 & Quantizer & XOUT & $\begin{aligned}= & G * F L O A T \\
& (I F I X(X 1+0.5)) \\
& \text { IF }(X 1 \cdot G E \cdot 0 \cdot 0) ; \\
= & G * F L O A T \\
& (I F I X(X 1-0.5)) \\
& \text { IF }(X 1 \cdot \operatorname{LT} \cdot 0 \cdot 0) .\end{aligned}$ & $\mathrm{X} 1$ & & & & \\
\hline 46 & $\operatorname{Ramp}$ & XOUT & $\begin{aligned}= & G^{*}(\text { TIME }-C I) \\
& \text { IF (TIME.GT.C1 }) ; \\
= & 0.0 \text { otherwise. }\end{aligned}$ & & & & $\mathrm{Cl}$ & \\
\hline
\end{tabular}




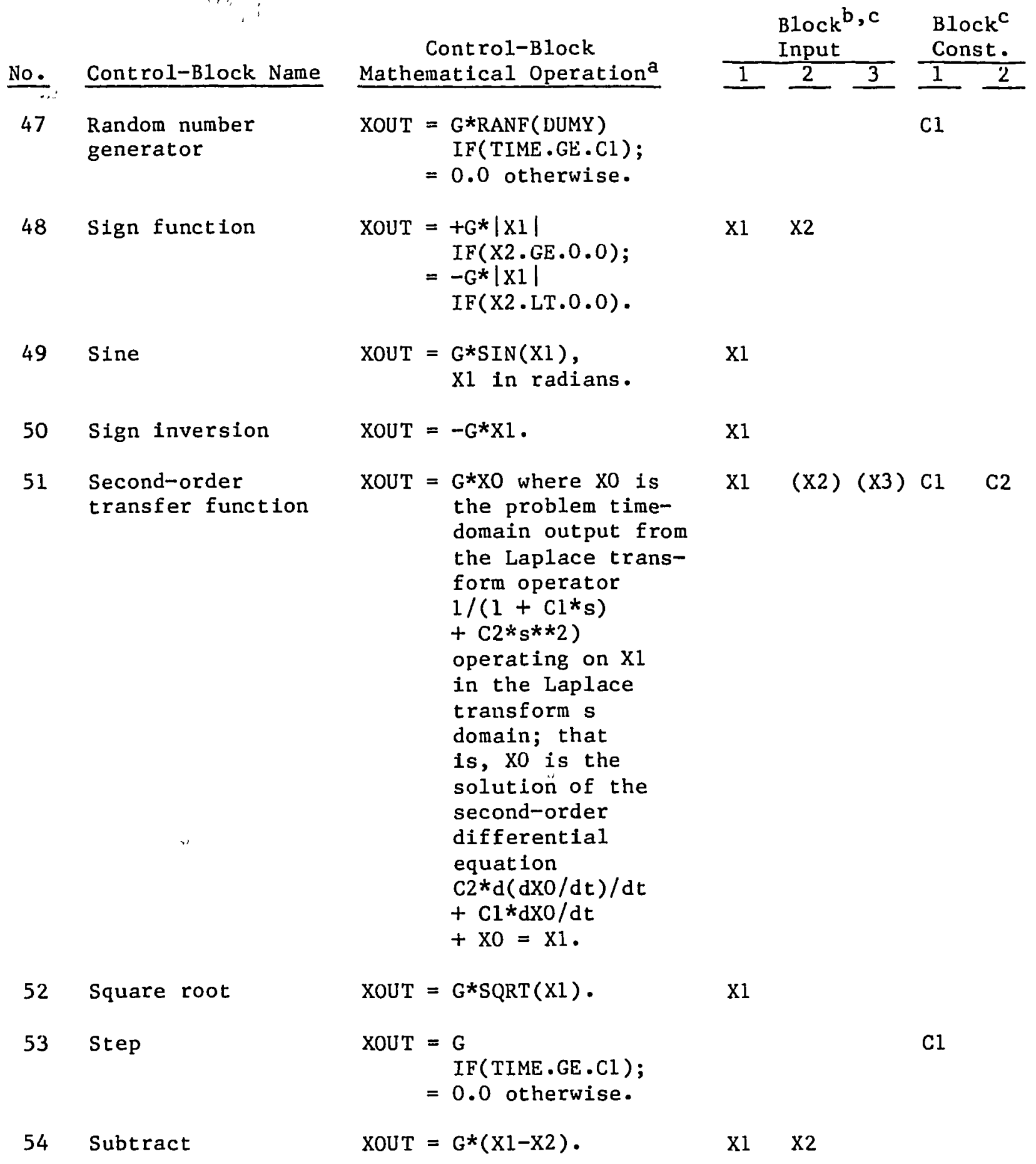


Control-Block

No. Control-Block Name Mathematical Operation ${ }^{a}$

55 Tangent

56 Sum constant

57 Sum three

58 Variable limiter

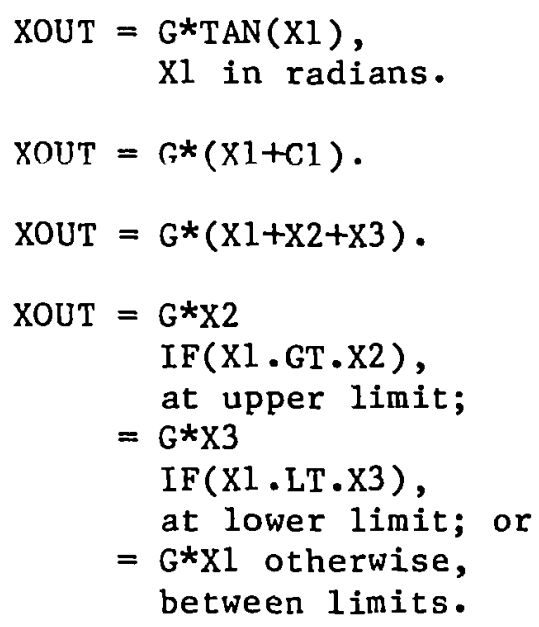

59 Weighted summer

XOUT $=G *(C 1 * X 1+C 2 * X 2)$.

$\mathrm{X} 1 \quad \mathrm{x} 2$

C1 C2

60 Exponentiate

XOUT $=G^{*}(\mathrm{X} 1 * * \mathrm{X} 2), \mathrm{X} 1>0 . \mathrm{X} 1 \mathrm{X} 2$

61 Zero-order hold

XOUT $=G * X 1$

IF (L2.EQ.1.0);

$\mathrm{X} 1 \quad \mathrm{~L} 2$

= XOUT otherwise.

100 Time delay 


\begin{tabular}{|c|c|c|c|c|c|c|c|}
\hline \multirow[b]{2}{*}{ No. } & \multirow[b]{2}{*}{ Control-Block Name } & \multirow{2}{*}{$\begin{array}{l}\text { Control-Block } \\
\text { Mathematical Operation a }\end{array}$} & \multicolumn{3}{|c|}{$\begin{array}{l}\text { Block }{ }^{b}, c \\
\text { Input }\end{array}$} & \multicolumn{2}{|c|}{$\begin{array}{l}\text { Block } \\
\text { Const. }\end{array}$} \\
\hline & & & 1 & 2 & 3 & 1 & 2 \\
\hline 101 & $\begin{array}{l}\text { Function of one } \\
\text { independent } \\
\text { variable }\end{array}$ & $\begin{aligned} \text { XOUT }= & \mathrm{G}^{*} \mathrm{f}_{\mathrm{n}}(\mathrm{X} 1) \\
& \text { The variable } \\
& \mathrm{n} \text { is the number } \\
& \text { of function } \\
& \text { table pairs } \\
& \text { of values to } \\
& \text { be input. }\end{aligned}$ & X1 & $\mathrm{n}$ & $(\mathrm{x} 3)$ & (C1) & \\
\hline 200 & PI Controller & 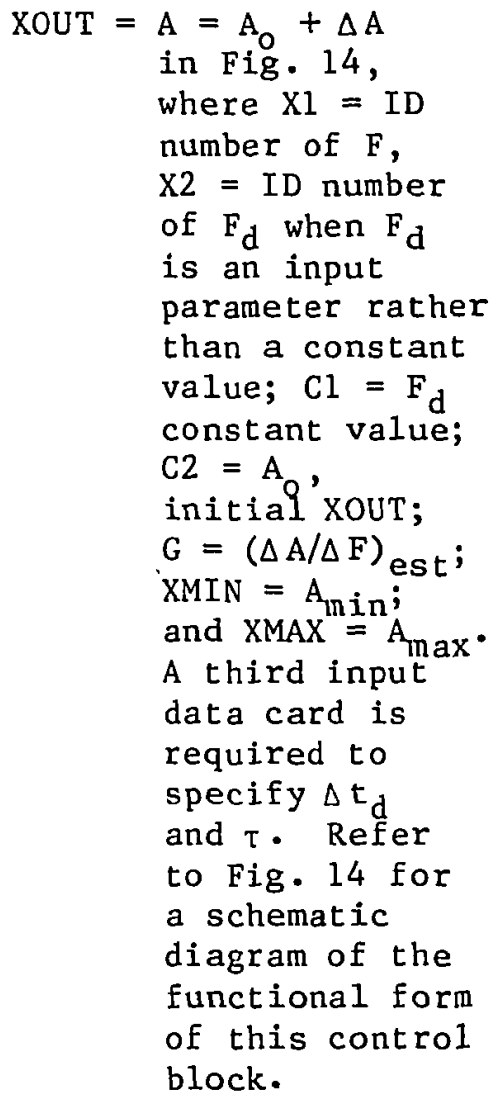 & $\mathrm{X} 1$ & $\mathrm{X} 2$ & & $\mathrm{Cl}$ & $\mathrm{C} 2$ \\
\hline
\end{tabular}


TABLE VIII (cont.)

Control-Block

No. Control-Block Name Mathematical Operationa

201 PID Controller

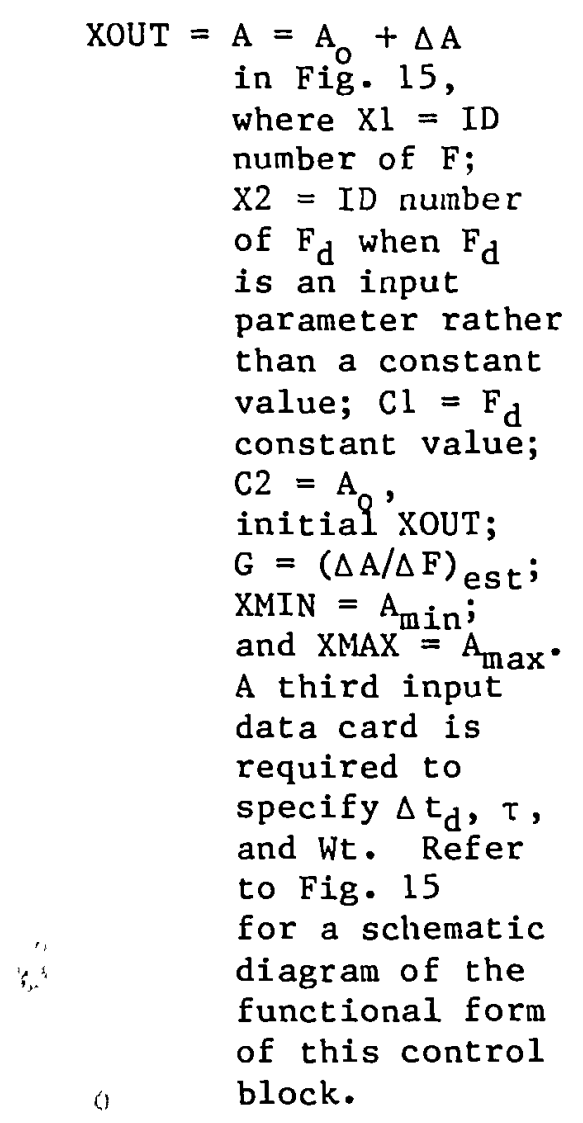

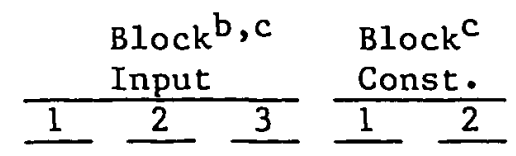

$\mathrm{X} 1 \quad \mathrm{X} 2 \quad(\mathrm{X} 3) \mathrm{C} 1$

$\mathrm{C} 2$

\footnotetext{
${ }^{a}$ XOUT appearing on the right-hand side of the defining equation indicates its value from the previous evaluation. $G$ is a constant gain factor applied to the XOUT operation result. XOUT appearing on the left-"hand side of the defining equation is constrained by constant lower and upper limits XMIN and XMAX in defining the contro1-block output value.
}

bAn "X" parameter indicates a continuous variable; an " $L$ " parameter indicates a logical (or discrete) parameter having a value of 0.0 or 1.0 only.

${ }^{c}$ Variables enclosed in parentheses are not input variables but are used internally by the control block for data storage. 


$$
\Delta A_{F I D}=(\Delta A / \Delta F)_{o s t}\left[\Delta F+(1 / \ddot{\Delta t d}) \int \Delta F d t+\overline{\Delta t_{0}(d \Delta F / d t)+\Delta F}\right]
$$

PID Control Block

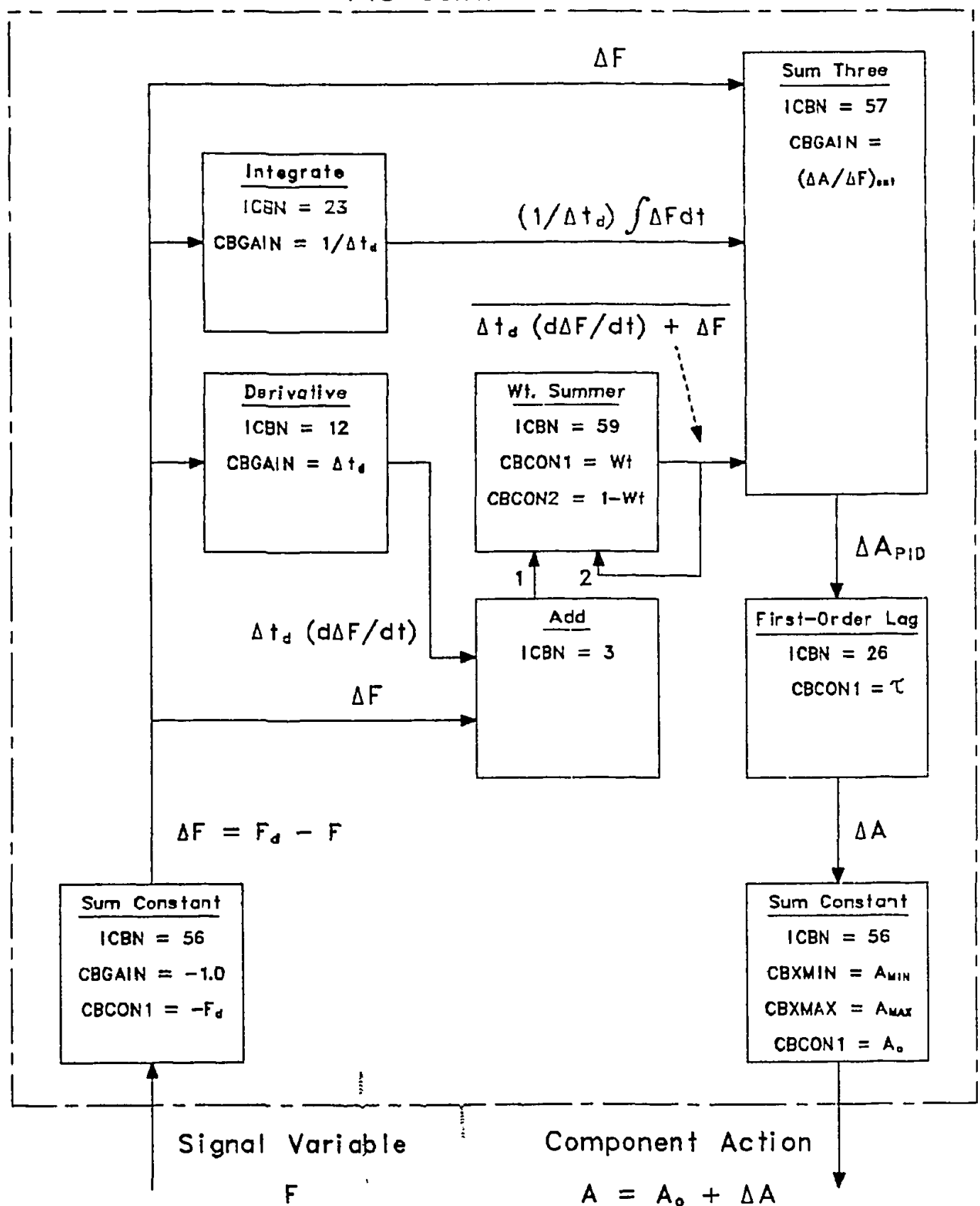

Fig. 14 .

Proportional plus integral controller diagram. 


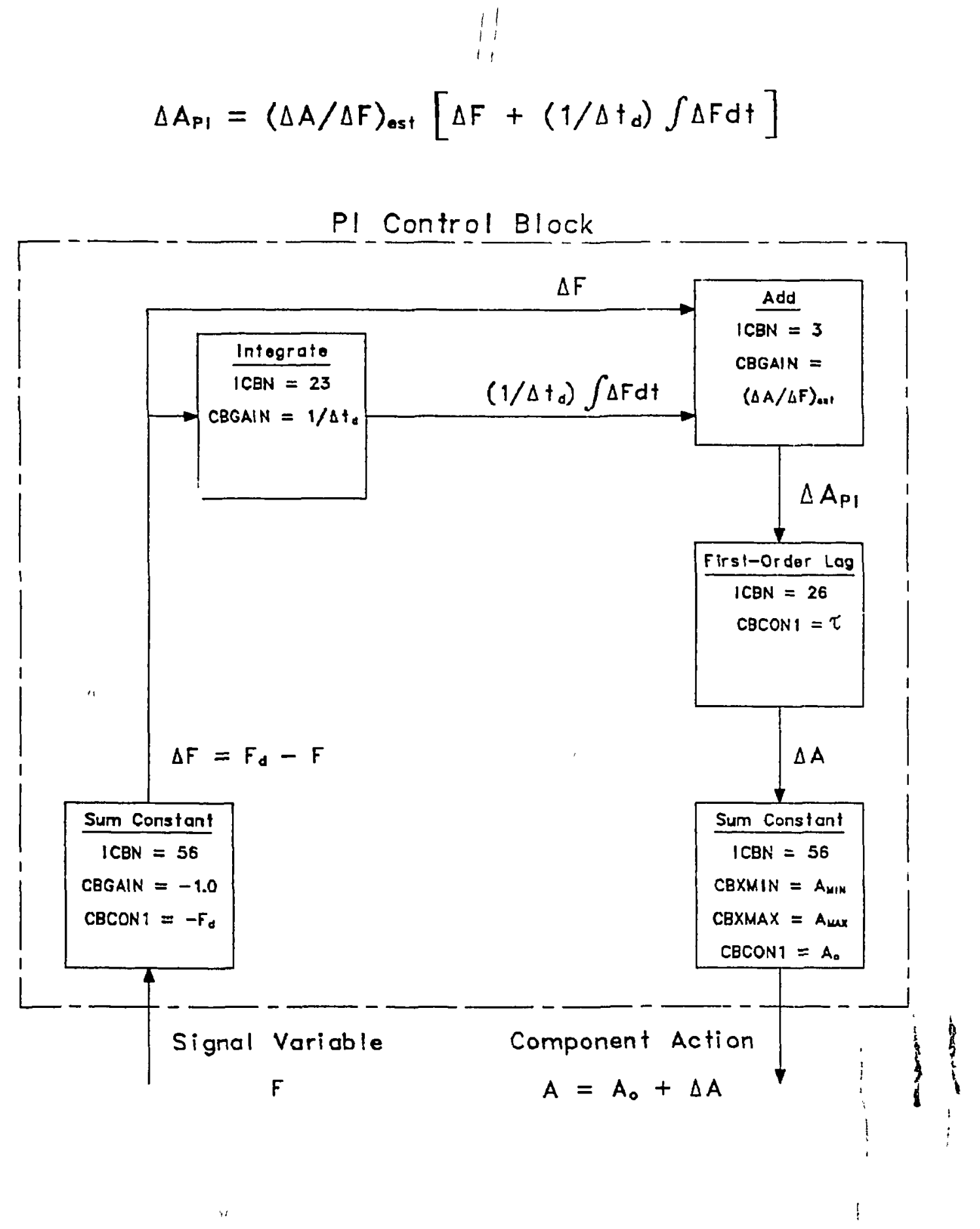

Fig. 15.

Proportional plus integral plus derivative controller diagram. 


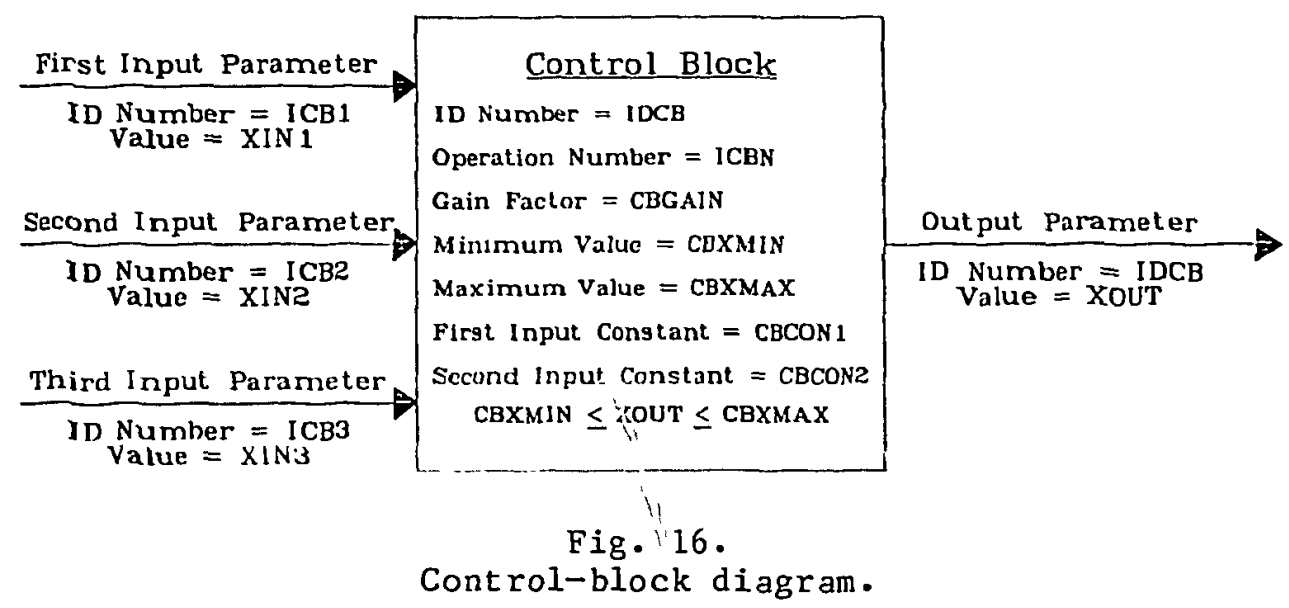

continuously or stepwise depending on the type of control-block operation. To have the output value 0.0 or 1.0 when it is $\operatorname{logical}$ (ICBN $=5,15,16,18,20$, $21,25,27,28,29,33,34,40,41,42$, and 43 ) requires that CBGAIN $=1.0$ be input to the control block.

TRAC-PF1 evaluates control blocks by having driver subroutine CBSET call subroutines CONBLK for ICBN $=1$ to 61 , DELAY for ICBN $=100$, and LININT for ICBN $=101$. Subroutines CONBLK and DELAY are modified forms of subroutines with the same names written by M. Giles and J. Milton for the TRAC-BDI computer program.45 Their control-block operations ICBN $=56$ (defining problem time) and 57 (setting trip status), which are done by signal variable ISVN $=0$ and trips in TRAC-PFl, have been replaced by operations that sum a constant to an input parameter and sum three input parameters. The rectangular integration control blocks $(I C B N=13,23,24$, and 31) have been further generalized to allow the user the option of selecting trapezoidal rule integration instead.

Before evaluating a control block, the signal variables and control blocks that define its input parameters need to be evaluated. When evaluating a control procedure with connected control blocks, the connecting arrows define the direction and order for evaluating the control blocks. Time lag produced by forward difference approximating the integrand (compared with evaluating the input parameters first using a backward difference approximation) has an unstabilizing effect. When the error to be corrected in the signal is increasing, corrective action is numerically delayed by a forward difference approximated integral controller. Eventually when enough 
corrective action has been applied to start decreasing the error signal, the forward difference approximated integral controller delays the removal of corrective action causing the error signal to overshoot its desired zero value. The inherent weakness of integral controllers to overshoot and produce an oscillatory response that can becone unstable 46 is enhanced by evaluating its integral with a forward difference approximation. Using a backward difference approximation has just the opposite effect. Its numerical difference error acts to reduce overshoot by the integral controller. This discussion only applies to the rectangular integration option (which is zeroorder accurate). When trapezoidal rule integration (which is first-order accurate) is used, the input parameters must be evaluated first for the control-block algorithm to evaluate correctly.

Consider the very useful proportional plus integral (PI) controller shown in Fig. 14. The proportional plus integral plus derivative (PID) controller shown in Fig. 15 is an enhancement of the PI controller that uses the derivative to control the rate of the controller adjustment. Both controllers are control blocks that define control procedures for a component actions. The input PWR system parameter $F$ and output component action $A$ could be a pressure to control a valve, a mass flow rate to control a pump, a fluid level to control a feedwater pump or valve, or a pressure to control a heater. The purpose of these controllers is to compare the system paraneter value with its desired value and to apply corrective action to return the system parameter to its desired value when it departs. They could be used to model an actual PI or PID electronic-circuit controller or to adjust a component action to maintain a quoted system parameter value.

The PI controller shown in Fig. 14 performs the following operations. In the lower-left corner of the figure, the sum constant control block subtracts from the PWR system parameter $F$ the desired value $F_{d}$ to define the $\Delta F$ error signal. The next three control blocks estimate the $\Delta A$ change in the component action from its initial value, which should cause $\Delta F$ to go to zero as the calculation proceeds. The integrate control block integrates $\Delta \mathrm{F}$ from the initial time to the present. Its integral represents the accumulated error in $F$. The add control block sums its $\Delta F$ (proportional) and $\int \Delta F d t$ (integral) input signals with appropriate $(\Delta A / \Delta F)$ est and $(\Delta A / \Delta F)_{\text {est }} / \Delta t_{d}$ gain factors to output the PI controller's estimated change in the component action 
that is required from its initial value. $(\Delta A / \Delta F)$ est $/ \Delta t_{d} * \Delta F d t$ corresponds to the $\Delta A_{P I}$ value that has accumulated up to the current time; $(\Delta A / \Delta F)$ est $t^{*} F$ corresponds to the desired instantaneous change in $\Delta A_{P I}$ at the current time that the PI controller estimates is needed to correct for the $\Delta F$ error still remaining. The first-order lag control block accounts for the fact that the $(\triangle A / \Delta F) e^{*} \Delta F$ instantaneous change in a component action may not be physically possible or numerically desirable. The inaximum rate of change in $\Delta A$ is constrained by the CBCON1 time constant, $\tau$, of the first-order lag control block. Through the value of $\tau$, the maximum adjustment rate of the hardware can be modeled. When an instantaneous change in $\Delta A_{P I}$ over a time step is desired, the first-order lag control block evaluates a slower change in $\Delta \mathrm{A}$ based on the first-order differential equation $\tau * d \Delta A / d t+\Delta A=\Delta A_{P I}$. The value of $\triangle A$ will lag the value of $\triangle A P I$ in time by an amount based on the magnitude of $\tau$. Finally, the sum constant control block in the lower-right corner of the figure sums the accumulated $\triangle \mathrm{A}$ change in the component action since the start of the calculation to the component-action initial value, $A_{0}$. For this controller, the output signal $A$ is applied directly to the component action by defining a component-action table with no entry pairs, NXXXTB $=0$.

5. Trips. Trips are ON-OFF logical switches for controlling the evaluation of component actions. When the controlling trip for a component action is $\mathrm{ON}$, the component action is evaluated from the definition of its component-action table. When the controlling trip is OFF, the component action is not evaluated; it is held constant at its initial value if its trip has never been ON or optionally at its last evaluated value when its trip was ON or at a user-defined input value for when its trip is OFF after being ON". Trips also can be used to implement special time-step data, generate restartdata dumps, and terminate problem execution at the time the trip is set oN.

Determining the ON or OFF set status of a trip is based on the value of its user-defined trip signal. Over the trip signal value range, the user defines two or four set-point values that delimit two or three subranges labeled with $O N$ or $O F F$ set status. When the value of the trip signal lies within a given subrange, the trip is assigned the set-status label of that subrange. Table IX shows five of the ten possible trip signal-range types that define set status. The $O N$ set status has two forms: $\mathrm{N}_{\text {forward }}$ and

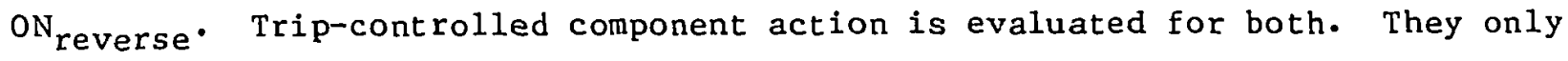


TABLE IX

TRIP SIGNAL-RANGE TYPES

ISRT

Signa1-Range

Type Number

Trip Signa1-Range Diagram With Subrange set Status

1

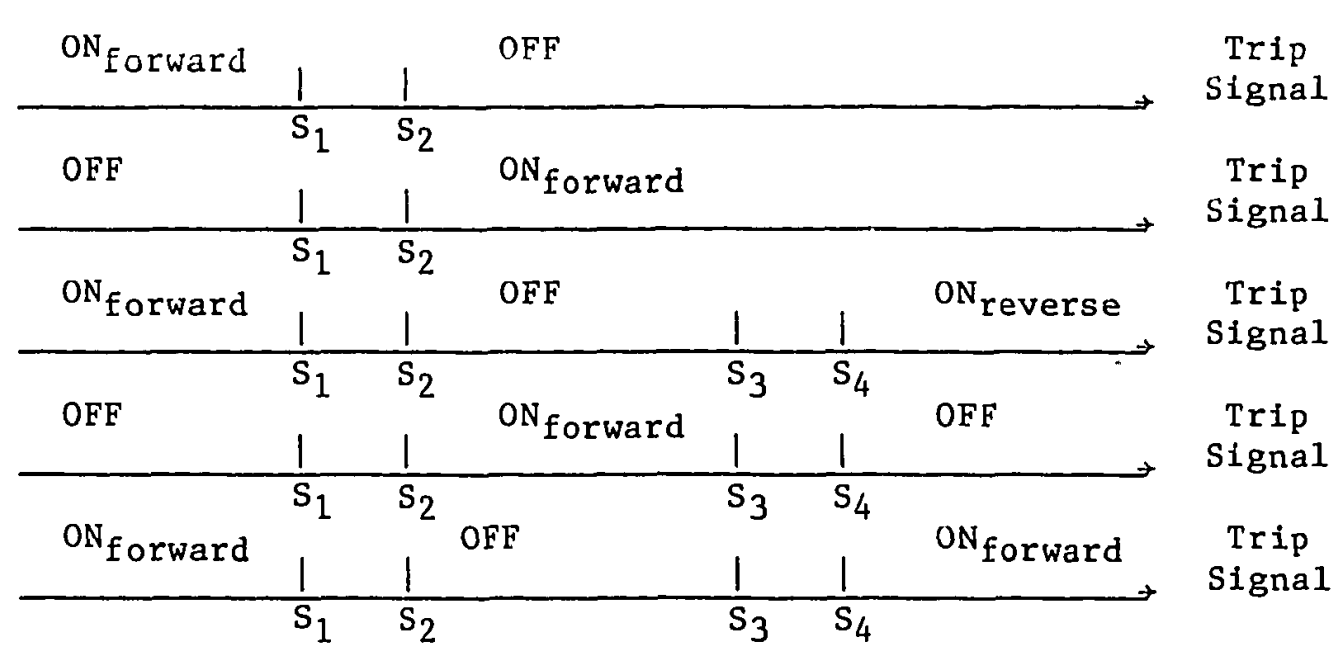

Making ISRT negative changes $\mathrm{ON}_{\text {forward }}$ to $\mathrm{ON}_{\text {reverse }}$ and $\mathrm{ON}_{\text {reverse }}$ to $\mathrm{ON}_{\text {forward }}$.

ISRT

Signal-Range

Type Number

Description of How the Trip Set Status Is Changed

1

When $\mathrm{ON}_{\text {forward }}$ trip is set to OFF when the trip signal $2 \mathrm{~S}_{2}$. When OFF, trip is set to $\mathrm{ON}_{\text {forward }}$ when the trip signal $\leq \mathrm{s}_{1}$.

2

When OFF, trip is set to $\mathrm{ON}_{\text {forward }}$ when the trip signal $2 \mathrm{~s}_{2}$. When $\mathrm{N}_{\text {forward }}$, trip is set to OFF when the trip signal $\leq s_{1}$.

When $\mathrm{ON}_{\text {forward, }}$ trip is set to OFF when trip signal $2 \mathrm{~s}_{2}$ and $\left\langle\mathrm{S}_{4}\right.$. When $\mathrm{ON}_{\text {forward, }}$ trip is set to $\mathrm{ON}_{\text {reverse }}$ when trip signal $2 \mathrm{~S}_{4}$. When OFF, the trip is set to $O N_{\text {forward }}$ when the trip signal $\leq \mathrm{s}_{1}$. When OFF, the trip is set to ON reverse when the trip signal $2 s_{4}$. When $\mathrm{ON}_{\text {reverse, }}$ trip is set to $\mathrm{OFF}$ when trip signal $\leq \mathrm{s}_{3}$ and $>\mathrm{S}_{1}$. When $\mathrm{ON}_{\text {reverse, }}$ trip is set to $\mathrm{ON}_{\text {forward }}$ when trip signal $\leq \mathrm{s}_{1}$.

When OFF (left), trip is set to ON $\mathrm{N}_{\text {forward }}$ when signal $2 \mathrm{~s}_{2}$ and $<\mathrm{s}_{4}$. When ON forward, trip is set to OFF when trip signal $\leq \mathrm{s}_{1}$ or $2 \mathrm{~S}_{4}$; When OFF( right), trip is set to $\mathrm{ON}_{\text {forward }}$ when signal $\bar{S} s_{3}$ and $>s_{1}$.

When $O N_{\text {forward }}\left(\right.$ left), trip is set to OFF when signal $2 s_{2}$ and $<s_{4}$. When OFF, trip is set to ON forward when trip signal $\leq s_{1}$ or $2 s_{4}$. When $\mathrm{ON}_{\text {forward }}$ ( $r i g h t$ ), trip is set to OFF when signal $s s_{3}$ and $>s_{1}$. 
differ with regard to the sign of the numerical value 1 for the on set status. The numerical values associated with the three set-status flags in Table IX are -1 for $O N_{\text {reverse, }} 0$ for $O F F$, and +1 for $\mathrm{ON}_{\text {forward }}$.

Input variable ISRT defines the signal-range type for a trip. Table IX shows the signal-range types for ISRT $=1,2,3,4$, and 5 . Assigning a negative sign to these ISRT values and changing $\mathrm{ON}_{\text {forward }}$ to $\mathrm{ON}_{\text {reverse }}$ and $\mathrm{ON}_{\text {reverse }}$ to $\mathrm{ON}_{\text {forward }}$ defines the other five types. The criteria to decide when the set status of a trip is to be changed are stated in the lower half of Table IX. The present set status of the trip determines which criterion is to be used. The criteria and signal-range type definitions are based on the requirement that the set-point values satisfy $S_{1}<S_{2}$ for ISRT $= \pm 1$ and \pm 2 and $s_{1}<s_{2}<s_{3}<s_{4}$ for ISRT $= \pm 3$, \pm 4 , and \pm 5 .

For ISRT $= \pm 1$ and \pm 2 , there are two set-status subranges. The trip signal needs to be equal or greater than the $S_{2}$ set-point value to change from the left to the right subrange set status. To change from the right to the left subrange set status, the trip signal needs to be equal or less than $s_{1}$. When the trip signal value lies between $s_{1}$ and $s_{2}$, the trip has the set status of the left or right subrange from which its signal cane.

For ISRT $= \pm 3, \pm 4$, and \pm 5 , there are three set-status subranges. Changing to either of the other two set-status labels is determined in much the same way as for a two-subrange signal-range type. The trip-signal value is compared with $S_{1}$ or $S_{2}$ with a decision to change to left or middle set status and compared with $S_{3}$ or $S_{4}$ with a decision to change to the middle or right set status. The trip-signal value crossing two subranges during a time step will result in the set-status criteria being satisfied twice even though the last criterion being satisfied is the only one edited by TRAC for the time step.

Defining a trip signal range to have more than three set-status subranges can be done by joining two or nore trips having the same trip signal and different set-point values. A trip-controlled trip (defined later in this section) is used to join them and to respond as if it were a four or more setstatus subrange trip. Whereas trips with three set-status subranges are defined directly by ISRT $= \pm 3, \pm 4$, and \pm 5 , they could be constructed by joining two trips having two set-status subranges with a trip-controlled trip. Three set-status subrange trips have been defined directly, however, because 
they are a convenient form for regulating a component action under trip control and save our having to define three trips to achieve that form.

Signa1-range type ISRT $= \pm 3$ is the form generally used to regulate a component action under trip control. The trip signal is the controlling parameter, and the component action is to be adjusted so that the trip signal lies within its desired value range from $S_{1}$ to $S_{4}$. When the trip signal falls below $S_{1}$, the left-subrange $O N$ set status has the component action evaluated in a manner that causes the trip signal value to increase. When the trip signal rises above $S_{4}$, the right-subrange $O N$ set status adjusts the component action in the opposite manner to cause the trip signal value to decrease. For this application, the component-action table needs to have its independent-variable form defined by the change in its IXXXSV variable value so that the ISET set-status value is applied as a factor to the independent variable of Table VI. This is done by defining the number of entry pairs in the component-action table to be negative, that is, $\operatorname{NXXXTB}<0$. As described above, the set-point values define the desired value range for the trip signal. The range can be as small or as large as the user feels is appropriate for constraining the trip-signal value based on trip control of the component action.

Each trip-signal set point is defined by a constant value with the possibility of a tabular function being applied to that value as a factor. The user inputs for each set point a constant set-point value to array variable $\operatorname{SETP}(I), \quad I=(1,2)$ or $I=(1,4) \quad$ [that satisfies $S_{1}<s_{2}$ or $\left.s_{1}<s_{2}<s_{3}<s_{4}\right]$, and an IO flag to array variable IFSP(I) to indicate if a tabular function factor is to be applied to the SETP(I) value. No such factor is applied when IFSP(I) $=0$; the set-point value is the constant $\operatorname{SETP}(I)$. Entering IFSP(I) $\neq 0$ defines the ID number for a set-point factor table of tabular data to be input after the trip and trip signal defining data. The set-point factor-table independent variable is defined by a signal variable or a control-block output variable. The variable value and not the change in the variable value defines the independent variable of the table. When a setpoint factor is to be applied to a set point, its table is linearly interpolated for each time step on the basis of the abscissa-coordinate signal-variable or control block output variable value. The input SETP(I) constant value is multiplied by the interpolated function value to determine 
the set-point value that is used. Set-point factor tables are useful when set points for regulating a component-action change on the basis of some system paraneter dependence. For example, the desired water level in the steamgenerator-secondary downcomer might depend on the reactor power level. A trip with varying water-level set-point values, dependent on the reactor power level, could be used to control the secondary-loop feedwater pump or valve.

Associated with each set point is a delay time defined by inpul array variable $\operatorname{DTSP}(I), I=(1,2)$ or $I=(1,4)$. The delay time provides a second criterion that needs to be satisfied before the trip set status is changed. The first criterion requires that the trip-signal value reach or cross the set-point value tested. Once that criterion is met, the second criterion then requires that the delay time of that set-point pass before changing the trip set status. With a delay time, the user is able to model the time necessary for the system hardware or the control room operator to process the controlling signal and initiate hardware action. Delay time should be defined with a nonnegative value (negative values are treated as zero). TRAC edits a message when each criterion is satisfied by a trip. For large delay times, it is possible for a number of first-criterion tests to be satisfied before their delay times pass. TRAC can handle up to five such delay-time pending setstatus changes stacking up before the next one is forced to replace the last one in the stack. When it does, a warning message is printed. For a delay time-pending set-status change to stack up, the time its first criterion was met (based on linear interpolation of the trip-signal value to equal the setpoint value) plus its delay time must be later than all pending times higher up in the stack. When that time is equal to or less than one or more pending times higher in the stack, they are replaced by this new entry. Through this mechanism, the user can prevent temporary spikes in a trip signal froin setting a trip $\mathrm{ON}$ and its component action being evaluated. The user does this by defining shorter delay times for the set points that turn a trip OFF than for the set points that turn a trip ON. The difference between these two delay times is the required time interval the trip signal value needs to stay in the ON subrange to prevent a satisfied OFF criterion that follows frou replacing its pending ON set-status change in the stack. 
There are three forms for defining the trip signal: a signal parameter, a signal expression, or a trip set-status signal. Two input variables define the trip signal: ITST defines the trip-signal type number and IDSG defines the trip-signal ID number. A "signal-parameter" trip is defined by ITST = 1 and has a signal-parameter trip signal. That signal parameter is a signal variable with ID number IDSG when IDSG >0 or a control-block output variable with ID number IDSG when IDSG < 0 . A "signal-expression" trip is defined by ITST $=2$ and has a signal-expression trip signal. The trip signal is an arithmetic expression operating on the values of one or more signal variables and control-block output variables. Its definition is input as trip-signalexpression input data with ID number IDSG. The form for that definition is described in the next paragraph. A trip-controlled trip (or "trip set-status signal" trip) is defined by ITST $=3$ and has a trip set-status signal. This signal is the sum or product of the set-status values of two or more other trips. The ID numbers of those trips are input as trip-controlled trip-signal data with ID number IDSG. The set-status values of those trips are to be summed if IDSG $>0$ or multiplied if IDSG $<0$ to evaluate the trip-controlled trip signal.

Evaluating a signal-expression trip signal involves evaluating an arithmetic expression with one to ten arithmetic subexpressions. Each subexpression has an arithmetic operator and two arguments. The arithmetic operators are addition, subtraction, multiplication, division, exponentiation, maximum value, minimum value, and absolute value. Argument values are defined by signal variables, control-block output variables, input constants, or an earlier evaluated subexpression in the list of subexpressions. The value of the signal expression is the value of the last subexpression in its list. Through its arithmetic operators, a signal expression allows signal variables from different components of the modeled PWR system to be combined in defining the trip signal. Signal variables that define a subexpression argument can be flagged in the signal-expression definition for reevaluation only at the tines its signal-expression trip is set $0 N$. In this way, signal variables evaluated at different times as well as in different components can be combined in defining a trip signal. 
The AND or OR logic of blocking or coincidence trips can be implemented with trip-controlled trips. Applying the AND logical operator defines a blocking trip that is $O N$ only if all the trips assigned to it are $O N$. The trip-controlled trip that has this form multiplies (IDSG $<0$ ) the set-status values of its assigned trips in defining the trip signal and uses a ISRT $=2$ signal-range type with $0<s_{1}<s_{2}<1$. It turns out, in this case, that the trip-controlled trip signal has the same value ( 0 or 1 ) as its subrange setstatus value. Applying the OR logical operator defines a coincidence trip that is $O N$ only if one or more of the assigned trips are $O N$. Its corresponding trip-controlled trip adds (IDSG $>0$ ) the set-status values of its assigned trips and uses the same signal-range type and set-point values as above. For a more general coincidence trip, we specify that a number $N$ or more (where $1 \leq \mathrm{N} \leq \mathrm{N}_{t}$ ) of the $\mathrm{N}_{t}$ assigned trips need to be $\mathrm{ON}$ for the coincidence trip to be $O N$. Within AND or OR logic, this requires logical expressions having logical subexpressions. For example, if $\mathrm{N}=2$ of $\mathrm{N}_{t}=3$ assigned trips need to be ON for the coincidence trip to be ON, its logical expression would need to have the form: (Trip \#1 AND Trip \#2) OK (Trip \#2 AND Trip \#3) OR (Trip \#3 AND Trip \#1). The form of this logical expression will be different for different values of $\mathrm{N}$ or $\mathrm{N}_{t}$. With trip-controlled trips, the required form is the suln of the set-status values of its assigned trips in all cases. The only difference is in defining the set points according to $(N-1)<s_{1}<s_{2}<N$. In this case, the trip-controlled trip signal value, which is the number of assigned trips that are $O N$, needs to be greater than $S_{2}$ (that is, the value $N$ or greater) to meet the criterion for setting the tripcontrolled trip ON.

The three trip-controlled trip examples above barely scratch the surface of the number of ways trip-controlled trips can be used to model AND/OR logical expressions. Switching from a ISRT $=2$ to a ISRT $=1$ signal-range type applies a NOT logical operator to the logical expression being modeled. Assigning a"trip-controlled trip to the definition of another trip-controlled trip signal provides further modeling flexibility. The above discussion assumes that the $\mathrm{ON}$ set status of the assigned trips is $\mathrm{ON}_{\text {forward }}$. Assigning

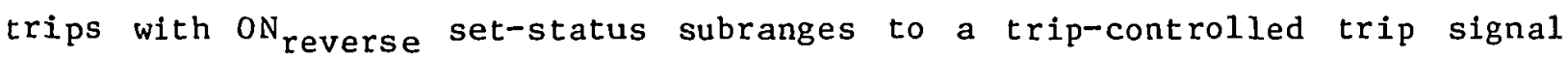
provides another dimension for modeling possible logical expressions. 
During input, trips are given ID numbers that assign them to the following functions:

1. to control component actions by defining their ID numbers to the general form variables IXXXTR in the component input data,

2. to define trip-controlled trip signals with their set-status values, and

3. to define the trip signals or set-status values by signal variables. Trip ID numbers of magnitude 1 to 9999 are allowed. A negative ID number defines a trip that is to be evaluated during steady-state and transient calculations. A positive ID number trip, on the other hand, is evaluated only during transient calculations. During steady-state calculations, the setstatus value specifled by input-variable ISET and an initialized trip signal value of $10^{20}$ define a positive-ID trip constant state. A positive-ID trip can be used as a blocking trip during a steady-state or transient calculation when the trip-evaluated set status is the opposite of its input ISET value.

6. Control-Parameter Evaluation. Signal variables, control blocks, and trips are assigned ID numbers when input by the user. Their ID numbers provide the means for referencing their values for use in the control procedure. Their ID numbers also define the order in which they are to be evaluated. Within each control-parameter type (signal variables, control blocks, and trips), parameters are evaluated in the order of increasing magnitude of their ID numbers. Thus, control blocks are ordered for evaluation with their ID numbers decreasing in negative value; trips are ordered with their ID numbers increasing in magnitude with either a plus or minus sign. This is done by subroutine ORDER after the control-parameter data are input, and the control-parameter restart data with ID numbers different from those input are added on. For example, a control-block input with ID number -5 would replace a control block with the same ID number from the restart file. Subroutine ORDER would reorder the list of control blocks so that control block ID $=-5$ would be placed before control blocks with IDs $<-6$ and after control blocks with IDs $>-5$. A trip input with ID'number 11 would replace a trip with the same ID from the restart file but not a trip with ID number -11. For the case of two trips having ID numbers with the same magnitude, that is, 11 and -11 , subroutine ORDER does not change their relative positions in the trip list. With trip $I D=11$ input and trip 
ID $=-11$ added on to the trip list from the restart file, trip ID $=-11$ would follow after trip ID $=11$ in the reordered list of trips. Subroutine ORDER is called once at the end of input (and restart) data processing to reorder the signal variable, control block, and trip lists. This saves having to search through ID numbers to determine that order when the control parameters are evaluated at the beginning of each time step.

Through ID numbers, the order for evaluating control parameters of a given type is defined. The order for evaluating control-parameter types is by default signal variables first, then control blocks, and finally trips. Since signal variables have almost all of their parameters defined by the modeled PWR systern, it is appropriate to evaluate them first. The input parameters for control blocks are signal variables and control blocks that, with proper ordering of control blocks, means control blocks should be evaluated after signal variables. Trip signals are defined by signal variables, control blocks, ard the set status of other trips. With proper ordering of trips, evaluation of trip signals should follow the evaluation of signal variables and control blocks.

In general, this approach is able to evaluate control-parameter values for the beginning of the time-step state of the system. There are a few situations, however, where such is not the case. Defining a trip signal or set status to be a signal variable (ISVN $=55$ or 56 ) will give its value at the beginning of the previous time step when the signal variables are evaluated before their trips. In this case, they should be evaluated after their trips. A control-block controller with an implicit feedback loop (Fig. 17) is another exception. To evaluate $x$ with the Add operator control block, $1 n(X)$ and thus $X$ need be known. By evaluating each of the two control blocks once, no matter which is evaluated first, the value of $X$ used to, evaluate $\ln X$ will be one time step behind the value that should be used. Accumulation of such error over many time steps can cause the value of $X$ to significantly lag its actual value in time. Instability can even result from that error. TRAC has not as yet been programmed to recognize such implicitloop structures in order to implement internally an iterative loop evaluation able to determine the consistent solution state. 


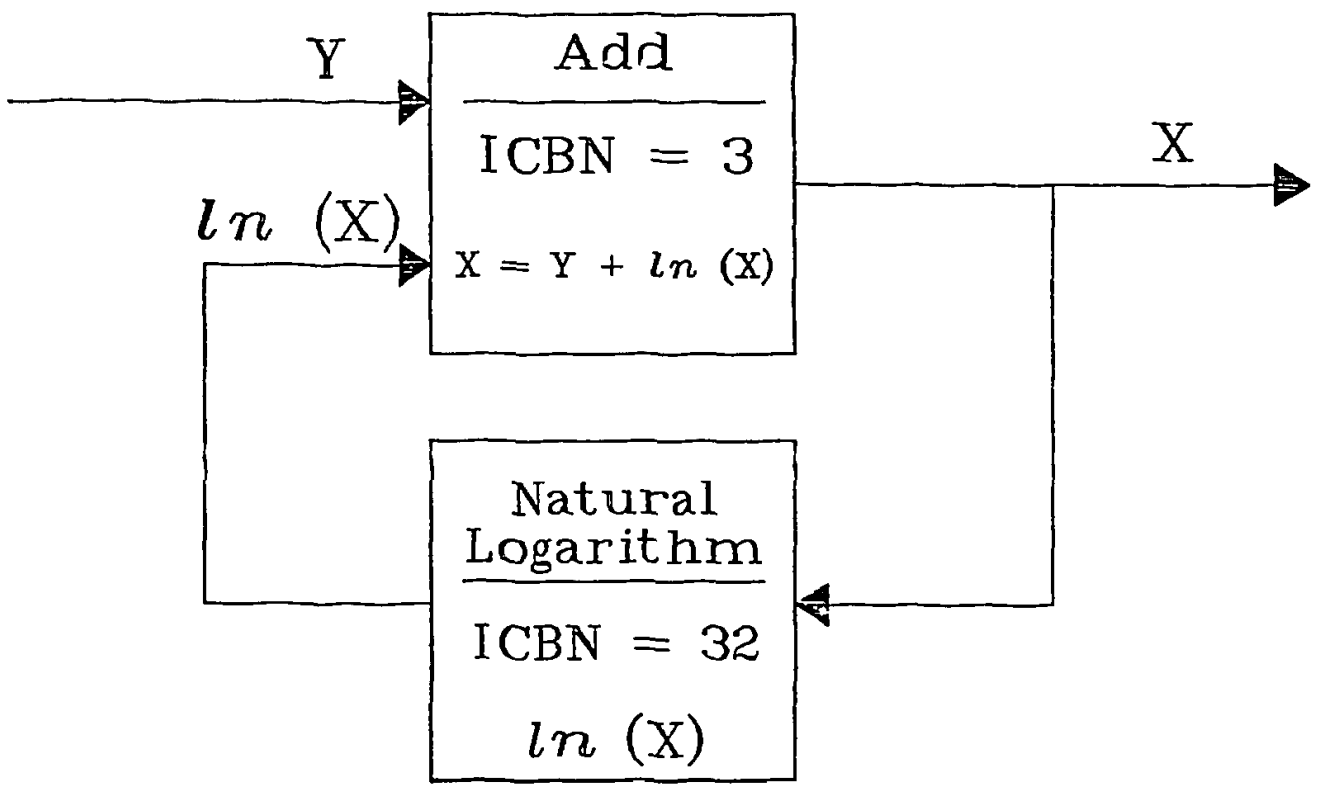

Fig. 17.

Implicit control-block loop example.

A multipass control-parameter evaluation procedure has been programued in TRAC to aid the user in addressing the above two situations. Within each evaluation pass, the user decides which signal variables, control blocks, and trips (in that order) are to be evaluated. Trip-signal and set-status signal variables can then be evaluated on a second control-parameter evaluation pass after their defining trips were evaluated on the first pass. The implicit control-block loop can be reevaluated with as many passes as the user decides are needed for convergence. On the last loop evaluation pass, all control blocks whose input parameters are affected by the loop evaluation would then be evaluated. During each of the passes, the user specifies which control parameters, if any, are to be evaluated for each type. Those parameters that are evaluated span a range of ID numbers that the user specifies. All control parameters with ID numbers in that range are evaluated during that pass. An example of such a procedure follows. Assume the following control parameters are to be evaluated:

1. signal variables with IDs 3 to 35 define system (component) parameters and signal variables with IDs 39 to 72 define signal and set status from trips with IDs \pm 7 to \pm 18 as well as system parameters. 
2. control blocks with IDs -1 to -12 require input parameters from signal variables with IDs 3 to 17 and provide input paraneters for control blocks in an irplicit loop, control blocks with IDs -13 to -17 are in an implicit loop for which five iterative evaluations are felt to be sufficient to converge on a consistent solution state and control blocks with IDs -18 to -42 require input parameters from the implicit loop control blocks and frotn signal variables.

3. trips with IDs $\leqslant 1$ to \pm 18 require signal variables with IDs 3 to 26 and control blocks with IDs -6 to -10 to define their signals and trips with IDs \pm 19 to \pm 30 require signal variables with IDs 18 to 72 and control blocks with IDs -5 to -40 to define their signals.

Evaluating these control parameters could be done with the five evaluation passes shown in Table $X$. After five passes (assuming convergence of the implicit loop evaluation) all control parameters would have values consistent with each other and the beginning of tine-step state of the system.

\section{TABLE $X$}

ID NUMBERS OF CONTROL PARAMETERS EVALUATED DURING EACH OF FIVE CONTROL-PARAMETER EVALUATION PASSES

\begin{tabular}{|c|c|c|c|}
\hline $\begin{array}{l}\text { Evaluation } \\
\text { Pass Number }\end{array}$ & $\begin{array}{c}\text { Signal } \\
\text { Variables }\end{array}$ & $\begin{array}{l}\text { Cont rol } \\
\text { Blocks }\end{array}$ & Trips \\
\hline 1 & 3 to 35 & -1 to -17 & \pm 1 to \pm 18 \\
\hline 2 & 39 to 72 & -13 to -17 & none \\
\hline 3 & none & -13 to -17 & none \\
\hline 4 & none & -13 to -17 & - none \\
\hline 5 & none & -13 to -42 & \pm 19 to \pm 30 \\
\hline
\end{tabular}


IV. COMPONENT MODELS

This section describes the TRAC-PFl/MODl component models. A physical description of each component is presented along with a typical TRAC noding diagram showing the conventions that are used to model the component. Mathematical models including finite-difference approximations are given only for those aspects of the component that are not covered in the basic hydrodynamics and heat-transfer descriptions (Sec. III). User options, restrictions on the use of the component, subroutines used by the component, and input/output information also are given. Detailed input specifications for each component are given in Sec. V.

A. Pipe

The PIPE component models the flow in a one-dimensional duct or pipe. A PIPE can be used alone in a problem, or it can be used as a connector between components to model a reactor system. The capability is provided to model area changes, wall heat sources, and heat transfer across the inner and outer wall surfaces. A wide selection of pipe materials is available within the code to represent the wall material in the wall-conduction calculation; additionally, the user can specify material properties in the input.

Figure 18 shows a typical noding diagram for a PIPE containing a venturi tube and an abrupt area change. The numbers within the PIPE indicate cell numbers, and those above indicate cell-boundary numbers. The geometry is specified by providing a volume and length for each cell and a flow area and hydraulic diameter at each cell boundary. The junction variables, JUNI and JUN2, provide reference numbers for connecting a pipe to other components. The numerical methods used to treat the thermal hydraulics in a PIPE are described in Sec. III.A.3.

Input options are available to allow for wall heat transfer, to use the full boiling curve, or to restrict heat transfer to forced convection, and to select wall-friction factors. Wall heat transfer can be onitted by setting the number of heat-transfer nodes (NODES) to zero. The CHF calculation can be bypassed by setting the input parameter ICHF to zero. Wall friction and losses caused by abrupt area changes are chosen by setting values of the input arrays, NFF and FRIC, at each cell interface. The choices for these arrays are described in the input specifications in Sec. V.D.5.e. 


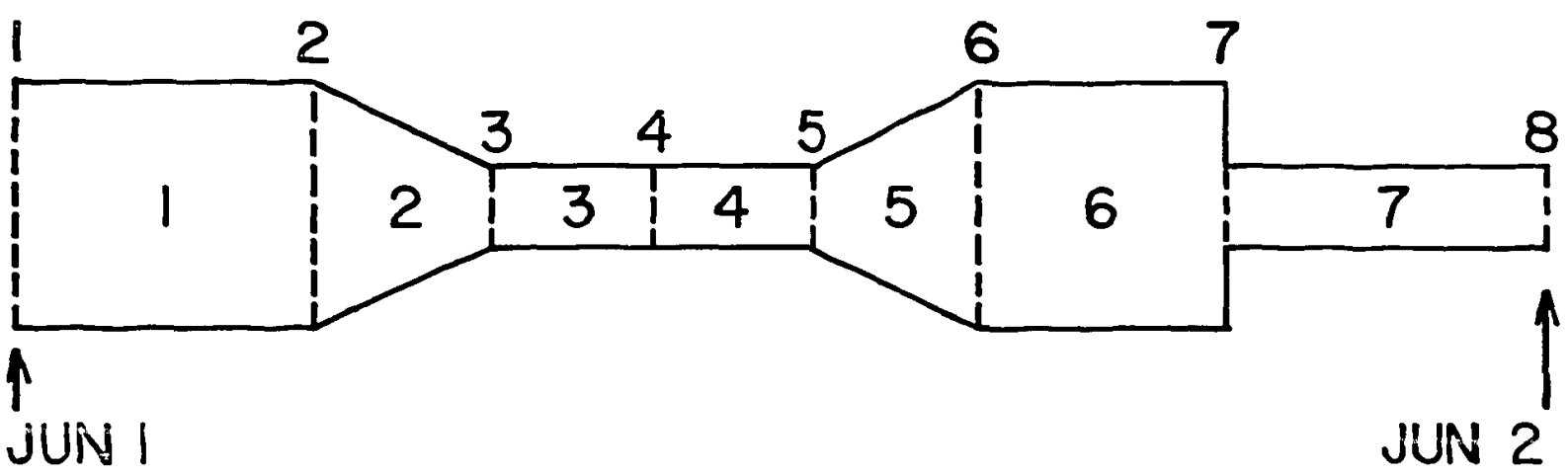

Fig. 18.

Pipe noding diagram.

By our setting NPOWTB to a nonzero number and NODES to zero, heat can be provided to a PIPE by a table of power vs time. The table provides total power, which is evenly distributed among all of the cells.

By setting parameter IACC to 1 or 2 (see PIPE input, Sec.V.C.5.e), a PIPE can be used to model an accumulator (ACCUM) component, discussed in Sec. IV.B.

Although a PIPE can be connected to any other component, including another PIPE, the user should keep the number of components to a minimum.

Detailed input for a PIPE module is processed by subroutines RPIPE and REPIPE. Subroutine RPIPE reads input data from the card input file. Subroutine REPIPE reads the corresponding data from the restart file. Initialization of the remaining variables is performed with subroutine IPIPE. This subroutine establishes the noding for wall heat transfer, sets the remaining fluid properties by calls to THERMO and FPROP, and inftializes the boundary data by a call to J1D.

During problem execution, the solution procedure is controlled by routines PIPE1, PIPE2, and PIPE3. At the beginning of each time step, PIPE1 calls PREPER that in turn calls FWALL for wall friction and orifice loss coefficients, MPROP for wall metal properties, HTPIPE for wall HTCs, and FEMOM to set up the stabilizer motion equations. Routine PIPE 1 also calls BKMOM for a final backward substitution on the motion equations. During the iterations for a time step, PIPE2 calls TF1D for the numerical hydrodynamics solution (see Sec. III.A.3) and JID to update boundary arrays. After a time step is 
completed successfully, PIPE3 calls CONSTB to set up the stabilizer mass and energy equations. Routine PIPE3 also calls POSTER that updates the wall temperatures with a call to CYLHT, computes new fluid properties (viscosity, heat capacity, and surface tension) with a call to FPROP, performs backward substitution on the stabilizer mass and energy equations and resets the boundary arrays with a call to J1D. If the time step fails to converge, THERMO is called to restore variables to their old values.

Output for a PIPE is managed by subroutine WPIPE. This subroutine prints the component number, junction numbers, iteration count, pressures, vapor fractions, saturation temperatures, liquid and vapor temperatures, liquid and vapor densities, liquid and vapor velocities, and wall friction factors. If wall heat transfer is included (NODES $\neq 0$ ), then information on the heat-transfer regime, liquid and vapor wall HTCs, interfacial HTC, heattransfer rate from the wall, wall temperature for critical heat flux, and wall temperature profiles also are provided.

B. Accumulator

An accumulator is a pressure vessel filled with ECC water and pressurized with nitrogen gas. During normal operation each accumulator is isolated from the reactor primary coolant system by a check valve. If the reactor-coolant-system pressure: falls below the accumulator pressure, the check valves open and ECC water is forced into the reactor coolant system.

An accumulator component may be simulated by an ACCUM module in TRAC. This module can only be connected at one junction to other 1 RAC components. This connection is at the highest-numbered cell. It is assumed that cell 1 is closed, as shown in the typical noding diagram in Fig. 19, and that the accumulator is not connected to a nitrogen pressure source. Therefore, the nitrogen pressure results from the expansion of the initial gas volume.

The procedures for data input, initialization of arrajs, advancement of time-dependent variables, and editing are similar to those given for a PIPE component (see Sec. IV.A). A sharp liquid-vapor interface is maintained by neglecting interfacial shear. In an accumulator the vapor-phase properties are those for nitrogen gas; therefore, interphase mass transfer cannot occur. The vapor-phase temperature minimum is not limited. Additionally, there is a phase separator at the accumulator discharge so that pure liquid is discharged. The accumulator walls are assumed to be adiabatic. 


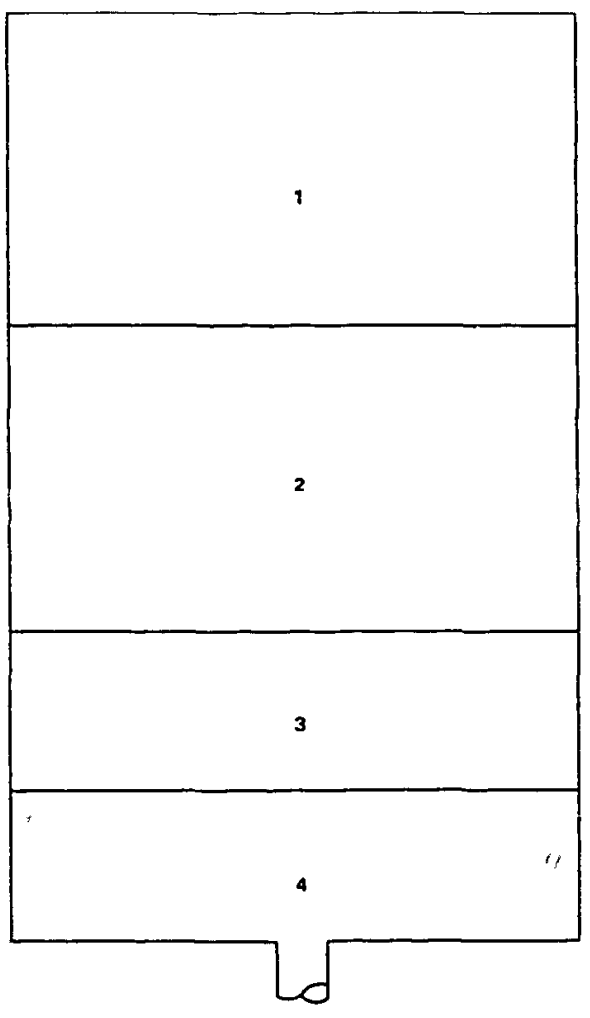

- JUNCTION

Fig. 19 .

Accumulator noding diagram.

The output edit is similar to that for a PIFE one-dimensional component with the addition of three variables specific to an accumulator.' These are (1) the discharge volumetric flow rate, (2) the total liquid volume discharged, and ( 3 ) the collapsed liquid level.

An accumulator also may be modeled with a PIPE component by setting the air partial pressures to the desired values. Thus, wall heat transfer can be included by setting NODES to a nonzero value. The air-steam vapor-phase temperature minimum is limited to $280 \mathrm{~K}$ by routine TIIERMO. An input switch activates the interface sharpener, (optional) phase separator at the discharge, and the additional edit as described above for the ACCUM component: The edit logic assumes that the component is vertical with the lowest-numbered cell at the top. 
C.

Break and Fill

The BREAK and FILL modules are used to impose boundary conditions at any one-dimensional component terminal junction. Consequently, these modules differ from the other component modules in that they do not model any system component per se and they do not perform any hydrodynamic or heat-transfer calculations. However, they are treated like any other component with respect to input, initialization, and identification procedures.

The BREAK module imposes a pressure boundary condition one cell away from its adjacent component, as shown in Fig. 20. The pressure boundary condition as well as the fluid properties associated with the BREAK may be specified as constants or functions of a system parameter defined through input BREAK tables. They also can be constant until a controlling trip is set ON and their evaluation is based on the BREAK tables. This module commonly is used to model the containment system in LOCA calculations.

A FILL module imposes a velocity boundary condition at the junction with its adjacent component, as shown in Fig. 21. For example, the ECC injection may be modeled with a FILL.

The velocity boundary condition as well as the fluid properties associated with a FILL are specified in one of three ways according to the FILL-type option selected. For the first type of FILL, the homogeneous fluid velocity and fluid properties are specified; for the second type, the mass

\section{BREAK COMPONENT}

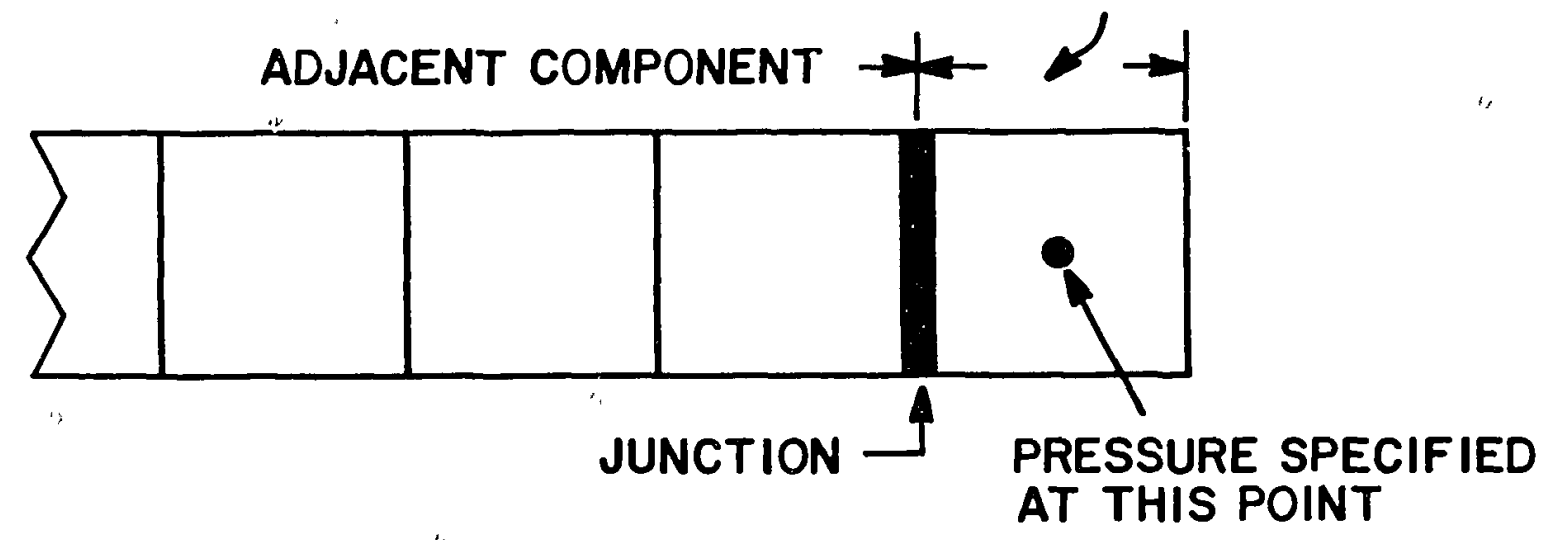

Fig. 20.

Break noding diagram. 


\section{FILL COMPONENT}

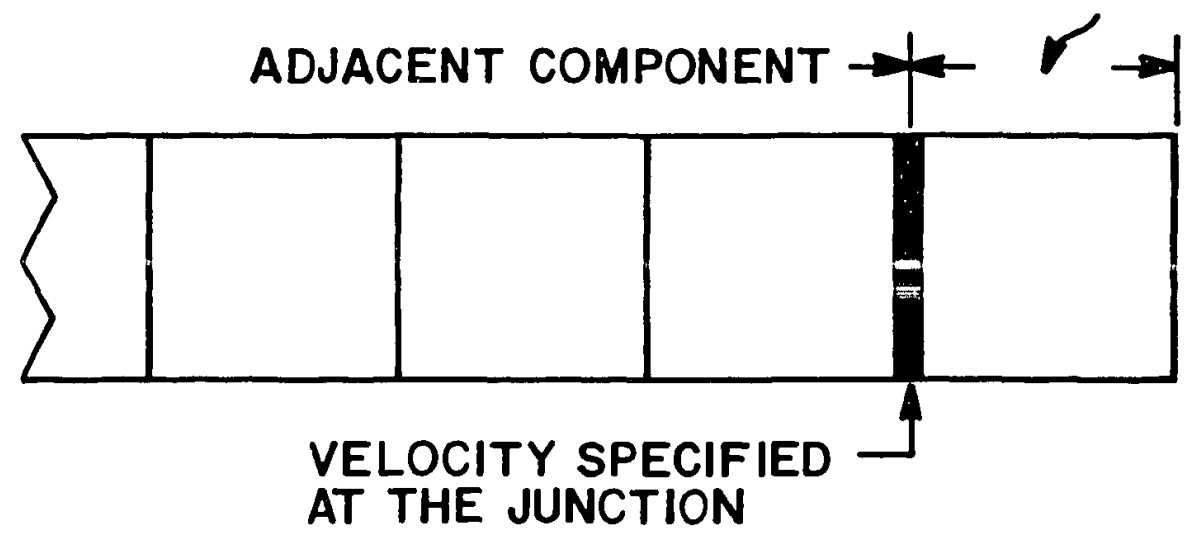

Fig. 21 .

Fill noding diagram.

flow and homogeneous fluid properties are specified; and for the third type, nonhomogeneous fluid velocities and fluid properties are specifled. For each type of FILL, the relevant parameters may be constant, interpolated from input FILL tables, or constant until a controlling trip is set on to require their evaluation from their tables. The independent variable of the table is a modeled-system parameter. When the table-evaluated FILL velocity or mass flow varies rápidly, using these values may lead to hydrodynamic instabilities. This situation may be avoided by using a weighted average of the previous value and the current table-determined value (see Sec. V.C.5.b).

The parameters needed to specify a FILL or a BREAK are described in Secs. V.C.5.b and V.C.5.d. It is suggested that the cell volume and length in a FILL or a BREAK be identical to those for the neighboring cell, of the adjacent component. The, pressure, void fraction, fluid temperatures, air partial pressure, and solute concentration specified for a FILL or a BREAK determine the properties of the fluid convected into the adjacent component if an inflow condition occurs. (By convention, inflow to the adjacent component corresponds to a positive FILL velocity and $a_{4}$ negative BREAK velocity.) A FILL or a BREAK may not be connected directly to the VESSEL component. 
D. Core

A CORE component is available in TRAC to analyze the reactor core in situations that do not demand a three-dimensional fluid-dynamics characterization.

This one-dimensional treatment can produce significant reduction in computer running time for a wide range of problems. The CORE component is a hybrid in nature. Wherever possible, we used the coding characteristic to the PIPE and VESSEL components.

The fluid-dynamics and exterior-wall conduction models are identical to those used by a PIPE component. Both are one-dimensional. In addition, any number of fuel rods may be introduced into the CORE component, although only the first (average) rod interacts thermally with the fluid. The rod heattransfer model is Identical to that used by the VESSEL component.

A rypical noding diagram for a CORE component is shown in Fig. 22 . Currently, connections can be made only at the first and last cells. Therefore, it has been necessary to model the upper and lower plenums using

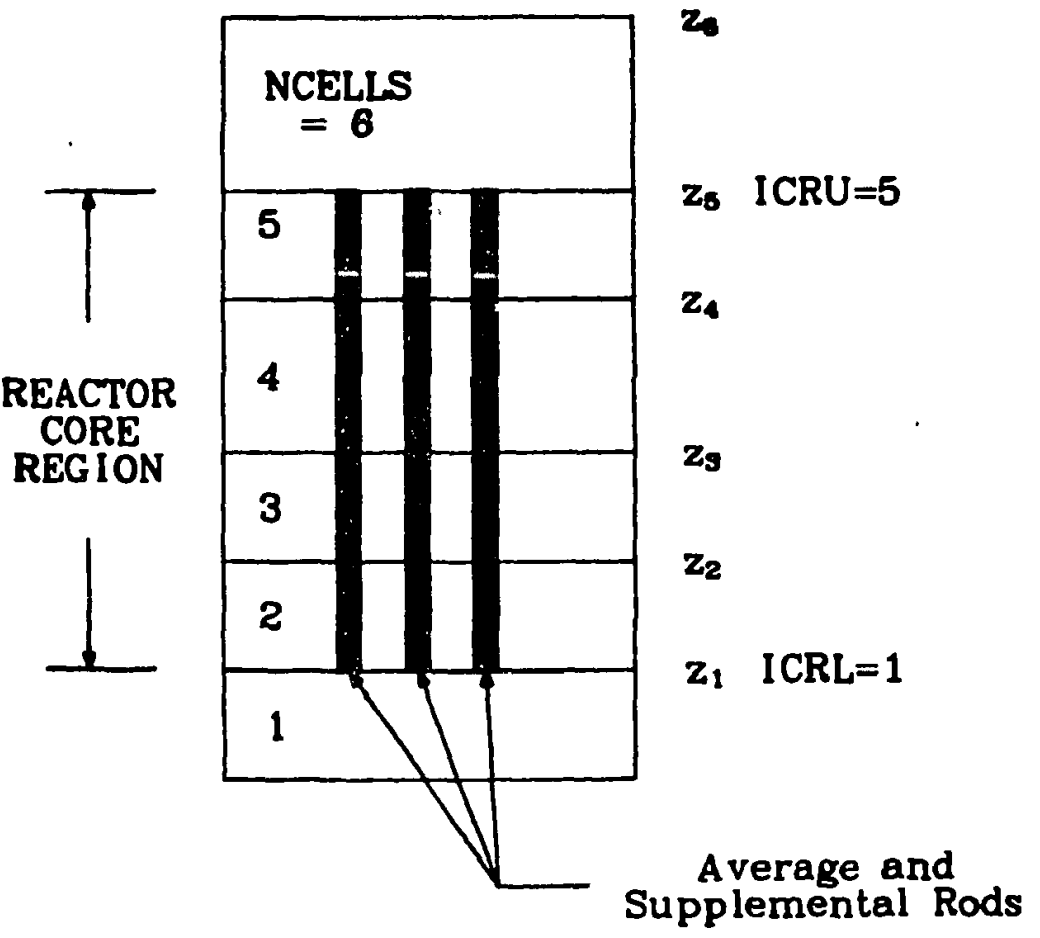

Fig. 22 .

Core noding diagram. 
other one-dimensional components. The vessel downcomer has been modeled by attaching a PIPE to the CORE component. Therefore, bypass effects are difficuit to model with this component. Detailed input specifications for a CORE component are provided in Sec. V.C.5.c. Input and output information is similar to that for the PIPE and VESSEL components.

E. Plenum

The PLENUM module, which models the thermal hydraulics of a large volume connected to an arbitrary number of one-dimensional components, is a singlecell component that acts like a momentum sink (for the case of flow out of the PLENUM through a given junction, the upstream velocity in the momentum-flux term is zero); however, all junction elevations and their orientations with respect to the PLENUM are preserved. There are single values for void fraction, liquid temperature, vapor temperature, and pressure in the PLENUM. At each junction, the code applies the standard one-dimensional, two-fluid momentum equations with the above mentioned restriction on the momentum-flux term. There is no requirement that the liquid and vapor velocities be equal at a given junction. The existence of a stratified flow is implied in the constitutive correlations if the mean fluid velocity in the PLENUM falls below a threshold value.

No junction data input is required for the PLENUM except for a list of the junction numbers connected to the component. The PLENUM requires one or more junctions. All pertinent junction information is obtained from the onedimensional modules connected to the PLENUM. The user specifies as many PLENUM-side cell lengths as junctions, such that each junction has its own associated cell length. The PLENUM does not require identical cell lengths. The code uses the cell lengths, as necessary, to calculate the velocity for each junction. In particular, the GRAV parameter at a given junction (input only in the adjacent component) must be calculated using the cell lengths from the adjacent cell and the PLENUM.

Currently, the code does not permit heat structures to be used with the PLENUM. Also, signal variables should not point to PLENUMs; if needed, the signal variables should point to an adjacent cell in one of the connecting, one-dimensional components. A PLENUM cannot be directly connected to BREAK, FILL, VESSEL, or other PLENUM components. 
PLENUM and PIPE data are processed similarly. Subroutine RPLEN reads the data from the input file (TRACIN), and subroutine REPLEN reads the data from the restart file (TRCRST). Subroutine IPLEN performs initialization of any remaining variables not provided by the input or restart files.

PIPE and PLENUM output edits also are similar.

Subroutines PLEN1, PLEN2, and PLEN3 perform the same functions for the PLENUM as PIPE1, PIPE2, and PIPE3 do for the PIPE.

F. Pressurizer

A PWR pressurizer is a large fluid reservoir that maintains the pressure within the reactor primary coolant system and compensates for changes in the coolant volume caused by system transients. During normal operation this reservoir contains the highest energy fluid in the primary system. It is usually maintained $\sim 50-60 \%$ full of saturated liquid that is pressurized by the saturated steam above it. The pressurizer controls the primary coolant loop pressure by a long surge line connected to one of the hot legs.

The PRIZER module simulates the pressurizer component. This module normally models the pressurizer itself with the surge line represented by $a$ PIPE or TEE component. Figure 23, a typical noding diagram, shows that a PRIZER coinponent may be connected at both junctions to other components. To calculate the collapsed liquid level we assume that cell 1 is at the upper end, which may be closed by connecting it to a zero-velocity FILL.* For steady-state calculations, the PRIZER module is replaced automatically by a BREAK equivalent on both sides. Thus, the thermal-hydraulic conditions in the PRIZER module during steady-state calculations remain unchanged except for the wall temperature, which is calculated from the conduction equation to obtain a steady-state wall temperature profile. The procedures for data input, injtialization of arrays, advancement of time-dependent variables, and editing are similar to those given for a PIPE component (see Sec. IV.A).

\footnotetext{
* If the top of the pressurizer is connected to a zero-velocity FILL, a void fraction of 1.0 should be input in the FILL component to invoke the interfacesharpener model in cell 1 of the PRIZER.
} 


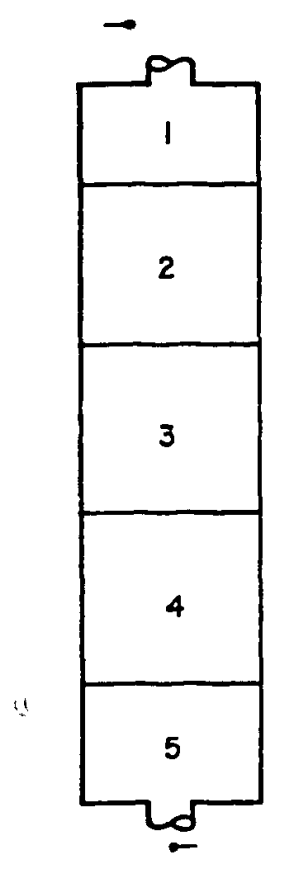

Fig. 23.

Pressurizer noding diagram.

The PRIZER component includes heater/sprayer logic to serve as a system pressure controller but not to simulate exactly the actual heater/sprayer system.** The user specifies a desired pressure set point, PSET, and the pressure deviation, DPMAX', at which the heater sprayers add or remove a maximum power of QHEAT. The power that is input to the pressurizer fluid is directly proportional to the difference between PSET and $P(1)$, the pressure in cell 1; that is,

$Q_{\text {input }}=$ QHEAT $\left[\frac{\text { PSET }-P(1)}{\text { DPMAX }}\right]$,

** The actual sprayer system can be modeled by setting up the TRAC model corresponding to the actual system spray configuration; the actual heater simulation can be achieved by modeling the bottom of the pressurizer by a separate one-cell PIPE component and by adding trip-controlled power into the coolant; 
with the absolute value of $Q_{\text {input }}$ less than or equal to QHEAT. This power is distributed over all fluid cells in the pressurizer, and the fraction of power input to the liquid in each cell is equal to the fraction of total liquid inass in that cell. Power is not added if the collapsed liquid level is less than the input parameter ZHTR. [The collapsed liquid level is calculated as follows: determine the total liquid volume in the PRIZER component; sequentially subtract from the total liquid volume the volume of the bottom cell (cell NCELLS), the next to the bottom cell (cell NCELLS-1), etc., until the cell that contains all of the remaining liquid volume is found; the collapsed liquid Ievel is the sum of the cell lengths $(\Delta x)$ of the filled cells plus a linearly interpolated (based on liquid fraction) $\Delta x$ from the partially filled cell.] If pressure control is not desired, then QHEAT is set to zero.

Wall friction coefficients are calculated in routine FWALL by specifying a friction correlation option, NFF, along with the additive friction factors, FRIC, for each cell edge. The homogeneous flow friction correlation option $(N F F=1)$ is suggested for a pressurizer.

The output edit for a PRIZER component is similar to a PIPE component with the addition of four variables specific to the pressurizer. They are (1) the discharge volumetric flow rate, (2) total liquid volume discharged, (3) collapsed liquid level, and (4) heater/sprayer power input to the pressurizer fluid.

G. Pump

The PUMP module describes the interaction of the system fluid with a centrifugal pump. The model calculates the pressure differential across the pump and its angular velocity as a function of the fluid flow rate and the fluid preperties. The model can treat any centrifugal pump and allows for the inclusion of two-phase effects.

The pump model is represented by a one-dimensional component with $N$ cells $(N>1)$. Figure 24 shows a typical noding diagram for the pump component. The pump momentum is modeled as a source between cells 1 and 2 . The source is positive for normal operation so that a pressure rise occurs from cell 1 to cell 2. Therefore, it is necessary to construct the cell noding such that the cell number increases in the normal flow direction. 


\section{NORMAL FLOW DIRECTION}
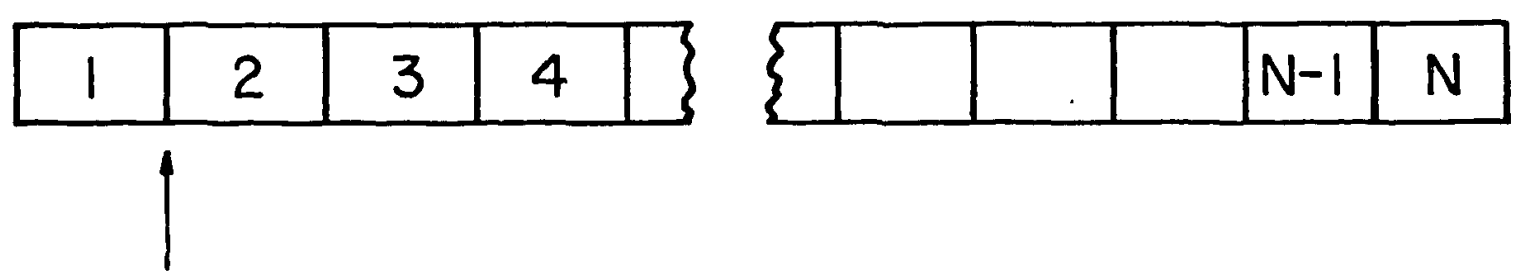

SMOM

Fig. 24 .

Pump noding diagram.

The following considerations were important in creating the PUMP module:

1. compatibility with adjacent components should be maximized,

2. choking at the pump inlet or outlet should be predicted automatically, and

3. the calculated pressure rise across the pump should agree with that measured at steady-state conditions.

The first two criteria precluded the use of a lumped-parameter model. The PUMP module, therefore, combines the PIPE module with pump correlations.

The pump model is identical to the one-dimensional pipe model except that the momentum equations between cells 1 and 2 are rewritten as

$\frac{v_{\ell}^{n+1}-v_{\ell}^{n}}{\Delta t}=\frac{\left[P_{1}^{n+1}-P_{2}^{n+1}+\Delta P^{n}+\left(\frac{\partial \Delta P}{\partial V}\right)^{n}\left(v_{\ell}^{n+1}-v_{\ell}^{n}\right)\right]}{\left(\varphi_{m}>\bar{\Delta} \bar{x}\right)}-g \cos \theta$

and

$\mathrm{v}_{\mathrm{g}:}=\mathrm{v}_{\ell}$ 
where $\Delta \mathrm{P}$ is the pressure rise through the pump evaluated from the pump correlation. The steady-state solution of Eq. (212) is

$\Delta \mathrm{P}=\mathrm{P}_{2}-\mathrm{P}_{1}+\mathrm{g} \cos \theta$,

which is the desired result. Friction does not enter explicitly into the pump motion equation. Therefore, additive friction is not allowed between cells 1 and 2 [FRIC $(2)=0.0$ ]

It is necessary to evaluate $\Delta \mathrm{P}$ and its derivative with respect to velocity for a pump cell only once each time step. The source is needed only in routines FEMOM and TFIDS1. This evaluation is performed by subroutine PUMPSR.

The pump correlation curves describe the pump head and torque response as a function of fluid volumetric flow rate and pump speed. Homologous curves (one curve segment represents a family of curves) are used for this description because of their simplicity. These curves describe, in a compact manner, all operating states of the pump obtained by combining positive or negative impeller velocities with positive or negative flow rates.

The following definitions are used in the subsequent development:

$\mathrm{H}=$ the pump head $=\frac{\Delta \mathrm{P}}{\rho}$,

$Q=$ the pump volumetric flow rate, and

$\Omega=$ the pump impeller angular velocity,

where $\Delta \mathrm{P}$ is the pressure rise across the pump and $\rho$ is the pump upstream mixture density. To allow one set of curves to be used for a variety of pumps, the following normalized quantities are used:

$\mathrm{h} \equiv \frac{\mathrm{H}}{\mathrm{H}_{\mathrm{R}}}$, 
$\mathrm{q} \equiv \frac{\mathrm{Q}}{\mathrm{Q}_{\mathrm{R}}}$, and

$\omega \equiv \frac{\Omega}{\Omega_{\mathrm{R}}}$,

where $H_{R}$ is the rated head (RHEAD) for the pump, $Q_{R}$ is the rated volumetric flow (RFLOW), and $\Omega_{R}$ is the rated pump speed (ROMEGA). The pump similarity relations 47 show that

$\frac{h}{\omega^{2}}=f\left(\frac{q}{\omega}\right)$

For small $\omega$ this correlation is not satisfactory, and the following combination of variables is used,

$\frac{\mathrm{h}}{\mathrm{q}^{2}}=\mathrm{f}\left(\frac{\mathrm{w}}{\mathrm{q}}\right)$

Correlation [Eq. (214)] is used in the range $0 \leq|q / \omega| \leq 1$ and results in two separate curves, one for $\omega>0$ and one for $\omega<0$. Correlation [Eq. (215)] is used in the range $0 \leq|w i q|<1$ and yields two separate curves, one for $q>0$ and one for $q<0$. The four resulting curve segments, as well as the curve selection logic used in TRAC, are shown in Table XI.

To account for two-phase effects on pump pefformance, the pump curves are divided into two separate regimes. Data indicate that two-phase pump. performance in the vapor-fraction range of $20-80 \%$ is degraded significantly in comparison with its performance at vapor fractions outside of this range. One set of curves describes the pump performance for single-phase fluid ( 0 or $100 \%$ vapor fraction), and another set describes the two-phase, fully degraded performance at some void fraction between 0 and $100 \%$. For single-phase conditions the curve segments for correflation [Eq. (214)] are input as HSP1 for $\omega>0$ and HSP4 for $\omega<0$, and correlation [Eq. (215)] curve segments are 
DEFINITIONS OF THE FOUR CURVE SEGMENTS THAT DESCRIBE THE HOMOLOGOUS PUMP HEAD CURVES ${ }^{\mathrm{a}}$

Curve Segment

1
4

2

3 $\underline{\mid \frac{q}{\omega}} \mid$

$\left[\begin{array}{l}\leq 1 \\ \leq 1\end{array}\right.$

$\left[\begin{array}{l}>1 \\ >1\end{array}\right.$ $\underline{\omega}$

$>0$

$<0$ $\underline{q}$

$$
1
$$

$\left.\begin{array}{l}>0 \\ <0\end{array}\right]$
Correlation

$\left\lceil\frac{\mathrm{h}}{\omega^{2}}=\mathrm{f}\left(\frac{\mathrm{q}}{\omega}\right)\right]$

$\left[\frac{\mathrm{h}}{\mathrm{q}^{2}}=\mathrm{f}\left(\frac{\omega}{\mathrm{q}}\right)\right]$

${ }^{a_{F o r}}$ the special case of both $\omega=0.0$ and $q=0.0$, the code sets $h=0.0$.

input as HSP2 for $q>0$ and HSP3 for $q<0$. The fully degraded version of correlation [Eq. (214)] is input as curve HTPl for $\omega>0$ and HTP4 for $\omega<0$. The fully degraded version of correlation [Eq. (215)] is input as HTP2 for $q>0$ and HTP3 for $q<0$.

The pump head at any vapor fraction is calculated from the relationship,

$H=H_{1}-M(\alpha)\left(H_{1}-H_{2}\right)$,

where

$\mathrm{H}=$ the total pump head,

\& $\mathrm{H}_{1}=\mathrm{h}_{1} \mathrm{H}_{\mathrm{R}}=$ the single-phase pump head ( $\mathrm{h}_{1}$ is the nondimensional

head from the single-phase homologous head curves),

$\mathrm{H}_{2}=\mathrm{h}_{2} \mathrm{H}_{\mathrm{R}}=$ the fully degraded pump head $\left(\mathrm{h}_{2}\right.$ is the nondimensional

head from they fully degraded homologous head curves),

$M=$ the head degradation multiplier (input as HDM), and

$\alpha=$ the vapor fraction.

To this point, no knowledge of density is required to calculate $H$ from the homologous head curves. The upstream mixture density is always used to convert the total pump head $\mathrm{H}$ to $\Delta \mathrm{P}$, the pressure rise through the pump, by the definition $\Delta \mathrm{P}=\rho \mathrm{H}$. 
The development of homologous torque curves parallels the previous development for homologous head curves. The dimensionless hydraulic torque is defined by

$\beta \equiv \frac{T_{h y}}{T_{R}}$,

where $T_{h y}$ is the hydraulic torque and $T_{R}$ is the rated torque (RTORK). The convention used is that a positive $\mathrm{T}_{\text {hy }}$ works to retard positive pump angular velocity. The dimensionless torque $\beta$ is correlated as either $\beta / \omega$ or $\beta / q$ just as the dimensionless head was correlated. For single-phase conditions the correlations yield the corresponding four curve segments TSP1, TSP2, TSP3, and TSP4. The fully degraded correlations produce four corresponding curves TTP1, TTP2, TTP3, and TTP4. The homologous torque curve segments are correlated in the same manner as the head curve segments shown in Table XI (replace $h$ with $\beta)$. For the special case of $\omega=q=0.0$, the $\operatorname{code} \operatorname{sets} \beta_{1}=\beta_{2}=0.0$.

The single-phase torque $T_{1}$ is dependent upon the fluid density and is calculated from

$\mathrm{T}_{1}=\beta_{1} \mathrm{~T}_{\mathrm{R}}\left(\frac{\rho}{\rho_{\mathrm{R}}}\right)$,

where $\beta_{1}$ is the dimensionless hydraulic torque from the single-phase homologous torque curves, $\rho$ is the pump upstream mixture density, and $\rho_{R}$ is the rated density (RRHO). The density ratio is needed to correct for the 
density difference between the pumped fluid and the rated condition. Similarly, the fully degraded torque $\mathrm{T}_{2}$ is obtained from

$T_{2}=\beta_{2} T_{R}\left(\frac{\rho}{\rho_{R}}\right)$

where $\beta_{2}$ is the dimensionless hydraulic torque from the fully degraded homologous torque curves. For two-phase conditions the impeller torque is calculated from

$T=T_{1}-N(\alpha)\left(T_{1}-T_{2}\right)$,

where $T$ is the total impeller torque and $N(\alpha)$ is the torque degradation multiplier (input as TDM).

In addition to the homologous riead and torque curves, the head and torque degradation multipliers defined in Eqs. (216) and (218) are required. These functions of void fraction are nonzero only in the vapor-fraction range where the pump head and torque are either partially or fully degraded.

The pump module treats the pump angular velocity as a constant (input) while the motor is energized. After a drive motor trip, the time rate of change for the pump angular velocity $\Omega$ is proportional to the sum of the moments acting on it and is calculated from the equation,

$I \frac{d \Omega}{d t}=-\sum_{i} T_{i}=-\left(T+T_{f}+T_{b}\right)$

$$
a
$$


where I is the combined impeller, shaft, and motor assembly moment of inertia (EFFMI); $T$ is the hydraulic torque on the impeller; $T_{f}$ is the torque caused by friction; and $T_{b}$ is the bearing and windage torque. We assume that $T_{f}$ and $T_{b}$ are

$\mathrm{T}_{\mathrm{F}}=\mathrm{C}_{1} \frac{\Omega|\Omega|}{\Omega_{\mathrm{R}}^{2}}$

and

$\mathrm{T}_{\mathrm{b}}=\mathrm{C}_{2} \frac{\Omega|\Omega|}{\Omega_{\mathrm{R}}^{2}}$

where $C_{1}$ and $C_{2}$ are input constants (TFRI and TFR2, respectively). The hydraulic torque $\mathrm{T}$ is evaluated using the homologous torque curves "and Eq. (218); it is a function of the volumetric flow, the "upstream void fraction, the upstream density, and the pump angular velocity. For time step $(n+1), E q \cdot(219)$ is evaluated explicitly,

$\Omega^{\ddot{n+1}}=\Omega^{\mathrm{n}}-\frac{\Delta \mathrm{t}}{\mathrm{I}}\left[\mathrm{T}(Q, \alpha, \rho, \Omega)+\left(\mathrm{C}_{1}+\mathrm{C}_{2}\right) \frac{\Omega^{n}\left|\Omega^{\mathrm{n}}\right|}{\Omega_{\mathrm{R}}^{2}}\right]$.

The wall heat transfer, wall friction, and CHF calculation options are the same for the PUMP module as for the PIPE module. In addition, $y^{\text {the }}$ following uptions are specified: pump type.(IPMPTY), motor action (IPM, TR)," reverse speed option (IRP), degradation option (IPM), and pump curve option' (OPTION). The input variables IPMPTR and NPMPTX specify, respectively, the trip number for the pump trip intiation and the number of pairs of points in the pump-speed table (array, SPTBL). If IPMPTR $=0$, no pump trip action occurs, and the pump runs for the entire calculation at the constant speed (OMEGAN) specified in the input. 
If the pump motor is energized (trip IPMPTR set OFF), the angular velocity is assumed to be the constant value specified (OMEGAN). If the motor is not energized (trip IPMPTR set ON), the pump speed is allowed to vary.

Two types of pimps are available. For pump type 1 (IPMPTY $=1$ ), the pump-speed variation is specified by the input table. The pump is energized initially at a constant speed specified by input (OMEGAN). Trip IPMPTR may initiate a pump trip, after which the pump speed is taken from a pump-speed table (array SPTBL). The independent variable for the pump-speed table may be elapsed time since trip initiation or any other signal variable, as discussed in Sec. V.B. For pump type 1 the torque calculation is not used. Pump type 2 (IPMPTY $=2$ ) is similar to option 1 except that a speed table is not input. Instead, the pump speed is calculated from Eq. (222) after a trip has occurred.

If the reverse speed option is specified $(I R P)=1$ ), the pump is allowed to rotate in both the forward and reverse directions. If reverse speed is not allowed $(I R P=0)$, the pump will rotate in the forward direction only. For this case, if negative rotation is calculated (after trip with pump type 2), the speed will be set to zero. If IRP $=0$ and a negative pump speed is encountered (i.e., from the speed table), error messages will be printed by subroutines PUMPD, PUMPX, and PUMPSR (this is a fatal error).

If the degradation option is turned on (IPM = 1 ), the degraded pump head and torque are calculated from Eqs. (216) and (218). If the degradation option is turned off (IPM $=0)$, only the single-phase head and torque homologous curves are used [equivalent to setting $M(\alpha)$ and $N(\alpha)$ to zero in Eqs. (216) and (218)].

The user may specify pump homologous curves in the input (OPTION $=0$ ) or alternatively may use the built-in pump curves (UPTION $=1$ or 2 ). The first set (OPTION = 1) of built-in pump curves is based on the Semiscale Mod-1 system pump. ${ }^{48-51}$ The Semiscale pump curves for single-phase homologous head (HSP), fully degraded two-phase homologous head (HTP), head degradation multiplier (HDM), single-phase homologous torque (TSP), and torque degradation 
multiplier (TDM) are provided in Figs. 25-29, respectively. The second set (OPTION = 2) of built-in pump curves is based on the Loss-of-Fluid Test (LOFT) system pump. 52 The LOFT pump curves for single-phase homologous head (HSP), fully degraded two-phase homologous head (HTP), head degradation multiplier (HDM), single-phase homologous torque (TSP), and torque degradation multiplier (TDM) are shown in Figs. 30-34, respectively. For lack of data, the fully degraded two-phase homologous torque curves (TTP) for both Semiscale and LOFT are zero. Where applicable, the curves are numbered corresponding to the conditions provided in Table XI.

Because these homologous curves are dimensionless, they can describe a variety of pumps by specifying the desired rated density, head, torque, volumetric flow, and angular velocity as input. We recommend that for full-scale PWR analyses, plant-specific pump curves be input; however, if such data are unavallable, the LOFT pump curves (OPTION $=2$ ) generally should be used.

There are several restrictions and limitations in the current version of the PUMP module. Because there is no pump motor torque vs speed model, the pump speed is assumed to be input if the motor is energized. Pump noding is restricted such that the cell numbers increase in the normal flow direction (NCELLS 2 2), the pump momentum source is located between cells 1 and 2 of the pump model, and the additive friction (loss coefficient) between cells 1 and 2 is $0.0[$ FRIC $(2)=0.0]$. A flow area change should not be modeled between cells 1 and 2. Finally, the head degradation multiplier $M(\alpha)$ and the torque degradation multiplier $N(\alpha)$ are assumed to apply to all operating states of the pump.

The PUMP module inpu: consists of the same geometric and hydrodynamic data and initial conditions that are required for the PIPE module. In addition, information specific to the PUMP is required, as described in the input specifications (Sec.V.C.5.g). The speed table (SPTBL) as well as the homologous pump curve arrays must be input in the following order:

$x(1), y(1), x(2), y(2), \ldots, x(n), y(n)$. 


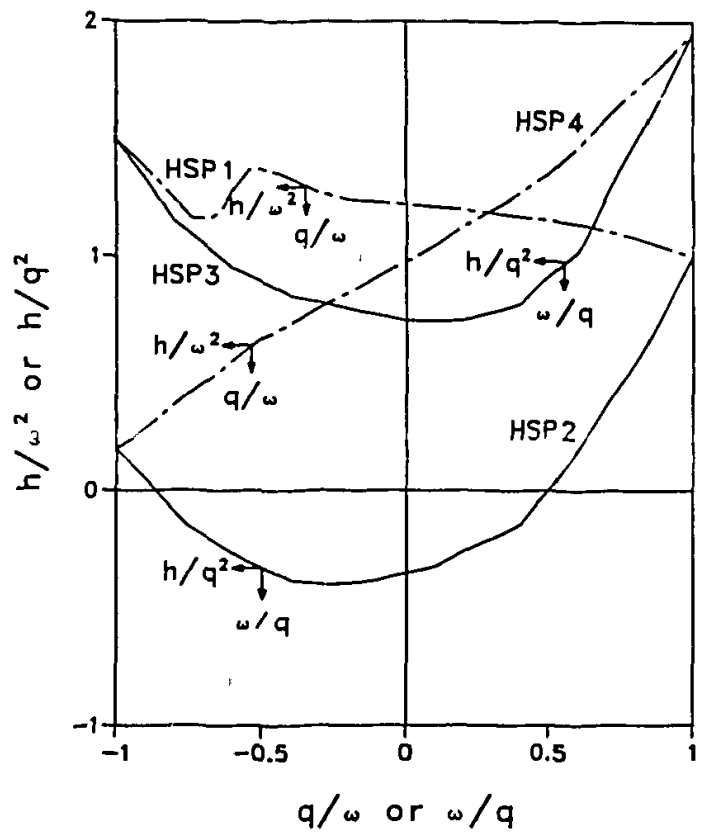

Fig. 25.

Semiscale single-phase homologous head curves.

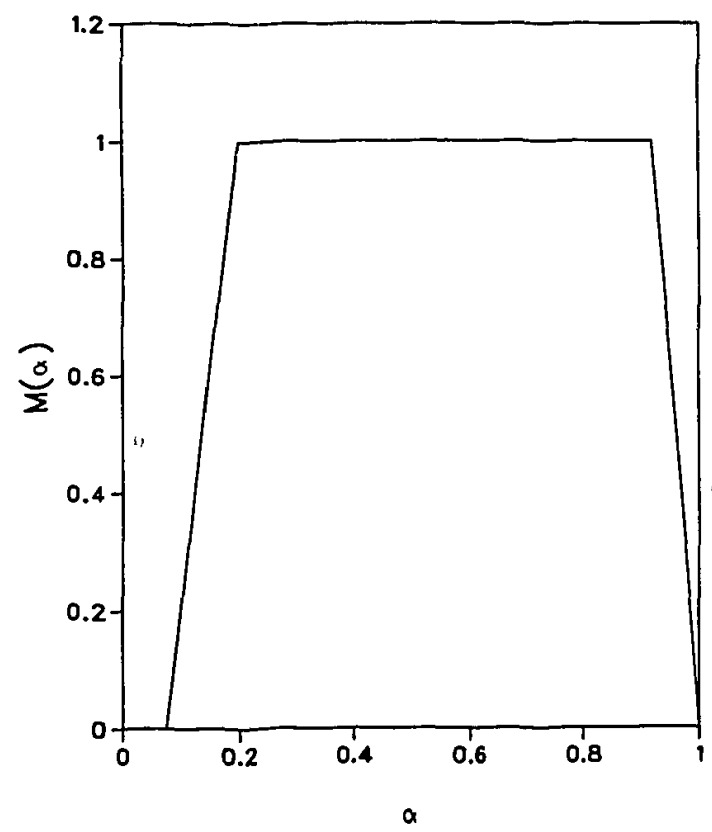

Fig. 27.

Semiscale head degradation multiplier curve.

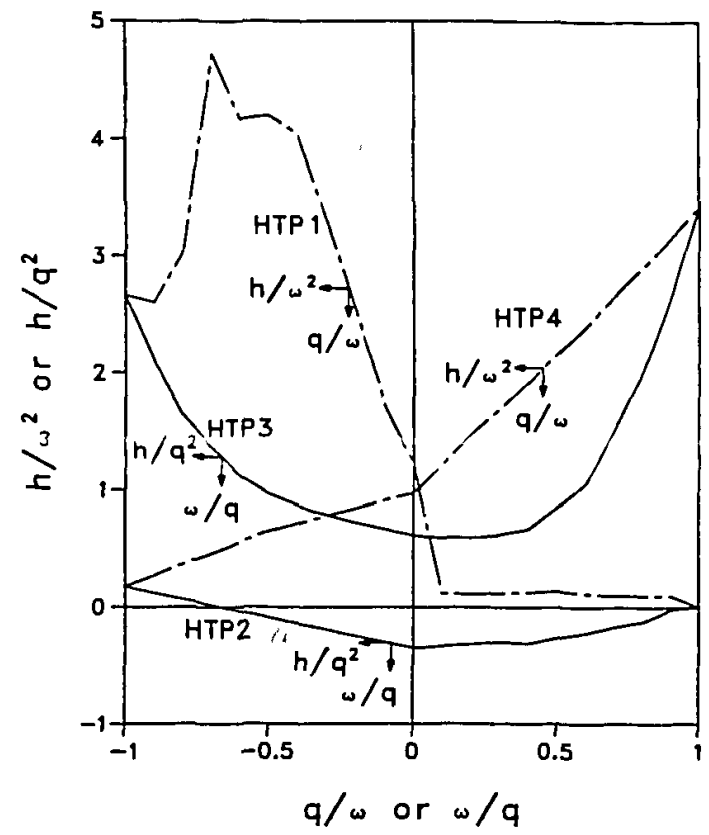

Fig. 26.

Semiscaie fully degraded homologous head curves.

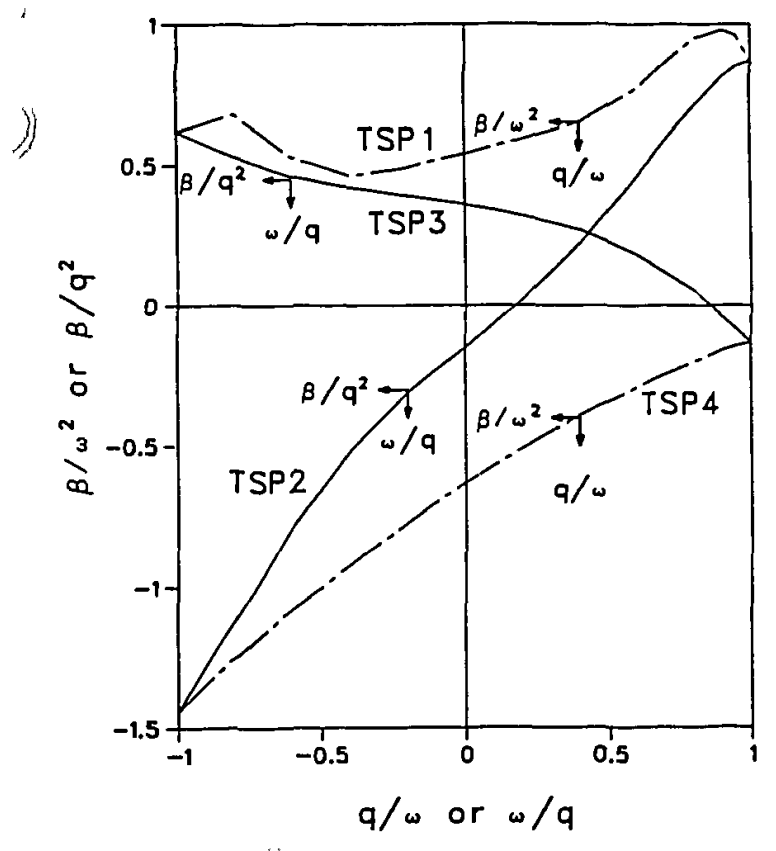

Fig. 28 .

Semiscale single-phase homologous torque curves. 


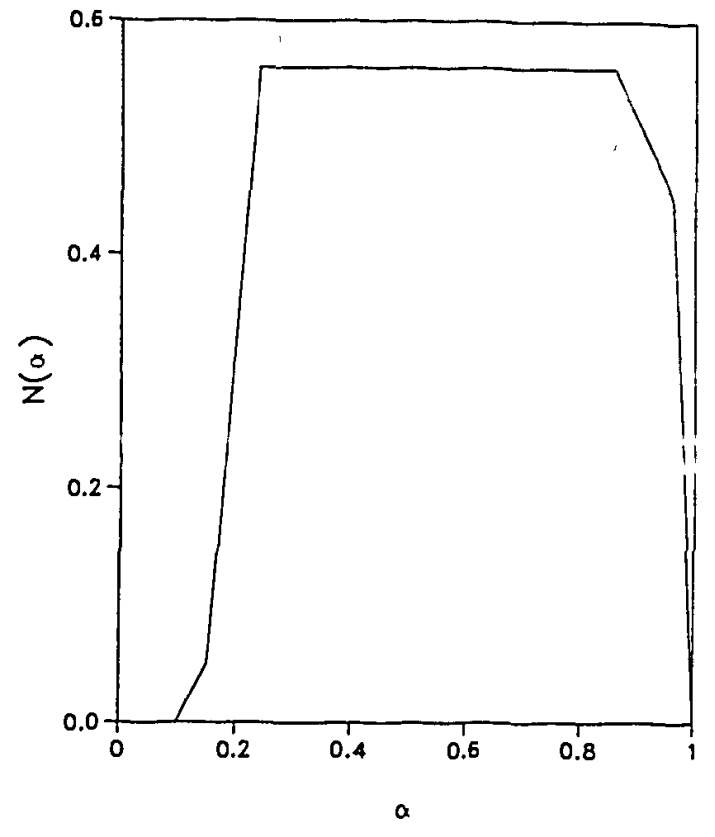

Fig. 29.

Semiscale torque degradation multiplier curve.

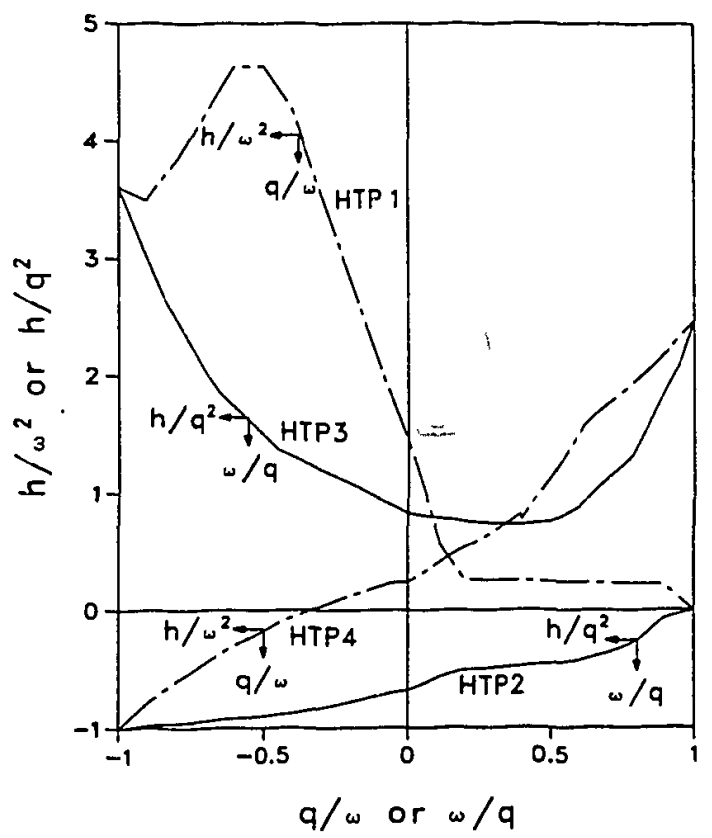

Fig. 31 . LOFT fully degraded homologous he'ad curves.

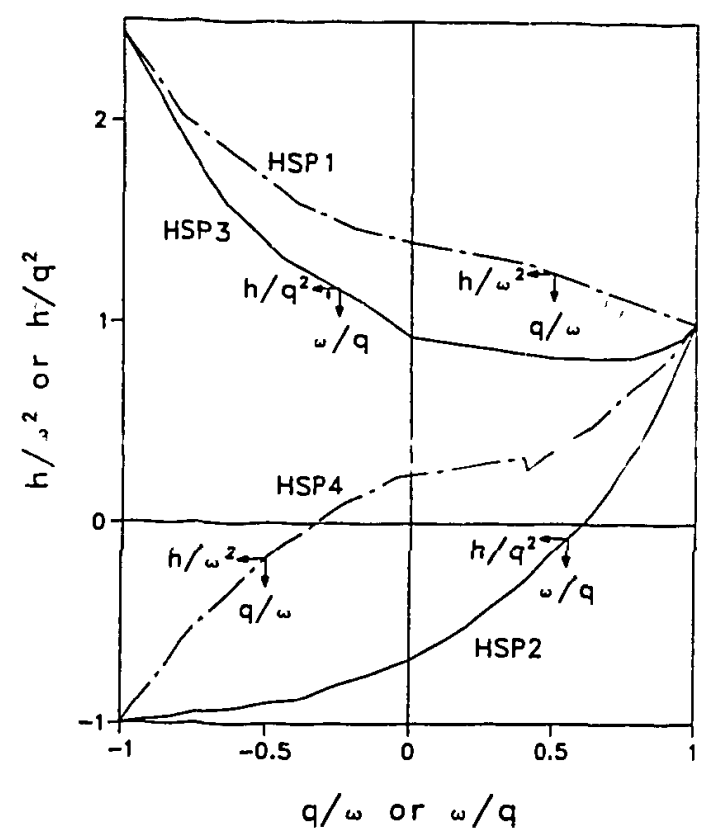

Fig. 30 . LOFT single-phase homologous head curves.

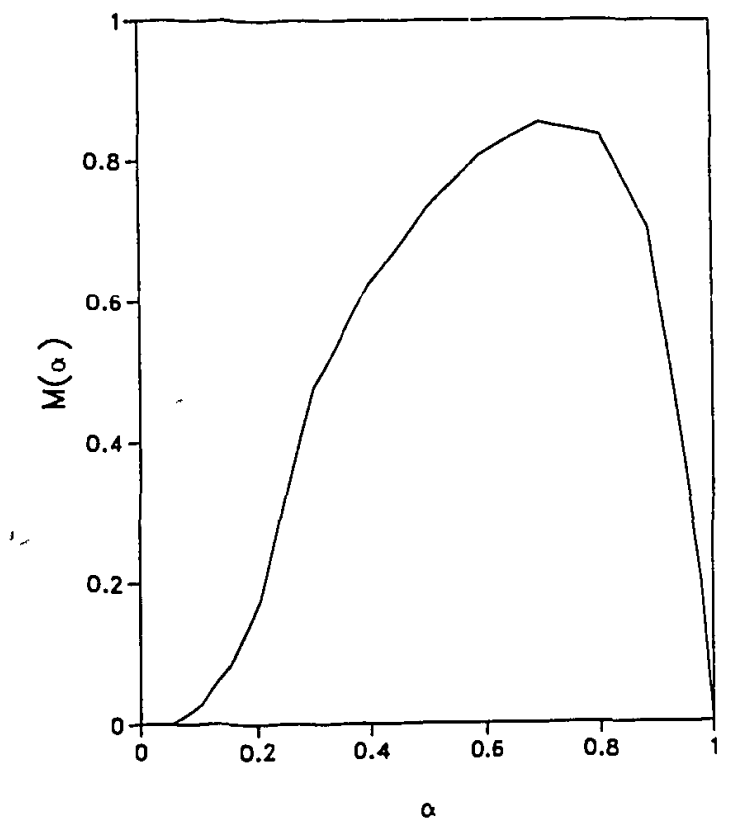

Fig. 32 .

LOFT head degradation multiplier curve. 


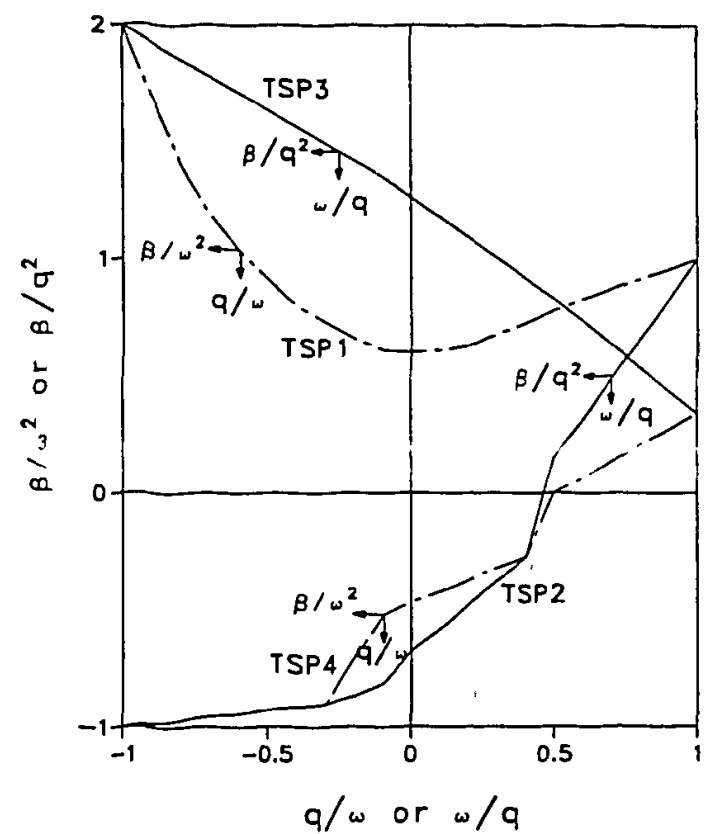

Fig. 33 .

LOFT single-phase homologous torque curves.

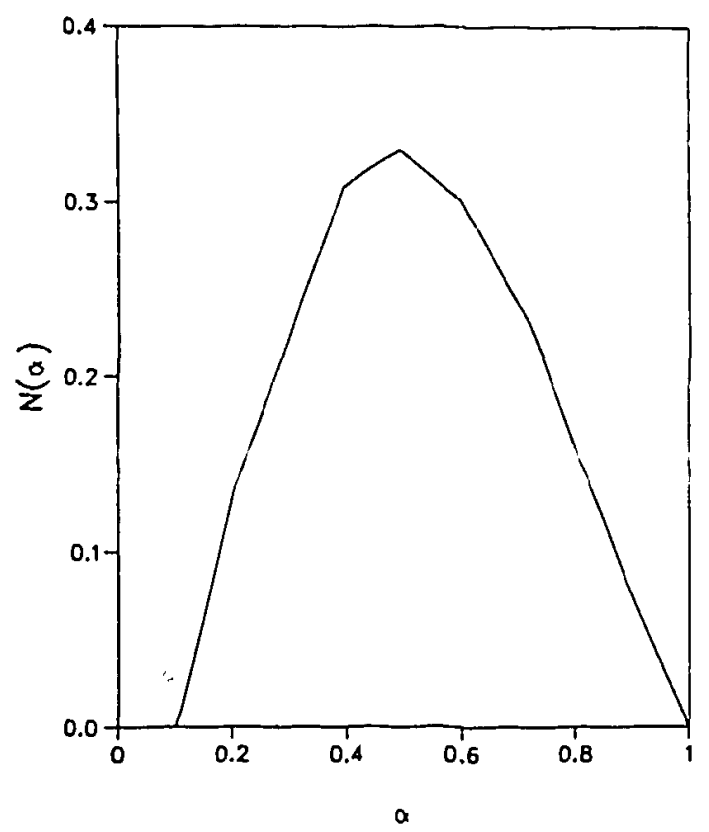

Fig. 34 .

LOFT torque degradation multiplier curve.

Here $x$ is the independent variable and $y$ is the dependent variable. Furthermore, the independent variables must increase monotonically on input, that is,

$x(1)<x(2)<\ldots<x(n-1)<x(n)$.

\section{Linear interpolation is used within the arrays.}

The pump data are processed in a manner similar to that for the PIPE component. Subroutine RPUMP reads data from the input file, and subroutine REPUMP reads data from the restart file. Subroutine RPUMP calls subroutines RDDIM and RDCRVS to read the pump curves; if the built-in pump curves are to be used, subroutine RDCRVS calls subroutine PUMPI to load the correct data. Subroutine IPUMP initializes the remaining variables.

Subroutines PUMP1, PUMP2, and PUMP3 perform the same functions for the PUMP component as PIPE1, PIPE2, and PIPE3 perform for the PIPE component. For the PUMP component, subroutine PREPER calls subroutine PUMPSR to calculate the momentum source and the pump speed. Subroutine PUMPSR calls subroutine PUMPX 
to calculate the pump head and pump torque. Subroutine PUMPX calls subroutine PUMPD to evaluate the homologous head and homologous torque from the pump curves.

H. Steam Generator

In the steam generator, energy is transferred from the high-temperature, high-pressure primary coolant to the secondary fluid so that steam is produced to drive the turbines. All steam-generator prinary flow paths are similar in that the reactor coolant enters a plenum, flows through a tube bundle where the coolant is in thermal contact with the secondary fluid, and discharges through an exit plenum. There are two basic designs, however, once-through and U-tube. In the once-through design the primary fluid enters an upper hemispherical head at the top of the steam generator, flows downward through the tube bundle, and exits through a lower hemispherical head and out the discharge nozzles. In the basic U-tube design, the lower plenum is divided into inlet and outlet plena by a vertical divider plate. The primary fluid enters the inlet plenum and flows into the tube region. The tubes of the $\mathrm{U}$-tube design are in the shape of inverted U's. The flow then exits through the outlet plenum.

Secondary flow paths differ remarkably between the different steam generator designs. The locations of the main feedwater, auxiliary feedwater, and steam outlet, for example, vary considerably. In once-through steam generator designs, the main feedwater typically enters a downcomer region, which may include a flow path to the tube bundle region (aspirated design), allowing bypass steam to mix with the inlet flow. In the aspirated designs, steam is drawn into the downcomer by the condensing caused by the cold feedwater. The saturated mixture enters the lower region of the tube bundle and flows countercurrent to the primary fluid. As the secondary flow progresses upward through the bundle, it passes through nucleate-boiling and film-boiling heat-transfer regimes. The secondary flow field is superheated in the top of the steam generator. Superheated steam exits through an outer annulus used to heat the steam generator shell and minimize thermal expansion between the tubes and the shell. In most once-through designs, the auxiliary feedwater enters the tube region at the top of the steam generator where it is forced into the center of the bundle.

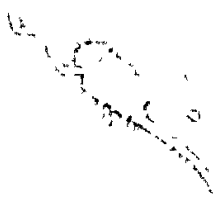


U-tube steam-generator designs are much more diverse. In the basic U-tube design, the subcooled main feedwater enters through a distribution ring at the top of an annular downcomer where it mixes with recirculating, saturated water from the moisture separators. The inixture descends through the downcomer and radially enters the tube bundle through a cylindrical gap at the bottom of the bundle. The secondary water is then heated by the reactor coolant as it moves up the bundle. The two-phase mixture enters two stages of moisture separators, which remove lhe liquid content directing it into the downcomer. Final drying of the steam may be accomplished using corrugated plate dryers. High-quality steam exits the top of the steam generator. Recirculation ratios (total mass flow to steam flow) vary with design and operating conditions. Typically, ratios of between 2.0 and 5.0 are realized. The auxiliary feedwater supply typically enters through the same nozzles as the main feedwater.

In a U-tube steam generator equipped with a counterflow preheater, the main feedwater enters the bottom of the steam generator through a small downcomer. The bottom of the steam generator is separated into two sections by a vertical divider plate between the hot and cold primary legs. The feedwater enters the semicylindrical section enclosing the cold leg of the tube bundle. This is the preheater or economizer region, which includes about one-third of the tube bundle height. In the preheater section, the flow progresses through a baffled region counter to the cold-leg flow direction and is heated to saturation. On exit from the preheater section, the secondary fluid mixes with the recirculated flow from the half-ring region enclosing the hot leg. This latter region is referred to as the evaporator section. The mixture progresses $u_{\mathrm{P}}$ the tube bundle and into the region containing the centrifugal moisture separators and dryers. The liquid removed in this region is directed into an annular downcomer. The downcomer directs flow either to the top of the preheater or through a gap under the preheater (thereby thermally separating the cold feedwater from the tube sheet) and into the evaporator region. Heat transfer in the evaporator section is by nucleate boiling. Most of the recirculated flow $(\sim 70 \%)$ is directed to the evaporator and a smaller amount to the top of the preheater. This flow split reduces the density differences between the hot- and cold-leg sides of the secondary at the preheater discharge level. 
The U-tube steam generator, equipped with a split-flow preheater differs from the counterflow preheater design only in the design of the preheater section. The feedwater enters through two nozzles that direct the flow to an upper and lower section of the preheater. Approximately $70 \%$ of the feedwater is directed to the upper preheater section where the fflow progresses through a baffled region counter to the reactor coolant flow direction. On exiting the upper frcheater, the flow then mixes with the evaporator flow. The remainder ( $30 \%$ of the inlet flow) progresses through the baffled region of the lowerpreheater section parallel to the primary flow and enters the bottom of the evaporator. This flow split prevents the introduction of cold feedwater onto the tube sheet. Although there is a $70 / 30$ split of feedwater entering the upper- and lower-preheater sections, leakage between the two sections results in about a $25 / 75$ split exiting the respective regions.

In the U-tube steam generator designs, the auxiliary feedwater may enter through the same nozzles as the main feedwater or through a top feedwater ring into the annular downcomer. When injected into the downcomer, the auxiliary feedwater maintains a degree of subcooling to promote an increased recirculation ratio and prevents. bolling of the recirculating flow, thereby providing im roved thermal-hydraulic conditions.

The TRAC steam-generator model has been provided with sufficient generality to allow the user the ability to construct the geometry pertinent to his analysis. All flow paths associated with the steam-generator component employ the one-dimensional two-fluid analysis. Furthermore, the standard onedimensional constitutive relations are used. Input is required for both a primary- and a secondary-flow path. It should be emphasized, however, that "What is referred to as primary or secondary steam-generator flow paths need not necessarily be connected to the primary or secondary reactor-flow loops. That is, because of the generality associated with the steam-generator secondary, it is possible, for example, to construct the entire component using the secondary flow path. When this is done, the primary component must be isolated (hydraulically and thermally) from the secondary components. As a second example, a tube rupture problem could be modeled using the primary steam-generator component to model the intact tubes. The secondary components could then be used to model the broken tube as well as the secondary steam-generator flow path. 
In the TRAC steam-generator model, the primary component may be either a one-dimensional PIPE or TEE. The primary component is used under typical conditions to represent the entire tube bank as a single, effective tube. Therefore, the volumes and flow areas for the primary fluid cells are based on all of the tubes within the tube bank; however, the hydraulic diameter is that of a single tube. The constitutive package (friction factors, interfacial quantities, etc.) used for the primary fluid-dynamic calculations is identical to that used for all of the TRAC one-dimensional (exvesse1) components. The number of fluid volumes (cells) used to model the steam-generator primary is user-specified (NCELL1). Typically, two of the cells model the inlet and the exit plena; the remaining NCELL1-2 cells are located in the tube bundle.

The TRAC program can model a tee connection on the primary flow path. This option allows the user to model a tube rupture problem, for example. When the primary-tee option is selected, the user must specify the cell on the primary side to which the connection is to be made (JCLP), the angle at which the connection is to be made (COSP), and the number of fluid cells used to model the flow field on the side tube connection (NCLP). The definitions of JCLP, COSP, and NCLP are equivalent to JCELL, COST, and NCELL2 for the TEE component (Sec. IV.H). Three external connections, including the primary-tee connection, may be made to the steam-generator primary side. Similar to all one-dimensional components, initial conditions and flow geometry for the primary flow path are input using the LOAD format.

Because secondary flow paths differ remarkably between steam-generator designs, the TRAC stieam-generator model allows the user to construct the secondary flow path pertinent to his analysis. Thus, once-through steam generators including aspirators or economizer sections and U-tube designs with counter- or split-flow preheaters and evaporator regions may be modeled. Downcomer and recirculation paths also may be included. An arbitrary number of external connections may be joined to the secondary flow path. Therefore, the number and the location of the main and auxiliary feedwater iñlets as well as the steam exit may be varied: A connection also may be made to the secondary flow path to model a tube rupture.

The secondary flow path $\mathrm{is}$ constructed using a minimum of 1 to a maximum of 10 PIPEs and/or TEEs. The user must specify the number (NSCMP) of secondary components necessary to model adequately the steam generator for his. 
analysis. For each component, the type (STYPE = PIPE or TEE), the number of cells (NCLS and NCLT), the çomponent number (NUMS), and the junction numbers (JSINT) are required. Secondary component and junction numbers that are unique for the entire system are suggested. Trips and signal variables that apply to the steam generator should refer to the component number NUM for the primary side and the appropriate NUMS for the secondary side. After the user specifies the global parameters necessary to construct the secondary flow, LOAD format is used to input the fluid-cell geometrics and initial conditions similar to the PIPE component.

A second level networking capability with a generalized data structure has been included to analyze the secondary steam-generator components. This permits the user the ability to effectively represent the secondary flo:" "pa' and include the heat structures important to the steam-generator behavior. Furthermore, this added feature reduces the size of the matrices necessary for obtaining solutions for the networks external to the steam-general component.

The steam-generator component provides the ability to connect fluid cells with generalized, one-dimensional structures in an arbitrary manner. Therefore, thermal communication across the tube walls, the downcomer, the tube shell, the plenum divider plates, and the tube sheets may be modeled. Only a one-dimensional, cylindrical geometry currently is available for the wall conduction analysis. Cartesian geometry may be approximated by specifying a large radius. The user is restricted further to using the same number of NODES to model all conduction paths.

The one-dimensional conduction paths are located throughout the steam generator by specifying an interior and exterior component number (ICMP and OCMP, respectively) and the interior and exterior cell number (ICELL and OCELL, respectively) for each conduction path. A zero is used for ICMP and OCMP to indicate a primary fluid cell.

The first node of each conduction element is the interior and the last $(i=$ NODES), the exterior. When referring to a cell located on the side tube of a TEE, the user should add the ceil number, counting the cell next to the main tube as one, to the number of cells on the main side. Examples of the values that should be assigned to these parameters for an aspirated oncethrough steam generator and for a U-tube steam generator are provided in Tables XII and XIII, respectively. Schematics for these two steam-generator 
TABLE XII

SPECIFICATION OF THE FLUID CELLS ASSOCIATED WITH THE CONDUCTION PATHS FOR AN ASPIRATED ONCE-THROUGH STEAM GENERATOR

\begin{tabular}{|c|c|c|c|c|}
\hline & ICMP & ICELL & OCMP & OCELL \\
\hline \multirow[t]{5}{*}{ Tube walls } & 0 & 2 & 31 & 1 \\
\hline & 0 & 3 & 31 & 2 \\
\hline & 0 & 4 & 31 & 3 \\
\hline & 0 & 5 & 32 & 1 \\
\hline & 0 & 6 & 32 & 2 \\
\hline \multirow{2}{*}{ Tube sheet } & 0 & 1 & 31 & 1 \\
\hline & 0 & 7 & 32 & 2 \\
\hline \multirow[t]{4}{*}{ Downcomer } & 31 & 1 & 30 & 3 \\
\hline & 31 & 2 & 30 & 2 \\
\hline & 32 & 1 & 32 & 4 \\
\hline & 32 & 2 & 32 & 3 \\
\hline
\end{tabular}

TABLE XIII

SPECIFICATION OF THE FLUID CELLS ASSOCIATED WITH THE CONDUCTION PATHS FOR A U-TUBE STEAM GENERATOR WITH A COUNTERFLOW PREHEATER

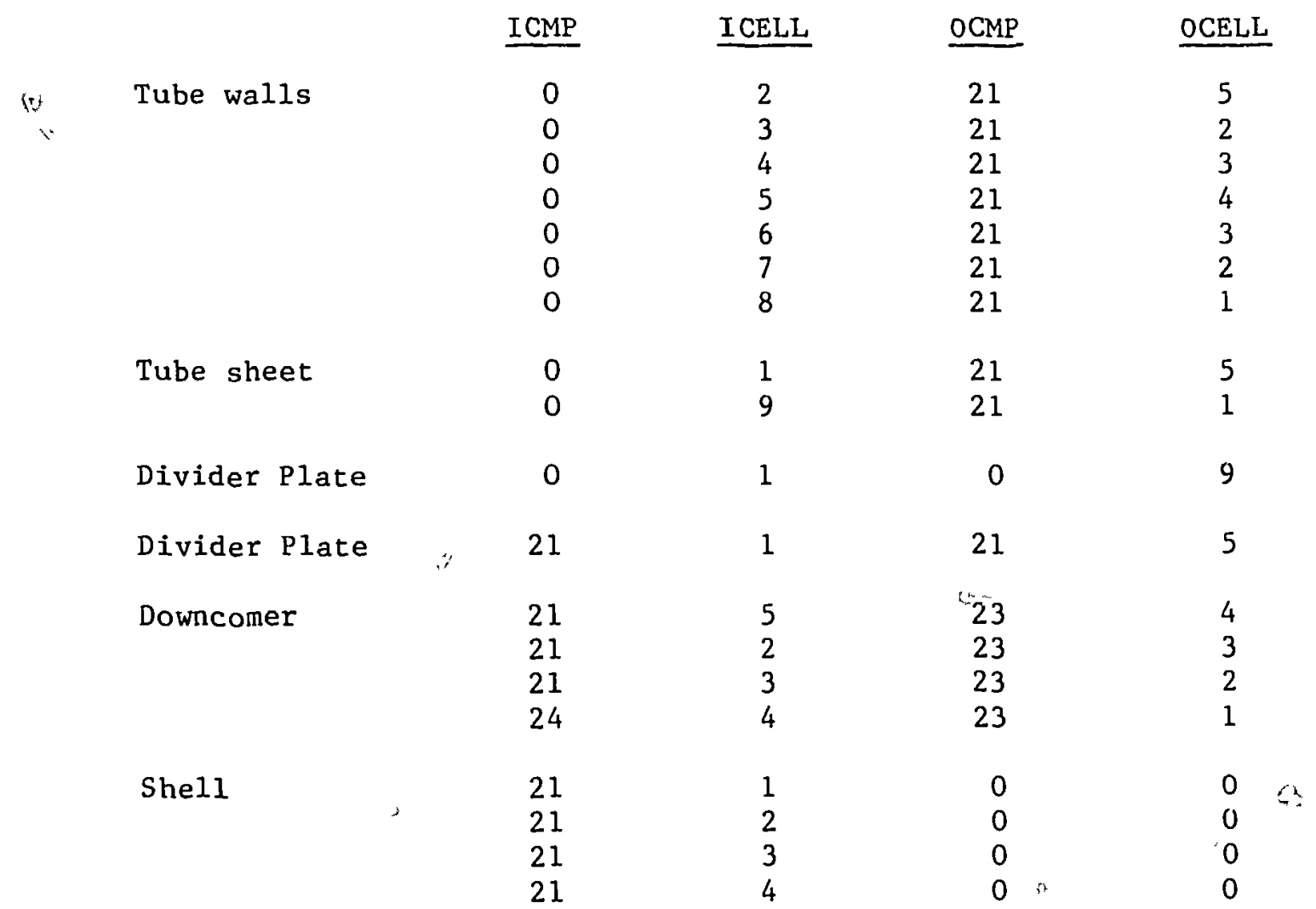


types are shown in Fig. 35. The only restriction to assigning fluid cells on each side of the conduction paths is that the same fluid cell cannot be assigned to both sides of the same conduction element. More than one conduction element may be associated with a given fluid cell. An external environment may be assigned to the exterior of a conduction path by setting both OCMP and OCELL to zero. The last node of that path then applies Newton's law as a boundary condition with the constant input values for the liquid and vapor IITCs and temperatures. The remaining conduclion path inpul defines the element material, geometry, and initial conditions. When more than one value is required for a parameter, the first value is associated with the interior (first node) of the element. The heat-transfer surface area for a conduction path used to describe the tubes represents the total surface area for áll tubes in the fluid cell under consideration. An internal check is made to assure the input values for the interior and exterior conduction path surface areas are consistent with the cylindrical geometry. The HTCs calculated on each side of the conduction element are based on the local fluid properties. The correlations are identical to those used for all one-dimensional components.

Output for the conduction paths is provided in two forms. The first provides information for each path. Temperature distributions as well as the fluid thermal state on each side of the element are available. Also, output is available on a fluid-cell basis, which allews the user insight into the energy available to each fluid cell from,the steam-generator structures. When more than one conduction element is in contact with a fluid cell, these (effective) properties are defined to conserve energy. The values provided include

(1) effective HTCs,

$$
h_{\ell \text { eff }}=\frac{\sum\left(h_{\ell i} A_{i}\right)}{A}, \text { and }
$$




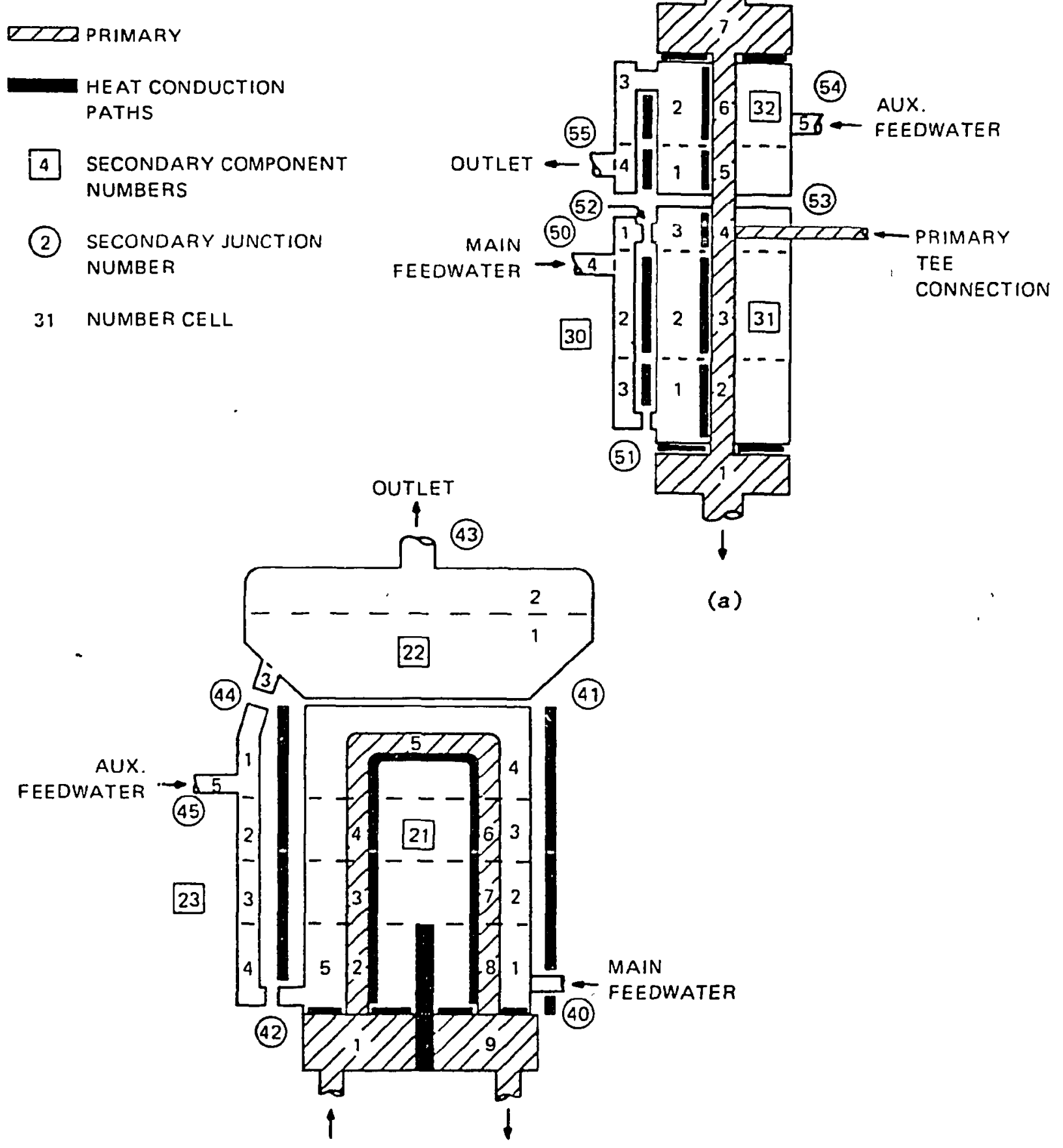

(b)

Fig. 35 .

TRAC steam-generator noding diagrams.

(a) Aspirated once-through steam generator and

(b) U-tube steam generator with a counterflow preheater. 
(2) effective wall temperatures,

$$
T_{\ell \text { eff }}=\frac{\sum\left(h_{\ell i} A_{i} T_{w i}\right)}{h_{\ell e f f}},
$$

where $A=\left\{A_{1}\right.$. The values are summed over all surfaces associated with the fluid cell. $i$ Similar relations apply to the vapor properties.

Input for the steam-generator component is processed by subroutines RSTGEN and RESTGN. RSTGEN obtains data from an input file (TRACIN), whereas RESTGN reads the data from a restart file (TRCRST). Initialization of any remaining variables not provided by the input or restart files is performed in the subroutine ISTGEN.

During problem execution, the solution procedure is controlled by subroutines STGEN1, STGEN2, and STGEN3. STGEN1 controls the prepass including calculation of wall friction and loss coefficients. Heat structure material properties and HTCs are obtained through a call to STGN1X. Solutions to the hydrodynamics equations are controlled by STGEN2. Fina1ly, a postpass is obtained using STGEN3. The postpass includes updating fluid properties and a call to STGN3X, which provides the heat-path conduction analysis. Output is provided to a dump file (TRCDUMP) through subroutine DSTGEN and an output file (TRCOUT) with WSTGEN.

I.

Tee

The TEE module models the thermal hydraulics of three piping branches, two of which lie along a common line, whereas the third enters at some angle $\beta$ from the main axis of the other two (Fig. 36). In TRAC-PF1, the tee is treated as two PIPEs, as indicated in Fig. 36. Beta is defined as the angle from the low-numbered end of PIPE 1 to PIPE 2. The low-numbered end of PIPE 2 always connects to PIPE 1 . The first PIPE extends from cell 1 to ce11 NCELL1 and connects to PIPE 2 at cell JCELL. The second PIPE begins at cell 1 and ends at ce11 NCELL2.

The connection is affected through mass, momentum, and energy source terms in PIPE 1. PIPE 2 sees the connection as boundary conditions from cell JCELL in PIPE 1. Liquid may be prevented from entering the TEE secondary by 


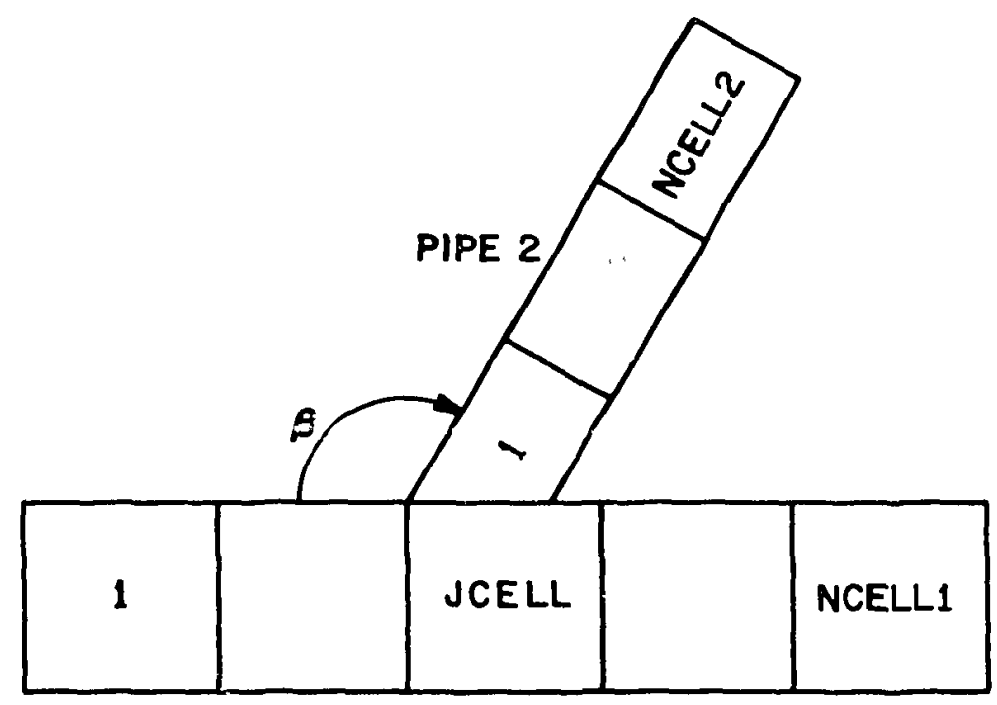

PIPE 1

Fig. 36 .

Tee noding diagram.

setting the value of FRIĆ at that junction to a value greater than $10^{20}$. However, no generalized separation model has been implemented. This FRICbased separator actually will work at any cell interface. Hence, caution should be exercised when introducing large values of FRIC at other locations in a system.

Because the TEE is modeled essentially as two interconnected PIPEs, the PIPE model description in Sec. IV.A should be referenced for additional information on the calculational sequence. Naturally, the sequence for a TEE includes separate calculations of the primary and secondary sides.

Detailed input specifications for a TEE component are given in Sec.V.C.5.1. Input and output information is very similar to that for a PIPE component except that two PIPEs are involved in a TEE component.

J. Turbine

In the turbine, energy is removed from the fluid as a result of work performed by the turbine. The model calculates the momenturn and energy losses and the angular velocity as a function of the fluid flow rate, fluid properties, and the turbine nozzle and blade geometry. These momentum and energy losses are subsequently subtracted from the TRAC momentum and energy equations respectively. 
Each TURB module represents an individual turbine stage. Thus the number of TURB components required in the TRAC model is equal to the total number of individual stages associated with the turbines rather than the number of turbines. However, the code internally recognizes the "family" of stages belonging to a particular turbine, which facilitates turbine governing and overall turbine performance calculations that depend on individual stage performanccs. All the data that are common among the family (i.e., data pertaining to the turbine rather than individual stages, such as, the generator efficiency or the generator power demand), are entered under stage 1, and the other stages of the family access these data from stage 1. Modeling an individual stage with a TURB component allows the user complete flexibility for modeling extraction, regeneration, reheating, etc., using the other TRAC input modules.

In both the impulse and reaction turbines, the thermodynamics of the internal processes is basically the same. In the impulse turbine, the expansion of steam takes place in the nozzles or in fixc $d$ blades, and the kinetic energy of steam is converted into mechanical work at the blades; whereas in the reaction turbine, the steam is allowed to expand in moving blades also, thereby transferring the reaction energy into mechanical work. Details of this process are discussed in many standard text books on thermodynamics. Salisbury, 53 in particular, discusses the subject comprehensively. The steam expansion process through a turbine stage is shown in Fig. 37. Line $A B$ represents isentropic expansion from pressure $p_{A}$ to pressure $P_{B}$. Because of friction and other irreversible losses, the actual state of steam after expansion is $B^{-}$and not $B$. Thus the steady-state energy equation gives

$\mathrm{h}_{\mathrm{A}}+\frac{\mathrm{v}_{\mathrm{A}}^{2}}{2}=\mathrm{h}_{\mathrm{B}}+\frac{\mathrm{v}_{\mathrm{B}}^{2}}{2}=\mathrm{h}_{\mathrm{B}^{-}}+\frac{\mathrm{v}_{\mathrm{B}^{-}}^{2}}{2}+\frac{\mathrm{P}}{\dot{\mathrm{m}}}$,

where $\dot{m}$ is the mass flow rate and $P$ is the stage work per second. The velocity $V_{B}$ is the ideal nozzle velocity, such that $P_{i d e a l}=\dot{\mathrm{m} V}{ }_{B}^{2} / 2$, and is easily obtained using $\mathrm{Eq}$. (223) along an isentrope. 

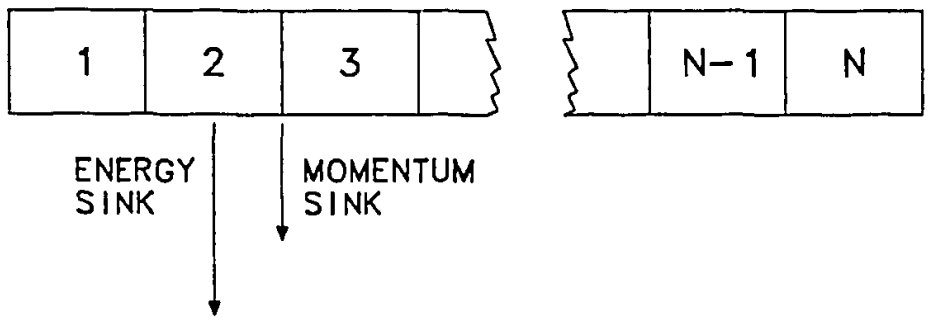

FLOW DIRECTION
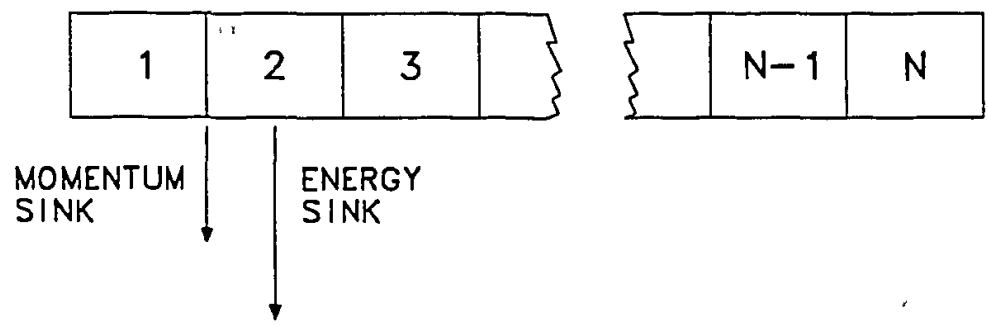

Fig. 37.

Steam expansion through a turbine stage.

The stage power, $P$, can therefore be calculated as

$\mathrm{P}=n_{\text {stg }} \mathrm{P}_{\text {ideal } 1}=n_{\text {stg }} \dot{\mathrm{m}} \frac{\mathrm{V}_{\mathrm{B}}^{2}}{2}$,

where $n_{\text {stg }}$ is the stage efficiency.

The final state $B^{-}$can be easily computed for a known $n$ stg under steady-state conditions using Eqs. (223), (224), the continuity equation, and the equation of state. This is the approach followed by Farman and Mot loch 54 in the RETRAN code. However, a preferable approach is to make use of the transient, two-phase TRAC hydrodynamic equations with appropriate sink terms in the momentum and energy equations. The momentum and energy terms corresponding to the turbine power are extracted as shown in a typical noding diagram in Fig. 38. The energy is always extracted from cell number 2, whereas the momentum is extracted either from the second or the third cell 


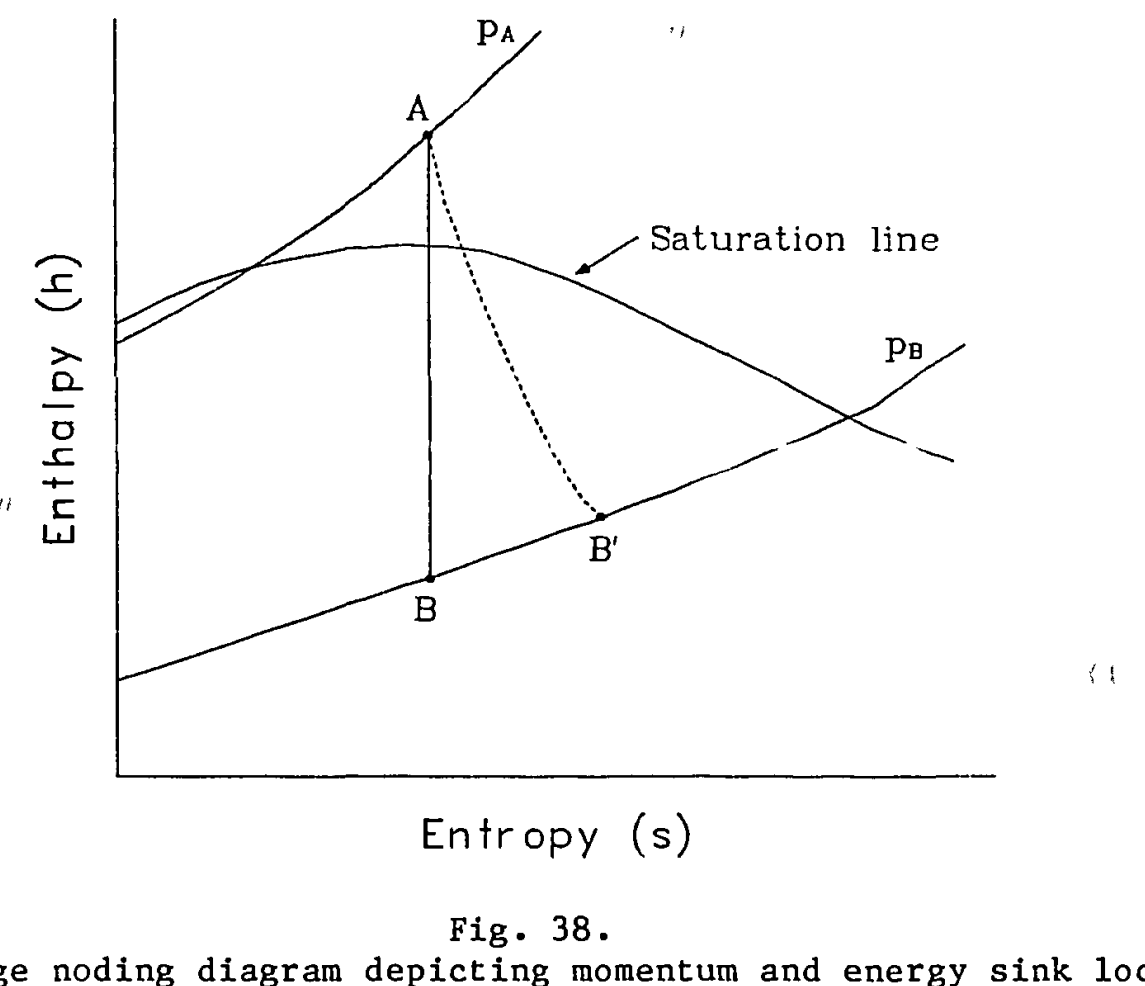

Turbine stage noding diagram depicting momentum and energy sink locations.

edge depending upon the flow direction. A minimum of three cells are required to model a TURB component. It is clear from Eq. (223) that the velocity at the turbine exit would have been $V_{B}$ had no work been done by the turbine. Owing to the turbine work, the exit velocity is $V_{B}$ - instead of $V_{B}$. Thus the momentum loss in the turbine stage is

$M=\dot{m}\left(V_{B}-V_{B^{-}}\right)=A_{j} \rho_{j} v_{j}\left(V_{B}-v_{B^{-}}\right)$

where $A$ is the area. Subscript $j$ refers to the momentum sink cell edge. Also, velocity $V_{B}$ - is the same as $v_{j}$, the actual cell-edge velocity calculated by the code after it accounts for the momentum loss. Therefore, writing Eq. (225) in terms of pressure loss, $\Delta p$, gives

$\Delta p=\rho_{j} v_{j}\left(v_{B}-v_{j}\right)$ 
Experience has shown that translating the above pressure loss into an equivalent FRIC results in much improved code stability than subtracting $\Delta \mathrm{p}$ from the momentum equation directly (with its associated derivative $\partial \Delta \mathrm{p} / \partial \mathrm{V}_{j}$, of course). Therefore, writing $\Delta p$ in terms of FRIC gives

$\Delta p=\operatorname{FRIC} \frac{\rho_{j} v_{j}^{2} 0.5\left(\Delta x_{j}+\Delta x_{j-1}\right)}{d_{j}}$,

where $d_{j}$ is the momentum source cell-edge diameter. Comparison of this equation with Eq. (226) leads to

FRIC $=\frac{v_{B}-v_{j}}{v_{j}} \frac{d_{j}}{0.5\left(\Delta X_{j}+\Delta x_{j-1}\right)}$.

Because the above value of FRIC is applied at either cell edge 2 or cell edge 3 (depending upon flow direction), the user Input additive FRIC values at these cell edges will be overwritten. Therefore FRIC(2) and FRIC(3) should always be input as 0.0 .

The energy loss that should be taken out of the total energy equation is given by Eq. (224). However, TRAC-PF1/MOD1 uses the thermal energy equation, which is obtained after combining the total energy equation with the momentum equation. Some simple algebraic substitutions lead to the following expression for the sink term for the thermal energy equation.

$P_{e n r}=P-\frac{V_{B}+V_{j}}{2} M=P-A_{j} \frac{V_{B}+V_{j}}{2} \Delta P$,

where $P_{\text {enr }}$ is the amount to be extracted irom the TRAC energy equation. The second term on the right hand side of Eq. (228) appears as a result of combining the momentum equation with the energy equation. 
Equations (227) and (228) can, therefore, be used to account for momentum and energy losses as a result of the power produced by a turbine stage provided the stage efficiency is known.

The stage efficiency is given by

$n_{\text {stg }}=n_{n b}-\phi_{m}-\phi_{\text {rem }}$,

where $n_{n b}$ is the nozzle-bucket efficiency to be obtained from the velocity diagram, $\phi_{m}$ is the molsture loss, and $\phi_{\text {rem }}$ are the remaining miscellaneous stage losses whose magnitudes are not easily definable. Term $\phi$ rem includes nozzle-erd loss, windage loss, dlaphragm-packing loss, etc. The moisture loss is included as a separate term because it is the most significant loss and its functional formulation is known. These losses are described in detail by Salisbury. Salisbury recommends the following expression for the molsture loss

$\phi_{\mathrm{m}}=1.15(1-\mathrm{x})$,

where $x$ is the thermodynamic quality of steam.

The nozzle-bucket efficiency is calculated as

$n_{n b}=\frac{\text { energy transfer to the blades }}{\text { avallable energy at the nozzle }}$

$$
=\frac{w \dot{m} \sum_{i=1}^{2 n} v_{a_{1}}}{\dot{m} \frac{v_{B}^{2}}{2}}=\frac{2 w}{v_{B}^{2}} \sum_{i=1}^{2 n} v_{a_{i}},
$$

where $W$ is the moving blade velocity, $v_{a}$ is the axial component of velocity relative to the moving blades, and $n$ is the number of rows of moving blades. A vector diagram for two rows of moving blades is presented in Fig. 39, which 
$\eta p$

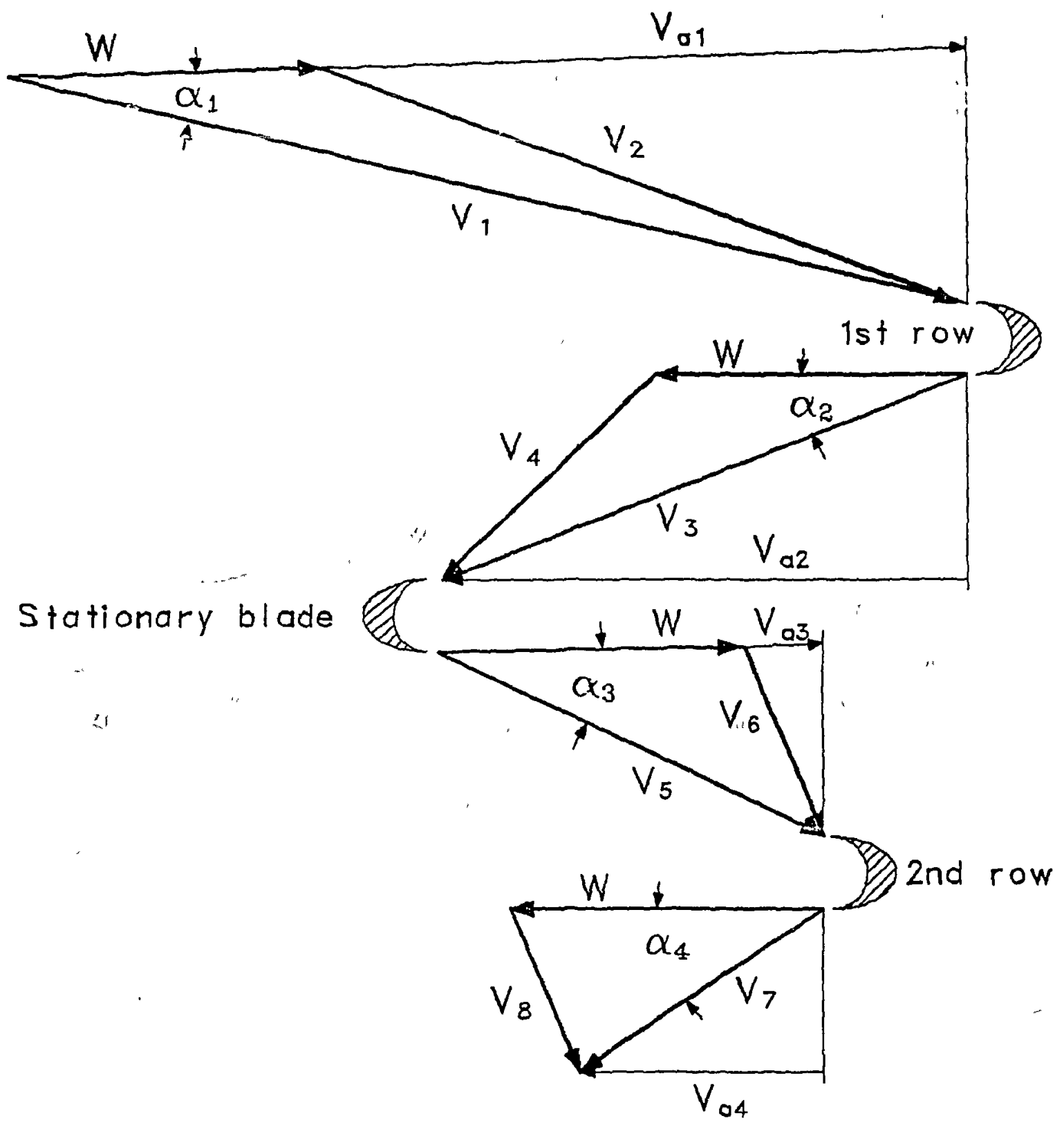

F1g. 39.

Velocity diagram for two rows of moving blades. 
can be extended to an arbitrary number of rows by our recursively appending a stationary blade row and a moving blade row in pairs to Fig. 39. There is no limit on the number of rows allowed in a TURB module. If. $r$ is the fraction of stage energy transferred by reaction, the velocity vectors can be written as follows:

$$
\begin{aligned}
& v_{1}=c_{n} v_{B}(1-r)^{1 / 2} \\
& v_{2}=\left(v_{1}^{2}+w^{2}-2 v_{1} w \cos \alpha_{1}\right)^{1 / 2}
\end{aligned}
$$$$
v_{3}=\left[\left(c_{b} v_{2}\right)^{2}+r \frac{c_{r}^{2}}{2 n-1} v_{B}^{2}\right]^{1 / 2}
$$$$
v_{4}=\left(v_{3}^{2}+W^{2}-2 v_{3} W \cos \alpha_{2}\right)^{1 / 2}
$$$$
v_{5}=\left[\left(c_{b} v_{4}\right)^{2}+r \frac{c_{r}^{2}}{2 n-1} v_{B}^{2}\right]^{1 / 2}
$$$$
\mathrm{v}_{6}=\left(\mathrm{v}_{5}^{2}+\mathrm{W}^{2}-2 \mathrm{v}_{5} \mathrm{~W} \cos \alpha_{3}\right)^{1 / 2}
$$$$
v_{7}=\left[\left(C_{b} v_{6}\right)^{2}+r \frac{c_{r}^{2}}{2 n-1} v_{B}^{2}\right]^{1 / 2}
$$$$
\mathrm{v}_{\mathrm{a}_{1}}=\mathrm{v}_{1} \cos \alpha_{1}-\mathrm{w}
$$$$
\mathrm{v}_{\mathrm{a}_{2}}=\mathrm{v}_{3} \cos \alpha_{2}
$$$$
v_{a_{3}}=v_{5} \cos \alpha_{3}-w
$$$$
\mathrm{v}_{\mathrm{a}_{4}}=\mathrm{v}_{7} \cos \alpha_{4}
$$

where $C_{n}$ is the nozzle velocity coefficient, $C_{b}$ is the bucket velocity coefficient, and $c_{r}^{2}$ is the fraction of total reaction energy actually delivered in all rows of blades. It is assumed that equal fractions of reaction energy are delivered in each blade row. 
Although the remaining losses,' $\phi_{\text {rem, }}$ required in Eq. (229) are not easily definable at off-design conditions, their magnitude is generally known at design conditions from the test data. In TRAC-PFl, the user is allowed an option of specifying the stage efficiency, nstg, at design conditions. If $n_{\text {stg }}$ is input, the code uses Eq. (229) to calculate $\phi_{\text {rem }}$ at design conditions. This value of $\phi_{\text {rem }}$ is then assumed constant throughout the transient. If the user does not input $\eta_{s t g}$ at design conditions, $\phi$ rem is assumed to be zero.

Thus, to summarize, a TURB module performs the following sequence of calculations.

1. With the upstream and downstream pressures and velocities from the previous time step, the ideal nozzle velocity, $v_{B}$, is calculated and we assume steam is an ideal gas.

2. With the bucket velocity, $W$, from the previous time step, $n_{n b}$ is calculated from Eqs. (231) and (232).

3. The stage efficiency, $\eta_{\text {stg, }}$, is then calculated from Eq. (229).

4. Stage power, $P$, is calculated from Eq. (224) with in from the previous time step.

5. An equivalent FRIC for the momentum equation is calculated from Eq. (227) with $\mathrm{V}_{j}$ from the previous time step.

6. The amount to be extracted from the energy equation, $P_{\text {enr }}$, is then calculated from Eq. (228).

So far we have discussed how each stage is handled by a TURB module, which represents an individual stage. In general, a turbine has more than one stage, and the governing is accomplished for the turbine as a whole and not for individual stages because only the total load on the turbine is known. Also, the angular speed among the "family" of stages' is the same because of direct coupling of the stages. Thus additional calculations that are common among the family of stages are required. These calculations are done after all the stages are processed, and these common data are stored under the first stage of the family.

Total turbine power is calculated as

$P_{\text {total }}=\sum_{i=1}^{m} P_{i}$, 
where $m$ is the total number of stages belonging to a turbine, i.e., the number of stages of the same family. However, the electrical generator power input is less than $P_{\text {total }}$ because of the friction at the bearings of the turbine-generator shaft. Therefore, the power input to the generator, Pgen, input' is

$\mathrm{P}_{\text {gen, input }}=\sum_{i=1}^{\mathrm{m}} \mathrm{P}_{\mathrm{i}}-\omega \mathrm{T}_{\mathrm{fric}}$,

where $\omega$ is the turbine-generator angular speed, and $\mathrm{T}_{\mathrm{fric}}$ is the frictional toíque given by

$T_{\text {fric }}=A+B \omega+C \omega^{2}$,

where $A, B$, and $C$ are the user input coefficients. The generator output is therefore

$P_{\text {gen, output }}=n_{\text {gen }}\left[\sum_{i=1}^{m} P_{i}-\omega T_{f r i c}\right]$,

where $n_{\text {gen }}$ is the generator efficiency, which is treated as constant. At design conditions, this output must be equal to the generator power demand. However, because of uncertainties in stage "power calculations, friction torque, and generator efficiency, this equality cannot be exactly satisfied. Thus a correction efficiency, $n_{\text {corr }}$; is defined, such that

$$
\eta_{\text {corr }}=\frac{\text { generator power demand at design conditions }}{\text { [ngen } \left.\left[\sum_{i=1}^{m} P_{i}-\omega T_{f r i c}\right]\right\}_{\text {design conditions }}} .
$$


This $\eta_{\text {corr }}$ is calculated during initialization and is used as a constant multiplier during the entire transient. Thus Eq. (236) modifies into

$P_{\text {gen,output }}=n_{\text {corr }} n_{\text {gen }}\left[\sum_{i=1}^{m} P_{i}-\omega T_{f r i c}\right]$.

At equilibrium conditions, this output should be equal to the generator power

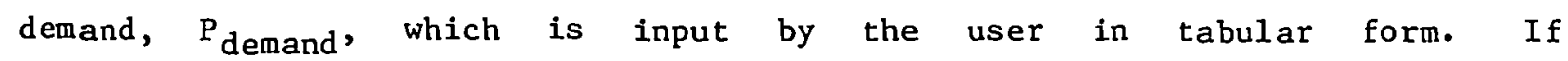
$\mathrm{P}_{\text {gen,output }}>\mathrm{P}_{\text {demand, }}$, the steam flow through the turbine should be reduced and vice versa. To accomplish this and to bypass the excess steam flow, two special options in the VALVE component have been implemented (see VALVE input description, Sec.V.C.5.k). An IVTY $=5$ option opens the valve with an increase in power demand. Such a valve could be added upstream of the turbine to control the steam supply to the turbine. Another option, IVTY $=6$, automatically closes the valve when the power demand increases. This valve option may be found useful in the bypass line. Figure 40 shows two typical turbines (a two-stage throttle governed and a three-stage bypass governed) without any extractions to illustrate the use of these special valve options. As the power demand increases, more steam has to be supplied to the turbine, and in case of a bypass governed turbine, some additional steam has to be supplied to lower stages. Thus the turbine valves use IVTY $=5$ option. However, as the steam flows through the turbines increase, the amount of steam bypassed must decrease. The turbine bypass valve therefore cán be modeled using IVTY $=6$ option. It might, however, be preferable to operate the turbine bypass valve using a controller in order to maintain a constant pressure in the steam generator.

The signal variable for the operation of these special type valves is defined as

$\mathrm{S}=\frac{\mathrm{P}_{\text {demand }}}{\mathrm{P}_{\text {gen,output }}}$

A value of $S>1.0$ "opens the IVTY $=5$ valve and closes the IVTY $=6$ valve, whereas $S<1.0$ operates these valves in the other direction.

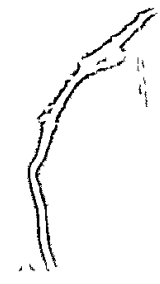




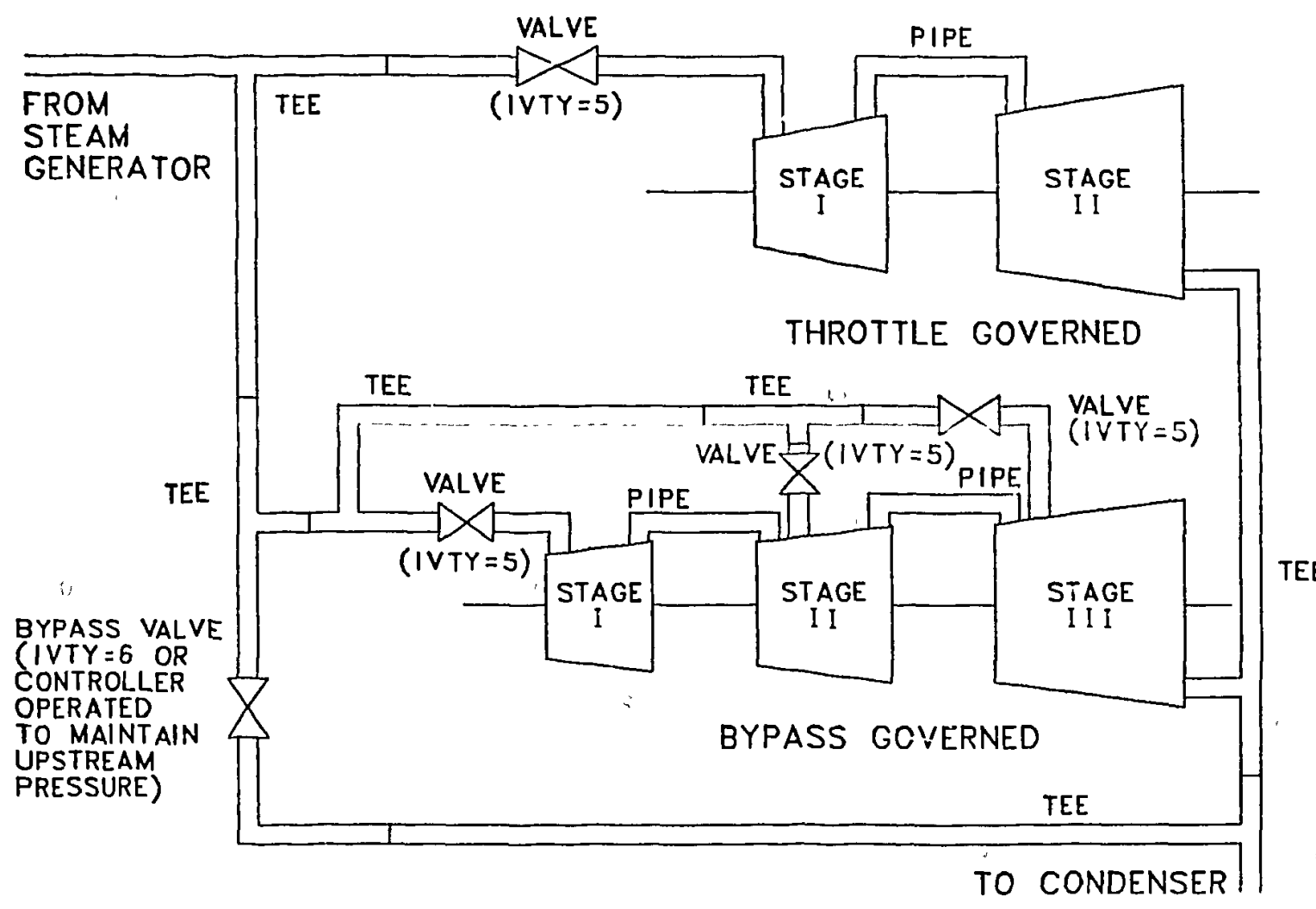

Fig. 40 .

A typical noding diagram showing the use of special valve options for modeling turbines.

The turbine speed is maintained constant as long as the plant is connected with the external load, i.e., $P_{\text {demand }} \neq 0.0$, to maintain constant grid frequency, To meet this power demand at the desired grid frequency, the steam supply to the turbine is adjusted as discussed above." However, the turbine is allowed to coast down at the receival of turbine trip signal (which is generated by tripping off the generator power demand). The turbine speed in this case is determined by the angular momentum balance

$I \frac{d \omega}{d t}=T_{\text {turb }}-T_{\text {fric }}-T_{\text {loss }}-T_{\text {demand }}$,

where $I$ is the turbine-generator assembly moment of inertia, $T_{\text {turb }}$ is the total turbine torque output from all stages, $\mathrm{T}_{10 s \mathrm{~s}}$ is the loss in torque as a 
result of generator losses, and $\mathrm{T}_{\text {demand }}$ is the torque due to external load. However, because the turbine is allowed to coast down only when the external load is zero, $\mathrm{T}_{\text {demand }}=0.0$. Also, because the generator is not producing any power, $\mathrm{T}_{\text {loss }}=0.0$. Thus $\mathrm{Eq} \cdot(239)$ simplifies to

$\mathrm{T} \frac{\mathrm{d} \omega}{\mathrm{dt}}=\frac{1}{\omega} \sum_{i=1}^{\mathrm{m}} \mathrm{P}_{i}-\mathrm{T}_{\text {Eric }}$

The TURB data are processed in a manner similar to that for the PIPE component. Subroutine RTURB reads data from the input file (TRACIN), and subroutine RETURB reads data from the restart file (TRCRST). Initialization of any remaining variables not provided by the input or restart files is performed in subroutine ITURB.

Subroutines TURB1, TURB2, and TURB3 perform the same functions for the TURB component as PIPE1, PIPE2, and PIPE3 perform for the PIPE component. For the TURB component, subroutine TURBI calls TRBPOW, which calculates turbine stage efficiency and power. Two additional subroutines, TRBPRE, and TRBPST, are called during the prepass and postpass stages, respectively, to perform calculations that are common for a family of different stages related to the same turbine.

Wall friction coefficients are calculated in subroutine FWALL by specifying a friction correlation option, NFF, along with the additive fraction factors, FRIC, for each cell edge. The homogeneous flow friction factor correlation option $(N F F=1$ ) is suggested for a TURB component. Also, as explained earlier in this section, FRIC(2) and FRIC(3) should be set to zero.

The output edit for a TURB component is similar to a PIPE component with the addition of stage efficiency, stage power, and stage mass flow. In addition, quantities pertaining to the entire turbine are printed under the

- TURB component modeling the first stage. They are: generator power output, turbine speed, and turbine signal value S defined by Eq. (238). 
K. Valve

The VALVE module is used to model various types of valves associated with light water reactors. The valve action is modeled by controlling the flow area and the hydraulic diameter at one cell interface of a onedimensional component as shown in Fig. 41. The valve action may not be located at a valve component junction unless that junction is connected to a BREAK.

Two methods are provided for specifying the valve flow area. The flow area FA can be computed directly from a flow area fraction (FAVLVE) according to

FA $=$ FAVLVE $\cdot$ AVLVE ,

where AVLVE is the input value for the fully open valve flow area. Alternatively, the flow area may be computed from the relative position (XPOS) of the valve stem if we assume a guillotine-type cut of circular cross section. The relative valve-stem position of XPOS $=1$ corresponds to a fully open valve with flow area AVLVE. In either case, the corresponding hydraulic diameter HD is computed from

hydraulic diameter $=\frac{4 F A}{P} \leq$ HVLVE,
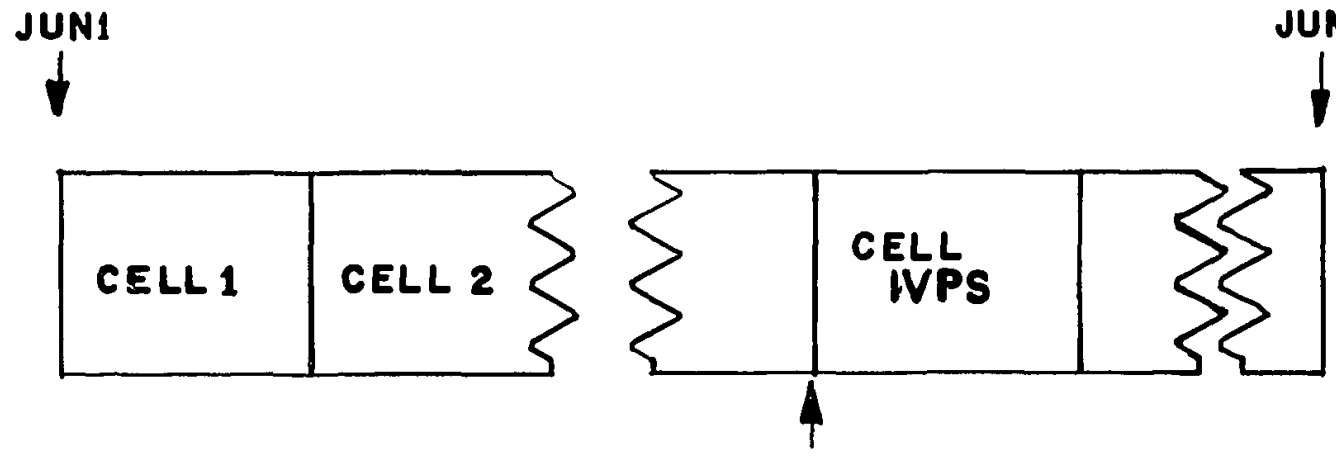

\section{FLOW AREA CONTROLLED \\ BY VALVE ACTION}

Fig. 41 .

Valve noding diagram. 
Where $P$ is the wetted perimeter of the guillotine-type cut opening and HVLVE is the input value for the fully open valve hydraulic diameter.

The FA fraction or valve stem position is entered as a constant or a tabular function defined by a table. Use of a table may be trip-initiated according to the control option selected. To increase the flexibility to model various types of valves, two valve tables may be input for tripcontrolled valves. The first table is used when the trip set status is $\mathrm{ON}_{\text {forward, }}$, and the second table is used when the trip set status is ON reverse (see Sec.V.C). The independent variable for the table is a modeled-system parameter defined by a signal variable or a control block.

Many different types of valves can be modeled because of the flexibility available to choose the independent variable for the valve action table and to implement table evaluation under trip control. Simple valves that either open or close on a trip may be modeled using an OFF-ON or ON-OFF type trip and a table that has relative time (since trip initiation) as the independent variable to obtain the desired rate of opening or closing. Valve leakage can be simulated by restricting the table minimum FA fraction or valve stem position to a value greater than zero. Simple valves can be used to model pipe breaks or the opening of rupture disks.

A simple check valve can be modeled by using a valve table with the appropriate pressure gradient as its indejendent variable. Alternatively, a check valve can be modeled as a trip-controlled valve with the pressure gradient used as the trip signal to trip on and the valve table used to control the rate of value movement.

A steam-flow control valve [or power-operated relief valve (PORV)] can be modeled using an $O N$ reverse ${ }^{-O F F-O N}$ forward trip to control it with the start closing pressure, end closing pressure, end opening pressure, and start opening pressure as the respective trip set points. The rate of opening ( $\mathrm{ON}_{\text {forward }}$ state) can be defined by the first value table and the rate of closing ( $O N_{\text {reverse }}$ state) by the second value table. The rate of opening and closing will be the same only if the first valve table is entered.

A PORV can be modeled by using a table with pressure as the independent variable and a step-like function for the flow area fraction or valve stem position. In this case, it is important that the step function not be too steep or the valve flow area may oscillate because of the coupling between the 
flow through the valve and the pressure variable. A bank of PORVs can be modeled with a single valve component in the same manner by our using a multistep function to simulate the multiple pressure set points corresponding to the various valves.

The input parameters for the VALVE module are defined in Sec. V.C.5.k.

L. Vesse1

The VESSEL module models a PWR vessel and its associated internals. The component is three-dimensional and uses a six-equation, two-fluid model to evaluate the flow through and around all internals of a PWR vessel including the downcomer, core, and upper and lower plena. Models incorporated into the VESSEL module are designed mainly for LOCA analysis, but the VESSEL module can be applied to other transient analyses as well. A mechanistic reflood model that can model quenching or dryout for an arbitrary number of quench fronts is included. The reactor power is modeled using point-reactor kinetics or by providing a power table in the problem input. Most of the detailed discussion of the fluid-dynamics, heat-transfer, and point-reactor kinetics equations and solution methods for the three-dimensional VESSEL module can be found in Sec. III. In this section, we discuss the VESSEL geometry and other important considerations.

A three-dimensional, two-fluid, thermal-hydraulic model in cylindrical coordinates describes the VESSEL flow. A regular cylindrical mesh, with variable mesh spacings in all three directions, encompasses the downcomer, core, and upper and lower plena of the VESSEL, as shown in Fig. 42. The user describes the mesh by specifying the radial, angular, and axial coordinates of the mesh-cell boundaries:

$$
\begin{aligned}
& r_{i} \quad i=1, \ldots, \text { NRSX ; } \\
& \theta_{j} \quad j=1, \ldots, \text { NTSX ; } \\
& \text { and } \\
& z_{k} \quad k=1, \ldots, \text { NASX ; }
\end{aligned}
$$


where NRSX is the number of rings, NTSX is the number of angular segments, and NASX is the number of axial levels. The point $\left(r_{i}, \theta_{j}, z_{k}\right)$ is a vertex in the coordinate mesh. Figure 43 illustrates the mesh construction. Mesh cells are formed as shown in Fig. 44 and identified by an axial level number and a cell number. For each axial level, the cell number is determined by counting the cells radially outward starting with the first angular segment and the innermost ring of cells, as shown in Fig. 43 . Figure 44 also shows the relative face numbering convention that is used in connecting other components

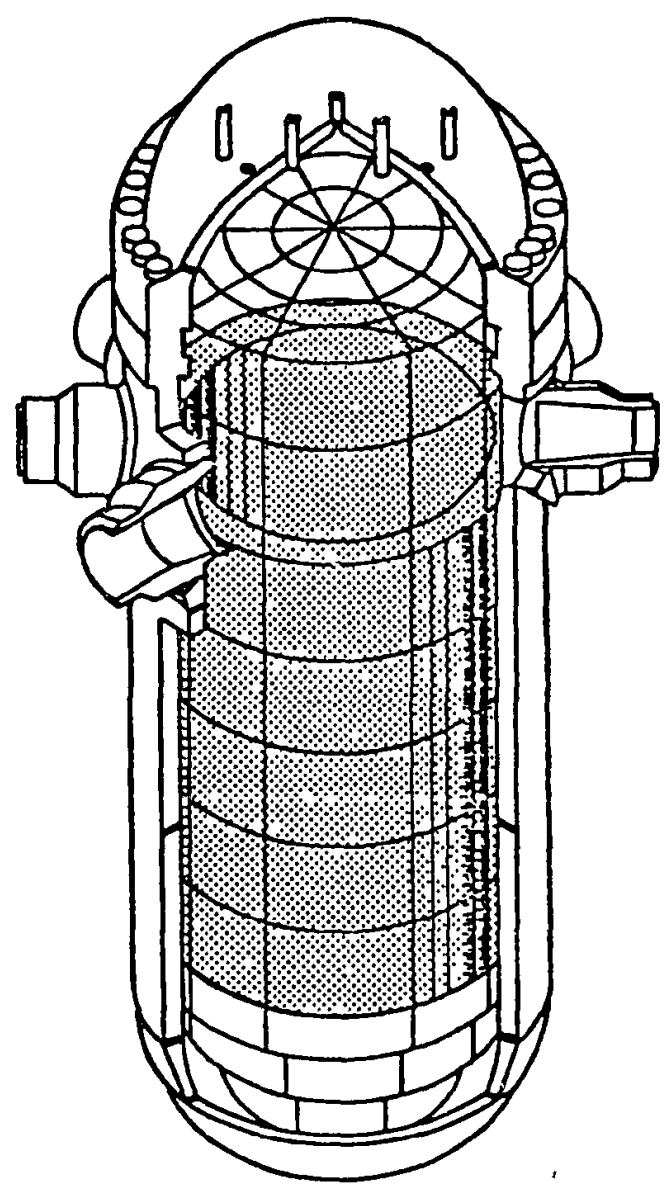

Fig. 42 .

Cell noding diagram for a typical PWR vessel.

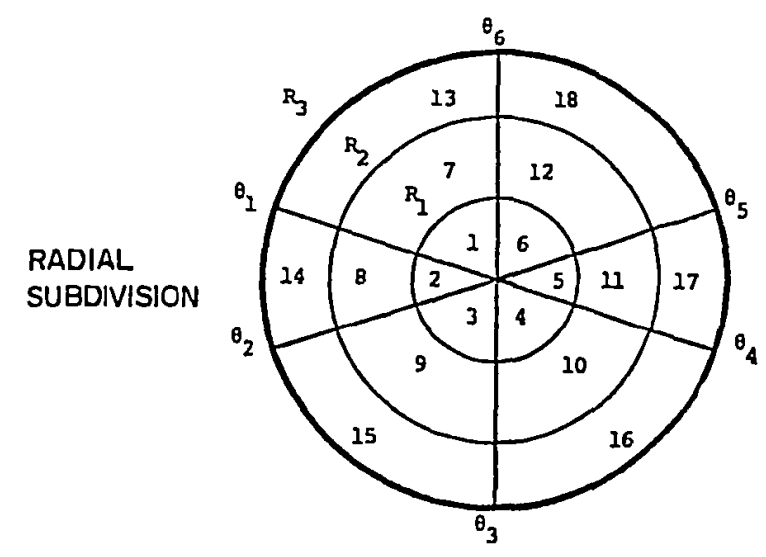

AXIAL

SUBDIVISION

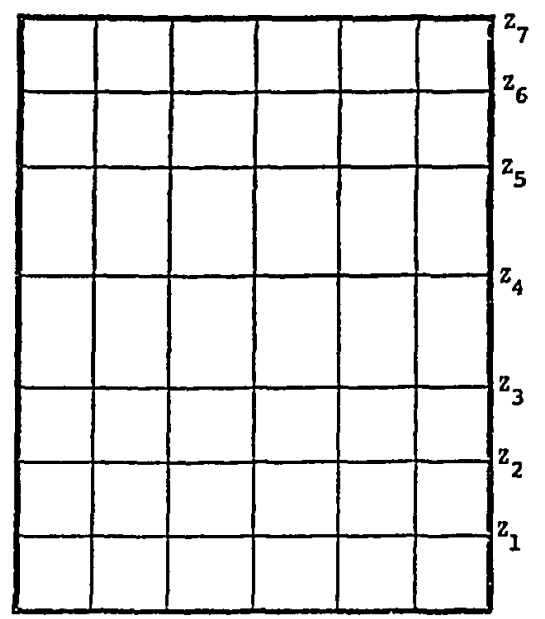

Fig. 43.

Vessel geometry, three-dimensional mesh construction with 3 rings, 6 angular segments, and 7 axial intervals. 


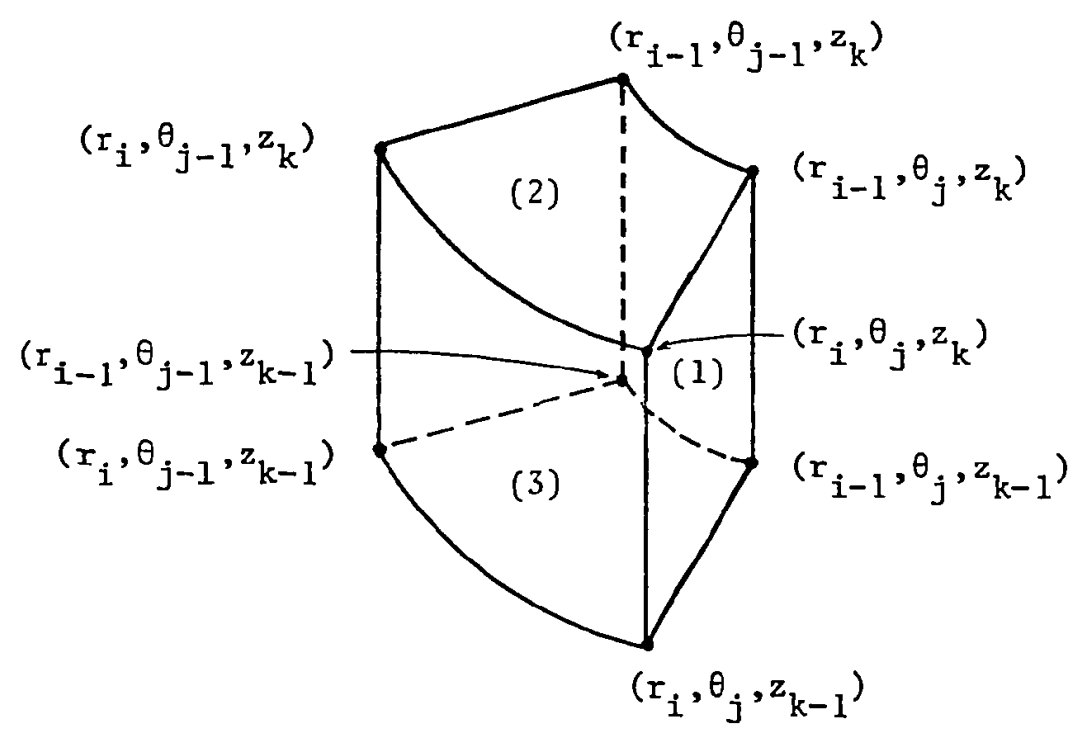

Fig. 44 .

Boundaries of a three-dimensional mesh cell. The face numbering convention also is shown. Faces 1,2 , and 3 are in the $\theta, z$, and $r$ directions, respectively.

to the vessel. Note that only three faces per mesh cell must be identified because the other faces will be defined by neighboring cells.

All fluid flow areas (on cell faces) and all fluid volumes are dimensioned so that the internal structure within the vessel can be modeled. Flow areas and fluid volumes are computed on the basis of the geometric mesh spacings and scaled according to factors supplied as input. The scaled volumes and flow areas then are used in the fluid-dynamics and heat-transfer calculations.

Flow restrictions and the volume occupied by the structure within each mesh cell are modeled through the use of these scale factors. For example, the downcomer walls are modeled by setting the appropriate flow area scale factors to zero. A feature is "provided to do this automatically in the code if the upper, lower, and radial downcomer position parameters (IDCU, IDCL, and IDCR) are specified as described in the input section (Sec.V.C.5.1). (The NAMELIST parameter IGEOM3 can be used to permit setting non-zero flow-area fractions in the downcomer walls to model leakage flow paths; then IDCU, IDCL, and IDCR can be set so that downcomer and lower plenum global parameters are written to the graphics file.) Flow restrictions such as the top and bottom 
core support plates require scale factors between zero and one. Figure 45 shows the cell faces that are scaled to model the downcomer and core supportplate flow restrictions.

Plumbing connections from other components to the VESSEL are made on the faces of the mesh cells. Only one connection per VESSEL cell is allowed, and each mesh cell in the VESSEL can have a component connected to it. Four input parameters are used to describe a connection: ISRL, ISRC, ISRF, and JUNS. The parameter ISRL defines the axial level in which the connection is made; ISRC is the mesh-cell number, as defined above; and ISRF is the face number, as defined in Fig. 44. If ISRF is positive, the connection is made on the face shown in the figure with the direction of positive flow inward into the cell. If ISRF is negative, the connection is made on the opposite face shown in the figure with the direction of positive flow also inward into the cell. (Face 1 is the azimuthal face at $\theta_{j}$, and face -1 is the azimuthal face at $\theta_{j-1}$. Face 2 is the axial face at $z_{k}$, and face -2 is the axial face at $z_{k-1}$. Face 3 is the radial face at $r_{i}$, and face -3 is the radial face at $r_{i-1}$ ) The parameter JUNS identifies the one-dimensional component junction connected to this cell face. Figure 46 shows two VESSEL pipe connections. Note that internal as well as external connections are allowed. The user is cautioned against connecting to the VESSEL any component with a flow area that differs greatly from the flow area of the mesh-cell face to which it is connected because this can cause anomalous pressure gradients. Such a situation can be avoided by proper adjustment of the VESSEL geometry coordinate spacings.

A VESSEL option models the Babcock and Wilcox vent valves that are located in the wall between the upper plenum and downcomer. These vent valves permit flow directly from the upper plenum to the downcomer and out the cold leg for a cold-leg break. They are modeled as fixed areas in the outer radial surface of a vessel cell with a variable FRIC term to model opening and closing.

The user specifies the cells that have vent valves by giving the axial level, cell number, and total area of vent valves for each cell with vent valves in the outer radial surface. The user also specifies for each cell with vent valves: (1) the pressure drop for the valves to be closed, DPCVN; (2) the pressure drop for the valves to be open, DPOVN; (3) the FRIC value when the valves are closed to model leakage, FRCVN; and (4) the FRIC value 


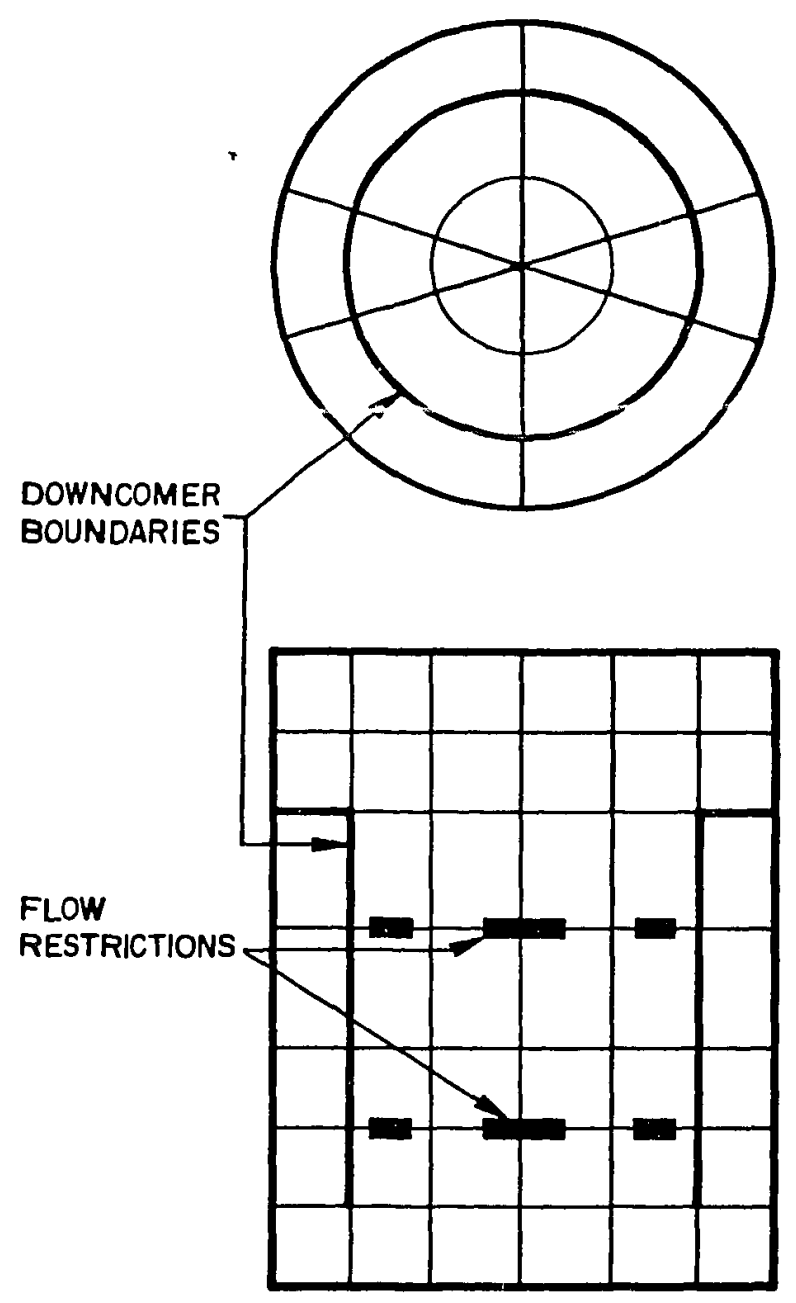

Fig. 45 .

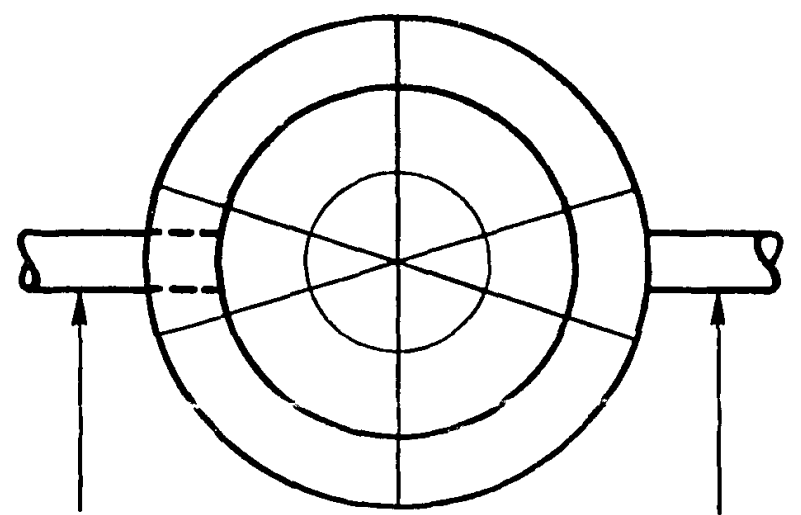

OUTLET
NOZZLE

INLET NOZZLE

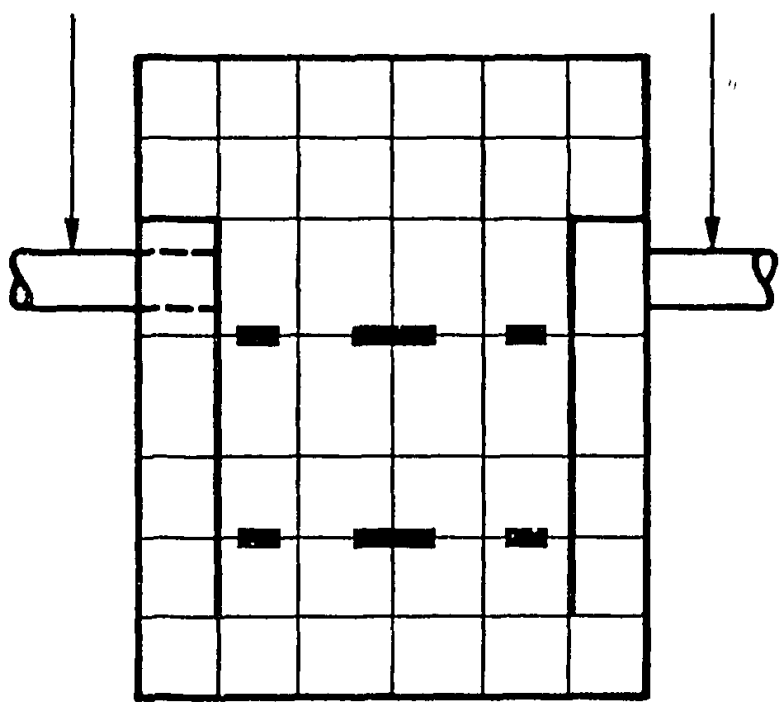

Fig. 46 .

Flow restrictions and downcomer modeling. Pipe connections to the vessel.

when the valves are open, FROVN. The pressure drop is defined as the pressure of the inner radial cell minus the pressure of the outer radial cell. The code uses FRCVN when the pressure drop is less than DPCVN, uses FROVN when the pressure drop is greater than DPOVN, and interpolates for pressure drops between DPCVN and DPOVN.

The reactor core region in the VESSEL is specified by the upper, lower, and radial core positional parameters (ICRU, ICRL, and ICRR). These parameters define, respectively, the upper, lower, and radial boundaries of the cylindrical core region. The example provided in Fig. 47 shows a possible configuration in which $I C R U=4, I C R L=2$, and $I C R R=2$. Each axial stack of 


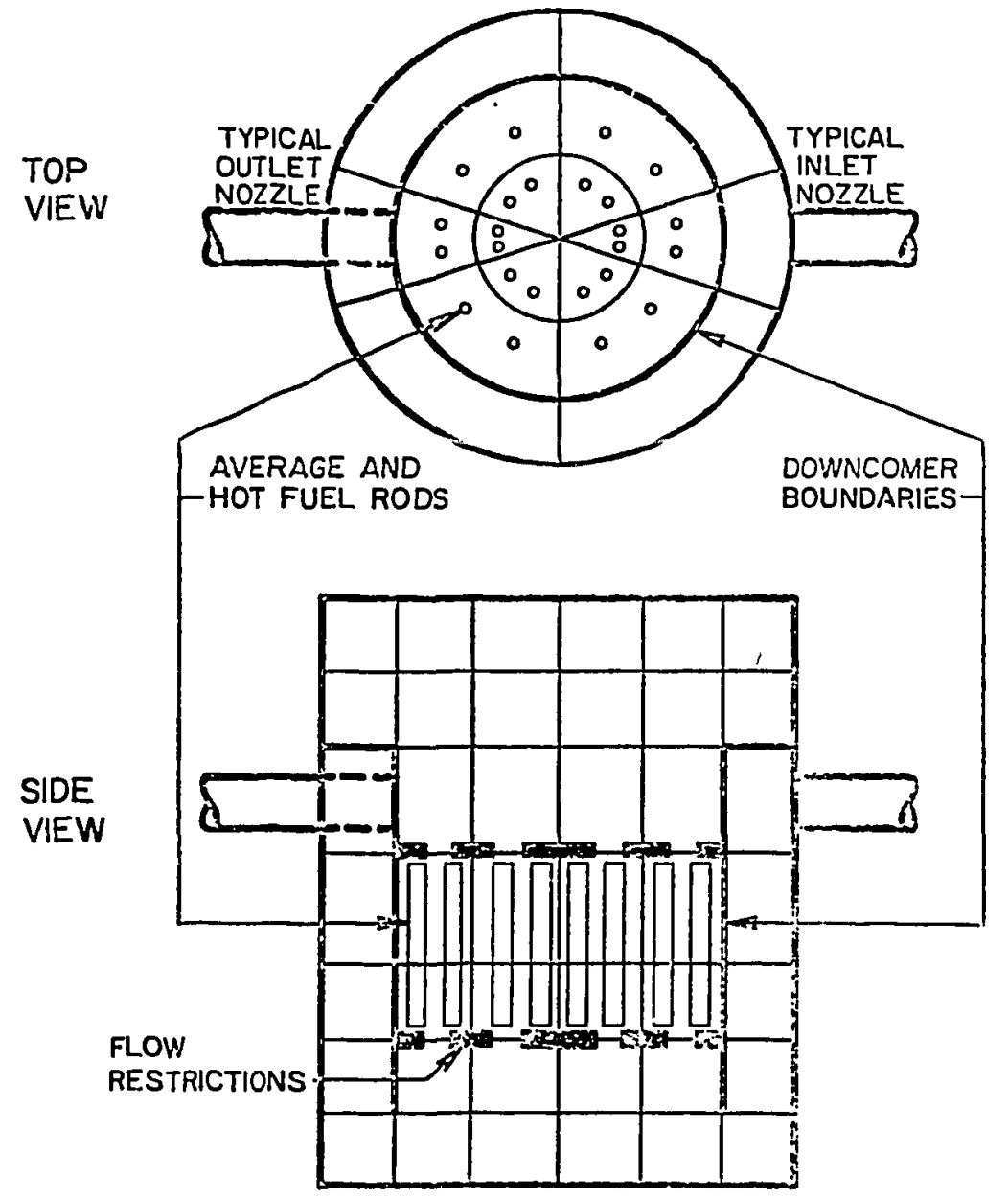

Fig. 47 .

Core region inside the vessel.

mesh cells in the core region can contain an arbitrary number of fuel rods. One average rod represents the average of the ensemble of rods in each axial stack of mesh cells, and its thermal calculation couples directly to the fluid dynamics. The thermal analysis of any supplemental rods does not feed back or couple directly to the fluid-dynamics analysis. However, the local fluid conditions in the mesh cell are used to obtain rod temperature histories for the additional rods. A fuel-cladding interaction treatment and a reflood treatment are available for these calculations and are described in Sec. III.B. 
Heat slabs of arbitrary thicknesses and surface areas can be defined in any mesh cell (including core regions) to model the heat capacity of structures within the vessel. A HTC is computed for each slab using the local fluid conditions. The temperature distribution is calculated based on a onedimensional conduction model (refer to Sec. III.B.1.b). The thermal response of the slab properties is included. Furthermore, the code can account for an arbitrary number of interfaces between dissimilar materials.

The total power level in the core may be specified in terms of either power or reactivity with optional reactivity feedback. The solution of the point-reactor kinetics equations used in the latter case is described in Sec. III.C. The power or reactivity can be constant, determined from a table, or constant until a trip and then determined from a table. The independent variable of the table may be any signal-variable form as described in Sec.V.C.4.b.

For trip-initiated table use, the independent variable of the table can be modified by a rate factor that is a (tabular) function of the departure of the trip signal from the set point that turns off the trip. Consequently, override control of the core power level can be simulated. The flexibility that is available for the selection of the trip signal, trip set points, and delay times as well as the choice for the independent variable and associated rate factor used to specify the power or reactivity permits accurate modeling of a large class of transient conditions.

The spatial power distribution in the core is specified by separate axial- and horizontal-plane power shapes, plus a radial power distribution within the fuel rods. These spatial distributions are specified in relative units at input and are held constant throughout a problem except for the axial power shape. Through input, one or more axial power shapes may be employed with any signal variable used to interpolate among them.

The power density in fuel rod node $i$ in cell $j$ on core level $k$ is given by the expression,

$$
P(i, j, k)=S \cdot P_{\text {tot }} \cdot \operatorname{RDPWR}(i) \cdot \operatorname{CPOWR}(j) \cdot \operatorname{ZPOWR}(k) \text {, }
$$


where $P_{\text {tot }}$ is the total core power level, $\operatorname{RDPWR}(i)$ is the relative power in fuel node $1, \operatorname{CPOWR}(j)$ is the relative power in cell $j, \operatorname{ZPOWR}(k)$ is the relative power at core elevation $k$, and $S$ is the scale factor that normalizes the three input relative power distributions. The scale factor $S$ is given by the expression,

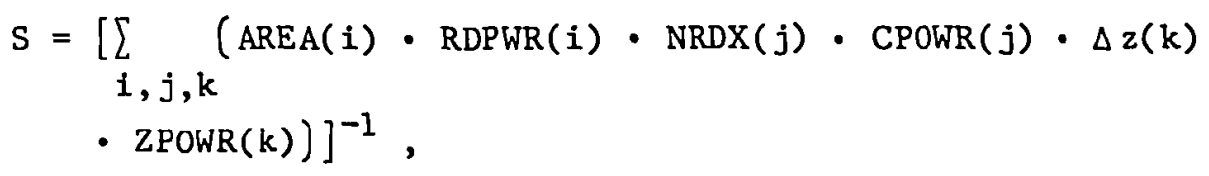

where $\operatorname{AREA}(i)$ is the cross-sectional area of fuel rod node $i, \operatorname{NRDX}(j)$ is the number of fuel rods in cell $j$, and $z(k)$ is the height of core axial level $k$. For the analyses of user-specified supplemental rods, the power density'in Eq. (224) is multiplied by an input power factor $\operatorname{RPKF}(j)$ to obtain the power density for each additional fuel rod. 
V. USER INFORMATION

This chapter describes how to set up a problem input data deck, obtain data dumps, restart problems from those dumps, and produce printer and graphics output files.

A. Input Organization and Format

The input deck is divided into six major sections: main, materialproperties, interactive control-panel, control-parameter, component, and timestep data. These data blocks are contained in the TRACIN file and are read in the above order (Fig. 48). The main-data information block contains general parameters including title cards for problem identification, restart and dump information, transient and steady-state information, problem size information, and problem convergence criteria. This data block always is present in TRACIN.

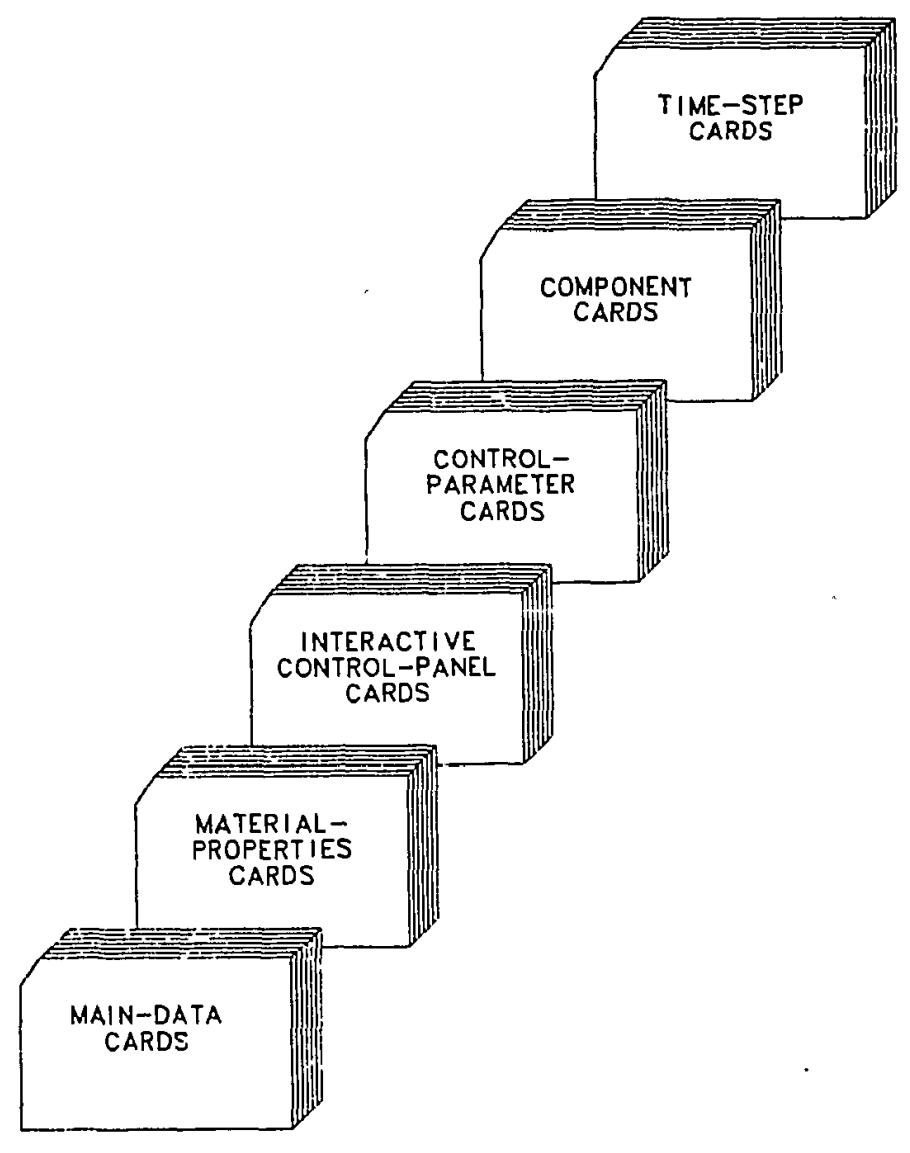

Fig. 48.

TRAC input deck organization. 
The user has the option to input material-properties data when the TRAC internally defined material properties are inappropriate. New properties may be entered for materials that TRAC does and does not model. These properties must be included in the TRACIN file for all restarts, bccause they are not written to the TRCDMP dump data file that becomes the renamed TRCRST restart data file.

The user inputs interactive control-panel data when he wishes to execute TRAC in the interactive mode. These data define the component locations for the PWR-system parameters that normally are monitored in the power-plant control room. Information that is not provided by the TRACIN file is obtained from the TRCRST restart data file. At present this capability is not maintained fully or documented; thus, it is not recommended for users outside of Los Alamos National Laboratory.

Control-parameter data define the control-procedure parameters that are used to control the PWR-system model: signal variables, control blocks, and trips as well as the order in which these three control-parameter types are evaluated. Signal variables define PWR-system parameters for control-block input, trip-signal definition, and component-action-table independent-variable definition. Control blocks are functional operators that operate on their input signals to produce an output signal. Their input signals are signal variables or the output signal from control blocks. A control block's output signal is used for the same applications as signal variables. Trips are ON-OFF switch controllers that provide a logic switch and specify when component actions are to be evaluated. The number of each control-parameter type is defined by the main data. When fewer of a particular controlparameter type are specified in TRACIN, the remainder comes frorn the TRCRST restart data file.

The component data block provides the main body of the input deck. This block contains a detailed description of every component in the problen unless the problem is reinitiated from a restart data dump. For restart problems, this block contains only additional or modified components. The rest of the component data is obtained from the restart file. There is a component data block in TRACIN unless all the component data are to be obtained from the restart file. 
The time-step data block specifies maximum and minimum time-step sizes, . edit frequencies, and the end of the problem. It always is present in TRACIN. All numeric input data are read into the code with either an E14.6 or I14 format statement, by the LOAD subroutine or as NAMELIST data. Standard FORTRAN statements are used for formatted and NAMELIST reads. The LOAD subroutine provides additional flexibility to input array data.

The user can create his TRACIN input deck in strict accordance with the input specifications described in Sec.V.C. (formatted input) or in free format. If formatted input is selected, all data and array loading operations must be entered in the card columns specified in Sec. V.C. "If free format is chosen, the input cards and the data on each card must adhere to the order specified in Sec. V.C, but the data do not have to be entered in the specified columns. In addition to the obvious convenience of not counting columns, free format gives the user greater flexibility in using comments to document his input deck and TRAC provides better diagnostics for input errors. The freeformat option is discussed in detail in Sec. V.E.

The following sections describe the dump/restart feature, the input data format in detail, the LOAD subroutine read formats, the free-format option, the NAMEIST format, and the TRAC output files. Appendix E provides a sanple input deck and a portion of this deck that was modified to illustrate various free-format possibilities.

B. Dump/Restart Feature

TRAC automatically generates a dump/restart data file named TRCDMP, which contains snapshots of the state of the system at various tines during problem execution. Any one of these snapshots, called dumps, may be used to initialize all or part of the system for subsequent calculations. The times when dumps are generated are determined by several criteria." The user may specify a dump interval on the time-step cards. A dump will be created whenever this time interval has elapsed since the last dump. These dumps are added sequentially to the end of the TRCDMP file. A dump also may be initiated by the user with one more designated trips. At the time the set status of any, of those trips is set $\mathrm{ON}$, a dump is added to the end of the TRCDMP file. This permits the restart of a problem when particular events of interest occur. 
In addition to these user-specified dumps, TRAC automatically generates dumps at various times. A dump is generated at the end of the initialization stage; another dump is generated at the end of the steady-state or transient calculation and at intermediate points in the calculation based upon the central-processor-unit (CPU) time utilized by the job.

To use a dump file to initialize a subsequent calculation, the name of the file must be changed from TRCDMP to TRCRST. The time-step number DSTEP of the particular dump desired must be specified on Main-Data Card 3. (A message containing this dump time-step number is sent to the print file TRCOUT whenever a dump is written.) If the time-step number specified is negative, TRAC will use the dump with the largest time-step number and overwrite the negative value of DSTEP (specified on Main-Data Card 3) with the time-step number taken from that dump.

Data retrieved from the selected dump depend on the information that already has been found in the TRACIN file. Any component not defined by the input deck (as determined from the component numbers listed in the IORDER array in the Main Data) is initialized from the restart dump. Also, any signal variable, control block, and trip (with an ID number that has not already been defined by the TRACIN file) in the dump will be initialized with its defined state from the dump.

C. TRAC-PFl Input Specifications

The TRAC input data are classified into six general data types:

(1) main,

(2) material properties,

(3) interactive control panel,

(4) control parameter,

(5) component, and

(6) time step. 
1. Main Data. The main parameters are 1 isted below in the order in which they are entered. This data block always must be supplied at the start of the input deck.

Main-Data Card 1. (Free format.) The first card of a TRACIN deck serves as the free-format $O N-O F F$ switch, indicating whether the following cards are in free or TRAC format. It must be present, containing either the string FREE (free format), TRAC (TRAC format), or both strings (FREE overrides TRAC). This card is in free format; up to 80 columns may be used; the control string(s) and documentation may appear anywhere on the card.

Main-Data Card 2. (Format 4I14) NUMTCR, IEOS, INOPT, NMAT

$\frac{\text { Columns }}{1-14} \quad \frac{\text { Variable }}{\text { NUMTCR }}$

$15-28$

IEOS

$29-42_{15}$ INOPT

43-56 NMAT

\section{Description}

Number of title cards to be read. Note: At least one title card must be supplied.

Air-water option.

$0=$ gas phase treated as steam-air mixture throughout system;

1 = gas phase treated as air throughout the system. Evaporation and condensation are inhibited.

Specification for including or excluding NAMELIST group INOPTS data after the title cards.

$0=$ NAMELIST group INOPTS data omitted after the title cards;

1 = NAMFLIST group INOPTS data inserted after the title cards.

Number of different materials for which heatconduction-related properties will be input. The material-properties data are not passed through the restart dump and, hence, must be in the input file for each restart.

Title Card(s). (Format 20A4) NUMTCR Cards

Columns

$1-80$
Description

Problem title information. 
NAMELIST Data Cards for Group INOPTS. (NAMELIST format) This section is included only if the variable INOPT $=1$. In this case one or more of the special input options described below may be specified. The format of this data is not checked during preprocessing and, therefore, should be entered carefully to avoid fatal input errors. The data are entered in columns 2-80 on one or more cards, beginning with "\$INOPTS" in column 2, and are terminated with a "\$" character. A more detailed discussion of the format for NAMELIST input data is included in Sec. V.F (also see local FORTRAN manual). The following variables are included in the NAMELIST group INOPTS, and one or more of them can be included in the NAMELIST data to select desired options. Variables omitted from the data retain their defaut status.

$\underline{\text { Variable }}$

ALP

CHM12, CHM22

CHM13, CHM23

CHM14, СHM24

CHM15, СHM25

HSTN

IADDED

ICFLOW

\section{Description}

Default value for initial void fractions. Used when ISTOPT is nonzero. (Real format.)

Subcooled and two-phase multipliers, respectively, for chokedflow multiplier set 2 (default $1.0,1.0$ ).

Subcooled and two-phase multipliers, respectively, for chokedflow multiplier set 3 (default $1.0,1.0$ ).

Subcooled and two-phase multipliers, respectively, for chokedflow multiplier set 4 (default $1.0,1.0$ ).

Subcooled and two-phase multipliers, respectively, for chokedflow multiplier set 5 (default $1.0,1.0$ ).

Default value for heat-slab temperatures in three-dimensional components. Used when ISTOPT is nonzero. (Real format.)

Switch that adds a status message to the TRCMSG file.

$\mathrm{N}=$ status inessage is added every Nth time step.

Choked-flow model controller.

$0=$ mode1 turned off;

$1=$ model using default multipliers turned on only for components connected to a BREAK (default).

2 = model using optional multipliers (NAMELIST variables CHM12, CHM22; CHM13, CHM23; CHM14, CHM24; and CHM15, CHM25) turned on at cell-edges indicated in component input. (Note that this option requires additional array data for all onedinensional hydrodynamics components.) 
Variable

IELV

IGAS

IGEOM3

IKFAC

IMFR

INVAN

Description

Switch that determines whether gravity (GRAV) terms or cellcentered elevations (ELEV), in meters, are to be input in the component data. When this option is selected, ELEV (dimensioned NCELLS) or cell-centered elevations should be input for GRAV array data cards in all components. In addition, a BREAK elevation BELV (BREAK component Card Number 5), a FILL elevation FELV (FILL component Card Number 4), and a VESSEL elevation shift SHELV (VESSEL component Card Number 13) are required.

0 = gravity terms must be input (default);

1 = cell-centered elevations must be input.

Flag for noncondensable gas.

$1=$ air (default);

2 = hydrogen.

Switch that determines, for all three-dimensional vessel components, if the areas between the downcomer and the inside of the vessel are to be set to zero.

$0=$ areas to be set to zero (default);

$1=$ the user enters the values of the area fractions used.

Switch that determines whether additive loss coefficients (FRIC) or $\mathrm{K}$ factors are to be input in the component data. When this option is selected, $\mathrm{K}$ factors (dimensioned NCELLS+1) must replace the FRIC array input cards in all components. To input $\mathrm{K}$ factors that depend on flow direction, include the NFRC1 or NFRC3 options.

$0=$ additive loss coefficients will be input (default);

1 = K factors will be input.

Switch that sends the phasic mass flows to the TRCGRF file.

1 = default;

3 = sends the phasic mass flows in the theta, radial, and axial directions.

Option that selects either the $\mathrm{T}_{\mathrm{CHF}}$ or $\mathrm{T}_{\mathrm{sat}}$ values for the inverted annular switch.

$0=\mathrm{T}_{\text {sat }}$ value is selected (all code assessment was performed with this default option);

$3=\mathrm{T}_{\mathrm{CHF}}$ value is selected. 
$\underline{\text { Variable }}$

IPOWR, TPOWR

IPRCPV

ISOLCN

\section{Description}

Variables that control core power initialization during steadystate calculations.

IPOWR $=-1$, TPOWR $=\infty$; core power is not turned on throughout the steady-state evaluation.

IPOWR $=-1, \quad$ TPOWR = specified; turns on core power at time TPOWR.

IPOWR $=0$, TPOWR $=\infty$ (defaults); turns on core power at time based on built-in logic. (When steady-state liquidvelocity-change ratio falls below a certain value or when the absolute value of the liquid velocity exceeds a certain value.)

IPOWR $=0$, TPOWR = specified; turns on core power at time TPOWR or at time based on built-in logic, whichever occurs first.

IPOWR $=1$, TPOWR = anything; turns on core power at the beginning of the steady state.

Switch that determines if control-panel-vector (CPV) parameters are processed.

$0=$ process $\mathrm{CPV}$ parameters;

$I=$ do not process $C P V$ parameters (default).

Solubility-parameters controller. (Solubility parameters characterize the dissolved material, which is followed by the solute tracker when ISOLUT $=1$ on Card Number 6.) **Warning** No input solubility parameters are saved on dump/restart; thus, these parameters must be input for each restart.

$0=$ use of default parameters for linear fit to solubility of orthoboric acid as a function of liquid temperature (default condition).

$l$ = input parameters for linear fit to solubility of solute as a function of liquid temperature. 
Variable

ISTOPT

NCRG

NDIA1

NDIA3
Description

Steady-state option that allows the user to input only once certain paraneters or default values that are used to initialize data arrays for other components. The default parameters also are input through the NAMELIST group INOPTS. The NAMELIST variables that can be assigned default values are ALP, VL, VV, TL, TV, TW, P, PA, QPPP, and HSTN. When ISTOPT is nonzero, the values of these variables are used to fill the corresponding arrays in all components except accumulators, pressurizers, valves, and steam-generator secondary sides. This option also can be used for transient calculations.

$0=$ option off (default);

$1=$ option on.

Those component arrays (excluding accumulators, pressurizers, valves, and steam-generator secondary sides), for which default values are included in the NAMELIST data, are filled with the default value. All cards that would contain data for defaulted arrays must be omitted from the input deck.

2 = option on.

Those component arrays (excluding accumulators, pressurizers, valves, and steam-generator secondary sides), for which default values are included in the NAMELIST data, are filled with the default value. Cards containing data for defaulted arrays must remain in the input deck but are overridden by the default value.

Switch that decides if ICRRG (the outermost radial ring in the core) is read af ter VESSEL Card Number 4.

0 = ICRRG is not read;

1 = ICRRG is read.

This variable can be used to force input of the heat-transfer diameter in addition to the hydraulic diameter, HD, to all onedimensional components. When it is set to 2 , the dimension of the HD array is doubled to $2 *($ NCELLS +1$)$. Input decks inust be changed so that (NCELLS+1) values end with an "E" for the hydraulic diameter. The next line begins an array of (NCELLS+1) values for the heat-transfer diameter.

This variable can be used to force input of the heat-transfer diameter in addition to the hydraulic diameter, HD, to all three-dimensional vessel components. When it is set to 2 , input decks must be changed to accommodate the added heattransfer diameter. The variables, $H D-T, H D-Z$, and $H D-R$, are input for the hydraulic diameter, followed immediately by a corresponding block containing the heat-transfer diameter. 
Variable

NFRC1

NFRC3

NOAIR

NORMDP

NORMRS

$\mathbf{P}$

PA
Description

This variable can be used to force input of forward and reverse loss coefficients to all one-dimensional components. When it is set to 2, the dimension of the FRIC array is doubled to $2 *$ (NCELLS+1). Input decks must be changed tc provide two consecutive arrays of loss coefficients, each dimensioned NCELLS+1. Each array is in LOAD format and inust be terminated with an "E." The first array provides loss coefficients that are used with positive velocities, and the second array provides loss coefficients used with negative velocities. To input these coefficients as $K$ factors, use the IKE 1 C option.

$1=$ standard FRIC input (default);

2 = two-way loss coefficients.

This variable can be used to force input of forward and reverse loss coefficients to all three-dimensional vessel components. When it is set to 2, input decks must be changed to accommodate the added reverse loss coefficients. The variables CFZL-T, CFZL-Z, CFZL-R, CFZV-T, CFZV-Z and CFZV-R are input as before to provide forward (positive velocity) loss coefficients. This block of input is inmediately followed by a corresponding block containing the reverse loss coefficients (RCFZL-T, RCFLL-Z, RCFZL-R, RCFZV-T, RCFZV-Z, RCFZL-R). To input these coefficients as standard $K$ factors, the IKFAC flag must be turned on.

Controls calculation of air partial pressure in one-dinensional components.

0 = air partial pressures solved for (less efficient when no air in system);

1 = air partial pressures set automatically to zero (default). (The air partial-pressure arrays must be entered even if NOAIR $=1$, in which case the arrays must be filled with O.0.)

Dump in the noninteractive mode.

$0=$ no (default if the code is interactive);

1 = yes.

Picks up restart from a normal noninteractive TRAC version.

$0=$ no (default if the code is interactive);

1 = yes.

Default value for initial pressures. Used when ISTOPT is nonzero. (Real format.)

Default value for initial air partial pressures. Used when ISTOPT is nonzero. (Real format.) 
Variable

QPPP

TL

TV

TW

VL

VV

\section{Description}

Default value for volumetric heat distribution in the walls of one-dimensional components. Used when ISTOPT is nonzero.- If this array is filled with the same nonzero constant, a uniform volumetric heat distribution results. (Real format.)

Default value for initial liquid temperatures. Used when ISTOPT is nonzero. (Real format.)

Default value for initial vapor temperatures. Used when ISTOPT is nonzero. (Real format.)

Default value for initial wall temperatures. Used when ISTOPT is nonzero. (Real format.)

Default value for initial liquid velocities. Used when ISTOPT is nonzero. (Real format.)

Default value for initial vapor velocities. Used when ISTOPT is nonzero. (Real format.)

Note: If ISOLCN $=0$ in the NAMELIST data, skip to Main-Data Card 3.

Solubility-Parameters Card. (Format 4E14.6) CNTLMN, CNMIN, CNTLMX, CNMAX

Columns Variable

1-14 CNTLMN

15-28 CNMIN

29-42 CNTLMX

43-56 CNMAX
Description

Minimum liquid temperature $(K)$ to use linear fit.

Solubility ( $\mathrm{kg}$ solute/ $\mathrm{kg}$ liquid) when liquid temperature at or below CNTLMN.

Maximum liquid temperature ( $K$ ) to use linear fit.

Solubility (kg solute/kg liquid) when liquid temperature at or above CNTLMX.

Main-Data Card 3. (Format I14,E14.6) DSTEP, TIMET

Columns Variable

1-14 DSTEP

15-28 TIMET
Description

Time-step number of dump to be used for restart. If DSTEP is less than zero, the last dump found will be used for restart.

Problem start time. If DSTEP or TIMET is less than zero, the time obtained from the retrieved dump overrides the TIMET input value. 


\begin{tabular}{|c|c|c|}
\hline Columns & Variable & Description \\
\hline $1-14$ & STDYST & $\begin{aligned} \text { Steady-state calculation indicator. } \\
0=\text { no steady-state calculation; } \\
1=\text { generalized steady-state calculation; or } \\
5=\text { static balance calculation (checks to see } \\
\text { if zero flow can be achieved with the } \\
\quad \text { pumps turned off and no heat transfer). }\end{aligned}$ \\
\hline $15-28$ & TRANSI & $\begin{array}{l}\text { Transient calculation indicator. } \\
0=\text { no transient calculation; } \\
1=\text { transient to be calculated. }\end{array}$ \\
\hline $29-42$ & NCOMP & $\begin{array}{l}\text { Number of components. Note that a steam- } \\
\text { generator component (Sec.V.C.5.h), including } \\
\text { all of its secondary-side components, should } \\
\text { be counted as one component. }\end{array}$ \\
\hline $43-56$ & NJUN & $\begin{array}{l}\text { Number of junctions excluding internal } \\
\text { junctions associated with the STGEN } \\
\text { component. }\end{array}$ \\
\hline $57-70$ & IPAK & $\begin{array}{l}\text { Water packing option. } \\
0=\text { off; } \\
1=\text { onl. }\end{array}$ \\
\hline
\end{tabular}

Main-Data Card 5. (Format 3E14.6) EPSO, EPSI, EPSS

\begin{tabular}{|c|c|c|}
\hline Columns & Variable & Description \\
\hline $1-14$ & EPSO & $\begin{array}{l}\text { Convergence criterion for outer iteration } \\
\left.\text { ( suggested value }=1.0 \times 10^{-3}\right) \text {. }\end{array}$ \\
\hline $15-28$ & EPSI & $\begin{array}{l}\text { Convergence criterion for vessel iteration } \\
\left.\text { ( suggested value }=1.0 \times 10^{-5}\right) \text {. }\end{array}$ \\
\hline $29-42$ & EPSS & $\begin{array}{l}\text { Convergence criterion for steady-state } \\
\text { calculation (suggested value }=1.0 \times 10^{-4} \text { ). }\end{array}$ \\
\hline Main-Data & Card 6. (Format 4I14) & OITMAX, IITMAX, SITMAX, ISOLUT \\
\hline Columns & Variable & Description \\
\hline $1-14$ & OITMÁXX & $\begin{array}{l}\text { Maximum number of outer iterations (suggested } \\
\text { value }=10 \text { ). }\end{array}$ \\
\hline $15-28$ & IITMAX & $\begin{array}{l}\text { Maximum number of vessel iterations } \\
\text { (suggested value }=50 \text {; set to zero for direct } \\
\text { inversion of the vessel matrix). }\end{array}$ \\
\hline
\end{tabular}




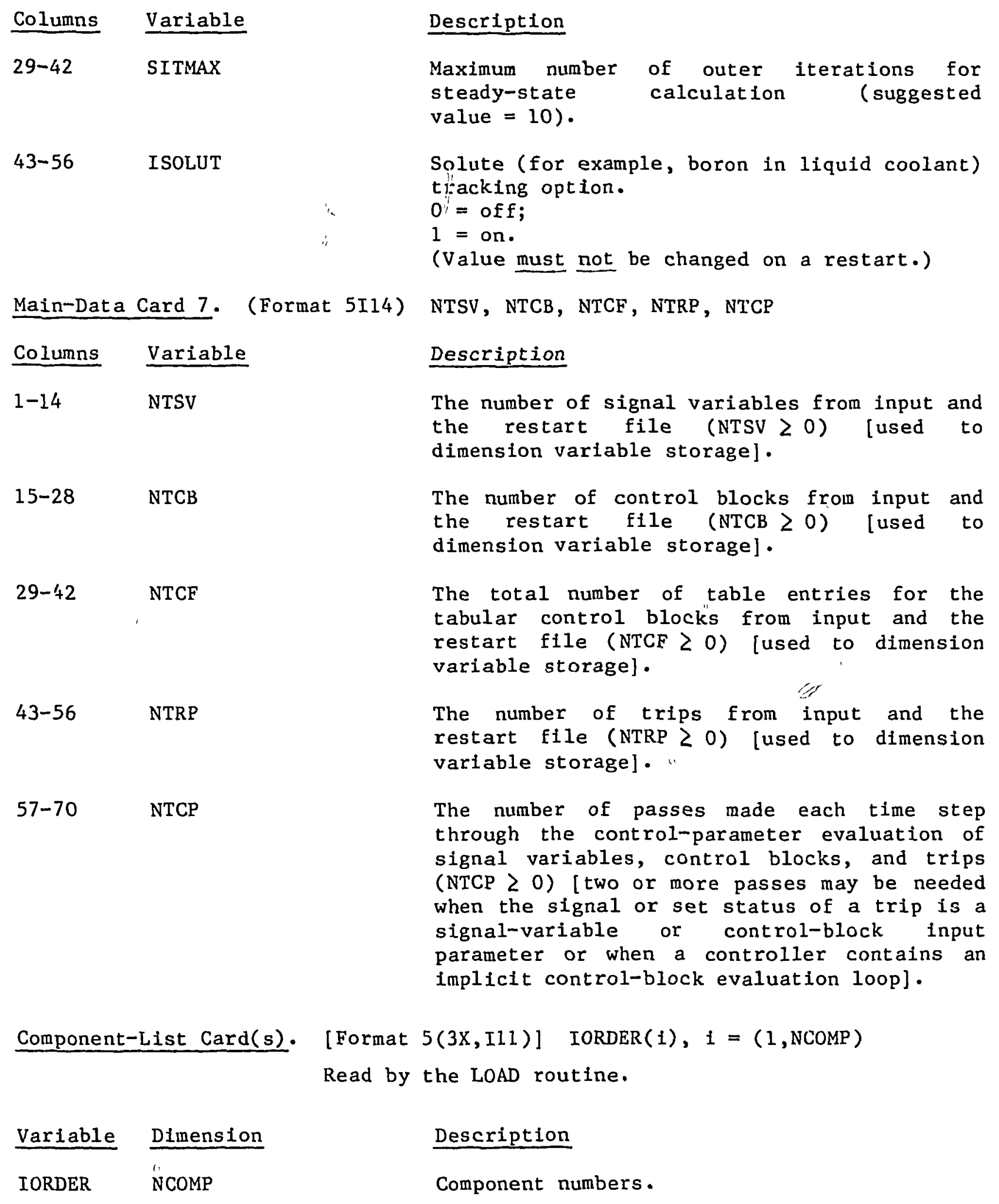


2. Material-Properties Data. If the TRAC internally defined material properties are inappropriate, the user may input his own data. Materialproperties data are required when NMAT $>0$ (word 4 on Main-Data Card 2). For each new material, the user must provide a unique material identifier (MATB > 50), the number of data-point sets provided for each material (PTBLN 22 ), and the data tables that specify the material properties ( $p, c_{p}$, $k$, and $\varepsilon$ ) as a function of temperature $(T)$. Linear interpolation is used to obtain property values for wall temperatures intermediate to those specified by the input tables. Extrapolation is not allowed (an error message results). The material-properties tables are not included in the dump; therefore, the tables must be included in TRACIN for a restart run.

Card Set 1. [Format 5(3X,Ill)] $\operatorname{MATB}(i), \quad i=(1$, NMAT). Read by the LOAD routine.

Variable Dimension

Description

MATB NMAT

New material identifiers, which require $\operatorname{MATB}(i) \geq 50, i=(1, \operatorname{MMAT})$. These are the material ID numbers used in the component input to reference the input material properties instead of the built-in properties.

Card Set 2. [Format 5(3X,I11)] $\operatorname{PTBLN}(i), i=(1$, NMAT). Read by the LOAD routine.

Variable Dimension

PTBLN NMAT

\section{Description}

Number of data-point sets provided for each of NMAT materials; material $\operatorname{MATB}(i)$ consists of PTBLN(i) data-point sets.

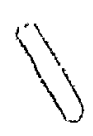


Card Set 3. Input NMAT data tables, one for each material MATB(i), $i=(1$, NMAT $)$. End each table with an E after the last entry.

Data Table i. [Format $5(3 X, E 11.5)] \quad \operatorname{PRPTB}(j, i), j=[1,5 * \operatorname{PTBLN}(i)] \cdot$ (Read by the LOAD routine:)

$\frac{\text { Variable }}{\operatorname{PRPTB}(i)} \quad \frac{\text { Dimension }}{5 * \operatorname{PTBLN}(i)}$

"I

\section{Description}

The material-properties data for material MATB(i-), each point of which consists of the five variables in order $T, p, c, k$, and $\varepsilon$ [where $T$ is the temperature $(K)^{2}, p$ is the density $\left(\mathrm{kg} \cdot \mathrm{m}^{-3}\right), c_{p}$ is the specific heat $\left(\mathrm{J} \cdot \mathrm{kg}^{-1} \cdot \mathrm{K}^{-1}\right), \mathrm{k}$ is the thermal conductivity $\left(\mathrm{W} \cdot \mathrm{m}^{-1} \cdot \mathrm{K}^{-1}\right)$, and $\varepsilon$ is the emissivity]. Each material MATB(i) consists of PTBLN(i) points with the values of $T$ increasing monotonically. The table array appears as $\mathrm{T}_{1}, \rho_{1}, \mathrm{c}_{\mathrm{p}_{1}}, \mathrm{k}_{1}, \varepsilon_{1}, \mathrm{~T}_{2}, \rho_{2}$, $c_{p}, 2, k_{2}, \varepsilon_{2}, \mathrm{~T}_{3}, \ldots, \mathrm{T}_{\operatorname{PTBLN}(i)}, \rho_{\operatorname{PTBLN}(i)}$, c p,PTBLN(i), $k_{\operatorname{PTBLN}(i)}, \varepsilon_{\operatorname{PTBLN}(i)}$, At least two points are required for each material, and the temperature range of the table must bound the range of application. Extrapolation is not allowed.

3. Interactive Contro1-Pane1-Vector Data. These data are input only when TRAC is evaluated in the interactive mode. The CPV data cards are read when IPRCPV $=0$ (an INOPTS NAMELIST paraneter). These cards also define the source of data for evaluating and displaying signal-variable parameters 1 through 15. Loop and mnemonic name data not supplied from TRACIN are obtained from the restart file.

Card Number 1. (Format E14.6,3I14) CPVINT, JCOMPS(1), JCOMPS(2), JCELL(2)

$\begin{array}{lll}\text { Columns } & \text { Variable } & \text { Description } \\ 1-14 & \text { CPVINT } & \text { CPV print interval. } \\ 15-28 & \text { JCOMPS(1) } & \text { Reactor-power component number. } \\ 29-42 & \text { JCOMPS(2) } & \text { Primary-pressure component number } . \\ 43-56 & \text { JCELL(2) } & \text { Primary-pressure cell number. }\end{array}$


Card Number 2. (Format 3II4, 2EI4.6) JCOMPS(3), JCELL(3), JCOMPS(4), RDIAM, Columns Variable $\quad$ Description

1-14 JCOMPS(3) Containment pressure and temperature component number.

15-28 JCELL(3) Containment pressure and temperature cell number.

29-42 JCOMPS(4) Pressurizer water level component number.

43-56 RDIAM , Refueling storage tank diameter (m).

57-70 RMASS Refueling storage tank water mass (kg).

Card Number 3. (Format 4II4) $\operatorname{JDISCH}(i), i=(1,4)$

\begin{tabular}{lll} 
Columns & Variable & Description \\
\cline { 2 - 3 } & JDISCH(i) & $\begin{array}{l}\text { Component number at refueling storage tank } \\
\text { discharge in loop } i \quad(1 \leq i \leq 4) .\end{array}$ \\
& component must be a FILL.
\end{tabular}

Card Number 4. (Format 5I14) LOOPCT, KPRESS, KTEMP, KFLOW, KLEVEL

\begin{tabular}{|c|c|c|}
\hline Columns & Variable & Description \\
\hline $1-14$ & LOOPCT & Number of loops in $t$ \\
\hline $15-28$ & KPRESS & $\begin{array}{l}\text { Pressure output unit } \\
0=\mathrm{MPa} \\
1=\text { psia. }\end{array}$ \\
\hline $29-42$ & KTEMP & $\begin{array}{l}\text { Temperature output } \\
0=\mathrm{K} \\
1={ }^{\circ} \mathrm{F}\end{array}$ \\
\hline $43-56$ & KFLOW & $\begin{array}{l}\text { Flow output units. } \\
0=\mathrm{kg} \cdot \mathrm{s}^{-1} \\
1=1 \mathrm{~b} \cdot \mathrm{s}^{-1}\end{array}$ \\
\hline $57-70$ & KLEVEL & $\begin{array}{l}\text { Level output units. } \\
0=\mathrm{m} \\
\mathrm{l}=\mathrm{ft}\end{array}$ \\
\hline
\end{tabular}


Loop Data Cards. Input from 0 to LOOPCT (word 1 on CPV Card Number 4 ) of the following card set. If fewer than LOOPCT card sets are input, conclude these data with a blank card or a card having an integer zero in column 14 for the first input parameter, LPNUM (a zero must be entered explicitly if the freeformat option is used). Data for the remaining loops will be obtained from the restart file.

Loop Card Number 1. (Format 5I14) LPNUM, ICOMPS(LPNUM,J), ICELLS(LPNUM,J), $J=(1,2)$

\begin{tabular}{|c|c|c|}
\hline Columns & Variable & Description \\
\hline $1-14$ & LPNUM & Loop ID number ( $1 \leq$ LPNUM $\leq$ LOOPCT). \\
\hline $15-28$ & ICOMPS ( LPNUM, I) & Hot-leg temperature component number. \\
\hline $29-42$ & ICELLS (LPNUM, 1) & Hot-leg temperature cell number. \\
\hline $43-56$ & ICOMPS ( LPNUM, 2) & Cold-leg temperature component number. \\
\hline $57-70$ & ICELLS ( IPNUM, 2) & Cold-leg temperature cell number. \\
\hline \multicolumn{3}{|l|}{ Loop Card } \\
\hline & ICELLS (IPNUM, & $, J), J=(3,4)$ \\
\hline Columns & Variable & Description \\
\hline $1-14$ & LOOPS (LPNUM) & $\begin{array}{l}\text { Number of physical loops represented by this } \\
\text { loop. }\end{array}$ \\
\hline $15-28$ & ICOMPS ( LPNUM; 3 ) & Primary-liquid mass-flow component number. \\
\hline $29-42$ & $\operatorname{ICELLS}(L P N U M, 3)$ & Primary-liquid mass-flow cell number. \\
\hline $43-56$ & ICOMPS (LPNUM, 4) & ECCS-liquid mass-flow component number. \\
\hline $57-70$ & ICELLS (LPNUM, 4) & ECCS-liquid mass-flow cell number. \\
\hline
\end{tabular}


Loop Card Number 3. (Format 5II4) ICOMPS(LPNUM,J), ICELLS(LPNUM, J), $J=(5,6)$, ICOMPS (LPNUM, 9)

$\begin{array}{ll}\text { Columns } & \text { Variable } \\ 1-14 & \text { ICOMPS (LPNUM , 5) } \\ 15-28 & \text { ICELLS (LPNUM, 5) } \\ 29-42 & \text { ICONPS (LPNUM, 6) } \\ 43-56 & \text { ICELLS (LPNUM, 6) } \\ 57-70 & \text { ICOMPS (LPNUM, 9) }\end{array}$

Description

Steam-generator secondary-side pressure component number.

Steam-generator secondary-side pressure cell number.

Steam-generator secondary-side stean massflow component number.

Steam-generator secondary-side steam massflow cell number.

Steam-generator secondary-side liquid-level component number.

Loop Card Number 4. (Format 4I14) ICOMPS(LPNUM,J), ICELLS(LPNUM,J), J = $(7,8$ )

$\begin{array}{ll}\text { Columns } & \text { Variable } \\ 1-14 & \text { ICOMPS (LPNUM, 7) } \\ 15-28 & \text { ICELLS (LPNUM, 7) } \\ 29-42 & \text { ICOMPS (LPNUM , 8) } \\ 43-56 & \text { ICELLS(LPNUM, 8) }\end{array}$

Description

Steam-generator secondary-side main-feedwater mass-flow component number.

Steam-generator secondary-side main-feedwater mass-flow cell number.

Steam-generator secondary-side auxiliaryfeedwater mass-flow component number.

Steam-generator secondary-side auxiliaryfeedwater mass-flow cell number. 
4. Contrij1-Parameter Data. Parameters and procedures used to control PWR-system operation are specified through the control-parameter data. Signal variables, control blocks, and trips are defined along with a multipass procedure for evaluating them at the start of each time step.

a. Multipass Control-Parameter Evaluation. Control parameters are evaluated in the following order: signal variables, control blocks, and trips. If a signal variable is to be evaluated after a control block or a trip or a control block is to be evaluated after a trip, two or more evaluation passes through the three control-parameter types are needed. For NTCP 22 evaluation passes (word 5 on Main-Data Card 7), the following Control-Parameter List Cards are input to define the subrange of parameters to be evaluated for each control-parameter type during each evaluation pass.

Contro1-Parameter List Cards. [Format 5(3X,Ill)] ISVl(i), ISV2(i), ICBl(i), $\operatorname{ICB} 2(i), \operatorname{ITP1}(i), \operatorname{ITP} 2(i), i=(1, \mathrm{NTCP}) . \quad \operatorname{Read}$ by the LOAD routine. (Indicated columns are only examples; instead, the user may wish to input each of the NTCP pass parameters on a set of two or three cards with the remaining fields on a card skipped by using an s.)

\begin{tabular}{|c|c|c|}
\hline Columns & Variable & Description \\
\hline $4-14$ & ISV1(1) & $\begin{array}{l}\text { The smallest signal-variable ID number } \\
\text { evaluated during the first control-parameter } \\
\text { evaluation pass [ } 1 \mathrm{~S} \text { ISVI(1)]. }\end{array}$ \\
\hline $18-28$ & ISV2(1) & $\begin{array}{l}\text { The largest signal-variable ID number } \\
\text { evaluated during the first control-parameter } \\
\text { evaluation pass [ISV1(1) S ISV2(1); input } \\
\text { Isv2(1) } 0 \text { if no signal variables are to be } \\
\text { evaluated during the } i=1 \text { first pass]. }\end{array}$ \\
\hline $32-42$ & ICB1 (1) & $\begin{array}{l}\text { The smallest (in absolute value) control- } \\
\text { block ID number evaluated during the first } \\
\text { control-parameter evaluation pass [ICB1(1) } \\
\leq-1 \text { ]. }\end{array}$ \\
\hline $46-56$ & ICB2 (1) & $\begin{array}{l}\text { The largest (in absolute value) control-block } \\
\text { ID number evaluated during the first control- } \\
\text { parameter evaluation pass [ICB2(1) S ICB1(1); } \\
\text { input ICB2( } 1 \text { ) } 0 \text { if no control blocks are to } \\
\text { be evaluated during the } i=1 \text { first pass]. }\end{array}$ \\
\hline
\end{tabular}




\begin{tabular}{|c|c|c|}
\hline Columns & Variable & Description \\
\hline $60-70$ & $\operatorname{ITP} 1(1)$ & $\begin{array}{l}\text { The smallest (in absolute value) trip ID } \\
\text { number evaluated during the first contro1- } \\
\text { parameter evaluation pass }[1 \leq|\operatorname{IPP}(1)|] \text {. }\end{array}$ \\
\hline $4-14$ & $\operatorname{ITP} 2(1)$ & $\begin{array}{l}\text { The largest (in absolute value) trip ID } \\
\text { number evaluated during the first control- } \\
\text { parameter evaluation pass [|ITP1(1)| } \\
\text { S |ITP2(1)|; input ITP2(1) = } 0 \text { if no trips } \\
\text { are Lo be evalualed during the } \mathrm{i}=1 \text { first } \\
\text { pass]. }\end{array}$ \\
\hline $18-28$ & $\operatorname{ISVI}(2)$ & $\begin{array}{l}\text { The smallest signal-variable ID number } \\
\text { evaluated during the second control-parameter } \\
\text { evaluation pass }[1 \leq \mathrm{ISV}(2)] \text {. }\end{array}$ \\
\hline $32-42$ & $\operatorname{ISV} 2(2)$ & Etc. \\
\hline
\end{tabular}

b. Signal-Variable Data. Signal-variable data are defined when NTSV $>0$ (word 1 on Main-Data Card 7). Signal variables define the input parameters for control blocks, the parameters for trip signals, the independent variable parameter for component-action tables, and the component action directly. Either NTSV or fewer signal variables are input. When fewer than NTSV signal variables are input, conclude the data with a blank card or a card having an integer zero for the first input parameter, IDSV. (A zero must be entered explicitly if the free-format option is used.) The remaining signal variables (for a total of NTSV) are obtained from the restart file. They are the signal variables on the restart file whose IDSV identification numbers differ fron those defined here on input. After all signal-variable data are read from input and obtained from the restart file, the signal variables are reordered with their ID numbers increasing monotonically. Each signal variable is defined by the following card.

Signal-Variable Card. (Format 5I14) IDSV, ISVN, ILCN, ICN1, ICN2 Columns Variable Description 1-14 IVSV

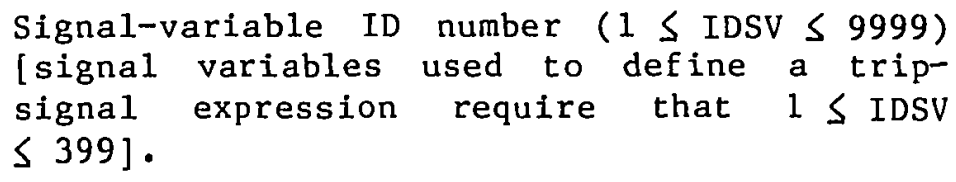
[signal variables used to define a tripsignal expression require that $1 \leq$ IDSV S 399]. 


\begin{tabular}{|c|c|c|}
\hline Columns & Variable & Description \\
\hline $15-28$ & ISVN & 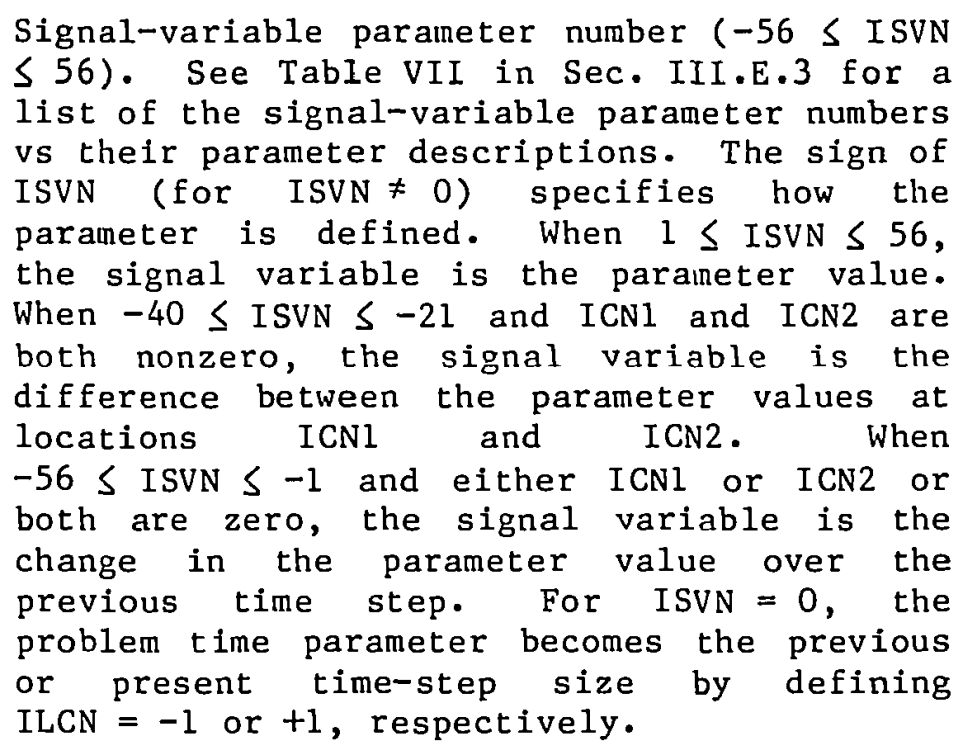 \\
\hline $29-42$ & ILCN & $\begin{array}{l}\text { The coolant loop number }(7 \leq \mid \text { ISVN } \leq 15) \text {, } \\
\text { the component number ( } 18 \leq \mid \text { ISVN } \leq 54) \text {, or } \\
\text { the trip ID number ( } 55 \leq \mid \text { ISVN| } 56) \text { of the } \\
\text { signal-variable paraineter. The variable ILCN } \\
\text { is not used when l } \leq \mid \text { ISVN| } 66 \text { or } \\
16 \leq \mid \text { ISVN| } \leq 17 \text {. }\end{array}$ \\
\hline $43-56$ & ICN 1 & $\begin{array}{l}\text { The cell number of the first location in } \\
\text { component ILCN where the signal-variable } \\
\text { parameter is defined when } 20 \leq \mid \text { ISVN } s 40 \text {. }\end{array}$ \\
\hline $57-70$ & ICN2 & $\begin{array}{l}\text { The cell number of the second location in } \\
\text { component ILCN where the signal-variable } \\
\text { parameter is defined when } 20 \leq \mid \text { ISVN| } \leq 40 \text {. }\end{array}$ \\
\hline
\end{tabular}

For the VESSEL and TEE (secondary side) components, $\mid$ ICN1| and $\mid$ ICN2 $\mid$ are defined by a composite number. For the VESSEL, it is the horizontal plane cell number times 1000 plus the axial level number. For the TEE secondary side, it is the total number of primary-side cells plus the secondary-side cell number. See Sec. III.E.3 for further information on defining cell and interface location composite numbers. 
Further information is provided by the numerical signs of ICN1 and ICN2 for defining the signal-variable value. For $21 \leq$ ISVN $S 40$, the signal variable is

(1) the parameter value in cell $|I C N 1|$ when ICN2 $=0$ or in cell |ICN2| when $\operatorname{ICN}=0$;

(2) the maximum parameter value between cells $\mid$ ICN1 $\mid$ and $|I C N 2|$ when ICN $1>0$ and ICN2 >0;

(3) the minimum parameter value between cells $\mid$ ICN1| and $\mid$ ICN2 $\mid$ when ICN $1<0$ and ICN2 $<0$; and

(4) the volume-averaged parameter value between cells |ICN1| and |ICN2| when ICN1 and ICN2 are of opposite sign.

When $-40 S$ ISVN $S-21$ and both ICN1 and ICN2 are nonzero, the signal variable is the difference between the paraneter value in cells $\mid$ ICN1| and |ICN2| (that is, the paraneter value in cell |ICN1| minus the parameter value in cell $|I C N 2|)$. If either ICN1 or ICN2 is zero when $-56 \leq$ ISVN $\leq-1$, the signa1 variable is the change in the parameter value in ce11 |ICN2| or |ICN1| over the previous time step.

c. Control-Block Data. Control-block data are defined when NTCB >0 (word 2 on Main-Data Card 7). Control blocks are mathematical functions that operate on one to three input parameters defined by signal variables and control blocks. The control-block output signal defines the input parameters for control blocks, the parameters for trip signals, the independent-variable parameter for component-action tables, and the component action directly. Either NTCB or fewer control blocks are input. When fewer than NTCB control blocks are input, conclude the data with a blank card or a card having an integer zero defining the first input parameter, IDCB (a zero must be entered explicitly if the free-format option is used). The remaining control blocks (for a total of NTCB) are obtained from the restart file. They are the control blocks on the restart file whose IDCB identification numbers differ from those defined on input. After all control-block data are read from input and obtained from the restart file, the control-blocks are reordered with the absolute value of their ID numbers (the value of -IDCB) Increasing monotonically. Each control block is defined by the following two cards. When 
the control block:; a tabular function of one independent variable $($ ICBN $=101)$, additića $\therefore$ data cards are needed to define the function table.

Control-Block Cards.

Card Nǘliber 1. (Format 5I14) IDCB, ICBN, ICB1, ICB2, ICB3

Columns Variable Description

1-14 IDCB

15-28 ICBN

Contro1-block ID number (-9999 S IDCB $\leq-1)$.

Control-block operation number ( $1 \leq \mathrm{ICBN}$ $S 61,100 \leq$ ICBN S 101, or $200 \leq$ ICBN S 201). See Table VIII in Sec. III.E.4 for the list of control-block operation numbers and the mathematical description of the operation each performs.

29-42 ICB1

ID number for the first input parameter to the control block. ICBI >0 defines a signal-variable parameter; ICBI $<0$ defines a control-block output parameter.

43-56 ICB2

ID number for the second input parameter to the control block. ICB2 $>0$ defines a signal-variable parameter; ICB2 <0 defines a control-block output parameter. For ICBN $=100$ or 101 , ICB2 is the number of entry pairs in the control block's function table.

57-70 ICB3

ID number for the third input parameter to the control block. ICB3 >0 defines a signa1-variable parameter; ICB3 $<0$ defines a control-block output parameter. . .

Card Number 2. (Format 5E14.6) CBGAIN, CBXMIN, CBXMAX, CBCON1, CBCON2

$\begin{array}{lll}\text { Columns } & \text { Variable } & \text { Description } \\ 1-14 & \text { CBGAIN } & \text { Control-block gain factor. } \\ 15-28 & \text { CBXMIN } & \begin{array}{l}\text { Control-block minimum value of its output } \\ \text { parameter. }\end{array} \\ 29-42 & \text { CBXMAX } & \begin{array}{l}\text { Control-block maximum value of its output } \\ \text { parameter }\end{array}\end{array}$

Note: If CBGAIN, CBXMIN, and CBXMAX all are defined to be zero or defined on a blank record (card), TRAC internally defines CBGAIN $=1.0, \quad$ CBXMIN $=-1.0 \times 10^{20}$, and $\mathrm{CBXMAX}=1.0 \times 10^{20}$. 


$\begin{array}{ll}\text { Columns } & \text { Variable } \\ 43-56 & \text { CBCON1 } \\ 57-70 & \text { CBCON2 }\end{array}$

Description

Control-block first constant.

Control-block second constant that is required by control blocks with operation numbers $\operatorname{ICBN}=11,30,51$, or 59. Control blocks with these operation numbers have XOUT initialized to zero. For all other operation numbers, CBCON2 defines the XOUT initial value for the control block.

If ICBN $\neq 101$ (word 2 on Control-Block Card Number 1), omit Card Number 3 .

Card Number 3. [Format 5(3X,I11)] $\operatorname{CFTAB}(i), i=(1, I C B 2 * 2)$ Read by the LOAD routine.

$\begin{array}{lll}\text { Variable Dimension } & \text { Description } \\ \text { CFTAB } & \text { ICB } 2 * 2 & \text { Control-block function table data; input } \\ & \text { (table s independent-variable value associ- } \\ & \text { ated with input paraneter ID number ICBl, } \\ & \text { function value defining the control-block } \\ & \text { output parameter) pairs }(*, *) .\end{array}$

Note: If ICBN $\neq 200$ or 201 (word 2 on Control-Block Card Number 1), omit Card Number 4 .

Card Number 4. (Format 3E14.6) CBDT, CBTAU, CBWT

Columns Variable Description

1-14 CBDT

The $\Delta t_{d}$ time constant that removes PI or PID controller error if convergence of the form $\Delta F(t)=\Delta F_{0} \exp \left[\left(t_{0}-t\right) / \Delta t_{d}\right]$ is assumed.

15-28 CBTAU

The $\tau$ time constant for the first-order lag function operation.

29-42 CBWT

The first constant Wt value for the weighted summer function operation when ICBN $=201$. 
d. Trip Data. Trip data are defined when NTRP $>0$ (word 4 on MainData Card 7). There are seven categories of trip-input data. The first category, Trip-Dimension Variables Card, is always input when NTRP $>0$. The five variables on this card and NTRP define the variable storage required for the remalning six trip-input data categories. In each remaining category, none, part, or all of the data can be input. Any data that are not input are obtained from the restart file.

Trip-Dimension Variables Card. (Format 5I14) NTSE, NTCT, NTSF, NTDP, NTSD

\begin{tabular}{|c|c|c|}
\hline Columns & Variable & Description \\
\hline $1-14$ & NTSE & $\begin{array}{l}\text { The number of different signal-expression } \\
\text { trip stgnals from input and the restart file } \\
\text { (NTSE } 20 \text { ) [used to dimension variable } \\
\text { storage]. }\end{array}$ \\
\hline $15-28$ & NTCT & $\begin{array}{l}\text { The number of different trip-controlled trip } \\
\text { signals from input and the restart file } \\
\text { (NTCT } 20 \text { ) [used to dimension variable } \\
\text { storage]. }\end{array}$ \\
\hline $29-42$ & NTSF & $\begin{array}{l}\text { The number of different set-point factor } \\
\text { tables referenced by trips from input and the } \\
\text { restart file (NTSF } \geq 0 \text { ) [used to dimension } \\
\text { variable storage]. }\end{array}$ \\
\hline $43-56$ & NTDP & $\begin{array}{l}\text { The number of trips from input and the } \\
\text { restart file that generate a restart dump and } \\
\text { possible problem termination when they are } \\
\text { set on (NTDP } 20 \text { ) [used to dimension variable } \\
\text { storage]. }\end{array}$ \\
\hline $57-70$ & NTSD & $\begin{array}{l}\text { The number of trip-controlled time-step data } \\
\text { sets from input and the restart file that are } \\
\text { used for time-step and edit control when } \\
\text { their defined trips are set oN (NTSD } 20 \text { ) } \\
\text { [used to dimension variable storage]. }\end{array}$ \\
\hline
\end{tabular}


Trip-Defining Variables Cards. Input from 0 to NTRP (word 4 on Main-Data Card 7) of the following card set. If fewer than NTRP card sets are input, conclude these data with a blank card or a card with an integer zero for the first input parameter, IDTP (a zero must be entered explicitly if the freeformat option is used). The remaining trips that have trip IDTP identification numbers different from those input, will be obtained from the restart file.

Card ivumber 1. (Formal 5I14) IDTP, ISRT, ISET, ITST, IDSG

Columns Variable Description

1-14 IDTP

The trip ID number ( $1 \leq \mid$ IDTP| $\leq 9999)$. Negative trip ID numbers have their trip set status evaluated during both the steady-state and transient calculations. Positive trip ID numbers have their trip set status evaluated only during the transient calculation with the input value of the trip set status, ISET, used during the steady-state calculation.

15-28 ISRT

if subranges and their delimiting set-point values for the eight possible values of ISRT $(1 \leq \mid$ ISRT $\mid \leq 4)$.

$29-42 \quad$ TSET

The initial trip set-status number (only used during steady state when IDTP $>0$ ).

$$
\begin{aligned}
-1 & =\mathrm{ON}_{\text {reverse }}, \\
0 & =\mathrm{OFF}, \text { or } \\
1 & =\mathrm{ON}_{\text {forward }} .
\end{aligned}
$$




\begin{tabular}{|c|c|c|}
\hline Columns & Variable & Description \\
\hline $43-56$ & ITST & $\begin{array}{l}\text { The trip-signal type number. } \\
\pm 1=\text { signal-variable trip, } \\
\pm 2 \text { = signal-expression trip, or } \\
\pm 3=\text { trip-controlled trip. } \\
\text { Making the ITST value negative omits editing } \\
\text { of trip-status changes (when the trip-signal } \\
\text { criterion is met and when the trip set status } \\
\text { is changed). }\end{array}$ \\
\hline $57-70$ & IDSG & $\begin{array}{l}\text { The ID number for the } \\
\text { trip-signal variable when ITST }= \pm 1 \\
\text { (IDSG }>0 \text { corresponds to IDSV in the } \\
\text { signal-variable data and IDSG }<0 \\
\text { corresponds to IDCB in the control- } \\
\text { block data), } \\
\text { trip-signal expression when ITST }= \pm 2 \\
\text { (IDSG corresponds to IDSE in the trip- } \\
\text { signal-expression data that follow), } \\
\text { or } \\
\text { trip-controlled trip signal when ITST }= \pm 3 \\
\text { (IDSG corresponds to IDTN in the trip- } \\
\text { controlled trip data that follow). }\end{array}$ \\
\hline Card Numb & 22. (Format 4E14.6) & $\operatorname{SETP}(i), i=(1, N S P)$ \\
\hline Variable & Dimension & Description \\
\hline SETP & NSP & 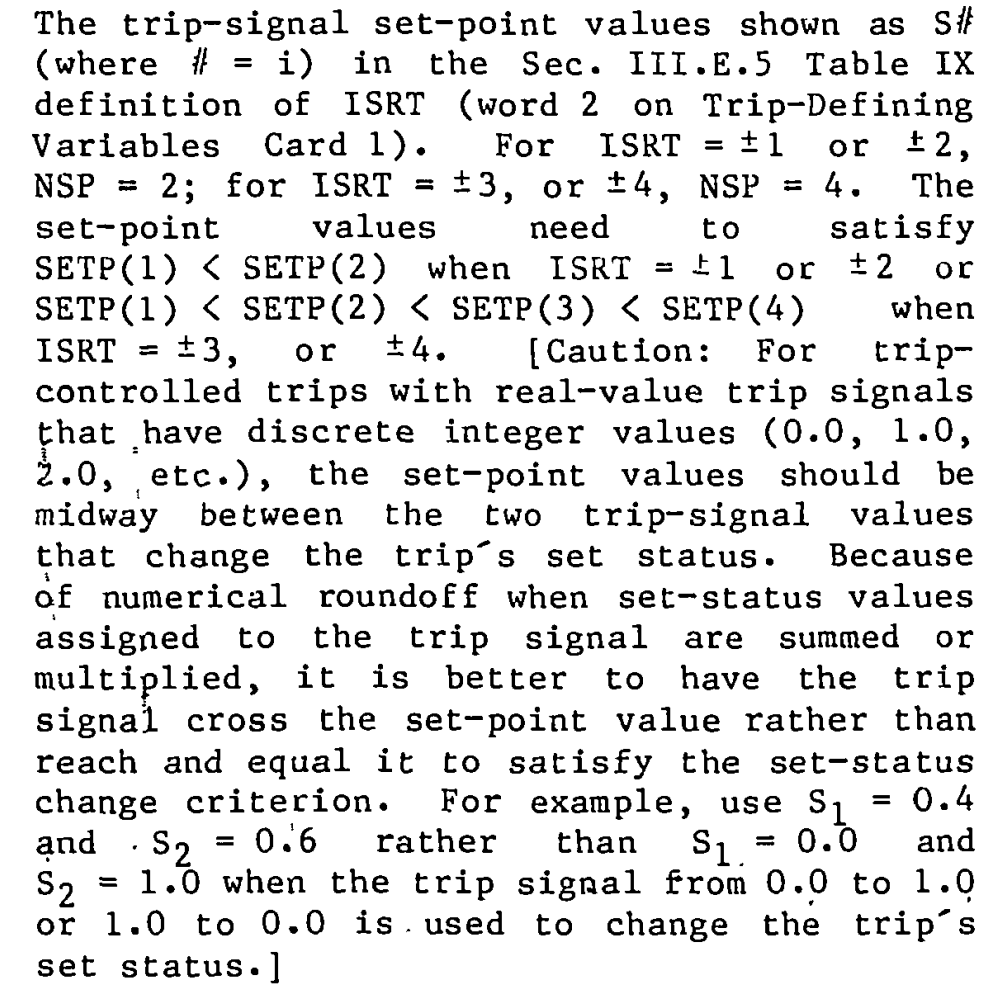 \\
\hline
\end{tabular}


Card Nunber 3. (Format 4E14.6) DTSP(i), $i=(1$, NSP)

$\begin{array}{lll}\text { Variable Dimension } & \text { Description } \\ \text { DTSP } & \text { NSP } & \text { The set-point delay times (s) after the trip } \\ & \text { signal crosses the set-point value to when } \\ & \text { the trip set status is changed. For } \\ & \text { ISRT }= \pm 1 \text { or } \pm 2, \text { NSP }=2 ; \text { for ISRT }= \pm 3 \text { or } \\ & \pm 4, N S P=4 .\end{array}$

Card Number 4. (Format 4Il4) IFSP(i), $i=(1, N S P)$

Variable Dimension $\quad$ Description

IFSP NSP

The set-point factor-table ID numbers. The variable IFSP(i) corresponds to IDFT defined on the Trip Set-Point Factor-Table Card Number 1 that follows. Input $\operatorname{IFSP}(i)=0$ when no factor table is to be applied to $\operatorname{SETP}(i)$; that is, the set-point value remains constant during the problem. For ISRT $= \pm 1$ or $\pm 2, \mathrm{NSP}=2 ;$ for $\operatorname{ISRT}= \pm 3$ or \pm 4 , NSP $=4$.

If none of the trips input have ITST $= \pm 2$ (word 4 on Trip-Defining Variables Card 1), omit the Trip-Signal-Expression Signal Cards.

Trip-Signal-Expression Signal Cards. Input the following card data for each different IDSG trip-signal identification number of trips with ITST $= \pm 2$ that are input.

Card Number 1. (Format 3I 14) IDSE, INSE, INCN

Columns Variable Description

1-14 IDSE

The trip-signal-expression signal ID number. This number corresponds to IUSG (word 5 on Trip-Defining Variables Card 1) for one or more of the input trips having ITST $= \pm 2$ ( 1 S $\mid$ IDSE $\mid$ 9999).

15-28 INSE

The number of subexpressions, defining the signal expression ( $1 \leq$ INSE $\leq 10$ ).

29-42 INCN

The number of different constants referenced in the subexpressions defining the signal expression ( $0 \leq$ INCN $\leq 5$ ). 
Input INSE cards of Card Number 2 to define the $J=(1$, INSE) arithmetic subexpressions.

Card Number 2. (Format 3I14) $\operatorname{ISE}(i, J), i=(1,3)$

$\begin{array}{ll}\text { Columns } & \text { Variable } \\ 1-14 & \operatorname{ISE}(1, \mathrm{~J}) \\ 15-28 & \operatorname{ISE}(2, \mathrm{~J}) \\ 29-42 & \operatorname{ISE}(3, \mathrm{~J})\end{array}$

Description

The arithnetic-operator ID number of the Jth arithmetic subexpression (see Table XIV).

The first argument ID number of the $J$ th arithmetic subexpression.

The second argument ID number of the $\mathrm{Jth}$ arithmetic subexpression.

TABLE XIV

ARITHMETIC-OPERATOR ID NUMBERS OF THE Jth ARITHMETIC SUBEXPRESSIONS

ID Number

1

2

3

4

5

6

7

8
Operator

Addition

Subtraction

Multiplication

Division

Exponentiation

Maximum value

Minimum value

Absolute value
Arithmetic Subexpression

(First argument ID number value)

+ (Second argument ID number value)

(First argument ID number value)

- (Second argument ID number value)

(First argument ID number value)

* (Second argument ID number value)

(First argument ID number value)

/ (Second argument ID number value)

(First argument ID number value)

** (Second argument ID number value)

$\operatorname{MAX}[($ First argument ID number value), (Second argument ID number value)]

MIN [(First argument ID number value), (Second argument ID number value)]

ABS(First argument ID number value) 
The first and second argument ID numbers define values that, when operated on by the arithmetic operator, give a value to their Jth arithmetic subexpression. There are four forms for the value of the first and second argument ID numbers. Their value is

(1) a signal-variable or control-block output value evaluated each time step when their ID number is a signal-variable ID number I S IDSV S 399 (word 1 of Signal-Variable Data) or a control-block ID number $-9999 \leq$ IDCB $\leq-1$ (word 1 of Control-Block Data);

(2) a signal-variable value evaluated initially and at time steps when the trip controlled by this signal expression is set to on reverse or $\mathrm{ON}_{\text {forward }}$ when their ID number is a signal-variable ID number IDSV plus 400 ( $400<$ IDSV $+400<800)$;

(3) a constant input on Card Number 3 that follows when its ID number is the ith subscript of $\operatorname{SCN}(i)$ plus $800(800<i+800<806)$; or

(4) the value of an earlier subexpression $j(0<j<J \leq$ INSE) when their ID number is $j$ plus $900(900<j+900<910)$.

For exainple, the signal expression,

$\max \left(\sqrt{(\operatorname{IDSV}=5)+(\operatorname{IDSV}=33)}, 1.0 \times 10^{-10}\right)$,

would be input as

$1 \quad 2 \quad 3 \quad 4 \quad 5$

$1234567890123456789012345678901234567890123456789012 \ldots$,

$\frac{12345678901234567890123456789012345678901234}{5}$

$5 \quad 901 \quad 801$

where $\operatorname{sCN}(1)=0.5, \operatorname{SCN}(2)=1.0 \times 10^{-10}, \operatorname{INCN}=2$, and INSE $=3$. 
Note: If INCN $=0$, omit Card Number 3 .

Card Number 3. (Format 5E14.6) $\operatorname{SCN}(1), i=(1, \mathrm{INCN})$

Variable Dimension Description

SCN INCN The constants used to evaluate the subexpressions.

If none of the trips being input have ITST $= \pm 3$ (word 4 on Trip-Defining

Variables Card 1), omit the Trip-Controlled Trip-Signal Cards.

Trip-Controlled Trip-Signal Cards. Input the following card data for each different IDSG trip-signal identification number of trips having ITST $= \pm 3$ that are input.

Card Number 1. (Format 2I14) IDTN, INTN

Columns Variable

1-14 IDTN

15-28 INTN

Card Number 2. (Format 5I14) $\operatorname{ITN}(i), i=(1, \operatorname{INTN})$

Variable Dimension

ITN INTN

Description

\section{Description}

The trip-controlled trip-signal ID number. This number corresponds to IDSG (word 5 on Trip-Defining Variables Card 1) for one or more of the input trips having ITST $= \pm 3$ ( 1 S $\mid$ ITST| S 9999).

The number of trip ID numbers whose ISET setstatus values (word 3 on Trip-Defining Variables Card 1) are summed (IDTN $>0$ ) or multiplied (IDTN < 0 ) to evaluate this tripcontrolled trip signal (2 S INTN $S 10$ ).

The trip ID numbers whose ISET set-status values are summed (IDTN $>0$ ) or multiplied (IDTN $<0$ ) to evaluate this trip-controlled trip signal. 
If all the trips have constant trip-signal set points because $\operatorname{IFSP}(i)=0$ (Trip-Defining Variables Card 4) was input for all the set points, omit the Trip Set-Point Factor-Table Cards.

Trip Set-Point Factor-Table Cards. Input the following card data for each different set-point factor-table ID number, IFSP(i), defined in the input trips.

Card Number 1. (Format 3I14) IDFT, IDSG, INFT

Columns Variable Description

1-14 IDFT The set-point factor-table ID number. This number corresponds to IFSP(1) [Trip-Defining Variables Card 4] for one or more trip set points.

15-28 IDSG

29-42 INFT

The signal-variable or control-block ID number defining the set-point factor-table independent variable. This number corresponds to one of the ID numbers in the list of signal variables (IDSG $>0$ ) or control blocks (IDSG < 0), either from input or from the restart file.

The number of set-point factor-table" entry pairs ( $2 \leq$ INFT $\leq 10)$.

Card Number 2. (Format 5E14.6) FT(i), $i=(1$, INFT*2).

Variable Dimension Description

FT INFT*2

Set-point factor-table data; input (tableindependent-variable value associated with parameter ID number IUSG, set-point-factor value) pairs $(*,-)$. 
If NTDP $S 0$ (word 4 on the Trip-Dimension Variables Card), omit the TripInitiated Restart-Dump and Problem-Termination Cards.

Trip-Initiated Restart-Dump and Problem-Termination Cards.

Card Number 1. (Format I14) NDMP

$\begin{array}{ll}\text { Columns Variable } & \text { Description } \\ \text { 1-14 NDNP } & \text { The total numbe of trips from the input file } \\ & \text { and the restart dump that generate a restart } \\ & \text { dump and possible problem termination when } \\ & \text { the trips are set to ON reverse or oN } \text { forward }_{\text {for }} \\ & (0 \leq \text { NDMP } S \text { NTDP). }\end{array}$

The input deck defines NDMP trips, and the NTDP-NDMP remaining trips will be obtained from the restart file. If NDMP $=0$, omit Card Number 2 because all NTDP trip ID numbers will be obtained from the restart file.

Card Number 2. (Format 5I14) $\operatorname{IDMP}(i), i=(1$, NDMP)

Variable Dimension Description

IDMP NDMP The absolute value of trip ID numbers that generate a restart data dump when the trip is set to ON reverse or $\mathrm{ON}_{\text {forward. If } \operatorname{IDMP}(i) \text { is }}$ given a negative sign, problem termination will occur after the restart dump is generated.

If NTSD $\leq 0$ (word 5 on the Trip-Dimension Variables Card), omit the TripInitlated Time-Step Data card set.

Trip-Initiated Time-Step Data Cards. Input from 0 to NTSD of the following card set. If fewer tl.an NTSD sets are input, conclude these data with a blank card or a card having an integer zero defining the first parameter, NDID (a zero must be entered if the free-format option is used).

Card Number 1. (Format 2I14) NDID, NTID

Columns Variable $\quad$ Description

1-14 NDID

The ID number for the following set of tripinitiated time-step data. 


$\begin{array}{lll}\text { Columns Variable } & \text { Description } \\ \text { 15-28 } & \text { NTID } & \text { The number of trip ID numbers that initiates } \\ & \text { the use of this time-step data set when any } \\ & \text { one of the trips is set to ON reverse or } \\ & \text { ONforward }(1 \leq N T S \leq 5) .\end{array}$

Card Number 2. (Format 5I14) $\operatorname{ITID(i),~} i=(1$, NTID)

Variable Dimension Description

ITID NTID The trip ID numbers that initiate use of this time-step data set when any one of the trips is set to ON reverse or ON $\mathrm{N}_{\text {forward }}$.

Card Number 3. (Format 4E14.6) DTMIN, DTMAX, DTEND, DTSOF

\begin{tabular}{|c|c|}
\hline Columns & Variable \\
\hline $1-14$ & DTMIN \\
\hline $15-28$ & DTMAX \\
\hline $29-42$ & DTEND \\
\hline $43-56$ & DTSOF \\
\hline
\end{tabular}

Description

The minimum time-step size (s).

The maximum time-step size (s).

The problem time interval (s) during which thiese time-step data are used.

The next time step ( $s$ ) [DTSOF >0] or the factor $|D T S O F|$ to be applied to the existing time step [DTSOF < 0] in defining the time step to be used at the start of the DTEND time interval when implementing these special time-step data.

Card Number 4. (Format 4E14.6) EDINT, GFINT, DMPIT, SEDINT

$\begin{array}{lll}\text { Columns } & \text { Variable } & \text { Description } \\ 1-14 & \text { EDINT } & \text { Print edit interval (s). } \\ 15-28 & \text { GFINT } & \text { Graphics edit interval (s). } \\ 29-42 & \text { DMPIT } & \text { Restart-dunp edit interval (s). } \\ 43-56 & \text { SEDINT } & \text { Short-print edit interval (s). }\end{array}$


Time-step data on Cards 3 and 4 replace the time-step data defined later in Sec. V.C.6 for a time interval DTEND (word 3 on Card Number 3) after any time-step data-set assigned trip is set to oN reverse or $\mathrm{ON}_{\text {forward }}$. This timestep data can be replaced by this or any other trip-controlled time-step data if any trip assigned to that tine-step data set is set to onreverse or $\mathrm{ON}_{\text {forward }}$ before the time interval DTEND of this set passes.

5. Component Data. Either NCOMP (word 3 on Main-Data Card 4) or fewer sets of component cards are input. The sets may be input in any order. If fewer than NCOMP sets are input, the end of the component data is marked by a single card containing the characters END in columns 1-3. In this case the remaining components are initialized from the TRCRST file. The format of each set depends on the component type. The following is the input format for the components. All velocities are positive in the direction of ascending cell number. Most of the subscripted component data variables are processed by the LOAD subroutine described in Sec.V.D. Additional information on preparing component input data can be found in Sec. IV where the component models are described. All tables that are entered as pairs of numbers $(x, y)$ must be supplied in ascending order of the independent variable $x$. In general, zerodimensioned arrays are not input.

Each component requires a junction number, JUN, for each of its connecting points. A junction is the connection point between two components. A pipe requires two junction numbers, one for each end. A unique junction number must be assigned to each connecting point and referenced by both components to be connected. For example, if two pipes are joined, then the junction numbers of the connecting end of each pipe must be the same. No component may "wrap around" and connect to itself and no junction may have only one component connected to it. Any of the single-ended components (BREAK; FILL, or ACCUM) nay be used to end a component line that is not a loop.

In the following input descriptions, the asterisk (*) indicates units of the defined variable and the hyphen (-) indicates dimensionless quantities. 
Accumulator Component (ACCUM)

a. Accumulator Component (ACCUM).

Card Number 1. (Format A10,4X,2I14,A30) TYPE, NUM, ID, CTITLE

Columns Varlable $\quad$ Description

1-5 TYPE Component type (ACCUM left-justified).

15-28 NUM Component ID number (must be unique for each component, $1 \leq \mathrm{NUM} \leq 999)$.

29-42 ID User ID number (arbitrary).

43-72 CTITLE Hollerith component description.

Card Number 2. (Format 3I14) NCELLS, JUN2, ICONC

\begin{tabular}{|c|c|c|}
\hline Columns & Variable & Description \\
\hline $1-14$ & NCELLS & Number of fluid cells. \\
\hline $15-28$ & JUN2 & $\begin{array}{l}\text { Junction number for junction adjacent to } \\
\text { cell NCELLS. This must be the accumulator } \\
\text { discharge. }\end{array}$ \\
\hline $29-42$ & ICONC & $\begin{array}{l}\text { Flag for input of solute in the coolant. } \\
\text { [Requires ISOLUT }=1 \text { (word } 5 \text { on Main-Data } \\
\text { Card 6)]. } \\
0=\text { no input; } \\
1=\text { dissolved solute only; } \\
2 \text { = both dissolved and plated-out solute. }\end{array}$ \\
\hline
\end{tabular}

ACCUM Array Cards. Seventeen sets of cards, one set for each of the following variables. Use LOAD format.

\begin{tabular}{|c|c|c|}
\hline Variable & Dimension & Description \\
\hline $\mathrm{DX}$ & NCELLS & Cell lengths (m). \\
\hline VOL & NCELLS & Ce11 volumes $\left(\mathrm{m}^{3}\right)$. \\
\hline FA & NCELLS+1 & Ce11-edge flow areas $\left(\mathrm{m}^{2}\right)$. \\
\hline FRIC & NCELLS+1 & $\begin{array}{l}\text { Additive loss coefficients }(-) \text { (See } \\
\text { variables IKFAC and NFRC1 in the NAMELIST } \\
\text { data for optional } \mathrm{K} \text { factors and reverse } \\
\text { loss-coefficlents, respectively.) }\end{array}$ \\
\hline
\end{tabular}


Note: Setting FRIC $>10^{20}$ at a cell edge invokes the steam-separator model (only the gas phase is allowed to flow through the cell edge); setting FRIC $<-10^{20}$ invokes the liquid-separator model (only the liquid is allowed to flow through the cell edge). If the reverse additive losscoefficient option (NFRCl $=2$ in the NAMELIST data) is chosen, steamand liquid-scparator models may be used separately in each forward and reverse direction.

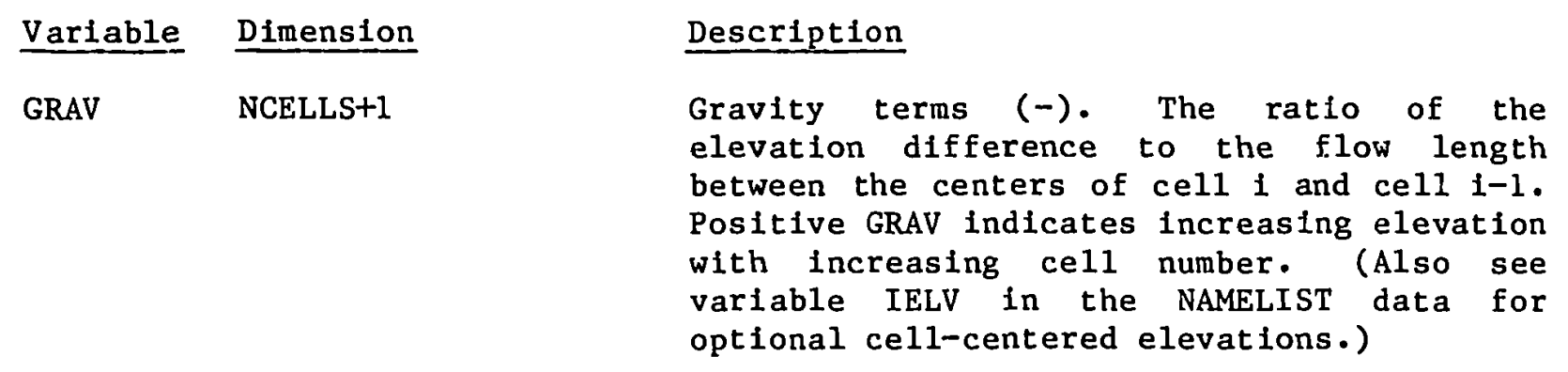

HD NCELLS+1

Hydraulic diameters ( $m$ ). (See variable NDIAl in the NAMELIST data for optional heat-transfer diameters.)

Note: If ICFLOW $=0$ or ICFLOW $=1$ (default) in the NAMELIST data, do not input array ICFLG.

ICFLG NCELLS+1

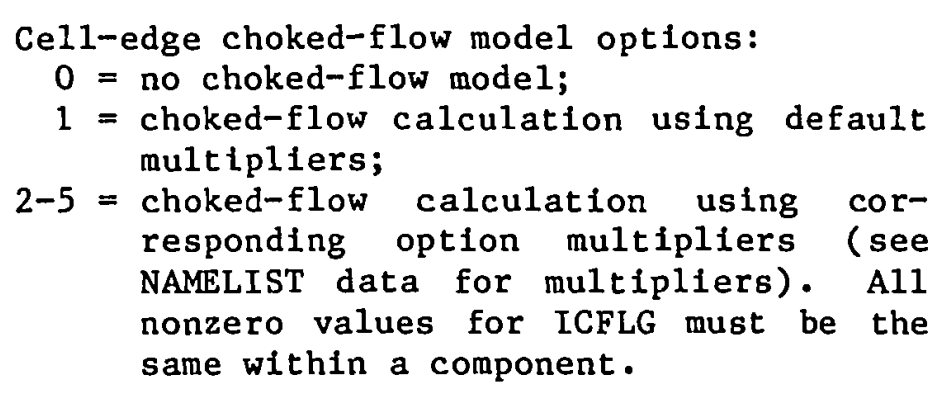

WARNING: Setting ICFLG >0 at adjacent, closely spaced cell-edges čan lead to numerical difficulties. 


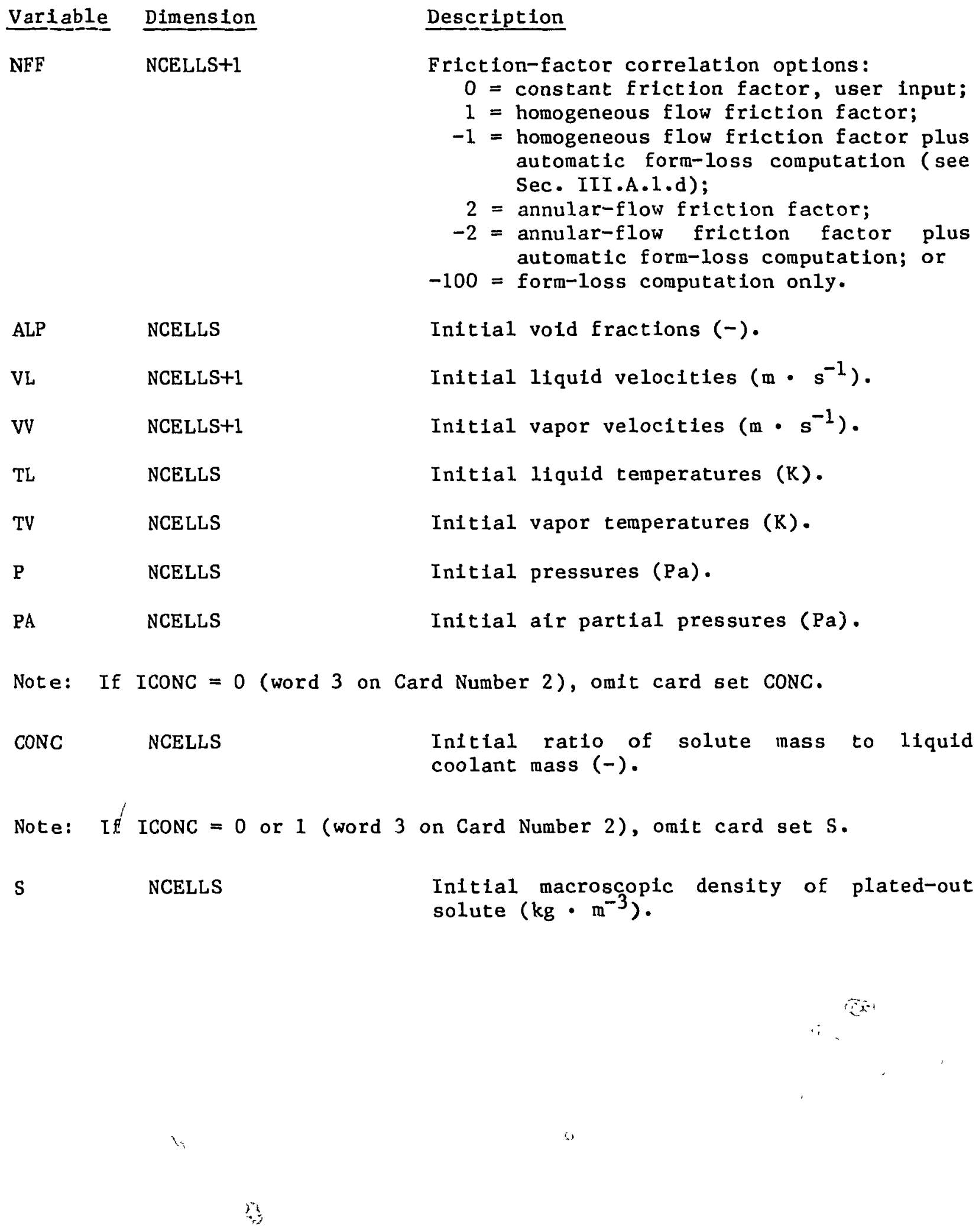


b. Break Component (BREAK).

Card Number 1- (Format A10,4X,2I14,A30) TYPE, NUM, ID, CTITLE

$\begin{array}{lll}\text { Columns } & \text { Variable } & \text { Description } \\ 1-5 & \text { TYPE } & \text { Component type (BREAK left-justif } \\ 15-28 & \text { NUM } & \begin{array}{l}\text { Component ID number (must be unicu } \\ \text { component, } 1 \leq \text { NUM } \leq 999) .\end{array} \\ 29-42 & \text { ID } & \text { User ID number (arbitrary). } \\ 43-72 & \text { CTITLE } & \text { Hollerith component description. }\end{array}$

Card Number 2. (Format 5I14) JUN1, IBTY, ISAT, IVDV, IOFF

\begin{tabular}{|c|c|c|}
\hline Columns & Variable & Description \\
\hline $1-14$ & JUNI & Junction number where break is located. \\
\hline $15-28$ & IB'TY & $\begin{aligned} & \text { Break-table type options: } \\
& 0=\text { no tables read; } \\
& 1=\text { read pressure table; } \\
& 2=\text { read pressure and temperature tables; } \\
& 3=\text { read above plus void fraction table; } \\
& 4= \text { read above plus air partial pressure } \\
& \text { table; } \\
& 5= \text { read above plus solute-to-coolant mass } \\
& \text { ratio table. (Requires ISOLUT = } 1 \text { on } \\
& \text { Main-Data Card } 6 . \text { ) }\end{aligned}$ \\
\hline $29-42$ & ISAT & 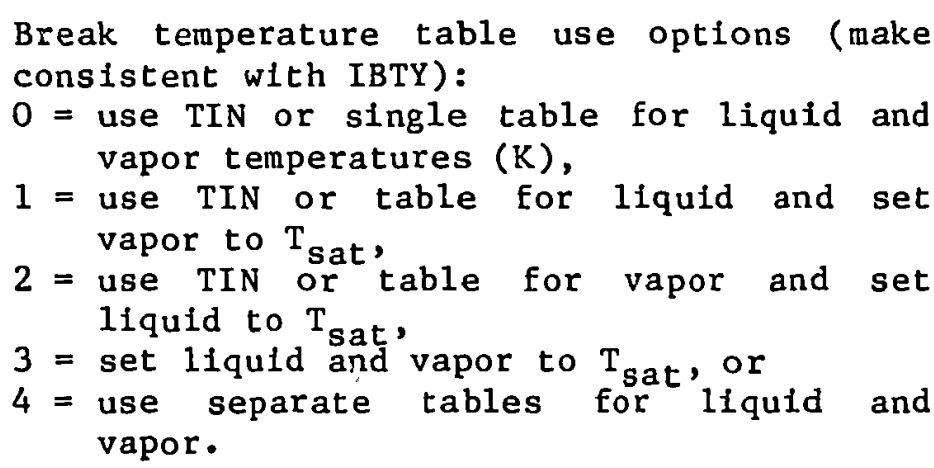 \\
\hline
\end{tabular}




\begin{tabular}{|c|c|c|}
\hline Columns & Variable & Description \\
\hline $43-56$ & IVDV & $\begin{array}{l}\text { Momentum transport during flow in from the } \\
\text { break ( } W V \text { term). } \\
0=\text { the velocity at the end of the break } \\
\text { opposite the junction, must be set to } \\
\text { the velocity at the break junction } \\
(V V=0) \text {. } \\
1=\text { the velocity at the end of the break } \\
\text { opposite the junction, must be set to } \\
\text { zero when computing } V V \text {. This is } \\
\text { recommended for breaks that model } \\
\text { connections to pressure-suppression } \\
\text { tanks or the atmosphere. }\end{array}$ \\
\hline $57-70$ & IOFF & $\begin{array}{l}\text { The break fluid-state option, which defines } \\
\text { the fluid state when the break table's } \\
\text { controlling-trip is ofF after being ON } \\
\text { [define IOFF when LBTR } \neq 0 \text { (word } 1 \text { on Card } \\
\text { Number } 3 \text { )]. } \\
0=\text { the last break table's interpolated } \\
\text { fluid state is held constant. } \\
1=\text { define the initial fluid state. } \\
2=\text { input the pressure to be used, but } \\
\text { maintain the fluid condition that } \\
\text { existed when the trip was set ofF. } \\
3=\text { input a complete fluid state definition } \\
\text { for when the controlling trip is OFF } \\
\text { after being oN. }\end{array}$ \\
\hline
\end{tabular}

Note: If IBTY $<1$ (word 2 on Card Number 2), omit Card Number 3 .

Card Number 3. (Format 5I14) IBTR, IBSV, NBTB, NBSV, NBRF

Columns Variable Description

1-14 IBTR

The trip ID number that controls evaluation of the break table ( $\mid$ IBTR $\mid<10000)$. Input IBTR $=0$ if there is no trip control.

15-28 IBSV

The break-table abscissa-coordinate variable ID number that defines the independent variable parameter for the IBTY $=1$ to 4 tables. IBSV > defines the ID number for a signal-variable parameter; IBSV $<0$ defines the ID number for a control-block output parameter. 
Columns Variable

29-42 NBTB

43-56 NBSV

57-70 NBRF

Card Number 4. (Format 5E14.6)

Columns Variable

1-14 DXIN

\section{Description}

The number of break-table pairs for break options IBTY $=1$ to 4 (defined by the absolute value of NBTB). NBTB $>0$ defines the independent-variable form to be the IBSV parameter value; NBTB $<0$ defines the independent-variable form to be the change in the IBSV parameter over the last time step times the trip set-status value ISET when the break table is trip controlled; $N B T B=0$ (for IBTY $=1$ only) defines the break pressure to be the IBSV parameter value.

The rate-factor table abscissi-coordinate variable ID number. NBSV > 0 defines the ID number for a signal-variable parameter; NBSV < 0 defines the ID number for a control-block output parameter; NBSV $=0$ when NBRF $\neq 0$ defines the difference between the trip signal and the set-point value that turns the trip OFF when the break table or tables are trip controlled.

The number of rate-factor table pairs (defined by the absolute value of NBRF). The rate factor is applied as a factor to the independent variable of the break table or tables when the rate factor is defined. No rate factor is defined when NBSV and NBRF are both zero. NBRF $>0$ defines the ratefactor table abscissa coordinate to be the NBSV parameter value; NBRF < 0 defines it to be the change in the NBSV parameter over the last ti e step times the trip set-status value ISET when IBTR $\neq 0$; NBRF $=0$ defines the rate factor to be the NBSV parameter value.

DXIN, VOLIN, ALPIN, TIN, PIN

\section{Description}

Break-cell length (m), generally the same as its neighboring cell in the adjacent component. Used only when the flow is from the break into the adjacent component and for stratified-flow calculations. 


\begin{tabular}{|c|c|}
\hline Columns & Variab \\
\hline $15-28$ & VOLIN \\
\hline $29-42$ & ALPIN \\
\hline $43-56$ & TIN \\
\hline $57-70$ & PIN \\
\hline
\end{tabular}

Card Number 5. (Format 5E14.6) Columns Variable $1-14 \quad$ PAIN

15-28 CONCIN

29-42 RBMX

43-56 POFF

57-70 BELV

\section{Description}

Break-cell volume $\left(\mathrm{m}^{3}\right)$, generally the same as its neighboring cell in the adjacent component. Used only for stratified-flow calculations.

Initial mixture void fraction (-) at the break.

Initial mixture temperature $(K)$ at the break.

Initial pressure ( $\mathrm{Pa}$ ) at the break.

PAIN, CONCIN, RBMX, POFF, BELV

Description

Initial air partial pressure $(\mathrm{Pa})$ at the break.

Ratio of initial solute mass to liquid mass at the break $(-)$ [used only if ISOLUT $=1$ (word 5 on Maln-Data Card 6)].

Maximum rate of change of the break pressure $\left(\mathrm{Pa} \cdot \mathrm{s}^{-1}\right)[0 \leq \mathrm{RBMX}]$.

Break pressure $(\mathrm{Pa})$ when the controllingtrip is set OFF after being ON [used only when IBTY $\geq 1$ and IOFF $\geq 2$ (words 2 and 5 on Card Number 2)].

Break cell-centered elevation ( $\mathrm{n}$ ) [used only to compute GRAV array when IELV = 1].

Note: If IBTY <.1 (word 2 on Card Number 2), IBTR $=0$ (word 1 on Card Number 3 ), or IOFF $\neq 3$ (word 5 on Card Number 2), omit Card Number 6 .

Card Number 6. (Format 5E14.6) ALPOFF, TLOFF, TVOFF, PAOFF, CONOFF

Columns Variable Description

1-14 ALPOFF

Break vold fraction (-) when the controlling-trip is set OFF after being $O N$ (input only when IBTY 23 ). 
Break Component (BREAK)

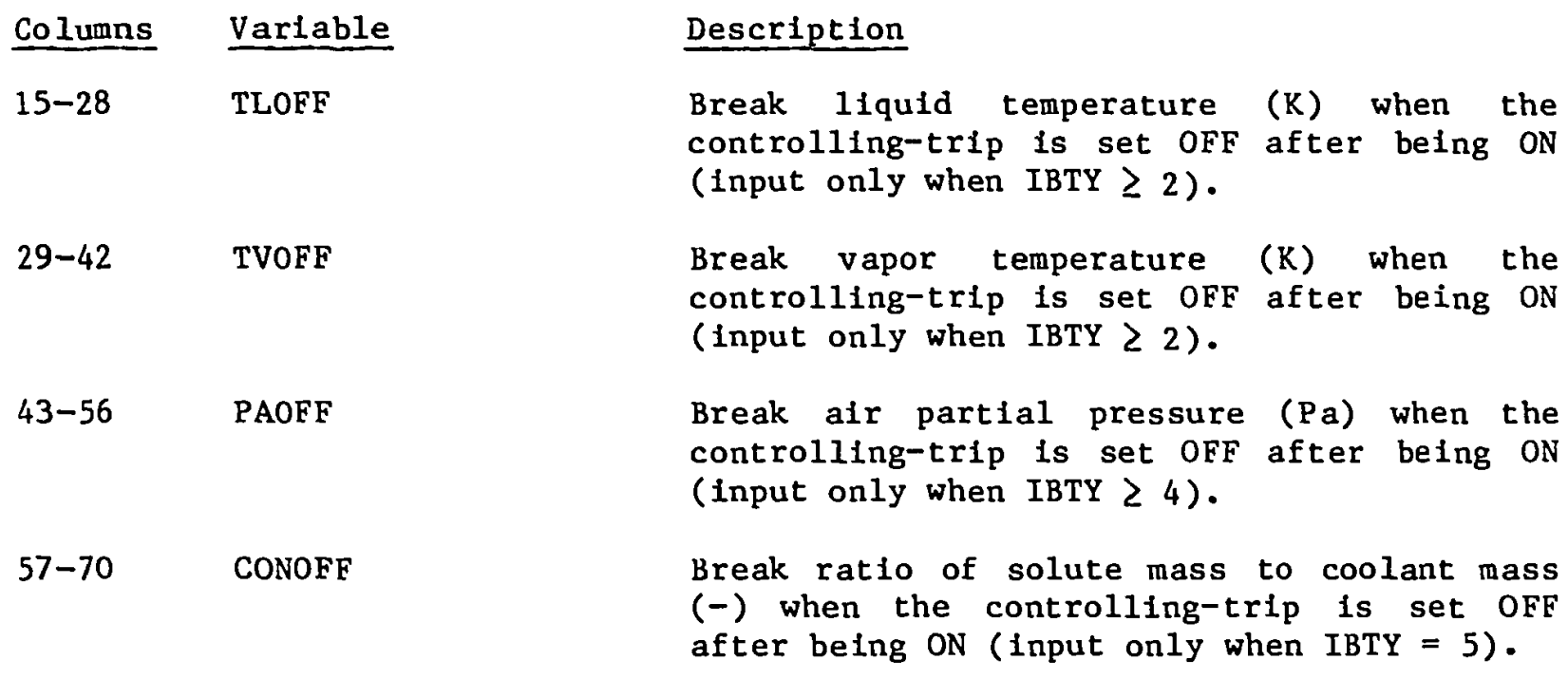

Note: If IBTY $<1$ (word 2 on Card Number 2) or NBTB $=0$ (word 3 on Card Number 3), omit Card Number 7 .

Card Number 7. (Format 5E14.6) PSCL, TLSCL, TVSCL, PASCL, CONSCL

Columns Variable Description

1-14 PSCL

Pressure scale factor. The dependent variable in the pressure table PTB is multiplied by this factor to obtain absolute pressure (input PSCL only if IBTY 21 ).

15-28 TLSCL

Liquid-temperature scale factor. The dependent variable in the liquid-temperature table TLTB is multiplied by this factor to obtain the absolute liquid temperature (K) [input TLSCL only if IBTY $Z 2$ ].

29-42 TVSCL

Vapor-temperature scale factor. The dependent variable in the vapor-temperature table TVTB is multiplied by this factor to obtain the absolute vapor temperature (K) [input TVSCL only if IBTY 22 and ISAT $=4$ ]

43-56 PASCL

Air partial pressure scale factor. The dependent variable in the air partial pressure table PATB is multiplied by this scale factor to obtain the absolute air partial pressure (Pa) [1nput PASCL only if IBTY 2 4] . 


$\begin{array}{lll}\text { Columns Variable } & \text { Description } \\ 57-70 & \text { CONSCL } & \text { Ratio of solute mass to liquid-mass scale } \\ & \text { factor. The dependent variable in the } \\ & \text { solute mass to liquid-mass ratio table } \\ & \text { ConCTB is multiplied by this scale factor to } \\ & \text { obtain the absolute ratio value (input } \\ & \text { CoNSCL only if IBTY }=5 \text { ). }\end{array}$

Note: If IBTY $<1$ (word 2 on Card Number 2) or NBTB $=0$ (word 3 on Card Number 3), omit the Break Array Cards.

BREAK Array Cards. Seven sets of cards, one set for each of the following variables. Use LOAD format.

$\begin{array}{lll}\text { Variable Dimension } & \text { Description } \\ \text { PTB } & \mid \text { NBTB } \mid * 2 & \text { Break pressure vs independent-variable-form } \\ & & \text { table. Input (independent-variable form } \\ & & \text { defined by IBSV and NBTB on Card Number } 3, \\ & \text { pressure) pairs (*,Pa). } 8\end{array}$

Note: If IBTY $<2$ (word 2 on Card Number 2), omit card set TLTB.

TLTB $\quad \mid$ NBTB $\mid * 2$

Break liquid temperature vs independentvariable-form table. Input (independentvariable form defined by IBSV and NBTB on Card Number 3, liquid temperature) pairs $(*, K)$.

Note: If IBTY $<2$ (word 2 on Card Number 2) or ISAT $\neq 4$. (word 3 on Card Number 2), omit card set TVTB.

TVTB $\quad \mid$ NBTB $\mid * 2$

Break vapor temperature vs independentvariable-form table. Input (independentvariable form defined by IBSV and NBTB on Card Number 3, vapor temperature) pairs $(*, K)$. 
Note: If IBTY $<3$ (word 2 on Card Number 2), omit card set ALPTB.

Varlable Dimension

ALPTB $\quad|\mathrm{NBTB}| * 2$
Description

Break void-fraction vs independent-variableform table. Input (Independent-variable form defined by IBSV and NBTB on Card Number 3 , vapor coolant volume fraction) pairs $(*,-)$.

Note: If IBTY $<4$ (word 2 on Card Number 2 ), omit card set PATb.

PATB $\quad \mid$ NBTB $\mid * 2$

Break air partial pressure vs independentvariable-form table. Input (independentvariable form defined by IBSV and NBTB on Card Number 3 , air partial pressure) pairs $(*, \mathrm{~Pa})$.

Note: If IBTY $<5$ (word 2 on Card Number 2), omit card set CONCTB.

CONCTB $\quad|\mathrm{NBTB}| * 2$

RFTB
$|\mathrm{NBRF}| * 2$
Break solute mass to liquid-mass ratio vs independent-variable-form table. Input (independent-variable form defined by IBSV and NBTB on Card Number 3, ratio of solute mass to liquid mass) pairs $[*,-]$.

Rate-factor table for the break function table's independent-variable form defined by IVSV and NBTB on Card Number 3. Input (independent-variable form defined by NBSV and NBRF on Card Number 3, rate factor to be applied to the break function table's independent/variable) pairs $(*,-)$. 

c. One-Dimensional Core Component (CORE).

Card Number 1. (Format A10,4X,2I14,A30) TYPE, NUM, ID, CTITLE

\begin{tabular}{|c|c|c|}
\hline Columns & Variable & Description \\
\hline $1-4$ & TYPE & Component type (CORE, left-justified). \\
\hline $15-28$ & NUM & $\begin{array}{l}\text { Component ID number (must be unique for each } \\
\text { component, I } \leq \text { NUM } \leq 999 \text { ). }\end{array}$ \\
\hline $29-42$ & ID & User ID number (arbitrary). \\
\hline $43-72$ & CTITLE & Hollerith component description. \\
\hline Card Num & r2. (Format 5I14) & NCELLS, JUN1, JUN2, MAT, ICHF \\
\hline Co lumins & Variable & Description \\
\hline $1-14$ & NCELLS & Number of fluid cells. \\
\hline $15-28$ & JUNl & $\begin{array}{l}\text { Junction number for junction adjacent to } \\
\text { cell } 1 \text { (assumed to be at the bottom of the } \\
\text { component). }\end{array}$ \\
\hline $29-42$ & JUN2 & $\begin{array}{l}\text { Junction number for junction adjacent to } \\
\text { cell NCELLS (assumed to be at the top of the } \\
\text { component). }\end{array}$ \\
\hline $43-56$ & MAT & $\begin{array}{l}\text { Material ID number of the wall (currently } \\
\text { not used). }\end{array}$ \\
\hline $57-70$ & ICHF & $\begin{array}{l}\text { CHF calculation flag. } \\
0=\text { no; } \\
1=\text { yes. }\end{array}$ \\
\hline
\end{tabular}

Card Number 3. (Format 3I14) ICONC, ICRU, ICRL

\begin{tabular}{|c|c|c|}
\hline Columns & Variable & Description \\
\hline $1-14$ & ICONC & $\begin{array}{l}\text { Flag for input of solute in the coolant. } \\
\text { [Requires IsoluT }=1 \text { (word } 5 \text { on Main-Data } \\
\text { Card 6).] } \\
0=\text { no input; } \\
1=\text { dissolved solute on } 1 \text {; } \\
2=\text { both dissolved and plated-out solute. }\end{array}$ \\
\hline $15-28$ & ICRU & $\begin{array}{l}\text { Power-region upper-boundary fluid-cell } \\
\text { number (input ICRU = NCELLS if boundary is } \\
\text { at JUN2). }\end{array}$ \\
\hline
\end{tabular}


Columns Variable

29-42 ICRL

Card Number 4. (Format 5I14) IRPWTY, NDGX, NDHX, NRTS, NHIST
Description

Neutronic-point-kinetics or reactor-power type option for defining programmed reactivity or reactor power (input parameters required for each option are shown in parentheses). Add 10 to the option value of IRPWTY if reactivity feedback is to be evaluated. For IRPWTY $>14$, reactivity feedback is evaluated and edited, but is not used because reactor power is defined directly.

IRPWTY

Option

1

2

\section{Reactor kinetics with constant REACT programmed reactivity} (RPOWRI, REACT).

Reactor kinetics with table lookup of programmed reactivity (RPOWRI, IRPWSV, NRPWTB, RPWTB ) .

Reactor kinetics with an initial zero-programmed reactivity and trip-initiated constant REACT progranmed reactivity (RPOWRI, IRPWTR, REACT).

Reactor kinetics with initial constant REACT programmed reactivity and trip-initlated table lookup of programmed reactivity (RPOWRI, REACT, IRPWTR, IRPWSV, NRPWTB, RPWTB).

Constant reactor power (RPOWRI).

Table lookup of reactor power (IRPWSV, NRPWTB, RPWTB).

Initial constant reactor power with trip-iniciated table lookup of reactor power (RPOWRI, IRPWTR, IRPWSV, NRPWTB, RPWTB) . 


\begin{tabular}{|c|c|c|}
\hline Columns & Variable & Description \\
\hline $15-28$ & NDGX & $\begin{array}{l}\text { The number of delayed-neutron groups. (If } \\
\text { NDGX } \leq 0 \text { is input when IRPWTY }=1,2,3 \text {, } 4 \text {, } \\
11 \text {, } 12,13 \text {, or } 14 \text {, the code defaults to } \\
6 \text { delayed-neutron groups with the delayed- } \\
\text { neutron constants defined internally; input } \\
\text { NDGX }=0 \text { when IRPWTY }=5,6,7,15,16 \text {, or } \\
17 .)\end{array}$ \\
\hline $29-42$ & NDHX & $\begin{array}{l}\text { The number of decay-heat groups. (If } \\
\text { NDHX } \leq 0 \text { is input when IRPWTY }=1,2,3,4 \text {, } \\
11,12,13 \text {, or } 14 \text {, the code defaults to } \\
11 \text { decay-heat groups with the decay-heat } \\
\text { constants defined internally; input NDHX =0 } \\
\text { when IRPWTY }=5,6,7,15,16 \text {, or } 17 . \text { ) }\end{array}$ \\
\hline $43-56$ & NRTS & $\begin{array}{l}\text { The number of time steps between edits of } \\
\text { the reactor power and reactivity feedback } \\
\text { changes (default value is NRTS }=10 \text { ). }\end{array}$ \\
\hline $57-70$ & NHIST & $\begin{array}{l}\text { The number of }(x, y) \text { pairs in the power- } \\
\text { history table. [Input NHIST } \geq 0 \text { when } \\
\text { IRPWTY }=1,2,3,4,11,12,13 \text {, or } 14 \text { (when } \\
\text { NHIST }=0 \text {, no power-history table will be } \\
\text { input; instead, delayed-neutron and decay- } \\
\text { heat concentrations will be input unless } \\
\text { NDGX } 0 \text { and NDHX } 0 \text { causing equilibrium } \\
\text { concentrations to be defined internally); } \\
\text { input NHIST } 0 \text { when IRPWTY } 5,6,7,15 \text {, } \\
16 \text {, or } 17 .]\end{array}$ \\
\hline
\end{tabular}

Note: If IRPWTY $=1,5,11$, or 15 (word 1 on Card Number 4), omit Card Number 5 .

Card Number 5. (Format 5I14) IRPWTR, IRPWSV, NRPWTB, NRPWSV, NRPWRF

Columns Variable Description

1-14 IRPWTR

The trip ID number that controls evaluation of the reactivity-power table (|IRPWTR| $<10000) \quad[0<\mid$ IRPWTR $\mid<10000$ when IRPWTY $=3,4,7,13,14$, or $17 ;$ IRPWTR $=0$ otherwise]. 
Columns " Variable

15-28 IRPWSV

29-42 NRPWTB

43-56 NRPWSV

\section{Description}

The reactivity-power table abscissacoordinate-variable ID number. The variable IRPWSV defines the independent-variable parameter for the reactivity-power table. IRPWSV $>0$ defines the ID number for a signa1-variable paraineter; IRPWSV $<0$ defines the ID number for a control-block output value $(0<\mid$ IRPWSV $\mid<1000$ when IRPWTY $=2,4,6,7,12,14,16$, or 17 ; IRPWSV $=0$ otherwise).

The number of reactivity-power table $(x, y)$ pairs (defined by the absolute value of NRPWTB). When NRPWTB >0, the Independentvariable form for the table is the IRPWSV parameter; when NRPWTB $<0$, the Independentvariable form for the table is the change in the IRPWSV parameter over the last time step times the trip set-status value ISET when the reactivity-power table is trip controlled; when NRPWTB $=0$, the reactivitypower table function is the IRPWSV parameter.

The rate-factor table abscissa-coordinatevariable ID number. NRPWSV defines the independent-variable parameter for the rate factor that is applied to the independent variable of the reactivity-power table. NRPWSV $>0$ defines the ID number for a signal-variable parameter; NRPWSV $<0$ defines the ID number for a control-block output value; NRPWSV $=0$ (when NRPWRF $\neq 0$ ) defines the difference between the trip signal and the set-point value that turns the trip OFF when the reactivity-power table is trip controlled. 
Columns Variable

57-70 NRPWRF

Card Number 6. (Format 5[14)
Description

The number of rate-factor table $(x, y)$ pairs (defined by the absolute value of NRPWRF). The rate factor is applied as a factor to the reactivity-power table's independent variable when the rate factor is defined. No rate factor is defined when NRPWSV and NRPWRF are both zero. NRPWRF $>0$ defines the rate-factor table abscissa coordinate to be the NRPWSV parameter; NRPWRF < 0 defines it to be the change in the NRPWSV parameter over the last time step times the trip setstatus value ISET when the reactivity-power table is trip controlled; NRPWRF $=0$ defines the rate factor to be the NRPWSV paraneter.

IZPWTR, IZPWSV, NZPWTB, NZPWSV, NZPWRF

Description

The trip ID number that controls evaluations of the axial power-shape table (|IZPWTR| $(10000)$ [input IZPWTR $=0$ when evaluation of the axial power-shape table is not trip controlled].

15-28 IZPWSV

29-42 NZPWTB
The axial power-shape table abscissacoordinate variable ID number. IZPWSV defines the Independent-variable parameter for the axial power-shape table. IZPWSV $>0$ defines the ID number for a signal-variable paraineter; IZPWSV < 0 defines the ID number for a control-block output parameter.

The number of axial power-shape table $[x, y(z)]$ pairs (defined by the absolute value of NZPWTB). Each pair consists of an abscissa-coordinate value $x$ and (ICRUICRL+1) [words 2 and 3 on Card Number 3] ordinate coordinate values of $y(z)$ defining the axial power shape. NZPWTB $>0$ defines the table's independent-variable form to be the IZPWSV parameter; NZPWPB < 0 defines the table's independent-vartable form to be the change in the IZPWSV parameter over the last time step times the trip set-status value ISET when the axial power-shape table is trip controlled; $N Z P W T B=0$ defines the reactor-kinetic stable function to be the IZPWSV parameter. 


Columns $\quad \frac{\text { Variable }}{\text { 43-56 }}$

57-70 NZPWRF

Card Number 7. (Format 5I14) IRFTR, NMWRX, NFCI, NFCIL, NZMAX
Description

Trip ID number for implementing the fuel-rod axial fine-mesh calculation during reflood (no axial fine-mesh calculation is performed if IRFTR $=0$ ).

Metal-water reaction option.

$0=$ off;

$1=$ on.

Fuel-cladding interaction (FCI) option $(N F C I=1$ performs the dynamic gap conductance calculation).

$0=$ off

$1=$ on. 


$\begin{array}{ll}\text { Columns } & \text { Variable } \\ \text { N3-56 } & \text { NFCIL } \\ 57-70 & \text { NZMAX }\end{array}$

Card Number 8. (Format 5I14) NRODS, NDRDS, NODES, NSLBS, NFBPWT

\section{Description}

Maximum number of FCI calculations per time step (input NFCIL $=1$ when NFCI $=1$ ).

Maximum number of rows of nodes used in the fuel-rod conduction calculation.

\begin{tabular}{|c|c|}
\hline Columns & Variabl \\
\hline $1-14$ & NRODS \\
\hline $15-28$ & NDRDS \\
\hline $29-42$ & NODES \\
\hline $43-56$ & NSLBS \\
\hline $57-70$ & NFBPWT \\
\hline
\end{tabular}

Card Number 9. (Format 5E14.6)

15-28 TNEUT

\section{Description}

Total number of calculational fuel rods.

Number of radial heat-transfer nodes in the fuel rods (input NDRDS $\geq 4$ if NRODS $>0$ fuel rods is spectfied; input NDRDS $^{\prime}=0$ if NRODS $=0$ ).

Number of heat-transfer nodes in the core wall. A value of 1 defaults to the lumpedparameter model. A value of 0 specifles no wall heat transfer.

Variable not used.

Flag option to replace the radial or axial power shape with a user-defined shape used to average the reactivity-feedback parameters over the core. (For the input value, add 1 for a radial shape or 2 for an axial shape.)

REACT, TNEUT, RPWOFF, RRPWMX, RPWSCL

Description

Initial programmed reactivity (IRPWTY $=1$, $2,4,11,12$, or 14) or trip-initiated programmed reactivity (IRPWTY $=3$ or 13) [REACT $=(k-1) \cdot \mathrm{k}^{-1}$ where $\mathrm{k}$ is the reactor multiplication constant; both REACT and $k$ have no units].

The prompt neutron IIfetime ( $s$ ) [TNEUT $\leq 0.0 \quad \therefore$ defaults internally to TNEUT $=1.625$ $\left.\times 10^{-5} \mathrm{~s}\right]$. 
$\frac{\text { Columns }}{29-42} \quad$ Variable

43-56 RRPWMX

57-70 RPWSCL

Card Number 10. (Format 5E14.6)

\begin{tabular}{|c|c|}
\hline Colunns & Variable \\
\hline $1-14$ & RPOWRI \\
\hline $15-28$ & ZPWIN \\
\hline
\end{tabular}

29-42 ZPWOFF

43-56 RZPWMX

57-70 QP3IN

Card Number 11. (Format 5E14.6)

$\frac{\text { Columns }}{1-14} \quad$ Variable

Description

Programmed reactivity $(-)$ [IRPWTY $=3,4$, 13,14 ] or reactor power (W) [IRPWTY $=7$ or 172

the reactivity-power cont trip is OFF after being ON (hold constant the last value of reactivity or power when the trip was ON when RPWOFF $\leq-1.0 \times 10^{19}$ ).

The maximum rate of change of programmed reactivity $\left(s^{-1}\right)$ or reactor power $\left(W \cdot s^{-1}\right)$ [RRPWMX $\geq 0.0$ ]:

Reactivity-power table scale factor for programmed reactivity or reactor power. The dependent variable in table RPWTB is multiplied by RPWSCL to obtain the absolute value.

RPOWRI, ZPWIN, ZPWOFF, RZPWMX, QP3IN

\section{Description}

Initial reactor power (W).

The axtal power-shape table's abscissacoordinate variable value corresponding to the initial axial power shape.

The axial power-shape table's abscissacoordinate variable value corresponding to the axial power-shape to be used when the fewer-shape table controlling trip is OFF after being ON (use the last evaluated axial power shape when the trip was ON when ZPWOFF $\leq-1.0 \times 10^{19}$ ).

The maximum rate of change of any $z$-interface value in the axial power shape $\left(s^{-1}\right)$ [RZPWMX 20.0$]$.

Power ( $W$ ) deposited in the wall and distributed according to the QPPP array.

RADIN, TH, HOUTL, HOUTV, TOUTL

\section{Description}

Inner radius (m) of the core wall. 


\begin{tabular}{|c|c|}
\hline Columns & Variable \\
\hline $15-28$ & $\mathrm{TH}$ \\
\hline $29-42$ & HOUTL \\
\hline $43-56$ & HOUTV \\
\hline $57-70$ & TOUTL \\
\hline Note: & $\begin{array}{l}\text { PIPE inpu } \\
\text { TL, HOUTV, }\end{array}$ \\
\hline \multicolumn{2}{|c|}{ Card Number 12.} \\
\hline Columns & Variable \\
\hline $1-14$ & TOUTV \\
\hline $15-28$ & PLDR \\
\hline $29-42$ & PDRAT \\
\hline $43-56$ & FUCRAC \\
\hline $57-70$ & HGAPO \\
\hline
\end{tabular}

Description

Core wall thickness (m).

HTC $\left(W \cdot \mathrm{m}^{-2} \cdot \mathrm{K}^{-1}\right)$ between outer boundary of the core wall and liquid outside the core wall.

HTC $\left(W \cdot \mathrm{m}^{-2} \cdot \mathrm{K}^{-1}\right)$ between outer boundary of the core wall and vapor outside the core wall.

Liquid temperature $(K)$ outside core wall.

Note: See PIPE input description, Sec. V.C.5.e, for further conments on the HOUTL, HOUTV, TOUTL, and TOUTV heat-transfer parameters.

Card Number 12. (Format 5E14.6) TOUTV, PLDR, PDRAT, FUCRAC, HGAPO

Description

Vapor temperature (K) outside core wall.

Pellet dish radius ( $m$ ) [no calculation of pellet dishing is performed if PLDR $=0.0$ ]

Fuel-rod pitch-to-diameter ratio (-).

Fraction of the fuel not cracked (used only if NFCI $=1$ on Card Number 7).

Fuel-rod gap conductance coefficient $\left(\mathrm{W} \cdot \mathrm{m}^{-2} \cdot \mathrm{K}^{-1}\right)$ [constant for NFCI $=0$ on Card Number 7; initial value otherwisel.

Card Number 13. (Format 3E14.6) DTXHT(1), DTXHT(2), DZNHT

$\frac{\text { Columns }}{1-14} \quad \frac{\text { Variable }}{\text { DTXHT(1) }}$

\section{Description}

Maximum $\Delta T(K)$ above which rows of nodes are inserted in the fuel-rod conduction calculation during reflood for the nucleate and transition boiling regimes [suggested value: $\operatorname{DTXHT}(1)=3.0]$.

15-28 DTXHT(2)

Maximum $\Delta T(K)$ above which rows of nodes are inserted in the fuel-rod conduction calculation during reflood for all boiling regimes except nucleate and transition [ suggested value: $\operatorname{DTXHT}(2)=10.0$ ] 
Columns Variable

29-42 DZNHT
Description

Minimum $\Delta \mathrm{Z}(\mathrm{m})$ below which no additional rows are inserted in the fuel-rod conduction calculation during the reflood calculation (this value comes from the diffusion number).

Note: If reactivity feedback is not evaluated (IRPWTY < 11 , word 1 on Card Number 4), omit Card Numbers 14-19. The reactivity-feedback input involves information on Card Numbers $14-19$ and tables RCTF, RCTC, RCAL, and RCBM in the CORE Common-Rod Array Cards that follow. Information and an example that are useful in the determination of the correct values for $\operatorname{IRCJTB}(I, J)$ on Card Numbers $14-17$ are provided with the tables.

Card Numbers 14-17. (Format 5I14/5I14/5I14/5I14) IRCJTB(I,J), I= $(1,4)$, $\mathrm{J}=(1,4)$

\begin{tabular}{|c|c|c|}
\hline Columns & Variable & Description \\
\hline $1-14$ & $\operatorname{IRCJTB}(1, J)$ & $\begin{array}{l}\text { The number of fuel-temperature } \mathrm{T}_{\mathrm{f}} \text { dependent } \\
\text { entries in the } \mathrm{Jth} \text { reactivity-coefficient } \\
\text { table }[1 \leq \operatorname{IRCJTB}(1, J)] \text {. }\end{array}$ \\
\hline $15-28$ & $\operatorname{IRCJTB}(2, J)$ & $\begin{array}{l}\text { The number of coolant-temperature } \mathrm{T}_{c} \text { depen- } \\
\text { dent entries in the Jth reactivity- } \\
\text { coefficient table }[1 \leq \operatorname{IRCJTB}(2, \mathrm{~J})] \text {. }\end{array}$ \\
\hline $29-42$ & $\operatorname{IRCJTB}(3, J)$ & $\begin{array}{l}\text { The number of vapor-coolant void-fraction } \alpha \\
\text { dependent entries in the Jth reactivity- } \\
\text { coefficient table }[1 \leq \operatorname{IRCJTB}(3, \mathrm{~J})] \text {. }\end{array}$ \\
\hline $43-56$ & $\operatorname{IRCJTB}(4, J)$ & $\begin{array}{l}\text { The number of boron } B \text { dependent entries in } \\
\text { the Jth reactivity-coefficient table } \\
{[1 \leq \operatorname{IRCJTB}(4, J)]}\end{array}$ \\
\hline
\end{tabular}


Columns Variable

57-70 $\operatorname{IBU}(\mathrm{J})$

\section{Description}

The boron unit's definition flag for the $\partial \mathrm{k} / \partial \mathrm{X}_{\mathrm{J}}$ reactivity coefficient's $\mathrm{x}_{\mathrm{J}}$ derivative parameter,

$$
x_{4}=\left\{{ }_{B_{r}}^{B_{m_{1}}}\right\} \text { for } \operatorname{IBU}(4)=\left\{\begin{array}{ccc}
0 & \text { or } 1 \\
-2 & \text { or }-1
\end{array}\right\} \text {, }
$$

and $f\left(T_{f}, T_{C}, \alpha, B\right)$ tabular dependence,

$$
B=\left\{\begin{array}{l}
B_{m_{r}} \\
\}
\end{array} \text { for } \operatorname{IBU}(J)=\left\{\begin{array}{lll}
-1 & \text { or } & 1 \\
-2 & \text { or } & 0
\end{array}\right\}\right. \text {, }
$$

where $B_{m}$ is the macroscopic density in the coolant-channel volume in $\mathrm{kg} \cdot \mathrm{m}^{-3}$ units, and $B_{r}$ is the mass ratio of parts boron per million parts liquid coolant in ppm units.

(

The $J=1$ card defines the fuel-temperature reactivity-coefficient table, the $J=2$ card defines the coolant-temperature reactivity-coefficient table, the $J=3$ card defines the vapor-coolant void-fraction reactivity-coefficient table, and the $\mathrm{J}=4$ card defines the boron reactivity-coefficient table. The reactivity coefficients are tabular functions of the four reactivity-feedback parameters, that is, $\mathrm{Jth}$ coefficient $=\mathrm{f}_{\mathrm{J}}\left(\mathrm{T}_{\mathrm{f}}, \mathrm{T}_{\mathrm{C}}, \alpha, \mathrm{B}\right), \mathrm{J}=1,2,3$, or 4 .

\begin{tabular}{|c|c|}
\hline Columns & Variable \\
\hline $1=14$ & IRCJFM(1) \\
\hline $15-28$ & IRCJFM(2) \\
\hline $29-42$ & IRCJFM(3) \\
\hline $43-56$ & $\operatorname{IRCJFM}(4)$ \\
\hline
\end{tabular}

Card Number 18. (Format 5I14) $\operatorname{IRCJFM}(J), J=(1,4), \operatorname{ISNOTB}$

\section{Description}

The form number for the fuel-temperature reactivity-coefficient type.

The form number for the coolant-temperature reactivity-coefficient type.

The form number for the vapor-coolant voidfraction reactivity-coefficient type.

The form number for the boron reactivitycoefficient type. 


\section{Columns Variable}

$57-70$

ISNOTB

\section{Description}

Flag that includes (excludes) the solute material in (from) the boron for the reactivity-feedback calculation.

$0=$ the solute is boron;

$1=$ the solute is not boron.

For the reactivity-coefficient forms, refer to Table IV on page 96 where $\mathrm{x}=\mathrm{T}_{\mathrm{f}}(\mathrm{K})$ for $\mathrm{J}=1, \mathrm{x}=\mathrm{T}_{\mathrm{C}}(\mathrm{K})$ for $\mathrm{J}=2, \mathrm{x}=\alpha$ for $\mathrm{J}=3, \mathrm{x}=\mathrm{B}_{\mathrm{r}}$ [ when $\operatorname{IBU}(4)=-2,-1]$, or $x=B_{m}[$ when $\operatorname{IBU}(4)=0,1]$ for $J=4$.

Card Number 19. (Format 5E14.6) POWEXP, BPPO, BPP1, BCRO, BCR1

Columns Variable

1-14 POWEXP

\section{Description}

The exponent value to which the cell values of the power distribution are raised in defining the weighting function for averaging the reactivity-feedback parameters over the core volume (suggested value: POWEXP $=2.0)$. The averaging process over the core for the reactivity-feedback parameter $\mathrm{X}$ is defined by

$$
\bar{x}=\frac{\sum_{i}^{c e 11 s} x_{i} m_{i} P_{i} \text { POWEXP }}{\sum_{i}^{c e l l s} m_{i} P_{i} \text { POWEXP }}
$$

where $P_{i}$ is the power in cell $i$. For $\mathrm{X}_{i}=\mathrm{Tf}_{i}$ or $\mathrm{T}_{c_{i}}, \mathrm{~m}_{i}$ is the mass of the fuel or coolant, respectively, in cell $i$; for $x_{i}=\alpha_{i}$ or $B_{i}, m_{i}=1$.

The zero-order coefficient of the firstorder polynomial $\mathrm{BPP}=\mathrm{BPPO}+\mathrm{BPPl} \mathrm{T}_{\mathrm{c}}$ that defines the effective (smeared and shielded) core-average concentration of burnablepoison pin boron in the coolant-channel volume $\left(\mathrm{kg} \cdot \mathrm{m}^{-3}\right)$. See Eq. (177). 
Columns Variable

29-42 BPP1

43-56 BCRO

57-70 BCR1

CORE Array Cards. Twenty sets of cards, one set for each of the following variables. Use LOAD format.

The first-order coefficient of the firstorder polynomial $\mathrm{BPP}=\mathrm{BPPO}+\mathrm{BPP} 1 \star_{\mathrm{T}} \mathrm{C}$ defining the effective (smeared and coreaverage concentration of burnable-poison pin boron in the coolant-channel volume $\left(\mathrm{kg} \cdot \mathrm{m}^{-3} \cdot \mathrm{K}^{-1}\right) \cdot \mathrm{T}_{\mathrm{c}}$ is the core-average coolant temperature. See Eq. (177).

The zero-order coefficient of the firstorder polynomial $\mathrm{BCR}=\mathrm{BCRO}+\mathrm{BCRl}$ o $_{\mathrm{PROG}}$ defining the effective (smeared and shielded) core-average concentration of control-rod pin boron in the coolant-channel volume $\left(\mathrm{kg} \cdot \mathrm{m}^{-3}\right)$. See Eq. (178).

The first-order coefficient of the firstorder polynomial $B C R=B C R 0+B C R 1 *_{P}$ PROG defining the effective (smeared and shielded) core-average concentration of the control-rod pin boron in the coolant-channel volume $\left(\mathrm{kg} \cdot \mathrm{m}^{-3}\right)$. PPROG is programmed reactivity and has no units. See Eq. (178).

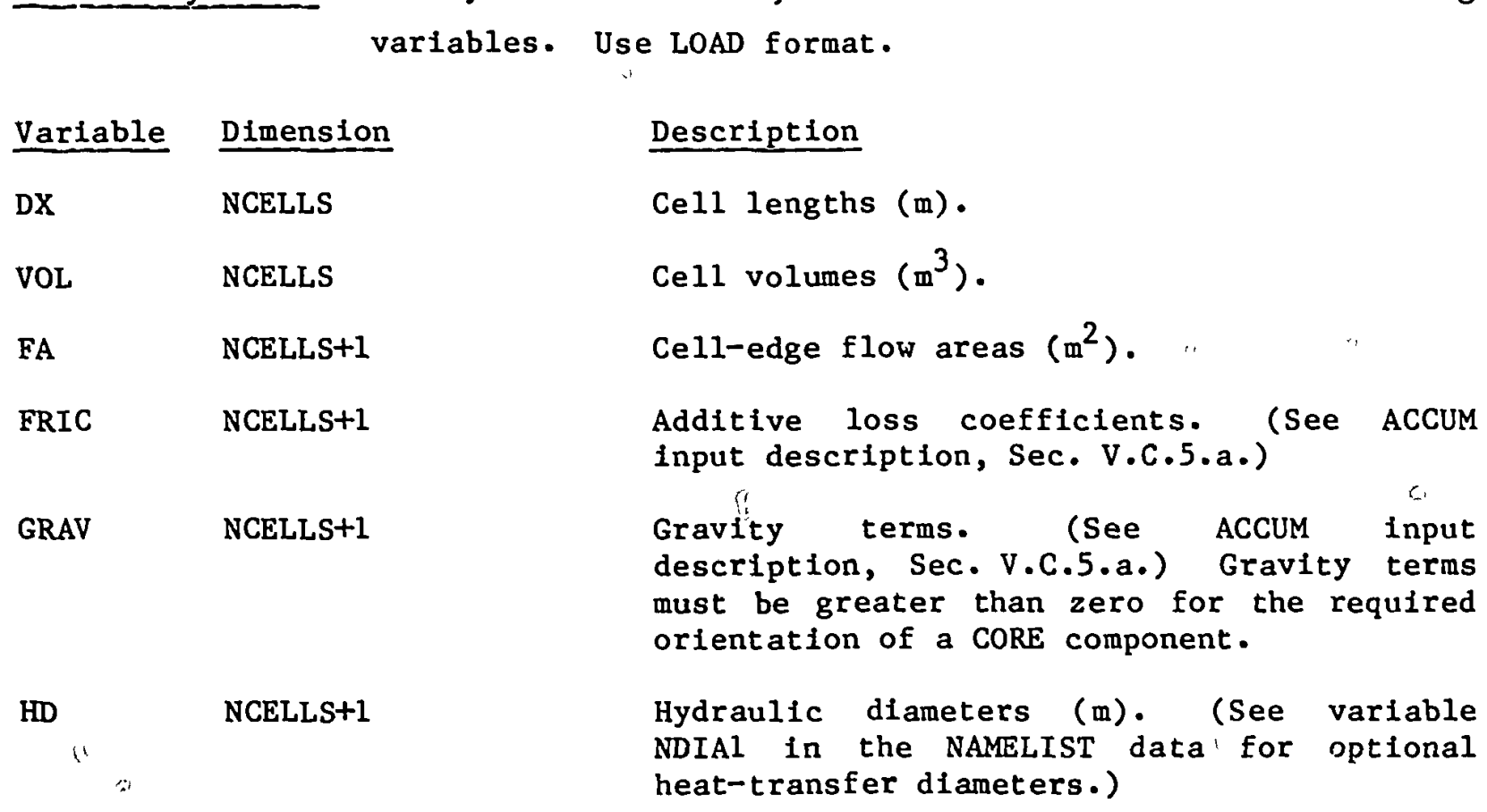


One-Dimensional Core Component (CORE)

Note: If ICFLOW $=0$ or ICFLOW $=1$ (default) in the NAMELIST data, do not input array ICFLG.

$\begin{array}{ll}\text { Variable } & \text { Dimension } \\ \text { ICFLG } & \text { NCELLS+1 } \\ \text { NFF } & \text { NCELLS+1 } \\ \text { ALP } & \text { NCELLS } \\ \text { VL } & \text { NCELLS+1 } \\ \text { VV } & \text { NCELLS+1 } \\ \text { TL } & \text { NCELLS } \\ \text { TV } & \text { NCELLS } \\ \text { P } & \text { NCELLS } \\ \text { PA } & \text { NCELLS }\end{array}$

\section{Description}

Cell-edge choked-flow model options. (See ACCUM input description, Sec. V.C.5.a.)

Friction-factor correlation options. (See ACCUM input description, Sec.V.C.5.a.)

Initial vold fractions (-).

Initial liquid velocities $\left(m \cdot s^{-1}\right)$.

Initial vapor velocities $\left(\mathrm{m} \cdot \mathrm{s}^{-1}\right)$.

Initial liquid temperatures (K).

Initial vapor temperatures (K).

Initial pressures ( $\mathrm{Pa}$ ).

Initial air partial pressures ( $\mathrm{Pa}$ ).

Note: If NODES $=0$ (word 3 on Card Number 8), do not enter arrays QPPP, MATID, and TW.

QPPP $\quad$ NODES*NCELLS

A nonnorpalized power protile $(-)$ in the core walls. Input for cell 1 , node 1 through NODES; then for cell 2, node 1 through NODES; etc. If the array is filled with the same nonzero constant, a uniform volumetric heat source for the wall results. The code will normalize the power proftle on a node-volume basis (normalized QPPP $(i)=$ $\left.\operatorname{QPPP}(i) /\left[\sum \operatorname{QPPP}(i) * \operatorname{VOL}(i)\right]\right)$. Filling the array 1 with zeros results in no power being deposited in the walis regardless ff the settings of QP3IN, QP3TB, etc. 
Variable Dimension

MATID NODES-1

\section{Description}

Array specifying material ID between radial nodes in wall. Dimension is 1 if NODES $=1$ and 0 if NODES $=0$. The following properties are built into the code:

$6=$ stalniess stecl, type 304 ;

7 = stainless steel, type 316 ;

8 = stainless steel, type 347 ;

9 = carbon steel, type A508;

10 = Incone1, type 718 ; or

12 = Inconel, type 600 .

(See, Sec. V.C.2 to input material properties.)

Note: Material-properties ID numbers $1-5$ and 11 are available only to the fuel-rod model.

TW

NODES*NCELLS

Inttial wall temperatures (K). Input in the isame order as QPPP.

Note: If ICONC $=0$ (word 1 on Card Number 3 ), omit card set CoNC.

CONC NCELLS

Initial ratio of solute mass to liquid mass $(-)$.

Note: If ICONC $=0$ or 1 (word 1 on Card Number 3 ), omit card set $S$.

S NCELLS

CORE Common-Rod Array Cards.

Variable

Dimension

$\mathrm{Z}$

NCELLS

RDPWR NDRDS

CPOWR $\quad 1$
Initial macroscopic density of plated-out solute $\left(\mathrm{kg} \cdot \mathrm{m}^{-3}\right)$. the following variables. Use LOAD format.

\section{Description}

Cumulative flow length (m) to the end of each cell from JUN1 (Card Number 2).

Relative radial power density at the node positions.

Relative power density for the average rod (suggested Input, 1.0). 


\begin{tabular}{|c|c|c|}
\hline Variable & Dimension & Description \\
\hline RPKF & NRODS-1 & $\begin{array}{l}\text { Rod power-peaking factors (relative to the } \\
\text { core average rod) for the additional rods. }\end{array}$ \\
\hline Note: & If $\mathrm{NZPWTB}=0$ on Card & Number 6 , omit card set ZPWTB. \\
\hline ZPWTB & $\begin{array}{l}: \text { NZPWTB } \mid * \\
{[1+(\text { ICRU-ICRL+1) }}\end{array}$ & $\begin{array}{l}\text { Axial power-shape vs independent-variable- } \\
\text { form table. Input [1ndependent variable de- } \\
\text { fined by IZPWSV and NZPWTB on Card Number } 6 \text {, } \\
\text { axial power-shape densities that have } \\
\text { (ICRU-ICRL+1) values] pairs }(*,-) \text {. The } \\
\text { relative axial power densities that define } \\
\text { the axial power shape are specified at the } \\
\text { cell interfaces from one end of the fuel rod } \\
\text { to the other. There are NZPWTB axial power } \\
\text { shapes being input with each having } \\
\text { (ICRU-ICRL+1) values. }\end{array}$ \\
\hline
\end{tabular}

Note: If NZPWRF or NZPWTB $=0$ on Card Number 6 , omit card set NZPWRF.

ZPWRF $\mid$ NZPWRF|*2 Rate-factor table for the axial power-shape table's independent variable. Input [independent-variable form defined by NZPWSV and NZPWRF on Card Number 6, rate factor to be applied to the axial power-shape table's independent variable] pairs $(*,-)$.

$\operatorname{RDX} \quad 1$

Number of fuel rods in the core (this is a real number, not an integer; fractional rods are allowed).

RADRD NDRDS Rod node radil (m) [cold] • 
Note: Only one MATRD element may have the value 3; and MATRD(1) and MATRD(NDRDS-1) cannot be 3 . Additional materlal properties can be input (Sec. V.C.2).

Variable Dimension

MATRD

NDRDS-1
Description

Fuel-rod-matertal ID numbers [dimension is 1 if NDRDS $=1$ (word 2 on Card Number 8 )]

ID

\section{Material Type}

Mixed oxide fuel

Zircaloy

Fuel-clad gap gases

Boron-nitrid o lation

Constantan/Nichrome heater wire

Stainless steel, type 304

Stainless steel, type 316

Stainless steel, type 347

Carbon steel, type A508

Inconel, type 718

Zircaloy dioxide

Inconel, type 600

Note: If NRPWTB $=0$ on Card Number 5, omit card set RPWTB́,

RPWTB $\mid$ NRPWTB $\mid * 2$

Programmed-reactivity or reactor-power vs independent-variable-form table. Input (independent-variable form defined by IRPWSV and NRPWTB on Card Number 5, programmed reactivity or reactor power as defined by IRPWTY) pairs (*,- or W).

Note: If NRPWTB $=0$ (word 3, Card Number 5) or NRPWRF $=0$ (word 5, Card Number 5), omit card set RPWRF.

RPWRF $\mid$ NRPWRF $\mid * 2$
Rate-factor table for the programmedreactivity or reactor-power table's independent variable. Input (independent-variable form defined by NRPWSV and NRPWRF on Card Number 5, rate factor to be applied to the progranmed-reactivity or reactor-power table $s$ independent variable) pairs $(*,-)$. 
Note: If IRPWTY < 11 (word 1, Card Number 4), omit card sets RCTF, RCTC, RCAL, and RCMB. The following four tables are not entered with $(x, y)$ pair values as is done for one-dimensional dependent tables. The general steps for entering tables RCTF, RCTC, RCAL, and RCBM are listed below.

1. Select the appropriate reactivity-coefficient form IRCJFM(J) and the appropriate boron units IBU(J) for the particular table J.

2. The user is allowed to specify only one set of points each for fuel temperature $T_{f}$, coolant temperature $T_{c}$, void fraction $\alpha$, and boron $B$. The numbers of unique points for each of the above parameters are defined by $\operatorname{IRCJTB}(1, J), \operatorname{IRCJTB}(2, J), \operatorname{IRCJTB}(3, J)$, and $\operatorname{IRCJTB}(4, \mathrm{~J})$. Each set of parameters must be increasing monotonically.

3. With $T_{C}, \alpha$, and $B$ constant, vary $T_{f}$ through its range and spècify the reactivity coefficients. Then, increment $T_{C}$, hold $\alpha$ and $B$ constant, and vary $T_{f}$ again. Continue incrementing $T_{c}$ until the range of $\mathrm{T}_{c}$ is covered.

4. Reset $T_{f}$ and $T_{c}$ to their first values, increment $\alpha$, and repeat step $3 .{ }^{2}$ Continue incrementing $\alpha$ until its range is covered.

5. Reset $T_{f}, T_{C}$, and $\alpha$ to their first values, increment $B$, and repeat steps 3 and 4 . Continue incrementing $B$ until its range is covered. At this point, all of the reactivity coefficients for table $J$ should be specified.

6. To enter the table, define $\operatorname{IRCJTB}(1, J)$ values of $T_{f}, \operatorname{IrCJTB}(2, J)$ values of $T_{1}, \operatorname{IRCJTB}(3, J)$ values of $\alpha$, and $\operatorname{IRCJTB}(4, J)$ values of $B$ in that order. The values for each $T_{f}, T_{f}, \alpha$, and $B$ should be increasing monotonically. Now, enter $\operatorname{IRCJTB}(1, J) * \operatorname{IrCJTB}(2, J)$ *IRCJTB $(3, \mathrm{~J})$ *IRCJTB $(4, \mathrm{~J})$ reactivity coefficients generated in steps 3-5 above in the same order in which they were generated. This completes table $\mathrm{J}$. 
Select $\operatorname{IRCJFM}(2)=1$ and $\operatorname{IBU}(2)=0$.

Reactor-kinetics-feedback data generated by steps 3-5 for a table with one value for $T_{f}$, two values for $T_{c}$, one value for $\alpha$, and two values for $B$.

$\begin{array}{lllcc}\begin{array}{l}\mathrm{T}_{f} \\ (\mathrm{~K})\end{array} & \begin{array}{l}\mathrm{T}_{\mathrm{c}} \\ (\mathrm{K})\end{array} & \underline{\alpha} & \begin{array}{c}\mathrm{B}_{\mathrm{r}} \\ (\mathrm{ppm})\end{array} & \begin{array}{c}(1 / \mathrm{k}) *\left(\partial \mathrm{k} / \partial \mathrm{T}_{\mathrm{c}}\right) \\ \left(\mathrm{K}^{-1}\right)\end{array} \\ & 293.0 & 0.0 & 700.0 & -4.8720 \times 10^{-5} \\ 0.0 & 616.5 & 0.0 & 700.0 & -1.1567 \times 10^{-3} \\ 0.0 & 293.0 & 0.0 & 2800.0 & -1.3944 \times 10^{-5} \\ 0.0 & 616.5 & 0.0 & 2800.0 & -6.0648 \times 10^{-4}\end{array}$

Because $T_{f}$ and $\alpha$ do not vary, a value of 0.0 may be used. The free-format input of table RCTC follows:

$$
\begin{aligned}
& 0.0 \mathrm{~s} \quad \text { * fuel temp } \\
& 293.0 \quad 616.5 \mathrm{~s} \quad \text { * coolant temp } \\
& 0.0 \mathrm{~s} \quad \text { * vold frac } \\
& 700.0 \quad 2800.0 \mathrm{~s} \quad * \text { boron conc } \\
& -4.8720 e^{-5} \quad-1.1567 e-3 \quad-1.3944 e-5 \quad-6.0648 e-4 e * \text { reactivity coef }
\end{aligned}
$$

When fewer than five values are specifled per record, the skip modifier "s" implies the data are continued on the next record.

Variable Dimension

RCT F

$\operatorname{IRCJTB}(1,1)+\operatorname{IRCJTB}(2,1)+$ $\operatorname{IRCJTB}(3,1)+\operatorname{IRCJTB}(4,1)+$ ( IRCJTB $(1,1)$ *IRCJTB $(2,1)$ * $\operatorname{IRCJTB}(3,1) * \operatorname{IRCJTB}(4,1))$

RCTC
$\operatorname{IRCJTB}(1,2)+\operatorname{IRCJTB}(2,2)+$ $\operatorname{IRCJTB}(3,2)+\operatorname{IRCJTB}(4,2)+$ (IRCJTB $(1,2)$ *IRCJTB $(2,2)$ * $\operatorname{IRCJTB}(3,2) * \operatorname{IRCJTB}(4,2))$

\section{Description}

The fuel-temperature reactivity-coefficient table. Input $\operatorname{IRCJTB}(1,1) \quad \mathrm{T}_{\mathrm{f}}$ values, $\operatorname{IRCJTB}(2,1) \quad T$ values, $\operatorname{IRCJTB}(3,1)$ a values, IRCJTB $(4,1)$ B values, and $\operatorname{IRCJTB}(1,1)$ * $\operatorname{IRCJTB}(2,1) *$ $\operatorname{IRCJTB}(3,1)$ *IRCJTB $(4,1)$ fue1-temperature reactivity-coefficient values that define the four-dimensional dependent table.

The coolant-temperature reactivitycoefficient table. 
One-Dimensional Core Component (CORE)

Variable Dimension

RCAL IRCJTB $(1,3)+\operatorname{IRCJTB}(2,3)+$ $\operatorname{IRCJTB}(3,3)+\operatorname{IRCJTB}(4,3)+$ (IRCJTB $(1,3) * \operatorname{IRCJTB}(2,3)$ * $\operatorname{IRCJTB}(3,3) * \operatorname{IRCJTB}(4,3))$

$\operatorname{RCBM} \operatorname{IRCJTB}(1,4)+\operatorname{IRCJTB}(2,4)+$ $\operatorname{IRCJTB}(3,4)+\operatorname{IRCJTB}(4,4)+$ (IRCJTB $(1,4) * \operatorname{IRCJTB}(2,4)$ * $\operatorname{IRCJTB}(3,4) * \operatorname{IRCJTB}(4,4))$
Description

The vapor-coolant void-fraction reactivity-coefficient table.

The boron reactivity-coefficient table.

Note: If power option IRPWTY $=5,6,7,15,16$, or 17 (word 1 on Card Number 4) is specified, omit card sets BETA, LAMDA, CDGN, LAMDH, EDH, CDHN, and PHIST.

Note: If NDGX $\leq 0$ (word 2 on Card Number 4), omit card sets BETA and LAMDA. The default 6 group delay-neutron constants will be defined internally.

BETA NDGX The effective delayed-neutron fraction.

LAMDA NDGX The delayed-neutron decay constant
$\left(s^{-1}\right)$.

Note: Use card set CDGN only if NHIST $=0$ and NDGX $>0$ on Card Number 4.

CDGN NDGX

The delayed-neutron precursor concentration (W).

Note: If NDHX $\leq 0$ (word 3 on Card Number 4), omit card sets LAMDH and EDH. The default 11 group decay-heat constants will be defined internally.

$\begin{array}{lll}\text { LAMDH } & \text { NDHX } & \text { Decay-heat decay constant }\left(s^{-1}\right) . \\ \text { EDH } & \text { NDHX } & \text { Effective decay-heat energy fraction. }\end{array}$

Note: Use card set CDHN only if NHIST $=0$ and NDGX $>0$ on Card Number 4.

CDHN NDHX The decay-heat concentration $(W \cdot s)$. 
Note: If NHIST $=0$ on Card Number 4 , omit card set PHIST.

Variable Dimension

PHIST NHIST*2

NFAX ICRU-ICRL

$\begin{array}{ll}\text { FPUO2 } & 1 \\ \text { FTD } & 1 \\ \text { GMIX } & 7\end{array}$

$\begin{array}{ll}\text { GMLES } & 1 \\ \text { PGAPT } & 1 \\ \text { PLVOL } & 1\end{array}$

PLVOL 1

\section{Description}

Power-history table. Use (present time minus past time, reactor-core prompt-fission power at that past time) pairs $(s, W)$. The first palr should be for the current power; that is, time interval 0.0 , with the time interval for subsequent pairs being positive and increasing monotonically for each pair.

Number of permanent fine-mesh intervals per coarse-mesh interval added at the start of the reflood calculation [the total number of heat-transfer rows per fuel rod must be less than NZMAX (word 5 on Card Number 7)]

Fraction of plutonium dioxide $\left(\mathrm{PuO}_{2}\right)$ in mixed-oxide fuel.

Fraction of theoretical fuel density.

Mole fraction of gap-gas constituents. Array is not used if NFCI $=0$, but must be input. Enter data for each gas in the order indicated.

Index Gas

$\begin{array}{ll}1 & \text { Helium } \\ 2 & \text { Argon } \\ 3 & \text { Xenon } \\ 4 & \text { Krypton } \\ 5 & \text { Hydrogen } \\ 6 & \text { Air/nitrogen } \\ 7 & \text { Water vapor }\end{array}$

Moles of gap gas per fuel rod (currently not used, but must be input).

Average gap-gas pressure ( $\mathrm{Pa}$ ) [not used if NFCI $=0$, but must be input].

Plenum volume $\left(\mathrm{m}^{3}\right)$ in each fuel rod above the pellet stack (currently not used, but must be input). 
One-Dimensional Core Component (CORE)

\begin{tabular}{|c|c|c|}
\hline Variable & Dimension & Description \\
\hline PSLEN & 1 & $\begin{array}{l}\text { Pellet-stack length (m) [currently not used, } \\
\text { but must be Input]. }\end{array}$ \\
\hline CLENN & 1 & $\begin{array}{l}\text { Total cladding length (m) [currently not } \\
\text { used, but must be input]. }\end{array}$ \\
\hline CORE Rod & Array Cards. & $\begin{array}{l}\text { Two sets of cards, one for each of the following } \\
\text { variables. Both card sets are input for each of NRoDS } \\
\text { (word } 1 \text { on Card Number } 8 \text { ) fuel rods, including the } \\
\text { average rod and each additional rod. Use LOAD format. }\end{array}$ \\
\hline Variable & Dimension & Description \\
\hline BURN & ICRU-ICRL+1 & Fuel burnup (MWD/MTU). \\
\hline RFTN & NDRDS* ( I CRU & Initlal fuel-rod temperatures $(K)$. \\
\hline
\end{tabular}




\section{d. Fill Component (FILL).}

Card Number 1. (Format A10,4X,2I14,A30) TYPE, NUM, ID, CTITLE

$\begin{array}{lll}\begin{array}{lll}\text { Columns } \\ 1-4\end{array} & \text { Variable } & \text { Description } \\ 15-28 & \text { NUM } & \text { " Component type (FILL left-justified). } \\ 29-42 & \text { ID } & \begin{array}{l}\text { Component ID number (must be unique for each } \\ \text { component, } 1 \leq N U M \leq 999) .\end{array} \\ 43-72 & \text { CTITLE } & \text { User ID number (arbitrary). }\end{array}$

Card Number 2. (Format 3I14) JUN1, IFTY, IOFF

Columns Variable

1-14 JUN1

15-28 IFTY
Description

Junction number where fill is connected.

Fill-type option:

$1=$ constant veloct $t y=$

2 = constant mass flow,

3 = constant generalized state,

4 = velocity vs independent-variable form,

5 = mass flow vs independent-variable form,

6 = generalized state vs independentvariable form,

7 = constant velocity unt 11 the controlling trip is set on then velocity vs independent-variable form,

$8=$ constant inass flow unt 11 the controlling trip is set $O N$ then mass flow $v s$ independent-variable form, or

9 = constant generalized state until the controlling trip is set ON then generalized state vs independentvariable form. 


\section{Columns Variable}

29-42 IOFF
Description

FILL fluid-state option defining the fluid state when the fill-table controlling trip is OFF after being ON [define IOFF when IFTY $=7,8$, or 9 (word 2 on Card Number 2)].

$0=$ the last fill-table interpolated fluid state is held constant;

1 = define the initial fluid state;

2 = input the velocity (IFTY $=7$ or 9) or mass flow (IFTY $=8$ ) to be used but maintain the fluid condition that existed when the trip was set OFF; or

3 = input a generalized fluid-state definition (IFTY $=9$ only).

Note: If IFTY $<4$ (word 2 on Card Number 2), omit Card Number 3.

Card Number 3. (Format 5I14) IFTR, IFSV, NFTB, NFSV, NFRF

Columns $\quad$ Variable

15-28 IFSV

29-42 NFTB

\section{Description}

The trip ID number for controlling evaluation of the fill table for fill-type options IFTY $=7,8$, or $9(\mid$ IFTR $\mid<10000)$ [ input IFTR $=0$ when IFTY $<7$ ]

The fill-table abscissa-coordinate variable ID number that defines the independentvariable parameter in the IFTY $=4$ to 9 tables. IFSV $>0$ defines the ID number for a signal-variable parameter; IFSV $<0$ defines the ID number for a control-block output parameter.

The number of fill-table $(x, y)$ pairs for fill-type options IFTY $=4$ to 9 (defined by the absolute value of NFTB). NFTB $>0$ defines the independent-variable form to be the IFSV parameter; NFTB $<0$ defines the independent-variable form to be the change in the IFSV parameter over the last time step times the trip set status value ISET when IFTY $=7,8$, or 9; NFTB $=0$ defines the FILL velocity or mass flow to be the IFSV parameter. 


$\frac{\text { Columns }}{43-56} \quad$ Variable

57-70 NFRF

Card Number 4. (Format 4E14.6)

$\begin{array}{ll}\text { Columns } & \text { Variable } \\ 1-14 & \text { TWTOLD } \\ 15-28 & \text { RFMX }\end{array}$

29-42 CONCIN

\section{Description}

The rate-factor table abscissa-conrdinate variable ID number. NFSV > 0 defines the ID number for a signal-variable parameter; NFSV $<0$ defines the ID number for a control-block output parameter; NFSV $=c$, (when NFRF $\neq 0$ ) defines the difference between the trip signal and the set-point value that turns the trip OFF when the fill table is trip controlled.

The number of rate-factor table $(x, y)$ pairs (defined by the absolute value of NFRF). The rate factor is applied as a factor to the fill-table independent variable when the rate factor is defined. No rate factor is defined when NFSV and NFRF are both zero. NFRF $>0$ defines the rate-factor table abscissa coordinate to be the NFSV parameter; NFRF < 0 defines it to be the change in the NFSV parameter over the last time step times the trip set-status value ISET when 1 FTY $=7,8$, or $9 ; \quad$ NFRF $=0$ defines the rate factor to be the NFSV parameter.

TWTOLD, RFMX, CONCIN, FELV

Description

The fraction of the previous fill fluid state that is averaged with the fill-tabledefined state to define the fill fluid state for this time step $(0.0 \leq$ TWTOLD $<1.0$; it is suggested that a value of 0.0 be used). To avold hydrodynamic instabilities, a value as large as 0.9 may be needed when the fill table depends on a parameter, such as the adjacent component pressure, that couples strongly to the fill velocity or a parameter that varies rapidly with time.

The maximum rate of change of fill velocity $\left(m \cdot s^{-2}\right)$ or mass flow $\left(\mathrm{kg} \cdot \mathrm{s}^{-2}\right)$ [0 $\leq$ RFMX].

The initial ratio of solute mass to liquidcoolant mass ( - ) in the FILL composition [ not used unless ISOLUT $=1$ (word 5) on Main-Data Card 6)]. 
Columns Variable

43-56 FELV

Card Number 5. (Format 5E14.6)

\begin{tabular}{|c|c|}
\hline Columns & Varial \\
\hline $1-14$ & DXIN \\
\hline $15-28$ & VOLIN \\
\hline i & \\
\hline $29-42$ & ALPIN \\
\hline $43-56$ & VLIN \\
\hline
\end{tabular}

$57-70 \quad$ TLIN

Card Number 6. (Format 5E14.6) PIN, PAIN, FLOWIN, VVIN, TVIN

$\begin{array}{ll}\text { Columns } & \text { Variable } \\ 1-14 & \text { PIN } \\ 15-28 & \text { PAIN } \\ 29-42 & \text { FLOWIN }\end{array}$

43-56 VVIN

Description
Fill cel1-centered elevation ( $m$ ) [used only to compute GRAV array when IELV $=1$ ]

DXIN, VOLIN, ALPIN, VLIN, TLIN

\section{Description}

Cell length (m), generally the same as its neighboring cell in the adjacent component. Currently not used.

Cell volume $\left(\mathrm{m}^{3}\right)$, generally the same as its neighboring cell in the adjacent component. Currently not used.

Initial coolant void fraction (-) for positive flow out of the fill.

Initial liquid velocity (m $\left.\cdot \mathrm{s}^{-1}\right)$; a positive value indicates flow into the adjacent component; a negative value indicates flow from the adjacent component (input for fill-type options IFTY $=1,3,7$ or 9 ).

Initial liquid temperature (K) for positive flow out of the fill.

Description

Initial fill pressure ( $\mathrm{Pa}$ ).

Initial fill air partial pressure ( $\mathrm{Pa}$.

Initial mass flow $\left(\mathrm{kg} \cdot \mathrm{s}^{-1}\right) ; a$ a positive value indicates flow into the adjacent component; a negative value indicates flow from the adjacent component (input for filltype options IFTY $=2$ or 8 ).

Initial vapor velocity $\left(\mathrm{m}, \mathrm{s}^{-1}\right)$; a positive value indicates flow into the adjacent component; a negative value indicates flow from the adjacent component (input for filltype options IFTY $=3$ or 9 ). 
Fi11 Component (FILL)

\begin{tabular}{|c|c|c|c|}
\hline Columns & Variable & Description & \\
\hline $57-70$ & TVIN & $\begin{array}{l}\text { Initial vapor temperature }(K) \text { for } \\
\text { flow (input for fill-type options } \\
\text { or } 9 \text { ). }\end{array}$ & $\begin{array}{l}\text { positive } \\
\text { IFTY }=3\end{array}$ \\
\hline
\end{tabular}

Note: If IFTY $<7$ (word 2 on Card Number 2) or IOFF 2 (word 3 on Card Number 2), omit Card Number 7 .

Card Number 7. (Format 4E14.6) FLWOFF, VLOFF, VVOFF, ALPOFF

\begin{tabular}{|c|c|c|}
\hline Columns & Variable & Description \\
\hline $1-14$ & FLWOFF & $\begin{array}{l}\left.\text { Mass flow ( } \mathrm{kg} \cdot \mathrm{s}^{-1}\right) \text { when the controlling } \\
\text { trip is set OFF after being ON (input when } \\
\text { IFTY }=8 \text { ). }\end{array}$ \\
\hline $15-28$ & VLOFF & $\begin{array}{l}\text { Liquid-coolant velocity }(\text { IFTY }=9) \text { or } \\
\text { coolant-mixture velocity }(\text { IFTY }=7) \text { when the } \\
\text { controlling trip is set OFF after being ON } \\
\text { (input when IFTY }=7 \text { or } 9 \text { ). }\end{array}$ \\
\hline $29-42$ & VVOFF & $\begin{array}{l}\text { Vapor-coolant velocity when the controlling } \\
\text { trip is set OFF after being ON (input when } \\
\text { IOFF }=3 \text { and IFTY }=9 \text { ). }\end{array}$ \\
\hline $43-56$ & ALPOFF & $\begin{array}{l}\text { Fluid void fraction ( }- \text { ) for positive flow } \\
\text { when the controlling trip is set OFF after } \\
\text { being ON (input when IOFF }=3 \text { and IFTY }=9 \text { ). }\end{array}$ \\
\hline
\end{tabular}

Note: If IFTY $\neq 9$ (word 2 on Card Number 2) or IOFF $\neq 3$ (word 3 on Card Number 2), omit Card Number 8 .

Card Number 8. (Format 5E14.6) TLOFF, TVOFF, POFF, PAOFF, CONOFF

Columns Variable Description

1-14 TLOFF

Liquid temperature (K) for positive flow when the controlling trip is set OFF after being $\mathrm{ON}$.

15-28 TVOFF

Vapor temperature $(K)$ for positive flow when the controlling trip is set OFF after being ON.

29-42 POFF

Fill pressure $(\mathrm{Pa})$ when the controlling trip is set $O F F$ after being $O N$. 


$\begin{array}{ll}\text { Columns } & \text { Variab1 } \\ 43-56 & \text { PAOFF } \\ 57-70 & \text { CONOFF }\end{array}$

Description

Fi1.1 air partial pressure ( $\mathrm{Pa}$ ) when the controlling trip is set OFF after being $O N$.

Ratio of solute mass to liquid-coolant mass $(-)$ when the controlling trip is set OFF after being oN.

Note: If IFTY $<4$ (word 2 on Card Number 2) or NFTB $=0$ (word 3 on Card Number 3 ), omit Card Number 9.

Card Number 9. (Format 2E14.6) VMSCL, VVSCL

\begin{tabular}{|c|c|c|}
\hline Columns & Variable & Description \\
\hline $1-14$ & VIMSCL & $\begin{array}{l}\text { Liquid-velocity (IFTY }=4,6,7 \text {, or } 9 \text { ) or } \\
\text { mass-flow (IFTY }=5 \text { or } 8 \text { ) scale factor. The } \\
\text { dependent variable in table VMTB is } \\
\text { multiplied by this factor to obtain the } \\
\text { absolute liquid velocity or absolute mass } \\
\text { flow. }\end{array}$ \\
\hline $15-28$ & VVSCL & $\begin{array}{l}\text { Vapor-velocity scale factor. The dependent } \\
\text { variable in table VVTB is multiplied by this } \\
\text { factor to obtain absolute vapor velocity } \\
\text { (input when IFTY }=6 \text { or } 9 \text { ). }\end{array}$ \\
\hline
\end{tabular}

Note: If IFTY $\neq 6$ and IFTY $\neq 9$ (word 2 on Card Number 2), omit Card Number 10 .

Card Number 10. (Format 5E14.6) TLSCL, TVSCL, PSCL, PASCL, CONSCL

\begin{tabular}{|c|c|c|}
\hline Columns & Variable & Description \\
\hline $1-14$ & TLSCL & $\begin{array}{l}\text { Liquid-temperature scale factor. } \\
\text { dependent variable in table TLTB is } \\
\text { multiplied by this factor to obtain absolute } \\
\text { liquid temperature. }\end{array}$ \\
\hline $15-28$ & TVSCL & $\begin{array}{l}\text { apor-temperature scale factor. The depen- } \\
\text { dent variable in table TVTB is multiplied by } \\
\text { this factor to obtain absolute vapor } \\
\text { temperature. }\end{array}$ \\
\hline $29-42$ & PSCL & $\begin{array}{l}\text { Pressure scale factor. The dependent } \\
\text { variable in table PTB is multiplied by this } \\
\text { factor to obtain absolute pressure. }\end{array}$ \\
\hline
\end{tabular}


Fill Component (FILL)

\begin{tabular}{|c|c|c|}
\hline Columns & Variable & Description \\
\hline $43-56$ & PASCL & $\begin{array}{l}\text { Air partial pressure scale factor. The de- } \\
\text { pendent variable in table PATB is multiplied } \\
\text { by this factor to obtain absolute air } \\
\text { partial pressure. }\end{array}$ \\
\hline $57-70$ & CONSCL & $\begin{array}{l}\text { Ratio of solute mas's to liquid-coolant mass } \\
\text { scale factor. The dependent variable in } \\
\text { table CONCTB is multiplied by this factor to } \\
\text { obtain the absolute solute mass to liquid- } \\
\text { coolant mass ratio. }\end{array}$ \\
\hline
\end{tabular}

Note: If IFTY $<4$ (word 2 on Card Number 2) or NFTB $=0$ (word 3 on Card Number 3), omit the Fil1 Array Cards.

FILL Array Cards. Nine sets of cards, one set for each of the following variables. Use LOAD format.

$\begin{array}{lll}\text { Variable Dimension } & \text { Description } \\ \text { VMTB } & \mid \text { NFTB } \mid * 2 & \text { Liquid velocity (IFTY }=4,6,7, \text { or 9) or mass } \\ & \text { flow (IFTY }=5 \text { or } 8 \text { ) vs independent- } \\ & \text { variable-form table. Input (independent- } \\ & \text { variable form, liquid velocfty or mass flow) } \\ & \text { pairs }(*, \mathrm{~m} \cdot \mathrm{s} \text { or } \mathrm{kg} \cdot \mathrm{s}) .\end{array}$

Note: If IFTY $\neq 6$ or 9 on Card Number 2 , omit card sets VVTB, TLTB, TVTB, ALPTB, PTB, and PATB.

\begin{tabular}{|c|c|c|}
\hline VVTB & $|\mathrm{NFTB}| * 2$ & $\begin{array}{l}\text { Vapor-velocity vs independent-variable-form } \\
\text { table. Input (independent-variable form, } \\
\text { liquid temperature) pairs }\left(*, m \cdot s^{-1}\right) \text {. }\end{array}$ \\
\hline TLTB & $|\mathrm{NFTB}| * 2$ & $\begin{array}{l}\text { Liquid-temperature vs independent-variable- } \\
\text { form table. Input (independent-variable } \\
\text { form, liquid temperature) pairs }(*, K) \text {. }\end{array}$ \\
\hline TVTB & $|\mathrm{NFTB}| * 2$ & $\begin{array}{l}\text { Vapor-temperature vs independent-variable- } \\
\text { form table. Input (independent-variable } \\
\text { form, vapor temperature) pairs }(*, K) \text {. }\end{array}$ \\
\hline ALPTB & $\mid$ NFTB $\mid * 2$ & $\begin{array}{l}\text { Coolant void-fraction vs independent- } \\
\text { variable-form table. Input (independent- } \\
\text { variable form, coolant void fraction).pairs } \\
(*,-) \text {. }\end{array}$ \\
\hline
\end{tabular}


Fill Component (FILL)

\begin{tabular}{|c|c|c|}
\hline Variabl & Dimension & Description \\
\hline PTB & $|\mathrm{NFTB}| * 2$ & $\begin{array}{l}\text { Pressure vs independent-variable-form table. } \\
\text { Input (Independent-variable form, pressure) } \\
\text { pairs }(*, \mathrm{~Pa}) \text {. }\end{array}$ \\
\hline PATB & $\mid$ NFTB $\mid * 2$ & $\begin{array}{l}\text { Air partial pressure vs independent- } \\
\text { variable-form table. Input (independent- } \\
\text { variable form, air partial pressure) pairs } \\
(*, \mathrm{~Pa}) \text {. }\end{array}$ \\
\hline Note: & $\begin{array}{l}\text { If } \text { ISOLUT }=0 \text { on Main-Data } \\
\text { omit card set CONCTB. }\end{array}$ & Card 6 or IFTY $\neq 6$ or 9 on Card Number 2 , \\
\hline CONCTB & $\mid$ NFTB $\mid * 2$ & $\begin{array}{l}\text { Ratio of solute mass to liquid-coolant mass } \\
\text { vs independent-variable-form table. Input } \\
\text { (ind?pendent-variable form, ratio of solute } \\
\text { mass to liquid-coolant mass) pairs }(*,-) \text {. }\end{array}$ \\
\hline RFTB & $|\mathrm{NFRF}| * 2$ & $\begin{array}{l}\text { Rate-factor table for the fill-table's inde- } \\
\text { pendent variable defined by IFSV and NFTB on } \\
\text { Card Number } 3 \text {. Input (Independent-varlable } \\
\text { form defined by NFSV and NFRF on Card } \\
\text { Number } 3 \text {, rate factor to be applied to the } \\
\text { fill-table Independent variable) pairs } \\
(*,-) \text {. }\end{array}$ \\
\hline
\end{tabular}


e. Pipe Component (PIPE).

Card Number 1. (Format A10,4X,2I14,A30) TYPE, NUM, ID, CTITLE

\begin{tabular}{|c|c|}
\hline Columns & Variable \\
\hline $1-4$ & TYPE \\
\hline $15-28$ & NUM \\
\hline $29-42$ & ID \\
\hline $43-72$ & CTITLE \\
\hline Card Nur & 2. (For \\
\hline Columns & Variable \\
\hline $1-14$ & NCELLS \\
\hline $15-28$ & NODES \\
\hline $29-42$ & JUN1 \\
\hline $43-56$ & JUN2 \\
\hline $57-70$ & MAT \\
\hline
\end{tabular}

\section{Description}

Component type (PIPE left-justified).

Component ID number (must be unique for each component, $1 \leq \mathrm{NUM} \leq 999$ ).

User ID number (arbitrary).

Hollerith component description.

NCELLS, NODES, JUN1, JUN2, MAT

\section{Description}

Number of fluid cells in the pipe component.

Number of radial heat-transfer nodes in the pipe wall. A value of 0 specifles no wall heat transfer.

Junction number for junction adjacent to cell 1 .

Junction number for junction adjacent to ce11 NCELLS.

Material ID number of the wall (currently not used).

Card Number 3. (Format 4I14) ICHF, ICONC, IACC, IPOW

\begin{tabular}{|c|c|c|}
\hline Columns & Variable & Description \\
\hline $1-14$ & ICHF & $\begin{array}{l}\text { CHF calculation flag. } \\
0=\text { no; } \\
1=\text { yes }\end{array}$ \\
\hline $15-28$ & ICONC & $\begin{array}{l}\text { Flag for input of solute in the coolant. } \\
\text { (Requires ISOLUT }=1 \text { on Main-Data Card } 6 . \text { ) } \\
0=\text { no input; } \\
1=\text { dissolved solute only; or } \\
2=\text { both dissolved and plated-out solute. }\end{array}$ \\
\hline
\end{tabular}


Columns Variable

29-42 IACC

43-56 IPOW

\section{Description}

Accumulator model flaz.

(See ACCUM module description, Sec. IV.B.)

$0=$ no accumulator logic;

1 = calculation of water level, volumetric flow, and liquid volume discharge, and addition of interface sharpener; or

2 = same as (1) plus the phase separator at JUN2 (gas phase is never allowed to flow out of component).

Presence of power deposited in the coolant flag.

$0=$ no;

1 = yes.

Note: If IPOW $=0$ (word 4 on Card Number 3), omit Card Number 4.

Card Number 4. (Format 5I14) IPOWTR, IPOWSV, NPOWTB, NPOWSV, NPOWRF

$\frac{\text { Columns }}{1-14} \quad \frac{\text { Variable }}{\text { IPOWTR }}$

15-28 IPOWSV

29-42 NPOWTB
Description

The trip ID number that controls evaluation of the power-to-fluid table (POWTB) [ $\mid$ IPOWTR $\mid<10000$ ] (input IPOWTR $=0$ if there is to be no trip control, and the table will be evaluated in the transient).

The independent-variable ID number for the power-to-fluid table (POWTB). IPOWSV $>0$ defines the ID number for a signal-variable parameter; IPOWSV $<0$ defines the ID number for a control-block output parameter.

The number of $(x, y)$ power-to-fluid table (POWTB) pairs (defined by the absolute value of NPOWTB). NPOWTB $>0$ defines the table independent-variable form to be the IPOWSV parameter; NPOWTB $<0$ defines the table independent-variable form to be the change in the IPOWSV parameter over the last time step times the trip set-status value ISET when the power-to-fluid table is trip controlled; NPOWTB $=0$ defines the power to be the IPOWSV parameter.

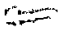




\begin{tabular}{|c|c|c|c|}
\hline Columns & Variable & & Description \\
\hline $43-56$ & NPOWSV & & $\begin{array}{l}\text { The independent-variable ID number for the } \\
\text { rate factor that is applied to the power-to- } \\
\text { fluid table (POWTB) independent variable. } \\
\text { NPOWSV }>0 \text { defines the ID number for a } \\
\text { signal-variable parameter; NPOWS }<0 \\
\text { defines the ID number for a control-block } \\
\text { output } \text { parameter; NPOWS }=0 \text { (when } \\
\text { NPOWRF } \neq 0 \text { ) defines the independent variable } \\
\text { to be the difference between the trip signal } \\
\text { and the set-point value that turns the trip } \\
\text { OFF when the power table is trip controlled. }\end{array}$ \\
\hline $57-70$ & NPOWRF & , & $\begin{array}{l}\text { The number of rate-factor table ( } x, y \text { ) pairs } \\
\text { (defined by the absolute value of NPOWRF). } \\
\text { The rate factor is applied to the power-to- } \\
\text { fluid table's (POWTB) independent variable } \\
\text { when the rate factor is defined. No rate } \\
\text { factor is defined when NPOWS and NPOWR are } \\
\text { both zero. NPOWRF }>0 \text { defines the rate- } \\
\text { factor table's independent variable to be } \\
\text { the NPOWSV parameter; NPOWRF < } 0 \text { defines it } \\
\text { to be the change in the NPOWSV parameter } \\
\text { over the last time step times the trip set- } \\
\text { status value ISET when the power table is } \\
\text { trip controlled; NPOWRF = } 0 \text { defines the rate } \\
\text { factor to be the NPOWSV parameter. }\end{array}$ \\
\hline
\end{tabular}

Note: If NODES $<1$ (word 2 on Card Number 2), omit Card Number 5.

Card Number 5. (Format 5I14) IQP3TR, IQP3SV, NQP3TB, NQP3SV, NQP3RF

$\begin{array}{lll}\text { Columns Variable } & \text { Description } \\ \text { 1-14 } & \text { IQP3TR } & \end{array}$


$\frac{\text { Columns }}{29-42} \quad \frac{\text { Variable }}{\text { NQP3TB }}$

43-56 NQP3SV

57-70 NQP 3RF

Card Number 6. (Format 5E14.6)

Columns Variable

1-14 RADIN

15-28 TH $\underline{\text { Description }}$

The number of $(x, y)$ power-to-wall table (POWTB) pairs (defined by the absolute value of NQP3TB). NQP3TB $>0$ defines the table's independent-variable form to be the IQP3SV parameter; NQP3TB < 0 defines the table's independent-variable form to be the change in the IQP3SV parameter over the last time step times the trip set-status value ISET when the power-to-wall table is trip controlled; NQP3TB $=0$ defines the power to the wall to be the IQP3SV parameter.

The indepeindent-variable ID number for the rate factor that is applied to the power-towall table's independent variable. NQP3SV $>0$ defines the ID number for a signal-variable parameter; NQP3SV < 0 defines the ID number for a control-block output parameter; $\quad$ NQP3SV $=0$ (when NQP3RF $\neq 0$ ) defines the independent variable to be the difference between the trip signal and the set-point value that turns the trip OFF when the power-to-wall table is trip controlled.

The number of $(x, y)$ rate-factor table pairs (defined by the absolute value of NQP3RF). The rate factor is applied to the power-towall (QP3TB) table's independent variable when the rate factor is defined. No rate factor is defined when NQP3SV and NQP3RF are both zero. NQP3RF>0 defines the ratefactor independent variable to be the NQP3SV parameter; NQP3RF < 0 defines it to be the change in the NQP3SV parameter over the last time step times the trip set-status value ISET when the power-to-wall table is trip controlled; NQP3RF $=0$ defines the rate factor to be the NQP3SV parameter.

RADIN, TH, HOUTL, HOUTV, TOUTL

Description

Inner radius ( $m$ ) of the pipe wall.

Pipe wall thickness (n). 


\begin{abstract}
Columns Variable
29-42 HOUTL

13-56 HOUTV

57-70 TOUTL

\section{Description}

HTC $\left(W \cdot \mathrm{m}^{-2} \cdot \mathrm{K}^{-1}\right)$ between outer boundary of pipe wall and liquid outside the pipe wall.

HTC ( $W \cdot \mathrm{m}^{-2} \cdot \mathrm{K}^{-1}$ ) between outer boundary of pipe wall and vapor outside the pipe wall.

Liquid temperature (K) outside the pipe wall.

Note: The four parameters, HOUTL, HOUTV, TOUTL, and TOUTV, allow flexibility in calculating possible heat losses from the outside of pipes. Typically, such heat losses are not important for fast transients or large-break LOCAs, and HOUTL and HOUTV can be set equal to zero. When heat losses are significant, they often can be approximated by a single HTC and a single external temperature.
\end{abstract}

Card Number 7. (Format 5E14.6) TOUTV, POWIN, POWOFF, RPOWMX, POWSCL

$\begin{array}{cll}\text { Columns Variable } & \text { Description } \\ \text { 1-14 } & \text { TOUTV } & \text { Vapor temperature (K) outside the pipe wall. } \\ 15-28 & \text { POWIN } & \text { Initial total power (W) deposited in the } \\ & \text { Eluid (not input when IPOW }=0 \text { ). The power } \\ & \text { is distributed linearly along the component. }\end{array}$


Note: If NODES $<1$ (word 2 on Card Number 2), omit Card Number 8 .

Card Number 8. (Format 4E14.6) QP3IN, QP3OFF, RQP3MX, QP3SCL

Columns $\quad \frac{\text { Variable }}{1-14}$

15-28 QP30FF

29-42 RQP3MX

43-56 QP3SCL

\section{Description}

Initial power ( $W$ ) deposited in the wall and distributed according to the QPPP array. If QP3IN > 0.0, it is the total power to the entire wall. When QP3IN $<0.0$ is used, the initial power to the wall in each cell is $|Q P 3 I N|$, anel the negative sign flags the power to the wall to be a cell-dependent vector of NCELLS (word 1 on Card Number 2) powers. Each $(x, y)$ point of the power-towall table (for QP3IN $<0.0$ ) has $1+$ NCELLS values [an independent-variable value $x$ and NCELLS power values $y(z)$ for cells 1 through NCELLS]. When the power-to-wall table is not being evaluated, the same power value of $|\mathrm{QP} 3 \mathrm{IN}|$ or QP3OFF (if QP3OFF $>-1.0 \times 10^{19}$ ) is applied at each of the NCELLS cells.

Power (W) to' the wall when the controlling trip is OFF after being ON (maintain the last tabie-evaluated power when the trip was $\mathrm{ON}$ if $\mathrm{QP} 3 \mathrm{OFF} \leq-1.0 \times 10^{19}$ ).

Maximum rate of change of the power to the wa11 ( $\left.W \cdot s^{-1}\right)$ [RQP3MX 20.0$]$.

Scale factor (-) for the power-to-wall table. The dependent variable in table QP3TB is multiplied by QP3SCL to obtain the absolute power to the wall.

PIPE Array Cards. Twenty-four sets of cards, one set. for each of the following variables. Use LOAD format.

$\begin{array}{lll}\text { Variable } & \text { Dimension } & \text { Description } \\ \text { DX } & \text { NCELLS } & \text { Cell lengths }(\mathrm{m}) . \\ \text { VOL } & \text { NCELLS } & \text { Cell volumes }\left(\mathrm{m}^{3}\right) . \\ \text { FA } & \text { NCELLS+1 } & \text { Cell-edge flow areas }\left(\mathrm{m}^{2}\right) .\end{array}$




\begin{tabular}{|c|c|c|}
\hline Variabl & Dimension & Description \\
\hline FRIC & NCELLS+1 & $\begin{array}{l}\text { Additive loss coefficients. (See ACCUM } \\
\text { input description, Sec.V.C.5.a.) }\end{array}$ \\
\hline GRAV & NCELLS+1 & $\begin{array}{l}\text { Gravity terms. (See } \\
\text { description, Sec. V.C.5.a.) }\end{array}$ \\
\hline HD & NCELLS+1 & $\begin{array}{l}\text { Hydraulic diameters ( } m \text { ). (See variable } \\
\text { NDIAl in the NAMELIST data for optional } \\
\text { heat-transfer diameters.) }\end{array}$ \\
\hline Note: & $\begin{array}{l}\text { If ICFLOW }=0 \text { or ICFLOW } \\
\text { input array ICFLG. }\end{array}$ & $=1$ (default) in the NAMELIST data, do not \\
\hline ICELG & NCELLS+1 & $\begin{array}{l}\text { Ce11-edge choked-flow model options. (See } \\
\text { ACCUM input description, Sec. V.C.5.a.) }\end{array}$ \\
\hline $\mathrm{NFF}$ & NCELLS+1 & $\begin{array}{l}\text { Friction-factor correlation options. (See } \\
\text { ACCUM input description, Sec. V.C.5.a.) }\end{array}$ \\
\hline ALP & NCELLS & Initial void fractions $(-)$. \\
\hline VL & NCELLS+1 & Initial liquid velocities $\left(\mathrm{m} \cdot \mathrm{s}^{-1}\right)$ \\
\hline VV & NCELLS+1 & Initial vapor velocities $\left(\mathrm{m} \cdot \mathrm{s}^{-1}\right)$. \\
\hline TL & NCELLS & Initial liquid temperatures $(K)$. \\
\hline TV & NCELLS & Initial vapor teinperatures $(K)$. \\
\hline $\mathrm{P}$ & NCELLS & Initial pressures $(\mathrm{Pa})$. \\
\hline $\mathrm{PA}$ & NCELLS & Initial air partial pressures $(\mathrm{Pa})$. \\
\hline
\end{tabular}


Note: If NODES $=0$ (word 2 on Card Number 2), do not enter arrays QPPP, MATID, and TW.

\begin{tabular}{|c|c|c|}
\hline Variable & Dimension & Description \\
\hline QPPP & NODES*NCELLS & $\begin{array}{l}\text { A nonnormalized power profile }(-) \text { in the } \\
\text { pipe walls. Input for cell } 1 \text {, node } 1 \\
\text { through NODES; then for cell } 2 \text {, node } 1 \\
\text { through NODES; etc. If the array is filled } \\
\text { with the same nonzero constant, a uniform } \\
\text { volunetric heat source for the wall results. } \\
\text { The code will normalize the power profile on } \\
\text { a node-volume basis (normalized QPPP(i) } \\
=\text { QPPP(i)/[ }[\text { QPPP( } i) * V O L(i)]) \text { Filling the } \\
\text { array with i zeros results in no power being } \\
\text { deposited in the walls regardless of the } \\
\text { settings of QP3IN, QP3TB, etc. }\end{array}$ \\
\hline MATID & NODES-1 & $\begin{array}{l}\text { Wall-material ID array specifying material } \\
\text { ID between nodes. Dimension is } 1 \text { if NODES } \\
=1 \text { and } 0 \text { if NODES }=0 \text {. (See available } \\
\text { built-in properties listed with array MATID } \\
\text { In the CORE input, Sec. V.C.5.c; or see } \\
\text { Sec. V.C.2 to input material properties.) }\end{array}$ \\
\hline TW & NODES*NCELLS & $\begin{array}{l}\text { Initial wall temperatures }(K) \text {. Input in the } \\
\text { same order as QPPP. }\end{array}$ \\
\hline
\end{tabular}

Note: If ICONC $=0$ (word 2 on Card Number 3 ), omit card set CONC.

CONC NCELLS Initial ratio of solute mass to 1iquid. coolant mass $(-)$.

Note: If ICONC $=0$ or 1 (word 2 on Card Number 3 ), omit card set $S$.

S NCELLS

Initial macrosçopic density of plated-out solute $\left(\mathrm{kg} \cdot \mathrm{m}^{-3}\right)$.

Note: If NPOWTB $=0$ on Card Number 4, omit card set POWTB.

POWTB $\quad \mid$ NPOWTB $\mid * 2$

Power-to-fluid vs independent-variable-form table. Input (independent-variable form defined by IPOWSV and NPOWTB on Card Number 4 , power) pairs $(*, W)$. The power is deposited directly into the fluid in the pipe with a uniform volumetric power density along the pipe length. 
Note: If NPOWRF or NPOWTB $=0$ on Card Number 4, omit card set POWRF.

Variable Dimension

POWRF $\quad \mid$ NPOWRF $\mid * 2$

\section{Description}

Rate-factor table for the POWTB table independent variable. Input (independentvariable form defined by NPOWSV and NPOWRF on Card Number 4, rate factor to be applied to the PCWTB table independent variable) pairs $(*,-)$.

Note: If NQP3TB $=0$ on Card Number 5, omft card set QP3TB.

QP3TB $\quad \mid$ NQP3TB $\mid * 2$

when $Q P 3 I N>0$;

$\mid$ NQP3TB $\mid *(1+$ NCELLS $)$

when QP3IN $<0$

\begin{abstract}
Power-to-wall vs independent-variable-form table. Input (independent-variable form defined by IQP3SV and NQP3TB on Card Number 5, power-to-wall) pairs $(*, W)$. If QP3IN >0.0, the dependent variable specifies the total power to the entire wal1; if QP3IN < 0.0 , the dependent variable is a vector specifying the power to the wall at each cell beginning with cell 1 .
\end{abstract}

Note: If $N Q P 3 T B$ or NQP3RF $=0$ on Card Number 5, omit card set QP3RF.

QP3RF $\quad|\mathrm{NQP3RF}| * 2$
Rate-factor table for the QP3TB table independent variable. Input (independentvariable form defined by NQP3SV and NQP3RF on Card Number 5, rate factor to be applied to the QP3TB table independent variable) pairs $(*,-)$. 
E. Plenum Component (PLENUM).

Card Number 1. (Format A6,8X,2I14,A30) TYPE, NUM, ID, CTrTLE

$\begin{array}{lll}\begin{array}{ll}\text { Columns } \\ 1-6\end{array} & \text { Variable } & \text { Description } \\ 15-28 & \text { TYPE } & \text { Component type (PLENUM left-justified). } \\ 29-42 & \text { ID } & \begin{array}{l}\text { Component ID number (must be urique for each } \\ \text { component, } 1 \leq \text { NUM } \leq 999) .\end{array} \\ 43-72 & \text { CTITLE } & \text { User ID number (arbitrary). }\end{array}$

Card Number 2. (Format 2114) NPLJN, ICONC

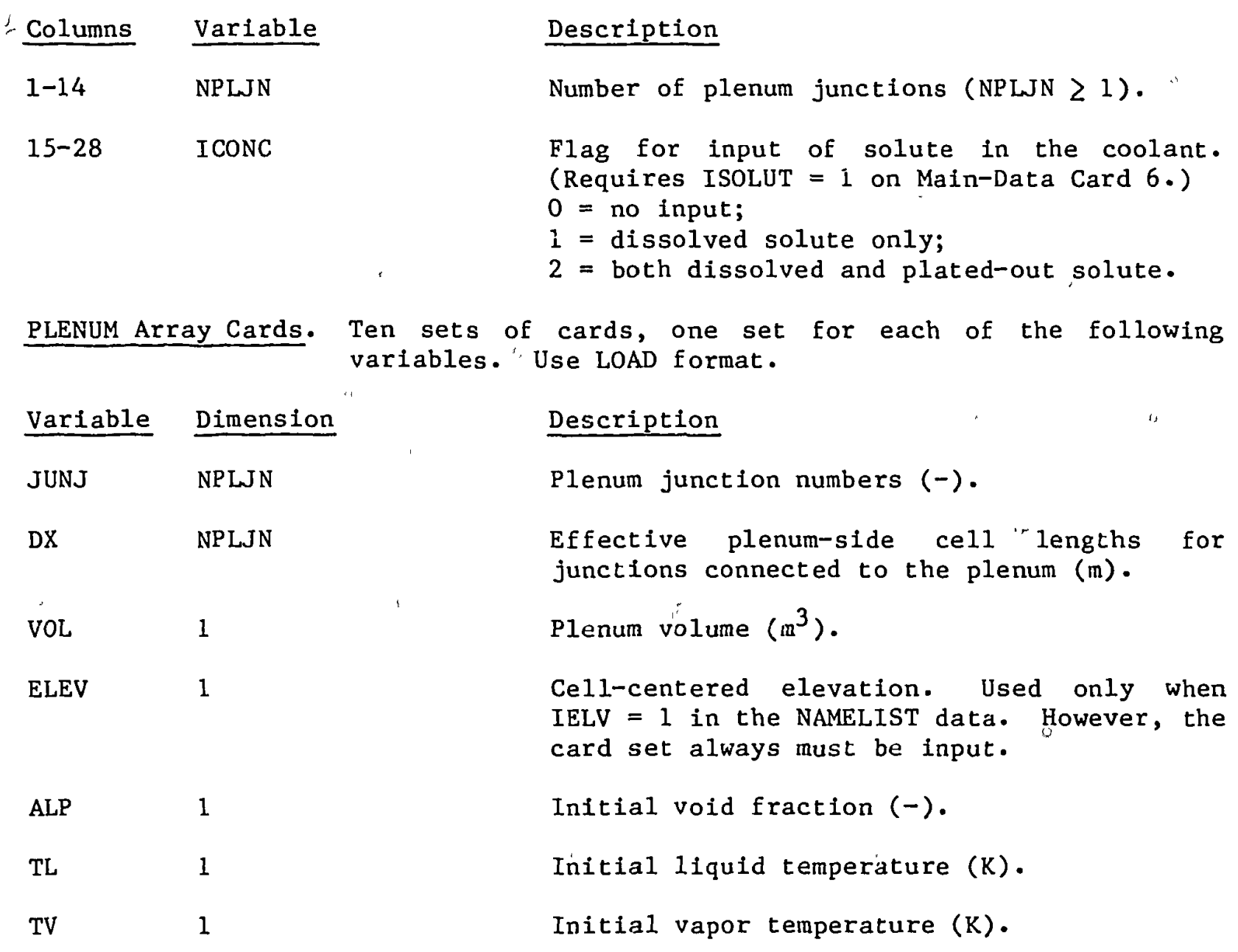


Plenum Component (PLENUM)

Variable Dimension

P $\quad 1$

PA 1

\section{Description}

Initial pressure $(\mathrm{Pa})$.

Initial air partial pressure ( $P$ a).

Note: If ICONC $=0$ (word 2 on Card Number 2), omit card set BOR.

BOR

1

Initial ratio of solute mass to liquid coolant mass $(-)$.

Note: If ICONC $=0$ or 1 (word 2 on Card Number 2), omit card set SOLID.

SOLID $\quad 1$

Initial macrosçopic density of plated-out solute (ikg $\cdot \mathrm{m}^{-3}$ ). 
g. Pressurizer Component (PRIZER).

Card Number 1. (Format AlO,4X,2I14,A30) TYPE, NUM, ID, CTITLE

\begin{tabular}{|c|c|c|}
\hline Columns & Variable & Description \\
\hline $1-6$ & TYPE & Component type (PRIZER left-justified). \\
\hline $15-28$ & NUM & $\begin{array}{l}\text { Component ID number (must be unique for each } \\
\text { component, } 1 \leq \text { NUM } \leq 999 \text { ). }\end{array}$ \\
\hline $29-42$ & ID & User ID number (arbitrary). \\
\hline $43-72$ & CTITLE & Hollerith component description. \\
\hline \multicolumn{2}{|c|}{ Card Number 2.} & NCELLS, NODES, JUN1, JUN2, MAT \\
\hline Columns & Variable & Description \\
\hline $1-14$ & NCELLS & $\begin{array}{l}\text { Number of fluid cells in the pressurizer } \\
\text { component. }\end{array}$ \\
\hline $15-28$ & NODES & $\begin{array}{l}\text { Number of radial heat-transfer nodes in the } \\
\text { pressurizer wall. A value of } 0 \text { specifies no } \\
\text { wall heat transfer. }\end{array}$ \\
\hline $29-42$ & JUN1 & $\begin{array}{l}\text { Junction number for junction adjacent to } \\
\text { cell 1. }\end{array}$ \\
\hline $43-56$ & JUN2 & $\begin{array}{l}\text { Junction number for junction adjacent to } \\
\text { cel1 NCELLS. This must be the pressurizer } \\
\text { discharge. }\end{array}$ \\
\hline $57-70$ & MAT & $\begin{array}{l}\text { Material ID number of the wall (not used at } \\
\text { present). }\end{array}$ \\
\hline
\end{tabular}

Card Number 3. (Format 2I14,E14.6) ICHF, ICONC, QP3IN

\begin{tabular}{lll} 
Columns & Variable & Description \\
\cline { 2 - 2 } $1-14$ & ICHF & CHF calculation flag. \\
& $0=$ no; \\
& $1=$ yes.
\end{tabular}




$\frac{\text { Columns }}{15-28} \quad \frac{\text { Variable }}{\text { ICONC }}$

29-42 QP3IN

Card Number 4. (Format 5E14.6) RADIN, TH, HOUTL, HOUTV, TOUTL

$\begin{array}{ll}\text { Columns } & \text { Variable } \\ 1-14 & \text { RADIN } \\ 15-28 & \text { TH } \\ 29-42 & \text { HOUTL } \\ 43-56 & \text { HOUTV } \\ & \\ 57-70 & \text { TOUTL }\end{array}$

Description

Flag for input of solute in the liquid coolant. [Requires ISOLUT $=1$ (word 5 on Main-Data Card 6).]

$0=$ no input;

1 = dissolved solute only; or

2 = both dissolved and plated-out solute.

Initial power ( $W$ ) deposited in the wall and distributed according to the QPPP array. If QP3IN > 0.0, it is the total power to the entire wall. When QP3IN $<0.0$ is used, the initial power to the wall in each cell is $\mid$ QP3IN|, and the negative sign flags the power to the wall to be a cell-dependent vector of NCELLS (word 1 on Card Number 2) powers. Each $(x, y)$ point of the power-towa11 table (for QP3IN $<0.0$ ) has 1+NCELLS values [an independent-variable value $x$ and NCELLS power values $\mathrm{y}(\mathrm{z})$ for cells 1 through NCELLS], When the power-to-wall table is not being evaluated, the same power value of |QP3IN] or QP3OFF (if QP3OFF >-1.0 $10^{19}$ ) is applied at each of the NCELLS cells.

\section{Description}

Inner radius (m) of the pressurizer wall.

Pressurizer will? thickness (m).

HTC $\left(W \cdot \mathrm{m}^{-2} \cdot \mathrm{K}^{-1}\right)$ between outer boundary of the pressurizer wall and liquid outside the pressurizer wall.

HTC $\left(W \cdot \mathrm{m}^{2} \cdot \mathrm{K}^{-1}\right)$ between outer boundary of the pressurizer wall and, vapor outside the pressurizer wall.

Liquid temperature (K) outside the pressurizer.

Note: See PIPE input description, Sec.V.C.5.e, for further comments on the HOUTL, HOUTV, TOUTL, and TOUTV heat-transfer parameters. 
Card Nunber 5. (Format 5E14.6) TOUTV, QHEAT, PSET, DPMAX, ZHTR

\begin{tabular}{|c|c|c|c|}
\hline Columns & Variable & & Description \\
\hline $1-14$ & TOUTV & & $\begin{array}{l}\text { Vapor temperature }(K) \text { outside } \\
\text { pressurizer. }\end{array}$ \\
\hline $15-28$ & QHEAT & & Total heater power $(W)$. \\
\hline $29-42$ & PSET & & $\begin{array}{l}\text { Pressure set point ( } \mathrm{Pa} \text { ) for heater/sprayer } \\
\text { control. }\end{array}$ \\
\hline $43-56$ & DPMAX & & $\begin{array}{l}\text { Pressure differential ( } \mathrm{Pa}) \text { at which } \\
\text { heater/sprayer has maximum power. }\end{array}$ \\
\hline $57-70$ & ZHTR & & Water level (m) for heater cutoff. \\
\hline PRIZER Arra & ay Cards. & $\begin{array}{l}\text { Twenty sets } \\
\text { variables. }\end{array}$ & $\begin{array}{l}\text { of cards, one set for each of the following } \\
\text { Use LOAD format. }\end{array}$ \\
\hline Variable & Dimension & & Description \\
\hline $\mathrm{DX}$ & NCELLS & & Cell lengths (m). \\
\hline VOL & NCELLS & & Cell volumes $\left(\mathrm{m}^{3}\right)$. \\
\hline FA & NCELLS+1 & & Ce11-edge flow areas $\left(\mathrm{m}^{2}\right)$. \\
\hline FRIC & NCELLS+1 & & $\begin{array}{l}\text { Additive loss coefficients }(-) \text {. (See ACCUM } \\
\text { input description, Sec. V.C.5.a.) }\end{array}$ \\
\hline GRAV & NCELLS+1 & & $\begin{array}{l}\text { Gravity terms (-). (See ACCUM } \\
\text { description, Sec. V.C.5.a.) }\end{array}$ \\
\hline $\mathrm{HD}$ & NCELLS+1 & & $\begin{array}{l}\text { Hydraulic diameters (m). (See variable } \\
\text { NDIA1 in the NAMELIST data for optional } \\
\text { heat-transfer diameters.) }\end{array}$ \\
\hline
\end{tabular}

Note: If ICFLOW $=0$ or ICFLOW $=1$ (default) in the NAMELIST data, do not input array ICFLG.

ICFLG NCELLS+1

Ce11-edge choked-flow model options. (See ACCUM input description, Sec. V.C.5.a.) 


$\begin{array}{ll}\text { Variable } & \text { Dimension } \\ \text { NFF } & \text { NCELLS+1 } \\ \text { ALP } & \text { NCELLS } \\ \text { VL } & \text { NCELLS+1 } \\ \text { VV } & \text { NCELLS+1 } \\ \text { TL } & \text { NCELLS } \\ \text { TV } & \text { NCELLS } \\ \text { P } & \text { NCELLS } \\ \text { PA } & \text { NCELLS }\end{array}$

Note: If NODES $=0$ (word 2 on Card Number 2), do not input arrays QPPP, MATID, and TW.

QPPP NODES*NCELLS

MATID NODES-1

\section{Description}

Friction-factor correlation options. (See ACCUM input description, Sec. V.C.5.a.) The value $\mathrm{NFF}=1$ is suggested for this component.

Initial void fractions (-).

Initial liquid velocities $\left(\mathrm{m} \cdot \mathrm{s}^{-1}\right)$.

Initial vapor velocities $\left(m \cdot s^{-1}\right)$.

Initial liquid temperatures $(K)$.

Initial vapor temperatures (K).

Initial pressures $(\mathrm{Pa})$.

Initial air partial pressures ( $\mathrm{Pa}$ ).
A nonnormalized power profile (-) in the pressurizer walls. Input for cell 1, node 1 through NODES; then for cell 2, node 1 through NODES; etc. If the array is filled with the same nonzero constant, a uniform volunetric heat source for the wall results. The code will normalize the power profile on a node-volume basis (normalized QPPP( 1 ) $\left.=\mathrm{QPPP}(i) /\left[\sum \mathrm{QPPP}(i) * \operatorname{VOL}(i)\right]\right)$. Filling the array with $i$ zeros results in no power being deposited in the walls regardless of the settings of QP3IN, QP3TB, etc.

Wal1-material ID array specifying material ID between nodes. Dimension is 1 if NODES $=1$ and 0 if NODES $=0$. (See available built-in properties listed with array MATID in the CORE input, Sec.V.C.5.c; or see Sec. V.C.2 to input material properties.)

Initial wall temperatures (K) (input in the same order as QPPP). 
Pressurizer Component (PRIZER)

Note: If ICONC $=0$ (word 2 on Card Number 3 ), omit card set CONC.

Variable Dimension Description

CONC NCELLS Initlal ratio of solute mass to liquid coolant mass $(-)$.

Note: If ICONC $=0$ or 1 (word 2 on Card Number 3 ), omit card set $S$.

S NCELLS

Initial macroscopic density of plated-out solute $\left(\mathrm{kg} \mathrm{m}^{-3}\right)$. 
h. Pump Component (PUMP).

Card Number 1. (Format A10,4X,2I14,A30) TYPE, NUM, ID, CTITLE

Columns Variable Description

1-4 TYPE

15-28 NUM

29-42 ID

$43-72$

CTITLE
Component type (PUMP left-justified).

Component ID number (must be unique for each component, $1 \leq$ NUM $\leq 999$ ).

User ID number (arbitrary).

Hollerith component description.

Card Number 2. (Format 5I14) NCELLS, NODES, JUN1, JUN2, MAT

$\begin{array}{ll}\begin{array}{ll}\text { Columns } \\ 1-14\end{array} & \begin{array}{l}\text { Variable } \\ \text { NCELLS }\end{array} \\ 15-28 & \text { NODES } \\ 29-42 & \text { JUN1 } \\ 43-56 & \text { JUN2 } \\ 57-70 & \text { MAT }\end{array}$

Description

Number of fluid cells in pump component (must be at least two).

Number of radial heat-transfer nodes in the wall. A value of 0 specifies no wall heat transfer.

Junction number for junction adjacent to cell 1 .

Junction number for junction adjacent to Ce11 NCELLS.

Material ID number of the wall (currently not used).

Card Number 3. (Format 5I14) ICHF, ICONC, IPMPTY, IRP, IPM

\begin{tabular}{|c|c|c|c|c|}
\hline Columns & Variable & Description & & \\
\hline $1-14$ & ICHF & $\begin{array}{l}\text { CHF calculation flag. } \\
0=\text { no; } \\
1=\text { yes. }\end{array}$ & & \\
\hline $15-28$ & ICONC & $\begin{array}{l}\text { Flag for input of } \\
\text { coolant. [Requires } \\
\text { Main-Data Card 6).] } \\
0=\text { no input; } \\
1=\text { dissolved solute } \\
2=\text { both dissolved an }\end{array}$ & $\begin{array}{l}\text { solute in } \\
\text { ISOLUT }=1 \\
\text { only; or } \\
\text { d plated-ou }\end{array}$ & $\begin{array}{l}\text { the liquid } \\
\text { (word } 5 \text { on }\end{array}$ \\
\hline
\end{tabular}




$\begin{array}{ll}\frac{\text { Columns }}{29-42} & \text { Variable } \\ \text { IPMPTY } \\ 43-56 & \text { IRP } \\ 57-70 & \text { IPM }\end{array}$

Card Number 4. (Format 5I14) IPMPTR, IPMPSV, NPMPTB, NPMPSV, NPMPRF
Description

The trip ID number that controls the pump speed (zero implies a constant pump speed) [|IPMPTR < 10000]. If the trip set status is OFF initially, the pump speed is defined by a signal variable or control block with ID number NPMPSD (word 5 on Card Number 9) or by a constant initial pump speed ONiEGAN (word 1 on Card Number 9 ) when NPMPSD $=0$.

The pump-table abscissa-coordinate variable ID number. IPMPSV defines the independentvariable parameter in the IPMPTY $=1$ pumpspeed table. IPMPSV $>0$ defines the ID number for a signal-variable parameter; IPMPSV $<0$ defines the ID number for a contro1block output parameter. ( (If IPMPTY $=2$ on Card Number 3, IPMPSV must be zero.)

The number of $(x, y)$ pump-speed table pairs (defined by the absolute value of NPMPTB). NPMPTB $>0$ defines the table's independentvariable form to be the IPMPSV parameter; NPMPTB $<0$ defines the table's independentvariable form to be the change in the IPMPSV parameter over the last time step times the trip set-status value ISET when the pumpspeed table is trip controlled; NPMPTB $=0$ defines the pump rotational speed to be the IPMPSV paraneter (if IPMPTY $=2$ on Card Number 3, NPMPTB must be zero). 


$\frac{\text { Columns }}{43-56} \quad$ Variable

57-70 NPMPRF
Description

The rate-factor table abscissa-coordinate variable ID number. NPMPSV defines the independent-variable parameter for the rate factor that is applied to the pump-speed Lable's independent variable. NPMPSV $>0$ defines the ID number for a signal-variable parameter; NPMPSV < 0 defines the ID number for a control-block output parameter; NPMPSV $=0$ (when NPMPRF 0 ) defines the difference between the trip signal and the set-point value that turns the trip OFF (if IPMPTY $=2$ on Card Number 3 , NPMPSV must be zero).

The number of $(x, y)$ rate-factor table pairs (defined by the absolute value of NPMPRF). The rate factor is applied to the pump-speed table's independent variable when the rate factor is defined. No rate factor is defined when NPMPSV and NPMPRF are both zero. NPMPRF $>0$ defines the rate-factor table's abscissa coordinate to be the NPMPSV parameter; NPMPRF < 0 defines it to be the change in the NPMPSV parameter over the last time step times the trip set-status value ISET when the pump-speed table is trip controlled; $N P M P R F=0$ defines the rate factor to be the NPMPSV parameter(if IPMPTY $=2$ on Card Number 3 , NPMPRF must be zero.)

Note: If NODES $<1$ (word 2 on Card Number 2), omit Card Number 5 .

Card Number 5. (Format 5I14) IQP3TR, IQP3SV, NQP3TB, NQP3SV, NQP3RF

Columns Variable

1-14 IQF3TR

\section{Description}

The trip ID number that controls evaluation of the power-to-wall table (QP3TB) $[\mid$ IQP3TR $\mid$ 10000]. (Input IQP3TR $=0$ if there is to be no trip control, and the table will be evaluated in the transient.) 
Columns

$15-28$

IQP3SV

$29-42$

NQP 3TB

43-56 NQP3SV

57-70 NQP3RF
Description

The independent-variable ID number for the power-to-wall table (QP3TB). IQP3SV >0 defines the ID number for a signal-variable parameter; IQP3SV < 0 defines the ID number for a control-block output parameter.

The number of $(x, y)$ power-to-wall table (POWTB) pairs (defined by the absolute value of NQP3TB). NQP3TB > 0 defines the table's independent-variable form to be the IQP3SV parameter; NQP3TB $<0$ defines the table-s independent-variable form to be the change in the IQP3SV parameter over the last time step times the trip set-status value ISET when the power-to-wall table is trip controlled; NQP3TB $=0$ defines the power to the wall to be the IQP3SV parameter.

The independent-variable ID number for the rate factor that is applied to the power-towall table's independent variable. NQP3SV $>0$ defines the ID number for a signal-variable paraneter; NQP3SV $<0$ defines the ID number for a control-block output parameter; $\quad$ NQP3SV $=0$ (when NQP3RF $\neq 0$ ) defines the independent variable to be the difference between the trip signal and the set-point value that turns the trip OFF when the power-to-wall table is trip cont rolled.

The number $(x, y)$ of rate-factor table pairs (defined by the absolute value of NQP3RF). The rate factor is applied as a factor to the power-to-wall table (QP3TB) independent variable when the rate factor is defined. No rate factor is defined when NQP3SV and NQP3RF are both zero. NQP3RF >0 defines the rate-factor table's independent variable to be the NQP3SV parameter; NQP3RF < 0 defines it to be the change in the NQP3SV parameter over the last time step times the trip set-status value ISET when the powerto-wall table is trip controlled; NQP3RF = 0 defines the rate factor to be the NQP3SV paraneter. 
Card Number 6. (Format 5E14.6) RADIN, TH, HOUTL, HOUTV, TOUTL

\begin{tabular}{|c|c|c|}
\hline Columns & Variable & Description \\
\hline $1-14$ & RADIN & Inner radius $(m)$ of the pump wall. \\
\hline $15-28$ & $\mathrm{TH}$ & Pump wall thickness (m). \\
\hline $29-42$ & HOUTL & $\begin{array}{l}\text { HTC }\left(\mathrm{W} \cdot \mathrm{m}^{-2} \cdot \mathrm{K}^{-1}\right) \text { between outer boundary } \\
\text { of the pump wall and liquid outside the pump } \\
\text { wall. }\end{array}$ \\
\hline $43-56$ & HOUTV & $\begin{array}{l}\text { HTC }\left(\mathrm{W} \cdot \mathrm{m}^{-2} \cdot \mathrm{K}^{-1}\right) \text { between outer boundary } \\
\text { of the pump wall and vapor outside the pump } \\
\text { wall. }\end{array}$ \\
\hline $57-70$ & TOUTL & $\begin{array}{l}\text { Liquid temperature }(K) \text { outside the pump } \\
\text { wall. }\end{array}$ \\
\hline
\end{tabular}

Note: See PIPE input description, Sec. V.C.5.e, for further comments on the HOUTL, HOUTV, TOUTL, and TOUTV heat-transfer parameters.

Card Number 7. (Format 4E14.6) TOUTV, EFFMI, TFR1, TFR2

\begin{tabular}{|c|c|}
\hline Columns & Description \\
\hline $1-14$ & Vapor temperature $(K)$ outside the pump wall. \\
\hline $15-28$ & Effective moment of inertia $\left(\mathrm{kg} \cdot \mathrm{m}^{2}\right)$. \\
\hline $29-42$ & $\begin{array}{l}\text { Frictional torque coefficient [Eq. (220)] } \\
(\mathrm{N} \cdot \mathrm{m}) .\end{array}$ \\
\hline $43-56$ & $\begin{array}{l}\text { Bearing and windage torque coefficient } \\
{[\mathrm{Eq} \cdot(221)](\mathrm{N} \cdot \mathrm{m}) .}\end{array}$ \\
\hline Note: & $\begin{array}{l}\text { Update TKINERTIA in version } 11.3 \text { adds a variable inertia calculation to } \\
\text { the PUMP component. The variable inertia calculation is used only if } \\
\text { EFFMI (Card Number } 7 \text { ) is negative. Then, the pump inertia is } \\
\text { calculated as follows. }\end{array}$ \\
\hline & $\begin{array}{l}\text { inertia }=\text { EFFMI } 1 \\
\text { if (IPMPS.eq.0.and.omega.1t.OMTEST) IPMPS }=1 \\
\text { if (IPMPS.eq.0) } \\
1 \text { inertia }=\text { AEFFMI*(omega/romega }) * * 2+\text { BEFFMI*(omega/romega) }\end{array}$ \\
\hline
\end{tabular}


The parameters in capitals are new input parameters. Parameter omega is the current pump speed, and parameter romega is the rated pump speed. The parameter inertia is the moment of inertia used to calculate the pump speed following the trip. The six new parameters (EFFMII, IPMPS, OMTEST, AEFFMI, BEFFMI, and CEFFMI) are defined below in the changes to the input description. Update TKINERTIA modifies common block PUMPVLTAB and subroutines RPUMP, REPUMP, and PUMPSR. These changes are transparent to the user if the variable pump inertia is not used. The changes to the PUMP component input description follow; these new cards are input after existing Card Number 7 only if EFFMI $<0.0$.

Card Number 7+1. (Format 5E14.6) EFFMI1, AEFFMI, BEFFMI, CEFFMI, OMTEST (Input this card only if variable EFFMI on Card Number 7 is negative.)

\begin{tabular}{|c|c|c|}
\hline Columns & Variable & Description \\
\hline $1-14$ & EFFMI1 & $\begin{array}{l}\text { The alternate effective moment of inertia } \\
\text { that is used after the pump speed falls } \\
\text { below OMTEST }\left(\mathrm{kg} \cdot \mathrm{m}^{2}\right) \text {. }\end{array}$ \\
\hline $15-28$ & AEFFMI & $\begin{array}{l}\text { The coefficient for the (OMEGA/ROMEGA)**2 } \\
\text { term j.n the calculation of the variable } \\
\text { moment of inertia }\left(\mathrm{kg} \cdot \mathrm{m}^{2}\right) \text {. }\end{array}$ \\
\hline $29-42$ & BEFFMI & $\begin{array}{l}\text { The coefficient for the (OMEGA/ROMEGA) term } \\
\text { in the calculation of the variable moment of } \\
\text { inertia }\left(\mathrm{kg} \cdot \mathrm{m}^{2}\right) \text {. }\end{array}$ \\
\hline $43-56$ & CEFFMI & $\begin{array}{l}\text { The constant term in the calculation of the } \\
\text { variable moment of inertia }\left(\mathrm{kg} \cdot \mathrm{m}^{2}\right) \text {. }\end{array}$ \\
\hline $57-70$ & OMTEST & $\begin{array}{l}\text { The pump speed below which EFFMIl (the } \\
\text { alternate effective moment of inertia) is } \\
\text { used (rad } s^{-1} \text { ). }\end{array}$ \\
\hline
\end{tabular}


Card Number $7+2$. (Format I14) IPMPS (Input this card only if variable EFFMI on Card Number 7 is negative.)

Columns Variable

1-14 IPMPS
Description

Flag indicates whether or not the pump speed previously has dropped below below OMTEST.

$0=$ the pump speed has been greater than OMTEST;

1 = the pump speed at some time has dropped below OMTEST.

Card Number 8. (Format 5E14.6) RHEAD, RTORK, RFLOW, RRHO, ROMEGA

Columns

$\underline{\text { Variable }}$

1-14 RHEAD

15-28 RTORK

29-42 RFLOW

43-56 RRHO

$57-70$

ROMEGA
Description

Rated head $\left(\mathrm{Pa} \cdot \mathrm{m}^{3} \cdot \mathrm{kg}^{-1}, \mathrm{~m}^{2} \cdot \mathrm{s}^{-2}, \mathrm{pr}\right.$

Rated torque $(\mathrm{N} \cdot \mathrm{m})$.

Rated volumetric flow $\left(\mathrm{m}^{3} \cdot \mathrm{s}^{-1}\right)$.

Rated density $\left(\mathrm{kg} \cdot \mathrm{m}^{-3}\right)$.

Rated pump speed ( $\left.\mathrm{rad} \cdot \mathrm{s}^{-1}\right)$.

Card Number 9. (Format 5E14.6I14) OMEGAN, OMGOFF, ROMGMX, OMGSCL, NPMPSD

\begin{tabular}{|c|c|c|}
\hline Columns & Variable & Description \\
\hline $1-14$ & OMEGAN & $\begin{array}{l}\text { Initial pump speed } \quad\left(\mathrm{rad} \cdot \mathrm{s}^{-1}\right) \text { when } \\
\text { NPMPSD }=0 .\end{array}$ \\
\hline $15-28$ & OMGOFF & $\begin{array}{l}\text { Pump speed ( } \mathrm{rad} \cdot \mathrm{s}^{-1} \text { ) when the controlling } \\
\text { trip is OFF after being ON (maintain the } \\
\text { last pump speed evaluated when the trip was } \\
\text { ON if OMGOFF } \leq-1.0 \times 10^{19} \text { ) [input only when } \\
\text { IPMPTR } \neq 0 \text { ]. }\end{array}$ \\
\hline $29-42$ & ROMGMX & $\begin{array}{l}\text { The maximum rate of pump-speed change } \\
\left.\text { (rad } \cdot s^{-2}\right) .\end{array}$ \\
\hline $43-56$ & OMGSCL & $\begin{array}{l}\text { Scale factor ( }- \text { ) for the pump-speed table. } \\
\text { The dependent variable in table PMPTB is } \\
\text { multiplied by OMGSCL to obtain absolute } \\
\text { rotational speed. }\end{array}$ \\
\hline
\end{tabular}


Columns Variable

57-70 NPMPSD

\section{Description}

The ID number of the signal-variable parameter or control-block parameter that defines the pump rotational speed initially when the controlling trip is OFF (input NPMPSD $=0$ if the initial pump speed is to be the constant OMEGAN).

Note: If NODES $<1$ (word 2 on Card Number 2), omit Card Number 10.

Card Number 10. (Format 4E14.6) QP3IN, QP30FF, RQP3MX, QP3SCL

\section{Columns Variable Description}

1-14 QP3IN

15-28 QP30FF

29-42 RQP3MX

43-56 QP3SCL
Initial power (W) deposited in the wall and distributed according to the QPPP array. If QP3IN >0.0, it is the total power to the entire wall. When QP3IN $<0.0$ is input, the initial power to the wall in each cell is |QP3IN| and the negative sign flags the power to the wall to be a cell-dependent vector of NCELLS (word 1 on Card Number 2) powers. Each $(x, y)$ point of the power-towall table (for QP3IN < 0.0 ) has 1+NCELLS values (an independent-variable value $x$ and NCELLS power values $\mathrm{y}(\mathrm{z})$ for cells 1 through NCELLS). When the power-to-wall table is not being evaluated, the same power value $\rho f$ $|\mathrm{QP} 3 \mathrm{IN}|$ or QP30FF (if QP30FF > $-1.0 \times 10^{19}$ is applied at each of the NCELLS cells.

Power ( $W$ ) to the wall when the controlling trip is OFF after being on inaintain the last table-evaluated power when the trip was $\mathrm{ON}$ if $\mathrm{QP} 30 \mathrm{FF} \leq-1.0 \times 10^{19}$ ).

The maximum rate of change of the power to the wall $\left(\mathrm{W} \cdot \mathrm{s}^{-1}\right)$ [RQP3MX $\geq 0.0$ ].

Scale factor $(-)$ for the power-to-wall table. The dependent variable in table QP3TB is multiplied by QP3SCL to obtain the absolute power to the wall. 
Card Number 11. (Format I14) OPTION

\begin{tabular}{lll} 
Columns Variable & Description \\
\cline { 2 - 3 } $1-14$ & OPTION & Pump curve option number. \\
& $0=$ user-specilied pump, input following; \\
& $1=$ use built-in Semiscale pump; or \\
& $2=$ use built-in LOFT pump.
\end{tabular}

Card Set 12 and Pump Curve Cards are needed only if OPTION $=0$. If OPTION $=1$ or 2 , skip to the Pump Array Cards. The user is referred to the pump model description in Sec. IV.F for definitions of the terms used below. Each homologous curve is divided into four curve segments. Each curve segment is denoted by the number appended to the curve name. The segments are defined by Table XI in Sec. IV.F.

Under certain conditions for OPTION $=0$, some curves do not need to be entered. However, to avoid confusion, we recommend that all curves be entered when OPTION $=0$. For IPMPTY $=1$ and IPM $=0$, curves HSP1 through HSP4 are required, and the remaining curves can be dummies. For IPMPTY $=1$ and IPM $=1$, curves HSPI through HSP4, HTPI through HTP4, and HDM are required, and the remaining curves can be dummies. For IPMPTY $=2$ and IPM $=0$, curves HSPl through HSP4 and TSPI through TSP4 are required, and the remaining curves can be dummies. For IPMPTY $=2$ and IPM $=1$, all curves are required. $\operatorname{NDATA}(I)$ must be greater than zero in Card Set 12.

Future code changes may restrict those curves that can be represented by dummy curves under various conditions.

Card Set 12. (Format 5I14) $\operatorname{NDATA}(I)(I=1,16)$, NHDM, NTDM

First Card.

$\begin{array}{lll}\text { Columns } & \text { Variable } & \text { Description } \\ 1-14 & \text { NDATA(1) } & \text { Number of pairs of points on the HSP1 curve. } \\ 15-28 & \text { NDATA(2) } & \text { Number of pairs of points on the HSP2 curve. }\end{array}$


Pump Component (PUMP)

Columns
$29-42$
$43-56$
$57-70$
Second Card.

\begin{tabular}{|c|c|}
\hline Columns & Variable \\
\hline $1-14$ & $\operatorname{NDATA}(6)$ \\
\hline $15-28$ & $\operatorname{NDATA}(7)$ \\
\hline $29-42$ & $\operatorname{NDATA}(8)$ \\
\hline $43-56$ & $\operatorname{NDATA}(9)$ \\
\hline $57-70$ & $\operatorname{NDATA}(10)$ \\
\hline \multicolumn{2}{|l|}{ Third Card. } \\
\hline Columns & Variable \\
\hline $1-14$ & $\operatorname{NDATA}(11)$ \\
\hline $15-28$ & $\operatorname{NDATA}(12)$ \\
\hline $29-42$ & $\operatorname{NDATA}(13)$ \\
\hline $43-56$ & NDATA $(14$ \\
\hline $57-70$ & NDATAC 15 \\
\hline
\end{tabular}

Fourth Card.

Columns Variable

1-14 NDATA(16)

15-28 NHDM

29-42 NTDM
Description

Number of pairs of points on the HSP3 curve.

Number of pairs of points on the HSP4 curve.

Number of pairs of points on the HTP1 curve.

\section{Description}

Number of pairs of points on the HTP2 curve. Number of pairs of points on the HTP 3 curve. Number of paris of points on the HTP 4 curve. Number of pairs of points on the TSP1 curve. Number of pairs of points on the TSP2 curve.

\section{Description}

Number of pairs of points on the TSP 3 curve. Number of pairs of points on the TSP4 curve. Number of pairs of points on the TTP1 curve. Number of pairs of points on the TTP 2 curve. Number of pairs of points on the TTP 3 curve.

\section{Description}

Number of pairs of points on the TTP4 curve. Number of pairs of points on the HDM curve. Number of pairs of points on the TDM curve. 
Pump Component (PUMP)

PUMP Curve Cards. Up to 18 sets of cards, one set for each curve listed in Card Set 12 that has nonzero data points. Use LOAD format. Data are entered in pairs $(x, y)_{i}, 1=(1$, NDATA $)$ where $x$ is the independent variable and $y$ is the dependent variable. The $x_{1}$ values must increase monotonically from -1.0 to 1.0 for the homologous curves and from 0.0 to 1.0 for the multiplier curves. If information for a particular curve does not exist or if you desire to input a simple dummy curve, we suggest that the four points $(-1.0,0.0,1.0$, 0.0 ) be used. [The suggested dummy for HDM and TDM is $(0.0,0.0,1.0,0.0)$.

\begin{tabular}{|c|c|c|c|}
\hline Variable & Dimension & Description & \\
\hline HSP 1 & $2 * \operatorname{NDATA}(1)$ & HSP1 curve & \\
\hline HSP 2 & $2 * \operatorname{NDATA}(2)$ & HSP2 curve & \\
\hline & & UCD? & $\begin{array}{l}\text { single-phase homologous } \\
\text { head curves. }\end{array}$ \\
\hline HSP 3 & $2 * \operatorname{NDATA}(3)$ & HSP3 curve & \\
\hline HSP 4 & $2 * \operatorname{NDATA}(4)$ & HSP4 curve & \\
\hline HTP1 & $2 * \operatorname{NDATA}(5)$ & HTP1 curve & \\
\hline HTP 2 & $2 * \operatorname{NDATA}(6)$ & HTP2 curve & \\
\hline & & & $\begin{array}{l}\text { fully degraded homolo- } \\
\text { gous head curves. }\end{array}$ \\
\hline HTP3 & $2 * \operatorname{NDATA}(7)$ & HTP3 curve & \\
\hline HTP4 & $2 * \operatorname{NDATA}(8)$ & HTP4 curve & \\
\hline TSP 1 & $2 * \operatorname{NDATA}(9)$ & TSP1 curve & \\
\hline TSP 2 & $2 * \operatorname{NDATA}(10)$ & TSP2 curve & \\
\hline & & & torque curves. \\
\hline TSP3 & $2 * \operatorname{NDATA}(11)$ & TSP3 curve & \\
\hline TSP4 & $2 * \operatorname{NDATA}(12)$ & TSP4 curve & \\
\hline
\end{tabular}




\begin{tabular}{|c|c|c|c|}
\hline Variab1e & & Dimension & Description \\
\hline TTP1 & & $2 * \operatorname{NDATA}(13)$ & TTP1 curve \\
\hline TTP2 & & $2 * \operatorname{NDATA}(14)$ & $\begin{array}{l}\text { fully degraded homolo- } \\
\text { gous torque curves }\end{array}$ \\
\hline $\operatorname{TTP} 3$ & & $2 * \operatorname{NDATA}(15)$ & TTP3 curve \\
\hline TTP4 & & $2 * \operatorname{NDATA}(16)$ & TTP4 curve \\
\hline HDM & & $2 *$ NHDM & Head degradation multiplier. \\
\hline TDM & & $2 * N T D M$ & Torque degradation multiplier. \\
\hline PUMP Ar & rray & $\begin{array}{l}\text { Cards. Twenty-four } \\
\text { following var }\end{array}$ & $\begin{array}{l}\text { sets of cards, one set for each of the } \\
\text { riables. Use LOAD format. }\end{array}$ \\
\hline Variabl & & Dimension & Description \\
\hline $\mathrm{DX}$ & & NCELLS & Ce11 lengths (m). \\
\hline VOL & & NCELLS & Cell volumes $\left(\mathrm{m}^{3}\right)$. \\
\hline FA & & NCELLS+1 & Cel1-edge flow areas $\left(\mathrm{m}^{2}\right)$ \\
\hline FRIC & & NCELLS+1 & $\begin{array}{l}\text { Additive loss coefficients (-) [FRIC(2) must } \\
\text { be } 0.0] \text { (See ACCUM input description, } \\
\text { Sec.V.C.5.a.) }\end{array}$ \\
\hline GRAV & & NCELLS+1 & $\begin{array}{l}\text { Gravity terms (-). (See ACCUM input } \\
\text { description, Sec. } \text { V.C.5.a.) }^{\text {il }}\end{array}$ \\
\hline HD & & NCELLS+1 & $\begin{array}{l}\text { Hydraulic diaméters (m). (See variable } \\
\text { NDIAl in the NAMELIST data for optional } \\
\text { heat-transfer diameters.) }\end{array}$ \\
\hline Note: & $\begin{array}{l}\text { If } \\
\text { inpu }\end{array}$ & $\begin{array}{l}\text { ICFLOW }=0 \text { or ICFLOW } \\
\text { ut array ICFLG. }\end{array}$ & $=1$ (default) in the NAMELIST data, do not \\
\hline ICFLG & & NCELLS+1 & $\begin{array}{l}\text { Cell-edge choked-flow model options. (See } \\
\text { ACCUM input description, Sec. V.C.5.a.) }\end{array}$ \\
\hline NFF & & NCELLS+1 & $\begin{array}{l}\text { Friction-factor correlation options. (See } \\
\text { ACCUM input description, Sec.V.C.5.a.) }\end{array}$ \\
\hline
\end{tabular}




$\begin{array}{ll}\text { Variable } & \text { Dimension } \\ \text { ALP } & \text { NCELLS } \\ \text { VL } & \text { NCELLS+1 } \\ \text { VV } & \text { NCELLS+1 } \\ \text { TL } & \text { NCELLS } \\ \text { TV } & \text { NCELLS } \\ \text { P } & \text { NCELLS } \\ \text { PA } & \text { NCELLS }\end{array}$

\section{Description}

Initial void fractions (-).

Initial liquid velocities $\left(m \cdot s^{-1}\right)$.

Initial vapor velocities $\left(m \cdot s^{-1}\right)$.

Initial liquid temperatures (K).

Initial vapor temperatures (K).

Initial pressures ( $\mathrm{Pa}$ ).

Initial air partial pressures $(\mathrm{Pa})$.

Note: If NODES $=0$ (word 2 on Card Number 2), do not enter arrays QPPP, MATID, and TW.

QPPP NODES*NCELLS

A nonnormalized power profile (-) in the pump walls. Input for cell 1 , node 1 through NODES; then cell 2, node 1 through NODES; etc. If the array is filled with the same nonzero constant, a uniform volumetric heat source for the wall results. The code will normalize the power profile on a nodevolume basis (normalized QPPP(i)/ $\left[\sum \mathrm{QPPP}(i)\right.$ *VOL(i)]). Filling the array withi zeros results in no power being deposited in the wall regardless of settings of QP3IN, QP3TB, etc.

MATID NODES-1

Wall-material ID array specifying material ID between nodes. Dimension is 1 if NODES $=1$ and 0 if NODES $=0$. (See available built-in properties listed with array MATID in the CORE input, Sec.V.C.5.c, or Sec. V.C.2 to input material properties.)

TW NODES*NCELLS

Initial wall temperatures $(K)$. Input in the same order as QPPP.

Note: If ICONC $=0$ (word 2 on Card Number 3 ), omit card set CONC.

CONC NCELLS

Intial ratio of solute mass to liquid coolant mass. 
Note: If ICONC $=0$ or 1 (word 2 on Card Number 3 ), omit card set $S$.

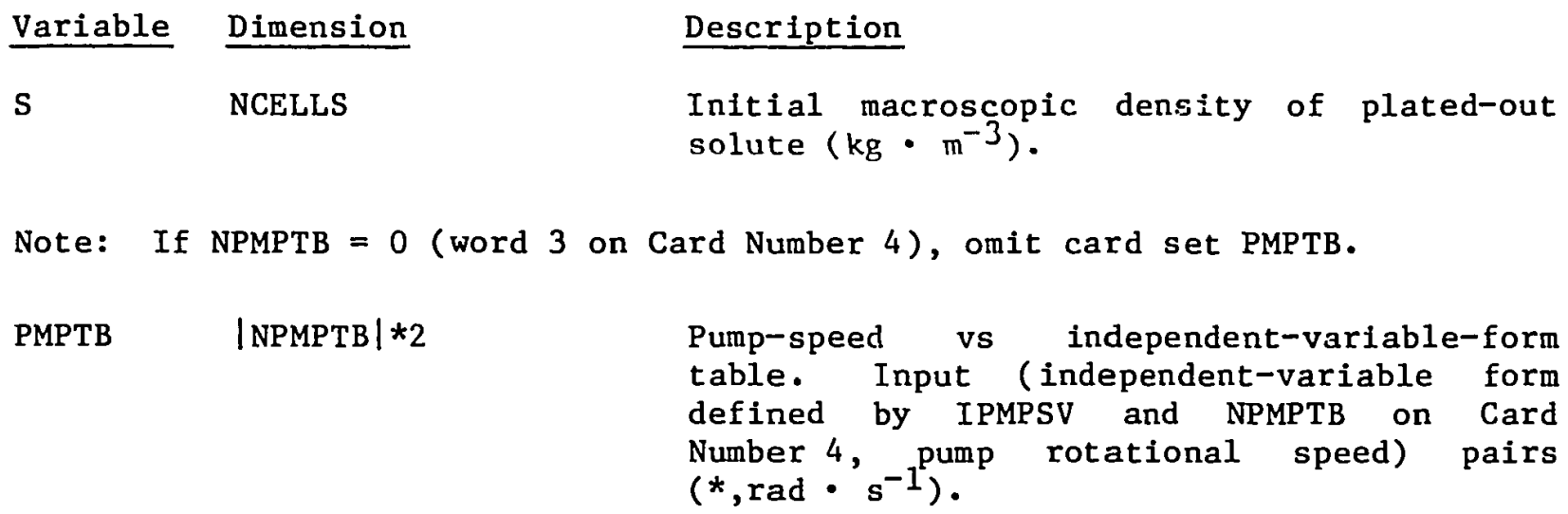

Note: If NPMPTB $=0$ (word 3 on Card Number 4) or NPMPRF $=0$ (word 5 on Card Number 4), omit card set PMPRF.

PMPRF $\quad \mid$ NPMPRF $\mid * 2$

Rate-factor table for the pump-speed table's independent variable. Input (independentvariable form defined by NPMPSV and NPMPRF on Card Number 4, rate factor to be applied to the pump-speed table's independent variable) pairs $(*,-)$.

Note: If $\mathrm{NQP} 3 \mathrm{~TB}=0$ (word 3 on Card Number 5), omit card set QP3TB.

QP3TB $\quad \mid$ NQP3TB $\mid * 2$

when $Q P 3 I N>0$

$\mid$ NQP3TB $\mid *(1+$ NCELLS $)$

when $Q P 3 I N<0$
Power-to-wall vs independent-variable-form table. Input (independent-variable form defined by IQP3SV and NQP3TB on Card Number 5 , power-to-wall pairs $(*, W)$. If QP3IN $>0.0$, the dependent variable specifies the total power to the entire wal1; if QP3IN < 0.0 , the dependent variable is a vector specifying the power to the wall at each cell beginning with cell 1 . 
Pump Component (PUMP)

Note: If NQP3TB $=0$ (word 3 on Card Number 5), or NQP3RF $=0$ (word 5 on Card Number 5), omit card set QF3RF.

Variable Dimension

QF3RF

\section{Description}

Rate-factor Lable for the QP3TB table"s independent variable. Input (indeperdentvariable form defined by NQP3SV and NCi23RF on Card Number 5, rate factor to be applied to the QP3TB table's independent variable) pairs $(*,-)$. 
i. Steam-Generator Component (STGEN).

General Input Data

The general input data, required to provide a global description of the steam-generator component, consist of the following cards.

Card Number 1. (Format A10,4X,2I14,A30) TYPE, NUM, ID, CTITLE

Columns Variable Description

1-5 TYPE Component type (STGEN left-justified).

15-28 NUM Component ID number (nust be unique for each component).

29-42 ID

User ID number (arbitrary).

43-72 CTITLE

Hollerith component description.

Card Number 2. (Format 5I14) NCELL1, NTUBE, NODES, NSCMP, MAT

Columns Variable Description

1-14 NCELL1

15-28 NTUBE

29-42 NODES

Number of fluid cells on the prinary side.

Number of one-dimensional conduction paths used to model the tube walls.

Number of radial heat-transfer nodes in the tube walls (NODES $\geq 1$ ). (Currently use NODES $=$ NDHT; see word 2 on Card Number 7.)

43-56 NSCMP

Number of components (pipes or tees) used to construct the secondary flow path.

57-70 MAT

Currently not used.

Card Number 3.. (Format 4I14) ICONC, ICHFl, JUN11, JUN12

$\begin{array}{lll}\text { Columns Variable } & \text { Description } \\ \text { 1-14 ICONC } & \text { Flag for input of solute in the primary } \\ & \text { liquid coolant. [Requires ISOLUT }=1 \\ & \text { (word } 5 \text { on Main-Data Card 6).] } \\ & 0=\text { no input; } \\ & 1=\text { dissolved solute only; or } \\ & 2=\text { both dissolved and plated-out solute. }\end{array}$


Columns Variable

15-28 ICHF1

29-42 JUN11

43-56 JUN12

Card Number 4. (Format 4I14) ICNC2, ICHF2, JUN21, JUN22
Description

Indicator for CHF calculation on the primary side.

$0=$ no CHF calculation;

$1=\mathrm{CHF}$ calculation.

Junction number for the junction adjacent to cell 1 on the primary side.

Junction number for the junction adjacent to cell NCELLl on the primary side.

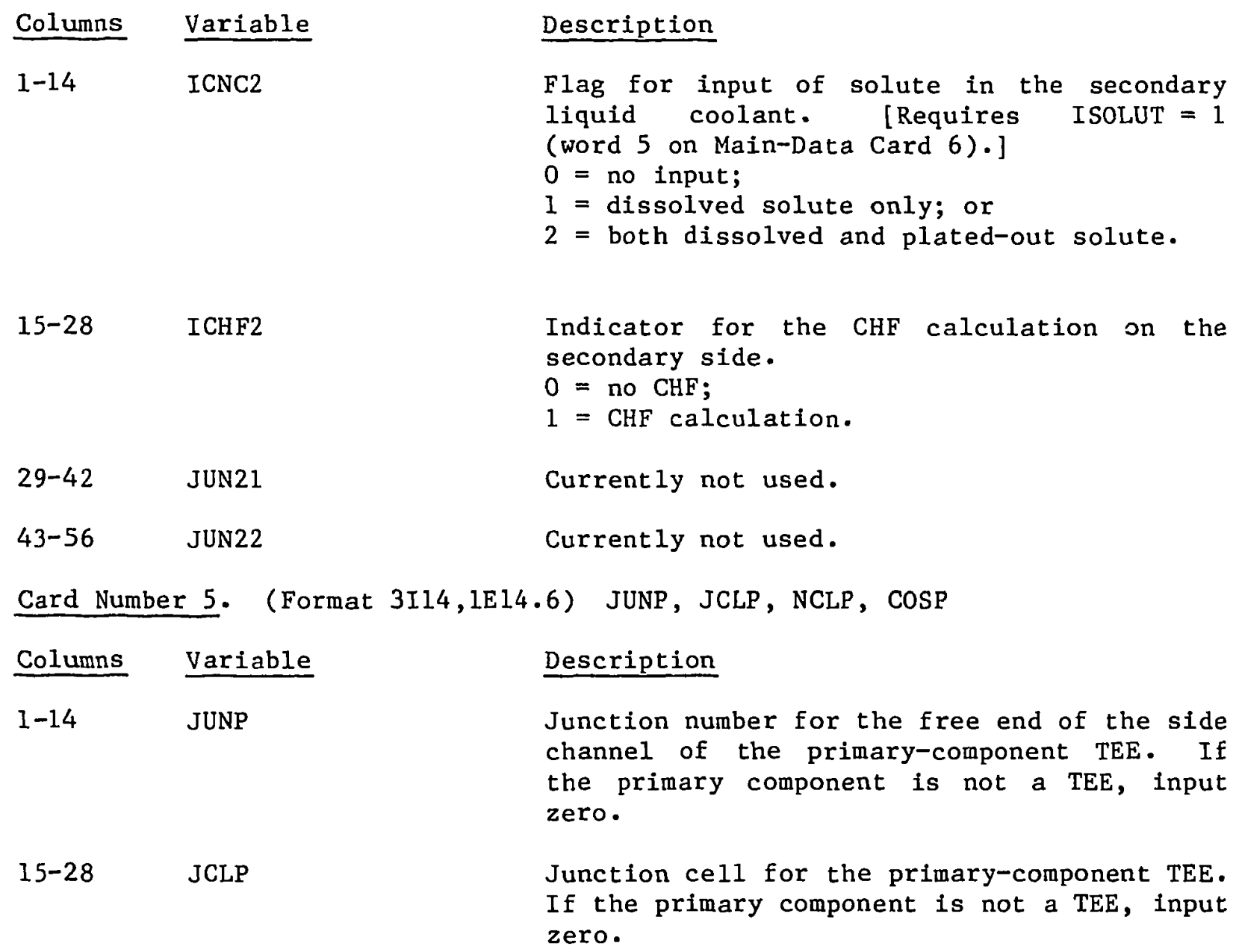




$\begin{array}{ll}\text { Columns } & \text { Variable } \\ 23-42 & \text { NCLP } \\ 43-56 & \text { CosP }\end{array}$

Card Number 6. (Format 3I14) JUNS1, JUNS2, NSJUN

\section{Description}

Number of cells on the side channel of the primary-component TEE. If NCLP $=0$, the primary-side component is a PIPE; if NCLP $>0$, the primary-side component is a TEE.

Cosine of the angle from the low-numbered side of the tnain channel to the side channel ior primary TEE. If the primary component is not a TEE, input zero.

\begin{tabular}{|c|c|c|}
\hline Columns & Variable & Description \\
\hline $1-14$ & JUNS1 & Currently not used. \\
\hline $15-28$ & JUNS2 & Currently not used. \\
\hline $29-42$ & NSJUN & $\begin{array}{l}\text { Total number of junctions associated with } \\
\text { the secondary-side components of the steam } \\
\text { generator. }\end{array}$ \\
\hline
\end{tabular}

Card Number 7.* (Format 2I14) NGHT, NDHT

Columns Variable Description

1-14 NGHT

Number of generalized heat-transfer surfaces (in addition to those used to model tube walls).

15-28 NDHT

Number of nodes used to model heat conduction in the generalized surfaces (NDHT $\geq 1$ ). (Currently use NDHT = NODES; see word 3 on Card Number 2.)

*This card is included to allow in the future for the capability to use different numbers of nodes in the tube walls and the generalized heat-transfer surfaces.

Card Number 8. (Format 3E14.6) RADIN, TH, QP3IN

Columns Variable Description

1-14 RADIN Currently not used. 
Columns Variable Description

15-28 TH Currently not used.

29-42 QP3IN

Power (W) deposited in the wall and distributed according to the QPPP array.

Secondary-Component Description Cards. NSCMP cards are required; each card is used to specify (A10,4X,2I14) STYPE, NCLS, NCLT. The secondary-component input data must be input in the order specified by this card set.

$\begin{array}{ll}\frac{\text { Columns }}{1-5} & \text { Variable } \\ 15-28 & \text { NTYPE } \\ 29-42 & \text { NCLT }\end{array}$

\section{Description}

Secondary-component type (PIPE or TEE). Number of fluid cells in the main tube. Number of side-leg fluid cells if STYPE $=$ TEE.

\section{Primary-Side Input Data}

The primary-side input data are required to specify the primary-side geometry and initial conditions. Seventeen sets of cards must be provided for each of the following variables (LOAD format is used). If the primary-side component is a TEE, input data for main channel here; side-channel data for a primary TEE is input after all the data for the secondary components and hefore the data for the generalized heat-conduction paths.

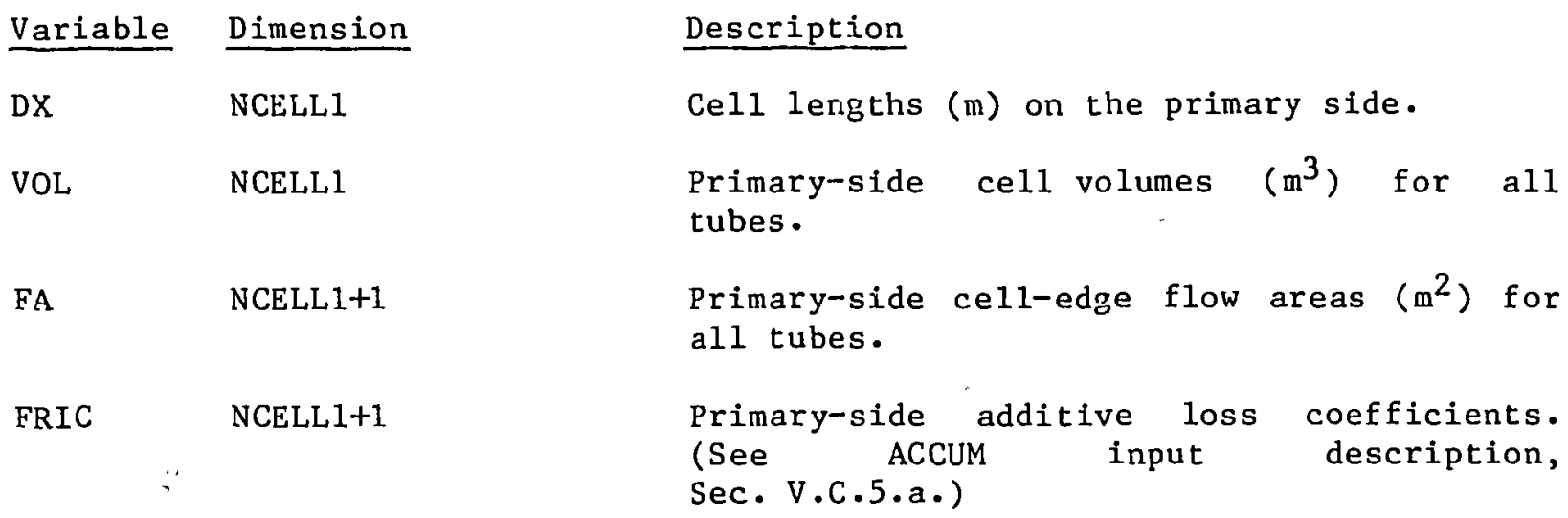




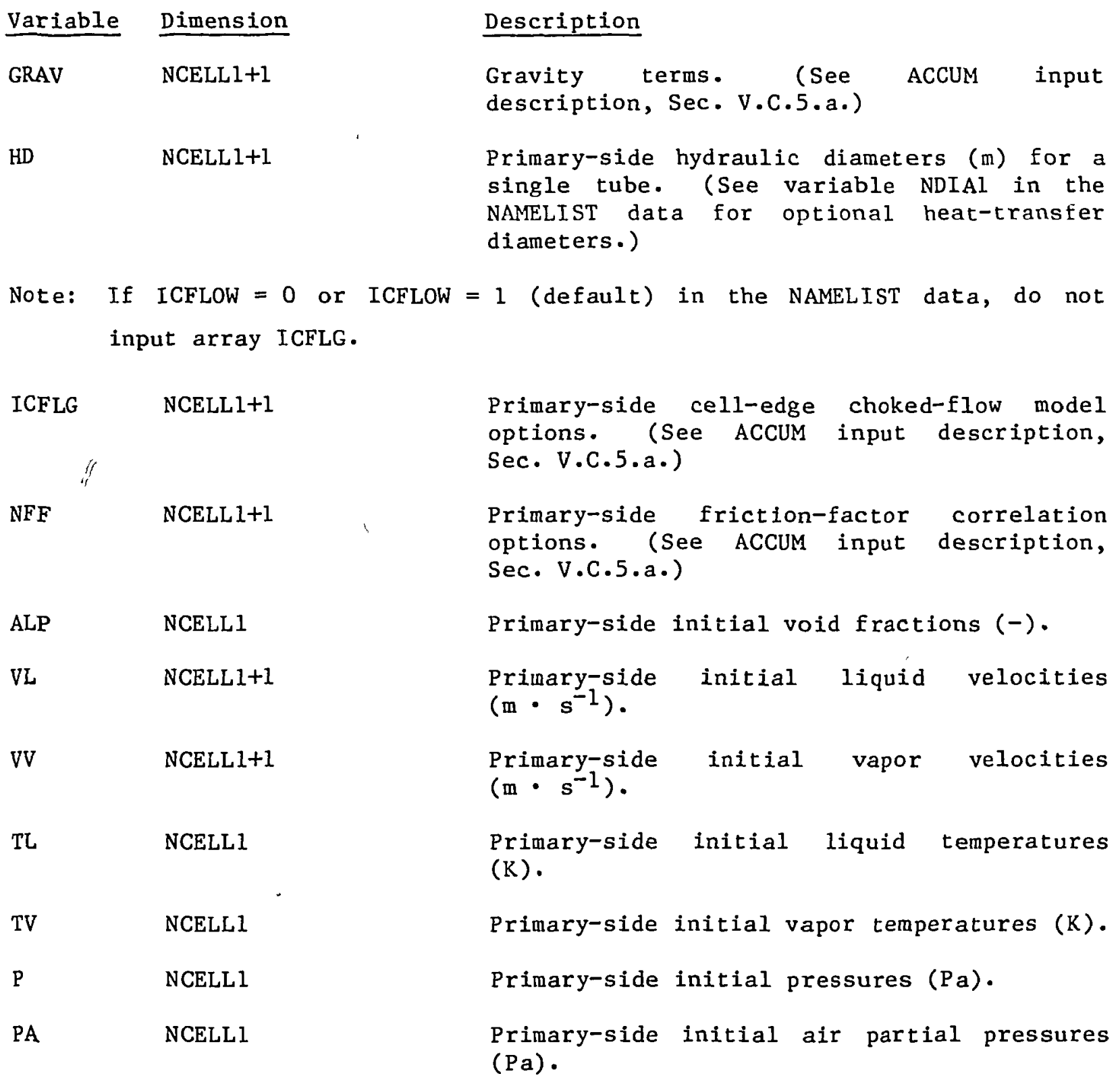

Note: If ICONC $=0$ (word 1 on Card Number 3 ), omit card set conc.

CONC NCELLl Primary-side initial solute mass to liquid mass ratio. 
Note: If ICONC $=0$ or 1 (word 1 on Card Number 3 ), omit card set $S$.

Variable Dimension Description

S NCELL1

Primary-side initial macroscopic density of plated-out solute $\left(\mathrm{kg} \cdot \mathrm{m}^{-3}\right)$.

Secondary-Side Input Data

The secondary-side input data are required to specify the secondary-side geometry and inftial conditions. NSCMP sets of cards are required. The secondary-component input data are read in the same order as provided in the secondary-component description cards (after Card Number 8).

Card Number 1. (Format 4I14) JSINT (3I14), NUMS (I14)

\begin{tabular}{|c|c|}
\hline Columns & Variable \\
\hline $1-14$ & $\operatorname{JSINT}(1, I)$ \\
\hline $15-28$ & $\operatorname{JSINT}(2, I)$ \\
\hline $29-42$ & $\operatorname{JSINT}(3, I)$ \\
\hline $43-56$ & NUMS (I) \\
\hline
\end{tabular}

Description

Junction number adjacent to cell 1 .

Junction number adjacent to cell NCLS(I).

Junction number for the free end of a TEE. If the particular component is not a TEE, input zero.

Secondary-component number. Secondarycomponent numbers should be unique for the problem under consideration to facilitate the use of trips and signal variables. This holds for the entire system, including other STGEN secondary sides.

Seventeen cards (or sets of cards) must be provided for each of the following variables to describe either the secondary PIPE component or the main channel of a secondary TEE component (LOAD format is used).

\begin{tabular}{|c|c|c|}
\hline Variable & Dimension & Description \\
\hline $\mathrm{DX}$ & $\operatorname{NCLS}(\mathrm{I})$ & Main-channel cell lengths (m). \\
\hline VOL & $\operatorname{NCLS}(I)$ & Main-channel volumes $\left(\mathrm{m}^{3}\right)$ \\
\hline FA & $\operatorname{NCLS}(I)+1$ & Main-channel cell-edge flow areas $\left(\mathrm{m}^{2}\right)$ \\
\hline FRIC & $\operatorname{NCLS}(I)+1$ & $\begin{array}{l}\text { Main-channel additive loss coefficients. } \\
\text { (See ACCUM input } \\
\text { Sec.V.C.5.a.) }\end{array}$ \\
\hline
\end{tabular}




\begin{tabular}{|c|c|c|}
\hline Variab] & Dimension & Description \\
\hline GRAV & $\operatorname{NCLS}(\mathrm{I})+1$ & $\begin{array}{l}\text { Main-channel gravity terms. (See ACCUM } \\
\text { input description, Sec. V.C.5.a.) }\end{array}$ \\
\hline HD & $\operatorname{NCLS}(\mathrm{I})+1$ & $\begin{array}{l}\text { Main-channel hydraulic diameters (m). (See } \\
\text { variable NDIAl in the NAMELIST data for } \\
\text { optional heat-transfer diametcrs.) }\end{array}$ \\
\hline Note: & $\begin{array}{l}\text { If } \text { ICFLOW }=0 \text { or } \\
\text { input array ICFLG. }\end{array}$ & ICFLOW $=1$ (default) in the NAMELIST data, do not \\
\hline ICFLG & $\operatorname{NCLS}(I)+1$ & $\begin{array}{l}\text { Main-channel cell-edge choked-flow model } \\
\text { options. (See ACCUM input description, } \\
\text { Sec.V.C.5.a.) }\end{array}$ \\
\hline NFF & $\operatorname{NCLS}(I)+1$ & $\begin{array}{l}\text { Main-channel friction-factor correlation } \\
\text { options. (See ACCUM input description, } \\
\text { Sec.V.C.5.a.) }\end{array}$ \\
\hline ALP & $\operatorname{NCLS}(I)$ & Main-channel initial void fractions $(-)$. \\
\hline VL & $\operatorname{NCLS}(I)+1$ & $\begin{array}{l}\text { Main-channel } \\
\left(\mathrm{m} \cdot \mathrm{s}^{-1}\right)\end{array}$ \\
\hline VV & $\operatorname{NCLS}(I)+1$ & initial vapor \\
\hline $\mathrm{TL}$ & $\operatorname{NCLS}(I)$ & $\begin{array}{l}\text { Main-channel initial liquid temperatures } \\
(\mathrm{K}) \text {. }\end{array}$ \\
\hline TV & $\operatorname{NCLS}(I)$ & Main-channel initial vapor temperatures $(K)$. \\
\hline $\mathbf{P}$ & $\operatorname{NCLS}(I)$ & Main-channel initial pressures $(\mathrm{Pa})$. \\
\hline PA & $\operatorname{NCLS}(\mathrm{I})$ & $\begin{array}{l}\text { Main-channel initial air partial pressures } \\
\text { (Pa). }\end{array}$ \\
\hline
\end{tabular}

Note: If ICNC2 $=0$ (word 1 on Card Number 4), omit card set CoNC.

CONC NCLS(I) Main-channel initial solute mass to liquid mass ratio.

Note: If ICNC2 $=0$ or 1 (word 1 on Card Number 4), omit card set $\mathrm{S}$.

S NCLS(I)

Main-channel initial macroscopic density of plated-out solute $\left(\mathrm{kg} \cdot \mathrm{m}^{-3}\right)$. 
If the secondary component is a TEE, the following input is required to model the side channel.

Card 1. $(E 14 \cdot 6, \mathrm{I} 14) \operatorname{Coss}(\mathrm{I}), \operatorname{JCLT}(\mathrm{I})$

Columns Variable Description

$1-14 \operatorname{cosS}(\mathrm{I})$

Cosine of the angle from the low-numbered side of the main channel to the side channel.

15-28 JCLT(I) Main-channel cell number to which the side channel is attached.

Seventeen cards (or sets of cards) must be provided to input the following variables to describe the side channel of a secondary TEE component (LOAD format is used).

\begin{tabular}{|c|c|c|}
\hline Variab & Dimension & Description \\
\hline $\mathrm{DX}$ & $\operatorname{NCLT}(I)$ & Side-channel cell lengths (m). \\
\hline VOL & $\operatorname{NCLT}(I)$ & Side-channel volumes $\left(\mathrm{m}^{3}\right)$. \\
\hline FA & $\operatorname{NCLT}(I)+1$ & Side-channel, cell-edge flow areas $\left(\mathrm{m}^{2}\right)$ \\
\hline FRIC & $\operatorname{NCLT}(I)+1$ & $\begin{array}{l}\text { Side-channel additive loss coefficients. } \\
\text { (See ACCUM input } \\
\text { Sec.V.C.5.a.) }\end{array}$ \\
\hline GRAV & $\operatorname{NCLT}(I)+1$ & $\begin{array}{l}\text { Side-channel gravity terms. (See ACCUM } \\
\text { input description, Sec.V.C.5.a.) }\end{array}$ \\
\hline $\mathrm{HD}$ & $\operatorname{NCLT}(I)+1$ & $\begin{array}{l}\text { Side-channel hydraulic diameters (m). (See } \\
\text { variable NDIAl in the NAMELIST data for } \\
\text { optional heat-transfer diameters.) }\end{array}$ \\
\hline Note: & $\begin{array}{l}\text { If ICFLOW }=0 \text { or ICFLOW } \\
\text { input array ICFLG. }\end{array}$ & $=1$ (defau1t) in the NAMELIST data, do not \\
\hline ICFLG & $\operatorname{NCLT}(I)+1$ & $\begin{array}{l}\text { Side-channel cell-edge choked-flow model } \\
\text { options. (See ACCUM input description, } \\
\text { Sec.V.C.5.a.) }\end{array}$ \\
\hline NFF & $\operatorname{NCLT}(I)+1$ & $\begin{array}{l}\text { Side-channel friction-factor correlation } \\
\text { options. (See ACCUM input description, } \\
\text { Sec.V.C.5.a.) }\end{array}$ \\
\hline
\end{tabular}


Steam-Generator Component (STGEN)

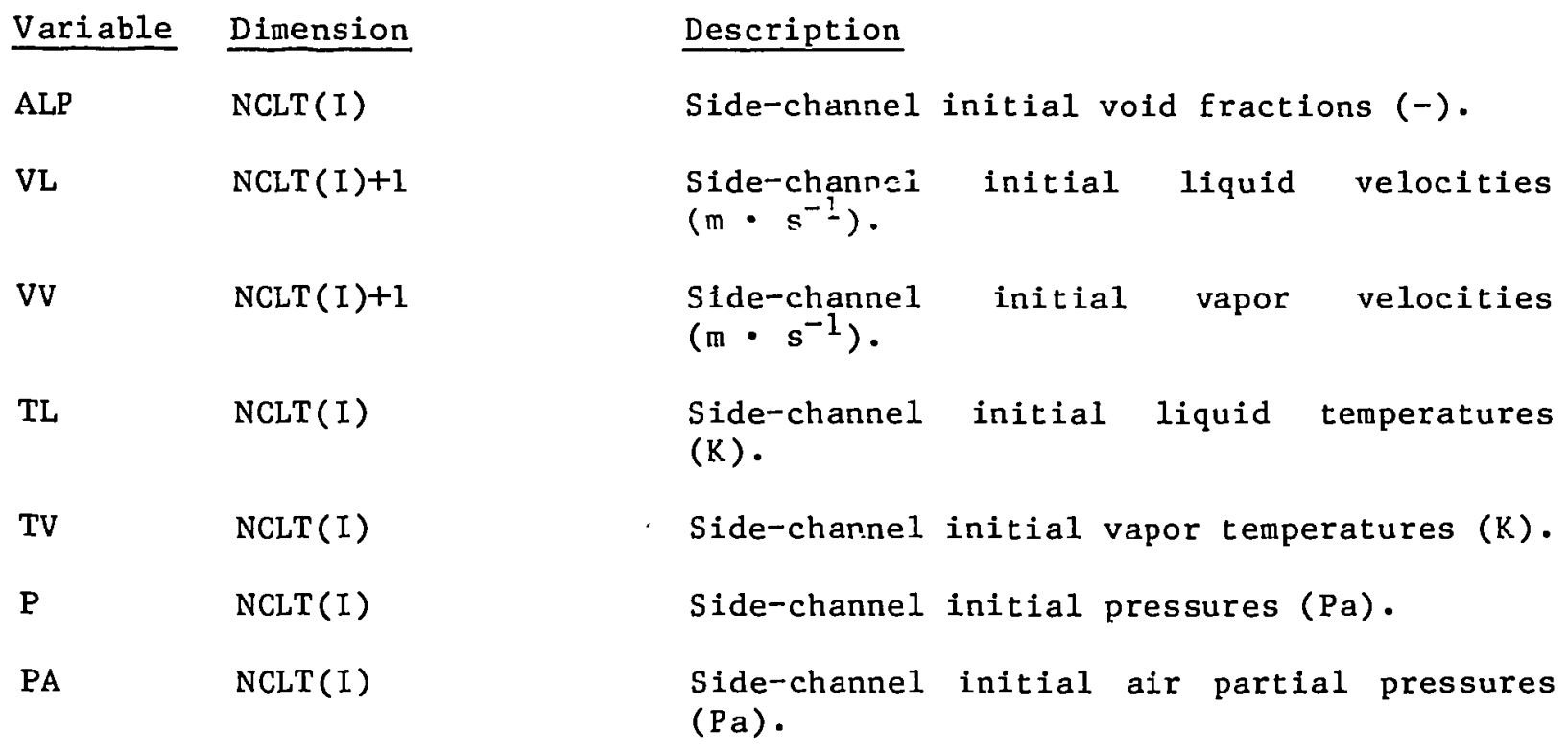

Note: If ICNC2 $=0$ (word 1 on Card Number 4), omit card set CONC.

CONC NCLT(I) Side-channel initial solute mass to liquid mass ratio.

Note: If ICNC2 $=0$ or 1 (word 1 on Card Number 4), omit card set $S$.

S NCLT(I) Side-channel initial macroscopic density of plated-out solute $\left(\mathrm{kg} \cdot \mathrm{m}^{-3}\right)$.

Note: This completes the input for a given secondary component. Repeat the secondary-side input data until NSCMP components have been entered.

Primary-Tee Side-Channel Input (NCLP $\neq 0$ )

If the primary component is a TEE, the following input data are required to specify the primary-tee side-channel geometry and initial conditions. Seventeen sets of cards must be provided for each of the following variables (LOAD format is used).

$\begin{array}{ll}\text { Variable Dimension } & \frac{\text { Description }}{\text { DX }} \quad \text { Primary-tee side-channel cell lengths (m). }\end{array}$ 


\begin{tabular}{|c|c|c|}
\hline Variab & Dimension & Description \\
\hline VOL & NCLP & Primary-tee side-channel volumes $\left(\mathrm{m}^{3}\right)$. \\
\hline FA & NCLP+1 & $\begin{array}{l}\text { Primary-tee side-channel cell-edge } \\
\text { areas }\left(\mathrm{m}^{2}\right) .\end{array}$ \\
\hline FRIC & NCLP+1 & $\begin{array}{l}\text { Primary-tee side-channel additive loss } \\
\text { coefficients. (See ACCUM input description, } \\
\text { Sec.V.C.5.a.) }\end{array}$ \\
\hline GRAV & NCLP+1 & $\begin{array}{l}\text { Primary-tee side-channel } \\
\text { (See } \text { gravity terms. } \\
\text { Sec.V.C.5.a.) }\end{array}$ \\
\hline $\mathrm{HD}$ & NCLP+1 & $\begin{array}{l}\text { Primary-tee side-channel hydraulic diameters } \\
\text { (m). (See variable NDIAl in the NAMELIST } \\
\text { data for optional heat-transfer diameters.) }\end{array}$ \\
\hline Note: & $\begin{array}{l}\text { If ICFLOW }=0 \text { or ICFLOW } \\
\text { input array ICFLG. }\end{array}$ & $=1$ (default) in the NAMELIST data, do not \\
\hline ICFLG & NCLP+1 & $\begin{array}{l}\text { Primary-tee side-channel cell-edge choked- } \\
\text { flow model options. (See ACCUM input } \\
\text { description, Sec.V.C.5.a.) }\end{array}$ \\
\hline NFF & NCLP+1 & $\begin{array}{l}\text { Primary-tee side-channel friction-factor } \\
\text { correlation options. (See ACCUM input } \\
\text { description, Sec.V.C.5.a.) }\end{array}$ \\
\hline ALP & NCLP & $\begin{array}{l}\text { Primary-tee side-channel } \\
\text { fractions }(-) .\end{array}$ \\
\hline VL & NCLP+1 & $\begin{array}{l}\text { Primary-tee side-channel } \\
\text { velocities }\left(\mathrm{m} \cdot \mathrm{s}^{-1}\right)\end{array}$ \\
\hline VV & NCLP+1 & $\begin{array}{l}\text { Primary-tee } \text { side-channel } \\
\text { velocities }\left(m \cdot \mathrm{s}^{-1}\right)\end{array}$ \\
\hline $\mathrm{TL}$ & NCLP & $\begin{array}{l}\text { Primary-tee side-channel } \\
\text { temperatures }(K)\end{array}$ \\
\hline $\mathrm{TV}$ & NCLP & $\begin{array}{l}\text { Primary-tee side-channel } \\
\text { temperatures }(K)\end{array}$ \\
\hline $\mathrm{P}$ & NCLP & $\begin{array}{l}\text { Primary-tee side-channel initial pressures } \\
\text { (Pa). }\end{array}$ \\
\hline
\end{tabular}


Variable Dimension

PA NCLP

\section{Description}

Primary-tee side-channel initial air partial pressures $(\mathrm{Pa})$.

Note: If ICONC $=0$ (word 1 on Card Number 3 ), omit card set CONC.

CONC NCLP

Primary-tee side-channel initial solute mass to liquid mass ratio.

Note: If ICONC $=0$ or 1 (word 1 on Card Number 3 ), omit card set $\mathrm{S}$.

$\mathrm{S} \quad \mathrm{NCLP}$

Primary-tee side-channel initial macroscopic density of plated-out solute $\left(\mathrm{kg} \cdot \mathrm{m}^{-3}\right)$.

Generalized Heat-Conduction-Path Input (NTUBE + NGHT $\neq 0$ )

$\cdots$ The heat-conduction input is required to specify heat-conduction paths between arbitrary fluid volumes internal to the steam-generator component. The same fluid volume cannot be specified on both sides of a heat-conduction path. Nineteen sets of cards are required (LOAD format is used).

Variable Dimension Description

ICMP (NTUBE+NGHT) Inner-wall component number (use zero for a primary cell).

ICELL (NTUBE+NGHT) Inner-wall fluid-cell number (ICELL $>0$ ). (See Sec. IV.G for a description of cell counting.)

OCMP* (NTUBE+NGHT)

Outer-wall component number (use zero for a primary cell).

OCELL $^{*} \quad$ (NTUBE+NGHT)

Outer-wall fluid cell number. If OCELL $=0$, the corresponding OCOMP must be zero. (See Section IV.G for a description of cell counting.)

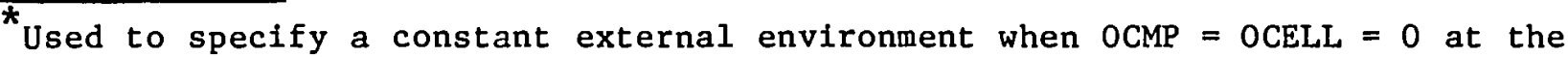
conditions of HOLG, HOVG, TOLG, and TOVG. These parameters are convenient for modeling heat losses from the system. Refer to Sec. IV.G.
} 


\begin{tabular}{|c|c|c|}
\hline Variable & Dimension & Description \\
\hline MATG & $(\mathrm{NDHT}-1) *(\mathrm{NTUBE}+\mathrm{NGHT})$ & $\begin{array}{l}\text { Material ID number specified at locations } \\
\text { between nodes for each conduction path. If } \\
\text { NDHT } 1 \text {, the dimension of this array is } \\
\text { (NTUBE I NGIT). Sce available built-in } \\
\text { properties listed with array MATID in the } \\
\text { CORE input, Sec. V.C.5.c, or Sec. V.C.2 to } \\
\text { input material properties. }\end{array}$ \\
\hline RADIG & (NTUBE+NGHT) & Conduction-path inner radius (m). \\
\hline $\mathrm{TH}$ & (NTUBE+NGHT) & Conduction-path thickness (m). \\
\hline QPPG & NDHT* (NTUBE+NGHT) & $\begin{array}{l}\text { Volumetric heat sources } W \cdot \mathrm{m}^{-3} \text { (currently } \\
\text { not used). }\end{array}$ \\
\hline TWGN & NDHT* (NTUBE+NGHT) & Initial wall temperatures $(\mathrm{K})$. \\
\hline HILG & (NTUBE+NGHT) & Currently not used. \\
\hline HIVG & (NTUBE+NGHT) & Currently not used. \\
\hline TILG & (NTUBE+NGHT) & Currently not used. \\
\hline TIVG & (NTUBE+NGHT) & Currently not used. \\
\hline WAIG & (NTUBE+NGHT) & $\begin{array}{l}\text { Inner-wall surface area }\left(\mathrm{m}^{2}\right) \\
>0.0] \cdot\end{array}$ \\
\hline $\mathrm{HOLG}^{*}$ & (NTUBE+NGHT) & $\begin{array}{l}\text { Exterior liquid heat-transfer coefficient } \\
\left(\mathrm{W} \cdot \mathrm{m}^{-2} \cdot \mathrm{K}^{-1}\right) .\end{array}$ \\
\hline HOVG* & (NTUBE+NGHT) & $\begin{array}{l}\text { Exterior vapor heat-transfer coefficient } \\
\left(\mathrm{W} \cdot \mathrm{m}^{-2} \cdot \mathrm{K}^{-1}\right) .\end{array}$ \\
\hline TOLG* & (NTUBE+NGHT) & External-environment liquid temperature (K). \\
\hline TOVG* & (NTUBE+NGHT) & External-environment vapor temperature $(K)$. \\
\hline WAOG & (NTUBE+NGHT) & $\begin{array}{l}\text { Outer-wa11 surface area }\left(\mathrm{m}^{2}\right) . \\
\operatorname{WAOG}(i)=\operatorname{WAIG}(i) *[1+\operatorname{TH}(i) / \operatorname{RADIG}(i)]) \text {. }\end{array}$ \\
\hline
\end{tabular}




\section{j. Tee Component (TEE).}

Card Number 1. (Format A10,4X,... $14, \mathrm{~A} 30$ ) TYPE, NUM, ID, CTITLE

\begin{tabular}{|c|c|c|}
\hline Columns & Variable & Description \\
\hline $1-3$ & TYPE & Component type (TEE left-justified). \\
\hline $15-28$ & NUM ., & $\begin{array}{l}\text { Component ID number (must be unique for each } \\
\text { component, } 1 \leq \text { NUM } \leq 999 \text { ). }\end{array}$ \\
\hline $29-42$ & ID & User ID number (arbitrary). \\
\hline $43-72$ & CTITLE & Hollerith component description. \\
\hline Card Nun & 2. (Format 3I14, & E14.6, I14) JCELL, NODES, MAT, COST, ICHF \\
\hline Columns & Variable & Description \\
\hline $1-14$ & JCELL & Junction cell number. \\
\hline $15-28$ & NODES & $\begin{array}{l}\text { Number of radial heat-transfer nodes in the } \\
\text { tee wall. A value of } 0 \text { specifies no wall } \\
\text { heat transfer. }\end{array}$ \\
\hline $29-42$ & MAT & $\begin{array}{l}\text { Material ID number of the wall (currently } \\
\text { not used). }\end{array}$ \\
\hline $43-56$ & $\cos T$ & $\begin{array}{l}\text { Cosine of the angle from the low-numbered } \\
\text { side of the main tube to the side tube. }\end{array}$ \\
\hline $57-70$ & I CHF & $\begin{array}{l}\text { CHF calculation flag. } \\
0=\text { no } \\
1=\text { yes. }\end{array}$ \\
\hline
\end{tabular}

Card Number 3. (Format 5I14) ICONC1, NCELL1, JUN1, JUN2, IPOW1

\begin{tabular}{|c|c|c|}
\hline Columns & Variable & Description \\
\hline $1-14$ & ICONCl & $\begin{array}{l}\text { Flag for input of solute in the main-tube } \\
\text { liquid coolant. [Requires ISOLUT =1 } \\
\text { (word } 5 \text { on Maln-Data Card 6).] } \\
0=\text { no input; } \\
1=\text { dissolved solute only; or } \\
2=\text { both dissolved and plated-out solute. }\end{array}$ \\
\hline $15-28$ & NCELL1 & Number of fluid cells in the tee main tube. \\
\hline $29-42$ & JUN1 & $\begin{array}{l}\text { Junction number for the junction adjacent to } \\
\text { cell } 1 \text {. }\end{array}$ \\
\hline
\end{tabular}




$\begin{array}{ll}\frac{\text { Columns }}{43-56} & \text { Variable } \\ 57-70 & \text { IPOW } 1\end{array}$

\section{Description}

Junction number for the junction adjacent to ce11 NCELL1.

Presence of power deposited in the tee maintube coolant flag.

0 = no;

1 = yes.

Note: If IPOW $1=0$ (word 5 on Card Number 3), omit Card Number 4.

Card Number 4. (Format 5I14) IPWTR1, IPWSV1, NPWTB1, NPWSV1, NPWRF1

Columns Variable

1-14 IPWTR1

15-28 IPWSV1

29-42 NPWTB1

\section{Description}

The trip ID number that controls evaluation of the power-to-fluid table (POWTB) for the tee main tube ( $\mid$ IPWTRI $\mid<10000$ ). (Input IPWTR1 $=0$ if there is to be no trip control, and the table will be evaluated in the transient.)

The independent-variable ID number for the power-to-fluid table (POWTB) for the tee main tube. IPWSVI $>0$ defines the ID number for a signal-variable parameter; IPWSVI < 0 defines the ID number for a control-block output parameter.

The number of $(x, y)$ power-to-fluid table's (POWTB) pairs for the tee main tube (defined by the absolute value of NPWTB1). NPWTBI $>0$ defines the table's independentvariable form to be the IPWSV1 parameter; NPWTBI < 0 defines the table's independentvariable form to be the change in the IPWSV1 parameter over the last time step times the trip set-status value ISET when the powerto-fluid table is trip controlled; NPWTBI $=0$ defines the power to be the IPWSVI parameter. 
$\frac{\text { Columns }}{43-56} \quad \frac{\text { Variable }}{\text { NPWSV1 }}$

57-70 NPWRF1

\section{Description}

The independent-variable ID number for the rate factor that is applied to the tee maintube power-to-fluid table's independent variable. NPWSV1 $>0$ defines the ID number for a signal-variable parameter; NPWSV1 < 0 defines the ID number for a control-block output parameter; NPWSV1 $=0$ (when NPWRF1 $\neq 0$ ) defines the independent variable to be the difference between the trip signal and the set-point value that turns the trip OFF when the power table is trip controlled.

The number of $(x, y)$ rate-factor table pairs (defined by the absolute value of NPWRF1). The rate factor is applied as a factor to the tee main-tube power-to-fluid (POWTB) table independent variable when the rate factor is defined. No rate factor is defined when NPWSV1 and NPWRFl are both zero. NPWRF1 $>0$ defines the rate-factor table's independent variable to be the NPWSV1 parameter; NPWRF1 $<0$ defines it to be the change in the NPWSV1 parameter over the last time step times the trip set-status value ISET when the tee main-tube power table is trip controlled; NPWRF1 = 0 defines the rate factor to be the NPWSV1 parameter.

Note: If NODES $<1$ (word 2 on Card Number 2), omit Card Number 5.

Card Number 5. (Format 5I14) IQPTR1, IQPSV1, NQPTB1, NQPSV1, NQPRF1

Columns Variable

1-14 IQPTR 1

\section{Description}

The trip ID number that controls evaluation of the power-to-wall table (QP3TB) for the tee main tube ( $\mid$ IQPTRI $\mid<10000)$. (Input IQPTR $1=0$ if there is to be no trip control, and the table will be evaluated in the transient.) 


$\frac{\text { Columns }}{15-28} \quad \frac{\text { Variable }}{\text { IQPSV1 }}$

29-42 NQPTB1

43-56 NQPSV1

57-70 NQPRF1
Description

The independent-variable ID number for the tee main-tube power-to-wall table (QP3TB). IQPSV1 $>0$ defines the ID number for a signal-variable parameter; IQPSV1 < 0 defines the ID number for a conlrol-block output parameter.

The number of $(x, y)$ power-to-wall table's (POWTB) pairs for the tee main tube (defined by the absolute value of NQPTB1). NQPTBI $>0$ defines the table's independentvariable form to be the IQPSVl parameter; NQPTB1 < 0 defines the table's independentvariable form to be the change in the IQPSV1 parameter over the last time step times the trip set-status value ISET when the powerto-wall table is trip controlled. NQPTB1 $=0$ defines the power to the wall to be the IQPSVI parameter.

The independent-variable ID number for the rate factor that is applied to the tee maintube power-to-wall table's independent variable. NQPSV1 $>0$ defines the ID number for a signal-variable parameter; NQPSV1 < 0 defines the ID number for a control-block output paraneter; NQPSVl $=0$ (when NQPRFl $\neq 0$ ) defines the independent variable to be the difference between the trip signal and the set-point value that turns the trip OFF when the power-to-wall table is trip is controlled.

The number of $(x, y)$ rate-factor table pairs (defined by the absolute value of NQPRF1). The rate factor is applied as a factor to the tee main-tube power-to-wall table's (QP3TB) independent variable when the rate factor is defined. No rate factor is defined when NQPSVI and NQPRF1 are both zero. NQPRF1 $>0$ defines the rate-factor table's independent variable to be the NQPSV1 parameter; NQPRF1< 0 defines it to be the change in the NQPSV1 parameter over the last time step times the trip set-status value ISET when the tee main-tube power-towall table is trip controlled; NQPRF1 =0 defines the rate factor to be the NQPSV1 parameter. 
Card Number 6. (Format 5E14.6) RADIN1, TH1, HOUTL1, HOUTV1, TOUTL1

\begin{tabular}{|c|c|c|}
\hline Columns & Variable & Description \\
\hline $1-14$ & RADIN1 & Inner radius ( $m$ ) of the tee main-tube wall. \\
\hline $15-28$ & THI & Wall thickness $(m)$ of the tee main tube. \\
\hline $29-42$ & HOUTL1 & $\begin{array}{l}\text { HTC }\left(W \cdot \mathrm{m}^{-2} \cdot \mathrm{K}^{-1}\right) \text { between outer boundary } \\
\text { of the tee main-tube wall and liquid outside } \\
\text { the tee main-tube wall. }\end{array}$ \\
\hline $43-56$ & HOUTV1 & $\begin{array}{l}\text { HTC }\left(W \cdot \mathrm{m}^{-2} \cdot \mathrm{K}^{-1}\right) \text { between outer boundary } \\
\text { of the tee main-tube wall and vapor outside } \\
\text { the tee main-tube wall. }\end{array}$ \\
\hline $57-70$ & TOUTLl & $\begin{array}{l}\text { Liquid temperature }(K) \text { outside the tee main- } \\
\text { tube wall. }\end{array}$ \\
\hline
\end{tabular}

Note: See PIPE input description, Sec.V.C.5.e, for further comments on the HOUTLl, HOUTVl, TOUTL1, and TOUTV1 heat-transfer parameters.

Card Number 7. (Format 5E14.6) TOUTV1, PWIN1, PWOFF1, RPWMX1, PWSCL1

\begin{tabular}{|c|c|c|}
\hline Columns & Variable & Description \\
\hline $1-14$ & TOUTVI & $\begin{array}{l}\text { Vapor temperature }(K) \text { outside the tee main- } \\
\text { tube wall. }\end{array}$ \\
\hline $15-28$ & PWIN1 & $\begin{array}{l}\text { Initial total power (W) deposited in the tee } \\
\text { main-tube fluid (not used when IPowl }=0 \text { ). } \\
\text { The power is distributed linearly along the } \\
\text { component. }\end{array}$ \\
\hline $29-42$ & PWOFF1 & $\begin{array}{l}\text { Total power (W) deposited in the tee main- } \\
\text { tube fluid when the controlling trip is OFF } \\
\text { after being ON (not used if IPOWl }=0 \text { or } \\
\text { IPWTRl :=0). If PWOFF } \leq-1.0 \times 10^{19} \text {, the } \\
\text { power to the fluid is held constant at the } \\
\text { last table-evaluated power when the trip was } \\
\text { ON. }\end{array}$ \\
\hline $43-56$ & RPWMX1 & $\begin{array}{l}\text { The maximum rate of change of the tee main- } \\
\text { tube power }\left(W \cdot s^{-1}\right) \text { deposited in the fluid } \\
\text { (RPWMX } \geq 0.0) \text { [not input if IPOWl }=0 \text { ]. }\end{array}$ \\
\hline
\end{tabular}


Columns Variable

57-70 PWSCL1

\section{Description}

Scale factor for the power-to-fluid table. The dependent variable in table POWTB1 is multiplied by PWSCLl to obtain absolute power deposited in the fluid (not used if IPOW1 $=0$ or NPWTBI $=0$ ).

Note: If NODES $<1$ (word 2 on Card Number 2), omit Card Number 8 .

Card Number 8. (Format 4E14.6) QPINl, QPOFFl, RQPMX1, QPSCL1

$\frac{\text { Columns }}{1-14} \quad \frac{\text { Variable }}{\text { QPINl }}$

15-28 QPOFFI

29-42 RQPMX1

43-56 QPSCL1

\section{Description}

Initial power (W) deposited in the tee maintube wall and distributed according to the QPPP array. If QPIN $>0.0$, it is the total power to the entire wall. When QPINl $<0$ is input, the power to the wall in each cell is $|Q P I N| \mid$, and the negative sign flags the power to the wall to be a cell-dependent vector of NCELL1 (word 2 on Card Number 3) powers. Each $(x, y)$ point of the power-towall table (for QPIN1 $<0.0$ ) has $1+$ NCELL1 values [an independent-variable value $x$ and NCELL1 power values $y(z)$ for cells 1 through NCELL1]. When the power-to-wall table is not being evaluated, the same power value of $\mid$ QPIN1| or QPOFF1 (if QPOFF1 > $-1.0 \times 10^{19}$ ) is applied at each of the NCELLl cells.

Power (W) to the tee main-tube wall when the controlling trip is OFF after being $O N$ (maintain the last table-evaluated power when the trip was $O N$ if QPOFFl $\left.\leq-1.0 \times 10^{19}\right)$.

The maximum rate of change of the power fo the wall for the tee main-tube (W. $s^{-I}$ ) [RQPMX1 20.0 ].

Scale factor (-) for the power-to-wall tiable for the tee main tube. The depenglent variable in table QP3TBI is multiplied by QPSCLl to obtain the absolute power to the wall. 
Card Number 9. (Format 4I14) ICONC2, NCELL2, JUN3, IPOW2

\section{Columns}

$1-14$

ICONC2

15-28 NCELL2

29-42 JUN3

43-56 IPOW2

\section{Description}

Flag for input of solute in the tee sidetube liquid coolant. [Requires ISOLUT $=1$ (word 5 on Main-Dala Card 6).]

$0=$ no input;

1 = dissolved solute only; or

2 = both dissolved and plated-out solute.

Number of fluid cells in the tee side tube.

Junction number at the free end of the side tube adjacent to cell NCELL2.

Presence of power deposited in the tee sidetube liquid coolant flag.

$0=$ no;

1 = yes.

Note: If IPOW2 $=0$ (word 4 on Card Number 9), omit Card Number 10.

Card Number 10. (Format 5I14) IPNTR2, IPWSV2, NPWTB2, NPWSV2, NPWRF2

Columns Variable Description

1-14 IPWTR2

15-28 IPWSV2
The trip ID number that controls evaluation of the power-to-fluid table (POWTB) for the tee side tube (|IPWTR2 $\mid<10000)$. (Input IPWTR2 $=0$ if there is to be no trip control, and the table will be evaluated in the transient.)

The independent-variable ID number for the tee side-tube power-to-fluid table (POWTB). IPWSV2 $>0$ defines the ID number for a signal-variable parameter; IPWSV2 < 0 defines the ID number for a control-block output parameter. 
Columns

$29-42$

Variable

NPWTB2

$43-56$

NPWSV2

$57-70$

NPWRF2

\section{Description}

The number of $(x, y)$ power-to-fluid table's (POWTB) pairs for the tee side tube (defined by the absolute value of NPWTB2). NPWTB2 $>0$ defines the table's independentvariable form to be the IPWSV2 parameter; NPWTB2 < 0 defines the table's independentvariable form to be the change in the IPWSV2 parameter over the last time step times the trip set-status value ISET when the tee side-tube power-to-fluid table is trip controlled; NPWTB2 $=0$ defines the power to be the IPWSV2 parameter.

The independent-variable ID number for the rate factor that is applied to the tee sidetube power-to-fluid table's independent variable. NPWSV2 $>0$ defines the ID number for a signal-variable parameter; NPWSV2 < 0 defines the ID number for a control-block output parameter; NPWSV2 $=0$ (when NPWRF2 $\neq 0$ ) defines the independent variable to be the difference between the trip signal and the set-point value that turns the trip OFF when the power table is trip controlled.

The number of $(x, y)$ rate-factor table pairs (defined by the absolute value of NPWRF2). The rate factor is applied as a factor to the tee side-tube power-to-fluid table's (POWTB) independent variable when the rate factor is defined. No rate factor is defined when NPWSV2 and NPWRF2 are both zero. NPWRF2 $>0$ defines the rate-factor table's independent variable to be the NPWSV2 parameter; NPWRF2 $<0$ defines it to be the change in the NPWSV2 parameter over the last time step times the trip set-status value ISET when the tee side-tube power table is trip controlled; NPWRF2 $=0$ defines the rate factor to be the NPWSV2 parameter. 
Note: If NODES $<1$ (word 2 on Card Number 2), omit Card Number 11.

Card Number 11. (Format 5I14) IQPTR2, IQPSV2, NQPTB2, NQPSV2, NQPRF2

$\frac{\text { Columns }}{1-14} \quad \frac{\text { Variable }}{\text { IQPTR2 }}$

15-28 IQPSV2

29-42 NQPTB2

\section{Description}

The trip ID number that controls evaluation of the power-to-wall table (QP3TB) for the tee side tube (|IQPTR2| 10000 ). (Input IQPTR2 $=0$ if there is to be no trip control, and the table will be evaluated in the transient.)

The independent-variable ID number for the tee side-tube power-to-wall table (QP3TB). IQPSV2 $>0$ defines the ID number for a signal-variable parameter; IQPSV2 <0 defines the ID number for a control-block output parameter.

The number of $(x, y)$ power-to-wall table's (POWTB) pairs for the tee side tube (defined by the absolute value of NQPTB2). NQPTB2 > 0 defines the table's independentvariable form to be the IQPSV2 parameter; NQPTB2 < 0 defines the table's independentvariable form to be the change in the IQPSV2 parameter over the last time step times the trip set-status value ISET when the powerto-wall table is trip controlled; NQPTB2 = 0 defines the power to the wall to be the IQPSV2 parameter.

The independent-variable ID number for the rate factor that is applied to the tee sidetube power-to-wall table's independent variable. NQPSV2 $>0$ defines the ID number for a signal-variable parameter; NQPSV2 <0 defines the ID number for a control-block output parameter; NQPSV2 $=0$ (when NQPRF2 $\neq 0$ ) defines the independent variable to be the difference between the trip signal and the set-point value that turns the trip OFF when the power-to-wall table is trip controlled. 
Columns Varieble

$57-70$
NQPRF2 . .

1.

Card Number 12. (Format 5E14.6)

$\begin{array}{ll}\text { Columns } & \text { Variable } \\ 1-14 & \text { RADIN2 } \\ 15-28 & \text { TH2 } \\ 29-42 & \text { HOUTL2 } \\ 43-56 & \text { HOUTV2 } \\ 57-70 & \text { TOUTL2 }\end{array}$

Description

The number of $(x, y)$ rate-factor table pairs (defined by the absolute value of NQPRF2). The rate factor is applied as a factor to the tee side-tube power-to-wall table (QP3TB) independent variable when the rate factor is defined. No rate factor is defined when NQPSV2 and NQPRF2 are both zero. NQPRF2 $>0$ defines the rate-factor table's independent variable to be the NQPSV2 parameter; NQPRF2 < 0 defines it to be the change in the NQPSV2 parameter over the last time step times the trip set-status value ISET when the tee side-tube power-towall table is trip controlled; NQPRF2 = 0 defines the rate factor to be the NQPSV2 parameter.

RADIN2, TH2, HOUTL2, HOUTV2, TOUTL2

\section{Description}

Inner radius (m) of the tee side-tube wall.

Wall thickness (m) of the tee side tube.

HTC $\left(W \cdot \mathrm{m}^{-2} \cdot \mathrm{K}^{-1}\right)$ between outer boundary of the tee side-tube wall and liquid outside the tee side-tube wall.

HTC $\left(W \cdot m^{-2} \cdot K^{-1}\right)$ between outer boundary of the tee side-tube wall and vapor outside the tee side-tube wall.

Liquid temperature $(K)$ outside the tee sidetube wall.

Note: See PIPE input description, Sec.V.C.5.e, for further comments on the HOUTL2, HOUTV2, TOUTL2, and TOUTV2 heat-transfer parameters.

Card Number 13. (Format 5E14.6) TOUTV2, PWIN2, PWOFF2, RPWMX2, PWSCL2

Columns Variable Description

1-14 TOUTV2 .

Vapor temperature $(K)$ outside the tee sidetube wall. 
$\frac{\text { Columns }}{15-28} \quad \frac{\text { Variable }}{\text { PWIN2 }}$

29-42 PWOFF2

43-56 RPWMX2

57-70 PWSCL2

\section{Description}

Initial total power (W) deposited in the tee side-tube fluid (not used when IPOW2 $=0$ ). The power is distributed linearly along the component.

Total power (W) deposited in the side-tube fluid when the controlling trip is OFF after being $O N$ (not used when IPOW2 $=0$ or IPWTR2 $=0$ ). If PWOFF2 $\leq-1.0 \times 10^{19}$, the power to the fluid is held constant at the last table-evaluated power when the trip was ON.

The maximum rate of change of the power tee side-tube power $\left(W \cdot \mathrm{s}^{-1}\right)$ deposited in the fluid (RPWMX1 $\geq 0.0) \quad[$ not used when IPOW2 $=0$ ].

Scale factor for the power-to-fluid table. The dependent variable in table POWTB2 is multiplied by PWSCL2 to obtain absolute power deposited in the fluid (not used if IPOW2 $=0$ or NPWTB2 $=0$ ).

Note: If NODES $<1$ (word 2 on Card Number 2), omit Card Number 14.

Card Number 14. (Format 4E14.6) QPIN2, QPOFF2, RQPNIX2, QPSCL2

Columns Variable

1-14 QPIN2

\section{Description}

Initial power ( $W$ ) deposited in the tee sidetube wall and distributed according to the QPPP array. If QPIN2 $>0.0$, it is the total power to the entire wall. When QPIN2 $<0.0$ is input, the initial power to the wall in each cell is |QPIN2|, and the negative sign flags the power to the wall to be a celldependent vector of NCELL2 (word 2 on Card Number 9) powers. Each $(x, y)$ point of the power-to-wall table (for QPIN2<0.0) has $1+$ NCELL2 values [an independent-variable value $x$ and NCELL2 power values $y(z)$ or cells 1 through NCELL2]. When the power-towal.1 table is not being evaluated, the same power value of $|Q P I N 2|$ or $Q P O F F 2>0.0$ (if QPOFF2 $>-1.0 \times 10^{19}$ ) is applied at each of the NCELL2 cells. 


\begin{tabular}{|c|c|c|}
\hline Columns & Variable & Description \\
\hline \multirow[t]{2}{*}{$15-28$} & QPOFF2 & $\begin{array}{l}\text { Power }(W) \text { to the tee side-tube wall when the } \\
\text { controlling trip is ofF after being oN } \\
\text { (maintain the last table-evaluated power }\end{array}$ \\
\hline & & $\begin{array}{l}\text { when the } 19 \text { trip was oN if } \\
\leq-1.0 \times\left(0^{19}\right) \text {. }\end{array}$ \\
\hline $29-42$ & RQPMX2 & $\begin{array}{l}\text { The maximum rate of change of the tee side- } \\
\left.\text { tube power to the wall ( } W \cdot s^{-1}\right) \text { [RQPMX2 } \\
20.0] \text {. }\end{array}$ \\
\hline $43-56$ & QPSCL2 & $\begin{array}{l}\text { Scale factor (-) for the tee side-tube } \\
\text { power-to-wall table. The dependent variable } \\
\text { in table QP3TB2 is multiplied by QPSCL2 to } \\
\text { obtain the absolute power to wall. }\end{array}$ \\
\hline
\end{tabular}

TEE Array Cards. Forty-eight sets of cards, one for each of the following variables. Use LOAD format.

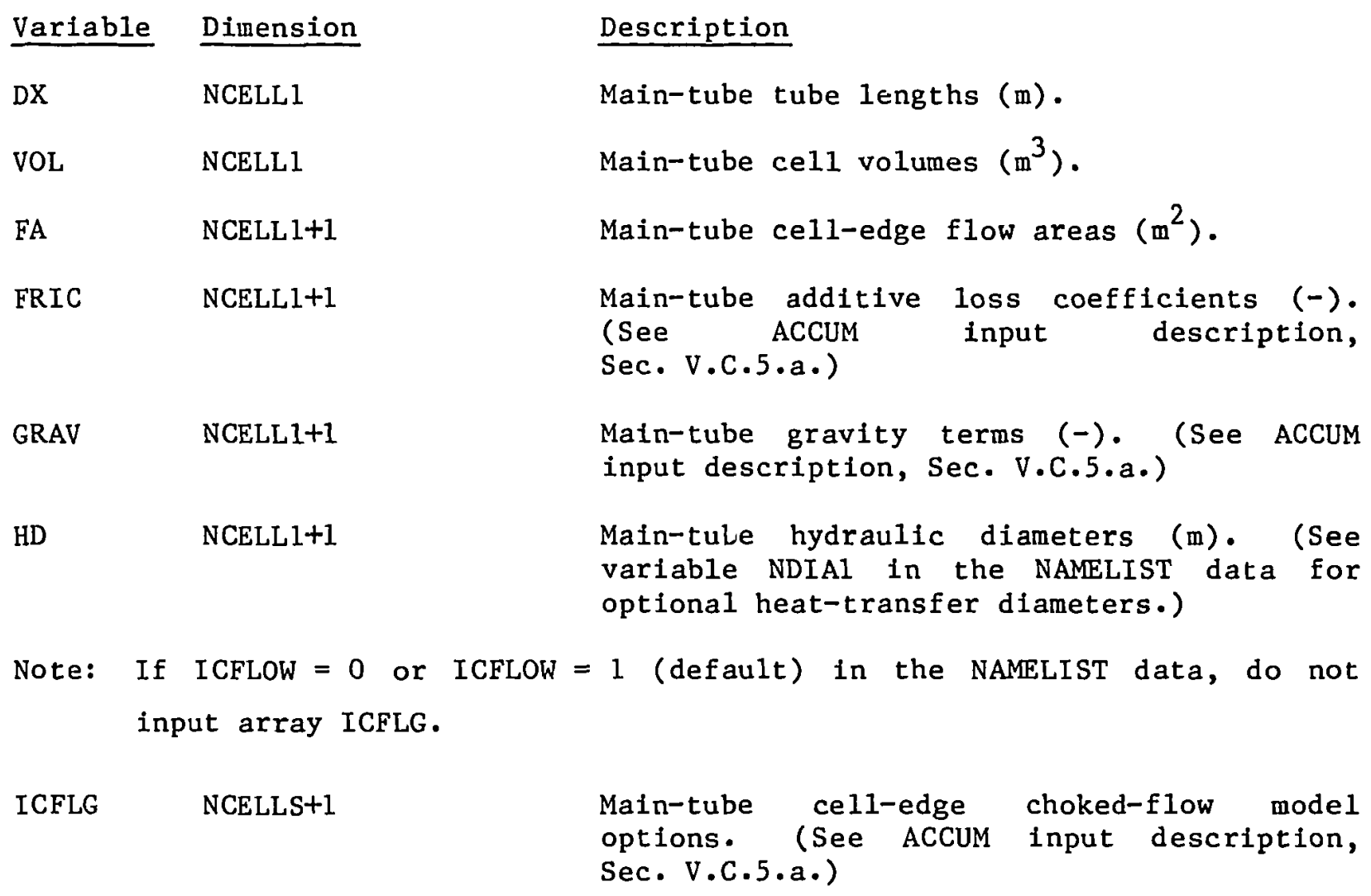




$\begin{array}{ll}\text { Variable } & \text { Dimensio } \\ \text { NFF } & \text { NCELL1+1 } \\ \text { ALP } & \text { NCELL1 } \\ \text { VL } & \text { NCELL1+1 } \\ \text { VV } & \text { NCELL1+1 } \\ \text { TL } & \text { NCELL1 } \\ \text { TV } & \text { NCELL1 } \\ \text { P } & \text { NCELL1 } \\ \text { PA } & \text { NCELL1 }\end{array}$

\section{Description}

Friction-factor correlation options for main tube (see ACCUM input description, Sec.V.C.5.a).

Main-tube initial void fractions (-).

Main-tube initial liquid velocities $\left(\mathrm{m} \cdot \mathrm{s}^{-1}\right)$.

Main-tube initial vapor velocities $\left(\mathrm{m} \cdot \mathrm{s}^{-1}\right)$.

Main-tube initial liquid temperatures $(K)$.

Main-tube initial vapor temperatures (K).

Main-tube initial pressures ( $\mathrm{Pa}$ ).

Main-tube Initial air partial pressures $(\mathrm{Pa})$.

Note: If NODES $=0$ (word 2 on Card Number 2), do not enter arrays QPPP, MATID, and TW.

QPPP NODES*NCELLS

A nonnormalized power profile ( - ) in the tee main-tube walls. Input for cell 1 , node 1 through NODES; then cell 2 , node 1 through NODES; etc. If the array is filled with the same nonzero constant, a uniform volumetric heat source for the wall results. The code will normalize the power profile on a nodevolume basis (normalized QPPP(i)/[ $[\mathrm{QPPP}(i)$ *VOL(i)]). Filling the array $i$ with zeros results in no power being deposited in the wall regardless of settings of QP3IN, QP3TB, etc.

MATID NODES-1

Wal1-material ID array specifying material ID between nodes in the tee-main tube. Dimension is 1 if NODES $=1$ and 0 if NODES $=0$ (see available built-in properties listed with array MATID in the CORE input, Sec.V.C.5.c, or see Sec.V.C.2 to input material properties). 


\begin{tabular}{|c|c|c|c|}
\hline Variab1 & & Dimension & Description \\
\hline $\mathrm{TW}$ & & NODES*NCELL1 & $\begin{array}{l}\text { Tee main-tube initial wall temperatures (K) } \\
\text { input in the same order as QPPP. }\end{array}$ \\
\hline Note: & If & ICONC1 $=0$ (word 1 on & Card Number 3), omit card set CONC. \\
\hline CONC & & NCELL1 & $\begin{array}{l}\text { Initial ratio of solute mass to liquid } \\
\text { coolant mass in the tee main tube. }\end{array}$ \\
\hline Note: & If & ICONC2 $=0$ or 1 (word & 1 on Card Number 9 ), omit card set $S$. \\
\hline $\mathbf{S}$ & & NCELL1 & $\begin{array}{l}\text { Initial macroscopic density of plated-out } \\
\text { solute in the tee side tube }\left(\mathrm{kg} \cdot \mathrm{m}^{-3}\right) \text {. }\end{array}$ \\
\hline Note: & If & NPWTB1 $=0$ (word 3 on & Card Number 4 ), omit card set POWTBl. \\
\hline POWTB1 & & $\mid$ NPWTB $1 \mid * 2$ & $\begin{array}{l}\text { Power-to-fluid vs independent-variable-form } \\
\text { table for the tee main tube. Input } \\
\text { (independent-variable form defined by IPWSVl } \\
\text { and NPWTB } 1 \text { on Card Number } 4 \text {, power) pairs } \\
(*, W) \text { The power is deposited directly into } \\
\text { the tee main-tube fluid with a uniform } \\
\text { volumetric power density along the tube } \\
\text { length. }\end{array}$ \\
\hline
\end{tabular}

Note: If NPWTB1 $=0$ (word 3 on Card Number 4) or NPWRF1 $=0$ (word 5 on Card Number 4), omit card set POWRFl.

POWRF1 $\quad \mid$ NPWRF1 $\mid * 2$

Rate-factor table for the tee main-tube POWTB table's independent variable. Input (independent-variable form defined by NPWSV1 and NPWRF1 on Card Number 4, rate factor to be applied to the POWTB table's independent variable) pairs $(*,-)$. 
Note: If NQPTB1 $=0$ (word 3 on Card Number 5), omit card set QP3TB1.

\begin{tabular}{|c|c|}
\hline Variable & Dimension \\
\hline QP3TB1 & $\mid$ NQPTB1 $\mid * 2$ \\
\hline & $\begin{array}{l}\text { when QPIN1 >0; } \\
\mid \text { NQPTB } 1 \mid *(1+\text { NCELL } 1) \\
\text { QPIN } 1<0\end{array}$ \\
\hline
\end{tabular}

\section{Description}

Power-to-wall independent-variable-form table for the tee main tube. Input (independent-variable form defined by IQPSV1 and when NQPTB1 on Card Number 5, power-to-wa11) pairs $(*, W)$. If QP3IN $>0.0$, the dependent variable specifies the total power to the entire wall; if QP3IN $<0.0$, the dependent variable is a vector specifying the power to the wall at each cell beginning with cell 1 .

Note: If $\mathrm{NQPTB1}=0$ (word 3 on Card Number 5) or NQPRF1 $=0$ (word 5 on Card Number 5), omit card set QP3RF1.

QP3RF1 |NQPRF1|*2

$\begin{array}{ll}\begin{array}{ll}\text { DX } \\ \text { VOL }\end{array} & \text { NCELL2 } \\ \text { FA } & \text { NCELL2 } \\ \text { FRIC } & \text { NCELL2+1 } \\ & \\ \text { GRAV } & \text { NCELL2+1 } 2+1 \\ & \\ \text { HD } & \text { NCELL2+1 }\end{array}$

Rate-factor table for the tee main-tube QP3TB table's independent variable. Input (independent-variable form defined by NQPSV1 and NQPRF1 on Card Number 5, rate factor to be applied to the QP3TB table's independent variable) pairs $(*,-)$.

Side-tube cell lengths (m).

Side-tube cell volumes $\left(\mathrm{m}^{3}\right)$.

Side-tube cell-edge flow areas $\left(\mathrm{m}^{2}\right)$.

Side-tube additive loss coefficients (-). (See ACCUM input description, Sec.V.C.5.a.)

Side-tube gravity terms (-). (See ACCUM input description, Sec.V.C.5.a.)

Side-tube hydraulic diameters (m). (See variable NDIAl in the NAMELIST data for optional heat-transfer diameters.)

Note: If ICFLOW $=0$ or ICFLOW $=1$ (default) in the NAMELIST data, do not input array ICFLG.

ICFLG NCELL2+1

Side-tube cell-edge choked-flow model options. (See ACCUM input description, Sec.V.C.5.a.) 
Tee Component (TEE)

\begin{tabular}{|c|c|c|}
\hline Variable & Dimension & Description \\
\hline NFF & NCELL2+1 & $\begin{array}{l}\text { Friction-factor correlation options for side } \\
\text { tube (see ACCUM input description, } \\
\text { Sec.V.C.5.a). }\end{array}$ \\
\hline ALP & NCELL2 & Side-tube initial void fractions $(-)$. \\
\hline VL & NCELL2+1 & $\begin{array}{l}\text { Side-tube } \\
\left(\mathrm{m} \cdot \mathrm{s}^{-1}\right)\end{array}$ \\
\hline VV & NCELL2+1 & $\begin{array}{l}\text { Side-tube } \\
\left(\mathrm{m} \cdot \mathrm{s}^{-1}\right)\end{array}$ \\
\hline $\mathrm{TL}$ & NCELL2 & Side-tube initial liquid temperatures (K). \\
\hline TV & NCELL2 & Side-tube initial vapor temperatures $(K)$. \\
\hline $\mathbf{P}$ & NCELL2 & Side-tube initial pressures $(\mathrm{Pa})$. \\
\hline PA & NCELL2 & initial air partial \\
\hline
\end{tabular}

Note: If NODES $=0$ (word 2 on Card Number 2), do not enter arrays QPPP, MATID, and TW.

QPPP NODES*NCELLS

A nonnormalized power profile $(-)$ in the tee side-tube walls. Input for cell 1 , node 1 through NODES; then cell 2 , node 1 through NODES; etc. If the array is filled with the same nonzero constant, a uniform volumetric heat source for the wall results. The code will normalize the power profile on a nodevolume basis (normalized QPPP(i)/[ $\sum$ QPPP (i) *VOL(i)]). Filling the array $i$ with zeros results in no power being deposited in the wall regardless of settings of QP3IN, QP3TB, etc.

MATID NODES-1

Wall-material ID array specifying material ID between nodes in the tee-side tube. Dimension is 1 if NODES $=1$ and 0 if NODES $=0$ ( listed with the array MATID in CORE infut, Sec.V.C.5.c, or see Sec.V.C.2 to input material properties). 
Variable Dimension

TW

NODES*NCELL2

Description

Tee side-tube initial wall temperatures $(K)$ input in the same order as QPPP.

Note: If ICONC2 $=0$ (word 1 on Card Number 9), omit card set CONC.

CONC NCELL2 Initial ratio of solute mass to liquid coolant mass in the tee side tube.

Note: If ICONC1 $=0$ or 1 (word 1 on Card Number 3 ), omit card set $\mathrm{S}$.

$\mathrm{S}$

NCELL2

Initial macroscopic density of plated-out solute in the tee main tube $\left(\mathrm{kg} \cdot \mathrm{m}^{-3}\right)$.

Note: If NPWTB2 $=0$ (word 3 on Card Number 10), omit card set POWTB2.

POWTB2 $\mid$ NPWTB2 $\mid * 2$

Power-to-fluid vs independent-variable-form table for the tee side tube. Input - (independent-variable form defined by IPWSV2 and NPWTB2 on Card Number 10, power) pairs $(*, W)$. The power is deposited directly into the tee side-tube fluid with a uniform volumetric power density along the tube length.

Note: If NPWTB2 $=0$ (word 3 on Card Number 10) or NPWRF2 $=0$ (word 5 on Card Number 10 ), omit card set POWRF.

POWRF $\quad \mid$ NPWRF2 $\mid * 2$

Rate-factor table for the tee side-tube POWTB table's independent variable. Input (independent-variable form defined by NPWSV2 and NPWRF2 on Card Number 10, rate factor to be applied to the POWTB table's independent variable) pairs $(*,-)$. 
Note: If NQPTB2 $=0$ (word 3 on Card Number 11), omit card set QP3TB2.

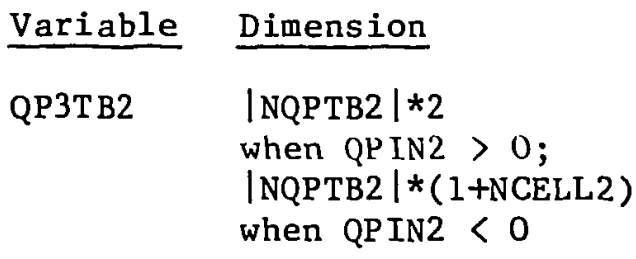

\section{Description}

Power-to-wall vs independent-variable form table for the tee side tube. Input (independent-variable form defined by IQPSV2 and IQPSV2 and NQPTB2 on Card Number 11 , powerto-wall) pairs $(*, W)$. If $Q P 3 I N>0.0$, the dependent variable specifies the total power to the entire wall; if QP3IN $<0.0$, the dependent variable is a vector specifying the power to the wall at each cell beginning with cell 1.

Note: If NQPTB2 $=0$ (word 3 on Card Number 11) or NQPRF2 $=0$ (word 5 on Card Number 11), omit card set QP3RF2.

QP3RF2 |NQPRF2 $\mid * 2$

Rate-factor table for the tee side-tube QP3TB table's independent variable. Input (independent-variable form, defined by NQPSV2 and NQPRF2 on Card Number 11, rate factor to be applied to the QP3TB table's independent variable) pairs $(*,-)$. 
Turbine Component (TURB)

k. Turbine Component (TURB). Each turbine stage is modeled as a separate TURB component.)

Card Number 1. (Format A10,4X,2I14,A30) TYPE, NUM, ID, CTITLE

Columns Variable

1-14 TYPE

15-28 NUM

29-42 ID

43-72 CTITLE

Card Number 2. (Format 5I14) NCELLS, JUNI, JUN2, ICONC, IPOW

Description component, $1 \leq$ NUM $\leq 99$ ).

User ID number (arbitrary).

1-14 NCELLS

15-28 JUN1

29-42 JUN2

43-56 ICONC

$57-70 \quad$ IPOW

Description

Component type (TURB left-justified).

Component ID number (must be unique for each

Hollerith component description.

Number of fluid cells in the component.

Junction number for junction adjacent to cell 1 .

Junction number for junction adjacent to Ce11 NCELLS.

Flag for input of solute in the liquid coolant. [Requires ISOLUT $=1$ (word 5 on Main-Data Card 6).]

0 = no input;

$1=$ dissolved solute only; or

2 = both dissolved and plated-out solute.

Power-to-fluid flag.

$0=$ no;

1 = yes. 
Note: If IPOW $=0$ (word 5 on Card Number 2), omit Card Numbers 3 and 4 .

Card Number 3. (Format 5I14) IPOWTR, IPOWSV, NPOWTB, NPOWSV, NPOWRF

\begin{tabular}{|c|c|c|}
\hline Columns & Variable & Description \\
\hline $1-14$ & IPOWTR & $\begin{array}{l}\text { The trip ID number that controls evaluation } \\
\text { of the power-to-fluid table (POWTB) } \\
\text { [|IPOWTR|<10000] (input IPOWTR }=0 \text { if } \\
\text { there is to be no trip control and the table } \\
\text { will be evaluated in the transient.) }\end{array}$ \\
\hline $15-28$ & IPOWSV & $\begin{array}{l}\text { The independent-variable ID number for the } \\
\text { power-to-fluid table (POWTB). IPOWSV }>0 \\
\text { defines the ID number for a signal-variable } \\
\text { parameter; IPOWS < } 0 \text { defines the ID number } \\
\text { for a control-block output parameter. }\end{array}$ \\
\hline $29-42$ & NPOWTB & $\begin{array}{l}\text { The number of ( } x, y \text { ) power-to-fluid table's } \\
\text { (POWTB) pairs (defined by the absolute value } \\
\text { of NPOWTB). NPOWTB }>0 \text { defines the table's } \\
\text { independent-variable form to be the IPOWSV } \\
\text { parameter; NPOWTB } 0 \text { defines the table's } \\
\text { independent-variable form to be the change } \\
\text { in the IPOWSV parameter over the last time } \\
\text { step times the trip set-status value ISET } \\
\text { when the power-to-fluid table is trip } \\
\text { controlled; NPOWTB = 0 defines the power to } \\
\text { be the IPOWSV parameter. }\end{array}$ \\
\hline $43-56$ & NPOWSV & $\begin{array}{l}\text { The independent-variable ID number for the } \\
\text { rate factor that is applied to the power-to- } \\
\text { fluid table s independent variable. } \\
\text { NPOWSV }>0 \text { defines the ID number for a } \\
\text { signal-variable parameter; NPOWSV } 0 \text { de- } \\
\text { fines the ID number for a control-block out- } \\
\text { put parameter; NPOWSV = } 0 \text { (when NPOWR } \neq 0 \text { ) } \\
\text { defines the independent variable to be the } \\
\text { difference between the trip signal and the } \\
\text { set-point value that turns the trip OFF when } \\
\text { the power table is trip controlled. }\end{array}$ \\
\hline
\end{tabular}




$\frac{\text { Columns }}{57-70} \quad \frac{\text { Variable }}{\text { NPOWRF }}$

Card Number 4. (Format 4E13.6)

Columns $\quad \frac{\text { Variable }}{1-14}$

15-28 POWOFF

29-42 RPOWMX

43-56 POWSCL

\section{Description}

The number of $(x, y)$ rate-factor table pairs (defined by the absolute value of NPOWRF). The rate factor is applied as a factor to the power-to-fluid table-s (POWTB) independent variable when the rate factor is defined. No rate factor is defined when NPOWSV and NPOWRF are both zero. NPOWRF $>U$ defines the rate-factor table's independent variable to be the NPOWSV parameter; NPOWRF < 0 defines it to be the change in the NPOWSV parameter over the last time step times the trip set-status value ISET when the power table is trip controlled; NPOWRF $=0$ defines the rate factor to be the NPOWSV parameter.

POWIN, POWOFF, RPOWMX, POWSCL

Description

Initial total power ( $W$ ) deposited in the turbine fluid (not used when IPOW $=0$ ). The power is distributed linearly along the component.

Total power ( $W$ ) deposited in the turbine fluid when the controlling trip is OFF after being ON (not used if IPOWTR $=0$ ). If POWOFF $\leq-1.0 \times 10^{19}$, the power to the fluid is held constant at the last table-evaluated power when the trip was $\mathrm{ON}$.

The maximum rate of change of power $\left(W \cdot s^{-1}\right.$ ) deposited in the turbine fluid (RPOWMX $\geq 0.0$ ) [not used when IPOW $=0$ ].

Scale factor for the power-to-fluid table. The dependent variable in the POWTB table is multiplied by POWSCL to obtain absolute power deposited in the fluid (not used when IPOW $=0$ or NPOWTB $=0$ ). 
Card Number 5. (Format 2I14, E14.6) ISTG, NEFCON, EFFDSN

\begin{tabular}{|c|c|c|}
\hline Columns & Variable & Description \\
\hline $1-14$ & ISTG & $\begin{array}{l}\text { Turbine stage number this TURB component } \\
\text { represents (if there is only one stage in } \\
\text { the turbine, ISTG }=1 \text { ). }\end{array}$ \\
\hline $15-28$ & NEFCON & $\begin{array}{l}\text { Flag to indicate whether the stage } \\
\text { efficiency is to be treated as constant. } \\
\text { NEFCON =0, the efficiency of the stage to } \\
\text { be computed internally. NEFCON }=1 \text {, the } \\
\text { stage efficiency to be treated as a } \\
\text { constant. }\end{array}$ \\
\hline $29-42$ & EFFDSN & $\begin{array}{l}\text { Stage efficiency at design conditions } \\
\text { (include windage loss, diaphragm-packing } \\
\text { loss, moisture loss, and nozzle-end loss; } \\
\text { thus, stage efficiency equals nozzle-bucket } \\
\text { efficiency minus all the losses listed } \\
\text { above). Note: If NEFCON }=1 \text {, EFFDSN value } \\
\text { will be used for stage efficiency under, all } \\
\text { conditions. If NEFCON }=0 \text {, the stage } \\
\text { efficiency at the off-design condition will } \\
\text { be calculated using EFFDS. If NEFCON } 0 \\
\text { and EFFDS } 0.0 \text {, the code calculates a } \\
\text { realistic value of EFFDS from quantities } \\
\text { input on Card Numbers } 6-8 \text { and Eard Set } 15 \text {. }\end{array}$ \\
\hline
\end{tabular}

Note: All the quantities entered on Card Number 6 should be consistent with the design efficiency, EFFDSN (word 3 on Card Number 5).

Card Number 6. (Format 5E14.6) PRES1, PRES2, QUALTY, SUPRHT, FLOW

$\begin{array}{lll}\text { Columns } & \text { Variable } & \text { Description } \\ 1-14 & \text { PRES1 } & \text { Stage upstream design pressure. } \\ 15-28 & \text { PRES2 } & \text { Stage downstream design pressure. } \\ 29-42 & \text { QUALTY } & \begin{array}{l}\text { Stage upstream design quality of steam (if } \\ \text { superheated, QUALTY }=1.0) .\end{array} \\ 43-56 & \text { SUPRHT } & \begin{array}{l}\text { Stage upstream degree of superheat of steam } \\ \text { at design conditions } \\ \text { SUPRHT }=0.0) .\end{array}\end{array}$




\begin{tabular}{|c|c|c|}
\hline Columns & Variable & Description \\
\hline $57-70$ & FLOW & Mixture mass flow at design conditions. \\
\hline Note: If 1 & NEFCON $\neq 0$ (word 2 on & Card Number 5), omit Card Numbers 7 and 8 \\
\hline Card Number & 7. (Format 5E14.6) & DIA, COEF1, COEF2, COF3SQ, REACTN \\
\hline Columns & Variable & Description \\
\hline $1-14$ & DIA & Pitch circle diameter of the buckets. \\
\hline $15-28$ & COEF1 & $\begin{array}{l}\text { Nozzle coefficient. The nozzle" exit } \\
\text { velocity is calculated by multiplying the } \\
\text { ideal isentropic velocity by COEFl. }\end{array}$ \\
\hline $29-42$ & COEF2 & $\begin{array}{l}\text { Bucket coefficient. Defined in the same } \\
\text { manner as COEFl. }\end{array}$ \\
\hline $43-56$ & COF3SQ & $\begin{array}{l}\text { Fraction of reaction energy actually } \\
\text { delivered in the entire stage [reaction loss } \\
\text { coefficient based on the energy (velocity }{ }^{2} \text { ) } \\
\text { instead of velocity]. }\end{array}$ \\
\hline $57-70$ & REACTN & $\begin{array}{l}\text { Degree of reaction at design conditions (if } \\
\text { impulse stage, REACTN }=0.0 \text { ). }\end{array}$ \\
\hline
\end{tabular}

Card Number 8. (Format I14) NROWS

Columns Variable

Description

1-14 NROWS

Number of rows of moving blades.

Note: If ISTG $\neq 1$ (word 1 on Card Number 5), omit Card Numbers 9 to 13 and Card Set Number 14 .

Card Number 9. (Format I14) NSTGES

Columns Variable Description

1-14 NSTGES

Total number of stages associated with the entire turbine. 
Turbine Component (TURB)

Card Number 10. (Format 5I14) ITRBTR, ITRBSV, NTRBTB, NTRBSV, NTRBRF

$\frac{\text { Columns }}{1-14} \quad \frac{\text { Variable }}{\text { ITRBTR }}$

15-28 ITRBSV

29-42 NTRBTB

43-56 NTRBSV

57-70 NTRBRF

\section{Description}

The trip ID number that controls the evaluation of the generator power-demand table ( $\mid$ ITRBTR $\mid<10000)$ [ITRBTR = 0 implies constant power demand].

The generator power-demand table abscissacoordinate variable ID number. ITRBSV $>0$ defines the ID number for a signal-variable parameter; ITRBSV < 0 defines the ID number for a control-block output parameter.

The number of $(x, y)$ generator power-demand table's pairs (defined by the absolute value of NTRBTB). NTRBTB >0 defines the table's independent-variable form to be the ITRBSV parameter; NTRBTB < 0 defines the table"s independent-variable form to be the change in the ITRBSV parameter over the last time step times the trip set-status value ISET; NTRBTB $=0$ defines the generator powerdemand to be the ITRBSV parameter.

The rate-factor table's abscissa-coordinate variable ID number. NTRBSV defines the independent-variable parameter for the ratefactor table that is applied as a factor to the generator power-demand table's independent variable. NTRBSV $>0$ defines the ID number for a signal-variable paraneter; NTRBSV $<0$ defines the ID number for a control-block output parameter; NTRBSV $=0$ (when NTRBRF $\neq 0$ ) defines the difference between the trip signal and the set-point value that turns the trip OFF.

The number of $(x, y)$ rate-factor table's pairs (defined by the absolute value of NTRBRF). NTRBRF >0 defines the rate-factor table's abscissa coordinate to be the NTRBSV parameter; NTRBRF < 0 defines it to be the change in the NTRBSV parameter over the last time step times the trip set-status value ISET; NTRBRF $=0$ defines the rate factor to be the NTRBSV parameter. 
Card Number 11. (Format 4E14.6) POWOP, POPOFF, RPOPMX, POPSCL

\begin{tabular}{|c|c|}
\hline Columns & Variabl \\
\hline $1-14$ & POWOP \\
\hline $15-28$ & POPOFF \\
\hline $29-42$ & RPOPMX \\
\hline $43-56$ & POPSCL \\
\hline
\end{tabular}

Description .

Initial generator operating power ( $W$ ).

Generator operating power (W) when the generator operating-power controlling trip is OFF after being ON (maintain the last table-evaluated generator operating power when the trip was $O N$ if POPUFF $\leq-1.0 \times 10^{19}$ ) [not input when ITRBTR $=0$ ].

The maximum rate of change of generator operating power $\left(W \cdot s^{-1}\right)$ [RPOPMX 20.0$]$.

Generator operating-power scale factor. The dependent variable in the generator operating-power TRBTB table is multiplied by POPSCL to obtain absolute generator operating power.

Card Number 12. (Format 4E14.6) POWD, OMEGD, OMEGOP, EFGEN

$\frac{\text { Columns }}{1-14} \quad$ Variable

Description

Generator design power (W) [must be consistent with parameters entered on Card Number 6 and EFFDSN entered on Card Number 5].

15-28 OMEGD

Turbine/generator design speed $\left(\mathrm{rad} \cdot \mathrm{s}^{-1}\right)$ [must be consistent with POWD, parameters entered on Card Number 6 , and EFFDSN entered on Card Number 5].

29-42 OMEGOP

Turbine/generator initial operating speed $\left(\mathrm{rad} \cdot \mathrm{s}^{-1}\right)$.

43-56 EFGEN

Generator efficiency.

Card Number 13. (Format 4E14.6) ATORK, BTORK, CTORK, INRTA

Columns Variable Description

1-14 ATORK

Turbine/generator friction torque zero-order coefficient ( $\mathrm{N} \cdot \mathrm{m}$ or $\mathrm{Pa} \cdot \mathrm{m}^{3}$ ) [torque is calculated as ATORK+BTORK $\star_{\omega}+\mathrm{CTORK}_{\omega}{ }^{2}$, where $\omega$ is the turbine speed]. 


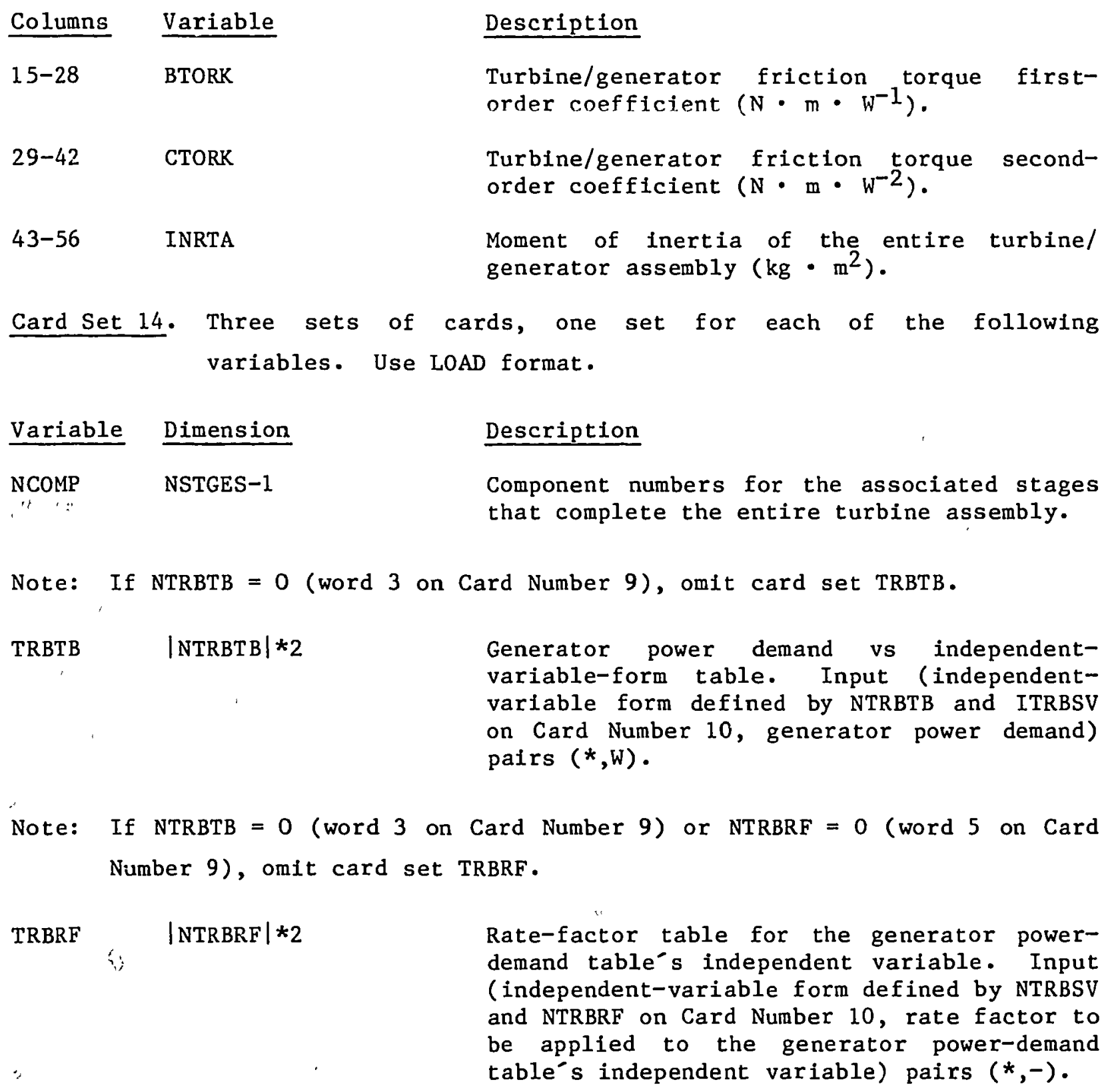

Card Set 14. Three sets of cards, one set for each of the following variables. Use LOAD format. variable form defined by NTRBTB and ITRBSV on Card Number 10, generator power demand) pairs $(*, W)$.

Note: If NTRBTB $=0$ (word 3 on Card Number 9) or NTRBRF $=0$ (word 5 on Card Number 9), omit card set TRBRF.

TRBRF ; $\mid$ NTRBRF $\mid * 2$

Rate-factor table for the generator powerdemand table's independent variable. Input (independent-variable form defined by NTRBSV and NTRBRF on Card Number 10, rate factor to be applied to the generator power-demand table $s$ independent variable) pairs $(*,-)$. 
Note: If NEFCON $\neq 0$ (word 2 on Card Number 5), omit Card Set 15.

Card Set 15. Use LOAD format.

Variable Dimension

ANGL $\quad$ NROWS*2

\section{Description}

Nozzle exit and blade angles (degrees). Input as follows: steam exit angle from the nozzle, first moving blade exit angle, first stationary blade exit angle, second moving blade exit angle, etc.

TURB Array Cards. Nineteen sets of cards, one set for each of the following variables. Use LOAD format.

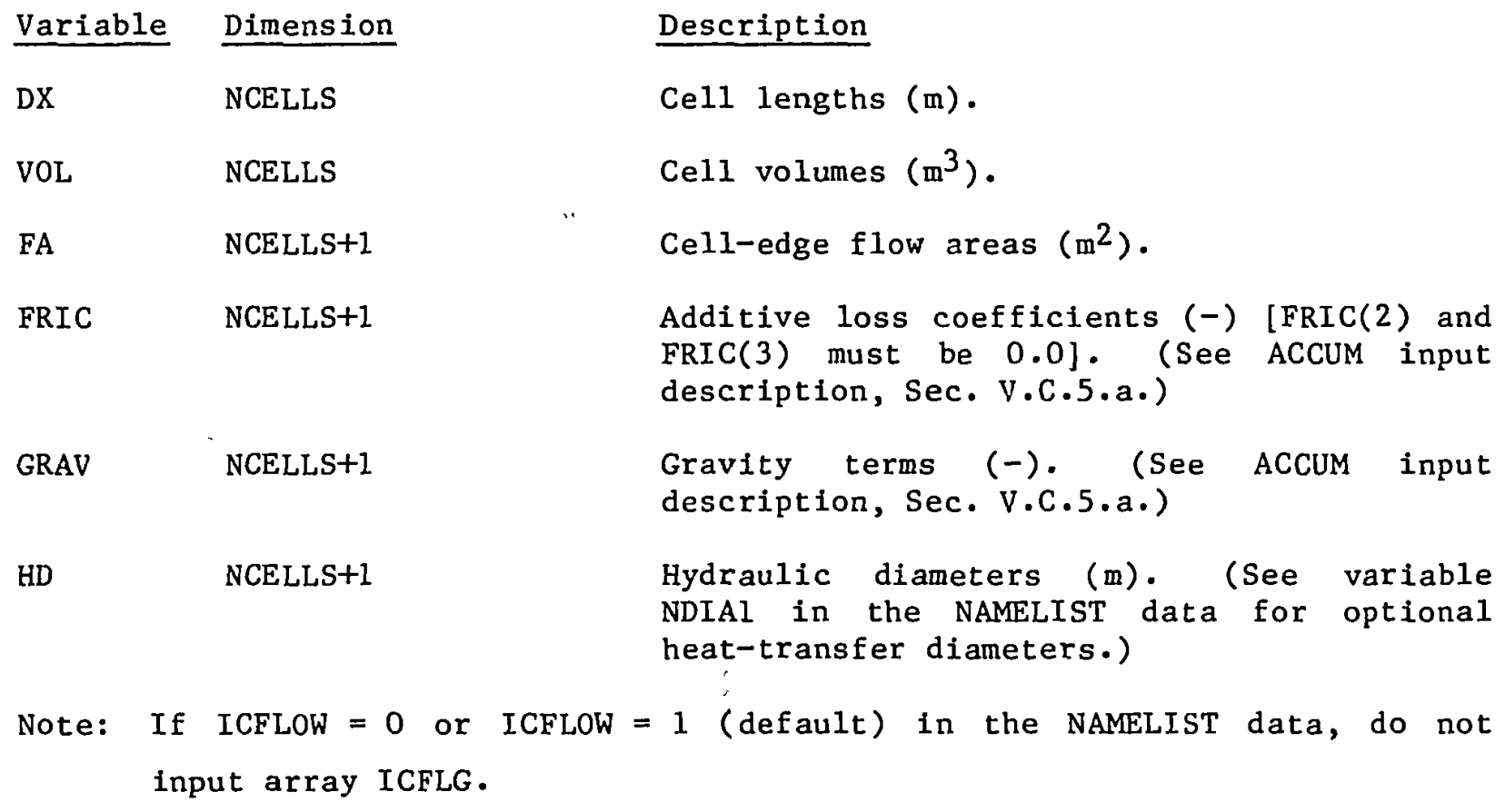

ICFLG NCELLS+1

Cell-edge choked-flow model options (see ACCuM input description, Sec. V.C.5.a). The value ICFLG $=0$ is suggested for this component.

NFF

NCELLS+1

Friction-factor correlation options (see ACCUM input description, Sec.V.C.5.a). The value $\mathrm{NFF}=1$ is suggested for this component. 
Turbine Component (TURB)

$\begin{array}{lll}\text { Columns } & \text { Variable } & \text { Description } \\ \text { ALP } & \text { NCELLS } & \text { Initial void fractions }(-) \cdot \\ \text { VL } & \text { NCELLS+1 } & \text { Initial liquid vclocitics }\left(m \cdot \mathrm{s}^{-1}\right) \text {. } \\ \text { VV } & \text { NCELLS+1 } & \text { Initial vapor velocities }\left(m \cdot \mathrm{s}^{-1}\right) . \\ \text { TL } & \text { NCELLS } & \text { Initial liquid temperatures }(\mathrm{K}) \cdot \\ \text { TV } & \text { NCELLS } & \text { Initial vapor temperatures }(\mathrm{K}) . \\ \text { P } & \text { NCELLS } & \text { Initial pressures }(\text { Pa) } . \\ \text { PA } & \text { NCELLS } & \text { Initial air partial pressures }(\mathrm{Pa}) .\end{array}$

Note: If ICONC $=0$ (word 4 on Card Number 2), omit card set CONC.

CONC NCELLS Initial ratio of solute mass to liquid coolant mass.

Note: If ICONC $=0$ or 1 (word 4 on Card' Number 2), omit card set s.

S NCELLS Initial macroscopic density of plated-out solute $\left(\mathrm{kg} \cdot \mathrm{m}^{-3}\right)$.

Note: If IPOW (word 5 on Card Number 2) or NPOWTB (word 3 on Card Number 3), omit card set POWTB.

POWTB $\quad \mid$ NPOWTB $\mid * 2$

Power-to-fluid vs independent-variable-form
table. Input (independent-variable form
defined by IPOWS and NPOWT on Card
Number 3, power) pairs (*, W). The power is
deposited directly into the fluid in the
turbine with a uniform volumetric power
density along the turbine length. 
Note: If IPOW $=0$ (word 5 on Card Number 2), NPOWTB $=0$ (word 3 on Card Number 3 ), or NPOWRF $=0$ (word 5 on Card Number 3 ), omit card set POWRF.

\section{Columns Variable}

POWRF $\mid$ NPOWRF $\mid * 2$

\section{Description}

Rate-factor table for the POWTB table's independent variable. Input (independentvariable form defined by NPOWSV and NPOWRF on Card Number 3, rate factor to be applied to the POWTB table's independent variable) pairs $(*,-)$. 


\section{Valve Component (VALVE).}

Card Number 1. (Format A10,4X,2I14,A30) TYPE, NUM, ID, CTITLE

$\begin{array}{lll}\text { Columns } & \text { Variable } & \text { Description } \\ 1-5 & \text { TYPE } & \text { Component type (VALVE left-justified). } \\ 15-28 & \text { NUM } & \begin{array}{l}\text { Component ID number (must be unique for each } \\ \text { component, } 1 \leq \text { NUM } \leq 999 \text { ). }\end{array} \\ 29-42 & \text { ID } & \text { User ID number (arbitrary). } \\ 43-72 & \text { CTITLE } & \text { Hollerith component description. }\end{array}$

Card Number 2. (Format 5I14) NCELLS, NODES, JUN1, JUN2, MAT

$\begin{array}{ll}\begin{array}{ll}\text { Columns } \\ 1-14\end{array} & \text { Nariable } \\ 15-28 & \text { NODES } \\ 29-42 & \text { JUN1 } \\ 43-56 & \text { JUN2 } \\ 57-70 & \text { MAT }\end{array}$

Description

Number of fluid cells (must be at least two).

Number of radial heat-transfer nodes in valve wall. A value of 0 spectfies no wall heat transfer.

Junction number for junction adjacent to cell 1 .

Junction number for junction adjacent to cell NCELLS.

Material ID number of the wall (currently not used).

Card Number 3. (Format 5I14) ICHF, ICONC, IVTY, IVPS, NVTB2

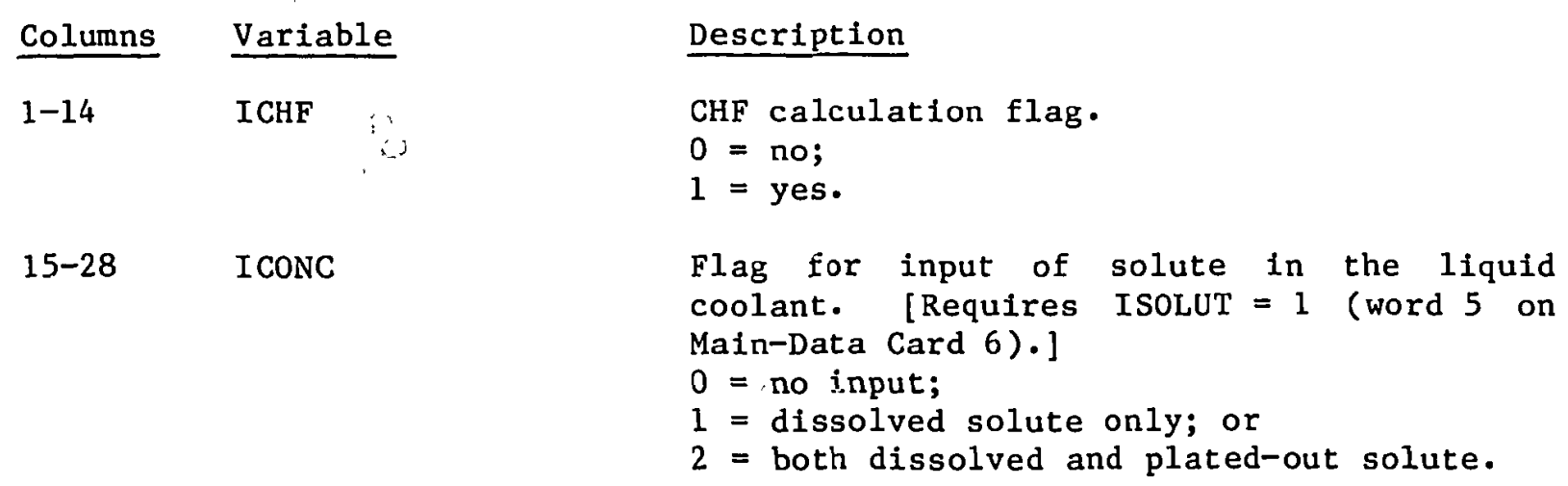


Valve Component (VALVE)

Columns Variable

$29-42$

$43-56$

IVPS

\section{Description}

Valve-type option.

$0=$ constant flow area;

$1=$ llow-dred fraclion vs independentvariable-form table is evaluated;

2 = relative valve-stem position $(0.0$ = fully closed, $1.0=$ fully opened) vs independent-variable-form table is evaluated;

3 = constant flow area until trip is set $O N$, then flow-area fraction vs independentvariable-form table is evaluated;

4 = constant relative valve-stem position until "trip is set oN, then relative valve-stem position vs independentvariable-form table is evaluated;

5 = valve is to be operated by a spectal turbine-component signal where increase in generator power demand opens the valve; or

6 = Similar to IVTY $=5$ except that an increase in generator power demand closes the valve.

The mesh-cell-interface number where the valve flow area is adjusted ( 1 < IVPS $<$ NCELLS+1 unless a BREAK component is at the VALVE component junction; then IVPS can equal 1 or NCELLS+1). 
Columns Variable

57-70 NVTB2

\section{Description}

The number of second valve table $(x, y)$ pairs (defined by the absolute value of NVTB2). Input NVTB2 $=0$ when IVTY $=0,1,2,5$, or 6. For IVTY $=3$ or 4 , NVTB2 $>0$ defines the table's independent-variable form to be the IVSV paramerer; NVTB2 $<0$ defines the table's independent-variable form to be the change in the IVSV parameter over the last time step times the trip set-status value ISET when the valve table is trip controlled; NVTB2 $=0$ (when NVTB1 $=0$ ) defines the valve flow-area fraction or relative valve-stem position to be the IVSV parameter value. When NVTB2 $=0$ and NVTBl $\neq 0$, no second valve table is defined. only the first valve table is used. When NVTB2 $\neq 0$, NVTB2 and NVTB1 have the same numerical sign, and the first valve table is used when the controlling trip is set to $\mathrm{ON}_{\text {forward, }}$ and the second valve table is used when the trip is set to ON reverse. NVTB1 $=0$, and NVTB2 $\neq 0$ is invalid.

Note: For valve-type option IVTY $=5$ or 6 , variables NVTB2 and IVSV, NVTBl, NVSV, and NVRF on Card Number 4 are defined by zero or left blank, and the arrays VTB1, VTB2, and VRF are not input.

Card Number 4. (Format 5I14) IVTR, IVSV, NVTBl, NVSV, NVRF

\section{Columns Variable}

$1-14 \quad$ IVTR

15-28 IVSV

\section{Description}

The trip ID number for valve-type option" when IVTY $=3$ or 4 (|IVTR $\mid<10000)$ or the component number of turbine stage 1 for valve-type option when IVTY $=5$ or 6 $(1 \leq \operatorname{IVTR} \leq 999)$.

The valve-table abscissa-coordinate variable ID number. IVSV defines the independentvariable parameter for the valve-type option IVTY $=1$ to 4 tables. IVSV $>0$ defines the ID number for a signal-variable parameter; IVSV $<0$ defines the ID number for a control-block output parameter. 
Columns Variable

29-42 NVTB1

43-56 NVSV

57-70 NVRF

\section{Description}

The number of first valve table $(x, y)$ pairs (defined by the absolute value of NVTBI). Input NVTB1 $=0$ when IVTY $=0,5$ or 6 . For IVTY $=2$ to 4 , NVTBI $>0$ defines the table $-\mathrm{s}$ independent-variable form to be the IVSV parameter; NVTB1 < 0 defines the table's independent-variable form to be the change in the IVSV parameter over the last time step times the trip set-status value ISET when the valve table is trip controlled; NVTB1 $=0$ defines the valve flow-area fraction or relative valve-stem position (depending on the value of IVTY) to be the IVSV parameter.

The rate-factor table abscissa-coordinate variable ID number. NVSV defines the independent-variable parameter for the rate factor that is applied to the first (and second when defined) valve table's independent variable. NVSV >0 defines the ID number for a signal-variable parameter; NVSV $<0$ defines the ID number for a control-block output parameter; NVSV $=0$ (when NVRF $\neq 0$ ) defines the difference between the trip signal and the set-point value that turns the trip OFF.

The number of rate-factor table $(x, y)$ pairs (defined by the absolute value of NVRF). The rate factor is applied as a factor to the first (and second when defined) valve table's independent variable when the rate factor is defined. No rate factor is defined when NVSV and NVRF are both zero. NVRF $>0$ defines the rate-factor table's abscissa coordinate to be the NVSV parameter; NVRF < 0 defines it to be the change in the NVSV parameter over the last time step times the trip set-status value ISET when the valve table is trip controlled; $N V R F=0$ defii:es the rate factor to be the NVSV parameter. 
Note: If NODES $<1$ (word 2 on Card Number 2), omit Card Number 5.

Card Number 5. (Format 5I14) IQP3TR, IQP3SV, NQP3TB, NQP3SV, NQP3RF

$\begin{array}{ll}\text { Columns } & \text { Variable } \\ 1-14 & \text { IQP3TR } \\ 15-28 & \text { IQP3SV } \\ 29-42 & \text { NQP3TB } \\ & \\ 43-56 & \text { NQP3SV }\end{array}$

\section{Description}

The trip ID number that controls evaluation of the power-to-wall table (QP3TB) [|IQP3TR $\mid<10000]$ (input IQP3TR $=0$ when there is to be no trip control, and the table will be evaluated in the transient.)

The independent-variable ID number for the power-to-wall table (QP3TB). IQP3SV >0 defines the ID number for a signal-variable parameter; IQP3SV < 0 defines the ID number for a control-block output parameter.

The number of $(x, y)$ power-to-wall table (POWTB) pairs (defined by the absolute value of NQP3TB). NQP3TB > 0 defines the table's independent-variable form to be the IQP3SV parameter; NQP3TB < 0 defines the table's independent-variable form to be the change in the IQP3SV parameter over the last time step times, the trip set-status value ISET when the power-to-wall table is trip controlled; NQP3TB $=0$ defines the power to the wall to be the IQP3SV parameter.

The independent-variable ID number for the rate factor that is applied to the power-towall table's independent variable. NQP3SV $>0$ defines the ID number for a signalvariable parameter; NQP3SV $<0$ defines the ID number for a control-block output parameter; NQP3SV $=0$ (when NQP3RF $\neq 0$ ) defines the independent variableil to be the difference between the trip signal and the set-point value that turns the trip OFF when the power-to-wall table is trip controlled.

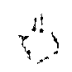


$\frac{\text { Columns }}{57-70} \quad \frac{\text { Variable }}{\text { NQP3RF }}$

Card Number 6. (Format 2I14) IVTROV, IVTYOV

\section{Description}

The trip ID number that overrides trip ID number IVTR (word 1 on Card Number 4) and opens ( $\mathrm{ON}_{\mathrm{forward}}$ ) or closes ( $\mathrm{ON}_{\text {reyerse }}$ ) the valve at the constant rate Rvov (word 2 on Card Number 7) when the trip is oN (input IVTROV $=0$ when IVTY $=5$ or 6 ) [|IVTROV] $<10000]$.

15-28 IVTYOV

Card Number 7 . (Format 4E14.6) RVMX, RVOV, FMINOV, FMAXOV

Columns $\quad$ Variable

Description

The maximum rate of valve flow-area fraction or relative valve-stem position adjustment $\left(s^{-1}\right)$.

15-28 RVOV

The rate of valve flow-area adjustment when the overriding trip IVTROV is ON $\left(\mathrm{s}^{-1}\right)$ [not used when IVTY $=5$ or 6 ]. 


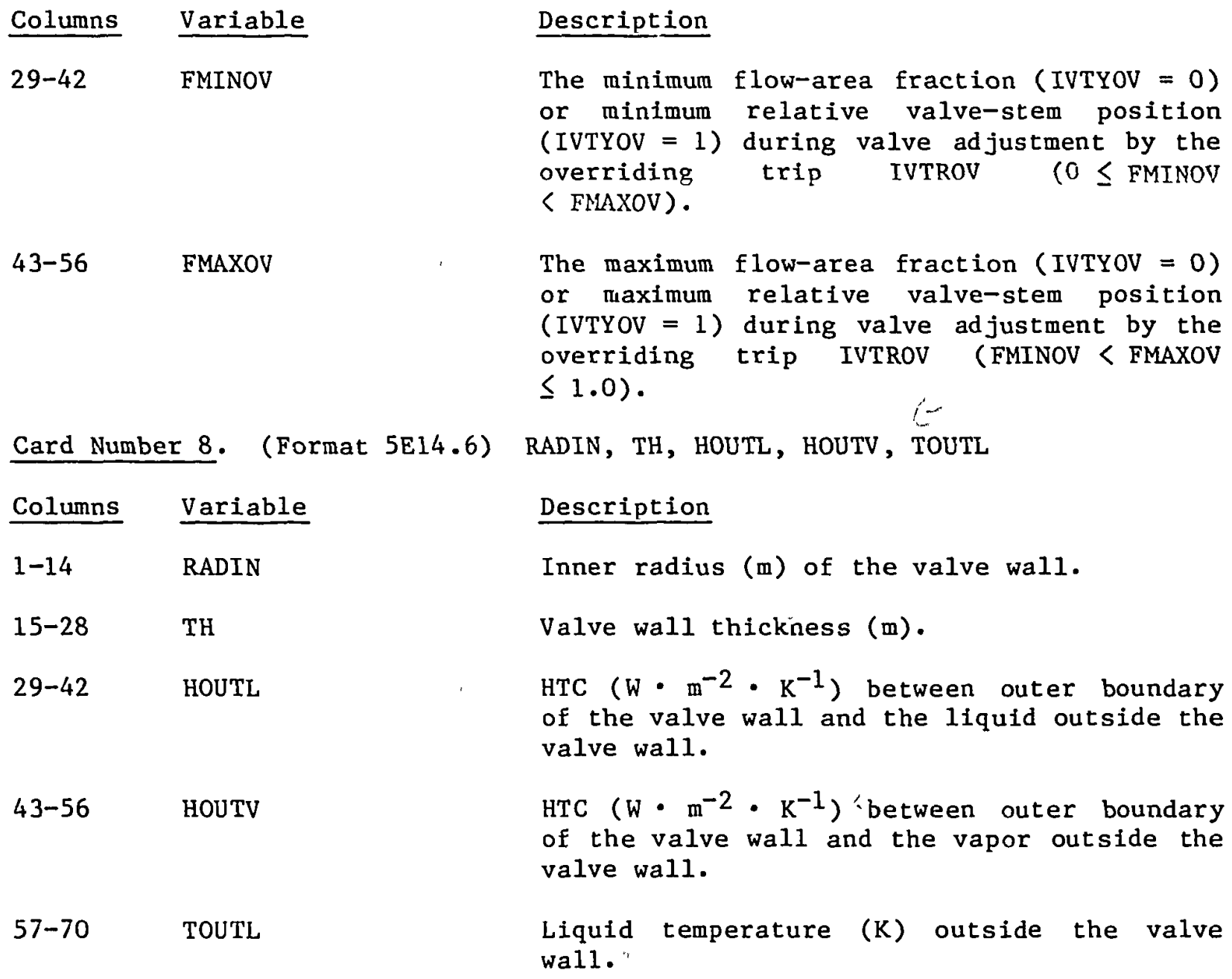

Note: See PIPE input description, Sec.V.C.5.e, for further comments on the HOUTL, HOUTV, TOUTL, and TOUTV heat-transfer parameters.

Card Number 9. (Format 5E14.6) TOUTV, AVLVE, HVLVE, FAVLVE, XPOS

$\begin{array}{ll}\frac{\text { Columns }}{1-14} & \text { Tariable } \\ 15-28 & \text { AVLVE }\end{array}$

Description

Vapor temperature (K) outside the valve wall.

Valve adjustable-interface flow area $\left(\mathrm{m}^{2}\right)$ when the valve is at a flow-area fraction or relative valve-stem position of 1.0 corresponding to $100 \%$ open. 
Valve Component (VALVE)

\begin{tabular}{|c|c|c|}
\hline Columns & Variable & Description \\
\hline $29-42$ & HVLVE & $\begin{array}{l}\text { "Valve adjustable-interface hydraulic diam- } \\
\text { eter }(m) \text { when the valve is } 100 \% \text { open. }\end{array}$ \\
\hline $43-56$ & FAVLVE & $\begin{array}{l}\text { Initial flow-area fraction at the valve } \\
\text { adjustable-interface IVPS (word } 4 \text { on Card } \\
\text { Number 3). If FAVLVE }\langle 0.0 \text { or }>1.0 \text {, FAVLVE } \\
\text { is evaluated based on the input XPos. }\end{array}$ \\
\hline $57-70$ & XPOS & $\begin{array}{l}\text { Initial relative valve-stem position at the } \\
\text { valve-adjustable interface IVPS }(0.0=\text { no } \\
\text { flow area, valve closed; } 1.0=\text { AVLVE flow } \\
\text { area, valve } 100 \% \text { opened). If } 0.0 \leq \text { FAVLVE } \\
\leq 1.0 \text { is input, a consistent value for XPOS } \\
\text { is evaluated in TRAC based on the valve stem } \\
\text { controlling a guillotine closure of a } \\
\text { circular flow-area cross section. 0ther- } \\
\text { wise, a consistent value of FAVLVE is evalu- } \\
\text { ated in TRAC based on } 0.0 \leq X P O S \leq 1.0 \text { that } \\
\text { is input. }\end{array}$ \\
\hline
\end{tabular}

Note: If NODES $<1$ (word 2 on Card Number 2), omit Card Number 10.

Card Number 10. (Format 4E14.6) QP3IN, QP30FF, RQP3MX, QP3SCL

Columns Variable

1-14 QP3IN

\section{Description}

Initial power ( $W$ ) deposited in the wall $1^{\prime \prime}$ and distributed according to the QPPP array. If QP3IN >0.0, it is the total power to the entire wall. When QP3IN $<0.0$ is input, the initial power to the wall in each cell is $|Q P 3 I N|$, and the negative sign flags the power to the will to be a cell-dependent vector of NCELLS (word 1 on Card Núber 2) powers. Each $(x, y)$ point of the power-towall table (for QP3IN $<0.0$ ) has $1+N C E L L S$ values [an independent-variable value $x$ and NCELLS power values $y(z)$ for cells 1 through NCELLS]. When the power-to-wall cable is not being evaluated, the same power value of $|\mathrm{QP} 3 I N|$ or QP3OFF (if QP3OFF $>-1.0 \times 10^{19}$ ) is applied at each of the NCELLS cells. 


$\begin{array}{ll}\text { Columns } & \text { Variable } \\ 15-28 & \text { QP3OFF } \\ 29-42 & \text { RQP3MX } \\ 43-56 & \text { QP3SCL }\end{array}$

Description

Power (W) to the wall when the controlling trip is OFF after being ON (maintain the last table-evaluated power when the trip was $O N$ if $Q P 3 O F F \leq-1.0 \times 10^{19}$ ).

The inaximum rate of change of the power to the wall (W. $\left.\mathrm{s}^{-1}\right)$ [RQP $\left.3 M X \geq 0.0\right]$.

The scale factor (-) for the power-to-wall table. The dependent variable in table QP3TB is multiplied by QP3SCL to obtain the absolute power to the wall.

VALVE Array Cards. Twenty-five sets of cards, one set for each of the following variables. Use LOAD format.

\begin{tabular}{|c|c|c|c|c|}
\hline Variab & & Dimension & & Descripticin \\
\hline $\mathrm{DX}$ & & NCELLS & & Cell lengths $(\boldsymbol{m})$. \\
\hline VOL & & NCELLS & & Ce1l volumes $\left(\mathrm{m}^{3}\right)$. \\
\hline FA & & NCELLS+1 & & Cel1-edge flow areas $\left(\mathrm{m}^{2}\right)$. \\
\hline FRIC & & NCELLS+1 & $=$ & $\begin{array}{l}\text { Additive loss coefficients }(-) \text { (See ACCUM } \\
\text { input description, Sec. V.C.5.a.) }\end{array}$ \\
\hline GRAV & & NCELLS+1 & & $\begin{array}{l}\text { Gravity terms (-). (See ACCUM input } \\
\text { description, Sec. V.C.5.a.) }\end{array}$ \\
\hline $\mathrm{HD}$ & & NCELLS+1 & & $\begin{array}{l}\text { Hydraulic diameters (m). (See variable } \\
\text { NDIAl in the NAMELIST data for optional } \\
\text { heat-transfer diameters.) }\end{array}$ \\
\hline Note: & If & $\begin{array}{l}\text { ICFLOW }=0 \text { or } \\
\text { ut array ICFLG. }\end{array}$ & ICFLOW & $=1$ (default) in the NAMELIST data, do not \\
\hline ICFLG & & NCELLS+1 & & $\begin{array}{l}\text { Cell-edge choked-flow model options. (See } \\
\text { ACCUM input description, Sec. V.C.5.a). }\end{array}$ \\
\hline NFF & & NCELLS+1 & & $\begin{array}{l}\text { Friction-factor correlation options. (See } \\
\text { ACCUM input description, Sec. V.C.5.a.) }\end{array}$ \\
\hline ALP & & NCELLS & & Initial void fractions $(-)$. \\
\hline
\end{tabular}




$\begin{array}{lll}\text { Variable } & \text { Dimension } & \text { Description } \\ \text { VL } & \text { NCELLS+1 } & \text { Initial liquid velocities }\left(m \cdot \mathrm{s}^{-1}\right) \text {. } \\ \text { VW } & \text { NCELLS+1 } & \text { Initial vapor velocities }\left(\mathrm{m} \cdot \mathrm{s}^{-1}\right) \\ \text { TL } & \text { NCELLS } & \text { Initial liquid temperatures }(\mathrm{K}) \cdot \\ \text { TV } & \text { NCELLS } & \text { Initial vapor temperatures }(\mathrm{K}) \cdot \\ \text { P } & \text { NCELLS } & \text { Initial pressures }(\mathrm{Pa}) \cdot \\ \text { PA } & \text { NCELLS } & \text { Initial air partial pressures }(\mathrm{Pa}) .\end{array}$

Note: If NODES $=0$ (word 2 on Card Number 2), do not enter arrays QPPP, MATID, and TW.

QPPP NODES*NCELLS

A nonnormalized power profile (-) in the valve walls. Input" for cell 1 , node 1 through NODES; then cell 2, node 1 through NODES; etc. If the array is filled with the same nonzero constant, a uniform volumetric heat source for the wall results. The code will normalize the power profile on a nodevolume basis (normalized QPPP(i)/[L QPPP(i) VOL(i)]). Filling the array withi zeros results in no power being deposited in the wall regardless of settings of QP3IN, QP3TB, etc.

MATID NODES-1

Wall-material ID array specifying material IV between nodes. Dimension is 1 if NODES $=1$ and 0 if NODES $=0$ (see available built-in properties listed with array MATID in the CORE input, Sec.V.C.5.c, or see Sec.V.C.2 to input material properties).

$\mathrm{TW}$ NODES*NCELLS Initial wall temperatures $(K)$ input in the same order as QPPP.

Note: If ICONC $=0$ (word 2 on Card Number 3 ), omit card set CONC.

CONC NCELLS Initial ratio of solute mass to liquid coolant mass. 
Valve Component (VALVE)

Note: If ICONC $=0$ or 1 (word 2 on Card Number 3 ), omit card set $S$.

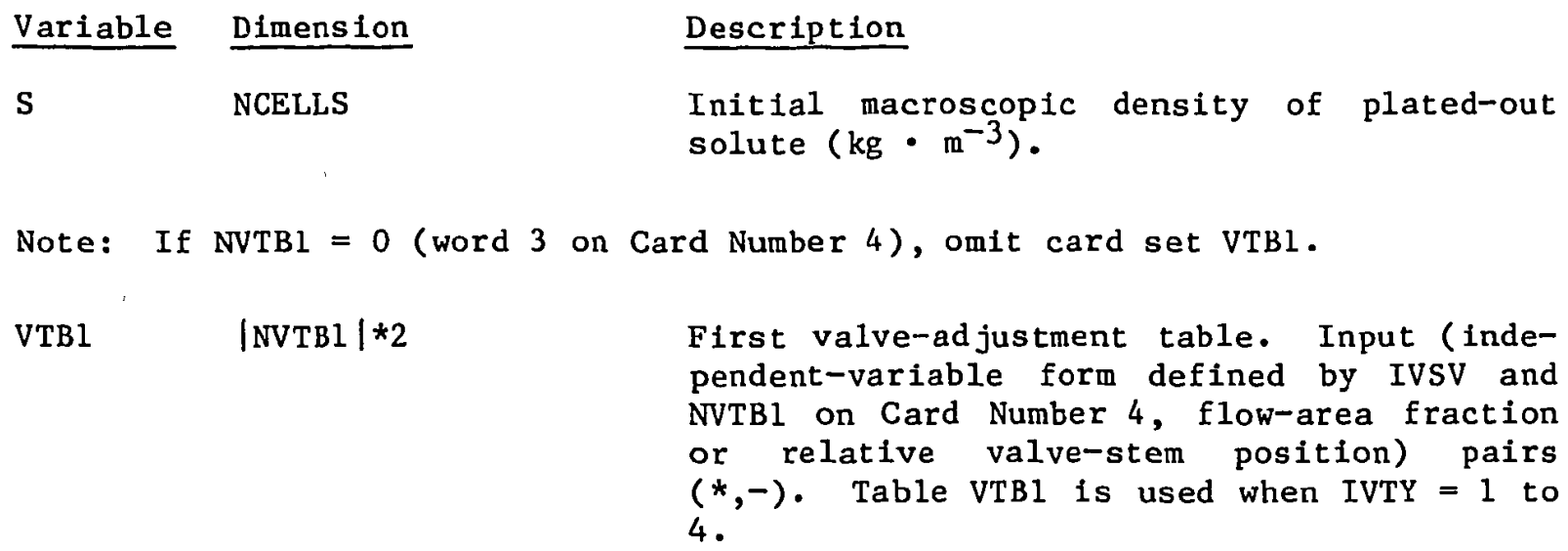

Note: If NVTB2 $=0$ (word 5 on Card Number 3 ), omit card set VTB2.

VTB2 $\mid$ NVTB2 $\mid * 2$

Second valve-adjustment table. Input (independent-variable form defined by IVSV on Card Number 4 and NVTB2 on Card Number 3 , flow-area fraction or relative valve-stem position) pairs $(*,-)$. If NVTB2 $\neq 0$ when IVTY $=3$ or 4 , define the flow-area fraction or relative valve-stem position values in the second valve-adjustment table to vary in the same direction as they do in the first valve-adjustment table; that is, if the flow-area fraction or relative valve-stem position increases in going from left to right in the first valve-adjustment table, define them to increase in going from left to right in the second valve-adjustment table as well.

Note: If NVTBL $=0$ (word 3 on Card Number 4) or NVRF $=0$ (word 5 on Card Number 4), omit card set VRF.

VRF $\quad|N V R F| * 2$

Rate-factor table for the first and second valve-adjustment table's independent variable. Input (independent-variable form defined by NVSV and NVRF on Card Number 4 , rate factor to be applied to the valveadjustment table's independent variable) pairs $(*,-)$. 
Note: If NQP3TB $=0$ (word 3 on Card Number 5), omit card set QP3TB.

$\begin{array}{ll}\text { Variable } & \frac{\text { Dimension }}{\text { QP3TB }} \\ & \mid \text { NQP3TB|*2 } \\ & \text { when QP2IN > ONO } \\ & \mid \text { NQP3TB|* (1+NCELLS })\end{array}$

\section{Description}

Power-to-wall independent-variable-form tab1e. Input (independent-variable form defined by IQP3SV and NQP3TB when QP3IN $<0$ on Card Number 5, power-to-wall) pairs $(*, W)$. If $Q P 3 I N>0.0$, the dependent variable specifies the total power to the entire wall; if QP3IN < 0.0 , the dependent variable is a vector specifying the power to the wall at each cell beginning with cell 1 .

Note: If NQP3RF $=0$ (word 3 on Card Number 5) or NQP3RF $=0$ (word 5 on Card Number 5), omit card set QP3RF.

QP3RF $\quad \mid$ NQP3RF $\mid * 2$

Rate-factor table for the QP3TB table's independent vartable. Input (Independentvariable form defined by NQP3SV and NQP3RF on Card Number 5, rate factor to be applied to the QP3TB table $e^{-s}$ independent variable) pairs $(*,-)$. 
m. Vesse1 Component (VESSEL).

Card Number 1. (Format A10,4X,2I14, A30) TYPE, NUM, ID, CTITLE

\begin{tabular}{|c|c|c|}
\hline Columns & Variable & Description \\
\hline $1-6$ & TYPE & Component type (VESSEL left-justified). \\
\hline $15-28$ & NUM & $\begin{array}{l}\text { Component ID number (must be unique for each } \\
\text { component, I S NUM } S 999 \text { ). }\end{array}$ \\
\hline $29-42$ & ID & User ID number (arbitrary). \\
\hline $43-72$ & CTITLE & Hollerith component description. \\
\hline
\end{tabular}

Card Number 2. (Format 5I14) NASX, NRSX, NTSX, NCSR, IVSSBF

\begin{tabular}{|c|c|c|}
\hline Columns & Variable & Description \\
\hline $1-14$ & NASX & Number of axial (z) segments (levels). \\
\hline $15-28$ & NRSX & Number of radial ( $r$ ) segments (rings). \\
\hline $29-42$ & NTSX & $\begin{array}{l}\text { Number of azimuthal }(\theta) \text { segments (sectors). } \\
\text { Setting NTSX }=1 \text { forces two-dimensional } \\
\text { Cartesian (slab) geometry in the VESSEL. }\end{array}$ \\
\hline $43-56$ & NCSR & $\begin{array}{l}\text { Number of cell-interface sources (component } \\
\text { connections). }\end{array}$ \\
\hline $57-70$ & IVSSBF & $\begin{aligned} & \text { Internal vessel boundary-condition flag; } \\
& 0= \text { no Internal boundary conditions } \\
& \text { (default); } \\
& 2= \text { first axial level acts as a FILL, last } \\
& \text { axial level acts as a BREAK; } \\
& 20= \text { first axial level acts as a BREAK, last } \\
& \text { axial level acts as a FILL; or } \\
& 22= \text { both the first and last axial levels } \\
& \text { act as BREAKS. }\end{aligned}$ \\
\hline
\end{tabular}


Card Number 3. (Format 5I14) IDCU, IDCL, IDCR, ICRU, ICRL

$\begin{array}{ll}\text { Columns } & \text { Varial } \\ 1-14 & \text { IDCU } \\ 15-28 & \text { IDCL } \\ 29-42 & \text { IDCR } \\ & \\ 43-56 & \text { ICRU } \\ & \\ 57-70 & \text { ICRL }\end{array}$

\section{Description}

Axial segment level at which the upper elevation is the downcomer upper-boundary elevation. If no downcomer is present, input IDCU $=0$ (this is the necessary and sufficient condition to indicate no downcomer as far as automatically setting flow areas to zero).

Axial-segment level at which the upper elevation is the downcomer lower-boundary elevation (if IDCU $=0$, input IDCL $=0$ ).

Radial-segment ring at which the outer radius forms the downcomer inner-radial boundary (if $I D C U=0$, input $I D C R=0$ ).

Axial-segment level at which the upper elevation is the core upper-boundary elevation (if no core is present, input ICRU $=0$ ).

Axial-segment level at which the upper elevation is the core lower-boundary elevation (if no core is present, input ICRL $=0$ ).

Card Number 4. (Format 5I14) ICRR, ILCSP, IUCSP, IUHP, ICONC

$\frac{\text { Columns }}{1-14} \quad$ Variable

15-28 ILCSP

$29-42 \quad$ IUCSP

\section{Description}

Radial-segment ring at which the outer radius forms the core outer radial boundary (if no core is present, input ICRR $=0$ ).

Axial-segment level at which the upper elevation is the lower-core support-plate elevation as used for graphics output [defaults to the value of ICRL (word 5 on Card Number 3) if ILCSP $=0$ ].

Axial-segment level at which :he upper elevation is the upper-core support-plate elevation as used for graphics output [defaults to the value of ICRU (word 4 on Card Number 3 ) if IUCSP $=0$ ].

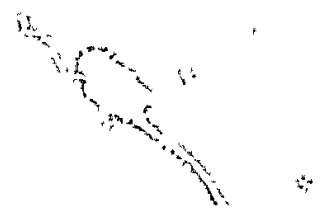


Columns Variable

43-56 IUHP

57-70 ICONC

\section{Description}

Axial-segment level at which the upper elevation is the upper-head support-plate elevation as used for graphics output [defaults to the value of IDCU (word 1 on Card Number 3 ) if IUHP $=0$ ].

Flag for input of solute in the liquid coolant. [Requires ISOLUT $=1$ (word 5 on Main-Data Card 6).]

$0=$ no input;

1 = dissolved solute only; or

2 = both dissolved and plated-out solute.

Card Number 5. (Format 4I14) NFFA, NFFR, NFFT, NVENT

\section{Description}

Axial friction-factor correlation option (input NFFA $=0$ ).

Radial friction-factor corrélation option (input NFFR $=0$ ).

Azimuthal friction-factor correlation option (input NFFT $=0$ ).

Number of vent valves in the VESSEL component. No vent valve or a maximum of one vent valve, per connection between cells is allowed; therefore, actual valves may have to be lumped together for each cell. 
Card Number 6. (Format 5I14) IRPWTY, NDGX, NDHX, NRTS, NHIST

$\begin{array}{ll}\text { Columns } & \text { Variable } \\ 1-14 & \text { IRPWTY }\end{array}$

\section{Description}

Neutronic point-kinetics or reactor-powertype option for defining programmed reactivity or reactor power (input parameters required for each option are shown in parentheses). Add 10 to the option value of IRPWTY if reactivity feedback is to be evaluated. For IRPWTY $>14$, reactivity feedback is evaluated and edited but is not used because reactor power is defined directly.

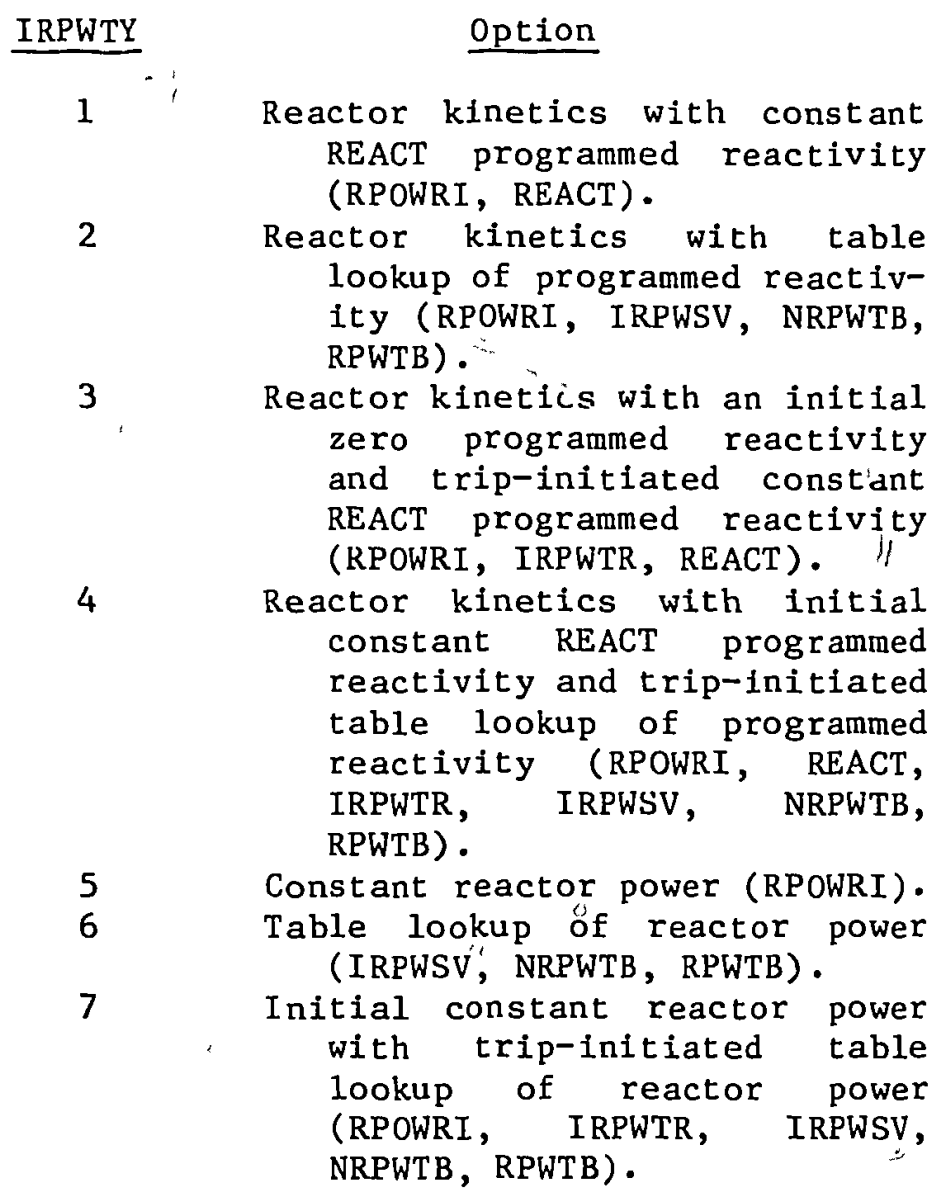


Columns Variable

15-28 NDGX

29-42 NDHX

43-56 NRTS

57-70 NHIST
Description

The number of delayed-neutron groups (if NDGX $\leq 0$ is input when IRPWTY $=1,2,3,4$, $11,12,13$, or 14 , the code defaults to 6 delayed-neutron groups with the delayedneutron constants defined internally; input NDGX $=0$ when IRPWTY $=5,6,7,15,16$, or 17).

The number of decay-heat groups (if NDHX $\leq 0$ is input when IRPWTY $=1,2,3,4,11,12$, 13 , or 14 , the code defaults to 11 decayheat groups with the decay-heat constants defined internally; input NDHX $=0$ when IRPWTY $=5,6,7,15,16$, or 17).

The number of time steps between edits of the reactor power and reactivity feedback changes (default value is NRTS $=10$ ).

The number of $(x, y)$ pairs in the powerhistory table [input NHIST 20 when IRPWTY $=1,2,3,4,11,12,13$, or 14 (when NHIST $=0$, no power-history table will be input; instead, delayed-neutron and decayheat concentrations wiil be input unless NDSX $\neq 0$ and NDHX $\neq 0$ causing equilibrium concentrations to be defined internally); input NHIST $=0$ when IRPWTY $=5,6,7,15$, 16 , or 17 ].

Note: If IRPWTY $=1,5,11$, or 15 (word 1 on Card Number 6), omit Card Number 7 .

Card Number 7. (Format 5I14) IRPWTR, IRPWSV, NRPWTB, NRPWSV, NRPWRF

Columns Variable

$1-14$

IRPWTR

\section{Description}

The trip ID number that controls evaluation of the reactivity-power table (|IRPWTR| $<10000) \quad[0<\mid$ IRPWTR $\mid<10000$ when IRPWTY $=3,4,7,13,14$, or $17 ;$ IRPWTR $=0$ otherwise]. 
Columns

$15-28$

$29-42$

$43-56$
Variable

IRPWSV

NRPWTB

NRPWSV

U

\section{Description}

The reactivity-power table's abscissa-coordinate variable ID number. IRPWSV defines the independent-variable parameter for the reactivity-power table. IRPWSV $>0$ defines the ID number for a signal-variable parameter; IRPWSV < 0 defines the ID number for a control-block output parameter $(0<\mid$ IRPWSV $\mid<10000$ when IRPWTY $=2,4,6$, $7,12,14,16$, or 17 ; IRPWSV = 0 otherwise).

The number of reactivity-power table $(x, y)$ (defined by the absolute value of NRPWTB). NRPWTB $>0$ defines the table's independentvariable form to be the IRPWSV parameter; "iRPWTB < 0 defines the table's independentvariable form to be the change in the IRPWSV parameter over the last time step times the trip set-status value ISET when the reactivity-power table is trip controlled; NRPWTB $=0$ defines the reactivity-power table's function to be the IRPWSV parameter. a)

The rate-factor table abscissa-coordinate variable ID number. NRPWSV defines the independent-variable parameter for the rate factor that is applied to the reactivitypower"s table independent variable. NRPWSV $>0$ defines the ID number for a signal-variable parameter; NRPWSV < 0 defines the ID number for a control-block output parameter; NRPWSV $=0$ (when NRPWRF $\neq 0$ ) defines the difference between the trip signal and the set-point value that turns the trip OFF when the reactivity-power table is trip controlled. 
Columns Variable

57-70 NRPWRF

Description

The number of rate-factor table $(x, y)$ pairs (defined by the absolute value of NRPWRF). The rate factor is applied as a ractor to the reactivity-power table's independent variable when the rate factor is defined. No rate factor is defined when NRPWSV and NRPWRF are both zero. NRPWRF $>0$ defines the rate-factor table's abscissa coordinate to be the NRPWSV parameter; NRPWRF $<0$ defines it to be the change in the NRPWSV parameter over the last time step times the trip set-status value ISET when the reactivity-power table is trip controlled; NRPWRF $=0$ defines the rate factor to be the NRPWSV parameter.

Card Number 8. (Format 5I14) IZPWTR, IZPWSV, NZPWTB, NZPWSV, NZPWRF

Columns Variable

1-14 IZPWTR

15-28 IZPWSV

29-42 NZPWTB

\section{Description}

The trip ID number that controls evaluations of the axial power-shape table (|IZPWTR| (10000) [input IZPWTR $=0$ when evaluation of the axial power-shape table is not trip cont rolled].

The axial power-shape table's abscissa-coordinate variable ID number. IZPWSV defines the independent-variable parameter for the axial power-shape table. IZPWSV $>0$ defines the ID number for a signal-variable parameter; IZPWSV < 0 defines the ID number for a control-block output parameter.

The number of axial power-shape table $(x, y, z)$ pairs (defined by the absolute value of NZPWTB). Each pair consists of an abscissa coordinate value $x$ and ICRU-ICRL+1 (Card Number 3 ) ordinate coordinate values of $y(z)$ defining the axial power shape. NZPWTB $>0$ defines the table's independentvariable form to be the IZPWSV parameter; NZPWTB $<0$ defines the table's independentvariable form to be the change in the IZPWSV parameter over the last time step times the trip set-status value ISET when the axial power-shape table is trip controlled. NZPWTB $=0$ defines the reactor-kinetics table's function to be the IZPWSV parameter. 


Columns
N3-56 Nariable
NZWSV
$57-70 \quad$ NZPWRF

Card Number 9. (Format 5I14) IRFTR, NMWRX, NFCI, NFCIL, NZMAX

$\begin{array}{ll}\frac{\text { Columns }}{1-14^{\circ}} & \text { IRFTR } \\ 15-28 & \text { NMWRX } \\ 29-42 & \text { NFCI }\end{array}$
controlled.

\section{Description} if IRFTR $=0$ ).

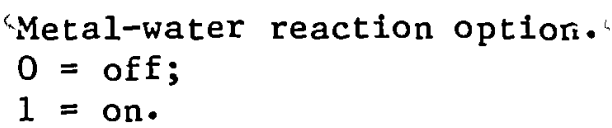

\section{Description}

The rate-factor table's abscissa-coordinate variable ID number. NZPWSV defines the independent-variable parameter for the rate factor that is appiied to the axial powershape table's independent variable. NZPWSV $>0$ defines the ID number for a signalvariable parameter; NZPWSV < 0 defines the ID number for a control-block output paraneter; NZPWSV $=0$ (when NZPWRF $\neq 0$ ) defines the difference between the trip signal and the set-point value that turns the trip OFF when the axial power-shape table is trip

The number of rate-factor table $(x, y)$ pairs (defined by the absolute value of NZPWRF). The rate factor is applied as a factor to the axial power-shape table's independent variable when the rate factor is defined. No rate factor is defined when NZPWSV and NZPWRF are both zero. NZPWRF $>0$ defines the rate-factor table's abscissa coordinate to be the NZPWSV parameter; NZPWRF $<0$ defines it to be the change in the NZPWSV parameter over the last time step times the trip set-status value ISET when the axial power-shape table is trip controlled; NZPWRF $=0$ defines the rate factor to be the NZPWSV. parameter.

Trip IV number for implementing the fuel-rod axial fine-mesh calculation during reflood (no axial fine-mesh calculation is performed

Fuel-clad interaction (FCI) option (NFCI $=1$ performs the dynamic gap conductance calculation).

$0=$ off

$1=$ on. 


$\begin{array}{ll}\text { Columns } & \text { Variabl } \\ 43-56 & \text { NFCIL } \\ 57-70 & \text { NZMAX }\end{array}$

Card Number 10. (Format 5I14) NRODS, NODES, NODHS, INHSMX, NFBPWT

\section{Description}

Maximum number of FCI calculations per time step (input NFCIL $=1$ when NFCI $=1$ ).

Maximum number of rows of nodes used in the fuel-rod conduction calculation. $\frac{\text { Columns }}{1-14} \quad \frac{\text { Variable }}{\text { NRODS }}$

15-28 NODES

29-42 NODHS

43-56 INHSMX

57-70 NFBPWT

Card Number 11. (Format 5E14.6) Columns Variable

1-14 REACT

\section{Description}

Total number of calculational fuel rods within the vessel (NRODS $\geq$ ICRR*NTSX, the number of core mesh cells in the horizontal plane).

Number of radial heat-transfer nodes in the fuel rods (input NODES $\geq 4$ if a core region with NRODS $>0$ fuel rods is specified; input NODES $=0$ if no core is specified).

The number of heat-transfer nodes in all the vessel slabs. A value of 1 defaults to the lumped-parameter model (NODHS 21 ).

The naximum number of interfaces between dissinilar materials in the vessel slabs (INHSMX 21 ; an internal check is made and an error message is printed for inconsistent values).

Flag option to replace the radial, axial, or horizontal-plane power shape with a userdefined shape used to average the reactivity-feedback parameters over the core. (For the input value, add 1 for a radial shape, 2 for a axial shape, and 4 for an horizontal-plane shape.)

REACT, TNEUT, RPWOFF, RRPWMX, RPWSCL

Description

Initial programmed reactivity (IRPWTY $=1$, $2,4,11,12,14$ ) or trip-initiated programmed reactivity, (IRPWTY $=3$ or 13) [REACT $=(k-1) \cdot k^{-i}$ where $k$ is the reactor multiplication constant; both REACT and $k$ have no units]. 


$\begin{array}{ll}\text { Columns } & \text { Variabl } \\ \text { 15-28 } & \text { TNEUT } \\ 29-42 & \text { RPWOFF } \\ 43-56 & \text { RRPWMX } \\ 57-70 & \text { RPWSCL }\end{array}$

Card Number 12. (Format 4E14.6)

$\begin{array}{lll}\text { Columns } & \text { Variable } \\ 1-14 & \text { RPOWRI } & \\ 15-28 & \text { ZPWIN } & \text { ". }\end{array}$

$29-42 . \quad$ ZPWOFF

43-56 RZPWMX

\section{Description}

The prompt neutron lifetime (s) [TNEUT $S 0.0$ defaylts internally to TNEUT $=1.625 \times 10^{-5} \mathrm{~s}$.

Programmed reactivity $(-)$ [IRPWTY $=3,4$, 13,14 ] or reactor power (W) [IRPWTY $=7$ or 17] when the reactivity-power controlling trip is OFF after being ON; hold constant the last evaluated value when the trip was $O N$ when RPWOFF $\leq-1.0 \times 10^{19}$.

The maximum rate of change of progrämmed reactivity $\left(s^{-1}\right)$ or reactor power $\left(W \cdot s^{-1}\right)$ [RRPWMX 20.0 ].

Reactivity-power table's scale factor for programmed reactivity or reactor power. The dependent variable in table RPWTB is multiplied by RPWSCL to obtain the absolute value.

RPOWRI, ZPWIN, ZPWOFF, RZPWMX

Description

Initial reactor power (W)...

The axial power-shape table $e^{-}$abscissacoordinate variable value corresponding to the initial axial power shape.

The axial power-shape table's abscissacoordinate variable value corresponding to the axial power shape to be used when the axial power-shape table's controlling trip is $O F F$ after being $O N$ (use the last evaluated axial power shape yhen the trip was $O N$ when ZPWOFF $\leq-1.0 \times 10^{19}$ ).

The maximum rate of change of any z-intefface value in the axial power shape $\left(s^{-1}\right)$ [RZPWMX 20.0$]$. 
Card Number 13. (Format 5E14.6) SHELV, PLDR, PDRAT, FUCRAC, HGAPO

\section{Columns Variable \\ 1-14 SHELV}

15-28 PLDR

29-42 PDRAT

43-56 FUCRAC

57-70 HGAPO

Card Number 14. (Format 3E14.6)

$\frac{\text { Columns }}{1-14} \quad \frac{\text { Variable }}{\operatorname{DTXHT}(1)}$

15-28 DTXHT(2)

29-42 DZNHT

\section{Description}

In the coordinate system in which the cellcentered elevations of the one-dimensional components are specified, SHELV is the elevation of the bottom of the vessel. This parameter is used only when IELV $=1$ in NAMELIST.

Pellet dish radius (m) [no calculation of pellet dishing is performed if PLDR $=0.0 \mathrm{]}$.

Fuel-rod pitch-to-diameter ratio $(-)$.

Fraction of the fuel not cracked (use only if $\mathrm{NFCI}=1$ on Card Number 9).

Fue1-rod gap conductance coefficient $\left(\mathrm{W} \cdot \mathrm{m}^{-2} \cdot \mathrm{K}^{-1}\right.$ ) [constant for $\mathrm{NFCI}=0$ on Card Number 9, initial value otherwise].

$\operatorname{DTXHT}(1), \operatorname{DTXHT}(2), \operatorname{DZNHT}$

\section{Description}

Maximum $\Delta T(K)$ above which rows of nodes are inserted in the fuel-rod conduction calculation during reflood for the nucleate and transition boiling regimes [suggested value: $\operatorname{DTXHT}(1)=3.0$ ]

Maximum $\Delta T(K)$ above which rows of nodes are inserted in the fuel-rod conduction calculation during reflood for all boiling regimes except nucleate and transition [ suggested value: $\operatorname{DTXHT}(2)=10.0$ ]

Minimum $\Delta \mathrm{Z}(\mathrm{m})$ below which no additional rows are inserted in the fuel-rod conduction calculation during the reflood calculation (this value comes from the diffusion number). 
Note: If reactivity feedback is not evaluated IRPWTY $<11$ (word 1 on Card Number 6), omit Card Numbers 15 to 17 .

Note: Card Number 15 has four cards input: the $J=1$ card defines the fuel temperature reactivity-coefficient table, the $J=2$ card defines the coolant temperature reactivity-coefficient table, the $J=3$ card defines the vapor coolant void-fraction reactivity-coefficieut table, and the $J=4$ card defines the boron material concentration reactivitycoefficient table.

Card Number 15. (Format 5I14/5I14/5I14/5I14) $\operatorname{IRCJTB}(I, J), I=(1,4), J=(1,4)$

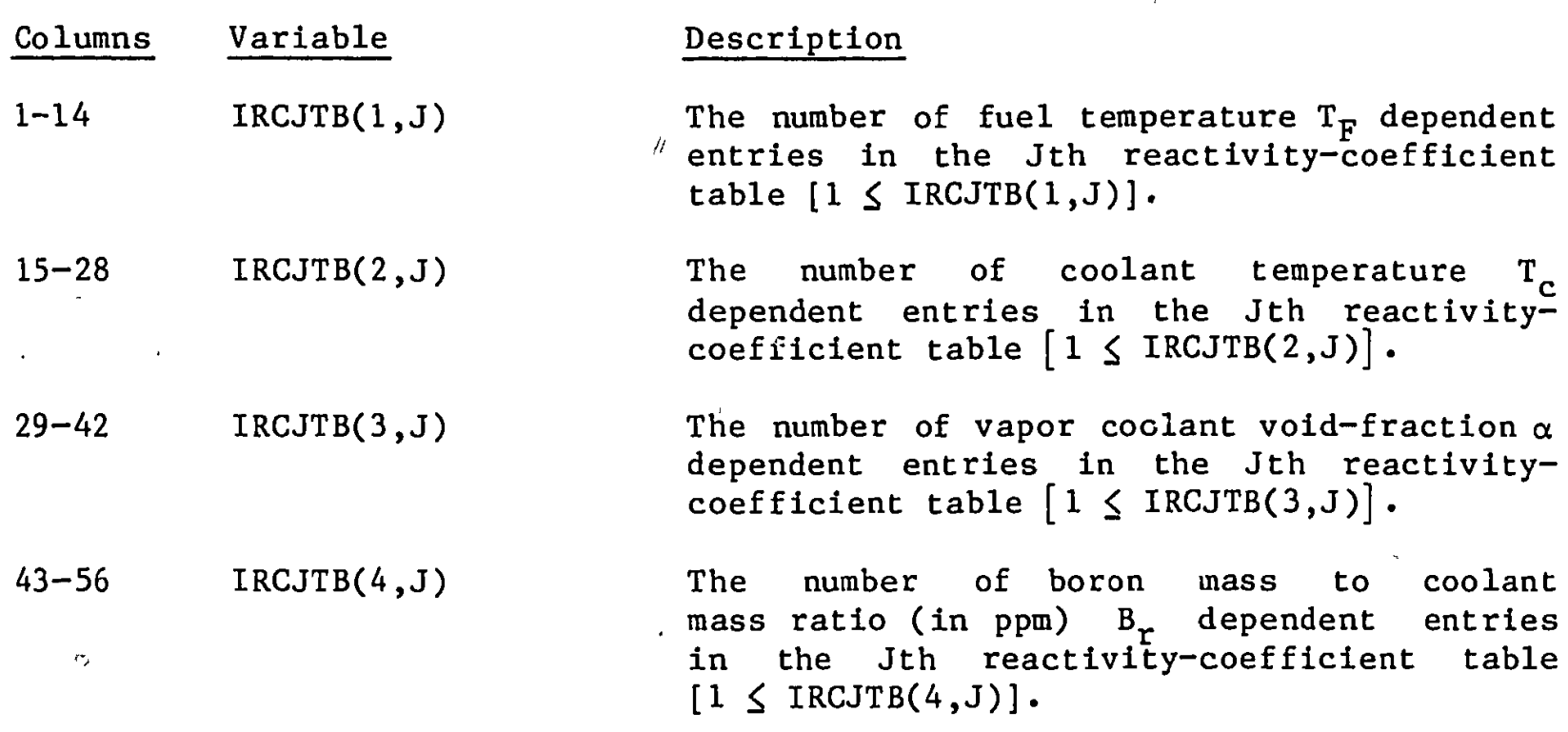


Columns Variable

57-70 IBU(J)
Description

The boron unit's definition flag for the $\partial \mathrm{k} / \partial \mathrm{X}_{\mathrm{J}}$ reactivity coefficient's $\mathrm{x}_{J}$ derivative parameter,

$$
x_{4}=\left\{\begin{array}{c}
B_{m} \\
B_{r}
\end{array} \text { for } \operatorname{IBU}(4)=\left\{\begin{array}{c}
0 \text { or } 1 \\
-2 \text { or }-1
\end{array}\right\}\right. \text {, }
$$

and $f\left(T_{f}, T_{C}, \alpha, B\right)$ tabular dependence,

$$
B=\left\{{ }_{B r}^{m_{m}}\right\} \text { for } \operatorname{IBU}(J)=\left\{\begin{array}{lll}
-1 & \text { or } & 1 \\
-2 & \text { or } & 0
\end{array}\right\} \text {, }
$$

where $B_{m}$ is the macroscopic density in the coolant-channel volume in $\mathrm{kg} \cdot \mathrm{in}^{-3}$ units, and $\mathrm{B}_{\mathrm{r}}$ is the mass ratio of parts boron per million parts liquid coolant in ppm units.

Note: On Card Number 16, the reactivity-coefficient type form numbers are defined as follows: $\operatorname{IRCJFM}(J)=0$ for $\partial k / \partial x$, IRCJFM(J) $=1$ for $(1 / k) * \partial k / \partial x=\partial R / \partial x, \operatorname{IRCJFM}(J)=2$ for $x * \partial k / \partial x$, and $\operatorname{IRCJFM}(J)=3$ for $(x / k) * \partial k / \partial x=x^{*} \partial R / \partial x$ where $x=T_{F}(K)$ for $J=1, x=T_{C}(K)$ for $J=2$, $x=\alpha$ for $J=3$, and $x=$ boron for $J=4$ (see variable IBU $(J)$, word 5 on Card Number 15, for boron units).

Card Number 16. (Format 5I14) IRCJFM(J), $J=(1,4)$, ISNOTB

Columns Variable

1-14 IRCJFM( 1 )

15-28 IRCJFM(2)

29-42 IRCJFM(3)

43-56 IRCJFM(4)
Description

The form number for the fuel temperature reactivity-coefficient type.

The form number for the coolant temperature reactivity-coefficient type.

The form number for the vapor coolant void fraction reactivity-coefficient type.

The form number for the boron material concentration reactivity-coefficient type. 


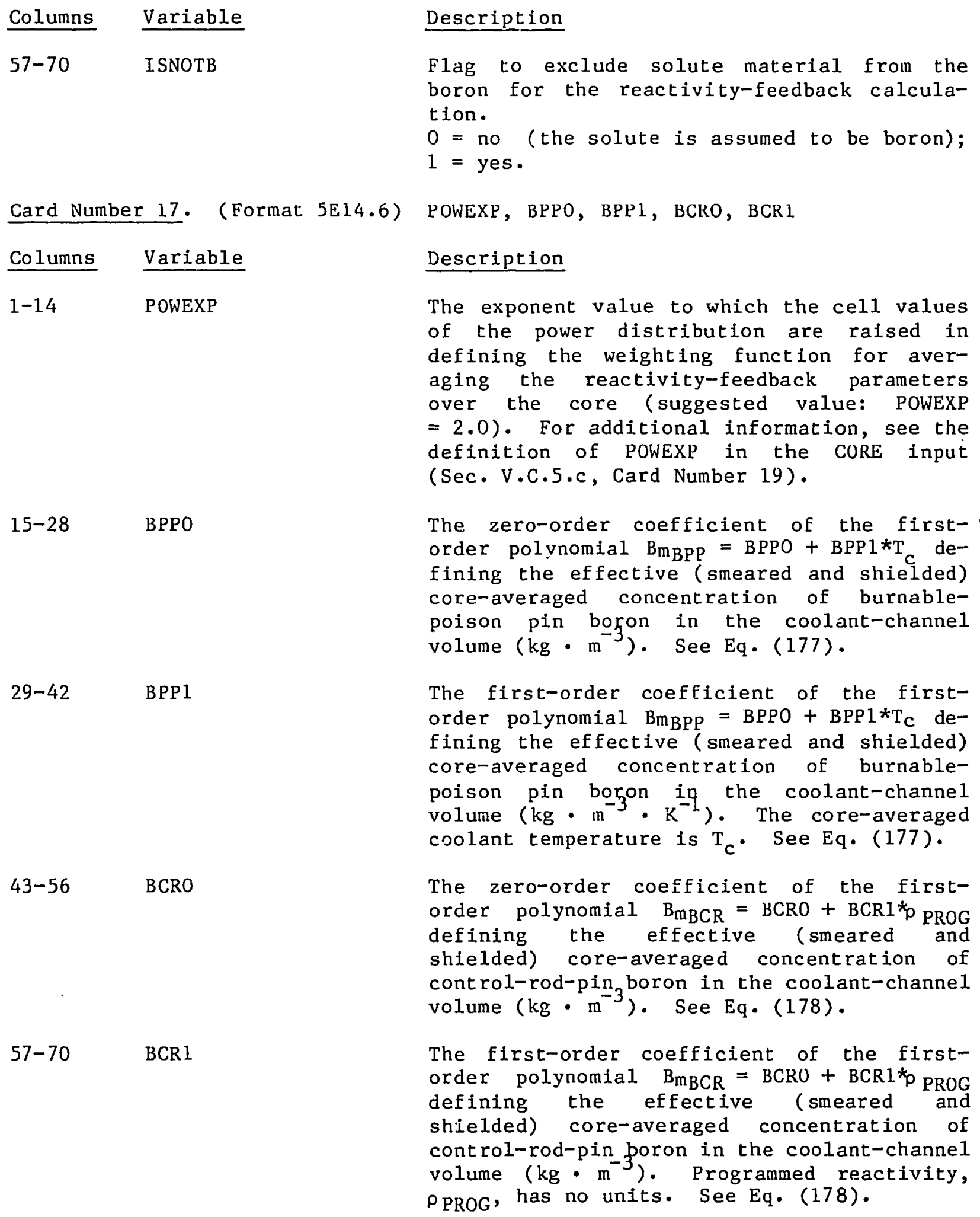


Vessel Component (VESSEL)

VESSEL Geometry Cards. Three sets of cards, one set for each of the following variables. Use $\mathrm{LOAD}$ format.

\begin{tabular}{|c|c|c|}
\hline Variable & Dimension & Description \\
\hline $\mathrm{z}$ & NASX & $\begin{array}{l}\text { Upper elevations (m) of axial-segment levels } \\
\text { (referenced to zero elevation at the bottom } \\
\text { interface of the first axial-segment level } \\
\text { in the vessel). }\end{array}$ \\
\hline RAD & NRSX & Outer radii (m) of radial-segment rings. \\
\hline $\mathrm{TH}$ & NTSX & $\begin{array}{l}\text { Theta angles (radians or degrees) at } \\
\text { azimuthal-segment ends. For NTSX }=1 \text {, the } \\
\text { VESSEL component is two-dinensional, and } x-y \\
\text { Cartesian (slab) geometry applies. }\end{array}$ \\
\hline
\end{tabular}

Omit the next two card types if NVENT $=0$ (word 4 on Card Number 5).

VESSEL Vent-Valve Location and Area Cards. (Format 2I14,E14.6) IZV, KV, AVENT One card per cell with the vent valve on the outer radial surface.

$\begin{array}{lll}\text { Columns } & \text { Variable } & \frac{\text { Description }}{1-14} \\ 15-28 & \text { KV } & \text { Axial-segnent level in the vessel. } \\ 15-42 & \text { AVENT } & \text { Horizontal-plane relative-cell number. } \\ & \begin{array}{l}\text { Total area ( } \mathrm{m}^{* * 2} \text { ) of the vent valve in outer } \\ \text { radial wall of the cell. }\end{array} \\ \text { VESSEL Vent-Valve Pressure-Drop and Friction-Loss Cards. }\end{array}$

(Format 4E14.6) DPCVN, DPOVN, FRCVN, FROVN One card per cell with the vent valve on the outer radial surface.

$\frac{\text { Columns }}{1-14} \quad \frac{\text { Variable }}{\text { DPCVN }}$

Description

Maximum pressure drop ( $\mathrm{Pa}$ ) between the inner and outer radial cells for the vent valve to be closed. 
Columns Variable

15-28 DPOVN

29-42 FRCVN

43-55 FROVN

VESSEL Source Cards.

Columns Variable

1-14 LISRL

15-28 LISRC

29-42 LISRF

43-56 LJUNS

\section{Description}

Minimum pressure drop ( $\mathrm{Pa}$ ) between the inner and outer radial cells for the vent valve to be opened.

Additive friction-loss coefficient for the vent valve in its closed position.

Additive friction-loss coefficient for the vent valve in its open position.

(Format 4I14) LISRL, LISRC, LISRF, LJUNS

One card for each of the NCSR (word 4 on Card Number 2) component-connection sources. See vessel description, Sec. IV.K.

Description

Axial-segment level number associated with the source.

Horizontal-piane relative-cell number assdiciated with the source.

Face number associated with the source.

1 = azimuthal direction;

2 = axial direction; or

3 = radial direction.

A positive numbe 'ndicates a connection to the top, outer iadius or second azimuthal face of the cell; a negative number indicates a connection to the bottom, inner radius or first azimuthal face of the cell.

Junction number associated "with the component source. 
Vessel Component (VESSEL)

VESSEL Core Cards. Thirty-one sets of cards, one for each of the following variables. Omit these cards if there is no core. Use LOAD format.

Note: See Sec. IV.K for precise definitions of the following parameters and the ordering convention: used for reading in the data. Many parameters are read in with dimension ICRR*NTSX (word 1 on Card Number 4 and word 3 on Card Number 2$)$. These parameters are supplied for each $(r, \theta)$ mesh cell in the horizontal-plane core region., Each such cell constitutes one of the axial channels in the core formed by a stack of mesh cell; with the same $(r, \theta)$ wesh boundaries. Each $(r, \theta)$ mesh cell encloses $a$ number of fuel rods and their associated coolant channels.

$\begin{array}{ll}\text { Variable } & \text { Dimension } \\ \text { RDPWR } & \text { NODES } \\ \text { CPOWR } & \text { ICRR*NTSX } \\ \text { IDROD } & \text { NRODS-ICRR*NTSX } \\ \text { RPKF } & \text { NRODS-ICRR*NTSX }\end{array}$

Description

Relative radial-power density at the node positions.

Relative power density in each $(r, \theta)$ mesh cell in the horizontal plane for the average fuel rod.

Horizontal-plane cell numbers where the additional fuel rods are placed.

Fuel-rod power-peaking factors (relative to the average fuel rod in the mesh cell) for the additional rods.

Note: If NZPWTB $=0$ (word 3 on Card Number 8 ), onit card set ZPWTB.

ZPWTB $\quad \mid$ NZPWTB $\mid *$

$(1+(I C R U-I C R L+1))$

Axial power-shape vs independent-variable form table. Input [independent-variable form defined by IZPWSV and NZPWTB on Card Number 8 , axial power-shape densities having (ICRU-ICRL+1) values] pairs $(*,-)$. The relative axial-power densities defining the axial-power shape are specified at the axial-segment level interfaces from one end of the fuel rod to the other. There are NZPWTB axial power shapes being input with each having (ICRU-ICRL+1) values. 
Note: If NZPWTB $=0$ (word 3 on Card Number 8) or NZPWRF $=0$ (word 5 on Card Number 8 ), omit card set ZPWRF.

\begin{tabular}{|c|c|c|}
\hline Variab] & Dimension & Description \\
\hline ZPWRF & $\mid$ NZPWRF $\mid * 2$ & $\begin{array}{l}\text { Rate-factor table for the axial power-shape } \\
\text { table-s independent variable. Input (inde- } \\
\text { pendent-variable form defined by NZPWSV and } \\
\text { NZPWR on Card Number } 8 \text {, rate factor to be } \\
\text { applied to the axial power-shape table } \\
\text { independent variable) pairs }(*,-) \text {. }\end{array}$ \\
\hline RDX & ICRR*NTSX & $\begin{array}{l}\text { Number of fuel rods in each }(r, \theta) \text { mesh cell } \\
\text { of the core (these are real numbers, not } \\
\text { integers; fractional rods are allowed). }\end{array}$ \\
\hline RADRD & NODES & Fuel-rod radii (m) [cold]. \\
\hline Note: & $\begin{array}{l}\text { Only one MATRD element } \\
\text { MATRD(NODES-1) cannot be } \\
\text { input (Sec.V.C.2). }\end{array}$ & $\begin{array}{l}\text { may have the value } 3 \text {; and } \operatorname{MATRD}(1) \text { and } \\
\text { 3. Additional material properties can be }\end{array}$ \\
\hline MATRD & NODES-1 & 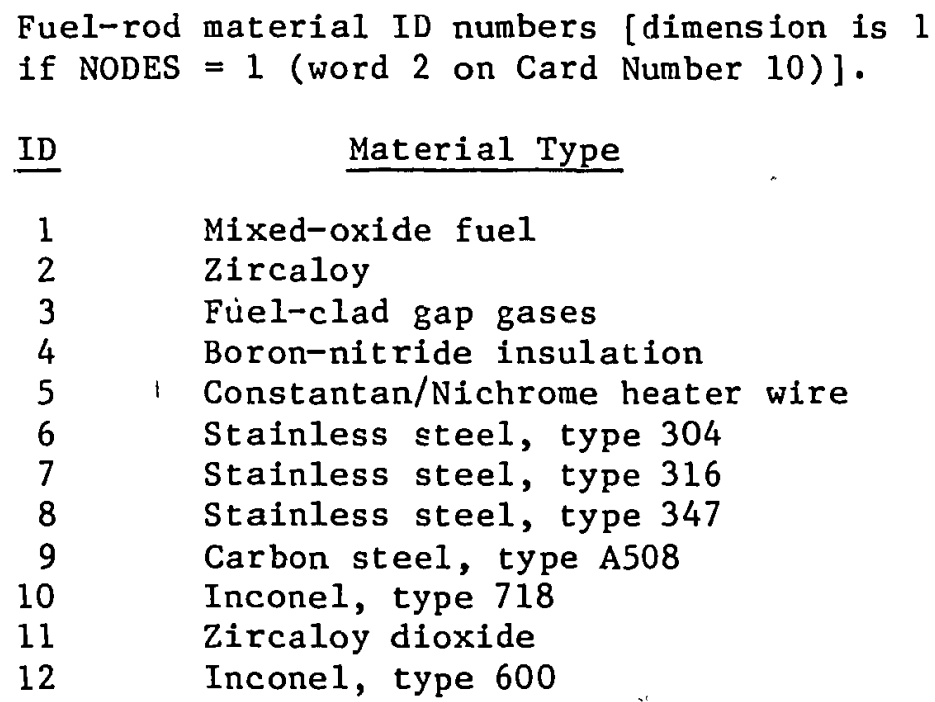 \\
\hline
\end{tabular}


Vessel Component (VESSEL)

Note: If NRPWTB $=0$ (word 3 on Card Number 7), omit card set RPWTB.

Variable Dimension

RPWTB $\mid$ NRPWTB $\mid * 2$

\section{Description}

Programmed-reactivity or reactor-power vs independent-variable-form table. Input (independent-variable form defined by IRPWSV and NRPWTB on Card Number 7, progranmed reactivity or reactor power as defined by IRPWTY) pairs $(*,-$ or $W)$.

Note: If NRPWTB $=0$ (word 3 on Card Number 7) or NRPWRF $=0$ (word 5 on Card Number 7), omit card set RPWRF.

RPWRF $\quad \mid$ NRPWRF $\mid * 2$

Rate-factor table for the programmedreactivity or reactor-power table's independent variable. Input (independentvariable form defined by NRPWSV and NRPWRF on Card Number 6, rate factor to be applied to the programmed-reactivity or reactor-power table's independent variable) pairs $(*,-)$.

Note: If IRPWTY < 11 (word 1 on Card Number 6), omit card sets RCTF, RCTC, RCAL, and RCBM. Additional information pertinent to these card sets is given in the note and example preceding the description of card set RCTF in the CORE input (Sec.V.C.5.c).

RCTF

$\operatorname{IRCJTB}(1,1)+\operatorname{IRCJTB}(2,1)+$ $\operatorname{IRCJTB}(3,1)+\operatorname{IRCJTB}(4,1)+$ ( $\operatorname{IRCJTB}(1,1) * \operatorname{IRCJTB}(2,1)$ * $\operatorname{IRCJTB}(3,1) * \operatorname{IRCJTB}(4,1))$

RCTC

$\operatorname{IRCJTB}(1,2)+\operatorname{IRCJTB}(2,2)+$ $\operatorname{IRCJTB}(3,2)+\operatorname{IRCJTB}(4,2)+$ (IRCJTB $(1,2) * \operatorname{IRCJTB}(2,2)$ * $\operatorname{IRCJTB}(3,2) * \operatorname{IRCJTB}(4,2))$
The fuel-temperature reactivity-coefficient table. Input $\operatorname{IRCJTB}(1,1) \mathrm{T}_{\mathrm{F}}$ values, $\operatorname{IRCJTB}(2,1) \quad T_{C}$ values, $\operatorname{IRCJTB}(3,1)$, a values, $\operatorname{IRCJ} J^{C} B(4,1) \quad B_{r}$ values, and IRCJTB $(1,1) * \operatorname{IRCJTB}(2,1)$ $*_{\operatorname{IRCJTB}}(3,1) *_{\operatorname{RCJTB}}(4,1)$ fuel-temperature reactivity-coefficient values that define the four-dimensionally dependent table. [Note: this table and the following three tables are not entered with $(x, y)$ pair values as is done for the onedimensionally dependent tables.]

The coolant-temperature reactivity-coefficient table. 
Variable Dimension

RCAL IRCJTB $(1,3)+\operatorname{IRCJTB}(2,3)+-$ $\operatorname{IRCJTB}(3,3)+\operatorname{IRCJTB}(4,3)+$ ( $\operatorname{IRCJ} T B(1,3) * \operatorname{IRCJTB}(2,3)$ * $\operatorname{IRCJTB}(3,3) * \operatorname{IRCJTB}(4,3))$

RCBM IRCJTB $(1,4)+\operatorname{IRCJTB}(2,4)+$ $\operatorname{IRCJTB}(3,4)+\operatorname{IRCJTB}(4,4)+$ ( IRCJTB $(1,4) * \operatorname{IRCJTB}(2,4)$ *

$\operatorname{IRCJTB}(3,4) * \operatorname{IRCJTB}(4,4))$

\section{Description}

The vapor-coolant void-fraction reactivity-coefficient table.

The boron-mass-concentration reactivitycoefficient table.

Note: If power option [IRPWTY $=5,6,7,15,16$, or 17 (word 1 on Card Number 6)] is specified, omit card sets BETA, LAMDA, CDGN, LAMDH, EDH, CDHN, and PHIST.

Note: If NDGX $\leq 0$ (word 2 on Card Number 6) was input, omit card sets BETA and LAMDA. The default 6 group delay-neutron constants will be defined internally.

$\begin{array}{ll}\text { BETA NDGX } & \text { The effective delayed-neutron fraction. } \\ \text { LAMDA NDGX } & \left.\text { The } e_{1}^{-1}\right) .\end{array}$

Note: Use card set CDGN only if NDGX $>0$ (word 2 on Card Number 6) and NHIST $=0$ (word 5 on Card Number 6 ) were input.

CDGN NDGX - The delayed-neutron precursor concentration $(W)$.

Note: If NDHX $\leq 0$ (word 3 on Card Number 6) was input, omit card sets LAMDH and EDH. The default 11 group decay-heat constants will be defined interna11y.

LAMDH NDHX

EDH NDHX
The decay-heat decay constant $\left(s^{-1}\right)$. The effective decay-heat energy fraction. 
Note: Use card set CDHN only if NDHX $>0$ (word 3 on Card Number 6) and NHIST $=0$ (word 5 on Card Number 6 ) were input.

Variable Dimension

CDHN NDHX

\section{Description}

The decay-heat concentration ( $-s)$.

Note: If NHIST $=0$ (word 5 on Card Number 6), omit card set PHIST.

PHIST

NFAX

FPUO2

FTD

GMIX

GMLES

ICRR*NTSX
NHIST*2 Power-history table. Input (present time minus past time, reactor-core prompt-fission power at that past time) pairs $(s, W)$ [the first pair should be for the present power, that is, the time interval 0.0 , with the time interval for subsequent pairs being positive and increasing monotonically for each pair].

ICRU-ICRL Number of permanent fine-mesh intervals per coarse-mesh interval added at the start of the reflood calculation [the total number of heat--ransfer rows per fuel rod must be less than NZMAX (word 5 on Card Number 9)].

ICRR*NTSX Fraction of plutonium dioxide $\left(\mathrm{PuO}_{2}\right)$ in mixed-oxide fuel.

ICRR*NTSX Fraction of theoretical fuel density .

ICRR*NTSX*7Mole fraction of gap-gas constituents. Array is not used if NFCI $=0$ but must be input. Enter data for each gas in the order indicated.

\begin{tabular}{cl} 
Index & \multicolumn{1}{c}{ Gas } \\
1 & Helium \\
2 & Argon \\
3 & Xenon \\
4 & Krypton \\
5 & Hydrogen \\
6 & Air/nitrogen \\
7 & Water vapor
\end{tabular}

Moles of gap gas per fuel rod (not used, but must be input). 


$\begin{array}{ll}\text { Variable } & \frac{\text { Dimension }}{\text { PGAPT }} \\ \text { ICRR*NTSX } \\ \text { PLVOL } & \text { ICRR*NTSX } \\ \text { PSLEN } & \text { ICRR*NTSX } \\ \text { CLENN } & \text { ICRR*NTSX }\end{array}$

VESSEL Level Cards. Thirty sets of cards, one for each of the following variables. Use IIOAD format. The 30 sets of cards are input for each axial segment level, or if desired, an entire level can be repeated by a single REPEAT LEVEL: card. (See description after the level data description.)

Note: The following parameters (dimensioned NRSX*NTSX defined on Card Number 2) are read in for each $(r ; \theta)$ mesh cell at each axial-segment level, that is, they extend over the entire vessel horizontal plane for each axial-segment level. Because a separate data set is read for each axial-segment level, these parameters are supplied for all, mesh cells . in the vessel.

$\begin{array}{ll}\text { Variable } & \frac{\text { Dimension }}{\text { NRSX*NTSX }} \\ \text { HSX } & \text { NODHS*NRSX*NTSX }\end{array}$

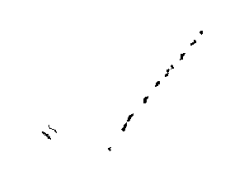

Q

\section{Description"}

Heat-slab area $\left(\mathrm{m}^{2}\right)$ [if no slab is desired in a cell, input 0.0 for the area]. o

Heat-slab node position (m). For each cell, HSX should increase monotonically; the first node sees the adiabatic boundary, and node NODHS (word 3 on Card Number 10) sees the fluid heat-transfer boundary. All NODHS values for a given cell are input together. The code assumes Cartesian geometry. When NODHS $=1$, HSX is the thickness of the heat slab. 
Vesse1 Component (VESSEL)

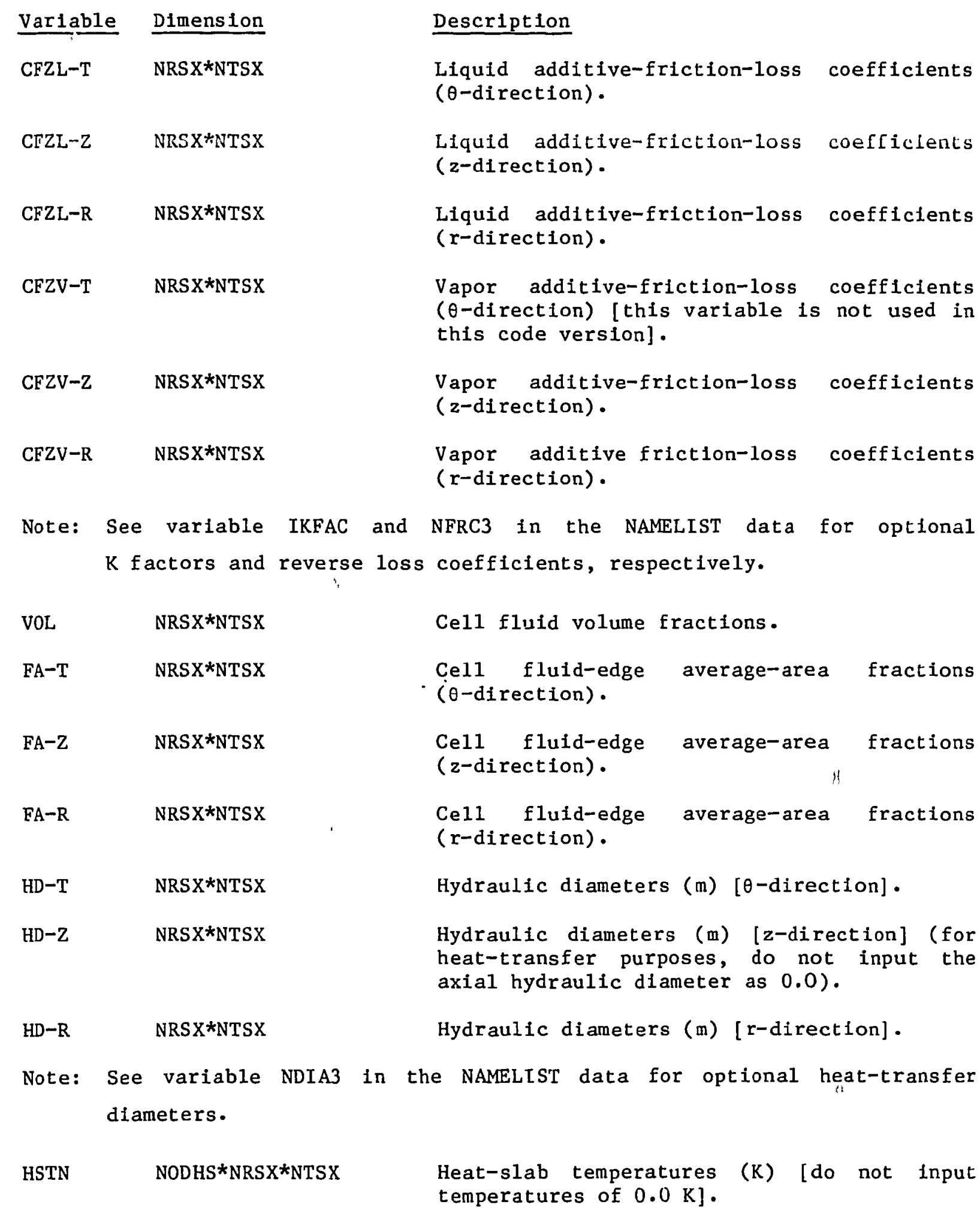




\begin{tabular}{|c|c|c|}
\hline Variable & Dimension & Description \\
\hline MATHS & $($ NODHS -1$) *$ NRSX $*$ NTSX & $\begin{array}{l}\text { Heat-slab material ID numbers specifyin } \\
\text { material between nodes. All materia } \\
\text { numbers must be defined. (See aval } \\
\text { built-in properties listed with array } \\
\text { in the CORE input, Sec. V.C.5.c, or } \\
\text { Sec. V.C.2 to input material propert } \\
\text { [Input (NODHS*NRSX*NTSX) when NODHS } \\
\text { NODHS }=0 \text { is not allowed.] }\end{array}$ \\
\hline ALPN & NRSX*NTSX & Vapor fraction $(-)$ \\
\hline VVN-T & NRSX*NTSX & Vapor veloc1ty $\left(m \cdot s^{-1}\right)[\theta-$ direction $]$. \\
\hline VVN $-\mathrm{Z}$ & NRSX*NTSX & Vapor velocity $\left(\mathrm{m} \cdot \mathrm{s}^{-1}\right)$ [z-direction] \\
\hline VVN-R & NRSX*NTSX & Vapor velocity $\left(m \cdot s^{-1}\right)$ [r-direction]. \\
\hline VLN-T & NRSX*NTSX & Liquid velocity $(\theta$-direction). \\
\hline VLN-Z & NRSX*NTSX & Liquid velocity (z-direction). \\
\hline VLN-R & NRSX*NTSX & Liquid velocity (r-direction). \\
\hline TVN & NRSX*NTSX & Vapor temperature (K). \\
\hline TLN & NRSX*NTSX & Liquid temperature $(K)$. \\
\hline PN & NRSX*NTSX & Pressure $(\mathrm{Pa})$ \\
\hline PAN & NRSX*NTSX & Air partial pressure ( $\mathrm{Pa})$. \\
\hline
\end{tabular}

Note: If ICONC $=0$ (word 5 on Card Number 4), omit card set CONC.

CONC NRSX*NTSX Initial ratio of solute mass to liquid coolant mass.

Note: If ICONC $=0$ or 1 (word 5 on Card Number 4), omit card set $\mathrm{S}$.

S NRSX*NTSX

Initial macroşopic density of plated-out solute $\left(\mathrm{kg} \cdot \mathrm{m}^{-3}\right)$. 
Vessel Component (VESSEL)

REPEAT LEVEL Card. (Format A12,2X,I14) This card can be used to repeat the data from one level to another. As many of these cards can be used as needed. Each REPEAT LEVEL card should be placed after the data for the preceding level and before the data for the next level. These cards may be used consecutively.

Columns Variable 1-12 AREP

15-18 NLEV

\section{Description}

The character string: "REPEAT, LEVEL"

The number of the level to be repeated. The value of NLEV must be greater than zero but less than the number of the level to be defined.

VESSEL Fuel-Rod Cards. Two sets of cards, one for each of the following variables. Both card sets are input for each of NRODS (word 1 on Card Number 10) fuel rods, including each average rod and each additional rod. Omit these cards if there is no core. Use LOAD format.

$\begin{array}{lll}\text { Viriable } & \text { Dimension } & \text { Description } \\ \text { BURN } & (\text { ICRU-ICRL+1) } & \text { Fuel burnup (MND/MTU). } \\ \text { RFTN } & \text { NODES*(ICRU-ICRL+1) } & \text { Initial fuel-rod temperatures (K). }\end{array}$


6. Time-Step Data. The last data block of input information is the time-step cards for directing the calculation and output edit. The problem time span is separated into time domains. Each domain (specified by two cards) may have different minimum and maximum time-step sizes and edit intervals. Any number of time domains may be input. The end of the calculation is specified by making the minimum time-step size a negative number. The format of each set of two time-step cards follows.

Card Number 1. (Format 4E14.6) DTMIN, DTMAX, TEND, RTWFP

$\begin{array}{ll}\frac{\text { Columns }}{1-14} & \text { Variable } \\ 15-28 & \text { DTMIN } \\ 29-42 & \text { TEND } \\ 43-56 & \text { RTWFP }\end{array}$

\section{Description}

Minimum allowable time-step size (s) for this time domain.

Maximum allowable time-step size (s) for this time domain.

End time ( $s$ ) of this time domain.

Ratio between heat-transfer and fluiddynamics time-step sizes. (Normally used only for steady-state calculations: suggested value $=10.0 ;$ a negative value of RTWFP forces the use of a scaling |RTWFP| even during a transient.)

Card Number 2. (Format 4E14.6) EDINT, GFINT, DMPINT, SEDINT

\begin{tabular}{|c|c|c|c|c|c|}
\hline Columns & Variable & Description & & & \\
\hline $1-14$ & EDINT & $\begin{array}{l}\text { Print-edit time interval (s) } \\
\text { domain. }\end{array}$ & for & this & time \\
\hline $15-28$ & GFINT & $\begin{array}{l}\text { Graphics-edit time interval } \\
\text { time domain. }\end{array}$ & (s) & for & this \\
\hline $29-42$ & DMPINT & $\begin{array}{l}\text { Restart-dump time interval (s) } \\
\text { domain. }\end{array}$ & for & this & time \\
\hline $43-56$ & SEDINT & $\begin{array}{l}\text { Short-print-edit time interval } \\
\text { time domain. }\end{array}$ & - (s) & for & this \\
\hline
\end{tabular}


The TRAC program uses the LOAD subroutine to read most subscripted array variables. The arrays may be read in floating point or integer format. The input card images for subscripted variables consist of up to six fields. The first five fields consist of an ( $A 1$ ), a repeat count (I2) [for operations $R$, $M$, and $I]$, and a floating point or integer data constant (E11.2 or III) [except for operatives $\mathrm{E}$ and $\mathrm{S}$ ]. The sixth field can be used for the operation $E$ only if the array data end in the fifth field. In formatted input decks, cards with an asterisk in column 1 are ignored and may be used for spacers or for comments.

Seven operations are defined. These operations and an explanation of each are listed below.

Operation

F

BLANK

$\mathrm{R}$

M

I

E

S

\section{Description}

Fill array starting at current data index with data constant No action

Repeat data constant I2 times

Multiple repeat. Repeat data constant $10 * 12$ tines

Interpolate between data constant and succeeding data constant with 12 points

End of data array (must be followed by at least one blank)

Skip to next card

Some restrictions in the use of the LOAD format are

1. end of data for an array must be signaled by $E$,

2. overstore or partial fill of an array is not allowed,

3. integer interpolation is not allowed, and

4. data for different arrays must be on different records.

Following are examples of the use of the options listed above to fill an array of dimension 11 with data.

EXAMPLE 1. Fill an integer array with a value of 61 .

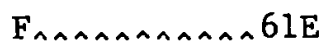

EXAMPLE 2. Use of the repeat option to fill an array with a value of 1.2 .

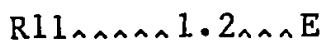


EXAMPLE 3. Use of the skip option.

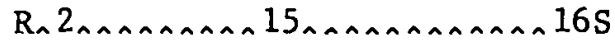

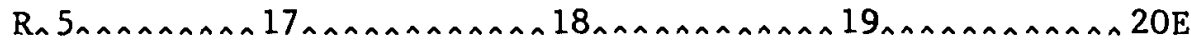

EXAMPLE 4. Use of the multiple repeat option to fill an array with 101 values.

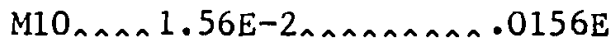

EXAMPLE 5. Use of the interpolation option to get points $1.0,2.0,3.0, \ldots, 11.0$.

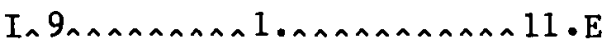

E. Free Format

Appendix E shows part of a TRACIN deck in free format that illustrates the points discussed here.

Section V.F gives the TRAC-PFI/MOD1" input specifications for formatted input. When the free-format option is chosen, TRAC-PF1/MODl internally converts a free-format TRACIN deck to a new deck in TRAC format that is written to a file called TRCINP. File TRCINP is read subsequently and automatically by the standard input routines. Therefore, to use the freeformat option, all cards must be kept in the same order as shown in Sec. V.C, and all variables must stay in the same order on those cards. Input records may be up to 80 columns long. All data that is not read by the LOAD routine must be delimited by at least one blank column. (However, data may start in column 1 or end in column 80.) Array data to be read by the LOAD routine may be blank delimited; delimited by any of the LOAD control characters, F, R, S, $I, M$, or $E$; or delimited by a control-character repeat count. (The integer zero should be entered explicitly.) As with the formatted input, free-format arrays read by the LOAD subroutine are limited to a maximum of five data entries per input card image. 
Note the following examples, all of which will run in free-format mode. Fค^^^^1.0E+07E

also TRAC format

F. 1. OE+07E

Fへ1.0E+07E

F1.0E+07E

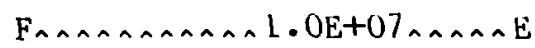

etc.

free format

R10^^^1.0E+07R,2^^^1.1E+07

also TRAC format; both modes read 10 repeats, 2 repeats

Rl^^^^1.0E+07

free-format reads 1 repeat; TRAC format (I2) reads 10 repeats

$\mathrm{R}, 10,1.0 \mathrm{E}+07, \cap \mathrm{R}, 2,1.1 \mathrm{E}+07$

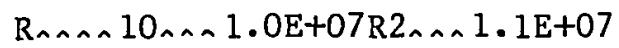

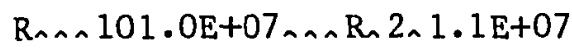

etc.

free format; al1 three read 10 repeats, 2 repeats

Note the Ran101.0E+07 example. The free-format parser scans to the first character after the $R$; it includes the next character in the repeat count if that character is a nonblank numeric. There is one exception to this rule: a situation such as $R_{n} 112.3456 \mathrm{E}+07$, where the control character is followed by one space, a two-digit repeat count, and a nonblank column. To facilitate conversion of existing TRAC-format decks, this field is assumed to indicate 1 repeat of $12.3456 \mathrm{E}+07$, not 11 of $2.3456 \mathrm{E}+07$. The following examples will give 11 repeats:

$R 11.2 .3456 E+07$

$\mathrm{R}, 11,2.3456 \mathrm{E}+07$

$R_{\wedge} \wedge 112 \cdot 3456 \mathrm{E}+07$

$\mathrm{R} 112.3456 \mathrm{E}+07$ 
The E character of the LOAD subroutine is recognized as part of the string "Enn" so the three columns after the $\mathrm{E}$ should be blank. The $\mathrm{E}$ can appear in column 78,79 , or 80 with any remaining columns blank.

The LOAD data must be 11 characters or less; non-LOAD data, 14 or less. The requirement to blank-delimit data carries with it the requirements that embedded and significant trailing blanks be entered explicitly as zero. The user of the free-format option also should note that to run previously formatted decks that have all-blank 14-column fields meant to represent zero, the zero must be entered explicitly. Of course, this is not necessary for trailing fields. In this regard, note for example the one-dimensional component variable IHYDRO that currently is not used by TRAC-PFI/MODI. A dummy value must be input for this variable.

The NAMELIST input currently is implemented using CDC-FTN-FORTRAN. The format is essentially free, but there are a few restrictions that are discussed in Sec. V.F.

1. Free-Format Comments, Problem Title Cards, and Hollerith Component Descriptions. Free-format TRACIN decks may be annotated with user comments. These comments must be delimited by asterisks (*s) in unbroken strings of any length. The first card of the deck is an exception to this requirenent. Comments and their delimiters are equivalent to blank columns. Should an input record have an odd number of comment delimiters (where *, **, ***, ****, etc. are all considered to be a single delimiter), everything on the record to the right of the last delimiter is considered a comment. The code will attempt to read information after even-numbered comment delimiters and before odd-numbered delimiters as input data. Entire records may be comments, for example, by making the first nonblank character an asterisk and not inserting any more comment delimiters on the line. Comments and comment cards may appear anywhere in the deck except

1. in and immediately before the problem title cards,

2. before Main Control Card Number 1, or

3. within NAMELIST group records (see additional comments on NAMELIST in Sec. V.C).

The NUMTCR title cards immediately following Main Control Card Number 2 (from which NUMTCR is read) are written to TRCINP exactly as they are read: asterisks, blank cards, and all. Blank and comment cards may appear between 
the first two main control cards and immediately after the NUMTCR title cards. Hollerith descriptions of individual components (the CTITLE information) are written to TRCINP, left-justified, starting in column 43. Asterisk strings in component descriptions are treated as comment delimiters.

2. Free-Format Input-Error Handling. The free-format option provides advantages over formatted runs in handling of many types of input errors. Free-format input error handling occurs during two stages, first as TRACIN is being converted to TRCINP and later as TRCINP is being read. In the first stage, when an error is detected (such as detectable errors that might arise from failure to blank-delimit data properly, for example, using 1.0 107 instead of $1.0 \mathrm{E} 07$ or $1.0 \mathrm{E}+07$ ) processing of the record in question is halted immediately, subroutine ERROR is called to send warning messages, and the record is flagged with '*ERROR' starting in column 75 before it is written to TRCINP. Currently, warning messages will stop after 50 cards have been detected in error, but the input processing will continue. In the second stage, urder most circumstances a fatal FORTRAN input error will not force an immediate program abort; the entire deck will be processed and appropriate error messages issued before program termination. An exception to this situation occurs in the case of fatal NAMELIST errors.

In situations where there are no errors detected but input problems are suspected, it is suggested that the user inspect the file TRCINP, where faulty records may be readily apparent.

F. NAMELIST Format

The NAMELIST statement is an extremely useful--but nonstandard--FORTRAN statement that can be used to input selectively values to subsets of groups of variables named in a program. The user-convenience features in TRAC available through NAMELIST input options are described in Sec.V.C, "TRAC-PF1 Input Specifications." The TRAC NAMELIST options are implemented using NAMELIST as described in the CDC-FTN-FORTRAN manual. (A somewhat more flexible NAMELIST is available at Los Alamos.) The reader is referred to the FTN manual for details. Here we point out that, although NAMELIST is essentially in free format, there are certain restrictions. At present these restrictions apply to a11 TRAC input decks, whether or not the TRAC free-format option is selected. 
The restrictions include the following (where Los Alamos-available extensions are indicated in parentheses).

1. Hollerith constants are not allowed (allowed).

2. The first column of all physical records is ignored [terminating dollar sign ( $\$$ ) can appear in any column].

3. There must be no embedded blanks in the string \$NAME, where NAME is a NAMELIST group name; there must be at least one trailing blank. The initial \$ must appear in column 2 .

4. Free-format *-delimited user comments are not allowed on NAMELIST cards. Also, free-format comment cards are not allowed among the physical records of a NAMELIST group record, although all-blank cards are allowed.

As an example, the following five cards might be used to input data for the NAMELIST group INOPTS (described in Sec. V.C).

$123456789 \ldots$

$\wedge$ \$INOPTS $\wedge$ IELV $=1, \ldots, \operatorname{IKFAC}_{\wedge}=\wedge 1$,

^ ^ISTOPT $=2$,

^ $A L P=0 ., V L=0 ., V V=0 ., T L=550 ., T V=550$.,

$\leadsto \sim \mathrm{P}=1.55 \mathrm{E}+07, \mathrm{PA}=0 ., \mathrm{QPPP}=0 ., \wedge \mathrm{TW}=5.5 \mathrm{E}+02, \mathrm{HSTN}=550$.,

$\sim$ \$END

G. Output Files

Figure 49 shows the files read and written by TRAC during a problem. We discussed the two input files, TRACIN and TRCRST, in Sec.V.A, the free-format option output-input file TRCINP in Sec. V.E, and the dump output file, TRCDMP, in Sec. V.B. This section describes the remaining three output files, TRCOUT, TRCGRF, and TRCMSG.

The TRCOUT file contains printer output. This file is produced with standard FORTRAN write statements contained in the various component module output subroutines.

Included are complete descriptions of the problem input file that was read by the code and the time edits that are produced with a frequency specified on the time-step cards. Each time edit includes a printout of results from each component in a problem. The component output includes pressures, temperatures, and other important results. The TRAC error messages, 


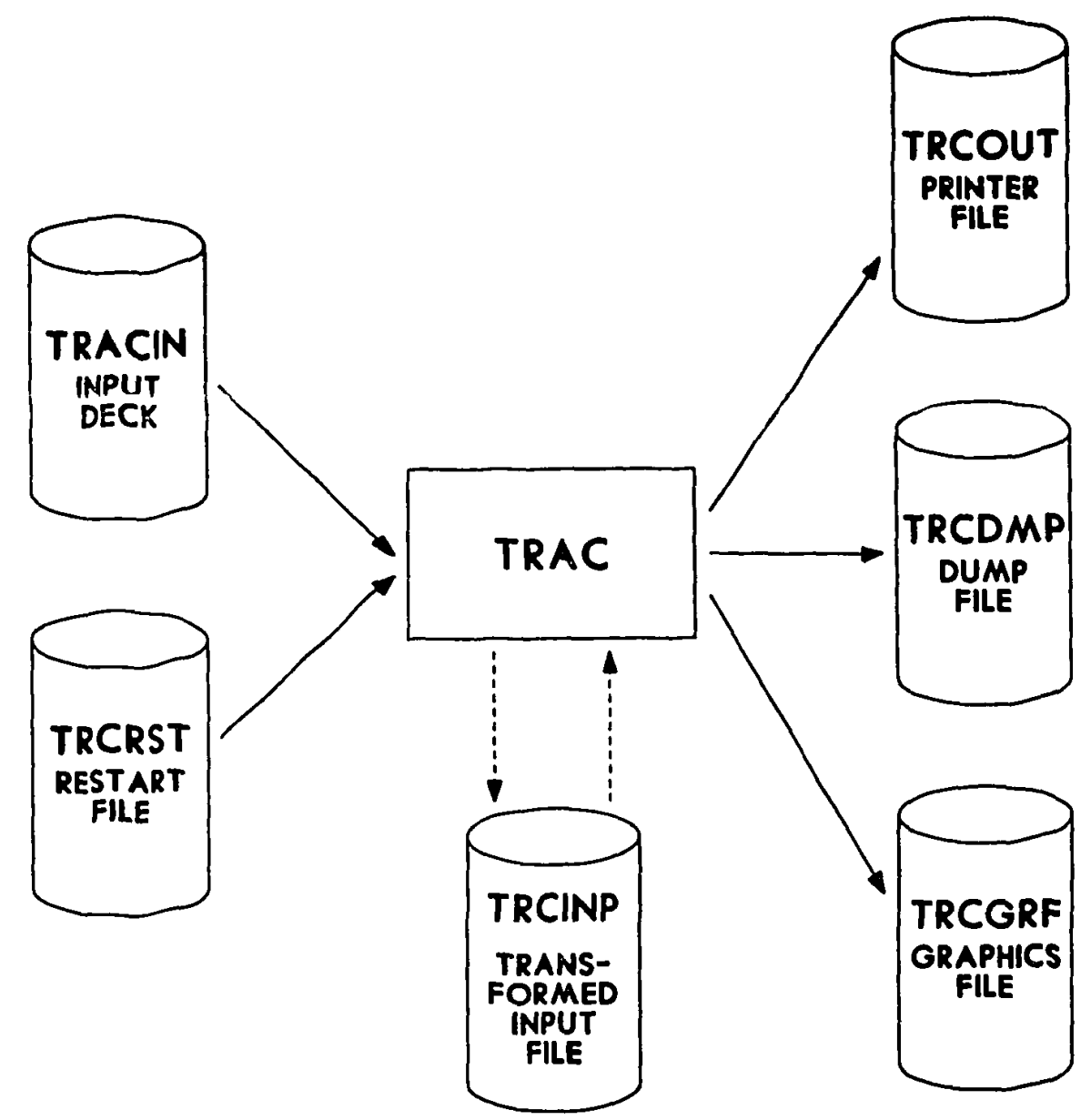

Fig. 49 .

TRAC input and output files.

if any, also are written in the TRCOUT file. Appendix F describes these messages. Section IV gives a more complete description of individual component output.

The TRCGRF file contains graphics output and is a structured binary file produced with unformatted write statements. This file structure is discussed in Sec. VI.C. A Livermore Time-Sharing System (LTSS) library computer code, GRIT, generates plots of the problem calculation from the TRCGRF file. A versatile graphics package TRAP/EXCON also has been developed to produce high-quality plots and movies from the TRCGRF file. This package is designed for the Los Alamos National Laboratory CDC 7600 computer and is documented separately. 
The TRCMSG file contains warning messages that are sometimes produced by various computational modules within TRAC. These warnings, written with formatted input/output $(I / 0)$, indicate difficulty with the progress of the problem. 
VI. PROGRAMMING DETAILS

A. Overall Code Organization

During TRAC development, much attention was paid to the programming techniques. The decision was made to strive for a code structure that minimizes the problems of maintaining and extending the code. In addition, we attempted to program in a manner that is understandable to knowledgeable persons in the LWR safety fleld and that reduces the difficulties of implementing TRAC at other sites and/or computers. We also recognized that we should take advantage of the Laboratory's efficlent computing facility. In this case, the system includes CDC 7600 computers running LTSS and Cray computers running CTSS. In conflict situations, we ranked the importance of these goals in the order in which they have been presented. Whenever possible, we segregated spectallzed coding to subprograms that perform specific, low-level service functions.

To attain these goals, our philosophy has been modularity. The TRAC program is modular in two Important ways. Because it analyzes reactor systems that consist of specific types of components, the program contains subroutines that treat each component type. The TRAC components are described in Sec. IV. This modularity simplifies both the subroutine programing and the data associated with particular components. For example, because fuel rods are associated only with the VESSEL and CORE components, no fuel-rod data are referenced nor are fuel-rod calculations performed by the subroutines that treat any other component.

Second, the TRAC program is functionally modular; that is, each TRAC subprogram performs a specific function. If the performance of a function requires modification, only those routines that perform that function must be altered. For example, if the dump/restart capability needs modification, only those routines that perform the dump/restart for the affected coimponents require changes. "Some low-level subprograms are used by all components, thereby strengthening this modularity. The most important low-level routines and a brief description of their functions are listed in Table XV. All TRAC subroutines are described in App. G.

In its simplest form, TRAC has no overlays, and all data reside in the main memory. One overlay structure is available, as is a split in data storage between small- and large-core memories. Some modifications of the 
TABLE XV

IMPORTANT LOW-LEVEL SUBPROGRAMS

Subprogram

BKMOM

BKSSTB

CHEN

CHF

CHOKE

CLEAR

CONBLK

CYLHT

ERROR

FEMOM

FF3D

FLUX

FPROP

FWALL

HTCOR

HTP IPE

HTVSSL

INNER

J1D
Description

Initiates backward substitution for stabilizing momentum equations.

Initiates backward substitution for stabilizing mass and energy equations.

Uses Chen correlation to evaluate the forced convection nucleate bolling HTC.

Evaluates the critical heat flux based on a local-conditions formulation.

Calculates choked phasic velocities and their derivatives.

Sets an array to a constant value.

Computes controller outputs.

Calculates temperature fields in a cylindrical annulus.

Processes error conditions.

Sets up stabilizing momentum equations:

Makes final pass update for all variables in three-dimensional vessel.

Calculates mass flow at boundary of one-dimensional components for use in mass inventory.

Calculates values for fluid enthalpy, transport properties, and surface tension.

Computes a two-phase friction factor.

Computes HTCs from material surfaces to vapor and liquid.

Averages velocteies $\|_{1}$ and generates HTCs for one-dimensional components.

Averages velocities and generates HTCs for the vessel.

Performs an inner iteration for a one-dimensional component.

Fills boundary array at component junctions. 
TABLE XV (cont.)

Subprogram

JUNSOL

MFROD

MPROP

POSTER

PRE PER

RDLCM

RKIN

RODHT

SAVBD

SCMLCM

SETBD

SLABHT

SOUND

STBME

TF1D

TFIDS

TF1DS1

TF1DS3

\section{Description}

Determines junction parameters for connecting and sequencing components.

Orders fuel-rod property selection and evaluates an average temperature for property evaluation.

Orders structure property selection and evaluates an average temperature for property evaluation.

Performs postpass calculation for a one-dimensional component.

Performs prepass calculation for a one-dimensional component.

Transfers data from LCM into SCM.

Integrateo the neutron point-kinetics equations.

Calculates the fuel-rod temperature field.

Moves boundary information into component arrays.

Transfers the fixed-length, varlable-length, and pointer tables to LCM.

Stores component information into boundary arrays.

Calculates the slab temperatures.

Calculates the homogeneous-equilibrium sound speed required in the choked-flow calculations.

Sets up stabilizing mass and energy equations.

Controls solution of the hydrodynamic equations for onedimensional components.

Linearizes the basic mass and energy equations and performs the initial eliminations on the linear equations.

Solves the basic motion equations for the new time velocity as a Iinear function of the new time pressure. It also evaluates the interfacial heat-transfer coefficients and areas.

Does the backward substitution for the equations obtained in TF1DS after the solution of the network matrix. 
TABLE XV (cont.)

Subprogram

Description

TF3DE

Evaluates constitutive relations for interfacial heat transfer and shear; makes an initial evaluation of new time velocities.

TF3DI Sets up the linearized three-dimensional finite-difference equations.

THERMO Calculates thermodynamic properties of water.

TRIP Returns status of a TRIP.

TRPSET Sets up trip status flags.

VOLV Calculates cell-averaged phase velocities for one-dimensional components.

WARRAY Writes a real array to TRCOUT.

WIARR Writes an integer array to TRCOUT.

WRLCM Transfers a given number of words from SCM to LCM.

overlay structure may be quickly obtained by changing cards flagged by the OVLAY definition in the Update (or Historian) source deck for TRAC.

Functional modularity within TRAC is taken a step further by its division into overlays. The use of an overlay structure originally was mandated by computer size limitations because the small CDC 7600 core memory, where all executable instructions must reside, is 1lafted to 65536 60-bit words. This division isolates functional subunits within TRAC. Figure 50 displays a calling tree of the TRAC overlays. T'able XVI briefly describes the function of each overlay. Overlays are loaded at Los Alamos by declaring the entry routine for each overlay, then satisfying all subsequent subroutine references from a global binary library of TRAC subroutines. The Cray version of TRAC does not need to be overlaid. However, we recommend that the user keep the input and initialization overlays to minimize memory charges.

The TRAC component modularity ran be seen in the data structure as well as in the program structure. We used the data modularity in a manner that can efficiently utilize a CDC 7600 computer. The CDC 7600 central memory is 
TABLE XVI

TRAC OVERLAYS

\begin{tabular}{|c|c|}
\hline Overlay & Description \\
\hline MNIN & $\begin{array}{l}\text { Controls overall flow of calculation. (The MAIN overlay also } \\
\text { contains many service routines used throughout the code.) }\end{array}$ \\
\hline INPUT & $\begin{array}{l}\text { Controls reading input and restart files and analyzes piping } \\
\text { loops. }\end{array}$ \\
\hline RDCOMP & Inputs and stores one-dimensional component data. \\
\hline RDCOM3 & Inputs and stores vessel data. \\
\hline RDREST & Reads and stores data from a restart dump file. \\
\hline INIT & Controls initialization of component data and graphics tables. \\
\hline ICMP & Initializes component data. \\
\hline IGRF & Initializes graphics table. \\
\hline DMPIT & Performs restart dumps. \\
\hline PREP & Controls prepass for all components. \\
\hline PREPLD & Performs the prepass calculations for one-dimensional components. \\
\hline PREP3D & $\begin{array}{l}\text { Performs prepass calculations for all three-dimensional vessel } \\
\text { components. }\end{array}$ \\
\hline TRIPS & $\begin{array}{l}\text { Evaluates signal variables and determines the set status of } \\
\text { trips. }\end{array}$ \\
\hline OUTER & Controls one complete outer iteration for all components. \\
\hline OUT1D & $\begin{array}{l}\text { Does an iteration on the basic finite-difference flow equations } \\
\text { for all one-dimensional components. }\end{array}$ \\
\hline OUT3D & Does an outer iteration on all vessel components. \\
\hline POST & Performs postpass for all components. \\
\hline EDIT & Adds an edit at the current time to the TRCOUT file. \\
\hline GRAF & Adds a graphics edit at the current time to the TRCGRF file. \\
\hline
\end{tabular}




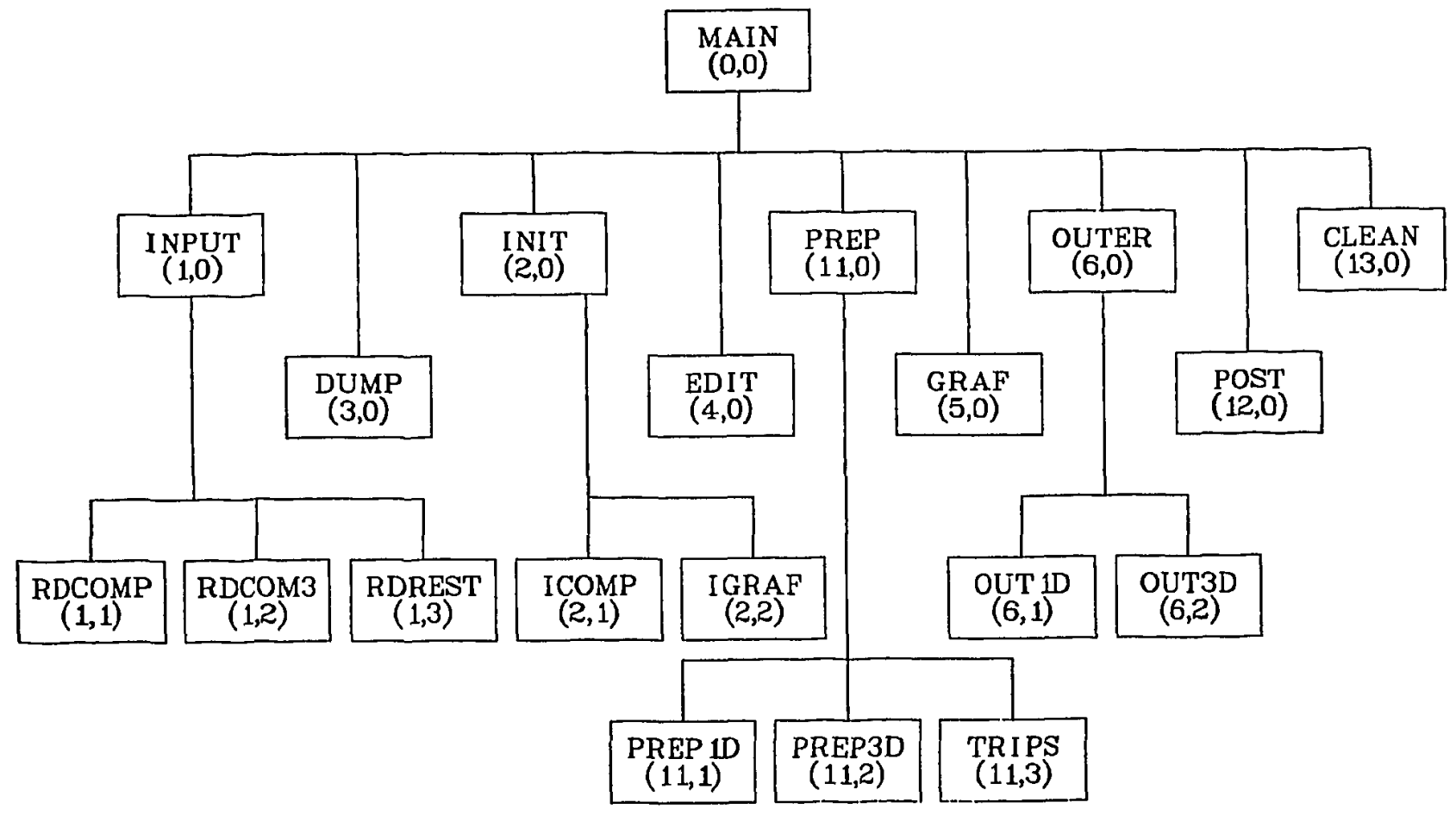

Fig. 50 .

TRAC overlay structure.

divided Into two segments: small core memory (SCM) and large core memory (LCM). The central processor unit (CPU) must retrieve the instruction stream from SCM, which is limited in size, but may retrieve data from either segment. Single-word accesses to SCM require between 27.5 and $275 \mathrm{~ns}$ to complete. Single-word accesses to the 512 000-word LCM require 1760 ns to complete. For transfers of large data blocks between SCM and LCM, the transfer time per word is as low as $27.5 \mathrm{~ns}$.

To take advantage of this feature, TRAC divides the data for each component into four blocks. These are the fixed-length table, the variablelength table, the pointer table, and the array data. The first three of these blocks are stored in SCM in the COMMON blocks, FLTAB, VLTAB, and PTAB, respectively. The structure of the FLTAB COMMON area is the same for all components. The variables in the VLTAB and PTAB COMMON areas differ from one component to another. Appendix I describes the fixed-length, variable-length, and pointer tables for each component. 
The array data are stored in SCM within the dynamic storage array. The location of individual arrays is determined by the value of variables in the pointer table. Dynamic storage of data arrays permits effective use of space for many different problems. The array data for all components are contained in the SCM blank COMMON dynamic area. The pointer tables for all onedimensional components have a common structure. The first 117 pointers locate the basic hydrodynamic, thermodynamic, and heat-transfer information and have the same interpretation for all one-dimensional components. The next 12 pointers locate data for wall heat transfer in those components that support the calculation. This common structure permits construction of lowlevel subroutines that can manipulate data for any one-dimensional component, for example, subroutine INNER in overiay OUTID.

When large-core memory is used, data for a particular component are stored in the dynamic SCM areas only while TRAC processes that component. At other times the data are retained in LCM. Two service subroutines, RDLCM and WRLCM, use the efficient block-transfer capability to transfer data to and from SCM as required. Processing of a component by TRAC begins with the transfer of its component data from LCM to SCM and the computation of the pointer variables based on the avallable space in the dynamic storage array. The processing of this component ends with the reverse data transfer from SCM to LCM. Figure 51 shows the relationship between SCM and LCM storage areas.

In addition to the data that refer to a particular component, TRAC uses many variables to describe the overall state of the calculation. These variables are grouped according to their use into several other COMMON areas. Appendix $H$ describes the structure of these COMMON areas, which are identical throughout TRAC.

The overall sequence of calculations is directed by the main program. Overlay INPUT always is invoked at the start of each TRAC execution to read component and control input data. The component data are initialized by overlay INIT. The reactor power level is set to zero at this point. The steady-state calculation (if requested) is performed by subroutine STEADY. Output operations are performed using the EDIT, GRAF, and DUMP overlays as required. During the steady-state calculation, the reactor power is turned on after the fluld flow rates have been established. This is to prevent high rod temperatures early in the steady-state calculation when the flow rates are 


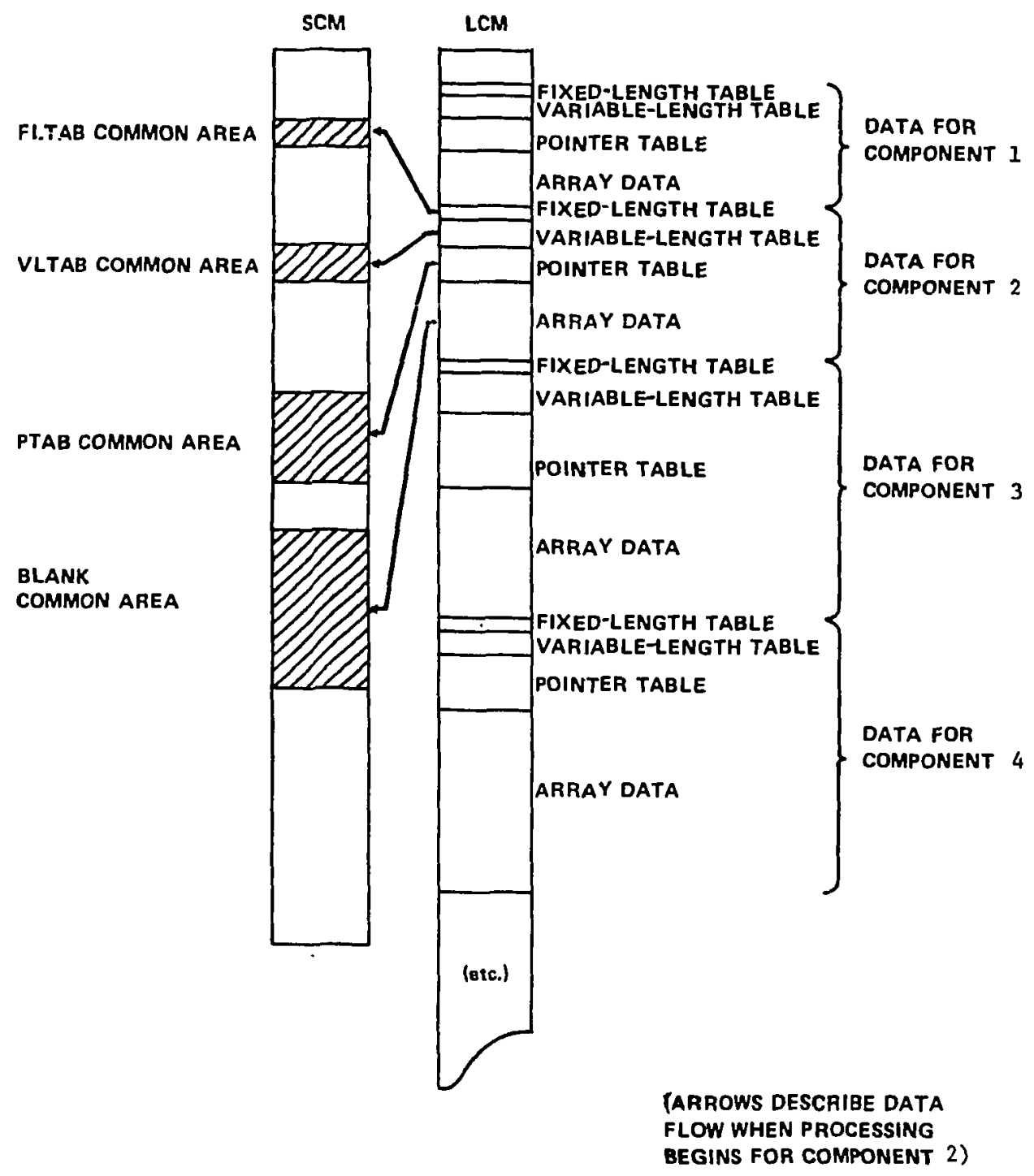

Fig. 51.

Relationships between SCM and LCM storage areas.

small. If no steady-state calculation is performed, the reactor power is turned on by subroutine STEADY in preparation for the transient calculation, which is performed by subroutine TRANS. Overlays EDIT, GRAF, and DUMP are invoked by TRANS to generate output as required. Overlay CLEAN is invoked to close all output files at the end of the problem or when a fatal error occurs. 
The processing of all TRAC input information is performed by the INPUT overlay and its sub-overlays RDCOMP, RDCOM3 and RDREST. This information is of two types: input data cards retrieved from the input file, TRACIN, and restart information from the problem restart file, TRCRST. In addition to obtaining the input data from the appropriate location, these overlays also organize the component data in memory, assign the array pointer variables for every component, allocate one fixed segment of line blank COMMON area, and analyze the problem loop structure.

Subroutine INPUT is the entry point for overlay INPUT and controls the input process. The main control parameters (see Sec.V.C.1) are read from the TRACIN file by subroutine INPUT. Using this information, INPUT allocates one fixed segment of the SCM blank COMMON area, as described in Table XVII. The remainder of the fixed segment of SCM is allocated by subroutine INIT. At the end of each of the overlays, INPUT and INIT, these fixed segments are moved to the end of the dynamic SCM area. (This is done to facilitate special handling of this area on the Laboratory computer.) The signal-variable and trip data from the TRACIN file are read and processed by subroutine RCNTL, which creates the CNTL array described in Fig. 52. Subroutine RDCOMP reads the onedimensional component data from the TRACIN file, assigns pointer values to the data, and then stores the data. RDCOM3 performs a similar function for the vessel component. Signal-variable, trip, and component data not contained in file TRACIN are retrieved from the restart file TRCRST by subroutine RDREST, which is analogous to RDCOMP, as described below. Finally, INPUT utilizes the subroutines ASIGN and SRTLP to fill the component LCM pointer array and to sort system components.

Subroutine RDCOMP invokes a component input routine to process each component. Table XVIII lists these routines. Input routines for onedimensional components utilize subroutine RCOMP to read data that are common to all one-dimensional components. Subroutine RDCOMP determines each component type by reading the first input card. When a component type "END" is encountered, RDCOMP knows that all component input has been read. The component input routines perform the following functions: read input cards for a component, store daita in the component data tables and write them to LCM, assign relative pointers for the component array data, and fill in the JUN 
FIXED SEGMENT ALLOCATIONS FOR THE BLANK COMMON AREA

\begin{tabular}{|c|c|c|}
\hline Pointer & Dimension & Array Description \\
\hline LTITLE & $20 *($ NUMTCR +2$)$ & $\begin{array}{l}\text { Problem title and version information } \\
\text { (stored using only the first four bytes of } \\
\text { each word). }\end{array}$ \\
\hline LORDER & NCOMP & $\begin{array}{l}\text { Component numbers stored in the order used } \\
\text { for iteration. }\end{array}$ \\
\hline LILCMP & NCOMP & $\begin{array}{l}\text { Component LCM pointers stored in the order } \\
\text { in which components were read. }\end{array}$ \\
\hline LNBR & NCOMP & $\begin{array}{l}\text { Component numbers stored in the order in } \\
\text { which components were read. }\end{array}$ \\
\hline LCOMPT & NCOMP & $\begin{array}{l}\text { Component LCM pointers stored in the order } \\
\text { used for iteration. }\end{array}$ \\
\hline LIITNO & NCOMP & $\begin{array}{l}\text { Number of inner iterations during the last } \\
\text { outer iteration for each component (in the } \\
\text { order used for iteration). }\end{array}$ \\
\hline LLCON & NCOMP & $\begin{array}{l}\text { Number of times each component was the last } \\
\text { to converge since last edit. }\end{array}$ \\
\hline LJUN & $8 *$ NJUN & $\begin{array}{l}\text { The JUN array for storage of the junction- } \\
\text { component pairs. }\end{array}$ \\
\hline LJSEQ & NJUN & $\begin{array}{l}\text { Junction numbers in the order in which } \\
\text { junctions occur in the junction-component } \\
\text { array. }\end{array}$ \\
\hline LVSI & NJUN & $\begin{array}{l}\text { Junction flow reversal indicators in the } \\
\text { order in which junctions occur in the } \\
\text { junction-component array. }\end{array}$ \\
\hline LBD & $56 *_{N J U N}$ & Boundary array data. \\
\hline LMAT & NMAT & $\begin{array}{l}\text { Pointer for additional material-property ID } \\
\text { number. }\end{array}$ \\
\hline LPTBLN & NMAT & $\begin{array}{l}\text { Pointer for the number of entry groups in } \\
\text { the user-defined material-property tables. }\end{array}$ \\
\hline LPRPTB & SNTAB & $\begin{array}{l}\text { Polnter to the user-defined } \\
\text { oroperty tables. }\end{array}$ \\
\hline
\end{tabular}


TABLE XVII (cont.)

\begin{tabular}{|c|c|c|}
\hline Pointer & Dimension & Array Description \\
\hline LCNTL & $\mathrm{KPTT}^{\mathrm{a}}$ & $\begin{array}{l}\text { Signal-variable, trip, and controller data } \\
\text { array described in Fig. } 52 \text {. }\end{array}$ \\
\hline LMSCT & NVCON & $\begin{array}{l}\text { Temporary storage for vessel pressure } \\
\text { changes adjaccnt to sources. }\end{array}$ \\
\hline LMCMSH & NVCON & $\begin{array}{l}\text { Storage for coarse-mesh number of vessel } \\
\text { source cells or absolute cell index if } \\
\text { direct vessel solution is used. }\end{array}$ \\
\hline LIVCON & NVCON+1 & $\begin{array}{l}\text { Pointer to network junction numbers that } \\
\text { connect to a vessel. }\end{array}$ \\
\hline LJOUT & $I T+1^{b}$ & $\begin{array}{l}\text { Storage area for pointers that locate the } \\
\text { beginning of each system loop within data } \\
\text { for IOU. }\end{array}$ \\
\hline LNVCNL & $\mathrm{IT}+1$ & $\begin{array}{l}\text { IA(LNVCNL+IL-1) points to the elements of } \\
\text { IVCON and IVLJN that begin the IL th loop. }\end{array}$ \\
\hline LLOOPN & $I T+1$ & $\begin{array}{l}\text { IA(LLOOPN+IL-1) gives the element of the } \\
\text { IORDER array that begins the IL th loop. }\end{array}$ \\
\hline LNSIGP & IT & $\begin{array}{l}\text { NSIGP (IL) is NSIG(IL) plus the number of } \\
\text { steam generators in loop IL. }\end{array}$ \\
\hline LNSIG & IT & $\begin{array}{l}\text { NSIG(IL) is the total number of components } \\
\text { excluding breaks and fills in a loop. }\end{array}$ \\
\hline LNJN & $I T$ & $\begin{array}{l}\text { NJN(IL) is the number of network junctions } \\
\text { in } 1 \text { oop IL. }\end{array}$ \\
\hline LIVLJN & NVCON+1 & $\begin{array}{l}\text { IVLJN(I) is the vessel junction number } \\
\text { corresponding to the network junction number } \\
\text { given by IVCON(I). }\end{array}$ \\
\hline LIOU & $\operatorname{IT} 2^{C}$ & $\begin{array}{l}\text { Network junction numbers for the junctions } \\
\text { of all components excluding breaks and } \\
\text { fills. }\end{array}$ \\
\hline LVRH & NJNT & $\begin{array}{l}\text { Storage for explicit information to evaluate } \\
\text { equations of motion at network junctions. }\end{array}$ \\
\hline
\end{tabular}


TABLE XVII (cont.)

$\begin{array}{ll}\text { Pointer } & \text { Dimension } \\ \text { LDVB } & \text { NJNT } \\ \text { LDREV } & \text { NJNT } \\ \text { LDREL } & \text { NJNT } \\ \text { LDRA } & \text { NJNT } \\ \text { LDRC } & \\ \text { LIDPCV } & \text { NVCON } \\ \text { LDPVC } & \text { Je } \\ \text { LOD } & 4 * N J N M X \\ & \text { JC }\end{array}$

Array Description

Storage for the right-hand side of the network junction equations or the changes in junction velocities.

Storage for righl-hand side of the vapor stabilizer equation.

Storage for right-hand side of the liquid stabilizer equation.

Storage for right-hand side of the air stabilizer equation.

Storage for the right-hand side of the solute concentration equation.

Pointers to coefficients stored in DPCV.

Locator that shows the beginning of coefficients to evaluate the derivatives of junction velocities with respect to vessel pressures.

Network junction coefficient matrix.

Temporary storage for intercomponent coupling information.

$a_{\text {Equals }} 7 * \mathrm{NTSV}+80 * \mathrm{NTRP}+38 * \mathrm{NTSE}+12 * \mathrm{NTCT}+23 * \mathrm{NTSF}+\mathrm{NTDP}+15 * \mathrm{NTSD}+10$.

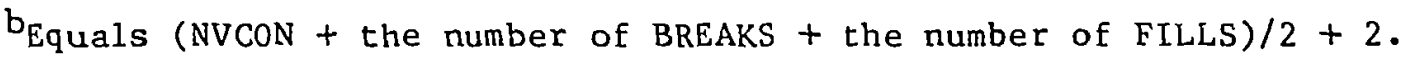

$\mathrm{C}_{\text {Equals }} \operatorname{MAXO}\left\{3,3^{*}[\right.$ IA(LJOUT + NLOOPS $\left.\left.)-1\right]\right\}$.

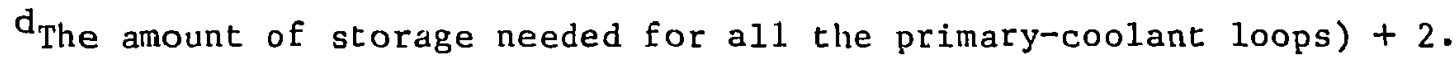

Equals product of the number of junctions in each loop and the number of vessel connections in each loop summed over all loops.

$f_{\text {Equals MAXO [NVCON*2*(JNVSSL }+1), N J N M X *(N J N M X+2)]}$. 


\begin{tabular}{|c|}
\hline KPTT \\
\hline NTSV \\
\hline NTRP \\
\hline NTCN \\
\hline NTSE \\
\hline NTCT \\
\hline NTSF \\
\hline NTDP \\
\hline NTSD \\
\hline $\begin{array}{l}\text { Signal- } \\
\text { Variable } \\
\text { Data }\end{array}$ \\
\hline $\begin{array}{l}\text { General } \\
\text { Trip } \\
\text { Data }\end{array}$ \\
\hline $\begin{array}{l}\text { Signal- } \\
\text { Expression } \\
\text { Trip } \\
\text { Data }\end{array}$ \\
\hline $\begin{array}{l}\text { Trip- } \\
\text { Controlled } \\
\text { Trip } \\
\text { Data }\end{array}$ \\
\hline $\begin{array}{l}\text { Trip } \\
\text { Set-Point } \\
\text { Factor } \\
\text { Tables }\end{array}$ \\
\hline $\begin{array}{l}\text { Trip, } \\
\text { Dump, } \\
\text { and } \\
\text { Termination } \\
\text { Data }\end{array}$ \\
\hline $\begin{array}{l}\text { Trip } \\
\text { Time-Step } \\
\text { Data }\end{array}$ \\
\hline
\end{tabular}

Length of trip data array

Number of signal variables

Number of trips

Number of controllers (currently not used)

Number of signal-expression trips

Number of trip-controlled trips

Number of set-point factor tables

Number of trips that generates restart

dumps and possible problem termination

Number of trip time-step data sets ."

$7 *$ NTSV entries

$80 *$ NTRP entries

$38 *$ NTSE entries

'12*NTCT entries'

$23 *$ NTSF entries

NTDP+l entries

$15 *$ NTSD entries

Fig. 52 .

Signal-variable and trip data. 
TABLE XVIII

\section{COMPONENT INPUT SUBROUTINES}

\begin{tabular}{l} 
Component Type \\
\hline ACCUM \\
BREAK \\
CORE \\
FILL \\
PIPE \\
PRIZER \\
PUMP \\
STGEN \\
TEE \\
TURB \\
VALVE \\
VESSEL
\end{tabular}

\begin{tabular}{l} 
Card Input \\
\hline RACCUM \\
RBREAK \\
RCORE \\
RFILL \\
RPIPE \\
RPRIZR \\
RPUMP \\
RSTGEN \\
RTEE \\
RTURB \\
RVLVE \\
RVSSL
\end{tabular}

Restart Input

REACCM

REBRK

RECORE

REFILL

REPIPE

REPRZR

REPUMP

RESTGN

RETEE

RETURB

REVLVE

REVSSL

array. The JUN array is doubly subscripted, JUN $(4,2 * N J U N)$. The second index indicates the order in which the junction-component pair was encountered during 1nput. The four values of the first index are described in Table XIX. The boundary array data are stored in a doubly dimensioned array, $B D(56, N J U N)$, whose second index indicates the order in which the junctions occur in the input data. The data in this array indicate the current condition of the adjacent component. Because both components connected to the junction use the same storage space, the JUN array reflects the state of the last of these components processed. The fluid properties are evaluated at one of three space points:

1. at the edge of the mesh cell closest to the junction,

2. at the mid-point of that mesh cell, or

3. at the other edge of that mesh cell.

The specific elements of the array are shown in Table XX.

Subroutine RDREST opens restart file TRCRST and finds the dump corresponding to the requested time-step number (Main-Data Card 3). (If the requested time step is negative, RDREST uses the last dump.) This dump then is used to Initialize the components and trips that were not found in the TRACIN file, with the component restart subroutines listed in Table XVIII. Restart subroutines for one-dimensional components utilize subroutine RECOMP to read data that are common to all one-dimensional components. The detailed 
TABLE XIX

FIRST INDEX FOR THE JUNCTION-COMPONENT PAIR ARRAY

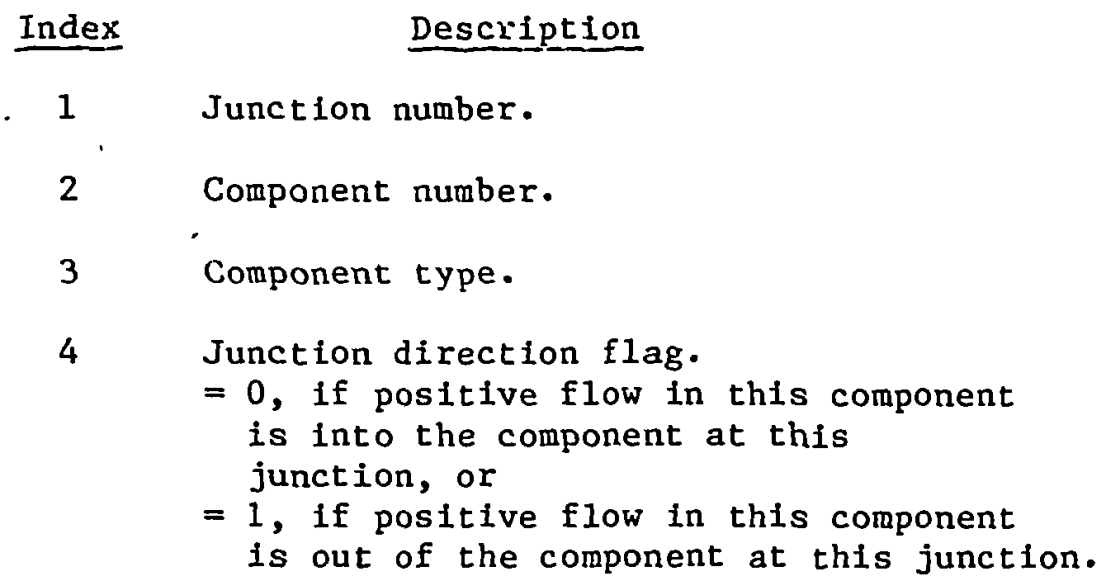

TABLE XX

BOUNDARY ARRAY DATA

Index

1

2

3

4

5

6

7

8

9

10

\section{Description}

Width of the adjacent mesh cell.

Volume of the adjacent mesh cell.

01d mixture density at space point 2 .

Product of new vapor density and vold fraction $\left(\alpha_{g}\right)$ at space point 2 .

Product of liquid density and liquid fraction $\left[(1-\alpha)_{l}\right]$ at space point 2 .

Sign multiplier used on velocities in the adjacent component for consistency.

Old void fraction at space point 2 .

old vapor density at space point 2 .

old liquid density at space point 2 .

New liquid velocity at space point 3 . 
TABLE XX (cont.)

Index

11

12

13

14

15

16

17

$\Rightarrow \quad 18$

19

20

21

22

23

24

26

27

28

30

\section{Description}

New vapor velocity at space point 3 .

Contribution to liquid momentum at space point 1 if there is a tee junction at point 2 .

Contribution to vapor momentum at space point 1 if there is a tee junction at point 2 .

old pressure at space point 2 .

New void fraction at space point 2 .

New vapor density at space point 2 .

New liquid density at space point 2 .

New stabilizer liquid velocity at space point 3 .

New stabilizer vapor velocity at space point 3 .

Further contribution to liquid momentum at point 1 if there is a tee junction at space point 2 .

Further contribution to vapor momentum at point 1 if there is a tee junction at space point 2 .

New pressure at space point 2 .

New liquid velocity at space point 1 .

New vapor velocity at space point 1 .

Surface tension at space point 2 .

Derivative of liquid velocity at point 1 with respect to pressure at space point 2 .

Derivative of vapor velocity at point 1 with respect to pressure at space point 2 .

New macroscopic liquid energy density $\left[(1-\alpha) \rho_{\ell} e_{\ell}\right]$ at space point 2 .

New macroscopic vapor energy density $\left(\alpha \rho_{g} e_{g}\right)$ at space point 2 .

Vapor viscosity at space point 2 . 
TABLE XX (cont.)

Index

31

32

33

34

35

36

37

38

39

40

41

42

43

44

45

46

47

48

49

50

\section{Description}

Liquid viscosity at space point 2 .

Flow area at space point 1 .

Hydraulic diameter at space point 1 .

old stabilizer liquid velocity at space point 3.

old stabilizer vapor velocity at space point 3 .

Component type of last component to enter data into this array.

Component number of last component to enter data into this array.

0ld bit flags for donor-cell logic and detecting crossings of the saturation line.

01d air density $\left(p_{a}\right)$ at space point 2 .

New macroscopic air density $\left(\alpha_{a}\right)$ at space point 2 .

01d macroscopic vapor density $\left(\alpha \rho_{g}\right)$ at space point 2 .

0ld macroscopic liquid density $\left[(1-\alpha) \rho_{\ell}\right]$ at space point 2 .

01d macroscopic vapor energy density $\left(\alpha \rho_{g} e_{g}\right)$ at space point 2 .

01d macroscopic liquid energy density $\left[(1-\alpha) \rho_{\ell} e_{\ell}\right]$ at space point 2 .

Void fraction at the beginning of the previous time step at space point 2 .

old macroscopic air density $\left(\alpha_{a}\right)$ at space point 2 .

01d partial pressure of afr at space point 2 .

$01 d$ vapor temperature at space point 2 .

01d liquid temperature at space point 2.

Vapor velocity averaged for space point 2 . 
TABLE XX (cont.)

Index

51

52

53

54

55

56

Description

Liquid velocity averaged for space point 2 .

Droplet interfacial-drag coefficient for space point 3 .

New bit flag information for space point 2.

Gravity vector at point 3 .

New solute concentration at point 2 .

New phase change rate at point 2 .

structure of the restart file is described in Sec. VI.F in conjunction with the dump capability.

Subroutine SRTLP sorts through the components of the system and groups them by loops that are isolated from one another by a vessel component. The IORDER array is rearranged to reflect this grouping and to provide a convenient order within each group for the flow network solution procedure. C. Component Initialization

The transient or steady-state calculation cannot be initiated directly from the input data. Many arrays and variables for each component are required that are not read by overlay INPUT. Overlay INIT Initializes these data based on the values of the input information. It also creates a table that supplies information to the graphics routines. The entry point subroutine, INIT, controls the inftialization process by calling ICOMP and IGRAF. Subroutine ICOMP completes the component data tables, and IGRAF Initializes the graphics capability.

Subroutine ICOMP checks the junction input data (stored in the junction component pair array JUN) to ensure that the system is configured properly, then fills in the JSEQ and VSI arrays in the fixed segment of the blank COMMON area (Table XVII). Then, ICOMP initializes the data for each one-dimensional component by transferring the component data from LCM to SCM, adjusting the array pointers to reflect the origin of the array data, invoking the appropriate component initialization subroutine (1isted in Table XXI), and then returning the inftialized data to LCM. For each three-dimensional 
component, ICOMP invokes subroutine CIVSSL. Subroutine CIVSSL transfers the component data from LCM to SCM; adjusts the array pointers; calls the intialization routine, IVSSL; and then returns the Initfalized data to LCM.

The component inftialization routines inftialize geometric and heattransfer arrays; inftialize fluid properties by calling subroutines THERMO and FPROP; and inttialize the junction data array, as described in Table XIX, by using subroutine JID. Other individual and array variables are initialized for specific components. Most of the one-dimensional component inftialization routines use subroutines COMPI and IPROP to initialize the data that are common among one-dimensional components.

The subroutine SETNET provides information to the individual components through the IOU array for passing on to the network junction matrix described in Sec. III.D.

The graphics initialization subroutine, IGRAF, creates the TRCGRF file; writes the header, catalog, and geometric data onto the file; and places the catalog in a LCM storage area. The catalog, which contains information about the data to be written on the TRCGRF file during the course of a problem, is constructed by the component graphics initialization routines listed in Table XXI. The data to be edited for each component are specified in these routines. Each datum type adds one entry to the catalog. This entry

TABLE XXI

COMPONENT INITIALIZATION SUBROUTINES

\begin{tabular}{lcc}
$\begin{array}{c}\text { Component } \\
\text { Type }\end{array}$ & $\begin{array}{c}\text { Initialization } \\
\text { Routine }\end{array}$ & $\begin{array}{c}\text { Graphics } \\
\text { Initialization } \\
\text { Routine }\end{array}$ \\
\hline ACCUM & IACCUM & IGACUM \\
BREAK & IBRK & IGBRAK \\
CORE & ICORE & IGCORE \\
FILL & IFILL & IGFILL \\
PIPE & IPIPE & IGPIPE \\
PRIZER & IPRIZR & IGPRZR \\
PUMP & IPUMP & IGPUMP \\
STGEN & ISTGEN & IGSTGN \\
TEE & ITEE & IGTEE \\
TURB & ITURB & IGTURB \\
VALVE & IVLVE & IGVLVE \\
VESSEL & IVSSL & IGVSSL
\end{tabular}


describes the data location and identifies it with the variable name contalning the data and a three-word Hollerith field. The catalog stored in LCM is interrogated later by subroutine GRAF to create each graphics edit. D. Transient Calculation

1. General. The transient calculation is directed by subroutine TRANS. The system state is advanced lhrough time by a sequence of prepass, outer iteration, and postpass calculations that TRANS requests by invoking overlay PREP, subroutine HOUT, and overlay POST, respectively. In these calculations one or more sweeps are made through all the components in the system. To provide the output requested by the user, TRANS invokes the EDIT, DUMP, and GRAF overlays by calling subroutine PSTEPQ.

Subroutine TRANS is structured as shown in Fig. 53. The major control variables within the time-step loop are: NSTEP, the current time-step number; TIMET, the time since the transient began; DELT, the size of the current time step; and OrTNO, the current outer 1teration number. The time-step loop begins with the selection of the time-step size, DELT, by subroutine TIMSTP. A prepass is performed for each component by overlay PREP. At this point, if the current time step is zero, TRANS calls in the EDIT overlay to print the system state at the beginning of the transient. Subroutine TRANS then calls subroutine HOUT that performs one or more outer iterations to solve the basic hydrodynamic equations. Each outer tteration is performed by overlay OUTER and corresponds to one iteration in a Newton solution procedure for the fully coupled difference equations for the flow network (see Sec. III.D.). The outer iteration loop normally completes when the outer iteration convergence criterion (EPSO on Main-Data Card 5) is met. This criterion is applied to the maximum fractional change in the pressures throughout the system during the last iteration.

The outer iteration loop alternatively may terminate when the number of outer iterations reaches a user-specified limit (OITMAX on Main-Data Card 6). In this case, TRAC restores the state of all components to that at the beginning of the time step, halves the time-step size (with the constraint that DELT be greater than or equal to DTMIN), and continues the calculation with the new time-step size. 


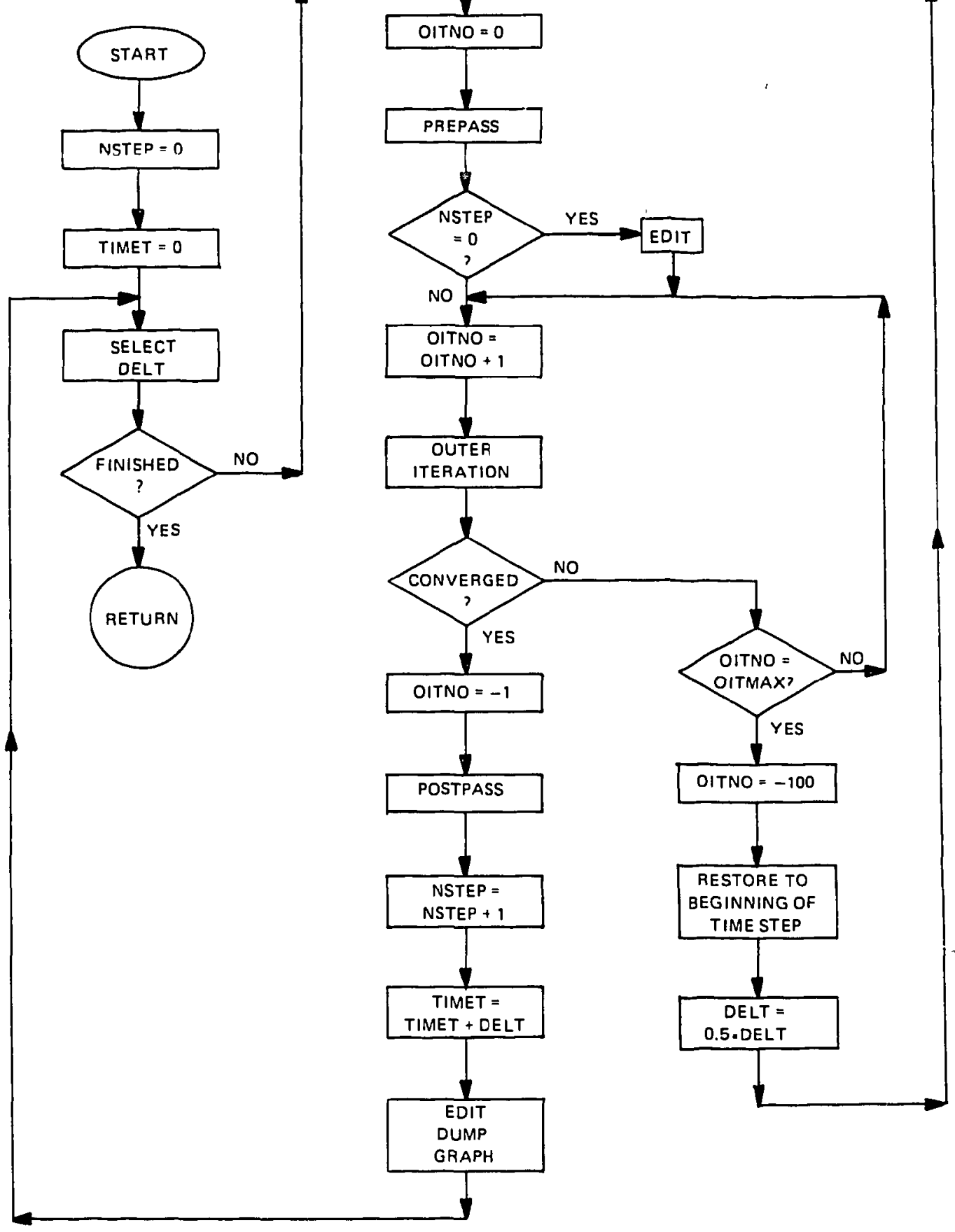

Fig. 53.

Flow diagram for transient calculations. 
When the outer iteration converges, TRANS calls in the POST overlay to perform a postpass. The time-step number then is incremented and TIMET is increased by DELT. A calculation is complete when TIMET reaches the last time specified on the time-step input cards.

2. Time-Step Selection and Output Control. The transient calculation interval is a sequence of time domains specified by the user on the timestep input cards. During each of these domains, the minimum and maximum timestep sizes and the edit, dump, and graphics intervals are fixed. When the EDIT, DUMP, and GRAF overlays are invoked, they calculate the time when the next output of the associated type is to occur. When TRANS finds that TIMET has reached or exceeded the indicated time, the corresponding output overlay is invoked again. Whenever a new time domain is reached, the output indicators are set to the requested time plus the new value of the appropriate interval.

Subroutine TIMSTP reads the time-step control cards and evaluates the size of the next time step. At the beginning of the transient, DELT is set to the minimum size specified for the first time domain. At other times, TIMSTP applies several algorithms, implemented in subroutine NEWDLT, to evaluate the size of the next time step. Subroutine TIMSTP then limits DELT to values between the minimum and maximum specified for this time step, unless the minimum time-step size is greater than the computed Courant limit in threedimensional regions. In this case, DELT is set equal to the Courant limit.

Two types of algorithms, inhibitive and promotional, are implemented in subroutine NEWDLT to evaluate the next tine step. The inhibitive algorithms Iimit the new time-step size to ensure stability and to reduce errors. The promotinnal algorithm increases the time-step size to improve computational efficiency.

Iteration counts are used by both the inhibitive and promotional algorithms. Both the number of outer iterations (MAXIT) and the number of vessel iterations (IIBIG) required for the previous time step are utilized. If MAXIT is less than four and IIBIG is below $70 \%$ of the maximum allowed number of vessel iterations (input as IITMAX), the promotional algorithm increases the time-step size (DELT) by $5 \%$. If MAXIT exceeds five, DELT is reduced by the factor 5/MAXIT; if IIBIG exceeds $0.7 *$ IITMAX+1, DELT is reduced by $(7 * I I T M A X+10) /(10 * I I B I G)$. 
If a new time-step size was estimated in this manner, NEWDLT then invokes the remaining inhibitive algorithms to reduce DELT, if needed for stability or accuracy. The Courant condition in three forms is applied to ensure stabllity. The three forms use the new, old, and extrapolated fluid velocities to evaluate the limit. The accuracy limits are based on the fractional changes in pressure and void fraction and the calculated error in the new total vessel mass.

During the last outer iteration calculation of the previous time step, the maximum changes in the pressure and void fraction over all fluid cells are evaluated. (Cells in which the void fraction is below $1 \%$ are not considered in evaluating the maximum fractional change of the void fraction.) The fractional pressure change ordinarily is limited by the outer convergence criterion, EPSO. If the maximum number of outer iterations is input as one, however, this is not the case. In this instance NEWDLT compares the maximum fractional pressure change (VARERM) to 0.1 . If the change exceeds $10 \%$, a maximum time-step size of $0.1 *$ DELT/VARERM is imposed. Similarly, if the maximum void-fraction change (DAMX) exceeds 0.5 , a maximum tine-step size of $0.5 *$ DELT/DAMX is used.

The error in the vessel mass inventory caused by the previous time step is evaluated by subtracting the mass at the previous time step and the net mass flow into the vessel from the new mass inventory. If the fractional mass error exceeds $0.01 \%$, another maximum time-step size is evaluated as $10^{-4} *_{\mathrm{DELT}}$ divided by the mass error.

Another maximum time-step size calculation is based on the maximum liquid, vapor, slab, and rod temperature changes during the last time step.

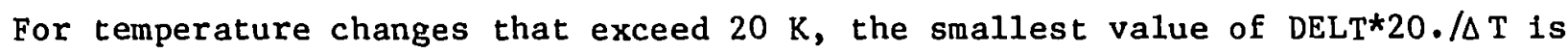
used.

The diffusion number for the rod conduction calculation, DIFMIN, also is used to evaluate, a maximum time-step size. It is evaluated as DIFMIN if DIFMIN is nonzero or $1.0 \times 10^{8}$ if DIFMIN is zero.

3. Prepass Calculations. The prepass calculation uses the system state at the completion of the previous time step to evaluate numerous quantities to be used during the outer iteration. The prepass begins by evaluating trip-signal variables and "determining the set position of all trips. The prepass then loops over all components in the system and performs 
an extra loop over all one-dimensional components. Each component begins the prepass by moving the values calculated during the last time step into the storage area for old time values. (See App. I for a varlables 1ist.) Next, wall and interfacial friction coefficients are calculated, and an initial forward elimination on the stabilizer motion equations is performed. For components that require heat-transfer calculations, the prepass also evaluates materlal properties and HTCs. A second pass through all one-dimensional components is required to do the backward substitution on the stabilizer equations of motion.

The prepass for vessel components can be more complex. Besides calculating material properties and HTCs for both average and hot rods, the prepass evaluates quench-front positions and fine-mesh properties if the reflood segment is under way, as indicated by the reflood flag NRFD.

The prepass calculation is controlled by overlay PREP, whose entry point routine has the same name. Subroutine PREP first processes all onedimensional components by calling PREP1D, which loads data for each component into SCM, invoking the appropriate component prepass subroutine (see Table XXII), then transfers the component data back to LCM. The prepass for all three-dimensional components is performed by PREP3D, which is called once by PREP after all one-dimensional components have been processed.

The one-dimensional component prepass routines utilize the common lowlevel routines SAVBD, PREPER, SVSET, TRPSET, CONBLK, and SETBD to avoid redundant coding. Subroutine SAVBD retrieves boundary data from adjacent components, stores it in the appropriate array locations, and moves dala for the last completed time step into the old time arrays. Subroutine PREPER evaluates wall friction by calling FWALL, evaluates interfacial friction coeffictents and begins solution of the stabilizer equations of motion by calling FEMOM, and uses subroutines MPROP and HTPIPE to evaluate material properties and HTCs. For a specific component any or all of these steps may occur under the control of the PREPER argument list.

Subroutine SVSET uses current values of system state variables to evaluate trip-signal variables. Subroutine TRPSET uses the current signal-variable values to set the status of trips. (This is in contrast to subroutine TRIP that interrogates the trip status in preparation for specific consequences of trips.) Subroutine CONBLK models system controllers by 
TABLE XXII

ITERATION SUBROUTINES

\begin{tabular}{llll}
$\begin{array}{c}\text { Component } \\
\text { Type }\end{array}$ & Prepass & Outer & Postpass \\
\cline { 1 - 1 } ACCUM & ACCUM1 & ACCUM2 & ACCUM3 \\
BREAK & BREAK1 & BREAK2 & BREAK3 \\
CORE & COREC1 & COREC2 & COREC3 \\
FILL & FILL1 & FILL2 & FILL3 \\
PIPE & PIPE1 & PIPE2 & PIPE3 \\
PRIZER & PRIZR1 & PRIZR2 & PRIZR3 \\
PUMP & PUMP1 & PUMP2 & PUMP3 \\
STGEN & STGEN1 & STGEN2 & STGEN3 \\
TEE & TEE1 & TEE2 & TEE3 \\
TURB & TURB1 & TURB2 & TURB3 \\
VALVE & VLVE1 & VLVE2 & VLVE3 \\
VESSEL & VSSL1 & VSSL2 & VSSL3
\end{tabular}

processing specified inputs through predefined control blocks. Subroutine SETED uses the information in the component data arrays to reset the boundáry data at both ends of a component.

Each three-dimensional component is processed by subroutine VSSLl, which PREP3D calls between loading and unloading the appropriate data areas. Subroutine VSSL1 uses subroutine RKIN to solve the reactor-kinetics problem; subroutine HTVSSL to evaluate fluid cell HTCs; and COREl to evaluate rod HTCs, fine-mesh properties, and quench-front positions.

4. Outer Iterations. The hydrodynamic state of the system is analyzed in TRAC by a sequence of Newton iterations that use full inversion of the linearized equations for each external loop and vessel at each iteration (see Sec. III.D). Throughout the sequence of iterations that constitute a time step (each called an outer iteration within TRAC), the properties evaluated during the prepass and the previous postpass remain fixed. These Include wall and rod temperatures, HTCs, wall friction factors, relative velocities, and quench-front positions. The remaining fluid properties are varied to obtain hydrodynamic model solutions.

Each call to overlay OUTER completes a single outer (Newton) iteration. Subroutine OUTER, which is the entry point routine of this overlay, controls the overall structure of an outer iteration, as presented in Fig. 54. Both 


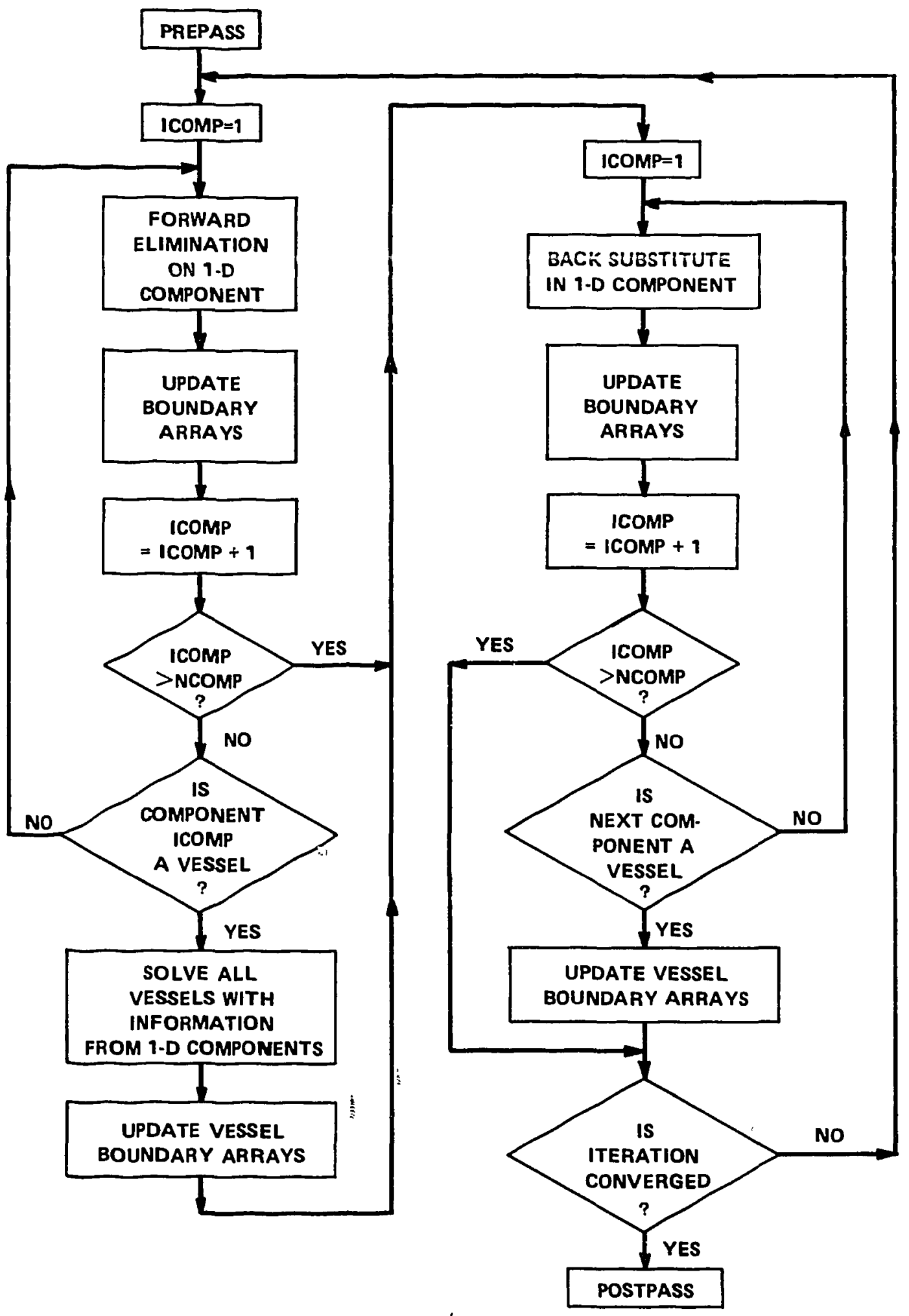

Fig. 54.

Outer iteration flow diagram. 
the forward-elimination and backward-substitution sweeps through the external loops are performed by subroutine OUTID and the associated outer iteration routines. The calculations that these routines perform are controlled by the common variable IBKS, which is set by subroutine OUTER. Subroutine OUT3D solves the hydrodynamic equations for all vessel components (IBKS $=0$ ) or updates boundary data (IBKS $=1$ ).

All one-dimensional components in a particular external loop are handled by a single call to subroutine OUTID. This routine loads the data blocks for a component into SCM, then invokes the appropriate component outer iteration subroutine (as listed in Table XXII), and returns the data to LCM. Subroutine OUT3D works in a similar manner, except that all three-dimensional components use the single subroutine VSSL2.

The outer iteration subroutines for one-dimensional components utilize subroutine INNER to perform common functions. Subroutine INNER retrieves boundary information from the boundary arrays, tests other boundary Information for consistency, calls subroutine TFID to perform the appropriate hydrodynamic calculation, and resets the boundary data arrays by calling subroutine JID. Subroutine TF1D invokes subroutines TF1DS1, TF1DS and TF1DS3 to solve the basic semi-implicit finite-difference equations.

Subroutine VSSL2 solves the appropriate problem (depending on the value of IBKS) for a single vessel component. Subroutines TF3DE and TF3DI are invoked to linearize the hydrodynamic equations. The linear system then is solved by one of two methods depending on the value of the input variable IITMAX. A value of IITMAX $=0$ causes direct inversion of the vessel matrix. If IITMAX >0, the system is solved by Gauss-Seidel iteration with coarse-mesh acceleration. Subroutine VELCK then is utflized to check for velocity sign changes. If any are found, TF3DI is called for the appropriate cells to relinearize the necessary equations.

Subroutine STDIR sets up the vessel matrix for direct inversion when IITMAX $=0$. Subroutine SOLVE is called to solve the linear system and then subroutine BACIT stores the new time pressures that were calculated. Subroutine ITRI is utilized to solve the system by Gauss-Seldel iteration when IITMAX > 0. Subroutines SREBAL and SOLVE are called to set up and solve the coarse-mesh equations for coarse-mesh rebalance. Next, subroutine BREBAL is invoked to apply the coarse-mesh scaling factor to the new time pressures. 
5. Postpass Calculations and Backup. After the system hydrodynamic state has been evaluated by a sequence of outer iterations, TRAC performs a postpass to solve the stabilizer mass and energy equations and to determine both mixture properties and wall and rod temperatures. These are based on the new fluid conditions. Overlay POST performs this postpass. The same overlay also implements the time-step backup procedure. Time-step backup is caused by faflure of the outer iteration process or extreme changes in void fraction. When fallure occurs, the outer iteration counter, OITNO, is set equal to -100, and overlay POST is invoked. Under these conditions, POST returns the component data arrays to their state at the beginning of the time step.

Subroutine POST is the entry point for this overlay. Subroutine POST first processes all one-dimensional components by loading the proper data into SCM, calling the appropriate component postpass subroutine (see Table XXII), and then returning the data to LCM. Then POST invokes subroutine POST3D to handle all three-dimensional components. Subroutine POST3D loads the vessel data areas, calls VSSL3, and returns the vessel data to LCM for each vessel in the problem.

The one-dimenstonal component postpass subroutines use the low-level routines SAVBD, POSTER, and SETBD to retrieve boundary conditions; to evaluate the stablizer equations, wall temperatures, mlxture properties, and transport properties; and to reset the boundary arrays, respectively.

The vessel processor, VSSL3, first must invoke subroutine FF3D to complete the hydrodynanic calculation, then call subroutine CORE 3 to evaluate rod temperatures.

When in the backup mode the postpass subroutine for each component moves data as necessary within the time-dependent array area, then uses subroutine THERMO to relnitialize the thermodynamic property arrays.

6. Vessel Data structure. All array data for any one-dimensional component are loaded into the core whenever that component is processed. Because the amount of array data is much larger for the three-dimensional VESSEL component, this is not possible for components of this type. Therefore, the array data for the VESSEL component are subdivided. There are three categories of VESSEL array data: component, level, and rod. 
The component data arrays describe the overall VESSEL state. These arrays are loaded into SCM before VESSEL processing begins and remain there throughout the VESSEL calculation.

The level data arrays contain fluid-dynamics and wall-temperature data organized by axial level within the VESSEL. A data management subroutine, MANAGE, is used by all VESSEL subroutines to load single levels into SCM and to replace them in LCM. There are never more than three levels of data in SCM at one time. These arrays are rotated through the SCM area during the fluiddynamics calculation by the VESSEL iteration subroutines.

The rod data arrays contain detailed information about the heat-transfer calculation in the fuel rods. These data are organized by fuel rod so that only data pertaining to the rod under study are in SCM at once. These data arrays are loaded into (and unloaded from) the blank COMMON area by calls to the MANAGE subroutine. Subroutines CORE1 and CORE3 coordinate the rod heattransfer calculations including the management of rod data.

In addition to solving the data space problem, the organization of the VESSEL array data improves the calculation efficiency by grouping data by their use. However, it introduces a communication problem between the fluiddynamics and heat-transfer calculations because some data must be in both the rod and the level arrays. This problem is resolved by a data transfer between the rod and the level data arrays. On a CDC 7600 this transfer is performed by direct LCM to LCM copies üsing subroutine LCMOVE during the prepass calculation.

E. Steady-State Calculations

Subroutine STEADY directs steady-state calculations. The calculation sequence of this subroutine is similar to that of the transient driver subroutine TRANS. Both STEADY and TRANS use subroutine HOUT to perform a group of outer iterations. The same sequence of iterations used for transient calculations also is used to advance the steady-state calculation. The main difference is the addition of steady-state convergence tests to STEADY. To provide output requested by the user, STEADY invokes the EDIT, DUMP, and GRAF overlays by calling subroutine PSTEPQ. These overlays are described in Sec. VI.F. Subroutine STEADY is called by the main program whether or not a steady-state" calculation has been requested. If no steady-state calculation 
is required, STEADY simply initializes the VESSEL power and returns control to the main program.

The steady-state calculation to be performed is determined by the value of the input variable STDYST on Main-Data Card 4, as described in the input specifications in Sec. V.C.1. The code sets the steady-state indication flag, ISTDY, to 1 and the transient calculation time, TIMET, to -1 to begin the steady-state calculations. In steady-state calculations the time variable is STIME instead of TIMET. These values of ISTDY and TIMET have the effects indicated in Table XXIII.

The time-step control in STEADY is identical to that implemented in TRANS. This includes the selection of the time-step size, the output timing, and the restarting of time steps if the outer iteration limit is exceeded. (In STEADY the input variable SITMAX, from Main-Data Card 6, is used as a delimiter in place of OITMAX.) The maximum normalized rates of change are calculated by subroutines TFIDS3 and FF3D. These rates and their locations in the system are transmitted to STEADY through the variables FMX and LOK in COMMON block SSCON. Tests for st'eady-state convergence are performed every 100 time steps and before every edit. The maximum normalized rates of change and their locations are included in the printed output, as shown in Table XXIV. The minimum value of the axial velocity (MINVEL) and its maximum normalized rate of change (FMXLVZ) in the vessel determine when the reactor power should be turned on. Once MINVEL exceeds $0.5 \mathrm{~m} / \mathrm{s}$ or FMXLVZ falls below 0.5 , the reactor power is set to the input value, RPOWRI (specified on Card

TABLE” XXIII

STEADY-STATE CALCULATION EFFECTS

Subroutine

PRIZR1

PRIZR2

PRIZR3

PUMP2

TIMSTP

TRPSET

\section{Effects}

Bypass calculation of fluid and thérmal conditions, and cause cataloging of mass flow through the system junctions.

Causes momentum source to be time averaged.

Multiplies time-step size for heat transfer by RTWFP. Causes trips with positive ID numbers not to be activated. 
EXAMPLE OF A STEADY-STATE CONVERGENCE EDIT

STEADY-STATE TIME-STEP NUMBER 114, CONVERGED IN 2 ITERATIONS •

$$
\text { TIME }=5.187 \times 10^{-2} \quad \text { DELT }=2.480 \times 10^{-3}
$$

\begin{tabular}{lccrr}
\multicolumn{1}{c}{ Variable } & Maximum Change Ratio & & Component & Cell \\
\cline { 2 - 4 } Pressure & $-5.228985 \times 10^{-1}$ & & 1 & 43 \\
Liquid velocity & $3.85848 \times 10^{1}$ & 14 & 1 \\
Vapor velocity & 0. & 0 & 0 \\
Void fraction & 0. & 0 & 0 \\
Liquid temperature & $-2.00387 \times 10^{-2}$ & 6 & 2 \\
Vapor temperature & $2.12964 \times 10^{-2}$ & 0 & 0 \\
Air pressure & 0. & 0 & 0
\end{tabular}

Number 12 of the vessel input data or Card Number 10 of the core input data). The generalized steady state completes when all normalized rates of change are below the user-specified convergence criterion, EPSS (on Main-Data Card 5) or when STIME reaches the end of the last time domain specified in the steadystate time-step input cards.

Both steady-state and transient calculations may be performed in one computer run. The end of the generalized steady-state time-step cards is signified by a single card containing a -1.0 in columns 11-14. The transient time-step input cards should follow Immediately. If the generalized steady state converges before reaching the end of the last time domain, the remaining sceady-state tine-step input cards are read so that the transient calculation proceeds correct $1 y$.

Figure 55 is a flow diagram for the steady-state calculation. 


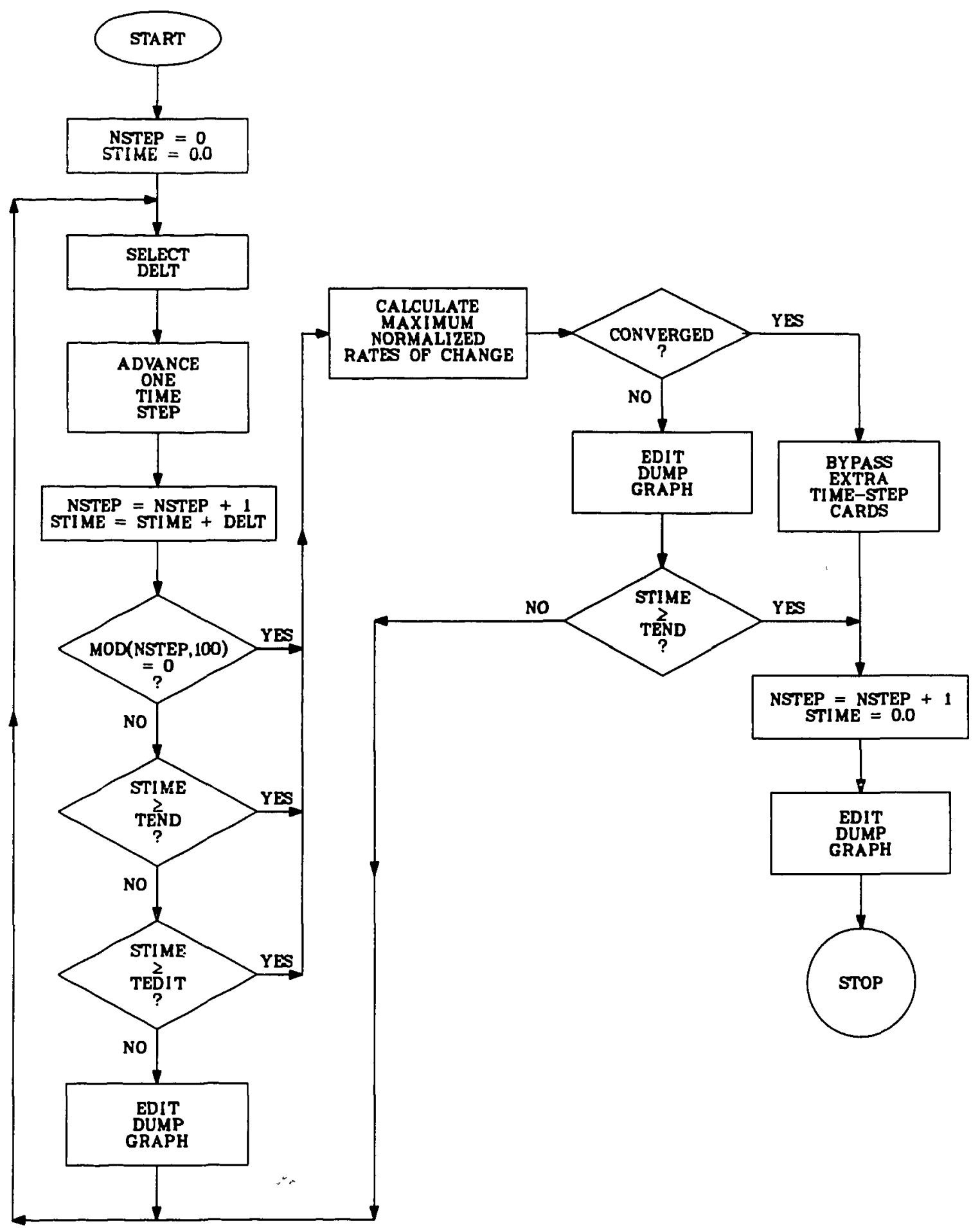

Fig. 55 .

Flow diagram for steady-state calculation. 
The TRAC program produces five output files: TRCOUT, TRCMSG, TRCGRF, TRCDMP, and TRCINP. The first of these files is in printer format and contains a user-oriented analysis of the calculation. During the input process, an input data description is placed in this file. At selected times during the calculation, overlay EDIT is invoked to add to this file a description of the current system state. The TRCGRF and TRCDMP files are binary files designed to allow analysis by graphics postprocessing programs and problem restart by TRAC, respectively. The TRCGRF file is created and the header, catalog, and geometric data are written into it during the initialization phase. File TRCDMP is created immediately thereafter by overlay DUMP. The TRCMSG file is in printer format and contains diagnostic messages concerning the progress of the calculation. File TRCINP is only created when the TRACIN input file is in free format. The data from TRACIN is written into file TRCINP in a form that can be read by the TRAC input routines. TRCINP is then used as the input file to TRAC.

Subroutine WCOMP, which is called by the entry point routine EDIT, directs the addition of a time-step edit to the TRCOUT file. Subroutine WCOMP writes general overall data first, then invokes lower level routines to describe the state of each component. For one-dimensional components, WCOMP directly calls the appropriate component edit subroutine. Table XXV lists the component edit subroutines. These routines invoke subroutine ECOMP to write the data common between one-dimensional components. For three-dimensional components, WCOMP calls subroutine CWVSSL, which loads the component data tables into SCM and then invokes the component edit subroutine WVSSL. The component edj.t routines add the data that are important for that component to the TRCOUT file in an appropriate format.

After Initialization by IGRAF, the time-edit data are added to the TRCGRF file by overlay GRAF. This overlay contains the single subroutine, GRAF, which uses the LCM graph data area. The TRCGRF file is a structured binary file written with unformatted write statements and containing information for graphics processing. 


\begin{tabular}{lcl}
$\begin{array}{c}\text { Component } \\
\text { Type }\end{array}$ & $\begin{array}{c}\text { First-Leve1 } \\
\text { Subroutine }\end{array}$ & $\begin{array}{l}\text { Lower-Leve1 } \\
\text { Subroutine }\end{array}$ \\
\cline { 3 - 4 } ACCUM & -- & WACCUM \\
BREAK & -- & WBREAK \\
CORE & -- & WCORE \\
FILL & -- & WFILL \\
PIPE & -- & WPIPE \\
PRIZER & -- & WPRIZR \\
PUMP & -- & WPUMP \\
STGEN & -- & WSTGEN \\
TEE & -- & WTEE \\
TURB & -- & WTURB \\
VALVE & -- & WVLVE \\
VESSEL & CWVSSL & WVSSL
\end{tabular}

Data contained on the TRCGRF file may be divided into four sections:

1. general information,

2. catalog information,

3. geometric information, and

4. time-edit data.

These data appear on the file in the above order, as shown in Fig. 56. The structure of the general information section of the file is given in Fig. 57 . This section contains title cards for problem identification and size information needed to describe the problem and the remainder of the file. The catalog section (Fig. 58) contains information that is used to describe the data stored in the time-edit section. The geometric section contains information relating to the cell structure of components. The time-edit section is made up of blocks of data as shown in Fig. 59. Individual arrays within each block are packed to save space. A block is written at each graphics edit taken during the course of a problem. The number of time-edit blocks written on the file is determined by the graphics edit frequency specified on the time-step cards. The last block is followed by a word "EOF" to signify the "end of file." 


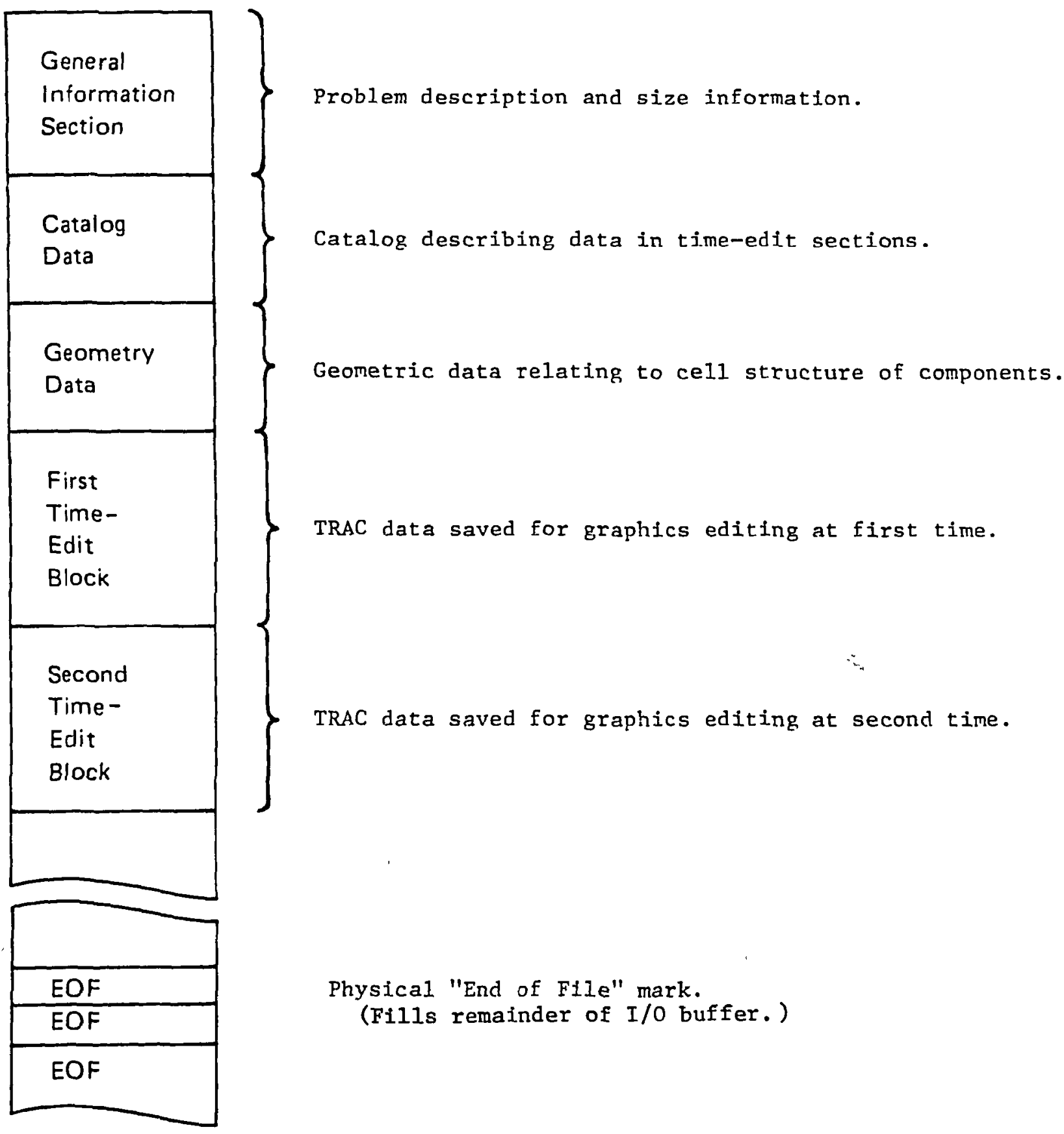

Fig. 56.

Overall graphics file structure. 


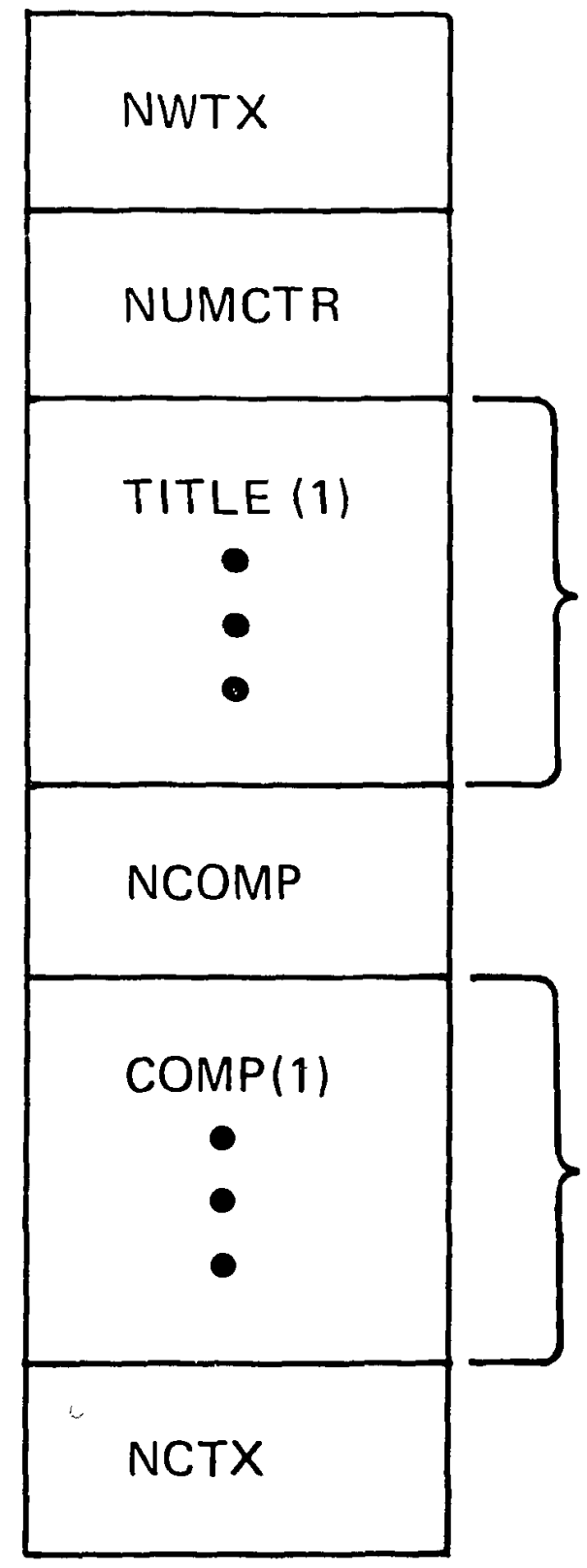

Length of each edit block (first word on file

Number of title cards

Title cards, 20*NUMCTR entries

Number of components

Component types, NCOMP entries

Number of catalog entries

Fig. 57 .

Structure of graphics file general information section. 


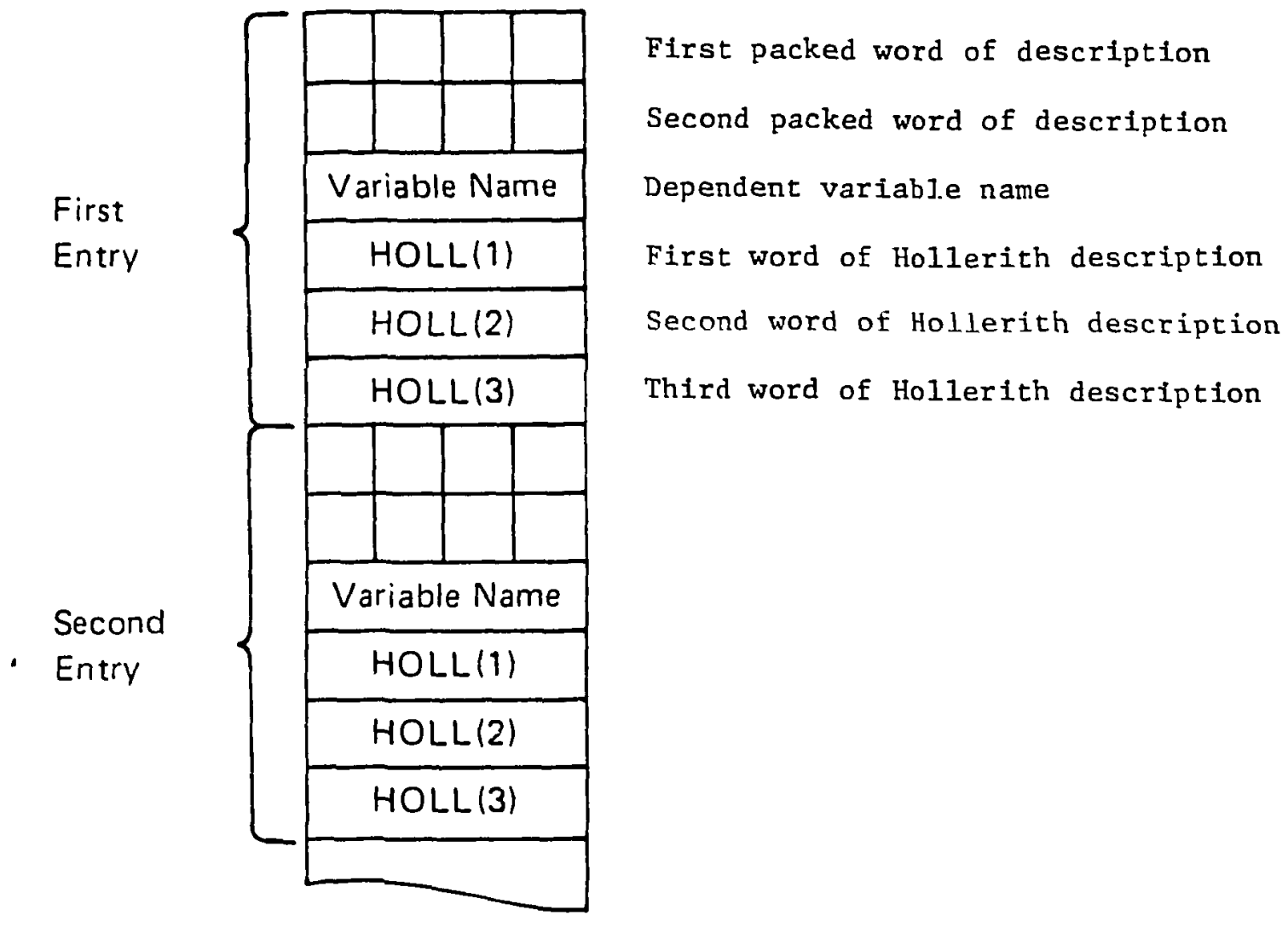

FIRST PACKED WORD OF DESCRIPTION

\begin{tabular}{|l|l|l|l|}
\hline ICOMP & NUM & ITYPE & NWRD \\
\hline
\end{tabular}

SECOND PACKED WORD OF DESCRIPTION

\begin{tabular}{|l|l|l|l|}
\hline ILRN & KPT & NSKIP & IPOS \\
\hline
\end{tabular}

\footnotetext{
ICOMP -- TRAC-assigned component number

NUM -- User-assigned component number

ITYPE -- Data type

NWRD -- Number of unpacked words stored

ILRN - Level or rod number

1) KPT - Relative pointer to data in TRCGRF

NSKIP - Data skip frequency

IPOS -- Relative pointer to where data were extracted from TRAC data base

Fig. 58 .

Structure of graphics file catalog. 


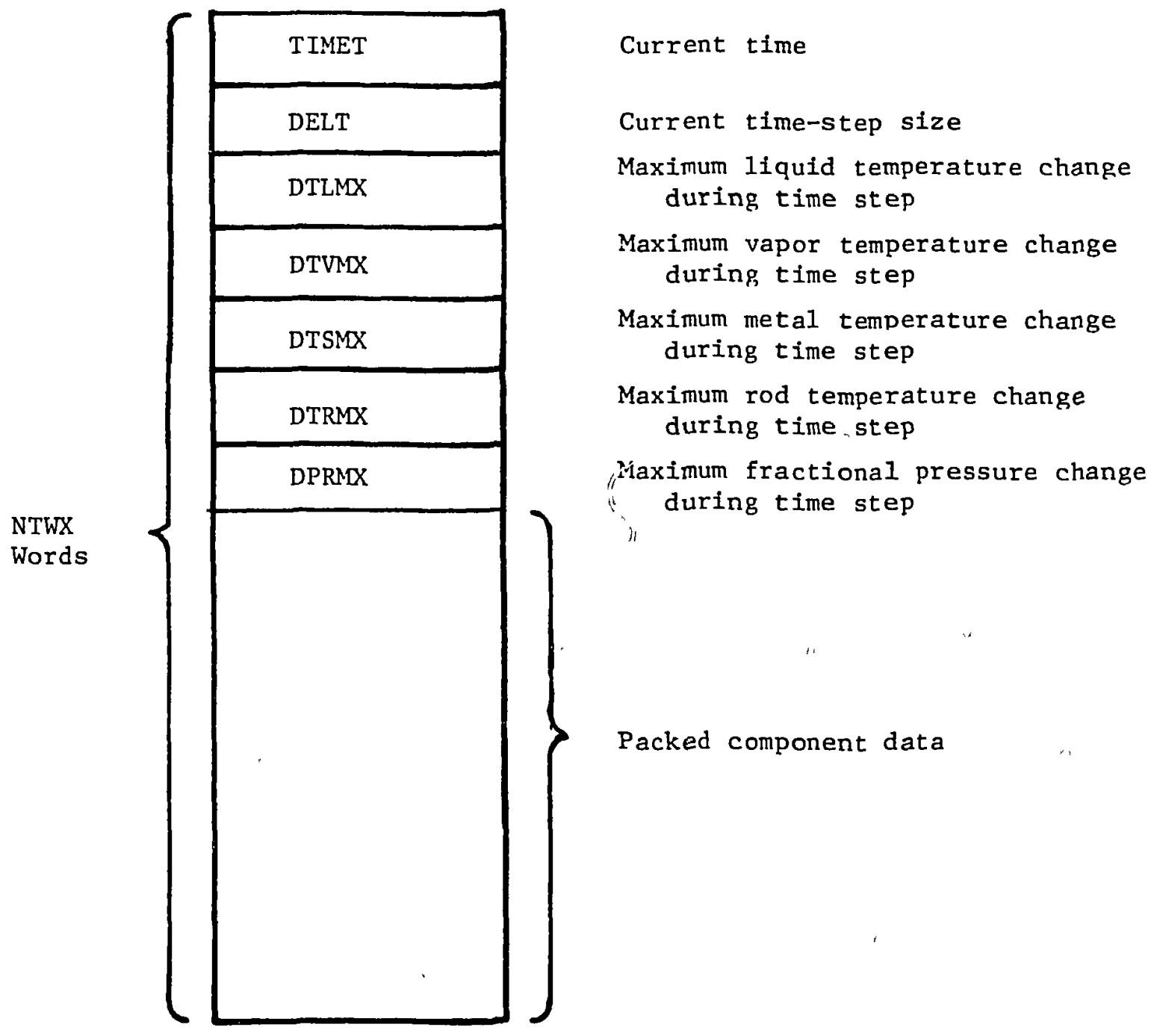

Fig. 59.

Structure of graphics file time-edit data section. 
The structure and lengths of the time-edit blocks are identical to one snother, minimizing the required catalog information. The catalog is made up of NCTX data entries with one catalog entry for each data type in a block. This relationship is displayed in Fig. 60. Each catalog entry contains six words that provide a data description and a pointer, relative to the beginning of the block, to a specific section in each block. A catalog entry may describe a single varlable or a data array. The word count also is included in the catalog. The data types stored are pressures, temperatures, void fractions, and other important system parameters.

The TRCDMP file is a structured binary file written with unformatted write statements. It contains sufficient data to restart the calculation from the current state, as described in Sec.VI.B. This file is created by a sequence of calls to overlay DUMP. The entry point subroutine, DMPIT, writes the dump header data and calls the component dump subroutines, which are listed in Table XXVI.

Figures 61-63 show the resulting file structure. This structure permits easy location of specific dumps and specific components within each dump. This reduces the effort required to restart the problem.

Figure 61 shows the overall dump-file structure with a general information section at the beginning followed by a series of time-edit blocks. A block is written at each dump edit taken during a problem. The number of time-edit blocks written on the file is determined by the dump-edit frequency specified on the time-edit cards. The last block is followed by the word "EOF" to signify the "end of file."

The structure of each time-edit block in the dump file is illustrated in Fig. 62. Data from each component is included in the component dump section shown at the bottom of the figure. Figure 63 shows a more detailed structure of the component dump section.

G. Storage Requirements

Although maximum use is made of dynamic storage allocation within TRAC, there are limitations on the complexity of problems that may be simulated. These limitations arise from the finite extent of the component data storage areas, as listed in Table XXVII. These limitations are imposed on the complexity of single components as well as on the system as a whole. 

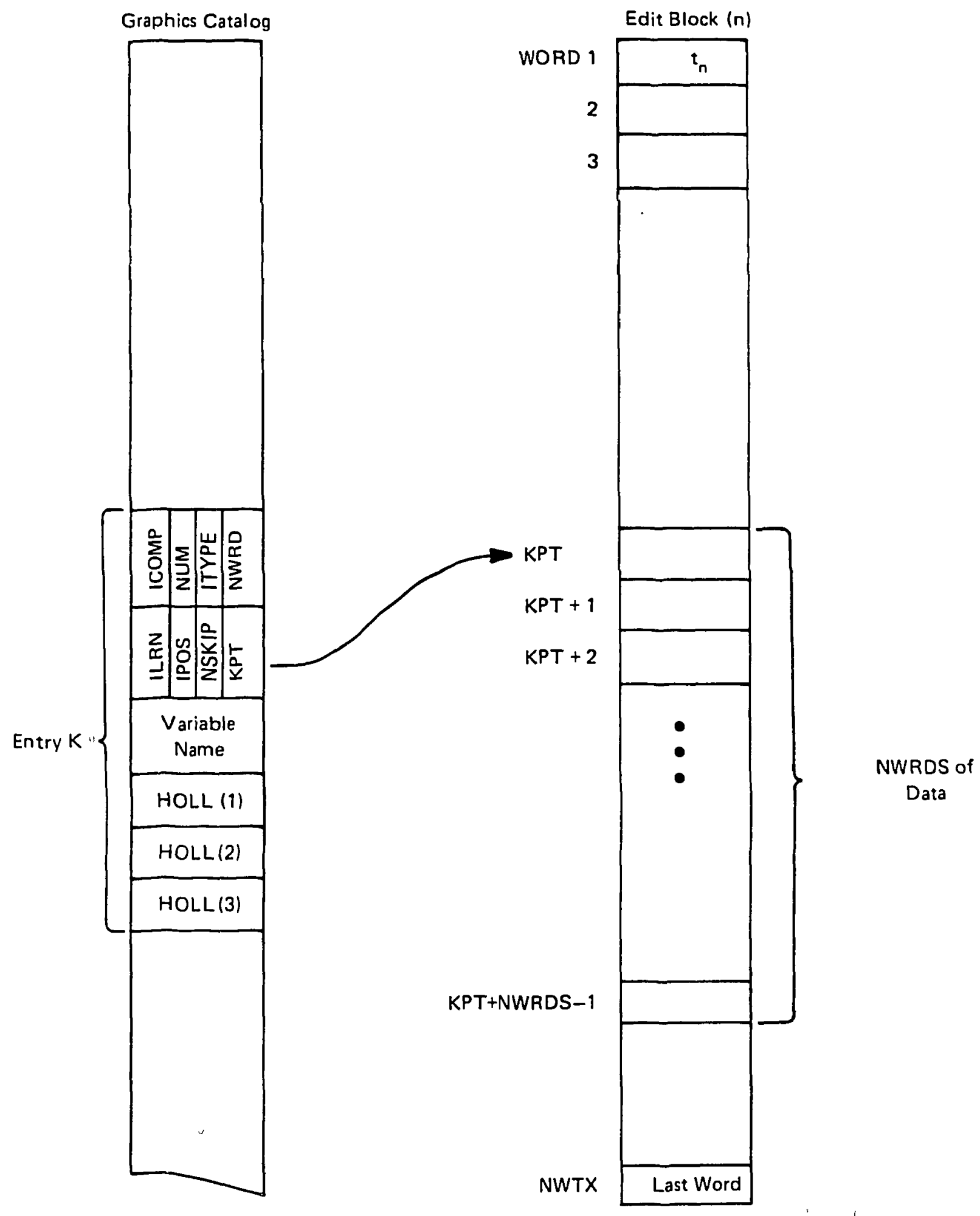

Fig. 60 .

Graphics file catalog and time-edit data correspondence. 


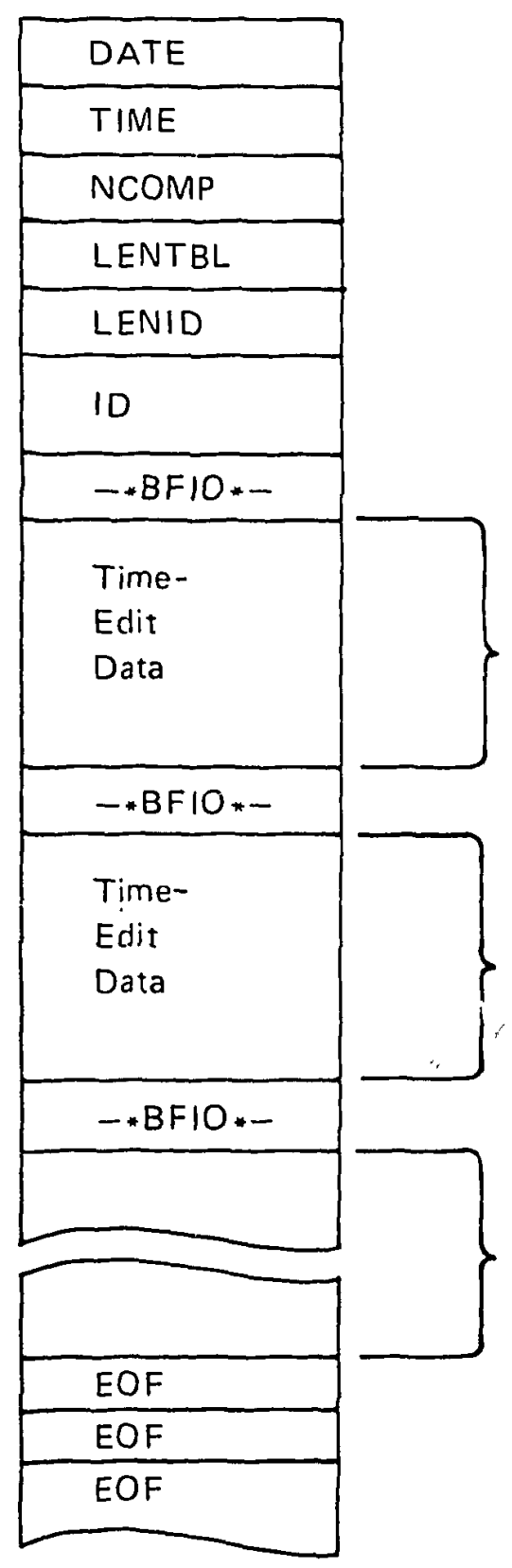

Date of file creation

Time of file creation

Number of components

Length of fixed-length table

Length of problem ID

Problem ID

Word indicating logical "end of file"

Dump at first time

Dump at second time

etc.

Physical "End of File" mark (Fills remainder of $1 / 0$ Buffer.) 


\begin{tabular}{|c|c|}
\hline ETIME & \multirow{23}{*}{ 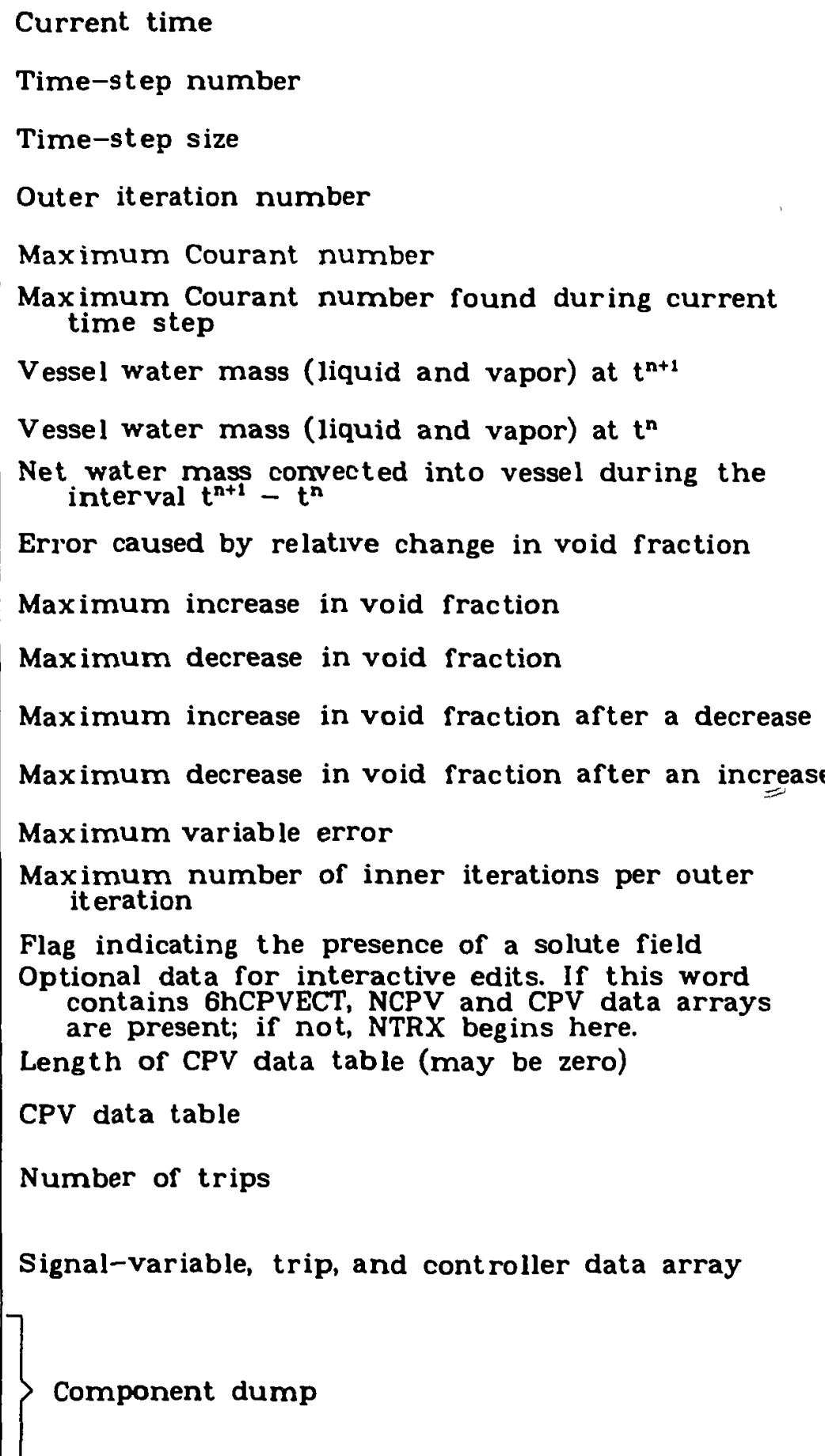 } \\
\hline NSTEP & \\
\hline DELT & \\
\hline OITNO & \\
\hline VMAXT & \\
\hline VMAXO & \\
\hline VMNEW & \\
\hline VMOLD & \\
\hline V $\mathbf{M}$ & \\
\hline DAMX & \\
\hline $\mathrm{DAU}$ & \\
\hline DAL & \\
\hline OAU & \\
\hline OAL & \\
\hline VERERM & \\
\hline II BIG & \\
\hline ISOLUT & \\
\hline CPV Flag & \\
\hline NCPV & \\
\hline CPV Data & \\
\hline NTRX & \\
\hline troller & \\
\hline & \\
\hline
\end{tabular}

Fig. 62 .

Structure of dump-file time-edit data section. 


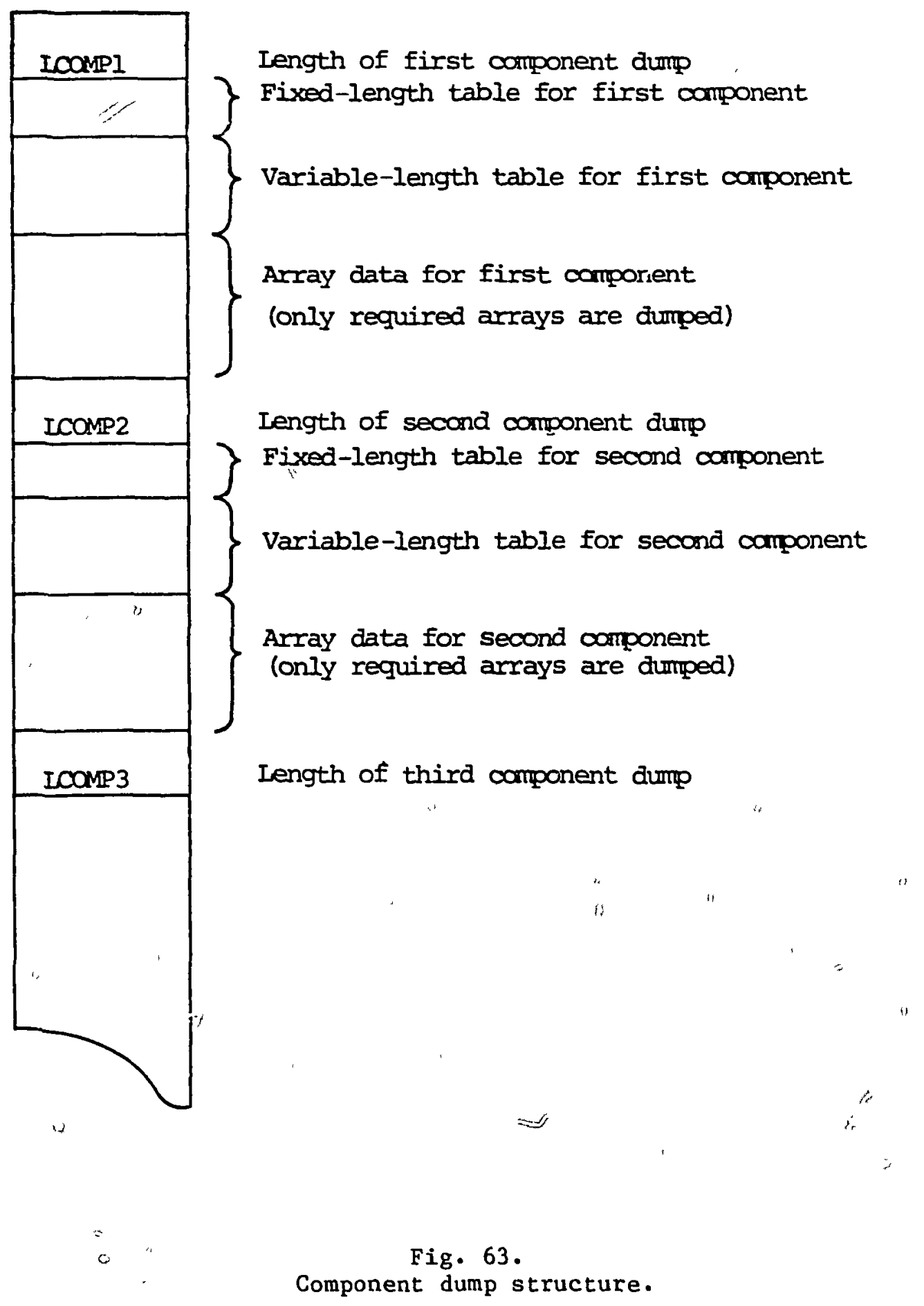


TABLE XXVI

COMPONENT DUMP SUBROUTINES

\begin{tabular}{|c|c|}
\hline $\begin{array}{c}\text { Component } \\
\text { Type }\end{array}$ & Subroutine \\
\hline ACCUM & DCOMP \\
\hline BREAK & DBRK \\
\hline CORE & LCORE \\
\hline FILL & DFILL \\
\hline PIPE & DCOMP \\
\hline PRIZER & DCOMP \\
\hline PUMP & DPUMP \\
\hline STGEN & DCOMP \\
\hline TEE & DCOMP \\
\hline TURB & DTURB \\
\hline VALVE & DVLVE \\
\hline VESSEL & DVSSL \\
\hline
\end{tabular}

TABLE XXVII

TRAC STORAGE ALLOCATIONS

$\begin{array}{lr}\text { Storage Area } & \text { Size (Words) } \\ \text { COMMON/LCMSP/ } & 13 i 071 \\ \text { BLANK COMMON } & 20000\end{array}$

Figure 64 displays the organization of the blank COMMON dynamic storage area in SCM. The fixed segment, which is described in Table XVII, contains information that is used by all system component subroutines and, therefore, must remain in SCM throughout the calculation.

The area that remains, marked as the component data area in Fig. 64, is available to each component when its data is in SCM. Each component type requires varying amounts of array space. Other than CORE, the required space for component types that are modeled with one-dimensional fluid dynamics is linear in the number of fluid cells, the number of heat-transfer nodes, and the product of these numbers. The CORE component requires additional space for storage of rod data. In any case, the space is assigned by the appropriate input routine (RPIPE, REPIPE, etc.), and the total amount of SCM 


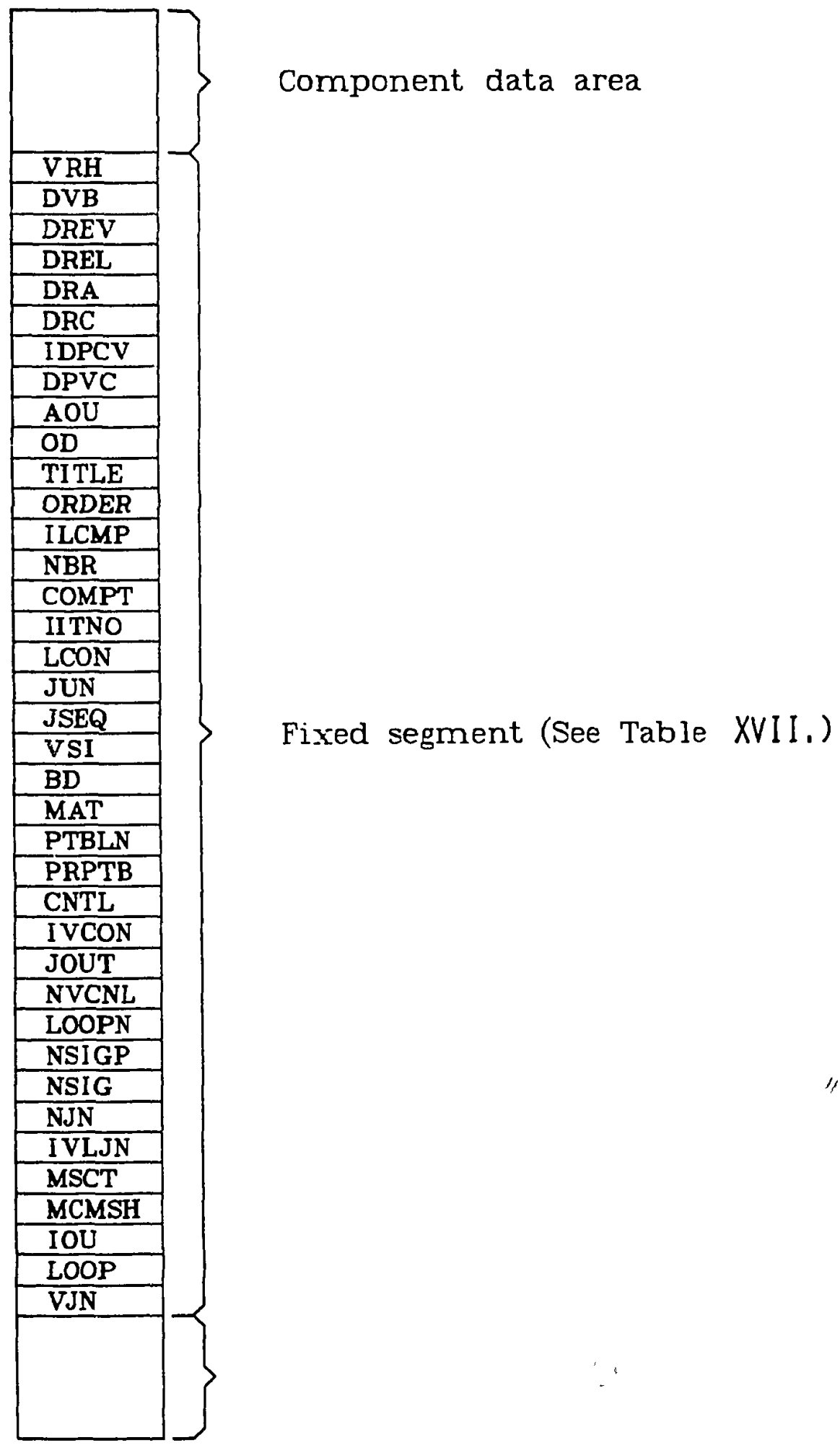

Fig. 64 .

Blank COMMON dynamic storage area organization. 
space required for each component is stored in the fixed-length table variable LENARR.

The array data for the VESSEL component are subdivided into three categories: component, level, and rod. The component data arrays describe the overall VESSEL state and remain in SCM throughout the VESSEL calculation. The variable LENARR contains the number of words of space in the component data area. The level and rod data arrays are transferred to SCM only when they are needed. The hydrodynamic variables for each axial level in the VESSEL are transferred between SCM and LCM as a unit. At certain points in the calculation, data for three distinct levels must be in SCM simultaneously. Therefore the SCM space required for each level is three times, its length

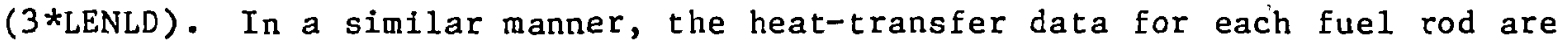
transferred between SCM and LCM as a unit. Because the data for only one rod must be in SCM at any point in the calculation, the rod data may extend over the avallable space in SCM. Storage requirements for each rod are given by LENRD. Level data and rod data do not reside in SCM simultaneously. All of the allocation of this space is accomplished in the subroutines RVSSL and REVSSL.

In addition to the component, ievel, and rod data arrays, NVCELL*(NVCELL+2) words of SCM space are needed for VESSEL components. This space is needed either for direct inversion of the vessel natrix when IITMAX $=0$ or for solving the system by Gauss-Seidel iteration with coarsemesh acceleration when IITMAX $>0$. If IITMAX' $=0$, then NVCELL is the total number of vessel cells. If IITMAX > 0, NVCELL is the total number of vessel coarse-mesh regions.

The finite extent of the LCM component storage area (COMMON block LCMSP) limits the total amount of component data that can be handled in a calculation. This amount is found by suming the SCM array requirements including all VESSEL levels and rods and adding the space required for the fixed-length, variable-length, and pointer tables for each component. The graphics catalog, discussed in Sec. VI.F, also is stored in this LCM block. This area requires one word for each component plus six words for every catalog entry. Figure 65 shows the organization of LCMSP. 


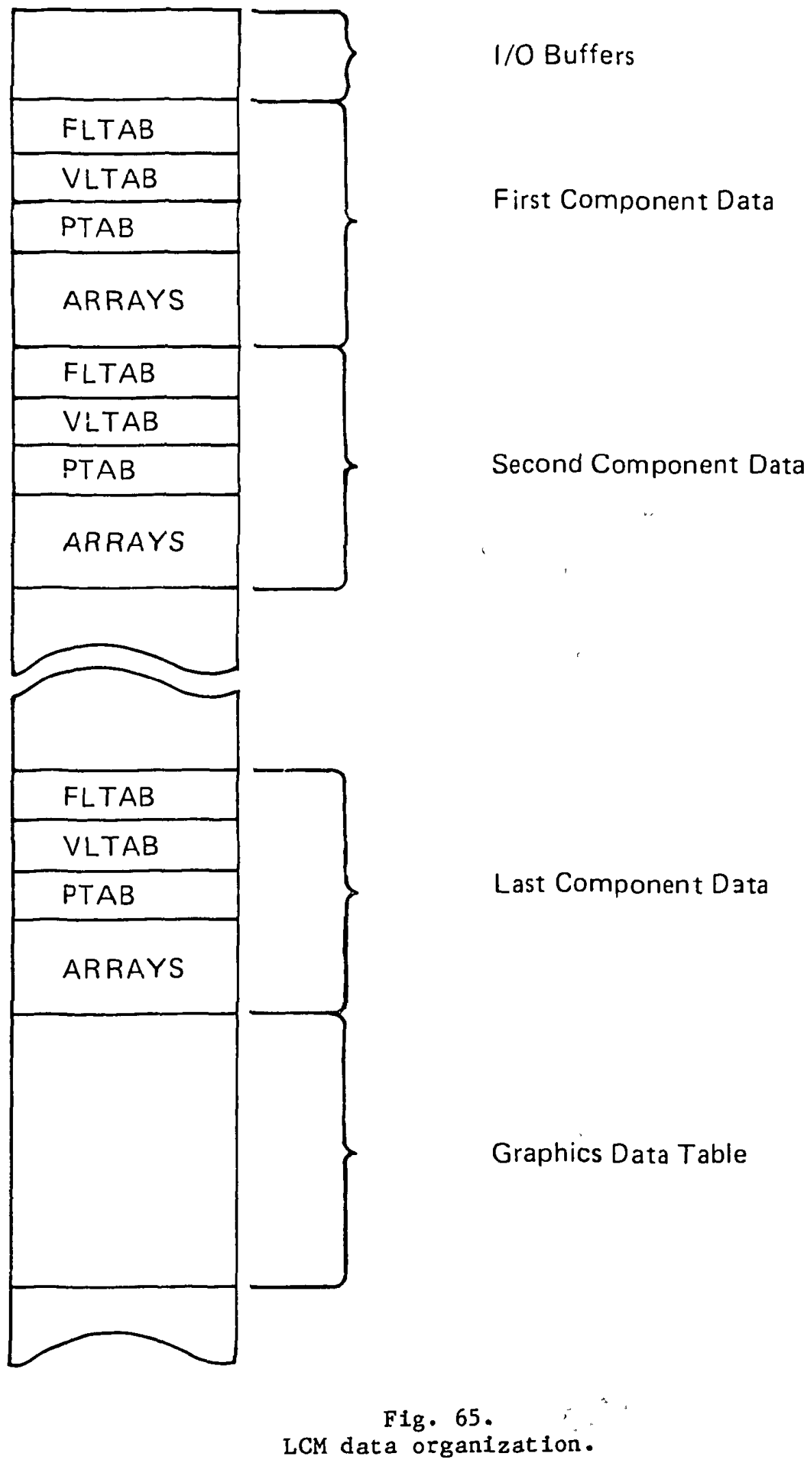




\section{H. TRAC for Various Machine Configurations}

At Los Alamos both our CDC 7600 and Cray versions of TRAC are maintained within a single CDC Update program library. Machine-dependent coding is isolated with Update *IF DEF cards. This capability was instalied in a way that makes conversion to other computers and operating systems much simpler than was possible with previous versions of TRAC.

The available definitions are summarized in Table XXVIII. For a CDC machine we recommend starting with the definitions 57600 , OVLAY, and TENB. For a Cray the appropriate definitions are CRAY, NOLCM, and EIGHTB. The variable dimension (VDM) definition is used at Los Alamos to implement dynamic run-time array storage, for both CDC (SCM and LCM storage) and Cray versions. The VDM option requires local operating system features to allocate memory. Currently, a static memory version is not in the coding for cray or other machines with large contiguous memories (that is, NOLCM machines). To implement VDM on the Los Alamos Crays, two system calls are used: one to obtain the code's field length, and another to expand memory as the input deck is read. An update is available at Los Alamos to implement static memory NOLCM versions. This update will be incorporated in TRAC in the near future. In the meantime, new NOLCM users should contact the Los Alamos TRAC group to obtain this update if they wish to use a static memory code.

When TRAC is implemented on a local computer, we recommend that any necessary changes be made with updates to the original program library and that the original sequence numbers for the code be retained. As corrections and minor improvements are made at Los Alamos, the Update decks will be based on the original update sequencing. These code changes will be made avallable to authorized users through a dial-up connection to a Los Alamos computer. 
TABLE XXVIII

TRAC DEFINITIONS

\section{Definition}

S7600

L7600

CRAY

OVLAY

FOURB

EIGHTB

TENB

NOLCM

HEX

VDM

LANL

INTERACT

\section{Description}

Coding is unique to the CDC 7600 Scope system.

Coding is unique to the CDC 7600 LTSS system.

Coding is unique to Cray computers.

On--code is overlaid. off--code is not overlaid (intended for CDC computers).

Coding is unique to a machine with four 8-bit bytes in a word.

Coding is unique to a machine with eight 8 -bit bytes in a word.

Coding is unique to a machine with ten 6-bit bytes in a word.

A machine with a contiguous memory (no LCM such as the CDC 7600 has).

On--use hexadecimal numbers. Off--use octal numbers.

Use variable-dimension memory.

On--codes are unique to Los Alamos.

On--create interactive version (currently not supported). 
APPENDIX A

\section{THERMODYNAMIC AND TRANSPORT FLUID PROPERTIES}

The thermodynamic and transport properties subroutines used in TRAC are based on polynomial fits to steam table data for water, and ideal gas behavior for the noncondensable gas component. Transport property fits were obtained from Ref. 55. The thermodynamic property routines are used by all TRAC component modules. Tables A-I through A-VI, which list the values of the constants, are given at the end of this appendix.

\section{THERMODYNAMIC PROPERTIES}

Subroutine THERMO supplies thermodynamic properties for TRAC. The Input variables are the total pressure, the partial pressure of the noncondensable gas component, and the liquid and gas-phase temperatures. The output variables include the saturation temperature corresponding to total pressure; the saturation temperature corresponding to the partial pressure of steam; the spectfic internal energies of liquid, gas phase, and noncondensable; the saturated liquid and steam enthalples corresponding to the partial pressure of steam; the liquid, gas-phase and noncondensable densities; the derivatives of saturation temperatures and saturation enthalpies with respect to pressure; and, finally, the partial derivatives of liquid, steam, and noncondensable internal energies and densities with respect to pressure (at constant temperature) and with respect to temperature (at constant pressure).

The range of validity for the thermodynamic properties supplied by THERMO is

$273.15 \mathrm{~K} \leq \mathrm{T}_{\ell} \leq 713.94 \mathrm{~K}$,

$273.15 \mathrm{~K} S \mathrm{~T}_{\mathrm{g}} \leq 3000.0 \mathrm{~K}$,

and

$1.0 \mathrm{~Pa} \leq \mathrm{p} \leq 45.0 \times 10^{6} \mathrm{~Pa}$. 
If THERMO is provided with data outside this range, it adjusts the data to the corresponding limit and issues a warning message.

Equations for the various properties used in THERMO are given below. Values of the constants are given in Tables A-I and A-II.

A. Saturation Properties

1. Relationship Between Saturation Temperature and Pressure. For $\mathrm{p}_{s}<90564.66 \mathrm{~Pa}$ and $\mathrm{T}_{s}<370.4251 \mathrm{~K}$, the enthalpy of evaporation $\mathrm{H}_{\ell \mathrm{v}}$ is represented accurately as a linear function of temperature, such that,

$h_{\ell v s}=3180619.59-2470.2120 \mathrm{~T}_{\mathrm{S}}$

The Clausius-Clapeyron equation, which assumes that stear is an ideal gas and neglects liquid volume compared to steam volume, can be written as

$\frac{\mathrm{dp}_{s}}{\mathrm{dT}_{s}}=\frac{h_{\ell v s} \mathrm{p}_{s}}{\mathrm{R}_{\mathrm{v}} \mathrm{T}_{\mathrm{s}}^{2}}$,

where $R_{v}$ is the gas constant for steam. Substituting for $h_{\ell} v s$ and integrating using the boundary condition $\mathrm{P}_{S}=24821 \mathrm{~Pa}$ at $\mathrm{T}_{S}=338 \mathrm{~K}$, gives

$p_{S}=24821\left(T_{s} / 338\right)^{-5.3512} \exp \left[20.387\left(T_{S}-338\right) / T_{s}\right]$.

TRAC calculations require determination of $T_{S}$ from given $p_{S}$, which can only be calculated by trial and error from the above equation. Instead of iterating on the above equation, a simplified logic for calcuiating $T_{s}$ is used that guarantees solution in a single iteration with an error of only a fraction of a percent. First, an approximate value of $T_{s}$ is calculated from

$\mathrm{T}_{\mathrm{s}, \text { approx }}=\frac{2263}{6.064-0.434 \ln \left(\mathrm{p}_{\mathrm{s}} / 100000\right)}$ 


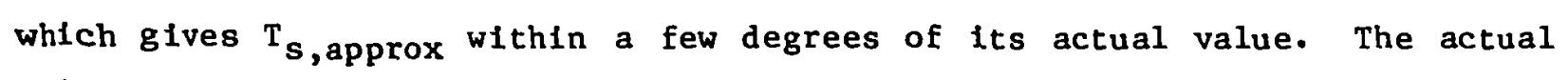
value of $T_{s}$ then is calculated by integrating the Clausius-clapeyron equation assuming constant $h_{\ell v s}$ between $T_{s}$, approx and $T_{s}$, which gives

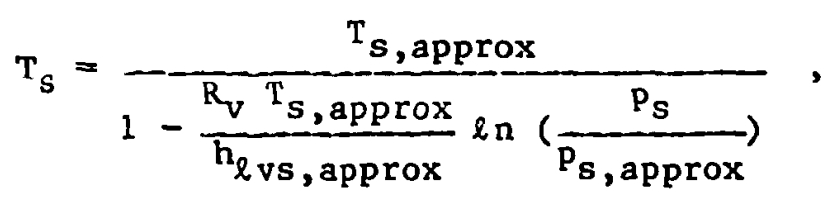

where $h_{\ell y s, a p p r o x}$ and $P_{S}$,approx are calculated corresponding to $T_{s, \text { approx }}$ using the equations given above. For $\mathrm{p}_{\mathrm{s}} 29.056466 \times 10^{4} \mathrm{~Pa}$ and $\mathrm{T}_{\mathrm{s}} \geq 370.4251 \mathrm{~K}$,

$$
p_{s}=\frac{1}{A_{14}}\left(\frac{T_{s}-C_{3}}{C_{1}}\right) C_{2}^{\frac{1}{2}}
$$

and

$\frac{\mathrm{dp}_{\mathrm{s}}}{\mathrm{dT} \mathrm{s}_{\mathrm{s}}}=\frac{\mathrm{p}_{\mathrm{s}}}{\mathrm{C}_{2}\left(\mathrm{~T}_{\mathrm{s}}-\mathrm{C}_{3}\right)}$

2. Internal Energy of Steam. For $\mathrm{p}_{\mathrm{v}} \leq 69738.3980 \mathrm{~Pa}$, where $\mathrm{p}_{\mathrm{v}}$ is the partial pressure of steam,

$e_{v s}=h_{v s}-\frac{p_{v}}{\rho_{v s}}=h_{v s}-R_{v} T_{s}$

and

$\frac{\mathrm{de}_{\mathrm{vs}}}{\mathrm{d \textrm {p } _ { \mathrm { v } }}}=\frac{\mathrm{dh}_{\mathrm{v} s}}{\mathrm{dp}_{\mathrm{v}}}-\mathrm{R}_{\mathrm{v}} \frac{\mathrm{dT}_{\mathrm{s}}}{\mathrm{d \textrm {p } _ { \mathrm { v } }}}$, 
where

$h_{v s}=-42.4793+4186.8\left(T_{s}-273.15\right)+h_{\ell v s}$

and

$\frac{d h_{v s}}{d p_{v}}=4186.8 \frac{d T_{s}}{d p_{v}}+\frac{d h_{\ell v s}}{d p_{v}}=(4186.8-2470.2120) \frac{d_{s}}{d p_{v}}$.

For $69738.3980<\mathrm{p}_{\mathrm{v}} \leq 88487.7383 \mathrm{~Pa}$,

$\mathrm{e}_{\mathrm{vs}} \doteq \mathrm{h}_{\mathrm{vs}}-\frac{\mathrm{p}_{\mathrm{v}}}{\rho_{\mathrm{vs}}}=\mathrm{h}_{\mathrm{vs}}-\mathrm{e}_{\mathrm{vs}}\left(\mathrm{\gamma}_{\mathrm{s}}-1\right)$

where

$\gamma_{s}=C_{9}+C_{10} P_{v}+C_{11} p_{v}^{2}$

Therefore,

$e_{v s}=\frac{h_{v s}}{Y_{s}}$

and

$\frac{d e_{v s}}{d p_{v}}=\frac{1}{\gamma_{s}}\left(\frac{d h_{v s}}{d p_{v}}-e_{v s} \frac{d \gamma_{s}}{d p_{v}}\right)$,

with $h_{v s}$ still given by the expression under $p_{v} \leq 69738.3980 \mathrm{~Pa}$ regime. For $88487.7383<\mathrm{p}_{\mathrm{v}} \leq \mathrm{C}_{21} \mathrm{~Pa}$,

$$
e_{v s}=c_{6}+\frac{c_{7}}{c_{8}+p_{v}}
$$


and

$\frac{\mathrm{de}_{\mathrm{vs}}}{\mathrm{dp}}=-\frac{c_{7}}{\left(c_{8}+p_{\mathrm{v}}\right)^{2}}$

For $\mathrm{P}_{\mathrm{v}}>\mathrm{C}_{21} \mathrm{~Pa}$,

$e_{v s}=c_{12}+\left(c_{14} p_{v}+c_{13}\right) p_{v}$

and

$\frac{\mathrm{de}_{\mathrm{vs}}}{\mathrm{dp}_{\mathrm{v}}}=\mathrm{C}_{13}+\mathrm{A}_{17 \mathrm{P}_{\mathrm{v}}}$

3. Heat Capacity of Steam at Constant Pressure. Although the heat capacity of steam is not an output variable from THERMO, it is required in subsequent steam property calculations. For $\mathrm{P}_{\mathrm{v}}<90564.66 \mathrm{~Pa}$,

$$
\begin{aligned}
C_{\text {pvs }}= & 1748.7825+3.5107478 \mathrm{~T}_{\mathrm{S}}-2.2509364 \times 10^{-2} \mathrm{~T}_{\mathrm{S}}^{2} \\
& +4.0515323 \times 10^{-5} \mathrm{~T}_{\mathrm{s}}^{3}
\end{aligned}
$$

and

$\frac{d c_{p v s}}{d p_{v}}=\frac{\mathrm{dT}_{s}}{\mathrm{dp}_{\mathrm{v}}}\left(3.5107478-4.5018729 \times 10^{-2} \mathrm{~T}_{\mathrm{s}}+1.2154597 \times 10^{-4} \mathrm{~T}_{\mathrm{s}}^{2}\right)$,

where $T_{s}$ is the saturation temperature corresponding to the partial pressure of steam. Fic $\mathrm{p}_{\mathrm{v}} \geq 90564.66 \mathrm{~Pa}$,

$c_{\text {pvs }}=\mathrm{C}_{52}+\mathrm{C}_{54} \mathrm{~T}_{1}+\mathrm{C}_{53} \mathrm{~T}_{1}^{2}+\frac{\mathrm{C}_{56}}{\mathrm{~T}_{1}}+\frac{\mathrm{C}_{55}}{\mathrm{~T}_{1}^{2}}$ 
and

$\frac{\mathrm{dc}_{\mathrm{pvs}}}{\mathrm{dP}_{\mathrm{v}}}=-\mathrm{A}_{15} \frac{\mathrm{dT}_{\mathrm{s}}}{\mathrm{dP}_{\mathrm{v}}}\left(\mathrm{C}_{54}+2 \mathrm{C}_{53} \mathrm{~T}_{1}-\frac{\mathrm{C}_{56}}{\mathrm{~T}_{1}^{2}}-2 \frac{\mathrm{C}_{55}}{\mathrm{~T}_{1}^{3}}\right)$,

where $\mathrm{T}_{1}=1-\mathrm{A}_{15} \mathrm{~T}_{\mathbf{S}}$.

4. Enthalpy of Steam. For $\mathrm{p}_{\mathrm{v}} \leq 88487.7383 \mathrm{~Pa}$, ,

$h_{v s}=-42.4793+4186.8\left(T_{s}-273.15\right)+h_{\ell \cdot v s}$

and

$\frac{d h_{v s}}{d p_{v}}=4186.8 \frac{\mathrm{dT}_{s}}{d p_{v}}+\frac{d h_{\ell v s}}{d p_{v}}=(4186.8-2470.2120) \frac{\mathrm{dT}_{s}}{d p_{v}}$.

For $\mathrm{P}_{\mathrm{v}}>88487.7383 \mathrm{~Pa}$,

$h_{v s}=e_{v s} \gamma_{s}$

and

$\frac{d h_{v s}}{d p_{v}}=r_{s} \frac{d e_{v s}}{d p_{v}}+e_{v s} \frac{d r_{s}}{d p_{v}}$

where (i)

$$
\begin{aligned}
\gamma_{s} & =c_{9}+C_{10} p_{v}+c_{11} p_{v}^{2}, \quad \text { for } 69738.3980<p_{v} \leq c_{21} \mathrm{~Pa}, \text { and } \\
& =c_{15}+C_{16} P_{v}+c_{17} p_{v}^{2}, \text { for } p_{v}>C_{21} \mathrm{~Pa} .
\end{aligned}
$$




\section{Density of Steam. The density of steam is calculated as}

$\rho_{\mathrm{vs}}=\frac{p_{\mathrm{v}}}{\left(\gamma_{s-1}\right) e_{\mathrm{vs}}}$

where

$r_{s}=\frac{h_{v s}}{e_{v s}}, \quad$ for $p_{v} \leq 69738.3980 \mathrm{~Pa}$

$=\mathrm{C}_{9}+\mathrm{C}_{10} \mathrm{P}_{\mathrm{v}}+\mathrm{C}_{11} \mathrm{p}_{\mathrm{v}}^{2}$, for $69738.3980<\mathrm{p}_{\mathrm{v}} \leq \mathrm{C}_{21} \mathrm{~Pa}$

$=\mathrm{c}_{15}+\mathrm{c}_{16} \mathrm{p}_{\mathrm{v}}+\mathrm{c}_{17} \mathrm{p}_{\mathrm{v}}^{2}$, for $\mathrm{p}_{\mathrm{v}}>\mathrm{C}_{21} \mathrm{~Pa}$.

\section{Enthalpy of Liquid.}

$$
h_{\ell s}=e_{\ell s}+\frac{p}{\rho_{\ell s}}
$$

and

$\frac{d h_{\ell s}}{d p}=\frac{d_{\ell s}}{d T_{s}} \frac{d T_{s}}{d p}+\frac{1}{\rho_{\ell s}}-\frac{p}{\rho_{\ell s}^{2}}\left[\left(\frac{\partial \rho_{\ell s}}{\partial p}\right)_{T}+\left(\frac{\partial \rho_{\ell s}}{\partial T}\right)_{p} \frac{d T_{s}}{d p}\right]$,

where $e_{\ell s}, \rho_{\ell s}$, and their derivatives are evaluated using the liquid equations given below. 


\section{B. Liquid Properties}

1. Internal Energy. The liquid internal energy is calculated by adding a correction term to the internal energy at saturation (corresponding to saturation pressure at liquid temperature), that is,

$e_{\ell}=e_{\ell}\left(T_{\ell}, P S L\right)+E L P$,

where PSL is the saturation pressure corresponding to $T_{\ell}$, and

$\operatorname{ELP}=(p-P S L)\left(\frac{\partial e_{\ell}}{\partial p}\right)_{T_{\ell}}$

where $\left(\partial e_{\ell} / \partial \mathrm{p}\right)_{\mathrm{T}_{\ell}}$ is calculated from the following expression.

$\left(\frac{\partial \mathrm{e}_{\ell}}{\partial \mathrm{p}}\right)_{\mathrm{T}_{\ell}}=\mathrm{C}_{\mathrm{ko}}\left[1-\exp \left(\mathrm{C}_{\mathrm{k} 4} \cdot \mathrm{PSL}\right)\right]+\mathrm{C}_{\mathrm{k} 2} \cdot \mathrm{PSL}^{2}$.

Therefore, the internal energy derivative with respect to $T_{\ell}$ is calculated as

$\left(\frac{\partial e_{\ell}}{\partial T_{\ell}}\right)_{P}=\left\{\frac{\partial}{\partial T_{\ell}}\left[e_{\ell}\left(T_{\ell}, P S L\right)\right]\right\}_{p}+E R T$,

where

$$
\begin{aligned}
& E R T=\left[\frac{\partial}{\partial T_{\ell}}(E L P)\right]_{p}=\left[C_{k o}\left[-1+\left(1-C_{k 4} p+C_{k 4} \cdot P S L\right) \exp \left(C_{k 4} \cdot P S L\right)\right]\right. \\
& \left.+C_{k 2}\left[2 p \cdot P S L-3 \cdot \mathrm{PSL}^{2}\right]\right\} \frac{\mathrm{d}}{\mathrm{dt} \mathrm{t}_{\ell}}(\mathrm{PSL})
\end{aligned}
$$


There are four temperature domains used in evaluating the liquid internal energy.

a. $\mathrm{T}_{\ell} \leq 370.4251 \mathrm{~K}$.

$$
e_{\ell}=-42.4793+4186.8 \cdot T L C+E L P
$$

and

$\left(\frac{\partial \mathrm{e}_{\ell}}{\partial \mathrm{T}_{\ell}}\right)_{\mathrm{p}}=4186.8+\mathrm{ERT}$,

where $\mathrm{TLC}=\mathrm{T}_{\ell}-273.15$.

$$
\text { b. } \quad 370.4251<\mathrm{T}_{\ell}<548.15 \mathrm{~K} \text {. }
$$$$
\mathrm{e}_{\ell}=\mathrm{ELCO}+\mathrm{ELC1} \cdot \mathrm{TLC}+\mathrm{ELC2} \cdot \mathrm{TLC}^{2}+\mathrm{ELC} \cdot \mathrm{TLC}^{3}+\mathrm{ELC} \cdot \mathrm{TLC}^{4}+\mathrm{ELP}
$$

and

$$
\begin{aligned}
& \left(\frac{\partial \mathrm{e}_{\ell}}{\partial \mathrm{T}_{\ell}}\right)_{\ell} \mathrm{P}=\mathrm{DELCO}+\mathrm{DELCl} \cdot \mathrm{TLC}+\mathrm{DELC} 2 \cdot \mathrm{TLC}^{2}+\mathrm{DELC} 3 \cdot \mathrm{TLC}^{3}+\mathrm{ERT} \\
& \text { c. } \quad 548.15 \mathrm{~S} \mathrm{~T}_{\ell} \leq 611.15 \mathrm{~K} \\
& \mathrm{e}_{\ell}=\mathrm{ELDO}+\mathrm{ELD} 1 \cdot \mathrm{TLC}+\mathrm{ELD} 2 \cdot \mathrm{TLC}^{2}+\mathrm{ELD} 3 \cdot \mathrm{TLC}^{3}+\mathrm{ELD}^{2} \cdot \mathrm{TLC}^{4}+\mathrm{ELP}
\end{aligned}
$$

and

$\left(\frac{\partial \mathrm{e}_{\ell}}{\partial \mathrm{T}_{\ell}}\right)_{\mathrm{p}}=\mathrm{DELDO}+\mathrm{DELD1} \cdot \mathrm{TLC}+\mathrm{DELD2} \cdot \mathrm{TLC}^{2}+\mathrm{DELD} 3 \cdot \mathrm{TLC}^{3}+\mathrm{ERT}$ 


$$
\text { d. } \quad \mathrm{T}_{\ell}>611.15 \mathrm{~K} \text {. }
$$

$$
e_{\ell}=E L E 0+E L E 1 \cdot T L C+E L E 2 \cdot T L C^{2}+E L E 3 \cdot T L C^{3}+E L E 4 \cdot T L C^{4}+E L P \text {, }
$$

and

$\left(\frac{\partial \mathrm{e}_{\ell}}{\partial \mathrm{T}_{\ell}}\right)_{\mathrm{p}}=\mathrm{DELE} 0+\mathrm{DELE} 1 \cdot \mathrm{TLC}+\mathrm{DELE} 2 \cdot \mathrm{TLC} \mathrm{C}^{2}+\mathrm{DELE} 3 \cdot \mathrm{TLC}^{3}+\mathrm{ERT} \cdot$

$$
\text { 2. Density. Define PBAR }=1.0 \times 10^{-5} \mathrm{p} \text { and } \mathrm{TLC}=\mathrm{T}_{\ell}-273.15 \text {. }
$$

There are three temperature domains.

$$
\text { a. } \quad \mathrm{T}_{\ell}>525.15 \mathrm{~K} .
$$

$$
\rho_{\ell}=1.43+\frac{1000}{\left(\mathrm{CVH} 1+\mathrm{CVH} 2 \cdot \mathrm{PBAR}+\mathrm{CVH} 3 \cdot \mathrm{PBAR}^{2}+\beta_{1} \cdot \mathrm{TLC}+\gamma_{1} \cdot \mathrm{TLC}^{2}\right)},
$$

$$
\begin{aligned}
\left(\frac{\partial \rho_{\ell}}{\partial \mathrm{p}}\right)_{\mathrm{T}_{\ell}}= & -\left(\rho_{\ell}-1.43\right)^{2} \cdot 1.0 \times 10^{-8}\{\mathrm{CVH} 2+2 \cdot \mathrm{CVH} 3 \cdot \mathrm{PBAR} \\
& \left.+\mathrm{TLC}(\mathrm{CVH} 5+2 \cdot \mathrm{CVH} 6 \cdot \mathrm{PBAR})+\mathrm{TLC}^{2}(\mathrm{CVH} 8+2 \cdot \mathrm{CVH} 9 \cdot \mathrm{PBAR})\right\},
\end{aligned}
$$

and

$\left(\frac{\partial \rho_{\ell}}{\partial \mathrm{T}_{\ell}}\right)_{\mathrm{P}}=-\left(\rho_{\ell}-1.43\right)^{2} \cdot 1.0 \times 10^{-3}\left(\beta_{1}+2 \cdot \gamma_{1} \cdot \mathrm{TLC}\right)$,

where

$\mathrm{B}_{1}=\mathrm{CVH} 4+\mathrm{CVH} 5 \cdot \mathrm{PBAR}+\mathrm{CVH} 6 \cdot \mathrm{PBAR}^{2}$

and

$\gamma_{1}=\mathrm{CVH} 7+\mathrm{CVH} 8 \cdot \mathrm{PBAR}+\mathrm{CVH} 9 \cdot \mathrm{PBAR}^{2}:$ 


$$
\begin{aligned}
& \text { b. } \quad \mathrm{T}_{\ell}<521.15 \mathrm{~K} \text {. } \\
& \rho_{\ell}=\frac{1000}{\left(\mathrm{CVL} 1+\mathrm{CVL} 2 \cdot \mathrm{PBAR}+\mathrm{CVL} 3 \cdot \mathrm{PBAR}^{2}+\beta_{1} \cdot \mathrm{TLC}+\gamma_{1} \cdot \mathrm{TLC}^{2}\right)}-2.01 \text {, } \\
& \left(\frac{\partial \rho_{\ell}}{\partial \mathrm{p}}\right)_{\mathrm{T}_{\ell}}=-\left(\rho_{\ell}+2.01\right)^{2} \cdot 1.0 \times 10^{-8}\{\mathrm{CVL} 2+2 \cdot \mathrm{CVL} 3 \cdot \mathrm{PBAR} \\
& \left.+\operatorname{TLC}(\mathrm{CVL} 5+2 \cdot \operatorname{CVL} 6 \cdot \mathrm{PBAR})+\mathrm{TLC}^{2}(\mathrm{CVL} 8+2 \cdot \mathrm{CVL} \cdot \mathrm{PBAR})\right\} \\
& \left(\frac{\partial \rho_{\ell}}{\partial \mathrm{T}_{\ell}}\right)_{\mathrm{p}}=-\left(\rho_{\ell}+2.01\right)^{2} \cdot 1.0 \times 10^{-3}\left(\beta_{1}+2 \cdot \gamma_{1} \cdot \mathrm{TLC}\right), \\
& \text { where } \\
& \beta_{1}=\mathrm{CVL} 4+\mathrm{CVL} 5 \cdot \mathrm{PBAR}+\mathrm{CVL} 6 \cdot \mathrm{PBAR}^{2} \\
& \text { and } \\
& \gamma_{1}=\mathrm{CVL} 7+\mathrm{CVL} 8 \cdot \mathrm{PBAR}+\mathrm{CVL} 9 \cdot \mathrm{PBAR}^{2} \cdot \\
& \text { c. } \quad 521.15 \mathrm{~K} \leq \mathrm{T}_{\ell} \leq 525.15 \mathrm{~K} \text {. An average of the functions in (a) and } \\
& \text { (b) above is used in this range. Call the two values } \rho_{\ell} a \text { and } \rho_{\ell b} \text {, then } \\
& \rho_{\ell}=\left(\frac{525.15-\mathrm{T}_{\ell}}{4.0}\right) \rho_{\ell \mathrm{b}}+\left(\frac{\mathrm{T}_{\ell}-521.15}{4.0}\right) \rho_{\ell \mathrm{a}}, \\
& \left(\frac{\partial \rho_{\ell}}{\partial \mathrm{p}}\right)_{\mathrm{T}_{\ell}}=\left(\frac{525.15-\mathrm{T}_{\ell}}{4.0}\right)\left(\frac{\partial \rho_{\ell \mathrm{b}}}{\partial \mathrm{p}}\right)_{\mathrm{T}_{\ell}}+\left(\frac{\mathrm{T}_{\ell}-521.15}{4.0}\right)\left(\frac{\partial \rho_{\ell \mathrm{a}}}{\partial \mathrm{p}}\right)_{\mathrm{T}_{\ell}} \text {, } \\
& \left(\frac{\partial \rho_{\ell}}{\partial \mathrm{T}_{\ell}}\right)_{\mathrm{p}}=\left(\frac{525.15-\mathrm{T}_{\ell}}{4.0}\right)\left(\frac{\partial \rho_{\ell b}}{\partial \mathrm{T}_{\ell}}\right)_{\mathrm{p}}+\left(\frac{\mathrm{T}_{\ell}-521.15}{4.0}\right)\left(\frac{\partial \rho_{\ell \mathrm{a}}}{\partial \mathrm{T}_{\ell}}\right)_{\mathrm{p}}+\frac{\rho_{\ell \mathrm{a}}-\rho_{\ell b}}{4.0} .
\end{aligned}
$$


d. Residual Void Correction. After evaluation in Secs. (a) through (c), $\rho_{\ell}$ and its derivatives are corrected to reflect a residual void fraction. In the following, the values calculated by (a) through (c) above are denoted by a tilde ( $\sim$ ).

$$
\text { 1. } \quad \mathrm{p} \geq 4.0 \times 10^{5} \mathrm{~Pa}
$$

$\left(\frac{\partial \rho_{\ell}}{\partial \mathrm{T}_{\ell}}\right)_{\mathrm{p}}=\left(1-\frac{1000}{\mathrm{p}}\right)\left(\frac{\partial \tilde{\rho}_{\ell}}{\partial \mathrm{T}_{\ell}}\right)_{\mathrm{p}}$,

$\left(\frac{\partial \rho_{\ell}}{\partial \mathrm{p}}\right)_{\mathrm{T}_{\ell}}=\left(1-\frac{1000}{\mathrm{p}}\right)\left(\frac{\partial \tilde{\rho}_{\ell}}{\partial \mathrm{p}}\right)_{\mathrm{T}_{\ell}}+\frac{1000 \tilde{\rho}_{\ell}}{\mathrm{p}^{2}}$,

and

$\rho_{\ell}=\left(1-\frac{1000}{p}\right) \tilde{\rho}_{\ell}$.

11. $\quad \mathrm{p}<4.0 \times 10^{5} \mathrm{~Pa}$.

$\left(\frac{\partial \rho_{\ell}}{\partial \mathrm{T}_{\ell}}\right)_{\mathrm{p}}=\left(0.995+6.25 \times 10^{-9} \mathrm{p}\right)\left(\frac{\partial \tilde{\rho}_{\ell}}{\partial \mathrm{T}_{\ell}}\right)_{\mathrm{p}}$,
$\left(\frac{\partial \rho_{\ell}}{\partial \mathrm{p}}\right)_{\mathrm{T}_{\ell}}=\left(0.995+6.25 \times 10^{-9} \mathrm{p}\right)\left(\frac{\partial \tilde{\rho}_{\ell}}{\partial \mathrm{p}}\right)_{\mathrm{T}_{\ell}}+6.25 \times 10^{-9} \tilde{\rho}_{\ell}$,

and

$\rho_{\ell}=\left(0.995+6.25 \times 10^{-9} \mathrm{p}\right) \tilde{\rho}_{\ell}$. 


\section{Vapor Properties}

1. Superheated Steam. $\left(\mathrm{T}_{\mathrm{g}}-\mathrm{T}_{\mathrm{S}}\right)>0$, where $\mathrm{T}_{\mathrm{S}}$ is the saturation temperature corresponding to partial pressure of steam.

The specific heat of steam at constant pressure is approximated as

$c_{p v}=\left(\frac{\partial h_{v}}{\partial T_{g}}\right)_{p_{v}}=\frac{c_{p v}, \text { idea1 }}{2}\left[1+\frac{T_{g}}{\left(T_{g}^{2}-\beta\right)^{1 / 2}}\right]$,

where

$B=T_{s}^{2}\left[1-\frac{1}{\left(A_{11} c_{p v s}-1\right)^{2}}\right]$.

Integrating along constant $\mathrm{p}_{\mathrm{v}}$ line gives

$h_{v}=h_{v s}+\frac{c_{p v}, 1 \text { dea } 1}{2}\left[\left(T_{g}-T_{s}\right)+\left(T_{g}^{2}-\beta\right)^{1 / 2}-\frac{T_{s}}{A_{11} c_{p v s}-1}\right] \cdot$

The internal energy of vapor is therefore calculated as

$$
\begin{aligned}
& e_{v}=e_{v s}+\frac{c_{p v}, i d e a 1}{2}\left[\left(T_{g}-T_{s}\right)+\left(T_{g}^{2}-\beta\right)^{1 / 2}-\frac{T_{s}}{A_{11} c_{p v s}-1}\right] \\
& -p_{v}\left(\frac{1}{\rho_{v}}-\frac{1}{\rho_{v s}}\right)
\end{aligned}
$$

The calculations are divided into two domains based on the value of $c_{p v s}$. 
a. $c_{\mathrm{pvs}}<c_{\mathrm{pv}, \text { ideal }}$. The steam density is calculated as

$$
\rho_{v}=\frac{\rho_{v}}{\left(\gamma_{s}-1\right) e_{v s}+R_{v}\left(T_{g}-T_{s}\right)}
$$

which gives

$$
e_{v}=e_{v s}+\frac{c_{p v, 1 d e a l}}{2}\left[\left(T_{g}-T_{s}\right)+\left(T_{g}^{2}-\beta\right)^{1 / 2}-\frac{T_{s}}{A_{11} c_{p v s}-1}\right]-R_{v}\left(T_{g}-T_{s}\right)
$$

Therefore,

$$
\begin{aligned}
& \left(\frac{\partial \rho_{v}}{\partial T_{g}}\right)_{P_{v}}=-\frac{\rho_{v} R_{v}}{\left(\gamma_{s}-1\right) e_{v s}+R_{v}\left(T_{g}-T_{s}\right)}=-\frac{\rho_{v}^{2} R_{v}}{P_{v}}, \\
& \left(\frac{\partial \rho_{v}}{\partial P_{v}}\right)_{T_{g}}=\rho_{v}\left\{\frac{1}{P_{v}}-\left[e_{v s} \frac{d \gamma_{s}}{d P_{v}}+\left(\gamma_{s}-1\right) \frac{d e_{v s}}{d P_{v}}-R_{v} \frac{d T_{s}}{d P_{v}}\right]\left[\frac{\cdot}{\left(\gamma_{s}-1\right)+R_{v}\left(T_{g}-T_{s}\right)}\right]\right\}, \\
& \left(\frac{\partial e_{v}}{\partial T_{g}}\right)_{P_{v}}=\frac{c_{p v, 1 d e a 1}}{\left(1-\frac{\beta}{n^{2}}\right)}-R_{v},
\end{aligned}
$$

and

$$
\left(\frac{\partial \mathrm{e}_{\mathrm{v}}}{\partial \mathrm{p}_{\mathrm{v}}}\right)_{\mathrm{Tg}}=-\frac{1}{2}\left[\left(\frac{\partial \mathrm{e}_{\mathrm{v}}}{\partial \mathrm{T}_{\mathrm{g}}}\right)_{\mathrm{p}_{\mathrm{v}}}+\mathrm{R}_{\mathrm{v}}\right]\left[\left(1-\frac{\beta}{\eta^{2}}\right) \eta_{\mathrm{p}}^{-}+\frac{1}{\eta} \frac{\mathrm{d} \beta}{\mathrm{d \textrm {p } _ { \mathrm { v } }}}\right],
$$


where

$$
\begin{aligned}
\eta^{\prime} & A_{11}\left[e_{v}-e_{v s}+R_{v}\left(T_{g}-T_{s}\right)\right]+T_{s}\left[1+\frac{1}{A_{11} c_{p v s}-1}\right], \\
\eta_{p}^{\prime} & =\left(\frac{\partial \eta}{\partial p_{v}}\right)_{g}-A_{11}\left(\frac{\partial e_{v}}{\partial P_{v}}\right)_{g} \\
& =-A_{11}\left[\frac{d e_{v s}}{d p_{v}}-R_{v} \frac{d T_{s}}{d P_{v}}\right]+\left[1+\frac{A_{11} c_{p v s}-1}{-1} \frac{d T_{s}}{d p_{v}}\right. \\
& -\frac{A_{11} T_{s}}{\left(A_{11} c_{p v s}-1\right)^{2}} \frac{d c_{p v s}}{d p_{v}},
\end{aligned}
$$

1. and

$\frac{d B}{d p_{v}}=\frac{2}{T_{s}}\left[B\left(\frac{d T_{s}}{d P_{v}}\right)+\frac{T_{s}^{3} A_{11}}{\left(A_{11} c_{p v s}-1\right)^{3}}\left(\frac{d c_{p v s}}{d p_{v}}\right)\right]$.

Note: If $\rho_{\mathrm{v}}$ is less than zero, the vapor density and its derivatives are superceded by

$$
\begin{aligned}
\rho_{v} & =\frac{p_{v}}{R_{v T_{g}}}, \\
\left(\frac{\partial v_{v}}{\partial T_{g}}\right)_{p_{v}} & =-\frac{\rho_{v}}{T_{g}}, \\
\text { and } & \\
\left(\frac{\partial \rho_{v}}{\partial p_{v}}\right)_{T_{g}} & =\frac{\rho_{v}}{P_{v}} ;
\end{aligned}
$$




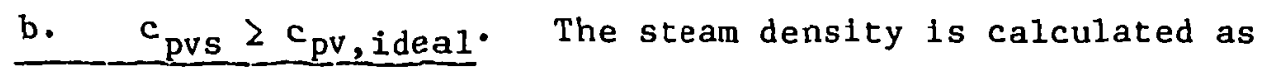

$\rho_{v}=\frac{\rho_{v}}{\left(\gamma_{s}-1\right) e_{v s}+C_{26}\left(e_{v}-e_{v s}\right)}$

which gives

$e_{v}=e_{v s}+A_{12}\left[\left(T_{g}-T_{s}\right)+\left(T_{g}^{2}-\rho\right)^{1 / 2}-\frac{T_{s}}{\left(A_{11} c_{p v s}-1\right)}\right]$.

Therefore,

$$
\begin{aligned}
\left(\frac{\partial \rho_{v}}{\partial T_{g}}\right)_{v}= & -\left(\frac{\partial e_{v}}{\partial T_{g}}\right)_{p_{v}}\left[\frac{C_{26} \rho_{v}}{\left(\gamma_{s}-1\right) e_{v s}+C_{26}\left(e_{v}-e_{v s}\right)}\right], \\
\left(\frac{\partial \rho_{v}}{\partial P_{v}}\right)_{T_{g}}= & \rho_{v}\left\{\frac{1}{p_{v}}-\left[e_{v s}\left(\frac{d \gamma s}{d p_{v}}\right)+\left(\gamma_{s}-C_{24}\right) \frac{d e_{v s}}{d p_{v}}\right]\right. \\
& \left.\times\left[\frac{1}{\left(\gamma_{s}-1\right) e_{v s}+C_{26}\left(e_{v}-e_{v s}\right)}\right]\right\}+\left(\frac{\partial \rho v}{\partial e_{v}}\right) P_{v}\left(\frac{\partial e_{v}}{\partial p_{v}} T_{g},\right. \\
\left(\frac{\partial e_{v}}{\partial T_{g}}\right)_{P_{v}}= & \frac{c_{v v}, 1 \text { deal }}{\left(1-\frac{\beta}{x^{2}}\right)},
\end{aligned}
$$

and

$\left(\frac{\partial \mathrm{e}_{\mathrm{v}}}{\partial \mathrm{p}_{\mathrm{v}}}\right)_{\mathrm{T}_{\mathrm{g}}}=-\frac{1}{2}\left(\frac{\partial \mathrm{e}_{\mathrm{v}}}{\partial \mathrm{T}_{\mathrm{g}}} \mathrm{P}_{\mathrm{v}}\left[\left(1-\frac{\beta}{\kappa^{2}}\right) \kappa_{\mathrm{p}}^{-}+\frac{1}{\kappa} \frac{\mathrm{d} \beta}{\mathrm{dp_{ \textrm {v } }}}\right]\right.$, 
where

$$
\begin{aligned}
k^{\prime}= & A_{13}\left(e_{v}-e_{v s}\right)+T_{s}\left[1+\frac{1}{\left(A_{11} c_{p v s}-1\right)}\right\}, \\
K_{p}^{\prime}= & \left(\frac{\partial K}{\partial P_{v}}\right)_{g}-A_{13}\left(\frac{\partial e_{v}}{\partial p_{v}} T_{g}\right. \\
= & -A_{13} \frac{d e_{v s}}{d p_{v}}+\left[1+\frac{A_{11} c_{p v s}-1}{d p_{v}}\right. \\
& -\frac{A_{11} T_{s}}{\left(A_{11} c_{p v s}-1\right)^{2}} \frac{d c_{p v s}}{d p_{v}},
\end{aligned}
$$

and

$$
\left(\frac{\partial \rho v}{\partial e_{v}}\right)_{v}=\frac{-C_{26} \rho v}{\left[\left(\gamma_{s}-1\right) e_{v s}+c_{26}\left(e_{v}-e_{v s}\right)\right]} .
$$

Note: If $\rho_{v}$ exceeds $0.9 \rho_{\ell}$, the vapor density and 1 ts derivatives are superceded by

$$
\begin{aligned}
\rho_{\mathrm{v}} & =0.9 \rho_{\ell}, \\
\left(\frac{\partial \rho_{\mathrm{v}}}{\partial \mathrm{T}_{\mathrm{g}}}\right)_{\mathrm{v}} & =0.9\left(\frac{\partial \rho_{\ell}}{\partial \mathrm{T}_{\ell}}\right)_{\mathrm{p}},
\end{aligned}
$$

and

$$
\left(\frac{\partial \rho_{v}}{\partial p_{v}}\right)_{T_{g}}=0.9\left(\frac{\partial \rho_{\ell}}{\partial p}\right)_{T_{\ell}}
$$


2. Subcooled Vapor. $\left(T_{g}-T_{S}\right) \leq 0$ The density and its derivatives are calculated in the same manner as for the superheated vapor case above. The internal energy and its derivatives are given below.

$$
\begin{aligned}
& e_{v}=e_{v s}+\left(T_{g}-T_{s}\right) \frac{c_{p v s}}{C_{24}} \text {, } \\
& \left(\frac{\partial \mathrm{e}_{\mathrm{v}}}{\left.\partial \mathrm{T}_{\mathrm{g}}\right)_{\mathrm{p}}}=\frac{\mathrm{c}_{\mathrm{pvs}}}{\mathrm{C}_{24}}\right. \\
& \left(\frac{\partial e_{v}}{\partial P_{v}}\right)_{T_{g}}=-\left(\frac{\partial e_{v}}{\partial T_{g}}\right)_{p_{v}}\left\{\frac{d T_{s}}{d p_{v}}-\left(\frac{c_{24}}{c_{p v s}}\right)\left[\frac{d e_{v s}}{d p_{v}}+\frac{\left(e_{v}-e_{v s}\right)}{c_{p v s}}\left(\frac{d c_{p v s}}{d p_{v}}\right)\right]\right\} \text {. } \\
& \text { 3. Noncondensable Gas (Air). } \\
& \text { a. Internal Energy. } \\
& e_{a}=c_{v a} T_{g} \text {, } \\
& \left(\frac{\partial \mathrm{e}_{\mathrm{a}}}{\left.\partial \mathrm{T}_{\mathrm{g}}\right)_{\mathrm{a}}}=\mathrm{c}_{\mathrm{va}}\right. \\
& \text { and } \\
& \left(\frac{\partial \mathrm{e}_{\mathrm{a}}}{\left.\partial \mathrm{p}_{\mathrm{a}}\right)_{\mathrm{g}}}=0.0\right.
\end{aligned}
$$




$$
\begin{array}{r}
\rho_{a}=\frac{\mathrm{P}_{a}}{\mathrm{R}_{\mathrm{a}} \mathrm{T}_{\mathrm{g}}}, \\
\left(\frac{\partial \rho_{\mathrm{a}}}{\left.\partial \mathrm{P}_{\mathrm{a}}\right)_{g}}=\frac{1}{\mathrm{R}_{\mathrm{a}} \mathrm{T}_{\mathrm{g}}},\right.
\end{array}
$$

and

$$
\left(\frac{\partial \rho_{a}}{\partial T_{g}}\right)_{a}=-R_{a} \rho\left(\frac{\partial \rho a}{\partial p_{a} T_{g}},\right.
$$

where $R_{a}$ is the gas constant for the noncondensable gas.

II. TRANSPORT PROPERTIES

Subroutine FPROP is used to obtain transport properties for water and the noncondensable gas. The input variables for this routine are the saturation temperature, pressure, enthalpies of each phase, vapor density, and the vapor temperature. The output transport varlables include the latent heat of vaporization, surface tension, constant pressure speciflc heat, viscosity, and thermal conductivity of steam, the liquid, and the noncondensable gas. The transport property calls are function calls within the FPROP subroutine. The polynomial equation fits for the transport properties used in FPROP are described. Note that the curve fits for $\sigma$ and $c_{p l}$ have been updated since TRAC-P1A. Values of the constants are given in Tables A-III through A-VI. "' 
A. Latent Heat of Vaporization

The latent heat of vaporization is calculated as

$h_{\ell v}=h_{v s}-h_{\ell s}$,

where $h_{v s}$ and $h_{\ell s}$ are calculated according to App. A.I.A.

B. Constant Pressure Specific Heats

Constants used in this section are given in Table A-III.

$c_{p \ell}=\left\{h_{\ell}\left[h_{\ell}\left(D_{O \ell}+D_{1 \ell} p\right)+\left(C_{0 \ell}+C_{1 \ell} p\right)\right]+B_{0 \ell}+B_{1 \ell} p\right\}^{-1}$

and

$c_{p v}=c_{1 g}+c_{2 g} T_{g}+\frac{c_{3 g} p}{\left(c_{5 g} T_{g}-c_{6 g}\right)^{2 \cdot 4}}+\frac{c_{4 g} p^{3}}{\left(c_{5 g} T_{g}-c_{6 g}\right)^{9}}$.

Because these values are used only for calculating heat-transfer coefficlents, these flts were chosen for simplicity and smoothness, and are not necessarily consistent with those derivable from the thermodynamic routines.

The specific heat of the noncondensable gas is assumed constant and is given in Table $\mathrm{A}-\mathrm{T}$. 
1. Liquid. Constants used in this section are given in Table A-IV. The evaluation of liquid viscosity is divided into three different enthalpy ranges. For $h_{\ell} \leq h_{1}$,

$$
\begin{aligned}
\mu_{\ell}= & {\left[A_{O \ell}+A_{1 \ell} x+A_{2 l} x^{2}+A_{3 \ell} x^{3}+A_{4 \ell} x^{4}\right] } \\
& -\left[B_{O \ell}+B_{1 \ell} n+B_{2 \ell} n^{2}+B_{3 \ell} n^{3}\right]\left(p-P_{1}\right),
\end{aligned}
$$

where

$x=\left(h_{\ell}-c_{0 n}\right) h_{0}$

and

$n=\left(h_{\ell}-e_{c 0 n}\right) e_{h o}$.

In the range $h_{1}<\bar{h} \leq h_{2}$,

$$
\begin{aligned}
\mu_{\ell}= & {\left[E_{0 \ell}+E_{1 \ell} h_{\ell}+E_{2 \ell} h_{\ell}^{2}+E_{3 \ell} h_{\ell}^{3}\right] } \\
& +\left[F_{0 \ell}+F_{1 \ell} h_{\ell}+F_{2 \ell} h_{\ell}^{2}+F_{3 \ell} h_{\ell}^{3}\right]\left(p-p_{i}\right) .
\end{aligned}
$$

For $h_{\ell}>h_{2}$,

$\mu_{\ell}=\left[D_{0 \ell}+D_{1 \ell} z+D_{2 \ell} z^{2}+D_{3 \ell} z^{3}+D_{4 \ell} z^{4}\right]$,

where

$$
z=\left(h_{\ell}-c_{n}\right) h_{00}
$$


2. Steam. Constants used in this section are given in Table $A-V$. Three vapor temperature ranges are used to represent the data.

a. $\mathrm{T}_{\mathrm{g}} \leq \mathrm{T}_{1}$

$\mu_{v}=\left[B_{1 g}\left(T_{g}-273.15\right)+C_{1 g}\right]-\rho_{v}\left[D_{1 g}-E_{1 g}\left(T_{g}-273.15\right)\right]$.

If $\mu_{\mathrm{v}}<10^{-7}$, it is set to that value.

b. $\quad \mathrm{T}_{1}<\mathrm{T}_{\mathrm{g}}<\mathrm{T}_{2}$.

$$
\begin{aligned}
\mu_{v}= & B_{1 g}\left(T_{g}-273.15\right)+C_{1 g}+\rho_{v}\left[F_{1 g}+F_{2 g}\left(T_{g}-273.15\right)\right. \\
& \left.+F_{3 g}\left(T_{g}-273.15\right)^{2}+F_{4 g}\left(T_{g}-273.15\right)^{2}\right]_{s} \\
& +\rho_{v}\left[G_{1 g}+G_{2 g}\left(T_{g}-273.15\right)+G_{3 g}\left(T_{g}-273.15\right)^{2}\right. \\
& \left.+G_{4 g}\left(T_{g}-273.15\right)^{3}\right]\left(A_{0 g}+A_{1 g}{ }^{\rho}+A_{2 g}{ }_{v}^{2}\right) \\
\text { c. } & T_{g} \geq T_{2} .
\end{aligned}
$$

$\mu_{v}=B_{1 g}\left(T_{g}-273.15\right)+C_{1 g}+\rho_{v}\left(A_{0 g}+A_{1 g} \rho_{v}+A_{2 g} \rho_{v}^{2}\right)$

3. Noncondensable Gas.

a. Alr. If the noncondensable gas is air, two ranges of $T_{g}$ are used.

i. $\quad \mathrm{T}_{\mathrm{g}} \leq 502.15 \mathrm{~K}$.

$\mu_{\mathrm{a}}=\mathrm{H}_{\mathrm{a} 1}+\mathrm{H}_{\mathrm{a} 2}\left(\mathrm{~T}_{\mathrm{g}}-273.15\right)+\mathrm{H}_{\mathrm{a} 3}\left(\mathrm{~T}_{\mathrm{g}}-273.15\right)^{2}$.

i1. $\quad \mathrm{T}_{\mathrm{g}}>502.15 \mathrm{~K}$.

$\mu_{a}=H_{b 1}+H_{b 2}\left(T_{g}-273.15\right)+H_{b 3}\left(T_{g}-273.15\right)^{2}$. 
b. Hydrogen. If the noncondensable gas is hydrogen, the vilscosity is calculated as

$\mu_{a}=H_{c 1}+H_{c 2} T_{g}+H_{c 3} T_{g}^{2}$.

D. Fluid Thermal Conductivities

The liquid thermal conductivity is given by

$k_{\ell}=A_{\ell 0}+A_{\ell 1} x_{k}+A_{\ell 2} x_{k}^{2}+A_{\ell 3} x_{k}^{3}$,

where

$\mathrm{x}_{k}=\frac{\mathrm{h}_{\ell}}{\mathrm{h}_{0}}$,

and the constants are given in Table A-VI.

The steam thermal conductivity is given by

$k_{v}=x_{1}+\rho_{v}\left[x_{2}+\frac{C_{\rho}}{\left(T_{g}-273.15\right)^{4.2}}\right]$,

where

$x_{1}=A_{g 0}+A_{g 1}\left(T_{g}-273.15\right)+A_{g 2}\left(T_{g}-273.15\right)^{2}+A_{g 3}\left(T_{g}-273.15\right)^{3}$

and

$\mathrm{x}_{2}=\mathrm{B}_{\mathrm{g} 0}+\mathrm{B}_{\mathrm{g} 1}\left(\mathrm{~T}_{\mathrm{g}}-273.15\right)+\mathrm{B}_{\mathrm{g} 2}\left(\mathrm{~T}_{\mathrm{g}}-273.15\right)^{2}$

However, the minimum permitted value for $k_{v}$ is $1.0 \times 10^{-4}$.

The thermal conductivity of the noncondensable gas is assumed constant and is given in Table A-VI. 
E. Surface Tension

The surface tension is given by

$\sigma=0.2358\left(1-0.625 \frac{647.3-\mathrm{T}_{\mathrm{s}}}{647.3}\right)\left(\frac{647.3-\mathrm{T}_{\mathrm{s}}}{647.3}\right) 1.256$.

\section{VERIFICATION}

The thermodynamic and transport property fits 17 used in TRAC have been compared with steam table data over a wide range of parameters. The agreement is satisfactory in the saturation region and in the superheated steam region for $\mathrm{p}<\mathrm{p}_{\mathrm{critica}}$ and $\mathrm{T}_{\mathrm{g}}<823.0 \mathrm{~K}$. For example, the saturation temperature corresponding to a given pressure is always calculated within a maximum error of $\pm 1 \mathrm{~K}$. However, in most situations the error is significantly smaller. The agreement also is good in the subcooled water region for $T_{\ell}<T_{\text {critical }}$.

Further verification was performed by comparing the TRAC polynomial fits with the WATER package 55 over a wider range of nonequilibrium [99 $\mathrm{K}$ of both superheat and subcooling (liquid superheat or vapor subcooling exceeding $99 \mathrm{~K}$ are highly unlikely in reactor applications)] for pressures up to $20 \mathrm{MPa}$. The comparisons showed good agreement for both the thermodynamic and transport properties throughout the saturation and nonequilibrium regions except for very extreme cases. However, at high degrees of subcooling or superheat, some minor inconsistencies were noticed. Because there are no data in these extreme cases, it is impossible to compare TRAC and the WATER package adequately.

In conclusion, for most TRAC applications the thermodynamic and transport property routines provide realistic values up to critical point. The simplified polynomial fits provide an efficient and low-cost method compared to other approaches such as steam table interpolation. 
TABLE A-I

POLYNOMIAL CONSTANTS FOR THERMO

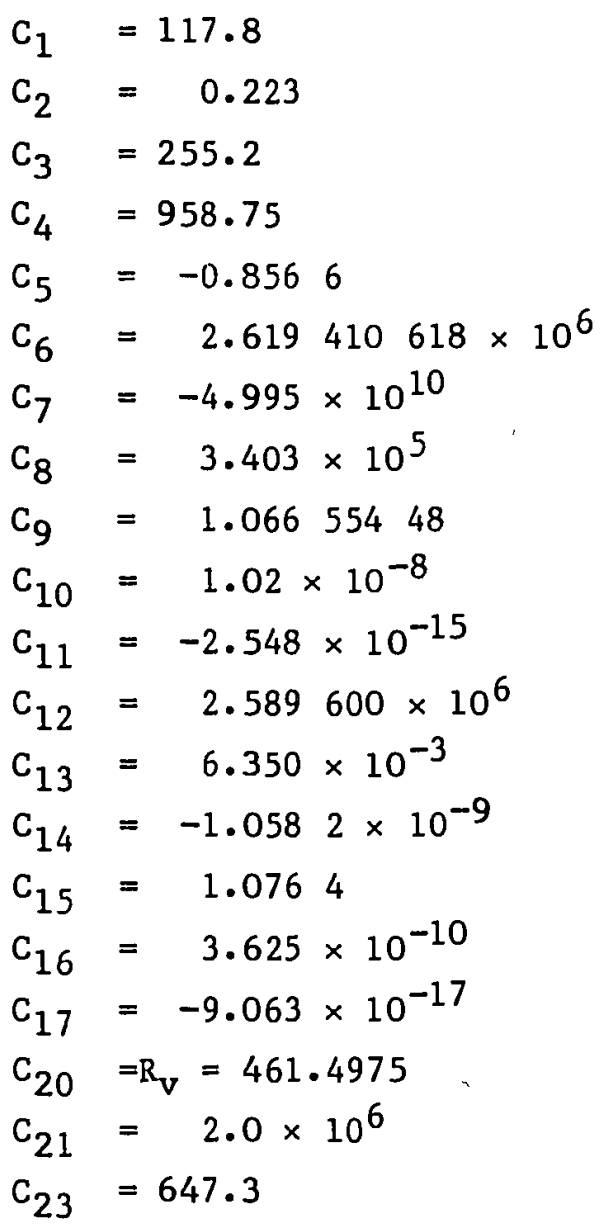


TABLE A-I (cont.)

$$
\begin{aligned}
& \text { ELCO }=1.75880 \times 10^{4} \\
& \text { ELC1 }=3.737452 \times 10^{3} \\
& \text { ELC2 }=3.934036 \\
& \text { ELC3 }=-0.015038190 \\
& \text { ELC4 }=3.011387 \times 10^{-5} \\
& \text { ELDO }=6.18527 \times 10^{6} \\
& \text { ELD1 }=-8.14547 \times 10^{4} \\
& \text { ELD2 }=4.46598 \times 10^{2} \\
& \text { ELD3 }=-1.04116 \\
& \text { ELD4 }=9.26022 \times 10^{-4} \\
& \text { CVH1 }=1.00213623 \\
& \text { CVH2 }=-5.632785 \times 10^{-5} \\
& \text { CVH3 }=-8.97130477 \times 10^{-9} \\
& \text { CVH4 }=-2.28287459 \times 10^{-5} \\
& \text { CVH5 }=4.76596787 \times 10^{-7} \\
& \text { CVH6 }=5.021313 \times 10^{-10} \\
& \text { CVH7 }=4.10115658 \times 10^{-6} \\
& \text { CVH8 }=-3.80398908 \times 10^{-9} \\
& \text { CVH9 }=-1.42199752 \times 10^{-12}
\end{aligned}
$$


DERIVED CONSTANTS FOR THERMODYNAMIC PROPERTIES GF WATER AND NONCONDENSABLE GAS

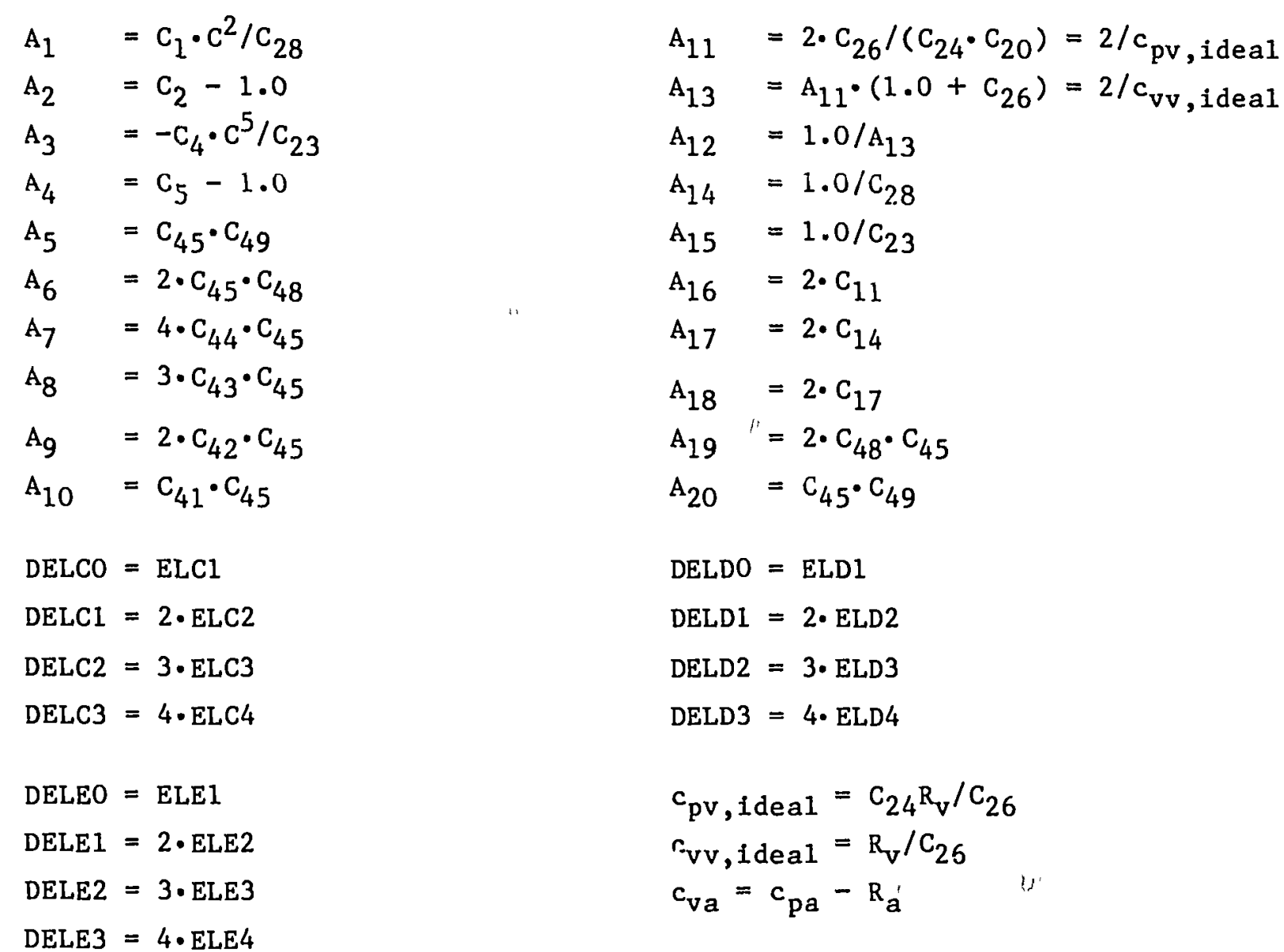


TABLE A-III

BASIC CONSTANTS FOR TRANSPORT PROPERTIES OF WATER AND AIR

$$
\begin{aligned}
& \mathrm{H}_{g 0}=2.739623397 \times 10^{6} \\
& \mathrm{H}_{\mathrm{g} 1}=3.758844554 \times 10^{-2} \\
& \mathrm{H}_{\mathrm{g} 2}=-7.163990945 \times 10^{-9} \\
& \mathrm{H}_{\mathrm{g} 3}=4.200231947 \times 10^{-16} \\
& \mathrm{H}_{\mathrm{g} 4}=9.850752122 \times 10^{-24}
\end{aligned}
$$

$$
\begin{array}{ll}
B_{0 \ell}=2.394907 \times 10^{-4} & { }^{B} 1 \ell=-5.196250 \times 10^{-13} \\
C_{0 \ell}=1.193203 \times 10^{-11} & C_{1 \ell}=2.412704 \times 10^{-18} \\
D_{0 \ell}=-3.944067 \times 10^{-17} & D_{1 \ell}=-1.680771 \times 10^{-24}
\end{array}
$$

$$
\begin{aligned}
& c_{1 g}=1.68835968 \times 10^{3} \\
& c_{2 g}=0.6029856 \\
& c_{3 g}=4.820979623 \times 10^{2} \\
& c_{4 g}=2.95317905 \times 10^{7} \\
& c_{5 g}=1.8 \\
& c_{6 g}=4.60 \times 10^{2}
\end{aligned}
$$


TABLE A-IV

\section{LIQUID VISCOSITY CONSTANTS}

$$
\begin{aligned}
& A_{0 \ell}=1.299470229 \times 10^{-3} \\
& A_{1 \ell}=-9.264032108 \times 10^{-4} \\
& A_{2 l}=3.81047061 \times 10^{-4} \\
& A_{3 l}=-8.219444458 \times 10^{-5} \\
& A_{4 l}=7.022437984 \times 10^{-6}
\end{aligned}
$$$$
D_{0 \ell}=3.026032306 \times 10^{-4}
$$$$
D_{1 \ell}=-1.836606896 \times 10^{-4}
$$$$
D_{2 \ell}=7.567075775 \times 10^{-5}
$$$$
D_{3 \ell}=-1.647878879 \times 10^{-5}
$$$$
\mathrm{D}_{4 \ell}=1.416457633 \times 10^{-6}
$$$$
F_{0 \ell}=-3.8063507533 \times 10^{-11}
$$$$
F_{1 \ell}=3.9285207677 \times 10^{-16}
$$$$
F_{2 \ell}=-1.2585799292 \times 10^{-21}
$$$$
F_{3 \ell}=1.2860180788 \times 10^{-27}
$$

$$
\begin{aligned}
& \mathrm{h}_{00}=3.892077365 \times 10^{-6} \\
& e_{\mathrm{cOn}}=5.53588 \times 10^{4} \\
& \mathrm{~h}_{1}=2.76 \times 10^{5}
\end{aligned}
$$

$$
\begin{aligned}
& \mathrm{B}_{0 \ell}=-6.5959 \times 10^{-12} \\
& \mathrm{~B}_{1 \ell}=-6.763 \times 10^{-12} \\
& \mathrm{~B}_{2 \ell}=-2.88825 \times 10^{-12} \\
& \mathrm{~B}_{3 \ell}=4.4525 \times 10^{-13}
\end{aligned}
$$

$E_{0 \ell}=1.4526052612 \times 10^{-3}$

$E_{1 \ell}=-6.9880084985 \times 10^{-9}$

$E_{2 \ell}=1.5210230334 \times 10^{-14}$

$E_{3 \ell}=-1.2303194946 \times 10^{-20}$

$h_{0}=8.581289699 \times 10^{-6}$

$c_{0_{n}}=4.265884 \times 10^{4}$

$p_{i}=6.894575293 \times 10^{5}$

$$
\begin{aligned}
& e_{\text {h0 }}=6.484503981 \times 10^{-6} \\
& c_{n}=4.014676 \times 10^{5} \\
& h_{2}=3.94 \times 10^{5}
\end{aligned}
$$




\section{VAPOR VISCOSITY CONSTANTS}

$$
\begin{aligned}
& A_{C g}=3.53 \times 10^{-8} \\
& A_{1 g}=6.765 \times 10^{-11} \\
& A_{2 g}=1.021 \times 10^{-14}
\end{aligned}
$$$$
F_{1 g}=-0.2885 \times 10^{-5}
$$$$
F_{2 g}=0.2427 \times 10^{-7}
$$$$
F_{3 g}=-0.6789333 \times 10^{-10}
$$$$
F_{4 g}=-0.6317037037 \times 10^{-13}
$$

$$
\begin{aligned}
& \mathrm{H}_{\mathrm{a} 1}=1.708 \times 10^{-5} \\
& \mathrm{H}_{\mathrm{a} 2}=5.927 \times 10^{-8} \\
& \mathrm{H}_{\mathrm{a} 3}=8.14 \times 10^{-11}
\end{aligned}
$$$$
\mathrm{H}_{\mathrm{cl}}=4.175 \times 10^{-6}
$$$$
\mathrm{H}_{\mathrm{c} 2}=1.588 \times 10^{-8}
$$$$
\mathrm{H}_{\mathrm{c} 3}=7.6705 \times 10^{-13}
$$

$$
\begin{aligned}
& B_{1 g}=0.407 \times 10^{-7} \\
& C_{1 g}=8.04 \times 10^{-6} \\
& D_{1 g}=1.858 \times 10^{-7} \\
& E_{1 g}=5.9 \times 10^{-20}
\end{aligned}
$$

$\mathrm{G}_{1 \mathrm{~g}}=176.0$

$G_{2 g}=-1.6$

$G_{3 g}=0.0048$

$G_{4 g}=-0.474074074 \times 10^{-5}$

$$
\begin{aligned}
& \mathrm{H}_{\mathrm{b} 1}=1.735 \times 10^{-5} \\
& \mathrm{H}_{\mathrm{b} 2}=4.193 \times 10^{-8} \\
& \mathrm{H}_{\mathrm{b} 3}=1.09 \times 10^{-11}
\end{aligned}
$$

$\mathrm{T}_{1}=573.15$

$\mathrm{T}_{2}=648.15$ 


\section{TABLE $A-V I$}

THERMAL CONDUCTIVITY CONSTANTS

$$
\begin{aligned}
& h_{0}=5.815 \times 10^{5} \\
& A_{\ell 0}=0.573738622 \\
& A_{\ell 1}=0.2536103551 \\
& A_{\ell 2}=-0.145468269 \\
& A_{\ell 3}=-0.01387472485 \\
& C=2.1482 \times 10^{5} \\
& A_{g 0}=1.76 \times 10^{-2} \\
& A_{g 1}=5.87 \times 10^{-5} \\
& A_{g 2}=1.04 \times 10^{-7} \\
& A_{g 3}=-4.51 \times 10^{-11} \\
& \mathrm{~B}_{\mathrm{g} 0}=1.0351 \times 10^{-4} \\
& B_{g 1}=0.4198 \times 10^{-6} \\
& \mathrm{~B}_{\mathrm{g} 2}=-2.771 \times 10^{-11} \\
& k_{a}=\left\{\begin{array}{ll}
0.0228 & \text { for air } \\
0.3094 & \text { fot hydrogen }
\end{array}\right\}
\end{aligned}
$$


APPENDIX B

MATERIAL PROPERTIES

\section{INTRODUCTION}

An extensive library of temperature-dependent material properties is incorporated in the TRAC code. The entire library is accessible by the vessel component; however, the ex-vessel components have access to structural material-property sets only. There are 12 sets of material properties that comprise the library; each set supplies values for thermal conductivity, specific heat, density, and spectral emissivity for use in the heat-transfer calculations. The first 5 sets and set 11 contain properties for nuclear heated or electrically heated fuel-rod simulation. Included are nuclear fuels, Zircaloy cladding, fuel-cladding gap gases, electrical heater-rod fllaments, electrical heater-rod insulating material, and Zircaloy dioxide. Sets 6-10 and 12 are for structural materials including stainless steels, carbon steel, and Inconels. In addition, fuel and cladding coefficients of thermal expansion obtained from MATPRO 56 subroutines FTHEX and CDTHEX are available when the gap-conductance thermal-expansion model is used.

Figure $B-1$ illustrates the calling tree for obtaining the property values. The subroutines MFPOD and MPROF are simple processors for calculating the average temperature and calling the appropriate subroutine based on the, user-specified material index. Subroutine FROD controls the fuel-cladding gap conductance and fuel-rod thermal conduction calculations. The material

indexes in the library are

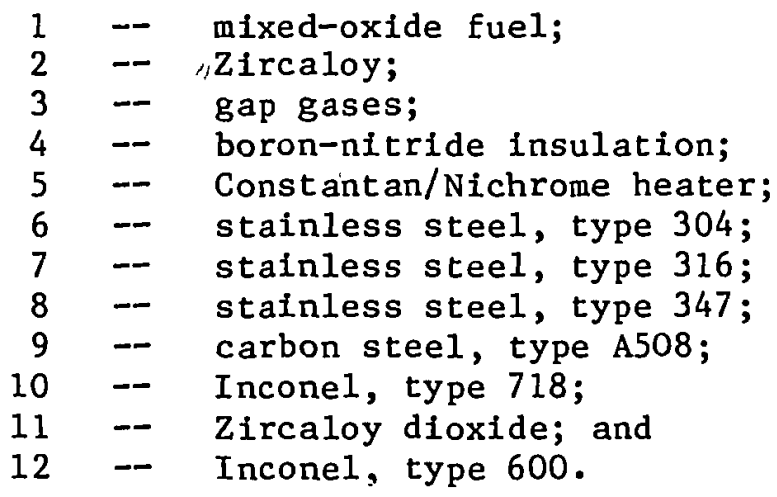

Gap-gas properties are calculated only when the dynamic fuel-cladding gap HTC option is used $($ NFCI $=1)$. 


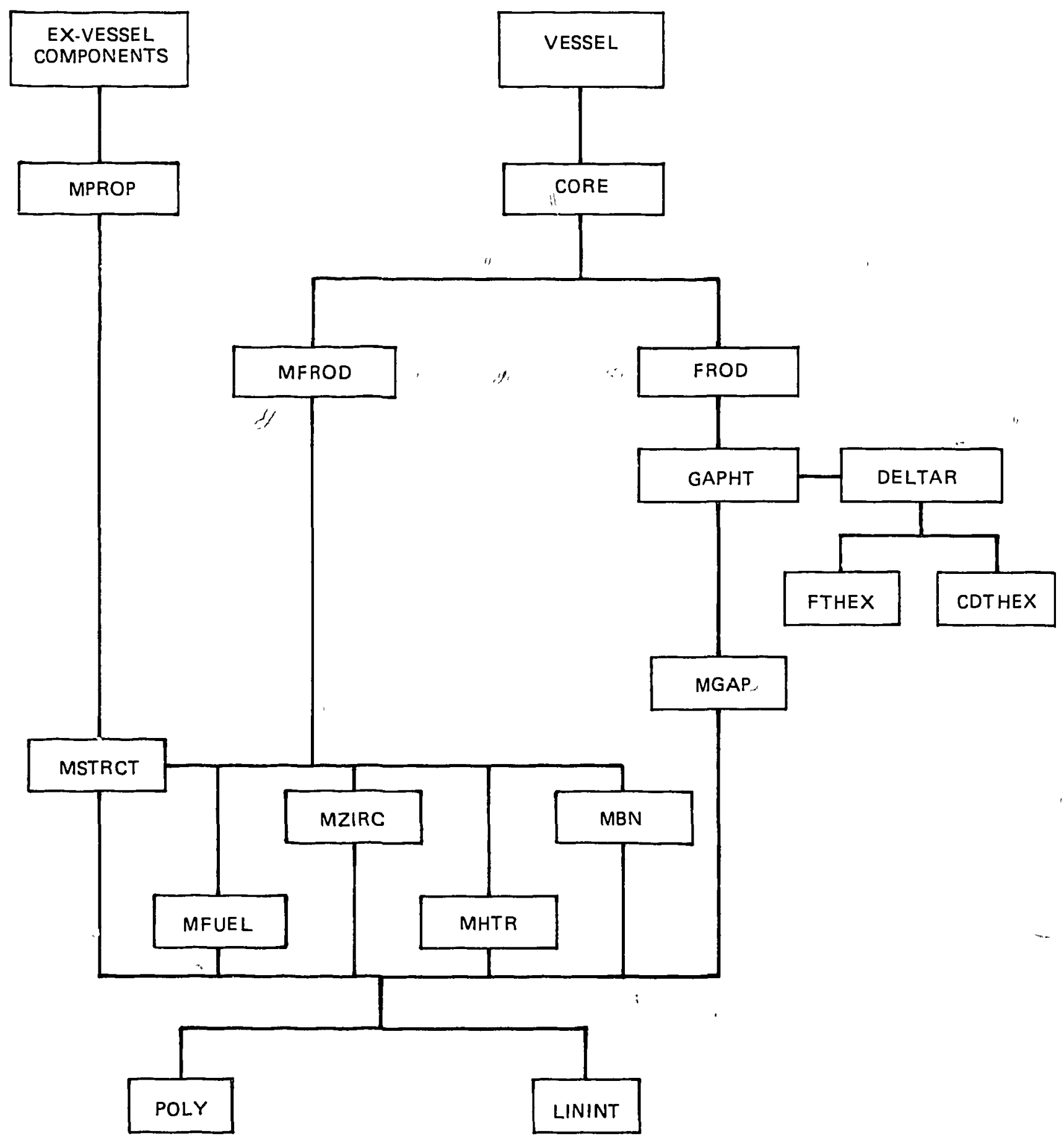

Fig. B-1.

Material-properties code organization. 
II. NUCLEAR FUEL MIXED-OXIDE PROPERTIES

Subroutine MFUEL calculates the properties for mixed-oxide $\left(\mathrm{UO}_{2}\right.$ and $\mathrm{PuO}_{2}$ ) nuclear fuels. Values obtained are influenced by three user-specified input variables: the fraction of theoretical density, the fraction of plutonium "oxide in the fuel, and the fuel burnup. Property changes upon melting are not included in this code version.

A. Density

The mixed-oxide "fuel density is calculated with a correction "factor to account for thermal expansion, which is assumed to be axisymmetric,

$$
\rho=\frac{d}{\left(1+3 \frac{\Delta L}{L}\right)} \text {, }
$$

where

$$
\begin{aligned}
\rho & =\text { density }\left(\mathrm{kg} / \mathrm{m}^{3}\right), \\
\mathrm{d} & =\mathrm{f}_{\mathrm{TD}}\left[\left(1-\mathrm{f}_{\mathrm{PuO}_{2}}\right) \rho_{\mathrm{UO}_{2}}+\mathrm{f}_{\left.\mathrm{PuO}_{2}{ }^{\rho} \mathrm{PuO}_{2}\right],}\right. \\
\mathrm{f}_{\mathrm{TD}} & =\text { fraction of theoretical fuel density }, \\
\mathrm{f}_{\mathrm{PuO}_{2}} & =\text { weight fraction of } \mathrm{PuO}_{2} \text { in the fuel, } \\
\rho_{\mathrm{UO}} & =1.097 \times 10^{4}, \\
\rho_{\mathrm{Pú}} & =1.146 \times 10^{4}, \\
\text { and } & \\
\frac{\Delta \mathrm{L}}{\mathrm{L}} & =1 \text { inear thermal expansion. }
\end{aligned}
$$


The value calculated for the Iinear thermal expansion is based on the MATPRO formulation, 56

$\frac{\Delta L}{L_{O}}=K_{1} T-K_{2}+K_{3} \exp \left(-E_{D} / k T\right)$,

where

$\frac{\Delta \mathrm{L}}{\mathrm{L}_{\mathrm{O}}}=1$ linear strain caused by thermal expansion (equal to $300 \mathrm{~K}$, unitless),

$\mathrm{T}=$ temperature $(\mathrm{K})$, and

${ }^{\prime}=$ Boltzmann's constant $\left(1.38 \times 10^{-23} \mathrm{~J} / \mathrm{K}\right)$.

The constants $K_{1}, K_{2}, K_{3}$, and $E_{D}$ are listed below.

\begin{tabular}{cccc} 
Constant & $\begin{array}{c}\text { Uranium } \\
\text { Dioxide }\end{array}$ & $\begin{array}{c}\text { P1utonium } \\
\text { Dioxide }\end{array}$ & Units \\
\cline { 2 - 3 } $\mathrm{K}_{1}$ & $1.0 \times 10^{-5}$ & $9.0 \times 10^{-6}$ & $\mathrm{~K}^{-1}$ \\
$\mathrm{~K}_{2}$ & $3.0 \times 10^{-3}$ & $2.7 \times 10^{-3}$ & Unitiess \\
$\mathrm{K}_{3}$ & $4.0 \times 10^{-2}$ & $7.0 \times 10^{-2}$ & Unitless \\
$\mathrm{E}_{\mathrm{D}}$ & $6.9 \times 10^{-20}$ & $7.0 \times 10^{-20}$ & $\mathrm{~J}$
\end{tabular}

B. Spectific Heat

. The mixed-oxide fuel specific heat correlations are taken from the MATPRO reports, 56,57

$c_{p}=15.496 \frac{b_{1} b_{4}^{2} \exp \left(b_{4} / T\right)}{T^{2}\left[\exp \left(b_{4} / T\right)-1\right]^{2}}+2 b_{2} T+\frac{b_{3} b_{5}}{b_{6} T^{2}} \exp \left(-b_{5} / b_{6} T\right)$, 
where $c=$ spectfic heat capacity $(\mathrm{J} / \mathrm{kg} \cdot \mathrm{K})$ and $\mathrm{T}=$ fuel temperature $(\mathrm{K})$. The constants $b_{1}$ through $b_{6}$ are 11sted below.

\begin{tabular}{ccc} 
Constants & $\begin{array}{c}\text { Urantum Dioxide } \\
(\text { Ref. 56) }\end{array}$ & $\begin{array}{c}\text { Mixed Oxides } \\
\text { (Ref. 57) }\end{array}$ \\
\cline { 2 - 3 } $\mathrm{b}_{1}$ & 19.145 & 19.53 \\
$\mathrm{~b}_{2}$ & $7.8473 \times 10^{-4}$ & $9.25 \times 10^{-4}$ \\
$\mathrm{~b}_{3}$ & $5.6437 \times 10^{6}$ & $6.02 \times 10^{6}$ \\
$\mathrm{~b}_{4}$ & 535.285 & 539.0 \\
$\mathrm{~b}_{5}$ & 37694.6 & 40100.0 \\
$b_{6}$ & 1.987 & 1.987
\end{tabular}

C. Thermal Conductivity

The mixed-oxide fuel thermal conductivity correlations are taken from the MATPRO report 56 and include porosity and density corraction factors. For $\mathrm{T}_{\mathrm{c}} \leq \mathrm{T}_{1}$,

$k=c\left[\frac{c_{1}}{c_{2}+T_{c}}+c_{3} \exp \left(c_{4} T_{c}\right)\right]$,

and for $\mathrm{T}_{\mathrm{c}}>\mathrm{T}_{1}$,

$k=c\left[c_{5}+c_{3} \exp \left(c_{4} T_{c}\right)\right]$,

where

$\mathrm{T}_{\mathrm{c}}=$ temperature $\left({ }^{\circ} \mathrm{C}\right)$,

$c .=100.0\left[\frac{1-\beta\left(1-f_{T D}\right)}{1-0.05}\right]$,

$B=c_{6}+c_{7} T_{c}$,

$\mathrm{f}_{\mathrm{TD}}=$ fraction of theoretical density . 
The constants $c_{1}$ through $c_{7}$ and $T_{1}$ are listed below.

\begin{tabular}{ccc} 
Constants & Uranium Dioxide & Mixed Oxides \\
\cline { 2 - 3 }$c_{1}$ & 40.4 & 33.0 \\
$c_{2}$ & 464.0 & 375.0 \\
$c_{3}$ & $1.216 \times 10^{-4}$ & $1.54 \times 10^{-4}$ \\
$c_{4}$ & $1.867 \times 10^{-3}$ & $1.71 \times 10^{-3}$ \\
$c_{5}$ & 0.0191 & 0.0171 \\
$c_{6}$ & 2.58 & 1.43 \\
$c_{7}$ & $-5.8 \times 10^{-4}$ & 0.0 \\
$T_{1}$ & 1650.0 & 1550.0
\end{tabular}

\section{Spectral Emissivity}

The mixed-oxide spectral emissivity is calculated as a function of temperature based on the MATPRO correlations.

The values for uranium-dioxide and mixed-oxide fuels are assumed equivalent:
$\varepsilon=0.8707$
for $T \leq 1000 \mathrm{C}$,
$\varepsilon=1.311-4.404 \times 10^{-4} \mathrm{~T}$
for $1000<\mathrm{T} \leq 2050 \mathrm{C}$,

and

$\varepsilon=0.4083$

for $\mathrm{T}>20500 \mathrm{C}$.

\section{ZIRCALOY CLADDING PROPERTIES}

Subroutine MZIRC calculates the properties for Zircaloy and oxidized Zircaloy cladding. The values obtained are for Zircaloy-4. Zircaloy-2 properties are assumed to be identical. The equations used are based on the correlations in the MATPRO report. 56 
A. Denstty

Zircaloy cladding exhibits an asymmetric thermal expansion behavior.

Thermal expansion is calculated in the radial and axial directions, and these effects are included in the density calculation,

$\rho=\frac{6.551 .4}{1+\left[2\left(\frac{\Delta \mathrm{L}}{\mathrm{L}}\right)_{\mathrm{r}}+\left(\frac{\Delta \mathrm{L}}{\mathrm{L}}\right)_{\mathrm{z}}\right]}$,

where

$\left(\frac{\Delta \mathrm{L}}{\mathrm{L}}\right)_{\mathrm{r}}=-2.373 \times 10^{-4}+6.721 \times 10^{-6} \mathrm{~T}_{\mathrm{c}}$

and

$\left(\frac{\Delta \mathrm{L}}{\mathrm{L}}\right)_{z}=-2.506 \times 10^{-5}+4.441 \times 10^{-6} \mathrm{~T}_{\mathrm{c}}$,

for $T S 1073.15$;

$\left(\frac{\Delta \mathrm{L}}{\mathrm{L}}\right)_{\mathrm{r}}=5.1395 \times 10^{-3}-1.12 \times 10^{-5}\left(\mathrm{~T}_{\mathrm{c}}-1073.15\right)$

and

$\left(\frac{\Delta L}{L}\right)_{z}=3.527 .7 \times 10^{-3}-1.06385 \times 10^{-5}\left(\mathrm{~T}_{\mathrm{c}}-1073.15\right)$,

for $1073.15<\mathrm{T} \leq 1273.15$;

$\left(\frac{\Delta \mathrm{L}}{\mathrm{L}}\right)_{\mathrm{r}}=-6.8 \times 10^{-3}+9.7 \times 10^{-6} \mathrm{~T}_{\mathrm{c}}$

and

$\left(\frac{\Delta \mathrm{L}}{\mathrm{L}}\right)_{\mathrm{z}}=-8.3 \times 10^{-3}+9.7 \times 10^{-6} \underset{\mathrm{T}}{\mathrm{T}}$,

for $\mathrm{T}_{\mathrm{c}}>1273.15$; and $\mathrm{T}_{\mathrm{c}}=$ temperature $\left({ }^{\circ} \mathrm{C}\right)$. 
B. Specific Heat

Because Zircaloy undergoes a phase change (alpha to beta) from 1090 to $1248 \mathrm{k}$, with a resultant sharp spike in the specific heat value during the transition, the specific heat is calculated by linear interpolation. Table B-I is used for $T S 1248 \mathrm{~K}$. For $\mathrm{T}>1248 \mathrm{~K}, \mathrm{c}_{\mathrm{p}}=356 \mathrm{~J} / \mathrm{kg} \cdot \mathrm{K}$. C. Thermal Conductivity

Four-term polynomials are used to calculate the Zircaloy and oxidized Zircaloy thermal conductivities. The Kelvin temperature is the independent variable; the polynomial constants are listed below.

\begin{tabular}{|c|c|c|}
\hline Constants & Zirconium & Zircontum Dioxlde \\
\hline$a_{0}$ & 7.51 & 1.96 \\
\hline$a_{1}$ & $2.09 \times 10^{-2}$ & $-2.41 \times 10^{-4}$ \\
\hline$a_{2}$ & $-1.45 \times 10^{-5}$ & $6.43 \times 10^{-7}$ \\
\hline$a_{3}$ & $7.67 \times 10^{-9}$ & $-1.95 \times 10^{-10}$ \\
\hline
\end{tabular}

The form of the polynomial used in this section and the subsequent material properties sections is

$y=a_{0}+a_{1} x+a_{2} x^{2}+\cdot \cdot \cdot+a_{m} x^{m} \cdot$

D. Spectral Emissivity

The emissivity of Zircaloy is temperature dependent, and the emissivity of Zircaloy oxide is temperature and time dependent. For simplicity, a constant value of $\varepsilon=0.75$ is used. 
IV. FUEL-CLADDING GAP-GAS PROPERTIES

Subroutine MGAP calculates values for the gap-gas mixture thermal conductivity that are used in predicting gap HTCs. The method is taken from the MATPRO report 56 and is based on calculating mixture values for seven possible constituent gases:

$$
k_{\text {gap }}=\sum_{i=1}^{n}\left(\frac{k_{i} x_{i}}{x_{i}+\sum_{\substack{j=1 \\ j \neq i}}^{n} \psi_{i j} x_{j}}\right),
$$

where

$k_{\text {gap }}=$ gap mixture thermal conductivity $(\mathrm{W} / \mathrm{m} \cdot \mathrm{K})$,

$\psi_{i j}=\phi_{i j}\left[1+2.41 \frac{\left(M_{i}-M_{j}\right)\left(M_{i}-0.142 M_{j}\right)}{\left(M_{i}+M_{j}\right)^{2}}\right]$,

$\phi_{i j}=\frac{\left[1+\left(\frac{k_{i}}{k_{j}}\right)^{1 / 2}\left(\frac{M_{i}}{M_{j}}\right)^{1 / 4}\right]^{2}}{2^{3 / 2}\left(1+\frac{M_{i}}{M_{j}}\right)^{1 / 2}}$,

$k_{i}=$ constituent gas thermal conductivity $(\mathrm{W} / \mathrm{m} \cdot \mathrm{K}), \mathrm{M}_{1}=$ constituent gas molecular weight, and $x_{i}=$ constituent gas mole fraction.

The seven constituent gases considered are helium, argon, xenon, krypton, hydrogen, air/nitrogen, and water vapor. Except for water vapor, their thermal conductivities are defined:

$k=a T^{b}$, 
where $\mathrm{T}=$ temperature $(\mathrm{K})$. The constants in the above thermal conductivity equation for various gases are listed below.

\begin{tabular}{lll}
\multicolumn{1}{c}{ Gas } & \multicolumn{1}{c}{$\mathrm{a}$} & $\mathrm{b}$ \\
helium & $3.36 \times 10^{-3}$ & 0.668 \\
argon & $3.421 \times 10^{-4}$ & 0.701 \\
xenon & $4.0288 \times 10^{-5}$ & 0.872 \\
krypton & $4.726 \times 10^{-5}$ & 0.923 \\
hydrogen & $1.6355 \times 10^{-3}$ & 0.8213 \\
air/nitrogen & $2.091 \times 10^{-4}$ & 0.846
\end{tabular}

For water vapor the following correlation is used:

$$
\begin{aligned}
k_{\text {steam }}= & \left(-2.8516 \times 10^{-8}+9.424 \times 10^{-10} \mathrm{~T}-6004 \times 10^{-14} \mathrm{~T}^{2}\right) \frac{\mathrm{P}}{\mathrm{T}} \\
& +\frac{1.009 \mathrm{p}^{2}}{\mathrm{~T}^{2}(\mathrm{~T}-273)^{4.2}}-84083 \times 10^{-3}-1.19998 \times 10^{-5} \mathrm{~T} \\
& -6.706 \times 10^{-8} \mathrm{~T}^{2}-4.51 \times 10^{-11} \mathrm{~T}^{3},
\end{aligned}
$$

where $p$ is the gap-gas pressure $\left(\mathrm{N} / \mathrm{m}^{2}\right)$.

When the gap dimension shrinks to the order of the gas mean-free path, a correction factor is applied to the light-gas thermal conductivities to account for the change in energy exchange between the gas and the surface. Again, if the MATPRO recommendations ${ }^{56}$ are used, the correction factor for hydrogen and helium is

$k=\frac{k_{1}}{1+f k_{1}}$, 
where

$f=\frac{0.2103 \mathrm{~T}_{g}}{\mathrm{P}_{\mathrm{g}} \lambda}$,

$\mathrm{T}_{\mathrm{g}}$ is the average gap-gas temperature $(\mathrm{K})$, and $\lambda$ is the characteristic fuel rms roughness equal to $4.389 \times 10^{-6} \mathrm{~m}$.

V. ELECTRICAL FUEL-ROD INSULATOR (BN) PROPERTIES

Subroutine MBN calculates values for boron-nitride insulators that are used in electrically heated nuclear fuel-rod simulators. Magnesium oxide insulators are assumed to have roughly equivalent values.

A. Density

A constant value of $2002 \mathrm{~kg} / \mathrm{m}^{3}$ from Ref. 58 is used.

B. Specific Heat

A four-term polynomial is used to calculate the specific heat. The independent variable is temperature in degrees Fahrenheit, and the constants, which are modifications of those reported in an EPRI report, 59 are 1 isted below.

$$
\frac{\mathrm{a}_{0}}{760.59} \quad \frac{\mathrm{a}_{1}}{1.7955} \quad \frac{\mathrm{a}_{2}}{-8.6704 \times 10^{-4}} \quad \frac{\mathrm{a}_{3}}{1.5896 \times 10^{-7}}
$$

C. Thermal Conductivity

The boron-nitride thermal conductivity calculation is based on a conversion to SI units of a curve fit reported in Ref. 60:

$k=25.27-1.365 \times 10^{-3} \mathrm{~T}_{\mathrm{f}}$,

where $k$ is the thermal conductivity $(W / m \cdot K)$ and $T_{f}$ is the temperature $\left({ }^{\circ} F\right)$. D. Spectral Emissivity

A constant value of unity is used for the boron-nitride spectral emissivity. 
VI. ELECTRICAL FUEL-ROD HEATER-COIL (CONSTANTAN) PROPERTIES

Subroutine MHTR calculates property values for Constantan heater colls as used in electrically heated nuclear fuel-rod simulators. We assume that Nichrome colls, used in some installations in place of Constantan, have similar properties. The correlations used are from Ref. 60 .

A. Density

A constant value of $8393.4 \mathrm{~kg} / \mathrm{m}^{3}$ is used.

B. Specific Heat

The specific heat is

$c_{p}=110 \mathrm{~T}_{\mathrm{f}}^{0.2075}$,

where $c_{p}$ is the specific heat $(\mathrm{J} / \mathrm{kg} \cdot \mathrm{K})$ and $\mathrm{T}_{\mathrm{f}}$ is the temperature $\left({ }^{\circ} \mathrm{F}\right)$. c. Thermal Conductivity

The thermal conductivity is

$\mathrm{k}=29.18+1.683 \times 10^{-3}\left(\mathrm{~T}_{\mathrm{f}}-100\right)$,

where $k$ is the thermal conductivity $(W / m \cdot K)$ and $T_{f}$ is the temperature $\left({ }^{\circ} F\right)$. D. Spectral Emissivity

A constant value of unity is used.

VII. STRUCTURAL MATERIAL PROPERTIES

Subroutine MSTRCT supplies property values for six types of structural materlals normally used in LWRs: stainless steel, type 304; stainless steel, type 316; stainless steel, type 347; carbon steel, type A508; and Inconel, types 600 and 718. These properties were obtained from Refs.60-62. A tabulation of the correlations used is given in Tables B-II through B-VII. 
TABLE B-I

SPECIFIC HEAT VS TEMPERATURE

\begin{tabular}{|c|c|}
\hline$I(K)$ & $c_{p}(\mathrm{~J} / \mathrm{kg} \cdot \mathrm{K})$ \\
\hline 300 & 281 \\
\hline 400 & 302 \\
\hline 640 & 381 \\
\hline 1090 & 375 \\
\hline 1093 & 502 \\
\hline 1113 & 590 \\
\hline 1133 & 615 \\
\hline 1153 & 719 \\
\hline 1173 & 816 \\
\hline 1193 & 770 \\
\hline 1213 & 619 \\
\hline 1233 & 469 \\
\hline 1248 & 356 \\
\hline
\end{tabular}


TABLE B-II

STRUCTURAL MATERIAL PROPERTIES

STAINLESS STEEL, TYPE 304

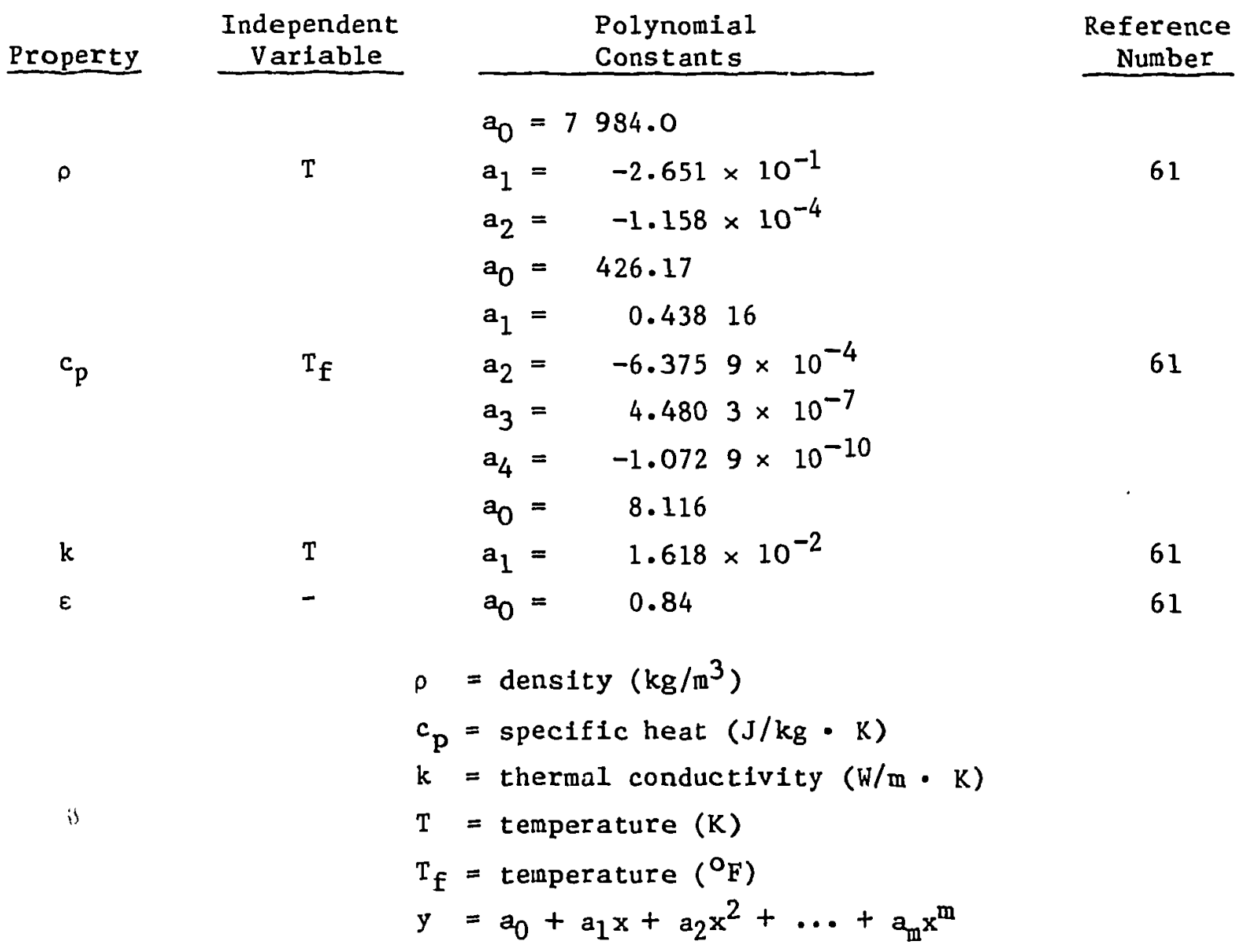




\section{STRUCTURAL MATERIAL PROPERTIES}

STAINLESS STEEL, TYPE 316

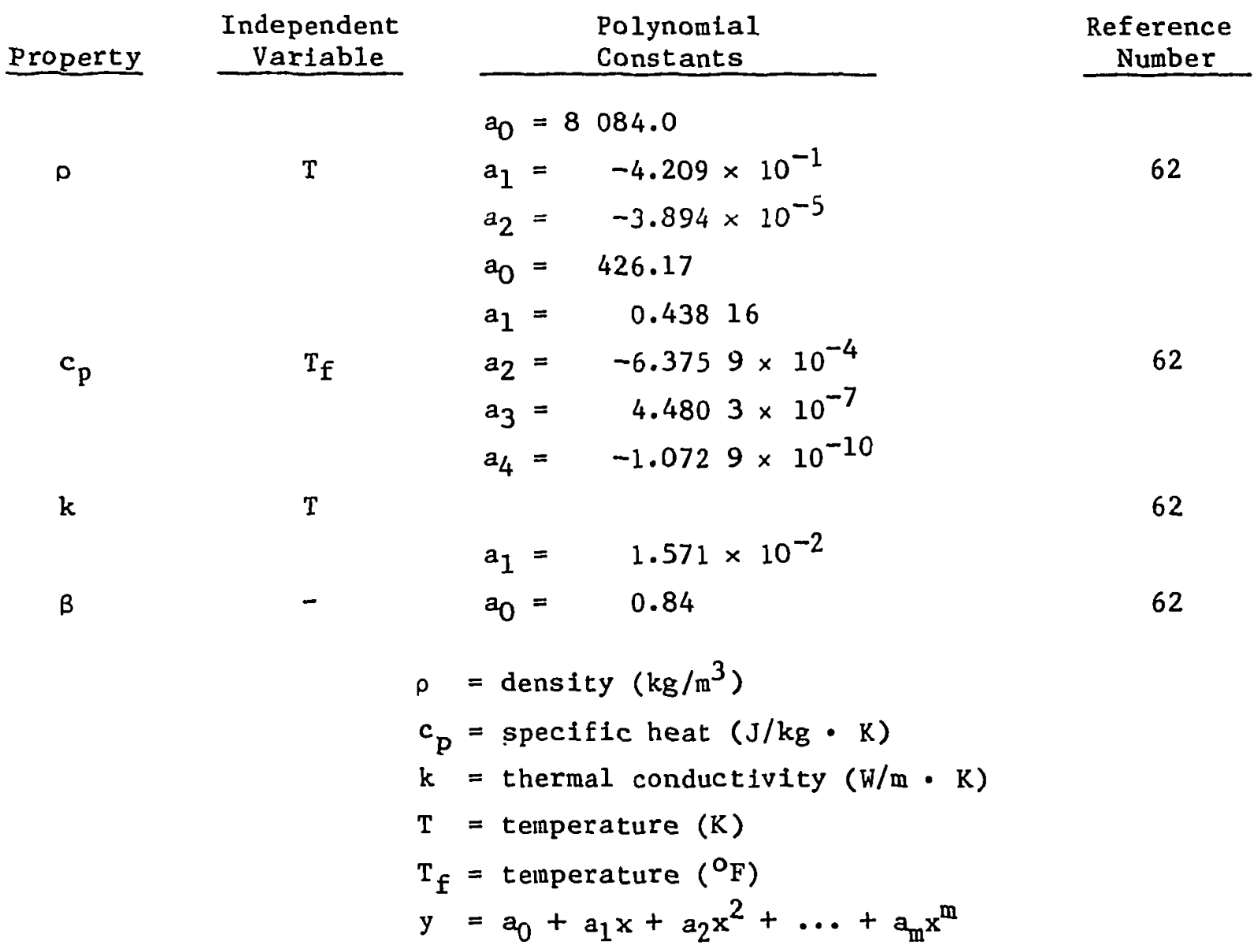


TABLE B-IV

\section{STRUCTURAL MATERIAL PROPERTIES}

STAINLESS STEEL, TYPE 347

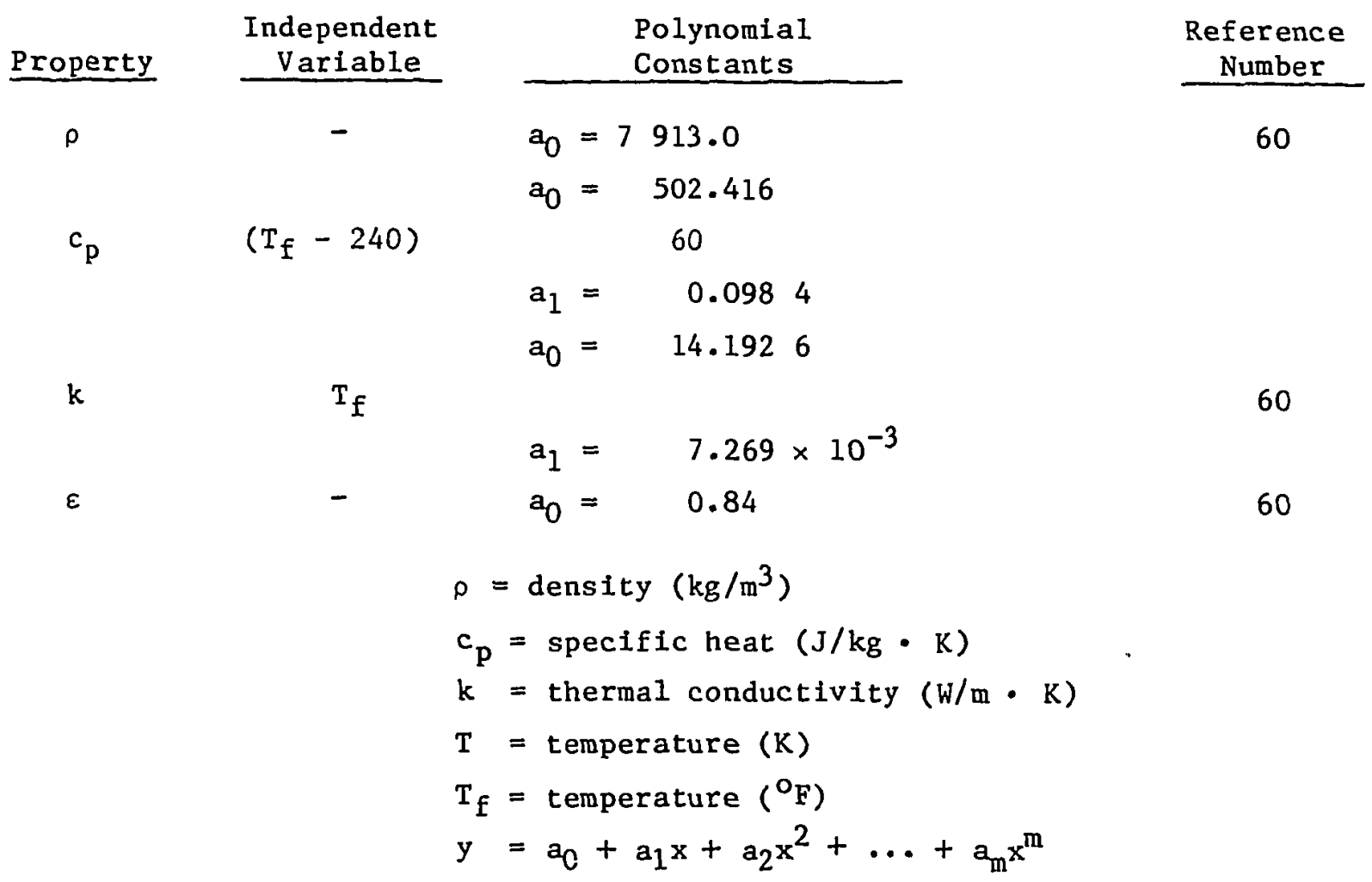


STRUCTURAL MATERIAL PROPERTIES

CARBON STEEL, TYPE 508

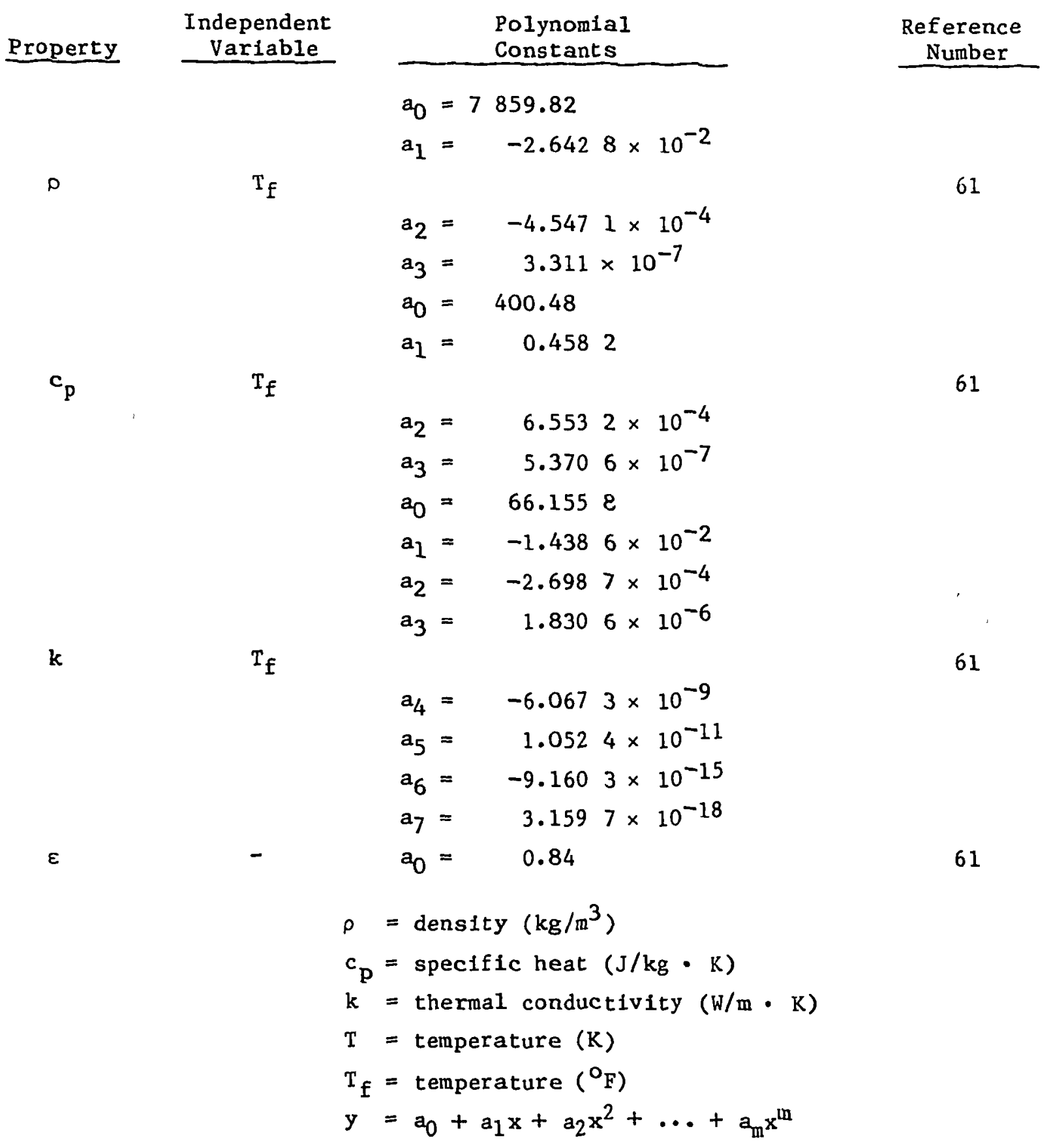


TABLE B-VI

STRUCTURAL MATERIAL PROPERTIES

INCONEL, TYPE 718

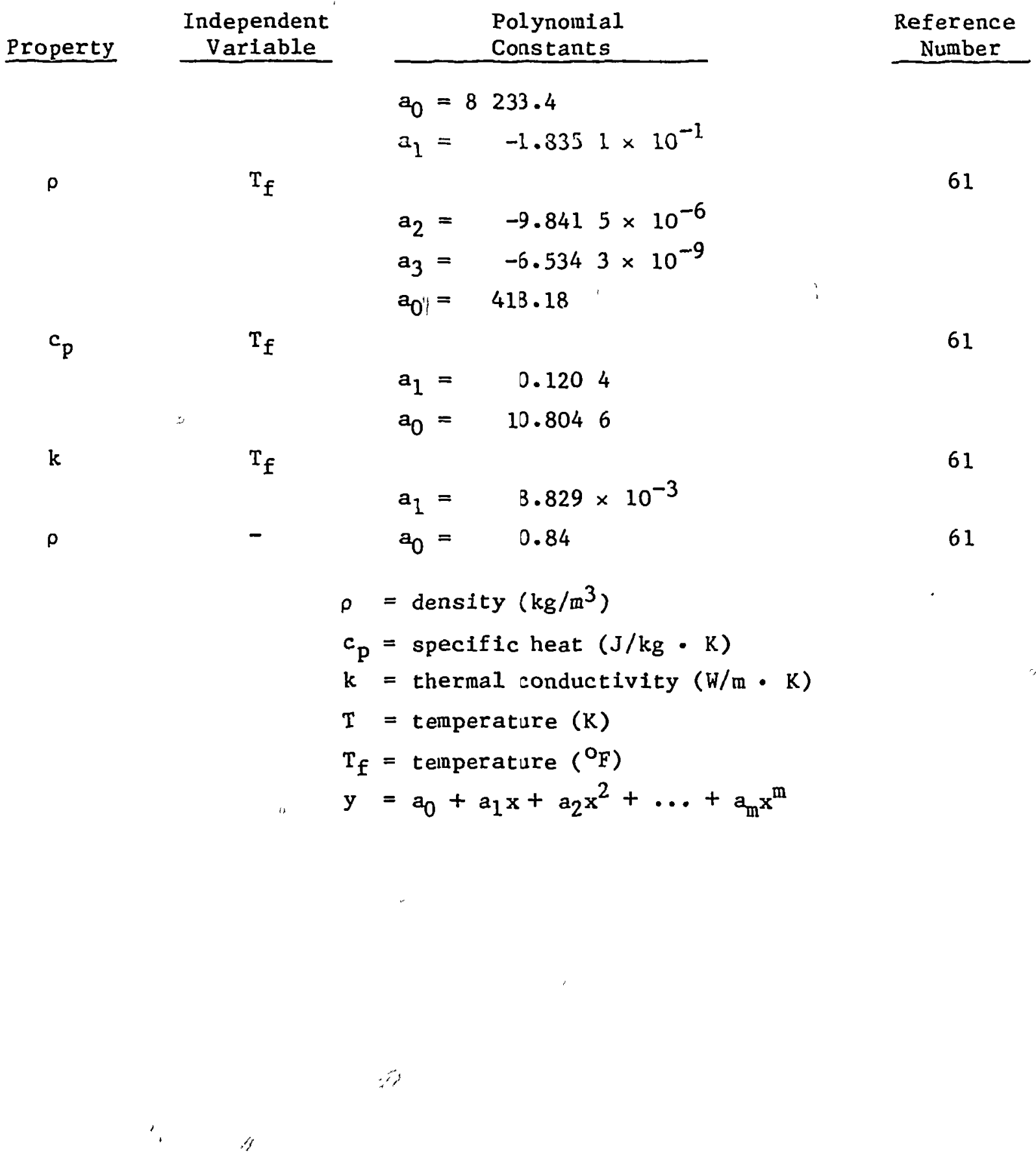


TABLE B-VII

STRUCTURAL MATERIAL PROPERTIES

INCONEL, TYPE $600^{\circ}$

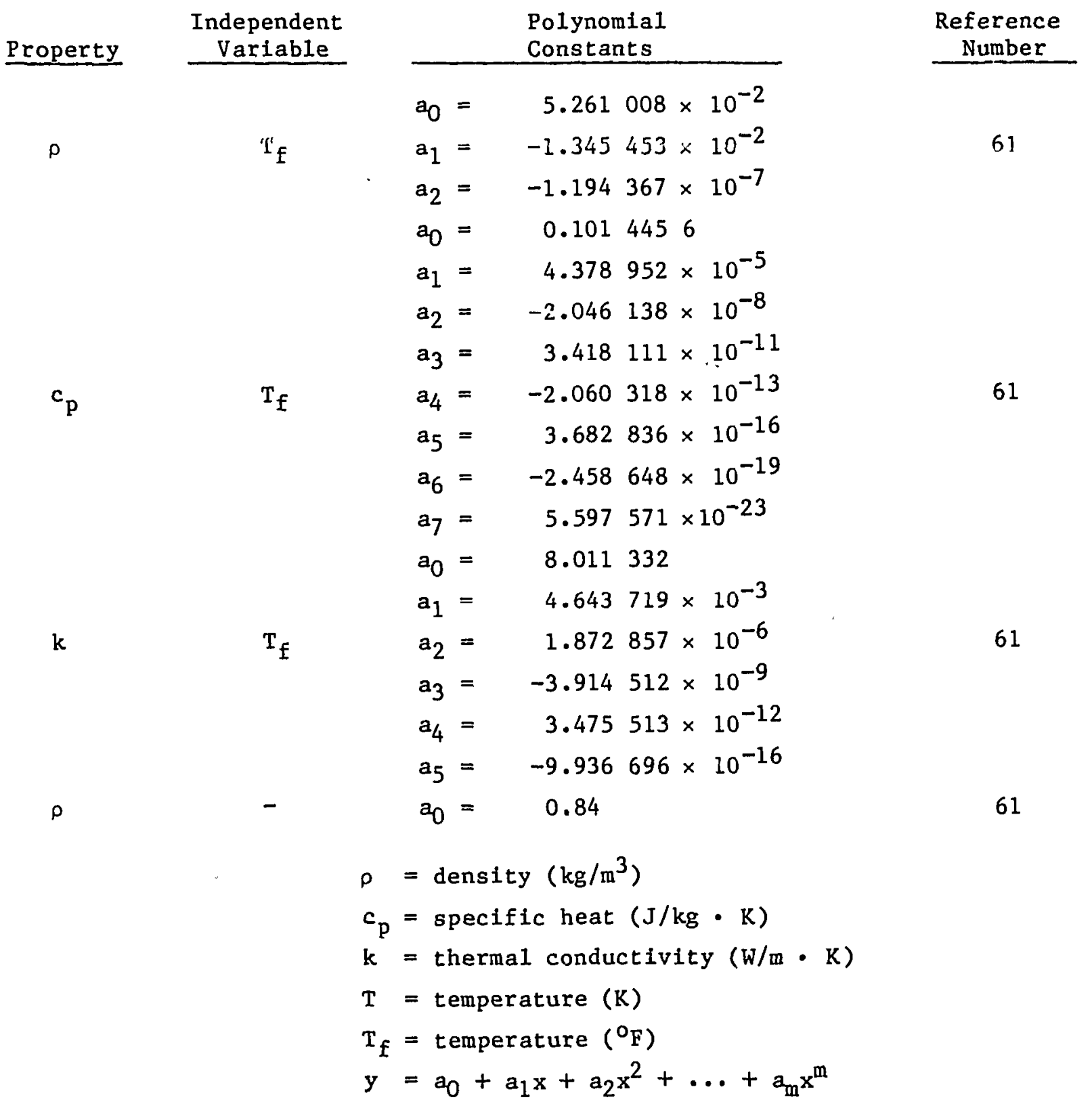

Inconel, type 600 coefficients are in British units; then $\rho$ is multiplied by $16.01846 ; c_{p}$, by $4.1868 \times 10^{-3}$; and $k$, by 1.729577 . 
APPENDIX C

SETS NUMERICAL METHOD

This appendix provides a simplified description of the numerical methods and solution procedures. Only simple single-phase flow equations are used, but generalization to the full TRAC equation set is relatively straightforward.

The SETS method is designed to propagate information needed for stability with minimal implicit coupling between spatial nodes. Information about pressure wave propagation is provided with a basic step, which is simply a semi-implicit equation set. A stabilizing step then is added to provide information about the transport of fluid density, energy, and momentum across cell boundaries. Because the stabilizing step is separated from the basic step, this approach has the further advantage that existing semi-implicit codes can be converted to the SETS method with relative ease.

\section{BASIC EQUATIONS}

To demonstrate the TRAC-PFI numerical methods, we consider a simplifled model for one-dimensional, single-phase flow in a pipe. The differential equations for this model are listed below.

$\frac{\partial \rho}{\partial t}+\nabla \cdot \rho V=0$

$\frac{\partial \rho \mathrm{e}}{\partial \mathrm{t}}+\nabla \cdot \rho \mathrm{eV}=-\mathrm{p} \nabla \cdot \mathrm{V}+\mathrm{h}\left(\mathrm{T}_{\mathrm{w}}-\mathrm{T}\right)$

and

$\frac{\partial V}{\partial t}+V \cdot \nabla V=-\frac{1}{\rho} \nabla p-K V|V|$

Here, $K$ is a wall friction coefficient that may be a function of velocity and fluid properties, $h$ is a heat-transfer coefficient multiplied by the heattransfer area per volume of fluid, and $T_{W}$ is a pipe wall temperature. 
A staggered spatial mesh is used for the finite-difference equations, with thermodynanic properties evaluated at the cell centers and the velocity evaluated at the cell edges. Only difference equations on the one-dimensional version of this mesh are demonstrated, but the generalization to two- and three-dimensional versions is not difficult. To ensure stability and to maintain consistency with differencing in previous TRAC verstons, flux terms at cell edges use donor-cell averages of the form

$$
\begin{array}{rlrl}
\langle Y V\rangle_{j+1 / 2} & =Y_{j} V_{j+1 / 2}, & V_{j+1 / 2} \geq 0 \\
& =Y_{j+1} V_{j+1 / 2}, \quad V_{j+1 / 2}<0 .
\end{array}
$$

Here $Y$ may be any state variable. Other forms of this average may maintain stability with higher-order spatial accuracy, but they have not been carefully studied. With this notation the one-dimensional finite-difference divergence operator is

$\nabla_{j} \cdot(Y V)=\frac{\left(A_{j+1 / 2}\langle Y V\rangle_{j+1 / 2}-A_{j-1 / 2}\langle Y V\rangle_{j-1 / 2}\right)}{V_{j} 1_{j}}$,

where $A$ is the area of the cell edge and $v^{\prime} l_{j}$ the cell volume. The term $W V$ becomes

$$
\begin{aligned}
v_{j+1 / 2} \nabla_{j+1 / 2} v & =\frac{v_{j+1 / 2}\left(v_{j+1 / 2}-v_{j-1 / 2}\right)}{\Delta x_{j+1 / 2}}, v_{j+1 / 2} \geq 0 \\
& =\frac{v_{j+1 / 2}\left(v_{j+3 / 2}-v_{j+1 / 2}\right)}{\Delta x_{j+1 / 2}}, v_{j+1 / 2}<0,
\end{aligned}
$$


where $\Delta x_{j+1 / 2}=0.5\left(\Delta x_{j}+\Delta x_{j+1}\right)$. This choice of $\Delta x_{j+1 / 2}$ for Eq. (C-6) is necessary for more accurate calculation of pressure drops in plpes modeled with a nonuniform mesh than is provided with a donor cell $\Delta x_{j+1 / 2}$.

For the flow model given by Eqs. $(C-1)-(C-3)$, the combination of basic and stabilizer equation sets can be written in several ways without significantly affecting the results. For example, the stabllizer step may precede the basic step for all equations, or the basic step may be done before the stabilizer step. When the SETS method is adapted to the two-fluid model for two-phase flow, several orderings of the difference equations can cause growing oscillations from feedback through interfacial friction terms. Some of these feedback problems are rather subtle and occur only when the onedimensional mesh forms a closed loop. One ordering that is always stable begins with the stabilizer step for the equations of motion, continues with a solution of the basic equation set for all equations, and ends with a stabilizer step for the mass and energy equations. For this ordering, the SETS finite-difference equations for Eqs. $(C-1)-(C-3)$ are

STABILIZER EQUATION OF MOTION

$$
\begin{aligned}
\frac{\left(\tilde{v}_{j+1 / 2}^{n+1}-v_{j+1 / 2}^{n}\right)}{\Delta t} & +v_{j+1 / 2}^{n}{ }_{j+1 / 2} \tilde{v}^{n+1} \\
& +\beta\left(\tilde{v}_{j+1 / 2}^{n+1}-v_{j+1 / 2}^{n}\right) \nabla_{j+1 / 2} \tilde{v}^{n} \\
& +\frac{1}{\langle p\rangle_{j+1 / 2}^{n} \Delta x_{j+1 / 2}}\left(p_{j+1}^{n}-p_{j}^{n}\right) \\
& +k_{j+1 / 2}^{n}\left(2 \tilde{v}_{j+1 / 2}^{n+1}-v_{j+1 / 2}^{n}\right)\left|v_{j+1 / 2}^{n}\right|=0,
\end{aligned}
$$

where

$$
\begin{array}{r}
\beta=0, \nabla_{j+1 / 2} \tilde{V}^{\tilde{n}}<0 \\
1, \quad \nabla_{j+1 / 2} \tilde{v}^{n}>0 ;
\end{array}
$$


BASIC EQUATIONS

$$
\begin{aligned}
\frac{\left(v_{j+1 / 2}^{n+1}-v_{j+1 / 2}^{n}\right)}{\Delta t} & +v_{j+1 / 2}^{n} \nabla_{j+1 / 2} \tilde{v}^{n+1} \\
& +\beta\left(v_{j+1 / 2}^{n+1}-v_{j+1 / 2}^{n} \nabla_{j+1 / 2} \tilde{v}^{n}\right. \\
& +\frac{1}{\langle p\rangle_{j+1 / 2}^{n} \Delta x_{j+1 / 2}}\left(\tilde{p}_{j+1}^{n+1}-\tilde{p}_{j}^{n+1}\right) \\
& +k_{j+1 / 2}^{n}\left(2 v_{j+1 / 2}^{n+1}-v_{j+1 / 2}^{n}\right)\left|v_{j+1 / 2}^{n}\right|=0 ;
\end{aligned}
$$

$\frac{\left(\tilde{\rho}_{j}^{n+1} \rho_{j}^{n}\right)}{\Delta t}+\nabla_{j} \cdot\left(\rho^{n_{V}^{n+1}}\right)=0 ;$

$\frac{\left(\tilde{p} \tilde{j}_{j}^{n+1} \tilde{e}_{j}^{n+1}-\rho{ }_{j}^{n} e_{j}^{n}\right)}{\Delta t}+\nabla_{j} \cdot\left(\rho^{n_{e} n^{n} V^{n+1}}\right)$

$$
+\tilde{p}_{j}^{n+1} \cdot j \quad\left(V^{n+1}\right)-\bar{h}_{j}^{n}\left(T_{w j}^{n}-\tilde{T}_{j}^{n+1}\right)=0 ;
$$

and

STABILIZER MASS AND ENERGY EQUATIONS

$$
\frac{\left(._{j}^{n+1}-\cdot n_{j}^{n}\right)}{t}+\cdot j \cdot\left(p^{n+1} v^{n+1}\right)=0 ;
$$

$\frac{\left(\rho_{j}^{n+1} e_{j}^{n+1}-\rho{ }_{j}^{n} e_{j}^{n}\right.}{\Delta t}+\nabla_{j} \cdot\left(\rho^{n+1} e^{n+1} V^{n+1}\right)$

$$
+\tilde{p}_{j}^{n+1} \nabla_{j} \cdot\left(V^{n+1}\right)-\overline{h_{j}^{n}}\left(T_{w j}^{n}-\tilde{T}_{j}^{n+1}\right)=0 .
$$


A tilde above a variable indicates that it is the result of an intermediate step and is not the final value for the time step.

Equations $(C-8)-(C-10)$ are very similar to a one-dimensional singlephase version of the difference equations used for the TRAC vessel component. These semi-implicit equations can be obtained by dropping all tildes from Eqs. $(C-8)-(C-10)$ and replacing the $W V$ and friction terms in Eq. (C-8) with $\mathrm{V}_{j+1 / 2}^{\mathrm{n}} \nabla_{j+1 / 2} \mathrm{~V}^{\mathrm{n}}$ and $\mathrm{K}^{\mathrm{n}} \mathrm{V}_{\mathrm{j}+1 / 2}^{\mathrm{n}+1}\left|\mathrm{~V}_{\mathrm{j}+1 / 2}^{\mathrm{n}}\right|$, respectively. In addition, the pressure work term for the three-dimensional energy equation is $p^{n} \nabla \cdot v^{n+1}$ rather than $\mathrm{p}^{\mathrm{n}+1} \nabla \cdot \mathrm{v}^{\mathrm{n}+1}$. This change in the work term was made because, in the three-dimensional numerics, only the linearized flow equations are solved, and the linearized form of $\mathrm{p}^{\mathrm{n}+1} \nabla \cdot \mathrm{v}^{\mathrm{n}+1}$ can produce nonphysical results for sudden, unexpected changes in pressure.

The heat-transfer term $\bar{h}^{n}\left(T_{w}^{n}-T^{n+1}\right)$ is written in this form to minimize coupling between the hydrodynamic and heat-transfer solution procedures. To maintain stability in regions of rapid change of $h, \bar{h}^{n}$ is an average of the heat-transfer coefficient evaluated at time level $n$ and the averaged coefficient from level $\mathrm{n}-1\left(\overline{\mathrm{h}}^{\mathrm{n}}=0.45 \mathrm{~h}^{\mathrm{n}}+0.55 \overline{\mathrm{h}}^{\mathrm{n}-1}\right)$.

The material Courant stability limit is eliminated by treatment of the terms $V \nabla V, \nabla \cdot \rho \mathrm{V}$, and $\nabla \cdot \rho \mathrm{eV}$ during the two steps. These are the terms involved in the information propagation that was discussed in the introduction. Additional robustness has been obtained with the particular form for the friction terms and the use of nonzero values of $\beta$ in the $W V$ terms. These special terms for friction and $W V$ are obtained by linearizing similar terms that are fully implicit in velocity $\left(k_{j+1 / 2}^{n} v_{j+1 / 2}^{n+1}\left|v_{j+1 / 2}^{n+1}\right|\right.$ and $\left.v_{j+1}^{n+1} / 2^{\nabla} j+1 / 2^{n+1}\right)$.

Equation (C-7) simply represents a tridiagonal linear systen in the unknown $\tilde{\mathrm{V}}^{\mathrm{n}+1}$ and is solved first (by TRAC in subroutines FEMON and BKSMOM). Next, the coupled nonlinear system given by Eqs. (C-8)-(C-10) is solved (in TF3DE, TF3DI, and FF3D for the three-dimensional and TF1DS1, TF1DS, and TF1DS3 for the one-dimensional equations). Once these equations are solved, $\mathrm{v}^{\mathrm{n}+1}$ is known; hence, Eqs. $(\mathrm{C}-11)$ and $(\mathrm{C}-12)$ are simple tridiagonal linear systeins, with unknowns $\rho_{j}^{n+1}$ and $\rho_{j}^{n+1} e_{j}^{n+1}$, respectively (solved by STBME and BKSSTB).

When this equation set is adapted to flow in complex piping networks, the pure tridiagonal structure is lost. However, the matrices are still sparse and easily solved (Sec. III.D). 
A standard linear stability analysis predicts unconditional stability for this set of difference equations, a result verified by a large number of computational test problems. However, at very large time steps, functional forms for the friction factor containing a strong velocity dependence can drive instabilities, as can a strong void-fraction dependence for interfacial friction in the two-fluid model. This is why the method is referred to as stability enhancing rather than unconditionally stable.

Because the basic form of the finite-difference operators (both spatial and temporal) is consistent between the two steps, the order of accuracy of the full SETS equations is the same (first order in space and time) as the basic semi-implicit Eqs. $(C-8)-(C-10)$. This consistency appears necessary to prevent feedback oscillations between the two steps. It also has the advantage of ensuring that, for modest time-step sizes, the results of any SETS calculation approach those of the basic semi-implicit equations.

The SETS method is especially valuable when applied to the full twofluid model for two-phase flow. For this model, the stabilizer equations add less than $20 \%$ to the computational cost per cell per step of the basic equation set. A fully implicit method multiplies this cost by a factor of 6 . The full finite-difference equations for the two-fluid model are given in Sec. III, including a useful variation on the finite-difference divergence operator.

II. SOLUTION OF THE BASIC EQUATION SET

The first step in the solution of the basic (semi-implicit) equation set rearranges the motion equation to obtain the new time velocity as a linear function of new time pressures. In TRAC this is accomplished in the subroutines TF3DE and TF1DS1. For Eq. (C-8), this step results in the relation

$$
v_{i}^{n+1}=\frac{v_{i}^{n}-\Delta t\left[v_{i}^{n} \nabla_{i}\left(\tilde{v}^{n+1}-\beta \tilde{v}^{n}\right)-k_{i}^{n} v_{i}^{n}\left|v_{i}^{n}\right|+\frac{\tilde{p}_{j+1}^{n+1}-\tilde{p}_{j}^{n+1}}{\zeta>_{i}^{n} \Delta x_{i}}\right]}{1+\Delta t\left(2 k_{i}^{n}\left|v_{i}^{n}\right|+\beta \nabla_{i} \tilde{v}_{n}\right)},
$$


where $i=j+1 / 2$. Given this relation, the derivatives of velocity with respect to pressure are

$\frac{d v_{j+1 / 2}^{n+1}}{d p_{j}^{n+1}}=\frac{\Delta t}{\langle\rho\rangle_{i}^{n} \Delta x_{i}\left(1+k_{i}^{n} \Delta t\left|v_{i}^{n}\right|+\Delta t \beta \nabla_{i} \tilde{v}^{n}\right)}$

and

$\frac{d v_{j+1 / 2}^{n+1}}{d p_{j+1}^{n+1}}=-\frac{d v_{j+1 / 2}^{n+1}}{d p_{j}^{n+1}}$

Equation ( $C-13)$ and thermodynamic equations giving $\rho(p, T)$ and $e(p, T)$ are substituted into Eqs. $(C-9)$ and $(C-10)$ to give a coupled system of nonlinear equations with unknowns $p_{j}^{n+1}$ and $T_{j}^{n+1}$ (tildes have been dropped to simplify notation). Solution of this system is obtained with a standard Newton 1teration. Given the latest estimates $\mathrm{p}_{j}^{-n+1}$ and $\mathrm{T}_{j}^{-\mathrm{n}+1}$ for pressures and temperatures, we assume the solution is

$p_{j}^{n+1}=p_{j}^{-n+1}+\delta p_{j}$

and

$T_{j}^{n+1}=T_{j}^{-n+1}+\delta T_{j}$ 
After substituting Eqs. $(C-13)-(C-17)$ into the basic equation set, making the necessary Taylor series expansions, and after discarding nonlinearterms in $\delta \mathrm{p}_{j}$ and $\delta \mathrm{T}_{j}$, we find the resulting linear mass and energy equations are

$$
\begin{aligned}
\left.\frac{\partial \rho}{\partial T}\right|_{j} ^{n+1} \delta T_{j} & +\left.\frac{\partial \rho}{\partial p}\right|_{j} ^{n+1} \delta p_{j}-\left(\frac{A_{j+1 / 2} \rho_{j+1 / 2}^{n} \Delta t}{v o l} \frac{d v_{j+1 / 2}^{n+1}}{d p_{j}^{n+1}}\right)\left(\delta p_{j+1}-\delta p_{j}\right) \\
& -\left(\frac{A_{j-1 / 2} \rho_{j-1 / 2}^{n} \Delta t}{v o v_{j}} \frac{d v_{j-1 / 2}^{n+1}}{d p_{j}^{n+1}}\right)\left(\delta p_{j}-\delta p_{j-1}\right) \\
& =\rho_{j}^{n}-\rho_{j}^{-n+1}-\Delta t \nabla_{j} \cdot\left(\rho^{n_{v}-n+1}\right)
\end{aligned}
$$

and

$$
\begin{aligned}
& \left(\left.\rho_{j}^{-n+1} \frac{\partial e^{-}}{\partial T}\right|_{j} ^{n+1}+\left.e_{j}^{-n+1} \frac{\partial \rho^{-}}{\partial T}\right|_{j} ^{n+1}+\Delta t \bar{h}_{j}^{n+1}\right) \delta T_{j} \\
& +\left(\left.\rho_{j}^{-n+1} \frac{\partial e^{-}}{\partial p}\right|_{j} ^{n+1}+\left.e_{j}^{-n+1} \frac{\partial \rho}{\partial p}\right|_{j} ^{n+1}+\Delta t \nabla_{j} \cdot v^{-n+1}\right) \delta p_{j} \\
& -\left(p_{j}^{-n+1}+\rho_{j+1 / 2}^{n} e_{j+1 / 2}^{n}\right)\left(\frac{A_{j+1 / 2} \Delta t}{v o l_{j}} \frac{d v_{j+1 / 2}^{n+1}}{d p_{j}^{n+1}}\right)\left(\delta p_{j+1}-\delta p_{j}\right) \\
& -\left(p_{j}^{-n+1}+\rho_{j-1 / 2}^{n} e_{j-1 / 2}^{n}\right)\left(\frac{A_{j-1 / 2} \Delta t}{v o 1} \frac{d v_{j}^{n+1}}{d p_{j}^{n+1}}\right)\left(\delta p_{j}-\delta p_{j-1}\right) \\
& =\rho_{j}^{n} e_{j}^{n}-\rho_{j}^{-n+1} e_{j}^{-n+1}-\Delta t\left[\nabla_{j} \cdot \rho^{n} e^{n} V^{-n+1}\right. \\
& \left.+p_{j}^{-n+1} \nabla_{j} \cdot v^{-n+1}-\bar{h}_{j}^{n}\left(T_{w, j}^{n}-T_{j}^{n+1}\right)\right] .
\end{aligned}
$$


The normal procedure for starting this linearization is to make an initial estimate for the new time pressure and temperature of $\mathrm{p}^{-\mathrm{n}+1}=\mathrm{p}^{\mathrm{n}}$ and $T^{-n+l}=T^{n}$. However, when SETS is used, an extra call to the thermodynamic subroutines can be saved by taking $\mathrm{p}^{-\mathrm{n}+1}=\tilde{\mathrm{p}}^{\mathrm{n}}$ and $\mathrm{T}^{-\mathrm{n}+1}=\tilde{\mathrm{T}}^{\mathrm{n}}$.

Equations $(\mathrm{C}-18)$ and $(\mathrm{C}-19)$ can be abbreviated as

$B_{j}\left(\begin{array}{l}\delta p_{j} \\ \delta T_{j}\end{array}\right)=\underline{b}_{j}+\underline{c}_{j}\left(\delta p_{j+1}-\delta p_{j}\right)-\underline{d}_{j}\left(\delta p_{j}-\delta p_{j-1}\right)$

where $B$ is a $2 \times 2$ matrix. For the one-dimensional numerics, this equation is multiplied by $B^{-1}$ (accomplished with a linear system solver), which yields

$-d_{i, j} \delta p_{j-1}+\left(1+d_{i, j}^{\prime}+c_{i, j}^{\prime}\right) \delta p_{j}-c_{i, j}^{i} \delta p_{j+1}=b_{i, j}$

and

$\delta T_{j}=b_{2, j}+c_{2, j}\left(\delta p_{j+1}-\delta p_{j}\right)-d_{2, j}\left(\delta p_{j}-\delta p_{j-1}\right)$,

where $\underline{b}^{-}=B^{-1} \underline{b}$, etc. All coefficients are stored, and then the set of equations represented by Eq. (21) is solved for all $\delta \mathrm{P}_{j}$. Finally," the known values for pressure variations are substituted into Eq. (C-22) to obtain temperature changes and used with Eqs. $(C-14)$ and $(C-15)$ to obtain updated velocities. Given these changes, the next estimates for new time pressures and temperatures are generated and used to obtain densities and energies. If changes in $\delta T$ and $\delta p$ are too large, these estimates are used to return to Eqs. $(C-16)$ and $(C-17)$ to relinearlze for another iteration of the onedimensional equations. As previously noted, the three-dimensional flow equations are not relinearized. In this case, time-step controls are used to assure the accuracy of a single linearization.

One-dimensional equation linearization and initial reduction are accomplished in TFIDS. The linear variations selected for the full two-fluid equation set are $\delta \mathrm{p}, \delta \mathrm{T}_{\ell}, \delta \mathrm{T}_{\mathrm{g}}, \delta \mathrm{P}_{\mathrm{a}}$, and $\delta \alpha$. The three-dimensional linearization, performed by TF3DI, is done somewhat differently. The variations obtained are $\delta \mathrm{p}, \delta \alpha, \delta(\alpha \mathrm{T} g)$, and $\delta\left((1-\alpha) \mathrm{T}_{\ell}\right)$, and the equations are rearranged so that solution is for $\delta P, \alpha^{n+1}, \alpha^{n+1} T_{g}{ }^{n+1}$, and $\left(1-\alpha^{n+1}\right) T_{\ell}^{n+1}$ 
rather than for the original variations. The partial pressure of air is eliminated from the three-dimensional equation set by algebraic manipulations with the noncondensable mass equation. Backward substitution to obtain new values of pressures, temperatures, and void fractions is performed by TFlDS 3 (one-dimensional) and FF3D (three-dimensional).

The principal difference in solution procedures between the one- and three-dimensional equation sets occurs in the actual solution for the linearized pressure [Eq. $(\mathrm{C}-21)]$. For one dimension, direct-solution techniques are always employed. If too many cells are present in the threedimensional mode1, an iterative method is applied. (See Sec. III.A.2.b.)

Note from the form of Eqs. $(C-18)$ and $(C-19)$ that the Jacobian for the system is reevaluated on each iteration. This reevaluation may not always be necessary but, for steam-water flows with phase change, it is often important for rapid convergence of the iteration. 
APPENDIX D

CHOKED-FLOW MODEL

The TRAC-PF1/MOD1 two-phase, two-component choked-flow model was developed from first principles using the characteristic analysis approach. The TRAC-PFI/MODl subcooled choked-flow model is a modified form of the Burnell model. In this appendix we discuss these choked-flow models and their implementation in TRAC-PF1/MODl. We compared the TRAC-PF1/MODI choked-flow models with the Burnell model for subcooled flow and with the homogeneousequilibrium model (HEM) for two-phase flow. These comparisons agree well $"$ under homogeneous conditions. TRAC-PF1/MODl results from models using the choking criteria agree well with those using a fine mesh (natural choking). Code-data comparisons between the separate-effects tests of the Marviken facility and the Edwards' blowdown experiment also are favorable.

I. INTRODUCTION

Because TRAC-PFI/MOD1 fluid-dynamics equations for one-dimensional components use a multistep procedure that allows the material Courant condition to be violated, the choking calculations can be done simply by using a sufficiently fine mesh for components with smooth area changes. However, the TRAC-PF1/MOD1 quasi-steady choked-flow model saves computational time because it allows a much coarser mesh. For components with abrupt area changes, a one-dimensional fine mesh can cause erroneous natural-choking results. For all such cases, a separate choking model is almost a necessity. Thus, a choking model not only improves computational efficiency but also accounts for effects such as sharp area changes, surface roughness, and threedimensional modeling, etc. Section D-II describes the TRAC-PF1/MOD1 chokedflow model. Section D-III compares this model with other conventional models and the test data. Section D-IV discusses the important conclusions. 
II. FEATURES

A. Two-Phase Flow Choking Criterion

The TRAC-PFI/MODl two-phase choking model is an extension of one developed by Ransom and Trapp $^{63}$ that incorporates an additional inert gas component. As suggested by Ransom and Trapp, we assume that thermal equilibrium exists between the phases. The validity of this assumption has not been investigated in the presence of an inert gas. However, this assumption is not an inherent feature of the TRAC-PF1/MOD1 model and can be changed easily, if necessary.

The two-fluid flow field under thermal equilibrium is described by the inert-gas continuity equation, the overall continuity equation, two phasic momentum equations, and the mixture energy equation. When the nondifferential source terms are omitted (because they do not enter into characteristic analysis), the equations are

$$
\frac{\partial}{\partial t}\left(\alpha \rho_{a}\right)+\frac{\partial}{\partial x}\left(\alpha_{a} v_{g}\right)=0,
$$

$$
\begin{aligned}
& \frac{\partial \rho_{m}}{\partial t}+\frac{\partial}{\partial x}\left(\rho_{m} v_{m}\right)=0, \\
& \alpha \rho_{g}\left[\frac{\partial v_{g}}{\partial t}+v_{g} \frac{\partial v_{g}}{\partial x}\right]+\alpha \frac{\partial p}{\partial x}
\end{aligned}
$$

$$
\begin{gathered}
+C \alpha(1-\alpha) \rho_{m}\left[\frac{\partial v_{g}}{\partial t}+v_{\ell} \frac{\partial v_{g}}{\partial x}-\frac{\partial v_{\ell}}{\partial t}-v_{g} \frac{\partial v_{\ell}}{\partial x}\right]=0, \\
(1-\alpha) \rho_{\ell}\left[\frac{\partial v_{\ell}}{\partial t}+v_{\ell} \frac{\partial v_{\ell}}{\partial x}\right]+(1-\alpha) \frac{\partial p}{\partial x} \\
\because \because v(1-\alpha) \rho_{m}\left[\frac{\partial v_{\ell}}{\partial t}+v_{g} \frac{\partial v_{\ell}}{\partial x}-\frac{\partial v_{g}}{\partial t}-v_{\ell} \frac{\partial v_{g}}{\partial x}\right]=0,
\end{gathered}
$$

and

$$
\frac{\partial}{\partial t}\left(\rho_{m} s_{m}\right)+\frac{\partial}{\partial x}\left[\alpha \rho_{g} V_{g} s_{g}+(1-\alpha) \rho_{\ell} V_{\ell} s_{\ell}\right]=0,
$$


where $C$ is the virtual mass coefficient; $s$ is the entropy; and subscripts a, $g, \ell$, and $m$ refer to the noncondensable gas, gas phase (air-vapor mixture), liquid, and air-vapor-liquid mixture, respectively. The last terms in Eqs. (D-3) and (D-4) represent interphasic force terms caused by relative acceleration. These terms are discussed in detail in Refs. 64 and 65 . Following Ransom and Trapp's formulation, the energy equation is written in the form of the mixture-specific entropy that is conserved for adiabatic flow (neglecting irreversibilities associated with interphasic mass transfer and relative phase acceleration). However, no basic difficulty in the analysis is experienced if the mixture energy equation is written in terms of the internal energy or the enthalpy.

In the thermal-equilibrium case, $\rho_{a}, \rho_{v}, \rho_{\ell}, s_{a}, s_{v}$, and $s_{\ell}$ are known functions of $\mathrm{P}_{a}$ and $\mathrm{p}_{\mathrm{v}}$. If we assume that Dalton's law of partial pressures applies, Eqs. $(D-1)-(D-5)$ can be written in terms of the five unknowns $p_{a}, p_{v}$, $\alpha, v_{g}$, and $v_{\ell}$. The matrix representation of these equations is

$A(\bar{U}) \frac{\partial \bar{U}}{\partial t}+B(\bar{U}) \frac{\partial \bar{U}}{\partial x}=0$,

where the $\bar{U}$ consists of $\mathrm{p}_{\mathrm{a}}, \mathrm{P}_{\mathrm{v}}, \alpha, \mathrm{V}_{\mathrm{g}}$, and $\mathrm{V}_{\ell}$. The characteristic roots, $\lambda_{i}$, of the above system of equations are defined as the roots of the fifth-order polynomial,

determinant $(A \lambda+B)=0$.

Choking occurs when the signal propagating with the largest velocity relative to the fluid is stationary; that is, the maximum value of the real part of a characteristic root, $\lambda_{i, r e, m a x}$ is zero. Equation $(D-7)$ is extremely difficult to solve analytically. Thus, TRAC-PF1/MODI obtains the characteristic roots of Eq. (D-7) numerically. This method advantageously maintains generality and facilitates computations under different assumftions. The next three paragraphs describe the calculational sequence for the TRAC-PF1/MOD1 two-phase choking criterion. 
1. The solution of Eq. (D-7) requires that $p_{a}, p_{v}, \alpha, \rho_{a}, \rho_{v}, \rho_{\ell}, s_{a}$, $s_{v}, s_{\ell}$, and their derivatives be specified at the cell edge where the choking criterion is applied. However, these quantities are known only at the cell center. Direct use of the cell-center quantities yields erroneous results caused by the presence of steep gradients near the choking plane. Therefore, an estimate of the thermodynamic state at the cell edge is necessary. This estimate is obtained by our assuming a constant entropy process between the cell center and the cell edge and by our iterating for the cell-edge pressure to maximize the mass flux (a classical technique used in generating the HEM tables). In addition to the thermodynamic state at the cell edge, this technique also gives the homogeneous-equilibrium sound speed, $a_{H E}$, that is used as a first estimate for the largest characteristic root. (When the nonhomogeneous effects are not dominant, the desired root is close to the homogeneous-equilibrium sound speed.)

2. If a constant phase slip $\left(v_{g} / V_{\ell}\right)$ is maintained, Eq. (D-7) yields intermediate time-level values of $v_{g}^{n+1 / 2}$ and $v_{l}^{n+1 / 2}$, so that $\lambda_{i, r e, \max }=0$. The intermediate time-level mixture velocity, $v_{m}^{n+1 / 2}$, then is compared with the current value of the mixture velocity, $v_{m}$. If $v_{m}^{n+1 / 2}<v_{m}$, the flow is choked.

3. By our using $v_{g}$ and $v_{T}$ when the flow is unchoked or $v_{g}^{n+1 / 2}$ and $\mathrm{V}_{\ell}^{\mathrm{n}+1 / 2}$ when the flow is choked, the solution of the TRAC-PF1/MOD1 hydrodynamic equations yields new time-step values, $v_{g}^{n+1}$ and $v_{\ell}^{n+1}$.

B. Subcooled-Flow Choking Criterion

During the subcooled blowdown phase, the fluid changes phase at the break because the containment pressure is much lower than the saturation pressure corresponding to the system fluid temperature. The transition from single- to two-phase flow, which is accompanied by discontinuous change in the fluid bulk modules, leads to a large discontinuity in the sound speed at the break. The physical process occurring during the subcooled blowdown phase is described briefly in the following paragraphs. Reference 64 discusses this phenomenon in greater detall.

Figure D-1 shows a converging-diverging nozzle. The pressure downstream is such that the vapor just begins to flash at the throat. The flow upstream of the throat is subsonic. However, because the sound speed is discontinuous when the fluid becomes saturated and because the mass conservation dictates 


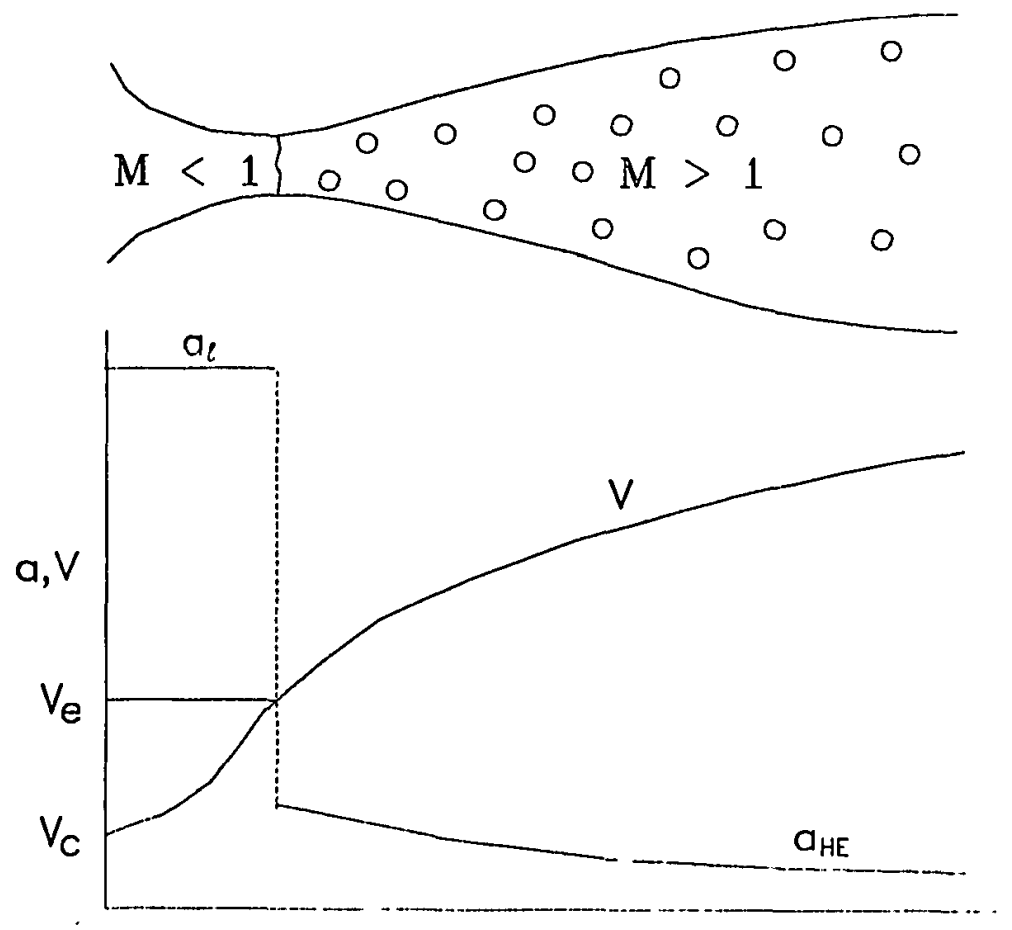

Fig. D-1.

Subcooled choking process when nucleation begins at the throat.

that the velocity just upstream of the throat must be equal to the velocity just downstream of the throat (where the fluid has only a miniscule void fraction), the flow is supersonic throughout the diverging section. Thus, the nozzle does not contain any point where the Mach number, $M$, equals one. Figure D-1 also shows the velocity profile and the sound speeds for this situation.

The velocity at the throat is calculated from Bernoulli's equation,

$v_{e}=\left[v_{c}^{2}+\frac{2\left(p_{c}-p_{e}\right)}{\rho_{m}}\right]^{1 / 2}$,

where subscripts $c$ and e refer to the cell-center (upstream) and the cell-edge (throat) conditions, respectively. The throat pressure, $\mathrm{P}_{e}$, is equal to the nucleation pressure, $P_{\text {nuc }}$, and can be considerably lower than the local 
saturation pressure, $P_{S}$, because thermal nonequilibrium causes nucleation delay.

Any further reduction in the downstream pressure does not affect the flow because the disturbance cannot move upstream where the flow is supersonic in the diverging section. Thus, Eq. (D-8) gives the throat velocity for a containment pressure lower than this pressure value (which is the case for most problems of interest in LWR applications). Next we consider the situation when the subcooled choked flow, as described above, exists initially, and the upstream pressure is lowered. As the upstream pressure decreases, the pressure at the throat remains equal to $p_{\text {nuc }}$, and again Eq. (D-8) can be used to calculate the throat velocity. However, the throat velocity decreases because the upstream pressure decreases. If the upstrean pressure is lowered further, a point is reached when $v_{e}$ equals $a_{H E}$. Any further reduction in the upstream pressure moves the point where $p$ equals $P_{\text {nuc }}$ upstream. In this case, the flow in the subcooled zone and the two-phase zone upstream of the throat is subsonic. The flow at the throat is sonic with $v_{e}$ equal to $a_{\mathrm{HE}}$; the flow in the diverging section is supersonic. If the upstream pressure is reduced further, the Pnuc point moves upstream until complete two-phase flow exists. Therefore, the maximum of the Burnell expression and the homogeneous-equilibrium sound speed give the subcooled choking criterion. Thus,

$v_{\ell}=\max \left\{a_{H E},\left[v_{c}^{2}+\frac{2\left(p_{c}-p_{e}\right)}{\rho_{m}}\right]^{1 / 2}\right\}$. 
We implemented a nucleation delay model, developed by Jones, 66 in TRAC-PFI/MODI to calculate the cell-edge pressure, $P_{e}$, from the saturation pressure, $P_{S}$,

$$
\begin{aligned}
P_{e} & =p_{s}-\max \left\{0.0,0.258 \frac{\sigma^{1.5}\left(\frac{T_{\ell}}{T_{c}}\right)^{13.76}\left[1+13.25\left(-\frac{1}{1.01325 \times 10^{11}} \frac{D p}{D t}\right)^{0.8}\right]^{0.5}}{\left(k T_{c}\right)^{0.5}\left(1-\frac{\rho_{g}}{\rho_{\ell}}\right)}\right. \\
& \left.-27(0.072)^{2}\left(\frac{A_{e}}{A_{c}}\right)^{2} \frac{\rho_{\ell} v_{e}^{2}}{2}\right\}
\end{aligned}
$$

where $\mathrm{D} / \mathrm{Dt}$ is the substantial derivative, $\mathrm{k}$ is the Boltzmann constant, $\mathrm{T}_{\mathrm{c}}$ is the critical temperature, and $\sigma$ is the surface tension.

The calculational sequence is similar to that for the two-phase model. The homogeneous-equilibrium sound speed is calculated by maximizing the mass flux, as described in Sec. D-II.A. The liquid-phase choking velocity, the only unknown quantity, is set explicitly by using Eq. (D-9), which gives $v_{\ell}^{n+1 / 2}$.

C. Single-Phase Vapor Choking Criterion

The single-phase vapor choking velocity is calculated by isentropically expanding the vapor to maximize the mass flux.

D. Transition Regimes

Because there is a discontinuity in the sound speed during the transition from liquid to two-phase flow, the flow during this transition regime must be analyzed carefully. In TRAC-PF1/MODl, this transition is handled by linear interpolation between the subcooled $(\alpha \leq 0.01)$ and the twophase $(\alpha \geq 0.1)$ regimes.

The transition from the two-phase to the vapor regime is smooth because the two-phase characteristic sulution approaches the homogeneous-equilibrium limit as $\alpha \rightarrow 1$. Thus, this transition is made by switching the calculational $\operatorname{logic}$ at $\alpha=0.999$. 
III. RESULTS

Section D-III.A compares the TRAC-calculated results with the test data from other conventional models under approximately homogeneous conditions to investigate the validity of the TRAC model under such conditions. Section D-III.B compares the TRAC-PF1/MOD1 choking calculations with the test data from models using fine mesh and from some separate-efects facilities.

A. Comparison of the TRAC-PF1/MODl Choked-Flow Model with Other Models

The primary requirement for an accurate choked-flow model is that it yield results that are close to the homogeneous-equilibrlum calculations when the flow approaches such a homogeneous limit, because the nonhomogeneous effects are only of secondary importance in most situations. Therefore, the homogeneous-equilibrium sound speed calculated by TRAC-PF1/MODl should agree with the true sound speed. Figure D-2 compares the TRAC-calculated homogeneous-equilibrium sound speed with that obtained from the tables of D. G. Hal1 67 for different void fractions at a 560-K saturation temperature. The agreement between the calculations and the tables is excellent.

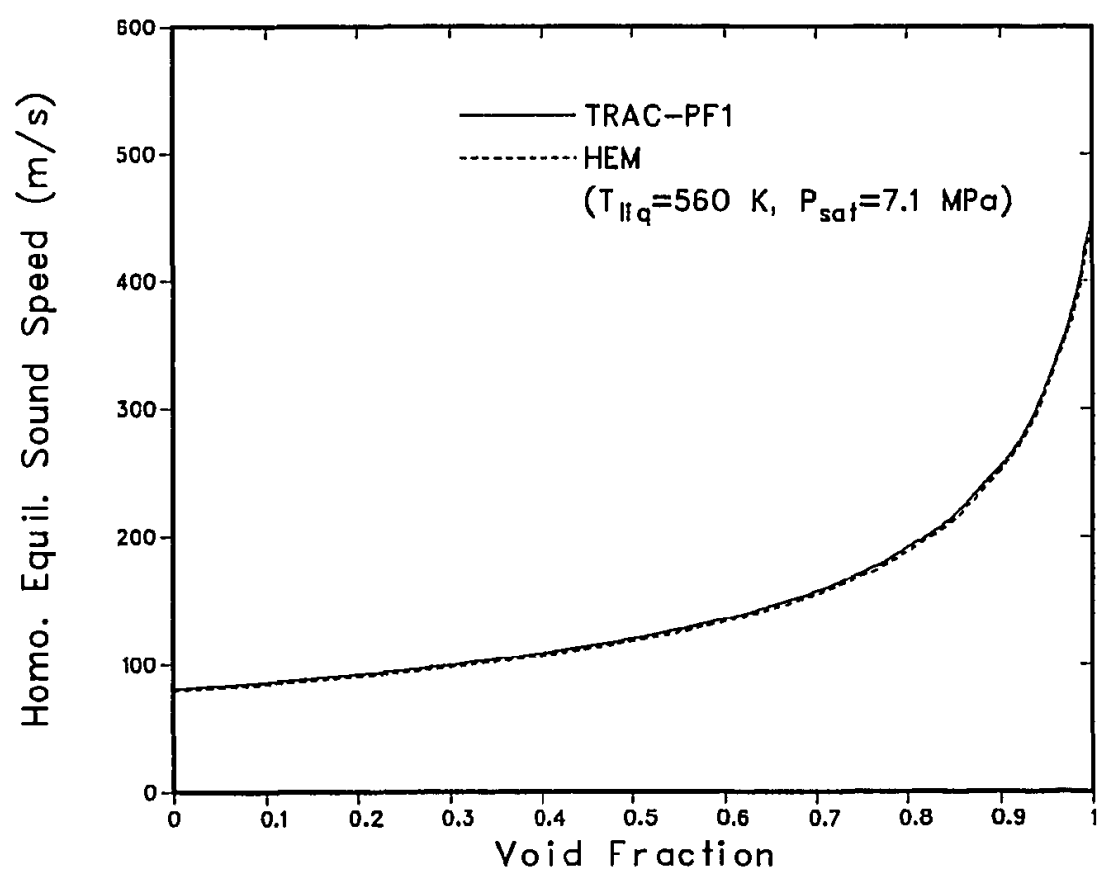

Fig. D-2.

Comparison of the TRAC-PFI/MOD1 two-phase homogeneous-equilibrium sound speed with that obtained from the HEM tables in Ref. 67. 
Figure D-3 comparas the TRAC-calculated subcooled critical flow with that from the Burne11: $\therefore$ del and from the HEM for stagnation pressures ranging from $7.1 \mathrm{MPa}$ (saturaté liquid) to $15.0 \mathrm{MPa}$ (subcooled liquid) at a constant 560-K temperature. The Burnell model is the modified-Burnell model from RELAP4/MOD6 (Ref. 68), that accounts for nucleation delay by an empirical expression. The HEM mass flux represents a lower limit on the mass flux. As desired, the TRAC-PF1/MOD1 model calculations give results that are similar to those for the Burnell model. The minor discrepancy between the TRAC-PF1/MODI choked-flow and the Burnell models is caused primarily by the difference between the nucleation-delay models.

Figure D-4 compares the TRAC-PF1/MOD1 two-phase critical-flow model calculations with the HEM data at 560-K saturation temperature. Again, the agreement is good. The TRAC-PF1/MODl calculated results differ from the HEM data because the nonhomogeneous effects are not accounted for in the HEM. Larger differences between the results obtained from the two models are expected when the upstream phasic velocities differ. (The flow upstream of the break was assumed to be stagnant for this calculation.)

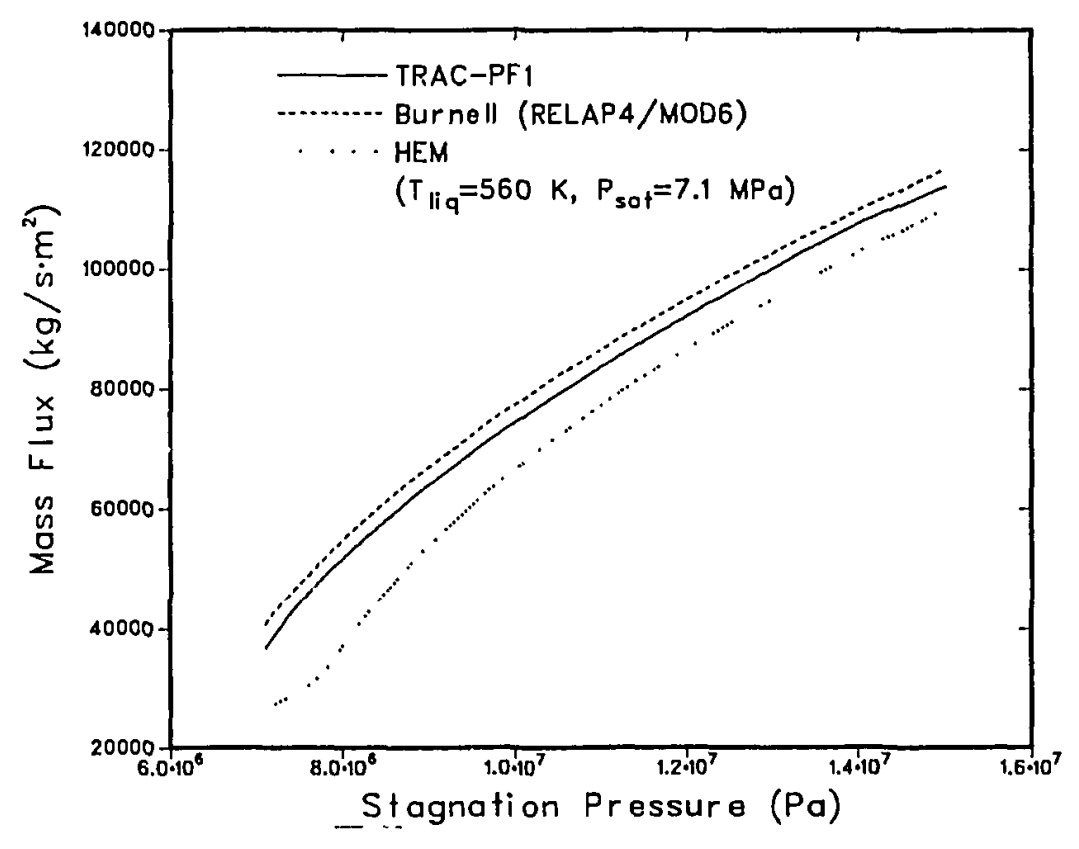

Fig. D-3.

Comparison of the subcooled critical mass fluxes. The TRAC-PFl/MODl calculations, the test data from the Burnell model, and the HEM tables in Ref. 67 were used. 


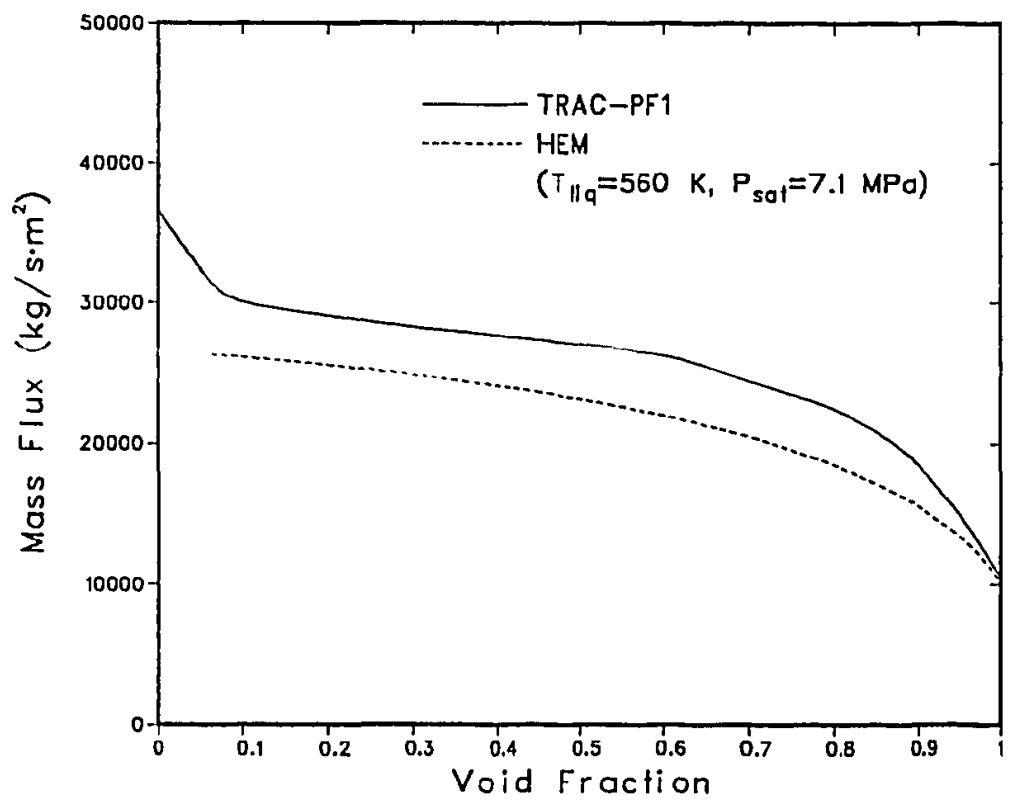

Fig. D-4 .

Comparison of the TRAC-calculated two-phase critical mass flux with the test data from the HEM tables in Ref. 67.

B. Comparison of the TRAC-PF1/MOD1 Choked-Flow Mode1 Calculations with the Fine-Mesh Calculations and the Test Data

A true test of the accuracy of a choking model is its ability to predict results similar to those obtained using an extremely fine mesh (natural choking) for geometries with smooth area changes. Therefore, the TRAC-PF1/MOD1 choking calculations are compared with the fine-mesh results and the test data from Tests 4 (Ref. 69) and 24 (Ref. 70) of the Marviken test factlity and the Edwards' blowdown experiment. 71

1. Marviken Test Facility. The Marviken full-scale critical-flow tests assess the ability of computer codes to predict large pressure-vessel blowdowns. The four major components of this facility area pressure vessel, originally designed to be part of the Marviken nuclear power plant; a discharge pipe; a test nozzle with the minimum flow area in the system; and a rupture-disk assembly. Figure D-5 shows the vessel that still includes part of the core superstructure and the moderator tank plus three gratings installed to eliminate vortex formation. Figure D-6 shows the other components. All elevations in both figures are measured relative to the 


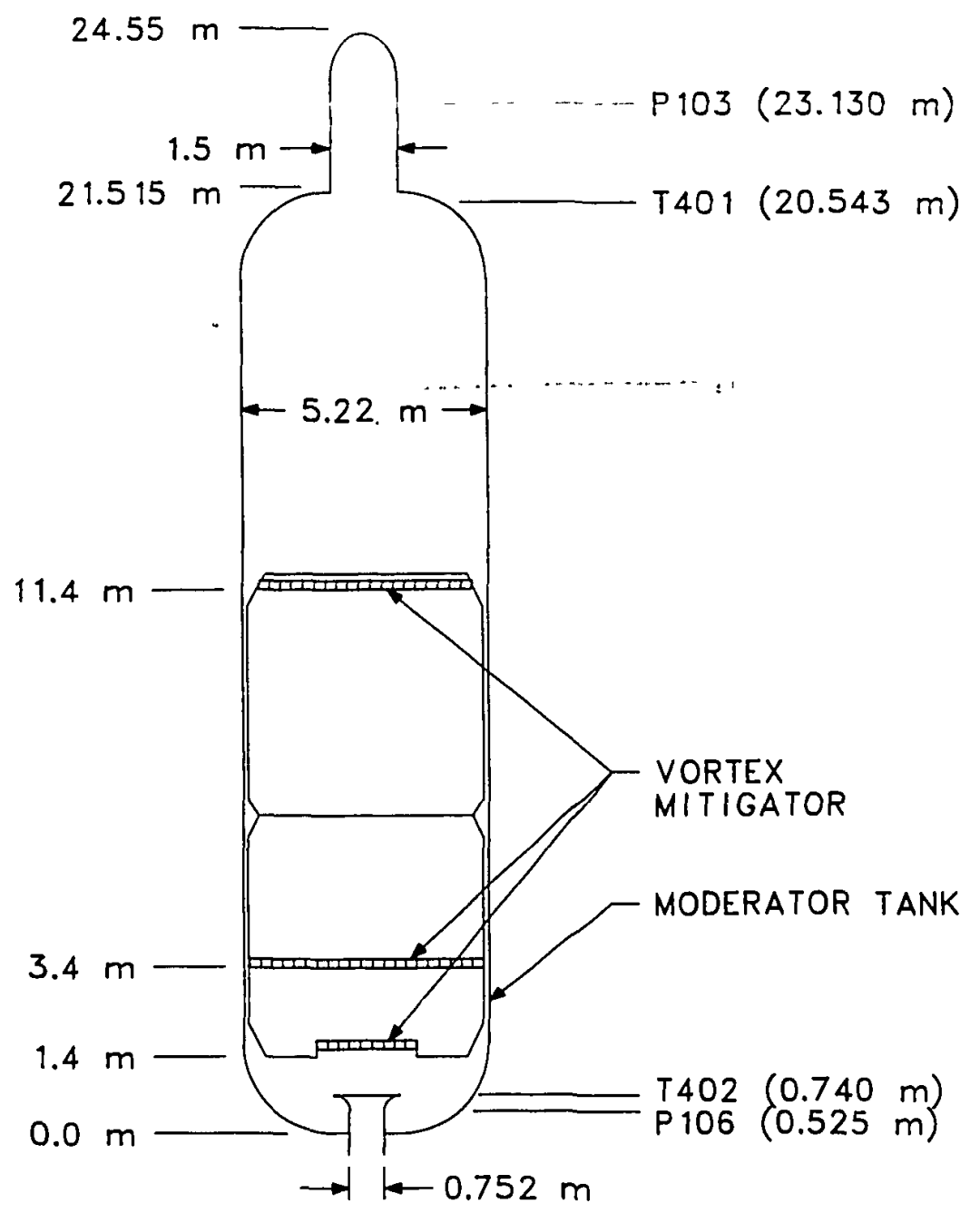

Fig. D-5.

Marviken pressure vessel. 


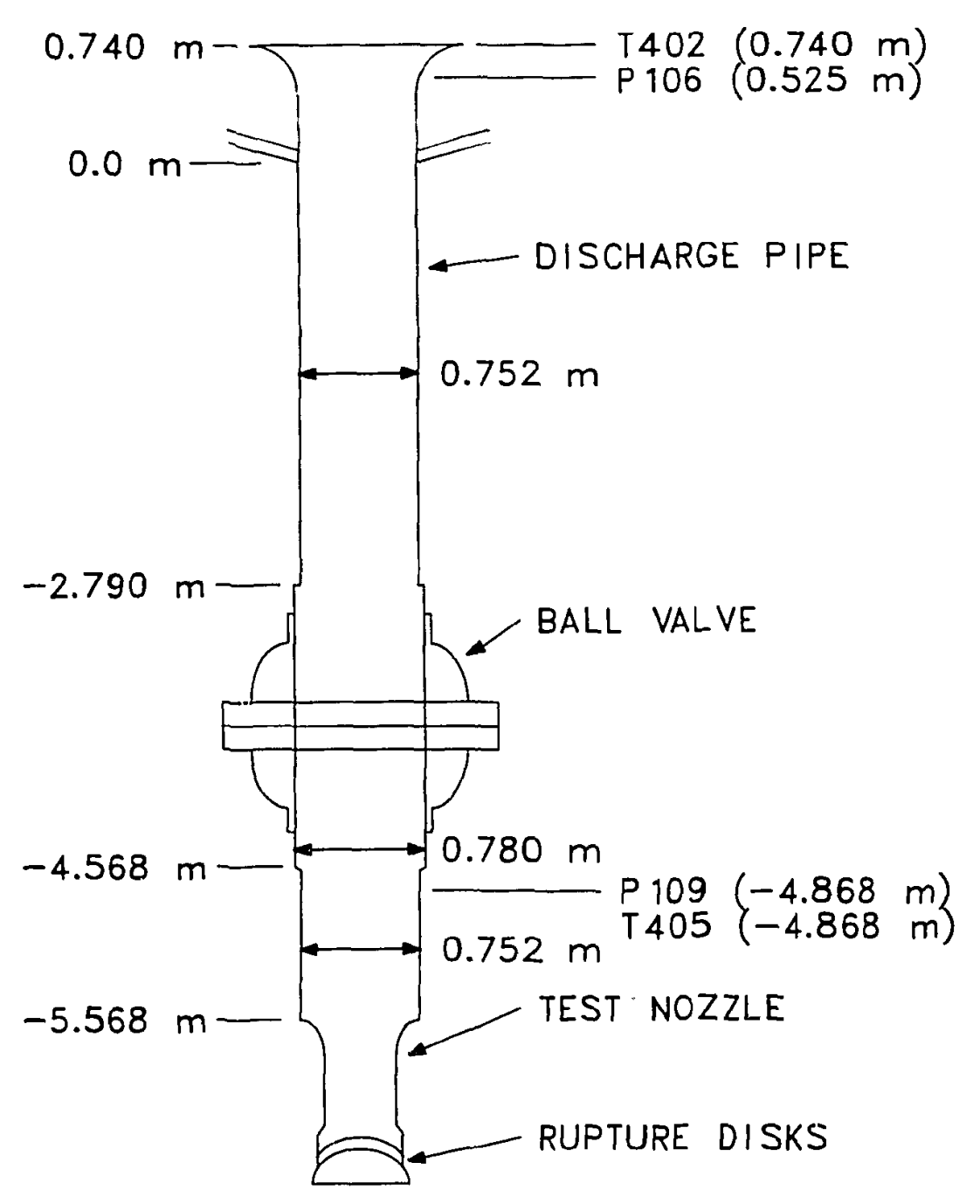

Fig. D-6.

Marviken discharge pipe, test nozzle, and rupture-disk assembly. 
vessel bottom. Pressure and temperature transducers are located along the vessel and the discharge pipe, as shown in Figs. D-5 and D-6. The signals from the various transducers are processed through a signal-conditioning unit with its channels connected to a pulse-code modulation system.

Before a test is run, the vessel is partially filled with deionized water and heated by removing water from the vessel bottom, passing it through an electric heater, and returning it to the steam dome at the vessel top. This procedure produces a complicated initial temperature distribution in the vessel. A saturated steam dome fills the vessel region above the initial water leve1. The test is initiated by releasing the rupture disks and is terminated by closing a ball valve in the discharge pipe. We specifically chose Marviken Tests 4 and 24 because Test 4 had the longest nozzle (and Test 24 , the shortest) in the entire test series.

The TRAC-PF1/MOD1 model for Marviken Tests 4 and 24 included four components. A zero-velocity FILL component modeled the vessel upper boundary. A PIPE component modeled the vessel above $2.6 \mathrm{~m}$, including the maximum diameter region plus the top cupola. Another PIPE component modeled the lower part of the vessel, the discharge pipe, the nozzle, and the rupture-disk assembly. A BREAK component provided a pressure boundary condition at the rupture-disk assembly lower boundary. For the fine-noding cases, the nozzles were modeled with 30 cells ( 15 in the converging section and 15 in the straight portion with a minimum cell length of $0.025 \mathrm{~m}$ ) for Test 4 and with 12 cells ( 5 in the converging section and 7 in the straight portion with a minimum cell length of $0.02 \mathrm{~m}$ ) for Test 24. When using the choked-flow model, the nozzles in both tests were modeled by only two cells, one in the converging section and the other simulating the entire straight section, with the choked-flow model invoked at the downstream edge of the second cell.

Figures D-7 and D-8 show the TRAC-PF1/MOD1 mass flows and pressure histories with the choking model and the fine noding compared with the test data. The measured mass flows are derived from velocity (pitot-static) and vessel differential-pressure measurements. The pitot-static data curve is valid throughout the transient, whereas the vessel differential-pressure curve is valid only after $5 \mathrm{~s}$. The choking calculations give almost identical results to those for the fine-mesh case. Both the choked-flow and the finemesh calculations also agree well with the te'st data except during the 


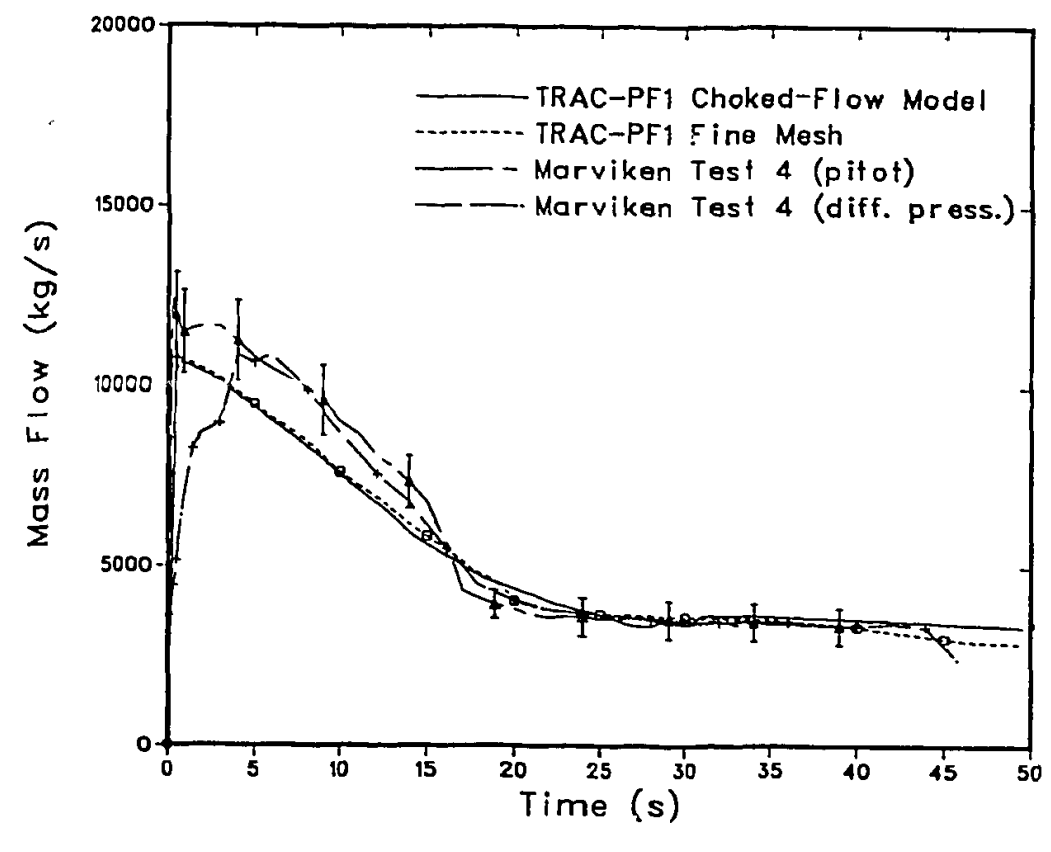

Fig. D-7.

Comparison of the nozzle mass flows for Marviken Test 4 between the test data and the TRAC-PFI/MODI models. The fine-mesh and choking criteria were used.

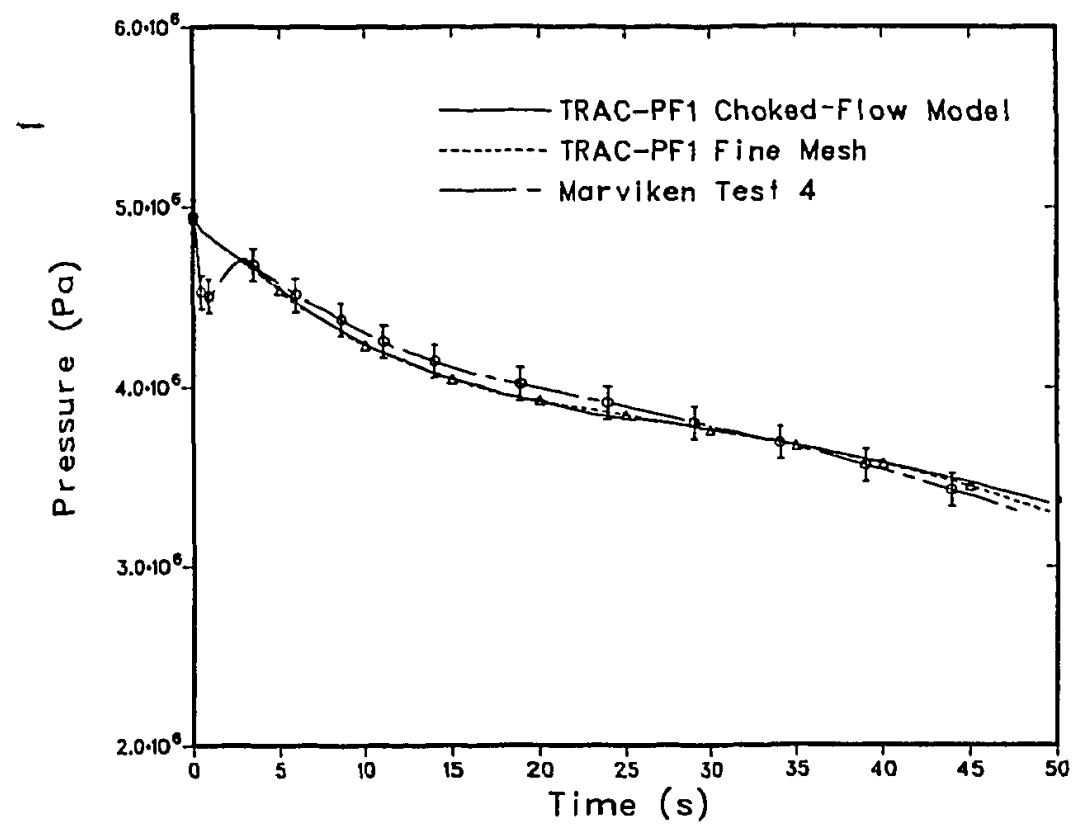

Fig. D-8.

Comparison of the system pressure histories for Marviken Test 4 between the test data and the TRAC-PF1/MOD1 models. The fine-mesh and choking criteria were used. 
subcooled blowdown phase when the nass flow is underpredicted by an average of $10 \%$. The dip in the measured pressure during the first $3 \mathrm{~s}$ of the transient indicates a significantly more pronounced nucleation delay than predicted by the TRAC-PF1/MOD1 model.

Figures $D-9$ and $D-10$ show the mass flows and pressure histories for Test 24. The agreement between the choking calculation and the results obtained from the fine-mesh case is not as good as for Test 4. This discrepancy is attributed to the predominance of nonequilibrium effects between the phases caused by the short nozzle length. These nonequilibrium effects are not modeled in the TRAC-PFI/MODI choking calculation. (The straight sections of the nozzles for Tests 4 and 24 , respectively, were 1.5 and $0.166 \mathrm{~m}$ long with L/D ratios of 2.95 and 0.33. )

To investigate the importance of nonequilibrium effects in Test 24 , we made a sensitivity run with the "frozen" assumption (using a seven-equation characteristic model without differential-type mass-transfer terms) in the characteristic solution. Figures $D-11$ and $D-12$ show the mass flows and the pressure histories for the new calculations. Using the choking model, we find the mass flow now is overpredicted and the pressure underpredicted compared with the mass flow and pressure from the fine-mesh results. The assumption that thermal equilibrium exists between the phases even in the absence of a noncondensable gas may not be valid in all situations.

2. Edwards ${ }^{-}$Blowdown Experiment. The Edwards' horizontal-pipe blowdown experiment studied depressurization phenomena of initially nonflowing subcooled water. The experimental apparatus consisted of a 4.096-m-long, straight steel pipe with a $0.073-\mathrm{m} 1 . \mathrm{d}$. The apparatus was designed for a maximum 17.24-MPa pressure at temperatures to $616.5 \mathrm{~K}$. The discharge end of the horizontal pipe was sealed with a $0.0127-$ m-thick glass disk.

The pipe was filled with demineralized water; a hydraulic pump and a control valve regulated the system pressure. The pipe was evacuated by a vacuum pump before it was filled with water. Before the glass disk was ruptured, the pipe was isolated from the supply tank to prevent the discharge of cold water into the pipe during blowdown. Pressure transducers were located at gauge stations GS-1 to GS-7, and a temperature transducer was located at GS-5 (Fig. D-13). Also provided at GS-5 were two aluminum alloy disk windows for transient void-fraction measurements, which used an $x$-ray 


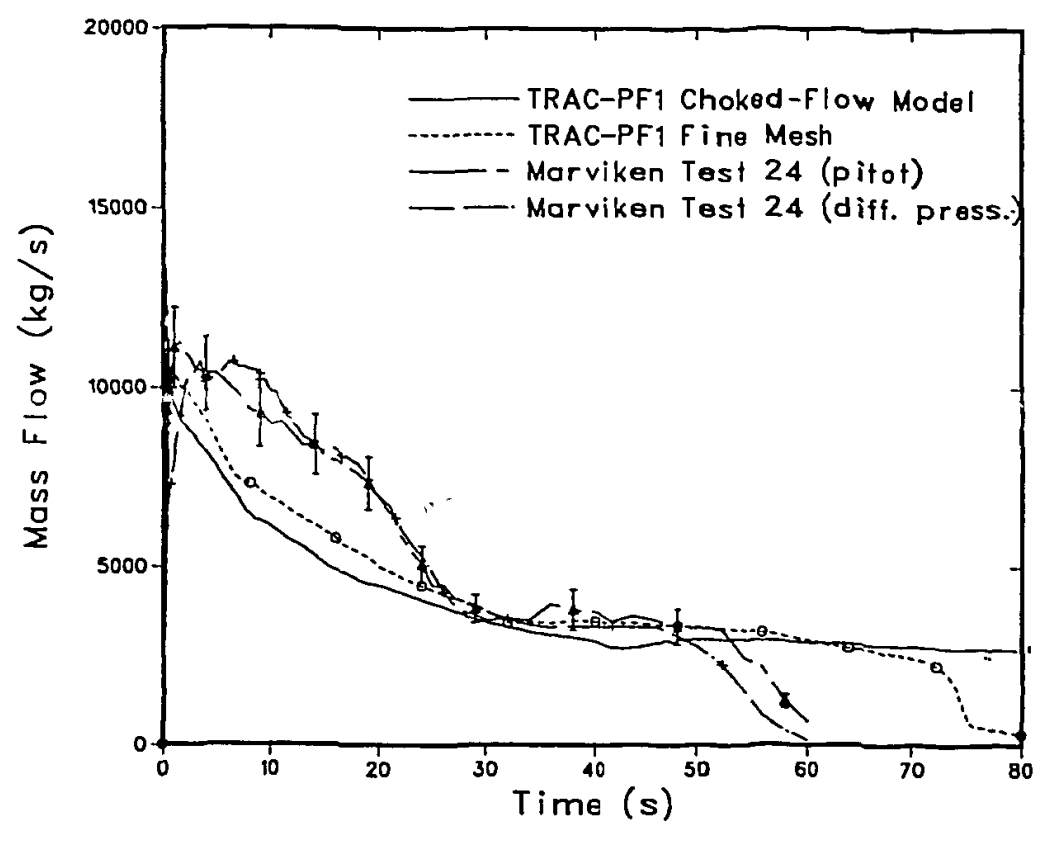

Fig. D-9.

is

Comparison of the nozzle mass flows for Marviken Test 24 between the test data and the TRAC-PF1/MOD1 models. The fine-mesh and choking criteria were used.

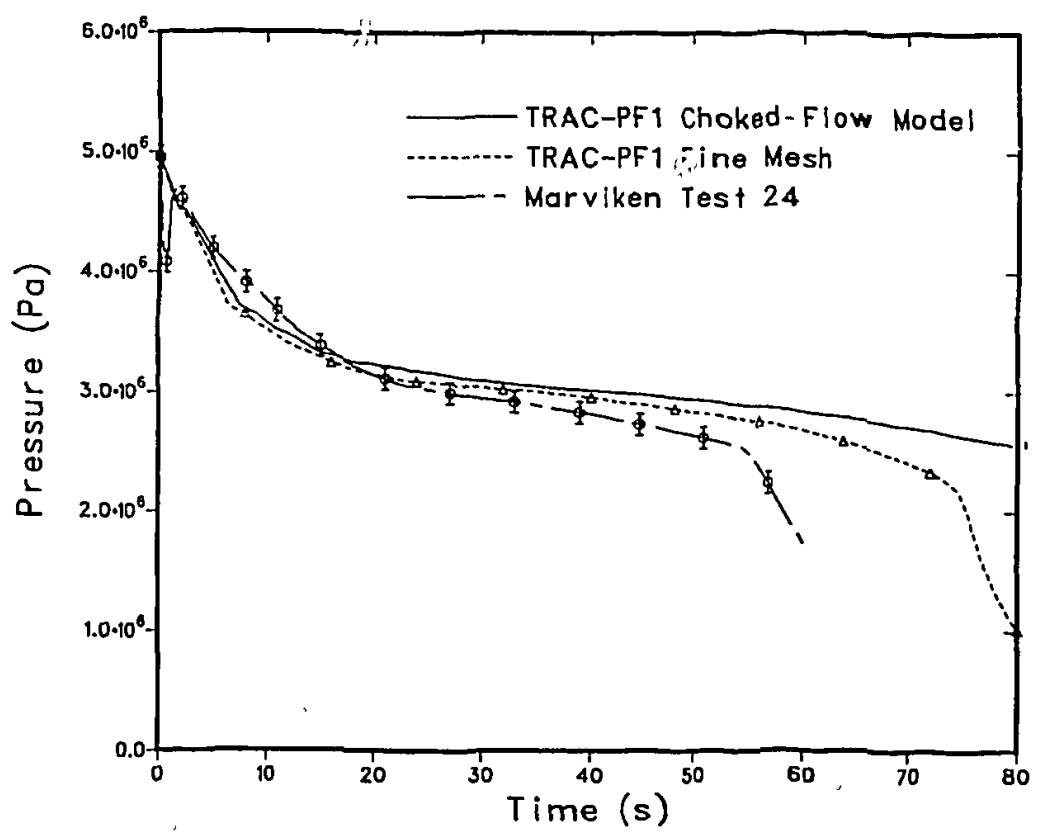

Fig. D-10.

Comparison of the system pressure historles for Marviken Test 24 between the test data and the TRAC-PFI/MODl models. The fine-mesh and choking criteria were used. 


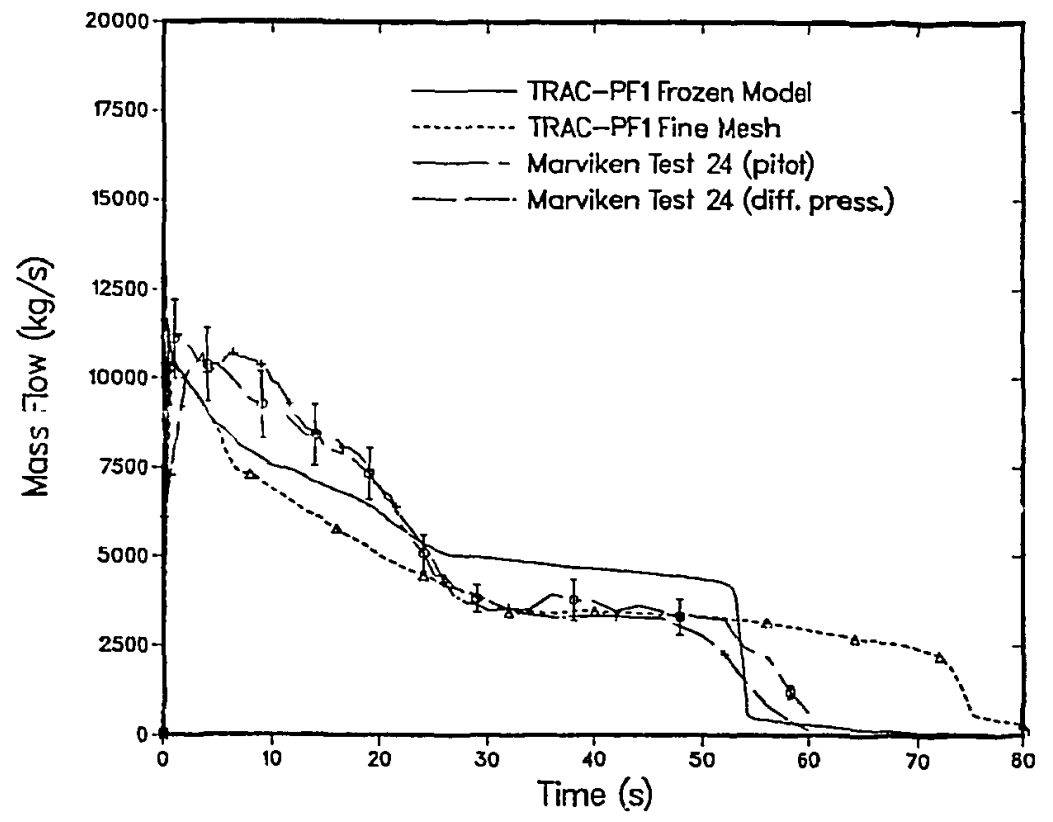

Fig. D-11.

Comparison of the nozzle mass flows for Marviken Test 24 betwee; the test data and the TRAC-PF1/MOD1 models. The fine-mesh and choking criteria obtained from the seven-equation frozen model were used.

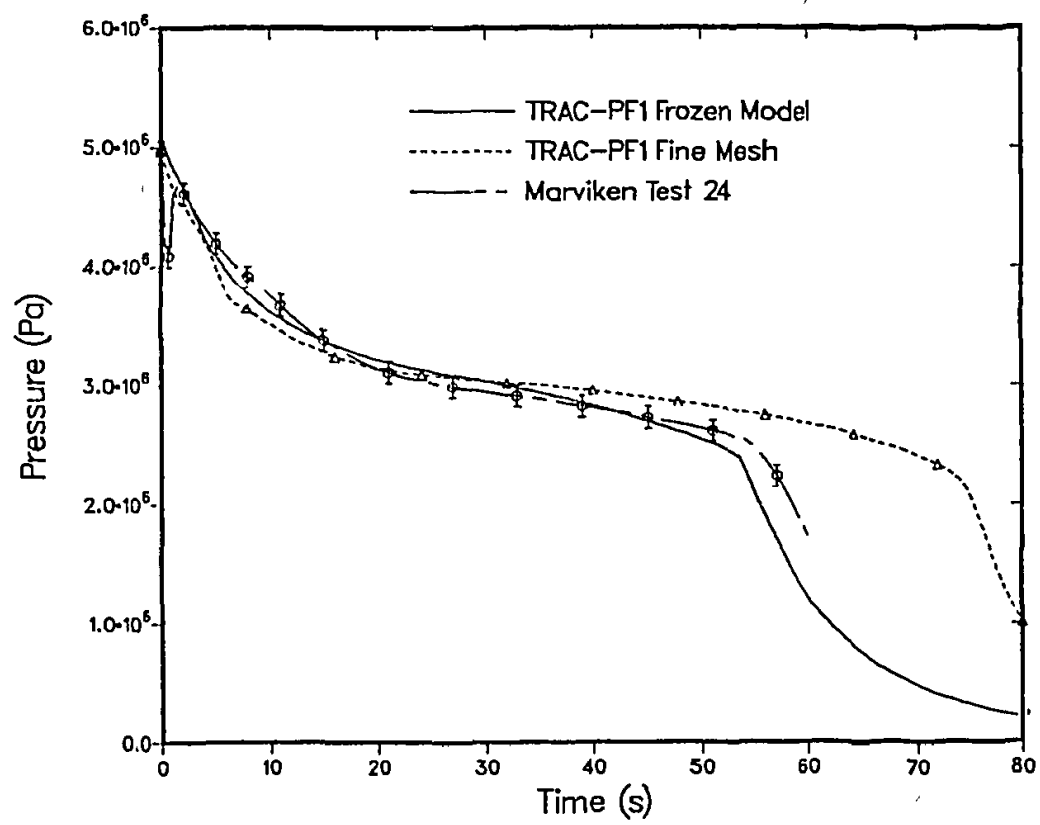

Fig. D-12.

Comparison of thesystem pressure histories for Marviken Test 24 between the test data and the TRAC-PF1/MOD1 models. The fine-mesh and choking criteria obtalned from the seven-equation frozen model were used. 


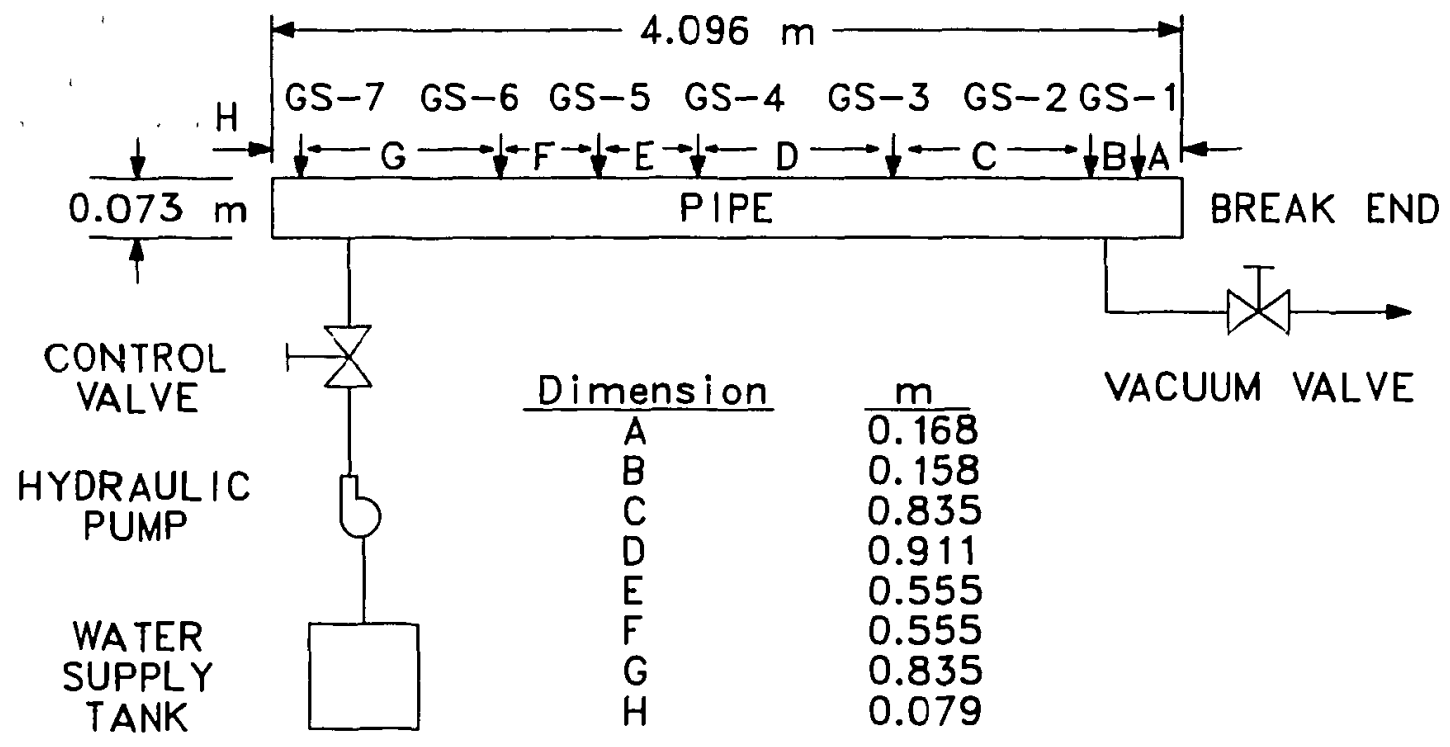

Fig. D-13.

Edwards' horizontal-pipe blowdown experiment (adapted from Ref. 71).

absorption system. The pipe was insulated and heated electrically. The operating procedure required that degassed water completely fill the pipe. The pipe was pressurized cold to $25 \%$ above the initial depressurization 7-MPa test pressure and checked for leaks. Next, the pressure was reduced to $3.45 \mathrm{MPa}$ and heat was applied gradually for $1.5 \mathrm{~h}$. During the heating of the water, the system pressure was maintained at $3.45 \mathrm{MPa}$ above the saturation pressure to prevent liquid flashing. The temperature variation along the pipe was limited by adjusting the voltage control for each heater. The system Initlally was brought to an approximately uniform 515-K temperature ${ }^{*}$ and 7-MPa pressure. Because the isolating valve between the pipe and storage tank was closed, the glass disk ruptured, and the dat were recorded automatically.

The TRAC-PF1/MOD1 model consisted of a zero-velocity FILL component to simulate the closed end of the pipe, two PIPE components coupled in series, and a BREAK component. Near the discharge end of the pipe, the minimum cell

\footnotetext{
* There is some uncertainty in the initial temperature profile. However, we used exactly identical initial conditions both in the choking and in the finemesh calculations to maintain the validity of the comparison between the two calculations.
} 
lengths were $0.00509 \mathrm{~m}$ for the fine-mesh case and $0.17325 \mathrm{~m}$ for the chokedflow model. The choking model was applied at the discharge end, which had the minimum cross-sectional area in the system. We had to estimate this area because fragments of glass remained intact during the experiment.

Figure D-14 compares the measured and calculated pressure histories near the middle of the pipe (GS-4). The agreement between the choking and the fine-mesh calculations again is good with the choking calculational results being closer to the data than the fine-mesh results. Although the discharge mass flow was not measured, the good pressure comparison between the calculations and the test data suggests good mass-flow calculations as well.

IV. CONCLUSIONS

The two-phase, two-component choked-flow model in TRAC-PF1/MOD1 was developed from first principles with a minimal amount of empiricism. The model assumes that thermal equilibrium exists between the phases in the presence or absence of an inert gas. The eigenvalues for the system of coupled differential equations are obtained numerically. This generality

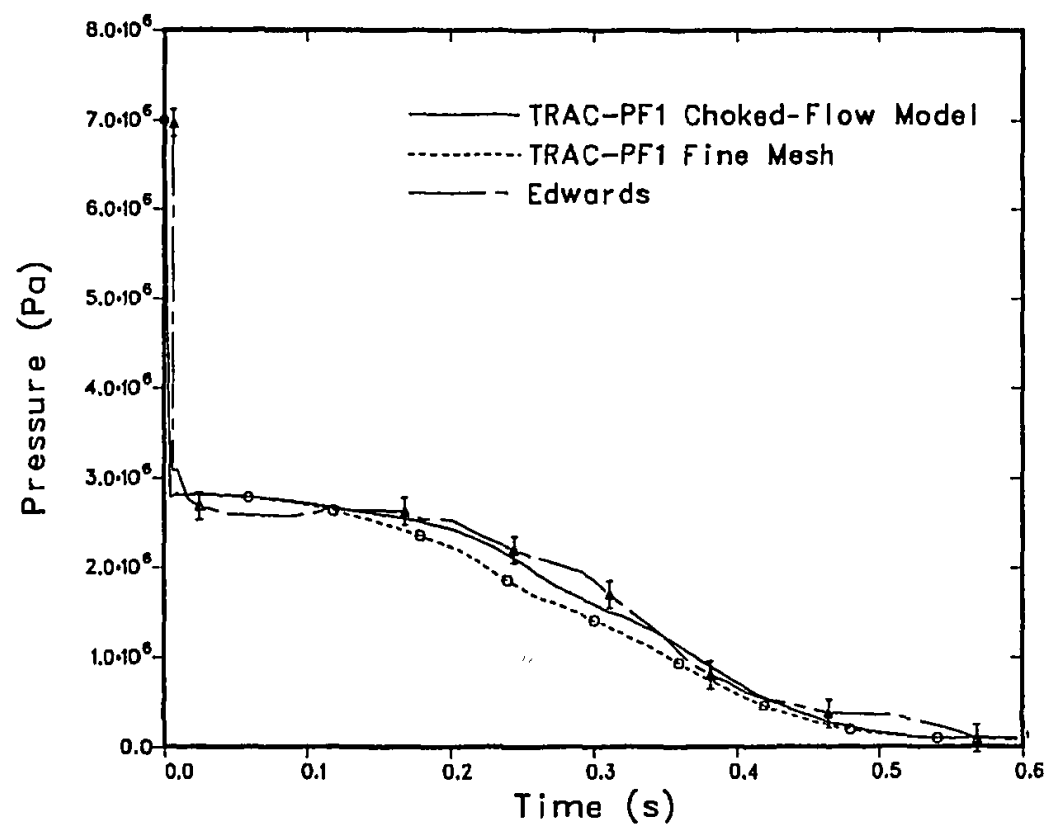

Fig. D-14.

Comparison of the system pressure histories for the Edwards' blowdown experiment between the test data and the TRAC-PF1/MOD1 calculations. The fine-mesh and choking criteria were used. 
gives the user the freedom to investigate and incorporate differential equations derived under different assumptions. The model yields results similar to those obtained using a fine mesh for components with smooth area changes. However, the quantitative agreement with the fine-mesh calculations is deficient for Marviken Test 24, which has a short nozzle, because the equilibrium assumption may be improper in that case. The results also compare well with other conventional models (the modified Burnell and the HEM). A good mass-flow comparison between the TRAC-PF1/MOD1 two-phase model and the HEM was obtained because the upstream fluid was stagnant, which gives mininal nonhomogeneous effects. However, for other two-phase situations, where the upstream liquid and vapor velocities differ significantly from each other, the nonhomogeneous effects may be very important. Comparisons of the TRAC-PF1/MOD1 calculations with the daca from the separate-effects Marviken tests and Edwards - blowdown experiment also were favorable. 


\section{APPENDIX E}

PWR SAMPLE PROBLEM

This PWR sample problem shows the setup of a large, complicated TRAC problem requiring extensive application of trips and control blocks. Anticipated transient experiment L6-1 (Ref. 72) conducted in the Loss-of-Fluid Test (LOFT) facility at Idaho National Engineering Laboratory was chosen to demonstrate the modeling techniques. Section E-I discusses the LOFT system briefly. Section E-II summarizes Test L6-1. Section E-III describes the TRAC one-dimensional model including the application of the powerful trip and control-block capability avallable in TRAC-PF1/MOD1. Section E-IV discusses the steady-state calculation. The transient calculation is delineated in Sec. E-V. Finally, Secs. E-VI.A and E-VI.B include listings of the steadystate and transient input decks, respectively. Section E-VI.C lists a threedimensional TRAC VESSEL input configuration that is equivalent to the onedimensional configuration used in Sec. E-VI.A.

\section{LOFT SYSTEM DESCRIPTION}

The LOFT facility is a 50-MW (thermal) PWR with instrumentation to measure and provide data on the thermal-hydraulic condition throughout the system. Operation of the LOFT system is typical of large [ 1000 MW (electric)] commerclal PWR operations. The facility consists of a reactor vessel with a nuclear core; an intact loop with an active steam generator, pressurizer, and two primary-coolant pumps (PCPs) connected in parallel; a broken loop with simulated pump, simulated steam generator, and two quickopening blowdown valve assemblies; a blowdown suppression system; and an emergency core-coolant (ECC) injection system that includes two low-pressure injection-system (LPIS) pumps, two high-pressure injection-system (HPIS) pumps, and two accumulators. An isometric drawing and a piping schematic of this system are shown in Figs. E-1 and E-2, respectively. Reference 72 contains additional details on the LOFT system. 


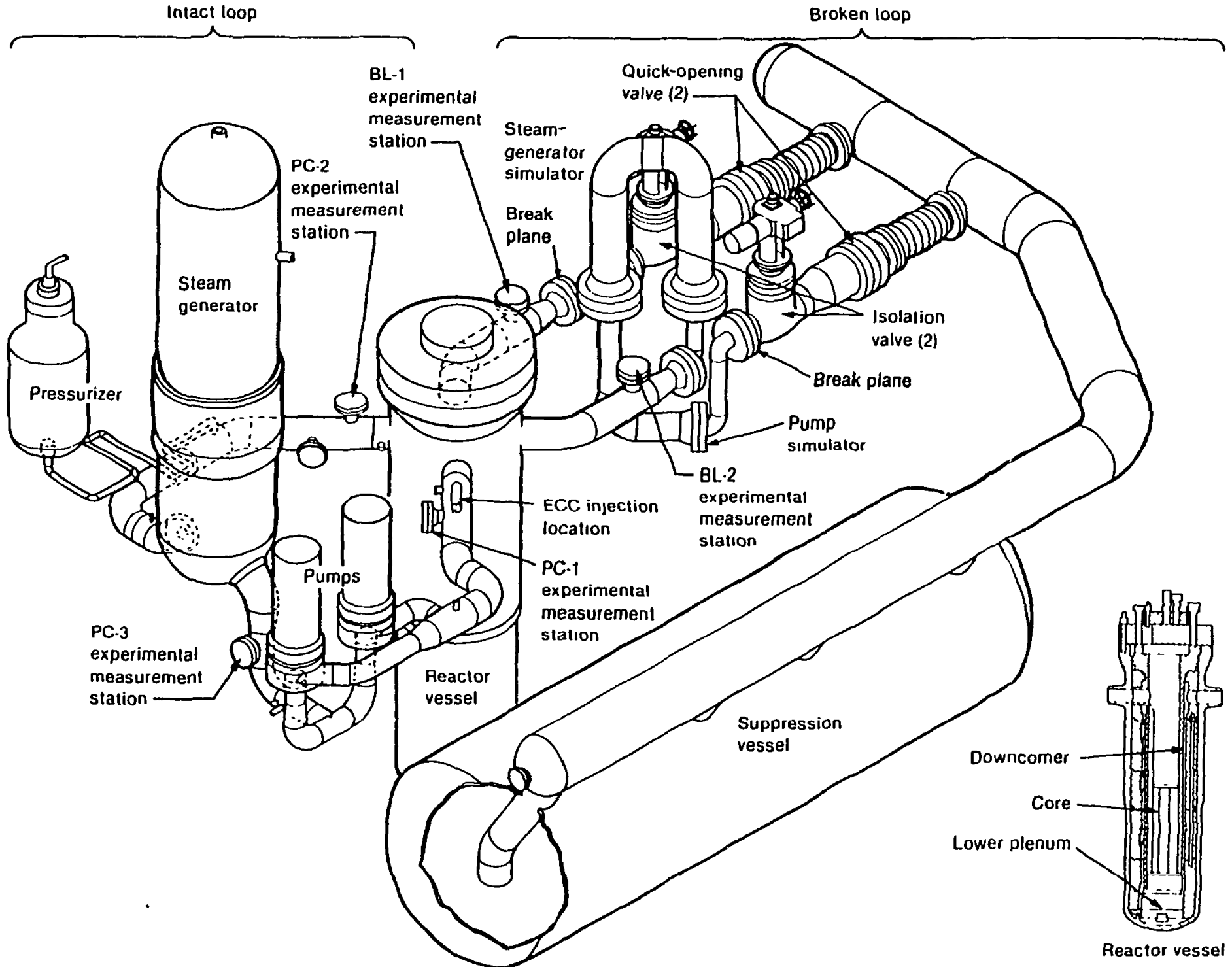

Fig. E-1.

LOFT facility configuration (Ref. 72). 


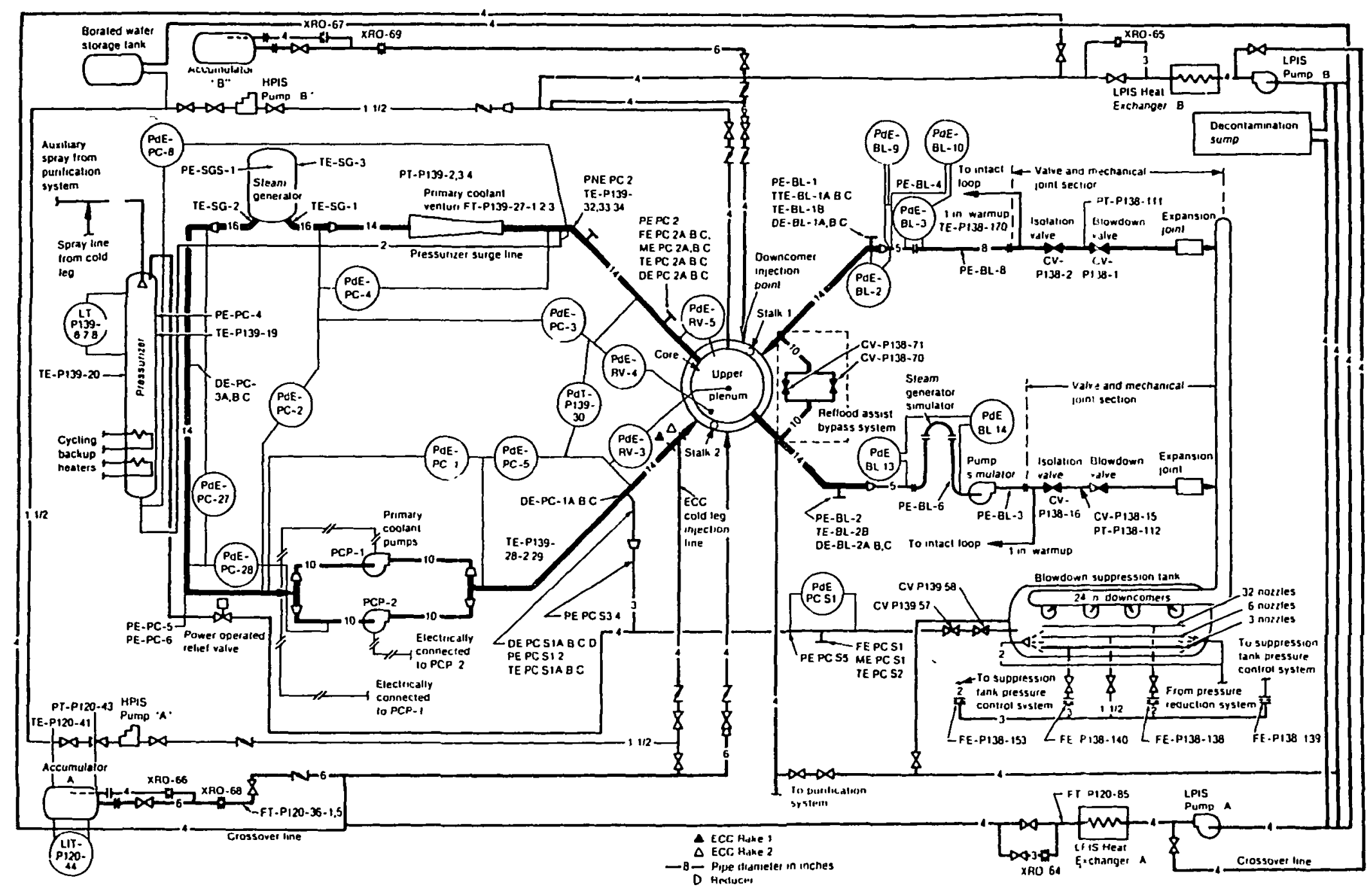

Fig. E-2.

LOFT piping schematic with instrumentation (Ref. 72). 
II.

TEST DESCRIPTION

Experiment L6-1 simulated a loss-of-steam load in a large PWR and was initiated by closing the main steam flow control valve (MSFCV). The pressurizer cycling heaters were on at experiment initiation but were shut off at $6.1 \mathrm{~s}$ as the primary-coolant system (PCS) pressure increased. The system pressure continued to rise because of impaired secondary-side heat removal capability. The pressurizer spray, which was initiated at $9.1 \mathrm{~s}$ to reduce PCS pressure, was continued until $30.4 \mathrm{~s}$. The PCS pressure continued to rise and caused the reactor to scram at $21.8 \mathrm{~s}$. Immediately after the reactor scram, the primary system started to depressurize. The MSFCV, which automatically began opening at $22.2 \mathrm{~s}$, reduced steam-generator secondary-side pressure and was closed at $40.6 \mathrm{~s}$. Low system pressure caused the pressurizer backup heaters to come on at $32.5 \mathrm{~s}$. The MSFCV again opened and closed automatically at $91.2 \mathrm{~s}$ and $104.4 \mathrm{~s}$, respectively, and was manually opened and closed starting at $312.6 \mathrm{~s}$. The pressurizer backup heaters turned off at $415.4 \mathrm{~s}$. The experiment was terminated at $700 \mathrm{~s}$.

The blowdown suppression system was not used in this experiment. We connected the broken loop to the intact loop through one-inch warm-up lines to prevent broken-loop stagnation.

III. TRAC ONE-DIMENSIONAL MODEL

Figures E-3,E-4, and E-5 show the loop, the steam generator, and the vessel arrangement, respectively. Figure E-6 shows an equivalent vessel configuration that uses the TRAC three-dimensional VESSEL component. An input listing for this three-dimensional model is given in Sec. E-VI.C. The junction and component numbers are shown in squares and circles, respectively. These junctions and component numbers can be used as guides when referring to the input listings in Sec. E-VI. The one-dimensional input model, which was used for the L6-1 calculation, consists of 39 components containing a total of 144 cells and 48 junctions. Table E-I lists the components. The input model corresponds to the LOFT hardware configuration with the following exceptions:

1. The pressure suppression system is not modeled because it was not used in the experiment.

2. The HPIS is represented by a FILL (component 17). The remaining ECC system is omitted from the TRAC model because there were no accumulator or LPIS injections in the L6 series of experiments. 

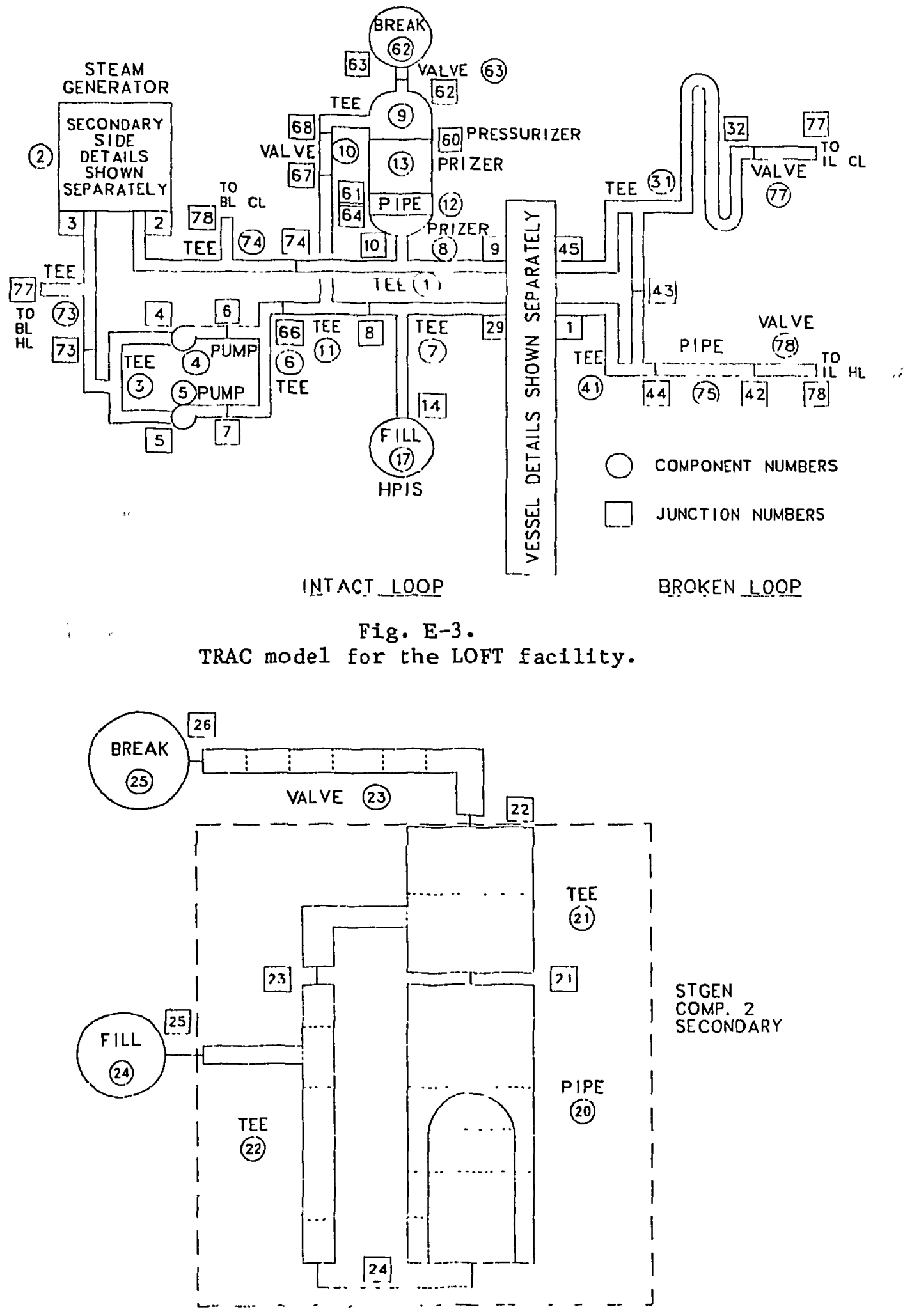

Fig. E-4.

"Steam-generator secondary-side noding for the LOFT facility. 

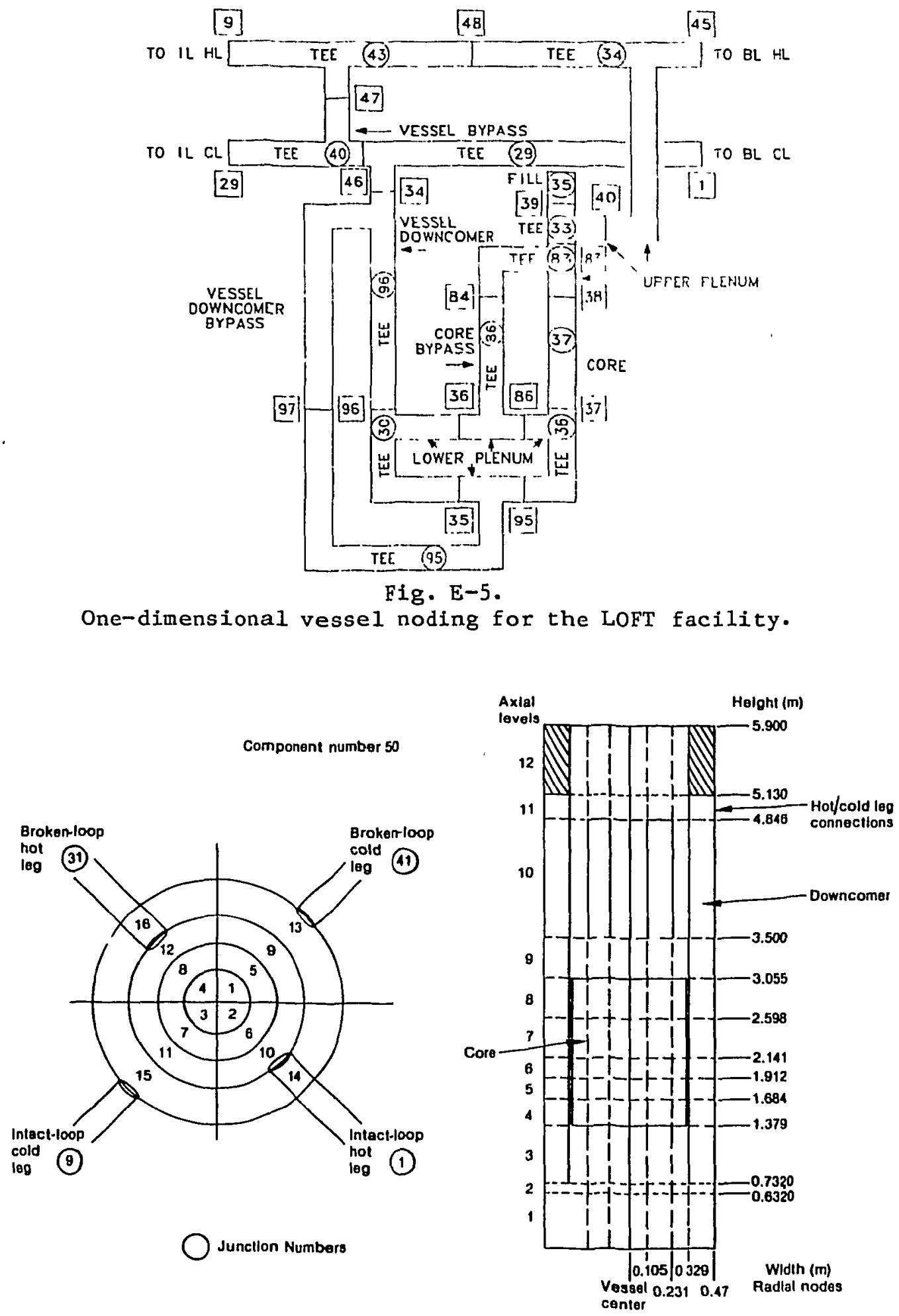

Three-dimensional vessel noding combining Fig. E-5.

Fig. E-6. combining all the components shown in 
TABLE E-I

TRAC MODEL COMPONENTS

\begin{tabular}{|c|c|c|c|c|}
\hline $\begin{array}{c}\text { Component } \\
\text { Number }{ }^{a} \\
\end{array}$ & $\begin{array}{l}\text { Component } \\
\text { Type } \\
\end{array}$ & Description & $\begin{array}{l}\text { Junction } \\
\text { Numbers } \\
\end{array}$ & $\begin{array}{c}\text { Number of } \\
\text { Fluid Cells } \\
\text { (Primary Side, } \\
\text { Secondary Side) } \\
\end{array}$ \\
\hline 1 & TEE & Intact-1oop hot leg & $9,74,10$ & 2,3 \\
\hline 2 & STGEN & Steam generator & $2,3,25,22$ & 10,13 \\
\hline 20 & PIPE & $\begin{array}{l}\text { Steam-generator } \\
\text { secondary-side riser }\end{array}$ & 24,21 & 5 \\
\hline 22 & TEE & $\begin{array}{l}\text { Steam-generator } \\
\text { secondary-side downcomer }\end{array}$ & $23,24,25$ & 4,1 \\
\hline 21 & TEE & $\begin{array}{l}\text { Steam-generator } \\
\text { secondary-side steam dowel }\end{array}$ & $21,22,23$ & 2,1 \\
\hline 3 & TEE & Pump suction & $4,5,73$ & 3,1 \\
\hline 4 & PUMP & Pump 2 & 4,6 & 2 \\
\hline 5 & PUMP & Pump 1 & 5,7 & 2 \\
\hline 6 & TEE & Pump discharge & $7,66,6$ & 2,1 \\
\hline 7 & TEE & Intact-loop cold leg & $8,29,14$ & 3,1 \\
\hline 8 & PRIZER & Pressurizer bottom section & 64,10 & 1 \\
\hline 9 & TEE & $\begin{array}{l}\text { Pressurizer top section } \\
\text { and spray }\end{array}$ & $60,62,68$ & 5,1 \\
\hline 10 & VALVE & $\begin{array}{l}\text { Pressurizer spray } \\
\text { control valve }\end{array}$ & 67,68 & 3 \\
\hline 11 & TEE & $\begin{array}{l}\text { Intact-loop cold leg and } \\
\text { pressurizer spray source }\end{array}$ & $66,8,67$ & 1,1 \\
\hline 12 & PIPE & $\begin{array}{l}\text { Pressurizer lower middle } \\
\text { section }\end{array}$ & 61,64 & 1 \\
\hline 13 & PRIZER & $\begin{array}{l}\text { Pressurizer upper } \\
\text { middle section }\end{array}$ & 60,61 & 6 \\
\hline 17 & F ILL & HPIS & 14 & 1 \\
\hline
\end{tabular}


TABLE E-I (cont.)

\begin{tabular}{|c|c|c|c|c|}
\hline $\begin{array}{l}\text { Component } \\
\text { Number } \\
\end{array}$ & $\begin{array}{l}\text { Component } \\
\text { Type } \\
\end{array}$ & Description & $\begin{array}{l}\text { Junctiof } \\
\text { Numbers }\end{array}$ & $\begin{array}{l}\text { (Primary Side, } \\
\text { Secondary Side) }\end{array}$ \\
\hline 23 & VALVE & Steam-line valve & 22,26 & 6 \\
\hline 24 & FILL & $\begin{array}{l}\text { steam-generator } \\
\text { secondary side }\end{array}$ & 25 & 1 \\
\hline 25 & BREAK & $\begin{array}{l}\text { Steam-generator secondary- } \\
\text { side pressure set point }\end{array}$ & 26 & 1 \\
\hline 29 & TEE & $\begin{array}{l}\text { Vessel inlet annulus and } \\
\text { broken-loop cold-leg } \\
\text { connection }\end{array}$ & $46,1,34$ & 1,1 \\
\hline 30 & TEE & Vessel lower plenum & $96,35,36$ & 2,1 \\
\hline 31 & TEE & Broken-loop hot leg & $45,32,43$ & 6,3 \\
\hline 33 & TEE & Vessel upper plenum & $83,39,40$ & 2,1 \\
\hline 34 & TEE & $\begin{array}{l}\text { Vessel upper plenum and } \\
\text { broken-loop hot-leg } \\
\text { connection }\end{array}$ & $48,45,40$ & 1,1 \\
\hline 35 & FILL & $\begin{array}{l}\text { Vessel upper-head } \\
\text { zero-velocity fill }\end{array}$ & 39 & 1 \\
\hline 36 & TEE & Vessel lower plenum & $95,37,86$ & 2,1 \\
\hline 37 & CORE & Nuclear reactor core & 37,38 & 4 \\
\hline 40 & TEE & $\begin{array}{l}\text { Vessel bypass and } \\
\text { intact-1oop cold-leg } \\
\text { connection }\end{array}$ & $29,46,47$ & 1,1 \\
\hline 41 & TEE & Broken-loop cold leg & $1,44,43$ & 2,2 \\
\hline 43 & TEE & $\begin{array}{l}\text { Vessel upper plenum and } \\
\text { intact-loop hot-leg } \\
\text { connection }\end{array}$ & $48,9,47$ & 1,1 \\
\hline 62 & BREAK & $\begin{array}{l}\text { Pressure-operated relief } \\
\text { valve pressure set point }\end{array}$ & 63 & 1 \\
\hline 63 & VALVE & $\begin{array}{l}\text { Pressure-operated } \\
\text { relief valve }\end{array}$ & 62,63 & 2 \\
\hline
\end{tabular}


TABLE E-I (cont.)

\begin{tabular}{|c|c|c|c|c|}
\hline \multirow[b]{2}{*}{$\begin{array}{c}\text { Component } \\
\text { Number }\end{array}$} & \multirow[b]{2}{*}{$\begin{array}{c}\text { Component } \\
\text { Type } \\
\end{array}$} & \multirow[b]{2}{*}{ Description } & \multirow[b]{2}{*}{$\begin{array}{l}\text { Junction } \\
\text { Numbers }\end{array}$} & $\begin{array}{l}\text { Number of } \\
\text { Fluid Cells }\end{array}$ \\
\hline & & & & $\begin{array}{r}\text { (Primary Side, } \\
\text { Secondary Side) } \\
\end{array}$ \\
\hline 73 & TEE & Pump suction & $73,3,77$ & 3,2 \\
\hline 74 & TEE & Intact-loop hot leg & $74,2,78$ & 2,2 \\
\hline 75 & PIPE & $\begin{array}{l}\text { Broken-loop cold-leg } \\
\text { outlet }\end{array}$ & 44,42 & 1 \\
\hline 77 & VALVE & $\begin{array}{l}\text { Broken-loop hot-leg } \\
\text { warm-up recirculation line }\end{array}$ & 77,32 & 2 \\
\hline 78 & VALVE & $\begin{array}{l}\text { Broken-loop cold-leg } \\
\text { warm-up recirculation line }\end{array}$ & 78,42 & 2 \\
\hline 83 & TEE & $\begin{array}{l}\text { Vessel upper plenum } \\
\text { and core bypass }\end{array}$ & $38,83,84$ & 2,2 \\
\hline 86 & TEE & $\begin{array}{l}\text { Vessel lower plenum and } \\
\text { core bypass }\end{array}$ & $86,36,84$ & 1,1 \\
\hline 95 & TEE & $\begin{array}{l}\text { Vessel lower plenum and } \\
\text { downcomer bypass }\end{array}$ & $35,95,97$ & 1,2 \\
\hline 96 & TEE & $\begin{array}{l}\text { Vessel downcomer and } \\
\text { downcomer bypass }\end{array}$ & $34,96,97$ & 6,6 \\
\hline
\end{tabular}

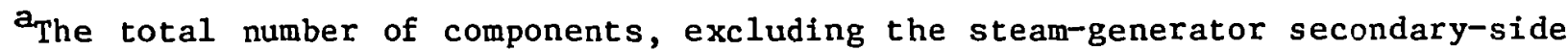
components, is 39 .

The total number of computational cells is 144 .

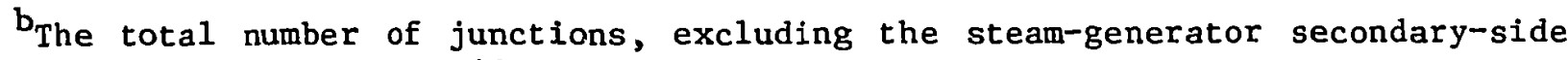
internal junctions, is 48 . 
It should be noted from Fig. E-3 that instead of modeling the pressurizer with a single PRIZER module, we modeled the top part of the pressurizer with a TEE, and the rest with two PRIZER modules with a PIPE in the middle. The TEE at the top was necessary to allow two connections, one for the spray and the other for the power-operated relief valve (PORV). (Note: The pressurizer spray option available in TRAC coes not simulate the true spray system. It is only a means of controlling the system pressurc. Therefore, for most practical problems of interest, the spray should be modeled by modeling the system hardware configuration accurately.) The pipe near the bottom was necessary for us to model the pressurizer heaters accurately, by utilizing the option for direct power into the coolant available in a PIPE module. [There were three problems in using the heater option available in the PRIZER module: (1) the pressurizer heater option does not control the heaters in the manner they were operated in the experiment; (2) the LOFT system has two banks of heaters, which could not be simulated using the pressurizer heater option; and (3) the pressurizer heater option would have equally distributed the energy everywhere in the liquid, whereas the energy is actually added only to the liquid near the bottom of the pressurizer.] More discussion on how the heaters are simulated exactly using trip-controlled direct power into the coolant will follow in this section.

Reference 73 reports that the LOFT spray valve (VALVE 10) has a leak area of $\sim 2.5 \%$ of its full-open value. The LOFT pressurizer is, therefore, never in a true steady-state condition because the heater and spray flow contributions do not exactly balance. To insure a steady state that is consistent with the experimental conditions at the start of the transient, we have added trip-controlled direct power into the coolant to the side leg of TEE 9 during the steady-state calculation to bring this cold spray liquid to saturation. This small heat contribution is terminated automatically at the start of the transient because the controlling trip goes of $f$. Therefore, this heater has no effect on the anticipated transient portion of the analysis.

The structural heat losses in the LOFT facility were reported by ollikkala ${ }^{74}$ as follows. A $6-\mathrm{kW}$ heat loss from the pressurizer, a 10-kW heat loss from the steam-generator secondary side, and a total of 174-kW heat loss from the primary system (excluding the pressurizer). These heat losses are incorporated in the TRAC model. With our assumption that the surrounding air 
temperature was $305 \mathrm{~K}$, we calculated average film coefficients based on outside surface areas of the pressurizer, the steam-generator secondary side, and the primary system.

The reactor power is modeled using point kinetics with reactivity feedback. The reactor kinetics parameters were determined from the experimental core power history during steady state.

All the automatic controls are modeled using the extensive trip and control capability available in TRAC-PF1/MODI, and they correspond to the actual system hardware configuration. A total of 24 signal variables, 21 trips, and 29 control blocks are required to model the entire LOFT control system. The trip set points needed for the modeling of the controls are listed in Table E-II. The sources of information for these set points include the best-estimate prediction, ${ }^{73}$ the quick-10ok report, ${ }^{75}$ the experiment data report, ${ }^{72}$ and the posttest analysis report. ${ }^{76}$ We resolved some of the discrepancies apparent in these sources among the set points by determining the actual test conditions from the experiment data report ${ }^{72}$ at times when the events actually took place. The important controls are described below. Refer to the input listing in Sec. E-VI.A and the TRAC input instructions, to follow these controls in detail.

A. Pressurizer Heaters

As discussed earlier in this section, the heaters are simulated by adding direct power into the coolant in PIPE 12. These heaters are easily controlled using only four trips. No control blocks are required. The two kinds of heaters in the LOFT facility are the $36-\mathrm{kW}$ cycling heaters that are first to come on at low-pressure signal and last to go off on high-pressure signal, and the 12-kW backup heaters that supplement the cycling heaters if their power is insufficient to maintain the system pressure. The cycling and backup heaters are controlled by trips 13 and 14, respectively. These trips, having positive IDs, are not evaluated during the steady state. However, a trip set status, ISET, of 1 is input for trip 13, which sets the trip to ONforward during steady state. The reason for doing this will soon be obvious.

The PIPE module allows only one power table to be input. One obvious approach to model the two banks of heaters would have been to split PIPE 12 into two separate PIPE components and input the two heater powers separately. 
MAJOR-TRIP SET POINTS FOR TEST L6-1

\section{Parameter}

Systom low-pressure scram (MPa)

Syctem high-pressure scram (MPa)

Intact-loop hot-leg high-temperature $\operatorname{scram}(\mathrm{K})$

Low primary-coolant mass flow ( $\mathrm{kg} / \mathrm{s})$

High core-averaged power scram (MW)

Steam-generator secondary-side low liquid-level scram (M)

Power-operated relief valve-opening set point (MPa)

Power-operated relief valve-closing set point (MPa)

Pressurizer spray on

Pressurizer spray off

Pressurizer cycling heaters on ( $M P a)$

Pressurizer cycling heaters of $f(M P a)$

Pressurizer backup heaters on ( $M P a)$

Pressurizer backup heaters off ( $\mathrm{MPa}$ )

HPIS on ( $\mathrm{MPa}$ )

HPIS off (MPa)

MSFCV opening set point before reactor scram (MPa)

MSFCV closing set point before reactor scram (MPa)

MSFCV opening set point after reactor scram

between 0 and $75 \mathrm{~s}$ (MPa)

6.99

between 75 and $200 \mathrm{~s}$ (MPa)

between $200 \mathrm{~s}$ and end of transient (MPa)

Set Point

14.36

15.77

583.3

433.5

51.5

2.0

16.70

16.56

15.24

14.90

14.75

14.93

14.62

14.80

13.297

15.500

5.425

5.315

6.9764

6.9464

MSFCV closing set point after reactor scram

between 0 and $75 \mathrm{~s}$ ( $\mathrm{MPa}$ )

6.7500

between 75 and $200 \mathrm{~s}$ (MPa)

6.6500

between $200 \mathrm{~s}$ and end of transient (MPa)

6.6000 
However, it is possible to model both banks of heaters using only one PIPE component as described below.

A trip-controlled trip, number -24 , is defined whose signal value is the sum of the ISET values of trips 13, 14, 7, and 7. (Trip 7, whose ISET $=-1$ during steady state, goes off at the start of the transient.) Note that a minus sign for trip -24 allows it to be evaluated during steady state. Trip $\cdot 24$ generates signal variable 23 , which is used as the independent variable of component 12 power table (see Card Number 4 for component 12 in the input).

During steady state, the ISET values of trips 13,14 , and 7 are 1 , 0 , and -1 , respectively. Therefore, signal variable 23 has a value of $1+0-1-1=-1$, and trip -24 set status is $\mathrm{ON}_{\text {reverse }}$ The power input from the table is, therefore, $398 \mathrm{~W}$, which is balanced by the heat loss to the environment from component 12 . This is done to achieve a true steady state in the component.

At the start of the transient, trip 7 turns off. However, because trip 13 has an initial ISET value of 1 , signal variable 23 has a value of 1 and trip -24 is $\mathrm{ON}_{\text {forward }}$. The power table gives a value of $36 \mathrm{~kW}$, equal to the' cycling heater power. Because the cycling heaters in the test were on at the initiation of the experiment, we input ISET $=1$ for trip 13 to achieve the destred result. Therefore, it is obvious from the power table that if both trips 13 and 14 are off, the power input is zero. If either one of them is on, the power input is $36 \mathrm{~kW}$, and if both of them are on, the power input is $48 \mathrm{~kW}$. The TRAC model, therefore, simulates the actual system because the trip set points prohibit trip 14 from turning on unless trip 13 is already on, or prohibit trip 13 from turning off unless trip 14 is off. In other words, the model works because the cycling heaters always turn on first and turn of $f$ last. As a result, the heater power is either $0,36 \mathrm{~kW}$, or $36+12=48 \mathrm{~kW}$, but never $12 \mathrm{~kW}$.

B. Pressurizer Liquid Leve1

The pressurizer liquid level is not a control parameter. However, this level is calculated in the models using control blocks $-1,-2$, and -3 , (control blocks are always designated by negative numbers to distinguish them from signal variables) for plotting purposes only. The control-block logic is shown in Fig. E-7. (Liquid level for a simple PRIZER module is calculated 
Signal Variables

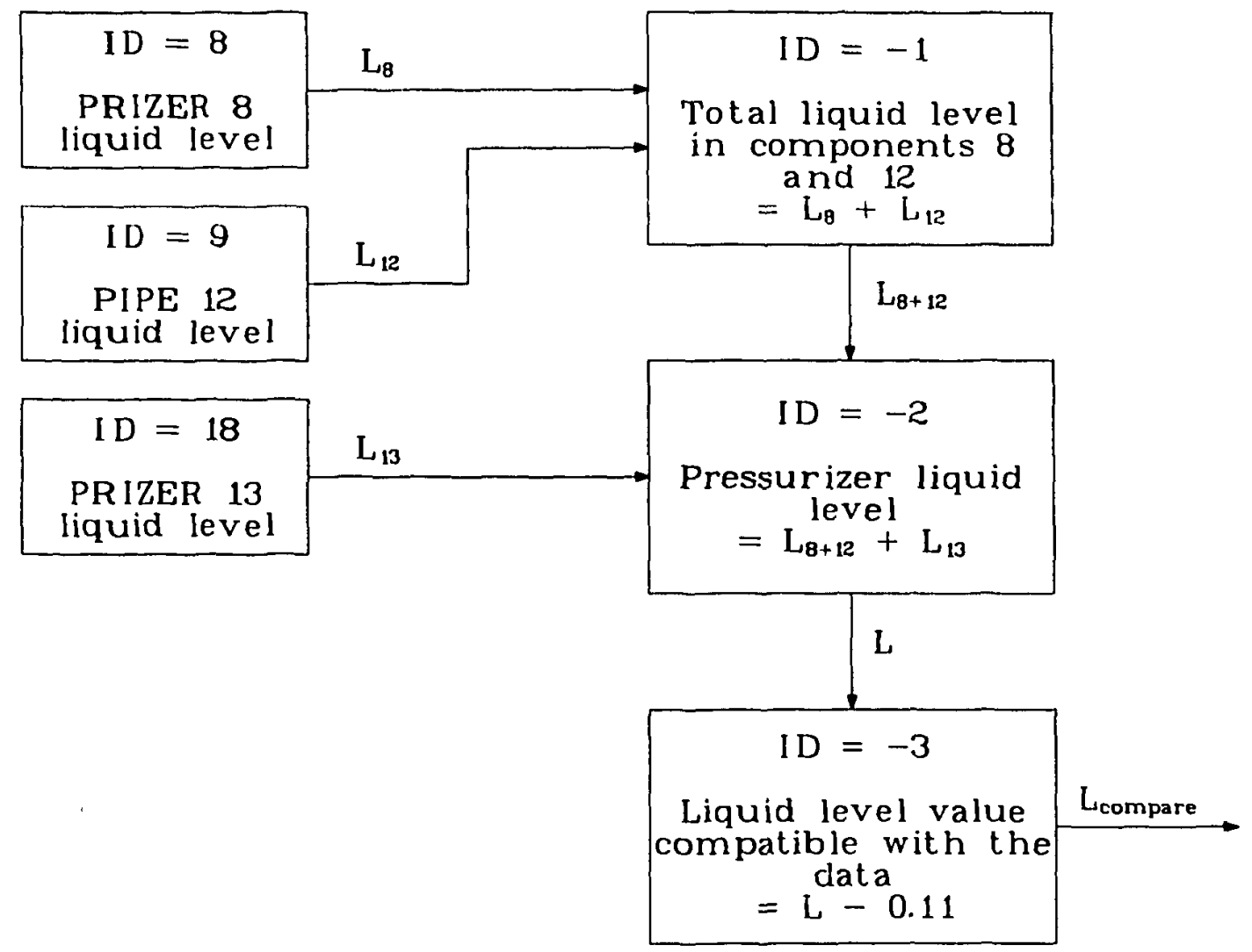

Fig. E-7.

Pressurizer liquid-level calculation using control blocks.

Yinternally by TRAC and output to the graphics file, without use of userdefined signal variables or control blocks.)

Control block -1 sums up the liquid levels in components 8 and 12 (signal variables 8 and 9). Control block -2 adds to this liquid level, the liquid level in component 13 (signal variable 18). There is no need to add component 9 liquid level because component 9 is always full of vapor. Finally, the calculated liquid level is offset by $-0.11 \mathrm{~m}$ in control block -3 . This is necessary because of slight differences in the actual pressurizer geometry and the geometry implied in the TRAC nodel (right circular cylinder). The value of -0.11 m-offset was estimated from the initial conditions, when equal initial masses of liquid in the test and the data led to a discrepancy of $0.11 \mathrm{~m}$. 
The pump speeds are controlled during steady state to achieve the desired coolant mass flow. Because the pumps were running during the transient, the pump speeds are not changed in the TRAC model after the end of steady state.

Figure E-8 shows a schematic of the macro pump controller. Control block -50 receives an intact-loop hot-leg mass-flow signal and sets up upper and lower limits to this mass-flow value to avold division by zero. Control block -55 generates the desired mass-flow value. Control block -60 calculates the ratio of the desired and actual mass flows: The new pump speed can be calculated as: present pump speed times the ratio of desired and actual mass flows. However, this approach was found to give oscillatory mass flows because of slow system response. To alleviate the problem, control block -62 is added, which along with control block -65 effectively results in $90 \%$ old, $10 \%$ new arithmetic averaging of old and new pump speeds. The sole purpose of control block -70 is to adjust the pump speed only during steady state. During steady state, control block -70 output is its input from control block -65. However, during the transient, control block -70 output is the value of signal variable 6 , which is the present pump speed. Therefore, the pump speed is not changed during the transient.

D. Steam-Generator Secondary-Side Feedwater Controller

The feedwater control provides the means for stable control of the fluid inventory in the steam-generator secondary side. The difference between the actual and desired levels provides the basic error signal for the system. The desired programmed level at zero power is $2.9464 \mathrm{~m}$ above the top of the tube sheet and is a $0.00508-m$ increment in level for each MW of core power.

A steam flow signal is required by the feedwater control system to obtain a satisfactory transient response, which reduces the steady-state level error that would occur without it.

A block diagram of the feedwater control system is shown in Fig. E-9. Control block -4 receives its input as the steam mass flow, and converts the input into an equivalent feedwater valve flow area fraction. Control block -5 converts this flow area fraction into an equivalent feedwater valve stem position, which is required to balance the steam flow exactly. Therefore, if 
Control Blocks

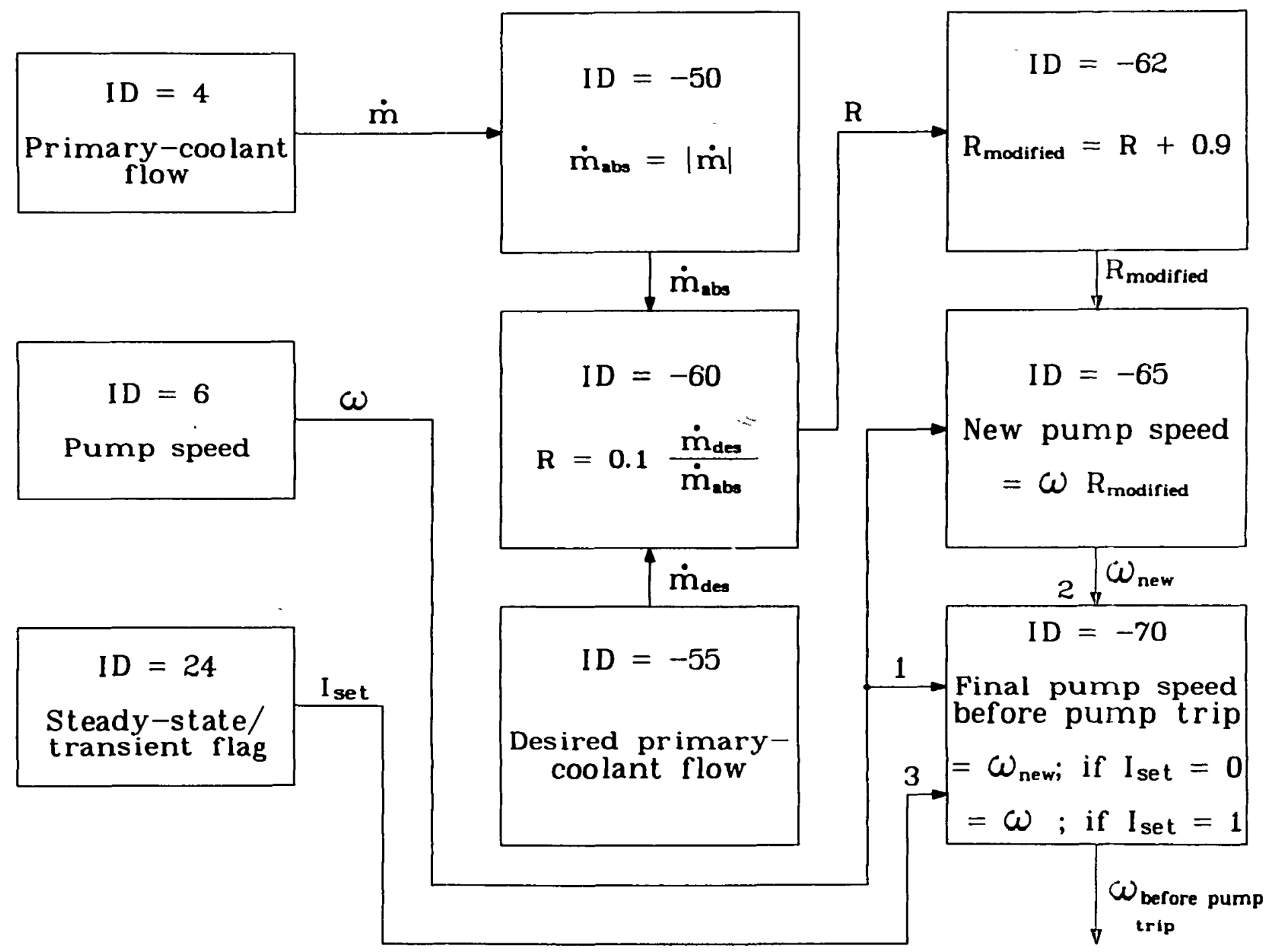

Fig. E-8.

Pump-speed control system. 
Signal Variables

Control Blocks

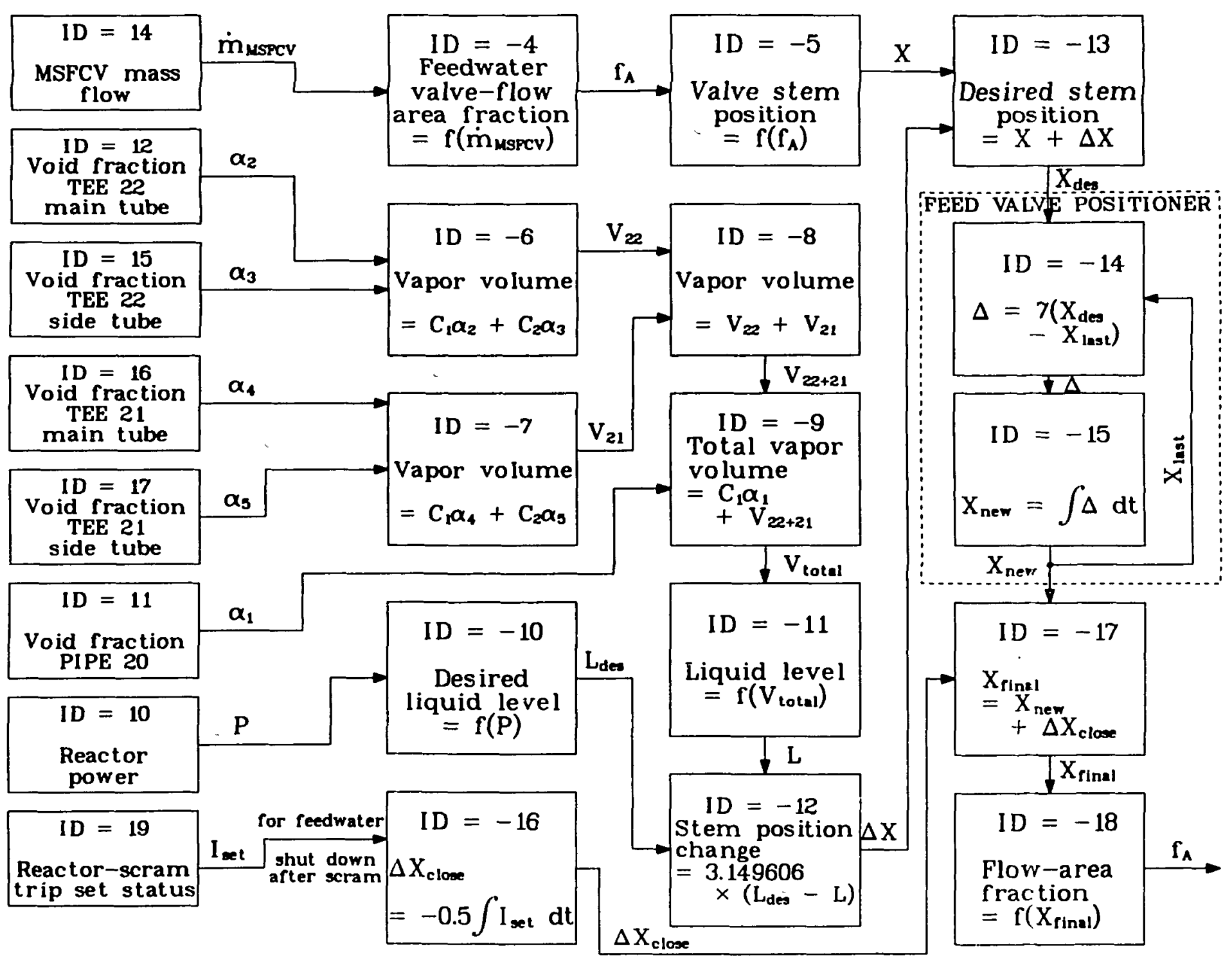

Fig. E-9.

Steam-generator secondary-side feedwater control system. 
the liquid level in the steam generator is below its desired value, the feedwater valve opening should be larger than the above value, and vice versa.

The liquid-level calculation is based on total liquid inventory in the steam-generator secondary side. Control blocks -6 through -9 calculate the total steam volume on the secondary side. A plot of the collapsed liquid level versus the liquid volume is available in the LOFT system description. 77 Reference 77 also reports that the total water volume at full power ( $50 \mathrm{MW}$ ) is $2.52 \mathrm{~m}^{3}$, which corresponds to an uncollapsed liquid level of $3.2004 \mathrm{~m}$; whereas, the liquid volume based on a collapsed liquid level of $3.2004 \mathrm{~m}$ is $3.8166 \mathrm{~m}^{3}$. Therefore, we generated a plot of uncollapsed liquid level versus the liquid volume from the collapsed liquid-level plot by multiplying its volume by a ratio of $2.52 / 3.8166$. Knowing the total secondary volume, we then converted this plot into the uncollapsed liquid level as a function of the total steam-generator secondary-side steam volume. Control block-11 uses this information to calculate the steam-generator secondary-side liquid level.

Control block -10 calculates the desired liquid level corresponding to the actual core power from the formula, liquid level $=2.9464+0.00508 \times$ core power in MW. Control block -12 calculates the error, desired liquid level minus actual liquid level, and translates the error into an equivalent valve stem position increment (or decrement) by using a gain factor of 3.149606 (Ref. 73). Control block -13 adds this value to the stem position required to just balance the steam flow. The output from control block -13 is, therefore, the desired stem position.

To stabilize the control of the liquid level, a feedback signal representative of the input is needed. The feedwater regulating-valve position is used as the feedback signal for the LOFT system. Control blocks $-1, j$ and -15 simulate this feedback. Control block -14 calculates the difference between the desired stem position and the current stem position, which is not allowed to exceed a magnitude of 0.05 (maximum rate of stem fraction movement is $0.05 / \mathrm{s}$ ). The integrator (control block-15), therefore, gives the value of the new stem position.

Control blocks -16 and -17 are added to the control system to account for the feedwater pump trip that occurs as soon as the reactor scram is initiated, which stops the feedwater flow within $2 \mathrm{~s}$. Finally, control block -18 changes the valve stem position into the flow area fraction. The 
actual feedwater mass flow as a function of the flow area fraction is input in FILL 24.

E. Steam-Generator Secondary-Side Steam-Flow Valve Controller

Automatic control of the steam-flow valve is provided to maintain the desired secondary-side pressure. However, different pressures are required to be maintained before and after the reactor scram. A block diagram of the steam-fliow valve control system is shown in Fig. E-10. Control block -41 outputs the trip set status (ISET) value of either trip -21 , which is used to, govern the pressure before the reactor scram, or trip 20, depending on whether trip 20 is on or off. The purpose of trip 20 is to start a forced valve action at the desired time. For Test L6-1, this action is to start closing the valve at the start of the transient. The output from control block -41 is, therefore, $0,-1$, or 1 , which requires the valve to remain stationary, closed, or open, respectively. Control block -42 outputs the signal from control block -41 before the reactor scram and trip 22 signal (trip 22 controls the secondary-side pressure) after reactor scram. A gain factor of 0.05 for control block -42 corresponds to the valve-stem fractional movement rate of 0.05 per second. Thus, control block -42 output is $0,-0.05$, or 0.05 , which determines whether the valve should remain stationary, start closing at a rate of 0.05 valve-stem fraction per second, or start opening at a rate of 0.05 valve-stem fraction per second. An integrator (control block -43) determines the new valve-stem position from its present value. Finally, control block -44 translates the valve-stem position into an equivalent flow area fraction.

It should be noted that trip 22, which controls the secondary pressure after the reactor scram, has associated trip set-point factor tables for both the upper and lower set points. These were necessary because the trip set points were observed to vary slightly during the experiment.

One final note on the control system is in order. As discússed above, this TRAC model has some signal variables that represent trip set-status or trip-signal values. Consequently, these signal variables cannot be evaluated before the trip data are processed. However, TRAC first processes the signal variables, then the control blocks, and finally the trips. Therefore, it was necessary to make two passes through the control parameters (cards 109-114 in Sec. E-VI.A). 


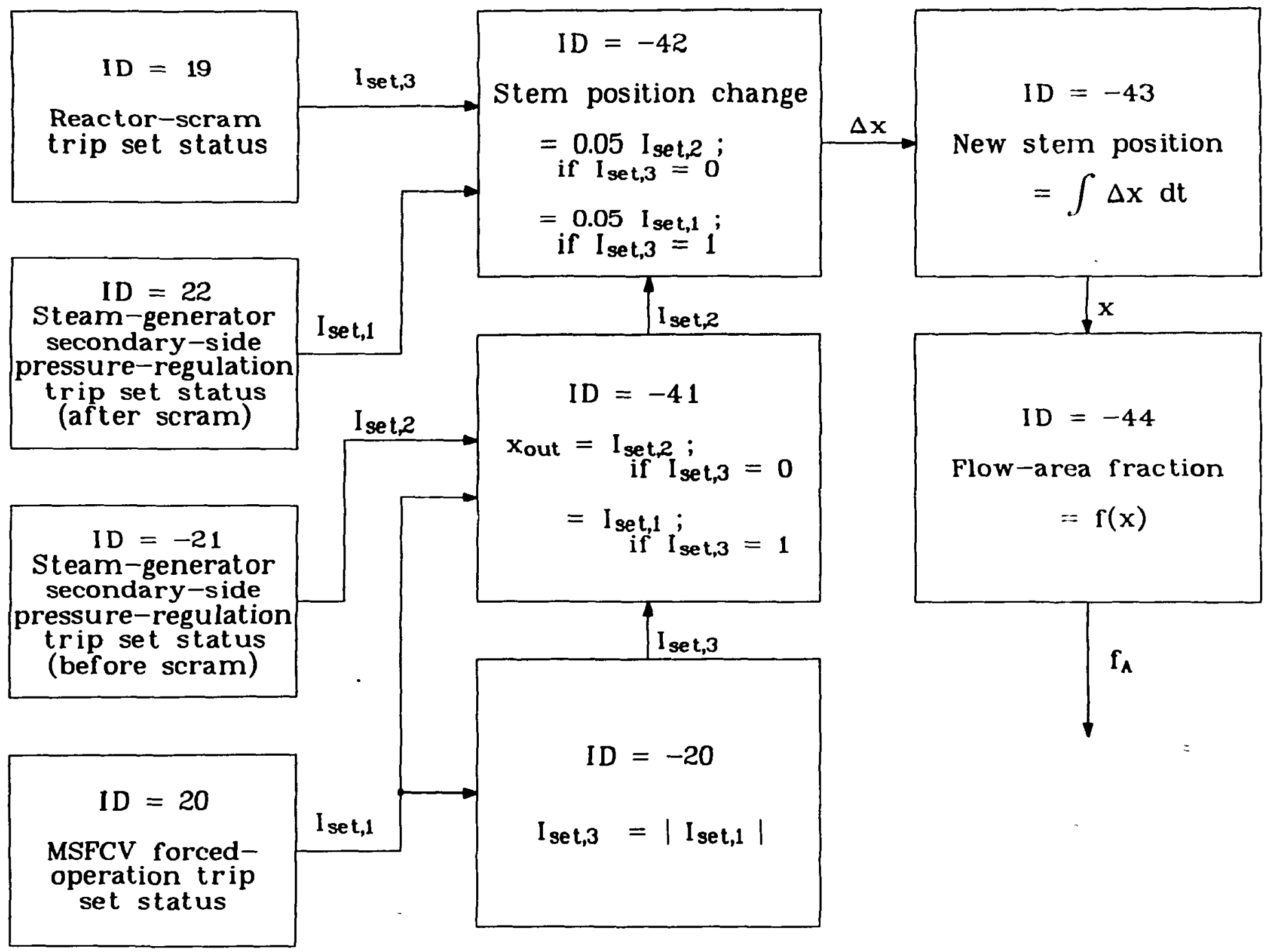


IV. STEADY-STATE CALCULATION

Based on the geometry and the noding described in Sec. E-III, we performed a steady-state calculation using the generalized steady-state TRAC option. The calculation took $109 \mathrm{~s}$ of Cray-1 CPU time to simulate $200 \mathrm{~s}$ of reactor steady-state time at an average $0.51-s$ time step. As noted in Sec. E-VI.A, all the initial velocities were zero. At the beginning of the calculation, the reactor power was automatically set to zero. At $0.02 \mathrm{~s}$, the liquid velocity in the core was large enough to turn on the core power. All major primary-system parameters, such as pressures, velocities, and temperatures, converged to their steady-state values in $\sim 30 \mathrm{~s}$. However, it took another $\sim 30 \mathrm{~s}$ for the secondary-side parameters to converge.

Figures E-11 through E-15 show the upper-plenum pressure, the pump number 1 speed (pump number 2 speed is identical to that for pump number 1), the primary-coolant mass flow, and the steam-generator inlet and outlet temperatures, respectively. As indicated earlier, these parameters are converged to their steady-state values by $\sim 30 \mathrm{~s}$. The pump-speed controller

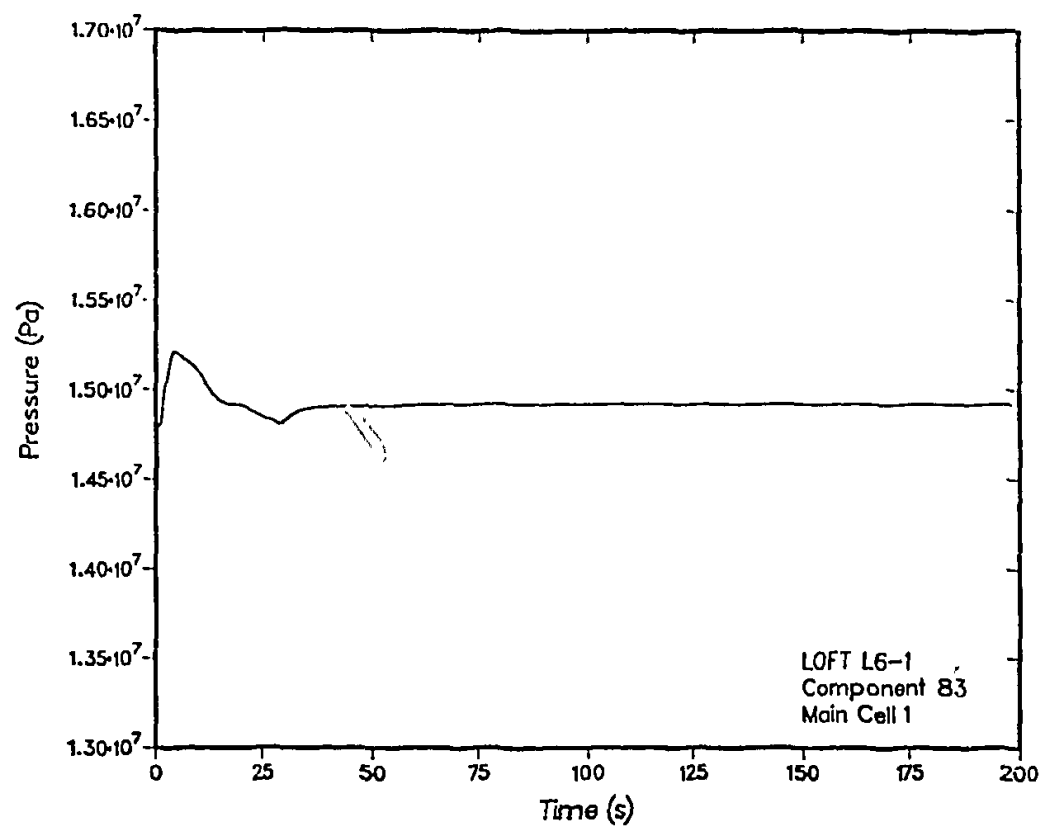

Fig. E-1i.

Steady-state upper-plenum pressure for Test L6-1. 


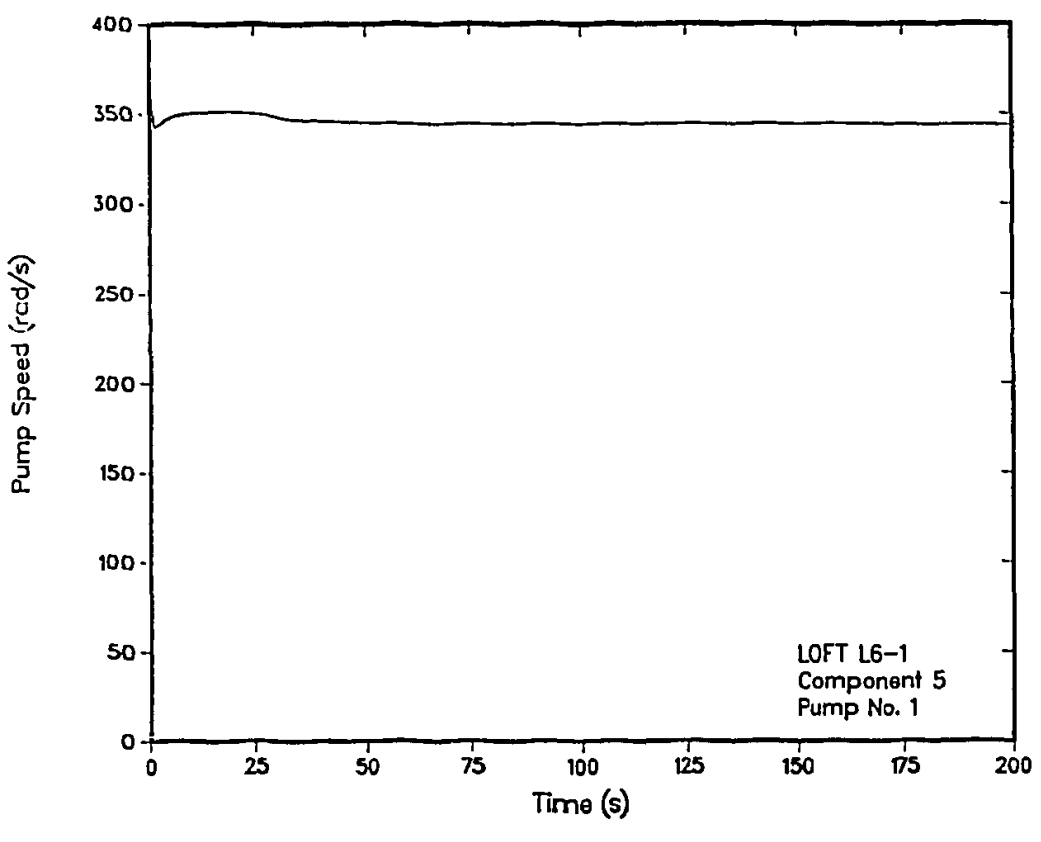

1) Fig. E-12.

Steady-state pump number 1 speed for Test L6-1.

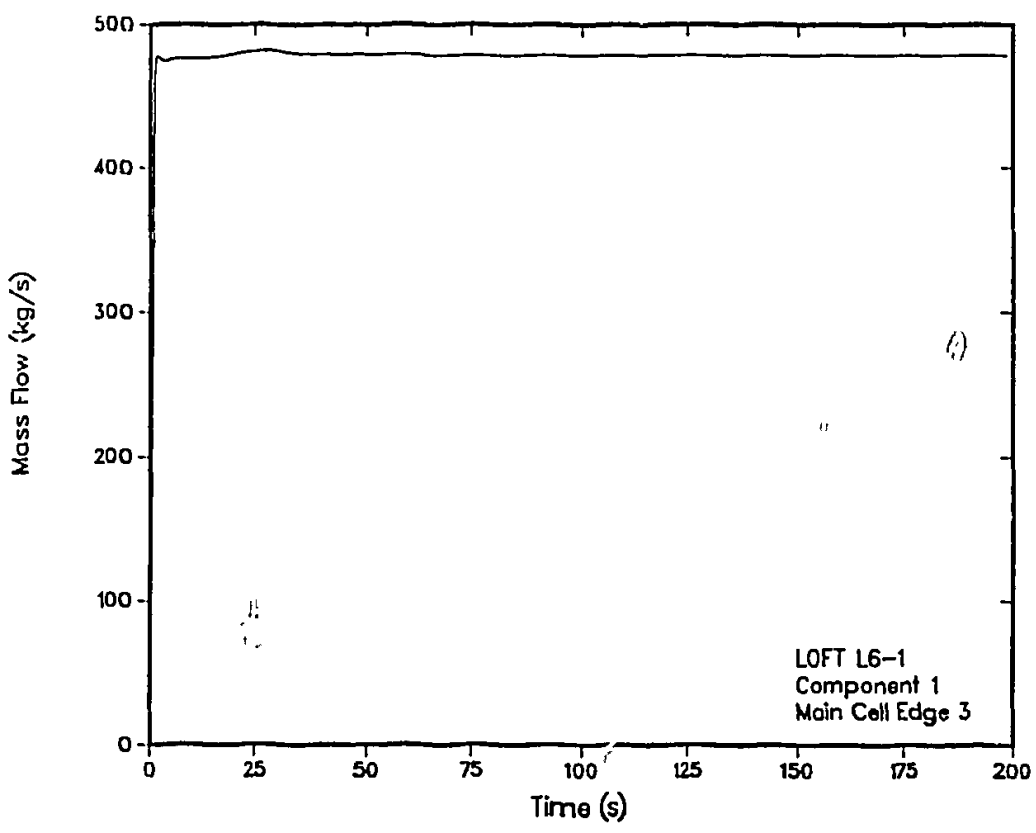

Fig. E-13.

Steady-state primary-coolant mass flow for Test L6-1. 


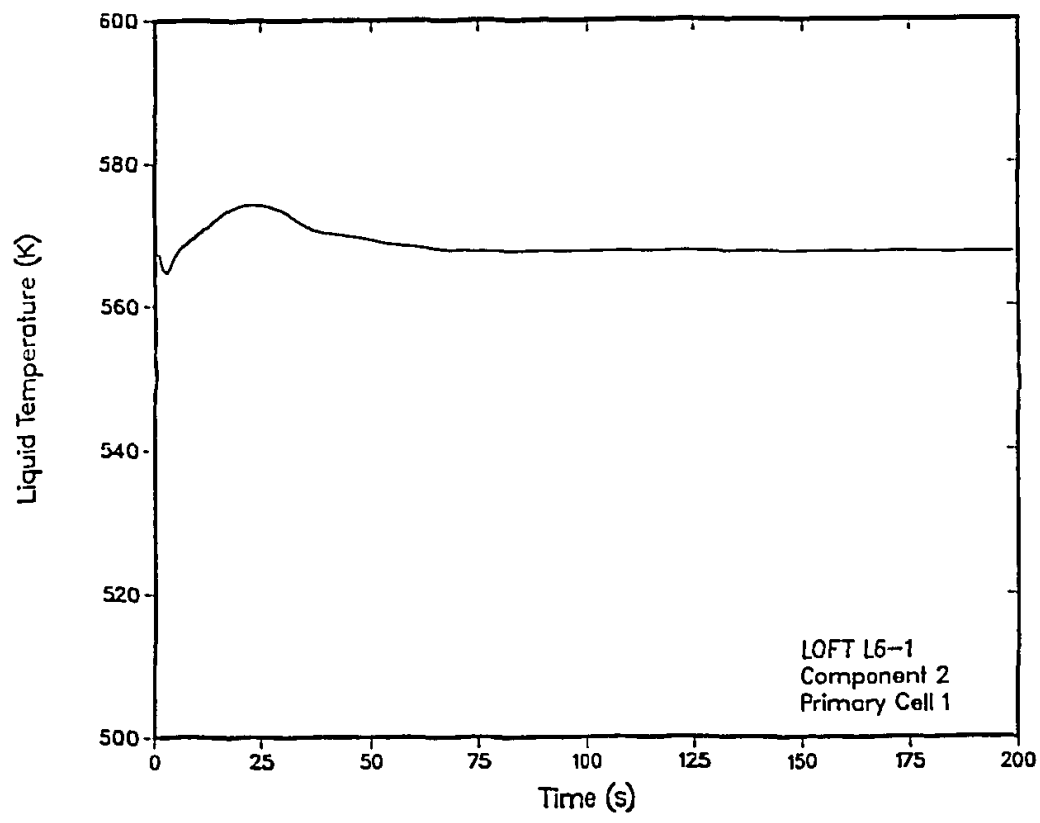

Fig. E-14.

Steady-state steam-generator primary-side inlet temperature for Test L6-1.

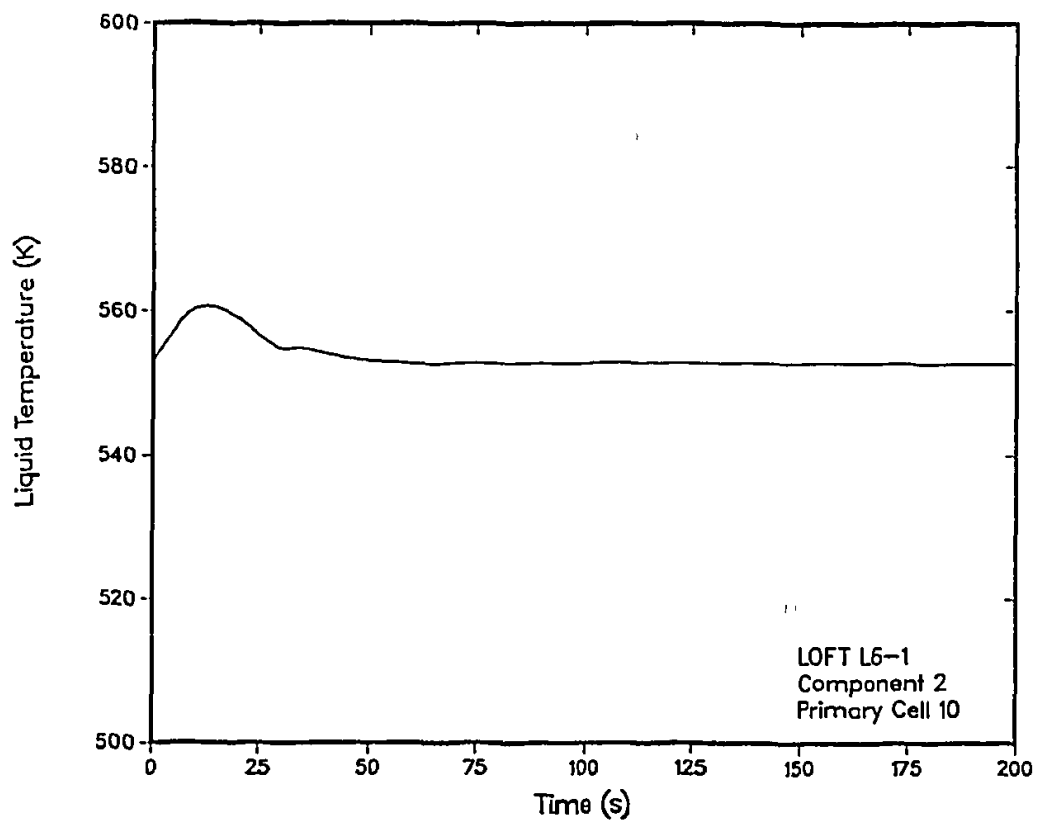

Fig. E-15.

Steady-state steam-generator primary-side outlet temperature for Test L6-1. 
performance is excellent and is able to bring the loop flow to its steadystate value within the first few seconds.

Secondary-side parameters are presented in Figs. E-16 through E-19. The pressure (Fig. F-16) reaches its steady-state value at $\sim 60 \mathrm{~s}$. The initial pressure spike near $10 \mathrm{~s}$ was caused by the steady state being initiated with the MSFCV fully closed, with this valve having a finite rate of opening. The secondary-side liquid level (Fig. E-17) is converged by $\sim 50 \mathrm{~s}$. However, as seen in Fig. E-18, it takes $\sim 10 \mathrm{~s}$ more for the feedwater flow to converge to a reasonably steady value. Even then the flow behavior is somewhat erratic. However, the feedwater controller works well, because its purpose is to maintain a steady liquid level. The feedwater flow oscillations have almost no impact on the rest of the system behavior. The steam flow also is converged in $\sim 60 \mathrm{~s}$ ( Fig. E-19).

\section{v. TRANSIENT CALCULATION}

The transient calculation was started from the last dump of the steadystate calculation. No new signal variables, controls, trips, or components were input at the start of the transient. This restart deck (Sec. E-VI.B),

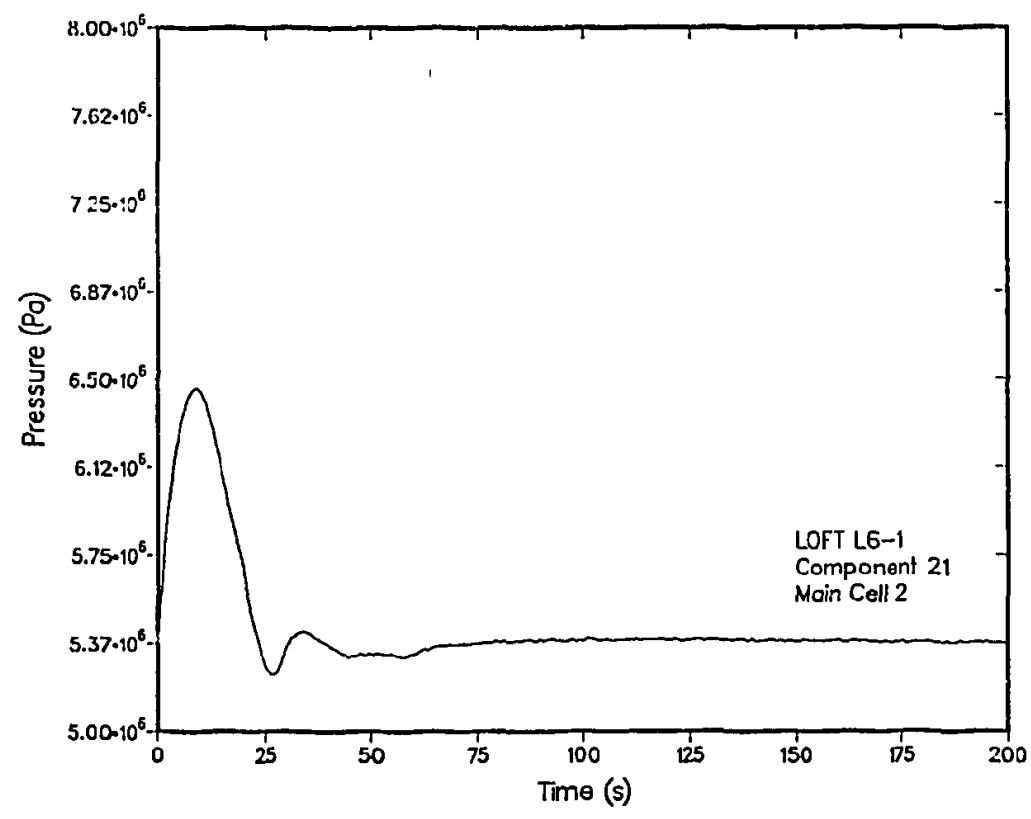
Fig. E-16.
Steady-state steam-generator secondary-side pressure for Test L6-1/\% 


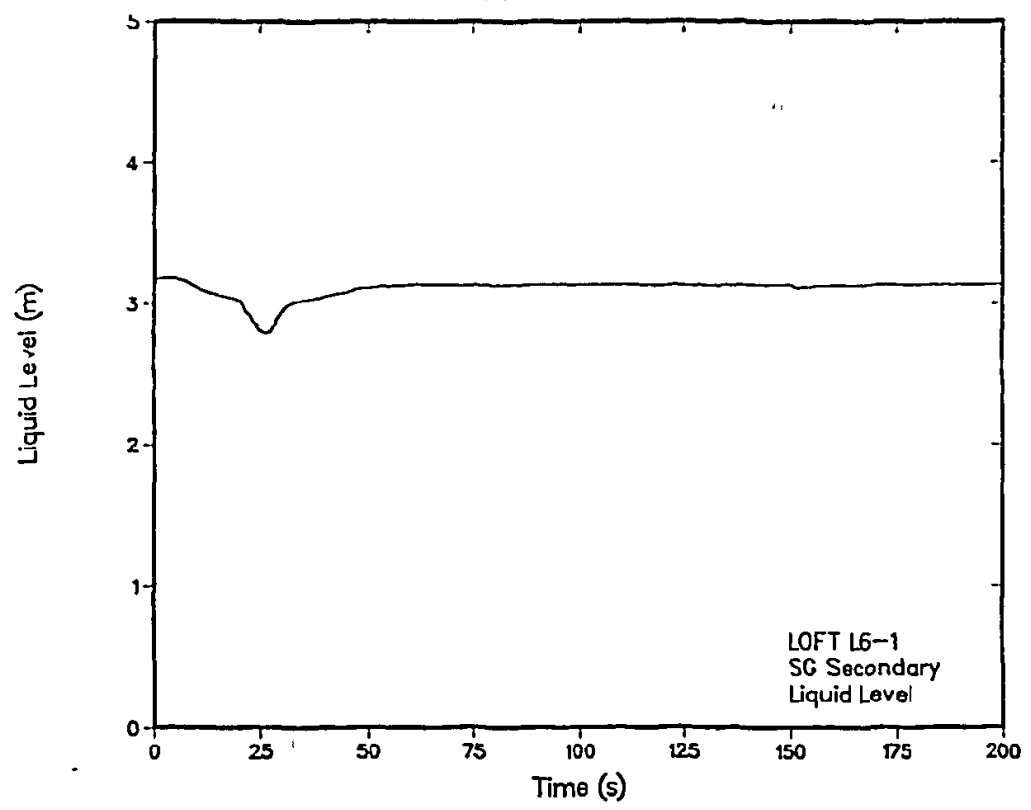

Fig. $\mathrm{E}-17$.

Steady-state steam-generator secondary-side liquid level for Test L6-l.

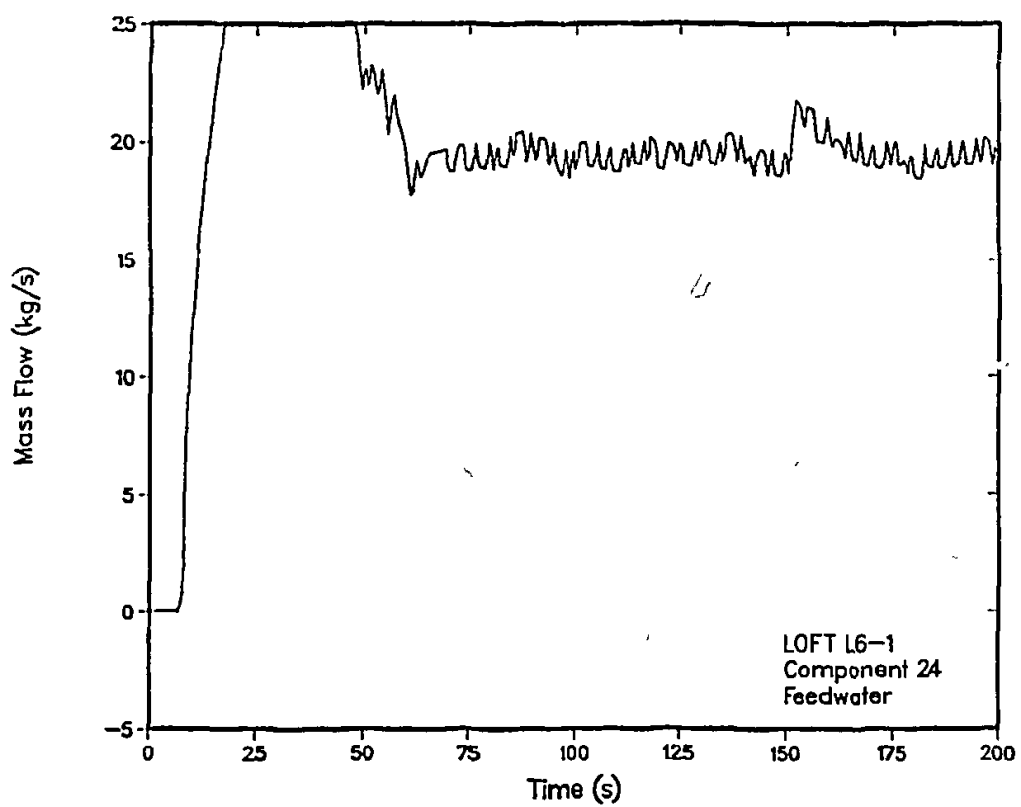

Fig. E-18.

Steady-state steam-generaţor secondary-side feedwater mass flow for Test L6-l. 


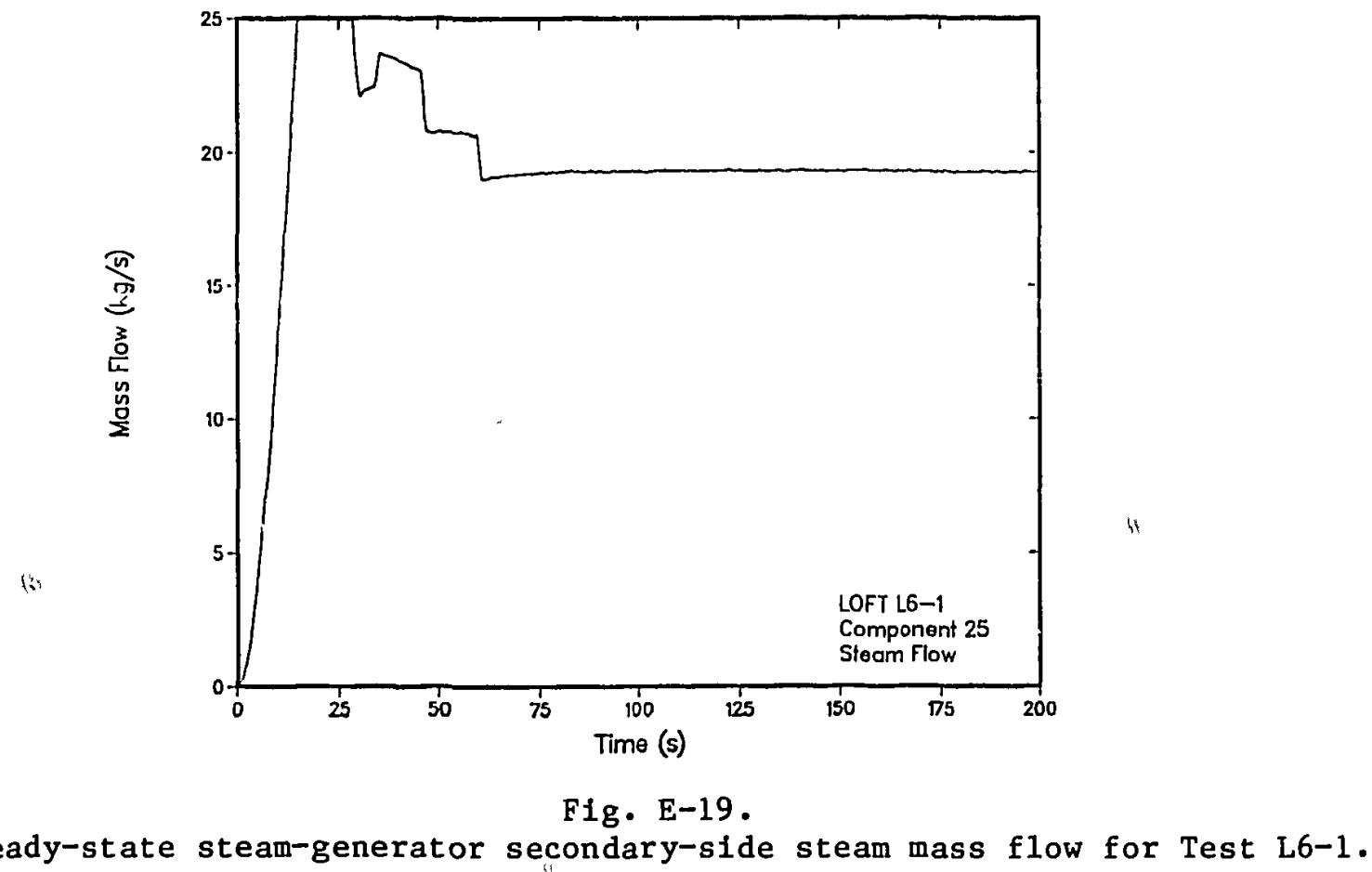

therefore, constitutes the minimum amount of input required during a restart. The Cray-1 CPU time required to simulate a 700-s system transient was $228 \mathrm{~s}$ at an average 0.78-s time step. The TRAC calculation, therefore, ran over three times faster than real time.

The initial conditions and specified test parameters used in Test L6-1 are listed in Table E-III. The TRAC steady-state calculation closely approximates the actual test conditions. Table E-IV lists the main events during the transient for the test and the calculation. The measured and calculated times agree well with" the exception of pressurizer heater operation. This discrepancy is caused by minor differences in the experimental and calculated system pressures.

The TRAC calculation was run up to $700 \mathrm{~s}$, but because most events of interest were over during the early part of the transient, the comparisons are presented between 0 and $250 \mathrm{~s}$ of the transient only for clarity in the figures. All the calculated parameters we compared with the experiment remained consistently within the measurement uncertainty in the 250- to 700-s time span. 
TABLE E-III

TEST L6-1 INITIAL CONDITIONS

Parameter

Core power (MW)

Pressurizer pressure (MPa)

Pressurizer liquid volume $\left(\mathrm{m}^{3}\right)$

Primary-coolant mass flow ( $\mathrm{kg} / \mathrm{s})$

Intact-loop cold-leg temperature (K)

Intact-loop hot-leg temperature (K)

Pump speeds ( $\mathrm{rad} / \mathrm{s}$ )

T.team-generator secondary-side pressure (MPa)

Steam-generator secondary-side liquid level (m)

Steam-generator secondary-side mass flow ( $\mathrm{kg} / \mathrm{s})$

\begin{tabular}{|c|c|}
\hline Actual & Calculated \\
\hline 36.9 & $36.9^{a}$ \\
\hline 14.78 & $14.78^{a}$ \\
\hline 0.63 & $0.63^{2}$ \\
\hline 478.5 & 478.6 \\
\hline 552.8 & 552.8 \\
\hline 567.5 & 567.6 \\
\hline 334 & $34 i^{b}$ \\
\hline $5 \cdot 37$ & $5.39^{c}$ \\
\hline 3.183 & $3.135^{d}$ \\
\hline 20.1 & 19.2 \\
\hline
\end{tabular}

$\mathrm{a}_{\text {Specified as input parameter. }}$

${ }^{b}$ Controlled to maintain the desired primary-coolant mass flow during the steady-state calculation.

${ }^{c}$ Controlled within maximum and minimum values of $5.425 \mathrm{MPa}$ and $5.315 \mathrm{MPa}$, respectively. (See Table E-II.)

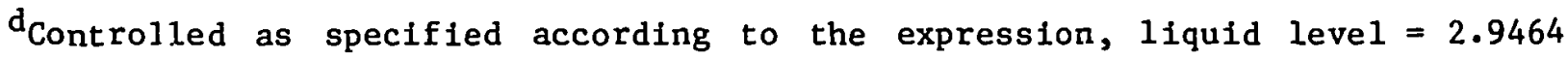
$+0.00508 \times$ core power in $\mathrm{MW}$, which gives a desired liquid level of $3.134 \mathrm{~m}$ for $36.9 \mathrm{MW}$ core power. However, the experimental liquid level was out of specification. 
TABLE E-IV

TEST L6-1 SEQUENCE OF EVENTS

\section{Event}

MSFCV started to close

Fressurizer cycling heaters off

Pressurizer spray on

Reactor scrammed

MSFCV started to open

Pressurizer spray of $f$

Pressurizer cycling heaters on

Pressurizer backup heaters on

MSFCV started to close

MSFCV started to open

MSFCV started to close

Pressurizer backup heaters off

MSFCV started to open

MSFCV started to close

Pressurizer backup heaters on

Pressurizer backup heaters off

Pressurizer cycling heaters off

Transient terminated

\begin{tabular}{|c|c|}
\hline $\begin{array}{c}\text { Experimental Time } \\
\text { (s) }\end{array}$ & $\begin{array}{c}\text { Calculated Time } \\
\text { (s) }\end{array}$ \\
\hline $2 \cdot 0^{a}$ & 2.0 \\
\hline 6.1 & 8.1 \\
\hline 9.1 & 10.7 \\
\hline 21.8 & 19.1 \\
\hline 22.2 & 22.1 \\
\hline 30.4 & 32.7 \\
\hline 31.4 & 34.4 \\
\hline 32.5 & 36.1 \\
\hline $33.2^{b}$ & 33.5 \\
\hline 91.2 & 94.0 \\
\hline $99.2^{b}$ & 100.7 \\
\hline$-c$ & 288.4 \\
\hline 312.6 & 314.4 \\
\hline $333.2^{b, d}$ & 321.6 \\
\hline$-c$ & 328.0 \\
\hline 415.4 & 399.8 \\
\hline$-c$ & 475.8 \\
\hline 700.0 & 700.8 \\
\hline
\end{tabular}

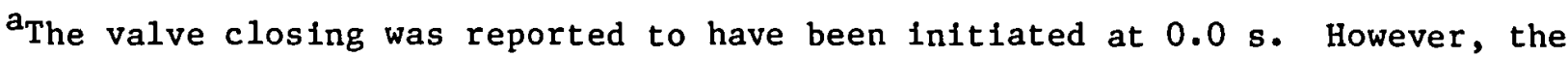
data show an $\sim 2-5$ time delay before any movement in the valve position was observed.

bonly the times when the valve was fully closed were reported in Ref. 72 . The times when the valve started to close were estimated from the times when the valve was fully closed, the maximum valve-stem position when the valve was open, and the valve-stem movement rate.

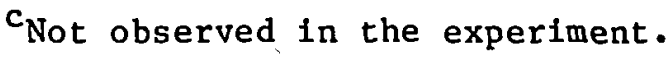

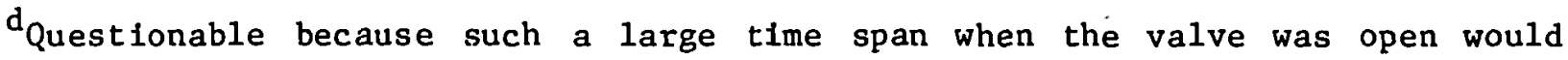
discharge several times the mass of steam actually observed to discharge in the experiment and would reduce the secondary-side pressure drastically.
} 
Figure E-20 shows a comparison between the calculated and experimental core powers. Because the calculated reactor scram occurred $2.7 \mathrm{~s}$ before that observed in the experiment (Table E-IV), the calculated power decay occurred earlier than the data. The power, though considerably within the data uncertainty, is somewhat overpredicted after the reactor scram. This is because the experimental curve lies within the deadband range of the detector. Thus, the actual discrepancy, if any, may be much smaller than that observed in Fig. E-20.

Figure E-21 compares the TRAC-calculated and the measured pressurizer pressures. The calculation is consistently, within the uncertainty of the measurement. At $\sim 10 \mathrm{~s}$, the pressurizer spray temporarily reduces the primaryside pressure, which is predicted by the calculation. The reactor scram also is calculated at about the right time $(20 \mathrm{~s})$. Both in the calculation and in the experiment, the reduced pressure turns off the pressurizer spray at $\sim 30 \mathrm{~s}$, and $\sim 3 \mathrm{~s}$ later the pressurizer heaters turn on. The pressure drop near $100 \mathrm{~s}$, which is caused by high secondary-side pressure, corresponds to the MSFCV opening. TRAC correctly and accurately predicts all events.

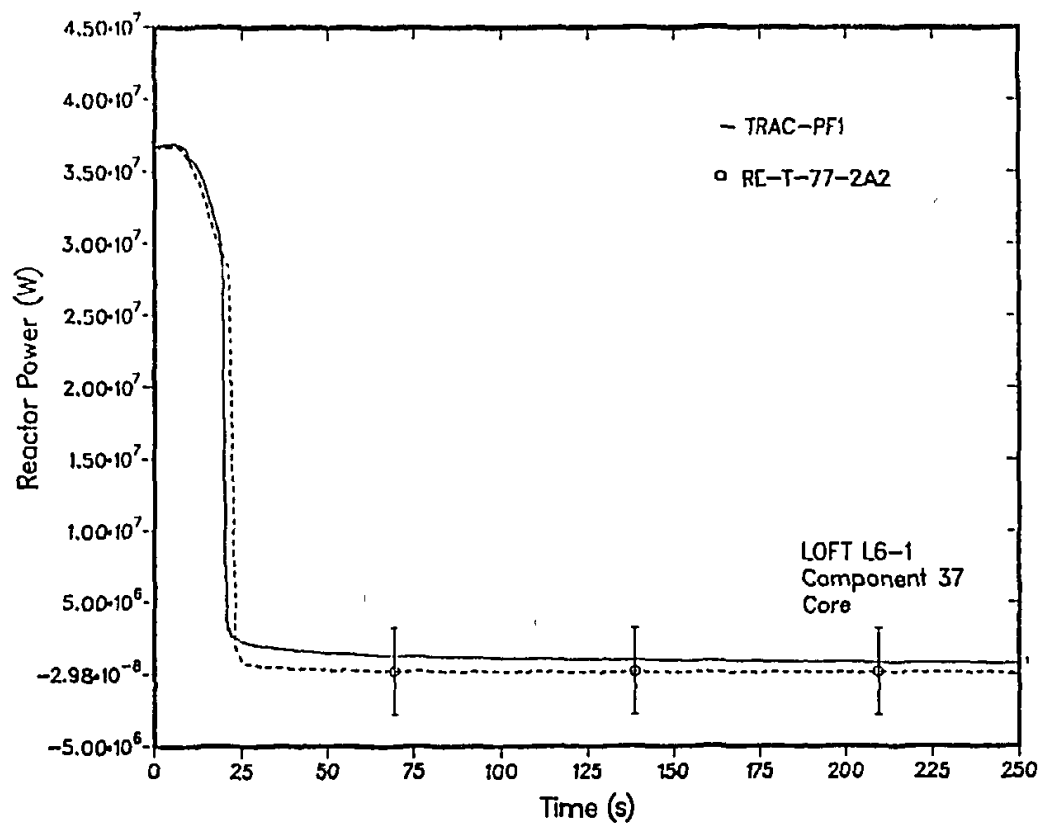

Fig. E-20.

Comparison of the TRAC-calculated with the measured core powers for Test L6-1. 


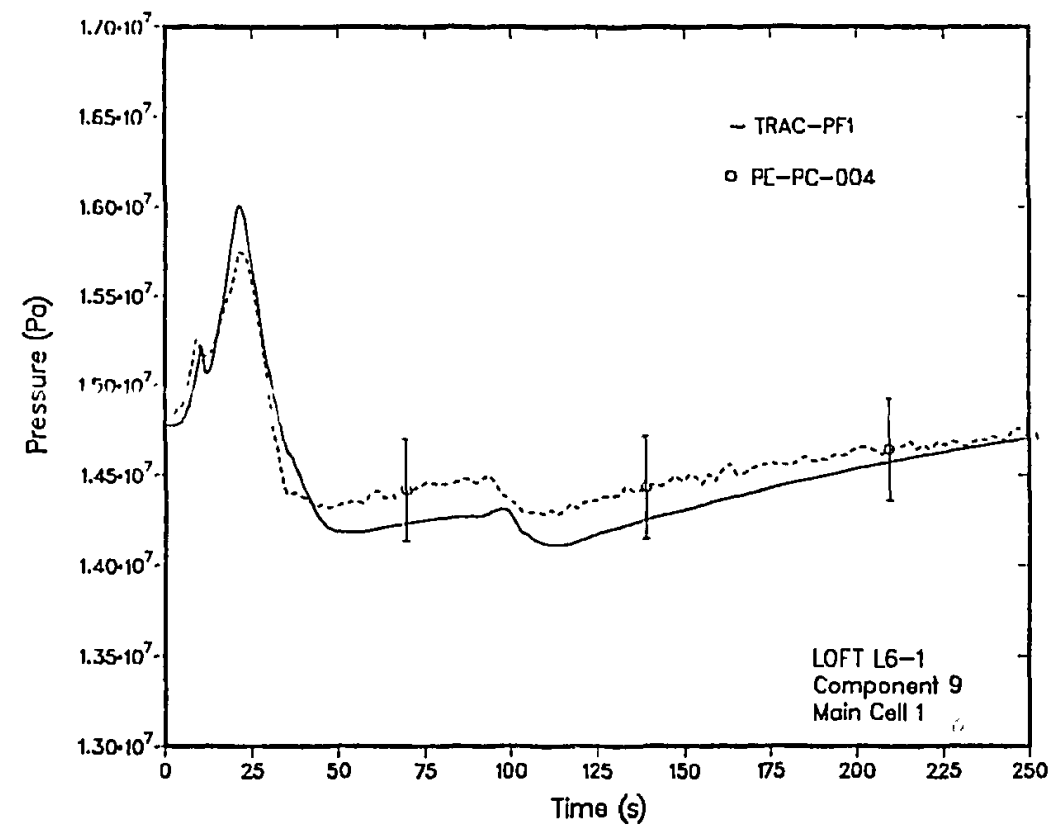

Fig. E-21.

Comparison of the TRAC-calculated with the measured pressurizer pressures for Test L6-1.

The primary-coolant mass-flow comparison between the calculation and the data is shown in Fig. E-22. The agreement is excellent. A small constant offset between the calculation and the data is caused by a difference between the published steady-state mass flow, 72 which was used in the pump-speed controller, and the data channel plotted.

Figure E-23 shows the steam-generator secondary-side feedwater flow comparison between the test data and the calculation. The feedwater controller correctly calculates the flow. Figures E-24 and E-25 show excellent agreement between the calculated and the measured steam-generator secondary-side steam mass flows and steam-generator secondary-side pressures, respectively. The steam-flow controller performance is, therefore, excellent. The apparent small discrepancy between the observed and calculated steam mass flows when the MSFCV is fully closed is caused by an unknown amount of leakage through the MSFCV. However, because the measured flow is within the deadband range of the instrument, the actual discrepancy may be insignificant. We did not model any leakage through the valve. Finally, Fig. E-26 compares the 


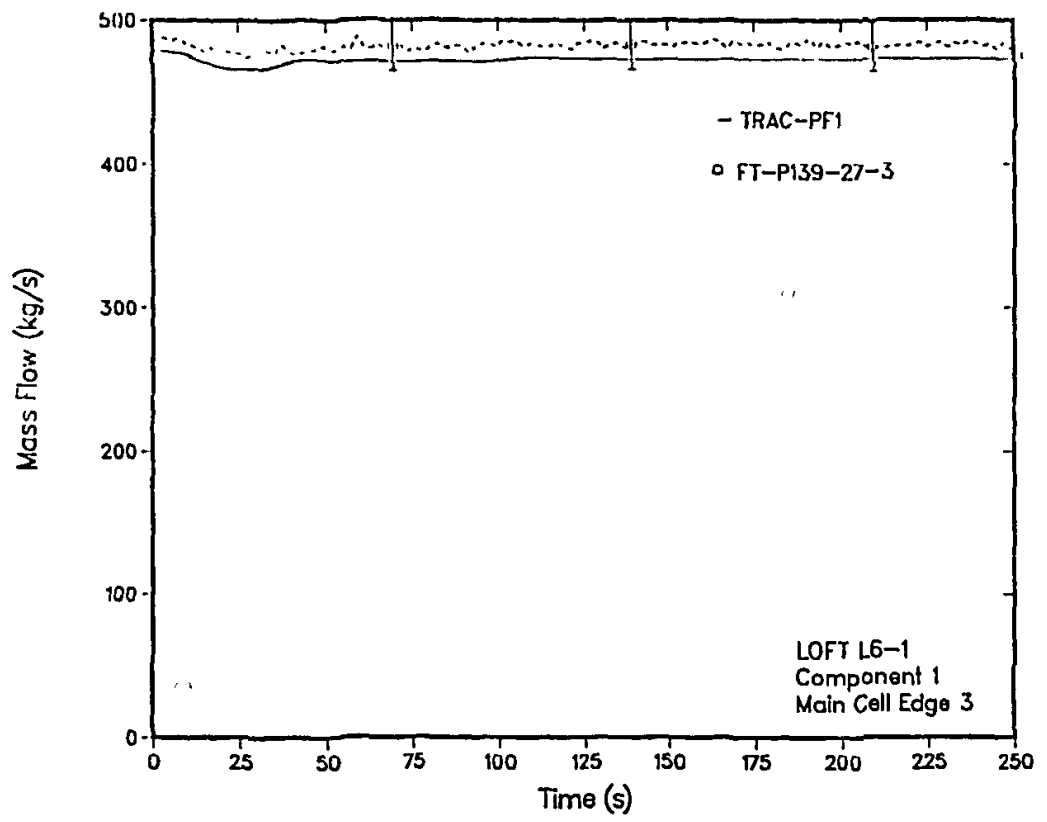

Fig. E-22.

Comparison of the TRAC-calculated with the measured primary-coolant mass flows for Test L6-1.

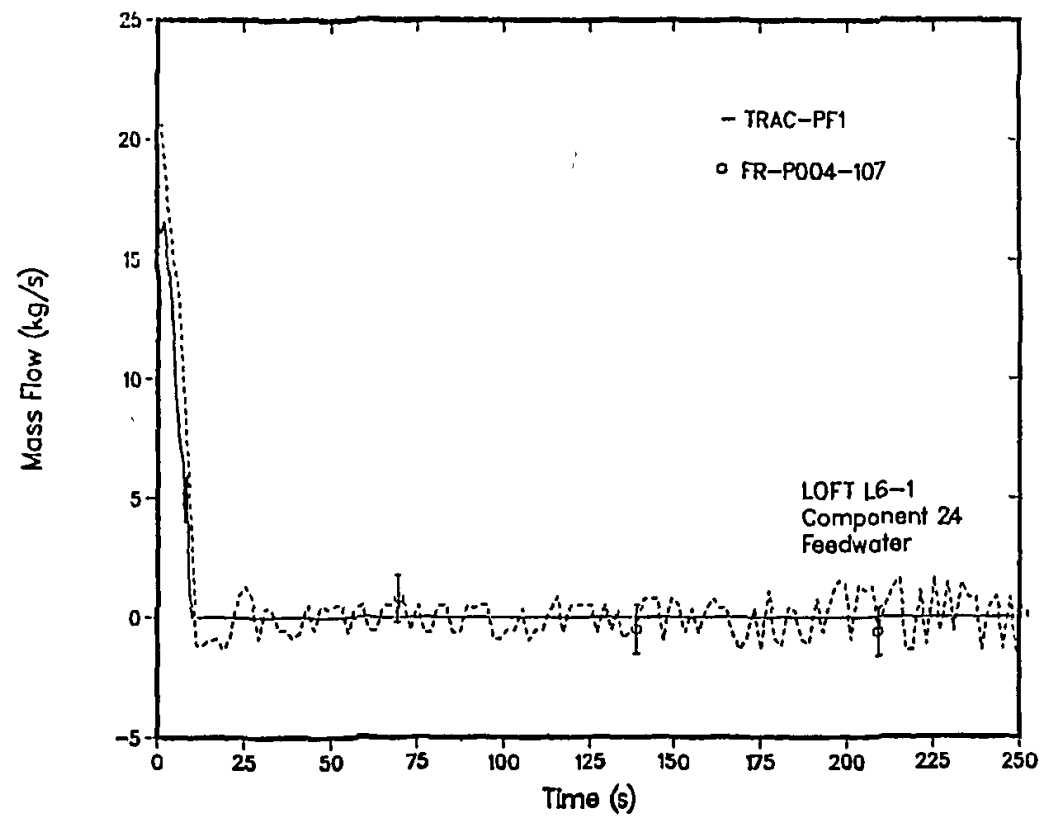

Fig. E-23.

Comparison of the TRAC-calculated with the measured steam-generator secondaryside feedwater mass flows for Test L6-1.

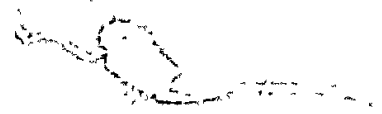




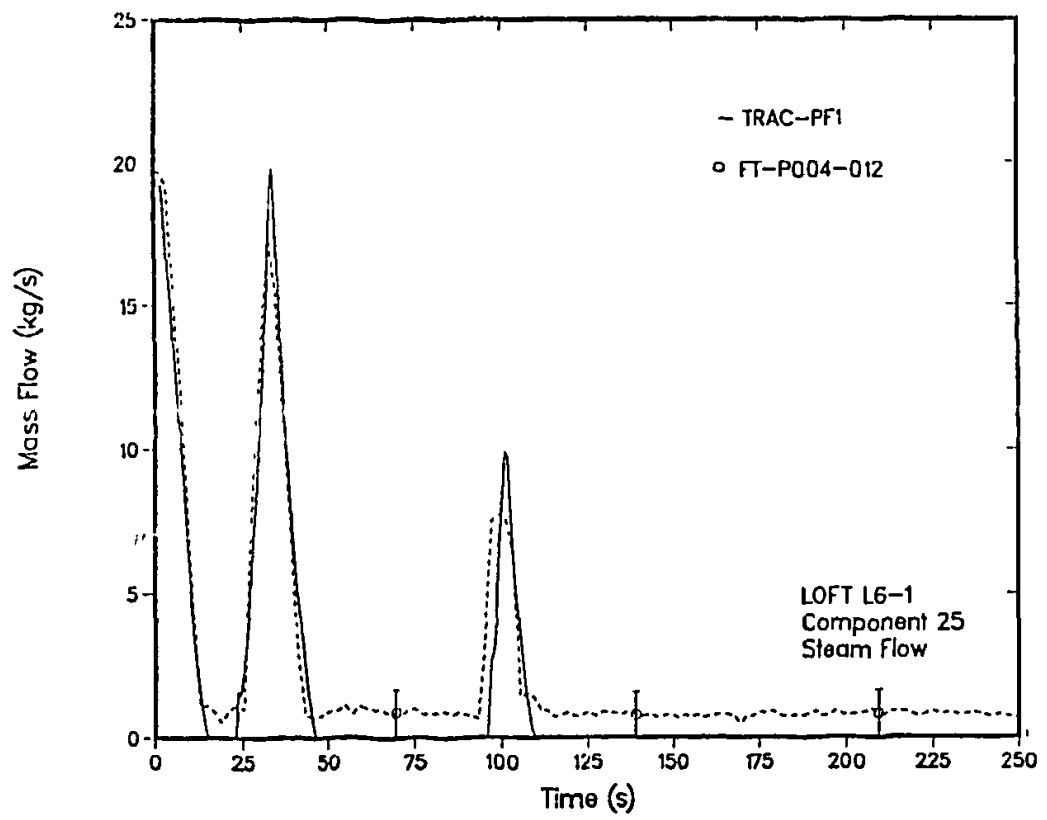

11

Fig. E-24.

Comparison of the TRAC-calculated with the measured steam-generator secondary-side steam mass flows for Test L6-1.

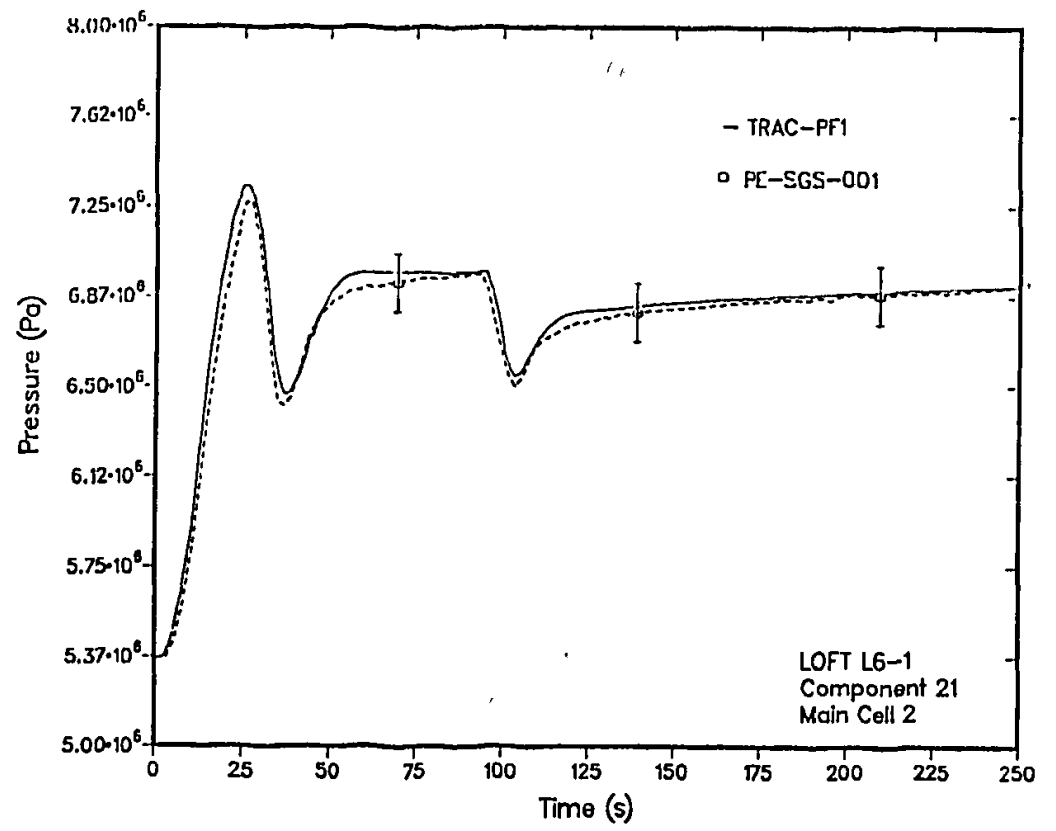

Fig. E-25.

Comparison of the TRAC;calculated with the measured secondary-side pressures for Test L6-1. 


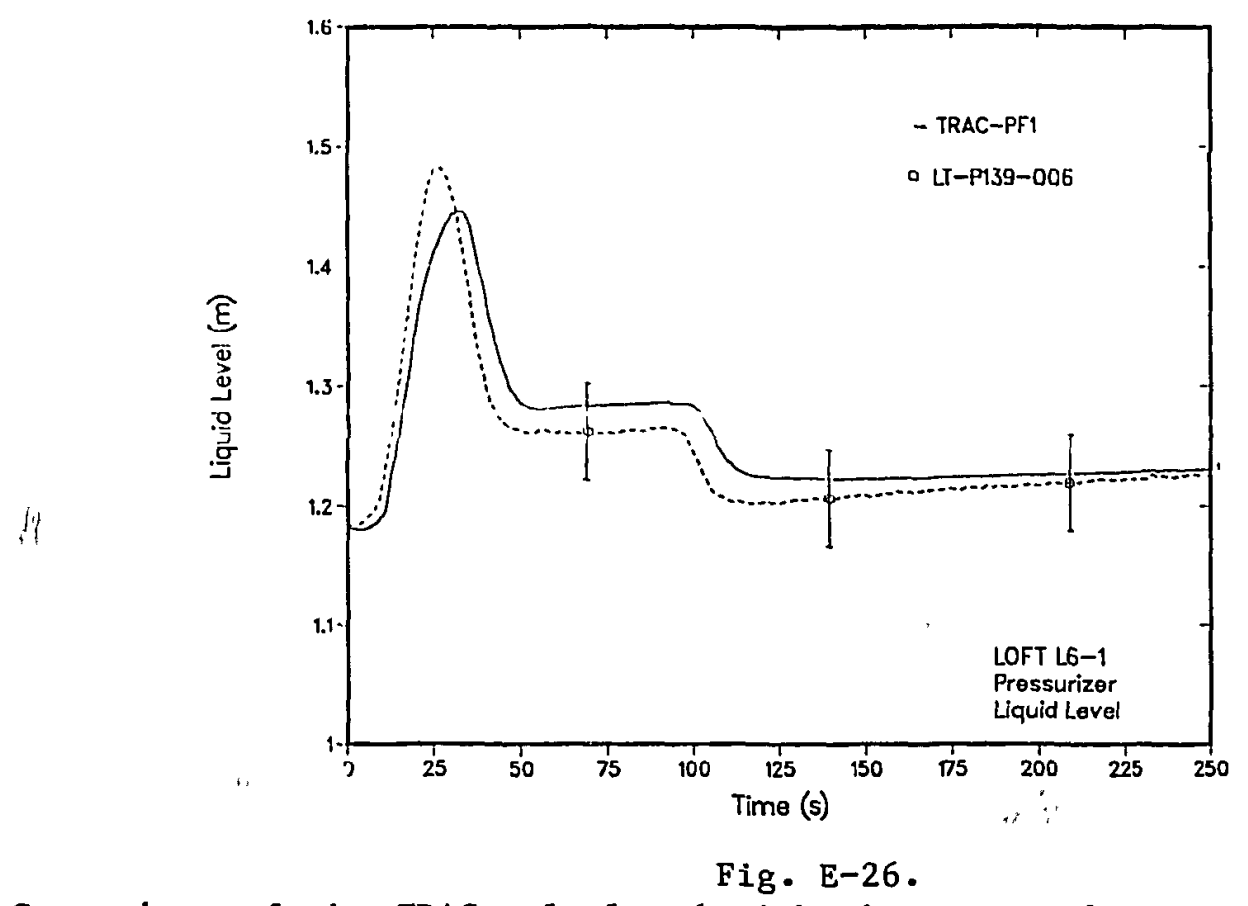

Comparison of the TRAC-calculated with the measured pressurizer liquid-level histories for Test L6-1.

TRAC-calculated and the measured liquid-level histories. The calculation is within the measurement uncertainty.

VI. INPUT LISTINGS

This section contains three separate input listings. The first is a complete steady-state one-dimensional input deck for the calculations described previously. The second is a restart input deck that begins the transient calculation. Finally, the third listing presents a threedimensional vessel input (to start a steady-state run) that is equivalent to the one-dimensional input. However, the three-dimensional VESSEL for this listing was extracted from a LOFT input deck used to model another transient (large-break (Test L2-3) and, therefore, represents identical geometry but not identical initial and boundary conditions. (Note there is no reactivity calculation in the input.) This listing demonstrates how the vessel can be modeled using the three-dimensional vessel-modeling capability. In this case, all the components shown in Fig. E-5 were combined into a single threedimensional VESSEL component as shown in Fig. E-6. A detailed description of 
another sample TRAC three-dimensional VESSEL is given in App. $C$ of the TRAC-PD2 users' manual. 78 The geometrical considerations given there also apply to TRAC-PF1/MOD1.

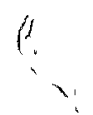

$=$ 
A. Steady-State One-Dimensional Input for LOFT $26-1$

$\begin{array}{llllllll}1 & 2 & 3 & 4 & 5 & 6 & 7 & 8\end{array}$

CARD 12345678901234567890123456789012345678901234567890123456789012345678901234567890

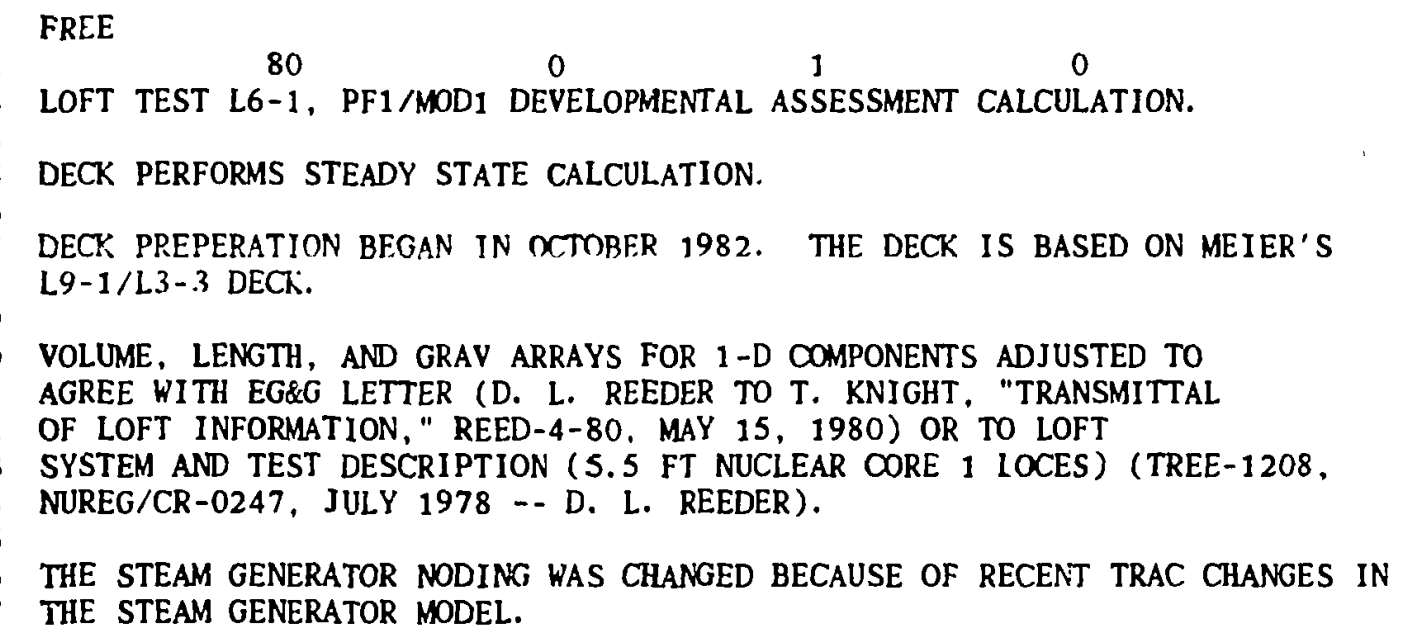


COMPROMISE TO THE FRAPCON CALCULATIONS (EG\&G, INFORMAL)

SHOWING GAP CLOSURE AND FUEL THERMAL CONDUCTIVITY DEGRADATION.

VESSEL VOLUMES CORRESPOND TO TABLE IX, P 121 OF TREE-1208

(D. L." REEDER, LOFT SYSTEM AND TEST DESCRIPTION). CORE AND UPPER

PLENUM HAVE COMPENSATING VOLUME ERRORS OF $1.9061 \mathrm{E}-4 \quad \mathrm{M}^{* *} 3$ (UPPER

PLENUM HIGH). THE DOWNCOMER AND LOWER PLENUM ALSO HAVE COMPENSATING

(BUT UNDETERMINED) VOLUME ERRORS. THE FLOW AREAS SHOULD BE

CHECKED FOR LARGE BREAK APPLICATIONS.

PRESSURE DROPS AROUND THE SYSTEM WERE CORRECTED PRIOR TO THE

L3-2 PRETEST PREDICTION. THE REFLOOD ASSIST BYPASS LINES WERE

CONNECTED AT THAT TIME.

THE SYSTEM HEAT LOSSES ARE ACCOUNTED FOR THROUGH HEAT TRANSFER

FROM THE EXTERNAL AREAS OF THE PIPING HEAT SLABS TO THE ENVIRONMENT

THROUGH HOUTV. THE SINK TEMPERATURE IS ASSUMED TO BE $305.37 \mathrm{~K}$.

IN ORDER TO ESTIMATE HOUTV. THE HEAT LOSSES ARE ASSUMED TO BE $174 \mathrm{KW}$

FROM THE PRIMARY SYSTEM (EXCLUDING THE PRESSURIZER), 6 KW FROM THE

PRESSURIZER, AND $10 \mathrm{KW}$ FROM THE STEAM GENERATOR SECONDARY.

(EGG-LOFT-5983, AUGUST 1982, P19).

COMPONENTS 31 AND 41 (BROKEN LOOP HOT AND COLD LEGS) HAVE

BEEN CONNECTED (AS IN L3-2) THROUGH THE REFLOOD ASSIST

BYPASS LINES WITH $5 \%$ FLOW (USED HD TO ACCOUNT FOR HYDRAULIC

LOSS IN WALL FRICTION WITH TWO-PHASE MULTIPLIER INSTEAD OF FRIC).

COMPONENT ORDER IN INPUT:

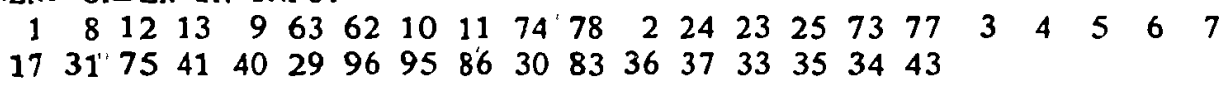

SINOPTS ICFLOW=2 S

NAMELIST DATA

\begin{tabular}{|c|c|c|c|c|c|c|}
\hline 0 & 0.000000 & & & & & ${ }^{* M C C} \quad 01$ \\
\hline 1 & 0 & 39 & 48 & & 1 & $* M O C \quad 02$ \\
\hline $1.00000 \mathrm{E}-3$ & $1.00000 \mathrm{E}-5$ & $1.00000 \mathrm{E}-4$ & 1. $00000 \mathrm{E}-3$ & & & ${ }^{*} \mathrm{MCC} \quad 03$ \\
\hline 10 & 0 & 25 & & & & *MCC 04 \\
\hline${ }^{*} \mathrm{NTSV} * 24$ & *NTCB* 29 & ${ }^{*}$ NTCF * 138 & *NTRP* 21 & *NTCP* & 2 & *MCC 05 \\
\hline 1 & 2 & 3 & 4 & & 5 & *IORDER \\
\hline 6 & 7 & 8 & 9 & & 10 & *IORDER \\
\hline 11 & 12 & $13 \mathrm{~S}$ & & & & *IORDER \\
\hline $17 \mathrm{~S}$ & & & & & & *IORDER \\
\hline 23 & 24 & $25 \mathrm{~S}$ & & & & *IORDER \\
\hline 29 & $30 \mathrm{~S}$ & & & & & *IORDER \\
\hline 31 & 33 & 34 & $35 S$ & & & *IORDER \\
\hline 36 & $37 "$ & $40 S$ & 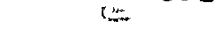 & & & * IORDER \\
\hline
\end{tabular}


101

102

103

104

105

106

107

108

109

110

111

112

113

114

115

116

117

118

119

120

121

122

123

124

125

126

127

128

129

130

131

132

133

134

135

$: 36$

137

138

139

, 140

141

142

143

144

145

146

147

148

149

150

$\begin{array}{ll}41 & 43 \mathrm{~S} \\ 62 & 63 \mathrm{~S} \\ 73 & 74 \\ 77 & 78 \mathrm{~S}\end{array}$

$18 \mathrm{~S}$

$-15 \mathrm{~S}$

$-24 \mathrm{~S}$

$24 \mathrm{~S}$

$-70 \mathrm{~S}$

$\mathrm{OE}$
* IORDER

* IORDER

* IORDER

* IORDER

* IORDER

* IORDER

* IORDER

* IORDER

* I SV

* ICB

* ITP

* ISV

* ICB

*ITP

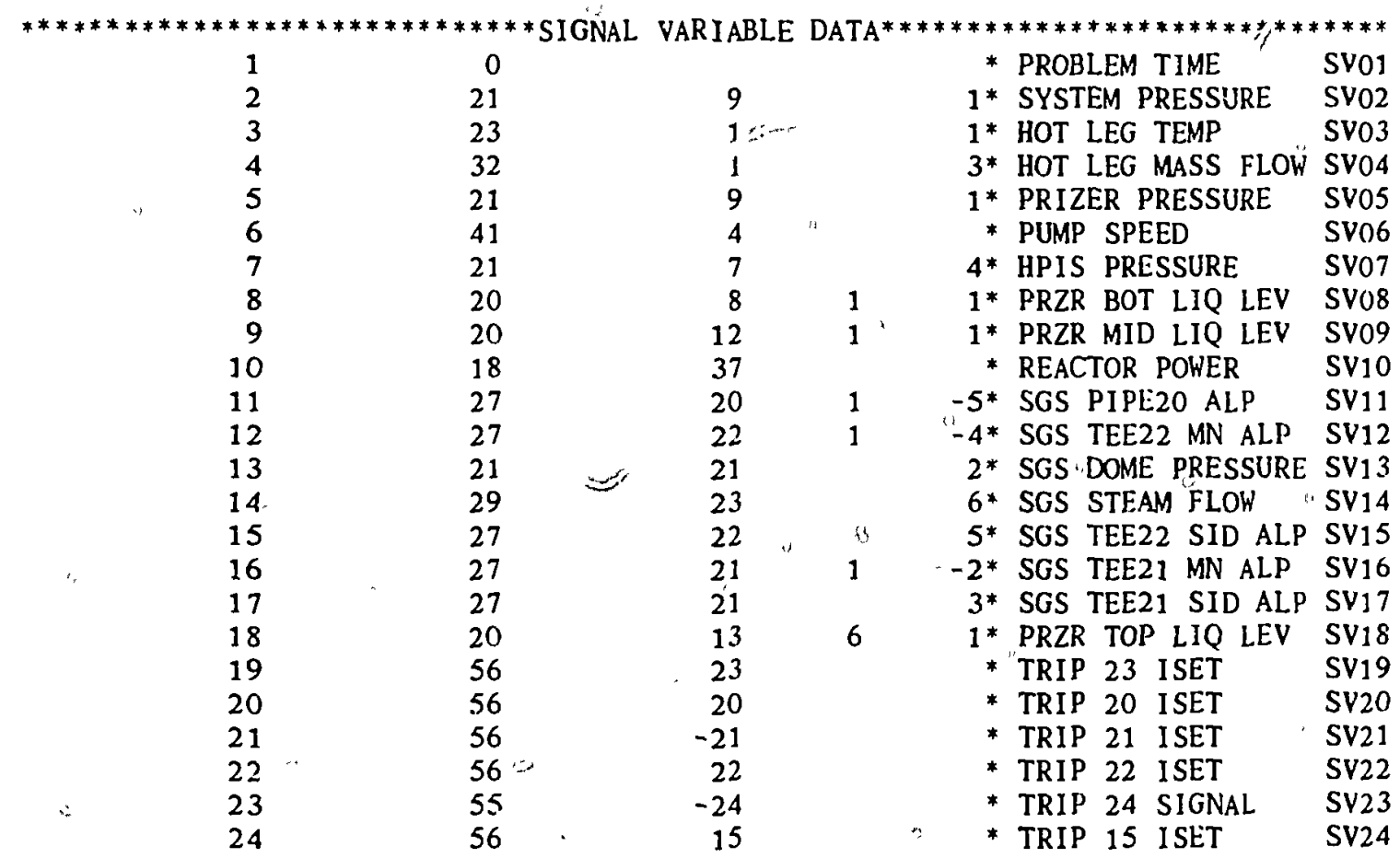

PRESSURIZER LIQUID LEVEL CALCULATION FOR PLOTTING

$\begin{array}{rrr}-1 & 3 & 8 \\ 1.000000 & 0.000000 & 1.350000\end{array}$

$-2$

1.000000

0.000000

1. $75000^{-1}$

18

${ }^{*} \mathrm{CBO} 01$

${ }^{*} \mathrm{CBO} 1$

$-3$

(3) 50

1.000000

0.000000

$1.640000^{-2}$

$-0.110000$

*CB02

* $\mathrm{CBO} 3$

SGS FEEDWATER PUMP MACRO CONTROLLER

${ }^{*} \mathrm{CBO} 3$ 


-4
1.000000
0.000000
13.720000
27.440000
999.000000
-5
1.000000

0.000000

0.2462 .00

0.391700

0.473500

0.568200

0.651500

0.719700

0.784100

0.897700

1.000000

$1.000000^{-6}$

1.000000
-7

1. 000000

1. $000000^{-8}$

$-9$

1. 000000 $-10$

1. 000000

0.000000 $-11$

1. 000000

0.000000

2. 266048

4. 150213

4. 835973

5. 405128

6.670213

$-12$

3. 149606 $-13$

1. 000000 $-14$

7.000000 $-15$

1. 000000 $-16$

$-0.500000$

1. 000000 $-18$
101

0.000000

0.0100100

0.391700

0.756200

1. $000000 \mathrm{E}$ 101

0.000000

0.000000

0.050000

0.136000

0. 200000

0.300000

0.400000

0.500000

0.600000

0.800000

1. $000000 \mathrm{E}$ 59

0.000000

59

0. 000000

$0.000000^{3}$

59

0.000000

101

2. 946400

2. 946400

101

0.000000

$5.610300 \mathrm{~S}$

5. 610300

3. 200400

2. 544000

1.886000

$0.000000 \mathrm{E}$

54
-8.390235

3

0.000000

$-0.050000$

23

0.000000
23

$-1.000000$

3
0.000000
101
14
1.000000

6.870000

20.580000

37.130000

1. -4

0.189400

0.337100

0.412900

$\therefore \quad 0.526500$

0.613600

0.689400

0.750000

0.848500

0.954500

12
1. 280323

1.280323
16

2.617779

3.898102

11

6.670213

3.200400

5. $0000 \mathrm{E}+07$

5.610300

2.594344

4. 417293

5.029433

5.840249

10.080000

$-12$

1.000000

$-13$

0.050000

$-14$

1.000000

19

0.000000

-15
0.634857

$-17$
7

0. $200000 \mathrm{~S}$

$0.577000 \mathrm{~S}$

$1.000000 \mathrm{~S}$

19

$0.025000 \mathrm{~S}$

$0.100000 \mathrm{~S}$

$0.150000 \mathrm{~S}$

$0.250000 \mathrm{~S}$

$0.350000 \mathrm{~S}$

$0.450000 \mathrm{~S}$

$\because 0.550000 \mathrm{~S}$

$0.700000 \mathrm{~S}$

$0.900000 \mathrm{~S}$

15
1.264108
17

2. 234813

$-7$

$-8$

2. 772111

3. $200400 \mathrm{E}$

10

4. $681000 \mathrm{~S}$

$2.946400 \mathrm{~S}$

2. $350000 \mathrm{~S}$

1. $246000 \mathrm{~S}$

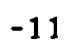

$-5$

$-15$

0.016215

0.382966

.

1.000000

${ }^{*} \mathrm{CBO} 4$

*CBO 4

*CBO 4

${ }^{*} \mathrm{CB} 04$

- CBO 4

*CBOA

${ }^{*} \mathrm{CB} 05$

*CB05

*CB05

*CB05

*CB05

* CBO5

${ }^{*} \mathrm{CB} 05$

${ }^{*} \mathrm{CBC5}$

*CB05

*CB05

*CBO5

*CB05

${ }^{*} \mathrm{CB} 06$

${ }^{*} \mathrm{CB} 06$

* $\mathrm{CB} 07$

${ }^{*} \mathrm{CB} 07$

*CB08

*CB08

*CB09

${ }^{*} \mathrm{CB} 09$

${ }^{*} \mathrm{CB} 10$

*CB10

${ }^{*} \mathrm{CB} 10$

${ }^{*} \mathrm{CB} 11$

${ }^{*} \mathrm{CB} 11$

*CB11

${ }^{*} \mathrm{CB} 11$

${ }^{*}$ CB 11

${ }^{*} \mathrm{CB} 11$

${ }^{*} \mathrm{CB} 11$

${ }^{*} \mathrm{CB} 11$

*CB12

*CB i2

${ }^{*} \mathrm{CB} 13$

${ }^{*} \mathrm{CB} 13$

${ }^{*} \mathrm{CB} 14$

${ }^{*} \mathrm{CB} 14$

*CB15

*CB 15

${ }^{*} \mathrm{CB} 16$

${ }^{*} \mathrm{CB} 16$

*CB17

*CB17

19

${ }^{*} \mathrm{CB} 18$ 


$\begin{array}{lrr}201 & & 1.000000 \\ 202 & \text { R02 } & 0.000000 \\ 203 & & 1.0000 \mathrm{E}-01 \\ 204 & & 4.1290 \mathrm{E}-01 \\ 205 & & 3.0000 \mathrm{E}-01 \\ 206 & & 6.5150 \mathrm{E}-01 \\ 207 & & 5.5000 \mathrm{E}-01 \\ 208 & & 8.4850 \mathrm{E}-01 \\ 209 & \text { R02 } & 1.0000 \mathrm{E}+00 \mathrm{E}\end{array}$

0.000000

2. 5000E-02

3. $3710 E-01$

2. $0000 \mathrm{E}-01$

5. 6820E-0?

4. 5000E-01

7. 5000E-01
8. $0000 \mathrm{E}-01$
0.806548

1. 8940E-01

1. 3600E-01

4. $7350 \mathrm{E}-01$

3. 5000E-01

6. 8940E-01

6. 0000 [- 01

8. 9770E-01
5. $0000 \mathrm{E}-02$

3. $9170 E-01$

2. 5000E-01

6. $1360 \mathrm{E}-01$

5. $0000 \mathrm{E}-01$

7. $8110 E-01$
9. $0000[-01$
2. $4620 \mathrm{E}-01$

1. $5000 \mathrm{E}-01$

5. $2650 E-01$

4. $0000 \mathrm{E}-01$

7. $1970 \mathrm{E}-01$

7. $0000 \mathrm{E}-01$

9. $5450 \mathrm{E}-01$
*CB1 8

${ }^{*} \mathrm{CB} 18$

${ }^{*} \mathrm{CB} 18$

${ }^{*} \mathrm{CB} 18$

*CB18

*CB18

*CB1 8

*CB 18

*CB18

SGS STEAM FLOW CONTROL VALVE MACRO CONTROLLER
*

-20
$1.0000 E+00$

$-41$

1. $0000 \mathrm{E}+00$

$-42$

5. $0000 E-02$ $-43$

1. $0000 E+00$

$-44$

1. $0000 E+00$

R02 0.000000

3. $0000 \mathrm{E}-01$

4. $4000 \mathrm{E}-01$

7. $0000 \mathrm{E}-01$

8. 8665E-01R02
0.000000

22
$-1.0000 \mathrm{E}+00$

22

$-5.0000 \mathrm{E}-02$
23

0.000000

101

0.000000

1. $0000 \mathrm{E}-01$

2. $4500 \mathrm{E}-01$

5. $7500 \mathrm{E}-01$

6. $6000 \mathrm{E}-01$

$1.0000 \mathrm{E}+00 \mathrm{E}$

PUMP SPEED MACRO CONTROLLER $\begin{array}{rr}-50 & 1 \\ 1.000000 & 1.0000 \mathrm{E}-20 \\ -55 & 9 \\ 1.000000 & 478.500000 \\ -60 & 14\end{array}$

0.100000

-62
1.000000

1.000
-65

1.000000 $-70$

1.000000
0.000000

0.900000

39

200.000000

200.000000
$1.0000 \mathrm{E}+00$

5. 22

$-42$

$1.0000 \mathrm{E}+00$

$1.0000 E+00$

7. $3360 E-02$

4. $0000 \mathrm{E}-01$

5. $1733 E-01$

8. $0000 \mathrm{E}-01$
$1.0000 \mathrm{E}+00$

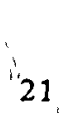

21

$-41$

12

2. $0000 E-01$

3. $4000 \mathrm{E}-01$

6. $0000 \mathrm{E}-01$

7. $7248 \mathrm{E}-01$
${ }^{*} \mathrm{CB} 20$

*CB20

$-20{ }^{*} \mathrm{CB} 41$

*CB41

$19{ }^{*} \mathrm{CB} 42$

${ }^{*} \mathrm{CB} 42$

${ }^{*} \mathrm{CB} 43$

${ }^{*} \mathrm{CB} 43$

${ }^{*} \mathrm{CB} 44$

-CB 44

1.5334E-01 *CB44

5. $0000 \mathrm{E}-01{ }^{*} \mathrm{CB} 44$

5. 4666E $-01 \quad{ }^{*} \mathrm{CB} 44$

$9.0000 \mathrm{E}-01$

${ }^{*} \mathrm{CB} 44$

*CB44

4
$1.00000 E+3$

478.500000

$-55$

0.200000

478. 500000

$-50$

*CB50

*CB50

*CB55

*CB55

*CB60

*CB60

1. 100000

0.900000

${ }^{*} \mathrm{CB} 62$

*CB65

500.000000

$500.000000^{6}$
${ }^{*} \mathrm{CB} 65$

$-65$

*CB70
24

\section{2}

SYSTEM LOW PRESSURE SCRAM TRIP

14. $3600 \mathrm{E}+6$

0

2

15. $7690 \mathrm{E}+6$

0.000000
14. $3610 \mathrm{E}+6$ 0.000000

15. $7700 \mathrm{E}+6$

0.000000
0.000000
0

1

1
*CB70 $\begin{array}{ll}0 & \text { *TRIP DIM } \\ 2 & \text { *TRIP01 } \\ & \text { *TRIPO1 } \\ & \text { *TRIP01 } \\ & \text { *TRIP01 } \\ 2 & \text { *TRIP02 } \\ & \text { *TRIP02 } \\ & \text { *TRIP02 }\end{array}$ 


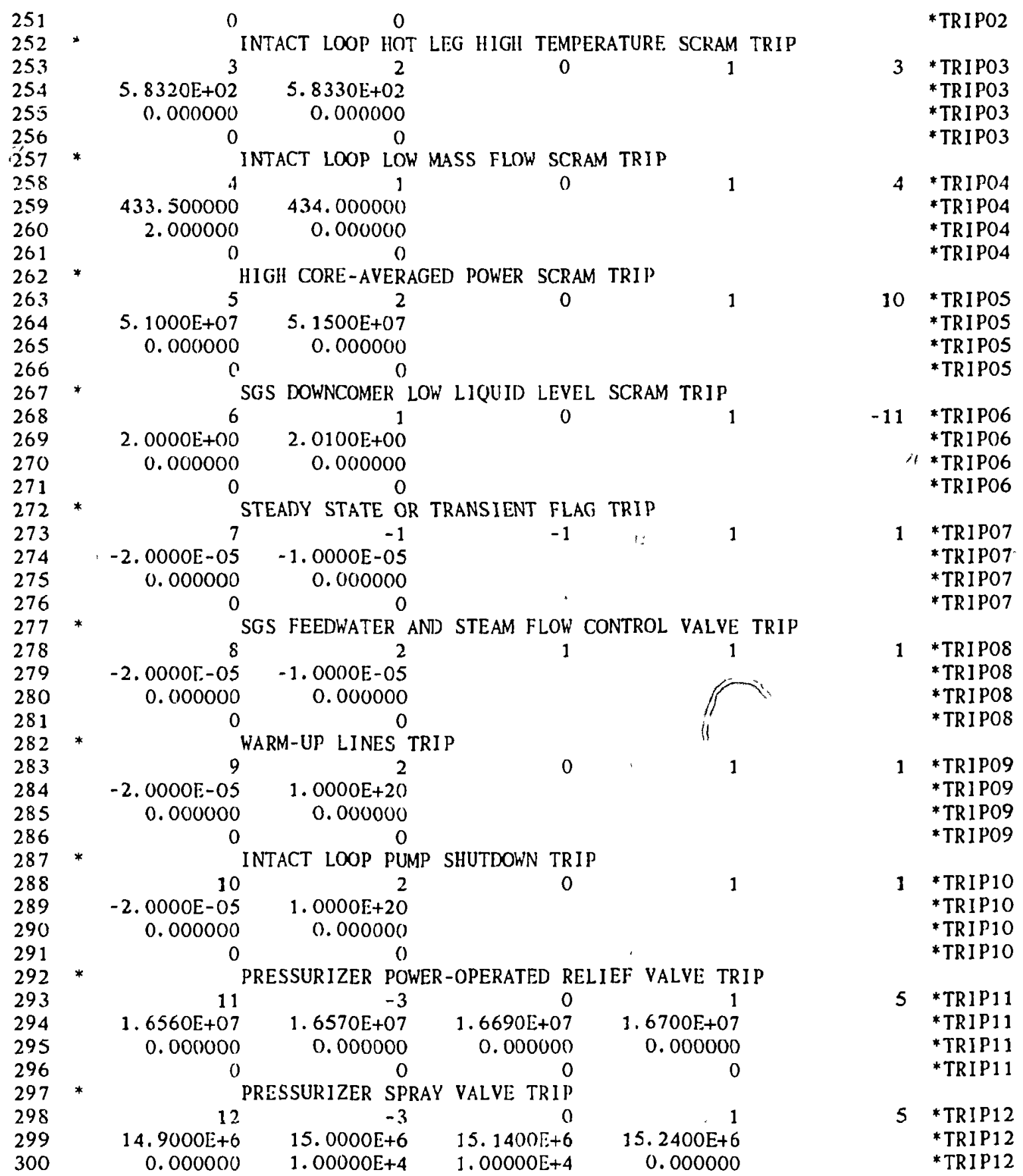




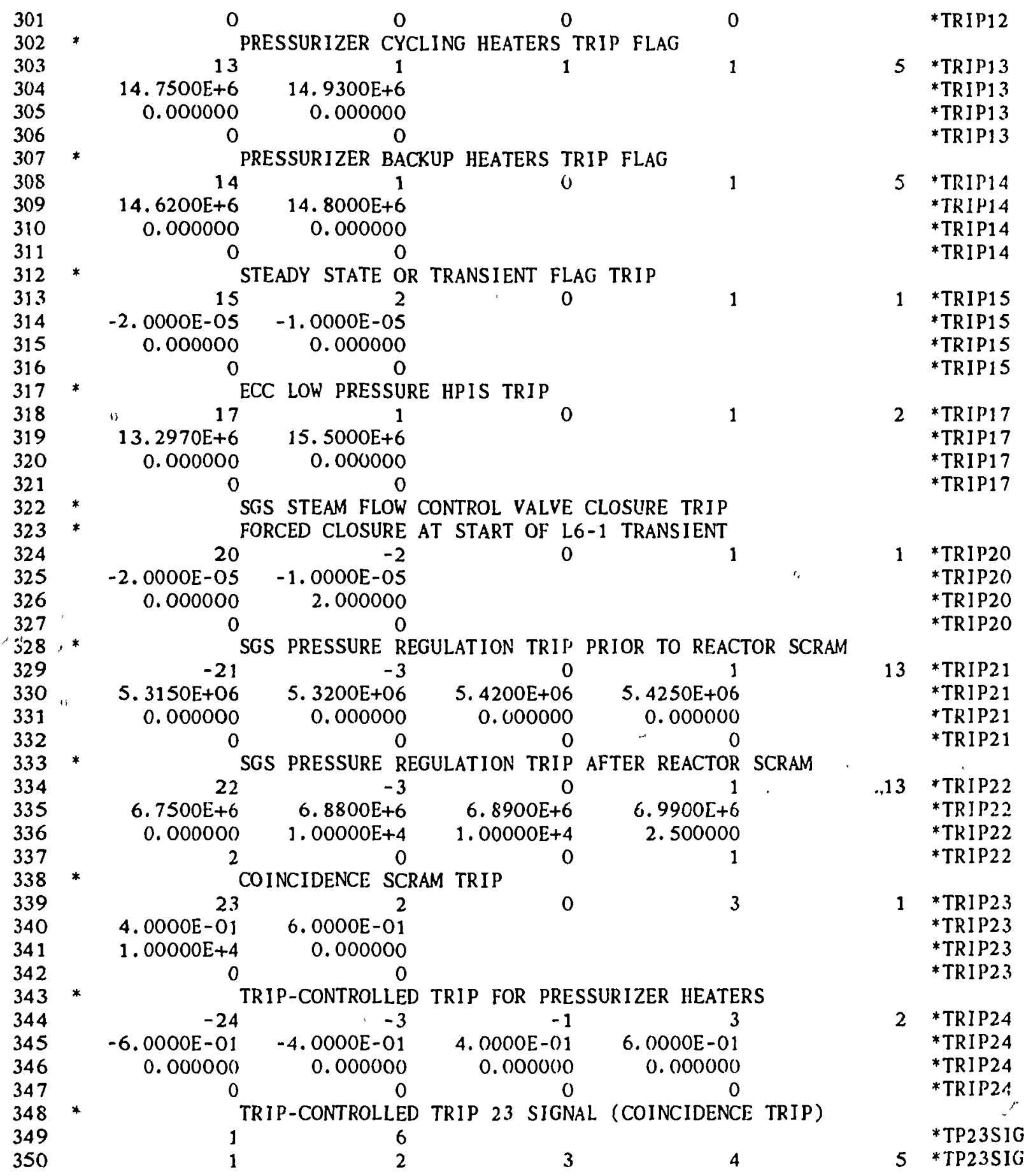




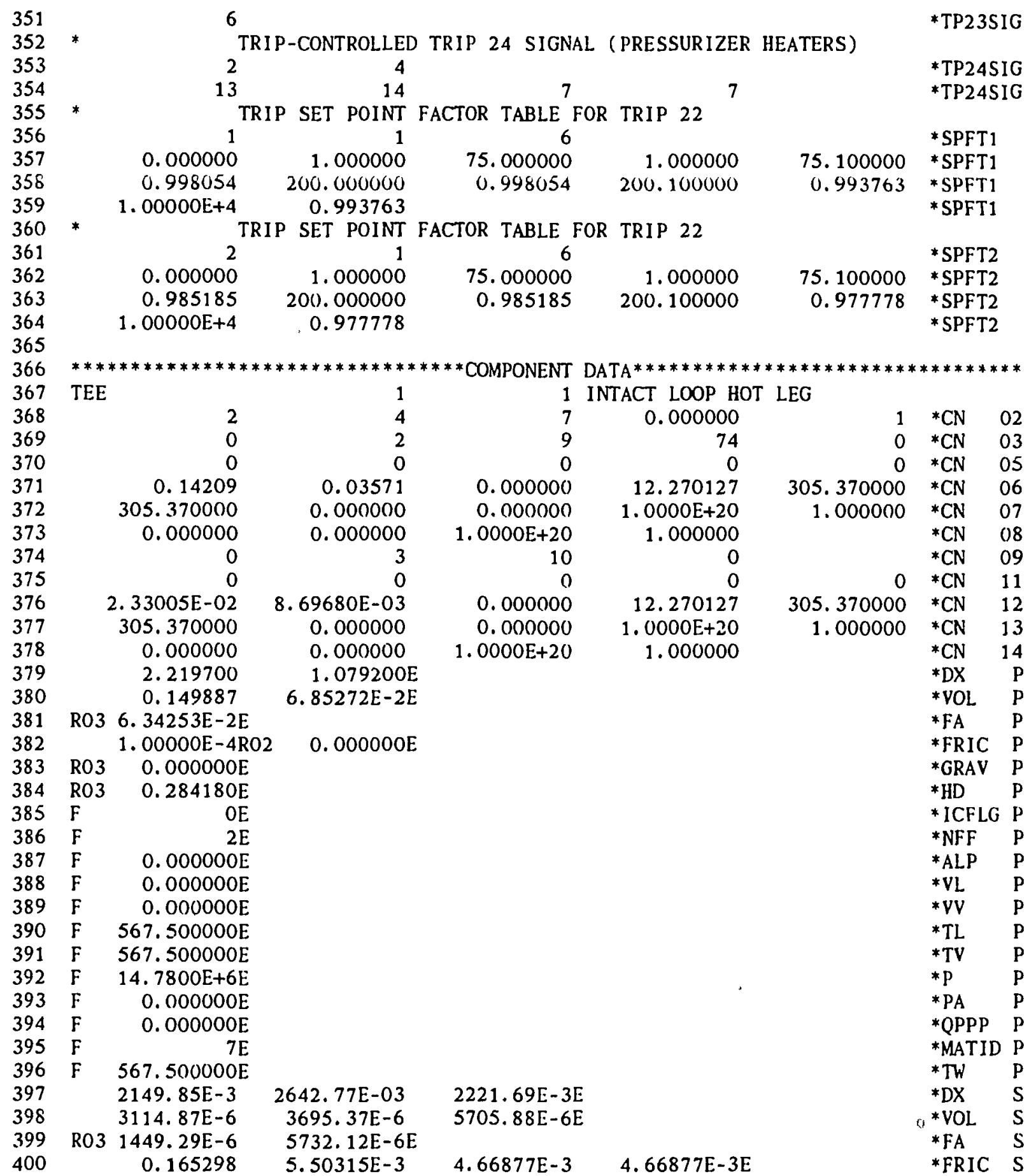




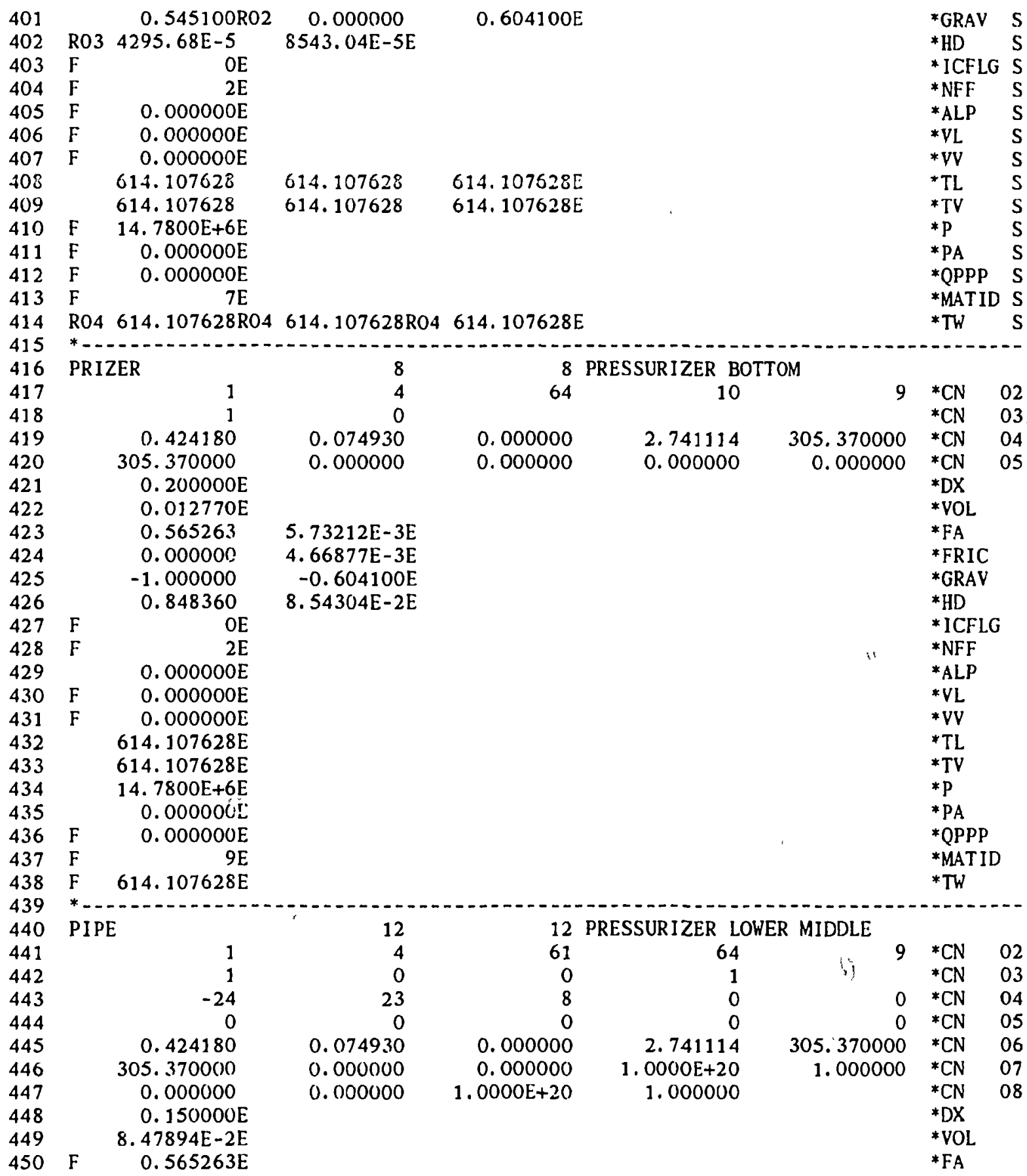




\begin{tabular}{|c|c|c|c|c|c|c|c|c|}
\hline 451 & $\mathrm{~F}$ & $0.000000 \mathrm{E}$ & & & & & \multicolumn{2}{|l|}{${ }^{*} \mathrm{FRIC}$} \\
\hline 452 & $F$ & $-1.000000 \mathrm{E}$ & & & & & * GRAV & \\
\hline 453 & $\mathrm{~F}$ & $0.848360 \mathrm{E}$ & & & & & \multicolumn{2}{|l|}{ *HD } \\
\hline 454 & $\mathrm{~F}$ & $\mathrm{OE}$ & & & & & \multicolumn{2}{|c|}{ *ICFLG } \\
\hline 455 & $\mathrm{~F}$ & $2 \mathrm{E}$ & & & & & \multicolumn{2}{|l|}{ *NFF } \\
\hline 456 & & $0.000000 \mathrm{E}$ & & & & & \multicolumn{2}{|l|}{ *ALP } \\
\hline 457 & $\mathrm{~F}$ & $0.000000 \mathrm{E}$ & & & & & \multicolumn{2}{|l|}{$\approx \mathrm{VL}$} \\
\hline 458 & $\mathrm{~F}$ & $0.000000 \mathrm{E}$ & & & & & \multicolumn{2}{|l|}{$* \mathrm{VV}$} \\
\hline 459 & & $614.107628 \mathrm{E}$ & & & & & \multicolumn{2}{|l|}{$* T L$} \\
\hline 460 & & $614.107628 \mathrm{E}$ & & & & & \multicolumn{2}{|l|}{$*$ TV } \\
\hline 461 & & $14.7800 \mathrm{E}+6 \mathrm{E}$ & & & & & \multicolumn{2}{|l|}{$* \mathrm{p}$} \\
\hline 462 & & $0.000000 \mathrm{E}$ & & & & & \multicolumn{2}{|l|}{ *PA } \\
\hline 463 & $\mathrm{~F}$ & $0.000000 \mathrm{E}$ & & & & & \multicolumn{2}{|l|}{ *QPPP } \\
\hline 464 & $\mathrm{~F}$ & $9 \mathrm{E}$ & & & & & \multicolumn{2}{|c|}{ *MATID } \\
\hline 465 & $\mathrm{~F}$ & $614.107628 \mathrm{E}$ & & & & & \multicolumn{2}{|c|}{$* T w$} \\
\hline 466 & & -1.250000 & 398.000000 & -0.750000 & $398.000000 \mathrm{~S}$ & & \multirow{2}{*}{\multicolumn{2}{|c|}{ *POWTB }} \\
\hline 467 & & -0.500000 & 0.000000 & 0.500000 & $0.000000 \mathrm{~S}$ & & *POWTB & \\
\hline 468 & & 0.750000 & $3.60000 \mathrm{E}+4$ & 1.250000 & 3. $60000 \mathrm{E}+4 \mathrm{~S}$ & & \multicolumn{2}{|c|}{ *POWTB } \\
\hline 469 & & 1.750000 & 4. $80000 E+4$ & 2.250000 & 4. $80000 E+4 E$ & & *POWT & \\
\hline 470 & * & $\ldots \ldots$ & $\cdots$ & $-\ldots$ & - & - & $\ldots-$ & $\cdots$ \\
\hline 471 & PR & I ZER & 13 & 13 & PRESSURIZER UPP & ER MIDDLE & & \\
\hline 472 & & 6 & 4 & 60 & 61 & 9 & ${ }^{*} \mathrm{CN}$ & 02 \\
\hline 473 & & 1 & 0 & & & & ${ }^{*} \mathrm{CN}$ & 03 \\
\hline 474 & & 0.424180 & 0.074930 & 0.000000 & 2.741114 & 305.370000 & ${ }^{*} \mathrm{CN}$ & 04 \\
\hline 475 & & 305.370000 & 0.000000 & 0.000000 & 0.000000 & 0.000000 & $*^{*} \mathrm{CN}$ & 05 \\
\hline 476 & & 0.400000 R05 & $0.200000 \mathrm{E}$ & 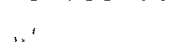 & & & *DX & \\
\hline 477 & & $0.202587 R 05$ & $0.113053 \mathrm{E}$ & & & & *VOL & \\
\hline 478 & $\mathrm{~F}$ & $0.565263 \mathrm{E}$ & 19 & & $\therefore$ & & $* F A$ & \\
\hline 479 & $\mathrm{~F}$ & $0.000000 \mathrm{E}$ & & & & & *FRIC & \\
\hline 480 & $\mathrm{~F}$ & $-1.000000 \mathrm{E}$ & & & & & *GRAV & \\
\hline 481 & $\mathrm{~F}$ & $0.848360 \mathrm{E}$ & & & & & *HD & \\
\hline 482 & $\mathrm{~F}$ & OE & a & . & & & ${ }^{*} \mathrm{ICFL}$ & \\
\hline 483 & $\mathrm{~F}$ & $2 \mathrm{E}$ & & & & & *NFF & \\
\hline 484 & & 1.000000 & $0.290325 R 04$ & $0.000000 \mathrm{E}$ & & & *ALP & \\
\hline 485 & $\mathrm{~F}$ & $0.000000 \mathrm{E}$ & & & & & $* \mathrm{VL}$ & \\
\hline 486 & $\mathrm{~F}$ & $0.000000 \mathrm{~L}$ & & & & & $* \mathrm{VV}$ & \\
\hline 487 & $\mathrm{~F}$ & $614.107628 \mathrm{E}$ & & & & - & ${ }^{*} \mathrm{TL}$ & \\
\hline 488 & $\mathrm{~F}$ & $614.107628 \mathrm{E}$ & & & & & $* T V$ & \\
\hline 489 & $\mathrm{~F}$ & $14.7800 \mathrm{E}+6 \mathrm{E}$ & & & & & $* \mathrm{P}$ & \\
\hline 490 & $\mathrm{~F}$ & $0.000000 \mathrm{E}$ & & & & & $* \mathrm{PA}$ & \\
\hline 491 & $\mathrm{~F}$ & $0.000000 \mathrm{E}$ & & & & & *QPPP & \\
\hline 492 & $\mathrm{~F}$ & $9 \mathrm{E}$ & & & & & *MATI & \\
\hline 493 & $\mathrm{~F}$ & $614.107628 \mathrm{E}$ & & & & & $* T W$ & \\
\hline 494 & & & & & & & & \\
\hline 495 & TEE & & 9 & 91 & PRIZER TOP AND & PRIZER SPRAY & & \\
\hline 496 & & 1 & 4 & 9 & 0.000000 & 1 & ${ }^{*} \mathrm{CN}$ & 02 \\
\hline 497 & & 0 & 5 & 60 & 62 & 0 & $* \mathrm{CN}$ & 03 \\
\hline 498 & & 0 & 0 & 0 & 0 & 0 & ${ }^{*} \mathrm{CN}$ & 05 \\
\hline 499 & & 0.424180 & 0.074930 & 0.000000 & 2.741114 & 305.370000 & ${ }^{*} \mathrm{CN}$ & 06 \\
\hline 500 & & 305.370000 & 0.000000 & 0.000000 & $1.0000 E+20$ & 1.000000 & ${ }^{*} \mathrm{CN}$ & 07 \\
\hline
\end{tabular}




$\begin{array}{lr}501 & 0.000000 \\ 502 & 0 \\ 503 & 7 \\ 504 & 0\end{array}$

505

506

507

508

509

510

511

512

513

514

515

516

517

518

519

520

521

522

523

524

525

526

527

528

529

530

531

532

533

534

535

536

537

538

539

540

541

542

543

544

545

546

547

548

549

550
0.023300

305. 370000

0.000000

$0.130732 \mathrm{R} 04$

0.066211

0.565263

F $\quad 0.000000 \mathrm{E}$

F $\quad 1.000000 E$

0.848360

$F \quad O E$

$\mathrm{F} \quad 2 \mathrm{E}$

$\mathrm{F} \quad 1.000000 \mathrm{E}$

F $\quad 0.000000 E$

F $\quad 0.000000 E$

F $\quad 614.107628 \mathrm{E}$

F $614.107628 \mathrm{E}$

F $\quad 14.7800 E+6 E$

F $\quad 0.000000 \mathrm{E}$

$\mathrm{F} \quad 0.000000 \mathrm{E}$

$\mathrm{F} \quad 9 \mathrm{E}$

F $614.107628 \mathrm{E}$

$\mathrm{F} \quad 0.046022 \mathrm{E}$

F $\quad 6.66991 \mathrm{E}-5 \mathrm{E}$

1. $44929 \mathrm{E}-4$

F $\quad 0.000000 E$

F $\quad-1.000000 E$

F 4. $29568 \mathrm{E}-2 \mathrm{E}$

$F \quad O E$

$\mathrm{F} 2 \mathrm{E}$

F $\quad 0.000000 E$

$\mathrm{F} \quad 0.000000 \mathrm{E}$

F $\quad 0.000000 \mathrm{E}$

F $614.107628 \mathrm{E}$

F $\quad 614.107628 \mathrm{E}$

$\mathrm{F} \quad 14.7800 \mathrm{E}+6 \mathrm{E}$

$F \quad 0.000000 E$

$F \quad 0.000000 E$

$\mathrm{F} \quad 6 \mathrm{E}$

F $\quad 614.107628 \mathrm{E}$

0.000000

50.000000

1. $00000 \mathrm{E}+4$
0.000000

1
1
0
0.008797
0.000000

0.000000

$0.100000 \mathrm{E}$

$1.0000 \mathrm{C}+20$

$$
68
$$

5

7. 30420E-4RU3 7.06858t-5t

1. $76715 \mathrm{E}-2 \mathrm{RO} 47.06858 \mathrm{E}-4 \mathrm{E}$

0.150000 RO4 3.00000E-2E

1. 44929E-3E

\begin{abstract}
0.000000
\end{abstract}
0.000000

$1.0000[+20$

$1.0000 \mathrm{E}+20$

1.000000

1.000000

1

0

0
20
00

.

$1.00000 \mathrm{E}+4 \quad{ }^{*} \mathrm{CN} \quad 13$

${ }^{*} \mathrm{CN} \quad 14$

*DX P

*VOL P

* FA P

*FRIC P

*GRAV P

*HD

* ICFLG P

*NFT P

*ALP P

*VL P

$* V V \quad P$

*TL $\quad \mathrm{P}$

*TV P

* $\mathrm{p} \quad \mathrm{P}$

*PA P

*QPPP P

*MATID P

*TW P

*DX S

*VOL S

*FA S

*FRIC S

* GRAV S

*HD S

*ICFLG S

*NFF $S$

*ALP S

*VL S

*VV S

*TL S

*TV S

*P $S$

*PA S

*QPPP S

*MATID $S$

*TW S

*POWTB2

* POWTB2

* POKT132

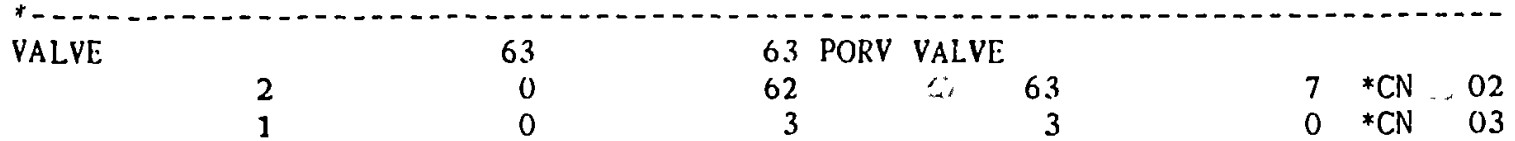




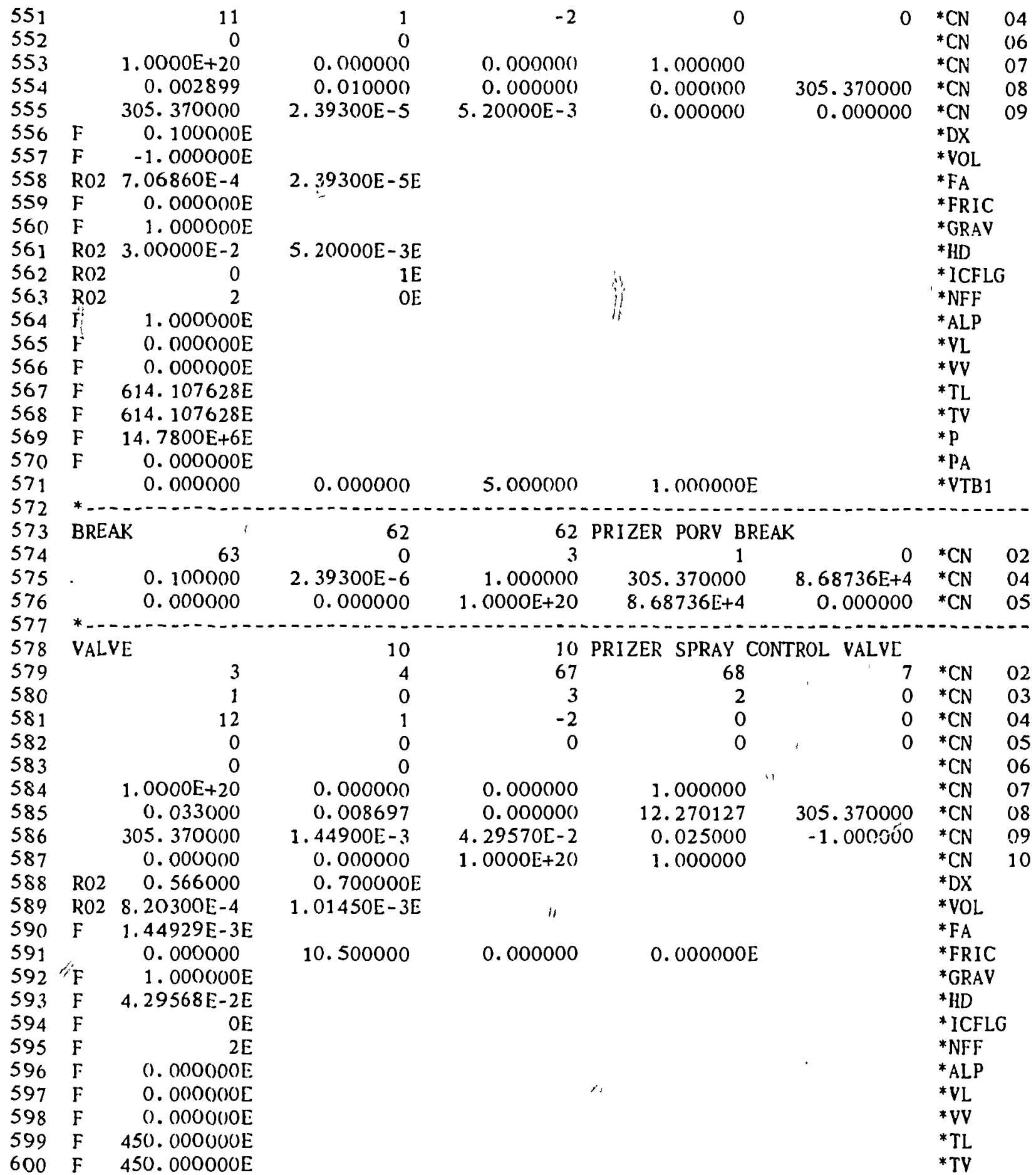




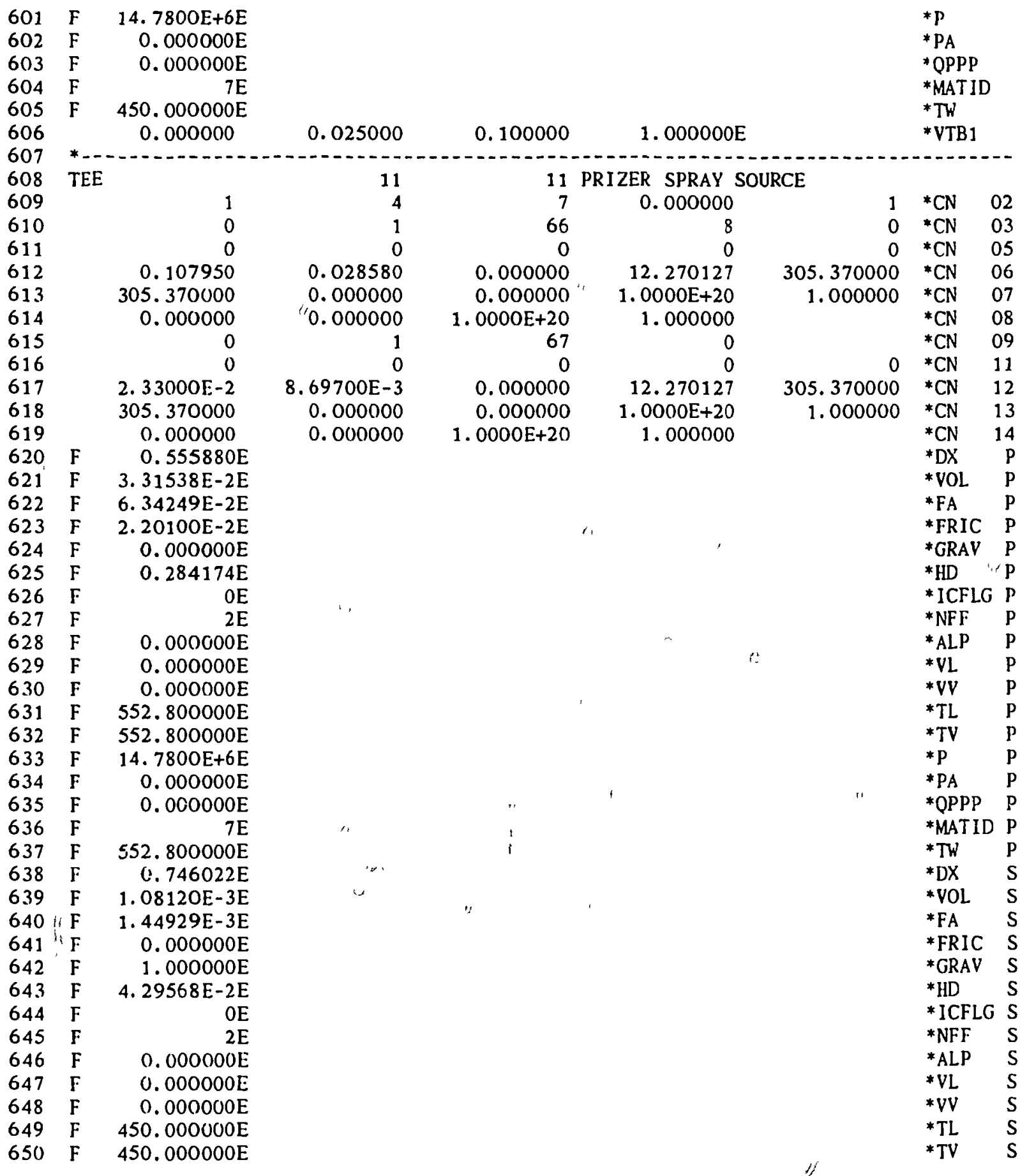


$\begin{array}{rlr}651 & \mathrm{~F} & 14.7800 \mathrm{E}+6 \mathrm{E} \\ 652 & \mathrm{~F} & 0.000000 \mathrm{E} \\ 653 & \mathrm{~F} & 0.000000 \mathrm{E} \\ 654 & \mathrm{~F} & 7 \mathrm{E} \\ 655 & \mathrm{~F} & 450.000000 \mathrm{E} \\ 656 & * & \\ 657 & \mathrm{TEE} & 1 \\ 658 & & 0 \\ 659 & & 0 \\ 660 & & 0.142090\end{array}$

$661 \quad 0.142090$

$662 \quad 305.370000$

$663 \quad 0.000000$

664

665

666

667

668

669

670

671

672

673

674

675

676

677

678

679

680

681

682

683

684

685

686

687

688

689

690

691

69

693

$694 \mathrm{~F}$

$695 \mathrm{~F}$

$696 \mathrm{~F}$

$697 \mathrm{~F}$

$698 \quad \mathrm{~F}$

$699 \quad F$

700

0
0

0.023300

305.370000

0.000000

4. $95548 \mathrm{E}-2$

R02 $6.34253 \mathrm{E}-2$

0.000000

0.000000

R02

0.284180

F $\quad 0.000000 \mathrm{E}$

F $\quad 0.000000 E$

F $\quad 0.000000 E$

F $567.500000 E$

F $\quad 567.500000 \mathrm{E}$

F $14.7800 E+6 E$

F $\quad 0.000000 \mathrm{E}$

F $\quad 0.000000 E$

$\mathrm{F} \quad 7 \mathrm{E}$

F. $567.500000 \mathrm{E}$

5. $000000 \mathrm{E}$

$\mathrm{F} \quad 7.24650 \mathrm{E}-3 \mathrm{E}$

R03 1. 44930E-3E

F $0.000000 E$

0.000000
$4.29600 E-2 E$
1.016000

$\mathrm{F} \quad \mathrm{OE}$

$0.000000,0.000000 \mathrm{E}$

$\begin{array}{ll}* \mathrm{P} & \mathrm{S} \\ =\mathrm{PA}_{\mathrm{A}} & \mathrm{S}\end{array}$

* QPPP S

*MATID S

*TW S

74

4

2

0.035710

0.000000

0.000000

2
0

0.008797

0.000000

0.000000

$1.625600 \mathrm{E}$

$0.111610 \mathrm{E}$

5. $16000 E-2 E$

5. $39400 \mathrm{E}-2$

0.000000

$0.256300 \mathrm{E}$

$0.118300 \mathrm{E}$

$0.423700 \mathrm{E}$

74 RECIR LINE FROM IL HL TO BL CL

$\begin{array}{ll}7 & 0.000000\end{array}$

71

0

0.000000

0.000000

1. $0000 \mathrm{E}+20$

78
0

0.000000

0.000000

1. $0000 \mathrm{E}+20$

2

12.270127

$1.0000 E+20$

1.000000

0.000000

$1.0000 \mathrm{E}+20$

1.000000 $\begin{array}{ccc}{ }^{*} \mathrm{CN} & 02 \\ 0 & { }^{*} \mathrm{CI} & 03 \\ 0 & { }^{*} \mathrm{CN} & 05\end{array}$

305. $370000{ }^{*} \mathrm{CN} \backslash 06$

$1.000000 * \mathrm{CN}: 07$

${ }^{*} \mathrm{CN} \quad 08$

*CN 09

${ }^{*} \mathrm{CN} \quad 11$

$305.370000{ }^{*} \mathrm{CN} \quad 12$

$1.000000 \quad{ }^{*} \mathrm{CN} \quad 13$

${ }^{*} \mathrm{CN} \quad 14$

*DX

*VOL P

*FA P

* FRIC P

*GRAV P

*HD P

*ICFLG P

*NFF- $\mathrm{P}$.

*ALP P

$* V L \quad P$

*VV P

*TL P

*TV P

*P $\quad \mathrm{P}$

*PA $\quad P$

*OPPP P

*MATID P

*TW P

*DX S

*VOL S

*FA S

*FRIC S

* GRaV S

*HD S

*ICFLG S

*NFF S

*ALPS

*VL S

*VV $S$

*TL S

*TV S

*P S 


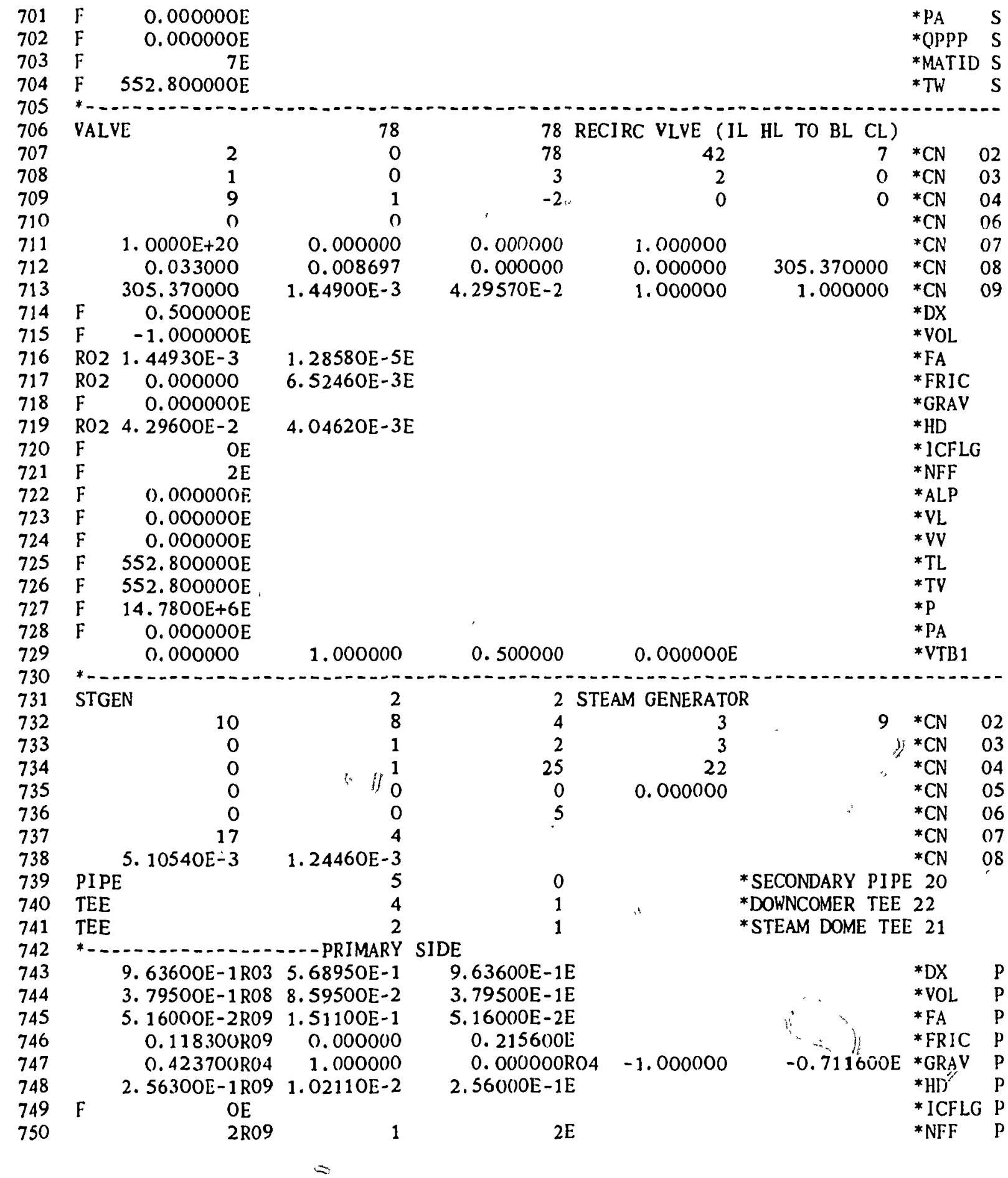




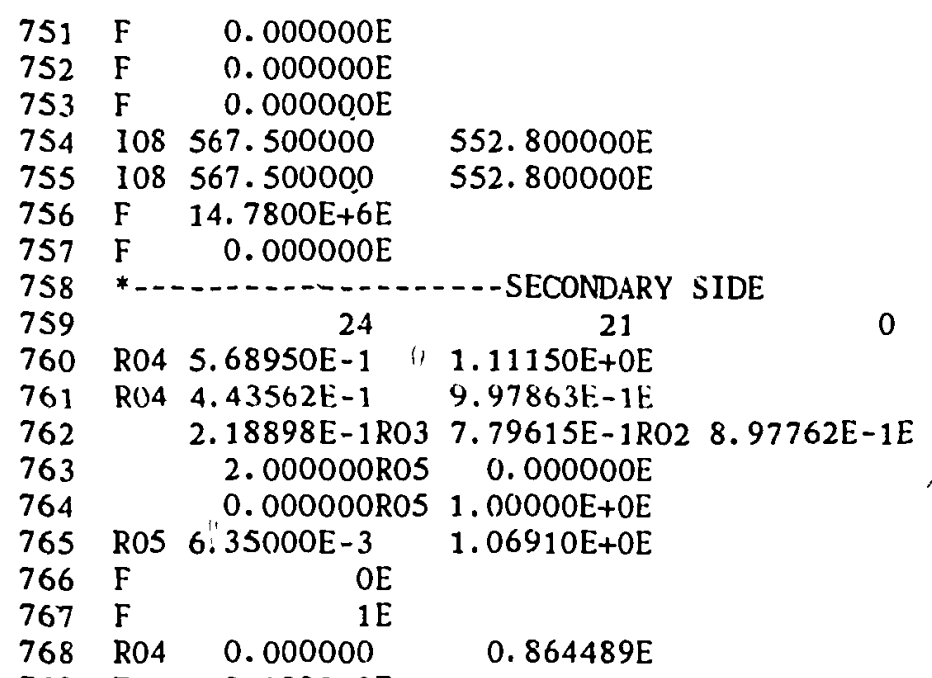

$0.796734 \mathrm{RO} O 2 \quad 0.000000 \mathrm{E}$
$5.68950 \mathrm{E}-1 \mathrm{E}$

1. $24544 \mathrm{E}-1 \mathrm{E}$

$0.000000 E$

6. $35000 \mathrm{E}-3 \mathrm{~F}$
1. $70685 \mathrm{E}+0$

3. $73632 \mathrm{E}-1$ $\begin{array}{ll}\text { *ALP } & \mathrm{P} \\ \text { *VL } & \mathrm{P} \\ \text { *VV } & \mathrm{P} \\ \text { *TL } & \mathrm{P} \\ \text { *TV } & \mathrm{P} \\ \text { *P } & \mathrm{P} \\ \text { *PA } & \mathrm{P}\end{array}$

20 *PIPE 20

*DX
*VOL
*FA
*FRIC
*GRAV
*HD
*ICFLC
*NFF
*ALP
*VL
*VV
*TL
*TV
*P
*PA

22 *TEE 22

$\begin{array}{ll}\text { *DX } & \mathrm{p} \\ \text { *VOL } & \mathrm{P} \\ \text { *FA } & \mathrm{p} \\ \text { *FRIC } & \mathrm{P} \\ \text { *GRAV } & \mathrm{P} \\ \text { *HD } & \mathrm{P} \\ \text { *ICFLG } & \mathrm{P} \\ \text { *NFF } & \mathrm{p} \\ \text { *ALP } & \mathrm{p} \\ \text { *VL } & \mathrm{p} \\ \text { *VV } & \mathrm{P} \\ \text { *TL } & \mathrm{P} \\ \text { *TV } & \mathrm{P} \\ \text { *P } & \mathrm{p} \\ \text { *PA } & \mathrm{P} \\ & \\ \text { *DX } & \mathrm{S} \\ \text { *VOL } & \mathrm{S} \\ \text { *FA } & \mathrm{S} \\ \text { *FRIC } & \mathrm{S} \\ \text { *GRAV } & \mathrm{S} \\ \text { *HD } & \mathrm{S} \\ \text { *ICFLG } & \mathrm{S} \\ \text { *NFF } & \mathrm{S}\end{array}$




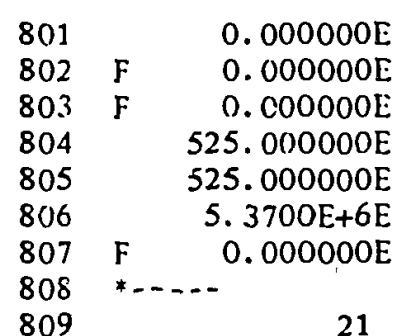

810 F $1.11150 E+0 E$

$8119.97863 \mathrm{E}-1$

812

813

814

815

816

817

818

819

820

821

822

823

824

825

826

827

828

829

830

831

832

833

834

835

836

837

838

839

840

841

842

843

844

845

846

847

848

849
850

R02 1.06910E+0

$\mathrm{F} O \mathrm{OL}$

$1 \mathrm{E}$

1. $000000 \mathrm{~F}$

$0.000000 E$

$0.000000 \mathrm{E}$

$541.571343 \mathrm{E}$

$541.571343 \mathrm{E}$

5. $3700 \mathrm{E}+6 \mathrm{E}$

F $\quad 0.000000 E$

0.000000

7. $41000 \mathrm{E}-1 \mathrm{E}$

3. $82966 \mathrm{E}-1 \mathrm{~F}$

5. $16842 \mathrm{E}-1 \mathrm{E}$

F $\quad 0.000000 E$

$-3.37626 \mathrm{E}-1 \mathrm{E}$

F 2.54000E-1E

F $\quad 0.000000 \mathrm{E}$

F $0.000000 E$

$541.571343 \mathrm{C}$

$541.571343 \mathrm{E}$

5. $3700 \mathrm{E}+6 \mathrm{E}$

F $\quad 0.000000 \mathrm{E}$

$\mathrm{RO} 8$

$\mathrm{RO3}$

R08
9. $97863 \mathrm{E}-1$
8. $97762 \mathrm{E}-1$
0.000000

F $1.000000 E$

$\mathrm{F} \quad O \mathrm{E}$

$1.000000 E$

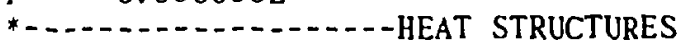

OR05
2

2

$1 \mathrm{RO2}$

1

1

2

20R05

1
22

1. $23695 \mathrm{E}+\mathrm{OE}$

1. $58904 \mathrm{E}+0$

1. $0000 \mathrm{E}+30$

4. $63292 \mathrm{E}-2 \mathrm{E}$

2. $44600 E-1 E$

2. $42900 \mathrm{E}-1 \mathrm{E}$

23
1
*ALP S

$* V L$ S

*VV S

*TL S

*TV S

*P $\quad S$

*PA S

21 *TEE 21

*DX P

*VUL P

*FA P

* FRIC P

* GRAV P

*HD

* ICFLG P

*NFF P

*ALP P

*VL P

*VV, $\quad \mathrm{P}$

*TL P

*TV P

*P P

*PA P

*DX a S

*VOL S

*FA S

*FRIC S

*GRAV S

S*HD

* ICFLG S

*NFF S

*ALP S

*VL S

*VV S

*TL S

*TV S

$* P \quad S$

*PA S

22R02

5

21E *ICMP

$6 *$ ICELL

* ICELL

* ICELL

$4 \mathrm{RO} 2 \quad 5 *$ ICELL

$4 \mathrm{~S}$

* ICELL

* ICELL

$\mathrm{ORO} 2 \quad \mathrm{OE} *$ OCMP

43 *OCELL 


\begin{tabular}{|c|c|c|c|c|c|c|c|c|}
\hline 851 & & 2 & $1 S$ & & \multicolumn{2}{|l|}{ *OCELL } \\
\hline 852 & $\mathrm{R} 02$ & 0 & 10R02 & \multirow{2}{*}{$\begin{array}{l}\text { OS } \\
2\end{array}$} & \multirow{2}{*}{\multicolumn{2}{|c|}{$1 \mathrm{~S}$}} & \multirow{2}{*}{\multicolumn{2}{|c|}{ *OCELL }} \\
\hline 853 & & $4 \mathrm{R} 03$ & 3 & & & & & \\
\hline 854 & R04 & OR02 & $\mathrm{OE}$ & \multirow[b]{2}{*}{ 6Rก6 } & \multirow{2}{*}{\multicolumn{2}{|c|}{$9 \mathrm{~S}$}} & \multicolumn{2}{|c|}{ *OCELL } \\
\hline 855 & R24 & $12 R 06$ & 9R03 & & & & \multicolumn{2}{|l|}{ *MATG } \\
\hline 856 & R18 & $6 R 18$ & $9 \mathrm{E}$ & & & & \multicolumn{2}{|l|}{ *MATG } \\
\hline 857 & R08 & $5.10540 \mathrm{E}-3$ & 0.685800 & $5.10540 \mathrm{E}-3$ & 10.000000 & 0.685800 & \multirow{2}{*}{\multicolumn{2}{|c|}{ *RADIG }} \\
\hline 858 & & 5. $10540 \mathrm{E}-3 \mathrm{R} 04$ & $0.644525 \mathrm{R} 02$ & $0.571500 R 06$ & $0.711200 \mathrm{E}$ & & & \\
\hline 859 & R08 & 1. $24460 \mathrm{E}-3$ & 0.088900 & $6.18435 E-3$ & 0.031750 & 0.088900 & \multicolumn{2}{|l|}{$* \mathrm{TH}$} \\
\hline 860 & & $6.18435 E-3 R 06$ & $0.012700 \mathrm{R} 06$ & $0.053975 \mathrm{E}$ & & & \multicolumn{2}{|l|}{$* T H$} \\
\hline 861 & $\mathrm{~F}$ & $0.000000 E$ & & & & & \multicolumn{2}{|l|}{ *QPPG } \\
\hline 862 & R32 & $541.571343 \mathrm{R} 12$ & 567.500000 R08 & $552.800000 R 48$ & $541.571343 E$ & & \multicolumn{2}{|l|}{$\begin{array}{l}* \text { TWGN } \\
* H U G\end{array}$} \\
\hline 863 & $F$ & $0.000000 E$ & & & & & \multirow{2}{*}{\multicolumn{2}{|c|}{ *HILG }} \\
\hline 864 & $F$ & $0.000000 E$ & & & & & & \\
\hline 865 & $\mathrm{~F}$ & $0.000000 E$ & & & & & \multicolumn{2}{|l|}{ *TILG } \\
\hline 866 & $\mathrm{~F}$ & $0.000000 E$ & & & & & \multicolumn{2}{|l|}{ *TIVG } \\
\hline 867 & R08 & 33.898952 & 1.388600 & 17.287714 & 0.738780 & 1.388600 & \multicolumn{2}{|l|}{ *WAIG } \\
\hline 868 & & $17.287714 \mathrm{RO} 4$ & 2. 304060 & 2.660813 & 2.848255 & 2.214599 & *WAIG & \\
\hline 869 & & 3.311234 & 7.627232 & 2.542411 & 4.966850 & $4.407869 E$ & *WAJG & \\
\hline 870 & R08 & 0.000000 R05 & $0.000000 \mathrm{R} 06$ & $0.000000 R 06$ & $0.000000 \mathrm{E}$ & & ${ }^{*} \mathrm{HOLG}$ & \\
\hline 871 & R08 & $0.000000 R 05$ & $0.000000 \mathrm{R} 06$ & $0.000000 \mathrm{R} 06$ & $1.580119 \mathrm{E}$ & & *HOVG & \\
\hline 872 & R08 & $0.000000 R 02$ & 305.370000 & $0.000000 R 02$ & 305.370000 RO6 & 0.000000 & *TOLG & \\
\hline 873 & R06 & $305.370000 E$ & & & & & *TOLG & \\
\hline 874 & R08 & 0.000000 RO2 & 305.370000 & $0.000000 \mathrm{R} 02$ & 305.370000 R06 & 0.000000 & *TOVG & \\
\hline 875 & R06 & $305.370000 \mathrm{E}$ & & & & & ${ }^{*}$ TOVG & \\
\hline 876 & R08 & 42.162876 & 1.568604 & 38.228926 & 0.738780 & 1. 668604 & *WAOG & \\
\hline 877 & & $38.228926 \mathrm{RO} 4$ & 2. 349460 & 2.719942 & 2.911549 & 2. 382671 & *WAOG & \\
\hline 878 & & 3. 562533 & 8.206085 & 2.735362 & 5.343799 & $4.742394 \mathrm{E}$ & *WAOG & \\
\hline 879 & & & 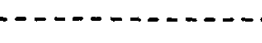 & - & $\ldots \ldots$ & $\ldots . . . .-1$ & 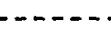 & $\cdots$ \\
\hline 880 & FILL & & 24 & $24 \mathrm{STI}$ & EAM GENERATOR & FEEDWATER & & \\
\hline 881 & & 25 & 8 & 0 & & & ${ }^{*} \mathrm{CN}$ & 02 \\
\hline 882 & & 8 & -18 & 6 & 0 & 0 & ${ }^{*} \mathrm{CN}$ & 03 \\
\hline 883 & & 0.000000 & $1.0000 E+20$ & 0.000000 & & & ${ }^{*} \mathrm{CN}$ & 04 \\
\hline 884 & & 2.000000 & $1.62146 \mathrm{E}-2$ & 0.000000 & 0.000000 & 479.000000 & ${ }^{*} \mathrm{CN}$ & 05 \\
\hline 885 & & 5. $3700 E+6$ & 0.000000 & 20.100000 & 0.000000 & 479.000000 & ${ }^{*} \mathrm{CN}$ & 06 \\
\hline 886 & & 1.000000 & 1.000000 & & & & ${ }^{*} \mathrm{CN}$ & 09 \\
\hline 887 & & 0.000000 & 0.000000 & 0.200000 & $6.870000 \mathrm{~S}$ & & *VMTB & \\
\hline 888 & & 0.391700 & 13.720000 & 0.577000 & $20.580000 \mathrm{~S}$ & & *VMTB & \\
\hline 889 & & 0.756200 & 27.440000 & 1.000000 & $37.130000 E$ & & *VMTB & \\
\hline$£ 90$ & & & & & DTH & & & \\
\hline 891 & VALV & & 23 & $23 \mathrm{ST}$ & EAM LJNE VALVE & & & \\
\hline 892 & & 6 & 0 & 22 & 26 & 7 & ${ }^{*} \mathrm{CN}$ & 02 \\
\hline 893 & & 1 & 0 & 3 & 6 & 0 & ${ }^{*} \mathrm{CN}$ & 0.3 \\
\hline 894 & & 8 & -44 & 0 & 0 & 0 & ${ }^{*} \mathrm{CN}$ & 04 \\
\hline 895 & & 0 & 0 & & & & ${ }^{*} \mathrm{CN}$ & 06 \\
\hline 896 & & $1.0000[+20$ & 0.000000 & 0.000000 & 1.000000 & & ${ }^{*} \mathrm{CN}$ & 07 \\
\hline 897 & & 0.121400 & 0.015100 & 0.000000 & 0.000000 & 305.370000 & ${ }^{*} \mathrm{CN}$ & 08 \\
\hline 898 & & 305.370000 & 4. $09446 \mathrm{E}-2$ & 0.228325 & 0.569 .330 & 0.620000 & ${ }^{*} \mathrm{CN}$ & 09 \\
\hline 899 & $\mathrm{~F}$ & $5.01191 \mathrm{E}$ & & & & & *DX & \\
\hline 900 & $\mathrm{~F}$ & $2.32198 E-1 E$ & & & & & $* \mathrm{VOL}$ & \\
\hline
\end{tabular}




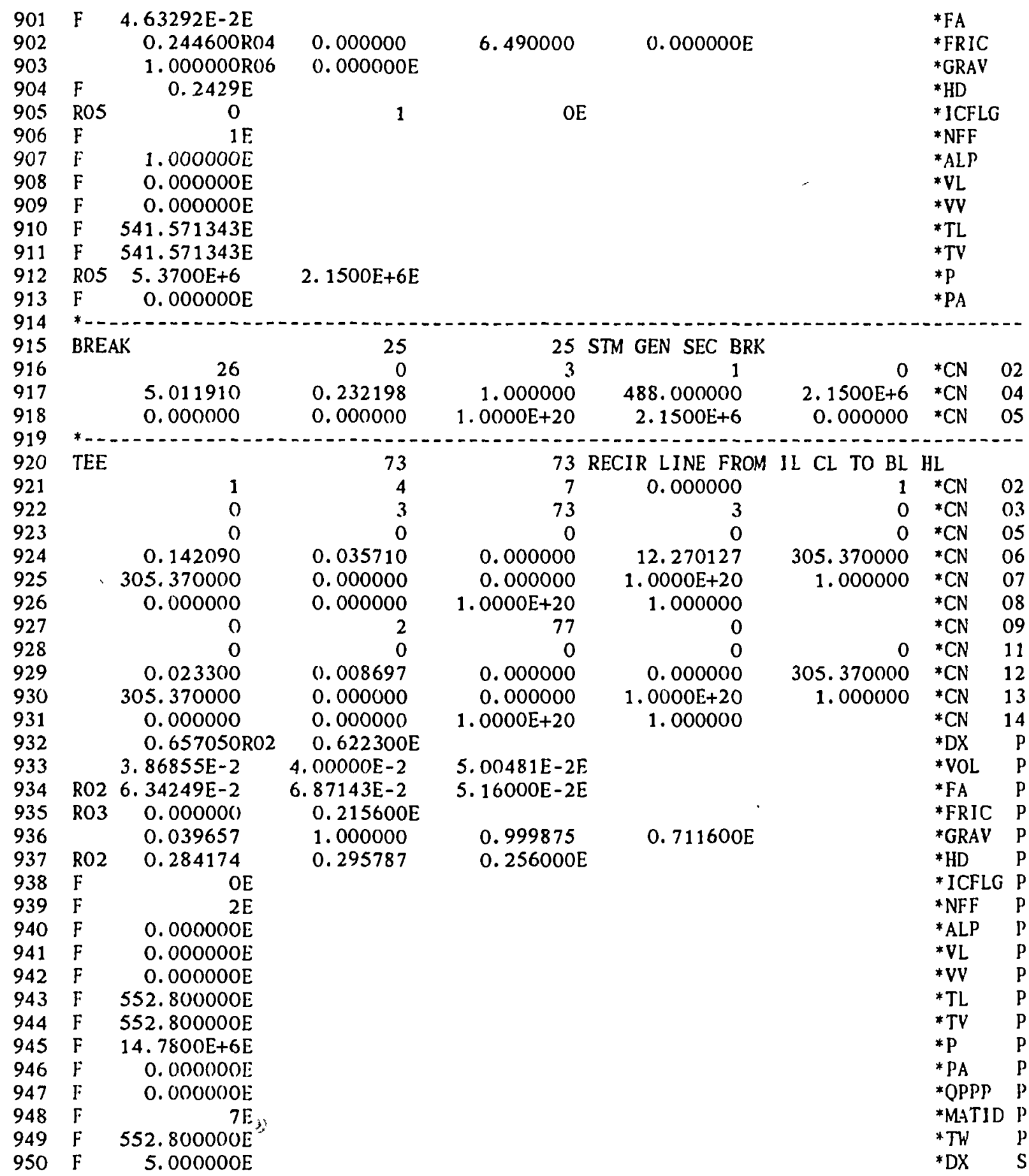


95

952 R03 1.44930E-3E

$953 \mathrm{~F} \quad 0.000000 \mathrm{E}$

$954 \quad 0.000000$

955 RO3 0.042960E

$956 \mathrm{~F} \quad \mathrm{OE}$

$957 \mathrm{~F} \quad 2 \mathrm{E}$

$958 \mathrm{~F} \quad 0.000000 \mathrm{E}$

$959 \mathrm{~F} \quad 0.000000 \mathrm{E}$

$960 \quad F$

961

962

963

964

965

966

967

968

969

970

971

972

973

974

975

976

977

978

$+979$

980

981

982

983

984

985

986

987

988

989

990)

991

992

993

994

995

996

997

998

999

1000

F $\quad 0.000000 \mathrm{E}$

F $\quad 567.500000 E$

F 567.500000E

I 14.7800E+6E

F $\quad 0.000000 E$

F $0.000000 E$

$\mathrm{F} \quad 7 \mathrm{E}$

567. 500000E

VALVE

2
1
9
0

1. $0000 \mathrm{E}+20$

0.033000

305. 370000

F $\quad 0.500000 \mathrm{E}$

RO2 1.44930E-3

R02 0.000000

F $\quad 0.000000 E$

RO2 4. 29600E-2

$\mathrm{F} \quad \mathrm{OE}$

F 2E

F $\quad 0.000000 \mathrm{E}$

F $0.000000 E$

F $\quad 0.000000 E$

F $\quad 567.500000 \mathrm{E}$

F $567.500000 \mathrm{E}$

F 14.7800E+6E

F $0.000000 E$

0.000000

TEE

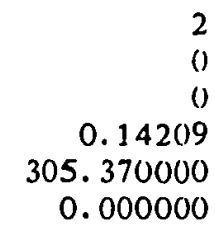

$0.253949 \quad 0.000000 \mathrm{E}$

77

0

0

T $-1.000000 \mathrm{E}$

0.000000

0.008697

1. $44900 \mathrm{E}-3$

$0.051956 \mathrm{E}$

$0.203800 \mathrm{E}$

$0.257201 \mathrm{E}$

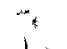

77

i. 3

$-2$

\begin{abstract}
0.000000
0.000000

4. 29570E-2
\end{abstract}

1. 000000

0.000000

1. 000000

305. 370000

(2) 1.000000

*VOL S

*FA S

*FRIC S

*GRAV S

*HD S

* ICFLG S

*NFF S

*ALP S

*VL S

*VV S

*TL S

*TV S

$* P \quad S$

*PA $\quad S$

*QPPP S

*MATID S

*TW S

ECIRC VLVE (IL CL TO BL HL)

$32 \quad 7 * \mathrm{CN} 02$

$2 \quad 0 * \mathrm{CN} \quad 03$

$0 \quad * \mathrm{CN} \quad 04$

*CN 06

*CN 07

*CN 08

*CN 09

*DX

*VOL

*FA

*FRIC

* GRAV

*HD

* ICFLG

*NFF

*ALP

$* V L$

*VV

*TL

*TV

*P

*PA

*VTB 1

$0.000000 \mathrm{E}$

\section{PUMP SUCTION}

$7 \quad 0.000000$

4

()

0
09

305. 370000

0.000000

0.03571

0.000000

0.000000

0.000000

0.000000

1. $0000 \mathrm{E}+20$

12. 270127

$1.0000 \mathrm{E}+20$

1.000000

$\begin{array}{rrr}1 & * \mathrm{CN} & 02 \\ 0 & * \mathrm{CN} & 03 \\ 0 & * \mathrm{CN} & 05 \\ 305.370000 & * \mathrm{CN} & 06 \\ 1.000000 & * \mathrm{CN} & 07 \\ & * \mathrm{CN} & 08\end{array}$




\section{CARD}

\section{1}

1002

1003

1004

1005

1006

1007

1008

1009

1010

1011

1012

1013

1014

1015

1016

1017

1018

1019

1020

1021

1022

1023

1024

1025

1026

1027

1028

1029

1030

1031

1032

1033

1034

1035

1036

1037

1038

3039

1040

1041

1042

1043

1044

1045

1046

1047

1048

1049

1050

$\begin{array}{cr}.0 & 1 \\ 0 & 0 \\ 0.14209 & 0.03571 \\ 305.370000 & 0.000000 \\ 0.000000 & 0.000000 \\ 6.35 \mathrm{E}-1 & 1.80340 \\ & 0.11438 \\ 2746.75 \mathrm{E}-5 & 0.5 \\ 3661.31 \mathrm{E}-5 \mathrm{R} 02 & 6342.49 \mathrm{E}-5 \\ \mathrm{~F} \quad 0.000000 \mathrm{E} & \end{array}$

$-0.62659$

$215.91 \mathrm{E}-3 \mathrm{R} 02 \quad 2841.74 \mathrm{E}-4$

$F$

F $2 \mathrm{E}$

$0.000000 \mathrm{E}$

$0.000000 \mathrm{E}$

$0.000000 \mathrm{E}$

$552.800000 \mathrm{E}$

$552.800000 E$

14. $7800 \mathrm{E}+6 \mathrm{E}$

$0.000000 E$

F $\quad 0.000000 E$

$\mathrm{F} \quad 7 \mathrm{E}$

F $\quad 552.800000 E$

$0.657050 \mathrm{E}$

3. $86855 \mathrm{E}-2 \mathrm{E}$

R02 6. 34249E-2E

R02 $0.000000 \mathrm{E}$

0.000000

R02 $0.284174 \mathrm{E}$

$F \quad O E$

$F \quad 2 E$

F $\quad 0.000000 E$

$\mathrm{F} \quad 0.000000 \mathrm{C}$

F $\quad 0.000000 E$

F $552.800000 E$

F 552.800000E

F $14.7800 \mathrm{E}+6 \mathrm{E}$

$F \quad 0.000000 E$

F $\quad 0.000000 \mathrm{E}$

$\mathrm{F} \quad 7 \mathrm{E}$

$F \quad 552.800000 E$

$0.039657 E$

PUMP

UMP

2
1

1
10

0.107950

305: 370000

941.540000

0.028580

$317.438(0) 0$

500.000000

$\begin{array}{rr}73 & 0 \\ 0 & 0 \\ 0.000000 & 12.270127 \\ 0.000000 & 1.0000 \mathrm{E}+20 \\ 1.0000 \mathrm{E}+20 & 1.000000 \\ 6.35 \mathrm{E}-1 \mathrm{~F} & \\ 2746.75 \mathrm{E}-5 \mathrm{E} & \\ 3.66131 \mathrm{E}-2 \mathrm{E} & \end{array}$

$\begin{array}{ccc} & * \mathrm{CN} & 09 \\ 0 & * \mathrm{CN} & 11\end{array}$

$305.370000 *$ CN 12

$1.000000{ }^{*} \mathrm{CN} \quad 13$

${ }^{*} \mathrm{CN} \quad 14$

*DX P

*VOL P

* Fa P

*FRIC P

* GRAV P

*HD P

* ICFLG P

*NFF $P$

*ALP P

*VL $\quad P$

*VV P

*TL P

*TV P

*P $\quad \mathrm{P}$

*PA P

*QPPP P.

*MATID P

*TW P

*DX S

*VOL S

*FA S

*FRIC S

*GRAV S

*HD S

*ICFLG S

*NFF $S$

*ALP S

*VL S

*VV S

*TL S

*TV S

*P S

*PA S

*QPPP $\mathrm{S}$

*MATID S

*TW S

PUMP NO. 2

4

1

$-11$

0
0.000000

3. 681250

0.315000
12.270127

231.186000

614.000000

$\begin{array}{rlr}7 & * \mathrm{CN} & 02 \\ 1 & * \mathrm{CN} & 03 \\ 0 & { }^{*} \mathrm{CN} & 04 \\ 0 & * \mathrm{CN} & 05 \\ 305.370000 & { }^{*} \mathrm{CN} & 06 \\ & { }^{*} \mathrm{CN} & 07 \\ 369.661000 & { }^{*} \mathrm{CN} & 08\end{array}$


CARD

1051

1052

1053

1054

1055

1056

1057

1058

1059

1060

1061

1062

1063

1064

1065

1066

1067

1068

1069

1070

1071

1072

1073

1074

1075

1076

1077

1078

1079

1080

1081

1082

1083

1084

1085

1086

1087

1088

1089

1090

1091

1092

1093

1094

1095

1096

1097

1098

1099

1100

\begin{tabular}{|c|c|c|c|c|c|c|c|}
\hline & 334.239321 & -1.000000 & $1.0000 E+20$ & 335.318435 & -70 & ${ }^{*} \mathrm{CN}$ & 09 \\
\hline & 0.000000 & 0.000000 & $1.0000 E+20$ & 1.000000 & & ${ }^{*} \mathrm{CN}$ & 10 \\
\hline & 2 & & & & & ${ }^{*} \mathrm{CN}$ & 11 \\
\hline $\mathbf{F}$ & $1352.13 E-3 E$ & & & & & *DX & \\
\hline F & $4955.48 \mathrm{E}-5 \mathrm{E}$ & & & & & *VOL & \\
\hline $\mathrm{F}$ & $3661.31 \mathrm{E}-5 \mathrm{E}$ & & & & & ${ }^{*} \mathrm{FA}$ & \\
\hline $\mathrm{r}$ & $0.000000 \mathrm{~L}$ & & & & & ${ }^{*} \mathrm{FRIC}$ & \\
\hline & $0.62659 \mathrm{RO} 2$ & $0.000000 \mathrm{E}$ & & & & *GRAV & \\
\hline $\mathrm{F}$ & $215.91 E-3 E$ & & & & & *HD & \\
\hline 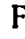 & $\mathrm{OE}$ & & & & & ${ }^{*}$ ICFLC & \\
\hline & 2 & 0 & $2 \mathrm{E}$ & & & ${ }^{*} \mathrm{NFF}$ & \\
\hline$F$ & $0.000000 \mathrm{E}$ & & & & & ${ }^{*}$ ALP & \\
\hline$F$ & $0.000000 \mathrm{E}$ & & & & & $* \mathrm{VL}$ & \\
\hline F & $0.000000 \mathrm{E}$ & & & & & *VV & \\
\hline $\mathbf{F}$ & $552.800000 \mathrm{E}$ & & & & & ${ }^{*} \mathrm{TL}$ & \\
\hline $\mathbf{F}$ & $552.800000 E$ & & & & & *TV & \\
\hline $\mathbf{F}$ & $14.7800 E+6 E$ & & & & & $* P$ & \\
\hline$F$ & $0.000000 \mathrm{E}$ & & & & & ${ }^{*} \mathrm{PA}$ & \\
\hline $\mathrm{F}$ & $0.000000 \mathrm{E}$ & & & & & ${ }^{*} \mathrm{QPPP}$ & \\
\hline $\mathbf{F}$ & $7 \mathrm{E}$ & & & & & *MATIL & \\
\hline & $552.800000 \mathrm{E}$ & & & & & ${ }^{*} T W$ & \\
\hline & 0.000000 & 1.000000 & 5.000000 & $0.645161 \mathrm{~S}$ & & *PMPTF & \\
\hline & 10.000000 & 0.435484 & 18.000000 & $0.225806 \mathrm{~S}$ & & *PMPTH & \\
\hline & 20.000000 & 0.129032 & 25.000000 & $0.064516 \mathrm{~S}$ & & * PMPTE & \\
\hline & 30.000000 & 0.044355 & 40.000000 & $0.024806 \mathrm{~S}$ & & *PMPTI & \\
\hline & 44.000000 & 0.019355 & 46.500000 & $0.000000 \mathrm{~S}$ & & *PMPTI & \\
\hline & 1.00000E+4 & $0.000000 \mathrm{E}$ & & & & *PMPTI & \\
\hline & & 5 & 5 & PUMP NO. 1 & & & \\
\hline & 2 & 4 & 5 & 7 & 7 & ${ }^{*} \mathrm{CN}$ & 02 \\
\hline & 1 & 0 & 1 & 1 & 1 & ${ }^{*} \mathrm{CN}$ & 03 \\
\hline & 10 & 4 & -11 & 0 & 0 & ${ }^{*} \mathrm{CN}$ & 04 \\
\hline & 0 & $i$ & 0 & 0 & 0 & ${ }^{*} \mathrm{CN}$ & 05 \\
\hline & 0.107950 & 0.028580 & 0.000000 & 12.270127 & 305.370000 & ${ }^{*} \mathrm{CN}$ & 06 \\
\hline & 305.370000 & 317.438000 & 3.681250 & 231.186000 & & ${ }^{*} \mathrm{CN}$ & 07 \\
\hline & 941.540000 & 500.000000 & 0.315000 & 614.000000 & 369.661000 & ${ }^{*} \mathrm{CN}$ & 08 \\
\hline & 334.239321 & -1.000000 & $1.0000 \mathrm{E}+20$ & 335. 318435 & -70 & ${ }^{*} \mathrm{CN}$ & 09 \\
\hline & 0.000000 & 0.000000 & $1.0000 E+20$ & 1.000000 & & ${ }^{*} \mathrm{CN}$ & 10 \\
\hline & & & & & & ${ }^{*} \mathrm{CN}$ & 11 \\
\hline$F$ & 1352. $13 \mathrm{E}-3 \mathrm{E}$ & & & & & $* D X$ & \\
\hline $\mathrm{F}$ & $4955.48 E-5 E$ & & & & & $*$ VOL & \\
\hline F & $3661.31 E-5 E$ & & & & & ${ }^{*} \mathrm{FA}$ & \\
\hline $\mathrm{F}$ & $0.000000 \mathrm{~F}$ & & & & & ${ }^{*} \mathrm{FRIC}$ & \\
\hline & $0.62659 \mathrm{R} 02$ & $0.000000 \mathrm{E}$ & & & & ${ }^{*}$ GRAV & \\
\hline$F$ & $215.91 \mathrm{E}-3 \mathrm{E}$ & & & & & ${ }^{*} \mathrm{HD}$ & \\
\hline $\mathbf{F}$ & $\mathrm{Ol}$ & & & & & ${ }^{*} \mathrm{ICFLC}$ & \\
\hline & 2 & 0 & $2 \mathrm{E}$ & & & ${ }^{*} \mathrm{NFF}$ & \\
\hline F & $0.000000 \mathrm{E}$ & & & & & *ALP & \\
\hline ] & $0.000000 \mathrm{E}$ & & & & & $* \mathrm{VL}$ & \\
\hline$F$ & $0.000000 \mathrm{E}$ & & & & & $* V V$ & \\
\hline
\end{tabular}


1101 F $\quad 552.800000 \mathrm{E}$

$1102 \mathrm{~F} \quad 552.800000 \mathrm{E}$

$1103 \mathrm{~F} \quad 14.7800 \mathrm{E}+6 \mathrm{E}$

$1104 \mathrm{~F} \quad 0.000000 \mathrm{E}$

$1105 \mathrm{~F} \quad 0.000000 \mathrm{E}$

$1106 \mathrm{~F} \quad 7 \mathrm{E}$

1107 F $\quad 552.800000 \mathrm{E}$

$1108 \quad 0.000000$

$1109 \quad 10.000000$

1110

1111

1112

1113

1114

1115

1116

1117

1118

1119

1120

1121

1122

1123

1124

1125

1126

1127

1128

1129

1130

1131

1132

1133

1134

1135

1136

1137

1138

1139

1140

1141

1142

1143

1144

1145

1146

1147

1148

1149

1150
20.000000

30.000000

44. 000000

1. $00000 \mathrm{E}+4$

TEE

2

0

0.10795

305. 370000

0.000000

0

0.10795

305. 370000

0.000000

130. $81 \mathrm{E}-2$

4785. 57E-5

$\mathrm{RO2} 3661.31 \mathrm{E}-5$

R02 0.000000

F $\quad 0.000000 \mathrm{E}$

RO2 215.91E-3

$F \quad O E$

$F \quad 2 E$

$0.000000 \mathrm{E}$

$0.000000 \mathrm{E}$

$0.000000 \mathrm{E}$

$552.800000 \mathrm{E}$

$552.800000 \mathrm{E}$

14. $7800 \mathrm{E}+6 \mathrm{E}$

$0.000000 \mathrm{E}$

o. $000000 \mathrm{E}$

$7 \mathrm{E}$

$552.800000 \mathrm{E}$

$542.92 \mathrm{E}-3 \mathrm{E}$

2543. $94 \mathrm{E}-5 \mathrm{I}$

$3661.31 \mathrm{~F}-5 \mathrm{E}$

0. OOOOUOE

$0.000000 \mathrm{E}$

215. $91 \mathrm{E}-3 \mathrm{E}$
1.000000

0.435484

0.129032

0.044355

0.019355

$0.000000 \mathrm{E}$
5.000000

18.000000

25.000000

40.000000

46. 500000
*TL

$*$ TV

*P

*PA

*QPPP

*MATID

*TW

* РMPTB

*PMPTB

* PMPTB

* PMPTB

* PMPTB

* PMPTB
$0.225806 \mathrm{~S}$

$0.064516 \mathrm{~S}$

$0.024806 \mathrm{~S}$

$0.000000 \mathrm{~S}$
6 PUMP DI SCHARGE

$7 \quad 0.000000$

7

0

0.02858

0.000000

0.000000

0.000000

1. $0000 \mathrm{E}+20$

1
0

0
0.02858

0.000000

0.000000

55. 588E-2E

3315. 38E-5E

$6342.49 E-5 E$

2. $201 \mathrm{E}-2 \mathrm{E}$

$0.284174 \mathrm{E}$
0.000000

0.000000

1. $0000 \mathrm{E}+20$
12. 270127

1. $0000 \mathrm{E}+20$

1.000000

12. 0

1. $0000 \mathrm{E}+20$

1. 000000

$\begin{array}{lll}1 & * \mathrm{CN} & 02 \\ 0 & { }^{*} \mathrm{CN} & 03 \\ 0 & * \mathrm{CN} & 05\end{array}$

$305.370000 * \mathrm{CN} \quad 06$

$1.000000{ }^{*} \mathrm{CN} \quad 07$

${ }^{*} \mathrm{CN} \quad 08$

*CN 09

$\begin{array}{rrr}0 & * \mathrm{CN} & 11\end{array}$

$1.000000 * \mathrm{CN} \quad 13$

*CN 14

*DX $\mathrm{P}$

*VOL P

*FA P

*FRIC, P

* GRaV P

*HD P

* ICFLG P

*NFF P

*ALP P

*VL P

*VV P

*TL P

*TV P

* $\mathrm{P} \quad \mathrm{P}$

*PA $\quad \mathrm{P}$

*QPPP P

* MATID p

*TW P

*DX S

* VOL S

*FA S

* FRIC S

* GRAV S

*IID S 
CARD $\quad 12345678901234567890123456789012345678901234567890123456789012345678901234567890$

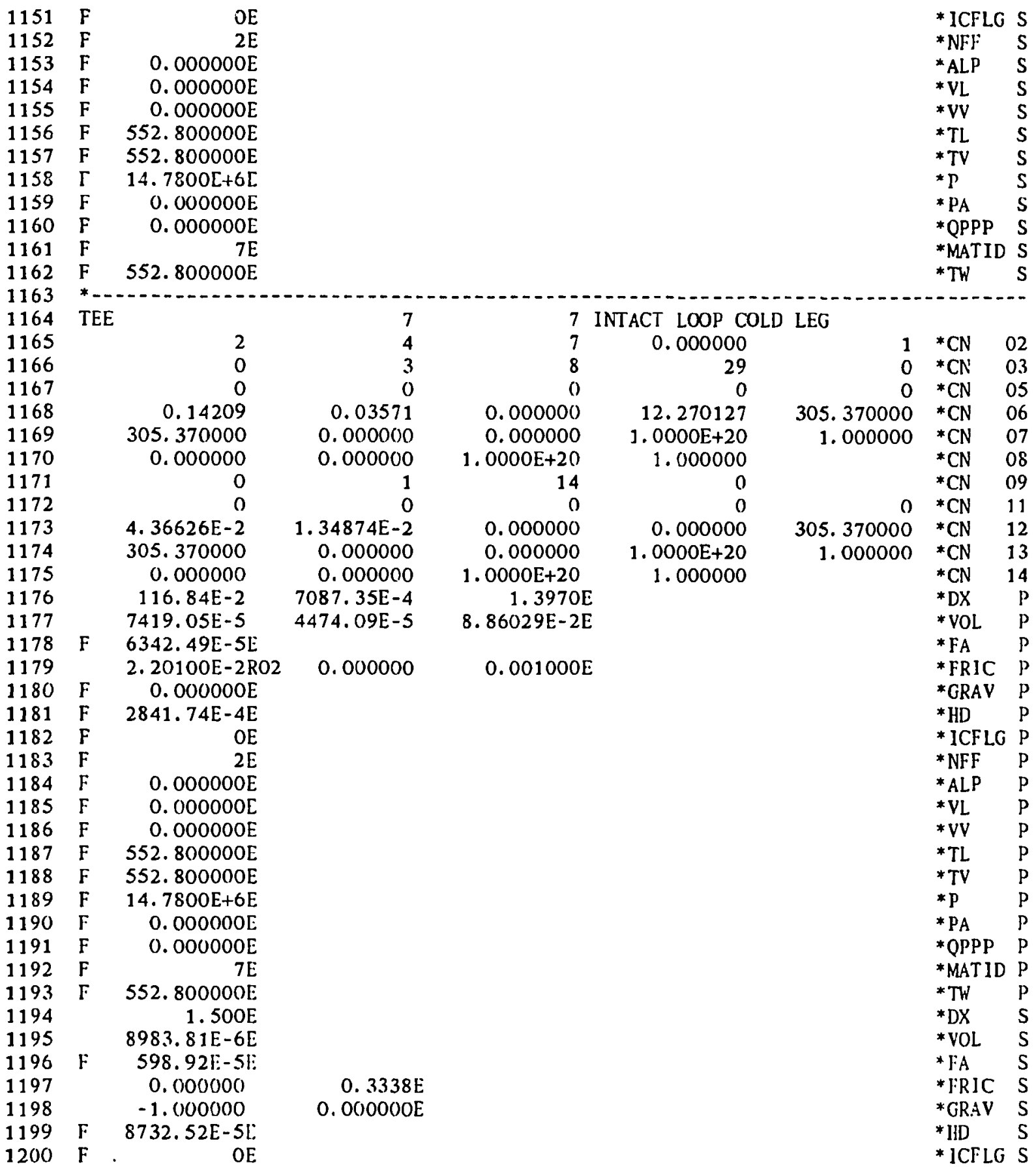




\begin{tabular}{|c|c|c|c|c|c|c|c|c|}
\hline 1201 & $F$ & $2 \mathrm{E}$ & & & & & $* N F F$ & $S$ \\
\hline 1202 & $F$ & $0.000000 \mathrm{E}$ & & & & & ${ }^{*} A L P$ & $S$ \\
\hline 1203 & $\mathrm{~F}$ & $0.000000 \mathrm{E}$ & & & & & $* V L$ & $S$ \\
\hline 1204 & $F$ & $0.000000 \mathrm{E}$ & & & & & $* V V$ & $S$ \\
\hline 1205 & $F$ & $500.000000 \mathrm{E}$ & & & & & $* T L$ & \\
\hline 1206 & $F$ & $500.000000 E$ & & & & & $* T V$ & $S$ \\
\hline 1207 & $\mathrm{~F}$ & $14.7800 \mathrm{E}+6 \mathrm{E}$ & & & & & $* P$ & S \\
\hline 1208 & $\mathrm{~F}$ & 0. OOOOOOE & & & & & ${ }^{*} \mathrm{PA}$ & \\
\hline 1209 & $\mathbf{F}$ & $0.000000 E$ & & & & & *QPPP & $S$ \\
\hline 1210 & $\mathrm{~F}$ & $7 E$ & & & & & *MATID & $S$ \\
\hline 1211 & $\mathbf{F}$ & $500.000000 \mathrm{E}$ & & & & & *TW & $S$ \\
\hline 1212 & & $\ldots$ & $--\infty$ & $-\cdots$ & 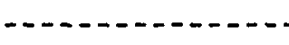 & & $\cdots$ & \\
\hline 1213 & Fl & & 17 & 171 & HPIS INJECTION & & & \\
\hline 1214 & & 14 & 8 & 1 & & & ${ }^{*} \mathrm{CN}$ & 02 \\
\hline 1215 & & 17 & 1 & -12 & 0 & 0 & ${ }^{*} \mathrm{CN}$ & 03 \\
\hline 1216 & & 0.000000 & $1.0000 \mathrm{E}+20$ & 0.000000 & & & ${ }^{*} \mathrm{CN}$ & 04 \\
\hline 1217 & & 1.500000 & $8.98381 \mathrm{E}-3$ & 0.000000 & 0.000000 & 375.000000 & ${ }^{*} \mathrm{CN}$ & 05 \\
\hline 1218 & & $14.7800 \mathrm{E}+6$ & 0.000000 & 0.000000 & 0.000000 & 375.000000 & ${ }^{*} \mathrm{CN}$ & 06 \\
\hline 1219 & & 1.000000 & 1.000000 & & & & ${ }^{*} \mathrm{CN}$ & 09 \\
\hline 1220 & & 0.000000 & 0.000000 & 0.200000 & $0.243750 \mathrm{~S}$ & & *VMTB & \\
\hline 1221 & & 1.600000 & 3.887500 & 20.600000 & $3.893750 \mathrm{~S}$ & & * VMTB & \\
\hline 1222 & & 21.600000 & 1.475000 & 22.600000 & $0.375000 \mathrm{~S}$ & & *VMTB & \\
\hline 1223 & & 23.600000 & 0.250000 & 26.600000 & $0.159375 \mathrm{~S}$ & & *VMTB & \\
\hline 1224 & & 33.600000 & 0.125000 & 39.600000 & $0.125000 \mathrm{~S}$ & & *VMTB & \\
\hline 1225 & & 40.100000 & 0.000000 & 1. $00000 E+4$ & $0.000000 \mathrm{E}$ & & *VMTB & \\
\hline 1226 & & $\ldots \ldots-\ldots$ & $-\ldots-n-\ldots$ & $-----\infty-\infty$ & 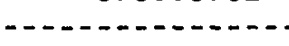 & $\ldots$ & $\ldots-$. & \\
\hline 1227 & TE & & 31 & 311 & BROKEN LOOP HOT & LEG & & \\
\hline 1228 & & 1 & 4 & 7 & 0.000000 & 1 & ${ }^{*} \mathrm{CN}$ & 02 \\
\hline 1229 & & 0 & 6 & 45 & 32 & 0 & ${ }^{*} \mathrm{CN}$ & 3 \\
\hline 1230 & & 0 & 0 & 0 & 0 & 0 & ${ }^{*} \mathrm{CN}$ & 5 \\
\hline 1231 & & 0.14209 & 0.03571 & 0.000000 & 12.270127 & 305.3 & ${ }^{*} \mathrm{CN}$ & 06 \\
\hline 1232 & & 305.370000 & 0.000000 & 0.000000 & $1.0000 \mathrm{E}+20$ & 1.000000 & ${ }^{*} \mathrm{CN}$ & 07 \\
\hline 1233 & & 0.000000 & 0.000000 & 1. $0000 E+20$ & 1.000000 & & ${ }^{*} \mathrm{CN}$ & 08 \\
\hline 12.34 & & 0 & 3 & 43 & 0 & & ${ }^{*} \mathrm{CN}$ & 09 \\
\hline 1235 & & 0 & 0 & 0 & 0 & 0 & ${ }^{*} \mathrm{CN}$ & 11 \\
\hline 1236 & & 0.10795 & 0.02858 & 0.000000 & 12.270127 & 305.370000 & ${ }^{\star} \mathrm{CN}$ & 12 \\
\hline 1237 & & 305.370000 & 0.000000 & 0.000000 & 1. $0000 E+20$ & 1.000000 & ${ }^{*} \mathrm{CN}$ & 13 \\
\hline 1238 & & 0.000000 & 0.000000 & 1. $0000 \mathrm{E}+20$ & 1.000000 & & ${ }^{*} \mathrm{CN}$ & 14 \\
\hline 1239 & & 2.548984 & 2. 408029 & 3.750796 & 2.168533 & 1.829369 & ${ }^{*} \mathrm{DX}$ & \\
\hline 1240 & & $2.001044 \mathrm{E}$ & & & & & ${ }^{*} \mathrm{DX}$ & \\
\hline 1241 & & $1.69381 \mathrm{E}-1$ & $9.8451 E-2$ & 3. $52044 \mathrm{E}-1$ & $9.5904 E-2$ & $6.0297 \mathrm{E}-2$ & *VOL & $\mathrm{P}$ \\
\hline 1242 & & $7.0984 \mathrm{E}-2 \mathrm{E}$ & & & & & *VOL & \\
\hline 1243 & & 6. $3425 \mathrm{E}-2$ & 6. $3425 E-2 \mathrm{RO} 2$ & 1. $07862 \mathrm{E}-1$ & $9.426 \mathrm{E}-3$ & 8. $365 E-3$ & $* F A$ & \\
\hline 1244 & & $5.1956 \mathrm{E}-2 \mathrm{E}$ & & & & - & *FA & \\
\hline 1245 & & 0.0001 & 0.000000 & 0.9026 & 0.9391 & 0.3710 & *FRIC & \\
\hline 1246 & & 0.0405 & $0.2038 \mathrm{E}$ & & & & *FRIC & \\
\hline 1247 & & 0.000000 & 0.1652 & 0.90507 & -0.8476 & -0.9399 & *GRAV & \\
\hline 1248 & & 0.6218 & $0.000 \mathrm{E}$ & & & & ${ }^{\star}$ GRAV & \\
\hline 1249 & & 0.284176 & 0.284176 & 0.370587 & 0.370587 & 0.109551 & *HD & \\
\hline 1250 & & 0.103201 & $0.257201 \mathrm{E}$ & & & & *HD & \\
\hline
\end{tabular}




\begin{tabular}{|c|c|c|c|c|c|c|c|}
\hline 1251 & $\mathrm{~F}$ & $O E$ & & & & & * ICFLG \\
\hline 1252 & $\mathrm{~F}$ & $2 \mathrm{E}$ & & & & & ${ }^{*} \mathrm{NFF}$ \\
\hline 1253 & $\mathrm{~F}$ & $0.000000 \mathrm{E}$ & & & & & *ALP \\
\hline 1254 & $\mathrm{~F}$ & $0.000000 \mathrm{E}$ & & & & & $* \mathrm{VL}$ \\
\hline 1255 & $\mathrm{~F}$ & $0.000000 \mathrm{E}$ & & & & & $* \mathrm{~W}$ \\
\hline 1256 & $\mathrm{~F}$ & $567.500000 \mathrm{E}$ & & & & & ${ }^{*} \mathrm{TL}$ \\
\hline 1257 & $\mathrm{~F}$ & $567.500000 \mathrm{E}$ & & & & & ${ }^{*} T V$ \\
\hline 1258 & $\mathrm{~F}$ & 14. $7800 E+6 E$ & & & & & $* \mathrm{p}$ \\
\hline 1259 & $\mathrm{~F}$ & $0.000000 \mathrm{E}$ & & & & & *PA \\
\hline 1260 & $\mathrm{~F}$ & $0.000000 \mathrm{E}$ & & & & & * QPPP \\
\hline 1261 & $\mathrm{~F}$ & $7 \mathrm{E}$ & & & & & *MATID \\
\hline 1262 & $\mathrm{~F}$ & $567.500000 \mathrm{E}$ & & & & & ${ }^{*} T W$ \\
\hline 1263 & & 1.3890 & 0.8140 & $5.1044 \mathrm{E}$ & & & ${ }^{*} \mathrm{DX}$ \\
\hline 1264 & & 0.0540 & 0.0314 & $0.1986 \mathrm{E}$ & & & *VOL \\
\hline 1265 & $\mathrm{~F}$ & $3.88 \mathrm{E}-2 \mathrm{E}$ & & & & & $* r_{A}$ \\
\hline 1266 & R03 & 0.000000 & $0.000000 E$ & & & & ${ }^{*} \mathrm{FR} I \mathrm{C}$ \\
\hline 1267 & & 0.000000 & 0.224200 & 0.191200 & $0.000000 \mathrm{E}$ & & ${ }^{*} \mathrm{GRAV}$ \\
\hline 1268 & R03 & 2. $223 \mathrm{E}-1$ & $1.410 \mathrm{E}-3 \mathrm{E}$ & & & & ${ }^{*} \mathrm{HD}$ \\
\hline 1269 & $\mathrm{~F}$ & $\mathrm{OE}$ & & & & & * JCFLG \\
\hline 1270 & $\mathrm{~F}$ & $2 \mathrm{E}$ & & & & $=$ & *NFF \\
\hline 1271 & $\mathrm{~F}$ & $0.000000 \mathrm{E}$ & & & & & $*^{*}$ ALP \\
\hline 1272 & $\mathrm{~F}$ & $0.000000 \mathrm{E}$ & & & & & $* \mathrm{VL}$ \\
\hline 1273 & $\mathrm{~F}$ & $0.000000 \mathrm{E}$ & & & & & $* v \mathrm{v}$ \\
\hline 1274 & $\mathrm{~F}$ & $552.800000 \mathrm{E}$ & & & & & ${ }^{*} \mathrm{TL}$ \\
\hline 1275 & $\mathrm{~F}$ & $552.800000 E$ & & & & & ${ }^{*} \mathrm{TV}$ \\
\hline 1276 & $\mathrm{~F}$ & 14. $7800 \mathrm{E}+6 \mathrm{E}$ & & & & & $* \mathrm{P}$ \\
\hline 1277 & $\mathrm{~F}$ & $0.000000 \mathrm{E}$ & & & & & $* \mathrm{PA}$ \\
\hline 1278 & $\mathrm{~F}$ & $0.000000 \mathrm{E}$ & & & ' & & *QPPP \\
\hline 1279 & $\mathrm{~F}$ & $7 \mathrm{E}$ & & & & & *MATID \\
\hline 1280 & $\mathrm{~F}$ & $552.800000 \mathrm{E}$ & & & & & ${ }^{*} \mathrm{TW}$ \\
\hline 1281 & & 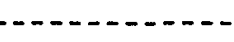 & & & & & \\
\hline 1282 & PIPE & & 75 & 75 & L. CL OUTLET $P$ & & \\
\hline 1283 & & 1 & 0 & 44 & 42 & 7 & ${ }^{*} \mathrm{CN}$ \\
\hline 1284 & & 1 & 0 & 0 & 0 & & ${ }^{*} \mathrm{CN}$ \\
\hline 1285 & & 0.958804 & 0.159919 & 0.000000 & 0.000000 & 305.370000 & ${ }^{*} \mathrm{CN}$ \\
\hline 1286 & & 305.370000 & 0.000000 & 0.000000 & $1.0000 E+20$ & 1.000000 & ${ }^{*} \mathrm{CN}$ \\
\hline 1287 & & $0.500000 E$ & & & & & $*^{\mathrm{DX}}$ \\
\hline 1288 & & $0.028705 E$ & & & & & $* \mathrm{VOL}$ \\
\hline 1289 & & 0.063427 & $1.28580 \mathrm{E}-5 \mathrm{E}$ & & & & $* \mathrm{FA}$ \\
\hline 1290 & & 0.017900 & $6.52460 \mathrm{E}-3 \mathrm{E}$ & & & & *FRIC \\
\hline 1291 & $\mathrm{~F}$ & $0.000000 \mathrm{E}$ & & & & & ${ }^{*} \mathrm{GRAV}$ \\
\hline 1292 & & 0.284180 & $4.04620 \mathrm{E}-3 \mathrm{E}$ & & & & $*_{\mathrm{HD}}$ \\
\hline 1293 & $\mathrm{~F}$ & $O E$ & & & & & *ICFLG \\
\hline 1294 & $\mathrm{~F}$ & $2 \mathrm{E}$ & & & & & ${ }^{*} \mathrm{NFF}$ \\
\hline 1295 & $\mathrm{~F}$ & $0.000000 \mathrm{E}$ & & & & & *ALP \\
\hline 1296 & $\mathrm{~F}$ & $0.000000 \mathrm{E}$ & & & & & $* \mathrm{VL}$ \\
\hline 1297 & $\mathrm{~F}$ & (0.000000E & & & & & $* \mathrm{VW}$ \\
\hline 1298 & $\mathrm{~F}$ & $552.800000 \mathrm{E}$ & & & & & $* \mathrm{TL}$ \\
\hline 1299 & $\mathrm{~F}$ & $552.800000 \mathrm{E}$ & & & & & $* T V$ \\
\hline 1300 & $\mathrm{~F}$ & 14. $7800 \mathrm{E}+6 \mathrm{E}$ & & & & & $* \dot{p}$ \\
\hline
\end{tabular}




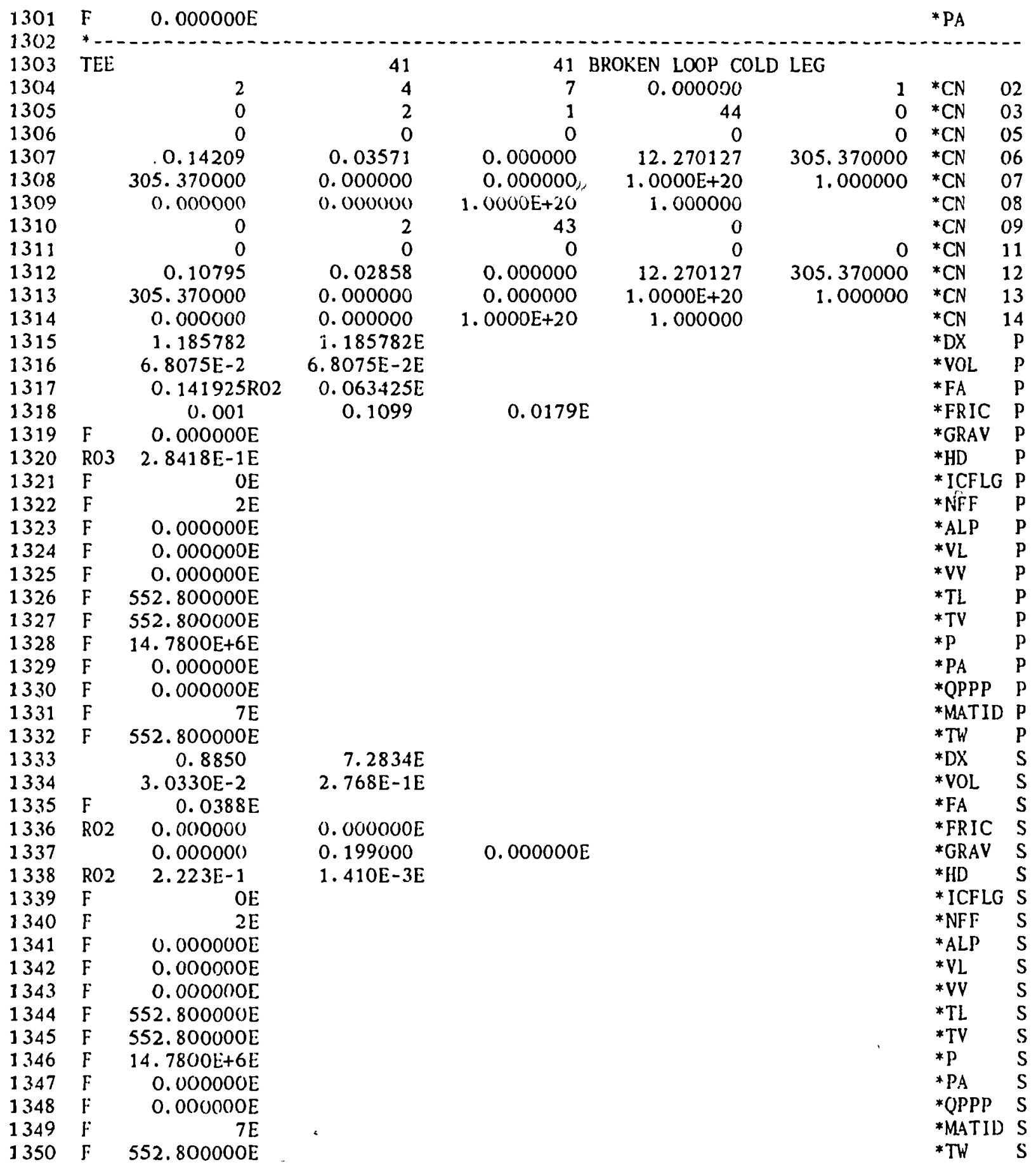




\begin{tabular}{|c|c|c|c|c|c|c|c|c|}
\hline $\begin{array}{l}1351 \\
1352\end{array}$ & $\mathrm{TE}$ & 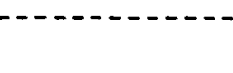 & 40 & 40 & VESSEL COLD LEG & & & \\
\hline 1353 & & 1 & 4 & 6 & 0.000000 & 1 & ${ }^{*} \mathrm{CN}$ & 02 \\
\hline 1354 & & 0 & 1 & 29 & 46 & 0 & ${ }^{*} \mathrm{CN}$ & 03 \\
\hline 1355 & & 0 & 0 & 0 & 0 & 0 & ${ }^{*} \mathrm{CN}$ & 05 \\
\hline 1356 & & 1. $4209 \mathrm{E}-01$ & $1.5907 \mathrm{E}-01$ & 0.000000 & 0.000000 & 305.370000 & ${ }^{*} \mathrm{CN}$ & 06 \\
\hline 1357 & & 305.370000 & 0.000000 & 0.000000 & 1. $0000 \mathrm{E}+20$ & 1.000000 & ${ }^{*} \mathrm{CN}$ & 07 \\
\hline 1358 & & 0.000000 & 0.000000 & $1.0000 E+20$ & 1.000000 & & $* \mathrm{CN}$ & 08 \\
\hline 1359 & & 0 & 1 & 47 & 0 & & ${ }^{*} \mathrm{CN}$ & 09 \\
\hline 1360 & & 0 & 0 & 0 & 0 & 0 & ${ }^{*} \mathrm{CN}$ & 11 \\
\hline 1361 & & 5. 0000E-02 & $5.0000 \mathrm{E}-02$ & 0.000000 & 0.000000 & 305.370000 & ${ }^{*} \mathrm{CN}$ & 12 \\
\hline 1362 & & 305.370000 & 0.000000 & 0.000000 & $1.0000 E+20$ & 1.000000 & ${ }^{*} \mathrm{CN}$ & 13 \\
\hline 1363 & & 0.000000 & 0.000000 & $1.0000 \mathrm{E}+20$ & 1.000000 & & $* \mathrm{CN}$ & 14 \\
\hline 1.264 & $\mathrm{~F}$ & 1. $0000 \mathrm{E}-01 \mathrm{E}$ & & & & & *DX & $\mathrm{P}$ \\
\hline 1365 & $\mathrm{~F}$ & $8.2790 \mathrm{E}-03 \mathrm{E}$ & & & & & *VOL & $P$ \\
\hline 1366 & & 0.063425 & $0.141925 \mathrm{E}$ & & & & $* F A$ & $\mathrm{P}$ \\
\hline 1367 & & 0.001000 & $0.000000 \mathrm{E}$ & & & & *FRIC & $\mathrm{P}$ \\
\hline 1368 & $\mathrm{~F}$ & $0.000000 E$ & & & & & *GRAV & $\mathrm{P}$ \\
\hline 1369 & $\mathrm{~F}$ & $2.8418 \mathrm{E}-01 \mathrm{E}$ & & & & & ${ }^{*} \mathrm{HD}$ & $\mathrm{P}$ \\
\hline 1370 & $\mathrm{~F}$ & $\mathrm{OE}$ & & & & & *ICFLG & $\mathrm{P}$ \\
\hline 1371 & $\mathrm{~F}$ & $2 E$ & & & & & $* N F F$ & $\mathrm{P}$ \\
\hline 1.372 & $F$ & $0.000000 \mathrm{E}$ & & & & & *ALP & $\mathrm{P}$ \\
\hline 1373 & $\mathrm{~F}$ & $0.000000 \mathrm{E}$ & - & & & & $* \mathrm{VL}$ & $\mathrm{P}$ \\
\hline 1374 & $\mathrm{~F}$ & $0.000000 E$ & & & & & *VV & $\mathrm{P}$ \\
\hline 1375 & $\mathrm{~F}$ & $552.800000 \mathrm{E}$ & & & & & ${ }^{*} \mathrm{TL}$ & $\mathbf{P}$ \\
\hline 1376 & $\mathrm{~F}$ & $552.800000 \mathrm{E}$ & & & & & $*$ TV & $\mathbf{P}$ \\
\hline 1377 & $\mathrm{~F}$ & $14.7800 \mathrm{E}+6 \mathrm{E}$ & & & & & $* P$ & P \\
\hline 1378 & $\mathrm{~F}$ & $0.000000 \mathrm{E}$ & & & & & $* P A$ & $\mathbf{P}$ \\
\hline 1379 & $\mathrm{~F}$ & $0.000000 \mathrm{E}$ & & & & & *QPPP & $P$ \\
\hline 1380 & $\mathrm{~F}$ & $6 E$ & & & & & *MAT ID & $P$ \\
\hline 1381 & $\mathrm{~F}$ & $552.800000 \mathrm{E}$ & & & & & ${ }^{*} T W$ & $\mathrm{P}$ \\
\hline 1382 & $F$ & 1. 0000E-01E & & & & & $* \mathrm{DX}$ & S \\
\hline 1383 & $\mathrm{~F}$ & $7.8540 E-04 E$ & & & & & *VOL & S \\
\hline 1384 & $\mathrm{~F}$ & $7.8540 \mathrm{E}-03 \mathrm{E}$ & & & & & $* F A$ & S \\
\hline 1385 & $F$ & $0.000000 \mathrm{E}$ & & & & & *FRIC & S \\
\hline 1386 & F & $0.000000 E$ & & & & & *GRAV & S \\
\hline 1387 & & $1.0000 \mathrm{~F}-01$ & $2.5150 \mathrm{E}-04 \mathrm{E}$ & & & & ${ }^{*} H D$ & S \\
\hline 1388 & $\mathrm{~F}$ & OE & & & & & *ICFLG & $S$ \\
\hline 1389 & $F$ & $2 E$ & & & & & *NFF & $\mathrm{S}$ \\
\hline 1390 & $\mathrm{~F}$ & $0.000000 \mathrm{E}$ & & & & & *ALP & S \\
\hline 1391 & $\mathrm{~F}$ & $0.000000 \mathrm{E}$ & & & & & $* V L$ & $\mathrm{~S}$ \\
\hline 1392 & $\mathrm{~F}$ & $0.000000 \mathrm{E}$ & & & & & $* \mathrm{VV}$ & S \\
\hline 1393 & $F$ & $552.800000 \mathrm{E}$ & & & & & $* T L$ & S \\
\hline 1394 & $\mathrm{~F}$ & $552.800000 \mathrm{E}$ & & & & & ${ }^{*}$ TV & S \\
\hline 1395 & $F$ & $14.7800 \mathrm{E}+6 \mathrm{E}$ & & & & & $* \mathrm{p}$ & $\mathrm{S}$ \\
\hline 1396 & $F$ & $0.0010000 \mathrm{~F}$ & & & & & ${ }^{*} \mathrm{PA}$ & S \\
\hline 1397 & $F$ & (1. OOOOOOE & * & & & & *QPPP & $\mathbf{S}$ \\
\hline 1398 & $\mathrm{~F}$ & $6 E$ & & & & & *MATID & $S$ \\
\hline 1399 & $\mathrm{~F}$ & $552.800000 \mathrm{E}$ & & & & & $* \mathrm{TW}$ & $\mathrm{S}$ \\
\hline 1400 & & & & & & & & \\
\hline
\end{tabular}




\begin{tabular}{|c|c|c|c|c|c|c|c|c|}
\hline 1401 & TEE & & 29 & 29 & VESSEL COLD LEG & AND DOWNCOMEI & & \\
\hline 1402 & & 1 & 10 & 6 & 0.000000 & 1 & ${ }^{*} \mathrm{CN}$ & 02 \\
\hline 1403 & & 0 & 1 & 46 & 1 & 0 & ${ }^{*} \mathrm{CN}$ & 03 \\
\hline 1404 & & 0 & 0 & 0 & 0 & 0 & ${ }^{*} \mathrm{CN}$ & 05 \\
\hline 1405 & & $1.4209 E-01$ & $1.5907 \mathrm{E}-01$ & 0.000000 & 0.000000 & 305.370000 & ${ }^{*} \mathrm{CN}$ & 06 \\
\hline 1406 & & 305.370000 & 0.000000 & 0.000000 & $1.0000 E+20$ & 1.000000 & ${ }^{*} \mathrm{CN}$ & 07 \\
\hline 1407 & & 0.000000 & 0.000000 & $1.0000 E+20$ & 1.000000 & & ${ }^{*} \mathrm{CN}$ & 08 \\
\hline 1408 & & 0 & 1 & 34 & 0 & & ${ }^{*} \mathrm{CN}$ & 09 \\
\hline 1409 & & 0 & 0 & 0 & 0 & 0 & ${ }^{*} \mathrm{CN}$ & 11 \\
\hline 1410 & & $9.2000 \mathrm{E}-01$ & 1. $3600 E-01$ & 0.000000 & 0.000000 & 305.370000 & ${ }^{*} \mathrm{CN}$ & 12 \\
\hline 1411 & & 305.370000 & 0.000000 & 0.000000 & $1.0000 \mathrm{E}+20$ & 1.000000 & ${ }^{*} \mathrm{CN}$ & 13 \\
\hline 1412 & & 0.000000 & 0.000000 & 1. $0000 E+20$ & 1.000000 & & ${ }^{*} \mathrm{CN}$ & 14 \\
\hline 1413 & $\mathrm{~F}$ & $0.70974 \mathrm{E}+0 \mathrm{E}$ & & & & & ${ }^{*} \mathrm{DX}$ & $\mathrm{p}$ \\
\hline 1414 & $\mathrm{~F}$ & $1.0073 \mathrm{E}-01 \mathrm{E}$ & & & & & ${ }^{*}$ VOL & $\mathrm{P}$ \\
\hline 1415 & $\mathrm{~F}$ & $0.141925 \mathrm{E}$ & & & & & ${ }^{*} \mathrm{FA}$ & $\mathrm{p}$ \\
\hline 1416 & & 0.000000 & 1. $00000 E-3 E$ & & & & ${ }^{*} \mathrm{FR} 1 \mathrm{C}$ & $\mathrm{P}$ \\
\hline 1417 & $F$ & $0.000000 \mathrm{E}$ & & & & & ${ }^{*} \mathrm{GRAV}$ & $p$ \\
\hline 1418 & $\mathrm{~F}$ & $2.8418 \mathrm{E}-01 \mathrm{E}$ & & & & & ${ }^{*} \mathrm{HD}$ & $\mathrm{P}$ \\
\hline 1419 & $\mathrm{~F}$ & $\mathrm{OE}$ & & & & & *ICFLG & $\mathrm{p}$ \\
\hline 1420 & $\mathrm{~F}$ & $2 \mathrm{E}$ & & & & & ${ }^{*} \mathrm{NFF}$ & $\mathbf{P}$ \\
\hline 1421 & $\mathrm{~F}$ & $0.000000 \mathrm{E}$ & & & & & ${ }^{*} \mathrm{ALP}$ & P \\
\hline 1422 & $\mathrm{~F}$ & $0.000000 \mathrm{E}$ & & & & & $* \mathrm{VL}$ & $P$ \\
\hline 1423 & $\mathrm{~F}$ & $0.000000 \mathrm{E}$ & & & & & $* \mathrm{VV}$ & $\mathrm{P}$ \\
\hline 1424 & $F$ & $552.800000 E$ & & & & & ${ }^{*} \mathrm{TL}$ & $\mathbf{p}$ \\
\hline 1425 & $\mathrm{~F}$ & $552.800000 \mathrm{E}$ & & & & & ${ }^{*} \mathrm{TV}$ & P \\
\hline 1426 & $F$ & $14.7800 E+6 E$ & & & & & $* P$ & $\mathrm{p}$ \\
\hline 1427 & $\mathrm{~F}$ & $0.000000 \mathrm{E}$ & & & & & ${ }^{*} \mathrm{PA}_{\mathrm{A}}$ & $\mathrm{P}$ \\
\hline 1428 & $\mathrm{~F}$ & 0.000000[ & & & & & ${ }^{*} \mathrm{QPPP}$ & $\mathrm{P}$ \\
\hline 1429 & $\mathrm{~F}$ & $6 E$ & & & & & *MATID & $\mathrm{P}$ \\
\hline 1430 & $\mathrm{~F}$ & $552.800000 \mathrm{E}$ & & & & & $*$ TW & $\mathrm{p}$ \\
\hline 14.31 & $\mathrm{~F}$ & $0.100000 \mathrm{E}$ & & & & & ${ }^{*} \mathrm{DX}$ & $\mathrm{S}$ \\
\hline 1432 & $\mathrm{~F}$ & $1.41925 E-2 E$ & & & & & *VOL & $\mathrm{S}$ \\
\hline 1433 & $F$ & $1.41925 \mathrm{E}-1 \mathrm{E}$ & $=$ & & & & ${ }^{*} \mathrm{FA}$ & $\mathrm{s}$ \\
\hline 1434 & $\mathrm{~F}$ & $0.000000 \mathrm{E}$ & & & & & *FRIC & $\mathrm{S}$ \\
\hline 1435 & $\mathrm{~F}$ & $-0.999850 \mathrm{E}$ & & & & & *GRAV & $\mathrm{S}$ \\
\hline 1436 & $\mathrm{~F}$ & $1.0000 E-01 E$ & & & & & *HD & $\mathrm{s}$ \\
\hline 1437 & $\mathrm{~F}$ & $\mathrm{OE}$ & & & & & ${ }^{*}$ ICTLG & $\mathrm{s}$ \\
\hline 1438 & $\mathrm{~F}$ & $2 \mathrm{E}$ & & & & & ${ }^{* N F F}$ & $\mathrm{~S}$ \\
\hline 1439 & $\mathrm{~F}$ & $0.000000 \mathrm{E}$ & & & & & *ALP & $S$ \\
\hline 1440 & $\mathrm{~F}$ & $0.000000 E$ & & & & & $* \mathrm{VL}$ & $S$ \\
\hline 1441 & $\mathrm{~F}$ & $0.000000 E$ & & & & & $=* \mathrm{VV}$ & $S$ \\
\hline 1442 & $\mathrm{~F}$ & $552.800000 \ddot{E}$ & & & & & $* T L$ & $S$ \\
\hline 1443 & $\mathbf{F}$ & $552.800000 \mathrm{E}$ & & & & & ${ }^{* T V}$ & $S$ \\
\hline 1444 & $\mathrm{~F}$ & $14.7800 E+6 E$ & & & & & $* \mathrm{p}$ & $S$ \\
\hline 1445 & $\mathrm{~F}$ & $0.000000 \mathrm{E}$ & & & & & $* \mathrm{PA}$ & $\mathrm{S}$ \\
\hline 1446 & $\mathrm{~F}$ & $0.0000001:$ & & & & & ${ }^{*} \mathrm{QPPP}$ & $S$ \\
\hline 1447 & $\mathrm{~F}$ & $6 \mathrm{E}$ & & & & & *MATID & $s$ \\
\hline 1448 & F & $552.800000 \mathrm{l}:$ & & & & & ${ }^{*} \mathrm{TW}$ & $\mathrm{S}$ \\
\hline & & & & & & & & \\
\hline 1450 & TEE & & 96 & 96 & CMR AND & ASS & & \\
\hline
\end{tabular}


$F$
1

0

0.940000

305.370000

0.000000

0
0

1. 480000

305.370000

0.000000

0. 795500

0. $381000 E$

0.117847

$5.05676 \mathrm{E}-2 \mathrm{E}$

$\mathrm{F}$

0.141925E

$0.000000 E$

$-0.999850 \mathrm{~F}$

$0.100000 \mathrm{E}$

F

$F$

$F$

$F$

$\mathrm{F}$

F

$F$

$F$

$F$

F

$552.800000 \mathrm{E}$

0.795500

$0.381000 \mathrm{E}$

$-1.000000 \mathrm{E}$

F $\quad 0.014760 E$

F $\quad 0.000000 \mathrm{E}$

0.000000 RO6 - $1.000000 \mathrm{E}$

$0.063500 \mathrm{E}$

0.895500

0.533000
0.000000

96

0.136000

0.000000

0.000000

0.127000

0.000000

0.000000

0.895500

0.132039

$-1.000000 \mathrm{E}$

0.000000

0.000000

1. $0000 \mathrm{E}+20$

97

0.000000

0.000000

1. $0000 \mathrm{E}+20$

0.533000

$7.07414 \mathrm{E}-2$

4. $37986 E-2$

0.000000

1. $0000 \mathrm{E}+20$

1. 000000

0.000000

1. $0000 \mathrm{E}+20$

1. 000000

0. 330000
0. 330000

5. $73364 E-2$

305.370000

1. 000000

0.432000

*DX P

DX P

*VOL P

*FA P

*FRIC P

*GRAV P

*HD P

* ICFLG P

*NFF P

*ALP P

*VL P

*VV P

*TL P

*TV P

* $P \quad P$

*PA $\quad P$

*QPPP P

* MATID P

* TW P

0.432000

*DX S

*VOL S

* FA S

*FRIC S

*GRAV S

*HD S

* ICF LG S

*NFF S

* ALP S

*VL S

*VV S

*TL S

*TV S

* P S

*PA S

*QPPP $\mathrm{s}$

*MATID $\mathrm{S}$

*TW S 


\begin{tabular}{|c|c|c|c|c|c|c|c|c|}
\hline 1502 & $\mathrm{TE}$ & & 95 & 95 & VSSL MID LP AND & DNCMR BYPASS & & \\
\hline 1503 & & 1 & 10 & 6 & 0.000000 & 1 & ${ }^{*} \mathrm{CN}$ & 02 \\
\hline 1504 & & 0 & 1 & 35 & 95 & 0 & ${ }^{*} \mathrm{CN}$ & 03 \\
\hline 1505 & & 0 & 0 & 0 & 0 & 0 & ${ }^{*} \mathrm{CN}$ & 05 \\
\hline 1506 & & 0.940000 & 0.136000 & 0.000000 & 0.000000 & 305.370000 & ${ }^{*} \mathrm{CN}$ & 06 \\
\hline 1507 & & 305.370000 & 0.000000 & 0.000000 & $1.0000 \mathrm{E}+20$ & 1.000000 & ${ }^{*} \mathrm{CN}$ & 07 \\
\hline 1508 & & 0.000000 & 0.000000 & $1.0000 E+20$ & 1.000000 & & ${ }^{*} \mathrm{CN}$ & 08 \\
\hline 1509 & & 0 & 2 & 97 & 0 & & ${ }^{*} \mathrm{CN}$ & 09 \\
\hline 1510 & & 0 & 0 & 0 & 0 & 0 & ${ }^{*} \mathrm{CN}$ & 11 \\
\hline 1511 & & 1.480000 & 0.127000 & 0.000000 & 0.000000 & 305.370000 & ${ }^{*} \mathrm{CN}$ & 12 \\
\hline 1512 & & 305.370000 & 0.000000 & 0.000000 & 1. $0000 E+20$ & 1.000000 & ${ }^{*} \mathrm{CN}$ & 13 \\
\hline 1513 & & 0.000000 & 0.000000 & $1.0000 E+20$ & 1.000000 & & ${ }^{*} \mathrm{CN}$ & 14 \\
\hline 1514 & & $0.100000 \mathrm{E}$ & & & & & $* \mathrm{DX}$ & $p$ \\
\hline 1515 & & $0.034005 \mathrm{E}$ & & & & & *VOL & $p$ \\
\hline 1516 & $\mathrm{~F}$ & $0.340050 \mathrm{E}$ & & & & $\because$ & $* \mathrm{FA}$ & $\mathrm{P}$ \\
\hline 1517 & $\mathbf{F}$ & $0.000000 \mathrm{E}$ & & · & & & *FRIC & $\mathrm{p}$ \\
\hline 1518 & $F$ & $0.000000 \mathrm{E}$ & & 1 & ' & & * GRAV & $\mathbf{P}$ \\
\hline 1519 & $F$ & $0.100000 \mathrm{E}$ & & & & & $* \mathrm{HD}$ & $\mathbf{P}$ \\
\hline 1520 & $\mathbf{F}$ & $\mathrm{OE}$ & & & & & * ICFLG & $P$ \\
\hline 1521 & $\mathbf{F}$ & $2 \bar{E}$ & & & & & *NFF & $\mathrm{p}$ \\
\hline 1522 & $\mathbf{F}$ & $0.000000 \mathrm{E}$ & & & & & *ALP & $\mathrm{P}$ \\
\hline 1523 & $\mathrm{~F}$ & $0.000000 E$ & & & & & $* \mathrm{VL}$ & $\mathrm{P}$ \\
\hline 1524 & $\mathrm{~F}$ & $0.000000 E$ & & & & & *VV & $\mathrm{P}$ \\
\hline 1525 & $\mathrm{~F}$ & $552.800000 \mathrm{E}$ & & & & & $*$ TL & $\mathrm{p}$ \\
\hline 1526 & $\mathbf{F}$ & $552.800000 \mathrm{E}$ & & & & & $* T V$ & $\mathrm{P}$ \\
\hline 1527 & $\mathbf{F}$ & $14.7800 \mathrm{E}+6 \mathrm{E}$ & & & & & $* P$ & $\mathrm{P}$ \\
\hline 1528 & $\mathrm{~F}$ & $0.000000 E$ & & & & & $* P_{A}$ & $\mathrm{P}$ \\
\hline 1529 & $\mathrm{~F}$ & $0.000000 \mathrm{E}$ & & & & & *QPPP & $\mathrm{P}$ \\
\hline 1530 & $\mathrm{~F}$ & $6 \mathrm{E}$ & & & & & *MATID & $\mathrm{P}$ \\
\hline 1531 & $\mathrm{~F}$ & $552.800000 E$ & & i & & & $* T W$ & $\mathrm{P}$ \\
\hline 1532 & & 0.432000 & $0.847000 \mathrm{E}$ & & & & $* \mathrm{DX}$ & S \\
\hline 1533 & $\mathrm{~F}$ & $-1.000000 \mathrm{E}$ & & & & & *VOL & $\mathrm{S}$ \\
\hline 1534 & $\mathrm{~F}$ & $0.014760 \mathrm{E}$ & & & & & ${ }^{*} \mathrm{FA}$ & $S$ \\
\hline 1535 & $\mathrm{~F}$ & $0.000000 \mathrm{E}$ & & & & & *FRIC & $S$ \\
\hline 1536 & & $0.000000 \mathrm{~F}$ & $1.000000 \mathrm{E}$ & & & & *GRAV & $\mathrm{S}$ \\
\hline 1537 & $F$ & $0.063500 \mathrm{E}$ & & & & & ${ }^{*} \mathrm{HD}$ & $\mathrm{S}$ \\
\hline 1538 & $\Gamma$ & $\mathrm{OE}$ & & & & & *ICFLG & $S$ \\
\hline 1539 & $F$ & $2 \mathrm{E}$ & & & & & *NFF & $S$ \\
\hline 1540 & $\mathrm{~F}$ & $0.000000 \mathrm{E}$ & & & & & ${ }^{*} A L P$ & $\mathrm{~S}$ \\
\hline 1541 & $\mathrm{~F}$ & $0.000000 \mathrm{E}$ & & & & & $* \mathrm{VL}$ & $S$ \\
\hline 1542 & $\mathrm{~F}$ & $0.000000 E$ & " & & & & *VV & $S$ \\
\hline 1543 & $\mathbf{F}$ & $552.800000 \mathrm{E}$ & $1+\infty$ & & & & ${ }^{*} \mathrm{TL}$ & S \\
\hline 1544 & $\mathrm{~F}$ & $552.800000 \mathrm{E}$ & & & & & $* T V$ & $S$ \\
\hline 1545 & $\mathrm{~F}$, & 14. $7800 E+6 E$ & & & & & $* \mathrm{P}$ & $S$ \\
\hline 1546 & $F$ & $0.000000 \mathrm{E}$ & & & & & $* P A$ & $S$ \\
\hline 1547 & $\mathbf{F}$ & $0.000000 \mathrm{E}$ & & & & & *QPPP & S \\
\hline 1548 & $\mathrm{~F}$ & $6 E$ & & & & & *MATID & $\mathrm{S}$ \\
\hline 1549 & $\mathrm{~F}$ & $552.800000 \mathrm{E}$ & & & & , & $* T W$ & $\mathrm{~s}$ \\
\hline 1550 & & $\ldots \ldots \ldots$ & - & & & & & \\
\hline
\end{tabular}


1551 TEE

1552

1553

1554

1555

1556

1557

1558

1559

1560

1561

1562

1563

1564

1565

1566

1567

1568

1569

1570

1571

1572

1573

1574

1575

1576

1577

1578

1579

1580

1581

1582

1583

1584

1585

1586

1587

1588

1589

1590

1591

1592

1593

1594

1595

1596

1597

1598

1599

1600

TEE

0.0

0. 331970

305. 370000

0.000000

0

1. 549400

305.370000

0.000000

$0.050000 \mathrm{E}$

2. $06605 \mathrm{E}-2 \mathrm{E}$

$0.413210 \mathrm{E}$

$0.000000 \mathrm{E}$

$0.000000 \mathrm{E}$

$0.100000 \mathrm{E}$

OE

$0.000000 \mathrm{E}$

$0.000000 \mathrm{E}$

$0.000000 E$

$552.800000 \mathrm{E}$

$552.800000 \mathrm{E}$

14. $7800 \mathrm{E}+6 \mathrm{E}$

$0.000000 \mathrm{E}$

$0.000000 \mathrm{E}$

$552.800000 \mathrm{E}$

$2.049500 \mathrm{E}$

$-1.000000 \mathrm{E}$

1. $24118 \mathrm{E}-2 \mathrm{E}$

$0.000000 \mathrm{E}$

$1.000000 \mathrm{E}$

$0.003500 E$

$O E$

$0.000000 \mathrm{E}$

$0.000000 \mathrm{E}$

$0.000000 \mathrm{E}$

$552.800000 \mathrm{E}$

$552.800000 \mathrm{E}$

14. $7800 \mathrm{E}+6 \mathrm{E}$

$0.000000 \mathrm{E}$

$0.000000 \mathrm{E}$

$552.800000 \mathrm{E}$
86 CORE BYPASS

$\begin{array}{rr}6 & 0.000000 \\ 86 & 36 \\ 0 & 0\end{array}$

$1.0000 \mathrm{E}+20$

0.0000

0.000000

$1.0000 \mathrm{E}+20$

84
0

0.016343

0.000000

0.000000

0.000000

0.000000

1. $0000 \mathrm{E}+20$
0.000000

1.000000

36

$\begin{array}{lll}1 & { }^{*} \mathrm{CN} & 02 \\ 0 & { }^{*} \mathrm{CN} & 03 \\ 0 & { }^{*} \mathrm{CN} & 05\end{array}$

$305.370000{ }^{*} \mathrm{CN} \quad 06$

$1.000000{ }^{*} \mathrm{CN} \quad 07$

* $\mathrm{CN} \quad 08$

*CN 09

$0 \quad{ }^{*} \mathrm{CN} \quad 11$

1. $0000 \mathrm{E}+20$

1.000000

$\begin{array}{rrr}05.370000 & { }^{*} \mathrm{CN} & 12 \\ 1.000000 & { }^{*} \mathrm{CN} & 13\end{array}$

*CN 14

*DX P

*VOL P

*FA P

*FRIC P

*GRAV P

*HD P

*ICFLG P

*NFF P

*ALP P

*VL P

*VV P

*TL P

*TV $\mathrm{p}$

$* P \quad P$

*PA P

*QPPP P

*MATID P

*TW P

*DX S

*VOL S

*FA S

*FRIC S

* GRAV S

* HD S

*ICFLG S

*NFF $S$

*ALP S

*VL S

$* V V \quad S$

*TL S

*TV $S$

$* P \quad S$

*PA $\quad S$

*QPPP $\mathrm{S}$

*MATID S

*TW $\mathrm{S}$ 


1601
1602
1603
1604
1605
1606
1607
1608
1609
1610
1611
1612
1613
1614
1615
1616
1617
1618
1619
1620
1621
1622
1623
1624
1625
1626
1627
1628
1629
1630
1631
1632
1633
1634
1635
1636
1637
1638
1639
1640
1641
1642
1643
1644
1645
1646
1647
1648
1649
1650

\begin{tabular}{|c|c|c|c|c|c|c|c|}
\hline & 1 & 10 & 6 & 0.000000 & 1 & ${ }^{*} \mathrm{CN}$ & 02 \\
\hline & 0 & 2 & 96 & 35 & 0 & ${ }^{*} \mathrm{CN}$ & 03 \\
\hline & 0 & 0 & 0 & 0 & 0 & ${ }^{*} \mathrm{CN}$ & 05 \\
\hline & $9.4000 \mathrm{E}-01$ & 1. $3600 \mathrm{E}-01$ & 0.000000 & 0.000000 & 305.370000 & ${ }^{*} \mathrm{CN}$ & 06 \\
\hline & 305.370000 & 0.000000 & 0.000000 & $1.0000 E+20$ & 1.000000 & ${ }^{*} \mathrm{CN}$ & 07 \\
\hline & 0.000000 & 0.000000 & $1.0000 E+20$ & 1.000000 & & ${ }^{*} \mathrm{CN}$ & 08 \\
\hline & 0 & 1 & 36 & 0 & & ${ }^{*} \mathrm{CN}$ & 09 \\
\hline & 0 & 0 & 0 & 0 & 0 & ${ }^{*} \mathrm{CN}$ & 11 \\
\hline & $5.0000 \mathrm{E}-02$ & $5.0000 E-02$ & 0.000000 & 0.000000 & 305.370000 & ${ }^{*} \mathrm{CN}$ & 12 \\
\hline & 305.370000 & 0.000000 & 0.000000 & 1. $0000 \mathrm{E}+20$ & 1.000000 & ${ }^{*} \mathrm{CN}$ & 13 \\
\hline & 0.000000 & 0.000000 & 1. $0000 E+20$ & 1.000000 & & ${ }^{*} \mathrm{CN}$ & 14 \\
\hline & 8. $4700 E-01$ & 4. $3200 \mathrm{E}-01 \mathrm{E}$ & & & & ${ }^{*} \mathrm{DX}$ & $\mathrm{P}$ \\
\hline & 1. $18892 \mathrm{E}-1$ & $1.5429 E-01 E$ & & & & $* \mathrm{VOL}$ & $\mathbf{p}$ \\
\hline $\mathrm{RO} 2$ & $1.41925 \mathrm{E}-1$ & 3. $4005 \mathrm{E}-01 \mathrm{E}$ & & & & $* \mathrm{FA}$ & $\mathbf{P}$ \\
\hline $\mathrm{F}$ & $0.000000 \mathrm{E}$ & & & & & *FRIC & $\mathbf{P}$ \\
\hline $\mathrm{RO} 2$ & -1.000000 & $0.000000 \mathrm{E}$ & & & & *GRAV & $\mathbf{p}$ \\
\hline $\mathrm{F}$ & 1. $0000 \mathrm{E}-01 \mathrm{E}$ & & & & & $* \mathrm{HD}$ & $\mathrm{p}$ \\
\hline $\mathrm{F}$ & $\mathrm{OE}$ & & & & & ${ }^{*}$ ICFLG & $\mathrm{P}$ \\
\hline$F$ & $2 \mathrm{E}$ & & & & & ${ }^{*} \mathrm{NFF}$ & $\mathrm{p}$ \\
\hline $\mathrm{F}$ & $0.000000 \mathrm{E}$ & & & & & ${ }^{*}$ ALP & $\mathrm{P}$ \\
\hline $\mathrm{F}$ & $0.000000 \mathrm{E}$ & & & & & $* \mathrm{VL}$ & $\mathrm{P}$ \\
\hline $\mathbf{F}$ & $0.000000 \mathrm{E}$ & & & & & $* V V$ & $P$ \\
\hline $\mathrm{F}$ & $552.800000 \mathrm{E}$ & & & & & $* \mathrm{TL}$ & $\mathbf{P}$ \\
\hline$F$ & $552.800000 \mathrm{E}$ & & & & & $* \mathrm{TV}$ & $\mathrm{P}$ \\
\hline $\mathrm{F}$ & 14. $7800 \mathrm{E}+6 \mathrm{E}$ & & & & & $* P$ & $\mathbf{P}$ \\
\hline $\mathrm{F}$ & $0.000000 E$ & & & & & $* \mathrm{PA}$ & $\mathbf{p}$ \\
\hline $\mathrm{F}$ & $0.000000 \mathrm{E}$ & & & & & ${ }^{*} \mathrm{QPPP}$ & $\mathrm{P}$ \\
\hline $\mathrm{F}$ & $6 \mathrm{E}$ & & & & & *MATID & $P$ \\
\hline$F$ & $552.800000 \mathrm{E}$ & & & & & *TW & $\mathrm{P}$ \\
\hline $\mathrm{F}$ & $1.0000 \mathrm{E}-01 \mathrm{E}$ & & & & & $* \mathrm{DX}$ & $\mathrm{S}$ \\
\hline$F$ & $4.1321 \mathrm{E}-02 \mathrm{E}$ & & & & & $* \mathrm{VOL}$ & $\mathrm{S}$ \\
\hline $\mathrm{F}$ & $4.1321 \mathrm{E}-01 \mathrm{E}$ & & & & & ${ }^{*} \mathrm{FA}$ & $\mathbf{S}$ \\
\hline $\mathrm{F}$ & $0.000000 \mathrm{E}$ & & & & & *FRIC & $\mathrm{S}$ \\
\hline $\mathrm{F}$ & $0.000000 \mathrm{E}$ & & & & & *GRAV & $\mathrm{s}$ \\
\hline $\mathrm{F}$ & 1. $0000 \mathrm{E}-01 \mathrm{E}$ & & & & & $* \mathrm{HD}$ & $\mathrm{S}$ \\
\hline $\mathrm{F}$ & $\mathrm{OE}$ & & & & & *ICFLG & 5 \\
\hline $\mathbf{F}$ & $2 E$ & & & & & $* \mathrm{NFF}$ & $\mathrm{S}$ \\
\hline $\mathrm{F}$ & $0.000000 \mathrm{E}$ & & 6 & & & *ALP & $\mathrm{S}$ \\
\hline $\mathrm{F}$ & $0.000000 \mathrm{E}$ & & & & & $* \mathrm{VL}$ & $\mathbf{S}$ \\
\hline $\mathrm{F}$ & $0.000000 \mathrm{E}$ & & & & & $* \mathrm{VV}$ & $\mathrm{s}$ \\
\hline $\mathrm{F}$ & $552.800000 \mathrm{E}$ & & & & & ${ }^{*} T L$ & $S$ \\
\hline $\mathrm{F}$ & $552.800000 \mathrm{E}$ & & & & & ${ }^{*} T \mathrm{~V}$ & S \\
\hline $\mathrm{F}$ & 14. $7800 \mathrm{E}+6 \mathrm{E}$ & & & & & $* \mathrm{P}$ & $\mathrm{S}$ \\
\hline $\mathrm{F}$ & $0.000000 \mathrm{E}$ & & & & & ${ }^{*} \mathrm{PA}$ & $\mathrm{s}$ \\
\hline $\mathrm{F}$ & $0.000000 E$ & & & & & ${ }^{*} \mathrm{QPPP}$ & $\mathrm{S}$ \\
\hline$\Gamma$ & $61:$ & & & & & *MATID & $\mathrm{s}$ \\
\hline $\mathrm{F}$ & $552.800000 \mathrm{E}$ & & & & & $* T W$ & $S$ \\
\hline TEE & & 83 & 83 & ER PLENUM & CORE BYPAS & & \\
\hline & 2 & 4 & 6 & 0.000000 & 1 & ${ }^{*} \mathrm{CN}$ & 02 \\
\hline
\end{tabular}




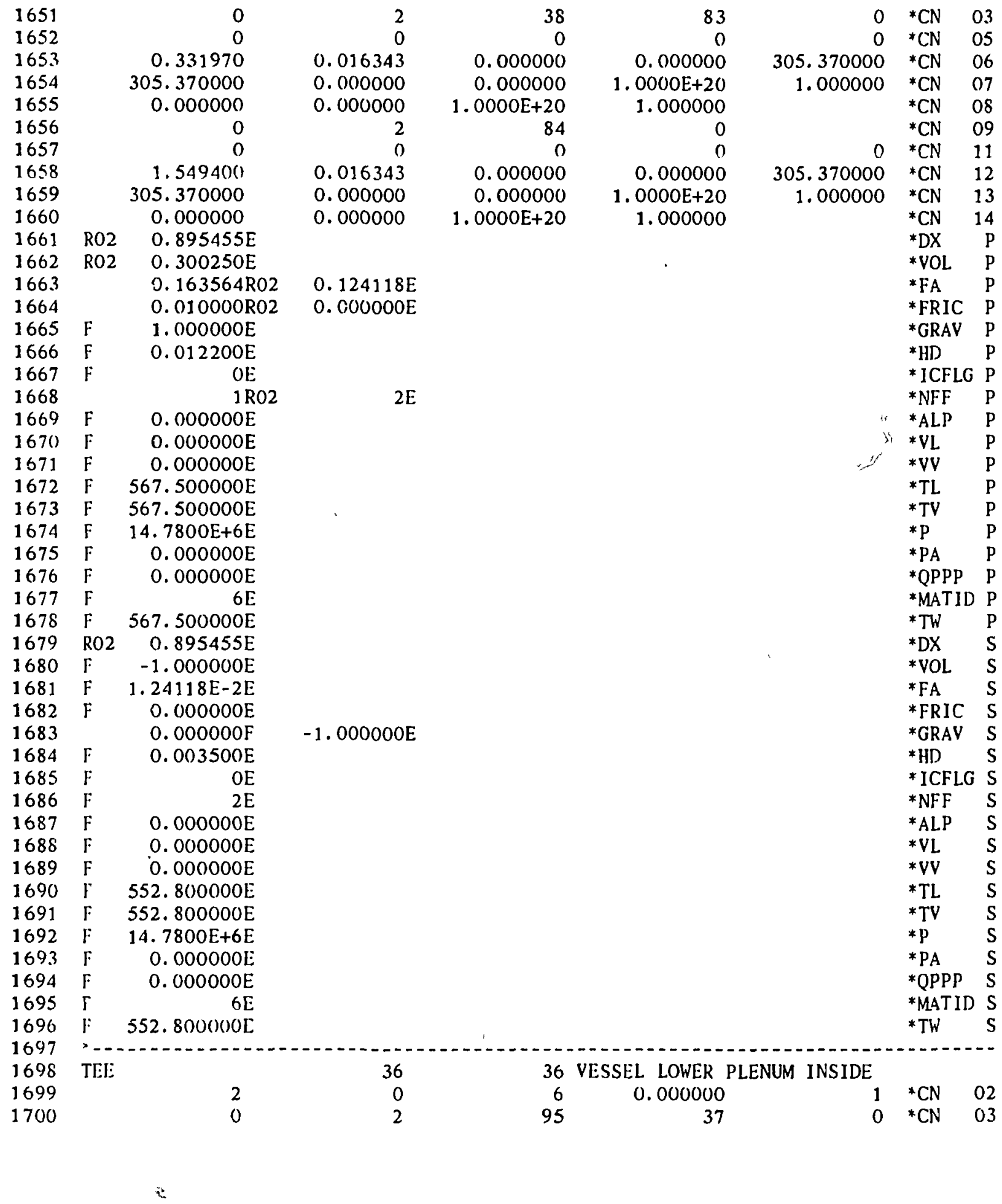




\begin{tabular}{|c|c|c|c|c|c|c|c|c|}
\hline 1701 & & 3. 3197: -01 & 4. 9029::-02 & 0.000000 & 0.000000 & 305.370000 & ${ }^{*} \mathrm{CN}$ & 06 \\
\hline 1702 & & 305.370000 & 0.000000 & 0.000000 & $1.0000 \mathrm{E}+20$ & 1.000000 & ${ }^{*} \mathrm{CN}$ & 07 \\
\hline 1703 & & 0 & 1 & 86 & 0 & & ${ }^{*} \mathrm{CN}$ & 09 \\
\hline 1704 & & $5.0000 \mathrm{E}-02$ & $5.0000 E-02$ & 0.000000 & 0.000000 & 305.370000 & ${ }^{*} \mathrm{CN}$ & 12 \\
\hline 1705 & & 305.370000 & 0.000000 & 0.000000 & $1.0000 E+20$ & 1.000000 & ${ }^{*} \mathrm{CN}$ & 13 \\
\hline 1706 & & 4. $3200 \mathrm{E}-01$ & $8.4700 \mathrm{E}-01 \mathrm{E}$ & & & & ${ }^{*} \mathrm{DX}$ & $\mathrm{P}$ \\
\hline 1707 & & 1. $46955 \mathrm{E}-1$ & 2. $31723 \mathrm{E}-1 \mathrm{E}$ & & & & *VOL & $\mathrm{p}$ \\
\hline 1708 & $\mathrm{RO2}$ & 3. $4005 \mathrm{I}:-01$ & $1.63564 \mathrm{E}-1 \mathrm{E}$ & & & & ${ }^{*} \mathrm{FA}$ & $\mathrm{P}$ \\
\hline 1709 & $\mathrm{R} 02$ & 0.000000 & $2.44366 \mathrm{E}-2 \mathrm{E}$ & & & & *FRIC & $\mathrm{P}$ \\
\hline 1710 & & $0.000000 R 02$ & 1. $0000 \mathrm{E}+00 \mathrm{E}$ & & & & ${ }^{*}$ GRAV & $\mathrm{P}$ \\
\hline 1711 & $\mathrm{R} 02$ & $1.0000 \mathrm{E}-01$ & 1. $2200 \mathrm{E}-02 \mathrm{E}$ & & & & $* \mathrm{HD}$ & $\mathrm{P}$ \\
\hline 1712 & $\mathrm{~F}$ & $O E$ & & & & & ${ }^{*}$ ICFLG & $P$ \\
\hline 1713 & $\mathrm{RO2}$ & 2 & $1 \mathrm{E}$ & & & & $* \mathrm{NFF}$ & $\mathrm{P}$ \\
\hline 1714 &.$F$ & $0.000000 \mathrm{E}$ & & & & & *ALP & $\mathrm{P}$ \\
\hline 1715 & $\mathrm{~F}$ & $0.000000 \mathrm{E}$ & & & & & $* V L$ & $\mathbf{P}$ \\
\hline 1716 & $\mathrm{~F}$ & $0.000000 \mathrm{E}$ & & & & & $* \mathrm{VV}$ & $P$ \\
\hline 1717 & $\mathrm{~F}$ & $552.800000 \mathrm{E}$ & & & & & $* \mathrm{TL}$ & $\mathrm{p}$ \\
\hline 1718 & $\mathrm{~F}$ & $552.800000 \mathrm{E}$ & & & & & $* T V$ & $\mathbf{P}$ \\
\hline 1719 & $\mathrm{~F}$ & $14.7800 E+6 E$ & & & & & $* \mathrm{P}$ & $\mathrm{P}$ \\
\hline 1720 & $\mathrm{~F}$ & $0.000000 \mathrm{E}$ & & & & & *PA & $P$ \\
\hline 1721 & $\mathrm{~F}$ & $0.5000 E-01 E$ & & & & & *DX & $\mathrm{S}$ \\
\hline 1722 & $\mathrm{~F}$ & 2. $06605 E-02 E$ & & & & & *VOL & $\mathrm{S}$ \\
\hline 1723 & $\mathrm{~F}$ & $4.1321 \mathrm{E}-01 \mathrm{E}$ & & & & & ${ }^{*} \mathrm{FA}$ & S \\
\hline 1724 & $\mathrm{~F}$ & $0.000000 \mathrm{E}$ & & & & & *FRIC & $S$ \\
\hline 1725 & $\mathrm{~F}$ & $0.000000 \mathrm{E}$ & & & & & ${ }^{*}$ GRAV & $S$ \\
\hline 1726 & $\mathrm{~F}$ & 1. $0000 \mathrm{E}-01 \mathrm{E}$ & & & & & $* \mathrm{HD}$ & S \\
\hline 1727 & $\mathrm{~F}$ & $\mathrm{OE}$ & & & & & ${ }^{* I C F L G}$ & $\mathrm{~s}$ \\
\hline 1728 & $\mathrm{~F}$ & $2 \mathrm{E}$ & & & & & ${ }^{* N F F}$ & S \\
\hline 1729 & $\mathrm{~F}$ & $0.000000 \mathrm{E}$ & & & & & *ALP & $S$ \\
\hline 1730 & $\mathrm{~F}$ & $0.000000 \mathrm{E}$ & & & & & $* V_{L}$ & S \\
\hline 1731 & $\mathrm{~F}$ & $0.000000 \mathrm{E}$ & & & & & $* \mathrm{VV}$ & $\mathrm{S}$ \\
\hline 1732 & $\mathrm{~F}$ & $552.800000 \mathrm{E}$ & & & & & $* \mathrm{TL}$ & $\mathrm{S}$ \\
\hline 1733 & $\mathrm{~F}$ & $552.800000 \mathrm{E}$ & & & & & $* \mathrm{TV}$ & $S$ \\
\hline 1734 & $\mathrm{~F}$ & 14. $7800 \mathrm{E}+6 \mathrm{E}$ & & & & & $* \mathrm{P}$ & $\mathrm{S}$ \\
\hline 1735 & $\mathrm{~F}$ & $0.000000 \mathrm{E}$ & & & & & $* \mathrm{P}_{\mathrm{A}}$ & $\mathrm{S}$ \\
\hline 1736 & & & & & & & & . \\
\hline 1737 & CORE & & 37 & 37 & NUCLEAR REACTOR & CORE & & \\
\hline 1738 & & 4 & 37 & 38 & 6 & 1 & $* \mathrm{CN}$ & 02 \\
\hline 1739 & & 0 & 4 & 0 & & & ${ }^{*} \mathrm{CN}$ & 03 \\
\hline 1740 & & 14 & 6 & 11 & 5 & & $* \mathrm{CN}$ & 04 \\
\hline 1741 & & 23 & 1 & -14 & 0 & 0 & ${ }^{*} \mathrm{CN}$ & 05 \\
\hline 1742 & & 0 & 1 & 1 & 0 & 0 & ${ }^{*} \mathrm{CN}$ & 06 \\
\hline 1743 & & 0 & 1 & 1 & 1 & 5 & ${ }^{*} \mathrm{CN}$ & 07 \\
\hline 1744 & & 3 & 10 & 4 & 0 & 0 & ${ }^{*} \mathrm{CN}$ & 08 \\
\hline 1745 & & 0.000000 & $1.6250 \mathrm{E}-05$ & $-1.0000 E+20$ & 1. $0000 E+00$ & $1.0000 E+00$ & $* \mathrm{CN}$ & 09 \\
\hline 1746 & & $3.6900 \mathrm{E}+07$ & 0.000000 & $0.0(0) 000$ & 0.000000 & & ${ }^{*} \mathrm{CN}$ & 10 \\
\hline 1747 & & 0.331970 & 0.016343 & 0.000000 & 0.000000 & 305.370000 & ${ }^{*} \mathrm{CN}$ & 11 \\
\hline 1748 & & 305.370000 & 0.000000 & 1. 334100 & 0.700000 & $1.00000 \mathrm{E}+3$ & ${ }^{*} \mathrm{CN}$ & 12 \\
\hline 1749 & & 0.000000 & 0.000000 & 0.000000 & & & ${ }^{*} \mathrm{CN}$ & 13 \\
\hline 1750 & & 12 & 1 & 1 & 1 & & ${ }^{*} \mathrm{CN}$ & 14 \\
\hline
\end{tabular}


1751

1752

1753

1754

1755

1756

1757

1758

1759

1760

1761

1762

1763

1764

1765

1766

1767

1768

1769

1770)

1771

1772

1773

1774

1775

1776

1777

1778

1779

1780

1781

1782

1783

1784

1785

1786

1787

1788

1789

1790

1791

1792

1793

1794

1795

1796

1797

1798

1799

1800

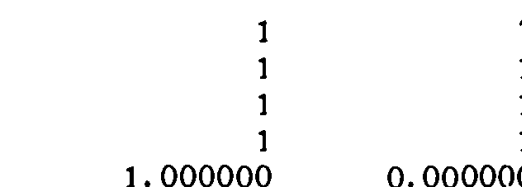

3. $8100 \mathrm{E}-01$

$7.8656 \mathrm{E}-02$

F $\quad 1.63564 \mathrm{E}-1 \mathrm{E}$

2. $44366 \mathrm{E}-2 \mathrm{RO} 3$

F $\quad 1.0000 E+00 E$

F $1.2200 \mathrm{E}-02 \mathrm{E}$

$F \quad O E$

$F \quad 1 E$

$F \quad 0.000000 E$

F $\quad 0.000000 \mathrm{E}$

F $\quad 0.000000 E$

552.800000

552.800000

F $\quad 14.7800 \mathrm{E}+6 \mathrm{E}$

F $\quad 0.000000 E^{\circ}$

$\mathrm{F} \quad 0.000000 \mathrm{E}$

$\mathrm{F} \quad 6 \mathrm{~F}$

R04 552. 800000R0 4

3. $8100 \mathrm{E}-01$

9. $5500 \mathrm{E}-01$

1.016000

F $\quad 1.0000 E+00 E$

1. $5000 \mathrm{E}+00$

0.000000

5. $0000 \mathrm{E}-02 \mathrm{E}$

F $\quad 1.3000 \mathrm{E}+03 \mathrm{E}$ 0.000000

3. $8725 \mathrm{E}-03$

$\mathrm{R} 06$

R02 0.000000

5. $0000 \mathrm{E}-01$

$-3.5600 \mathrm{E}-02$

$1.0000 \mathrm{E}+00$

$-1.6550 \mathrm{E}-01$

1. $5000 \mathrm{E}+(10$

2. $9300 \mathrm{E}+02$

1. $0000 \mathrm{E}+03$

2. $0000 \mathrm{E}+0.3$

$-6.2700 \mathrm{E}-05$

-3. $4000 \mathrm{E}-05$

$-2.4000 E-05$

0.000000

5. 6090[:+02

1. $0000 \mathrm{OH}+02$

$-2.0500 \mathrm{E}-04$

9. $6000 \mathrm{E}-01$

5. $0000 \mathrm{E}-01 \mathrm{E}$

1. $9000 \mathrm{E}-01$

7. 7449E-04

4. $6622 \mathrm{E}-0.3$

2. $0000 \mathrm{E}-01$

$-1.0160 E-02$

8. $0000 \mathrm{E}-01$

$-1.4740 \mathrm{E}-01$

1. $3000 E+00$

$-1.6840 \mathrm{E}-01 \mathrm{E}$

4. $0000 \mathrm{E}+02$

1. $2000 \mathrm{E}+03$

2. $2000 E+03$

$-5.3700 E-05$

$-3.1000 E-05$

-2.2900 I: -05 I:

2. $9300 \mathrm{E}+02$

5. $8870 E+02$

8. $0000 \mathrm{E}+02$

$-3.2300 \mathrm{E}-() 4$
0.000000

2. $9100 E-01$

6. $0075 \mathrm{E}-02$

0.000000

557.700000

557.700000

562.600000

0.000000

4. $7100 \mathrm{E}-01$

9. $7236 \mathrm{E}-02$
562.600000

0.651693

1. $00000 \mathrm{E}-2 \mathrm{E}$

5. $3300 \mathrm{E}-01 \mathrm{E}$

1. $10035 \mathrm{E}-1 \mathrm{E}$

$567.500000 \mathrm{E}$

$567.500000 \mathrm{E}$

557.700000R04 562.600000R04 567.500000E

$6.7200 \mathrm{E}-01 \quad 1.1430 \mathrm{E}+00$

9. $6500 \mathrm{E}-01$

$1.060000 R 03$

$0.000000 E$

8. 0000E-01

1. 5489E-03

4. $7422 \mathrm{E}-03$

3R02 $2 \mathrm{E}$

$-5.1000 E-04$

6. $0000 \mathrm{E}-01$

$-5.3000 \mathrm{E}-02$

1. $1000 \mathrm{E}+00$

$-1.6700 \mathrm{E}-01$

5. $0000 \mathrm{E}+02$

1. $4000 \mathrm{E}+0.3$

0.000000

$-4.8000 \mathrm{E}-05$

$-2.8700 E-05$

3. $9430 \mathrm{~F}:+(12$

6. $16501:+02$

1. $5000 \mathrm{E}+(03$

$-5.0800 \mathrm{E}-04$

1. $6760 \mathrm{E}+00 \mathrm{E}$

9. $7500 \mathrm{E}-01$

1. $0000 \mathrm{E}+00$

2. 3235E-03

5. $0508 \mathrm{E}-03$

3. $0000 \mathrm{E}-01$

$-2.0300 \mathrm{E}-02$

9. $0000 \mathrm{E}-01$

$-1.5820 \mathrm{E}-01$

1. $4000 \mathrm{E}+00$

6. $5000 \mathrm{E}+02$

1. $6000 \mathrm{E}+03$

0.000000

$-4.2100 E-05$

$-2.6800 \mathrm{E}-0.5$

4. $7460 \mathrm{E}+02$

0.0000100

${ }^{*} \mathrm{CN} \quad 14$

${ }^{*} \mathrm{CN} \quad 14$

${ }^{*} \mathrm{CN} \quad 14$

*CN 15

$8.690000 \quad{ }^{*} \mathrm{CN} \quad 16$

*DX

*VOL

*FA

*FRIC

*GRAV

*HD

* ICFLG

*NFF

*ALP

*VL

*VV

*TL

*TV

*P.

*PA

*QPPP

*MATID

*TW

$* \mathrm{Z}$

9. 9000E-01 *RDPWR

* RDPWR

*CPOWR

*RPKF

6. 3000E-01 *ZPOWR

*ZPOWR

*NRDX

3. 0979E-03 *RADRD

5. 3594E-03E *RADRD

*MATRD

$-1.8100 \mathrm{E}-03 \quad{ }^{*} \mathrm{RPWTB}$

7. 0000E-01 *RPWTB

$-8.9300 \mathrm{E}-02 \quad$ *RPWTB

$1.2000 \mathrm{E}+00 \quad$ *RPWTB

$-1.6770 \mathrm{E}-01 \quad *$ RPWTB

$8.0000 \mathrm{E}+02$

1. $8000 \mathrm{E}+03 \quad *$ RCTF

$0.000000 *$ RCTF

$-3.8000 \mathrm{E}-05 \quad *$ RCTF

$-2.5300 \mathrm{E}-05 *$ RCTF

* RCTF

$5.3320 \mathrm{E}+02 \quad * \mathrm{RCTC}$

$-6.0000 \mathrm{E}+02 \quad *$ RCTC

2. 2000E+03 -7.2400E-05 *RCTC

$-7.1900 \mathrm{E}-04$

$-1.0590 \mathrm{E}-03$

${ }^{*}$ RCTC 


1801
1802
1803
1804
1805
1806
1807
1808
1809
1810
1811
1812
1813
1814
1815
1816
1817
1818
1819
1820
1821
1822
1823
1824
1825
1826
1827
1828
1829
1830
1831
1832
1833
1834
1835
1836
1837
1838
1839
1840
1841
1842
1843
1844
1845
1846
1847
1848
1849
1850

$-1.6090 \mathrm{E}-03$

$-6.0700 \mathrm{E}-04$

$-1.8600 \mathrm{E}-(04$

$-2.9200 \mathrm{E}-05$

$-5.9300 \mathrm{E}-04$

$-1.6500 \mathrm{E}-04$

R05 $0.000000 E$

ROS $0.000000 \mathrm{~F}$

0.000169

$0.000247 \mathrm{E}$

3.870000

$0.012700 \mathrm{E}$

9. $9163 \mathrm{E}+07$

4. $4156 \mathrm{E}+10 \mathrm{E}$

1. $7720 \mathrm{E}+00$

4. $8100 \mathrm{E}-05$

7. $5850 \mathrm{E}-10 \mathrm{E}$

2. $9900 \mathrm{E}-03$

6. $4500 \mathrm{E}-03$

5. 7000E-04E

6. $2263 \mathrm{E}+04$

4. $0742 \mathrm{E}+09$

6. 8247E+08E

$F$

F $\quad 0.000000 \mathrm{E}$

F $\quad 9.3000 \mathrm{E}-01 \mathrm{E}$

9. $8101 \mathrm{E}-01$

7.0991E-03

F $\quad 1.0000 E+00 E$

F 3.1300E+05E

F $\quad 0.000000 \mathrm{E}$

F $\quad 0.000000 E$

F $\quad 0.000000 E$

F $\quad 0.000000 \mathrm{E}$

F 552.800000E

F $\quad 0.000000 \mathrm{E}$

F $552.800000 \mathrm{E}$

F $\quad 0.000000 E$

F $552.800000 \mathrm{E}$

TEE

0.000000

$0.000000 \mathrm{E}$

$-2.7600 \mathrm{E}-04$

0.000832

1. 400000

1. $3495 \mathrm{E}+09$

5. $7740 \mathrm{E}-01$

5. $3440 E-06$

8. 2500E-03

2. $3100 \mathrm{E}-03$

5. $2723 E+05$

2. $5550 \mathrm{E}+09$
$-1.6000 \mathrm{E}-04$

$-1.3770 \mathrm{E}-0.3$

$-4.9600 \mathrm{E}-04$

$-1.2100 \mathrm{E}-04$

$-1.6600 \mathrm{E}-05$

$-4.4400 \mathrm{E}-04$

$8.4818 E+06$

1. $9468 \mathrm{~F}+09$

1. $9276 \mathrm{E}+10$

6. 7430E-02

5. $7260 \mathrm{E}-07$

1. 5500E-02

1. $6400 \mathrm{E}-03$

1. $9350 \mathrm{E}-02$

8. 5000E-04

1. $1486 \mathrm{E}+08$

1. $0161 \mathrm{E}+09$

6. $2140 \mathrm{E}-03$

1. $0360 \mathrm{E}-07$

4. 7390E-04

2. $9590 \mathrm{E}-08$

1. $1650 \mathrm{E}-02$

4. 3000E-04

9. $0869 \mathrm{E}+08$

$5.1462 \mathrm{~F}+08$

1. $010.3 E-02$

$1.7830 \mathrm{E}-03$

0.000000

*.TD

*GMIX

* GMIX

*GMLES

*PGAPT

*PLVOL

*PSLEN

*CLENN

*BURN

* RFTN

*BURN

*RFTN

*BURN

*RFTN

$\begin{array}{rr} & 33 \\ 1 & 4 \\ 0 & 2 \\ 0 & 0 \\ 3.3197 \mathrm{E}-01 & 4.9029 \mathrm{E}-02 \\ 305.3700(0) & 0.000000 \\ 0.00000) & 0.000000 \\ 0 & 1 \\ 0 & 0 \\ 1.4209 \mathrm{E}-01 & 4.9029 \mathrm{E}-02\end{array}$

33

2

0

00

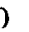

1

$1.4209 E-01$
4. $9029 E-02$

33 VESSEL UPPER PLENUM

6

83

0

0.000000

0.000000

1. $0000 \mathrm{E}+20$

40

0.000000
0.000000

39

0.000000

1. $0000 \mathrm{E}+20$

$1.00(0)(00)$

0

0.000000

$\begin{array}{rll}1 & * \mathrm{CN} & 02 \\ 0 & { }^{*} \mathrm{CN} & 03 \\ 0 & { }^{*} \mathrm{CN} & 05 \\ 305.370000 & { }^{*} \mathrm{CN} & 06 \\ 1.000000 & { }^{*} \mathrm{CN} & 07 \\ & { }^{*} \mathrm{CN} & 08 \\ & { }^{*} \mathrm{CN} & 09 \\ 0 & { }^{*} \mathrm{CN} & 11 \\ 305.370000 & { }^{*} \mathrm{CN} & 12\end{array}$




\begin{tabular}{|c|c|c|c|c|c|c|c|c|}
\hline 1851 & & 305.370000 & 0.000000 & 0.000000 & 1.0000E+20 & 1.000000 & ${ }^{*} \mathrm{CN}$ & 13 \\
\hline 1852 & & 0.000000 & 0.000000 & $1.0000 E+20$ & 1.000000 & & ${ }^{*} \mathrm{CN}$ & 14 \\
\hline 1853 & & $2.8418 \mathrm{~L}-01$ & 7. $7000 \mathrm{E}-(01 \mathrm{E}$ & & & & *DX & $\mathbf{P}$ \\
\hline 1854 & & 3. $52978 \mathrm{E}-2$ & $2.58172 \mathrm{E}-1 \mathrm{E}$ & & & & $* \mathrm{VOL}$ & $\mathbf{p}$ \\
\hline 1855 & & $0.124118 \mathrm{RO} 2$ & $0.124458 E$ & & & & ${ }^{*} \mathrm{FA}$ & \\
\hline 1856 & $F$ & 0.000000 : & & & & & ${ }^{*}$ FRIC & $\mathbf{P}$ \\
\hline 1857 & $\mathrm{~F}$ & 1. $0000 \mathrm{E}+00 \mathrm{E}$ & & & & & ${ }^{*} \mathrm{GRAV}$ & \\
\hline 1858 & $\mathrm{~F}$ & 1. $2200 \mathrm{~F}-02 \mathrm{E}$ & & & & & *HD & 1 \\
\hline 1859 & $\mathrm{~F}$ & $\mathrm{OE}$ & & & & & *ICFLG & \\
\hline 1860 & $\mathrm{~F}$ & $2 \mathrm{E}$ & & $\because$ & & & *NFF & $\mathrm{p}$ \\
\hline 1861 & $\Gamma$ & $0.000000 E$ & & & & & ${ }^{* A L P}$ & \\
\hline 1862 & $\mathrm{~F}$ & $0.000000 \mathrm{E}$ & & & & & $* \mathrm{VL}$ & \\
\hline 1863 & $\mathrm{~F}$ & $0.000000 E$ & & & & & $* V \bar{v}$ & \\
\hline 1864 & $\mathrm{~F}$ & $567.500000 \mathrm{E}$ & & & & & ${ }^{*} \mathrm{TL}$ & \\
\hline 1865 & $\mathrm{~F}$ & $567.500000 E$ & & & & & $* T V$ & \\
\hline 1866 & $\mathrm{~F}$ & $14.7800 E+6 E$ & & & & & ${ }^{*} \mathrm{P}$ & \\
\hline 1867 & $\mathrm{~F}$ & $0.000000 E$ & & & & & *PA & \\
\hline 1868 & $\mathrm{~F}$ & $0.000000 \mathrm{E}$ & & & & & ${ }^{*} \mathrm{QPPP}$ & \\
\hline 1869 & $\mathrm{~F}$ & $6 E$ & & & & & *MATID & \\
\hline 1870 & $\mathrm{~F}$ & $567.500000 \mathrm{E}$ & & & & & $* T W$ & \\
\hline 1871 & $\mathrm{~F}$ & $0.109737 \mathrm{E}$ & & & & & *DX & \\
\hline 1872 & $\mathrm{~F}$ & 1. $36577 \mathrm{E}-2 \mathrm{E}$ & & & & & $* \mathrm{VOL}$ & \\
\hline 1873 & $\mathrm{~F}$ & $0.124458 \mathrm{~F}$. & & & & & $* \mathrm{FA}$ & \\
\hline 1874 & $\mathrm{~F}$ & $0.000000 E$ & & & & & *FRIC & \\
\hline 1875 & $F$ & $0.000000 E$ & & & & & *GRAV & \\
\hline 1876 & $\mathrm{~F}$ & $2.8418 E-01 E$ & & & & & *HD & \\
\hline 1877 & $\mathrm{~F}$ & OF: & & & & & ${ }^{*}$ ICFLG & \\
\hline 1878 & $\mathrm{~F}$ & $2 \mathrm{E}$ & & & & & $* \mathrm{NFF}$ & \\
\hline 1879 & $\mathrm{~F}$ & $0.000000 E$ & & & & & *ALP & \\
\hline 1880 & $\mathrm{~F}$ & $0.000000 \mathrm{E}$ & & & & & $* \mathrm{VL}$ & \\
\hline 1881 & $\mathrm{~F}$ & $0.000000 \mathrm{E}$ & & & & & *VV & \\
\hline 1882 & $\mathrm{~F}$ & $567.500000 E$ & & & & & ${ }^{*} \mathrm{TL}$ & \\
\hline 1883 & $\mathrm{~F}$ & $567.500000 \mathrm{E}$ & & & & & $* T V$ & \\
\hline 1884 & $\mathrm{~F}$ & $14.7800 \mathrm{E}+6 \mathrm{E}$ & & & "i & & $* \mathbf{p}$ & \\
\hline 1885 & $\mathrm{~F}$ & $0.000000 \mathrm{E}$ & & & & & $* P A$ & \\
\hline 1886 & $\Gamma$ & $0.000000 \mathrm{E}$ & & & & & ${ }^{*} \mathrm{QPPP}$ & \\
\hline 1887 & $\mathrm{~F}$ & & & & & & *MATID & \\
\hline 1888 & $\mathrm{~F}$ & $567.500000 \mathrm{E}$ & & & & & ${ }^{*} \mathrm{TW}$ & \\
\hline 1889 & & & & & & & & \\
\hline 1890 & FIL & & 35 & 35 & VESSEL HEAD & & & \\
\hline 1891 & & 39 & 1 & 0 & & & ${ }^{*} \mathrm{CN}$ & 02 \\
\hline 1892 & & 0.000000 & 1. $0000 \mathrm{E}+20$ & 0.000000 & & & ${ }^{*} \mathrm{CN}$ & \\
\hline 1893 & & 0.770000 & 0.258172 & 0.000000 & 0.000000 & 567.500000 & ${ }^{*} \mathrm{CN}$ & 05 \\
\hline 1894 & & 14. $7800 E+6$ & 0.000000 & 0.000000 & 0.000000 & 567.500000 & ${ }^{*} \mathrm{CN}$ & \\
\hline 1895 & & & & & +2 & & $\cdots$ & \\
\hline 1896 & TEE & & 34 & 34 & VLSSLL HOT LEG & CONNECTION & & \\
\hline 1897 & & 1 & () & 6 & 0.000000 & 1 & ${ }^{*} \mathrm{CN}$ & \\
\hline 1898 & & 0 & 1 & 48 & 45 & 0 & ${ }^{*} \mathrm{CN}$ & \\
\hline 1899 & & 1. 4209: -01 & $4.9029 \mathrm{I}-02$ & 0.000000 & 0.000000 & 305.370000 & ${ }^{*} \mathrm{CN}$ & 06 \\
\hline 1900 & & 305.370000 & 0.000000 & 0.000000 & $1.0000 \mathrm{E}+20$ & 1.000000 & ${ }^{*} \mathrm{CN}$ & \\
\hline
\end{tabular}




\begin{tabular}{|c|c|c|c|c|c|c|c|c|}
\hline 1901 & & 0 & 1 & 40 & 0 & & ${ }^{*} \mathrm{CN}$ & 09 \\
\hline 1902 & & 1. $4209 \mathrm{E}-01$ & 4. $9029 \mathrm{E}-02$ & 0.000000 & 0.000000 & 305.370000 & ${ }^{*} \mathrm{CN}$ & 12 \\
\hline 1903 & & 305.370000 & 0.000000 & 0.000000 & 1. $0000 \mathrm{E}+20$ & 1.000000 & ${ }^{*} \mathrm{CN}$ & 13 \\
\hline 1904 & $\mathrm{~F}$ & $4.0000 \mathrm{E}-01 \mathrm{E}$ & & & & & *DX & $\mathrm{p}$ \\
\hline 1905 & $\mathrm{~F}$ & $2.6087 \mathrm{E}-02 \mathrm{E}$ & & & & & *VOL & $\mathrm{P}$ \\
\hline 1906 & $\mathrm{~F}$ & 6. $3425 \mathrm{E}-02 \mathrm{E}$ & & & & & *FA & $\mathrm{p}$ \\
\hline 1907 & & 0.000000 & $0.000100 \mathrm{E}$ & & & & *FRIC & $\mathrm{P}$ \\
\hline 1908 & $\mathrm{~F}$ & $0.000000 \mathrm{E}$ & & & & & ${ }^{*} \mathrm{GRAV}$ & $\mathrm{D}$ \\
\hline 1909 & $\mathrm{~F}$ & $2.8418 \mathrm{E}-01 \mathrm{E}$ & & & & & *HD & $\mathbf{p}$ \\
\hline 1910 & $\mathrm{~F}$ & $\mathrm{OE}$ & & & & & ${ }^{*}$ ICTLG & \\
\hline 1911 & $\mathrm{~F}$ & $2 E$ & & & & & $* N F F$ & $\mathrm{P}$ \\
\hline 1912 & $\mathrm{~F}$ & $0.000000 \mathrm{E}$ & & & & & ${ }^{*} \mathrm{ALP}$ & $\mathrm{p}$ \\
\hline 1913 & $\mathrm{~F}$ & $0.000000 \mathrm{E}$ & & & & & ${ }^{*} \mathrm{VL}$ & \\
\hline 1914 & $\mathrm{~F}$ & $0.000000 \mathrm{E}$ & & & & & *VV & $\mathbf{P}$ \\
\hline 1915 & $\mathrm{~F}$ & $567.500000 \mathrm{E}$ & & & & & ${ }^{*} \mathrm{TL}$ & $\mathbf{p}$ \\
\hline 1916 & $\mathrm{~F}$ & $567.500000 \mathrm{E}$ & & & & & *TV & \\
\hline 1917 & $\mathrm{~F}$ & $14.7800 \mathrm{E}+6 \mathrm{E}$ & & & & & $* \mathrm{p}$ & \\
\hline 1918 & $F$ & $0.000000 \mathrm{E}$ & & & & & ${ }^{*} \mathrm{PA}$ & \\
\hline 1919 & $F$ & $0.109737 \mathrm{E}$ & & & & & ${ }^{*} \mathrm{DX}$ & \\
\hline 1920 & $\mathrm{~F}$ & 1. $36577 \mathrm{E}-2 \mathrm{E}$ & & & & & $*$ VOL & \\
\hline 1921 & $\mathrm{~F}$ & $0.124458 \mathrm{E}$ & & & & & ${ }^{*} \mathrm{FA}$ & \\
\hline 1922 & $\mathrm{~F}$ & $0.000000 \mathrm{E}$ & & & & & ${ }^{*}$ FRIC & \\
\hline 1923 & $\mathrm{~F}$ & $0.000000 \mathrm{E}$ & & & & & ${ }^{*} \mathrm{GRAV}$ & \\
\hline 1924 & $\mathrm{~F}$ & $2.8418 \mathrm{E}-01 \mathrm{E}$ & & & & & $* \mathrm{HD}$ & \\
\hline 1925 & $\mathrm{~F}$ & $\mathrm{OE}$ & & & & & ${ }^{*}$ ICFLG & \\
\hline 1926 & $\mathrm{~F}$ & $2 \mathrm{E}$ & & & & & ${ }^{*} \mathrm{NFF}$ & \\
\hline 1927 & $\mathrm{~F}$ & $0.000000 \mathrm{E}$ & & & & & $*$ ALP & \\
\hline 1928 & $\mathrm{~F}$ & $0.000000 \mathrm{E}$ & & & & & $* \mathrm{VL}$ & \\
\hline 1929 & $\mathrm{~F}$ & $0.000000 \mathrm{E}$ & & & & & *VV & \\
\hline 1930 & $\mathrm{~F}$ & $567.500000 E$ & & & & & ${ }^{*} \mathrm{TL}$ & \\
\hline 1931 & $F$ & $567.500000 \mathrm{E}$ & & & & & $* T V$ & \\
\hline 1932 & $\mathrm{~F}$ & $14.7800 E+6 E$ & & & & & $* \mathrm{p}$ & \\
\hline 1933 & $\mathrm{~F}$ & $0.000000 \mathrm{E}$ & & & & & $* P A$ & \\
\hline 1934 & & & & & 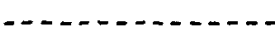 & & & \\
\hline 1935 & TEE & & 43 & 43 & VESSEL HOT LEG & & & \\
\hline 1936 & & 1 & 4 & 6 & 0.000000 & 1 & ${ }^{*} \mathrm{CN}$ & \\
\hline 1937 & & 0 & 1 & 48 & 9 & 0 & ${ }^{*} \mathrm{CN}$ & \\
\hline 1938 & & 0 & 0 & 0 & 0 & 0 & ${ }^{*} \mathrm{CN}$ & \\
\hline 19.39 & & $1.4209 \mathrm{E}-01$ & $4.9029 \mathrm{E}-02$ & 0.000000 & 0.000000 & 305.370000 & ${ }^{*} \mathrm{CN}$ & \\
\hline 1940 & & 305.370000 & 0.000000 & 0.000000 & $1.0000 E+20$ & 1.000000 & ${ }^{*} \mathrm{CN}$ & 07 \\
\hline 1941 & & 0.000000 & 0.000000 & $1.0000 E+20$ & 1.000000 & & ${ }^{*} \mathrm{CN}$ & \\
\hline 1942 & & 0 & 1 & 47 & 0 & & ${ }^{*} \mathrm{CN}$ & \\
\hline 1943 & & 0 & 0 & 0 & 0 & 0 & ${ }^{*} \mathrm{CN}$ & \\
\hline 1944 & & $5.0000 \mathrm{E}-02$ & $5.0000 \mathrm{E}-02$ & 0.000000 & 0.000000 & 305.370000 & ${ }^{*} \mathrm{CN}$ & \\
\hline 1945 & & 305.370000 & 0.000000 & 0.000000 & $1.0000 E+20$ & 1.000000 & ${ }^{*} \mathrm{CN}$ & \\
\hline 1946 & & 0.0000000 & 0.000000 & 1. $0000 E+20$ & 1.000000 & & ${ }^{*} \mathrm{CN}$ & \\
\hline 1947 & $\mathrm{~F}$ & 1.000OE-01E & & & & & ${ }^{*} \mathrm{DX}$ & \\
\hline 1948 & $F$ & $6.5218 E-03 E$ & & : & & & ${ }^{*} \mathrm{VOL}$ & \\
\hline 1949 & $\mathrm{~F}$ & $6.3425 \mathrm{E}-02 \mathrm{E}$ & & & & & $* \mathrm{FA}_{\mathrm{A}}$ & \\
\hline 1950 & & 0.000000 & $0.000100 \mathrm{E}$ & & & & *FRIC & \\
\hline
\end{tabular}




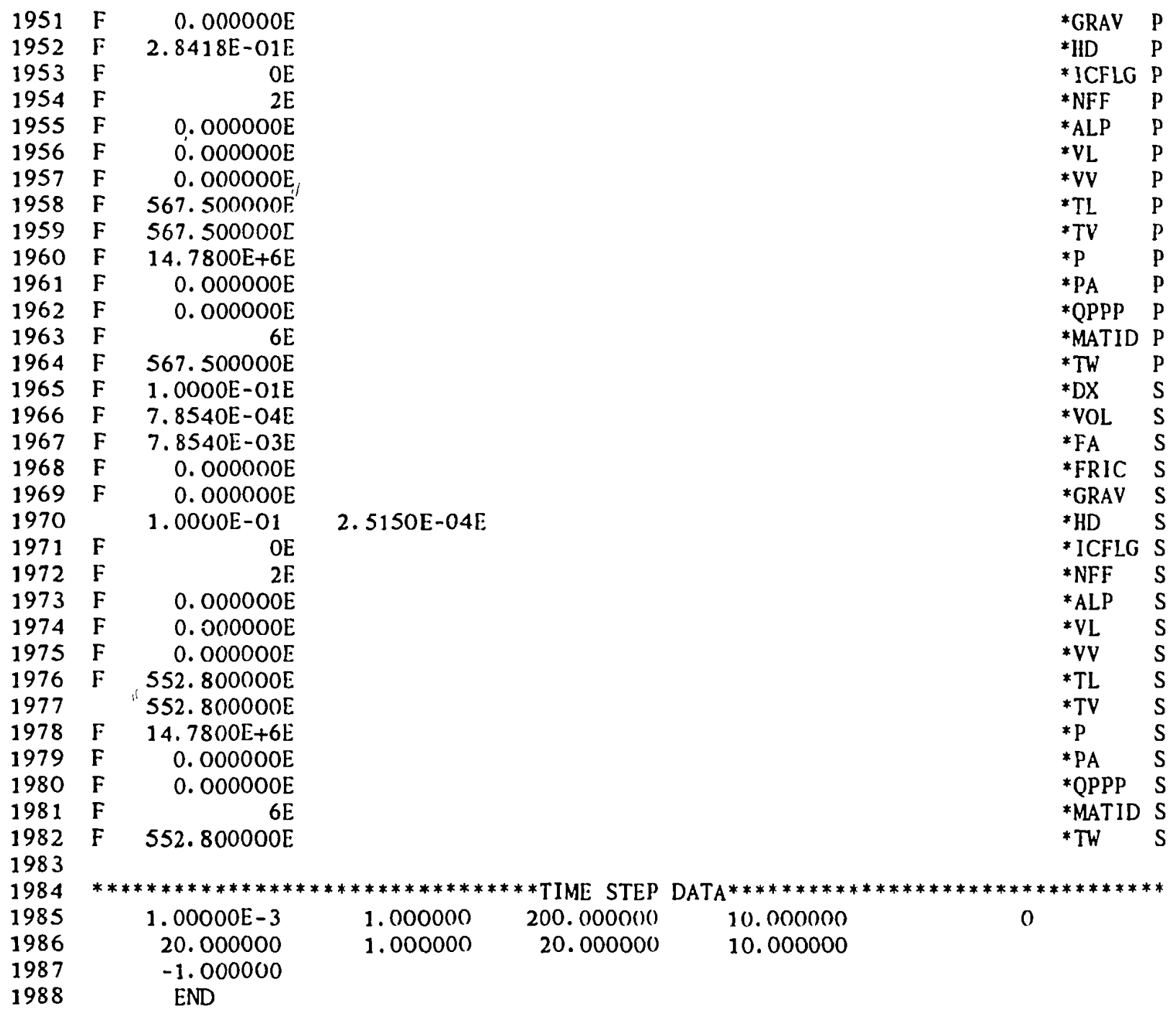


129

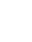

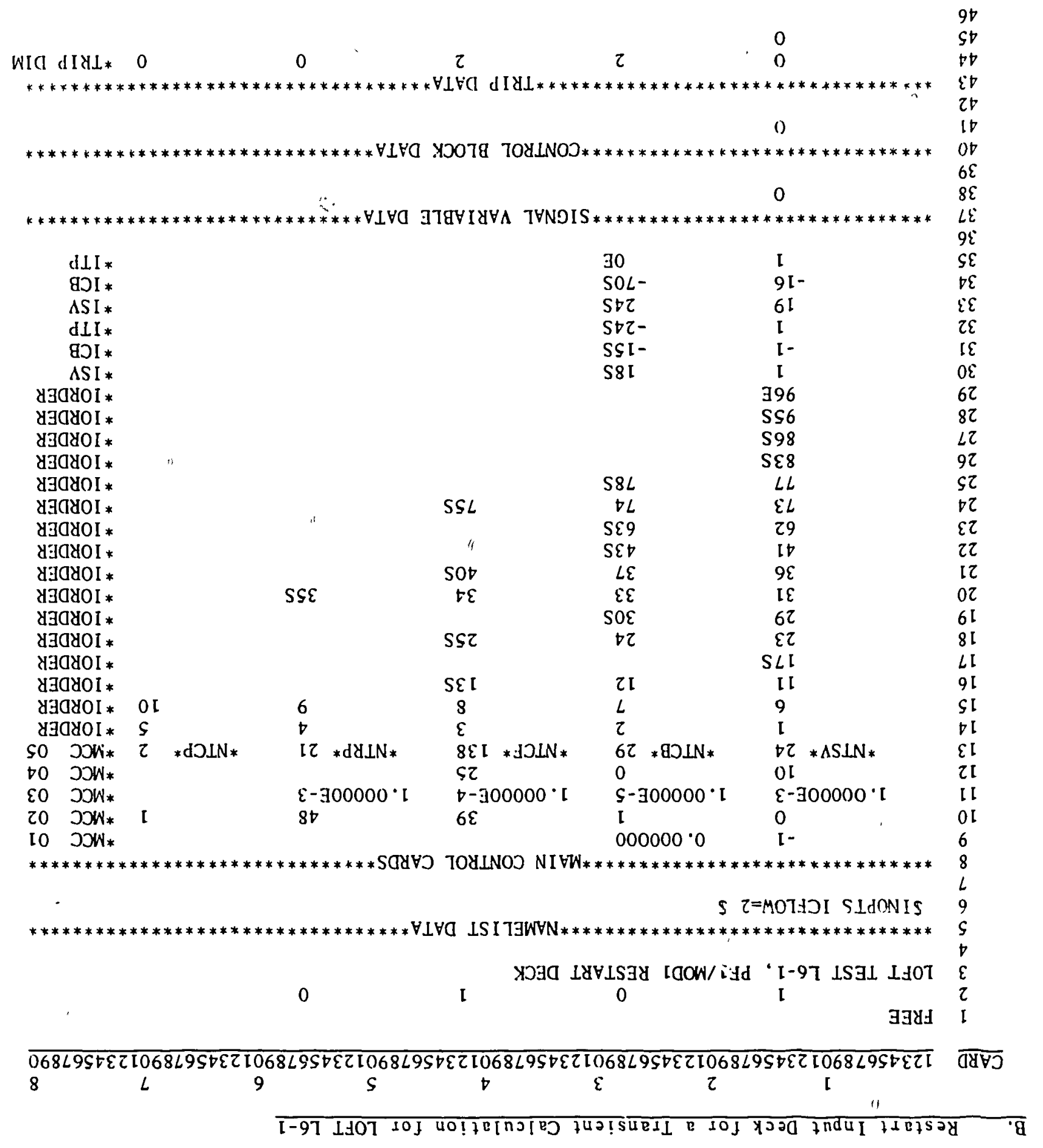




\section{CARD 12345678901234567890123456789012345678901234567890123456789012345678901234567890}

47

48

49

50

51

52

53

54

55

56
END

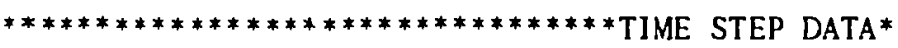

1. $00000 \mathrm{E}-3$

10.000000

$1.00000 \mathrm{E}-3$

25. 000000

$-1.000000$

END
1.000000

0.500000

250.000000

10.000000

10.000000

$1.000000 \quad 700.000000 \quad 10.000000$

1. 000000
10.000000 
C. LOFT Three-Dimensiona] VESSEL Input

This input corresponds to the vessel conponents shown in Fig. E-5.

$$
\begin{array}{llllllll}
1 & 2 & 3 & 4 & 5 & 6 & 7 & 8
\end{array}
$$

CARD 12345678901234567890123456789012345678901234567890123456789012345678901234567890

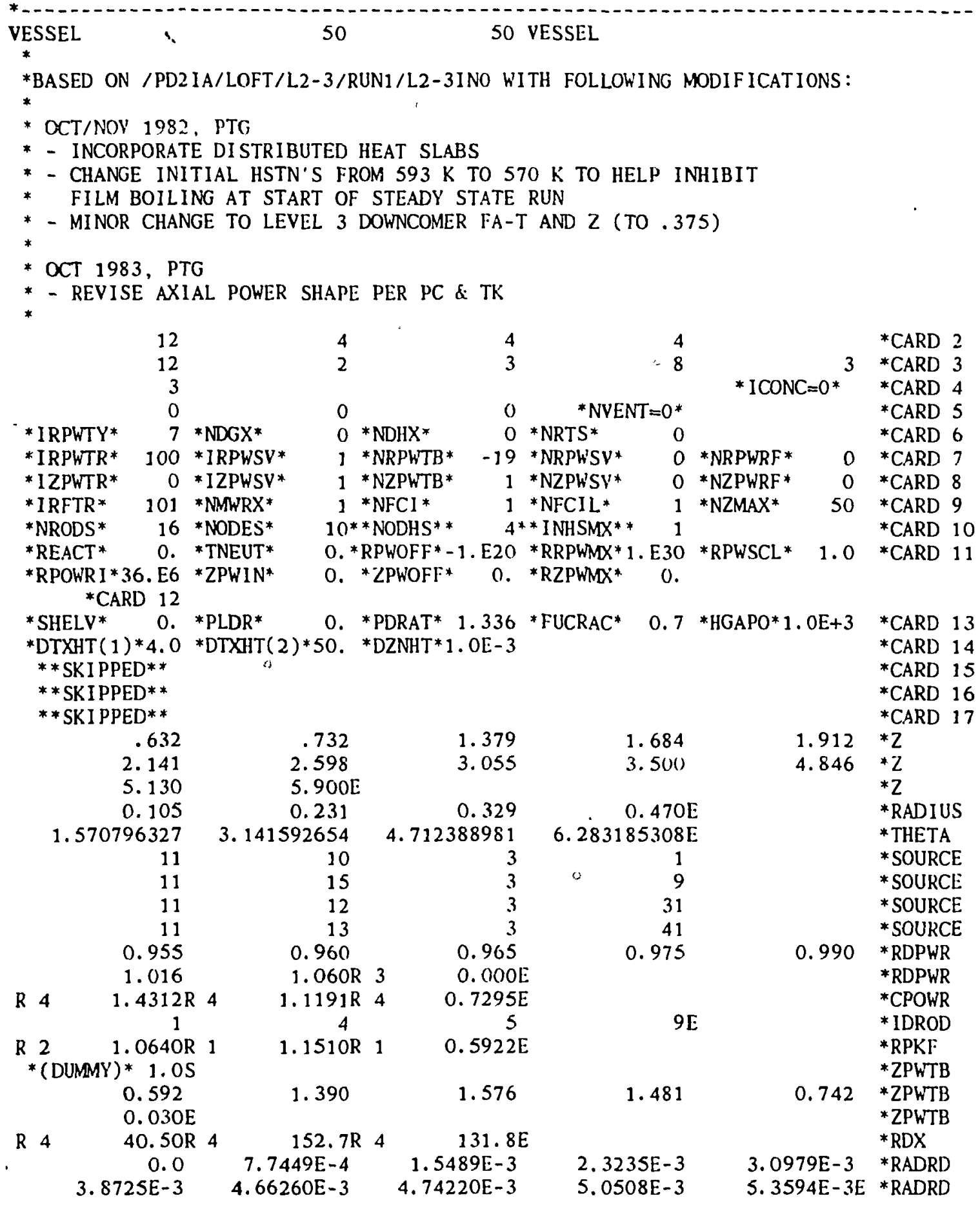

4. $66260 \mathrm{E}-3 \quad 4.74220 \mathrm{E}-3$

2. $3235 \mathrm{E}-3$

$5.0508 \mathrm{E}-3$

3. 0979E-3 *RADRD

5. $3594 \mathrm{E}-3 \mathrm{E} *$ RADRD 
R 6

$\begin{array}{rrr}51 & \mathrm{R} 6 & 1 \\ 52 & & 0.0 \\ 53 & & 1.0 \\ 54 & & 5.0 \\ 55 & & 15.0 \\ 56 & & 50.0 \\ 57 & & 100.0 \\ 58 & & 150.0 \\ 59 & & 300.0 \\ 60 & & 500.0 \\ 61 & & 1000.0 \\ 62 & \mathrm{~F} & 0 \mathrm{E} \\ 63 & \mathrm{~F} & 0.0 \mathrm{E} \\ 64 & \mathrm{~F} & 0.93 \mathrm{E}\end{array}$

$659.81010 \mathrm{E}-1$

$667.09910 \mathrm{E}-3$

$67 \quad 9.81010 \mathrm{E}-1$

$68 \quad 7.09910 \mathrm{E}-3$

69

70

71

72

73

74

75

76

77

78

79

80

81

82

83

84

85

86

87

88

89

90

91

92

$93 \mathrm{~F}$

$94 \mathrm{R} 4$

95

96

97

98

99

100

9. $81010 \mathrm{E}-1$

7. $09910 \mathrm{E}-3$

9. $81010 \mathrm{~L}-1$

7. 09910 T. -3

9. $81010 \mathrm{E}-1$

7. $09910 \mathrm{E}-3$

9. $81010 \mathrm{E}-1$

7. $09910 \mathrm{E}-3$

9. $81010 \mathrm{E}-1$

7. $09910 \mathrm{E}-3$

9. $81010 \mathrm{E}-1$

7. $09910 \mathrm{E}-3$

9. $81010 \mathrm{E}-1$

7. $09910 \mathrm{E}-3$

9. $81010 \mathrm{E}-1$

7. $09910 \mathrm{E}-3$

9. $81010 \mathrm{E}-1$

7. $09910 \mathrm{E}-3$

9. $81010 \mathrm{OE}-1$

7. $09910 \mathrm{E}-3$

I $1.0 \mathrm{E}$

$1 . \mathrm{OE}$

$0 . \mathrm{OE}$

$0 . \mathrm{OE}$

$0.0087 R^{4}$

0.0

0.0

0.0

0.0

0.0

$36.00 \mathrm{E}+6$

$3 R 2$

2. $16.3 \mathrm{E}+6$

1. $937 \mathrm{E}+6$

1. $687 \mathrm{E}+6$

1. $364 \mathrm{E}+6$

1. $184 \mathrm{E}+6$

1. $112 \mathrm{E}+6$

9. $330 \mathrm{E}+5$

7. $895 \mathrm{E}+5$

6. $459 E+5 E$

$2 \mathrm{E}$

0.1

2. 0

10.0

20.0

75.0

125.0

200.0

400.0

600.0

2. $512 \mathrm{C}+6 \mathrm{~S}$

2. $081 \mathrm{E}+6 \mathrm{~S}$

1. $794 \mathrm{E}+6 \mathrm{~S}$

1. $615 \mathrm{E}+6 \mathrm{~S}$

1. $256 \mathrm{E}+6 \mathrm{~S}$

1. $148 \mathrm{E}+6 \mathrm{~S}$

1. $041 \mathrm{E}+6 \mathrm{~S}$

8. $612 \mathrm{E}+5 \mathrm{~S}$

7. $536 \mathrm{E}+5 \mathrm{~S}$

* MATRD

*RPWTB

* RPWTB

* RPWTB

*RPWTB

*RPWTT3

* RPWTB

RPWTB

*RPWTB

* RPWTB

* RPWTB

*NFAX

*FPUO2

*FTI

$0.00000 \mathrm{E}+0$

$1.01030 \mathrm{E}-2 \quad 1.78300 \mathrm{E}-3$

$0.00000 E+0$

*GMIX

$0.00000 E+0 S$

$0.00000 \mathrm{E}+0$

$0.00000 \mathrm{E}+0 \mathrm{~S}$

$0.00000 \mathrm{E}+0$

$0.00000 \mathrm{E}+0 \mathrm{~S}$

$0.00000 E+0$

$0.00000 \mathrm{~F}+\mathrm{OS}$

$0.00000 \mathrm{E}+0$

$0.00000 \mathrm{E}+0 \mathrm{~S}$

$0.00000 \mathrm{E}+0$

$0.00000 \mathrm{E}+0 \mathrm{~S}$

$0.00000 \mathrm{E}+1)$

$0.00000 E+0 S$

$0.00000 \mathrm{E}+0$

$0.00000 \mathrm{E}+0 \mathrm{~S}$

$0.00000 \mathrm{E}+0$

$0.00000 \mathrm{E}+0 \mathrm{~S}$

$0.00000 \mathrm{E}+0$

$0.00000 \mathrm{E}+0 \mathrm{~S}$

$0.00000 \mathrm{E}+0$

$0.00000 \mathrm{E}+0 \mathrm{~S}$

$0.00000 \mathrm{E}+0$

$0.00000 \mathrm{E}+0 \mathrm{E}$

$1.01030 E-2 \quad 1.78300$ E- 3

$0.00000 E+0$

* GMIX

$1.01030 \mathrm{E}-2 \quad 1.78300 \mathrm{E}-3$

$0.00000 \mathrm{E}+0$

$1.01030 \mathrm{E}-2 \quad 1.78300 \mathrm{E}-3$

$0.00000 \mathrm{E}+\mathrm{O}$

GMIX

*GMIX

*GMIX

*GMIX

$1.01030 \mathrm{E}-2 \quad 1.78300 \mathrm{E}-3$

$0.00000 E+0$

* GMIX

*GMIX

$1.01030 \mathrm{E}-2 \quad 1.78300 \mathrm{E}-3$

$0.00000 E+0$

*GMIX

*GMIX

1.01030E-2 1.78.300E:-3

$0.00000 E+0$

* GMIX

*GMIX

$1.01030 \mathrm{E}-2 \quad 1.78300 \mathrm{E}-3$

$0.00000 E+0$

*GMIX

$1.01030 \mathrm{E}-2 \quad 1.78300 \mathrm{E}-3$

$0.00000 E+O$

*GMIX

*GMIX

$1.01030 \mathrm{E}-2 \quad 1.78300 \mathrm{E}-3$

$0.00000 E+0$

*GMIX

*GMIX

$1.01030 \mathrm{E}-2 \quad 1.78300 \mathrm{E}-3$

$0.00000 E+0$

*GMIX

1.01030E-2 1.78300E-3

$0.00000 \mathrm{E}+0$

*GMIX

*GMIX

*GMIX

*GMIX

* GMLES

* PGAPT

*PLVOL

$0.0333 \mathrm{R} 4$

$0.0431 R 4$

0. $344 \mathrm{E}$

0. 09

(). 09

(). 1

$0.105 \mathrm{~S}$

0. 1

o. 1055

). 09

0.09

0. 09

0.09

0. 1

0. $105 \mathrm{~S}$

0. 1

o. $105 \mathrm{~S}$

0.1

$0.105 \mathrm{~S}$

0.1

$0.105 \mathrm{~S}$

*PSLEN

*CLENN

*HSA LEVEL 1

*HSX

*HSX

*HSX

* IISX

*HSX

*HSX 


\begin{tabular}{|c|c|c|c|c|c|c|}
\hline 101 & & 0.0 & 0.09 & 0.1 & $0.105 \mathrm{~S}$ & *HSX \\
\hline 102 & & 0.0 & 0.09 & 0.1 & $0.105 S$ & *IISX' \\
\hline 103 & & 0.0 & 0.09 & 0.1 & 0.1055 & *IISX \\
\hline 104 & & 0.0 & 0.09 & 0.1 & $0.105 \mathrm{~S}$ & ${ }^{*} \mathrm{HSX}$ \\
\hline 105 & & 0.0 & 0.09 & 0.1 & 0.1055 & ${ }^{*} \mathrm{HSX}$ \\
\hline 106 & & 0.0 & 0.09 & 0.1 & 0.1055 & *HSX \\
\hline 107 & & 0.0 & 0.09 & 0.1 & 0.1055 & ${ }^{*} \mathrm{HSX}$ \\
\hline 108 & & 0.0 & 0.09 & 0.1 & $0.105 \mathrm{~S}$ & *HSX \\
\hline 109 & & 0.0 & 0.09 & 0.1 & $0.105 \mathrm{~S}$ & ${ }^{*}$ HSX \\
\hline 110 & & 0.0 & 0.09 & 0.1 & $0.105 E$ & *IISX \\
\hline 111 & $\mathrm{~F}$ & $0.0 \mathrm{~T}$ & & & & ${ }^{*} \mathrm{CFZL}-\mathrm{T}$ \\
\hline 112 & $r$ & $0.0 E$ & & & & ${ }^{*} \mathrm{CFZL}-\mathrm{Z}$ \\
\hline 113 & $\mathrm{~F}$ & $0.0 E$ & & & & ${ }^{*} \mathrm{CFZL}-\mathrm{R}$ \\
\hline 114 & $\mathrm{~F}$ & $0.0 \mathrm{E}$ & & & & ${ }^{*} \mathrm{CFZV}-\mathrm{T}$ \\
\hline 115 & $F$ & $0 . \mathrm{OE}$ & & & & ${ }^{*} \mathrm{CF} \cdot \mathrm{ZV}-\mathrm{Z}$ \\
\hline 136 & $\mathrm{~F}$ & $0.0 \mathrm{E}$ & & & & ${ }^{*} \mathrm{CFZV}-\mathrm{R}$ \\
\hline 117 & $\mathrm{~F}$ & 1. OE & & & & *VOL \\
\hline 118 & $F$ & $1 . \mathrm{OE}$ & & & & ${ }^{*} \mathrm{FA}-\mathrm{T}$ \\
\hline 119 & $\mathrm{~F}$ & 1.OE & & & ? & *FA $-Z$ \\
\hline 120 & $\mathbf{F}$ & $1.0 \mathrm{E}$ & & & & $* F A-R$ \\
\hline 121 & $\mathrm{~F}$ & $0.1 \mathrm{E}$ & & & & *HD-T \\
\hline 122 & $\mathrm{~F}$ & $0.1 \mathrm{E}$ & & & & *HD $-\mathrm{Z}$ \\
\hline 123 & $\mathrm{~F}$ & $0.1 E$ & & & & *HD-R \\
\hline 124 & $\mathrm{~F}$ & $5.70000 \mathrm{E}+2 \mathrm{E}$ & & & & *HSTN \\
\hline 125 & $\mathrm{~F}$ & $6 \mathrm{E}$ & & & & *MATHS \\
\hline 126 & $\mathrm{~F}$ & $0.0 \mathrm{E}$ & & & & *ALPN \\
\hline 127 & $\mathrm{~F}$ & 0.01 . & & & & *VVN-T \\
\hline 128 & $F$ & $0.0 E^{\circ}$ & & & & $* V V N-Z$ \\
\hline 129 & $F$ & $0.0 \mathrm{E}$ & & & & *VVN-R \\
\hline 130 & $\mathbf{F}$ & $0.0 \mathrm{E}$ & & & & $* V L N-T$ \\
\hline 131 & $\mathrm{~F}$ & $0.0 \mathrm{E}$ & & & & $* V L N-Z$ \\
\hline 132 & $F$ & $0.0 E$ & & & & $* V L N-R$ \\
\hline 133 & $F$ & 6. $15000 \mathrm{E}+2 \mathrm{E}$ & & & & *TVN \\
\hline 134 & $F$ & $5.93000 E+2 E$ & $"$ & & & ${ }^{*}$ TLN \\
\hline 135 & $\mathrm{~F}$ & 1. $50600 \mathrm{E}+7 \mathrm{E}$ & & & & $* \mathrm{PN}$ \\
\hline 136 & $\mathrm{~F}$ & $0 . \mathrm{OE}$ & & & & *PAN \\
\hline 137 & $\mathrm{R} 12$ & $0.0 R 4$ & $0.0956 \mathrm{E}$ & & & *IISA LEVEL 2 \\
\hline 138 & & 0.0 & 0.09 & 0.1 & $0.105 \mathrm{~S}$ & *ISSX \\
\hline 139 & & 0.0 & 0.09 & 0.1 & $0.105 \mathrm{~S}$ & *HSX \\
\hline 140 & & 0.0 & 0.09 & 0.1 & $0.105 \mathrm{~S}$ & ${ }^{*}$ HSX \\
\hline 141 & & 0.0 & 0.09 & 0.1 & $0.105 \mathrm{~S}$ & ${ }^{*} H S X$ \\
\hline 142 & & 0.0 & 0.09 & 0.1 & $0.105 \mathrm{~S}$ & ${ }^{*} \mathrm{HSX}$ \\
\hline 143 & & 0.0 & 0.09 & 0.1 & $0.105 \mathrm{~S}$ & *HSX \\
\hline 144 & & 0.0 & 0.09 & $0 . i^{\prime}$ & $0.105 \mathrm{~s}$ & *HSX \\
\hline 145 & & 0.0 & 0.09 & 0.1 & $0.105 \mathrm{~S}$ & *HSX \\
\hline 146 & & (). 0 & 0.09 & (). 1 & $0.105 \mathrm{~S}$ & * HSX \\
\hline 147 & & 0.0 & 0.09 & 0.1 & $0.105 \mathrm{~S}$ & ${ }^{*} \mathrm{ISX}$ \\
\hline 148 & & 0.0 & 0.09 & 0.1 & (1. $105 \mathrm{~S}$ & *HSX \\
\hline 149 & " & 0.0 & 0.09 & 0.1 & $0.105 \mathrm{~S}$ & *HSX \\
\hline 150 & & 0.0 & 0.09 & 0.1 & 0.1055 & ${ }^{*}$ HSX \\
\hline
\end{tabular}




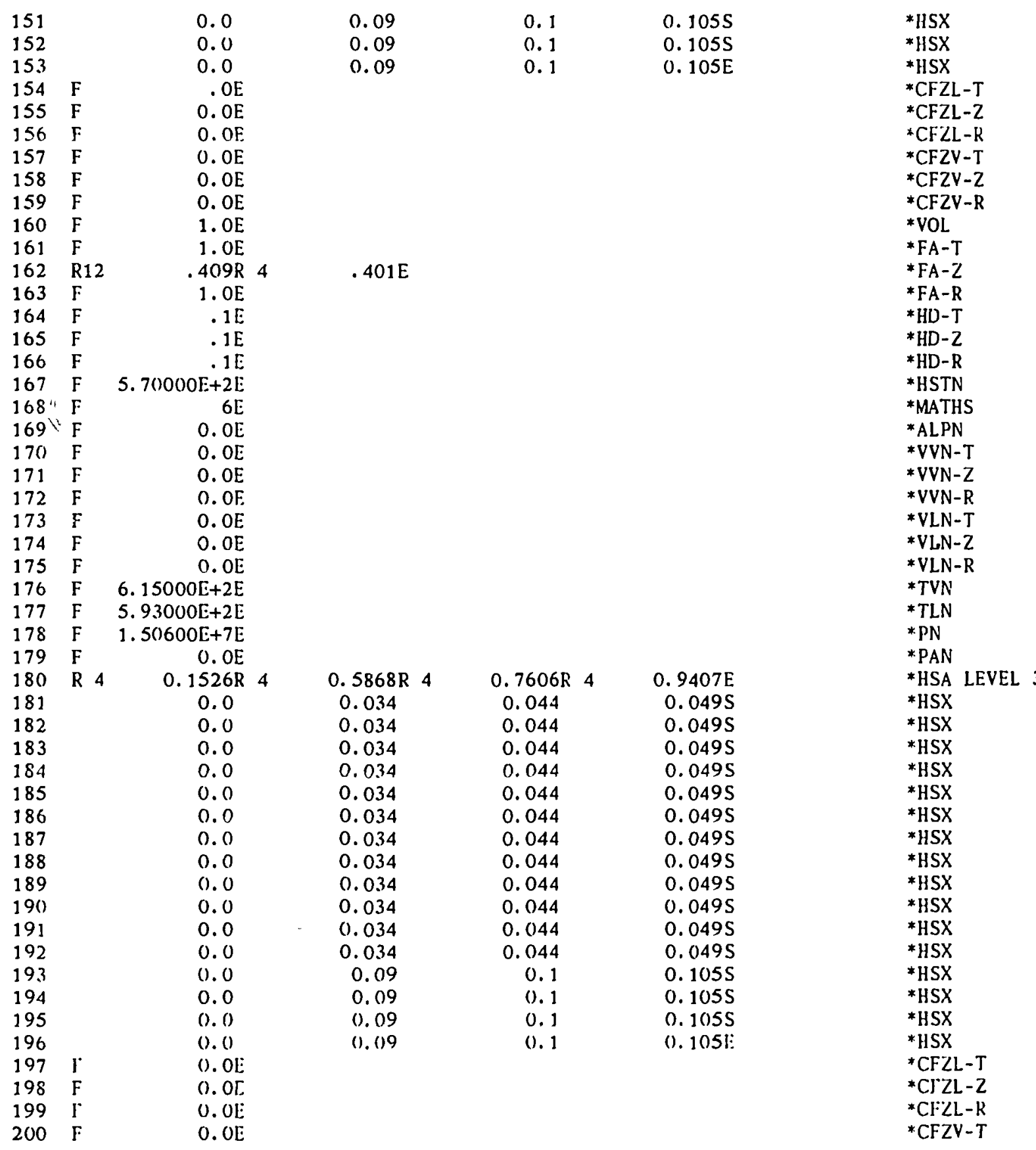




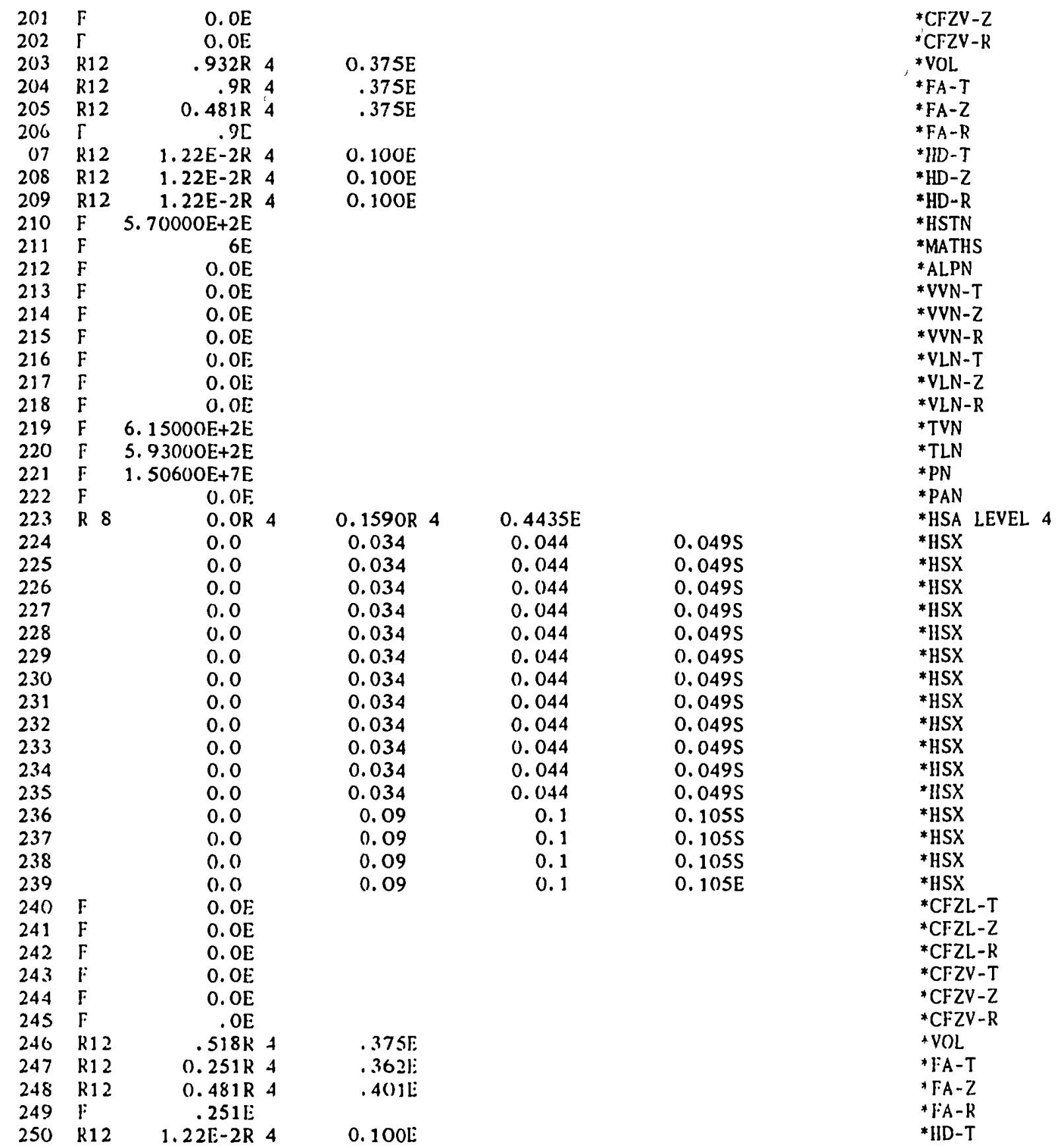




\begin{tabular}{|c|c|c|c|c|}
\hline 251 & $\mathrm{R} 12$ & 1. $22 \mathrm{E}-2 \mathrm{R}$ & 4 & $0.100 \mathrm{E}$ \\
\hline 252 & $\mathrm{R} 12$ & 1. $22 \mathrm{E}-2 \mathrm{R}$ & 4 & $0.100 \mathrm{E}$ \\
\hline 253 & $\mathrm{~F}$ & 5. $70000 E+2 E$ & & \\
\hline 254 & $\Gamma$ & $6 E$ & & \\
\hline 255 & $\mathrm{~F}$ & $0.0 \mathrm{E}$ & & \\
\hline 256 & $\mathrm{~F}$ & $0 . \mathrm{OE}$ & & \\
\hline 257 & F & $0.0 \mathrm{C}$ & & \\
\hline 258 & $\mathrm{~F}$ & $0.0 \mathrm{E}$ & & \\
\hline 259 & $\mathrm{~F}$ & $0.0 \mathrm{E}$ & & \\
\hline 260 & $\mathrm{~F}$ & $0.0 \mathrm{E}$ & & \\
\hline 261 & $\mathrm{~F}$ & $0.0 \mathrm{E}$ & & \\
\hline 262 & $F$ & $6.15000 E+2 E$ & & \\
\hline 263 & $\mathrm{~F}$ & $5.93000 E+2 E$ & & \\
\hline 264 & $\mathrm{~F}$ & i. $50600 E+7 E$ & & \\
\hline 265 & $\mathrm{~F}$ & $0.0 \mathrm{E}$ & & \\
\hline 266 & R 8 & $0.0 R$ & 4 & $0.1189 \mathrm{R} \quad 4$ \\
\hline 267 & & 0.0 & & 0.034 \\
\hline 268 & & 0.0 & & 0.034 \\
\hline 269 & & 0.0 & & 0.034 \\
\hline 270 & & 0.0 & & 0.034 \\
\hline 271 & & 0.0 & & 0.034 \\
\hline 272 & & 0.0 & & 0.034 \\
\hline 273 & & 0.0 & & 0.034 \\
\hline 274 & & 0.0 & & 0.034 \\
\hline 275 & & 0.0 & & 0.034 \\
\hline 276 & & 0.0 & & 0.034 \\
\hline 277 & & 0.0 & & 0.034 \\
\hline 278 & & 0.0 & & 0.034 \\
\hline 279 & & 0.0 & & 0.09 \\
\hline 280 & & 0.0 & & 0.09 \\
\hline 281 & & 0.0 & & 0.09 \\
\hline 282 & & 0.0 & & 0.09 \\
\hline 283 & $\mathrm{~F}$ & $0.0 E$ & & \\
\hline 284 & $\mathrm{~F}$ & $0.0 \mathrm{E}$ & & \\
\hline 285 & $\mathrm{~F}$ & $0.0 \mathrm{E}$ & & \\
\hline 286 & $\mathrm{~F}$ & $0.0 E$ & & \\
\hline 287 & F & $0.0 E$ & & \\
\hline 288 & $\mathrm{~F}$ &. $\mathrm{OF}$ & & \\
\hline 289 & $\mathrm{R} 12$ & $.518 \mathrm{R}$ & 4 & $.375 \mathrm{E}$ \\
\hline 290 & R12 & $0.251 R$ & 4 & $.362 E$ \\
\hline 291 & R12 & $0.481 \mathrm{R}$ & 4 & $.401 \mathrm{E}$ \\
\hline 292 & $\ddot{r}$ & $.251 \mathrm{E}$ & & \\
\hline 293 & $\mathrm{R} 12$ & 1. $22 \mathrm{E}-2 \mathrm{R}$ & 4 & $0.100 \mathrm{E}$ \\
\hline 294 & R12 & 1. $22 \mathrm{E}-2 \mathrm{R}$ & 4 & $0.100 \mathrm{E}$ \\
\hline 295 & R12 & 1. $22 \mathrm{E}-2 \mathrm{R}$ & 4 & $0.100 \mathrm{E}$ \\
\hline 296 & r & 5. $70000 \mathrm{E}+2 \mathrm{E}$ & & \\
\hline 297 & F & $6 E$ & & \\
\hline 298 & $\Gamma$ & 0.01. & & \\
\hline 299 & $\mathrm{~F}$ & $0.0 \mathrm{OE}$ & & \\
\hline 300 & F & $0.0 E$ & & \\
\hline
\end{tabular}

\section{$0.3315 \mathrm{E}$}

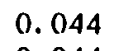

0.044

0.044

0.044

0.044

0.044

0.044

0.044

0.044

0.044

0.044

0.044

0.1

0.1

0.1

0.1

$0.049 \mathrm{~S}$

$0.049 \mathrm{~S}$

$0.049 \mathrm{~S}$

$0.049 \mathrm{~S}$

$0.049 \mathrm{~S}$

0.0495

$0.049 \mathrm{~S}$

$0.049 \mathrm{~S}$

$0.049 \mathrm{~S}$

$0.049 \mathrm{~S}$

$0.049 \mathrm{~S}$

$0.049 \mathrm{~S}$

$0.105 \mathrm{~S}$

$0.105 \mathrm{~S}$

$0.105 \mathrm{~S}$

0. 105E
*HD-Z

*HD-R

* HSTN

*MATHS

*ALPN

*VVN-T

*VVN-Z

*VVN-R

*VLN-T

*VLN-Z

*VLN-R

*TVN

*TLN

*PN

*PAN

* HSA leVEl 5

*HSX

*HSX

*HSX

*HSX

*HSX

*HSX

*HSX

*HSX

*HSX

*HSX

*HSX

*HSX

*HSX

*HSX

*HSX

*HSX

*CFZL-T

*CFZL-Z

*CIZL-R

*CFZV - T

*CFZV $-Z$

*CFZV-R

*VOL

*FA-T

*FA-Z

*FA-R

*HD-T

*HD-Z

*HD-R

* HSTN

*MATHS

*ALPN

*VVN-T

*VVN-Z 


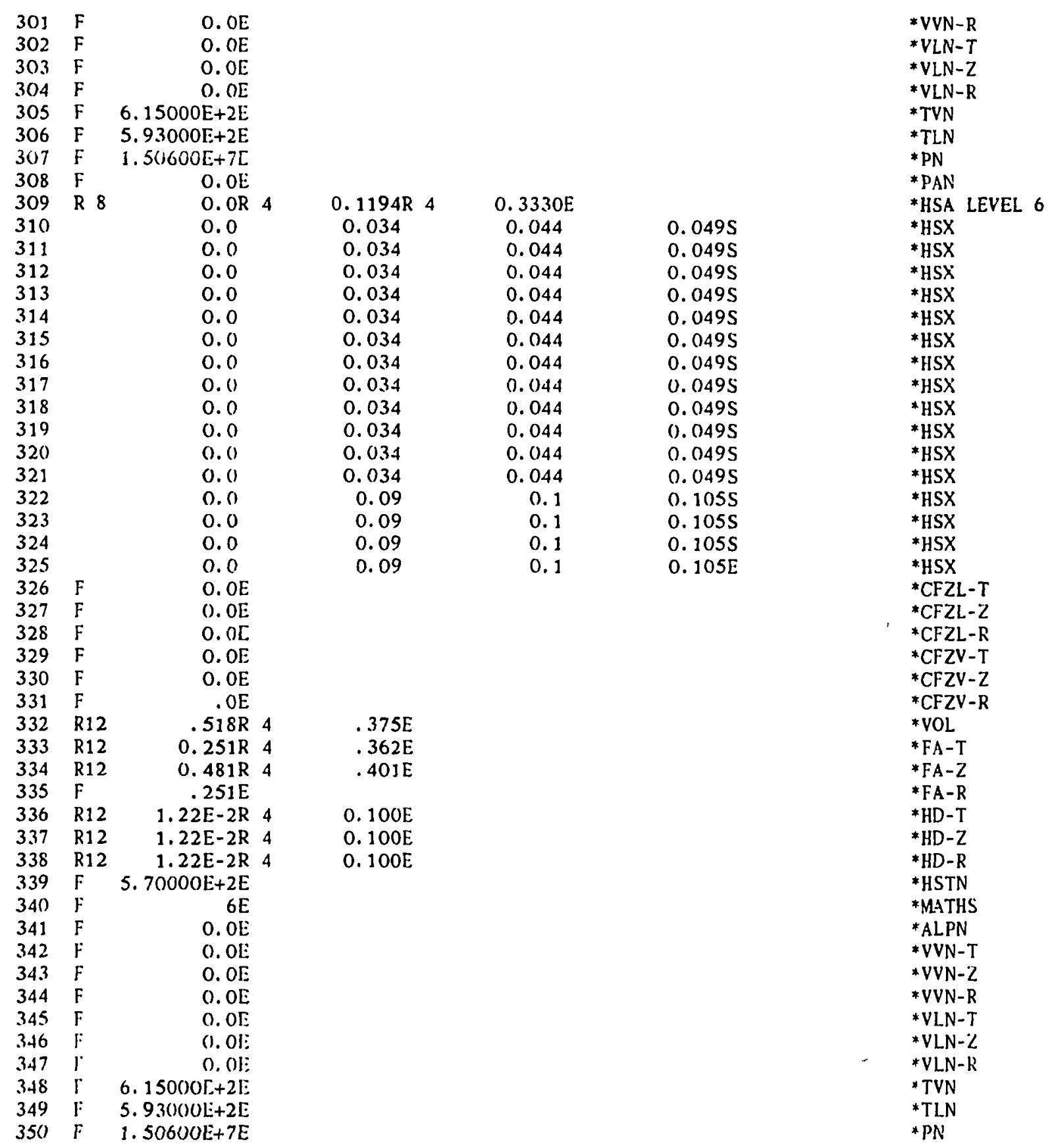




\begin{tabular}{|c|c|c|c|c|c|c|}
\hline 351 & $\mathrm{~F}$ & $0.0 E$ & & & & ${ }^{*} \mathrm{PAN}$ \\
\hline 352 & R 8 & $0.0 \mathrm{~K} 4$ & $0.2383 R$ & $0.6645 \mathrm{~F}$ & & ${ }^{*}$ HSA LEVEL 7 \\
\hline 35.3 & & 0.0 & 0.034 & 0.044 & 0.0495 & ${ }^{*} \mathrm{HSX}$ \\
\hline 354 & & 0.0 & 0.034 & 0.044 & $0.049 \mathrm{~S}$ & ${ }^{*} \mathrm{HSX}$ \\
\hline 355 & & 0.0 & 0.0 .34 & 0.044 & $0.049 \mathrm{~S}$ & ${ }^{*} \mathrm{HSX}$ \\
\hline 356 & & 0.0 & 0.034 & 0.044 & $0.049 \mathrm{~S}$ & *HSX \\
\hline 357 & & 0.0 & 0.034 & 0.044 & 0.0495 & *HSX \\
\hline 358 & & 0.0 & 0.034 & 0.044 & $0.049 \mathrm{~S}$ & ${ }^{*}$ HSX \\
\hline 359 & & 0.0 & 0.034 & 0.044 & $0.049 \mathrm{~S}$ & ${ }^{*}$ HSX \\
\hline 360 & & 0.0 & 0.034 & 0.044 & $0.049 \mathrm{~S}$ & ${ }^{*} \mathrm{HSX}$ \\
\hline 361 & & 0.0 & 0.034 & 0.044 & 0.0495 & ${ }^{*} \mathrm{HSX}$ \\
\hline 362 & & 0.0 & 0.034 & 0.044 & $0.049 \mathrm{~S}$ & ${ }^{*} \mathrm{HSX}$ \\
\hline 363 & & 0.0 & 0.034 & 0.044 & $0.049 \mathrm{~S}$ & $*$ HSX \\
\hline 364 & & 0.0 & 0.034 & 0.044 & $0.049 \mathrm{~S}$ & ${ }^{*} \mathrm{HSX}$ \\
\hline 365 & & 0.0 & 0.09 & 0.1 & $0.105 \mathrm{~S}$ & ${ }^{*} \mathrm{HSX}$ \\
\hline 366 & & 0.0 & 0.09 & 0.1 & $0.105 \mathrm{~S}$ & *HSX \\
\hline 367 & & 0.0 & 0.09 & 0.1 & $0.105 \mathrm{~S}$ & ${ }^{*}$ HSX \\
\hline 368 & & 0.0 & 0.09 & 0.1 & $0.105 \mathrm{E}$ & ${ }^{*}$ HSX \\
\hline 369 & $\mathrm{~F}$ & $0 . \mathrm{OE}$ & & & & ${ }^{*} \mathrm{CFZL}-\mathrm{T}$ \\
\hline 370 & $\mathrm{~F}$ & $0.0 \mathrm{E}$ & & & & ${ }^{*} \mathrm{CrZL}-\mathrm{Z}$ \\
\hline 371 & $\mathrm{~F}$ & $0.0 \mathrm{E}$ & & & & ${ }^{*} \mathrm{CFZL}-\overline{\mathrm{R}}$ \\
\hline 372 & $\mathrm{~F}$ & $0.0 \mathrm{E}$ & & & & ${ }^{*} \mathrm{CFZV}-\mathrm{T}$ \\
\hline 373 & $\mathrm{~F}$ & $0.0 \mathrm{E}$ & & & & ${ }^{*} \mathrm{CFZV}-\mathrm{Z}$ \\
\hline 374 & $\mathrm{~F}$ &. $\mathrm{OE}$ & & & & ${ }^{*} \mathrm{CF} Z \mathrm{~V}-\mathrm{R}$ \\
\hline 375 & R12 & $.518 R_{4}$ & $.375 \mathrm{E}$ & & & *VOL \\
\hline 376 & $\mathrm{R} 12$ & $0.251 R_{4}$ & $.362 \mathrm{E}$ & & & $* F A-T$ \\
\hline 377 & $\mathrm{R} 12$ & $0.481 \mathrm{R}_{4}$ & $.401 \mathrm{E}$ & & & $* F A-Z$ \\
\hline 378 & $F$ & .251[ & & & & $* \Gamma A-R$ \\
\hline 379 & $\mathrm{R} 12$ & 1. $22 \mathrm{E}-2 \mathrm{R} \quad 4$ & $0.100 \mathrm{E}$ & & & ${ }^{*} \mathrm{HD}-\mathrm{T}$ \\
\hline 380 & $\mathrm{R} 12$ & 1. $22 \mathrm{E}-2 \mathrm{R} \quad 4$ & $0.100 \mathrm{E}$ & & & ${ }^{*} H D-Z$ \\
\hline 381 & R12 & 1. $22 \mathrm{E}-2 \mathrm{R} \quad 4$ & $0.100 \mathrm{E}$ & & & *HD-R \\
\hline 382 & $\mathrm{~F}$ & $5.70000 E+2 E$ & & & & *HSTN \\
\hline 383 & $\mathrm{~F}$ & $6 \mathrm{E}$ & & & & *MATHS \\
\hline 384 & $\mathrm{~F}$ & $0.0 \mathrm{E}$ & & & & *ALPN \\
\hline 385 & $\mathrm{~F}$ & $0.0 \mathrm{E}$ & & & & $* \mathrm{VVN}-\mathrm{T}$ \\
\hline 386 & $\mathrm{r}$ & $0.0 \mathrm{E}$ & & & & *VVN-Z \\
\hline 387 & $\mathrm{~F}$ & $0.0 \mathrm{E}$ & & & & *VVN-R \\
\hline 388 & $\mathrm{~F}$ & $0.0 \mathrm{E}$ & & & & *VLN-T \\
\hline 389 & $\mathrm{~F}$ & $0.0 \mathrm{E}$ & & & & *VLN-Z \\
\hline 390 & $\mathrm{~F}$ & $0.0 \mathrm{E}$ & & & & *VLN-R \\
\hline 391 & $\mathrm{~F}$ & $6.15000 E+2[$ & & & & *TVN \\
\hline 392 & $\mathrm{~F}$ & $5.93000 \mathrm{E}+2 \mathrm{E}$ & & & & $*$ TLN \\
\hline 39.3 & $\mathrm{~F}$ & 1.50600L+7E & & & & *PN \\
\hline 394 & $\mathrm{~F}$ & $0.0 \mathrm{E}$ & & & & * PAN \\
\hline 395 & $\mathrm{R} 8$ & $0.0 R_{4}$ & $0.2383 R$ & $0.6645 \mathrm{E}$ & & *HSA LEVEL 8 \\
\hline 396 & & 0.0 & 0.034 & 0.044 & $0.049 \mathrm{~S}$ & * ISX \\
\hline 397 & & 0.0 & 0.034 & 0.044 & (1. 0495 & $H S X$ \\
\hline 398 & & 0.0 & 0.034 & 0.044 & 0.0495 & ${ }^{*} \mathrm{HSX}$ \\
\hline 399 & & 0.0 & 0.034 & 0.044 & 0.0495 & *HSX \\
\hline 400 & & 0.0 & 0.034 & 0.044 & $0.049 \mathrm{~S}$ & *IISX \\
\hline
\end{tabular}




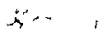

CARD 123456789012345' 90123456789012345678901234567890123456789012345678901234567890

\begin{tabular}{|c|c|c|c|c|c|c|}
\hline 401 & & $0.0 \ldots+2$. & 0.034 & 0.044 & $0.049 \mathrm{~S}$ & *IISX \\
\hline 402 & & 0.0 & 0.034 & 0.044 & $0.049 \mathrm{~S}$ & ${ }^{*}$ HSX \\
\hline 40.3 & & 0.0 & 0.034 & 0.044 & 0.0495 & *IISX \\
\hline 404. & & 0.0 & 0.034 & 0.044 & $0.049 \mathrm{~S}$ & *HSX \\
\hline 405 & & 0.0 & 0.034 & 0.044 & $0.049 \mathrm{~S}$ & ${ }^{*} \mathrm{HSX}$ \\
\hline 406 & & 0.0 & 0.034 & 0.044 & $0.049 \mathrm{~S}$ & *IISX \\
\hline 107 & & 0,0 & 0.0 .34 & 0.044 & $0.049 \mathrm{~S}$ & ${ }^{*} \mathrm{HSX}$ \\
\hline 408 & & 0.0 & 0.09 & 0.1 & $0.105 \mathrm{~S}$ & ${ }^{*} \mathrm{HSX}$ \\
\hline 409 & & 0.0 & 0.09 & 0,1 & $0.105 \mathrm{~S}$ & *HSX \\
\hline 410 & & 0.0 & 0.09 & 0.1 & $0.105 \mathrm{~S}$ & *HSX \\
\hline 411 & & 0.0 & 0.09 & 0.1 & $0.105 \mathrm{E}$ & *HSX \\
\hline 412 & $F$ & $0.0 \mathrm{~F}$ & & & & ${ }^{*} \mathrm{CFZL}-\mathrm{T}$ \\
\hline 413 & $F$ & 0.0 : & & & & ${ }^{*} \mathrm{CFZL}-\mathrm{Z}$ \\
\hline 414 & $F$ & $0.0 \mathrm{E}$ & & & & ${ }^{*} \mathrm{CF} Z \mathrm{~L}-\mathrm{R}$ \\
\hline 415 & $F$ & $0.0 E$ & & & & ${ }^{*} \mathrm{CFZV}-\mathrm{T}$ \\
\hline 416 & $\Gamma$ & $0.0 \mathrm{O}$ & & & & ${ }^{*} \mathrm{CFZV}-\mathrm{Z}$ \\
\hline 417 & $\xi$ &.$O E$ & & & & ${ }^{*} \mathrm{CF} Z V-\mathrm{R}$ \\
\hline 418 & $\mathrm{R} 12$ & $.518 \mathrm{R} 4$ & $.375 E$ & & & *VOL \\
\hline 419 & $\mathrm{R} 12$ & $0.251 \mathrm{R}^{4}$ & $.362 E$ & & & $* F A-T$ \\
\hline 420 & $\mathrm{R} 12$ & $0.481 \mathrm{R} 4$ & $.401 \mathrm{E}$ & & & $* F A-Z$ \\
\hline 421 & $F$ & $.251 E$ & & & & $* F A-R$ \\
\hline 422 & $\mathrm{R} 12$ & $1.22 \mathrm{E}-2 \mathrm{R} \quad 4$ & $0.100 \mathrm{~F}$ & & & $* H D-T$ \\
\hline 423 & $\mathrm{R} 12$ & 1. $22 E-2 R \quad 4$ & $0.100 \mathrm{E}$ & & & ${ }^{*} \mathrm{HD}-\mathrm{Z}$ \\
\hline 424 & $\mathrm{R} 12$ & 1. $22 E-2 R \quad 4$ & $0.100 E$ & & & ${ }^{*} \mathrm{HD}-\mathrm{R}$ \\
\hline 425 & $\mathrm{~F}$ & $5.70000 E+2 E$ & & & & ${ }^{*}$ HSTN \\
\hline 426 & $\Gamma$ & $6 \mathrm{E}$ & & & & *MATHS \\
\hline 427 & r & $0.0 \mathrm{E}$ & & & & ${ }^{* A L P N}$ \\
\hline 428 & $\mathbf{F}$ & $0.0 E$ & & & & *VVN-T \\
\hline 429 & $F$ & $0 . \mathrm{OE}$ & & & & $*$ VVN-Z \\
\hline 430 & $\mathrm{~F}$ & $0.0 \mathrm{E}$ & & & & $*$ VVN-R \\
\hline 431 & $\mathrm{~F}$ & $0.0 \mathrm{E}$ & & & & *VLN-T \\
\hline 432 & $F$ & $0.0 \mathrm{E}$ & & & & *VLN-Z \\
\hline 433 & $\mathrm{~F}$ & $0.0 \mathrm{E}$ & & & & $* V L N-R$ \\
\hline 434 & $\mathrm{~F}$ & $6.15000 E+2 E$ & & & & *TVN \\
\hline 435 & $F$ & $5.93000 E+2 E$ & & & & $*$ TLN \\
\hline 436 & $F$ & 1. $50600 \mathrm{E}+7 \mathrm{~F}$ & & & & $* \mathrm{PN}$ \\
\hline 437 & $\mathrm{~F}$ & O. OE: & & & & *PAN \\
\hline 438 & R 4 & $0.7208 R \quad 4$ & $2.7708 \mathrm{R} \quad 4$ & $.232 \mathrm{R}_{4}$ & $1.2243 \mathrm{E}$ & *HSA LEVEL 9 \\
\hline 439 & & 0.0 & .00073 & .00147 & $.0022 \mathrm{~S}$ & ${ }^{*}$ HSX \\
\hline 440 & & 0.0 & .00073 & .00147 & $.0022 \mathrm{~S}$ & *HSX \\
\hline 441 & & 0.0 & .06073 & .00147 & $.0022 \mathrm{~S}$ & ${ }^{*}$ HSX \\
\hline 442 & & 0.0 & .00073 & .00147 & .00225 & ${ }^{*}$ HSX \\
\hline 443 & & 0.0 & .00073 & .00147 & $.0022 \mathrm{~S}$ & *HSX \\
\hline 444 & & 0.0 & .00073 & .00147 & $.0022 S$ & *IISX \\
\hline 445 & & 0.0 & .00073 & .00147 & $.0022 \mathrm{~S}$ & *HSX \\
\hline 446 & & 0.0 & $.00(173$ & $.001+7$ & $.0022 S$ & ${ }^{*}$ HSX \\
\hline 447 & & 0.0 & .034 & .044 & $.049 S$ & *HSX \\
\hline 448 & & 0.0 & .034 & .044 & .0495 & $* H S X$ \\
\hline 449 & & 0.0 & .034 & .044 & .0495 & ${ }^{*} \mathrm{HSX}$ \\
\hline 450 & & 0.0 & .034 & .044 & $.049 \mathrm{~S}$ & *HSX \\
\hline
\end{tabular}




\begin{tabular}{|c|c|c|c|c|c|c|c|}
\hline 451 & & 0.0 & 0.09 & & 0.1 & $0.105 \mathrm{~S}$ & *HSX \\
\hline 452 & & 0.0 & 0.09 & & 0.1 & $0.105 \mathrm{~S}$ & ${ }^{*} \mathrm{HSX}$ \\
\hline 45.3 & & 0.0 & 0.09 & & 0.1 & $0.105 \mathrm{~S}$ & ${ }^{*} \mathrm{HSX}$ \\
\hline 454 & & 0.0 & 0.09 & & 0.1 & $0.105 \mathrm{E}$ & *HSX \\
\hline 455 & $\mathrm{~F}$ & $0.0 E$ & & & & & ${ }^{*} \mathrm{CFZL}-\mathrm{T}$ \\
\hline 456 & R 4 & $0.0 \mathrm{R} 4$ & 1. $2 \mathrm{R}$ & 4 & $0.5 \mathrm{R} 4$ & $0.0 E$ & ${ }^{*} \mathrm{CFZL}-\mathrm{Z}$ \\
\hline 457 & F & $0.0 F$ & & & & & ${ }^{*} \mathrm{CF} 2 \mathrm{~L}-\mathrm{R}$ \\
\hline 458 & $\mathrm{~F}$ & $0.0 \Sigma$ & & & & & ${ }^{*} \mathrm{CFZV}-\mathrm{T}$ \\
\hline 459 & R 4 & $0.0 \mathrm{R} 4$ & 1. $2 \mathrm{R}$ & 4 & $0.5 \mathrm{R} 4$ & $0.0 \mathrm{E}$ & ${ }^{*} \mathrm{CFZV}-\mathrm{Z}$ \\
\hline 460 & $\mathrm{~F}$ & $0 . \mathrm{OE}$ & & & & & ${ }^{*} \mathrm{CFZV}-\mathrm{R}$ \\
\hline 461 & $\mathrm{R} 12$ & $0.951 \mathrm{R} 4$ & $.375 \mathrm{E}$ & & & & $*$ VOL \\
\hline 462 & $\mathrm{R} 12$ & $0.366 \mathrm{R}^{4}$ & $.362 \mathrm{E}$ & & & & *FA-T \\
\hline 46.3 & R 4 & $0.85 \mathrm{R} 8$ & $0.366 \mathrm{R}$ & 4 & $0.4010 E$ & & ${ }^{*} \mathrm{FA}-\mathrm{Z}$ \\
\hline 464 & $F$ & $0.3660 \mathrm{E}$ & & & & & ${ }^{*} \mathrm{FA}-\mathrm{R}$ \\
\hline 465 & R12 & 1. $22 \mathrm{E}-2 \mathrm{R} \quad 4$ & $0.100 E$ & & - & & ${ }^{*} \mathrm{HD}-\mathrm{T}$ \\
\hline 466 & $\mathrm{R} 12$ & $1.22 \mathrm{E}-2 \mathrm{R} \quad 4$ & $0.100 \mathrm{~F}$ & & & & ${ }^{*} \mathrm{HD}-\mathrm{Z}$ \\
\hline 467 & $\mathrm{R} 12$ & 1. $22 \mathrm{E}-2 \mathrm{R} \quad 4$ & $0.100 \mathrm{E}$ & & & & ${ }^{*} \mathrm{HD}-\mathrm{R}$ \\
\hline 468 & $F$ & 5. $70000 E+2 E$ & & & & & ${ }^{*} \mathrm{HSTN}$ \\
\hline 469 & $\mathrm{~F}$ & $6 E$ & & & & & *MATHS \\
\hline 470 & $F$ & $0.0 E$ & & & & & *ALPN \\
\hline 471 & $F$ & $0.0 \mathrm{E}$ & & & & & *VVN-T \\
\hline 472 & $F$ & $0.0 \mathrm{E}$ & & & & & *VVN-Z \\
\hline 473 & $F$ & $0.0 \mathrm{E}$ & & & & & *VVN-R \\
\hline 474 & $F$ & $0.0 E$ & & & & & $* V_{4} N-T$ \\
\hline 475 & $F$ & $0.0 \mathrm{E}$ & & & & & *VLN-Z \\
\hline 476 & $\mathrm{~F}$ & $0.0 \mathrm{E}$ & & & & & *VLN-R \\
\hline 477 & $\mathrm{~F}$ & 6. $15000 \mathrm{E}+2 \mathrm{E}$ & & & & & *TVN \\
\hline 478 & F & $5.93000 E+2 E$ & & & & & *TLN \\
\hline 479 & $F$ & 1. $50600 \mathrm{E}+7 \mathrm{E}$ & & & & & *PN \\
\hline 480 & $\mathrm{~F}$ & $0.0 E$ & & & & & *PAN \\
\hline 481 & R 4 & $0.6641 \mathrm{R}^{4}$ & $2.5528 \mathrm{R}$ & 4 & $.702 R_{4}$ & 1. $2228 \mathrm{E}$ & ${ }^{*}$ HSA LEVEL 10 \\
\hline 482 & & 0.0 & .0005 & & .001 & $.0015 S$ & ${ }^{*} \mathrm{HSX}$ \\
\hline 483 & & 0.0 & .0005 & & .001 & $.0015 S$ & ${ }^{*}$ HSX \\
\hline 484 & & 0.0 & .0005 & & .001 & $.0015 S$ & ${ }^{*} \mathrm{HSX}$ \\
\hline 485 & & 0.0 & .0005 & & .001 & $.0015 S$ & ${ }^{*} \mathrm{HSX}$ \\
\hline 486 & & 0.0 & .0005 & & .001 & $.0015 \mathrm{~S}$ & ${ }^{*} \mathrm{HSX}$ \\
\hline 487 & & 0.0 & .0005 & & .001 & $.0015 S$ & ${ }^{*} \mathrm{HSX}$ \\
\hline 488 & & 0.0 & .0005 & & .001 & $.0015 S$ & ${ }^{*} \mathrm{HSX}$ \\
\hline 489 & & 0.0 & .0005 & & .001 & $.0015 S$ & ${ }^{*} \mathrm{HSX}$ \\
\hline 490 & & 0.0 & .0 .34 & & .044 & .0495 & *HSX \\
\hline 491 & & 0.0 & .034 & & .044 & $.049 S$ & *HSX \\
\hline 492 & & 0.0 & .034 & & .044 & $.049 \mathrm{~S}$ & ${ }^{*}$ HSX \\
\hline 493 & & 0.0 & .034 & & .044 & $.049 \mathrm{~S}$ & ${ }^{*} \mathrm{HSX}$ \\
\hline 494 & & 0.0 & 0.09 & & 0.1 & $0.105 \mathrm{~S}$ & ${ }^{*} \mathrm{HSX}$ \\
\hline 495 & & 0.0 & 0.09 & & 0.1 & $0.105 S$ & *HSX \\
\hline 496 & .. & 0.0 & 0.09 & & (1). 1 & $0.105 \mathrm{~S}$ & ${ }^{*}$ HSX \\
\hline 497 & & 0.0 & 0.09 & & (). 1 & $0.105 \mathrm{E}$ & ${ }^{\star}$ HSX \\
\hline 498 & F & 0. or: & & & & & ${ }^{*} \mathrm{CF} Z \mathrm{~L}-\mathrm{T}$ \\
\hline 499 & F & $0.01:$ & & & & & ${ }^{*} \mathrm{CF} \mathrm{ZL}-\mathrm{L}$ \\
\hline 500 & $\mathrm{~F}$ & $0.0 \mathrm{E}$ & & & & & ${ }^{*} \mathrm{CFZL}-\mathrm{R}$ \\
\hline
\end{tabular}




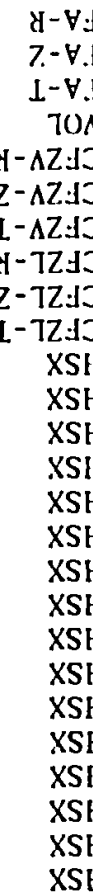

I I T3A

NVd *

$\mathrm{Nd}$ *

NTL*

NAL*

y-NTA*

$\mathrm{Z}-\mathrm{N} 7 \mathrm{~A}$.

L-NTA*

d-NAS*

$\mathrm{Z}-\mathrm{N} \Lambda \Lambda *$

L-NAS*

Nd7F*

SHLVW*

NLSH*

$\mathrm{y}-\mathrm{CH} *$

$\mathrm{Z}-\mathrm{OH} *$

1 - $\mathrm{UH} *$

$y-\forall I *$

2- $-\mathrm{I}_{\mathrm{I}}$.

L- $\forall$ J*

TOA*

$y-\Delta Z s J$

$\mathrm{Z}-\Lambda \mathrm{Z} \mathrm{AJ}$ *

L-AZ:JJ*

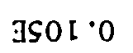

SSO I 0

SSOI ${ }^{\circ} 0$

SSOI '0

S6to $0^{\circ}$

$5600^{\circ}$

S6r0 $0^{\circ}$

S6t0 $0^{\circ}$

SS $100^{\circ}$

SS $100^{\circ}$

SS $100^{\circ}$

SS $100^{\circ}$

SS $100^{\circ}$

SS 100.

SS $100^{\circ}$

SS ז00.

$30059^{\circ} \cdot$
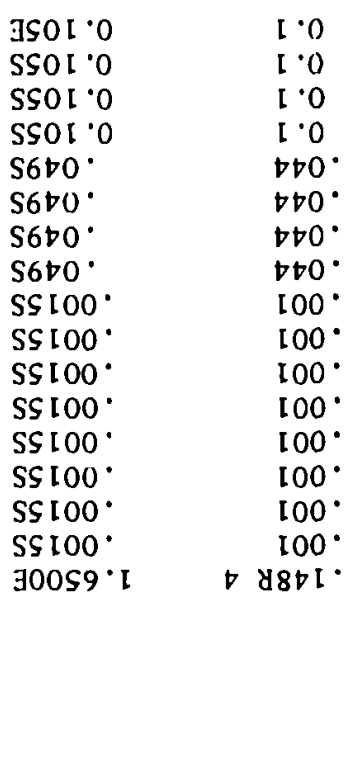

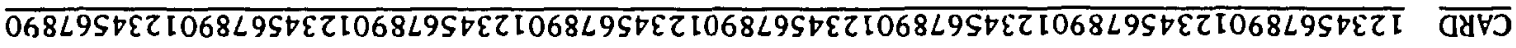

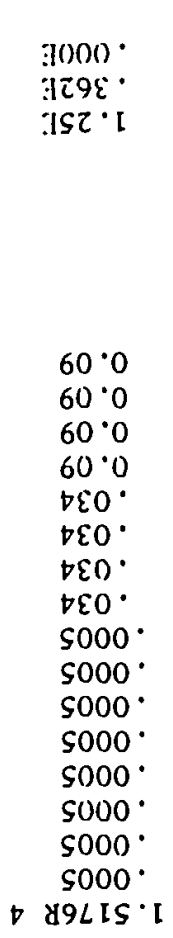

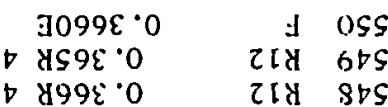

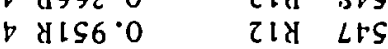

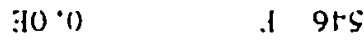

$30^{\circ} 0 \quad \&$ StS

$30^{\circ} 0 \quad \& \quad \forall t S$

$30^{\circ} 0 \quad$ a $\varepsilon \forall \subseteq$

$30^{\circ} 0 \quad \pm \quad 2 \forall S$

30.0

$0.0 \quad 0$ Ob

$0.0 \quad 6 \varepsilon S$

$0^{\circ} 0 \quad 8 \varepsilon S$

$0^{\circ} 0 \quad$ LES

$0^{\circ} 0 \quad 9 \varepsilon S$

0.0 SES

$0.0 \quad t \varepsilon S$

$0^{\circ} 0 \quad \varepsilon \varepsilon S$

$0^{\circ} 0 \quad$ ZES

0.0 IES

$0^{\circ} 0 \quad 0 \varepsilon S$

$0.0 \quad 625$

$0.0 \quad 825$

$0.0 \quad$ LIS

$0^{\circ} 0 \quad 925$

$0.0 \quad$ SZS

$\forall$ ४ $8 \nabla 6 \varepsilon^{\circ} \quad \forall$ y $\forall Z S$

$30^{\circ} 0$ I $\varepsilon 2 S$

$\exists L+100905 \cdot 1$ I 225 $\exists Z+.3000 \varepsilon 6^{\circ} \mathrm{S}$ id L $2 S$ $3 z+3000 S I \cdot 9$ \& UZS $300^{\circ} 0$ \& 6 IS $30^{\circ} 0$ I 8 [S $300^{\circ} 0$ I $L$ IS $30^{\circ} 0$ \& 9 IS 30.0 I SIS $\exists 0^{\circ} 0$ I $t$ [S $\exists 0^{\circ} 0$ I $\varepsilon[S$ 39 $\mathrm{d}$ IIS $\exists z+30000 L$ 's I IIS

$300[\cdot 0 \quad \forall y z-3 z z \cdot 1$ zIy 0 IS 300L $0 \quad \forall \mathrm{d} z-] \tau \tau \cdot\left[\quad \tau\left[\mathrm{d}\right.\right.$. $605^{\circ}$

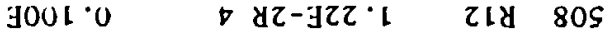

$30000^{\circ} 0$ \& LOS

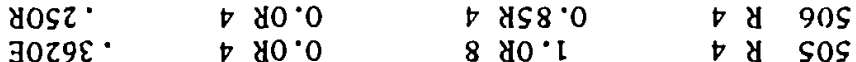

उSLE. 30.0 \& $\varepsilon 0 S$ 30.0 I 205 $30 \%$ \& LOS 


\section{CARD 12345678901234567890123456789012345678901234567890123456789012345678901234567890}

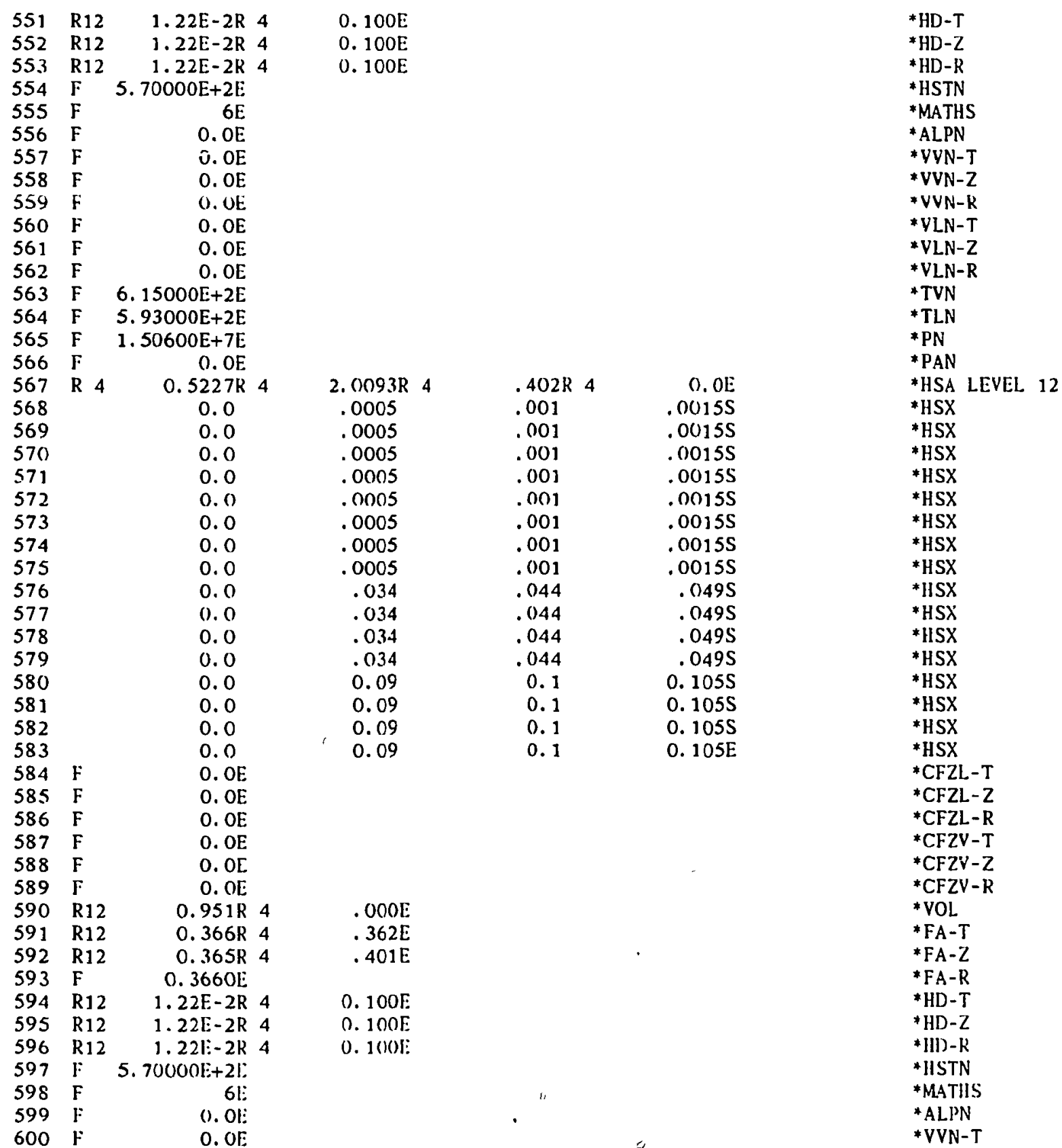




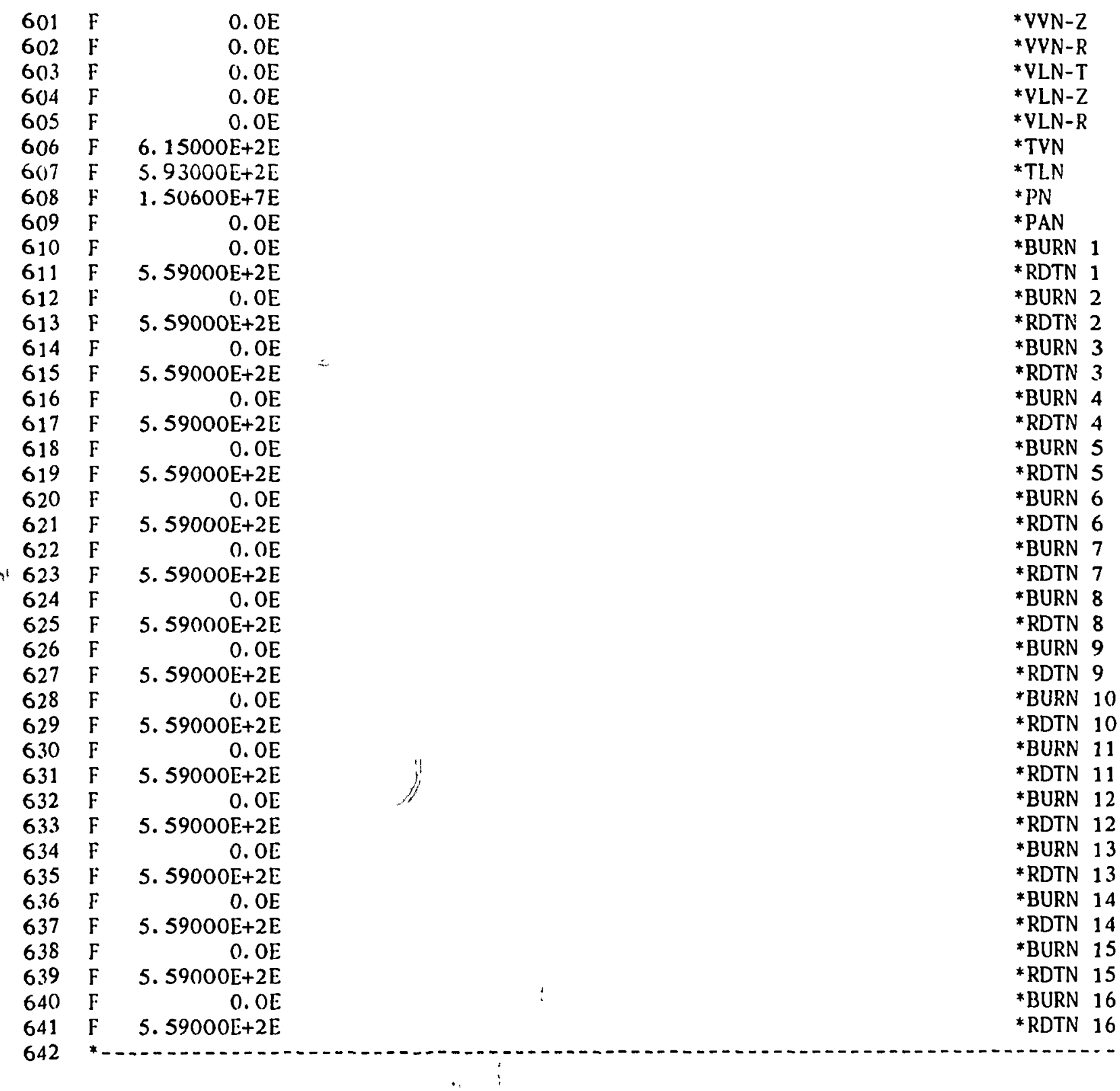


APPENDIX F

TRAC ERROR MESSAGES

Subroutine ERROR handles errors diagnosed by TRAC. The subroutine uses the level number associated with each error listed below to determine its course of action.

Leve1

\section{Actions}

1, 3 Fatal error, stop problem.

2 Nonfatal error, continue problem.

4 Fatal error, add dump to the TRCDMP file, then stop problem.

$-4 \quad$ Problem stopped by user.

The error messages are written to the TRCOUT and TRCMSG files and to the terminal. The message begins with the name of the subroutine, bounded by asterisks $(* . . *$ ), which detected the error. Because of this format and because implementation of TRAC on various computers differs, we have used the subroutine name to alphabetize the following list of error messages. If an error message occurs that is not found in the following list, we suggest that you inspect the coding in the subroutine listed in the message for more detail.

Subroutine Leve1 Message

BFIN 1 DATA SET EOF ERROR

BFIN 1 DATA SET TYPE ERROR

$\begin{array}{lll}\text { BFOUT } & 1 & \text { DATA } \\ & \text { SET TYPE ERROR }\end{array}$

BITS

1 ILLEGAL BIT SPECIFIED
Explanation

An illegal end-of-file was found when the data were read.

An error occurred when the data were read in the binary format.

An error occurred when the data were written in a binary format.

An attempt was made to set bit beyond the word length. 


$\begin{array}{lcl}\begin{array}{l}\text { Subroutine } \\ \text { BREAKX }\end{array} & \begin{array}{l}\text { Level } \\ \text { BK TABLE LOOKUP ERROR }\end{array} \\ \text { CBSET } & 1 & \begin{array}{l}\text { C-BLOCK ID NOT FOUND } \\ \text { TO SET NFLG }\end{array}\end{array}$

CBSET $\quad 1 \quad$ C-BLOCK ID NOT FOUND TO SET OFLG

CBSET

CBSET

CBSET

CHBD

$\mathrm{CHF}$

CHKSR

CHKSR
1 ERROR IN TABLE LOOKUP

1 CNTL . BLK. ID NOT FOUND

1 SIG. VAR. ID NOT FOUND

2 BOUNDARY ERROR DETECTED

1 TCHF FAILED TO CONVERGE

2 TWO VESSEL SOURCES LOCATED IN ONE CELL

2 VESSEL SOURCE POSITION ERROR

\section{Explanation}

An error exists in interpolating a break table.

The first input parameter ID number for the control block could not be found in the list on control blocks so that it could be flagged with this control block's new flag.

The first input parameter ID number for this control block could not be found in the list of control blocks so that it could be flagged with this control block's old flag.

One of the control-block input parameters has a negative ID number that could not be found in the list of control blocks.

An error was detected by subroutine LININT while it was linearly interpolating in the control-block FNGl table.

One of the control-block input parameters has a positive ID number that could not be found in the list of signal variables.

Adjacent components have mismatched geometry.

The calculation failed to converge on a unique critical heat-flux wall temperature.

The VESSEL input specifies two sources in one fluid cell.

The specified source position is Impossible. 
$\begin{array}{lcl}\text { Subroutine } & \text { Level } & \frac{\text { Message }}{\text { CHOKE }} \\ 2 & \begin{array}{l}\text { CHARACTERISTIC SOLUTION } \\ \text { DID NOT CONVERGE }\end{array}\end{array}$

CHOKE 2 CONVERGENCE FAILED IN GREV

CHOKE

2 LARGEST CHARACTERISTIC ROOT WAS COMPLEX

CHOKE

2 NEGATIVE DFLDP CALCULATED, ASSUMED ZERO

CHOKE

CHOKE

CHOKE

CIVSSL
2 NEGATIVE DFVDP CALCULATED ASSUMED ZERO

H

2 ONLY APPROXIMATE SOLUTION OBTAINED

SEARCH FAILED

1 CONNECTIONS COMPUTED AFTER VESSEL
2 QUICK SOLUTION
Explanation

The two-phase characteristic solution using a quicksolution search was bounded, but complete convergence could not be obtained within allowed iterations.

The system's routine GREV has trouble calculating all the eigen values of the two-phase characteristic solution.

An informative message "is printed under debug mode only.

The calculated derivative $\partial V_{\ell} / \partial \rho$ was negative because the round-off errors should always be $>0.0$. Therefore, the derivative was set to 0.0 .

The calculated derivative $\partial v_{g} / \partial \rho$ was negative because the round-off errors should be >0.0. Therefore; the derivative was set to 0.0 .

The normal two-phase choking solution maintains constant phasic slip. However, because of convergence problems, this condition could not be satisfied, but rather the relative velocity between the phases was approximately maintained.

An informative message is printed under debug mode oniy.

The component calculational sequence must compute the connections before the vesse1. 


\begin{tabular}{|c|c|c|}
\hline Subroutine & Leve1 & Message \\
\hline CIVSSL & 1 & IORDER PROBLEM \\
\hline CIVSSL & 1 & JUNCTION PROBLEM \\
\hline CIVSSL & $I$ & $\begin{array}{l}\text { VESSEL CONNECTED TO } \\
\text { A FILL }\end{array}$ \\
\hline CIVSSL & 1 & $\begin{array}{l}\text { VESSEL CONNECTED TO } \\
\text { BREAK }\end{array}$ \\
\hline CONBLK & 1 & $\begin{array}{l}\text { BAD CNTL-BLOCK } \\
\text { OPERATION NUMBER }\end{array}$ \\
\hline
\end{tabular}

$\begin{array}{lll}\text { CONBLK } & 1 & \text { IMPROPER LLAG } \\ \text { BLOCK CONSTANTS }\end{array}$

$\begin{array}{lll}\text { CONBLK } & 1 & \text { IMPROPER SOTF } \\ \text { BLOCK CONSTANTS }\end{array}$

$\begin{array}{lll}\text { CONBLK } & 1 & \text { INVALID CNTL-BLOCK } \\ & \text { INPUT VALUES }\end{array}$

CONBLK 1 INVALID DEADFUNCTION CONSTANTS

CONBLK I NONPOSITIVE LAG TIME

$\begin{array}{lll}\text { CONVRT } & 1 \text { ARRAY DIMENSIONS EXCEEDED }\end{array}$

\section{Explanation}

The calculational sequence must compute the component connected to the vessel before it calculates the vessel.

A component adjacent to the VESSEL cannot be found.

A VESSEL cannot be connected to a FILL.

A VESSEL cannot be connected to a BREAK.

A control-block operation number does not lie between 1 and 61 .

The lead-lag transfer function control block 30 has a first constant that is "negative or a second constant that is zero or negative.

The second-order transfer function control block 51 has a first constant that is negative or a second constant that is zero or negative.

A control block is defined with invalid input parameter values.

The dead control block 11 has a second constant that is less than the first constant.

The first-order LAG transfer function control block 26 has a first constant that is zero or negative.

The s subroutine, which extracts a vessel deck for modification and re-input, assumes that there are no more than 25 axial, radial, or theta zones. 
Subroutine Level

CORECL

1

REACTOR POWER INITIALIZED

COREC3

CTAIN1

CTAIN2

CTAIN3

DELAY

MPIT

DMPIT

ELGR

Message
Explanation

The reactor core's total power is turned on to its input initial value.

Insufficient SCM is available for this CORE.

Containment component will be in a future TRAC version.

Containment component will be in a future TRAC version.

Containment component will be in a future TRAC version.

An error was detected by subroutine LININT when it tried to linearly interpolate the time delay table on a control block 100 .

File TRCDMP could not be created.

An invalid component type was encountered.

The input value of a form loss was such that when the code converted it into an equivalent FRIC, the FRIC value exceeded $10^{20} \cdot$ A FRIC value exceeding $10^{20}$ w111 invoke the steam separator model at the cell edge under consideration. Obviously, such was not the user's intention, otherwise the user would have input a form-loss value exceeding $10^{20}$ in the first place.

An illegal overlay sequence exists. 


\begin{tabular}{|c|c|c|c|}
\hline Subroutine & Leve1 & Message & Explanation \\
\hline EVFXXX & 1 & NEED LOCAL DIM.GT.50 & $\begin{array}{l}\text { Local array FXXXo is dimen- } \\
\text { sloned } 50 ; \text { for components } \\
\text { with more than } 50 \text { mesh cells, } \\
\text { subroutine EVFXXX cannot } \\
\text { evaluate a QPPP factor for } \\
\text { each mesh cell. }\end{array}$ \\
\hline EVFXXX & 1 & TABLE LOOKUP ERROR & $\begin{array}{l}\text { Subroutine LININT encountered } \\
\text { an error while trying to } \\
\text { linearly interpolate the com- } \\
\text { ponent-action table value for } \\
\text { the situation when the con- } \\
\text { trolilng trip is oFF after } \\
\text { being oN. }\end{array}$ \\
\hline EVLTAB & 1 & CNTL. BLOCK NOT FOUND & $\begin{array}{l}\text { The negative ID number that } \\
\text { defines the independent vari- } \\
\text { able of the component-action } \\
\text { table was not specified in } \\
\text { the control-block list. }\end{array}$ \\
\hline EVLTAB & 1 & SIGNAL VAR. NOT FOUND & $\begin{array}{l}\text { The positive ID number that } \\
\text { defines the independent vari- } \\
\text { able of the component-action } \\
\text { table was not specifled in } \\
\text { the signal-variable list. }\end{array}$ \\
\hline EVLTAB & 1 & TABLE LOOKUP ERROR & $\begin{array}{l}\text { Subroutine LININT found an } \\
\text { error when evaluating the } \\
\text { component-action table. }\end{array}$ \\
\hline FILLX & 1 & $\begin{array}{l}\text { GENSTATE FILL TABLE LOOKUP } \\
\text { ERROR }\end{array}$ & $\begin{array}{l}\text { There are zero entries in the } \\
\text { FILL table. }\end{array}$ \\
\hline GETBIT & 1 & ILLEGAL BIT SPECIFIED & $\begin{array}{l}\text { The specified bit position is } \\
\text { either too small or too } \\
\text { large. }\end{array}$ \\
\hline GRAF & 1 & DATA TYPE ERROR & $\begin{array}{l}\text { There is an invalid data type } \\
\text { in the graphics catalog. }\end{array}$ \\
\hline GRAF & 1 & SCM OVERFLOW & $\begin{array}{l}\text { There is insufficient SCM for } \\
\text { packing graphics data. }\end{array}$ \\
\hline GRFPUT & 2 & $\begin{array}{l}\text { ERROR IN GRAPHICS } \\
\text { OUTPUT }\end{array}$ & $\begin{array}{l}\text { Integer is too large to be } \\
\text { packed into a 15-bit word. }\end{array}$ \\
\hline
\end{tabular}




\begin{tabular}{|c|c|c|c|}
\hline Subroutine & Level & Message & Explanation \\
\hline HOUT & 1 & $\begin{array}{l}\text { OUTER ITERATION DID NOT } \\
\text { CONVERGE }\end{array}$ & $\begin{array}{l}\text { The outer-iteration procedure } \\
\text { failed three consecutive } \\
\text { times. }\end{array}$ \\
\hline ICOMP & 1 & FATAL INPUT ERRORS & $\begin{array}{l}\text { An error was encountered dur- } \\
\text { ing component data initiali- } \\
\text { zation causing JFLAG . NE. } 0 \\
\text { at the end of subroutine } \\
\text { ICOMP. }\end{array}$ \\
\hline ICOMP & 1 & $\begin{array}{l}\text { FRICTION LOSS HIGHER } \\
\text { THAN TURBINE OUTPUT } \\
\text { OUTPUT }\end{array}$ & $\begin{array}{l}\text { The friction torque coeffi- } \\
\text { clents specified for the tur- } \\
\text { bine are so large that the } \\
\text { friction loss exceeds the } \\
\text { normal design power from all } \\
\text { stages. }\end{array}$ \\
\hline ICOMP & 1 & $\begin{array}{l}\text { INCONSISTENT JUNCTION } \\
\text { NUMBERS }\end{array}$ & $\begin{array}{l}\text { Inconsistent specification of } \\
\text { junction numbers was made. }\end{array}$ \\
\hline ICOMP & 1 & JUNCTION COUNT ERROR & $\begin{array}{l}\text { The number of junctions spec- } \\
\text { ified is inconsistent with } \\
\text { the number found. }\end{array}$ \\
\hline ICOMP & 1 & JUNCTION NUMBERS WRONG & $\begin{array}{l}\text { The junctions are assigned } \\
\text { incorrectly. }\end{array}$ \\
\hline ICOMP & 1 & SCM OVERFLOW & $\begin{array}{l}\text { Insufficlent SCM is available } \\
\text { to load problem. }\end{array}$ \\
\hline ICOMP & 1 & $\begin{array}{l}\text { TURBINE STAGES } \\
\text { INCONSISTENT WITH INPUT }\end{array}$ & $\begin{array}{l}\text { The user specified component } \\
\text { numbers of the associated } \\
\text { turbine stages under stage } 1 . \\
\text { This specification is not } \\
\text { consistent with the other } \\
\text { TURB components input. }\end{array}$ \\
\hline ICOMP & 1 & UNRECOGNIZED COMPONENT & $\begin{array}{l}\text { The component type was not } \\
\text { recognized. }\end{array}$ \\
\hline ICOMP & 1 & $\begin{array}{l}\text { WRONG TURB COMPONENT } \\
\text { NUMBER ON VALVE }\end{array}$ & $\begin{array}{l}\text { The VALVE component for IVTY } \\
\text { option of } 5 \text { or } 6 \text { requires a } \\
\text { TURB component number. Th1s } \\
\text { number is inconsistent with } \\
\text { the TURB components input. }\end{array}$ \\
\hline
\end{tabular}




\begin{tabular}{|c|c|c|c|}
\hline Subroutine & Leve1 & Message & Explanation \\
\hline ICORE & 1 & BAD FUEL-ROD POWER SUM & $\begin{array}{l}\text { The input parameters for the } \\
\text { fuel-rod geometry, number, } \\
\text { and power distribution are } \\
\text { invalid. A nonpositive, un- } \\
\text { normalized power from all } \\
\text { fuel rods was evaluated. }\end{array}$ \\
\hline IGRAF & 1 & $\begin{array}{l}\text { COMPONENT TYPE NOT } \\
\text { RECOGNIZED }\end{array}$ & $\begin{array}{l}\text { An invalid component type was } \\
\text { encountered. }\end{array}$ \\
\hline IGRAF & 1 & $\begin{array}{l}\text { GRAPHICS FILE ALLOCATION } \\
\text { FAILURE }\end{array}$ & $\begin{array}{l}\text { An I/O error occurred while } \\
\text { allocated space was sought } \\
\text { for graphics file. }\end{array}$ \\
\hline IGRAF & 1 & $\begin{array}{l}\text { NO LCM SPACE FOR GRAPH } \\
\text { CATALOG }\end{array}$ & $\begin{array}{l}\text { Insufficient LCM is avail- } \\
\text { able. }\end{array}$ \\
\hline IGRAF & 1 & $\begin{array}{l}\text { NO SCM SPACE FOR } \\
\text { GRAPH CATALOGUE }\end{array}$ & $\begin{array}{l}\text { Problem too large to run with } \\
\text { current code. User must } \\
\text { reduce nodes or alter code. }\end{array}$ \\
\hline IGRAF & 1 & SCM OVERFLOW & $\begin{array}{l}\text { Insufficient SCM is avail- } \\
\text { able. }\end{array}$ \\
\hline INPUT & 1 & DUPLICATE COMPONENT NUMBERS & $\begin{array}{l}\text { Two components with the same } \\
\text { number were found in the } \\
\text { TRACIN file. }\end{array}$ \\
\hline INPUT & 1 & FATAL INPUT ERROR(S) & $\begin{array}{l}\text { A fatal input error was found } \\
\text { when an input or restart file } \\
\text { was read. }\end{array}$ \\
\hline INPUT & 1 & FILE TRACIN DOES NOT EXIST & $\begin{array}{l}\text { The input deck does not } \\
\text { exist. }\end{array}$ \\
\hline INPUT & 1 & $\begin{array}{l}\text { INOPTS NAMELIST DATA } \\
\text { NOT FOUND }\end{array}$ & $\begin{array}{l}\text { The NAMELIST option was spec- } \\
\text { ified; however, the NAMELIST } \\
\text { data for group INOPTS are not } \\
\text { in the TRACIN file. }\end{array}$ \\
\hline INPUT & 1 & NO SPACE FOR BUFFERS & $\begin{array}{l}\text { Insufficient LCM is avallable } \\
\text { for } \mathrm{I} / 0 \text { buffers. }\end{array}$ \\
\hline INPUT & 2 & SCM OVERFLOW & $\begin{array}{l}\text { Insufficient SCM is avallable } \\
\text { for this problem. The user } \\
\text { must radice the number of } \\
\text { nodes or redo the INPUT } \\
\text { overlay. }\end{array}$ \\
\hline
\end{tabular}




$\begin{array}{lcc}\text { Subroutine } & \text { Leve1 } & \frac{\text { Message }}{\text { INPUT }} \\ 2 & \begin{array}{l}\text { SOLUBILITY PARAMETERS } \\ \text { NOT REASONABLE }\end{array}\end{array}$

INPUT

INPUT

IOVLY

IOVIY

IOVLY

IOVLY

ISTGEN

ISTGEN

IVLVE
I TOO MANY CELLS FOR DIRECT INVERSION $($ LIMIT $=100)$

1 VERSION INFORMATION EXCEEDS LCM

1 A-ARRAY OVERFLOW

1 ERROR INITIALIZING SCM

I SCM SPACE TOO SMALL FOR OVERLAY

12 JNCTS OF 1 CELL COMP. EXT.

2 JUNCTION ERROR

1 INVALID VALVE LOCATION
Explanation

The solubility parameters entered for option ISOLCN do not define a reasonable linear relationship between solubility and temperature or may generate negative solubilities.

The user requested direct inversion of a VESSEL matrix when the VESSEL has more than 100 cells. This limit may be bypassed on machines with larger memories.

The version information exceeds its allocated LCM space.

The main SCM data array is too small. This message occurs only for the CDC 7600 version with a fixed A-dimenston $(\sim 25000$ words). Routine IOVLY directly issues diagnostic statistics. The global problem data are started at the $h i g h$ end of $A$, and the available space is smaller than the total dimension of A.

Subroutine SETSCM found an error.

Insufficient SCM is available for this overlay.

Code cannot find two internal secondary junction numbers that have the same value.

Error in the specification of the secondary internal junction numbers of the steam generator.

The valve interface where the flow area is adjustable does not lie between two cells within the VALVE component. 
Subroutine

IVSSL

Leve1

1

BAD FUEL-ROD POWER SUM

JFIND

LOAD

LOAD

LOAD

LOAD

LOAD
I JUNCTION PROBLEM

2 ARRAY FILLED BUT OPERATION END NOT FOUND

2 DATA OVERFLOWED ARRAY REPEAT COUNT RESET TO ONE

2 INPUT ERROR ENCOUNTERED

ON CARD NO. XXXX, REST

OF COMPONENT SKIPPED
LOAD

LOAD

UNEXPECTED COMPONENT
ENCOUNTERED

LOAD

LOAD
Explanation

The input parameters for the fuel-rod geometry, number, and power distribution are invalid. A nonpositive, unnormalized power from all fuel rods was evaluated.

A junction number could not be located in the junction sequence array.

When the array data were read, an $E$ was not specified after the array was filled. The user also should check for an additional incorrect entry for this array.

When the array data were read, a repeat operation overfilled the array.

The array-reading routine found an error flag on a card set by the free-format inputoption preprocessor routine. Execution of TRAC stops after the entire input deck is processed.

When the array data for a component were loaded, data for an additional component or an "END" card was specified.

When the array data were loaded, NAMELIST data were found.

When an integer array was read, an interpolation operation was specified.

Insufficient data were entered to fill an array. 


\begin{tabular}{lcl}
$\begin{array}{l}\text { Subroutine } \\
\text { LOAD }\end{array}$ & 2 & \multicolumn{1}{c}{ Message } \\
& $\begin{array}{l}\text { OPERATION END ENCOUNTERED } \\
\text { BUT INTERPOLATION } \\
\text { INCOMPLETE }\end{array}$ \\
LOAD & 2 & $\begin{array}{l}\text { REAL DATA ENCOUNTERED } \\
\text { IN INTEGER } \triangle \text { RRAY }\end{array}$ \\
LOAD & 2 & $\begin{array}{l}\text { REPEAT COUNT LESS THAN } \\
\text { ONE, COUNT RESET TO ONE }\end{array}$ \\
LOAD & 2 & $\begin{array}{l}\text { REPEAT LEVEL CARD } \\
\text { MISPLACED }\end{array}$
\end{tabular}

LOAD

LOAD

LOAD

LOCPMP

LOCTEE

LOCTRB

LOCVLV
2 UNDEFINED OPERATION REPEAT COUNT SET TO ONE

2 UNEXPECTED END-OF-FILE REACHED

2 ZERO OR FEWER INTERPOLATIONS-OOPERATION TREATED AS BLANK

1 VARIABLE NAME NOT RECOGNIZED

1 VARIABLE NAME NOT RECOGNIZED

1 VARIABLE NAME NOT RECOGNIZED

1 VARIABLE NAME NOT RE.COGNIZED

\section{Explanation}

When the array data were read, an end flag (E) was specified before both endpoints of an interval to be interpolated were read.

Real data were found in an integer array.

When the array data were read, a repeat count of less than one was found.

When the array data were read, a repeat-level card was found.

When the array data were read, an undefined load operation was specified.

When the array data were read, an unexpected end-offile was found.

When the array data were read, an interpolation count of less than one was specified.

A programming error occurred when the user tried to locate the position of a TURBINE variable in its common block.

A programming error occurred when the user tried to locate the position of a TURBINE variable in its common block.

A programming error occurred when the user tried to locate the position of a TURBINE variable in its common block.

A programming error occurred when the user tried to locate the position of a VALVE variable in its common block. 
Subroutine

LPCON

LPCON

LPCON

MAIN

MANAGE

MANAGE

MODCMP

MODCMP

MODIFY

MSTRCT

MSTRCT

NXTCMP

ORIENT

OUTID

\section{Leve1}

1

MISSING VESSEL JUNCTION

1 NO PUMP IN PRINCIPAL LOOP

1 NO STGEN IN PRINCIPAL LOOP

1 NO SPACE FOR VERSION INFORMATION

1 LEVEL PROBLEM

1 SCM MEMORY OVERFLOW

3 ERROR CHANGING COMPONENT

3 TYPE NOT RECOGNIZED

-4 HALTED FOR MANUAL RESTART

2 INCORRECT TABULAR MAT. I.D.

2 TEMPERATURE OUTSIDE TABLE RANGE

1 END-OF-FILE REACHED WHEN SEARCHING FOR NEXT COMPONENT

1 NO JUNCTION MATCH

1 COMPONENT TYPE NOT RECOGNIZED

\section{Explanation}

An Invalid vessel junction number was found in the vesse1 junction data area.

No pump was found in the loop description.

No stcam generator was found in the loop description.

Insufficient LCM is available for version information.

The requested core level or rod number does not exist.

Insufficient SCM is available.

An error occurred when a component was modifled. This will be preceded by a more specific error message ( $1 n-$ teractive mode only).

The component type specifled is not available in TRAC.

A component has been modified using the interactive mode.

A wall-material identifier could not be located.

Wall temperature is outside range of the tabular material data.

When the data for a new component were read, an end-offile was found.

A junction connected to a tee or steam generator in the JUN array was not found.

Invalid component type was encountered. 


\begin{tabular}{|c|c|c|}
\hline Subroutine & Level & Message \\
\hline OUT3D & 1 & $\begin{array}{l}\text { COMPONENT TYPE } \\
\text { NOT RECOGNIZED }\end{array}$ \\
\hline OUTER & 1 & FATAL ERROR \\
\hline POST & 1 & $\begin{array}{l}\text { COMPONENT TYPE NOT } \\
\text { RECOGNIZED }\end{array}$ \\
\hline POST3D & 1 & $\begin{array}{l}\text { COMPONENT TYPE NOT } \\
\text { RECOGNIZED }\end{array}$ \\
\hline POSTER & 1 & NO SCM SPACE FOR CYLHT \\
\hline PRCINT & 2 & ABORTED BY CONTROLLER \\
\hline & $"$ & \\
\hline PRCINT & 2 & INTERRUPTED--CONTINUED \\
\hline PRCINT & -4 & STOPPED BY REQUEST \\
\hline
\end{tabular}

PREINP

2-1 INPUT ERROR DETECTED IN TRACIN $\begin{array}{lll}\text { PREP3D } & 1 & \begin{array}{l}\text { COMPONENT TYPE NOT } \\ \text { RECOGNIZED }\end{array}\end{array}$

$\begin{array}{lll}\text { PUMPD } & 1 & \text { CANNOT LOCATE HEAD CURVE }\end{array}$

\section{Explanation}

Invalid component type was encountered.

A fatal error occurred.

Invalid component type was encountered.

Invalid component type was encountered.

Insufficient SCM is available.

The user aborted this run (interactive mode only). No cleanup is done.

The user continued the program after an interruption (interactive mode only).

The user stopped the run (interactive mode only). Run terminates normally.

The free-format input-option preprocessor routine found an input error. Possible causes include a missing positive character (for example, I.0.E. 07), the omission of the first (format-option switch) card, or a simple typographical error. An immediate fatal error occurs if the first card is incorrect. In all other cases, a flag is set that stops execution after the entire deck is processed.

Invalid component type was encountered.

Invalid component type was encountered.

The PUMP regime is outside the data base. 


\begin{tabular}{|c|c|c|c|}
\hline Subroutine & Leve1 & Message & Explanation \\
\hline PUMPD & 1 & CANNOT LOCATE TORQUE CURVE & $\begin{array}{l}\text { The PUMP regime is outside } \\
\text { the data base. }\end{array}$ \\
\hline PUMPSR & 1 & ERROR IN ROUTINE PUMPX & $\begin{array}{l}\text { An error was encountered when } \\
\text { a pump head or torque was } \\
\text { evaluated. }\end{array}$ \\
\hline PUMPSR & 1 & INSUFFICIENT SCM SPACE & $\begin{array}{l}\text { Insufficient SCM is available } \\
\text { for PUMP since calculations. }\end{array}$ \\
\hline PUMPSR & 1 & PUMP SPEED NOT FOUND & $\begin{array}{l}\text { The signal-variable or con- } \\
\text { trol-block ID number NPMPSD } \\
\text { that defines the initial pump } \\
\text { speed directly could not be } \\
\text { found in the signal-variable } \\
\text { or control-block list of ID } \\
\text { numbers. }\end{array}$ \\
\hline RIMACH & 1 & I OUT OF BOUNDS & $\begin{array}{l}\text { The number of machine con- } \\
\text { stants (required for the de- } \\
\text { termination "of eigenvalues) } \\
\text { should be at Jeast } 1 \text { but } \\
\text { should not exceed } 5 \text {. This } \\
\text { number is out of bounds. }\end{array}$ \\
\hline RACCUM & 2 & $\begin{array}{l}\text { VLT EXCEEDS ITS LIMIT } \\
\text { SEE TRCOUT }\end{array}$ & $\begin{array}{l}\text { Instructions were given in } \\
\text { the TRCOUT file to increase } \\
\text { LENDIM in BLKDAT and to } \\
\text { change dimension VLT in } \\
\text { GENVLT. }\end{array}$ \\
\hline RBREAK & 2 & $\begin{array}{l}\text { ERROR IN TABLE } \\
\text { SPECIFICATIONS }\end{array}$ & $\begin{array}{l}\text { Incompatible BREAK options } \\
\text { were selected. }\end{array}$ \\
\hline RBREAK & 2 & $\begin{array}{l}\text { IBTY INCONSISTENT WITH } \\
\text { ISOLUT }\end{array}$ & $\begin{array}{l}\text { A solute-concentration table } \\
\text { cannot be used at a BREAK } \\
\text { unless the solute-tracker } \\
\text { flag (ISOLUT) is set to one. }\end{array}$ \\
\hline RBREAK & 2 & LCM OVERFLOW & $\begin{array}{l}\text { Insufficient LCM is available } \\
\text { for this BREAK. }\end{array}$ \\
\hline RBREAK & 2 & $\begin{array}{l}\text { PAIN MUST NOT BE } \\
\text { GREATER THAN PIN }\end{array}$ & $\begin{array}{l}\text { The air partial pressure at } \\
\text { the BREAK may not exceed the } \\
\text { total pressure at the BREAK. }\end{array}$ \\
\hline RBREAK & 2 & SCM OVERFLOW & $\begin{array}{l}\text { Insufficient SCM is available } \\
\text { for this BREAK. }\end{array}$ \\
\hline
\end{tabular}




\begin{tabular}{|c|c|c|}
\hline Subroutine & Leve1 & Message \\
\hline RCOMP & 2 & ICFLG MUST BE .LE.5 \\
\hline RCOMP & 2 & $\begin{array}{l}\text { ICONC \& ISOLUT } \\
\text { ARE INCONSISTENT }\end{array}$ \\
\hline RCOMP & 2 & $\begin{array}{l}\text { INCONSISTENT } \\
\text { VALUES FOR ICFLG }\end{array}$ \\
\hline RCOMP & 2 & $\begin{array}{ll}\text { NEGATIVE } & \text { FRIC. GE. } \\
-1.0 E+20 & \text { NOT ALLOWED }\end{array}$ \\
\hline RCOMP & 2 & $\begin{array}{l}\text { NFF MUST BE } 0,1,2,-1, \\
-2, \text { OR }-100\end{array}$ \\
\hline RCOMP & 2 & $\begin{array}{l}\text { PA MUST NOT BE GREATER } \\
\text { THAN } P\end{array}$ \\
\hline$\Delta$ & & \\
\hline RCORE & 2 & $\begin{array}{l}\text { INCONSISTENT INIT } \\
\& \text { TABLE REACT-POWER }\end{array}$ \\
\hline
\end{tabular}

RCORE

RCORE

RCORE

RCORE
2 INCONSISTENT Z LENGTHS

2 LCM OVERFLOW

2 NZMAX . LT. NCRZ+1 +SUM NFAX(I)

4

2 SCM OVERFLOW

\section{Explanation}

Only five sets of multipliers are allowed in the chokedflow model.

Solute concentrations were entered before the ISOLUT option was selected.

A11 nonzero values for ICFLG for each component must be the same.

An additive friction-factor of less than $-10^{20}$ can be used to select the liquidseparator model. In all other cases, the additive friction factor must be positive.

The only NFF options in TRAC are $0,1,2,-1,-2$, or -100 .

The air partial pressure may not exceed the total pressure for a hydrodynamic cell.

The reactivity-power-table's interpolated value for the independent-variable value of 0.0 differs from the initial reactivity or power value.

The input values for the height of each core level are not increasing monotonically.

Insufficient LCM is available for this CORE.

The number of rows of rod conduction nodes is less than the minimum specified for reflood calculations.

Insufficient SCM is avallable for this CORE. 


\begin{tabular}{|c|c|c|c|}
\hline Subroutine & Level & Message & Explanation \\
\hline RCORE & 2 & $\begin{array}{l}\text { VLT EXCEEDS ITS LIMIT } \\
\text { SEE TRCOUT }\end{array}$ & $\begin{array}{l}\text { Instructions were given in } \\
\text { the TRCOUT file to increase } \\
\text { LENDIM in BLKDAT and to } \\
\text { change the dimension in } \\
\text { GENVLT. }\end{array}$ \\
\hline RCNTL & 2 & $\begin{array}{l}\text { \# OF SET PT. FAC. TABLES } \\
\text { GT. } 25 \text { DIM. }\end{array}$ & $\begin{array}{l}\text { The number of set-point fac- } \\
\text { tor tables is greater than } \\
\text { the local dimension of array } \\
\text { IFSP (25), which stores the } \\
\text { set-point factor-table ID } \\
\text { numbers. }\end{array}$ \\
\hline RCNTL & 2 & $\begin{array}{l}\text { \# OF T.S.E. OR T.C.T. } \\
\text { GT. } 25 \text { DIM. }\end{array}$ & $\begin{array}{l}\text { The number of trip-signal ex- } \\
\text { pressions or trip-controlled } \\
\text { trips is greater than the } \\
\text { local dimension of array ISEN } \\
\text { (25) or ITCN ( } 25) \text {. These } \\
\text { arrays are used to store the } \\
\text { signal ID numbers that are } \\
\text { compared to the signal ID } \\
\text { numbers in the input. }\end{array}$ \\
\hline RCNTL & 2 & $\begin{array}{l}\text { BAD CONTROL-BLOCK TABLE } \\
\text { STORAGE }\end{array}$ & $\begin{array}{l}\text { The total number of control- } \\
\text { block FNG1 table values ex- } \\
\text { ceeds the number of values } \\
\text { specified by NTCF (Main- } \\
\text { Control Card Number 5). }\end{array}$ \\
\hline RCNTL & 2 & $\begin{array}{l}\text { BAD SIG. EXP. } \\
\text { OPERATOR }\end{array}$ & $\begin{array}{l}\text { The arithmetic-operator ID } \\
\text { number for a subexpression } \\
\text { within the slgnal-expression } \\
\text { definition has an invalid } \\
\text { input value of less than one } \\
\text { or greater than eight. }\end{array}$ \\
\hline RCNTL & 2 & BAD TRIP ID DEFINITION & $\begin{array}{l}\text { A trip ID has an absolute } \\
\text { value that is } 0 \text { or greater } \\
\text { than } 9999 \text {. }\end{array}$ \\
\hline RCNTL & 2 & $\begin{array}{l}\text { INVALID TRIP SET STATUS } \\
\text { DEFINED }\end{array}$ & $\begin{array}{l}\text { The trip set-status variable } \\
\text { ISET has an invalid input } \\
\text { value. }\end{array}$ \\
\hline RCNTL & 2 & $\begin{array}{l}\text { SET POINT FACT. } \\
\text { TAB. \# PAIRS.GT.10 }\end{array}$ & $\begin{array}{l}\text { The set-point factor table } \\
\text { has more than } 10 \text { data pairs. }\end{array}$ \\
\hline
\end{tabular}




\begin{tabular}{|c|c|c|}
\hline Subroutine & Level & Message \\
\hline RCNTL & 2 & $\begin{array}{l}\text { SET PT.FAC.TABLE } \\
\text { DIM. TOO SMALL }\end{array}$ \\
\hline
\end{tabular}

RCNTL 2 TRIP-CONTROL TRIP

$\begin{array}{lll}\text { RCN'CL } & 2 \text { TRIPS DOING DUMPS }\end{array}$ DIM. TOO" SMALL

RCNTL

f) RCPVEC

RCPVEC

2 BAD LOOP NUMBER ASSUMED

$\begin{array}{lll}\text { RDCOMP } & 1 & \begin{array}{l}\text { COMPONENT TYPE NOT } \\ \text { RECOGNIZED }\end{array} \\ \text { RDDIM } & 2 & \begin{array}{l}\text { ILLEGAL PUMP CURVE } \\ \text { OPTION }\end{array}\end{array}$

RDREST

1 COMPONENT DATA NOT FOUND

RDREST
2 BAD LOOP COUNT GIVEN FOR CONTROL PANEL VECTOR

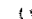

TRIP SIGNAL EXP. DIM. TOO SMALL
1 DUMP TIME NOT FOUND ON RESTART FILE

\section{Explanation}

The number of set-point factor-table parameters is less than the storage allocated for such parameters by variable NTSF.

The number of trip-controlled trif-signal parameters is less than the storage allocated for such parameters by variable NTCT.

The number of trip IDs that produce restart dumps is greater than the storage allocated for such parameters by variable NTDF.

The number of trip signalexpression parameters is greater than the storage allocated for such parameters by variable NTSE.

An incorrect number of reactor coolant loops was specified. The number must be 1 , 2,3 , or 4 .

The interactive-edit input specifies a loop that does not exist.

An invalid component type was specified.

An illegal PUMP option" was specified on PUMP Card Number 9 .

Data for a specific component were not found in the input or the restart file.

The restart dump at the time specified in the input file is not in the restart file. 


\begin{tabular}{|c|c|c|}
\hline Subroutine & Level & Message \\
\hline RDREST & 1 & FILE TRCRST DOES NOT EXIS? \\
\hline RDREST & 1 & $\begin{array}{l}\text { ISOLUT FROM TRCRST } \\
\text { AND TRACIN DIFFER }\end{array}$ \\
\hline RDREST & 1 & NO DUMPS ON FILE \\
\hline RDREST & 1 & $\begin{array}{l}\text { RESTART FILE INCOMPATIBLE } \\
\text { WITH THIS PROBLEM }\end{array}$ \\
\hline RDREST & 1 & RESTART: FILE OPEN ERROR \\
\hline RDREST & 1 & $\begin{array}{l}\text { TYPE NOT RECOGNIZED IN } \\
\text { RESTART }\end{array}$ \\
\hline REACCM & 2 & POINTER TABLE MISMATCH \\
\hline
\end{tabular}

READI 1 INPUT ERROR-ENCOUNTERED

READI

READI

READI

READI

\section{UNEXPECTED LOAD DATA}

1 INPUT ERROR--NEW

COMPONENT OR END WAS ENCOUNTERED UNEXPECTEDLY

1 INPUT ERROR--REAL DATA ENCOUNTERED IN INTEGER FIELD

2 INPUT ERROR--UNEXPECTED NAMELIST DATA ENCOUNTERED

2 REPEAT LEVEL CARD MISPLACED
Explanation

Component data were omitted from the input deck, and a restart dump file to initialize the missing components cannot be found.

The solute-tracking options in the input and the restartfile data differ. The ISOLUT parameter must be set either to ON or to OFF in both files.

Incomplete dumps are specified in the TRCRST file.

The restart file cannot be used with this TRAC version.

An I/O error occurred when the restart file was opened.

An invalid component type was specified.

The accumulator (ACCUM) pointer table does not match the restart-file data.

A load operation was found when integer data in I14 format were read.

Data for a new component were found before all of the data for the current component were read.

Real data were found when integer data in $I 14$ format were read.

When integer data in I14 format were read, NAMELIST data were found.

A repeat-level card was found when integer data in I14 format were read. 


\begin{tabular}{|c|c|c|c|}
\hline Subroutine & Leve1 & Message & Explanation \\
\hline READI & 1 & $\begin{array}{l}\text { UNEXPECTED END-OF-FILE } \\
\text { REACHED }\end{array}$ & $\begin{array}{l}\text { An end-of-file was found when } \\
\text { integer data in Il } 4 \text { format } \\
\text { were read. }\end{array}$ \\
\hline READR & 1 & $\begin{array}{l}\text { INPUT ERROR--NEW COMPONENT } \\
\text { WAS ENCOUNTERED UNEXPECTEDLY }\end{array}$ & $\begin{array}{l}\text { Data for a new component were } \\
\text { found before all of the data } \\
\text { for the current component } \\
\text { were read. }\end{array}$ \\
\hline READR & 1 & $\begin{array}{l}\text { INPUT ERROR--UNEXPECTED } \\
\text { LOAD DATA ENCOUNTERED }\end{array}$ & $\begin{array}{l}\text { A load operation was found } \\
\text { when reading nonarray real } \\
\text { data in E14.6 format. }\end{array}$ \\
\hline READR & 2 & $\begin{array}{l}\text { INPUT ERROR-OUNEXPECTED } \\
\text { NAMELIST DATA ENCOUNTERED }\end{array}$ & $\begin{array}{l}\text { When reading real data in } \\
\text { E } 14.6 \text { format, NAMELIST data } \\
\text { were found. }\end{array}$ \\
\hline READR & 2 & REPEAT LEVEL CARD MISPLACED & $\begin{array}{l}\text { A REPEAT LEVEL card was found } \\
\text { when reading real data in } \\
\text { E14.6 format. }\end{array}$ \\
\hline READR & 1 & $\begin{array}{l}\text { UNEXPECTED END-OF-FILE } \\
\text { REACHED }\end{array}$ & $\begin{array}{l}\text { An end-of-file was found when } \\
\text { reading real data in E } 14.6 \\
\text { format. }\end{array}$ \\
\hline REBRK & 1 & $\begin{array}{l}\text { ERROR IN TABLE } \\
\text { SPECIFICATIONS }\end{array}$ & $\begin{array}{l}\text { An error stopped the pro- } \\
\text { cessing of input data. }\end{array}$ \\
\hline REBRK & 1 & FATAL ERROR & $\begin{array}{l}\text { An error stopped the pro- } \\
\text { cessing of the input data. }\end{array}$ \\
\hline REBRK & 2 & LCM OVERFLOW & $\begin{array}{l}\text { Insufficient LCM is available } \\
\text { for the BREAK data from the } \\
\text { restart file. }\end{array}$ \\
\hline REBRK & 2 & POINTER TABLE MISMATCH & $\begin{array}{l}\text { The BREAK pointer table does } \\
\text { not match the restart-file } \\
\text { data. }\end{array}$ \\
\hline REBRK & 2 & SCM OVERFLOW & $\begin{array}{l}\text { Insufficient SCM is available } \\
\text { for the BREAK data from the } \\
\text { restart file. }\end{array}$ \\
\hline RECNTL & 1 & $\begin{array}{l}\text { CONTROL BLOCKS EXCEED } \\
\text { DIMENSION }\end{array}$ & $\begin{array}{l}\text { The amount of control-block } \\
\text { data in the input and the re- } \\
\text { start files exceeds its stor- } \\
\text { age allocation on Main- } \\
\text { Control Card 5. }\end{array}$ \\
\hline
\end{tabular}


$\begin{array}{lcl}\text { Subroutine } & \text { Leve1 } & \frac{\text { Message }}{\text { RECNTL }} \\ 1 & \begin{array}{l}\text { CONTROL PARA. STORAGE } \\ \text { TOO SMALL }\end{array}\end{array}$

RECNTL 1 NUMBER TRIPS

EXCEED DIMENSION

RECNTL 1 SET-PT-FACTOR TABLES

EXCEED DIM

RECNTL 1 SIG. VARIABLES

EXCEED DIMENSION

$7^{6}$

$\begin{array}{lll}\text { RECNTL } & 1^{/ /} \text {TIME STEP DATA } \\ & \text { EXCEED DIMENSION }\end{array}$

RECNTL 1 TOO MANY DMP TRIPS FROM RESTART

RECNTL

1

TOO MANY SETPOINT-FACTOR TABLES

RECNTL
1 TOO MANY SP. TIME STEP DATA SETS
Explanation

The variable storage that was allocated by the input data for the signal variables, control blocks, and trips is too small to contain the remaining data from the restart file.

The number of trips with different ID numbers from the input and the restart files exceeds the input datastorage dimension.

The number of different setpoint factor-table ID numbers in the input and the restart files exceeds the input datastorage dimension.

The number of signal variables with different ID numbers in the input and the restart files exceeds the input data-storage dimension.

The number of trip-controlled time-step data sets with different ID numbers in the input and the restart files exceeds the input datastorage dimension.

The number of trip ID numbers in the input and the restart files exceeds the input datastorage dimension. These trip ID numbers when set to ON generate restart dumps.

The number of set-point factor tables in the restart file exceeds the input datastorage dimension.

The number of trip-controlled time-step data sets in the restart file exceeds the input data-storage dimension. 


\begin{tabular}{lcl} 
Subroutine & Leve1 & \multicolumn{1}{c}{ Message } \\
RECNTL & 1 & $\begin{array}{l}\text { TOO MANY TRIPS } \\
\text { GENERATING DUMPS }\end{array}$ \\
RECNTL & 1 & $\begin{array}{l}\text { TOO MANY TRIP-SIG. } \\
\text { EXPRESSIONS }\end{array}$
\end{tabular}

RECNTL 1 . TRIP-SIGNAL EXPS . EXCEED DIMEN. $\begin{array}{ll}\text { RECNTL } 11 & \text { TRP-CONT-TRP SIGNALS } \\ \text { EXCEED DIM. }\end{array}$

RECORE

RECORE

$\begin{array}{lcc}\text { RECORE } & 2 & \text { SCM OVERFLOW } \\ \text { REFILL } & 1 & \text { FATAL ERROR } \\ \text { REFILL } & 2 & \text { LCM OVERFLOW }\end{array}$

REFILL

2 POINTER TABLE MISMATCH

REFILL
2 POINTER TABLE MISMATCH

2 LCM OVERFLOW
Explanation

The number of trip ID numbers in the restart file exceeds the input data-storage dimension. These trip ID numbers when set to ON generate restart dumps.

The number of signal expressions in the restart file exceeds the input datastorage dimension.

The number of signal-expression ID numbers in the input and the restart files exceeds the input data-storage dimension.

The number of trip-controlled trip ID numbers with different ID numbers in the input and the restart files exceeds the input data-storage dimension.

Insufficient LCM is available for this CORE.

The CORE pointer table does not match the restart-file data.

Insufficient SCM is available for this CORE.

An error stopped the processing of the input data.

Insufficient LCM is available for the FILL data from the restart file.

The FILL pointer table does not match the restart-file data.

Insufficient SCM is available for this FILL. 


\begin{tabular}{|c|c|c|c|}
\hline Subroutine & Leve 1 & Message & Explanation \\
\hline REPIPE & 2 & POINTER TABLE MISMATCH & $\begin{array}{l}\text { The PIPE pointer table does } \\
\text { not match the restart-file } \\
\text { data. }\end{array}$ \\
\hline REPRZR & 2 & POINTER TABLE MISMATCH & $\begin{array}{l}\text { The pressurizer (PRIZER) } \\
\text { pointer table does not match } \\
\text { the restart-file data. }\end{array}$ \\
\hline REPUMP & 2 & POINTER TABLE MISMATCH & $\begin{array}{l}\text { The PUMP pointer table does } \\
\text { not match the restart-file } \\
\text { data. }\end{array}$ \\
\hline REPUMP & 1 & SCM OVERFLOW & $\begin{array}{l}\text { Insufficient SCM is available } \\
\text { for the PUMP data from the } \\
\text { restart file. }\end{array}$ \\
\hline RETEE & 2 & POINTER TABLE MISMATCH & $\begin{array}{l}\text { The TEE pointer table does } \\
\text { not match the restart-file } \\
\text { data. }\end{array}$ \\
\hline RETURB & 2 & POINTER TABLE MISMATCH & $\begin{array}{l}\text { The restart pointers do not } \\
\text { match the original pointers. } \\
\text { Probably a newer code version } \\
\text { with updated pointers was } \\
\text { used for the restart, whereas } \\
\text { an older version was used for } \\
\text { the previous run. }\end{array}$ \\
\hline REVLVE & 2 & POINTER TABLE MISMATCH & $\begin{array}{l}\text { The VALVE pointer table does } \\
\text { not match the restart-file } \\
\text { data. }\end{array}$ \\
\hline REVSSL & 2 & LCM OVERFLOW & $\begin{array}{l}\text { Insufficient LCM is available } \\
\text { for the VESSEL data ferom the } \\
\text { restart file. }\end{array}$ \\
\hline REVSSL & 2 & POINTER TABLE MISMATCH & $\begin{array}{l}\text { The VESSEL pointer table does } \\
\text { not match the restart-file } \\
\text { data. }\end{array}$ \\
\hline REVSSL & 2 & SCM OVERFLOW & $\begin{array}{l}\text { Insufficient SCM is available } \\
\text { for the VESSEL data from the } \\
\text { restart file. }\end{array}$ \\
\hline RFILL & 2 & BAD FILL TYPE OPTION & $\begin{array}{l}\text { An illegal FILL option was } \\
\text { specified on FILL Card Num- } \\
\text { ber } 2 \text {. }\end{array}$ \\
\hline RFILL & 2 & BAD TRIP ID DEFINITION & $\begin{array}{l}\text { An incorrect trip ID of }-1 \text {, } \\
<-9999 \text {, or }>9999 \text { was speci } \\
\text { fied. }\end{array}$ \\
\hline
\end{tabular}




\begin{tabular}{|c|c|c|}
\hline Subroutine & Level & Message \\
\hline RFILL & 2 & $\begin{array}{l}\text { IFSV }=0 \\
\text { WHEN IFTY.GT.3 }\end{array}$ \\
\hline RFILL & 2 & $\begin{array}{l}\text { INCONSISTENT INIT AND } \\
\text { TABLE FLOW }\end{array}$ \\
\hline$"$ & & \\
\hline RFILL & 2 & LCM OVERFLOW \\
\hline RFILL & 2 & $\begin{array}{l}\text { PAIN MUST NOT BE } \\
\text { GREATER THAN PIN }\end{array}$ \\
\hline RFILL & 2 & SCM OVERFLOW !! \\
\hline RFILL & 2 & $\begin{array}{l}\text { VLT EXCEEDS ITS LIMIT } \\
\text { SEE TRCOUT }\end{array}$ \\
\hline
\end{tabular}

RPIPE 2 INCONSISTENT INIT \& TABLE POWER

RPIPE

RPIPE

$\mathrm{RPPH}$
1 CONVERGENCE FAILURE EVALUATING PUMP SPEED"

\section{Explanation}

A FILL table, based on IFTY $>4$, cannot be defined because no table independentvariable ID number was specified.

The initial values for the FILL table and for the FILL initial state are not equal.

Insufficient LCM is available for the FILL data from the input file.

The air partial pressure is greater than the total pressure in a FILL.

Insufficient SCM is available for the FILL data from the input file.

Instructions were given in the TRCOUT file to increase LENDIM in BLKDAT and to change the dimension in GENVLT.

The initial values for the PIPE power-to-fluid table POWTB and for the PIPE powerto-fluid variable POWIN are not equal.

The initial values for the PIPE power-to-wall table QP3TB and for the PIPE powerto"wall variable QP3IN are not equal.

Instructions were given in the TRCOUT file to increase LENDIM in BLKDAT and to change the dimension in GENVLT.

The PUMP speed to achieve the needed head could not be calculated. 


\section{Subroutine Leve1}

RPRIZR

2 VLT EXCEEDS ITS LIMIT SEE TRCOUT

RPUNP

2 BAD TRIP ID DEFINITION

RPUMP

2 FRIC(2) .NE. 0 .

RPUMP

RPUMP ，

RPUMP

RPUMP

RPUMP

RPUMP

RPUMP

RPUMP
2 NCELLS.LT. 2

2 NPMPSV.NE.O

2 INCONSISTENT INIT

\& TABLE QPPPF

2 INCONSISTENT INIT

\& TABLE SPEED

2 IPMPSV.NE.O

$2 \quad$ NPMPRF . NE. 0

2 NPMPTB.NE. 0

2 PUMP TYPE NOT RECOGNIZED
Explanation

Instructions were given in the TRCOUT file to increase LENDIM in BLKDAT and to change the dimension in GENVLT.

An incorrect trip ID of -1 , $<-9999$, or $>9999$ was specified.

The value for FRIC(2) must be 0.0 in the PUMP.

The initial values for the power-to-wall table QP3TB and for the power-to-wall variable QP3IN are not equal.

The initial values for the PUMP-speed table and for the PUMP speed are not equal.

The independent-variable ID number for the PUMP-speed table should not be defined for PUMP-type 2 .

An incorrect number of PIPE fluid cells was specified. The PIPE must have at least two fluid cells.

The number of the rate-factor table's entry pairs should be zero for PUMP-type 2 .

The independent-variable ID number for the rate-factor table assigned to the PUMPspeed table should not be defined for PUMP-type 2 .

The number of the PUMP-speed table's entry pairs should be zero, for PUMP-type 2.

An incorrect PUMP type was specified. 


\begin{tabular}{|c|c|c|c|}
\hline Subroutine & Level & Message & Explanation \\
\hline RPUMP & 2 & QPPP-F TABLE PARAM BAD & $\begin{array}{l}\text { The power-to-wall table QP } 3 \mathrm{~TB} \\
\text { is defined but its indepen- } \\
\text { dent-variable ID number is } \\
\text { zero. }\end{array}$ \\
\hline RPUMP & 2 & SPEED TABLE PARAM. BAD & $\begin{array}{l}\text { The signal-variable ID number } \\
\text { for lhe rolational-speed- } \\
\text { table's independent variable } \\
\text { for PUMP-type } 1 \text { is invalid. } \\
\text { The PUMP-type variable IPMPTY } \\
\text { must be either } 1 \text { or } 2 \text {. }\end{array}$ \\
\hline RPUMP & 2 & $\begin{array}{l}\text { VLT EXCEEDS ITS LIMIT } \\
\text { SEE TRCOUT }\end{array}$ & $\begin{array}{l}\text { Instructions were given in } \\
\text { the TRCOUT file to increase } \\
\text { LENDIM in BLKDAT and to } \\
\text { change the dimension in } \\
\text { GENVLT. }\end{array}$ \\
\hline RSTGEN & 2 & GEN.H.T. IDI .EQ. IDO & $\begin{array}{l}\text { The same fluld volume was } \\
\text { specifled for both sides of } \\
\text { the same heat-transfer sur- } \\
\text { face in the steam generator. }\end{array}$ \\
\hline RSTGEN & 2 & H.T. I.D.ERROR & $\begin{array}{l}\text { The component number (ICMP or } \\
\text { OCMP) for a heat-transfer } \\
\text { surface in the steam gener- } \\
\text { ator is incorrect. }\end{array}$ \\
\hline RSTGEN & 2 & JUNS2 ERROR & $\begin{array}{l}\text { The value of JUNS2 is } \\
\text { incorrect; if NSCMP }=1 \text { or } \\
\text { JUNS1 =0, then JUNS2 }=0 \text {. }\end{array}$ \\
\hline RSTGEN & 2 & NODES . NE . NDHT & $\begin{array}{l}\text { The number of nodes in the } \\
\text { steam-generator tubes and in } \\
\text { the additional heat-transfer } \\
\text { surfaces must be equal. }\end{array}$ \\
\hline RSTGEN & 2 & NO. EXT $\cdot$ JUN. $\cdot$ LT $\cdot 2$ & $\begin{array}{l}\text { The number of external junc- } \\
\text { tions on the steam-generator } \\
\text { secondary side is incorrect. } \\
\text { The number must be two or } \\
\text { more. }\end{array}$ \\
\hline RSTGEN & 2 & NSCMP ERROR & $\begin{array}{l}\text { The number of secondary } \\
\text { internal components is } \\
\text { incorrect; the limits are } \\
1<\text { NSCMP }<10 .\end{array}$ \\
\hline RSTGEN & 2 & TYPE ERROR & $\begin{array}{l}\text { The secondary component is } \\
\text { not a PIPE or TEE. }\end{array}$ \\
\hline
\end{tabular}




\begin{tabular}{|c|c|c|}
\hline Subroutine & Level & Message \\
\hline RSTGEN & 2 & $\begin{array}{l}\text { VLT EXCEEDS ITS LI } \\
\text { SEE TRCOUT }\end{array}$ \\
\hline RSTGEN & 2 & WALL AREAS INCONSI \\
\hline RTEE & 2 & $\begin{array}{l}\text { INCONSISTENT INIT } \\
\& \text { TABLE POWERI }\end{array}$ \\
\hline
\end{tabular}

RTEE 2 INCONSISTENT INIT \& TABLE POWER2

RTEE

RTEE

RTURB

RTURB
2 INCONSISTENT INIT \& TABLE QPPPFI

2 INCONSISTENT INIT \& TABLE QPPPF2

2 \#STAGES.LT.1

2 INCONSISTENT INIT AND TABLE GEN POWER
Explanation

Instructions were given in the TRCOUT file to increase LENDIM in BLKDAT and to change the dimension in GENVLT.

The internal and external wall areas for a generalized heat transfer are inconsistent with cylindrical geometry; thus, the energy will be calculated incorrectly in the wall-conduction calculation.

The TEE primary-side initial values for the power-to-fluid table POWTB and for the power-to-fluid variable PWIN1 are not equal.

The TEE secondary-side initial values for the power-tofluid table POWTB and for the power-to-fluid variable PWIN2 are not equal.

The TEE primary-side initial values for the power-to-wall table QP3TB and for the power-to-wall variable QPINl are not equal.

The TEE secondary-side initial values for the power-towall table QP3TB and for the power-to-wall variable QPIN2 are not equal.

A turbine (TURB) must have at least one stage.

The turbine power table's initial power and the initial operating power POWOP have different values. 


\begin{tabular}{|c|c|c|c|}
\hline Subroutine & Leve1 & Message & Explanation \\
\hline RTURB & 2 & NCELLS .LT. 3 & $\begin{array}{l}\text { The number of cells in the } \\
\text { turbine component (TURB) is } \\
\text { incorrect; this component }\end{array}$ \\
\hline & & & $\begin{array}{l}\text { must have at least three } \\
\text { cells. }\end{array}$ \\
\hline RTURB & 2 & NROWS . GT . 2 & $\begin{array}{l}\text { A maximum of two rows of } \\
\text { moving blades is allowed in a } \\
\text { turbine stage. }\end{array}$ \\
\hline RTURB & 2 & POWER TABLE PARAM. BAD & $\begin{array}{l}\text { Direct power into the coolant } \\
\text { table is defined, but its } \\
\text { independent-variable ID num- } \\
\text { ber is zero. }\end{array}$ \\
\hline RTURB & 2 & $\begin{array}{l}\text { VLT EXCEEDS ITS LIMIT } \\
\text { SEE TRCOUT }\end{array}$ & $\begin{array}{l}\text { Instructions were given in } \\
\text { the TRCOUT file to increase } \\
\text { LENDIM in BLKDAT and to } \\
\text { change the dimension in } \\
\text { GENVLT. }\end{array}$ \\
\hline RVLVE & 2 & BAD FIRST VALVE TABLE & $\begin{array}{l}\text { A second VALVE table is de- } \\
\text { fined, but a first. VALVE } \\
\text { table is not defined. }\end{array}$ \\
\hline RVLVE & 2 & BAD QPPPF TABLE SIGNAL & $\begin{array}{l}\text { The QPPP-factor table is de- } \\
\text { fined, but its independent- } \\
\text { variable ID is zero. }\end{array}$ \\
\hline RVLVE & 2 & $\begin{array}{l}\text { BAD OVERRIDING TRIP } \\
\text { VALVE ADJUST RATE }\end{array}$ & $\begin{array}{l}\text { The overriding trip's VALVE } \\
\text { adjustment rate is negative. }\end{array}$ \\
\hline RVLVE & 2 & $\begin{array}{l}\text { BAD OVERRIDING TRIP } \\
\text { VALVE RANGE }\end{array}$ & $\begin{array}{l}\text { The overriding trip's minimum } \\
\text { and maximum VALVE closure } \\
\text { states do not lie between } 0 \\
\text { and } 1 \text {. }\end{array}$ \\
\hline RVLVE & 2 & BAD OV TRIP VALVE TYPE & $\begin{array}{l}\text { An overriding trip is de- } \\
\text { fined, but its VALVE type } \\
\text { IVTYOV is not } 0 \text { or } 1 .\end{array}$ \\
\hline RVLVE & 2 & BAD TRIP ID DEFINITION & $\begin{array}{l}\text { The trip ID number is greater } \\
\text { than the maximum allowed } \\
\text { value of } 9999 \text {. }\end{array}$ \\
\hline RVLVE & 2 & BAD VALVE TABLE DEFINE & $\begin{array}{l}\text { The number of pair entries in } \\
\text { the first VALVE table is in- } \\
\text { consistent with the VALVE op- } \\
\text { tion IVTY value. }\end{array}$ \\
\hline
\end{tabular}




$\begin{array}{lcc}\text { Subroutine } & \text { Leve1 } & \frac{\text { Message }}{\text { RVLVE }} \\ \text { RVLVE } & 2 & \begin{array}{c}\text { BAD VALVE TABLE } \\ \text { MAX ADJUST RATE }\end{array} \\ & 2 & \text { BAD VALVE TABLE SIGNAL }\end{array}$

RVLVE

2 BAD VALVE TABLE TRIP

RVLVE

2 BAD VALVE TYPE OPTION

RVLVE

2 FAVLVE \& XPOS INVALID

$\begin{array}{ll}\text { RVLVE } & 2 \text { INCONSISTENT INIT } \\ & \& \text { FIRST TABLE }\end{array}$

RVLVE

RVLVE

RVLVE

RVSSL
2 INCONSISTENT INIT \& SECOND TABLE

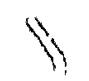

2 INCONSISTENT INIT

\& TABLE QPPPF

2 VLT EXCEEDS ITS LIMIT SEE TRCOUT

2 BAD TRIP ID DEFINITION

\section{Explanation}

The VALVE's maximum adjustment rate is negative.

The signal variable ID number defining the VALVE table's independent variable is inconsistent with the VALVE option IVTY value.

A nonzero trip ID number is
input when the VALVE type op-
tion indicates no trip con-
trol, or a zero trip ID num-
ber is input when the VALVE
is to be trip controlled.

The VALVE option parameter IVTY has an input value outside the 0 to 6 defined range.

The input values of FAVLVE and XPOS are both outside their 0 to 1 physical range.

The first VALVE table's initial value and the initial VALVE closure state (FAVLVE or XPOS) have different values.

The second VALVE table's initial value and the initial VALVE closure state (FAVLVE or XPOS) have different values.

The QPPP-factor table's initial value and the initial QPPP factor QFPIN have different values.

in

In the TRCOUT file instructions are given to increase, LENDIM in BLKDAT and change the dimension in GENVIT.

A trip ID number was either input equal to -1 , less than -9999, or greater than 9999. 


\begin{tabular}{|c|c|c|}
\hline Subroutine & Level & Message \\
\hline RVSSL & 2 & CORE ENTIRELY BLOCKED \\
\hline RVSSL & 2 & $\begin{array}{l}\text { ICONC \& ISOLUT ARE } \\
\text { INCONSISTENT }\end{array}$ \\
\hline RVSSL & 2 & $\begin{array}{l}\text { ILLEGAL REPEAT } \\
\text { LEVEL NUMBER USED }\end{array}$ \\
\hline RVSSL & 2 & ILLEGAL VALUE FOR IVSSBF \\
\hline RVSSL & 2 & $\begin{array}{l}\text { INCONSISTENT CORE } \\
\text { DEFINING DATA }\end{array}$ \\
\hline
\end{tabular}

RVSSL f

RVSSL

RVSSL

RVSSL

RVSSL
2 INCONSISTENT INIT \& TABLE REACT-POWER

2 INCONSISTENT LEVEL ELEVATIONS

2 INCONSISTENT

REACT-POWER TABLE
Explanation

The core flow area in the axial direction is zero at a VSSL level causing axial flow blockage through the core.

The input values for ICONC and ISOLUT are not consistent with each other.

An illegal level number was read from a REPEAT LEVEL card.

The only legal values for IVSSBF are $0,2,20$, and 22 .

Indicates either NASX < IDCU or NRSX < IDCR or IDCL > IDCU when a downcomer region is present (IDCU $\neq 0$, IDCL $\neq 0$, IDCR $\neq 0$ ).

The core region model parameters ICRU, ICRL, ICRR, NODES, NCRX, and NCRZ are defined inconsistent ly.

The reactivity-power table at its relative abscissa coordinate value of 0.0 and the initial reactivity or power have different values.

Indicates input values for which $\mathrm{Z}(\mathrm{I}+1)<\mathrm{Z}(\mathrm{I})$. a)

The IRPWTY reactor-power-type option parameters and the IRPWSV defined presence or absence of a reactor-power table are inconsistent.

The IRPWTY reactor-power type option parameter and the IRPWTR defined trip control option are inconsistent.

Indicates input values for which $R(I+1)<R(I)$. 


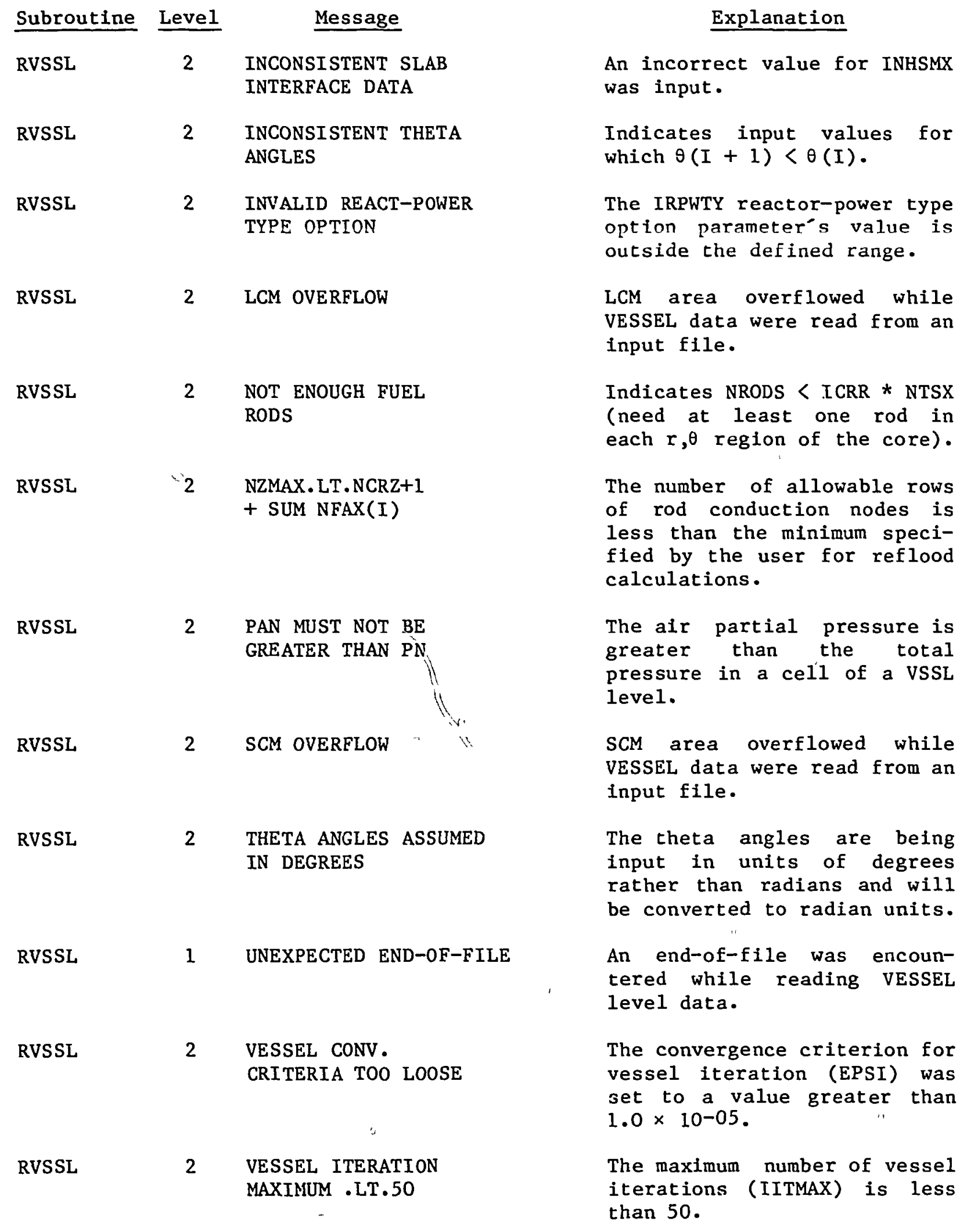


Subroutine Leve1

RVSSL

2 VLT EXCEEDS ITS LIMIT SEE TRCOUT

SCMLCM

2 LCM OVERFLOW

SCMLCM

2 SCM OVERFLOW

SETCMP

SETCMP

$\vdots$

SETCPV

SGEEV

SGEEV
2 CONTAINMENT MODULE
NOT IMPLEMENTED

2 TURBINE MODULE

NOT IMPLEMENTED

1 SPECIFICATION ERRORS

FORCE TERMINATION

1 JOB.NE. $0,{ }^{\prime A N D ~ N} \cdot$.GT . LDV
Explanation

In the TRCOUT file, instructions are given to increase LENDIM in BLKDAT and change the dimension in GENVLT.

Insufficient LCM is available for storing component data."

Insufficient SCM is available for reading in component array data.

A control panel vector input card has specified a containment component.

This version of the interactive facility does allow use of the turbine component in control panel vector input.

An error has been discerned while central panel vector input cards were processed. A flag is set so TRAC will halt after all input cards have been processed.

The user has requested eigenvector calculation in addition to the eigenvalues. However, the leading dimension of the array $V$, where eigenvectors are stored, is less than the order of the input matrix $A$.

The leading dimension of array $V$, where eigenvectors are stored, should be equal to the order of the input matrix A. If this is not the case, the elements of $A$ are rearranged.
OF A OTHER THAN N

BY $N$ INPUT ELEMENTS

HAVE BEEN CHANGED 


\begin{tabular}{|c|c|c|}
\hline Subroutine & Level & Message \\
\hline SGEEV & 2 & $\begin{array}{l}\text { LDA. LT . LDV, ELEMENTS } \\
\text { OF V OTHER THAN THE } \\
\text { THE N BY N OUTPUT ELEMENT } \\
\text { HAVE BEEN CHANGED }\end{array}$ \\
\hline SGEEV & 1 & $\mathrm{~N} \cdot \mathrm{GT} \cdot \mathrm{LDA}$ \\
\hline SGEEV & 1 & N .LT. 1 \\
\hline SOUND & 2 & $\begin{array}{l}\text { CANNOT CONVERGE ON PAIR } \\
\text { AT THE CELL EDGE }\end{array}$ \\
\hline & & $\therefore$ \\
\hline SOUND & 2 & $\begin{array}{l}\text { CANNOT FIND POSITIVE } \\
\text { FLOW PRESSURE }\end{array}$ \\
\hline
\end{tabular}

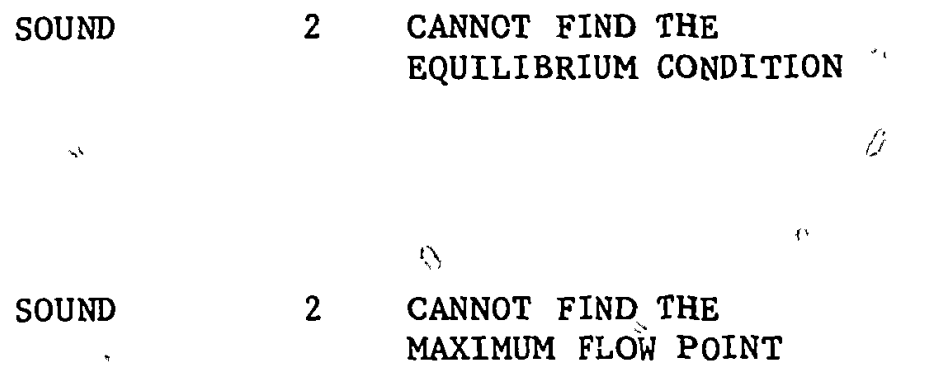

SOUND 2 SOUND SPEED SOLUTION DID NOT CONVERGE

\section{Explanation}

The leading dimension of array $\mathrm{V}$, where eigenvectors are stored, should be equal to the order of the input matrix A. If this is not the case, the elements of $A$ are rearranged.

The order of the input matrix $A$ exceeds the leading dimension of $A$.

The order of the input matrix $A$ is less than 1.

Cannot converge on the noncondensable gas partial pressure while the conditions at the cell edge are estimated from the cell-center values.

When the flow is maximized by iteration of the cell-edge pressure, no physically realistic pressure value gives positive flow. This should never happen except under some extreme nónequilibrium conditions.

Calculating the thermodynamic equilibrium condition in the presence of a noncondensable requires an iterative type solution, which did not converge.

When the flow is maximized by iteration of the cell-edge pressure, the decrease in pressure (down to physically realistic values) always keeps increasing the flow. Thus, the choking condition is never determined.

The iterative solution, for determining; the choking point by iterating on the cell-edge pressure while maximizing the mass flux, did not converge. 


$\begin{array}{lcl}\begin{array}{l}\text { Subroutine } \\ \text { SRCHCL }\end{array} & 2 & \begin{array}{l}\text { Level } \\ \text { LIST IN COMPONENT }\end{array} \\ \text { SRTLP } & 1 & \text { SRTLP FAILURE } \\ & 1 & \text { STEADY STATE NOT CONVERGED } \\ \text { STEADY } & 1 & \begin{array}{l}\text { INSUFFICIENT SPACE FOR } \\ \text { CYLHT }\end{array} \\ \text { STGN3X } & 1 & \text { TRIP ID NOT FOUND } \\ \text { SVSET } & 1 & \end{array}$

SVSET3 1 NEED LOCAL DIM.GT.50

$\begin{array}{lll}\text { TEMPL } & 1 & \text { TOO MANY ITERATIONS }\end{array}$

$\begin{array}{lll}\text { THERMO } & 2 & \text { LIQUID TEMP } \\ & \text { LIMIT EXCEEDED }\end{array}$

THERMO

THERMO
(T)

2 PRESSURE LIMIT EXCEEDED

2 VAPOR TEMP LIMIT EXCEEDED

\section{Explanation}

A component number specified on a central panel vector input card is not in the component list (interactive only).

SRTLP failed because of errors in component input data related to junction numbers. See file TRCOUT for additional clues.

The problem did not reach a steady state within the specifled time domains.

Insufficient SCM is avai1able.

The trip ID number assigned to a signal variable defining the trip-signal or set-status value could not be found in the list of trip ID numbers.

There are more than 50 local values in subroutine SVSET3 that need to be saved for the VESSEL component while two or more axial levels are searched for signal-variable evaluation.

The temperature cannot be evaluated from values of the enthalpy and pressure.

The liquid temperature in some cell has fallen below $280 \mathrm{~K}$ or has risen above $647 \mathrm{~K}$.

The pressure in some cell has fallen below $1.0 \times 10^{3}$ or risen above $1.9 \times 10^{7}$.

The vapor temperature in some cell has fallen below $280 \mathrm{~K}$. 


\begin{tabular}{lcl} 
Subroutine & Level & \multicolumn{1}{c}{ Message } \\
TIMCHK & 2 & $\begin{array}{l}\text { TERMINATING DUE TO TIME } \\
\text { LIMIT }\end{array}$ \\
TIMSTP & 4 & $\begin{array}{l}\text { CANNOT REDUCE TIME STEP } \\
\text { EURTHER }\end{array}$
\end{tabular}

TKIP

TRPSET

TRPSET

TRPSET

1 EXP. SIGNAI NOT FOUND

TRPSET

TRPSET

TRPSET

1 CNTL SIGNAL NOT FOUND

$\begin{array}{lll}\text { TRPSET } & 1 & \begin{array}{l}\text { SET-P-FACTOK TABLE ID } \\ \text { NOT FOUND }\end{array} \\ \text { TRPSET } & 1 & \text { SIGNAL EXP. NOT FOUND } \\ \text { TRPSET } & 1 & \text { S-P-FAC TABLE CON BLK } \\ \text { NOT FOUND }\end{array}$


Subroutine

TRPSET

TRPST

TRPSE $i$

TRPSET

TRPET

TRPSET

VLVEX

VSSL 1

\section{Explanation}

The signal-variable ID number that defines the set-pointfactor table's independent variable was not found in the list of signal-variable ID numbers.

There are too many delay time pending set-status changes for a trip.

The trip-controlled trip signal ID number that defines the trip-controlled trip signal could not be found in the list of trip-controlled tripsignal ID numbers.

A signal-variable ID number that defines the trip-signal / could not be found in the list of signal-vartable ID numbers.

The turbine has been overloaded so much that with the governing valve fully open, it still cannot meet the power demand. This situation could sometimes temporarily occur under high1y transient conditions when the steam flow through the turbine has not caught up with the power demand.

The reactor "core"s total power is turned on to its input initial value. 


\section{APPENDIX G}

TRAC SUBPROGRAMS

Name

ACCM1X

ACCMBD

ACCUM1

ACCUM2

ACCUM3

ALLBLK

AS IGN

ASTPLN

AUXPLN

BACIT

BALANC

BALBAK

BDP L,EN

BFALOC

BFCLOS

BFIN

BFOUT

BITS

BKMOM

BKSMOM
Function

Fvaluates accumulator (ACCUM) water level.

Sets boundary array for the accumulator (ACCUM) component.

Controls accumulator (ACCUM) prepass.

Controls accumulator (ACCUM) outer iteration.

Controls accumulator (ACCUM) postpass.

Tests for all blanks in specified substring of string.

Assigns the component LCM pointers according to the iteration number.

Calculates mass and energy fluxes at the PLENUM junctions during postpass.

Calculates mass and energy fluxes at the PLENUM junctions during the outer iteration.

Initiates backwărd substitution after direct vessel matrix inversion.

Support subroutine for SGEEV.

Support subroutine for SGEEV.

Fills the PLENUM boundary array.

Allocates files and buffers "for buffered I/O.

Empties buffers and closes file.

Initiates binary input subroutine.

Initiates binary output subroutine.

Manages bitt address flags.

Initiates backward substitution for stabilizing momentum equations.

Backward substitution for stabilizing momentum equations. 
Name

BKSST B

BLKDATA

BREAKL

BREAK2

BREAK3

BREAKX

BREBAL

CBSET

CDTHEX

CELLAV

CHBD

CHBSAV

CHBSET

CHEN

$\mathrm{CHF}$

CHF 1

CHKBD

CHKSR

CHOKE

Initiates backward substitution for stabilizing mass and energy equations.

Initializes common variables in a block data statement.

Controls BREAK prepass.

Controls BREAK outer iteration.

Controls BREAK postpass.

Evaluates BREAK pressure, temperature, and void fraction.

Initiates backward substitution after coarse-mesh rebalance in the VESSEL.

Controls the evaluation of control blocks ICBl to ICB2 where subroutine CBSE'C previously was called ICBP times.

Calculates the diametral thermal expansion of Zircaloy as a function of temperature.

Calculates cell-averaged quantities that are required for the interphasic heat-transfer calculation for one-dimensional components.

Checks boundary data.

Transfers selected BD array data into the $A$ array required for the accumulator phase-separation model.

Stores data in the BD array temporarily to check for the consistency in the junction data.

Uses Chen correlation to evaluate the forced convection nucleate boiling HTC.

Evaluates the CHF based on a local-conditions formulation.

Applies Biasi CHF correlation.

Checks for the consistency in the boundary-array data during inttialization.

Checks VESSEL source locations.

Establishes the choked phasic velocities and their derivatives. 
Name

CIVSSL

CLEAN

CLEAR

COMPI

CONBLK

CONC

CONSTB

CONVRT

CORE I

CORE3

CORECI

COREC2

COREC3

CORGEO

CORROD

CPLL

CPVEC3

CPVPRT

CPVV1

CTAIN I
Function

Transfers vessel data from LCM to SCM so that the remaining data can be initialized.

Closes TRAC output files.

Sets an array to a constant value.

Performs various i-array loading tasks common to most oncdimensional components.

Computes all 61 types of control-block outputs that do not require table storage (that is, except for "DLAY" and FNG 1").

Returns maximum solubility (kg solute/kg water) for species ISPEC at pressure $P$ and water temperature TL.

Drives subroutine STBME.

Takes absolute areas and converts them into fractional areas.

Evaluates rod HTCs and tracks quench fronts.

Evaluates rod temperature distributions.

Controls CORE prepass.

Controls CORE outer iteration.

Controls CORE postpass.

Writes out the geometric data for a one-dimensional CORE.

Writes out the rod data for a one-dimenstonal core.

Calculates specific heat of liquid water as a function of enthalpy and pressure.

Evaluates the control-panel vector parameters.

Prints the control-panel status, which is called from PSETQ.

Calculates specific heat of water vapor as a function of temperature and pressure.

Controls containment prepass. 


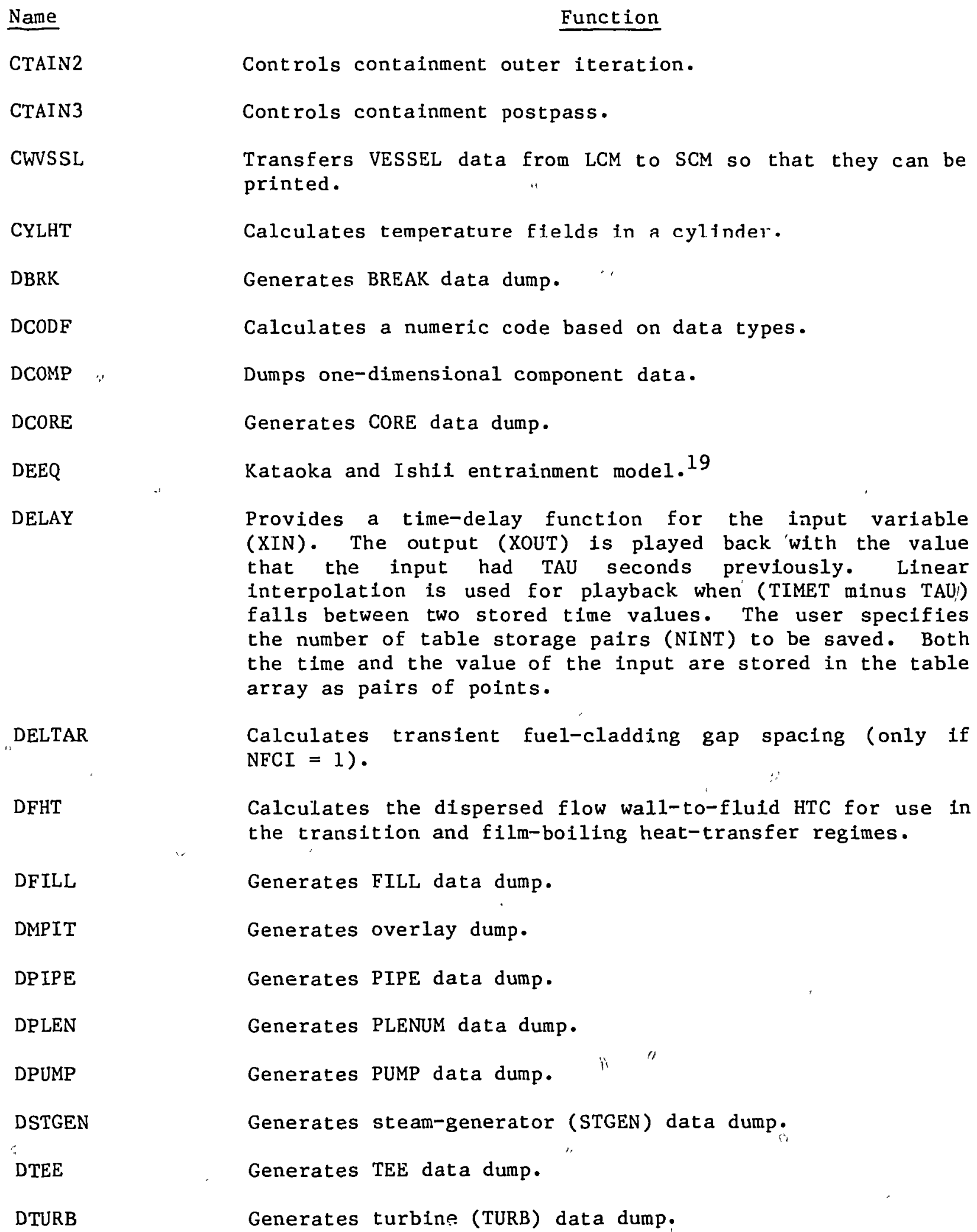


DVLVE

DVSSL

ECOMP

EDIT

ELGR

ENABIN

ENDDMP

ENDGRF

EOVLY

ERRGET

ERROR

ERRTRP

ESTGEN

ETEE

EVALDF

EVFXXX

EVLTAB

EXPAND

FAXPOS

FEMOM

FF3D
Generates VALVE data dump.

Generates VESSEL data dump.

Writes hydrodynamic and heat-transfer information for onedimensional components to output file.

Begins entry routine for overlay edit.

Converts cell elevations to the slope between cells and converts $\mathrm{K}$ factors to TRAC friction coefficients.

Enables and processes (CTRL-E)I interrupts.

Empties dump buffers and closes dump file.

Empties graphics buffers and closes graphics file.

Closes overlay bookkeeping.

Sets error trap indicators.

Processes different kinds of error conditions.

Processes trapped errors.

Evaluates steam-generator tee parameters on explicit pass.

Evaluates tee parameters on explicit pass.

Evaluates the absolute difference between XOLD and XNEW.

Evaluates the XXX component-action function.

Interpolates the function value $F$ from the tabular data based on the value of the table's independent variable: a signal variable (NVAR.GT.0), a control block (NVAR.LT.0), or a trip-signal difference DELSV (NVAR.EQ.0).

Adds rows of conduction nodes within the vessel rods during reflood.

Evaluates the flow-area fraction, FA, on valve-stem position, XPOS for the VALVE.

Sets up stabilizing momentum equations.

Makes final páss update for all variables in threedimensional VESSEL. 


\section{FILL1}

FILL2

FILL3

FILLX

FLUX

FNMESH

FPROP

FROD

FTHEX

EWALL

GAPHT

GETBIT

GETCRV :

GRAF

GRFGET

GRFPUT

GVSSL1

GVSSL2

HEV

HOUT
Controls FILL prepass.

Controls FILL outer iteration.

Controls FILL postpass.

Evaluates postpass FILL velocity.

Calculates mass flow at the boundary of a one-dimensional component for use in mass inventory.

Initializes the supplemental user-specified rows of conduction nodes within the vessel rods at the start of reflood.

Calculates values for fluid enthalpy, transport properties, and surface tension.

Calculates temperature profiles in nuclear or electrically heated fuel rods.

Calculates the fuel linear thermal-expansion coefficient for uranium-dioxide and mixed-oxide fuels.

Computes a two-phase friction factor.

Calculates fuel-cladding gap HTC.

Retín value of bit $\mathrm{N}$ of word $\mathrm{B}$.

Gets appropriate pump curves from data base.

Edits graphics data during transient.

Returns entries in graphics catalog block.

Places entries in graphics catalog block.

Calculates integrated vessel parameters for graphics purposes.

Calculates average values for vessel graphics (integrated values calculated in subroutine GVSSLl).

Calculates the heat of evaporation of liquid corresponding to a given temperature for low pressures.

Controls the outer iteration logic for a complete "time step. 
HQR

HQR2

HTCOR

HTIF

HTP IPE

HTVSSL

HUNTS

HVF ILM

IACCUM

IBRK

ICHL

ICMP

ICMPR

ICOMP

ICORE

IDEL

IFILL

IGACUM

IGBRAK
Support subroutine for SGEEV.

Support subroutine for SGEEV.

Computes HTCs.

Calculates the interphasic heat transfer for the zerodimensional and one-dimensional components.

Averages velocities and generates HTCs for one-dimensional components.

Averages velocities and generates HTCs for the vessel.

Searches character string for specified search string.

Calculates the vapor HTC that is the maximum of the Bromley, natural-convection, and the Douga11-Rohsenow coefficients.

Initializes the accumulator (ACCUM) data arrays that are not input from cards.

Initializes the BREAK data arrays that are not input from cards.

Returns character at given position in string (leftjustified, blank-filled).

Overlay controls the intialization of component data.

Logically compares a real variable with an integer variable.

Controls the routines that initialize component data.

Initializes the CORE data arrays that are not input from cards.

Searches specified substring of string for any one character in a set of specified characters.

Initializes the FILL data arrays that are not input from cards.

Supplies accumulator (ACCUM) data for graphics.

Supplies BREAK data for graphics. 
Name

IGCOMP

IGCORE

IGF ILL

IGPIPE

IGP LEN

IGPRZR

IGPUMP

IGRAF

IGRF

IGSTGN

IGSVCB

IGTEE

IGTURB

IGVLVE

IGVSS2

IGVSSL

INDEL

INIT

INNER

INPUT

IOVLY

IP IPE
Supplies graphic output information for most one-dimensional components to the graphics COMMON block.

Specifies graphics data for one-dimensional CORE.

Supplies FILL data for graphics.

Supplies PIPE data for graphics.

Outputs FLT and VLT data.

Supplies pressurizer (PRIZER) data for graphics.

Supplies PUMP data for graphics.

Initializes graphics variables and writes a header to the graphics file.

Controls the creation of the graphics dictionary.

Supplies steam-generator (STGEN) data for graphics.

Obtains the signal-variable values.

Supplies TEE data for graphics.

Supplies turbine (TURB) stage data for graphics.

Supplies VALVE data for graphics.

Returns the VESSEL variable-length table's variables NASX and LENLD.

Supplies VESSEL data for graphics.

Searches specified substring of string for first nonoccurrence of any one character in a set of specified characters.

Supplies entry routine for overlay INIT.

Performs an inner iteration for a one-dimensional component.

Supplies entry routine for overlay INPUT.

Initializes overlay bookkeeping.

Initializes the PIPE data arrays that are not input from cards. 
Name

IPLEN

IPRIZR

IPROP

IPUMP

ISORT

ISTGEN

ITEE

ITRI

ITURB

IVLVE

IVSSL

JID

JFIND

JUNSOL

JVALUE

LCMOVE

LEVEL

LININT

\section{Function}

Loads the PLENUM arrays that are needed, but not input, to start a problem.

Initializes the pressurizer (PRIZER) data arrays that are not input from cards.

Calls subroutines THERMO, FPROP, and MTXPRP for most onedimensional components.

Initializes the PUMP data arrays that are not input froln cards.

Sorts a list of integers in ascending order.

Initializes the steam-generator-(STGEN) data arrays that are not input from cards.

Initializes the TEE data arrays that are not input from cards.

Gives iterative solution of reduced, linearized threedimensional finite-difference equations.

Loads the arrays that are not input but that are needed to start a problem.

Initializes the VALVE data arrays that are not input from cards.

Initializes the VESSEL data arrays that are not input from cards.

Fills boundary array at component junctions.

"

Locates junctions in junction sequence array.

Determines junction parameters for connecting and sequencing components.

Converts one character of a string to a binary number; $0-9$ returned as binary mode; blank, as binary 0; all others, as less than 0 .

Copies data from'one part of LCM to another.

Uses a curve fit to obtain the water level in a cylindrical pipe as a function of the void fraction.

Performs linear interpolation on arrays.<smiles>C1=C[CH-]CC=C1</smiles> 
Name

LINT4D

LOAD

LOCF

LOCPMP

LOC'IEE

LOCTRB

LOCVLV

MACCUM

MANAGE

MAP IN

MBN

MBREAK

MCORE

MCTAIN

MFILL

MFROD

MFUEL

MGAP

MHTR

\section{Function}

Linearly interpolates a function table with zero to four independent variables.

Reads in specially formatted input data.

Locates the variable address.

Calculates the required relative variable location in a common block for the VALVE.

Calculates the required relative variable location in a common block for the TEE.

Calculates the required relative variable location in a common block for the TURB.

Calculates the required relative variable location in a common block for the VALVE.

Controls modification of accumulator (ACCUM) components.

Performs all level and rod data management operations for it the vessel.

Converts data types [for the nuclear plant analyzer (NPA) only].

Calculates values for electrically heated nuclear fuel-rod insulator properties.

Controls modification of a BREAK.

Allows changes to a CORE.

Allows changes to containment components.

Controls modification of a FILL.

orders fuel-rod property selection and evaluates an average temperature for property evaluation.

Calculates values for uranium-dioxide and uranium-plutonium dioxide properties.

Calculates values for the thermal conductivity of the gapgas mixture.

Calculates values for electrically heated fuel-rod heater coil properties. 
Name

MIXPRP

MODCMP

MODCPV

MODCTL

MODIFY

MODSV

MODTRP

MP IPE

MPLEN

MPRIZR

MPROP

MPUMP

MSTGEN

rs

MSTRCT

MTEE

MTURB

MVALVE

MVSSL

MWRX

MZIRC

NEWDLT

NXTCMP

\section{Function}

Calculates mixture properties from those of separate phases.

Controls modification of components.

Allows modification of the control-panel vector specification cards.

Modifies and writes out the main control cards.

Control program for the interactive modification.

Allows modification of the signal-variable cards.

Allows modification of the trip data cards.

Controls the modification of a PIPE.

Controls modification of a PLENUM.

Controls modification of pressurizer (PRIZER) components.

Orders structure property selection and evaluates an average temperature for property evaluation.

Controls modification of a PUMP.

Controls modification of steam-generator (STGEN) compunents.

Calculates properties for certain types of steel.

Cont rols modification of a TEE.

Controls modification of a turbine (TURB).

Controls modification of a VALVE.

Controls modification of a VESSEL.

Calculates the Zircaloy steam reaction in the cladding at high temperatures.

Calculates properties for Zircaloy 4 .

Evaluates prospective new time increment.

Finds the beginning of data for the next component. 
Name

ORIEER

y

ORTHES

ORTRAN

OUTID

OUT3D

OUTER

PACKIT

PIPE1

PIPE1X

PIPE2

PIPE3

PLEN I

PLEN2

PLEN3

PMPP

POST

POST3D

POSTER

PRCINT

PRCNPA

\section{Function}

Rearranges the signal-variable, control-block, and trip ID numbers in ascending order based on their absolute value and searches for the DO loop index values for each controlparameter evaluation pass through the signal variables, control blocks, and trips.

Support subroutine for SGEEV.

Support subroutine for SGEEV.

Controls outer calculation for one-dimensional components.

Controls outer calculation for a VESSEL.

Controls outer calculation for one time step.

Packs data from one array into another.

Controls pipe prepass.

Calculates liquid volume discharged ( $\left.q_{q u t}\right)$, collapsed liquid level ( $z$ ), and volumetric flow rate $\left(v_{\text {flow }}\right)$; assumes vertical component with low-numbered cell at top.

Controls PIPE outer iteration.

Controls PIPE postpass.

Performs the prep stage calculation for the PLENUM timestep initialization.

Controls PLENUM outer iteration.

Controls PLENUM postpass.

Calculates PUMP enthalpy, pressure, and density.

Controls postpass calculation for one time step.

Controls postpass calculation for the VESSEL.

Performs postpass calculation for one-dimensional components.

Processes interrupts.

Processes NPA commands that affect TRAC execution during an NPA simulation. 
Name

PREINP

PREP

PREP1D

PRE P3D

PREPER

PRIZR1

PRIZR2

PRIZR3

PRZRIX

PRZR3X

PSTEPQ

PUMP1

PUMP2

PUMP3

PUMPD

PUMPI

PUMPSR

PUMPX

R1MACH

RACCUM

RBREAK
Function

Converts free-format TRACIN deck to format used by TRAC input subroutine.

Controls prepass calculation for one time step.

Controls the one-pass calculation for one-dimensicnal components.

Controls prepass calculation for three-dimensional components.

Performs prepass calculation for one-dimensional components.

Controls pressurizer (PRIZER) prepass.

Controls pressurizer (PRIZER) outer iteration.

Controls pressurizer (PRIZER) postpass.

Evaluates pressurizer (PRIZER) water level and heater/sprayer source.

Evaluates mass change during steady-state calculation.

Controls printing, dumping, and graphing of data at the completion of a time step.

Controls PUMP prepass.

Controls PUMP outer iteration.

Controls PUMP postpass.

Calculates head and torque from PUMP curves.

Supplies built-in PUMP characteristics.

Evaluates PUMP momentum and energy source.

Calculates PUMP head and torque.

Support subroutine for SGEEV.

Reads accumulator (ACCUMí data input file and creates pointer table for these data.

Reads BREAK data from input file and creates a pointer table for these data. 
Name

RCNTL

RCOMP

RCORE

RCPVEC

RDCOM3

RDCOMP

RDCRDS

RDCRVS

RDDIM

RDLCM

RDREST

REACCM

READI

READR

REBRK

RECNTL

RECOMP

RECORE

RECPV

RE FILL

Function

Reads in signal-variable, trip, and controller input data.

Reads data common to most one-dimensional components from input files and writes these data to output file.

Rcads CORE data from input file and creates a pointer table for these data.

Processes the control-panel vector input cards.

Controls reading of three-dimensional VESSEL data fron input file.

Controls reading of component data from input file.

Reads time-step cards until DTMIN $<0$ is encountered.

Reads PUMP curves from input file.

Reads number of points on PUMP curves from input file.

Moves data from LCM to SCM.

Controls reading of component data from a restart dump file.

Reads accumulator (ACCUM) data from a restart dump and creates a pointer table for these data.

Reads integer data in 114 format.

Reads real data in E14.6 format.

Reads BREAK data from a restart dump and creates a pointer table for these data.

Reads the signal-variable, trip, and controller data from the restart file.

Reads data from disk common to most one-dimensional components (used by subroutine RESTART).

Reads CORE data from a restart dump and creates a pointer table for these data.

Adds the restart file control-panel vector data that was not specified on input and prints it out.

Reads FILL data from a restart dump and creates a pointer table for these data. 
Name

REPIPE

REPLEN

REPRZR

REPUMP

i)

RESTGN

RETEE

RETURB

REVLVE

REVSSL

RFDBK

RFILL

RHOLIQ

RKIN

RODHT

RPIPE

RPLEN

RPRIZR

Reads PIPE data from a restart dump and creates a pointer table for these data.

Reads PLENUM data from a restart dump and creates a pointer table for these data.

Reads pressurizer (PRIZER) data from a restart dump and creates a pointer table for these data.

Reads PUMP data from a restart dump and creates a pointer table for these data.

Reads steam-generator (STGEN) data from a restart dump and creates a pointer table for these data.

Reads TEE data from la restart dump and creates a pointer table for these data.

Reads turbine (TURB) stage data from a restart dump and creates a pointer table for these data.

Reads VALVE data from a restart dump and creates a pointer table for these data.

Reads VESSEL data from a restart dump and creates a pointer table for these data.

Evaluates the reactor core reactivity feedback caused by changes in the fuel temperature, coolant temperature, and coolant void from the beginning of the previous time step.

Reads FILL data from input file and creates a pointer table for these data.

Calculates values of liquid density and its derivatives.

Integrates the neutron point-kinetics equations.

Calculates the fuel-rod temperature field.

Reads PIPE data from the input file and creates a pointer table for these data.

Reads PLENUM data from the input file and creates a pointer table for these data.

Reads pressurizer (PRIZER) data from input file and sets up a pointer table for these data. 
Name

RPUMP

RSTGEN

RTEE

RTURB

RVLVE

RVSSL

SIDPTR

SATDER

SATPRS

SATTMP

SAVBD

SCLTBL

SCMLCM

SCOPY

SCOPYM

SEDIT

SETBD

SETBRK

SETCMP

\section{Function}

Reads PUMP data from input file and creates a pointer table for these data.

Reads steam-generator (STGEN) data from input file and creates pointer tables for these data.

Reads TEE data from input file and creates a pointer table for these data.

Reads turbine (TURB) stage data from input file and creates a pointer table for these data.

Reads VALVE data from input file and creates a pointer table for these data.

Reads VESSEL data from input file and creates a pointer table for these data.

Sets pointers for one-dimensional components.

Calculates the derivative of saturation temperature of vapor with respect to pressure.

Calculates saturation pressure of vapor corresponding to a given temperature.

Calculates saturation temperature of vapor corresponding to a given pressure.

Moves boundary information into component arrays.

Scales input table according to scale factor passed by input routine.

Checks for overflow. Transfers fixed-length, variablelength, and pointer tables to LCM. Adjusts pointers.

Support subroutine for SGEEV.

Support subroutine for SGEEV.

- Writes short edit to TRCOUT file.

Stores component information in boundary arrays.

Sets special pointers for a break.

Determines the component type and sets the LCM pointer for control-panel vector parameter IPARAM for component ICOMP. 
Name

SETCOR

SETCPV

SETEOS

SETFIL

SETLCM

SETNET

SETPMP

SETPRZ

SETSCM

SETVSL

SGEDI

SGEEV

SGEFA

SGESL

SGTBC

SHIFTB
Function

Sets special pointers for a CORE.

Sets up the control-panel vector pointers. A11 errors encountered by subordinate routines are treated as warning errors. If an error would inhibit further processing, IERRFL is set to 1 . At the end of this routine a fatal error is issued if IERREL is not 0 . This allows checking of all parameters in a single pass.

Sets the equation of state constants.

Sets special pointers for a fill.

Monitors use of LCM dynamic area.

Provides the information needed to set up the network solution matrices.

Sets the volume flow pointer for a PUMP.

Sets special pointers for a pressurizer (PRIZER).'

Monitors use of SCM dynamic area.

Sets special polnters for a VESSEL.

Computes the determinant of a watrix using the factors computed by SGEFA.

Computes the eigenvalues and eigenvectors of a general real matrix.

Factors a real matrix by Gaussian elimination. SGEFA is usually called by SGECO, but it can b... a saving in time if RCOND is not\% ...eeded. (time for $\mathrm{SGECO})=(1+9 / \mathrm{N}) *$ (time for SGEFA)

Solves the real system $A * X=B$ or $\operatorname{TRANS}(A) * X=B$ using the factors computed by SGECO or SGEFA.

Boundary cell calculations for the special case where the - TEE junction cell is the first or last cell in a main steam-generator pipe. This logic duplicates the logic in subroutine TEEl after the call to BKMOM.

Translates the table's abscissa coordinate values so that the function value $F$ in the table corresponds to an abscissa coordinate value of 0.0 . 
Name

SHRINK

SIGMA

SLABHT

SMOVE

SMOVEN

SOUND

SPLIT

SRCHCL

SREBAL

SRTLP

STBME

ST BMPL

STDIR

STEADY

STGABD

STGEN1

STGEN2

STGEN3

STGNIX

STGN3X

STGNTX

Removes rows of conduction nodes within the VESSEL rods during reflood.

Returns surface tension of water as a function of pressure.

Calculates the slab temperatures.

Moves a character from one string to another.

Moves a specified number of characters froin one string to another.

Homogeneous equilibrium sound speed calculation.

Reads appropriate data from PUMP curves.

Searches the component list for component number ICOMP. It returns the component LCM pointer in IBASE. If the component is not found, it sets IERR to one and returns -1 in IBASE.

Sets up coarse-mesh rebalance in the VESSEL.

Sorts components into loops and reorders them for the network solution.

Sets up the stabilizing mass and energy equations.

Sets up the stabilizing mass and energy equations for the PLENUM.

Sets up direct inversion of the VESSEL matrix.

Generates a steady-state solution.

Finds the void fractions in adjacent cells within a steam generator to use in heat-transfer averaging.

Controls steam-generator (STGEN) prepass.

Controls steam-generator (STGEN) outer iteration.

Controls steam-generator (STGEN) postpass.

Evaluates HTCs for steam-generator (STGEN) secondary.

Performs steam-generator (STGEN) heat-transfer calculation.

Computes needed quantities on prepass. 
Name

STGPTH

STPCLS

STPMSG

STPRD

STPWRT

STRLER

SVSET

SVSET 1

SVSET3

TEE1

TEE 1X

TEE2

TEE3

TF1D

IFIDS

TF1DS1

TFIDS3

TF3DE

TF3DI

TFPLBK
Function

Initializes TRAC communication to the NPA controller.

Closes disk file used for NPA controller communication.

Checks for message from the NPA controller.

Reads message from the NPA controller.

Writes riessage to the NPA controller.

Providés error message for NPA controller communication failure (reserved for future use).

Evaluates location-independent $\quad(0=\text { ISUN }<17)^{\prime}$ "signal variables.

Evaluates signal variables with locations defined in the one-dimensional components.

Evaluates signal variables with locations defined in the three-dimensional VESSEL.

Controls TEE prepass.

Calculates suurce for TEE side-leg hydrodynamics.

Controls TEE outer iteration.

Controls TEE postpass.

Drives one-dimensional hydrodynamics routines.

Solves the hydrodynamic equations for the one-dimensional two-fluid pipe model.

Sets up initial velócity approximations and their pressure derivatives for the one-dimensional two-fluid pipe model.

Performs the backward substitution for the one-dimensional two-fluid pipe model.

Evaluates constitutive relitions for interfacial shear and heat transfer; makes an evaluation of new time velocities.

Sets up the linearized three-dimensional finite-difference equations.

Performs the backward substitution for the basic difference equations for the PLENUM (similar to TFlDS3 for the other one-dimensional components). 
TFPLN

THCL

THCV

THERMO

TIMCHK

TIMSTP

TMS FB

TRAC

TRANS

TRANSF

TRBPOW

TRBPRE

TRBPST

TRIP

TRIPS

TRISLV

TRPSET

TURBI i)

Solves the basic hydrodynamic equations for the PLENUM (similar to TFIDS for the other one-dimensional components).

Returns thermal conductivity of water as a function of pressure and enthalpy.

Returns thermal conductivity of steam as a function of pressure and enthalpy.

Calculates thermodynamic properties of water.

Checks elapsed time to see whether certain functions should be performed.

Sets up time-step and time-edit interval times.

Calculates the minimum stable film-boiling temperature $\left(\mathrm{T}_{\mathrm{min}}\right)$.

Supplies MAIN program.

Controls overall calculation for each time step.

Transfers data from the steam-generator internal network matrix to the loop network matrix.

Calculates the efficiency and power output of a turbine stage.

Calculates the data pertaining to the entire turbinegenerator set (common/sum all stages) during the prep stage.

Calculates the data pertaining to the entire turbinegenerator set (common/sum all stages) during the post stage.

Returns status of a trip.

Evaluates the control parameters for the beginning of the time-step system state.

Solves linear system of the form $A * X=B$ where $A$ is tridiagonal.

Sets up trip status flags.

Performs the prep stage calculation for the turbine stage component time-step initialization. 
TURB2

TURB3

UNPKIT

VALUE

VELCK

VISCL

VISCV

VLEVEL

VLVE 1

VLVE 2

VLVE 3

VLVEX

VOLFA

VOLV

VSLGEO

VSLLEV

VSLROD

VSSL1

VSSL2

VSSL 3

WACCUM

Controls turbine stage outer iteration.

Controls turbine stage postpass.

Unpacks data packed by subroutine PACKIT.

Converts an Ascii string to its binary value.

Checks for incorrect donor-celling in subroutine VSSL caused by velocity sign changes.

Evaluates viscosity of water as a function of pressure and enthalpy.

Evaluates viscosity of steam as a function of pressure and enthalpy.

Indicates the beginning of a certain level in a VESSEL.

Controls VALVE prepass.

Controls VALVE outer iteration.

Controls VALVE postpass.

Evaluates the value of the flow-area change action.

Calculates cell volume flow areas.

Calculates cell-averaged phase velocities for onedimensional components.

Writes the geometry cards for the VESSEL on the restart input file.

Writes the level data cards for the VESSEL on the restart input file.

Writes the rod data cards for the VESSEL to the restart.. input file.

Performs prepass calculations for VESSEL dynamics.

Performs inner iterations for VESSEL dynamics.

Performs postpass calculations for VESSEL dynamics.

Writes selected accumulator (ACCUM) data to output file TRCOUT: 
WARRAY

WBREAK

WCOMP

WCORE

WFILL

WIARR

WPIPE

WPLEN

WPRIZR

WPUMP

WRCOMP

WRITEE

WRITEI

WRITER

WRLCM

WSTGEN :

WTEE

WTURB

WVLVE

WVSSL
Writes a real array to output file TRCOUT.

Writes selected BREAK data to output file TRCOUT.

Controls the writing of selected component data to output file TRCOUT.

Writes selected CORE data to output file TRCOUT.

Writes selected FILL data to output file TRCOUT.

Writes an integer array to output file TRCOUT.

Writes selected PIPE data to output file TRCOUT.

Writes selected PLENUM quantities to the printer.

Writes selected pressurizer (PRIZER) data to output file TRCOUT.

Writes selected PUMP data to output file TRCOUT.

Writes data common to one-dimensional" components to output files.

This subroutine dóes not combine numbers as does subroutine $R$. It merely puts an $E$ at the end of the data for a variable to make it compatible with the LOAD subroutine of the TRAC code.

Takes integers and puts them into format compatible with the LOAD subroutine of TRAC.

Takes real numbers and puts them into format compatible with the LOAD subroutine of TRAC.

Transfers a given number of words from SCM to LCM.

Writes selected steam-generator (STGEN) data to output file TRCOUT.

Writes selected TEE data to output file TRCOUT.

Writes selected quantities to the printer for a turbine (TURB) stage component.

Writes selected VALVE data to output file TRCOUT.

Writes selected VESSEL data to output file TRCOUT. 
APPENDIX H

DESCRIPTION OF COMMON-BLOCK VARIABLES

DECK BKCNTRL

COMMON/BKCTRL/IPREIT, LBCKV, LREIT , LREITV

INTEGER VARIABLES:

IPREIT: Flag to print messages on forced reiteration.

LBCKV: $\quad$ If .TRUE., then variable forces a time-step back-up.

LREIT: IE 'TRUE', then variable forces a reiteration.

LPEITV: If .TRUE., then variable forces a reiteration.

COMMON/DONR/I'TDON, JDONP , NCOMDP

INTEGER VARIABLES :

ITDON: " If flow reversals occur for OITNO>ITDON, the time step is backed up.

JDONP: $\quad$ Cell number in NCOMDP.

NCOMDP: $\quad$ Component number of flow reversal forcing back-up.

DECK BKPOST

COMMON/BKPOST/BKPALL , BKPALU, BKPSTA, BKPSTP , BKPSTT

COMMON/BKPOST/IBKPST, JBKPST , LBKPST

REAL VARIABLES :

BKPALL: Maximum lower limits on void fraction such that a back-up is forced if the vold fraction lies within these inits.

BKPALU: "Maximum upper limits on void fraction such that a back-up is forced if the void fraction lies within
these limits.

BKPSTA: Void-fraction variation that is allowed in the POST stage. If the void-fraction change exceeds BKPSTA, back-up is forced. 
BKPSTP: Maximum fractional pressure change that is allowed in the POST stage. If the fractional pressure change exceeds BKSTP, back-up is forced.

'BKPSTT: Maximum variation in liquid and vapor temperatures that is allowed in the POST stage. If the temperature change exceeds BKPSTT, back-up is forced.

INTEGER VARIABLES :

IBKPST: Component forces back-up.

JBKPST: $\quad$ Cel1 number.

LBKPST: Logical variable. If .TRUE., then a time-step back-up is forced from POST.

COMDECK BLANKCOM

COMMON A(25000)

A(25000): Dynamic SCM storage area. This array is dimensioned to other sizes or dynamically dimensioned for certain conditional directives imposed during compilation.

COMDECK CDBLKS

COMMON/CODEBK/CBNAM, ILEV , MNXILV, MAXLEN, MAXLN3, MLNVMT

DIMENSION CBNAM(5)

INTEGER VARIABLES :

CBNAM: Array containing the names of the overlays currently in core.

ILEV: $\quad$ The number of overlays currently in core.

MAXILV: Maximum amount of SCM storage needed for threedimensional components when only one level of data is required.

MAXLEN: Maximum amount of SCM storage needed to process any one-dimensional component.

MAXLN3: Maximum amount of SCM storage needed to process any three-dimensional component.

MLNVMT: The amount of SCM space required to solve the vessel matrix. 


\section{COMDECK CFLOW}

COMMON/CFLOW/ CHM1, CHM2, CHMLT1 , CHMLT2

COMMON/CFLOW/ICFLOW, IHOR, IHORG

DIMENSION CHM1(5), CHM2(5)

REAL VARIABLES :

i.

CHM1: Array of choked-flow multipliers on the subcooled Hlow.

CHM2: Array of choked-flow multipliers on the two-phase flow.

CHMLT1: Multiplier on the subcooled flow.

CHMLT2: Multiplier on the two-phase flow.

INTEGER VARIABLES :

ICFLOW: $\quad$ Choked-flow mode controller.

$0=$ model turned off,

1 = model using default multipliers turned on only for components connected to a BREAK (default condition), or

2 = model using óptional multipliers turned on at cell edges indicated in component input (note that this option requires additional array data for all onedimensional hydrodynamic components).

IHOR: $\quad$ Drag controller.

$0=$ uses dispersed drag only,

$1=$ (default) uses stratified drag in one dimension if conditions are met, or

2 = always uses stratified drag.

IHORG: Stratified flow regime controller.

$0=$ turns off stratified flow regime including head gradient force,

$1=$ (default) uses normal stratified flow, or

2 = turns off head gradient force. 


\section{COMDECK CHECKS}

COMMON/ CHECKS / DTEND , DTSTRT , HDUMP , HEDIT , HGRAF , HSEDIT

COMMON/CHECKS/NALT, NDID

REAL VARIABLES :

DTEND: Time interval during which the special time-step data are used.

DTSTRT: A constant initially defined to be -1.0 that can be used to force a calculation to begin with a specified time-step size ( $\triangle$ TSTRT when DTSTRT has been set greater than zero).

HDUMP: $\quad$ Saved value of the next restart/dump edit time from the regular time-step data when the special time-step data are used.

HEDIT: Saved value of the next long edit time from the regular time-step data when the special time-step data are used.

HGRAE: Saved value of the next graphics edit time from the regular time-step data when the special time-step data are used.

HSEDIT: Saved value of the next short edit time from the regular time-step data when the special time-step data are used.

INGGER VARIABLES:

NALT: Constant used to determine if void-fraction adjustments are needed when the interfacial drag is calculated at a one-dimensional junction connected to a BREAK.

NDID: $\quad$ ID number of the spectal time-step data that are being used.

\section{COMDECK CHGALP}

COMMON/ CHGALP /DAL , DAU , OAL , OAU , XDAL , XDAU , XOAL , XOAU

COMMON/ CHGALP / JDAL , JDAU , JOAL , JOAU , NDAL , NDAU, NOAL , NOAU

REAL VARIABLES :

DAL: $\quad$ Maximum decrease in void fraction. 


DAU:
OAL: $\begin{aligned} & \text { Maximum increase in vold fraction over the time step. } \\ & \text { following an increase. } \\ & \text { OAU: }\end{aligned}$

INTEGER VARIABLES :

$\begin{array}{ll}\text { JDAL: } & \text { Cel1 where DAL occurred. } \\ \text { JDAU: } & \text { Cel1 where DAU occurred. } \\ \text { JOAL: } & \text { Cel1 where OAL occurred. } \\ \text { JOAU: } & \text { Cell where OAU occurred. } \\ \text { NDAL: } & \text { Component where DAU occurred. } \\ \text { NDAU: } & \text { Component where OAL occurred. } \\ \text { NOAL: } & \text { Component where OAU occurred. } \\ \text { NOAU: } & \end{array}$

COMDECK COMCOM

COMMON/CC/IMAX, IMIN, JFLAG

INTEGER VARIABLES :

IMAX: Last component (TRAC-assigned component number) in a loop to control the outer calculation for onedimensional components.

IMIN :

First component (TRAC-assigned component number) in a loop to control the outer calculation for onedimensional components.

JFLAG: Flag that indicates an error in the outer calculation. 
COMDECK CONSTANT

COMMON/CONST/PI, GC ,ZERO, ONE , ERSALP

REAL VARIABLES :

PI: $\quad$ Constant $\pi(3.1415926535898)$.

GC: Gravitational constant $\left(9.80665 \mathrm{~m} \cdot \mathrm{s}^{-2}\right)$.

ZERO: $\quad$ Real constant zero.

ONE : $\quad$ Real constant one.

EPSALP : Void-fraction cutoff for thermodynamic vapor properties.

COMDECK CONTRLLR

COMMON/CONTRL/DAMMC , DAMX, DELT , DELTHT, DIFMIN, DPRMX , DTLMX, DTMAX, DTMIN, DTO, DTRMX, DTSMX , DTVMX, EPS1, EPS2, EPSI , EPSO, EPSP, EPSS, ETIME, HTLOSI , HTLOSO, ODELT , TEND , TIMEC , TIMET , VARER, VCMN , VCMX, VMAXO, VMAXT , VMCON, VMNEW, VMOLD

COMMON/CONTRL/DSTEP, IADDED, ICCMX, ICMP, ICP , IEOS, IFPREP , IGEOM3, IITMAX, IM100, IM100X, IMFR, INVAN, IPAK, IPAKON, IPKPMP, IRSTFL, ISOLUT, ISSFLG, ISTDY, ISTTC , ITMIN , ITPAKO , KCCMX, LCMPTR , LEVSTG , NCMN, NCMX , NCRG , NDIA1 , NDIA3 , NFRC1 , NFRC3 , NITAV , NITMN , NITMX, NIVSMN, NIVSMX, NSEND, NSEO, NSMN, NSMX, NSPL , NSPU, NSSO, NSTP , NSVSMN, NSVSMX , OITMAX, SITMAX, STDYST, TRANSI

REAL VARIABLES :

DAMMC: Maximum void-fraction change during time step.

DAMX: $\quad$ Error caused by relative change in void fraction.

DELT: Current time increment for advancement of finitedifference equations.

DELTHT: Heat-transfer time-step size.

DIFMIN: Minimum diffusion number required for stability of the nod conduction solution.

DPRMX :

Maximum pressure change during the current time step.

DTLMX:

Maximum liquid-temperature change during time step.

DTMAX:

Maximum allowable time-step size for time interval. 


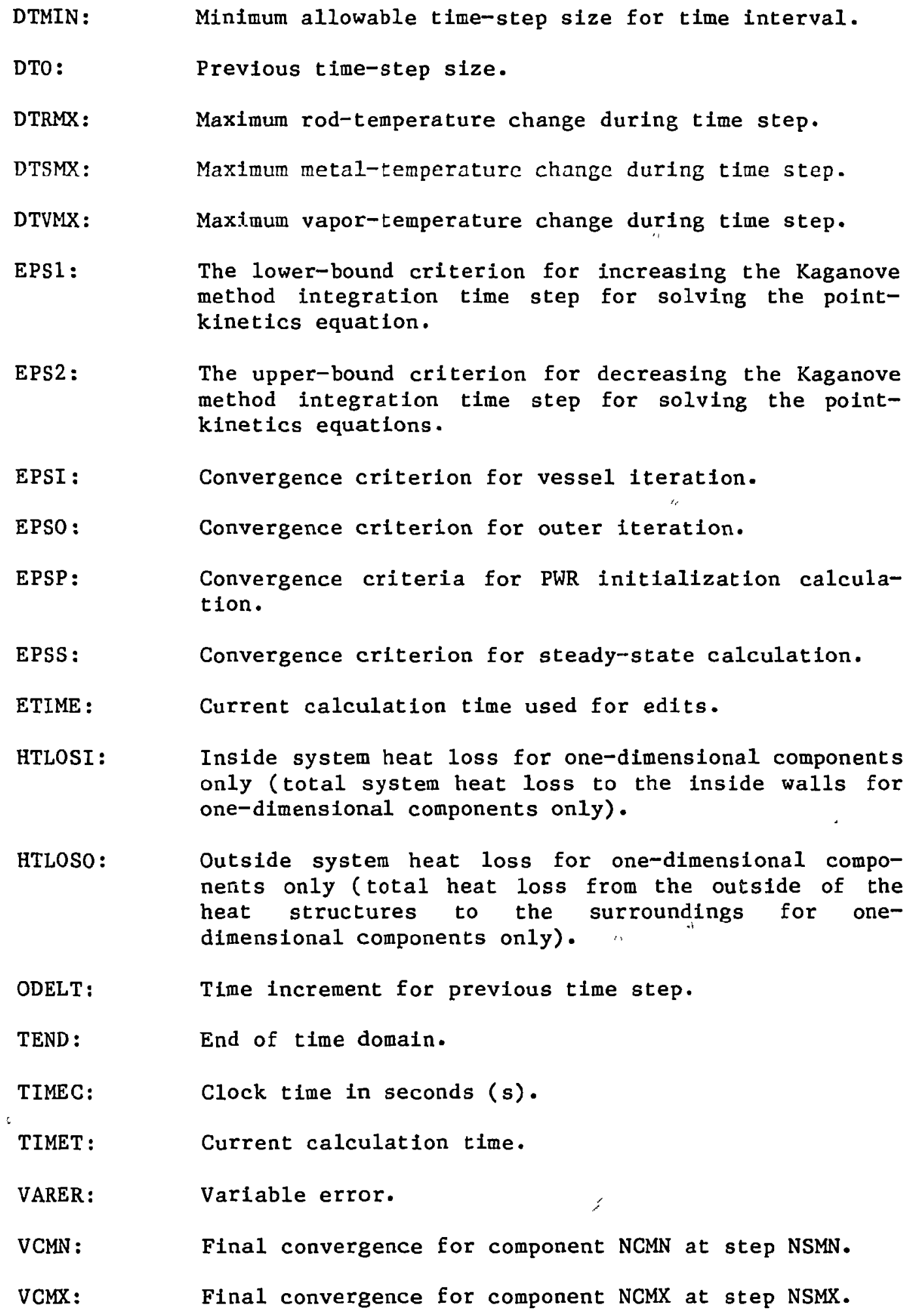

HTLOSO: Outside system heat loss for one-dimensional components only (total heat loss from the outside of the heat structures to the surroundings for onedimensional components only).

ODELT: Time increment for previous time step.

TEND :

End of time domain.

TIMEC: $\quad$ Clock time in seconds (s).

TIMET: $\quad$ Current calculation time.

VARER: $\quad$ Variable error.

VCMN : $\quad$ Final convergence for component NCMN at step NSMN.

VCMX: $\quad$ Final convergence for component NCMX at step NSMX. 

VMAXO:
Maximum Courant number found during time step.
VMAXT: Maximum Courant number.
VMCON: Net water mass (liquid plus vapor) convected into
VMNEW: Vessel water mass (1iquid plus vapor) at $\left(t^{n+1}\right)$.
VMOLD: Vessel water mass (liquid plus vapor) at $\left(t^{n}\right)$.

\section{INTEGER VARIABLES :}

DSTEP: $\quad$ Time-step number of dump to be used for restart.

IADDED: Time-step interval for printing calculation summary to the terminal. (Zero suppresses this print.)

ICCMX: Component number in the IORDER array with the most severe time-step limit for stability.

ICMP: $\quad$ Component indicator.

ICP: Temporary pointer to next free location in the dynamic storage area for component data.

IEOS:

Afr-water option flag.

0 = steam-air-water;

1 = air-water (no steam present).

IFPREP: $\quad$ Flag that indicates sections of PREPER to be executed (nonzero only for one-dimensional cores).

IGEOM3: Vessel geometry flag.

$0=$ (default) flow areas between the downcomer and inside of the vessel set to zero;

$1=$ flow areas between the downcomer and inside of the vessel maintained at the user input values.

Note: The vent valve option overrides IGEOM3 $=1$ option in cells that have vent valve connections.

IITMAX: Maximum number of vessel iterations.

IM100: Flag that indicates if back-up occurred on previous time-step (used for mass check on $\log i c$ ).

IM100X: Flag that indicates whether previous time step that failed was obtained from a restart. 
IMFR :

Calculates the theta, axial, and radial mass flows $(\mathrm{kg} / \mathrm{s})$ for both liquid and vapor, and adds them to the graphics. This option is invoked by a new NAMELIST variable IMFR.

1 = (default) one-dimensional vessel mass flow;

3 = three-dimensional vessel mass flow.

INVAN :

Flag to select either $T_{C H F}$ or $T_{\text {sat }}$ for control of the inverted annular flow regime.

IPAK:

Flag that indicates water-packing option.

$0=$ off;

$1=$ on.

IPAKON: Flag that indicates if water-packing logic is on during time step.

IPKPMP: $\quad$ Flag that indicates if water-packing corrections are made at a pump source.

$0=$ (default) water-packing corrections are not made at a pump source;

1 = water-packing corrections are made at a pump source.

IRSTFL: Flag to control dump generation in the interactive mode.

$0=$ normal operation;

1 = termination with a dump because the interactive mode is requesting a component modification.

ISOLUT: $\quad$ Flag that turns on solute tracking option.

ISSFLG: $\quad$ Flag that controls editing in steady state.

ISTDY: $\quad$ Flag that indicates type of calculation.

$0=$ transient;

1 = steady state.

ISTTC: $\quad$ Static check flag.

0 = normal mode;

$1=a$ static balance check was requested when STDYST $=5$ was input.

ITMIN : Minimum stable film-boiling option flag.

ITPAKO: Iteration number for which water packing was detected.

KCCMX: Cell in above component that limits stability.

LCMPTR: Pointer to end of component data for last component read. 
LEVSTG :

NCMN :

NCMX:

NCRG:

NDIA1 :

NDIA3:

NFRC1 :

NFRC3:

NITAV:

NITMN :

NITMX:

NIVSMN :

NIVSMX:
Void-fraction averaging flag.

$0=$ default, no void-fraction averaging is performed in HTCOR in the steam-generator secondaries;

1 = special void-fraction averaging is performed in HTCOR for steam-generator secondaries.

Position in IORDER array for component that was last to converge at step NSMN.

Position in IORDER array for component that was last to converge at step NSMX.

NAMELIST variable (not documented elsewhere and overwritten to zero) that could be used to force the input of ICRRG (see the VESSEL variable-length table, App. I). Logic is incomplete.

Heat-transfer diameter option for one-dimensional components.

$1=$ (default) no heat-transfer diameter input for onedimensional components;

2 = heat-transfer diameter input for one-dimensional components.

Heat-transfer diameter option for three-dimensional components.

$1=$ (default) no heat-transfer diameter input for three-dinensional components;

3 = heat-transfer diameter input for three-dimensional components.

Set to 2 if forward and reverse loss coefficients are to be input for one-dimensional components.

Set to 2 if forward and reverse loss coefficients are to be input in the vessel.

Average number of outer iterations since the last edit.

Minimum number of outer iterations since the last edit.

Maximum number of outer iterations since the last edit.

Minimum number of vessel iterations required since the last edit.

Maximum number of vessel iterations required since the last edit. 


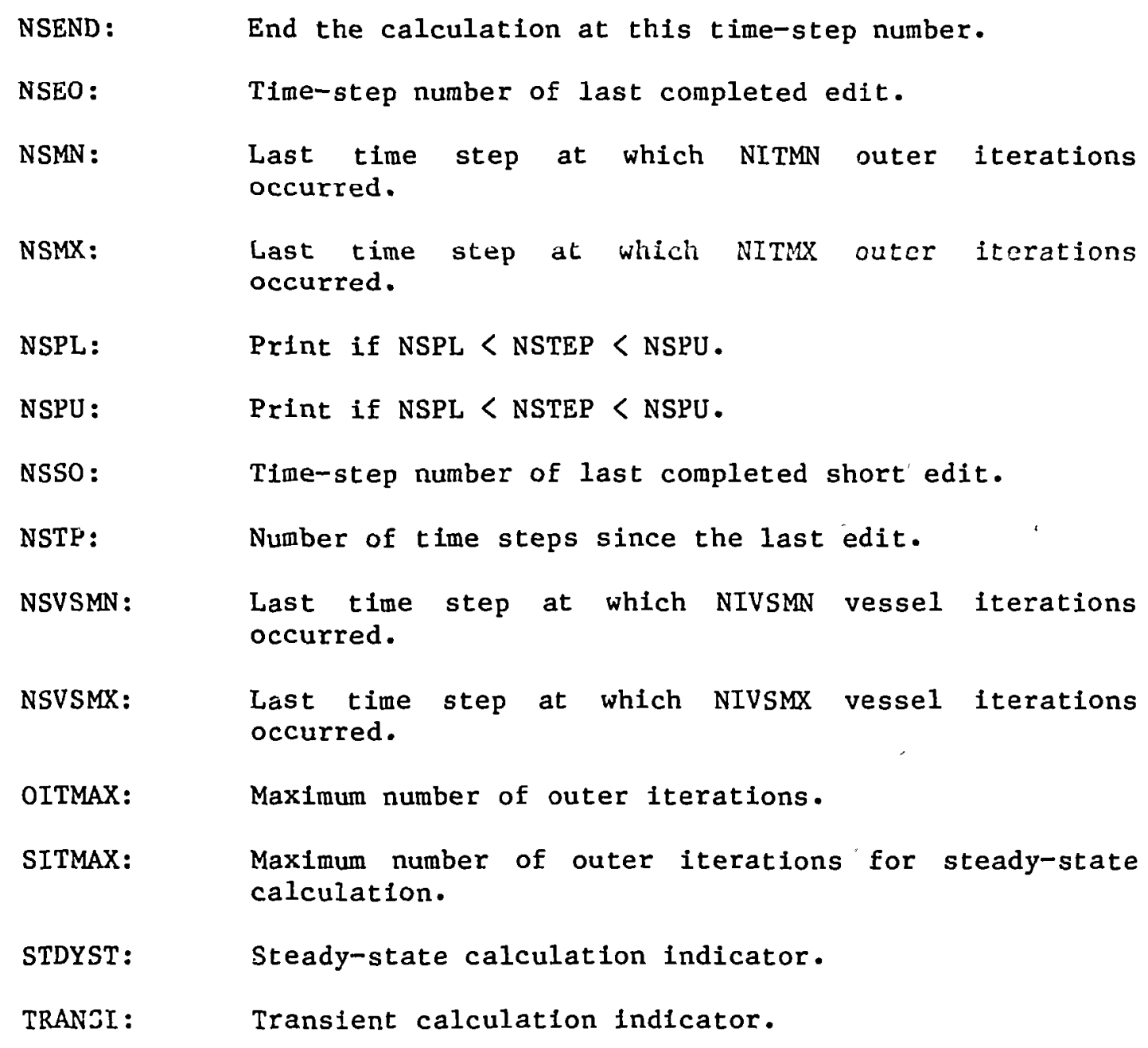

COMDECK CPVECT

COMMON/CPVECT/CPVINT, RDIAM, RMASS , RSTMLT , TCPVPR, VCPVLP , VCPVPR COMMON/CPVECT/IHTRIG , INTFLG, IPRCPV , KFLOW, KLEVEL , KPRESS , KTEMP, LÁSOG, LCPV LP, LCPV PR , NCPVP , NORMDP , NORMRS

$\operatorname{DIMENSION} \operatorname{LCPVLP}(4,9), \operatorname{LCPVPR}(9), \operatorname{VCPVLP}(4,9), \operatorname{VCPVPR}(6)$

REAL VARIABLES:
CPVINT:
Print Interval for control-panel vector.
RDIAM:
Refueling storage tank diameter.
RMASS :
Initial refueling storage tank water mass $(\mathrm{kg})$.
RSTMLT:
Multiplier for converting refueling storage tank water mass to water level.


TCPVPR:

VCPVLP :

VCPVPR:

INTEGFR VARTABLES :

IHTRIG :

INTFLG :

IPRCPV :

KFLOW :

KLEVEL :

KPRESS:

KTEMP :

LASOG:

LCPVLP :

LCPVPR :

NCPVP :

NORMDP :

NORMRS :
Time of the last control-panel vector printout.

Control-panel vector loop parameter values.

Control-panel vector primary parameter values.
Terminal (TTY) heading print trigger.

Flag that indicates an interrupt message has been received.

Control-panel vector processing flag.

$0=$ control-panel vector processing selected;

1 = control-panel vector processing not selected.

Flow output units.

$0=\mathrm{kg} / \mathrm{s}$;

$1=1 \mathrm{~b} / \mathrm{s}$.

Level output units.

$0=\mathrm{m}$;

$1=\mathrm{ft}$.

Pressure output units.

$0=\mathrm{MPa}$;

$1=$ psi.

Temperature output units.

$0=\mathrm{K}$;

$1={ }_{F}$.

Length of each interactive graphics edit.

LCM pointers to variables used in evaluating controlpanel vector loop parameters.

LCM pointers for variables used in evaluating controlpanel vector primary parameters.

Number of interactive graphic dumps.

Flag that indicates a dump in the noninteractive mode. $0=$ no dump occurs, default if code is interactive;

1 = dump occurs.

Flag that indicates if a restart was obtained from a normal noninteractive TRAC version.

$0=$ no restart, default if code is interactive;

1 = a restart was obtained. 


\section{COMDECK CPVINP}

COMMON / CPV INP / ICELLS , ICOMPS , JCELL , JCOMPS , JDISCH , LOOPCT , LOOPID , LOOPS

$$
\begin{aligned}
\text { DIMENSION } & \operatorname{ICELLS}(4,8), \operatorname{ICOMPS}(4,9), \operatorname{JCELL}(3), \operatorname{JCOMPS}(4), \operatorname{JDISCH}(4), \\
\operatorname{LOOPID}(4), \operatorname{LOOPS}(4) & \text {, }
\end{aligned}
$$

\section{INTEGER VARIABLES :}

$\operatorname{ICELLS}(\mathrm{L}, \mathrm{I})$ : Cel1 number in loop $\mathrm{L}$ where the control-panel vector parameter $I$ is located.

$I=1$, hot-leg temperature;

$=2$, cold-leg temperature;

$=3$, primary-liquid mass flow;

$=4$, ECCS liquid mass flow;

$=5$, steam-generator secondary-side pressure;

$=6$, steam-generator secondary-side steam-mass flow;

$=7$, steam-generator secondary-side main-feedwater mass flow; and

$=8$, steam-generator secondary-side auxiliaryfeedwater mass flow.

ICOMPS(L,I): Component number in loop $\mathrm{L}$ where the control-panel vector parameter $I$ is located. $I=1$ through 8 defined in $\operatorname{ICELLS}(\mathrm{L}, \mathrm{I})$.

$I=9$, steam-generator secondary-side water level.

JCELL(I): Cell number where the control-panel vector global parameter $I$ is located.

$I=2$, primary pressure; and

$=3$, containment pressure and temperature.

JCOMPS(I): Component number where, the control-panel vector global parameter $I$ is located. $I=2$ and 3 defined in $\operatorname{ICELL}(I)$.

$I=1$, reactor power; and

$=4$, pressurizer water level.

JDISCH(L): Component number for refueling storage tank discharge in loop L (should be a FILL).

LOOPCT: Number of loops in this model ( $1 \leq$ LOOPCT $\leq 4)$.

LOOPID(L): Loop identification for loop L.

LOOPS(L): Number of physical loops in loop L. 
COMDECK DEFVAL

COMMON/DEFVAL/ALPQ, HSTNQ, PQ, PAQ, QPPPQ , TLQ , TVQ, TWQ, VLQ, VVQ

COMMON/DEFVAL/ISTOPT

REAL VARIABLES:

ALPQ: Default value for initial void fractions input through NAMELIST and used to specify void fractions when ISTOPT $=1$ or 2 .

HSTNQ: Default value for initial VESSEL heat-slab temperatures input through NAMELIST and used to specify the VESSEL heat-slab temperatures when ISTOPT $=1$ or 2 .

PQ: Default value for initial pressures input through NAMELIST and used to specify pressures when ISTOPT $=1$ or 2 .

PAQ: Default value for initial air partial pressures input through NAMELIST and used to specify air partial pressures when ISTOPT $=1$ or 2 .

QPPPQ: Default value for initial volumetric heat sources in pipe walls input through NAMELIST and used to specify volumetric heat sources when ISTOPT $=1$ or 2 .

TLQ: Default value for initial liquid temperatures input through NAMELIST and used to specify liquid temperatures when ISTOPT $=1$ or 2 .

TVQ: Default value for initial vapor temperatures input through NAMELIST and used to specify vapor temperatures when ISTOPT $=1$ or 2 .

TWQ: Default value for initial wall temperatures input through NAMELIST and used to specify wall temperatures when ISTOPT $=1$ or 2 .

VLQ: Default value for initial liquid velocities input through NAMELIST and used to specify liquid velocities when ISTOPT $=1$ or 2 .

VVQ: Default value for initial vapor velocities input through NAMELIST and used to specify vapor velocities when ISTOPT $=1$ or 2 . 
INTEGER VARIABLE:

ISTOPT: Default-value input option.

COMDECK DF1DC

COMMON/DF IDC/ A11111, C1A, ClAV , C2A , C2AV , CT , CTP, DVJP, FL1 , FL2, FV1 , FV2, HAVLV, QTP , R1L , RIV , R2L , R2V , SO1, SO2, SALT, SAVT, SSAC, SSE, SSMC , SSMOM, SSVC, SSVE, VJS , ZZZZZZ

COMMON/DF IDC/IO1, IO2, IO3, IACC2, IBKS, ICLFLG, ICME , ICORL , ICORU, IIO1, IIO2, IIO3, IL, ISFLG , ISLB , ISRB , IVPVLV , JSTART , LP INDX, MSC , NC2 , NJN, NSTG, NTEE

REAL VARIABLES :

A11111: Dummy variable that provides a known start to the COMMON block.

CiA: Fraction of liquid velocity at the left face of the tee junction cell that contributes "to the momentum transfer into the tee side leg.

ClAV: Vapor velocity fraction, at the left face of the tee junction cell that contributes to the momentum transfer into the tee side leg.

C2A :

Fraction of liquid velocity at the right face of the tee junction cell that contributes to the momentum transfer into the tee side leg.

- C2AV: Vapor velocity fraction at the right face of the tee junction cell that contributes to the inomentum transfer into the tee side leg.

CT :

Momentum source coefficient.

CTP :

Momentum source coefficient.

DVJP: $\quad$ Pressure derivative of source velocity.

FL1: Temporary storage for liquid mass-flow corrections for mass conservation checks at low-numbered cell face.

FL2:

Temporary storage for liquid mass-flow corrections for mass conservation checks at high-numbered cell face.

FV1: Temporary storage for vapor mass-flow corrections for mass conservation checks at low-numbered cell face.

FV2 :

Temporary storage for vapor mass-flow corrections for mass conservation checks at high-numbered cell face. 
HAVLV: Temporary storage for the hydraulic diameter when the valve is open.

QTP :

Total direct power input.

R1L:

Coefficient for the liquid momentum source term at the left edge of the tee junction cell.

RIV : Coefficient for the vapor momentum source term at the left edge of the tee junction cell.

R2L: $\quad$ Coefficient for the liquid momentum source term at the right edge of the tee junction cell.

R2V: Coefficlent for the vapor momentum source term at the right edge of the tee junction cell.

S01: $\quad$ Sign of $\operatorname{IOU}(1$, current component).

S02: $\quad$ Sign of $\operatorname{IOU}(2$, current component).

SALT: Source term to liquid for compressible work.

SAVT: $\quad$ Source term to vapor for compressible work.

SSAC: $\quad$ Air source.

SSE : $\quad$ Energy source.

SSMC : Mass source.

SSMOM: Momentum source to left-hand cell boundary.

SSVC: $\quad$ Vapor mass source.

SSVE: $\quad$ Vapor energy source.

VJS: $\quad$ Source velocity.

ZzZzZZ: Dummy variable that provides a known end to the COMMON block.

INTEGER VARIABLES :
I01:
$\operatorname{ABS}(\operatorname{IOU}(1$, current component)).
I02:
$\operatorname{ABS}(\operatorname{IOU}(2$, current component))
I03:
IOU( 3 , current component) [always positive]
IACC2 :
Flag for pipe used to model accumulator. 
IBKS :

ICLFLG :

ICME :

ICORL :

ICORU :

II01:

II02:

II03:

IL:

ISFLG :

ISL̇B:

ISRB :

JSTART :

LPINDX:

MSC:

NC2:

NJN :

NSTG :

NTEE :
Indicator for network solution.

Flag used by the steam-generator component in the postpass to instruct the numerical stabllizer whether or not the second junction of a secondary component is connected to an external component.

Component index for referencing IOU array.

Core lower boundary.

Core upper boundary.

I01 plus a displacement for the current loop.

I02 plus a loop displacement.

I03 plus a loop displacement.

Loop number index.

Flag that indicates if the calculations are being performed for a steam-generator component.

Left-hand boundary switch.

Right-hand boundary switch.

Ce1l number at left end of one-dimensional segment.

Loop index that indicates the loop in the system.

Cell number for source terms.

Cell number that begins a tee and steam-generator tee secondary.

Number of network matrix junctions.

Counter for steam generators.

Counter for tees. 
COMDECK DIDDLE

COMMON/DIDDLE/ AFCT , ALPBCT, ALPCC,ALPLVL, ALPLVU, ALPSHL , ALPSHU, CALV2, CBMIN, ENCUT , ENFAC1 , ENFAC2 , ENMIN, FAREA1 , FAREAH, FAREAV , FMD1 , FMD2, FSE5, VDRPF , SCINAN, VDRPMX, VECLCT, VECVCT, VINTF, VLVCMX, VRBCUT, VRTCUT

COMMON/DIDDLE/NIFSLB

REAL VARIABLES :

AFCT :

Area scaling for waves on inverted annular interface.

ALPBCT :

Lower bound on bubble void fraction used to compute interfacial heat-transfer rates (and resulting $\Gamma$ ) when boiling.

ALPCC: Void fraction that gives the minimum value for the bubble condensation rate.

ALPLVL: Lowest value of the maximum adjacent void fraction allowed for calculating a plug interfacial area.

ALPLVU: Highest value of the minimum adjacent void fraction allowed for calculating a plug interfacial area.

ALPSHL: Void below which the interface sharpener is off.

ALPSHU: Void above which the interface sharpener is completely on.

CALV2: The constant a in Eq. (82), the liquid-side HTC for superheated drops.

CBMIN : Minimum allowed mean bubble diameter.

ENCUT: Minimum droplet entrainment fraction.

ENFACl: Scaling factor for minimum entrainment velocity.

ENFAC2: $\quad$ Scaling factor for entrainment correlation exponent.

ENMIN: $\quad$ Variable not implemented.

FAREAl: $\quad$ Scale factor for one-dimensional plug flow condensation area.

FAREAH: $\quad$ Scale factor for three-dimensional separated plug flow horizontal condensation area.

FAREAV: $\quad$ Scale factor for three-dimensional separated plug flow vertical condensation area. 
FMD1 :

Factor scaling the gravity void-fraction adjustment. If both are zero, there is no manometer damping.

FMD2 :

Factor scaling the gravity void-fraction adjustment. If both are zero, there is no manometer damping.

FSE5: Scale factor for pool entrainment.

SCINAN : $\quad$ Scale factor for inverted annular drag.

VDRPF : Scale factor in the expression for the limiting circulation velocity in a drop.

VDRPMX: Maximum allowed internal c1rculation velocity in a drop.

VECLCT: $\quad$ Lowest allowed liquid speed when computing interfacial coefficients.

VECVCT: Lowest allowed vapor speed when computing interfacial coefficients.

VINTF: $\quad$ Factor in expression for mean circulation speed in a moving drop.

VLVCMX: Maximum liquid velocity used for the condensation ALV correlation.

VRBCUT: Minimum allowed relative velocity for computing ALV during bolling.

VRTCUT: Minimum allowed relative speed for computing interfacial coefficients (except during boiling).

INTEGER VARIABLES :

NIFSLB: If nonzero, then slabs should be used to test for inverted annular flow.

COMDECK DIDDLH

COMMON/DIDDLH/ AFLML, AFLMU, ALP2 , ALP3 , ALPBR , AL.PCUT , ALOW, AUP , FDFHL , HGF , HGVMN COMMON/DIDDLH/ IHTAV, IHTCN, NSHTCN

REAL VARIABLES :

AFLML: Vold fraction below which Bromley film boiling contributes fully to the liquid.

AFLMU: Void fraction above which no Bromley coefficient is added to the liquid. 
ALP2: Void above which vapor is in forced convection.

ALP3: Void above which there is no liquid heat transfer.

ALPBR: Void above which liquid convection is linearly faired of $f$ and vapor connection is faired on.

ALPCUT: Void above which nucleate boiling is not permicted (if other criteria are met).

ALOW: $\quad$ Lowest value of $\alpha$ in adjacent cells.

AUP: $\quad$ Highest value of $\alpha$ in adjacent cells.

FDFHL: A scaling factor for the wall to droplet heat-transfer correlation.

HGF : Function of nucleate-boiling heat transfer, which contributed to subcooled boiling.

HGVMN : Cutoff velocity for condensation used to suppress subcooled nucleate boiling.

INTEGER VARIABLES :

IHTAV: Variable is normally $1 . "$ If IHTAV is 0 , then there is no time averaging of HTCs.

IHTCN: Variable is normally 0 . If set to 1 , then HTCs are forced to remain constant.

NSHTCN: Variable is normally 10000 000. If NSTEP > NSHTCN, then IHTCH is set to 1 (for debugging only).

COMDECK DIMNSION

COMMON/DIMEN/IFREE , JNVSSL, LAST , LENBD, LENDIM, LENTBL, LFREE, LLAST, LVER , NCOMP, NCOMPT, NJNMX, NJNT, NJUN, NLOOPS , NMVS SL , NPX, NSTGJ , NTHM, NTHMID, NTHM3D , NUMTCR , NVCON

INTEGER VARIABLES:

IFREE: $\quad$ First free word in the dynamic storage area.

JNVSSL: Maximum number of vessel junctions in a loop.

LAST: $\quad$ Last word in the dynamic storage area.

LENBD: $\quad$ Length of boundary data array for each junction.

LENDIM: Variable that dimensions the component variable-length tables. 


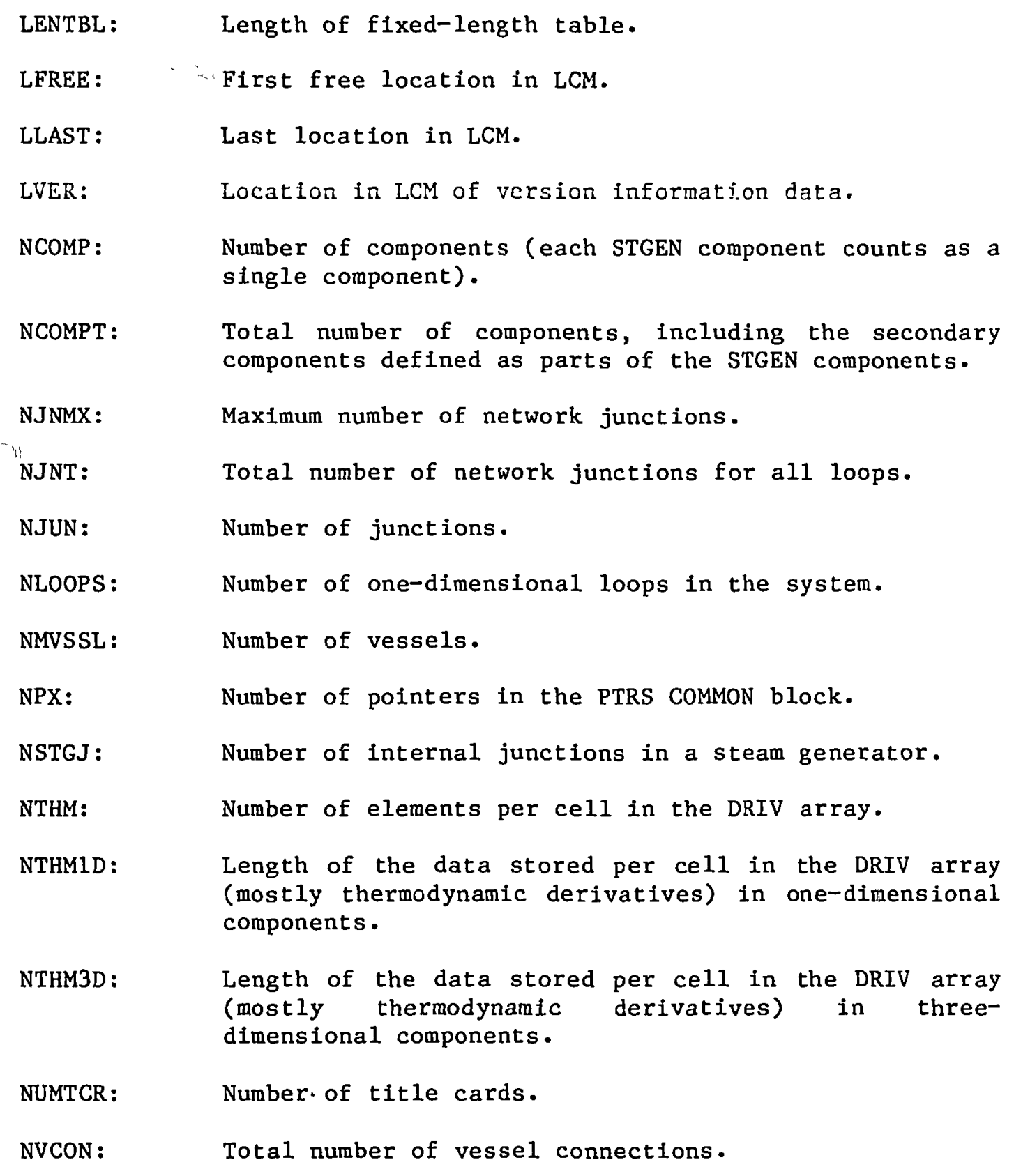


COMDECK DLIMIT

COMMON/DLIM/DELAMX , DELCMX, DELDMX, DELEMX, DELPMX, DELRMX, DELVMX, DELXMX, DTBKUP , FPMAX, FXMAX , GXMAX

COMMON/DLIM/NLIM

DIMENSION $\operatorname{NLIM(8)}$

REAL VARIABLES:

DELAMX:

DELCMX:

DELDMX:

DELEMX:

DELPMX :

DELRMX:

DELVMX:

DELXMX:

DTBKUP :

FPMAX:

$\dddot{2}$

FXMAX:

GXMAX :
Time-step limit caused by void-fraction change.

Time-step limit caused by maximum changes in pressures and temperatures.

Time-step limit caused by numerical considerations in the rod heat transfer.

Time-step limit caused by vessel mass errors.

Estimated time-step size that results in a $20 \%$ change in core power.

Time-step limit caused by final value of the percentage variation in pressure from iteration to iteration.

Material Courant stability limit (computed only in vessels).

The maximum time-step size permitted for adjustment of VALVE components.

Desired time-step size for repeating a time step when the back-up is detected by subroutine HOUT.

Maximum fractional change (0.2) in core power per time step.

Valve adjustment algorithm parameter $(0.4)$.

Maximum limit on the change in the VALVE flow-area fraction over a time step.

INTEGER VARIABLES :

$\operatorname{NLIM(8):~Array~that~stores~the~number~of~times~each~of~the~}$ time-step limits controls the time-step size since the last major edit. 
COMDECK DMPCTRL

COMMON/ CTRLDP / DMPF LG , DMP INT , LTDUMP , TDUMP

COMMON/CTRLDP / ICTRLD, NSDO

DIMENSION ICTRLD(8)

REAL VARIABLES :

DMPINT: Dump interval for time domain.

LTDUMP: $\quad$ CPU time when last dump was taken.

TDUMP: Calculation time when next dump will be taken.

INTEGER VARIABLES :

DMPFLG: Flag that signals whether the dump output file has been initialized.

$0=$ uninitialized;

1 = initialized.

ICTRLD(8): Array that contains buffering information about the dump output file.

NSDO: $\quad$ Time-step number of last completed dump.

COMDECK ELVKF

COMMON/ELVKF/IELV, I INL , IKFAC

INTEGER VARIABLES :

IELV: Input option switch that allows user to input cellcentered elevations for gravity term.

IINL: Index for the two passes through INIT.

IKFAC: Input option switch that allows user to input $K$ factors for additive loss coefficients. 
COMDECK ERRCON

COMMON /ERRCON/DARA ,DARL, DARV ,DDVL, DDVV , DTLL, DTLLM, DTLU , DTLUM, DTVL, DTVLM, DTVU, DTVUM, TIMDL , TIMDU

COMMON/ERRCON/ICHGA, IDARA, IDARL, IDARV , IDDVL, IDDVV, IDTLL , IDTLU, IDTVL, IDTVU, ILRE IT , IPTEST , JDARA, JDARL, JDARV , JDDVL, JDDVV, JDTLI, JDTLU, JDTVL, JDTVU, JPTEST , NSDL, NSDU

REAL VARIABLES :

DARA:

Maximum change in $\alpha \rho_{a}$ *

DARL :

Measure of the maximum difference in $(1-\alpha)_{\ell}$ between the basic and stabilizer steps.

DARV :

Measure of the maximum difference in $\alpha \rho \mathrm{g}$ between the basic and stabilizer steps.

DDVL: $\quad$ Measure of the maximum difference in $V_{\ell}$ between the basic and stabilizer steps.

DDVV: Measure of the maximum difference in $\mathrm{v}_{\mathrm{g}}$ between the basic and stabilizer steps.

DTLL: Largest decrease in $\mathrm{T}_{\ell}$ for current iteration.

DTLLM: DTVLM and DTLLM are limits on DTVL and DTLL beyond which another iteration must be performed.

DTLU: $\quad$ Largest increase in $\mathrm{T}_{\ell}$ for current iteration.

DTLUM: DTVLM and DTLLM are limits on DTVL and DTLL beyond which another iteration must be performed.

DTVL: $\quad$ Largest decrease in vapor temperature in a given iteration.

DTVLM: DTVLM and DTLLM are limits on DTVL and DTLL beyond which another iteration must be performed.

DTVU: $\quad$ Largest increase in $\mathrm{T}_{\mathrm{g}}$ for current iteration.

DTVUM: DTVLM and DTLLM are limits on DTVL and DTLL beyond which another iteration must be performed.

TIMDL: If TIMDL S TIMET S TIMDU, details of DARV, etc., should be printed.

TIMDU: If TIMDL $\leq$ TIMET $\leq$ TIMDU, details of DARV, etc., should be printed. 


\section{INTEGER VARIABLES :}

ICHGA:

IDARA :

IDARL :

IDARV :

IDDVL:

IDDVV :

IDTLL:

IDTLU:

IDTVL:

IDTVU:

ILREIT:

IPTEST :

JDARA :

JDARL :

JDARV :

JDDVL:

JDDVV:

JDTLL:

JDTLU :

JDTVL:

JDTVU:

JPTEST:

NSDL :
Flag to print maximum void-fraction changes to the message file.

Component where DARA occurred.

Component where DARL occurred.

Component where DARV occurred.

Component where DDVL occurred.

Component where DDVV occurred.

Component where DTLL occurred.

Component where DTLU occurred.

Component where DTVL occurred.

Component where DTVU occurred.

Flag that allows reiteration messages when equation set changes.

Component with maximum $|\delta \mathrm{p} / \mathrm{p}|$.

Cell where DARA occurred.

Cell where DARL occurred.

Cel1 where DARV occurred.

Ce11 where DDVL occurred.

Cell where DDVV occurred.

Ce11 where DTLL occurred.

Ce11 where DTLU occurred.

Cell where DTVL occurred.

Ce11 where DTVU occurred.

Cell with maximum $|\delta \mathrm{p} / \mathrm{p}|$.

If NSDL $\leq$ NSTEP $\leq$ NSDU, a detailed diagnostic of DARV, ? etc., should be printed to TRCMSG. 
NSDU: $\quad$ If NSDL $\leq$ NSTEP $\leq$ NSDU, a detailed diagnostic of DARV, etc., should be printed to TRCMSG.

\section{FIXED-LENGTH TABLES}

The structure of the fixed-length data tables is shown below and is identical for all TRAC components. Refer to Sec. VI for a detailed discussion of the TRAC data base.

\section{COMDECK FIXEDLT}

COMMON/FLTAB/HTLSCI, HTLSCO

$$
==-\infty
$$

COMMON/FLTAB/CTITLE ; ICFLG , ID , IREST, LENARR, LENFV, LENFV2, LENPTR, LENVLT, LEXTRA, LFV, LFVN, NCELLT , NODES, NRVLT , NUM, NUMBM1, NUMBM2, NUMBM3, NUMBN1 , NUMBN2, NUMBN3 , TYPE

DIMENSION CTITLE(3)

REAL VARIABLES :

HTLSCI: Inside component heat loss.

HTLSCO: Outside component heat loss.

INTEGER VARIABLES :

CTITLE(3): Component description.

ICFLG: Cell-edge choked-flow model options.

IV: Component identification.

IREST: Component restart indicator.

LENARR: Length of array block.

LENFV: " Length of fundamental variables.

LENFV2: Length of fundamental variables for which old time and new time values are the same at the start of the OUTER code block.

LENPTR: $\quad$ Length of pointer table.

LENVLT: $\quad$ Length of variable-length table.

LEXTRA: Length of nonstandard dump for components.

LFV: Relative position of old fundamental variables. 


$\begin{array}{ll}\text { LFVN: } & \text { Relative position of new fundamental variables. } \\ \text { NCELLT: } & \text { Total number of cells. } \\ \text { NODES: } & \text { Number of heat-transfer nodes. } \\ \text { NRVLT: } & \text { Number of real variables in each component VLT COMMON } \\ & \text { block. } \\ \text { NUM: } & \text { Component number. } \\ \text { NUMBMl : } & \text { Indices to access large numerals for printing compo- } \\ & \text { nent NUM and IORDER. } \\ \text { NUMBM2: } & \begin{array}{l}\text { Indices to access large numerals for printing compo- } \\ \text { nent NUM and IORDER. }\end{array} \\ \text { NUMBM3: } & \text { Indices to access large numerals for printing compo- } \\ \text { nent NUM and IORDER. } & \text { Indices to access large numerals for printing compo- } \\ \text { NUMBN1: } & \text { nent NUM and IORDER. } \\ \text { NUMBN2: } & \text { Indices to access large numerals for printing compo- } \\ \text { nent NUM and IORDER. } & \text { Indices to access large numerals for printing compo- } \\ \text { NUMBN3: } & \text { nent NUM and IORDER. } \\ \text { TYPE: } & \text { Component type. }\end{array}$

COMDECK FIXUM

COMMON/FIXUM/NOAIR, NSMEC, NTHRMC , NVTC

INTEGER VARIABLES :

$\begin{array}{ll}\text { NOAIR: } & \text { Variable that turns off air field calculations. } \\ \text { NSMEC: } & \begin{array}{l}\text { Variable that turns of stabilizer mass and energy } \\ \text { equations }\end{array} \\ \text { NTHRMC: } & \text { Variable that turns off (debugs) basic equation set. } \\ \text { NVTC: } & \text { Variable that turns off stabilizer motion equations. }\end{array}$




\section{COMDECK GRAPHICS}

COMMON/GRAPH/EDINT , GF INT , SEDINT , TEDIT , TGRAF , TSEDIT

COMMON/GRAPH/I I BUF F, ICTRLG, IPKG , KP, LCAT , LCMGCT , LENCAT , NCTX, NSGO, NWTX

[NUCLEAR PLANT ANALYZER (NPA) VARIABLES LISTED BELOW CURRENTLY ARE INTENDED ONLY FOR IN-HOUSE LOS ALAMOS USE.]

COMMON/GRAPH/EDTBLK, NBLKOUT, IGROUT , IBLK

DIMENSION ICTRLG(8)

REAL VARIABLES:

EDINT: $\quad$ Print edit interval for time domain.

GFINT: $\quad$ Graphics edit interval for time domain.

SEDINT: $\quad$ Interval for short edits.

TEDIT: $\quad$ Time of next print edit.

TGRAF: $\quad$ Time of next graphics edit.

TSEDIT: $\quad$ Time for next short edit.

INTEGER VARIABLES :

IBLK: Counter for number of data-edit blocks.

IBUFF: Length of graphics buffer.

ICTRLG(8): Array that contains buffering information about the graphics output file.

IGROUT: Controller that maintains the address for NPA random disk writes.

IPKG: $\quad$ Graphics file packing density.

KP: $\quad$ Pointer in graphics catalog block.

LCAT: $\quad$ Address of graphics catalog in SCM.

LCMGCT: Address of graphics catalog in LCM.

LENCAT: Number of words in each catalog entry. $\quad \approx$

NBLKOUT: Counter for the number of words written to buffer in current graphics data edit. 
NCTX: Number of graphics catalog entries.

NSGO: Time-step number of last completed graphics edit.

NWTX: Number of words written to disk per graphics edit.

LOGICAL VARIABLE:

EDTBLK: Flag that is set to TRUE when graphics file is written to start of the first data edit.

COMDECK HOLERITH

COMMON/HOLL/ACCUMH, BREAKH, COREH, CTAINH, FILLH, PIPEH, PLENH, PRIZRH, PUMPH, STGENH, TEEH, TURBH, VALVEH, VSSLH

INTEGER VARIABLES :

$\begin{array}{ll}\text { ACCUMH: } & \text { Hollerith representation of word "ACCUM." } \\ \text { BREAKH: } & \text { Hollerith representation of word "BREAK." } \\ \text { COREH: } & \text { Hollerith representation of word "CORE." } \\ \text { CTAINH: } & \text { Hollerith representation of word "CTAIN." } \\ \text { FILLH: } & \text { Hollerith representation of word "FILL." } \\ \text { PIPEH: } & \text { Hollerith representation of word "PIPE." } \\ \text { PLENH: } & \text { Hollerith representation of word "PLENUM." } \\ \text { PRIZRH: } & \text { Hollerith representation of word "PRIZER." } \\ \text { PUMPH: } & \text { Hollerith representation of word "PUMP." } \\ \text { STGENH: } & \text { Hollerith representation of word "STGEN." } \\ \text { TEEH: } & \text { Hollerith representation of word "TEE." } \\ \text { TURBH: } & \text { Hollerith representation of word "TURB." } \\ \text { VALVEH: } & \text { Hollerith representation of word "VALVE." } \\ \text { VSSLH: } & \text { Hollerith representation of word "VESSEL." }\end{array}$


COMDECK HOLLR

COMMON/HOLLR/HPLEN, HPRIZR, VSS

REAL VARIABLES:

$\begin{array}{ll}\text { HPLEN : } & \text { Real Hollerith PLENUM component name. } \\ \text { HPRIZR: } & \text { Real Hollerith PRIZER component name. } \\ \text { VSS: } & \text { Real Hollerith VESSEL component name. }\end{array}$

COMDECK IOUNITS

COMMON/UNITS/CARD, IBFADD, IBFADG, IBFADR, IBFLND, IBFLNG, IBFLNR, IDOUT, IGOUT, IMOU T, IN, INPROC, IODONE, IOERR, IOSKIP, IOUT, IRSTRT , ITTY, LCMCPD

INTEGER VARIABLES :

CARD(9): Variable that contains the current input card in character format.

IBFADD: $\quad$ Pointer to beginning of dump LCM buffer.

IBFADG: Pointer to beginning of graphics LCM buffer.

IBFADR: Polnter to beginning of restart LCM buffer.

IBFLND: $\quad$ Length of dump buffer.

IBFLNG: $\quad$ Length of graphics buffer.

IBFLNR: Length of restart buffer.

IDOUT: I/O unit number for dump output file (currently set to unit 12).

IGOUT: I/O unit number for graphics output file (currently set to unit 11 ).

IMOUT: I/O unit number for warning messages (currently set to unit 7).

IN: I/O unit number for input to TRAC (initially set to unit 5 to point to file TRCINP; if the input does not invoke free format, IN is changed to 1 to point to file TRACIN).

INPROC: Flag used during input that indicates whether component data is being processed. 

IODONE: Flag that indicates if the current input card has been read.
IOERR: Input error flag.
IOSKIP: Flag that turns input processing off and on.
IOUT: I/O unit number for printed output file (currently set to unit 6 ).
IRSTRT: I/O unit number for restart input file (currently set to unit 13$)$.
ITTY: I/O unit number for terminal output (currently set to unit 59).
LCMCPD: $\quad$ Storage for the beginning address for reading from or writing to LCM with calls to subroutines RDLCM and WRLCM.

COMDECK ITERSTAT

COMMON/ISTAT/VARERM, VERR

COMMON/ISTAT/IIBIG, IIFAIL, IITNO, IITV, IOTT, NSTEP , OITNO

REAL VARIABLES :

VARERM: Maximum variable error.

VERR: Velocity error at component junction.

INTEGER VARIABLES :

IIBIG: Maximum number of inner iterations per outer iteration.

IIFAIL: Flag that indicates failure of hydrodynamics to converge.

IITNO: Inner iteration number.

IITV: Variable that passes the vessel inner iteration count.

IOTT: Temporary storage for IITNO.

NSTEP: $\quad$ Number of time steps taken.

OITNO: $\quad$ Outer iteration number. 
COMDECK JUNCTION

COMMON/JUNCT/JMATCH , JPTR

INTEGER VARIABLES :

JMATCH: Counts the number of bad junction numbers detected during the network trace in SRTLP.

JPTR: $\quad$ Number of junction-component pafrs.

COMDECK LCMSPACE

COMMON/LCMSP/ALCM(131071)

ALCM(131071): Dynamic LCM storage area.

COMDECK MDFCOM

COMMON/MDFCOM/ICLIST(100), INPT, INRST

DIMENSION ICLIST(100)

INTEGER VARIABLES :

ICLIST(100): Component number 11st that is used during interactive deck modifications.

INPT: Unit number for the Input deck that is to be interactively modified.

INRST: Unit number for the restart input deck, which contains interactively modified components.

COMDECK MEMORY

COMMON/TIMER/ADATE, ATIME , CPTIME , CPUT, SYSTIM, TIMEI , TIMIO, TLTSS

COMMON/TIMER/NSTEPT

REAL VARIABLES:

ADATE: Storage for the date obtalned from a call to the system routine DATE.

ATIME: : Storage for the time obtalned from a call to the system routine DATE.

CPTIME: $\quad$ Storage for the CPU time obtained from a call to the system routine TIMING. 

CPUT: Storage for the cumulative CPU time from previous jobs in a Iinked series of calculations; CPUT is set to 0.0 at time 0.0 .
SYSTIM: Storage for the system time obtained from a call to the system routine TIMING.
TIMEI: Storage for the time limit of the current job and obtained from a call to the system routine GETJTL.
TIMI0: Storage for the I/O time obtained from a call to the system routine TIMING.
TLTSS: $\quad$ Storage for the charge time obtained from a call to the system routine SECOND.

INTEGER VARIABLE:

NSTEPT: Storage for the cumulative number of time steps from previous jobs in a linked series of calculations; NSTEPT is set to 0 at time 0.0 .

COMDECK NMFAIL

COMMON/NMFAIL/S3

COMMON/NMFAIL/IFTP, ITFL1, NFL1, NFL3

REAL VARIABLE:

S3: $\quad$ offset on liquid super heat used in interfacial area ad justment.

\section{INTEGER VARIABLES :}

IFTP: Flag that prevents thermal failure messages if a message has come from TF1SD3 or FF3D.

ITFLl: Iteration number of the last TF1DS3 failure.

NFL1: Total number of TFIDS3 fallures in the current time step.

NFL3: Total number of FF3D failures in the current time step. 
COMDECK NPA

[NUCLEAR PLANT ANALYZER (NPA) VARIABLES LISTED BELOW CURRENTLY ARE INTENDED ONLY FOR IN-HOUSE LOS ALAMOS USE.]

COMMON/NPA/CONNPA, DTDUMP, DTEDIT , DTNPA, SRATIO, TGEDIT , TIMNPA, TPAUSE, TPDUMP, TRCNPA, TSTOP, VALNPA, ZNAME

COMMON/NPA/ IACT , IFD, NACT , NFNPA, NPACOM, NTSNPA

DIMENSION TIMNPA(100), VALNPA(100), ZNAME (6), NAPCOM(100)

REAL VARIABLES:

CONNPA:

Hollerith data name for the NPA executive "master" program connected to the process-to-process (PTP) data path.

DTDUMP: $\quad$ Time edit, defined interactively by the the NPA user, between dump (restart) edits to file TRCDMP.

DTEDIT: $\quad$ Time interval, defined interactively by the NPA user, between large edits to file TRCOUT.

DTNPA: Time error (0.1 s) allowed to implement an NPA user command before the user is prompted by the system to decide if a back-up procedure should be performed to implement the command action at the correct problem time.

SRATIO: Maximum speed ratio of the problem time-step size to the computer time required to evaluate the time step.

TGEDIT: $\quad$ Problem time after which the next NPA user-defined graphics edit to file TRCGRF will occur.

TIMNPA(100): Array that defines the problem time when a pending NPA user-defined action will be implemented.

TPAUSE: $\quad$ Problem time when the next NPA user-defined pause in the TRAC calculation will occur.

TPDUMP: $\quad$ Problem time after which the next NPA user-defined dump (restart) edit to file TRCDMP will occur.

TRCNPA: Hollerith data name for the TRAC "slave" program connected to the PTP data path.

TSTOP: $\quad$ Problem time specified by the NPA user after which the TRAC calculation will terminate. 
VALNPA(100): Array that defines the desired value of an NPA usercontrolled action that is pending at time TIMNPA.

ZNAME(6): Array containing a Hollerith data message that communicates NPA user commands to TRAC or TRAC warning messages to the NPA executive program.

\section{INTEGER VARIABLES :}

IACT : Flag that is changed from 0 to 1 when an additional graphics edit and dump (restart) edit will be performed before an NPA user-defined component-action adjustment is performed.

IFD: $\quad$ Number of PTP data paths.

NACT: $\quad$ Number of pending NPA user-defined commands stored in arrays TIMNPA, VALNPA, and NPACOM.

NFNPA: $\quad$ Flag that is changed from 0 to 1 when file PTPDSK will not be destroyed at the end of the TRAC run.

NPACOM(100): Array that defines the component number to which a pending NPA user-defined action will be applied at time TIMNPA.

NTSNPA: Number of TRAC time steps (2) between TRAC status messages that are being sent to the NPA executive program.

COMDECK PIPEPT

COMMON / PTAB/LPOWRF, LPOWTB, LQP3RF, LQP3TB , PTDUM

INTEGER VARIABLES :

LPOWRF: A-array pointer variable for the rate-factor table that is applied to the independent variable of the reactivity/power table.

LPOWTB: A-array pointer variable for the reactivity/power definition table.

LQP3RF: A-array pointer variable for the rate-factor table that is applied to the independent variable for the $q^{\prime \prime-}$ source table.

LQP3TB: A-array pointer variable for the $\mathrm{q}^{\cdots}$ source table.

PTDUM: Dummy array that accounts for the remaining unused variables in the PTAB COMMON block. 
COMDECK POINTERS

-----GENERAL POINTERS-----

COMMON/PTRS/I11111, LBD, LCNTL , LCOMPT , LDRA , LDRC , LI ITNO , LIJVS , LILCMP , LIOU, LISVF, LIVCON, LIVLJN, LJOUT, LJ SEQ, LJUN, LLCON, LLOOPN, LMA'T B , LMCMSH, LMSCT, LNBR, LNJN, LNSIG, LNSIGP, LNVCNL . LORDER, LPRPTB, LPTBLN, LTITLE, LVSI

-----NETWORK SOLUTION POINTERS------

COMMON/PTRS/JAOL, JAOV , JDRA , JDRC , JDREL , JDREV , JDRL , JDRV , JN JUN , JOD, LAOL , LAOU, LATJV, LDPVC, LDPVCV , LDREL, LDREV , LDRL , LDRV , LDVB , LIDPCV, LOD, LVRH

- - COMBINATION OF UNSHIFTED POINTERS AND ARRAY LENGTHS-----

COMMON/PTRS/LENFXD, LFXD, LVMAT , LVSSC , LVS SIP , NMAT, NVCELL , NZZZZZ

DIMENSION IPT(62)

GENERAL POINTERS:

I11111: Dummy variable that provides a known start to the COMMON block.

LBD: $\quad$ Boundary data array, described in Table xx of Sec. VI.

LCNTL: Signal-varlable, trip, and controller data array described in Fig. 52 of Sec. VI.

LCOMPT: Component LCM pointers stored in the order used for iteration.

LDRA: Storage for right-hand side of the air stabilizer equation.

LDRC: Pointers for network variables for the solute tracking option.

LIITNO: Number of Inner Iterations during the last outer iteration for each component (in the order used for iteration).

LIJVS: Pointer for a temporary array that contains a list of all junction numbers that link to a vessel.

LILCMP: $\quad$ Component LCM pointers stored in the order in which components were read.

LIOU: Network junction numbers for the junctions of all components excluding BREAKs and FILLs. 
LISVF: $\quad$ Pointer to an array of flags indicating whether or not a particular component is used to evaluate one or more signal variables $(-1$, no signal variable; +1 , signal variable). This array uses the same order in which the component data are processed.

LIVCON: Pointer to network junction numbers that connect to a vessel.

LIVLNN: IVLJN(I) is the vessel junction number that corresponds to the network junction number given by $\operatorname{IVCON}(I)$.

LJOUT: $\quad$ Storage area for pointers that locate the beginning of each system loop within data for IOU.

LJSEQ: Junction numbers in the order in which junctions occur in the junction-component array.

LJUN: $\quad$ Junction-component pair array, described in Table XIX.

LLCON: Number of times each component was the last to converge since last edit.

LLOOPN: IA(LLOOPN+IL-1) gives the element of the IORDER array that begins the $I L^{\text {th }}$ loop.

LMATB: $\quad$ Pointer for additional material-property ID number.

LMCMSH: $\quad$ Storage for number of coarse-mesh vessel source cells or absolute cell index if direct vessel solution is used.

LMSCT: Temporary storage for vessel pressure changes adjacent to sources.

LNBR: $\quad$ Component numbers stored in the order in which components were read.

LNJN: $\quad$ NJN(IL) is the number of network junctions in loop IL.

LNSIG: NSIG(IL) is the total number of components excluding BREAKs and FILLs in a loop.

LNSIGP: $\quad$ NSIGP(IL) is NSIG(IL) plus the number of steam generators (STGENs) in loop IL.

LNVCNL: IA(LNVCNL+IL-1) points to the elements of IVCON and IVLJN that begin the $\mathrm{IL}^{\text {th }}$ loop.

LORDER: Component numbers stored in the order used for: iteration. 
LPRPTB: Pointer to user-defined material-property tables.

LPTBLN: $\quad$ Pointer for the number of entry groups in the userdefined material-property table.

LTITLE: Problem title and version information (stored using only the first four bytes of each word).

LVSI: Junction flow reversal indicators in the order in which junctions occur in the junction-component array.

NETWORK SOLUTION POINTERS:

LAOL: Variable to rework solution of ARL, AREL, and VLT (contains rework matrix).

LAOU: $\quad$ Network junction coefficient matrix.

LAOV: Variable to rework solution of ARV, AREV, and VVT (contains rework matrix).

LDPVC: Locator that shows the beginning of coefficients to evaluate the derivatives of junction velocities with respect to vessel pressures.

LDPVCV: Pointer for reordered coupling coefficients between the vessel and the one-dimensional network solution.

LDREL: $\quad$ Storage for right-hand side of the liquid stabilizer equation.

LDREV: Storage for right-hand side of the vapor stabilizer equation.

LDRL: Variable to rework solution of ARL and VLT (contains right-hand side of linear equations).

LDRV: Variable to rework solution of ARV and VVT (contains right-hand side of linear equations).

LDVB: $\quad$ Storage for the right-hand side of the network junction equations or the changes in junction velocities.

LIDPCV: $\quad$ Pointers to coefficients stored in DPCV.

LOD: " Temporary storage for intercomponent coupling information.

LVRH: $\quad$ Storage for explicit information to evaluate equations of motion at network junctions. 
JAOL:

Pointer for steam-generator internal network solution matrix.

JAOV:

Pointer for steam-generator internal network solution matrix.

JDRA: $\quad$ Pointer for steam-generator internal network solution vector.

JDRC:

Pointer for steam-generator internal network solution vector.

JDREL :

Pointer for steam-generator internal network solution vector.

JDREV: $\quad$ Network vector internal to the steam generator.

JDRL: $\quad$ Pointer for steam-generator internal network solution vector.

JDRV: $\quad$ Pointer for steam-generator internal network solution vector.

JNJUN: Temporary storage location used to define the number of junctions in the current network solution procedure.

$\mathrm{J}$

Pointer for steam-generator internal network solution matrix.

COMBINATION OF UNSHIFTED POINTERS AND ARRAY LENGTHS:

LENFXD :

Length of data that always remains in the SCM array A.

LFXD:

First word address in the A array of the data defined by LENFXD.

LVMAT: Vessel matrix storage for coarse-mesh rebalance or direct inversion.

LVSSC: $\quad$ Right-hand side of equation associated with LVMAT.

LVSSIP: $\quad$ Pivoting information for LVMAT.

NMAT :

Number of additional material-property tables provided by the user.

NVCELL: Total number of coarse regions when iteration is used on the vessel or total number of cells in all vessels if direct solution is used. 
NZZZZZ: Dummy integer that provides a known end to the COMMON block.

COMDECK PSE

COMMON / PSE/NP ICMP , NPSE , NPSE1 , NPSE3 , NPSIZ , NPSJ , NP SK, NPSV1

INTEGER VARIABLES:

NPICMP: Component number in TFIDS (pause) if NSTEP $=$ NPSEl.

NPSE: $\quad$ Pause in TRANS if NSTEP $=$ NPSE.

NPSEl: $\quad$ Pause in TFlDS if NSTEP = NPSE1. The cell number is NPSJ, and the component number is NPICMP.

NPSE3: $\quad$ Pause in TF3DI if NSTEP $=$ NPSE3. The cell index $K$ is NPSK, and the second level is NPSIZ.

NPSIZ: Leve1 in TF3DI (pause) if NSTEP = NPSE3.

NPSJ: Cell number in TFIDS (pause) if NSTEP = NPSE1.

NPSK: $\quad$ Cell index in TF3DI (pause) if NSTEP = NPSE3.

NPSV1:,$\ldots$ Pause in TFIDS1 if NSTEP = NPSE1. The cell number is

COMDECK RESTART! NPSJ, and the component number is NPICMP.

COMMON/RSTART/DDATE, DDTIME

COMMON/RSTART/DLNFLT, DNCOMP, ICTRLR

DIMENSION ICTRLR(8)

REAL VARIABLES :

DDATE: $\quad$ Date restart file was created.

DDTIME: $\quad$ Time restart file was created.

INTEGER VARIABLES:

DLNFLT: Length of fixed-length tables read from restart file.

DNCOMP: Number of components in the restart file.

ICTRLR(8): Array that contains buffering information about the restart file. 
COMDECK SIGNAL

COMMON/SIGNAL/CPV , DSV

DIMENSION CPV (42), DSV(2)

REAL VARIABLES:

CPV(42): Control-panel vector for storing the values of signal-variable parameter numbers 1 through 6 for the global parameters and 7 through 15 for up to four coolant loops.

DSV(2): Dummy signal-variable vector for storing the values of signal-variable parameter numbers 16 and 17.

COMDECK SOLCON

COMMON/SOLCON/CNC, CNMAX, CNMIN, CNT, CNTLMN, CNTLMX

REAL VARIABLES:

CNC: Constant term ( $\mathrm{kg}$ solute/kg liquid) in linear fit to solubility.

CMMAX: Solubility (kg solute/kg liquid) when liquid temperature is at or above CNTLMX.

CNMIN : Solubility ( $\mathrm{kg}$ solute/kg liquid) when : liquid temperature is at or below CNTLMN.

CNT: Coefficient of liquid temperature (kg solute/ $\mathrm{kg}$ liquid $\mathrm{k}$ ) in linear fit.

CNTLMN: Minimum liquid temperature $(K)$ to use linear fit.

CNTLMX: Maximum liquid temperature $(K)$ to use linear fit.

COMDECK STDYERR

COMMON/SSCON/EPS , EPSPOW, FMAX, FMXLVZ, MAXFLN, MINVEL , RTWFP , STIME, TPOWR

COMMON/SSCON/IPOVEL, IPOWR, LOK, NCORES, NEF

DIMENSION $\operatorname{FMAX}(7), \operatorname{LOK}(7,2)$

REAL VARIABLES :

EPS: Band on steady-state time for editing and terminating the problem. 


\begin{tabular}{|c|c|}
\hline EPSPOW: & $\begin{array}{l}\text { Core power to be initialized when the liquid velocity } \\
\text { steady-state change ratio is less than or equal to } \\
\text { EPSPOW. }\end{array}$ \\
\hline $\operatorname{FMAX}(7):$ & Maximum normalized errors. \\
\hline FMXLVZ: & $\begin{array}{l}\text { Maximum normalized rate of change for axial vessel } \\
\text { velocities. }\end{array}$ \\
\hline MAXFLN : & $\begin{array}{l}\text { Maximum one-dimensional mass flow at this steady-state } \\
\text { convergence test. }\end{array}$ \\
\hline MINVEL : & Minimum liquid velocity for initializing core power. \\
\hline RTWFP : & $\begin{array}{l}\text { Ratio of heat-transfer to fluid-dynamics time-step } \\
\text { sizes. }\end{array}$ \\
\hline STIME : & Current calculation time for steady state. \\
\hline TPOWR: & $\begin{array}{l}\text { Steady-state time (s) when the rod power should be } \\
\text { turned on. }\end{array}$ \\
\hline
\end{tabular}

INTEGER VARIABLES:

$\begin{array}{ll}\text { IPOVEL: } & \begin{array}{l}\text { Flag that initializes core power based on liquid } \\ \text { velocity in core. } \\ \text { O = liquid velocity in core less than or equal to } \\ \text { MINVEL; }\end{array} \\ & 1=\text { liquid velocity in core greater than MINVEL. } \\ \text { IPOWR: } & \text { Flag that turns on the power in steady state. } \\ \text { LOK }(7,2): & \text { Location of maximum normalized errors. } \\ \text { NCORES: } & \text { Total number of core and vessel components. } \\ \text { NEF: } & \text { Number of time steps between steady-state checks. }\end{array}$

COMDECK SYSSUM

COMMON/SYSSUM/ALQCOR, ALQPRZ, ALQUP , CORWM , PMX, TLMX, TLNCOR, TRODMX, TSHCOR, TSNCOR, TVMX, VOLCOR, XLQCOR, XTSHCR

COMMON/SYSSUM/JPMX, JTLMX, JTVMX, NPMX, NTLMX, NTVMX

REAL VARIABLES:
ALQCOR:
Mean core liquid fraction.
ALQPRZ:
Mean pressure liquid fraction. 


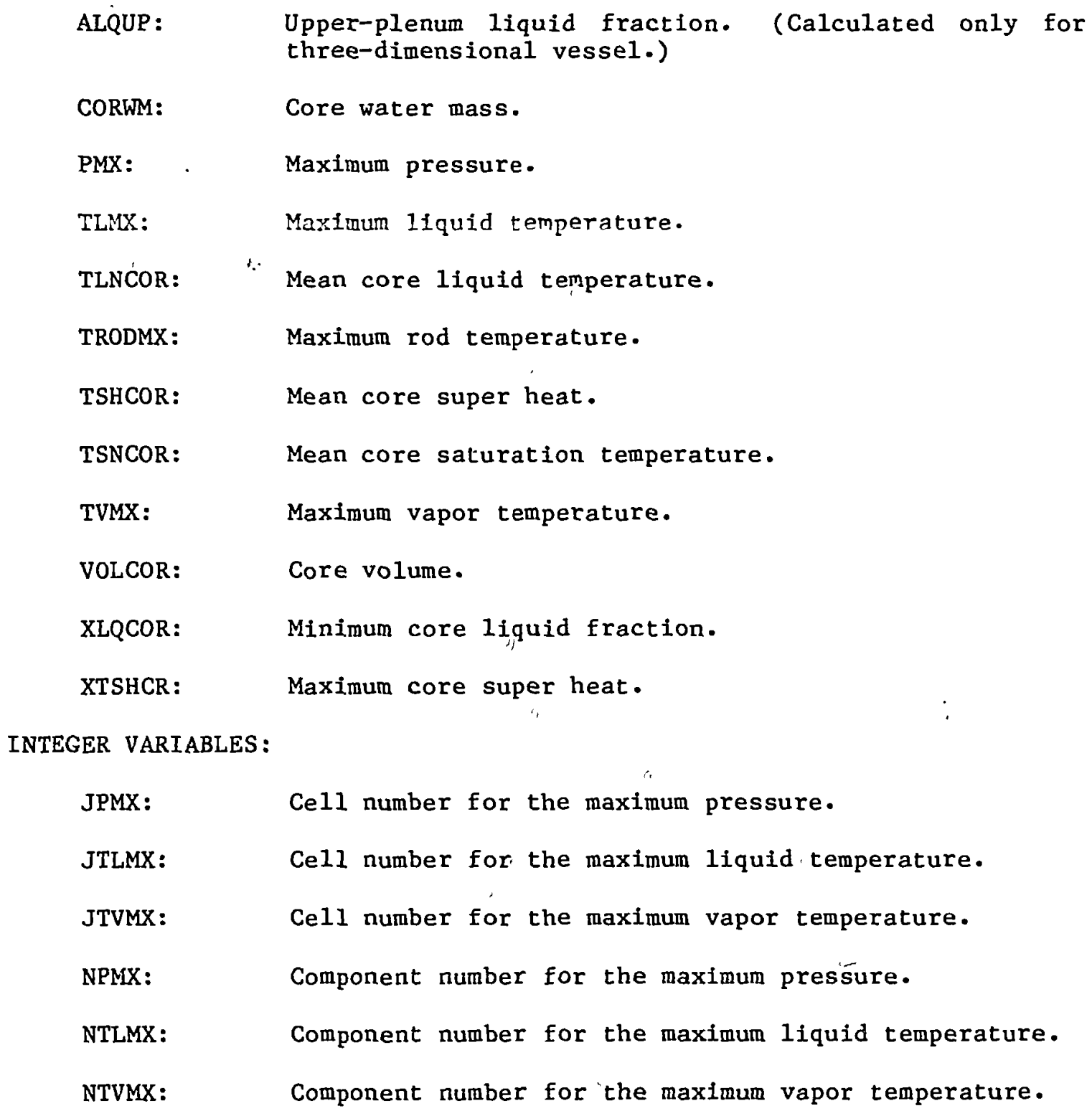




$\begin{array}{ll}\text { KABSO: } & \begin{array}{l}\text { Storage offset to obtain an absolute cell number when } \\ \text { multiple vessels are used. }\end{array} \\ \text { KCMSH: } & \text { offset for coarse-mesh indexing with multiple vessels. } \\ \mathrm{KL}: & \begin{array}{l}\text { Displacement of level (IZ-1) from level (IZ) in A3D } \\ \text { array. }\end{array} \\ \mathrm{KU}: & \begin{array}{l}\text { Displacement of level (IZ+1) from level (IZ) in A3D } \\ \text { array }\end{array} \\ \text { ORG: } & \text { Origin of level (IZ) data A3D array. }\end{array}$

COMDECK TOTALS

COMMON/TOTALS/TLEN, TVOL

TLEN : $\quad$ Total length of a component section.

TVOL: $\quad$ Total volume of a component section.

COMDECK TSATCN

COMMON/TSATCN/AEOS14,CEOS1, CEOS2, CEOS3, CEOSLP

COMMON/TSATCN/IGAS, ILIQ

DIMENSION CEOSLP(40)

REAL VARIABLES:

AE0S14: Constant in expression for saturation temperature ca1culation for intermediate pressures.

CEOS1: $\quad$ Constant, in expression for saturation temperature, set in routine THERMO, calculation for the intermediate pressures.

CE0S2: Constant in expression for saturation temperature, calculation for the intermediate pressures.

CE0S3: Constant in expression for saturation temperature, calculation for intermediate pressures.

CiOSLP(40): Equation-of-state array for low pressures. Defined in subroutine SETEOS. 
INTEGER VARIABLES :
IGAS: $\quad$ Flag for noncondensable gas.
$1=$ air
2 = hydrogen.

ILIQ: $\quad$ Variable not implemented.

COMDECK TWOSTEP

COMMON/TWOSTP/NPSFE, NPSME, NTSPRN

INTEGER VARIABLES :

NPSFE: $\quad$ Pause in FEMOM if NSTEP = NPSE1. The cell number is NPSJ, and the component number is NPICMP.

NPSME: $\quad$ Pause in STBME if NSTEP = NPSE1. The cell number is NPSJ, and the component number is NPICMP.

NTSPRN: $\quad$ Flag for printing extra stabilizer step information to TRCOUT.

COMDECK VCKDAT

COMMON/VCKDAT/DONTOL

COMMON/VCKDAT/IPRVCK, ISKIP , ITVKMX

REAL VARIABLES :

DONTOL: Tolerance for density difference requiring re-donorcelling in VESSEL.

INTEGER VARIABLES:

IPRVCK: $\quad$ Flag to print information about re-donor-celling in VESSEL (normally set to 0 for no print).

ISKIP: Flag to skip re-donor-cell logic in VESSEL component (normally set to 0 for no skip).

ITVKMX: Maximum iteration count to check for need to re-donorcell in VESSEL. 
COMDECK WEBNUM

COMMON/WE BNUM/ALVFCP , ALVFCS , BMIN , CHTFCP , CHTFCS , CHTI BC , CHTI BH , CNDFC , DMIN, PENTL, PENTU, VLSPR, VVLOW, VVUP, WEB, WED, WEDU

COMMON/WE BNUM/ICHVOL

REAL VARIABLES:

ALVFCP: Multiplier on ALV for low-velocity vertical components.

ALVFCS: $\quad$ Multiplíer on ALV under spray conditions.

BMIN : $\quad$ Minimum allowed bubble size.

CHTFCP: Multiplier on CHTI for low-velocity vertical components.

CHTFCS: Multiplier on CHTI under spray conditions.

CHTIBC: Vapor bubble interfacial HTC when TV > TSAT.

CHTIBH: Vapor bubble interfacial HTC when TV < TSAT.

CNDFC: $\quad$ Condensation rate scaling factor.

DMIN : $\quad$ Minimum allowed drop size.

PENTL : $\quad$ Lower bound on entrained $\alpha$.

PENTU : Upper bound on entrained $\alpha$.

VLSPR: Lower limit on quantity $(1-\alpha) V_{\ell}$ at the top of the cell above which spray condition 1 s assumed to exist.

VVLOW: $\quad$ Lower limit on vapor velocity for special condensation model for low-velocity vertical components.

VVUP: $\quad$ Upper limit on vapor velocity for special condensation for low-velocity vertical components. Note: For liquid velocity greater than VLUP, regular condensation model is used. For liquid velocity less than VLLOW, the special condensation model is used. For liquid velocity between VLLOW and VLUP, a linear interpolation between the two models is used.

WEB: $\quad$ Bubble Weber number.

WED: Droplet Weber number. 
WEDU :

Droplet Weber number during core upflow (not implemented).

INTEGER VARIABLES :

ICHVOL: Flag that invokes a minimum value on the interface HTC.

$0=$ has no effect, normal;

1 = sets the minimum to the cell volume times $1.0 \times 10^{7}$.

COMDECK XVAR

COMMON/XVAR/NXI, NX4, NX5

INTEGER VARIABLES :

NX1: The SX1-NX5 variables are reserved for code testing purposes.

NX4: The SX1-NX5 variables are reserved for code testing purposes.

NX5: Variable set to 1 to bypass coarse-mesh rebalance in the VESSEL.

COMDECK XVOL

COMMON/XVOL/BGSS, DAWL, DAXVL, DAXVU, DGSS , FREV

COMMON/XVOL/IFVT, IFVTU, LDAX

REAL VARIABLES :

BGSS :

DAWL :

DAXVL:

DAXVU:

DGSS :

FREV :

INTEGER VARIABLES :

IFVT:

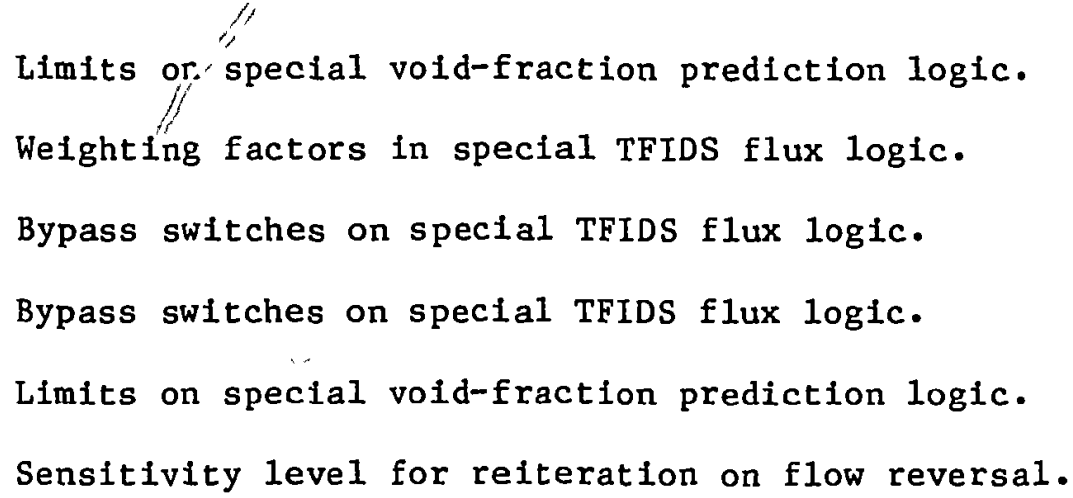

\footnotetext{
Flag for setting velocities passed to TFIDS for special flux logic.
} 
IFVTU:

Time velocity controller.

$0=$ the XVSET logic uses the old time velocity;

$1=$ the XVSET logic uses the the new time velocity.

LDAX:

Bypass switches on special TFIDS flux logic. 
APPENDIX I

COMPONENT DATA TABLES

I. GENERAL POINTER TABLES

The polnter tables for one-dimenstonal components (described below) use four general sets of pointers DUALPT, HYDROPT, INTPT, and . HEATPT. A. DUALPT

These pointers refer to variables whose values are stored at both old and new time values.

\section{INTEGER VARIABLES}

\begin{tabular}{|c|c|c|c|c|}
\hline Word(s) & Name & Array & Dimension & Description \\
\hline 1 & LALP & ALP & NCELLS & old vapor fractions. \\
\hline 2 & LALPD & ALPD & 0 & $\begin{array}{l}\text { Variable not currently imple- } \\
\text { mented. }\end{array}$ \\
\hline 3 & LALPDN & ALPDN & 0 & $\begin{array}{l}\text { Variable not currently imple- } \\
\text { mented. }\end{array}$ \\
\hline 4 & LALPN & ALPN & NCELLS & New vapor fraction. \\
\hline 5 & LALV & ALV & NCELLS & $\begin{array}{l}\text { Old value of liquid-to-interface } \\
\text { HTC times interfacial area (ALV). }\end{array}$ \\
\hline 6 & LALVN & ALVN & NCELLS & $\begin{array}{l}\text { New value of liquid-to-interface } \\
\text { HTC times interfacial area (ALV). }\end{array}$ \\
\hline 7 & LARA & ARA & NCELLS & old stabilizer value for $\alpha \rho_{a^{*}}$ \\
\hline 8 & LARAN & ARAN & NCELLS & New stabllizer value for $\alpha \rho a^{*}$ \\
\hline 9 & LAREL & AREL & NCELLS & $\begin{array}{l}\text { old stabilizer } \\
(1-\alpha) \rho_{\ell} e_{\ell}\end{array}$ \\
\hline 10 & LARELN & ARELN & NCELLS & $\begin{array}{l}\text { New stabilizer } \\
(1-\alpha) \rho_{\ell} e_{\ell}\end{array}$ \\
\hline 11 & LAREV & AREV & NCELLS & old stabilizer value for $\alpha \rho_{v} e_{v}$ \\
\hline 12 & LAREVN & AREVN & NCELLS & New stabilizer value for $\alpha \rho_{v} e_{v}$ \\
\hline 13 & LARL & ARL & NCELLS & $\begin{array}{l}\text { old stabilizer } \\
(1-\alpha) \rho_{\ell}\end{array}$ \\
\hline
\end{tabular}




\begin{tabular}{|c|c|c|c|c|}
\hline Word(s) & Name & Array & Dimension & Description \\
\hline 14 & LARLN & ARLN & NCELLS & $\begin{array}{lll}\text { New stabilizer } & \text { value } & \text { for } \\
(1-\alpha) \rho_{\ell} \cdot & \end{array}$ \\
\hline 15 & LARV & ARV & NCELLS & old stabilizer value for $\alpha p_{v^{*}}$ \\
\hline 16 & LARVN & ARVN & NCELLS & New stab1lizer value for $\alpha \rho v^{\prime}$ \\
\hline 17 & LBIT & BIT & NCELLS+1 & Bit flags from previous time step. \\
\hline 18 & LBITN & BITN & NCELLS+1 & Bit flags for current time step. \\
\hline 19 & LCIF & CIF & 2 (NCELLS+1) & old Interfacial drag coefficients. \\
\hline 20 & LCIFN & CIFN & $2($ NCELLS+1) & New interfacial drag coefficients. \\
\hline 21 & LCONC & CONC & NCELLS*ISOLUT & $\begin{array}{l}\text { Old boron mass to coolant mass } \\
\text { ratio. ISOLUT }=0 \text { or } 1 .\end{array}$ \\
\hline 22 & LCONCN & CONC & NCELLS*ISOLUT & $\begin{array}{l}\text { New boron mass to coolant mass } \\
\text { ratio. ISOLUT }=0 \text { or } 1 .\end{array}$ \\
\hline $23-25$ & $\operatorname{LD}(3)$ & $\mathrm{D}$ & 0 & $\begin{array}{l}\text { Variable not currently imple- } \\
\text { mented. }\end{array}$ \\
\hline $26-28$ & $\operatorname{LDN}(3)$ & DN & 0 & $\begin{array}{l}\text { Variable not currently imple- } \\
\text { mented. }\end{array}$ \\
\hline 29 & LEA & EA & NCELLS & old air internal energy. \\
\hline 30 & LEAN & EAN & NCELLS & New air internal energy. \\
\hline 31 & LEL & EL & NCELLS & old liquid internal energy. \\
\hline 32 & LELN & ELN & NCELLS & New liquid internal energy. \\
\hline 33 & LEV & EV & NCELLS & old vapor internal energy. \\
\hline 34 & LEVN & EVN & NCELLS & New vapor internal energy. \\
\hline 35 & LGAM & GAM & NCELLS & $\begin{array}{l}\text { O1d vapor generation rate per unit } \\
\text { volume. }\end{array}$ \\
\hline 36 & LGAMN & GAMN & NCELLS & $\begin{array}{l}\text { New vapor generation rate per unit } \\
\text { volume. }\end{array}$ \\
\hline 37 & LHIL & HIL & NCELLS & $\begin{array}{l}\text { New HTC between inside wall and } \\
\text { liquid. }\end{array}$ \\
\hline 38 & LHILO & HILO & NCELLS & $\begin{array}{l}\text { Old HTC between inside wall and } \\
\text { liquid. }\end{array}$ \\
\hline
\end{tabular}




\begin{tabular}{|c|c|c|c|c|}
\hline Word(s) & Name & Array & Dimension & Description \\
\hline 39 & LHIV & HIV & NCELLS & $\begin{array}{l}\text { New HTC between inside wall and } \\
\text { vapor. }\end{array}$ \\
\hline 40 & LHIVO & HIVO & NCELLS & $\begin{array}{l}\text { o1d HTC between inside wall and } \\
\text { vapor. }\end{array}$ \\
\hline 41 & LHLV & HLV & NCELLS & $\begin{array}{l}\text { Old value of vapor-to-interface } \\
\text { HTC times interfacial area (CHTI). }\end{array}$ \\
\hline $\begin{array}{c}42 \\
"\end{array}$ & LHLVN & HLVN & NCELLS & $\begin{array}{l}\text { New value of vapor-to-interface } \\
\text { HTC times interfacial area (CHTI). }\end{array}$ \\
\hline 43 & $\mathrm{LP}$ & $P$ & NCELLS & Old pressure. \\
\hline 44 & LPA & PA & NCELLS & old air partial pressure. \\
\hline 45 & LPAN & PAN & NCELLS & New air partial pressure. \\
\hline 46 & LPN & PN & NCELLS & New pressure. \\
\hline 47 & LROA & ROA & NCELLS & old air densities. \\
\hline 48 & LROAN & ROAN & NCELLS & New air density. \\
\hline 49 & LROL & ROL & NCELLS & $01 d$ liquid densities. \\
\hline 50 & LROLN & ROLN & NCELLS & New" liquid density. \\
\hline 51 & LROV & ROV & NCELLS & 01d vapor densities. \\
\hline 52 & LROVN & ROVN & NCELLS & New vapor density. \\
\hline 53 & LS & S & NCELLS*ISOLUT & $\begin{array}{l}\text { Old boron solute mass plated on } \\
\text { structure surface. ISOLUT }=0 \text { or } \\
1 \text {. }\end{array}$ \\
\hline 54 & LSN & $\mathrm{SN}$ & NCELLS*ISOLUT & $\begin{array}{l}\text { New boron solute mass plated on } \\
\text { structure surface. ISOLUT }=0 \text { or } \\
\text { l. }\end{array}$ \\
\hline 55 & LTD & $\mathrm{TD}$ & 0 & $\begin{array}{l}\text { Variable not currently } \\
\text { mented. }\end{array}$ \\
\hline 56 & LTDN & TDN & 0 & $\begin{array}{l}\text { Variable not currently } \\
\text { mented. }\end{array}$ \\
\hline 57 & LTL & TL & NCELLS & 01d liquid temperature. \\
\hline 58 & LTLN & TLN & NCELLS & New liquid temperature. \\
\hline
\end{tabular}




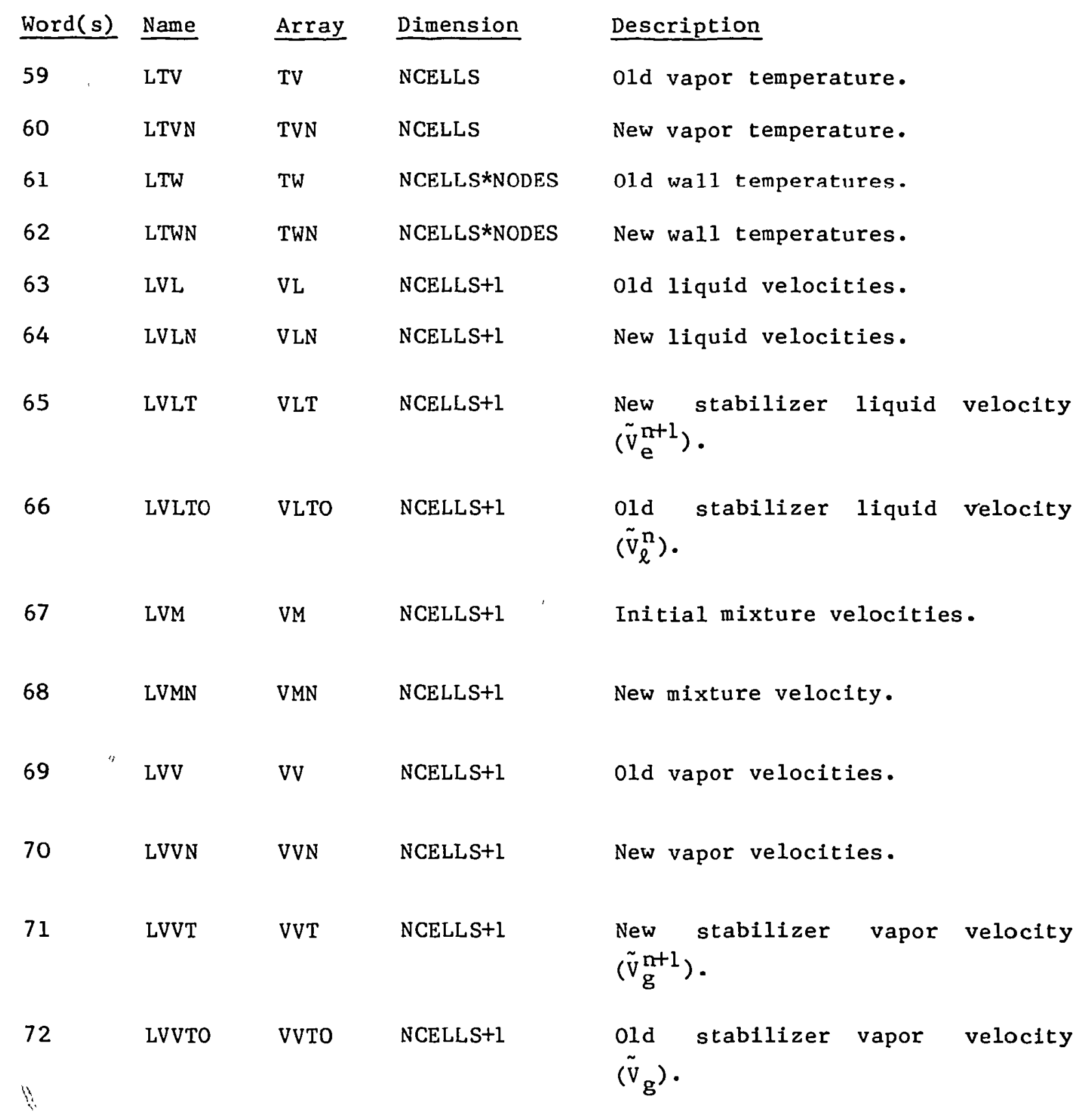




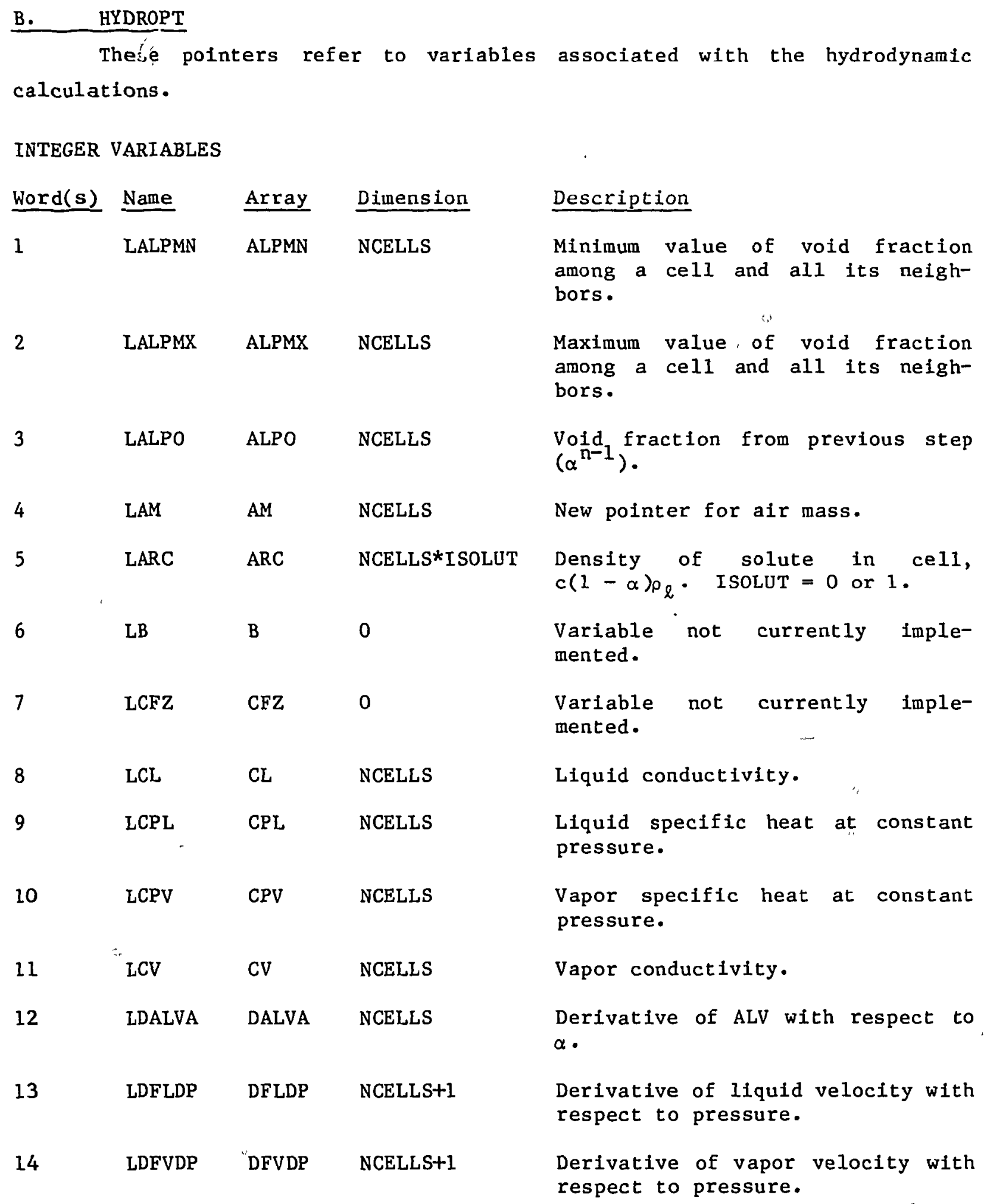




\begin{tabular}{|c|c|c|c|c|}
\hline Word (s) & Name & Array & Dimension & Description \\
\hline 15 & LDRIV & DRIVI & $19 *($ NCELLS +1$)$ & $\begin{array}{l}\text { Storage array for thermodynamic } \\
\text { derivatives and enthalpies. }\end{array}$ \\
\hline 16 & LDX & $\mathrm{DX}$ & NCELLS & Cell length in flow direction. \\
\hline 17 & LELEV & ELEV & NCELLS*IELV & $\begin{array}{l}\text { The pointer for the array } \\
\text { containing cell-centered eleva- } \\
\text { tions. It is used only if IELV is } \\
\text { set to } 1 \text { in the NAMELIST input. }\end{array}$ \\
\hline 18 & LFA & FA & NCELLS+1 & Cell-edge flow area. \\
\hline 19 & LFAVOL & FAVOL & NCELLS & $\begin{array}{l}\text { Cell flow area used in choked-flow } \\
\text { model. }\end{array}$ \\
\hline 20 & LFINAN & FINAN & NCELLS & Inverted annular regime factor. \\
\hline 21 & LFRIC & f) FRIC & $\begin{array}{l}\text { ( NCELLS+1) } \\
\text { * NFRC1 }^{\text {NFR }}\end{array}$ & Additive friction factors. \\
\hline 22 & LFSMLT & FSMLT & NCELLS & $\begin{array}{l}\text { Interphasic area multiplier during } \\
\text { condensation. }\end{array}$ \\
\hline 23 & LGRAV & GRAV1 & NCELLS+1 & Gravitation terms (cosine theta). \\
\hline 24 & LGRVOL & GRAV1 & NCELLS & Cell-averaged GRAV. \\
\hline 25 & $\mathrm{LH}(1)$ & WFHF & NCELLS+1 & $\begin{array}{l}\text { Weighting factor for stratified- } \\
\text { flow regime. }\end{array}$ \\
\hline 26 & $\mathrm{LH}(2)$ & $S I * D X$ & NCELLS+1 & Stratified interfacial area. \\
\hline 27 & $\mathrm{LH}(3)$ & DHLDZ & NCELLS+1 & $\begin{array}{l}\text { Gravitational head force caused by } \\
\text { alpha gradient. }\end{array}$ \\
\hline 28 & LHD & HD & $\begin{array}{l}(\text { NCELLS+1) } \\
\star_{\text {NDIAl }}\end{array}$ & Hydraulic diameters. \\
\hline 29 & LHFG & HFG & NCELLS & Latent heat of vaporization. \\
\hline 30 & LHGAM & HGAM & NCELLS & $\begin{array}{l}\text { Continuation of heat transfer to } \\
\text { subcooled boiling. }\end{array}$ \\
\hline 31 & LHLA & HLA & NCELLS & $\begin{array}{l}\text { Sum of all products of liquid HTC } \\
\text { with heat-transfer area. }\end{array}$ \\
\hline 32 & iLATW & HLATW & NCELLS & $\begin{array}{l}\text { Similar to LHLA except that the } \\
\text { product includes wall temperature. }\end{array}$ \\
\hline
\end{tabular}




\begin{tabular}{|c|c|c|c|c|}
\hline Word $(s)$ & Name & Array & Dimension & Description \\
\hline 33 & LHVA & HVA & NCELLS & $\begin{array}{l}\text { Sum of all products of vapor HTC } \\
\text { with heat-transfer area (similar } \\
\text { to LHLA). }\end{array}$ \\
\hline 34 & LHVATW & HVATW & NCEZLS & $\begin{array}{l}\text { Similar to LHVA except that the } \\
\text { product includes wall temperature. }\end{array}$ \\
\hline 35 & LQP3F & QP3F & NCELLS & $\begin{array}{l}\text { QPPP factor applied to the wall } \\
\text { heat source. }\end{array}$ \\
\hline 36 & LQPPP & QPPP & NODES *NCELLS & Wall heat source. \\
\hline 37 & LRHS & RHS & NCELLS & $\begin{array}{l}\text { Right-hand side of the vapor con- } \\
\text { tinuity and energy equations. }\end{array}$ \\
\hline 38 & LRMEM & RMEM & 0 & $\begin{array}{l}\text { Variable not currently imple- } \\
\text { mented. }\end{array}$ \\
\hline 39 & LRMVM & RMVM & NCELLS+1 & $\begin{array}{l}\text { Mixture density times mixture } \\
\text { velocity. }\end{array}$ \\
\hline 40 & LROM & ROM & NCELLS & Mixture density. \\
\hline 41 & LRVMF & RVMF & NCELLS+1 & Pointer for vapor mass flow. \\
\hline 42 & LSIG & SIG & NCELLS & Surface tension. \\
\hline 43 & LTRID & TRID & $6 *($ NCELLS+1) & $\begin{array}{l}\text { Storage for stabilizer } \\
\text { system. }\end{array}$ \\
\hline 44 & LTSAT & TSAT & NCELLS & Saturation temperature. \\
\hline 45 & LTSSN & TSSN & NCELLS & $\begin{array}{l}\text { Saturation temperature for steam } \\
\text { pressure. }\end{array}$ \\
\hline $46^{k}$ & LVISL & VISL & NCELLS & Liquid viscosity. \\
\hline 47 & LVISV & VISV & NCELLS & Vapor viscosity. \\
\hline 48 & LVLALP & VLALP & NCELLS & $\begin{array}{l}\text { Liquid mass flux that enters the } \\
\text { cell from the cell edges located } \\
\text { above the cell. }\end{array}$ \\
\hline 49 & LVLVC & VLVC & NCELLS & $\begin{array}{l}\text { Liquid velocity at a neighboring } \\
\text { cell edge where the donor-celled } \\
\text { liquid fraction is maximum. }\end{array}$ \\
\hline 50 & LVLVOL & VLVOL & NCELLS & $\begin{array}{l}\text { Cell liquid velocity used in } \\
\text { choked-flow model. }\end{array}$ \\
\hline
\end{tabular}




\begin{tabular}{|c|c|c|c|c|}
\hline Word(s) & Name & Array & Dimension & Description \\
\hline 51 & LVLX & VLX & 0 & $\begin{array}{l}\text { Variable not currently imple- } \\
\text { mented. }\end{array}$ \\
\hline 52 & LVOL & VOL & NCELLS & Cell volumes. \\
\hline 53 & LVR & VR & NCELLS+1 & Relative velocity. \\
\hline 54 & LVVVOL & VVVOL & NCELLS & $\begin{array}{l}\text { Cell vapor velocity used in } \\
\text { choked-flow model. }\end{array}$ \\
\hline 55 & LVVX & VVX & 0 & $\begin{array}{l}\text { Variable not currently imple- } \\
\text { mented. }\end{array}$ \\
\hline 56 & LWA & WA & NCELLS & Wall areas. : \\
\hline 57 & LWAT & WAT & NCELLS & Total heat-transfer area. \\
\hline 58 & LWFL & WFL & NCELLS+1 & Wáll friction factor for liquid. \\
\hline 59. & LWFV & WFV & NCELLS+1 & Wall friction factor for vapor. \\
\hline C. & \multicolumn{4}{|c|}{ INTPT } \\
\hline \multicolumn{3}{|c|}{ These pointers refer $t$} & to variables wit & th integer values. \\
\hline Word (s) & Name & Array & Dimension & Description \\
\hline 1 & LIDR & IDR & NCELLS & Heat-transfer regime. \\
\hline 2 & LMATID & MATID & NODES-1 & Material identifications. \\
\hline D." & HEATPT & $\mathrm{NFF}$ & NCELLT+1 & Friction-correlation options. \\
\hline \multicolumn{5}{|c|}{$\begin{array}{l}\text { These pointers } \\
\text { fer calculations. }\end{array}$} \\
\hline Word(s) & Name & Array & Dimension & Description \\
\hline 1 & LCPW & $\mathrm{CPW}$ & $\begin{array}{l}\text { (NODES-1) } \\
\text { *NCELLS }\end{array}$ & Specific heat of wall. \\
\hline 2 & LCW & $\mathrm{CW}$ & $\begin{array}{l}\text { (NODES-1) } \\
\text { *NCELLS }\end{array}$ & Wall conductivity. \\
\hline 3 & LDR & $\begin{array}{c}\mathrm{DR} \\
0\end{array}$ & NODES-1 & Radial mesh size. \\
\hline
\end{tabular}




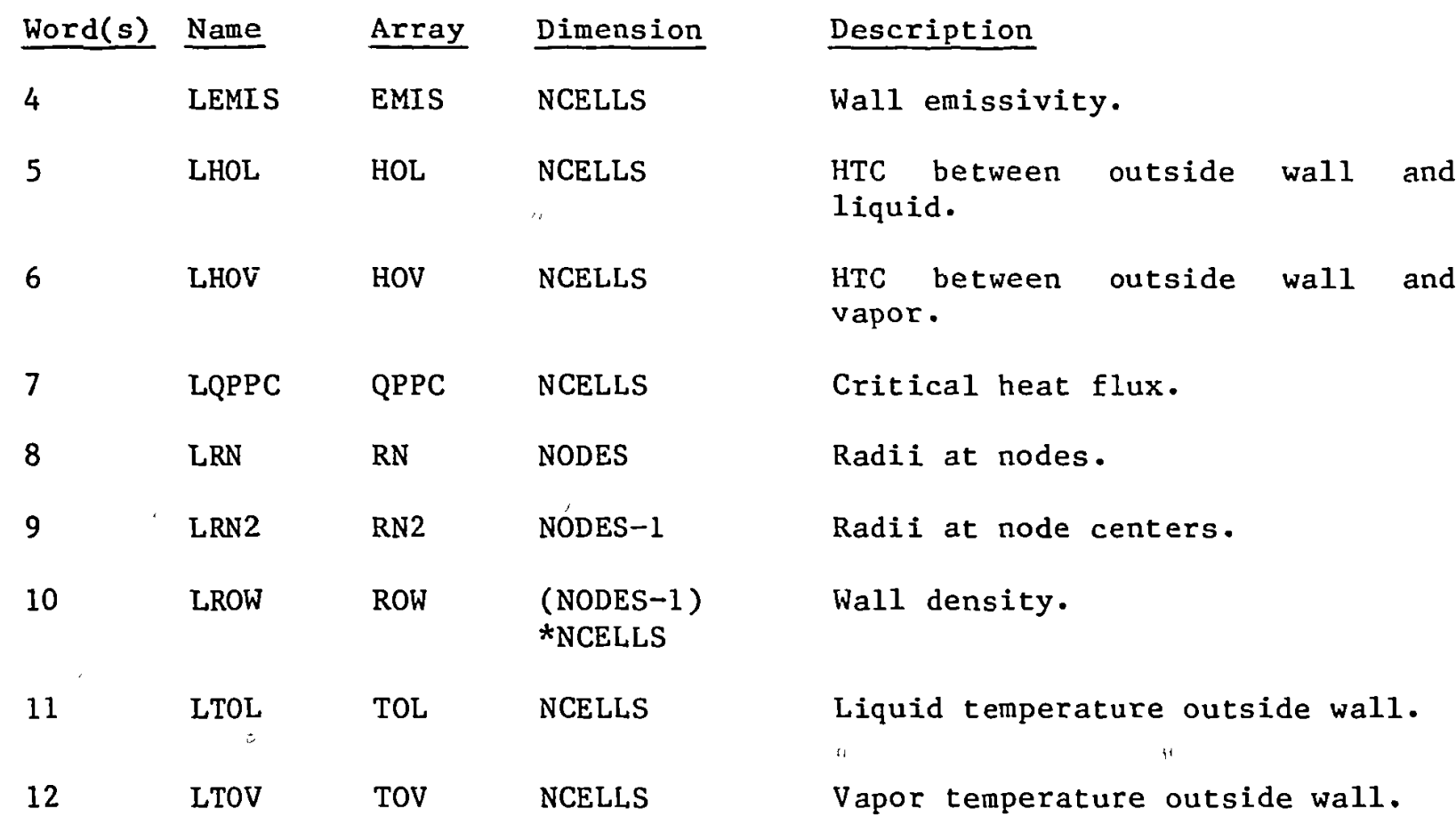

II. ACCUMULATOR MODULE

A. ACCUMVLT-ACCUM Variabie-Length Table

REAL VARIABLES

\begin{tabular}{|c|c|c|}
\hline Position(s) & Parameter & Description \\
\hline 1 & AAl111 & $\begin{array}{l}\text { Dummy variable that provides a known start to } \\
\text { the COMMON block. }\end{array}$ \\
\hline 2 & BSMASS & Time-integrated mass flow from component. \\
\hline $3-4$ & FL(2) & $\begin{array}{l}\text { Liquid mass-flow corrections for mass-conserva- } \\
\text { tion checks. }\end{array}$ \\
\hline 5 & FLOW & Volume flow rate at discharge. \\
\hline $6-7$ & FV(2) & $\begin{array}{l}\text { Vapor mass-flow corrections for mass-conserva- } \\
\text { tion checks. }\end{array}$ \\
\hline 8 & QINT & Initial water volume in accumulator. \\
\hline 9 & QOUT & $\begin{array}{l}\text { Volume of liquid that has been discharged from } \\
\text { the accumulator. }\end{array}$ \\
\hline 10 & $\mathrm{z}$ & Water height above discharge. \\
\hline
\end{tabular}




$\begin{array}{lll}\text { Position(s) } & \text { Parameter } & \text { Description } \\ 11 & 211111 & \begin{array}{l}\text { Dummy variable that provides a known end to the } \\ \text { COMMON block. }\end{array}\end{array}$

INTEGER VARIABLES

12

13

14

15

16

17

18

19

20

21

22

B.

\begin{tabular}{lllll} 
Word $(\mathrm{s})$ & Name & Array & Dimension & Description \\
\cline { 4 - 5 } $1-72$ & DUALPT & -- & -- & General pointer table. \\
$73-131$ & HYDROPT & - & - & General pointer table. \\
$132-134$ & INTPT & -- & - & General pointer table. \\
135 & LBD1 & BD1 & LENBD & Dummy BD1 array. \\
136 & LQPPL & QPPL & NCELLT & Heat flux from wall to liquid. \\
$137-317$ & PTDUM & -- & -- & Dummy variabie.
\end{tabular}

Dummy variable that provides a known start to the COMMON block.

Iteration index of adjacent component.

Indicator for presence of boron in the coolant.

Indicator that accumulator has emptied.

Indicator for velocity update at JUN1 (equal to zero).

Indicator for velocity update at JUN2.

Junction sequence number at cell NCELLS of the accumulator.

Junction number of the junction at cell NCELLS. Number of fluid cells.

Type of adjacent component at JUN2.

Dummy variable that provides a known end to the COMMON block.
2I1111

my variable. 
C. ACCDATA--ACCUM Data Table

This data table includes the following COMMON blocks: BLANKCOM and FIXEDLT, both defined in App. H; ACCUMVLT, defined in Sec. II.A; and ACCUMPT, defined in Sec. II.B.

III. BREAK MODULE

A. BREAKVLT--BREAK Variable-Length Table

REAL VARIABLES

Position(s) Parameter Déscription

1

2

3

4

5

6

7

8

9

10

11

12

13

14
AAl111

ALPOFF

BSA

BSMASS

BXA

BXMASS

CONOFE

PAOFF

POFF

RBMX

TIN

TLOFF

TUOFF

211111
Dummy variable that provides a known start to the COMMON block.

Coolant void fraction when the trip is OFF after it was $\mathrm{ON}$.

Total air from break.

Time-integrated mass flow from break.

Air mass flow from break.

Current mass flow from break.

Ratio of boron mass to coolant mass when the trip is OFF after it was ON.

Air partial pressure when the trip is OFF after it was $\mathrm{ON}$.

Coolant pressure when the trip is OFF after it was $\mathrm{ON}$.

Maximum rate of change of pressure at the break.

Fluid temperature at the break.

Liquid temperature when the trip is OFF after it was $\mathrm{ON}$.

Vapor temperature when the trip is OFF after it was ON.

Dummy variable that provides a known end to the COMMON block. 
INTEGER VARIABLES

\begin{tabular}{|c|c|c|}
\hline Position(s) & Parameter & Description \\
\hline 15 & IA1111 & $\begin{array}{l}\text { Dummy variable that provides a known start to } \\
\text { the COMMON block. }\end{array}$ \\
\hline 16 & IBE & $\begin{array}{l}\text { Last interpolated interval in the rate-factor } \\
\text { table. }\end{array}$ \\
\hline 17 & IBP & $\begin{array}{l}\text { Last interpolated interval in the break compo- } \\
\text { sition parameter tables. }\end{array}$ \\
\hline 18 & IBSV & $\begin{array}{l}\text { Break-table abscissa-coordinate varlable ID } \\
\text { number. }\end{array}$ \\
\hline 19 & IBTR & $\begin{array}{l}\text { Trip ID number that controls evaluation of the } \\
\text { break tables. }\end{array}$ \\
\hline 20 & IBTY & Break-table input option. \\
\hline 21 & ICJ & Iteration index of adjacent component. \\
\hline 22 & INEXTI & Implicitness level of adjacent component. \\
\hline 23 & IOFF & $\begin{array}{l}\text { Fluid-state option when the trip is } O F F \text { after } \\
\text { it was ON. }\end{array}$ \\
\hline 24 & IONOFF & Number of time steps the trip is oN. \\
\hline 25 & ISAT & Break-table use option. \\
\hline 26 & JS 1 & Junction sequence number. \\
\hline 27 & JUN1 & Junction number where break is located. \\
\hline 28 & NBRF & Number of pairs in the rate-factor table. \\
\hline 29 & NBSV & $\begin{array}{l}\text { Rate-factor table's abscissa-coordinate vari- } \\
\text { able ID number. }\end{array}$ \\
\hline 30 & NBTB & Number of pairs for each break table. \\
\hline 31 & TYPE 1 & Type of adjacent component at JUNl. \\
\hline 32 & $\mathrm{ZI} 1111$ & $\begin{array}{l}\text { Dummy variable that provides a known end to the } \\
\text { COMMON block. }\end{array}$ \\
\hline
\end{tabular}




\begin{tabular}{|c|c|c|c|c|}
\hline Word(s) & Name & Array & Dimension & Description \\
\hline $1-72$ & DUALPT & -- & -- & General pointer table. \\
\hline $73-131$ & HYDROPT & -- & -- & General pointer table. \\
\hline 132 & LALPTB & ALPTB & $|\mathrm{NBTB}| * 2$ & Void fraction table. \\
\hline 133 & LCONTB & CONTB & $|\mathrm{NBTB}| * 2$ & $\begin{array}{l}\text { Ratio of boron mass to coolant } \\
\text { mass table. }\end{array}$ \\
\hline 134 & LPATB & PTAB & $|\mathrm{NBTB}| * 2$ & Air partial pressure table. \\
\hline 135 & LPTB & РTB & $|\mathrm{NBTB}| * 2$ & Pressure table. \\
\hline 136 & LRFTB & RFTB & $|\mathrm{NBRF}| * 2$ & Rate-factor table. \\
\hline 137 & LTLTB & TLTB & $|\mathrm{NBTB}| * 2$ & Liquid temperature table. \\
\hline 138 & LTVTB & TVTB & $|\mathrm{NBTB}| * 2$ & Vapor temperature table. \\
\hline $139-317$ & PTDUM & -- & - & Dummy variable. \\
\hline
\end{tabular}

C. BRKDATA--BREAK Data Table

This data table includes the following COMMON blocks: BLANKCOM and FIXEDLT, both defined in App. H; BREAKVLT, defined in Sec. III.A; and BREAKPT, defined in Sec. III.B.

IV. CORE MODULE

A. COREVLT--CORE Variable-Length Table

REAL VARIABLES

Position(s) Parameter Description

1

2

3
AAl11 1

AMH2

BCRO
Dummy variable that provides a known start to the COMMON block.

Hydrogen mass generated from metal-water reaction.

Zero-order coefficient of the first-order polynomial that defines the effective coreaveraged" concentration of control-rod pin boron. 


\begin{tabular}{|c|c|c|}
\hline Position(s) & Parameter & Description \\
\hline 4 & BCR1 & $\begin{array}{l}\text { First-order coefficient of the first-order } \\
\text { polynomial that defines the effective core- } \\
\text { averaged concentration of control-rod pin } \\
\text { boron. }\end{array}$ \\
\hline 5 & BEFF & Total delayed neutron fraction. \\
\hline 6 & BPPO & $\begin{array}{l}\text { Zero-order coefficient of the first-order } \\
\text { polynomial that defines the effective core- } \\
\text { averaged concentration of burnable-poison pin } \\
\text { boron. }\end{array}$ \\
\hline 7 & BPP1 & $\begin{array}{l}\text { First-order coefficient of the first-order } \\
\text { polynomial that defines the effective core- } \\
\text { averaged concentration of burnable-poison pin } \\
\text { boron. }\end{array}$ \\
\hline 8 & BSMASS & Total fluid mass for core. \\
\hline 9 & DRFB & $\begin{array}{l}\text { Reactivity-feedback change in } K \text { over last time } \\
\text { step. }\end{array}$ \\
\hline 10 & DRI & $\begin{array}{l}\text { Estimated change in reactivity over the pre- } \\
\text { vious time step. }\end{array}$ \\
\hline 11 & DRIO & $\begin{array}{l}\text { old value of ORI equals the old value of the } \\
\text { power or reactivity-estimate correction. }\end{array}$ \\
\hline $12-13$ & DTNHT(2) & $\begin{array}{l}\text { Delta temperature minimums used in reflood cal- } \\
\text { culation. }\end{array}$ \\
\hline 14 & DTPK & $\begin{array}{l}\text { The Kaganove-method integration time step for } \\
\text { solving the point-kinetics equations. }\end{array}$ \\
\hline $15-16$ & $\operatorname{DTXHT}(2)$ & $\begin{array}{l}\text { Delta temperature maximums used in reflood } \\
\text { calculation. }\end{array}$ \\
\hline 17 & DZNHT & Delta $z_{\text {min }}$ used in reflood calculation. \\
\hline 18 & ENEFF & Total decay heat fraction. \\
\hline 19 & EXTSOU & $\begin{array}{l}\text { Thermal power ( } W \text { ) produced by external source } \\
\text { neutrons in the reactor core. }\end{array}$ \\
\hline $20-21$ & $\mathrm{FL}(2)$ & $\begin{array}{l}\text { Liquid mass-flow corrections for mass conserva- } \\
\text { tion checks. }\end{array}$ \\
\hline 22 & FUCRAC & Fraction of uncracked fuel. \\
\hline
\end{tabular}




\begin{tabular}{|c|c|c|}
\hline Position(s) & Parameter & Description \\
\hline $23-24$ & FV (2) & $\begin{array}{l}\text { Vapor mass-flow corrections for mass conserva- } \\
\text { tion checks. }\end{array}$ \\
\hline 25 & HGAPO & Rod gap-conductance coefficient (MATRD $=3$ ). \\
\hline 26 & HOUTL & $\begin{array}{l}\text { HTC between outer boundary of core wall and } \\
\text { liquid. }\end{array}$ \\
\hline 27 & HOUTV & $\begin{array}{l}\text { HTC between outer boundary of core wall and } \\
\text { vapor. }\end{array}$ \\
\hline 28 & PDRAT & Rod pitch-to-diameter ratio. \\
\hline 29 & PLDR & $\begin{array}{l}\text { Pellet dish radius. (PLDR }=0.0 \text {, no calcula- } \\
\text { tion of pellet dishing.) }\end{array}$ \\
\hline 30 & POWEXP & $\begin{array}{l}\text { Exponent value to which the power distribution } \\
\text { is raised and that deftnes the weighting } \\
\text { function for averaging the reactivity-feedback } \\
\text { parameters over the core volume. }\end{array}$ \\
\hline 31 & QP3 IN & Initial QPPP factor. \\
\hline 32 & QRDTOT & Total rod heat flux. \\
\hline 33 & QSLTOT & Total slab heat flux. \\
\hline 34 & RADIN & Inner radius of core wall. \\
\hline 35 & REAC & $\begin{array}{l}\text { Reactivity feedback at the beginning of the } \\
\text { previous time step. }\end{array}$ \\
\hline 36 & REACN & $\begin{array}{l}\text { Reactivity-feedback estimate at the end of the } \\
\text { present time step. }\end{array}$ \\
\hline 37 & REACT & $\begin{array}{l}\text { Total reactivity at the beginning time of the } \\
\text { present time step. }\end{array}$ \\
\hline 38 & RMCK & $\begin{array}{l}\text { Reactor multiplication constant at } \\
\text { beginning time of the present time step. }\end{array}$ \\
\hline 39 & RMCKN & $\begin{array}{l}\text { Reactor multiplication constant estimate at the } \\
\text { ending time of the present time step. }\end{array}$ \\
\hline 40 & RPOWPF & The prompt fission power. \\
\hline 41 & RPOWR & old reactor power. \\
\hline 42 & RPOWRI & Initial reactor power. \\
\hline
\end{tabular}




\begin{tabular}{|c|c|c|}
\hline Position(s) & Parameter & Description \\
\hline 43 & RPOWRN & New reactor power. \\
\hline 44 & RPOWRO & 01d reactor power in the previous time step. \\
\hline 45 & RPWOFF & $\begin{array}{l}\text { Programmed reactivity or reactor power when the } \\
\text { controlling trip is OFF after it was ON. }\end{array}$ \\
\hline 46 & RRPWMX & $\begin{array}{l}\text { Maximum rate of change of programmed reactivity } \\
\text { or reactor power. }\end{array}$ \\
\hline 47 & RZPWMX & $\begin{array}{l}\text { Maximum rate of change of the axial power } \\
\text { shape. }\end{array}$ \\
\hline 48 & SDT & $\begin{array}{l}\text { The time interval (s) since the last reactivity } \\
\text { change printout. }\end{array}$ \\
\hline 49 & STIMET & $\begin{array}{l}\text { The problem time }(s) \text { at which the last reac- } \\
\text { tivity change was summed to varlable storage } \\
\text { for late printout. }\end{array}$ \\
\hline 50 & TH & Thickness of core wall. \\
\hline 51 & TNEUT & Neutron generation time. \\
\hline 52 & TouTL & Liquid temperature outside core. \\
\hline 53 & TOUTV & Vapor temperature outside core. \\
\hline 54 & TRAMAX & Average-rod peak-cladding temperature. \\
\hline 55 & TRHMAX & Maximum supplemental rod temperature. \\
\hline 56 & ZPWIN & $\begin{array}{l}\text { Axial power shape table's abscissa-coordinate } \\
\text { variable value corresponding to the initial } \\
\text { axial power shape. }\end{array}$ \\
\hline 57 & ZPWOFF & $\begin{array}{l}\text { Axial power shape table's abscissa-coordinate } \\
\text { variable value corresponding to the axial power } \\
\text { shape to use when the controlling trip is OFF } \\
\text { after it was oN. }\end{array}$ \\
\hline 58 & Z11111 & $\begin{array}{l}\text { Dummy variable that provides a known end to the } \\
\text { COMMON block. }\end{array}$ \\
\hline
\end{tabular}

INTEGER VARIABLES

59

IA111

Dummy variable that provides a known start to the COMMON block. 


\begin{tabular}{|c|c|c|}
\hline Position(s) & Parameter & Description \\
\hline $60-63$ & $\operatorname{IBU}(4)$ & $\begin{array}{l}\text { Boron-unit flag for the Jth reactivity coeffi- } \\
\text { cient. }\end{array}$ \\
\hline 64 & ICHF & CHF calculation option. \\
\hline 65 & ICJ1 & Iteration index of adjacent component at JUN1. \\
\hline 66 & ICJ2 & Iteration index of adjacent component at JUN2. \\
\hline 67 & ICONC & Indicator for presence of boron in the coolant. \\
\hline 68 & ICRL & Core lower-boundary segment number, $Z$ (ICRL). \\
\hline 69 & ICRU & Core upper-boundary segment number, $\mathrm{z}$ (ICRU). \\
\hline 70 & IONOFF & $\begin{array}{l}\text { Number of time steps the reactivity-power } \\
\text { table's controlling trip has been oN. }\end{array}$ \\
\hline $71-74$ & $\operatorname{IRC}(4)$ & $\begin{array}{l}\text { Number of values that defines the argument- } \\
\text { number reactivity-coefficient table. }\end{array}$ \\
\hline $75-78$ & $\operatorname{IRCJEM}(4)$ & $\begin{array}{l}\text { Reactivity-coefficient form number for the } \\
\text { argument-number reactivity-feedback parameter. }\end{array}$ \\
\hline $79-94$ & $\operatorname{IRCJTB}(4,4)$ & $\begin{array}{l}\text { The number of first argument reactivity- } \\
\text { feedback parameter-value entries for the second } \\
\text { argument reactivity-coefficient table. }\end{array}$ \\
\hline 95 & IRF & $\begin{array}{l}\text { Last interpolated interval number in the rate- } \\
\text { factor table for the reactivity-power table. }\end{array}$ \\
\hline 96 & IRFTR & $\begin{array}{l}\text { Trip ID number that controls evaluation with } \\
\text { the reflood axial fine mesh in the fuel rod. }\end{array}$ \\
\hline 97 & IRP & $\begin{array}{l}\text { Last interpolated interval number in the } \\
\text { reactivity-power table. }\end{array}$ \\
\hline 98 & IRPWSV & $\begin{array}{l}\text { The signal-variable ID number that defines the } \\
\text { power or reactivity table's independent vari- } \\
\text { able. }\end{array}$ \\
\hline 99 & IRPWTR & Reactor-kinetics trip ID number. \\
\hline 100 & IRPWTY & Reactor-kinetics option flag. \\
\hline 101 & ISNOTB & $\begin{array}{l}\text { A flag variable that is defined if the solute } \\
\text { is boron for the reactivity-feedback } \\
\text { calculation. } \\
0=\text { solute is boron, } \\
1=\text { solute is not boron. }\end{array}$ \\
\hline
\end{tabular}




\begin{tabular}{|c|c|c|}
\hline Position(s) & Parameter & Description \\
\hline 102 & ISOLLB & Indicator for velocity update at JUN1. \\
\hline 103 & ISOLRB & Indicator for velocity update at JUN2. \\
\hline 104 & $I Z F$ & $\begin{array}{l}\text { Last interpolated interval number in the rate- } \\
\text { factor table for the axial power-shape table. }\end{array}$ \\
\hline 105 & IZP & $\begin{array}{l}\text { Last interpolated interval number in the axial } \\
\text { power-shape table. }\end{array}$ \\
\hline 106 & IZPWSV & $\begin{array}{l}\text { Axial power-shape table's abscissa-coordinate } \\
\text { variable ID number. }\end{array}$ \\
\hline 107 & IZPWTR & $\begin{array}{l}\text { Trip ID number that controls evaluation of the } \\
\text { axial power-shape table. }\end{array}$ \\
\hline 108 & JS1 & Junction sequence number at cell 1 of the core. \\
\hline 109 & JS2 & $\begin{array}{l}\text { Junction sequence number at cell NCELLS of the } \\
\text { core. }\end{array}$ \\
\hline 110 & JUN1 & Junction number of the junction at cell 1 . \\
\hline 111 & JUN2 & Junction number of the junction at cell NCELLS. \\
\hline 112 & LENFVG & $\begin{array}{l}\text { Length of power data stored at both the old and } \\
\text { new times. }\end{array}$ \\
\hline 113 & LENGEN & Length of the general data. \\
\hline 114 & LENRD & Length of the rod data. \\
\hline 115 & LFVG & Relative position of old-time power data. \\
\hline 116 & LFVGN & Relative position of new-time power data. \\
\hline 117 & LFVR & Relative position of old-time rod data. \\
\hline 118 & LFVRN & Relative position of new-time rod data. \\
\hline 119 & LNFVR & $\begin{array}{l}\text { Length of rod data stored at both old and new } \\
\text { times. }\end{array}$ \\
\hline 120 & LNPTRR & Number of rod-data pointers. \\
\hline 121 & MAT & Material identification. \\
\hline 122 & NCELLS & Total number of fluid cells. \\
\hline
\end{tabular}




\begin{tabular}{|c|c|c|}
\hline Position(s) & Parameter & Description \\
\hline 123 & NCRZ & Number of axial mesh cells. \\
\hline 124 & NDGX & Number of delayed neutron groups. \\
\hline 125 & NDHX & Number of decay heat groups. \\
\hline 126 & NDRDS & Number of rod heat-transfer nodes. \\
\hline 127 & NDSLB & Number of slab heat-transfer nodes (unused). \\
\hline 128 & NFBPWT & $\begin{array}{l}\text { Flag that defines the spatial distribution used } \\
\text { to weight the averaging of the reactivity-feed- } \\
\text { back parameters over the reactor-core volume. }\end{array}$ \\
\hline 129 & NFCI & $\begin{array}{l}\text { Fuel-cladding interaction (FCI) flag. } \\
0=\text { no calculation, } \\
1=\text { calculation. }\end{array}$ \\
\hline 130 & NFCIL & $\begin{array}{l}\text { Upper limit on number of FCI calculations per } \\
\text { time step. }\end{array}$ \\
\hline 131 & NFUEL & Number of nodes in fuel pellet. \\
\hline 132 & NINT & $\begin{array}{l}\text { Number of interfaces between dissimilar mate- } \\
\text { rials in rods. }\end{array}$ \\
\hline 133 & NMWRX & $\begin{array}{l}\text { Metal-water reaction flag. } \\
0=\text { no calculation, } \\
1 \text { = calculation. }\end{array}$ \\
\hline 134 & NONOFF & $\begin{array}{l}\text { The number of time steps the trip-controlling } \\
\text { evaluation of the axial power-shape table has } \\
\text { been oN. }\end{array}$ \\
\hline 135 & NRAMAX & $\begin{array}{l}\text { Cell number of the average-rod peak-cladding } \\
\text { temperature (always } 1 \text { ). }\end{array}$ \\
\hline 136 & NRFD & $\begin{array}{l}\text { Reflood flag. } \\
0=\text { takes no action, } \\
1=\text { turns on fine-mesh flag. }\end{array}$ \\
\hline 137 & NR!IMAX & $\begin{array}{l}\text { Cell number of the supplemental rod peak- } \\
\text { cladding temperature. }\end{array}$ \\
\hline 138 & NRODS & Number of computational rods. \\
\hline 139 & NRPWRF & $\begin{array}{l}\text { The number of power } \\
\text { table entries. }\end{array}$ \\
\hline
\end{tabular}




\begin{tabular}{|c|c|c|}
\hline Position(s) & Parameter & Description \\
\hline 140 & NRPWSV & $\begin{array}{l}\text { Reactivity-power rate-factor table }{ }^{-} \text {s abscissa- } \\
\text { coordinate variable ID number. }\end{array}$ \\
\hline 141 & NRPWTB & $\begin{array}{l}\text { Number of entry pairs in the reactivity-power } \\
\text { table. }\end{array}$ \\
\hline 142 & NRTS & $\begin{array}{l}\text { The number of time steps the programmed- } \\
\text { reactivity and reactivity-feedback changes are } \\
\text { summed for printout. }\end{array}$ \\
\hline 143 & NSET & $\begin{array}{l}\text { The previous reflood fine-mesh trip set } \\
\text { position status value. }\end{array}$ \\
\hline 144 & NSLBS & Number of computational slabs (unused). \\
\hline 145 & NZMAX & $\begin{array}{l}\text { Maximum number of rows of heat-transfer nodes } \\
\text { used in reflood calculation. }\end{array}$ \\
\hline 146 & NZPWRF & $\begin{array}{l}\text { Number of entry pairs in the axial power-shape } \\
\text { rate-factor table. }\end{array}$ \\
\hline 147 & NZPWSV & $\begin{array}{l}\text { Axial power-shape rate-factor table's abscissa- } \\
\text { coordinate variable ID number. }\end{array}$ \\
\hline 148 & NZPWTB & $\begin{array}{l}\text { Number of axial power shapes in the axial } \\
\text { power-shape table. }\end{array}$ \\
\hline 149 & TYPE1 & Type of adjacent component at JUN1. \\
\hline 150 & TYPE2 & Type of adjacent component at JUN2. \\
\hline 151 & $\mathrm{ZI} 1111$ & $\begin{array}{l}\text { Dummy variable that provides a known end to the } \\
\text { COMMON block. }\end{array}$ \\
\hline
\end{tabular}

B. COREPT--CORE Pointer Table

$\begin{array}{lllll}\text { Word }(s) & \text { Name } & \text { Array } & \text { Dimension } & \\ 1-72 & \text { DUALPT } & -- & - & \text { Description } \\ 73-131 & \text { HXDROPT } & -- & -- & \text { General pointer table. } \\ 132-134 & \text { INTPT } & - & -- & \text { General pointer table. } \\ 135-146 & \text { HEATPT } & -- & -- & \text { General pointer table. }\end{array}$

GLOBAL DATA POINTERS

147 LBETA BETA NDGX Delayed neutron group fraction. 


\begin{tabular}{|c|c|c|c|c|}
\hline Word(s) & Name & Array & Dimension & Description \\
\hline 148 & LCDG & CDG & NDGX & $\begin{array}{l}\text { Old concentration of delayed neu- } \\
\text { tron groups. }\end{array}$ \\
\hline 149 & LCDGN & CDGN & NDGX & $\begin{array}{l}\text { New concentration of delayed neu- } \\
\text { tron groups. }\end{array}$ \\
\hline 150 & $\mathrm{LCDH}$ & $\mathrm{CDH}$ & NDHX & $\begin{array}{l}\text { old concentration of decay heat } \\
\text { groups. }\end{array}$ \\
\hline 151 & LCDHN & CDHN & NDHX & $\begin{array}{l}\text { New concentration of decay heat } \\
\text { groups. }\end{array}$ \\
\hline 152 & LCLEN & CLEN & 1 & old total cladding length. \\
\hline 153 & LCLENN & CLENN & 1 & New total cladding length. \\
\hline 154 & LCPOWR & CPOWR & 1 & Relative rod power density. \\
\hline 155 & LEDH & EDH & NDHX & $\begin{array}{l}\text { Energy yield fraction of decay } \\
\text { heat groups. }\end{array}$ \\
\hline 156 & LFPU02 & FPUO2 & 1 & $\begin{array}{l}\text { Fraction of plutonium oxide in } \\
\text { mixed-oxide fuel fraction. }\end{array}$ \\
\hline 157 & LFTD & FTD & 1 & Fuel theoretical density. \\
\hline 158 & LGMIX & GMIX & 7 & $\begin{array}{l}\text { Mole fraction of gap-gas constitu- } \\
\text { ents. }\end{array}$ \\
\hline 159 & LGMLES & GMLES & 1 & Moles of gap gas. \\
\hline 160 & LLAMDA & LAMDA & NDGX & $\begin{array}{l}\text { Delay constant of neutron delayed } \\
\text { groups. }\end{array}$ \\
\hline 161 & LLAMDH & LAMDH & NDHX & $\begin{array}{l}\text { Delay constant of decay } \\
\text { groups. }\end{array}$ \\
\hline 162 & LMATRD & MATRD & NDM1 & Rod material identification. \\
\hline 163 & LNFAX & NFAX & NCRZ & Rod fine-mesh noding factor. \\
\hline 164 & $\underset{r}{\operatorname{LNRDX}}$ & NRDX & 1 & Number of rods in volume, \\
\hline 165 & LPGAPT & PGAPT & 1 & Gap total gas pressure. \\
\hline 166 & LPLVOL & PLVOL & 1 & Rod plenum volume. \\
\hline 167 & LPSLEN & PSLEN & 1 & Pellet stack length. \\
\hline
\end{tabular}




\begin{tabular}{|c|c|c|c|c|}
\hline Word(s) & Name & Array & Dimension & Description \\
\hline 168 & LRADRD & RADRD & NDRDS & Rod node radius (cold). \\
\hline 169 & LRCAL & RCAL & $\begin{array}{l}\sum \operatorname{IRCJTB}(i, 3)+ \\
\pi_{i} \operatorname{IRCJTB}(i, 3)\end{array}$ & $\begin{array}{l}\text { Coolant void-fraction reactivity- } \\
\text { coefficient table. The symbol } \pi \text { i } \\
\text { indicates the product of the } \\
\text { following variable taken over the } \\
i \text { subscript. }\end{array}$ \\
\hline 170 & LRCBM & RCBM & $\begin{array}{l}\sum \operatorname{IRCJTB}(1,4)+ \\
\pi_{i} \operatorname{IRCJTB}(i, 4)\end{array}$ & $\begin{array}{l}\text { Boron reactivity-coefficient } \\
\text { table. The symbol } \pi \text { indicates } \\
\text { the product of the following } \\
\text { variable taken over the i } \\
\text { subscript. }\end{array}$ \\
\hline 171 & LRCN & $\mathrm{RCN}$ & 4 & $\begin{array}{l}\text { Reactivity-coefficient values at } \\
\text { the beginning of the previous time } \\
\text { step. }\end{array}$ \\
\hline 172 & LRCTC & $\mathrm{RCTC}$ & $\begin{array}{l}\sum \operatorname{IRCJTB}(i, 2)+ \\
\pi_{i} \operatorname{IRCJTB}(i, 2)\end{array}$ & $\begin{array}{l}\text { Coolant temperature reactivity- } \\
\text { coefficient table. The symbol } \pi i \\
\text { indicates the product of the } \\
\text { following variable taken over the } \\
i \text { subscript. }\end{array}$ \\
\hline 173 & LRCTF & RCTF & $\begin{array}{l}\sum \operatorname{IRCJTB}(i, 1)+ \\
\pi_{i} \operatorname{IRCJTB}(i, 1)\end{array}$ & $\begin{array}{l}\text { Fuel temperature reactivity- } \\
\text { coefficient table. The symbol } \pi \\
\text { indicates the product of the } \\
\text { following variable taken over the } \\
i \text { subscript. }\end{array}$ \\
\hline 174 & LRDPWR & RDPWR & NDRDS & Rod relative radial power density. \\
\hline 175 & LRPKF & RPKF & NRODS & Rod power peaking factor \\
\hline 176 & LRPWRF & RPWRF & $\mid$ NRPWRF $\mid * 2$ & $\begin{array}{l}\text { Rate-factor table for the power or } \\
\text { reactivity table. }\end{array}$ \\
\hline 177 & LRPWTB & RPWTB & $|\mathrm{NRPWTB}| * 2$ & Rèactivity power table. \\
\hline 178 & LRS & RS & $\begin{array}{l}\text { NDRDS*MOD } \\
(\text { NFBPWT }, 2)\end{array}$ & $\begin{array}{l}\text { Pointer variable for the fuel-rod } \\
\text { radial shape weight function used } \\
\text { to average reactivity-feedback } \\
\text { parameters over the core volume. } \\
{[\operatorname{MOD}(N, 2)=N-(N / 2) * 2]}\end{array}$ \\
\hline 179 & LSRP & SRP & 15 & $\begin{array}{l}\text { Summed programmed and feedback- } \\
\text { reactivity changes. }\end{array}$ \\
\hline 180 & LXN & $\mathrm{XN}$ & 4 & $\begin{array}{l}\text { New reactivity-feedback parameter } \\
\text { values. }\end{array}$ \\
\hline
\end{tabular}




\begin{tabular}{|c|c|c|c|c|}
\hline Word(s) & Name & Array & Dimension & Description \\
\hline 181 & LXO & xo & 4 & $\begin{array}{l}\text { old reactivity-feedback parameter, } \\
\text { values. }\end{array}$ \\
\hline 182 & LZ & $\mathrm{Z}$ & NCELLS & Axial segment upper elevation \\
\hline 183 & LZPW & $\mathrm{ZPW}$ & ICRU-ICRL+1 & $\begin{array}{l}\text { Last interpolated axial power } \\
\text { shape. }\end{array}$ \\
\hline 184 & LZPWRF & ZPWRF & $|\mathrm{NZPWRF}| * 2$ & $\begin{array}{l}\text { Axial Fower-shape rate-factor } \\
\text { table. }\end{array}$ \\
\hline 185 & LZPWTB & ZPWTB & $\begin{array}{l}\mid \text { NZPWTB } \mid \\
((\text { ICRU-ICRL })+2)\end{array}$ & Relative axial power-shape table. \\
\hline 186 & LzS & $\mathrm{ZS}$ & $\begin{array}{l}((\text { ICRU-ICRL }) \\
+1) *(\text { NFBPWT } / 2)\end{array}$ & $\begin{array}{l}\text { Pointer variable for the axial } \\
\text { shape. }\end{array}$ \\
\hline ROD DATA & POINTERS & & & \\
\hline 187 & LBURN & BURN & $\mathrm{NCRZ}+1$ & Fuel burnup. \\
\hline 188 & LCND & CND & $\begin{array}{l}\text { NDRDS* } \\
(\text { NCRZ+1) }\end{array}$ & Rod conductivity. \\
\hline 189 & LCNDR & CNDR & $\begin{array}{l}\text { NINT* } \\
(\text { NCRZ+1) }\end{array}$ & $\begin{array}{l}\text { Rod heat conductivity at right of } \\
\text { interface. }\end{array}$ \\
\hline 190 & LCPDR & CPDR & $\begin{array}{l}\text { NINT** } \\
\left(N^{\prime \prime} Z^{\prime \prime}+1\right)\end{array}$ & $\begin{array}{l}\text { Rod heat capacity at specific } \\
\text { heat. }\end{array}$ \\
\hline 191 & LCPND & CPND & $\begin{array}{l}\text { NDRDS* } \\
(\text { NCRZ+1) }\end{array}$ & Rod specific heat. \\
\hline 192 & LDRLDT & DRLDT & NCRZ & $\begin{array}{l}\text { Derivative of liquid density with } \\
\text { respect to liquid temperature. }\end{array}$ \\
\hline 193 & LDRVDT & DRVDT & NCRZ & $\begin{array}{l}\text { Derivative of vapor density with } \\
\text { respect to vapor temperature. }\end{array}$ \\
\hline 194 & LDRZ & $\mathrm{DRZ}$ & NCRZ +1 & $\begin{array}{l}\text { Old zirconium dioxide reaction, } \\
\text { depth. }\end{array}$ \\
\hline 195 & LDRZN & DRZN & NCRZ+1 & $\begin{array}{l}\text { New zirconium dioxide reaction } \\
\text { depth. }\end{array}$ \\
\hline 196 & LEMISR & EMISR & $\begin{array}{l}\text { NDRES* } \\
(\text { NCRZ+1) }\end{array}$ & Rod emissivity. \\
\hline $197:$ & LHDR & HDR & NCRZ & Rod bundle hydraulic diameter. \\
\hline
\end{tabular}




\begin{tabular}{|c|c|c|c|c|}
\hline Word(s) & Name & Array & Dimension & Description \\
\hline 198 & LHGAP & HGAP & NCRZ+1 & Gap conductance. \\
\hline 199 & LHLVR & HLVR & NCRZ & Interfacial HTC. \\
\hline 200 & LHRFL & HRFL & NZMAX & Fine-mesh liquid HTC. \\
\hline 201 & LHRFV & HRFV & NZMAX & Fine-mesh vapor HTC. \\
\hline 202 & LHRLL & HRLL & NCRZ+1 & Liquid HTC. \\
\hline 203 & LHRLV & HRLV & NCRZ+1 & Vapor HTC. \\
\hline 204 & LIDHT & IDHT & NZMAX & Heat-transfer cell identifier. \\
\hline 205 & LIDRGR & IDRGR & $\mathrm{NCRZ+2}$ & Flow regime flag. \\
\hline 206 & LIHTF & IHTF & NXMAX & $\begin{array}{l}\text { Fine-mesh heat-transfer regime } \\
\text { flag. }\end{array}$ \\
\hline 207 & LNOHT & NOHT & 1 & $\begin{array}{l}\text { Number of rows of heat-transfer } \\
\text { nodes for each rod. }\end{array}$ \\
\hline 208 & LPGAP & PGAP & NCRZ+1 & Gap local gas pressure. \\
\hline 209 & LPINT & PINT & $\mathrm{NCRZ}+1$ & Pellet-cladding contact pressure. \\
\hline 210 & LPLDV & PLDV & NCRZ & Pellet dish volume. \\
\hline 211 & LQWRX & QWRX & NCRZT1 & Metal-water reaction heat source. \\
\hline 212 & LRADR & RADR & $\begin{array}{l}\text { NDRDS* } \\
(\text { NCRZ+1) }\end{array}$ & 01d radial node positions. \\
\hline 213 & LRDHLO & RDHLO & NCRZ & $\begin{array}{l}\text { Variable not currently imple- } \\
\text { mented. }\end{array}$ \\
\hline 214 & LRDHLR & RDHLR & NCRZ & Liquid HTC. \\
\hline 215 & LRDHVO & RDHVO & NCRZ & $\begin{array}{l}\text { Variable not currently imple- } \\
\text { mented. }\end{array}$ \\
\hline 216 & LRDHVR & RDHVR & NCRZ & Vapor HTC. \\
\hline 217 & LRDTLR & $\begin{array}{c}\text { RDTLR } \\
\text { D }\end{array}$ & NCRZ & $\begin{array}{l}\text { Average-rod wall temperature seen } \\
\text { by liquid. }\end{array}$ \\
\hline 218 & LRDTVR & RDTVR & NCRZ & $\begin{array}{l}\text { Average-rod wall temperature seen } \\
\text { by vapor. }\end{array}$ \\
\hline 766 & $\int_{\rightarrow+\infty}$ & & $\vartheta$ & $\therefore$ \\
\hline
\end{tabular}




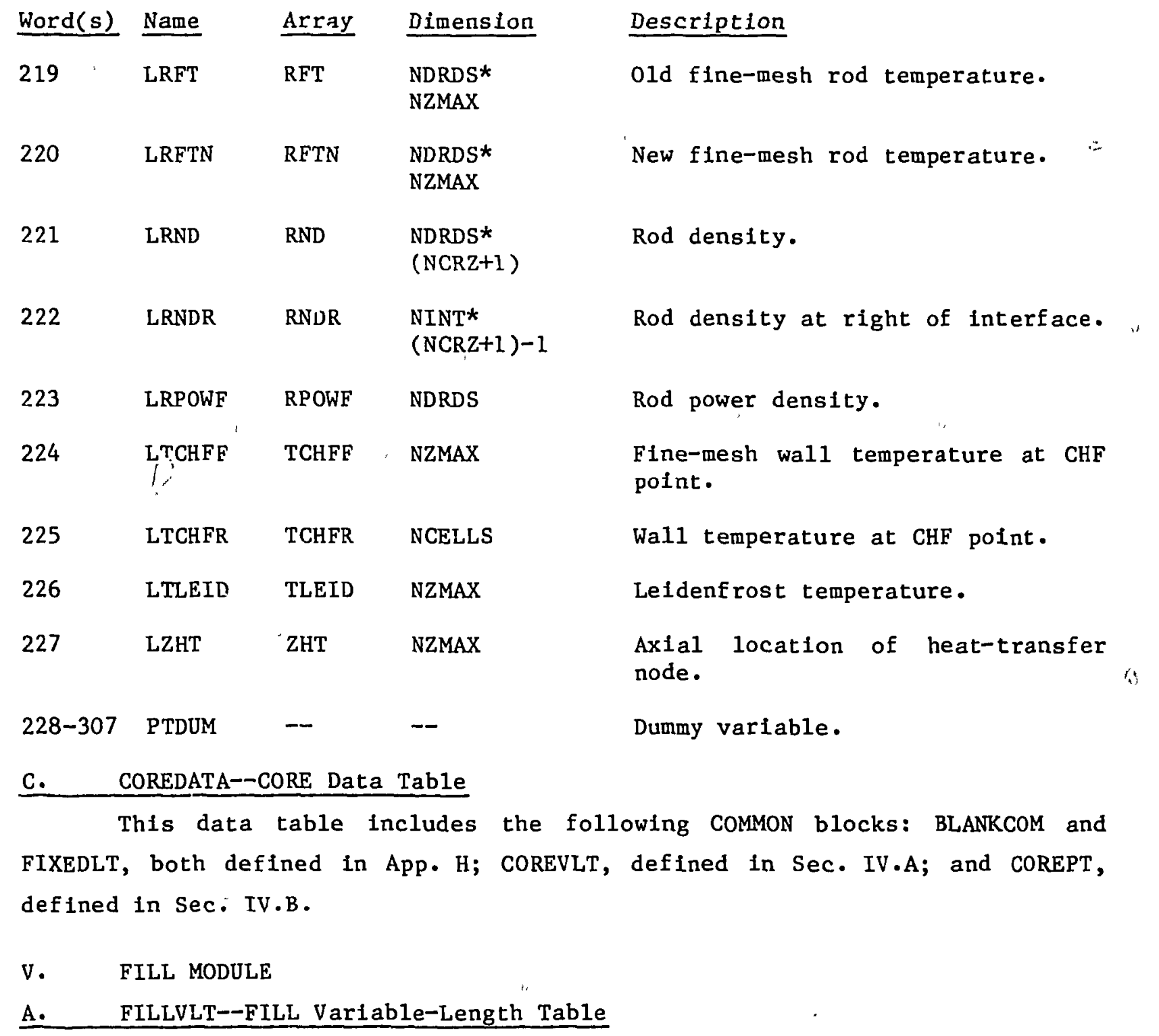

REAL VARIABLES

Position(s) Parameter Description

1 AAllil Dummy variable that provides a known start to the COMMON block.

2

3
ALPOFF

CONOFF
Coolant void fraction when the trip is oFF after it was $0 \mathrm{~N}$.

Ratio of boron mass to coolant mass when the trip is $O F F$ after it was $O N$. 


\begin{tabular}{|c|c|c|c|}
\hline Position(s) & & Parameter & Description \\
\hline 4 & & FLOWIN & $\begin{array}{l}\text { Initial mass flow into or from adjacent compo- } \\
\text { nent. }\end{array}$ \\
\hline 5 & & FLWOFF & $\begin{array}{l}\text { Coolant mass flow when the trip is OFF after it } \\
\text { was ON. }\end{array}$ \\
\hline 6 & & FSMASS & Time-integrated mass-flow rate out of fill. \\
\hline 7 & & FXMASS & Current mass-flow rate out of fill. \\
\hline 8 & & PAOFF & $\begin{array}{l}\text { Air partial pressure when the trip is OFF after } \\
\text { it was ON. }\end{array}$ \\
\hline 9 & & POFF & $\begin{array}{l}\text { Coolant pressure when the trip is OFF after it } \\
\text { was ON. }\end{array}$ \\
\hline 10 & & RFMX & $\begin{array}{l}\text { Maxinum rate of change of fill velocity or mass } \\
\text { flow. }\end{array}$ \\
\hline 11 & & TLOFF & $\begin{array}{l}\text { Liquid temperature when the trip is } O F F \text { after } \\
\text { it was ON. }\end{array}$ \\
\hline 12 & & TVOFF & $\begin{array}{l}\text { Vapor temperature when the trip is OFF after it } \\
\text { was ON. }\end{array}$ \\
\hline 13 & & TWTOLD & $\begin{array}{l}\text { The fraction of a previous fill fluid dynamic- } \\
\text { state parameter that is averaged with the fill } \\
\text { table s defined parameter and that defines the } \\
\text { fill parameter value for this time step } \\
(0.0 \leq \text { TWTOLD }(1.0) \text {. }\end{array}$ \\
\hline 14 & & VLOFF & $\begin{array}{l}\text { Liquid velocity when the trip is } \mathrm{OFF} \text { after it } \\
\text { was } \mathrm{ON} \text {. }\end{array}$ \\
\hline 15 & $\Rightarrow$ & VVOFF & $\begin{array}{l}\text { Vapor velocity when the trip is } O F F \text { after it } \\
\text { was ON. }\end{array}$ \\
\hline 16 & & 211111 & $\begin{array}{l}\text { Dummy variable that provides a known end to the } \\
\text { COMMON block. }\end{array}$ \\
\hline
\end{tabular}

INTEGER VARIABLES

17

18

19
IA1111

ICJ

IFF
Dummy variable that provides a known start to the COMMON block.

Iteration index of adjacent component

Last interpolated interval in the rate-factor
table. 


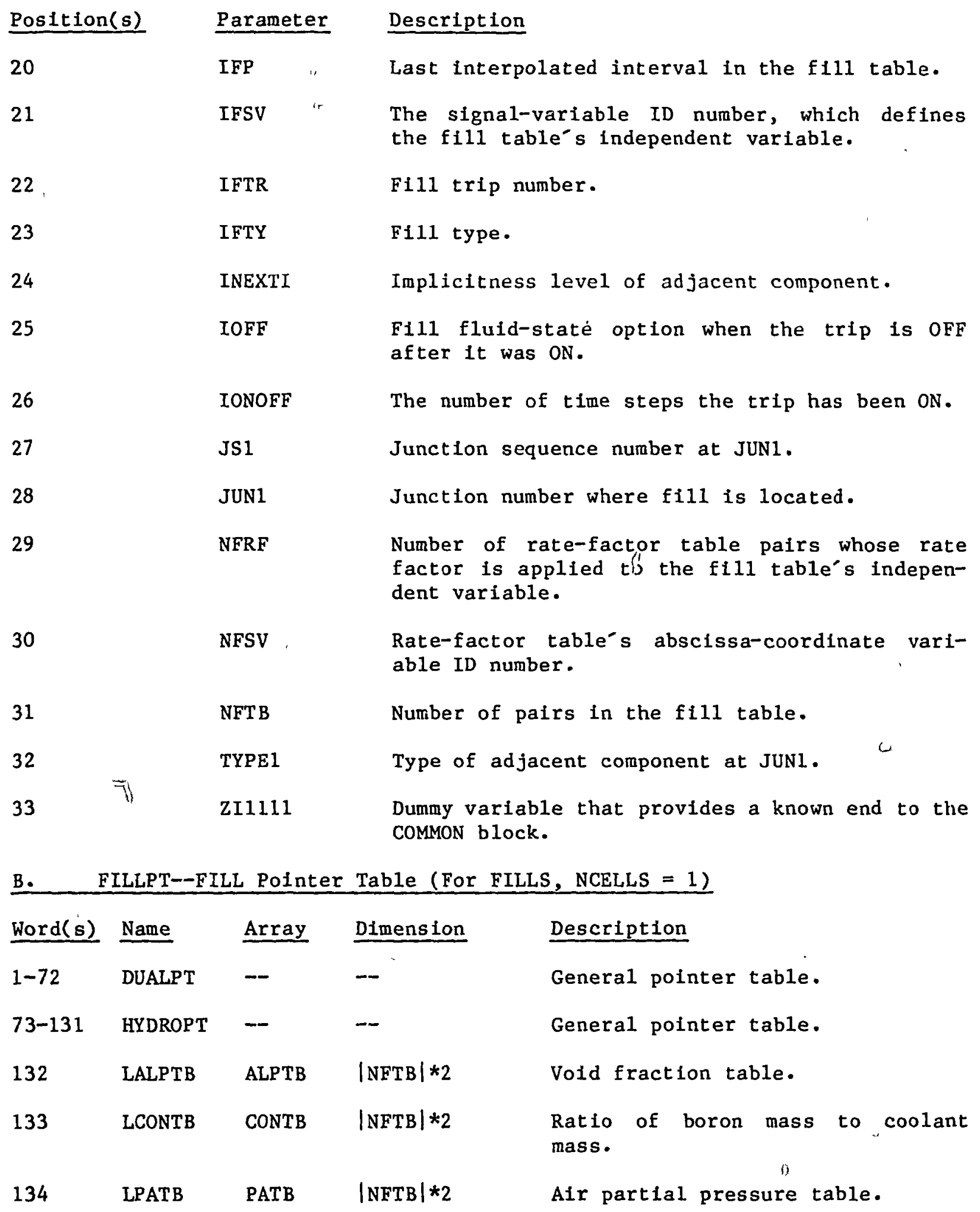




\begin{tabular}{|c|c|c|c|c|}
\hline Word(s) & Name & Arráy & Dimension & Description \\
\hline 135 & LPTB & РTB & $\mid$ NFTB $\mid * 2$ & Pressure table. \\
\hline 136 & LRFTB & RFTB & $|\mathrm{NFRF}| * 2$ & $\begin{array}{l}\text { The pointer for the fill } \\
\text { factor table. }\end{array}$ \\
\hline 137 & LTLTB & TLTB & $\mid$ NFTB $\mid * 2$ & Liquid temperature table. \\
\hline 138 & LTVTB & TVTB & $\mid$ NFTB $\mid * 2$ & Vapor temperature table. \\
\hline 139 & LVMTB & VMTB & $\mid$ NFTB $\mid * 2$ & Liquid velocity table. \\
\hline 140 & LVVTB & VVTB & $\mid$ NFTB $\mid * 2$ & Vapor velocity table. \\
\hline $141-317$ & PTDUM & $-\infty$ & -- & Dummy variable. \\
\hline
\end{tabular}

C. FILLDATA--FILL Data Table

This data table includes the following COMMON blocks: BLANKCOM and FIXEDLT, both defined in App. H; FILLVLT, defined in Sec. V.A; and FILLPT, defined in Sec. V.B.

VI. PIPE MODULE

A. 2 PIPEVLT--PIPE Variable-Length Table

REAL VARIABLES

Position(s) Parameter Description

1

2

3

4

$=5-6$

$7-8$

9
AAl111

BSMASS

CPOW

ENINP

FL(2)

$\mathrm{FV}(2)$

HOUTL
Dummy variable that provides a known start to the COMMON block.

Time-integrated mass flow from pipe.

Special pipe power input.

Total (time-integrated) energy directly input to the pipe.

Liquid mass-flow corrections for mass conservation checks.

Vapor mass-flow corrections for mass conservation checks.

HTC between outer boundary of pipe wall and liquid. 


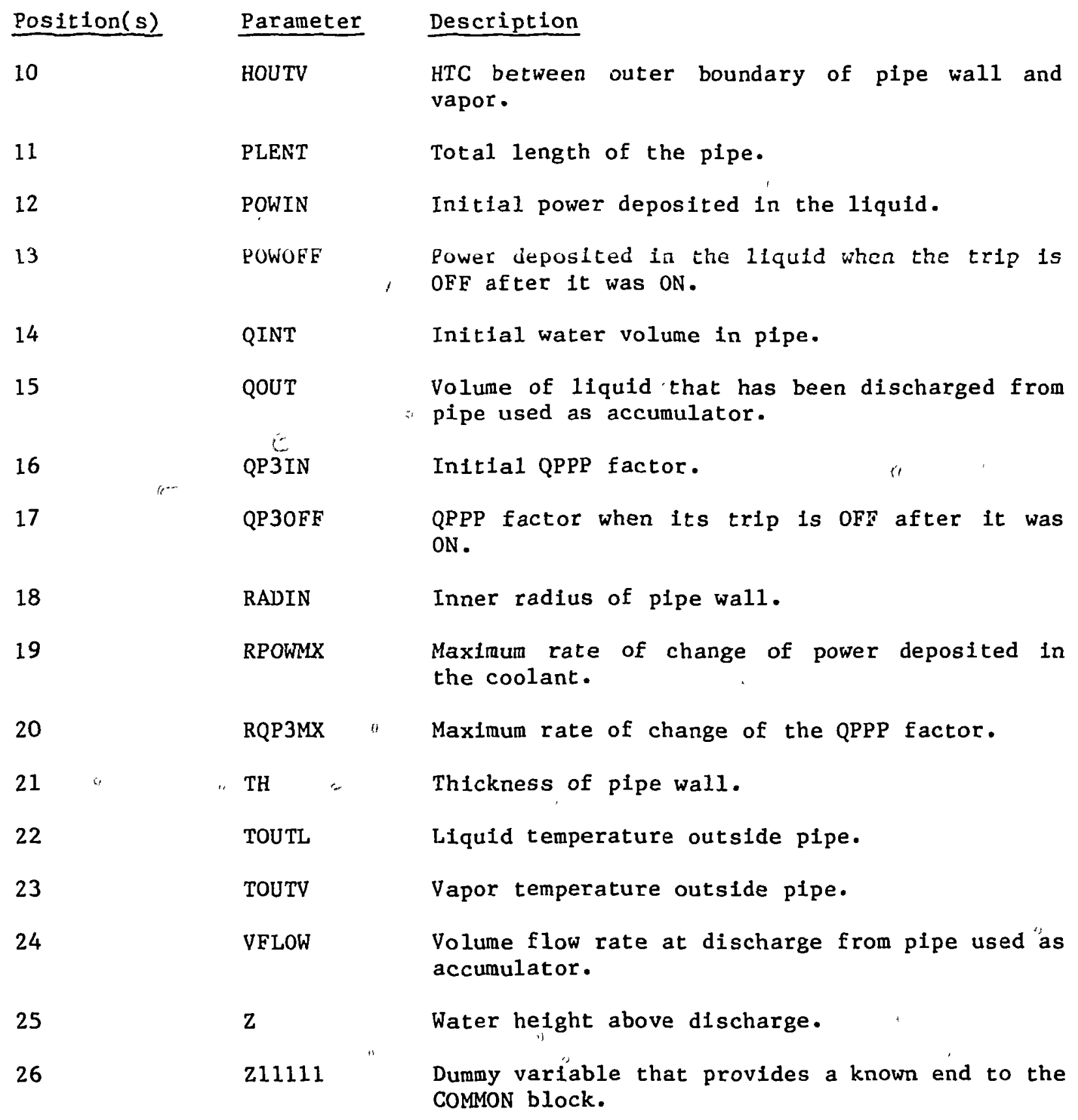

INTEGER VARIABLES

Position(s) Parameter Description

27

IAl111 Dummy variable that provides a known start to the COMMON block.

28 IACC Pipe accumulator option switch. 


\begin{tabular}{|c|c|c|}
\hline Position(s) & Parameter & Description \\
\hline 29 & ICHF & CHF calculation oftion. \\
\hline 30 & ICJ1 & Iteration index of adjacent component at JUNl. \\
\hline 31 & ICJ 2 & Iteration index of adjacent compónent at JUN2. \\
\hline 32 & ICONC & Indicator for presence of boron in the coolant. \\
\hline 33 & IONOFF & $\begin{array}{l}\text { Number of time steps the power deposited in the } \\
\text { coolant trip has been ON. }\end{array}$ \\
\hline 34 & $\begin{array}{c}\text { IPF } \\
\theta^{\prime}\end{array}$ & $\begin{array}{l}\text { Last interpolated interval in the power } \\
\text { deposited in the coolant's rate-factor table. }\end{array}$ \\
\hline 35 & IPOW & $\begin{array}{l}\text { Indicalor for presence of power deposited in } \\
\text { the coolant. }\end{array}$ \\
\hline 36 & IPOWSV & $\begin{array}{l}\text { Power deposited in the coolant table's } \\
\text { abscissa-coordinate variable ID number. }\end{array}$ \\
\hline 37 & IPOWTR & $\begin{array}{l}\text { Trip ID number that controls evaluation of the } \\
\text { power deposited in the coolant table. }\end{array}$ \\
\hline 38 & IPP & $\begin{array}{l}\text { Last interpolated interval in the power } \\
\text { deposited in the coolant table. }\end{array}$ \\
\hline 39 & IQF & $\begin{array}{l}\text { Last interpolated interval in the QPPP factor } \\
\text { table's rate-factor table. }\end{array}$ \\
\hline 40 & IQP & $\begin{array}{l}\text { Last interpolated interval in the QPPP factor } \\
\text { table. }\end{array}$ \\
\hline 41 & IQP3SV & $\begin{array}{l}\text { QPPP factor table's abscissa-coordinate } \\
\text { variable ID number. }\end{array}$ \\
\hline 42 & IQP3TR & $\begin{array}{l}\text { Trip ID number that controls evaluation of the } \\
\text { QPPP factor table. }\end{array}$ \\
\hline 43 & ISOLLB & Indicator for velocity update at JUN1. \\
\hline 44 & ISOLRB & Indicator for velocity update at JUN2. \\
\hline 45 & JS1 & Junction sequence number at cell 1 of the pipe. \\
\hline 46 & JS2 & $\begin{array}{l}\text { Junction sequence number at cell NCELLS of the } \\
\text { pipe. }\end{array}$ \\
\hline 47 & JUN1 & Junction number of the junction at cell 1 . \\
\hline
\end{tabular}




\begin{tabular}{|c|c|c|}
\hline Position(s) & Parameter & Description \\
\hline 48 & JUN2 & Junction number of the junction at cell NCELLS. \\
\hline 49 & MAT & Material identification. \\
\hline $50 "$ & NCELLS & Number of fluid cells. \\
\hline 51 & NONOFF & $\begin{array}{l}\text { Number of time steps the QPPP factor table's } \\
\text { controlling trip has been ON. }\end{array}$ \\
\hline 52 & NPOWRF & $\begin{array}{l}\text { Number of pairs in the power deposiled in the } \\
\text { coolant table-s rate-factor table. }\end{array}$ \\
\hline 53 & NPOWSV & $\begin{array}{l}\text { Power deposited in the coolant rate-factor } \\
\text { table's abscissa-coordinate variable ID number. }\end{array}$ \\
\hline 54 & NPOWTB & Length of pipe power table. \\
\hline 55 & NQP3RF & $\begin{array}{l}\text { Number of pairs in the QPPP factor table's } \\
\text { rate-factor table. }\end{array}$ \\
\hline $56^{\prime \prime}$ & NQP3SV & $\begin{array}{l}\text { QPPP factor rate-factor table's abscissa- } \\
\text { coordinate variable ID number. }\end{array}$ \\
\hline 57 & NQP3TB & Number of pairs in the QPPP factor table. \\
\hline 58 & TYPE1 & Type of adjacent component at JUNI. \\
\hline 59 & TYPE2 & Type of adjacent component at JUN2. \\
\hline 60 & $\mathrm{zi}: 111$ & $\begin{array}{l}\text { Dummy variable that provides a known end to the } \\
\text { COMMON block. }\end{array}$ \\
\hline
\end{tabular}

B. PIPEPT--PIPE Polnter Table

\begin{tabular}{|c|c|c|c|c|}
\hline Word (s) & Name & Array & Dimension & Description \\
\hline $1-72$ & DUALPT & -- & - & General pointer table. \\
\hline $73-131$ & HYDROPT & 二- & - & General pointer table. \\
\hline $132-134$ & INTPT & -- & - & General pointer table. \\
\hline $135-146$ & HEATPT & - & - & General pointer table. \\
\hline 147 & LPOWRF & POWRF & $\mid$ NPOWRF $\mid * 2$ & $\begin{array}{l}\text { Rate-factor tables for the power } \\
\text { deposited in the coolant tables. }\end{array}$ \\
\hline 148 & LPOWTB & POWTB & $\mid$ NPOWTB $\mid * 2$ & $\begin{array}{l}\text { Power deposited in the coolant } \\
\text { table. }\end{array}$ \\
\hline
\end{tabular}




$\begin{array}{lllll}\text { Word(s) } & \text { Name } & \text { Array } & \text { Dimension } & \text { Description } \\ 149 & \text { LQP3RF } & \text { QP3RF } & \mid \text { NQP3RF|*2 } & \begin{array}{l}\text { Rate-factor table for the QPPP } \\ \text { factor tables. }\end{array} \\ 150 & \text { LQP3TB } & \text { QP3TB } & \mid \text { NQP3TB|*2 } & \text { QPPP factor tables. } \\ 151-317 & \text { PTDUM } & -- & -- & \text { Dummy variable. }\end{array}$

C. PIPEDATA-PIPE Data Table

This data table includes the following COMMON blocks: BLANKCOM and FIXEDLT, both defined in APp. H; PIPEVLT, defined in Sec. VI.A; and PIPEPT, defined in Sec. VI.B.

VII. „PLENUM MODULE

The plenum data are all contained in the PLENDATA COMMON block that consists of the following COMMON blocks: DIMNSION, IOUNITS, JUNCTION, FIXEDLT, and BLANKCOM, all defined in APp. $\mathrm{H}$; VLTAB, defined in Sec. VII.A; and PTAB, defined in Sec. VII.B. "

A. VLTAB--PLENUM Variable-Length Table

REAL VARIABLES

\begin{tabular}{|c|c|c|}
\hline Position(s) & Parameter & Description \\
\hline 1 & AA1111 & $\begin{array}{l}\text { Dummy variable that provides a known start to } \\
\text { the COMMON block. }\end{array}$ \\
\hline 2 & BL & $\begin{array}{l}\text { Temporary storage for liquid mass conservation } \\
\text { checks. }\end{array}$ \\
\hline 3 & BSMASS & Time-integrated mass flow from pienum. \\
\hline 4 & $\mathrm{BV}$ & $\begin{array}{l}\text { Temporary storage for liquid mass conservation } \\
\text { checks. }\end{array}$ \\
\hline 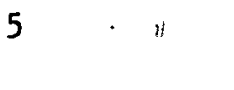 & FLXA & $\begin{array}{l}\text { Total air mass flow into the plenum during a } \\
\text { time step. }\end{array}$ \\
\hline 6 & FLXAL & $\begin{array}{l}\text { Total liquid volumetric flow into the plenum } \\
\text { during a time step. }\end{array}$ \\
\hline 7 & FLXAV & $\begin{array}{l}\text { Total vapor (gas phase) volumetric flow into } \\
\text { the plenum during a time step. }\end{array}$ \\
\hline 8 & FLXC & $\begin{array}{l}\text { Total solute mass flow into the plenum during a } \\
\text { time step. }\end{array}$ \\
\hline
\end{tabular}




\begin{tabular}{|c|c|c|}
\hline Position(s) & Parameter & Description \\
\hline 9 & FLXEL & $\begin{array}{l}\text { Total liquid internal energy flow into the } \\
\text { plenum during a time step. }\end{array}$ \\
\hline 10 & FLXEV & $\begin{array}{l}\text { Total vapor internal energy flow into the } \\
\text { plenum during a time step. }\end{array}$ \\
\hline 11 & FLXL & $\begin{array}{l}\text { Total liquid mass flow into the plenum during a } \\
\text { time step. }\end{array}$ \\
\hline 12 & FLXV & $\begin{array}{l}\text { Total vapor mass flow into the plenum during a } \\
\text { time step. }\end{array}$ \\
\hline 13 & $\mathrm{KXCL}$ & $\begin{array}{l}\text { Temporary storage for the right-hand side of } \\
\text { the liquid, stabilizer mass and energy } \\
\text { equations. }\end{array}$ \\
\hline 14 & $\mathrm{RXCV}$ & $\begin{array}{l}\text { Temporary storage for the right-hand side of } \\
\text { the vapor stabilizer mass and energy equations. }\end{array}$ \\
\hline 15 & $X I$ & $\begin{array}{l}\text { Gross total liquid volumetric flow from the } \\
\text { plenum during a time step. }\end{array}$ \\
\hline 16 & $\mathrm{XV}$ & $\begin{array}{l}\text { Gross total vapor volumetric flow from the } \\
\text { plenum during a time step. }\end{array}$ \\
\hline 17 & $\mathrm{Z} 11111$ & $\begin{array}{l}\text { Dummy variable that provides a known end to the } \\
\text { COMMON block. }\end{array}$ \\
\hline
\end{tabular}

INTEGER VARIABLES

18

19

20

21

22

23

24
IA1111

K

ICONC

IPOW

LENAAR

NPLJN

ZI1111
Dummy variable that provides a known start to the COMMON block.

Address location that designates the location of the array data variables.

Indicator for the presence of boron in the coolant.

Indicator for the presence of power deposited in the coolant.

Lengths of the array data that are referenced using pointers.

Number of plenum junctions.

'Dummy variable that provides a known end to the COMMON block. 
B. PTAB--PLENUM Pointer Table

\begin{tabular}{|c|c|c|c|}
\hline Word (s) & Name & Array & Dimensicn \\
\hline 1 & L11111 & -- & 1 \\
\hline 2 & LALW & ALW & NPLJN \\
\hline 3 & LAVW & AVW & NPLJN \\
\hline 4 & LDBND & DBND & $5 *_{\text {NPLJN }}$ \\
\hline & ". & "* & \\
\hline 5 & LDFLDP & DFLDP & NPLJN \\
\hline 6 & LDFVDP & DFVDP & NPLJN \\
\hline 7 & LDNFL & DONFL & NPLJN \\
\hline
\end{tabular}

8

LDNFV DONFV NPLJN

9

LDX

DX

NPLJN

10

LFAVOL FAVOL NPLJN

11

LGRAV

GRAV

NPLJN

12

LIOJ

IOJ

NPLJN

13

LJUNJ

JUNJ

NPLJN

\section{Description}

Dummy variable that provides a known start to the COMMON block.

Temporary storage for the righthand side of the liquid stabilizer mass and energy equations.

Temporary storage for the righthand side of the vapor stabilizer mass and energy equations.

Donor-cell quantities $\alpha \rho_{\mathrm{v}}$, $(1-\alpha) \rho_{\ell}, \alpha \rho_{v} e_{v},(1-\alpha) \rho_{\ell} e_{\ell}$, and $\alpha_{a}$

Derivative of the liquid velocity with respect to pressure.

Derivative of the vapor velocity with respect to pressure.

Donor-cell flag for liquid.

$0.0=$ indicates flow into the plenum,

$1.0=$ indicates flow from the plenum.

Donor-cell flag for vapor.

$0.0=$ indicates flow into the plenum,

$1.0=$ indicates flow from the plenum.

Effective plenum-side cell lengths for junctions connected to the plenum (required in the solution of the momentum equations).

Cell flow area used in the chokedflow model.

Gravitational "terms (cosine theta).

Network junction numbers.

Plenum junction numbers. 


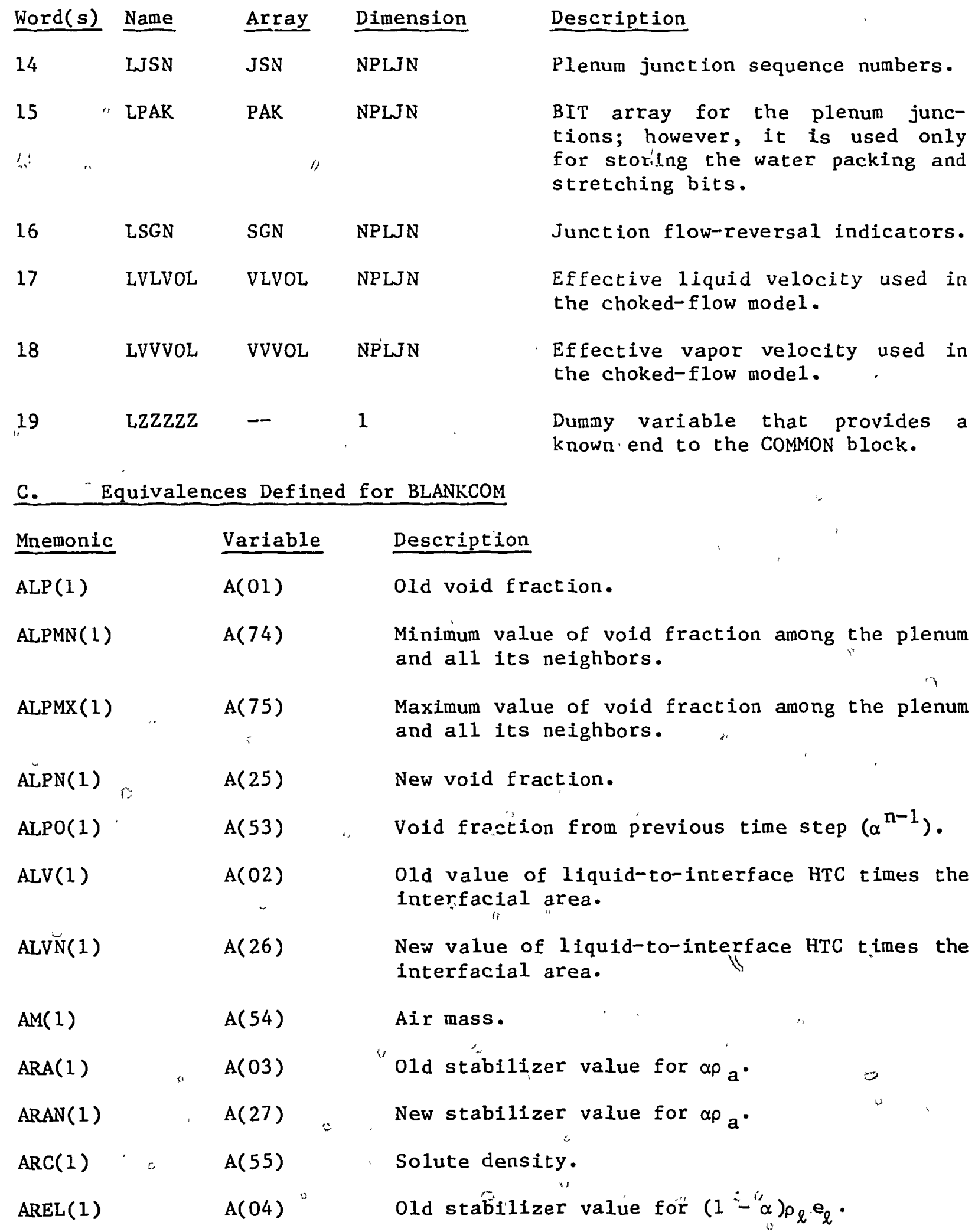




\begin{tabular}{|c|c|c|c|}
\hline Mnemonic & & Variable & Description \\
\hline $\operatorname{ARELN}(1)$ & & $A(28)$ & New stabilizer value for $(1-\alpha) p_{\ell} e_{\ell}$. \\
\hline $\operatorname{AREV}(1)$ & & $A(05)$ & old stabilizer value for $\alpha \rho_{\mathrm{v}} \mathrm{e}_{\mathrm{v}}$. \\
\hline $\operatorname{AREVN}(1)$ & & $A(29)$ & New stabilizer value for $\alpha \rho v_{v} e_{v}$. \\
\hline $\operatorname{ARL}(1)$ & & $A(06)$ & old stabilizer value for $(1-\alpha)_{\ell}$. \\
\hline $\operatorname{ARLN}(1)$ & & $A(30)$ & New stabilizer value for $(1-\alpha)_{\ell}$. \\
\hline $\operatorname{ARV}(1)$ & & $A(07)$ & old stabilizer value for $\alpha \rho v$ \\
\hline $\operatorname{ARVN}(1)$ & & $A(31)$ & New stabilizer value for $\alpha \rho_{v^{*}}$ \\
\hline $\operatorname{BIT}(1)$ & & $A(22)$ & old bit flag. \\
\hline $\operatorname{BITN}(1)$ & & $A(46)$ & New bit flag. \\
\hline BOR(1) & & $A(08)$ & old boron-to-coolant mass ratio. \\
\hline $\operatorname{BORN}(1)$ & & $A(32)$ & New boron-to-coolant mass rátio. \\
\hline $\operatorname{CHTI}(1)$ & 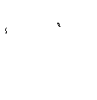 & $A(12)$ & $\begin{array}{l}\text { old value of vapor-to-interface HTC times the } \\
\text { interfacial area. }\end{array}$ \\
\hline $\operatorname{CHTIN}(1)$ & & $A(36)$ & $\begin{array}{l}\text { New value of vapor-to-interface HTC times the } \\
\text { interfactal area. }\end{array}$ \\
\hline $\operatorname{CL}(1)$ & 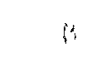 & $A(49)$ & Liquid conductivity. \\
\hline $\operatorname{CPL}(1)$ & & $A(50)$ & Liquid specific heat at constant pressure. \\
\hline $\operatorname{CPV}(1)$ & & $A(51)$ & Liquid specific heat at constant volume. \\
\hline $\mathrm{CV}(1)$ & . & $\mathrm{A}(52)$ & Vapor conductivity. \\
\hline $\operatorname{DALP}(1)$ & & $A(57)$ & Weighting factor XVSET. \\
\hline $\operatorname{DALVA}(1)$ & & $A(68)$ & Derivative of ALV with respect to $\alpha$. \\
\hline $\operatorname{DELDP}(1)$ & & $\mathrm{A}(80)$ & $\begin{array}{l}\text { Derivative of the liquid internal energy with } \\
\text { respect to pressure at constant temperature. }\end{array}$ \\
\hline $\operatorname{DELDT}(1)$ & & $A(82)$ & $\begin{array}{l}\text { Derivative of the liquid internal energy with } \\
\text { respect to temperature at constant pressure. }\end{array}$ \\
\hline $\operatorname{DEVAP}(1)$ & & $A(94)$ & $\begin{array}{l}\text { Derivative of air internal energy with respect } \\
\text { to pressure at constant temperature. }\end{array}$ \\
\hline
\end{tabular}




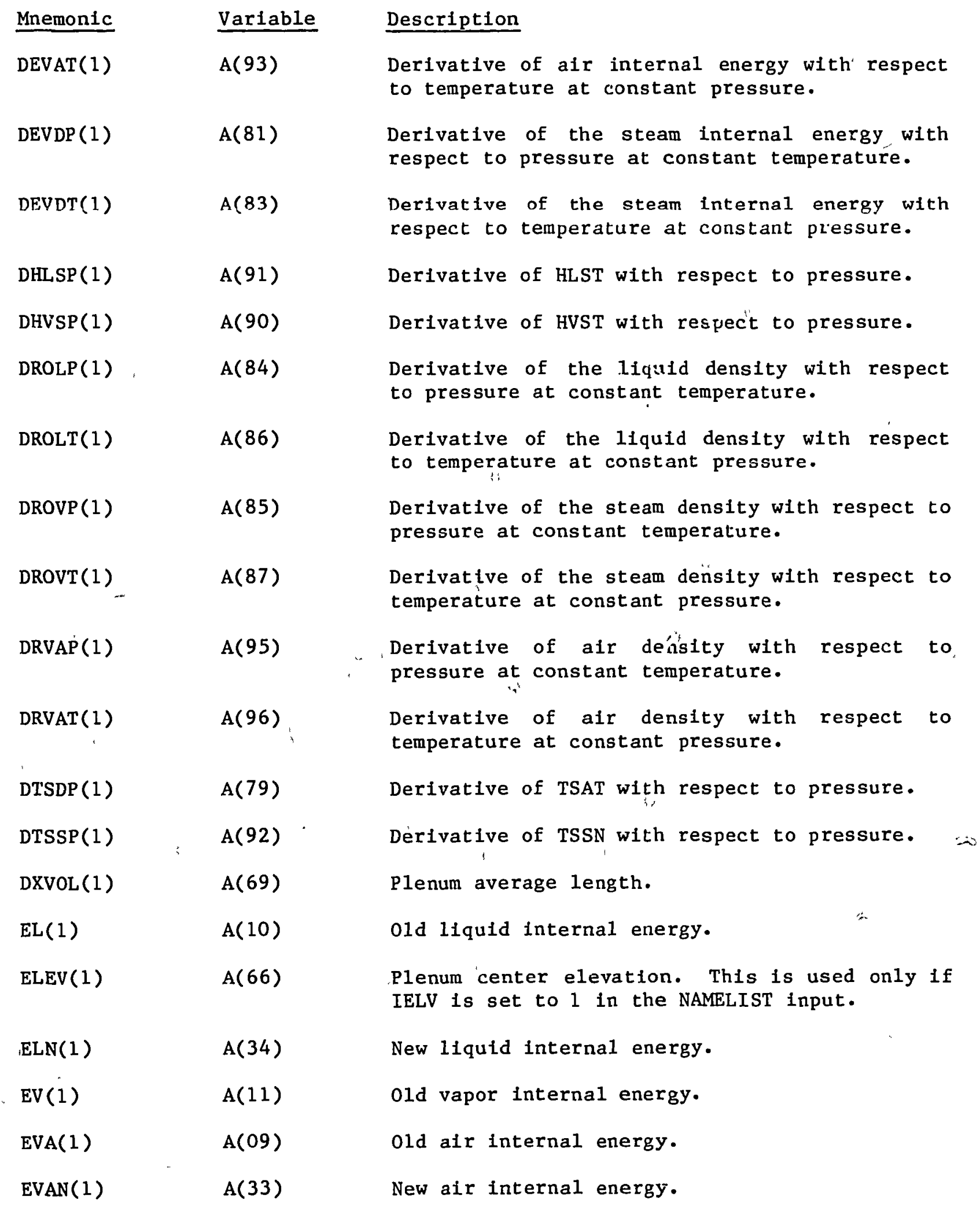




\begin{tabular}{|c|c|c|}
\hline Mnemonic & Variable & Description \\
\hline $\operatorname{EVN}(1)$ & $A(35)$ & New vapor internal energy. \\
\hline FASMLT( 1$)$ & $A(77)$ & Interphase area multiplier during condensation. \\
\hline FAVOL (1) & $A(70)$ & Plenum average flow área. \\
\hline GAM( 1$)$ & $A(13)$ & Old vapor generation rate per unlt volume. \\
\hline $\operatorname{GAMN}(1)$ & $A(37)$ & New vapor generation rate per unit volume. \\
\hline GRAVOL(1) & $A(73)$ & Plenum average GRAV. \\
\hline HFG( 1 ) & $A(56)$ & Latent heat of vaporization. \\
\hline $\operatorname{HIL}(1)$ & $A(23)$ & old HTC between inside wall and liquid. \\
\hline $\operatorname{HILN}(1)$ & $A(47)$ & New HTC between inside wall and liquid. \\
\hline $\operatorname{HIV}(1)$ & $A(24)$ & 01d HTC between inside wall and vapor. \\
\hline $\operatorname{HIVN}(1)$ & $A(48)$ & New HTC between inside wall and vapor. \\
\hline HLST ( 1$)$ & $A(89)$ & $\begin{array}{l}\text { Saturated liquid enthalpy (at TSSN and total } \\
\text { pressure). }\end{array}$ \\
\hline HVST (1) & $A(88)$ & $\begin{array}{l}\text { Saturated steam enthalpy (at TSSN and steam } \\
\text { partial pressure). }\end{array}$ \\
\hline$P(1)$ & $A(14)$ & 01d pressure. \\
\hline $\operatorname{PA}(1)$ & $A(15)$ & old áir partial pressure. \\
\hline $\operatorname{PAN}(1)$ & $A(39)$ & New air partial pressure. \\
\hline $\mathrm{PN}(1)$ & $A(38)$ & New pressure. \\
\hline $\mathrm{QP3F}(1)$ & $A(67)$ & QPPP factor applied to the wall heat source. \\
\hline ROL (1) & $A(17)$ & old liquid density. \\
\hline $\operatorname{ROLN}(1)$ & $A(41)$ & New liquid density. \\
\hline $\operatorname{ROM}(1)$ & $A(58)$ & Mixture density. \\
\hline $\operatorname{ROV}(1)$ & $A(18)$ & Old vapor density. \\
\hline $\operatorname{ROVA}(1)$ & $A(16)$ & old air density. \\
\hline ROVAN (1) & $A(40)$ & New air density. \\
\hline
\end{tabular}




\begin{tabular}{|c|c|c|}
\hline Mnemonic & Variable & Description \\
\hline $\operatorname{ROVN}(1)$ & $A(42)$ & New vapor density. \\
\hline SIG (1) & $A(59)$ & Surface tension. \\
\hline $\operatorname{SOLID}(1)$ & $A(19)$ & $\begin{array}{l}\text { old boron solute mass plated on structure } \\
\text { surface. }\end{array}$ \\
\hline SOLIDN(1) & $A(43)$ & $\begin{array}{l}\text { New boron solute mass plated on structure } \\
\text { surface. }\end{array}$ \\
\hline $\operatorname{TL}(1)$ & $A(20)$ & 01d 1iquid temperature. \\
\hline $\operatorname{TLN}(1)$ & $A(44)$ & New liquid temperature. \\
\hline $\operatorname{TSAT}(1)$ & $A(60)$ & $\begin{array}{l}\text { Saturation temperature corresponding to total } \\
\text { vapor pressure. }\end{array}$ \\
\hline $\operatorname{TSSN}(1)$ & $A(61)$ & $\begin{array}{l}\text { Saturation temperature corresponding to steam } \\
\text { partial pressure. }\end{array}$ \\
\hline $\operatorname{TV}(1)$ & $A(21)$ & Old vapor temperature. \\
\hline $\operatorname{TVN}(1)$ & $A(45)$ & New vapor temperature. \\
\hline VISL(1) & $A(62)$ & Liquidd viscosity. \\
\hline $\operatorname{VISV}(1)$ & $\mathrm{A}(63)$ & Vapor viscosity. \\
\hline VLALP(1) & $\mathrm{A}(78)$ & $\begin{array}{l}\text { Maximum value of the liquid mass flux entering } \\
\text { the plenum from junctions located above the } \\
\text { plenum. }\end{array}$ \\
\hline $\operatorname{VLVC}(1)$ & $A(76)$ & $\begin{array}{l}\text { Absolute value of the liquid velocity at a } \\
\text { junction where the donor-celled liquid fraction } \\
\text { is maximum. }\end{array}$ \\
\hline VLVOL(1) & $A(71)$ & Plenum average liquid velocity. \\
\hline VOL(1) & $A(64)$ & Plenum volume. \\
\hline VVVOL(1) & $A(72)$ & Plenum average vapor velocity. \\
\hline WA(1) & $A(65)$ & Wall area. \\
\hline
\end{tabular}


III. PRESSURIZER MODULE

A. PRIZEVLT--PRIZER Variable-Length Table

REAL VARIABLES

Position(s) Parameter Description

1

2

3

4

5

6-7

8

$9-10$

11

12

13

14

15

16

17

18

19

20
AA1111

BSMASS

BSMSSP

BXMASS

DPMAX

FL(2)

FLOW

FV(2)

HOUTL

HOUTV

PSET

QHEAT

QIN

QINT

QOUT

QP3IN

RADIN

TH
Dummy variable that provides a known start to the COMMON block.

Time-integrated mass flow from pressurizer.

Current mass-flow rate during transient.

Current mass-flow , rate during steady state.

Differential pressure at which heaters have maximum power.

Liquid mass-flow corrections for mass conservation checks.

Volume flow rate at discharge.

Vapor mass-flow corrections for mass conservation checks.

HTC between outer boundary of pressurizer wall and 1iquid.

HTC between outer boundary of pressurizer wall and vapor.

Pressurizer pressure set point for heater-spray control.

Total heater power.

Heater power being input to water.

Initial water volume in pressurizer.

Volume of liquid that has discharged from the pressurizer.

Intial QPPP factor.

Inner radius of pressurizer wall.

Th1ckness of pressurizer wall. 


\begin{tabular}{|c|c|c|}
\hline Position(s) & Parameter & Description \\
\hline 21 & TOUTL & Liquid temperature outside pressurizer. \\
\hline 22 & TOUTV & Vapor temperature outside pressurizer. \\
\hline 23 & $\mathrm{Z}$ & Water height above discharge. \\
\hline 24 & ZUTR & Water height for heater cutoff. \\
\hline 25 & Z11111 & $\begin{array}{l}\text { Dummy variable that provides a known end to the } \\
\text { COMmoN block. }\end{array}$ \\
\hline
\end{tabular}

INTEGER VARIABLES

26

27

28

29

30

31

32

33

34

35

36

37

38

39

40
IA1111

ICHF

ICJ

ICONC

ICT 1

IUV1

IU์?

JS1

JS2

JUN1

JUN2

MAT

NCELLS

TYPE1

TYPE2
Dummy variable that provides a known start to the COMMON block.

CHF calculation option.

Iteration index of adjacent component.

Indicator for presence of boron in the coolant.

The sequence number (position in the IORDER array) of the component next to the junction of the pressurizer. This variable is computed but not used.

Indicator for velocity update at JUN1 (equal to zero).

Indicator for velocity update at JUN2.

Junction sequence number at cell 1 of the pressurizer.

Junction sequence number at cell NCELLS of the pressurizer.

Junction number of the junction at cell 1 .

Junction number of the junction at cell NCELLS.

Material ID number of the pressurizer wall.

Number of fluid cells.

Type of adjacent component at JUNl.

Type of component adjacent at JUN2. 


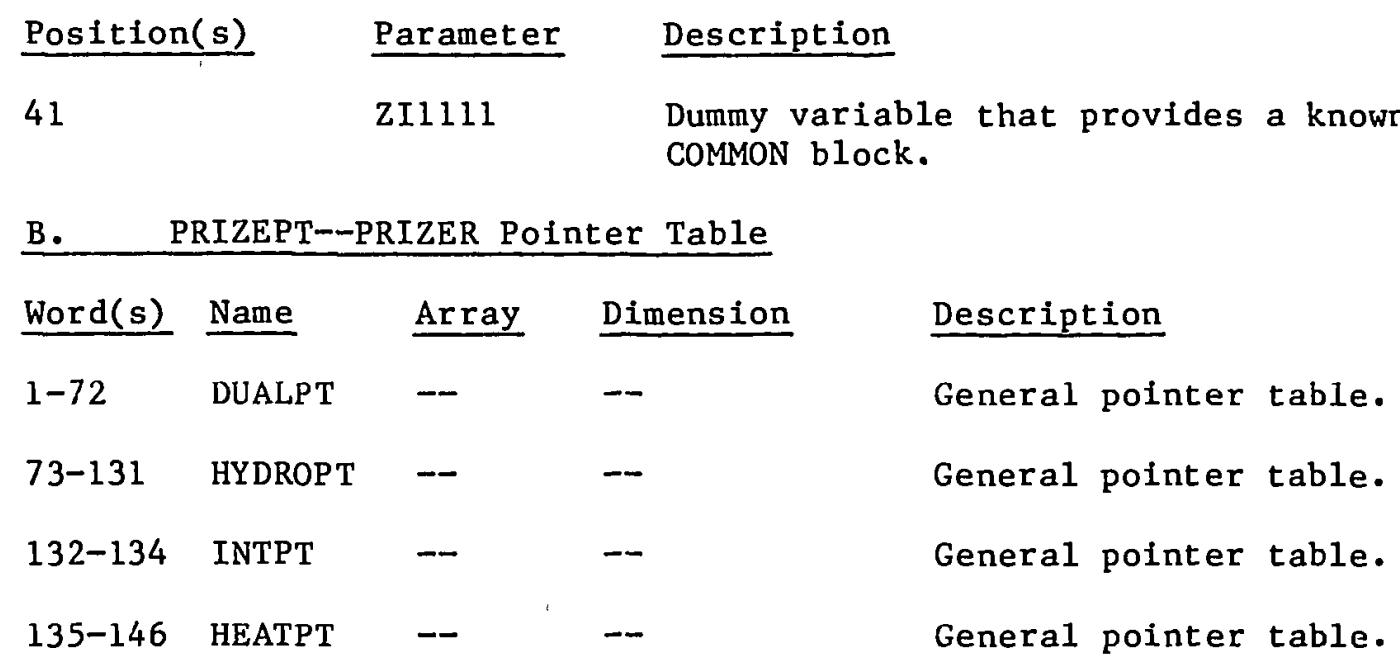

C. PRZDATA--PRIZER Data Table

This data table includes the following COMMON blocks: BLANKCOM and FIXEDLT, both defined in App. H; PRIZEVLT, defined in Sec.VIII.A; and PRIZEPT, defined in Sec. VIII.B.

IX. PUMP MODULE

A. PUMPVLT--PUMP Variable-Length Table

REAL VARIABLES

Position(s) Parameter Description

1

AA1111 Dummy variable that provides a known start to the COMMON block.

2

AEFFMI . The coefficient for the (OMEGA/ROMEGA)**2 term in the calculation of the variable moment of inertia $\left(\mathrm{kg} \cdot \mathrm{m}^{2}\right)$.

3

ALPHA

Pump void fraction.

4

ALPHAO

Void fraction used on previous time step for pump head calculation.

5

BEFFMI

The coefficient for the (OMEGA/ROMEGA) term in the calculation of the variable moment of inertia $\left(\mathrm{kg} \cdot \mathrm{m}^{2}\right)$.

BSMASS

Time-integrated mass flow from pump.

CEFFMI

The constant term in the calculation of the variable moment of inertia $\left(\mathrm{kg} \cdot \mathrm{m}^{2}\right)$. 


\begin{tabular}{|c|c|c|}
\hline Position(s) & Parameter & Description \\
\hline 8 & DELP & Delta $P$ across pump. \\
\hline 9 & DSMOM & Derivative of pump head.with velocity. \\
\hline 10 & EFFMI & Moment of inertia. \\
\hline 11 & EFFMII & The alternate effective moment of inertia. \\
\hline $12-13$ & FL(2) & $\begin{array}{l}\text { Liquid mass-flow corrections for mass conserva- } \\
\text { tion checks. }\end{array}$ \\
\hline 14 & FLOW & Pump volumetric flow rate. \\
\hline $15-16$ & $\mathrm{FV}(2)$ & $\begin{array}{l}\text { Vapor mass-flow corrections for mass conserva- } \\
\text { tion checks. }\end{array}$ \\
\hline 17 & HEAD & Pump head. \\
\hline 18 & HOUTL & $\begin{array}{l}\text { HTC between outer boundary of pump wall and } \\
\text { liquid. }\end{array}$ \\
\hline 19 & HOUTV & $\begin{array}{l}\text { HTC between outer boundary of pump wall and } \\
\text { vapor. }\end{array}$ \\
\hline 20 & MFLOW & Pump mass-flow rate. \\
\hline 21 & OMEGA & Angular velocity at old time. \\
\hline 22 & OMEGAN & Angular velocity at new time. \\
\hline 23 & OMGOFF & $\begin{array}{l}\text { Pump rotational speed when its controlling trip } \\
\text { is OFF after it was ON. }\end{array}$ \\
\hline 24 & OMTEST & $\begin{array}{l}\text { The PUMP speed below which EFFMI1 (the } \\
\text { alternate effective moment of inertia) is used } \\
\text { (rad } \cdot s^{-1} \text { ). }\end{array}$ \\
\hline 25 & QP3IN & Initiai QPPP factor. \\
\hline 26 & QP30FF & $\begin{array}{l}\text { QPPP factor when its controling trip is OFF } \\
\text { after it was ON. }\end{array}$ \\
\hline 27 & RADIN & Inner radius of wall. \\
\hline 28 & RFLOW & Rated flow. \\
\hline 29 & RHEAD & Rated head. \\
\hline 30 & RHO & Pump mixture density. \\
\hline
\end{tabular}




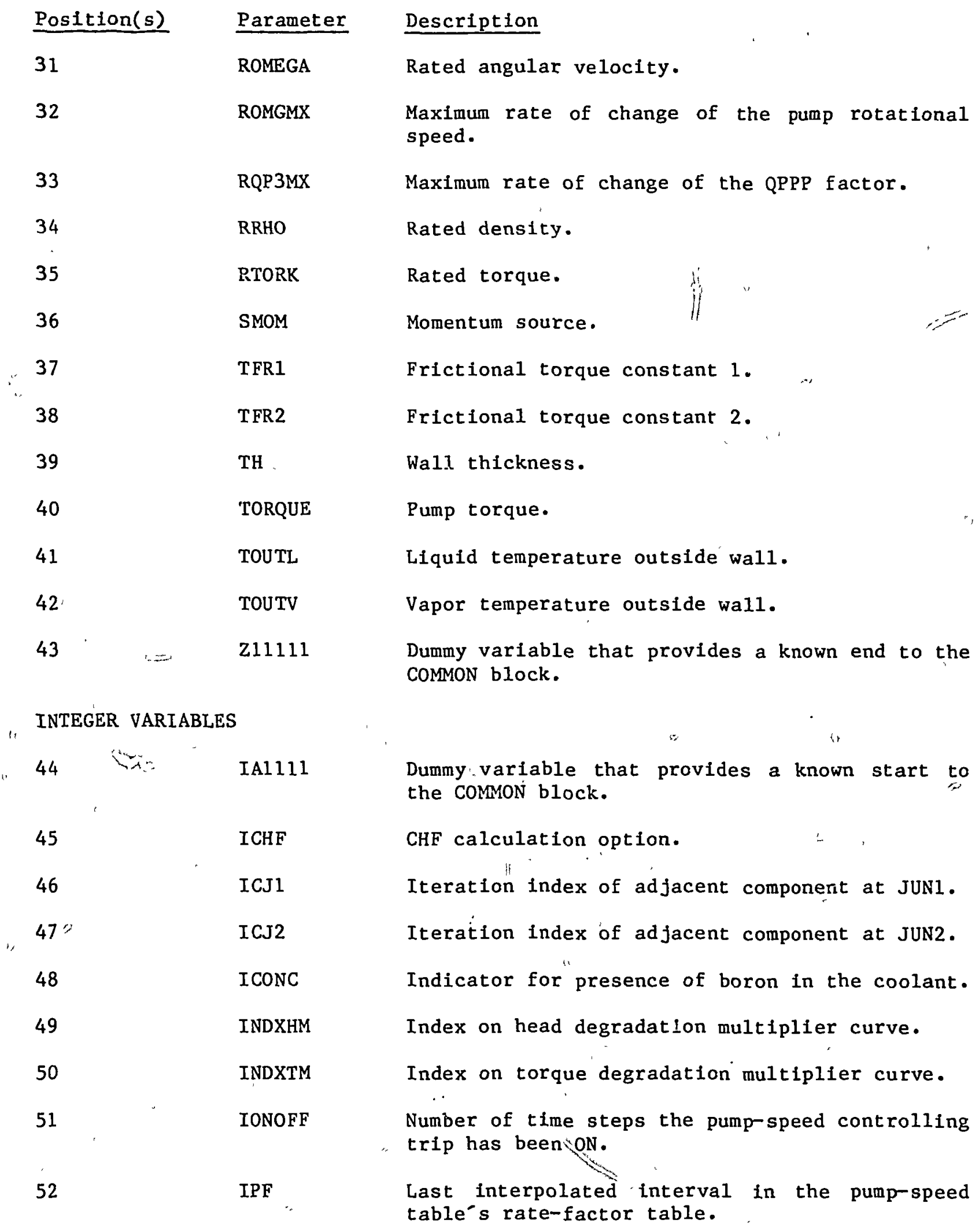




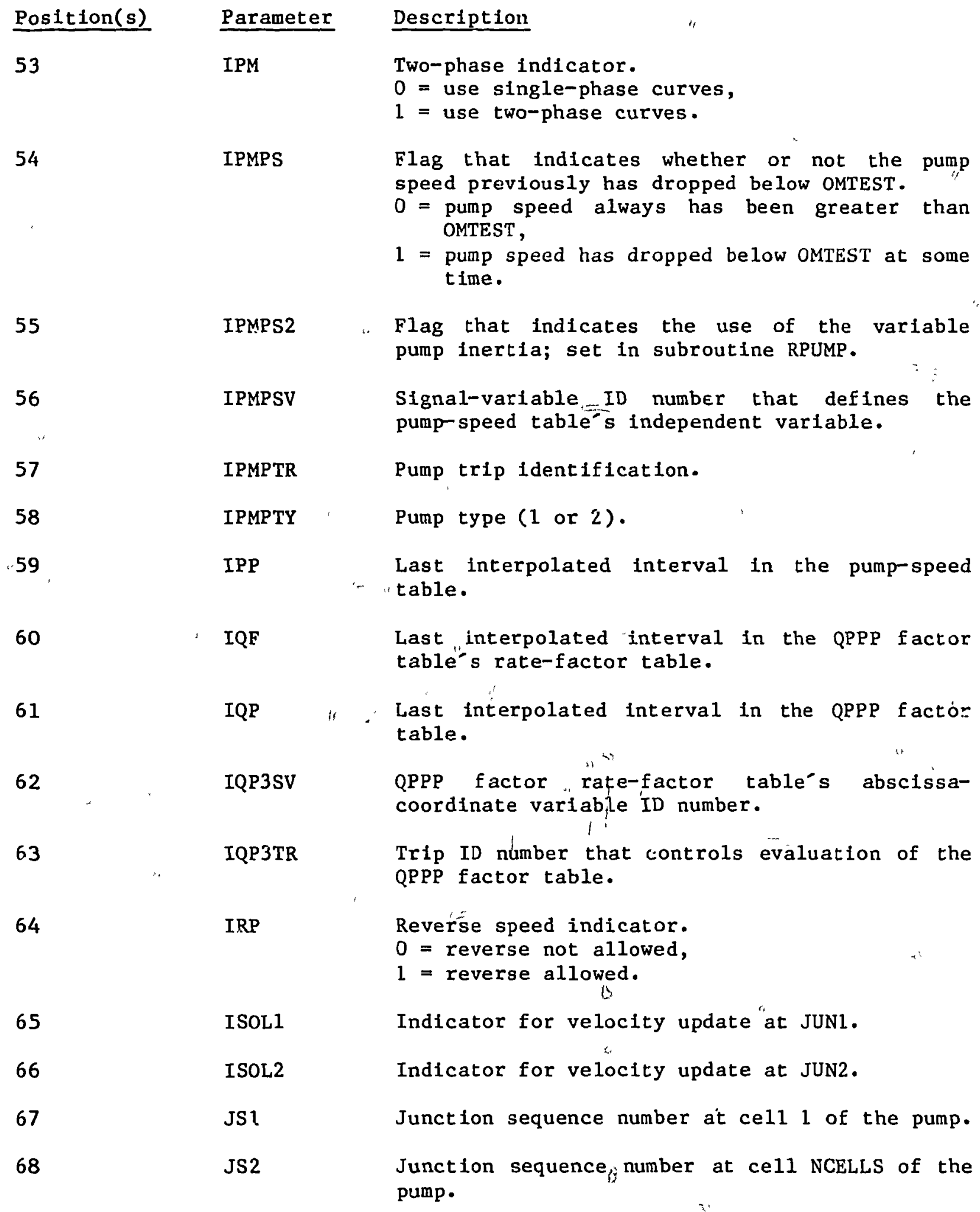




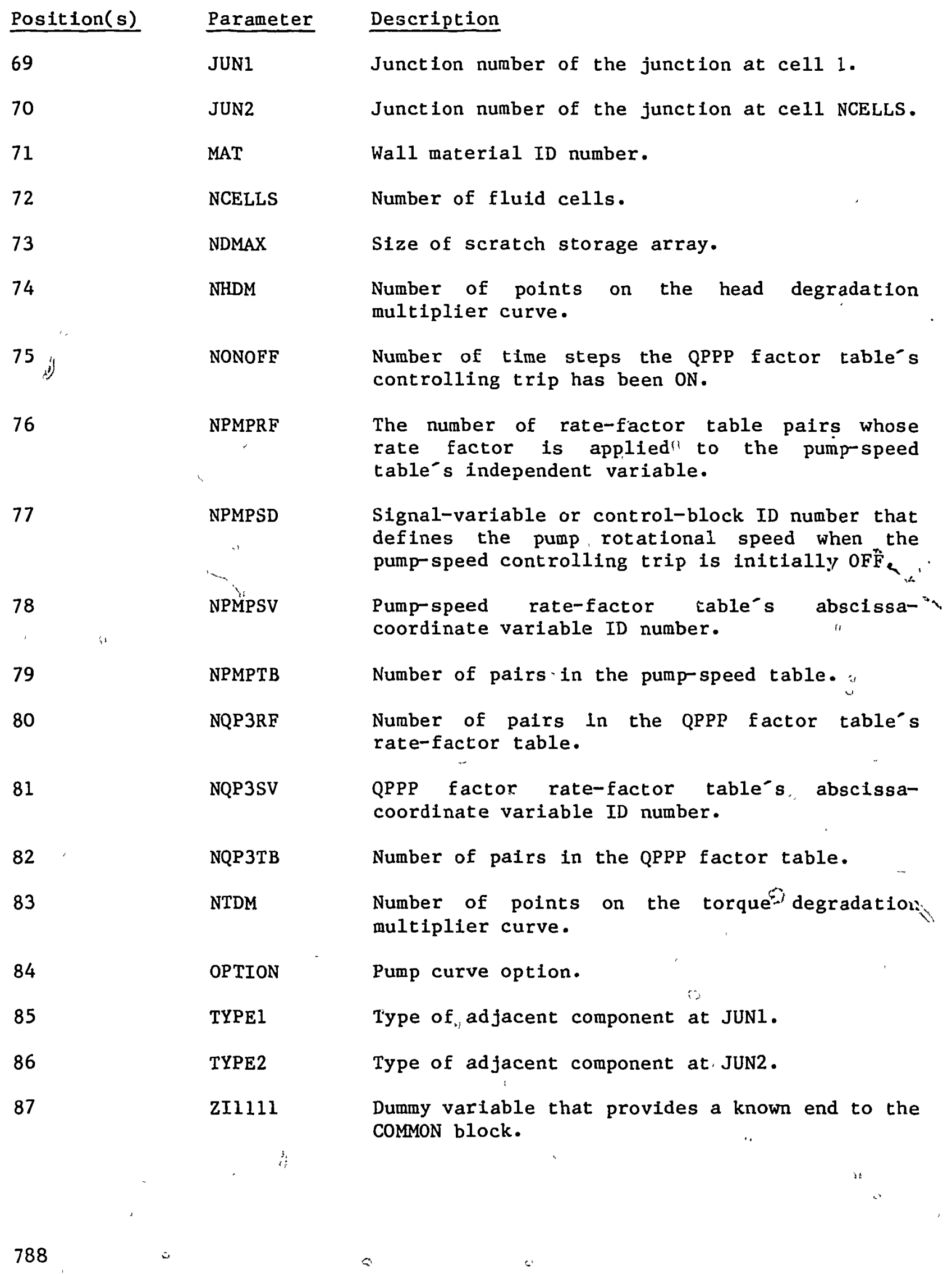




\begin{tabular}{|c|c|c|c|c|c|c|c|}
\hline Word(s) & Name & Array & & Dimension & & Description & \\
\hline $1-72$ & DUALPT & -- & " & $-\bar{n}$ & - & General pointer table. & \\
\hline $73-131$ & HYDROPT & -- & & -- & & General pointer table. & \\
\hline $132-134$ & INTPT & -- & & -- & & General pointer table. & \\
\hline $135-146$ & HEATPT & -- & & -- & & G'eneral pointer table. & \\
\hline HEAD AND & \multicolumn{3}{|c|}{ TORQUE TABLE } & & & 4 & \\
\hline 147 & LHSP1 & HSPl & & $2 * \operatorname{NDATA}(1)$ & & Single-phase head curve 1 . & \\
\hline 148 & LHSP2 & HSP2 & & $2 * \operatorname{NDATA}(2)$ & & Single-phase head curve 2 . & \\
\hline 149 & LHSP3 & HSP3 & & $2 * \operatorname{NDATA}(3)$ & & Single-phase head curve 3 . & \\
\hline 150 & LHSP4 & HSP4 & & 2.*NDATA(4) & & Single-phase head curve 4 . & 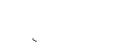 \\
\hline 151 & LHTP1 & HTPl & . & $2 * \operatorname{NDATA}(5)$ & & Two-phase head curve 1 . & \\
\hline 152 & LHTP2 & HTP2 & & $2 * \operatorname{NDATA}(6)$ & & Two-phase head curve 2 . & \\
\hline 153 & LHTP3 & HTP 3 & & $2 * \operatorname{NDATA}(7)$ & & Two-phase head curve 3 . & \\
\hline 154 & LHTP4 & HTP 4 & & $2 * \operatorname{NDATA}(8)$ & & Two-phase head curve 4 & \\
\hline 155 & LTSP1 & TSP1 & & $2 * \operatorname{NDATA}(9)$ & & Single-phase torque curve 1. & 妾 \\
\hline 156 & LTSP2 & TSP2 & & $2 * \operatorname{NDATA}(10)$ & & Single-phase torque curve 2 . & \\
\hline 157 & LTSP3 & TSP3 & & $2 * \operatorname{NDATA}(11)$ & & Single-phase torque curve 3 . & \\
\hline 158 & LTSP4 & TSP4 & 11 & $2 * \operatorname{NDATA}(12)$ & $=$ & Single-phase torque curve 4 . & \\
\hline 159 & LTTP1 & TTP1 & & $2 * \operatorname{NDATA}(13)$ & & Two-phase torque curve 1 . & \\
\hline 160 & LTTP2 & TTP2 & & $2 * \operatorname{NDATA}(14)$ & & Two-phase torque curve 2 . & \\
\hline 161 & LTTP3 & TTP3 & & $2 * \operatorname{NDATA}(15)$ & & Two-phase torque curve 3 . & ¿ \\
\hline 162 & LTTP4 & TTP4 & & $2 * \operatorname{NDATA}(16)$ & & Two-phase torque curve 4 . & \\
\hline MISCELLAI & NEOUS TAB & & & & & . & \\
\hline 163 & LBD4 & BD4 & $\checkmark$ & LENBD & & Dummy variable. & \\
\hline 164 & LHDM & HDM & & $2 *$ NHDM & & Head degradation multiplier & curve. \\
\hline
\end{tabular}




\begin{tabular}{|c|c|c|c|c|}
\hline Word(s) & Name & Array & Dimension & Description \\
\hline 165 & LIDXCS & IDXCS & 16 & Curve set index array. \\
\hline 166 & LNDATA & NDATA & 16 & $\begin{array}{l}\text { Number of sets of points in head } \\
\text { and torque curves. }\end{array}$ \\
\hline 167 & LPMPRF & PMPRE & $\mid$ NPMPRF $\mid * 2$ & $\begin{array}{l}\text { Rate-factor table for the pump- } \\
\text { speed table. }\end{array}$ \\
\hline 168 & LPMPTB & PMPTB & $\mid$ NPMPTB $\mid * 2$ & Pump rotational speed table. \\
\hline 169 & LQP3RF & QP3RF & $|\mathrm{NQP} 3 \mathrm{RF}| * 2$ & $\begin{array}{l}\text { Rate-factor table for the QPPP } \\
\text { factor table. }\end{array}$ \\
\hline 170 & LQP3TB & QP3TB & $|\mathrm{NQP} 3 \mathrm{~TB}| * 2$ & QPPP factor table. \\
\hline 171 & LTDM & TDM & $2 * N T D M$ & degradation \\
\hline $172-317$ & PTDUM & - & -- & Dummy varlable. \\
\hline
\end{tabular}

C. PUMPDATA--PUMP Data Table

This data table includes the following COMMON blocks: BLANKCOM and FIXEDLT, both defined in App. H; PUMPVLT, defined in Sec. IX.A; and PUMPPT, defined in, Sec. IX.B.

X. STEAM-GENERATOR MODULE

A. STGENVLT--STGEN Variable-Length Table

REAL VARIABLES

\begin{tabular}{|c|c|c|}
\hline Position(s) & Parameter & Description \\
\hline 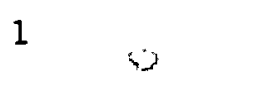 & AAl111 & $\begin{array}{l}\text { Dummy variable that provides a known start to } \\
\text { the COMMON block. }\end{array}$ \\
\hline 2 & BSMSS1 & Time-integiated mass flow from primary side. \\
\hline 3 & BSMSS2 & "Time-integrated"mass flow from secondary side. \\
\hline 4 & CA1P & $\begin{array}{l}\text { Fraction of liquid velocity at the right face } \\
\text { of the primary tee junction cell that contri- } \\
\text { butes to the momentum transfer into the primary } \\
\text { tee side leg. }\end{array}$ \\
\hline 5 & CAlVP & $\begin{array}{l}\text { Fraction of vapor velocity at the right face of } \\
\text { the primary tee junction cell that contributes } \\
\text { to the momentum transfer into the primary tee } \\
\text { side leg. }\end{array}$ \\
\hline
\end{tabular}




\begin{tabular}{|c|c|c|c|c|}
\hline Positis & & Parameter & & Description \\
\hline 6 & & CAP & & $\begin{array}{l}\text { Fraction of liquid velocity at the left face of } \\
\text { the primary tee junction cell that contributes } \\
\text { to the momentum transfer into the tee side leg. }\end{array}$ \\
\hline 7 & & CAVP & & $\begin{array}{l}\text { Fraction of vapor velocity at the left face of } \\
\text { the primary tee junction cell that contributes } \\
\text { to the momentum transfer into the primary tee } \\
\text { side leg. }\end{array}$ \\
\hline 8 & & $\operatorname{cosp}$ & & $\begin{array}{l}\text { Cosine of the angle measured from the low- } \\
\text { numbered side of the primary tube to the } \\
\text { primary tee tube. }\end{array}$ \\
\hline $9-12$ & & FL(4) & & $\begin{array}{l}\text { iiquid mass-flow corrections for mass conserva- } \\
\text { tion checks. }\end{array}$ \\
\hline $13-16$ & & $\mathrm{FV}(4)$ & & $\begin{array}{l}\text { Vapor mass-flow corrections for mass conserva- } \\
\text { tion checks. }\end{array}$ \\
\hline 17 & & HTLSGI & & $\begin{array}{l}\text { Heat loss on the inside of a general surface } \\
\text { (HAAT). }\end{array}$ \\
\hline 18 & & HTLSGO & & $\begin{array}{l}\text { Heat loss on the outside of a general surface } \\
\text { (HAAT). }\end{array}$ \\
\hline 19 & & QP3IN & & Initial QPPP factor. \\
\hline 20 & & RADIN & & Inner radius of a tube wall. \\
\hline 21 & & RTILP & & $\begin{array}{l}\text { Coefficient for the liquid momentum source term } \\
\text { at the left edge of the primary junction cell. }\end{array}$ \\
\hline 22 & - & RT2LP & & $\begin{array}{l}\text { Coefficient for the liquid momentum source term } \\
\text { at the right edge of the primary tee junction } \\
\text { cell. }\end{array}$ \\
\hline 23 & & RTIVP & & $\begin{array}{l}\text { Coefficient for the vapor momentum source term } \\
\text { at the left edge of the primary tee junction } \\
\text { cell. }\end{array}$ \\
\hline 24 & . & RT2VP & ¿ & $\begin{array}{l}\text { Coefficient for the upper momentum source term } \\
\text { at the right edge of the primary tee junction. }\end{array}$ \\
\hline 25 & 0 & TH & & Tube wall thickness. \\
\hline 26 & & Z11111 & & $\begin{array}{l}\text { Dummy variable that provides a known end to the } \\
\text { COMMON block. }\end{array}$ \\
\hline
\end{tabular}


INTEGER VARIABLES

Position(s)

27

28

29

30

31

32
Parameter

IAl111

I CHF 1

I CHF2

ICNC2

ICONC

ISOLP

ISVLB1

ISVLB2

ISVRB1

ISVRB2

JCLP

JINL

JOTL

JS11

JS 12

JSP

JUN1 1

\section{Description}

Dummy variable that provides a known start to the COlMON block.

Indicator for a CHF calculation on the primary side.

$0=$ no CHF calculation,

1 = CHF calculation.

Indicator for a CHF calculation on the secondary side.

$0=$ no $\mathrm{CHF}$ calculation,

$1=\mathrm{CHF}$ calculation.

Presence of boron in the secondary flag.

$0=$ no boron present,

1 = boron present.

Presence of boron in the primary flag

$0=$ no boron present,

1 = boron present.

Indicator for velocity update at JUNP.

Indicator for velocity update at JUN11.

Indicator for velocity update at JUN21.

Indicator for velocity update at JUN12.

Indicator for velocity update at JUN22.

Junction cell index of a primary tee connection.

Junction cell index for the secondary inlet junction.

Junction cell index for the secondary outlet junction.

Junction sequence number at JUN1.

Junction sequence number at JUN2.

Junction sequence number at JUNP.

Junction number adjacent to cell 1 on primary side. 


\begin{tabular}{|c|c|c|}
\hline Position(s) & Parameter & Description \\
\hline 44 & JUN12 & $\begin{array}{l}\text { Junction number adjacent to cell NCELLl on } \\
\text { primary side. }\end{array}$ \\
\hline 45 & JUNP & $\begin{array}{l}\text { Junction number of the high-numbered-cell end } \\
\text { of a primary tee. }\end{array}$ \\
\hline 46 & LGHT & $\begin{array}{l}\text { Location in the pointer table of the old time } \\
\text { generalized heat-transfer vartables. }\end{array}$ \\
\hline 47 & LGHTN & $\begin{array}{l}\text { Location in the pointer table of the new time } \\
\text { gereralized heat-transfer variables. }\end{array}$ \\
\hline 48 & LNGHT & $\begin{array}{l}\text { Length of the variables of the generalized } \\
\text { heat-transfer information for which old and new } \\
\text { time values are stored. }\end{array}$ \\
\hline 49 & MAT & Material identification for tubes. \\
\hline 50 & NCELL1 & $\begin{array}{l}\text { Number of fluid cells on tube side (primary } \\
\text { stde) excluding the side leg if the primary } \\
\text { component is a tee. }\end{array}$ \\
\hline 51 & NCELL2 & $\begin{array}{l}\text { Number of fluid cells on shell side (secondary } \\
\text { side). }\end{array}$ \\
\hline 52 & NCELLS & $\begin{array}{l}\text { Total number of fluid cells (NCELL1 } \\
+ \text { NCELL2 }+1 \text { ) excluding, if the primary } \\
\text { component is a tee, the cells on the primary } \\
\text { tee side leg. }\end{array}$ \\
\hline 53 & NCLP & Number of cells on the primary tee. \\
\hline $54-63$ & $\operatorname{NCLS}(10)$ & $\begin{array}{l}\text { Number of cells on the primary side of a } \\
\text { secondary component. }\end{array}$ \\
\hline $64-73$ & $\operatorname{NCLT}(10)$ & $\begin{array}{l}\text { Number of cells on the side leg of a secondary } \\
\text { component (a TEE). }\end{array}$ \\
\hline 74 & NDHT & $\begin{array}{l}\text { Number of nodes used in the second set of heat } \\
\text { conduction paths. }\end{array}$ \\
\hline 75 & NGHT & $\begin{array}{l}\text { Number of heat conduction paths used to model } \\
\text { structures in the second set of generalized } \\
\text { heat-transfer volumes. }\end{array}$ \\
\hline, 76 & NITJN & $\begin{array}{l}\text { Number of junctions internal to the secondary } \\
\text { flow path (includes the junctions). }\end{array}$ \\
\hline r & NODMX & $\begin{array}{l}\text { Maximum number of nodés for heat conduction } \\
\text { paths (maximum of NODES and NOHT). }\end{array}$ \\
\hline
\end{tabular}




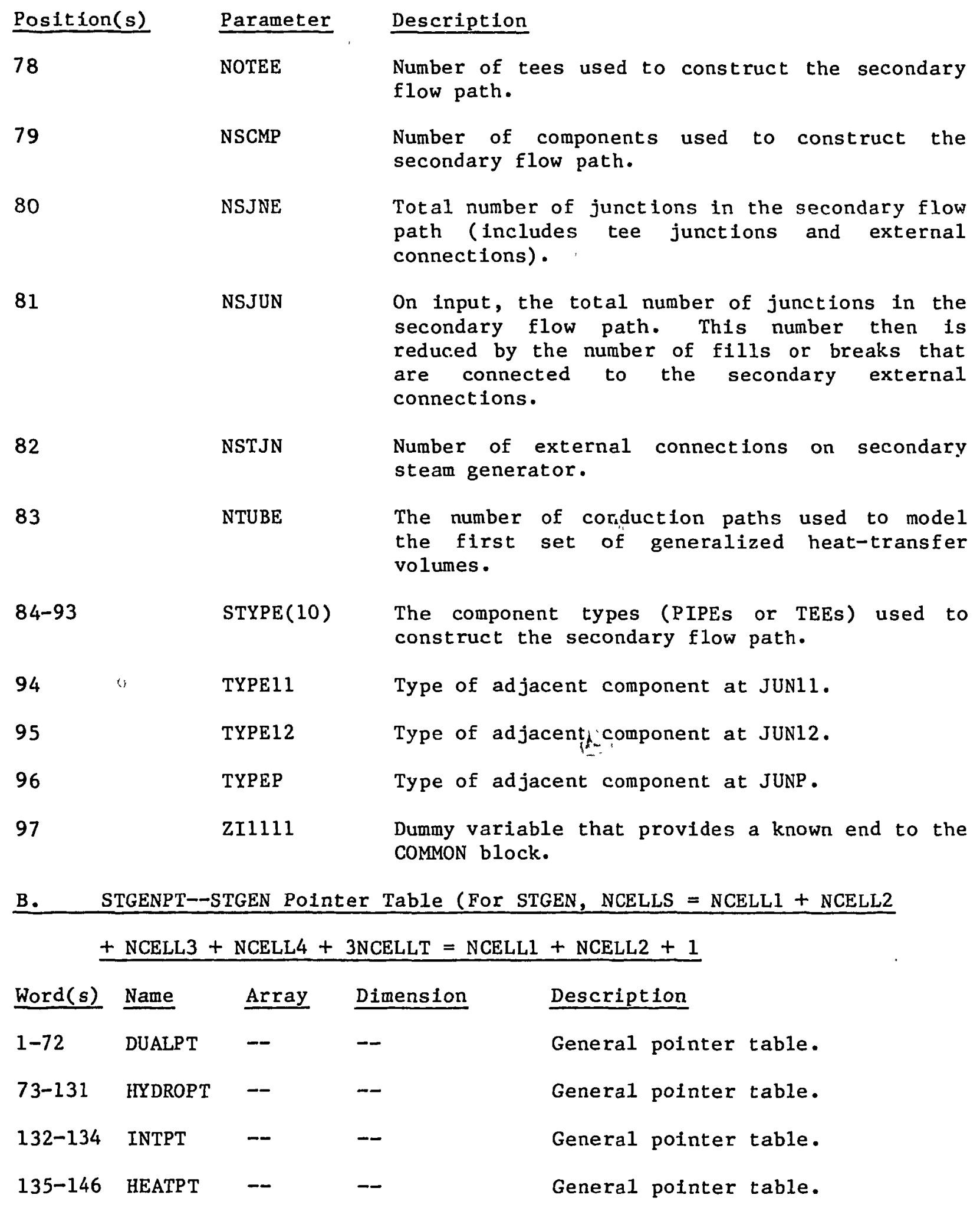




\begin{tabular}{|c|c|c|c|c|}
\hline Word(s) & Name & Array & Dimension & Description \\
\hline 147 & LQPPL & QPPL & NCELLS-1 & $\begin{array}{l}\text { Heat flux }\left(\mathrm{W} \cdot \mathrm{m}^{-3}\right) \text { from wall to } \\
\text { liquid. }\end{array}$ \\
\hline 148 & LQPPV & QPPV & NCELLS-1 & $\begin{array}{l}\text { Heat flux }\left(w \cdot \mathrm{m}^{-3}\right) \text { from wall to } \\
\text { vapor. }\end{array}$ \\
\hline \multicolumn{5}{|c|}{ COMPONENT POINTERS } \\
\hline 149 & LCAS & CAS & NSCMP & $\begin{array}{l}\text { Source term for a fluid cell } \\
\text { containing a side tee junction. }\end{array}$ \\
\hline 150 & LCA1S & CAls & NSCMP & $\begin{array}{l}\text { Source term for a fluid cell that } \\
\text { "contains a side tee junction. }\end{array}$ \\
\hline 151 & LCAIVS & CA1VS & NSCMP & $\begin{array}{l}\text { Source term for a fluid cell that } \\
\text { contains a side tee function. }\end{array}$ \\
\hline 152 & LCAVS & CAVS & NSCMP & $\begin{array}{l}\text { Source term for a fluid cell that } \\
\text { contains a side tee junction. }\end{array}$ \\
\hline 153 & LCoss & coss & NSCMP & $\begin{array}{l}\text { Cosine of the angle measured from } \\
\text { the low-numbered side of the } \\
\text { primary tube to the side tube for } \\
\text { a secondary TEE component. }\end{array}$ \\
\hline 154 & LFLS & FLS & $4 *$ NSCMP & Liquid volumetric flow rate. \\
\hline 155 & LFVS & FVS & $4 *$ NSCMP & Vapor volumetric flow rate. \\
\hline 156 & LJCLSC & JCLSC & NSJUN & $\begin{array}{l}\text { Secondary cell where secondary } \\
\text { junction is located. }\end{array}$ \\
\hline 157 & LJCLT & JCLT & NSCMP & $\begin{array}{l}\text { Fluid cell number that contains a } \\
\text { side-leg tee connection. }\end{array}$ \\
\hline 158 & LJNPOS & JNPOS & NSJUN & $\begin{array}{l}\text { Junction location parameter } \\
\text { (Inflow, outflow, or tee). }\end{array}$ \\
\hline 159 & LJNS & JNS & $3 *$ NSCMP & $\begin{array}{l}\text { Junction numbers for components } \\
\text { that compose the steam-generator } \\
\text { secondary side. }\end{array}$ \\
\hline 160 & LJNSC & JNSC & NSJUN & $\begin{array}{l}\text { Secondary external junction number } \\
\text { (from input). }\end{array}$ \\
\hline 161 & LJSINT & JSINT & $4 *$ NSCMP & $\begin{array}{l}\text { Sequence numbers for the junctions } \\
\text { that compose the steam-generator } \\
\text { secondary-side flow path. }\end{array}$ \\
\hline
\end{tabular}




\begin{tabular}{|c|c|c|c|}
\hline Word (s) & Name & Array & Dimension \\
\hline 162 & LJSSN & JSSN & NSJUN \\
\hline 163 & LJUNS & JUNS & $\begin{array}{l}\text { 8*(NSJUN } \\
\text {-NOTEE) }\end{array}$ \\
\hline 164 & LNCMSC & NCMSC & NSJUN \\
\hline 165 & LNCTS & NCTS & NSJUN \\
\hline 166 & LNSOL & NSOL & $4 *$ NSCMP \\
\hline 167 & LNUMS & NUMS & NSCMP \\
\hline 168 & LRTILS & RTILS & NSCMP \\
\hline 169 & LRT2LS & RT2LS & NSCMP \\
\hline 170 & LRT1VS & RTIVS & NSCMP \\
\hline 171 & LRT2VS & RT2VS & NSCMP \\
\hline 172 & LVSS & Vss & NSJUN \\
\hline
\end{tabular}

Description

BD array for the external junction.

An array that contains junction information for the components that compose the steam-generator secondary-side flow path.

Secondary component number that contains the junction.

A vector that contains the junction numbers for the components that comprise the steam-generator secondary side.

An array that contains information about the method of connecting secondary-side steam-generator components.

User-specified steam-generator secondary-side component numbers.

A source term for a fluid cell that contains a side tee junction.

A source term for a fluid cell that contains a side tee junction.

A source term for a fluid cell that contains a side tee junction.

A source term for a fluid cell that contains a side tee junction.

An array that contains information about the method of connecting secondary-side steam-generator components.

HEAT-TRANSFER POINTERS

$\begin{array}{llllll}173 & \text { LCPWG } & \text { CPWG } & \text { NGHT*NDHM1 } & \begin{array}{l}\text { Wall } \\ \left(\mathrm{J} \cdot \mathrm{kg}^{-1} \cdot \mathrm{K}^{-1}\right) \cdot\end{array} \\ 174 & \text { LCWG } & \text { CWG } & \text { NGHT*NDHM1 } & \text { Wall conductivity }\left(\mathrm{W} \cdot \mathrm{m}^{-1} \cdot \mathrm{K}^{-1}\right) . \\ 175 & \text { LDRG } & \text { DRG } & \text { NGHT*NDHM1 } & \begin{array}{l}\text { Internodal spacing }(\Delta \mathrm{r} \text { in m } \\ \text { between nodes in the wall. }\end{array}\end{array}$




\begin{tabular}{|c|c|c|c|c|}
\hline Word(s) & Name & Array & Dimension & Description \\
\hline 176 & LEMSG & EMSG & NGHT & Wall emissivity. \\
\hline 177 & LHILG & HHILG & NGHT & $\begin{array}{l}\text { HTC }\left(W \cdot \mathrm{m}^{-2} \cdot \mathrm{K}^{-1}\right) \text { to the liquid } \\
\text { on the interior of a wall. }\end{array}$ \\
\hline 178 & LHILGN & HILGN & NGHT & $\begin{array}{l}\left.\text { New time HTC ( } \cdot \mathrm{m}^{-2} \cdot \mathrm{K}^{-1}\right) \text { to } \\
\text { the liquid on the interior of a } \\
\text { wall. }\end{array}$ \\
\hline 179 & LHIVG & HIVG & NGHT & $\begin{array}{l}\text { HTC }\left(W \cdot \mathrm{m}^{-2} \cdot \mathrm{K}^{-1}\right) \text { to the vapor } \\
\text { on the interior of a wall. }\end{array}$ \\
\hline 180 & LHIVGN & HIVGN & NGHT & $\begin{array}{l}\text { New time HTC }\left(W \cdot \mathrm{m}^{-2} \cdot \mathrm{K}^{-1}\right) \text { to } \\
\text { the vapor on the interior of a } \\
\text { wall. }\end{array}$ \\
\hline 181 & LHOLG & HOLG & NGHT & $\begin{array}{l}\text { HTC }\left(\mathrm{W} \cdot \mathrm{m}^{-2} \cdot \mathrm{K}^{-1}\right) \text { to the liquid } \\
\text { on the exterior of a wall. }\end{array}$ \\
\hline 182 & LHOLGN & HOLGN & NGHT & $\begin{array}{l}\text { New time HTC }\left(W \cdot \mathrm{m}^{-2} \cdot \mathrm{K}^{-1}\right) \text { to } \\
\text { the liquid on the exterior of a } \\
\text { wall. }\end{array}$ \\
\hline 183 & "LHOVG & HOVG & NGHT & $\begin{array}{l}\text { HTC }\left(W \cdot \mathrm{m}^{-2} \cdot \mathrm{K}^{-1}\right) \text { to the vapor } \\
\text { on the exterior of a wall. }\end{array}$ \\
\hline 184 & LHOVGN & HOVGN & NGHT & $\begin{array}{l}\text { New time HTC }\left(W \cdot \mathrm{m}^{-2} \cdot \mathrm{K}^{-1}\right) \text { to } \\
\text { the vapor on the exterior of a } \\
\text { wall. }\end{array}$ \\
\hline 185 & LIDGHI & IDGHI & NGHT & $\begin{array}{l}\text { Heat-transfer regime flag for the } \\
\text { Interior of a wall. }\end{array}$ \\
\hline 186 & LIDGHO & IDGHO & NGHT & $\begin{array}{l}\text { Heat-transfer regime flag for the } \\
\text { exterior of a wall. }\end{array}$ \\
\hline 187 & LIDGI & IDGI & NGHT & $\begin{array}{l}\text { Fluid cell identifier for heat } \\
\text { transfer with the interior of a } \\
\text { wall. }\end{array}$ \\
\hline 188 & LIDGO & IDGO & NGHT & $\begin{array}{l}\text { Fluid cell identifler for heat } \\
\text { transfer with the exterior of a } \\
\text { wall. }\end{array}$ \\
\hline 189 & LMATG & MATG & NGHT*NDHMl & Wall material identifier. \\
\hline 190 & LQPPG & QPPG & NGHT*NODMX & $\begin{array}{l}\text { Wall volumetrif heat flux distri- } \\
\text { bution }\left(\mathrm{W} \cdot \mathrm{m}^{-3}\right) \text {. }\end{array}$ \\
\hline
\end{tabular}




\begin{tabular}{|c|c|c|c|c|}
\hline Word(s) & Name & Array & Dimension & Description \\
\hline 191 & LRADIG & RADIG & NGHT & Wall interior radius (m). \\
\hline 192 & LRNG & RNG & NGHT*NODMX & $\begin{array}{l}\text { Radial locations }(m) \text { of the wall } \\
\text { heat-transfer nodes. }\end{array}$ \\
\hline 193 & LRN2G & RN2G & NGHT*NDHMI & $\begin{array}{l}\text { Radial locations (m) of the heat } \\
\text { conduction cell edges. }\end{array}$ \\
\hline 194 & LROWG & ROWG & NGHT*NDHMl & Wall density $\left(\mathrm{kg} \cdot \mathrm{m}^{-3}\right)$. \\
\hline 195 & LTHG & THG & NGHT & Wall thickness (m). \\
\hline 196 & LTILG & TILG & NGHT & $\begin{array}{l}\text { Liquid temperature }(\mathrm{K}) \text { of the } \\
\text { fluid interior to the wall. }\end{array}$ \\
\hline 197 & LTIVG & TIVG & NGHT & $\begin{array}{l}\text { Vapor temperature }(\mathrm{K}) \text { of the fluid } \\
\text { interior to the wall. }\end{array}$ \\
\hline 198 & LTOLG & TOLG & NGHT & $\begin{array}{l}\text { Liquid temperature }(\mathrm{K}) \text { of the } \\
\text { fluid exterior to the wall. }\end{array}$ \\
\hline 199 & LTOVG & TOVG & NGHT & $\begin{array}{l}\text { Vapor temperature }(K) \text { of the fluid } \\
\text { exterior to the wall. }\end{array}$ \\
\hline 200 & LTWG & TWG & NGHT*NODMR $-b$ & Wall temperature distribution $(K)$. \\
\hline 201 & LTWGN & TWGN & NGHT*NODMX & $\begin{array}{l}\text { New time wall temperature distri- } \\
\text { bution }(K) \text {. }\end{array}$ \\
\hline 202 & LWAIG & WAIG & NGHT & Interior wall surface area $\left(\mathrm{m}^{2}\right)$. \\
\hline 203 & LWAOG & WAOG & NGHT & Exterior wall surface area $\left(m^{2}\right)$. \\
\hline NETWORK & POINTERS & & & 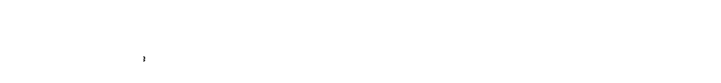 \\
\hline 204 & LAOLS & AOLS & $\begin{array}{l}\text { NSJUN* } \\
(\text { NSJUN+I) }\end{array}$ & Steam-generator network matrix. \\
\hline 205 & LAOUS & AOUS & NSJUN*NSJUN & Steam-generator network matrix. \\
\hline 206 & LAOVS & AOVS & NSJUN*NSJUN & Steam-generator network matrix. \\
\hline 207 & LBDP & $\mathrm{BDP}$ & LENBD & $\begin{array}{l}\text { Boundary array for the primary tee } \\
\text { connection. }\end{array}$ \\
\hline 208 & LBDS & BDS & NSJUN*LENBD & $\begin{array}{l}\text { Boundary arrays for the steam- } \\
\text { generator secondary components. }\end{array}$ \\
\hline 209 & LDRAS & DRAS & NSJUN & Steam-generator network vector. \\
\hline
\end{tabular}




\begin{tabular}{|c|c|c|c|c|}
\hline Word(s) & Name & Array & Dimension & Description \\
\hline 210 & LDRCS & DRCS & NSJUN & Steam-generator network vector. \\
\hline 211 & LDRELS & DRELS & NSJUN & Steam-generator network vector. \\
\hline 212 & LDREVS & DREVS & NSJUN & Steam-generator network vector. \\
\hline 213 & LDRLS & DRLS & NSJUN & Steam-generator network vector. \\
\hline 214 & LDRVS & DRVS & NSJUN & Steam-generator network vector. \\
\hline 215 & LDVBS & DVBS & NSJUN & Steam-generator network vector. \\
\hline 216 & LIOJS & IIOJS & NSJUN & $\begin{array}{l}\text { Vector that contains information } \\
\text { regarding the association of the } \\
\text { steam-generator junctions and the } \\
\text { external network matrix. }\end{array}$ \\
\hline 217 & LIOUS & IOUS & NSJUN & $\begin{array}{l}\text { Vector that contains information } \\
\text { regarding the sequence numbers of } \\
\text { the steam-generator secondary } \\
\text { junctions. }\end{array}$ \\
\hline 218 & LODS & ODS & $4 *$ NSJUN & Steam-generator network matrix. " \\
\hline 219 & LVRHS & VRHS & NSJUN & Steam-generator network matrix. \\
\hline $220-308$ & PTDUM & - & -- & Dummy variable. \\
\hline
\end{tabular}

C. STGNDATA--STGEN Data Table

This data table includes the following COMMON blocks: BLANKCOM and FIXEDLT, both defined in App. H; STGENVLT, defined in Sec.X.A; and STGENPT, defined in Sec. X.B.

XI. . , TEE MODULE

A. TEEVLT--Variable-Length Table

REAL VARIABLES

Position(s) Parameter Description

1

2

3
AAl111

BSMASS

$\mathrm{CA}$
Dummy variable that provides a known start to the COMMON block.

Time-integrated mass flow from tee.

Fraction of liquid velocity at the left face of the tee junction cell that contributes to the momentum transfer into the tee side leg. 


\begin{tabular}{|c|c|c|c|}
\hline Position(s) & & Parameter & Description \\
\hline 4 & & $\mathrm{CAl}$ & $\begin{array}{l}\text { Fraction of liquid velocity at the right face } \\
\text { of the tee junction cell that contributes to } \\
\text { the momentum transfer into the side leg. }\end{array}$ \\
\hline 5 & & CAlV & $\begin{array}{l}\text { Fraction of vapor velocity at the right face of } \\
\text { the tee junction cell that contributes to the } \\
\text { momentum transfer into the tee side leg. }\end{array}$ \\
\hline 6 & & $\mathrm{CAV}$ & $\begin{array}{l}\text { Fraction of vapor velocity at the left face of } \\
\text { the tee junction cell that contributes to the } \\
\text { momentum transfer into the tee side leg. }\end{array}$ \\
\hline 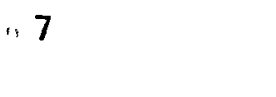 & & $\operatorname{cosT}$ & $\begin{array}{l}\text { Cosine of the angle from the low-numbered } \\
\text { segment to the secondary tube. }\end{array}$ \\
\hline 8 & & ENIN1 & $\begin{array}{l}\text { Total (time-integrated) energy iirectly input } \\
\text { to the primary. }\end{array}$ \\
\hline 9 & & ENIN2 & $\begin{array}{l}\text { Total (time-integrated) energy directly input } \\
\text { to the secondary. }\end{array}$ \\
\hline $10-13$ & & FL(4) & $\begin{array}{l}\text { Liquid mass-flow corrections for mass conserva- } \\
\text { tion checks. }\end{array}$ \\
\hline $14-17$ & & FV (4) & $\begin{array}{l}\text { Vapor mass-flow corrections for mass conserva- } \\
\text { tion checks. }\end{array}$ \\
\hline 18 & & HOUTL1 & $\begin{array}{l}\text { HTC to liquid at the outer boundary of the } \\
\text { primary tube wall. }\end{array}$ \\
\hline 19 & & HOUTL2 & $\begin{array}{l}\text { HTC to liquid at the outer boundary of the } \\
\text { secondary tube wall. }\end{array}$ \\
\hline 20 & & HOUTV1 & $\begin{array}{l}\text { HTC to vapor at the outer boundary of the } \\
\text { primary tube wall. }\end{array}$ \\
\hline 21 & $\because$ & HOUTV2 & $\begin{array}{l}\text { HTC to vapor at the outer boundary of the } \\
\text { secondary tube wall. }\end{array}$ \\
\hline 22 & & POWR 1 & Power per length added to the tee primary. \\
\hline 23 & & POWR2 & Power per length added to the tee secondary. \\
\hline 24 & & PWIN1 & $\begin{array}{l}\text { Initial power deposited in the coolant of the } \\
\text { tee primary tube. }\end{array}$ \\
\hline 25 & & PWIN2 & $\begin{array}{l}\text { Initiaid power deposited in the coolant of the } \\
\text { tee secondary tube. }\end{array}$ \\
\hline
\end{tabular}




\begin{tabular}{|c|c|c|}
\hline Position(s) & Parameter & Description \\
\hline 26 & PWOFF1 & $\begin{array}{l}\text { Power deposited in the coolant of the tee } \\
\text { primary tube when its controlling trip is OFF } \\
\text { after it was oN. }\end{array}$ \\
\hline 27 & PWOFF2 & $\begin{array}{l}\text { Power deposited in the coolant of the tee } \\
\text { secondary tube when its controlling trip is OFF } \\
\text { after it was ON. }\end{array}$ \\
\hline 28 & QPINI & Initial QPPP factor in the tee primary tube. \\
\hline 29 & QPIN2 & Intial QPPP factor in the tee secondary tube. \\
\hline 30 & QPOFE1 & $\begin{array}{l}\text { QPPP factor for the tee primary tube when its } \\
\text { controlling trip is OFF after it was ON. }\end{array}$ \\
\hline 31 & QPOFF2 & $\begin{array}{l}\text { QPPP factor for the tee secondary tube when its } \\
\text { controlling trip is OFF after it was ON. }\end{array}$ \\
\hline 32 & RADIN1 & Inner radius of the primary tube. \\
\hline 33 & RADIN2 & Inner radius of the secondary tube. \\
\hline 34 & RPWMX1 & $\begin{array}{l}\text { Maximum rate of change of power deposited in } \\
\text { the coolant for the tee primary tube. }\end{array}$ \\
\hline 35 & RPWMX2 & $\begin{array}{l}\text { Maximum rate of change of power deposited in } \\
\text { the coolant for the tee secondary tube. }\end{array}$ \\
\hline 36 & $\mathrm{RQPMX1}$ & $\begin{array}{l}\text { Maximum rate of change of the QPPP factor for } \\
\text { the tee secondary tube. }\end{array}$ \\
\hline 37 & $\mathrm{RQPMX} 2$ & $\begin{array}{l}\text { Maximum rate of change of the QPPP factor for } \\
\text { the tee secondary tube. }\end{array}$ \\
\hline 38 & RT1L & $\begin{array}{l}\text { Coefficient for the liquid momentum source term } \\
\text { at the left edge of the junction cell. }\end{array}$ \\
\hline 39 & $\operatorname{RTIV}$ & $\begin{array}{l}\text { Coefficient for the vapor momentum source term } \\
\text { at the left edge of the tee junction cell. }\end{array}$ \\
\hline 40 & RT2L & $\begin{array}{l}\text { Coefficient for the liquid momentum source term } \\
\text { at the right edge of the tee junction cell. }\end{array}$ \\
\hline 41 & $\mathrm{RT} 2 \mathrm{~V}$ & $\begin{array}{l}\text { Coefficient for the upper momentum source term } \\
\text { at the right edge of the tee junction. }\end{array}$ \\
\hline 42 & THI & Wa11 thickness of the primary tube. \\
\hline 43 & TH2 & Wail thickness of the secondary tube. \\
\hline
\end{tabular}




\begin{tabular}{|c|c|c|}
\hline Position(s) & Parameter & Description \\
\hline 44 & TLEN1 & Length of the primary. \\
\hline 45 & TLEN2 & Length of the secondary. \\
\hline 46 & TOUTL1 & $\begin{array}{l}\text { Temperature of liquid outside the primary tube } \\
\text { wall. }\end{array}$ \\
\hline 47 & TOUTL2 & $\begin{array}{l}\text { Temperature of liquid outside the secondary } \\
\text { tube wall. }\end{array}$ \\
\hline 48 & TOUTV1 & $\begin{array}{l}\text { Temperature of vapor outside the primary tube } \\
\text { wall. }\end{array}$ \\
\hline 49 & TOUTV2 & $\begin{array}{l}\text { Temperature of vapor outside the secondary tube } \\
\text { wall. }\end{array}$ \\
\hline 50 & 211111 & $\begin{array}{l}\text { Dummy variable that provides a known end to the } \\
\text { COMMON block. }\end{array}$ \\
\hline
\end{tabular}

INTEGER VARIABLES

51

52

53

54

55

56

57

58

59
IA1111

ICHF

ICJ 1

ICJ 2

ICJ 3

ICONCl

ICONC2

IONOF1

IONOF2
Dummy variable that provides a known start to the COMMON block.

CHF calculation option.

Iteration index of adjacent component to tee at JUN1.

Iteration index of adjacent component to tee at JUN2.

Iteration index of adjacent component to tee at JUN3.

Indicator for presence of boron in the coolant of the tee primary tube.

Indicator for presence of boron in the coolant of the tee secondary tube.

Number of time steps the power deposited in the coolant table for the tee primary tube's controlling trip has been ON.

Number of time steps the power deposited in the coolant table for the tee secondary tube's controlling trip has been $\mathrm{ON}$. 


\section{Position(s)}

60

61

64

65

66
Parameter

IPFI

IPF2

IPOWI

IPOW2

IPP1

IPP2

IPWSV1

IPWSV2

IPWTR 1

IPWTR2

IQF 1

IQF 2

IQP1
Description

Last interpolated interval number of the ratefactor table for the power deposited in the coolant of the tee primary tube.

Last interpolated interval number of the ratefactor table for the power deposited in the coolant of the tee secondary tube.

Indicator for presence of power deposited in the coolant of the tee primary tube.

Indicator for presence of power deposited in the coolant of the tee secondary tube.

Last interpolated interval number of the power deposited in the coolant table for the tee primary tube.

Last interpolated interval number of the power deposited in the coolant table for the tee secondary tube.

Power deposited in the coolant table's abscissa-coordinate variable ID number for the tee primary tube.

Power deposited in" the coolant table's abscissa-coordinate variable 'ID number for the tee secondary tube.

Trip ID number that controls evaluation of the power deposited in the coolant table for the tee primary tube.

Trip ID number that controls evaluation of the power deposited in the coolant table for the tee secondary tube.

Last interpolated interval number of the ratefactor table for the QPPP factor table of the tee primary tube.

Last interpolated interval number of the ratefactor table for the QPPP factor of the tee secondary tube.

Last interpolated interval number of the QPPP factor table for the tee primary tube. 


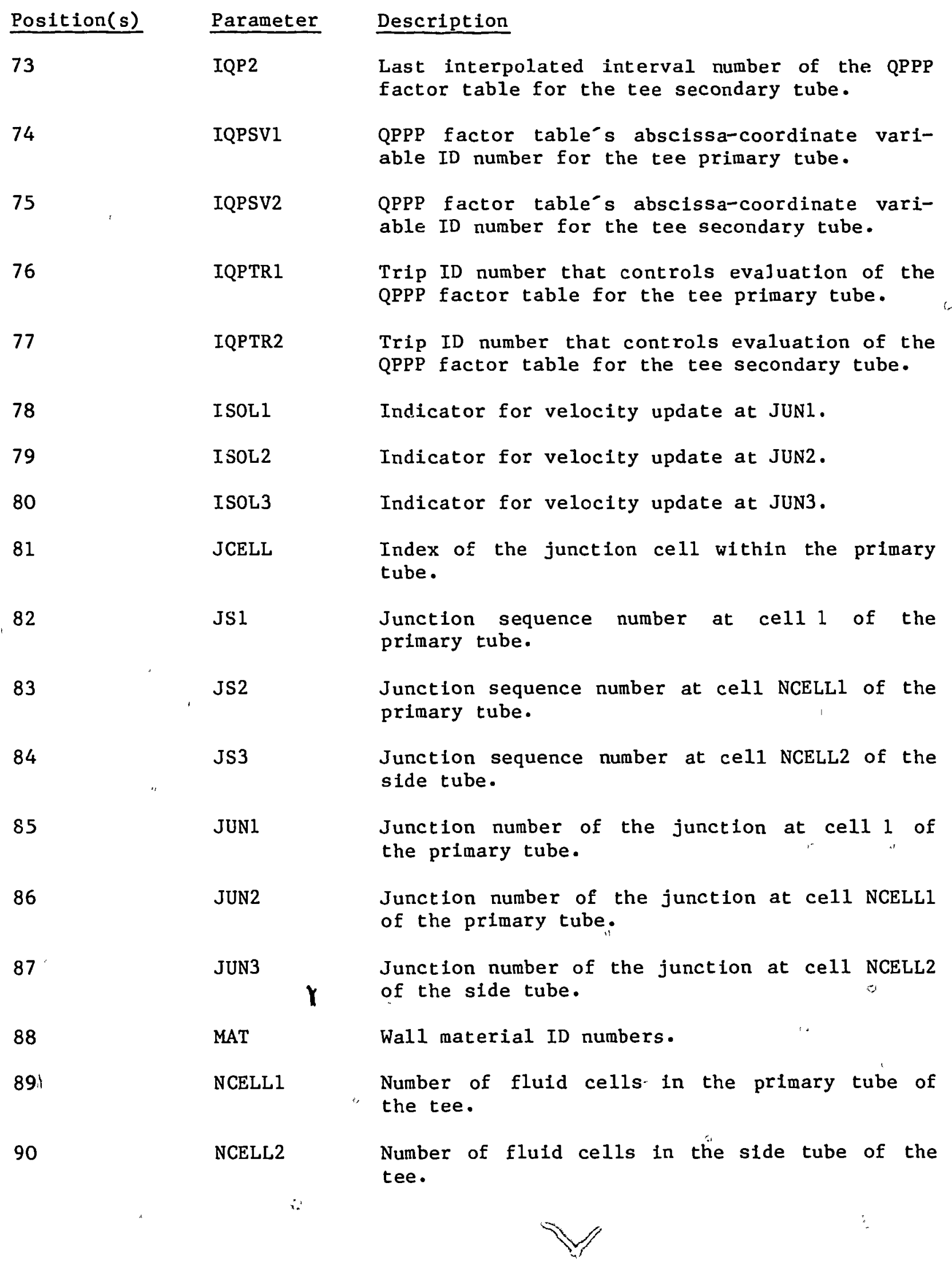




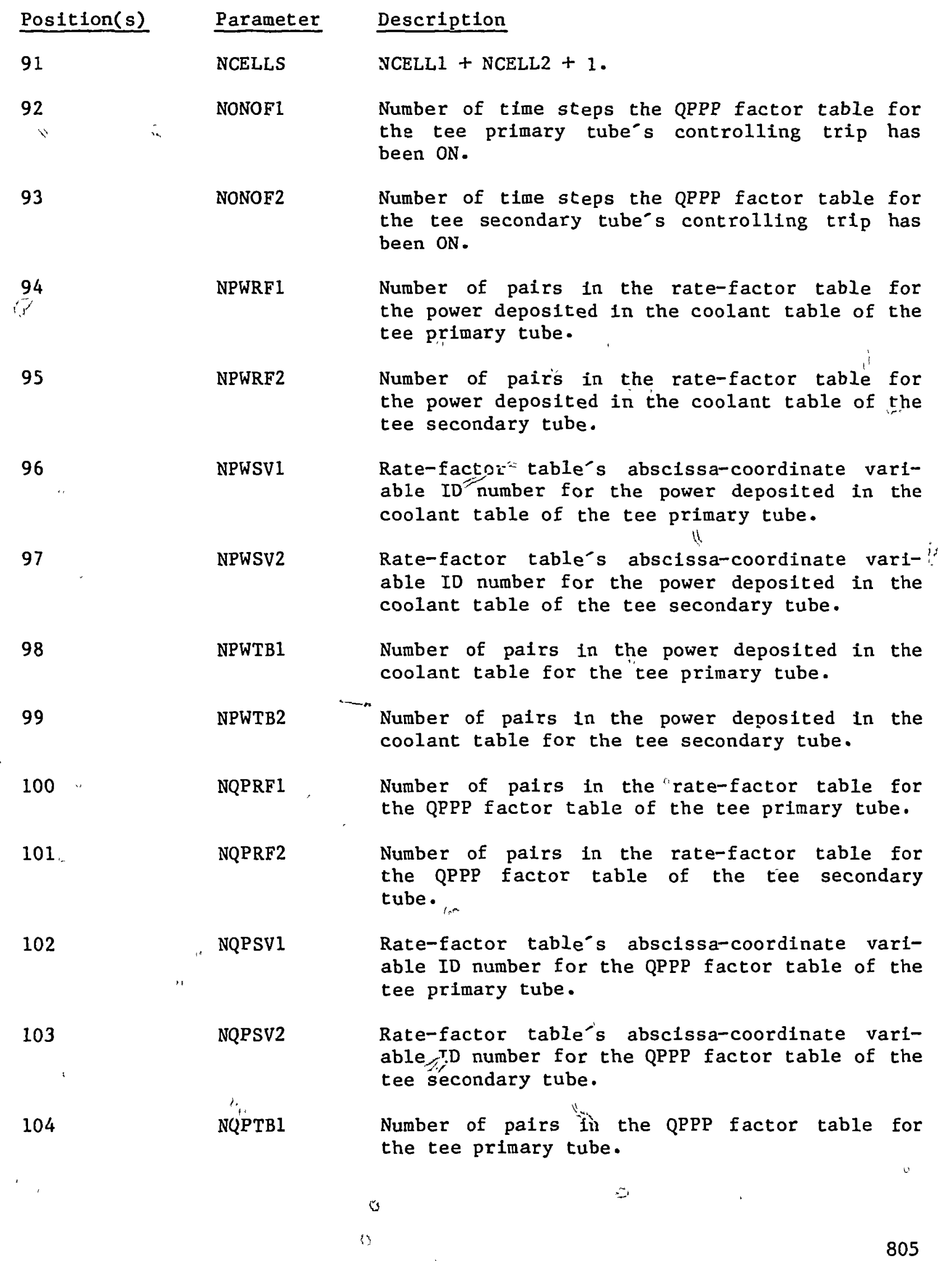




\begin{tabular}{|c|c|c|c|c|}
\hline \multicolumn{2}{|c|}{ Position(s) } & Parameter & Description & \\
\hline 105 & & NQPTB2 & $\begin{array}{l}\text { Number of } \\
\text { the tee sec }\end{array}$ & $\begin{array}{l}\text { pairs in the QPPP factor table for } \\
\text { :ondary tube. }\end{array}$ \\
\hline 106 & & TYPEI & Type of adj & jacent component at JUN1. \\
\hline 107 & & TYPE2 & Type of adj & jacent component at JUN2. \\
\hline 108 & & TYPE 3 & Type of adj & jacent component at JUN3. \\
\hline 109 & $\cdots d_{t}^{\prime \prime}$ & ZI1111 & $\begin{array}{l}\text { Dummy varia } \\
\text { COMMON bloc }\end{array}$ & $\begin{array}{l}\text { ble that provides a known end to the } \\
\text { k. }\end{array}$ \\
\hline B. & EEPT-TEE & Pointer & Table (For TEE, & NCELLS $=$ NCELLL $1+$ NCELL $2+1)$ \\
\hline Word(s) & Name & Array & Dimension & Description \\
\hline $1-72$ & DUALPT & - & -- & General pointer table. \\
\hline $73-131$ & HYDROPT & - & - & General pointer table. \\
\hline $132-134$ & INTPT & - & -- & General pointer table. \\
\hline $135-146$ & HEATPT & -- & -- & General pointer table. \\
\hline 147 & LBD4 & BD4 & LENBD & Dummy BD4 array. \\
\hline 148 & LPOWRF & POWRF & $\begin{array}{l}(\mid \text { NPWRFI } \mid \\
+\mid \text { NPWRF } 2 \mid) * 2\end{array}$ & $\begin{array}{l}\text { Rate-factor tables for the power } \\
\text { deposited in the coolant tables. }\end{array}$ \\
\hline 149 & LPOWTB & POWTB & $\begin{array}{l}(\mid \text { NPWTB1 } \mid \\
+\mid \text { NPWTB2 } \mid) * 2\end{array}$ & $\begin{array}{l}\text { Power deposited in the coolant } \\
\text { table. }\end{array}$ \\
\hline 150 & LQP3RF & QP3RF & $\begin{array}{l}(|\mathrm{NQPRF} 1| \\
+|\mathrm{NQPRF} 2|) * 2\end{array}$ & $\begin{array}{l}\text { Rate-factor table for the QPPP } \\
\text { factor tables. }\end{array}$ \\
\hline 151 & LQP3TB & QP3TB & $\begin{array}{l}(\mid \text { NQPTB1 } \mid \\
+\mid \text { NQPTB2 } \mid) * 2\end{array}$ & QPPP factor tables. \\
\hline $152-317$ & PTDUM & -- & -- & Dummy variable. \\
\hline
\end{tabular}


C. TEEDATA--TEE Data Table

This data table includes the following COMMON blocks: BLANKCOM and FIXEDLT, both defined in App. H; TEEVLT, defined in Sec. XI.A; and TEEPT, defined in Sec. XI.B.

XII. TURBINE MODULE

A. TURBNVLT--TURB Variable-Length Table

REAL VARIABLES

Position(s) Parameter Description

1

2

3

4

5

6

7

8

9

10

11

12

13

14

15

16-17
AA1111

ALPHAI

ALPHA2

AR

BSMASS

COEF 1

COEF2

COF3SQ

CP

CPOW

DIA

DSMOM

EFFDSN

EFFSTG

ENINP

FL(2)
Dummy variable that provides a known start to the COMMON block.

Upstream void fraction.

Downstream void fraction.

Area ratio (bucket exit area/nozzle exit area).

Time-integrated mass flow from turbine.

Nozzle coefficient.

Bucket coefficient.

Fraction of reaction energy actually delivered in the stage.

Specific heat at constant pressure.

Special turbine input.

Bucket centerline diameter.

Derivative of SMOM with respect to velocity.

Stage efficiency at design conditions.

Stage efficiency.

Total (time-integrated) energy directly input to the turbine.

Liquid mass-flow corrections for mass conservation checks. 


\begin{tabular}{|c|c|c|}
\hline Position(s) & Parameter & Description \\
\hline 18 & ELODIR & $\begin{array}{l}\text { F1ow direction flag. } \\
-1.0=\text { indicates normal flow direction is from } \\
\text { JUN2 to JUN1, } \\
1.0=\text { indicates normal flow direction is from } \\
\text { JUN1 to JUN2. }\end{array}$ \\
\hline 19 & FLOW & Mass-flow rate. \\
\hline $20-21$ & FV (2) & $\begin{array}{l}\text { Vapor mass-flow corrections for mass conserva- } \\
\text { tion checks. }\end{array}$ \\
\hline 22 & GAMMA & Isentropic exponent of expansion. \\
\hline 23 & PHIREM & $\begin{array}{l}\text { Remaining losses (rotation or diaphragm- } \\
\text { packing). }\end{array}$ \\
\hline 24 & PLENT & Total length of the turbine stage. \\
\hline 25 & POWIN & Initial power in the coolant. \\
\hline 26 & POWDSN & Stage power output at design conditions. \\
\hline 27 & POWOFF & $\begin{array}{l}\text { Power in the coolant when the controllifig trip } \\
\text { is OFF after it was ON. }\end{array}$ \\
\hline 28 & POWSTG & Stage power output. \\
\hline 29 & PRES 1 & Upstream pressure. \\
\hline 30 & PRES2 & Downstream pressure. \\
\hline 31 & QUALTY & Thermodynamic quality of steam. \\
\hline 32 & REACTN & Degree of reaction at design conditions. \\
\hline 33 & RHOL 1 & Upstream liquid density. \\
\hline 34 & RHOL2 & Downstream liquid density. \\
\hline 35 & RHOM1 & Upstream mixture density. \\
\hline 36 & RHOM2 & Downstream mixture density. \\
\hline 37 & RHOV1 & Upstream steam density. \\
\hline 38 & RHOV2 & Downstream steam density. \\
\hline 39 & RPOWMX & $\begin{array}{l}\text { Maximum rate of change of the power deposited } \\
\text { in the coolant. }\end{array}$ \\
\hline
\end{tabular}




\begin{tabular}{|c|c|c|}
\hline Position(s) & Parameter & Description \\
\hline 40 & SMOM & $\begin{array}{l}\text { Source term in the momentum equation (head } \\
\text { gain). }\end{array}$ \\
\hline 41 & SUPRHT & Upstream degree of superheat of steam. \\
\hline 42 & TEMPL1 & Upstream liquid temperature. \\
\hline 43 & TEMPL2 & Downstream liquid temperature. \\
\hline 44 & TEMPV1 . & Upstream steam temperature. \\
\hline 45 & TEMPV2 & Downstream steam temperature. \\
\hline 46 & VELL1 & Upstream liquid velocity. \\
\hline 47 & VELL2 & Downstream liquld velocity. \\
\hline 48 & VELM1 & Upstream mixture velocity. \\
\hline 49 & VELM2 & Downstream mixture velocity. \\
\hline 50 & VELV1 & Upstream steam velocity. \\
\hline 51 & VELV2 & Downstream steam velocity. \\
\hline 52 & VSTAG & Stagnation velocity. \\
\hline 53 & Z11111 & $\begin{array}{l}\text { Dummy variable that provides a known end to the } \\
\text { COMMON block. }\end{array}$ \\
\hline
\end{tabular}

INTEGER VARIABLES

54

55

56

57

58

59

60
IA1111

ICJ1

ICJ2

ICONC

IONOFF

IPF

IPOW
Dummy variable that provides a known start to the COMMON block.

Iteration index of adjacent component at JUN1. Iteration Index of adjacent component at JUN2.

Presence of boron dissolved in the liquid coolant flag.

Number of tine steps the power deposited in the coolant trip has been $\mathrm{ON}$.

Power deposited in the coolant table's ratefactor table interval last interpolated.

Presence of power in the coolant flag. 


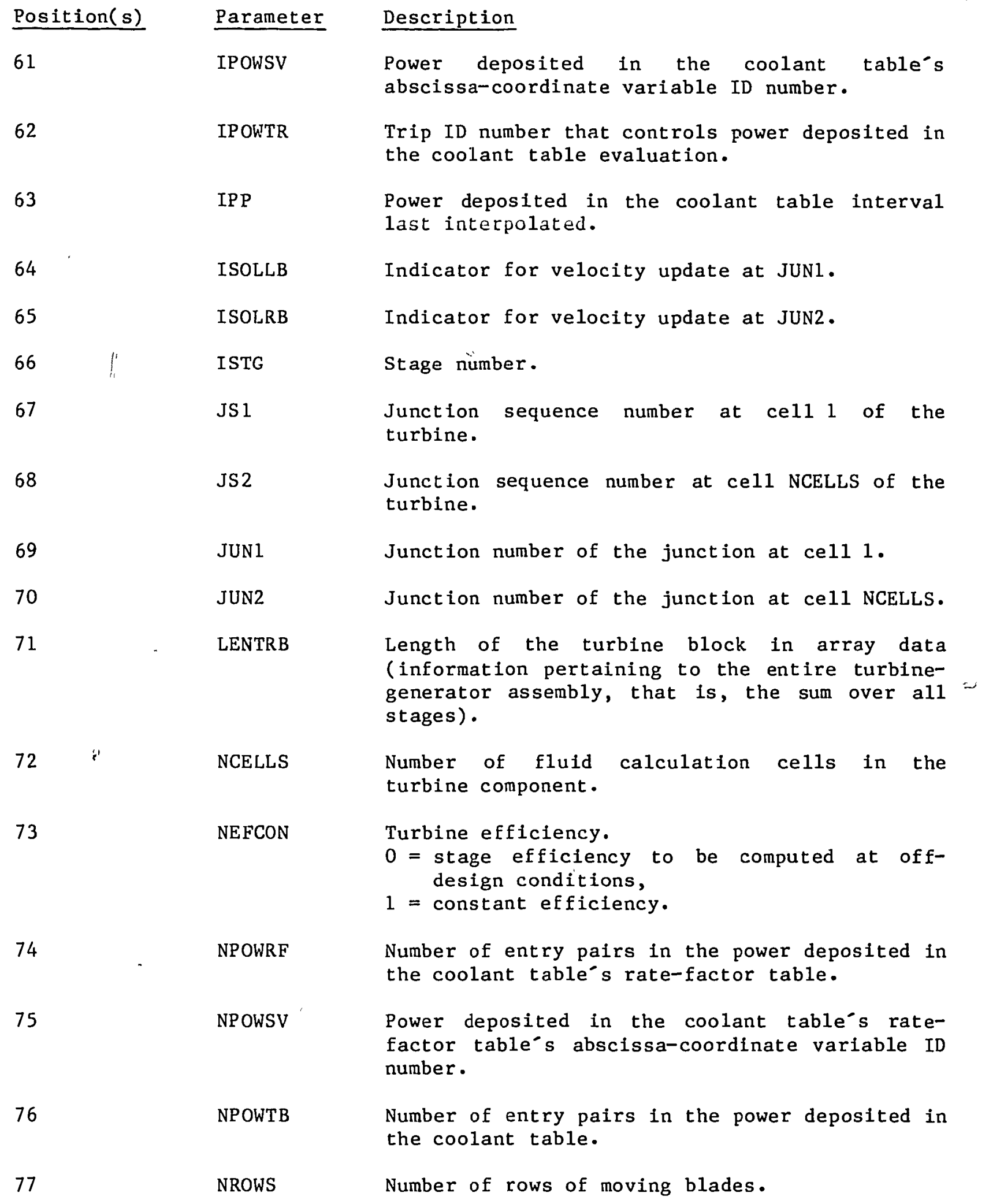




$\begin{array}{lll}\text { Position(s) } & \text { Parameter } & \text { Description } \\ 78 & \text { TYPE1 } & \text { Type of adjacent component at JUN1. } \\ 79 & \text { TYPE2 } & \text { Type of adjacent component at IUN2. } \\ 80 & \text { ZI1111 } & \begin{array}{l}\text { Dummy variable that provides a known end to the } \\ \text { COMMON block. }\end{array}\end{array}$

B. TURBPT--TURB Pointer Table

\begin{tabular}{|c|c|c|c|c|}
\hline Word(s) & Name & Array & Dimension & Description \\
\hline $1-72$ & DUALPT & $-\infty$ & -- & General pointer table. \\
\hline $73-131$ & HYDROPT & -- & -- & General pointer table. \\
\hline $132-134$ & INTPT & -- & -- & General pointer table. \\
\hline $135-146$ & HEATPT & -- & -- & General pointer table. \\
\hline 147 & LANGL & ANGL & NROWS 2 & Pointer for blade angles. \\
\hline 148 & LPOWRF & POWRF & $\mid$ NPOWRF $\mid * 2$ & $\begin{array}{l}\text { Pointer variable address for the } \\
\text { power deposited in the coolant } \\
\text { table-s rate-factor table. }\end{array}$ \\
\hline 149 & LPOWTB & POWTB & $\mid$ NPOWTB $\mid * 2$ & $\begin{array}{l}\text { Pointer variable address for the } \\
\text { power deposited in the coolant } \\
\text { table. }\end{array}$ \\
\hline 150 & LTURB & TURB & -1 & $\begin{array}{l}\text { Absolute LCM address for the } \\
\text { turbine data common among all } \\
\text { stages. }\end{array}$ \\
\hline $151-314$ & PTDUM & -- & -- & Dummy variable. \\
\hline
\end{tabular}

C. TURBDATA--TURB Data Table

This data table includes the following COMMON blocks: BLANKCOM and FIXEDLT, both defined in App.H; TURBNVLT, defined in Sec. XII.A; and TURBPT, defined in Sec. XII.B. 
XIII. VALVE MODULE

A. VALVEVLT--VALVE Variable-Length Table

REAL VARIABLES

\begin{tabular}{|c|c|c|}
\hline Position(s) & Parameter & Description \\
\hline 1 & AAl 111 & $\begin{array}{l}\text { Dummy variable that provides a known start to } \\
\text { the COMMON block. }\end{array}$ \\
\hline 2 & AVLVE & Valve open flow area. \\
\hline 3 & BSMASS & Time-integrated mass flow from the valve. \\
\hline 4 & FAVLVE & $\begin{array}{l}\text { Fraction of the fully open flow area AVLVE to } \\
\text { which the adjustable valve cross section is } \\
\text { set. }\end{array}$ \\
\hline $5-6$ & FL(2) & $\begin{array}{l}\text { Liquid mass-flow corrections for mass conserva- } \\
\text { tion checks. }\end{array}$ \\
\hline 7 & FMAXOV & $\begin{array}{l}\text { Maximum flow area fraction or relative valve } \\
\text { stem position during valve adjustment by the } \\
\text { overriding trip. }\end{array}$ \\
\hline 8 & FMINOV & $\begin{array}{l}\text { Minimum flow area fraction or relative valve } \\
\text { stem position during valve adjustment by the } \\
\text { overriding trip.l }\end{array}$ \\
\hline $9-10$ & FV (2) & $\begin{array}{l}\text { Vapor mass-flow corrections for mass conserva- } \\
\text { tion checks. }\end{array}$ \\
\hline 11 & HOUTL & $\begin{array}{l}\text { HTC between outer boundary of "valve wall and } \\
\text { liquid. }\end{array}$ \\
\hline 12 & HOUTV & $\begin{array}{l}\text { HTC between outer boundary of valve wall and } \\
\text { vapor. }\end{array}$ \\
\hline 13 & HVLVE & Valve open hydraulic diameter. \\
\hline 14 & QP3IN & Initial QPPP factor. \\
\hline 15 & QP3OFF & $\begin{array}{l}\text { QPPP factor when the controlling trip is OFF } \\
\text { after it was ON. }\end{array}$ \\
\hline 16 & RADIN & Inner radius of pipe wall. \\
\hline 17 & RQP 3MX & $\begin{array}{l}\text { Maximum allowed rate of change of the QPPP } \\
\text { factor }(s) \text {. }\end{array}$ \\
\hline
\end{tabular}




\begin{tabular}{|c|c|c|}
\hline Position(s) & Parameter & Description \\
\hline 18 & RVMX & $\begin{array}{l}\text { Maximum rate of change of valve flow area } \\
\text { fraction or relative valve stem position. }\end{array}$ \\
\hline 19 & RVOV & $\begin{array}{l}\text { Rate of change of valve flow area fraction or } \\
\text { relative valve stem position when controlled by } \\
\text { the overriding trip being on. } \\
\text {, }\end{array}$ \\
\hline 20 & $\mathrm{TH}$ & Thickness of pipe wall. \\
\hline 21 & TOUTL & Liquid temperature outside valve. \\
\hline 22 & TOUTV & Vapor temperature outside valve. \\
\hline 23 & XPOS & $\begin{array}{l}\text { Variable flag for valve operation in progress. } \\
0=\text { no moveinent, } \\
1=\text { opening movenent. }\end{array}$ \\
\hline
\end{tabular}

211111 Dummy variable that provides a known end to the COMMON block.

INTEGER VARIABLES

25

26

27

28

29

30

31

32

33

34

35
[A1111

ICHF

ICJI

IC.J2

ICONC

IONOFF

IQF

IQP

IQP3SV

IQP3TR

ISOLLB
Dumny variable that provides a known start to the COMMON block.

CHF calculation option.

I'teration index of adjacent component at JUN1.

Iteration index of adjacent component at JUN2.

Indicator for presence of boron in the coolant.

Number of time steps the valve table's controlling trip has been ON.

Last interpolated interval number in the racefactor table for the QPPP factor table.

Last interpolated interval number in the QPPP factor table.

QPPP factor table's abscissa-coordinate variable ID number.

Trip ID number that controls evaluation of the QPPP factor table.

Indicator for velocity update at JUN1: 


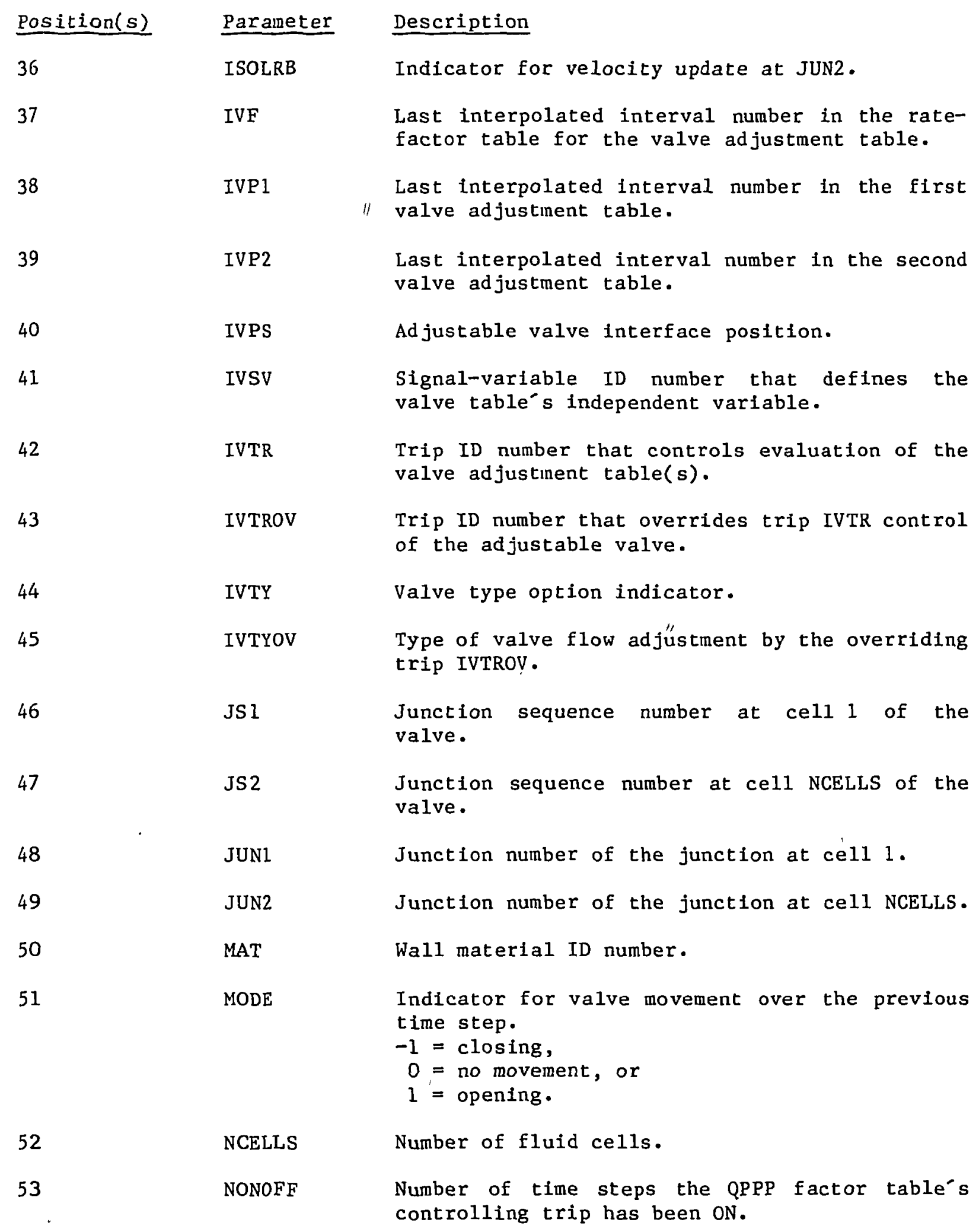




\begin{tabular}{|c|c|c|c|}
\hline \multicolumn{2}{|c|}{ Position(s) } & Parameter & Description \\
\hline 54 & & NQP3RF & $\begin{array}{l}\text { Number of pairs in the rate-factor table for } \\
\text { the QPPP factor table. }\end{array}$ \\
\hline 55 & & NQP3SV & $\begin{array}{l}\text { Rate-factor table-s abscissa-coordinate vari- } \\
\text { able ID number for the QPPP factor table. }\end{array}$ \\
\hline 56 & & NQP3TB & "Number of pairs in the QPPP factor table. \\
\hline 57 & & NVRF & $\begin{array}{l}\text { Number of rate-factor table pairs whose rate } \\
\text { factor is applied to the valve table's } \\
\text { independent variable. }\end{array}$ \\
\hline 58 & & NVSV & $\begin{array}{l}\text { Rate-factor table's abscissa-coordinate vari- } \\
\text { able ID number for the valve adjustment } \\
\text { table(s). }\end{array}$ \\
\hline 59 & & NVTB1 & $\begin{array}{l}\text { Number of pairs in the first valve adjustment } \\
\text { table. }\end{array}$ \\
\hline 60 & & NVTB2 & $\begin{array}{l}\text { Number of pairs in the second valve adjustment } \\
\text { table. }\end{array}$ \\
\hline 61 & 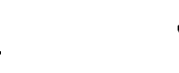 & TYPE 1 , & Type of adjacent component at JUNl. \\
\hline 62 & & TYPE2 & Type of adjacent component at JUN2. \\
\hline 63 & & ZI1111 & $\begin{array}{l}\text { Dummy variable that provides a known end to the } \\
\text { COMMON block. }\end{array}$ \\
\hline B. & LVEPT--VA & ALVE Pointer & Table \\
\hline Word(s) & Name & Array & Description \\
\hline $1-72$ & DUALPT & - & General pointer table. \\
\hline $73-131$ & HYDROPT & -- & General pointer table. \\
\hline $132-134$ & INTPT & -- & General pointer table. \\
\hline $135-146$ & HEATPT & - & General pointer table. \\
\hline 147 & LQP3RF & QP3RF & $\begin{array}{l}\text { Rate-factor table for the QPPP } \\
\text { factor table. }\end{array}$ \\
\hline 148 & LQP3TB & QP3TB & QPPP factor table. \\
\hline 149 & LVRF & VRF & $\begin{array}{l}\text { Rate-fictor table for the valve } \\
\text { adjustment table(s). }\end{array}$ \\
\hline
\end{tabular}




\begin{tabular}{lllll}
\multicolumn{1}{l}{ Word(s) } & Name & Array & Dimension & Description \\
150 & LVTB1 & VTB1 & $\mid$ NVTB1|*2 & First valve adjustment table. \\
151 & LVTB2 & VTB2 & $\mid$ NVTB2|*2 & Second valve adjustment table. \\
$152-317$ & PTDUM & - & - & Dummy variable.
\end{tabular}

C. VLVEDATA--VALVE Data Table

This data table includes the following COMMON blocks: BLANKCOM and FIXEDLT, both defined in App. H; VALVEVLT, defined in Sec. XIII.A; and VLVEPT, defined in Sec. XIII.B.

XIV. VESSEL MODULE

A. VSSELVLT--VESSEL Variable-Length Table

REAL VARIABLES

Position(s) Parameter $\quad \underline{\text { Description }}$

1

2

3

4

5

6

7

BPP 1

AA11 11

AMH2

BCRO

BCR 1

BE FF

BPPO

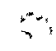

Dummy variable that provides a known start to the COMMON block.

Hydrogen mass generated from metal-water reaction.

Zero-order coefficient of the first-order polynomial that defines the effective coreaveraged concentration of control-rod pin boron.

First-order coefficient of the first-order polynomial "that defines the effective coreaveraged concentration of control-rod pin boron.

Total delayed neutron fraction.

Zero-order coefficient of the first-orjer polynomial that defines the effective coreaveraged concentration of burnable-poison pin boron.

First-order coefficient of the first-order polynomial that defines the effective coreaveraged concantration of burnable-poison pin boron. 


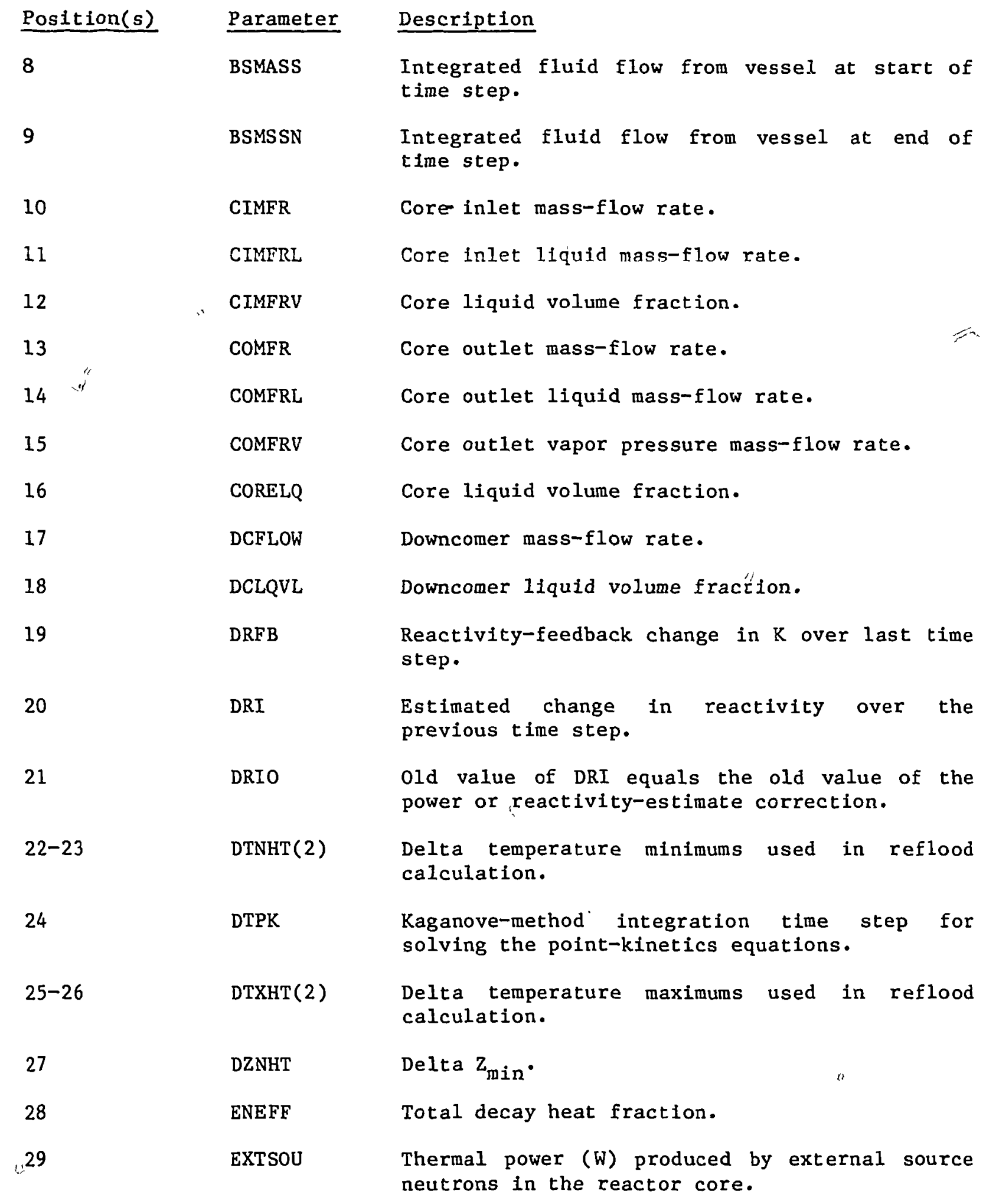




\begin{tabular}{|c|c|c|}
\hline Position(s) & Parameter & Description \\
\hline 30 & FUCRAC & Fraction of uncracked fuel. \\
\hline 31 & HGAPO & Rod gap-conductance coefficient (MATRD $=3$ ). \\
\hline 32 & PCORE & Average core pressure. \\
\hline 33 & PDC & Downcomer average pressure. \\
\hline 34 & PDRAT & Rod pitch-to-diameter ratio. \\
\hline 35 & PLDR & $\begin{array}{l}\text { Pellet dish radius. } \\
0.0=\text { no pellet-dish calculation, } \\
1.0=\text { pellet-dish calculation. }\end{array}$ \\
\hline 36 & PLP & Lower-plenum average pressure. \\
\hline 37 & POWEXP & $\begin{array}{l}\text { Exponent value to which the power distribution } \\
\text { is raised to define the weighting function for } \\
\text { averaging the reactivity-feedback parameters } \\
\text { over the core volume. }\end{array}$ \\
\hline 38 & PUP & Upper-plenum average pressure. \\
\hline 39 & QRDTOT & Total rod heat flux. \\
\hline 40 & QSLTOT & Total slab heat flux. \\
\hline 41 & REAC & $\begin{array}{l}\text { Reactivity feedback at the beginning of the } \\
\text { previous time step. }\end{array}$ \\
\hline 42 & REACN & $\begin{array}{l}\text { Reactivity-feedback estimate at the end of the } \\
\text { present time step. }\end{array}$ \\
\hline 43 & REACT & $\begin{array}{l}\text { Total reactivity at the beginning of the } \\
\text { present time step. }\end{array}$ \\
\hline 44 & RMCK & $\begin{array}{l}\text { Reactor multiplication constant at the } \\
\text { beginning of the present time step. }\end{array}$ \\
\hline 45 & RMCKN & $\begin{array}{l}\text { Reactor multiplication constant estimate at the } \\
\text { end of the present time step. }\end{array}$ \\
\hline 46 & RPOWPF & Prompt fission power. \\
\hline 47 & RPOWR & old reactor power. \\
\hline 48 & RPOWRI & Initial reactor power. \\
\hline 49 & RPOWRN & New reactor power. \\
\hline
\end{tabular}




\begin{tabular}{|c|c|c|}
\hline Position(s) & Parameter & Description \\
\hline 50 & RPOWRO & old reactor power of the previous time step. \\
\hline 51 & RPWOFF & $\begin{array}{l}\text { Programmed reactivity or reactor power when th } \\
\text { controlling trip is OFF after it was ON. }\end{array}$ \\
\hline 52 & RRPWMX & $\begin{array}{l}\text { Maximum rate of change of programmed reactivi } \\
\text { or reactor power. }\end{array}$ \\
\hline 53 & RZPWMX & $\begin{array}{l}\text { Maximum rate of change of the axial pow } \\
\text { shape. }\end{array}$ \\
\hline 54 & SD'T & $\begin{array}{l}\text { Time interval (s) since the last reactivi } \\
\text { change printout. }\end{array}$ \\
\hline 55 & SHELV & $\begin{array}{l}\text { Addition to the input ressel } \mathrm{Z} \text { coordinates } \\
\text { get elevations for computing GRAV in o } \\
\text { dimension. }\end{array}$ \\
\hline 56 & STIMET & $\begin{array}{l}\text { Problem time (s) at which the last reactivit } \\
\text { change was summed to variable storage for late } \\
\text { printout. }\end{array}$ \\
\hline 57 & TCILMF & Integrated core inlet liquid mass flow $(\mathrm{kg})$. \\
\hline 58 & TCIVMF & Integrated core inlet vapor mass flow $(\mathrm{kg})$. \\
\hline 59 & TCOLMF & Integrated core outlet liquid mass flow ( $\mathrm{kg})$. \\
\hline 60 & TCORE & Average core temperature. \\
\hline 61 & TCOVMF & Integrated core outlet "vapor mass flow $(\mathrm{kg})$. \\
\hline 62 & TDC & Downcomer average liquid temperature. \\
\hline 63 & TLP & Lower-plenum average temperature. \\
\hline 64 & TNEUT & Neutron generation time. \\
\hline 65 & TRAMAX & Average-rod peak-cladding temperature. \\
\hline 66 & TRHSAX & Maximum supplemental rod temperature. \\
\hline 67 & TSCORE & Average core saturation temperature. \\
\hline 68 & TSDC & Downcomer average saturation temperature. \\
\hline 690 & TSLP & Lower-plenum average saturation temperature. \\
\hline 70 & TSUP & Upper-plenum average liquid temperature. \\
\hline
\end{tabular}




\begin{tabular}{|c|c|c|}
\hline Position(s) & Parameter & Description \\
\hline 71 & TUP & Upper-plenum average liquid temperature. \\
\hline 72 & VBMASS & Mass counter for internal break sources. \\
\hline 73 & VBMSSN & Mass counter for internal break sources. \\
\hline 74 & VCORE & Total liquid mass in the core. \\
\hline 75 & VDCLQ & Total liquid mass in the downcomer. \\
\hline 76 & VFMASS & Mass counter for internal fill source. \\
\hline 77 & VFMSSN & Mass counter for internal fill source. \\
\hline 78 & VLCORE & Core liquid mass. \\
\hline 79 & VLPLIQ & Lower-plenum liquid volume fraction. \\
\hline 80 & VLPLM & Lower-plenum liquid mass. \\
\hline 81 & VLPLQ & Total liquid mass in the lower plenum. \\
\hline 82 & VLQMSS & Total liquid mass in the vessel. \\
\hline 83 & VOLDC & Downcomer volume. \\
\hline 84 & VOLLP & Lower-plenum volume. \\
\hline 85 & VOLUP & Upper-plenum volume. \\
\hline 86 & VSFLOW & Vessel mass flow. \\
\hline 87 & VUPLIQ & Upper-plenum liquid volume fraction. \\
\hline 88 & VUPLM & Upper-plenum liquid mass. \\
\hline 89 & ZPWIN & $\begin{array}{l}\text { Axial power-shape table's abscissa-coordinate } \\
\text { variable value corresponding to the initial } \\
\text { axial power shape. }\end{array}$ \\
\hline 90 & ZPWOFF & $\begin{array}{l}\text { Axial power-shape table's abscissa-coordinate } \\
\text { variable value that corresponds to the axial } \\
\text { power shape that is used when the controlling } \\
\text { trip is OFF after it was oN. }\end{array}$ \\
\hline 91 & 211111 & $\begin{array}{l}\text { Dummy variable that provides a known end to the } \\
\text { COMMON block. }\end{array}$ \\
\hline
\end{tabular}


92

93-96

97

98

99

100

101

102

103

104

105

106

107

108

$109-112$

$113-116$

$117-132$

133
IA1111

$\operatorname{IBU}(4)$

ICONC

ICRL

ICRR

ICRRG

ICRU

IDCL

IDCR

IDCU

IITOT

ILCSP

INHSMX

IONOFF

$\operatorname{IRC}(4)$

$\operatorname{IRCJFM(4)}$

IRCJTB $(4,4)$

IRF
Dummy variable that provides a known start to the COMMON block.

Boron-unit flag for the Jth reactivity coefficient.

Presence of boron in the coolant flag.

Core lower-boundary segment number, Z(ICRL).

Core outer radial-boundary segment number, RAD(ICRR).

Outermost radial ring in the core. Currently defaults to ICRR.

Core upper-boundary segment number, Z(ICRU).

Downcomer lower-boundary segment number, $\mathrm{Z}$ (IDCL).

Downcomer radial-boundary segment number, RAD (IDCR).

Downcomer upper-boundary segment number, $\mathrm{Z}$ (IDCU).

Inner iteration counter.

Lower-core support-plate axial segment number.

Number of interfaces between dissimilar materials in the vessel slabs.

Number of time steps the reactivity-power table"s controlling trip has been on.

Number of values that defines the argument number reactivity-coefficient table.

Form number of the reactivity coefficient for the argument number reactivity-feedback parameter.

Number of first argument reactivity-feedback parameter value entries for the second argument reactivity-coefficient table.

Last interpolated interval number in the ratefactor table for the reactivity-ppwer table. 


\begin{tabular}{|c|c|c|}
\hline Position(s) & Parameter & Description \\
\hline 134 & IRFTR & $\begin{array}{l}\text { Trip ID number that controls evaluation with } \\
\text { the reflood axial fine mesh in the fuel rod. }\end{array}$ \\
\hline 135 & IRP & $\begin{array}{l}\text { Last interpolated interval number in the } \\
\text { reactivity-power table. }\end{array}$ \\
\hline 136 & IRPWSV & $\begin{array}{l}\text { Reaclivity-power table's abscissa-coordinate } \\
\text { variable ID number. }\end{array}$ \\
\hline 137 & IRPWTR & $\begin{array}{l}\text { Trip ID number that controls evaluation of the } \\
\text { reactivity-power table. }\end{array}$ \\
\hline 138 & IRPWTY & Reactor-kinetics option indicator. \\
\hline 139 & ISNOTB & $\begin{array}{l}\text { A flag variable that is defined if the solute } \\
\text { is boron for the reactivity-feedback calcula- } \\
\text { tion. } \\
0=\text { solute is boron, } \\
1=\text { solute is not boron. }\end{array}$ \\
\hline 140 & IUCSP & Upper-core support-plate axial segment number. \\
\hline 141 & IUHP & Upper head-plate axial segment number. \\
\hline 142 & IVSSBF & $\begin{aligned} & \text { Internal vessel boundary condition. } \\
& 0= \text { no internal boundarles (default); } \\
& 2= \text { first axial level acts like a fill, last } \\
& \text { axial level acts like a break; } \\
& 20= \text { first axial level acts like a break, last } \\
& \text { axial level acts like a fill; } \\
& 22= \text { both the first and last axial levels act } \\
& \text { like breaks. }\end{aligned}$ \\
\hline 143 & IZBK & Switch for backup on water pack. \\
\hline 144 & IZBK2 & Switch for re-donor-cell logic. \\
\hline 145 & IZNX & Variable used in water-packing logic. \\
\hline 146 & IZF & $\begin{array}{l}\text { Last interpolated interval number in the rate- } \\
\text { factor table for the axial power-shape table. }\end{array}$ \\
\hline 147 & IZP & $\begin{array}{l}\text { Last interpolated interval number in the axial } \\
\text { power-shape table. }\end{array}$ \\
\hline 148 & IZPWSV & $\begin{array}{l}\text { Axial power-shape table's abscissa-coordinate } \\
\text { variable ID number. }\end{array}$ \\
\hline 149 & IZPWTR & $\begin{array}{l}\text { Trip ID number that controls evaluation of the } \\
\text { axial power-shape table. }\end{array}$ \\
\hline
\end{tabular}




\begin{tabular}{|c|c|c|}
\hline Position(s) & Parameter & Description \\
\hline 150 & LENLD & Length of level data. \\
\hline 151 & LENRD & Length of rod data. \\
\hline 152 & LFVL & $\begin{array}{l}\text { Relative position of old fundamental variables } \\
\text { of level data. }\end{array}$ \\
\hline 153 & LFVNL & $\begin{array}{l}\text { Relative position of new fundamental variables } \\
\text { of level data. }\end{array}$ \\
\hline 154 & LFVNR & $\begin{array}{l}\text { Relative position of new fundamental variables } \\
\text { of rod data. }\end{array}$ \\
\hline 155 & LFVR & $\begin{array}{l}\text { Relative position of old fundamental variables } \\
\text { of rod data. }\end{array}$ \\
\hline 156 & LNFVL & Length of fundamental variables of level data. \\
\hline 157 & LNFVR & Length of fundamental variables of rod length. \\
\hline 158 & LNPTRL & Number of level data pointers. \\
\hline 159 & LNPTRR & Number of pointers of rod data. \\
\hline 160 & NASX & Number of axial segments (levels). \\
\hline 161 & NCELLS & Total number of fluid cells. \\
\hline 162 & NCLX & Number of fluid cells per level. \\
\hline 163 & NCRX & Maximum number of core volumes per level. \\
\hline 164 & NCRXX & Total number of core volumes. \\
\hline 165 & NCRZ & Number of core levels. \\
\hline 166 & NCSK & Number of cell sources (connections). \\
\hline 167 & NDGX & Number of delayed neutron groups. \\
\hline 168 & NDHX & Number of decay heat groups. \\
\hline 169 & NFBPWT & $\begin{array}{l}\text { Flag that defines the spatial distribution used } \\
\text { to weight the averaging of the reactivity-feed- } \\
\text { back parameters over the reactor-vessel volume. }\end{array}$ \\
\hline 170 & NFCI & $\begin{array}{l}\text { FCI flag. } \\
0=\text { no calculation, } \\
1=\text { calculation. }\end{array}$ \\
\hline
\end{tabular}




\begin{tabular}{|c|c|c|}
\hline Position(s) & Parameter & Description \\
\hline 171 & NFCIL & Limit on FCI calculations per time step. \\
\hline 172 & NFFA & Axial friction-factor correlation option. \\
\hline 173 & NFFR & Radial friction-factor correlation option. \\
\hline 174 & NFFT & Theta friction-factor correlation option. \\
\hline 175 & NFUEL & Number of nodes in fuel pellet. \\
\hline 176 & NINT & $\begin{array}{l}\text { Number of interfaces between dissimilar mate- } \\
\text { rials in rods. }\end{array}$ \\
\hline 177 & NMWRX & $\begin{array}{l}\text { Metal-water reaction flag. } \\
0=\text { no calculation, } \\
1=\text { calculation. }\end{array}$ \\
\hline 178 & NODHS & Number of nodes in the heat slab. \\
\hline 179 & NONOFF & $\begin{array}{l}\text { Number of time steps the trip-controlling } \\
\text { evaluation of the axial power-shape table has } \\
\text { been ON. }\end{array}$ \\
\hline 180 & NRAMAX & $\begin{array}{l}\text { Location of average-rod peak-cladding tempera- } \\
\text { ture used in reflood calculation. }\end{array}$ \\
\hline 181 & NRFD & $\begin{array}{l}\text { Reflood flag. } \\
0=\text { takes no action, } \\
1=\text { turns on fine-mesh flag if it is off. }\end{array}$ \\
\hline 182 & NRHMAX & $\begin{array}{l}\text { Location of supplemental rod peak-cladding } \\
\text { temperature. }\end{array}$ \\
\hline 183 & NRODS & Number of computational rods. \\
\hline 184 & NRPWRF & $\begin{array}{l}\text { Number of rate-factor table pairs whose rate } \\
\text { factor is applied to the power or reactivity } \\
\text { table's independent variable. }\end{array}$ \\
\hline 185 & NRPWSV & $\begin{array}{l}\text { Reactivity-power rate-factor table's abscissa- } \\
\text { coordinate variable ID number. }\end{array}$ \\
\hline 186 & NRPWTB & $\begin{array}{l}\text { Number of entry pairs in the reactivity-power } \\
\text { table. }\end{array}$ \\
\hline 187 & NRSX & Number of radial segments. \\
\hline 188 & NRTS & $\begin{array}{l}\text { Number of time steps over which the programmed } \\
\text { reactivity and reactivity-feedback changes are } \\
\text { summed for printout. }\end{array}$ \\
\hline 82 & & \\
\hline
\end{tabular}




\begin{tabular}{|c|c|c|c|c|}
\hline \multicolumn{2}{|c|}{ Position(s) } & \multicolumn{2}{|r|}{ 'escription } & \\
\hline 189 & & NSET & $\begin{array}{l}\text { Libsolute valu } \\
\text { step. }\end{array}$ & $\begin{array}{l}\text { ue "of the reflood fine axial mesh } \\
\text { tus number during the previous time }\end{array}$ \\
\hline 190 & & NTSX & Number of the & eta segments. \\
\hline 191 & & NVENT & $\begin{array}{l}\text { Number of } c \\
\text { radial surfac }\end{array}$ & $\begin{array}{l}\text { cells with vent valves in outer } \\
\text { ce. }\end{array}$ \\
\hline 192 & & NZMAX & $\begin{array}{l}\text { Maximum numbe } \\
\text { used in reflo }\end{array}$ & $\begin{array}{l}\text { er of rows of heat-transfer nodes } \\
\text { ood calculation. }\end{array}$ \\
\hline 193 & & NZPWRF & $\begin{array}{l}\text { Number of ent } \\
\text { rate-factor } t\end{array}$ & $\begin{array}{l}\text { try pairs in the axial power-shape } \\
\text { table. }\end{array}$ \\
\hline 194 & & NZPWSV & $\begin{array}{l}\text { Axial power-s } \\
\text { coordinate va }\end{array}$ & $\begin{array}{l}\text { shape rate-factor table's abscissa- } \\
\text { ariable ID number. }\end{array}$ \\
\hline 195 & & NZPWTB & $\begin{array}{l}\text { Number of a } \\
\text { power-shape } t\end{array}$ & $\begin{array}{l}\text { axial power shapes in the axial } \\
\text { table. }\end{array}$ \\
\hline 196 & & ZI1111 & $\begin{array}{l}\text { Dummy variabl } \\
\text { COMMON block. }\end{array}$ & le that provides a known end to the \\
\hline B. & SSLPT $-V$ & SSEL Point & er Table & \\
\hline COMMON & ATA POIN & CERS & & \\
\hline Word $(s)$ & Name & Array & Dimension & Description \\
\hline 1 & LAVENT & AVENT & NVENT & Pointer for vent valve AEARS. \\
\hline 2 & LBE'TA & BETA & NDGX & Delayed neutron group fraction. \\
\hline 3 & LCDG & CDG & NDGX & $\begin{array}{l}\text { old concentration of delayed neu- } \\
\text { tron groups. }\end{array}$ \\
\hline 4 & LCDGN & CDGN & NDGX & $\begin{array}{l}\text { New concentration of delayed neu- } \\
\text { tron groups. }\end{array}$ \\
\hline 5 & $\mathrm{LCDH}$ & $\mathrm{CDH}$ & NDHX & $\begin{array}{l}\text { old concentration of decay heat } \\
\text { groups. }\end{array}$ \\
\hline 6 & LCDHN & CDHN & NDHX & $\begin{array}{l}\text { New concentration of decay heat } \\
\text { groups. }\end{array}$ \\
\hline 7 & LCLEN & CLEN & NCRX & old total cladding length. \\
\hline 8 & LCLENN & CLENN & NCRX -1 & New total cladding length. \\
\hline
\end{tabular}




\begin{tabular}{|c|c|c|c|c|}
\hline Word(s) & Name & Array & Dimension & Description \\
\hline 9 & LCPOWR & CPOWR & NCRX & Relative power per rod. \\
\hline 10 & LDPCVN & DPCVN & NVENT & $\begin{array}{l}\text { Pointer for vent valve maximum } \Delta \mathrm{P} \\
\text { to be closed. }\end{array}$ \\
\hline 11 & LDPOVN & DPOVN & NVENT & $\begin{array}{l}\text { Pointer for vent valve minimum } \Delta \mathrm{P} \\
\text { to be open. }\end{array}$ \\
\hline 12 & LDR & DR & NRSX & Radial segment lengths (delta R). \\
\hline 13 & LDTH & DTH & NTSX & $\begin{array}{l}\text { Theta segment length (delta } \\
\text { theta). }\end{array}$ \\
\hline 14 & LDVLDP & DVLDP & NCSR & $\begin{array}{l}\text { Derivative of liquid source velo- } \\
\text { city with respect to pressure. }\end{array}$ \\
\hline 15 & LDVVDP & DVVDP & NCSR. & $\begin{array}{l}\text { Derivative of vapor source velo- } \\
\text { city with respect to pressure. }\end{array}$ \\
\hline 16 & LDZ & DZ & NASX & Axtal segment lengths (delta $Z$ ). \\
\hline 17 & LEDH & EDH & NDHX & $\begin{array}{l}\text { Energy yield fraction of decay } \\
\text { heat groups. }\end{array}$ \\
\hline 18 & LFPUO2 & FPUO2 & NCRX & $\begin{array}{l}\text { Fraction of plutonium oxide in } \\
\text { mixed-oxide fuel fraction. }\end{array}$ \\
\hline 19 & LFRCVN & FRCVN & NVENT & $\begin{array}{l}\text { Pointer for vent valve FRIC value } \\
\text { when closed. }\end{array}$ \\
\hline 20 & LFROVN & FROVN & NVENT & $\begin{array}{l}\text { Pointer for vent valve FRIC value } \\
\text { when open. }\end{array}$ \\
\hline 21 & LFTD & FTD & NCRX & $\begin{array}{l}\text { Fuel density (fraction of theoret- } \\
\text { ical). }\end{array}$ \\
\hline 22 & LGMIX & GMIX & $\operatorname{NCRX} * 7$ & $\begin{array}{l}\text { Mole fraction of gap-gas constitu- } \\
\text { ents. }\end{array}$ \\
\hline 23 & LGMLES & GMLES & NCRX & Moles of gap gas. \\
\hline 24 & LHS & HS & $\begin{array}{l}\text { NCRX* } \\
(\mathrm{NFBPWT} / 4)\end{array}$ & $\begin{array}{l}\text { Pointer variable for the hori- } \\
\text { zontal plane shape weight function } \\
\text { used. }\end{array}$ \\
\hline 25 & LICJ & ICJ & NCSR & $\begin{array}{l}\text { Adjacent component associated with } \\
\text { source. }\end{array}$ \\
\hline 26 & LIDROD & IDROD & NRODS & Cell identifier for rods. \\
\hline
\end{tabular}




\begin{tabular}{|c|c|c|c|c|}
\hline Word (s) & Name & Array & Dimension & Description \\
\hline 27 & LISOLB & ISOLB & NCSR & Indicator for velocity update. \\
\hline 28 & LISRC & ISRC & NCSR & $\begin{array}{l}\text { Relative cell number associated } \\
\text { with source. }\end{array}$ \\
\hline 29 & LISRF & ISRF & NCSR & $\begin{array}{l}\text { Face number associated with } \\
\text { source. }\end{array}$ \\
\hline 30 & LISRL & ISRL & NCSR & $\begin{array}{l}\text { Level number associated with } \\
\text { source. }\end{array}$ \\
\hline 31 & LIZREP & IZREP & NASX & $\begin{array}{l}\text { Indicator for levels to be re- } \\
\text { donor-celled. }\end{array}$ \\
\hline 32 & LJSN & JSN & NCSR & $\begin{array}{l}\text { Junction sequence number associ- } \\
\text { ated with source. }\end{array}$ \\
\hline 33 & LJUNS & JUNS & NCSR & $\begin{array}{l}\text { Junction number associated with } \\
\text { source. }\end{array}$ \\
\hline 34 & LLAMDA & LAMDA & NDGX & Decay constant of delayed groups. \\
\hline 35 & LLAMDH & LAMDH & NDHX & $\begin{array}{l}\text { Decay constant of decay heat } \\
\text { groups. }\end{array}$ \\
\hline 36 & LLOCVN & LOCVN & NVENT & Pointer for vent valve location. \\
\hline 37 & LMATRD & MATRD & NODES-1 & Rod material identification. \\
\hline 38 & LMSC & MSC & NCSR & Absolute cell number of source. \\
\hline 39 & LNFAX & NFAX & NCRZ & Rod fine-mesh noding factor. \\
\hline 40 & LNRDX & NRDX & NCRX & Number of rods in volume. \\
\hline 41 & LNSRL & NSRL & NASX & Number of sources on level. \\
\hline 42 & LPGAPT & PGAPT & NCRX & Gap total gas pressure. \\
\hline 43 & LPLVOL & PLVOL & NCRX & Rod plenum volume. \\
\hline 44 & LPSLEN & PSLEN & NCRX & Pellet stack length. \\
\hline 45 & LPSNEW & PSNEW & NCSR & New source pressure. \\
\hline 46 & LPSOLD & PSOLD & NCSR & old source pressure. \\
\hline 47 & LRAD & RAD & NRSX & Radial segment outer radil. \\
\hline
\end{tabular}




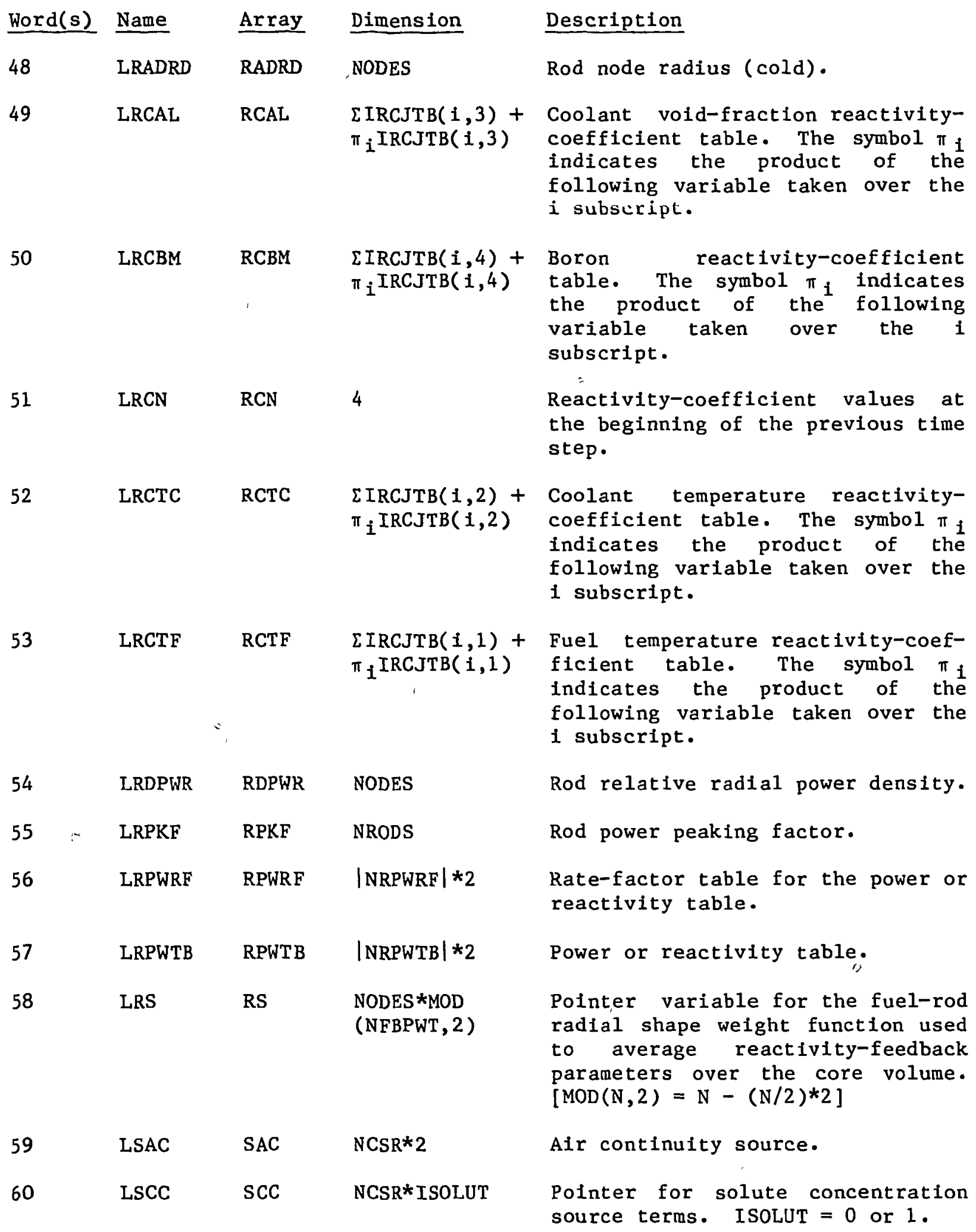




\begin{tabular}{|c|c|c|c|c|}
\hline Word(s) & Name & Array & Dimension & Description \\
\hline 61 & LSLC & SLC & $N C S R * 2$ & Liquid continulty source. \\
\hline 62 & LSLE & SLE & $\mathrm{NCSR} * 2$ & Liquid energy source. \\
\hline 63 & LSMOML & SMOML & $\mathrm{NCSR} * 6$ & Liquid momentum source. \\
\hline 64 & LSMOMV & SMOMV & $\mathrm{NCSR} * 6$ & Vapor momentum source. \\
\hline 65 & LSRP & SRP & 15 & $\begin{array}{l}\text { Summed programmed and feedback- } \\
\text { reactivity changes. }\end{array}$ \\
\hline 66 & LSVC & SvC & $\mathrm{NCSR} * 2$ & Vapor continuity source. \\
\hline 67 & LSVE & SVE & $\mathrm{NCSR} * 2$ & Vapor energy source. \\
\hline 68 & LTH & $\mathrm{TH}$ & NTSX & Theta segment angle. \\
\hline 69 & LVELSL & VELSL & NCSR & Liquid source velocity. \\
\hline 70 & LVELSV & VELSV & NCSR & Vapor source velocity. \\
\hline 71 & LXN & $\mathrm{XN}$ & 4 & $\begin{array}{l}\text { New reactivity-feedback parameter } \\
\text { values. }\end{array}$ \\
\hline 72 & LXO & xo & 4 & $\begin{array}{l}\text { old reactivity-feedback parameter } \\
\text { values. }\end{array}$ \\
\hline 73 & $\mathrm{Lz}$ & $z$ & NASX & Axial segment upper elevation. \\
\hline 74 & LZPW & $\mathrm{ZPW}$ & NCRZP1 & $\begin{array}{l}\text { Last interpolated axial power } \\
\text { shape. }\end{array}$ \\
\hline 75 & LZPWRF & ZPWRF & $|\mathrm{NZPWRF}| * 2$ & $\begin{array}{l}\text { Axial power-shape rate-factor } \\
\text { table. }\end{array}$ \\
\hline 76 & LZPWTB & ZPWTB & $\begin{array}{l}\mid \text { NZPWTB } \mid * \\
\text { NCRZP2 }\end{array}$ & Relative axial power-shape table. \\
\hline 77 & LZS & ZS & $\begin{array}{l}\text { NCRZP1 * }(\text { MOD } \\
(\text { NFBPWT }, 4) / 2)\end{array}$ & $\begin{array}{l}\text { Pointer variable for the axial- } \\
\text { direction shape. }[\operatorname{MOD}(N, 2)=N \\
-(N / 2) * 2]\end{array}$ \\
\hline
\end{tabular}

LEVEL DATA POINTERS

$\begin{array}{lllll}78 & \text { LAID1 } & \text { AID1 } & \text { NCLX*IDROP } & \begin{array}{l}\text { Variable not } \\ \text { mented. }\end{array} \\ 79 & \text { LAID1N } & \text { AID1N } & \text { NCLX*IDROP } & \begin{array}{l}\text { Variable } \\ \text { mented. }\end{array}\end{array}$




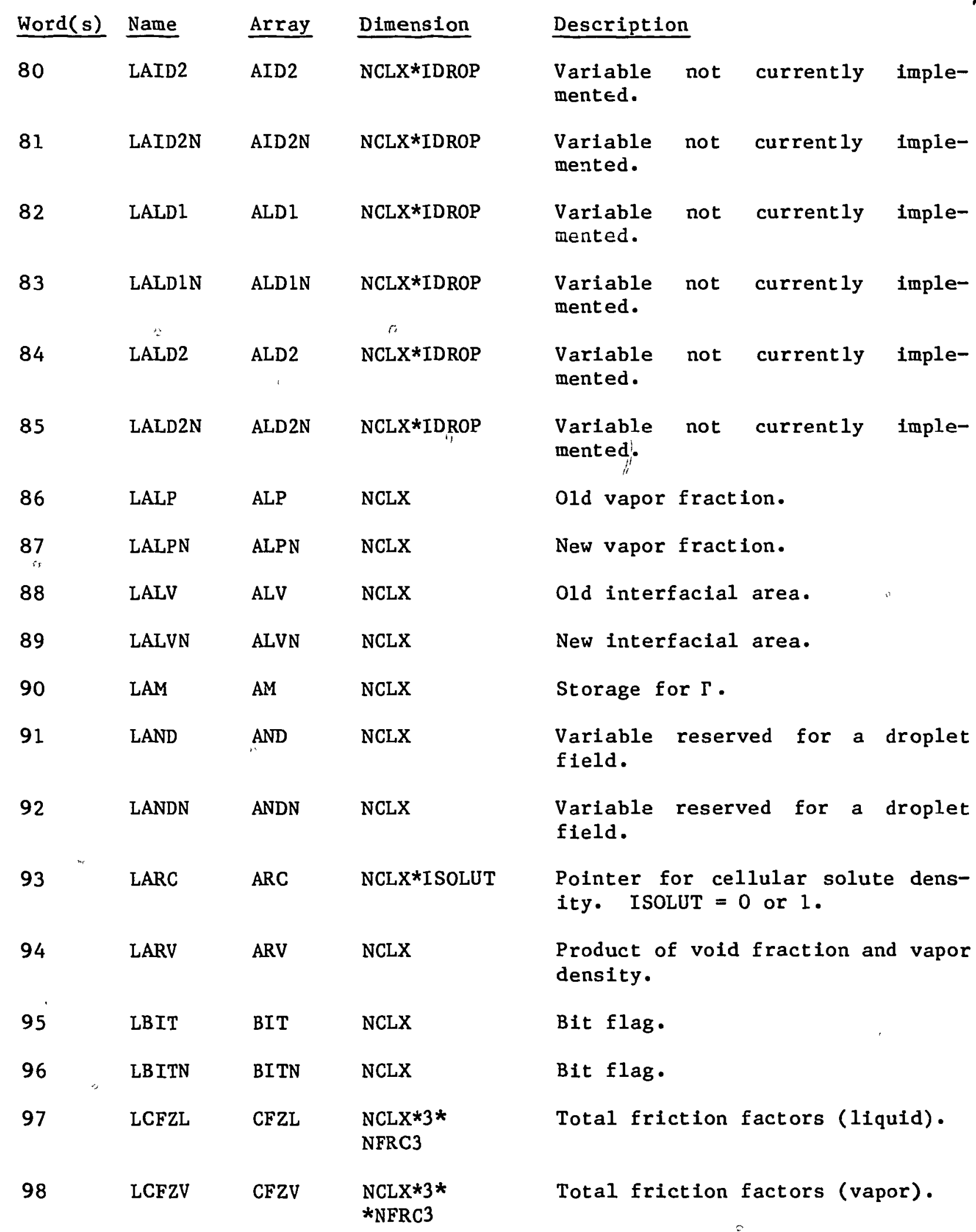




\begin{tabular}{|c|c|c|c|c|}
\hline Word(s) & Name & Array & Dimension & Description \\
\hline 99 & LCL & $\mathrm{CL}$ & NCLX & Liquid conductivity. \\
\hline 100 & LCNHS & CNHS & NCLX*NODHS & Slab conductivities. \\
\hline 101 & LCNHSN & CNHSN & INHSMX *NCLX & $\begin{array}{l}\text { Slab conductivities at a material } \\
\text { interface. }\end{array}$ \\
\hline 102 & LCONC & CONC & NCLX*ISOLUT & $\begin{array}{l}\text { New ratio of boron mass to coolant } \\
\text { mass. ISOLUT }=0 \text { or } 1 \text {. }\end{array}$ \\
\hline 103 & LCONCO & CONCO & NCLX*ISOLUT & $\begin{array}{l}\text { O1d ratio of boron mass to coolant } \\
\text { mass. ISOUUT }=0 \text { or } 1 \text {. }\end{array}$ \\
\hline 104 & LCPHS & CPHS & NCLX*NODHS & Slab specific heats. \\
\hline 105 & LCPHSN & CPHSN & INHSMX *NCLX & $\begin{array}{l}\text { Slab specific heat at a material } \\
\text { interface. }\end{array}$ \\
\hline 106 & LCPL & CPL & NCLX & $\begin{array}{l}\text { Liquid specific heat at constant } \\
\text { pressure. }\end{array}$ \\
\hline 107 & LCPV & $\mathrm{CPV}$ & NCLX & $\begin{array}{l}\text { Vapor specific heat at constant } \\
\text { pressure. }\end{array}$ \\
\hline 108 & $\mathrm{LCV}$ & $\mathrm{CV}$ & NCLX & Vapor conductivity. \\
\hline 109 & LDLL & DLL & $\operatorname{NCLX} * 3$ & $\begin{array}{l}\text { Derivative used in momentum update } \\
\text { (vapor). }\end{array}$ \\
\hline 110 & LDRIV & DRIV & NTHM3D*NCLX & $\begin{array}{l}\text { Variable array for thermodynamic } \\
\text { derivatives, enthalpies, and tem- } \\
\text { porary storage for matrix inver- } \\
\text { sions. }\end{array}$ \\
\hline 111 & LDROP & DROP & $\operatorname{NCLX} * 4$ & Droplet field storage area. \\
\hline 112 & LDVD1 & DVD1 & NCLX*3*IDROP & $\begin{array}{l}\text { Variable not currently imple- } \\
\text { mented. }\end{array}$ \\
\hline 113 & LDVD2 & DVD2 & NCLX*3*IDROP & $\begin{array}{l}\text { Variable not currently imple- } \\
\text { mented. }\end{array}$ \\
\hline 114 & LDVV & DVV & $\mathrm{NCLX} * 3$ & $\begin{array}{l}\text { Derivative used in momentum update } \\
\text { (liquid). }\end{array}$ \\
\hline 115 & LEA & EA & NCLX & old air energy. \\
\hline 116 & LEAN & EAN & NCLX & New air energy. \\
\hline
\end{tabular}




\begin{tabular}{|c|c|c|c|c|}
\hline Word(s) & Name & Array & Dimension & Description \\
\hline 117 & LEL & EL & NCLX & old liquid internal energy. \\
\hline 118 & LELN & ELN & NCLX & New liquid internal energy. \\
\hline 119 & LEMHS & EMHS & NCLX*NODHS & Slab emissivities. \\
\hline 120 & LEV & EV & NCLX & old vapor internal energy. \\
\hline 121 & LEVN & EVN & NCLX & New vapor ini.ernal energy. \\
\hline 122 & LFA & FA & $\operatorname{NCLX} * 3$ & Cell-edge fluid areas. \\
\hline 123 & LFAG & FAG & NCLX*3 & Cell-edge g eometric areas. \\
\hline 124 & LFINAN & FINAN & NCLX & Inverted annular regime factor. \\
\hline 125 & LFRCIN & "FRCIN & $\mathrm{NCLX} * 3$ & New interfacial friction factors. \\
\hline 126 & LFRICI & FRICI & NCLX*3 & old interfacial friction factors. \\
\hline 127 & LFRICL & FRICL & $\mathrm{NCLX} * 3$ & Friction multipliers (liquid). \\
\hline 128 & LFRICV & FRICV & NCLX*3 & Friction multipliers (vapor). \\
\hline 129 & LGAM & GAM & $\operatorname{NCLX}$ & Storage for $\Gamma$. \\
\hline 130 & LGAMN & GAMN & NCLX & Storage for $\Gamma$. \\
\hline 131 & LGCOND & GCOND & NCLX & Vapor zondensation rate. \\
\hline 132 & LHLA & HLA & NCLX & $\begin{array}{l}\text { Sum of all products of liquid HTC } \\
\text { with heat-transfer area. }\end{array}$ \\
\hline 133 & LHLATW & HLATW & NCLX & $\begin{array}{l}\text { Similar to LHLA except that the } \\
\text { product includés wall temperature. }\end{array}$ \\
\hline 134 & LGEVAP & GEVAP & NCLX & Liquid evaporation rate. \\
\hline 135 & LGRAV & GRAV & NCLX & Gravitation terins. \\
\hline & & --ITEMS & BELOW ARE REPEATED & FOR EACH LEVEL-- \\
\hline 136 & LHD & HD & $\begin{array}{l}\text { NCLX*3* } \\
\text { NDIA3 }\end{array}$ & Hydraulic diameters. \\
\hline 137 & LHFG & HFG & NCLX & Latent heat of vaporization. \\
\hline 138 & LHGAM & HGAM & NCLX & Storage for the subcooled boiling. \\
\hline
\end{tabular}




\begin{tabular}{|c|c|c|c|c|}
\hline Word(s) & Name & Array & Dimension & Description \\
\hline 139 & LHLV & $\mathrm{HLV}$ & NCLX & old interfacial HTC. \\
\hline 140 & LHLVN & HLVN & NCLX & New interfacial HTC. \\
\hline 141 & LHSA & HSA & NCLX & Heat-slab area. \\
\hline 142 & LHSHL & HSHL & NCLX & Slab HTC (liquid). \\
\hline 143 & LHSHLO & HSHLO & NCLX & $\begin{array}{l}\text { Variabie not currently imple- } \\
\text { mented. }\end{array}$ \\
\hline 144 & LHSHV & HSHV & NCLX & Slab HTC (vapor). \\
\hline 145 & LHSHVO & HSHVO & NCLX & $\begin{array}{l}\text { Variable not currently imple- } \\
\text { mented. }\end{array}$ \\
\hline 146 & LHST & HST & NCLX*NODHS & Old heat-slab temperatures. \\
\hline 147 & LHSTN & HSTN & NCLX*NODHS & New heat-slab temperatures. \\
\hline 148 & LHSX & HSX & NCLX*NODHS & $\begin{array}{l}\text { Spacing of heat-transfer nodes in } \\
\text { the slabs. }\end{array}$ \\
\hline $149^{\prime \prime}$ & LHVA & HVA & NCLX & $\begin{array}{l}\text { Sum of all products of vapor HTC } \\
\text { with heat-transfer area (similar } \\
\text { to LHLA). }\end{array}$ \\
\hline 150 & LHVATW & HVATW & NCLX & $\begin{array}{l}\text { Similar to LHVA except that the } \\
\text { product includes wall temperature. }\end{array}$ \\
\hline 151 & LICMSH & $\mathrm{ICMSH}$ & NCLX & $\begin{array}{l}\text { Index to associated coarse-mesh } \\
\text { region. }\end{array}$ \\
\hline 152 & LIDRGS & IDRGS & NCLX & Slab heat-transfer regime flag. \\
\hline 153 & LIHSN & IHSN ". & NCLX & Heat-slab number. \\
\hline 154 & LISRN & ISRN & NCSR & Source numbers on level. \\
\hline 155 & LMATHS & MATHS & NCLX*NHSMI & Slab material ID numbers. \\
\hline 156 & LMFRL & MFRL & NCLX*IMFR & Liquid mass flow. \\
\hline 157 & LMFRV & MFRV & NCLX*IMFR & Vapor mass flow." \\
\hline 158 & LP & $\mathrm{P}$ & NCLX & 01d pressure. \\
\hline 159 & LPA & $\mathrm{PA}$ & NCLX & old air partial pressure. \\
\hline
\end{tabular}




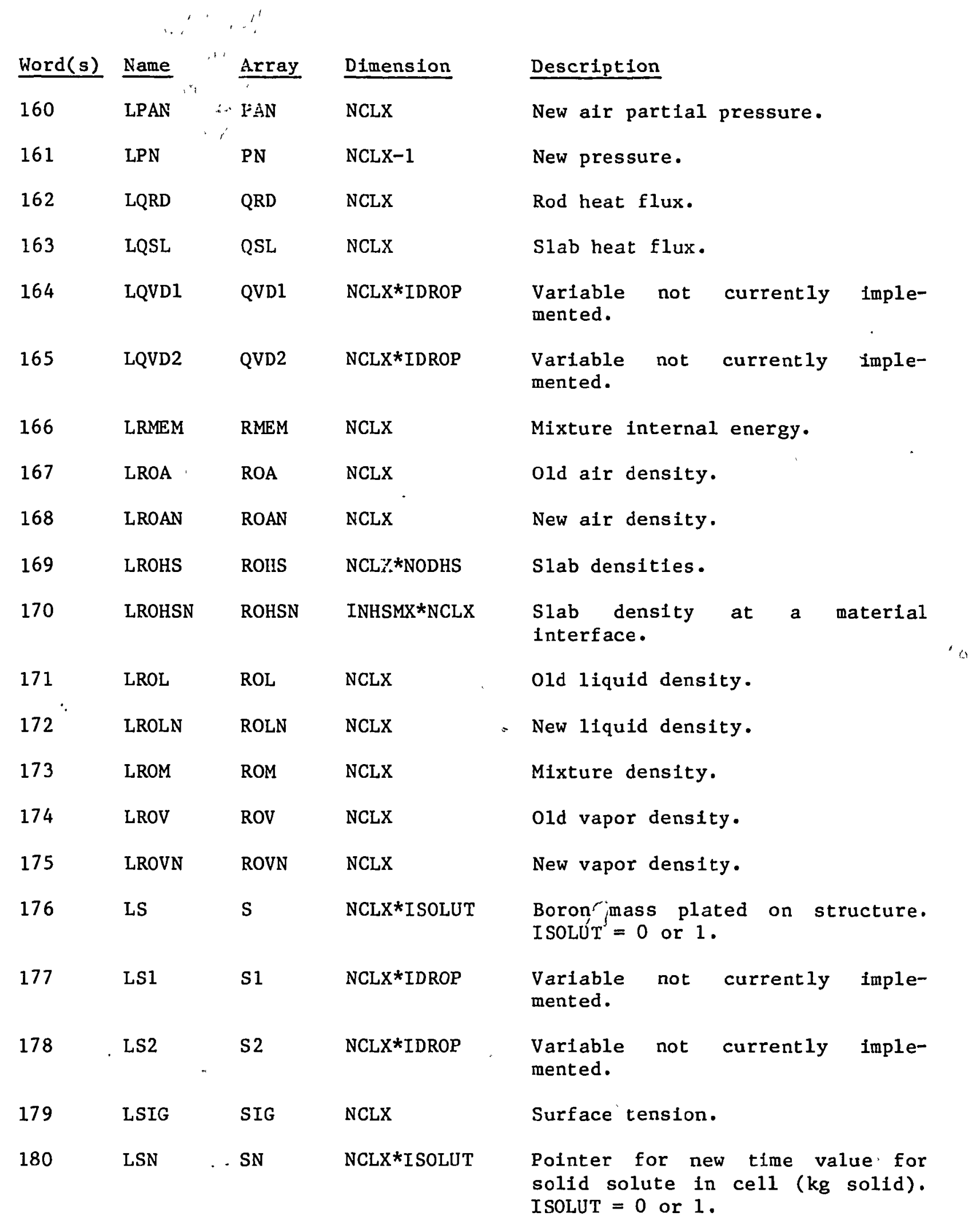




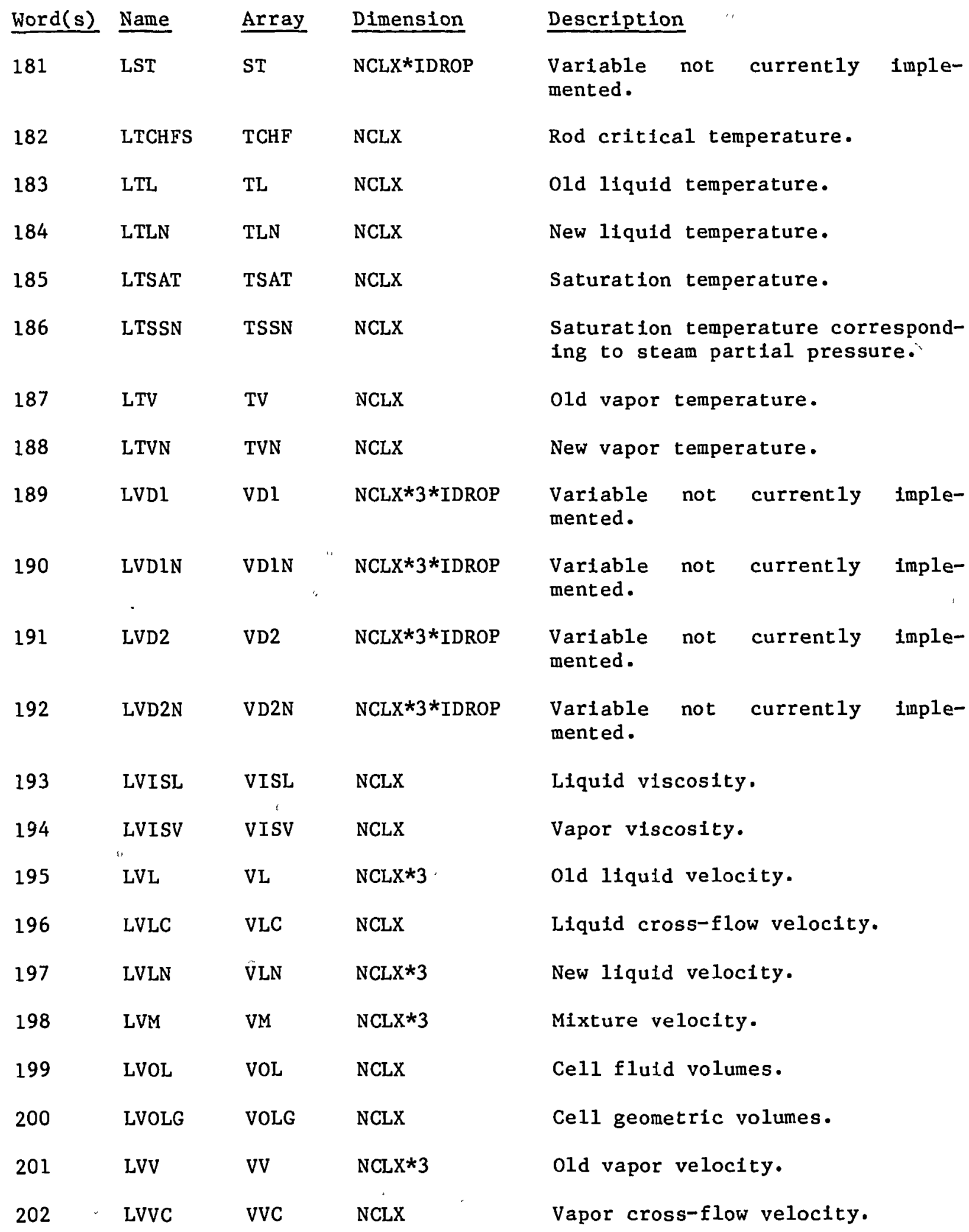




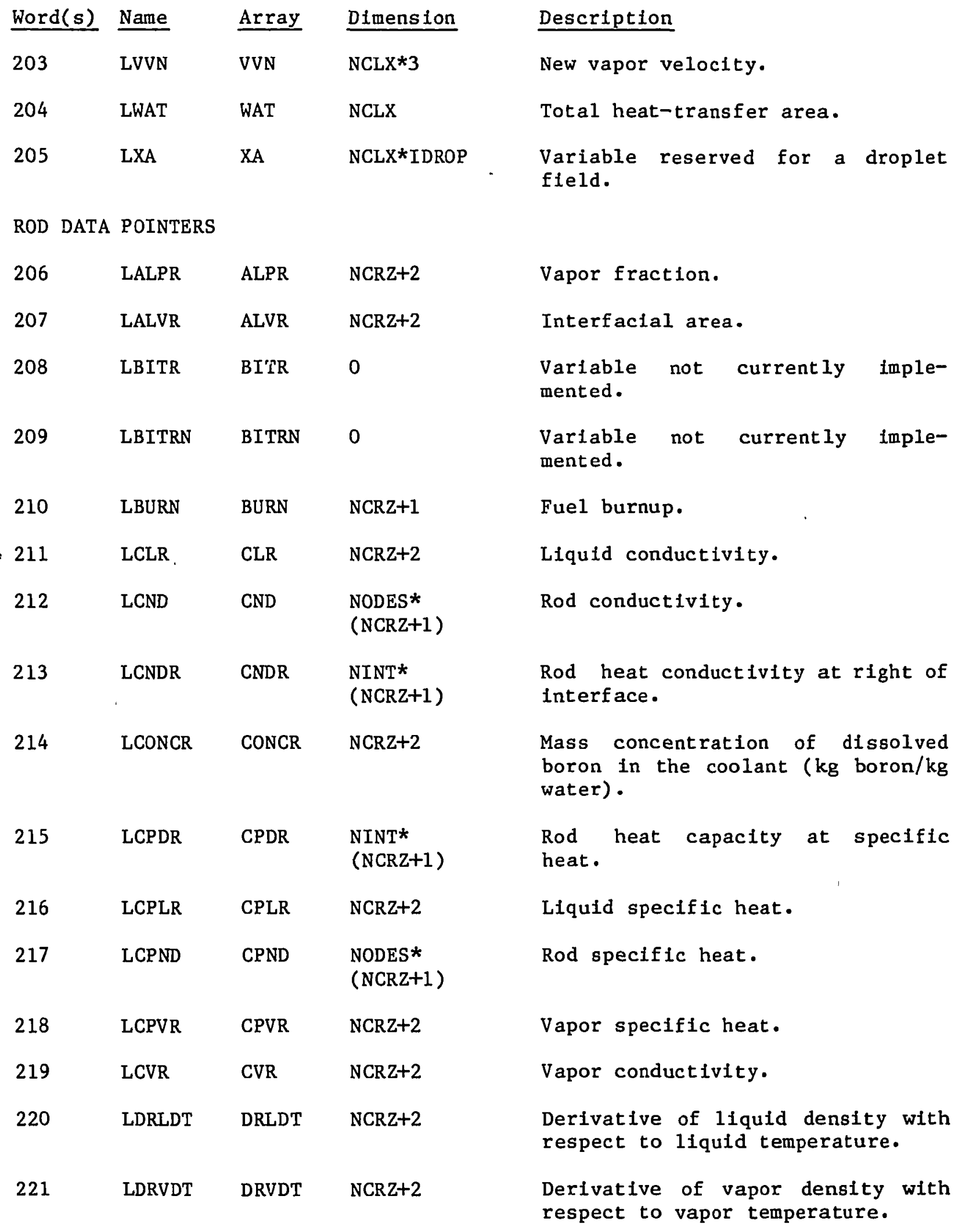




\begin{tabular}{|c|c|c|c|c|}
\hline Word (s) & Name & Array & Dimension & Description \\
\hline 222 & LDRZ & DRZ & NCRZ+1 & $\begin{array}{l}\text { old zirconium dioxide reaction } \\
\text { depth. }\end{array}$ \\
\hline 223 & LDRZN & DRZN & NCRZ+1 & $\begin{array}{l}\text { New zirconium dioxide reaction } \\
\text { depth. }\end{array}$ \\
\hline 224 & LEAR & EAR & NCRZ+2 & $\begin{array}{l}\text { Specific internal energy of the } \\
\text { noncondensable gas component. }\end{array}$ \\
\hline 225 & LELR & ELR & NCRZ+2 & old liquid internal energy. \\
\hline 226 & LEMIS & EMIS & $\begin{array}{l}\text { NODES* } \\
(\text { NCRZ+1) }\end{array}$ & Rod emissivity. \\
\hline 227 & LEVR & EVR & NCRZ+2 & old vapor internal energy. \\
\hline 228 & LFINAR & FINAR & NCRZ & old vapor internal energy. \\
\hline 229 & LHDR & $\mathrm{HDR}$ & NCRZ +2 & Rod-bundle hydraulic diameter. \\
\hline 230 & LHFGR & HFGR & NCRZ+2 & Latent heat of vaporization. \\
\hline 231 & LHGAMR & HGAMR & NCRZ & $\begin{array}{l}\text { Fine-mesh contribution to sub- } \\
\text { cooled bolling. }\end{array}$ \\
\hline 232 & LHGAP & HGAP & NCRZ+1 & Gap conductance. \\
\hline 233 & LHLAR & HLAR & NCRZ & $\begin{array}{l}\text { Sum of all products of the liquid } \\
\text { HTC and the heat-transfer area. }\end{array}$ \\
\hline 234 & LHLATR & HLATR & NCRZ & $\begin{array}{l}\text { Sum of all products of the liquid } \\
\text { HTC, the heat-transfer area, and } \\
\text { the wall temperature. }\end{array}$ \\
\hline 235 & LHLSR & HLSR & NCRZ+2 & $\begin{array}{l}\text { Specific enthalpy of the liquid } \\
\text { phase at saturation (corresponding } \\
\text { to saturation temperature at par- } \\
\text { tial pressure of steam. }\end{array}$ \\
\hline 236 & LHLVR & HLVR & NCRZ+2 & Interfacial HTC. \\
\hline 237 & LHRFL & HRFL & NZMAX & Fine-mesh liquid HTC. \\
\hline 238 & LHRFV & HRFV & NZMAX & Fine-mesh vapor HTC. \\
\hline 239 & LHRLL & HRLL & NCRZ+1 & Liquid HTC. \\
\hline 240 & LHRLV & HRLV & NCRZ+1 & Vapor HTC. \\
\hline
\end{tabular}




\begin{tabular}{|c|c|c|c|c|}
\hline Word(s) & Name & Array & Dimension & Description \\
\hline 241 & LHVAR & HVAR & NCRZ & $\begin{array}{l}\text { Sum of all products of the vapor } \\
\text { HTC and the heat-transfer area. }\end{array}$ \\
\hline 242 & LHVATR & HVATR & NCRZ & $\begin{array}{l}\text { Sum of all products of the vapor } \\
\text { HTC, the heat-transfer area, and } \\
\text { the wall temperature. }\end{array}$ \\
\hline 243 & LHVSR & HVSR & NCRZ+2 & $\begin{array}{l}\text { Specific enthalpy of the steam } \\
\text { (not gas) at saturation (at } \\
\text { partial pressure of steam and } \\
\text { saturation temperature). }\end{array}$ \\
\hline 244 & LIDHT & IDHT & NZMAX & Heat-transfer cell identifier. \\
\hline 245 & LIDRGR & IDRGR & NCRZ+2 & Flow-regime flag. \\
\hline 246 & LIHTF & IHTF & NZMAX & $\begin{array}{l}\text { Fine-mesh heat-transfer } \\
\text { flag. }\end{array}$ \\
\hline 247 & LNOHT & NOHT & 1 & $\begin{array}{l}\text { Number of rows of heat-transfer } \\
\text { nodes for each rod. }\end{array}$ \\
\hline 248 & LPAR & PAR & $\mathrm{NCRZ}+2$ & $\begin{array}{l}\text { Partial pressure of the noncon- } \\
\text { densable gas component. }\end{array}$ \\
\hline 249 & LPGAP & PGAP & NCRZ+1 & Gap local gas pressure. \\
\hline 250 & LPINT & PINT & NCRZ+1 & Pellet-cladding contact pressure. \\
\hline 251 & LPLDV & PLDV & NCRZ & Pellet dish volume. \\
\hline 252 & LPR & PR & NCRZ+2 & Coolant pressure. \\
\hline 253 & LQWRX & QWRX & NCRZ+1 & Meta1-water reaction heat source. \\
\hline $254:$ & LRADR & RADR & $\begin{array}{l}\text { NODES* } \\
(\mathrm{NCRZ}+1)\end{array}$ & old radial node positions. \\
\hline 255 & LRADRN & RADRN & $\begin{array}{l}\text { NODES* } \\
(\mathrm{NCRZ}+1)\end{array}$ & New radial node positions. \\
\hline 256 & LRDHLO & RDHLO & NCRZ & $\begin{array}{l}\text { Variable not currently } \\
\text { mented. }\end{array}$ \\
\hline 257 & LRDHLR & RDHLR & NCRZ & Liquid HTC. \\
\hline 258 & LRDHVO & RDHVO & NCRz & $\begin{array}{l}\text { Variable not, currently } \\
\text { mented. }\end{array}$ \\
\hline
\end{tabular}




\begin{tabular}{|c|c|c|c|c|}
\hline Word(s) & Name & Array & Dimension & Description \\
\hline 259 & LRDHVR & RDAVR & NCRZ & Vapor HTC. \\
\hline 260 & LRDTLR & RDTLR & NCRZ & $\begin{array}{l}\text { Average-rod wall temperature seen } \\
\text { by liquid. }\end{array}$ \\
\hline 261 & LRDTVR & RDTVR & NCRZ & $\begin{array}{l}\text { Average-rod wall temperature seen } \\
\text { by vapor. }\end{array}$ \\
\hline 262 & LRFT & RFT & NODES*NZMAX & old fine-mesh rod temperatures. \\
\hline 263 & LRFTN & RFTN & NODÉS*NZMAX & New fine-mesh rod temperatures. \\
\hline 264 & LRND & RND & $\begin{array}{l}\text { NODES* } \\
(\text { NCRZ+1) }\end{array}$ & Rod density. \\
\hline 265 & LRNDR & RNDR & $\begin{array}{l}\text { NINT* } \\
(\operatorname{NCRZ}+1)-1\end{array}$ & $\begin{array}{l}\text { Rod density at right of } \\
\text { interface. }\end{array}$ \\
\hline 266 & LROAR & ROAR & NCRZ+2 & $\begin{array}{l}\text { Density of the noncondensable gas } \\
\text { component. }\end{array}$ \\
\hline 267 & LROLR & ROLR & NCRZ+2 & Liquid density. \\
\hline 268 & LROMR & ROMR & NCRZ+2 & Mixture density. \\
\hline 269 & LROVR & ROVR & NCRZ+2 & Vapor density. \\
\hline 270 & LRPOWF & RPOWE & NODES & Rod power density. \\
\hline 271 & LSIGR & SIGR & $\mathrm{NCRZ}+2$ & Surface tenston. \\
\hline 272 & LSR & SR & NCRZ+2 & $\begin{array}{l}\text { Amount of plated out boron in cell } \\
\left(\mathrm{kg} \text { boron } \mathrm{m}^{-3}\right) \text {. }\end{array}$ \\
\hline 273 & LTCHFF & TCHFF & NZMÁX & $\begin{array}{l}\text { Fine-mesh wall temperature at } \mathrm{CHF} \\
\text { point. }\end{array}$ \\
\hline 274 & LTCHFR & TCHFR & NCRZ & Wa11 temperature at CHF point. \\
\hline 275 & LTLR & TLR & $\mathrm{NCRZ}+2$ & Liquid temperature. \\
\hline 276 & LTSATR & TSATR & NCRZ+2 & Saturation temperature. \\
\hline 277 & LTSSNR & TŚSSNR & NCRZे+2 & $\begin{array}{l}\text { Saturation temperature corre- } \\
\text { sponding to partial pressure of } \\
\text { steam. }\end{array}$ \\
\hline 278 & LTVR & TVRR & NCRZ +2 & Vapor temperature. \\
\hline
\end{tabular}




\begin{tabular}{|c|c|c|c|c|}
\hline Word(s) & Name & Array & Dimension & Description \\
\hline 279 & LVISLR & VISLR & $\mathrm{NCRZ}+2$ & Liquid viscosity. \\
\hline 280 & LVISVR & VISVR & NCRZ+2 & Vapor viscosity. \\
\hline 281 & LVLCR & VLCR & NCRZ+2 & Liquid cross-flow velocity. \\
\hline 282 & LVL2R & VLZR & NCRZ+2 & Axial liquid velocity. \\
\hline 283 & LVMZR & VMZR & NCRZ+2 & Axial mixture velocity. \\
\hline 284 & LVOLR & VOLR & $\mathrm{NCRZ}+2$ & $\begin{array}{l}\text { Pointer variable for the fluid } \\
\text { volume in axial mesh cells associ- } \\
\text { ated with the fuel rod. }\end{array}$ \\
\hline 285 & LVVCR & VVCR & NCRZ+2 & Vapor cross-flow velocity. \\
\hline 286 & LVVZR & VVZR & NCRZ+2 & Axial vapor velocity. \\
\hline 287 & LWATR & WATR & NCRZ & Total rod heat-transfer area. \\
\hline 288 & LZHT & $\mathrm{ZHT}$ & NZMAX & $\begin{array}{l}\text { Axial location of heat-transfer } \\
\text { node. }\end{array}$ \\
\hline $289-300$ & PTDUM & -- & - & Dummy variable. \\
\hline C. & LDAT & SEL & a Table & \\
\hline
\end{tabular}


REFERENCES

1. G. Kocomustafaogullari, "Thermo-Fluid Dynamics of Separated Two-Phase Flow," Ph.D. Thesis, School of Mechanical Engineering, Georgia Institute of Technology, At lanta, Georgia (December 1971).

2. M. Ishi1, Thermo-Fluid Dynamic Theory of Two-Phase Flow, Collection de 1a Direction des Etudes et Recherches D”Electricite de France, Eyrolles, Paris (1975).

3. F. H. Harlow and A. A. Amsden, "A Numerical Fluid Dynamics Calculation Method for All Flow Speeds," J. Comput. Phys. 8 , 197 (1971).

4. F. H. Harlow and A. A. Amsden, "KACHINA: An Eulerian Computer Program for Multifield Fluid Flows," Los Alamos Scientific Laboratory report LA-5680 (December 1975).

5. D. R. Liles and W. H. Reed, "A Semi-Implicit Method for Two-Phase Fluid Dynamics," J. Comput. Phys. 26, No. 3, 390-407 (1978).

6. J. H. Mahaffy, "A Stability-Enhancing Two-Step Method for One-Dimensional Two-Phase Flow," Lịs Alamos Scientific Laboratory report LA-7951-MS (August 1979).

7. J. H. Mahaffy, "A Stability-Enhancing Two-Step Method for Fluid Flow Calculations," J. Comput. Phys. 46, 329-341 (1982).

8. J. G. Collier, Convective Boiling and Condensation (McGraw-Hill Book Company, New York, New York, 1972).

9. W. M. Rohsenow and H. Y. Chot, Heat, Mass, and Momentum Transfer (Prentice-Hall Inc., Englewood Clifís, New Jersey, 1961).

10. C. W. Hirt and N. C. Romero, "Application of a Drift-Flux Model To Flashing In Straight Pipes," Los Alamos Scientific Laboratory report LA-6005-MS (July 1975).

11. G. W. Govier and A. Aziz, The Flow of Complex Mixtures in Pipes (Van Nostrand-Rheinhold Co., New York, New York, 1972).

12. B. S. Massey, Mechanics of Fluids (D. Van Nostrand Co., New York, New York, 1968).

13. V. L. Streeter, Fluid Mechanics (McGraw-Hill Book Company, New York, New York, 1966).

14. "Flow of Fluids," Crane Company, Technical Paper 409 (May 1942).

15. S. Lekach, "Development of a Computer Code for Thermal Hydraulics of Reactors ('THOR)," Brookhaven National Laboratory Quarterly Progress report BNL-19978 (1975). 
-16. C. J. Crowley, J. A. Block, and C. N. Cary, "Downcomer Effects in a 1/15-Scale PWR Geometry: Experimental Data Report," Creare, Inc. report NUREG-0281 (May 1977).

17. W. C. Rivard and M. D. Torrey, "Numerical Calculation of Flashing from Long Pipes Using a Two-Field Model," Los Alamos Scientific Laboratory report LA-6104-MS (November 1975).

18. K. Lee and D. J. Ryley, "The Evaporation of Water Droplets in Superheated Steam," ASME paper 68-HT-11 (1968).

19. I. Kataoka and M. Ishii, "Mechanism and Correlation of Droplet Entrainment and Deposition in Annular Two-Phase Flow," Argonne National Laboratory report ANL-82-44, NUREG/CR-2885 (July 1982).

20. G. B. Wallis, One-Dimensional Two-Phase Flow (McGraw-Hill Book Inc. New York, New York, 1969) Nov. 1978-0ct. 1979 (January 1980).

21. V. P. Isachenko, "Heat Transfer in Condensation in Turbulent Jets," Teploenergetika No. 2, pp. 7-10 (1976).

22. Y. Taitel and A. E. Dukler, "A Model for Predicting Flow Regime Transitions in Horizontal and Near Horizontal Gas-Liquid Flow," AIChE Journal 22, No. 1, 47-55 (1976).

23. P. J. Roache, Computational Fluid Dynamics, (Hermosa Publishers, Albuquerque, NM, $1 \overline{972) . ~}$

24. G. D. Spriggs, "Heat Slab Formulation for TRAC Input," Q-7-83-568, Los Alamos National Laboratory document to "TRAC Distribution (December 16, 1982, Revised January 1, 1983).

25. G. Yadigaroglu, "The Reflooding Phase of the LOCA in PWRs. Part I: Core Heat Transfer and Fluid Flow," Nucl. Saf. 19 (1978).

26. "Reactor Safety Research Progran, Quarterly Report for the Period July 1--September 30, 1978," Battelle Pacific Northwest Laboratories report PNL-2653-3, NUREG/CR-0546 (1978).

27. L. S. Tong and J. Weisman, Thermal Analysis of Pressurized Water Reactors, Second Edition (American Nuclear Soclety, LaGrange Park, Illinois, 1979).

28. P. E. MacDonald and J. Weisman, "Effect of Pellet Cracking on Light Water Reactor Fuel Temperatures," Nuc1. Technol. 31, 357-366 (1976).

29. B. A. Boley and J. H. Weiner, Theory of Thermal Stresses (John Wiley and Sons, Inc., New York, New York, 1960).

30. J. V. Cathcart, "Quarterly Progress Report on the Zirconium Metal-Water Oxidation Kinetics Program," Oak Ridge National Laboratory report ORNL/NUREG/TM-41 (August 1976). 
31. "MATPRO-Version 11: A Handbook of Materials Propertles for Use in the Analysis of Light Water Reactor Fuel Rod Behavior," Idaho National Engineering Laboratory report TREE-1280, NUREG/CR-0497 (February 1979).

32. J. C. Chen, "A Correlation for Boiling Heat Transfer of Saturated Fluids in Convective Flow," ASME paper 63-HT-34 (1963).

33. J. P. Holman, Heat Transfer, Third Edition (McGraw-Hill Book Company, New York, New York, 1972).

34. T. A. Bjornard and P. Griffith, "PWR Blowdown Heat Transfer," in Thermal and Hydraulic Aspects of Nuclear Reactor Safety, Vol. 1 (American Society of Mechanical Engineers, New York, New York, 1977), pp. 17-41.

35. L. A. Bromley, "Heat Transfer in Stable Film: Boiling," Chem. Eng. Prog. 46, 221-227 (May 1950).

36. S. S. Kutateladze, "Heat Transfer During Film Boiling," in Heat Transfer in Condensation and Boiling, Atomic Energy Commission report AEC-TR-3770 (1952).

37. R. P. Forslund and W. M. Rohsenow, "Dispersed Flow Film Boiling," J. Heat Trans. 90, 399-407 (November 1968).

38. W. H. McAdams, Heat Transmission, Third Edition (McGraw-Hill Book Company, New York, New York, 1954).

39. R. S. Dougall and W. M. Rohsenow, "Film Boiling on the Inside of Vertical Tubes with Upward Flow of the Fluid at Low Qualities," Massachusetts Institute of Technology Mechanical Engineering report 9079-26 (1963).

40. L. Biasi, G. C. Clerici, S. Garribba, R. Sala, and A. Tozzi, "Studies on Burnout: Part 3," Energ. Nucl. (Milan) 14, 530-536 (1967).

41. F. B. Hildebrand, Introduction to Numerical Analysis (McGraw-Hill Book Company, New York, New York, 1974).

42. R. E. Henry, "A Correlation for the Minimum Film Boiling Temperature," AIChE Symposium Series 138, 81-90 (1974).

43. J. J. Kaganove, "Numerical Solution of the One-Group Space-Independent Reactor Kinetics Equations for Neutron Density Given the Excess Reactivity," Argonne National Laboratory report AN,-6132 (1960).

44. "American National Standard for Decay Heat Power in Light Water Reactors," American Nuclear Society publication ANSI/ANS-5.1 (1979).

45. M. M. Giles and J. D. Milton, TRAC-BD1 Control System Model, TRAC-BWR Completion report WR-CD-81-056 (November 1981).

46. H. L. Harrison and J. G. Bollinger, Introduction to Automatic Control (International Textbook Company, Scranton, Pennsylvania, 1964), p. 144 . 
47. V. L. Streeter and E. B. Wylie, Hydraulic Transients (McGraw-Hill Book Company, New York, New York, 1967), pp. 151-160.

48. "RELAP4/MOD5: A Computer Program for Transient Thermal-Hydraulic Analysis of Nuclear Reactors and Related Systems," Vol. 1, Idaho National Engineering Laboratory report ANCR-NUREG-1335 (September 1976).

49. D. J. Olson, "Experiment Data Report for Single- and Two-Phase SteadyState Tests of the 1-1/2-Loop MOD-1 Semiscale System Pump," Aerojet Nuclear Company report ANCR-1150 (May 1974).

50. G. G. Loomis, "Intact Loop Pump Performance During the Semiscale MoD-1 Isothermal Test Series," Aerojet Nuclear Company report ANCR-1240 (October 1975).

51. D. J. Olson, "Single- and Two-Phase Performance Characteristics of the MOD-1 Semiscale Pump Under Steady-State and Transient Fluid Conditions," Aerojet Nuclear Company report ANCR-1165 (October 1974).

52. Douglas L. Reeder, "LOFT System and Test Description (5.5-Ft. Nuclear Core 1 LOCES)," EG\&G Idaho, Inc. report TREE-1208, NUREG/CR-0247, pp. 161-166 (July 1978).

53. J. Kenneth Salisbury, Steam Turbines and their Cycles (Robert E. Krieger Publishing Company, Huntington, New York, 1974).

54. R. Fi' Farman and C. G. Motloch, "RETRAN Turbine and Condensing Heat Transfer Models," proc. 1st Int., RETRAN Conf,, Seattle, Washington, September 22-24, 1980, Electric Power Research Institute report EPRI WS-80-150, pp. 5-1 through 5-25.

55. W. A. Coffman and L. L. Lynn, "WATER: A Large Range Thermodynamic and Transport Water Property FORTRAN-IV Computer Program," Bettis Atomic Power Laboratory report WAPD-TM-568 (December 1966).

56. D. L. Hagrman, G. A. Reymann, and R. E. Mason, "MATPRO-Version 11 (Revision 1): A Handbook of Material Properties for Use in the Analysis of Light Water Reactor Fuel Rod Behavior," EG\&G Idaho, Inc. report TREE-1280, Rev."1, NUREG/CR-049 (February 1980).

57. "MATPRO-Version 09: A Handbook of Materials Properties for Use in the Analysis of Light Water Reactor Fuel Rod Behavior," Idaho National Engineering Laboratory report TREE-NUREG-1005 (December 1976). w.

58. Y. S. Touloukian, Editor, Thermophysical Properties of High Temperature Solid Materials (MacMillan Co., New York, 1967).

59. "A Prediction of the SEMISCALE Blowdown Heat Transfer Test S-02-8 (NRC Standard Problem Five)," Electric Power Research Institute report EPRI-NP-212 (October 1976). 
60. W. L. Kirchner, "Reflood Heat Transfer In A Light Water Reactor," US Nuclear Regulatory Commission report NUREG-0106, Vols. I and II (August 1976).

61. J. C. Spanner, Editor, "Nuclear Systems Materials Handbook--Vo1. 1 Design Data," Hanford Engineering Development Laboratory report TID-26666 (1976).

62. "Properties for LMFBR Safety Analysis," Argonne National Laboratory report ANL-CEN-RSD-76-1 (1976).

63. V. H. Ransom and J. A. Trapp, "The RELAP5 Choked Flow Model and Application to a Large Scale Flow Test," Proceedings of the ANS/ASME/NRC International Topical Meeting on Nuclear Reactor Thermal-Hydraulics, Saratoga Springs, New York, 0ctober 5-8, 1980, pp. 799-819.

64. "RELAP5/MOD1 Code Manual, Volume 1: System Models and Numerical"Methods," Idaho National Engineering Laboratory report EGG-2070 DRAFT, Rev. 1, NUREG/CR-1826 (March 1981).

65. R. T. Lahey, Jr., "RPI Two-Phase Flow Modeling Program," presented at the Fifth Water Reactor Safety Research Information Meeting, Washington, DC (November 7-11, 1977).

66. 0. C. Jones, Jr., "Flashing Inception in Flowing Liquids," Brookhaven National Laboratory report BNL-NUREG-51221 (1980).

67. Douglas G. Hall and Linda S. Czapary, "Tables of Homogeneous Equilibrium Critical Flow Parameters for Water in SI Units," Idaho National Engineering Laboratory report EGG-2056 (September 1980).

68. "RELAP 4/MOD6, A Computer Program for Transient Thermal-Hydraulic Analysis of Nuclear Reactors and Related Systems," Idaho National Engineering Laboratory report CDAP TR 003 (January 1978).

69. "Results from Test 4, The Marviken Full-Scale Critical Flow Tests," Joint Reactor Safety Experiments in the Marviken Power Station, Sweden, Marviken report MXC-204 (September 1979).

70. "Results from Test 24, The Marviken Full-Scale Critical Flow Tests," Joint Reactor Safety Experiments in the Marviken Power Station, Sweden, Marviken report MXC-224 (September 1979).

71. A. R. Edwards and T. P. O-Brien, "Studies of Phenomena Connected with the Depressurization of Water Reactors," J. Br. Nucl. Energ. Soc. 9 , April 1970, pp. 125-135.

72. Doyle L. Batt and Janice M. Carpenter, "Experiment Data Report for LOFT Anticipated Transient Experiments L6-1, L6-2, and L6-3," Idaho National Engineering Laboratory report EGG-2067, NUREG/CR-1797 (December 1980).

73. Clinton D. Keeler, "Best Estimate Prediction for LOFT Nuclear Experiments L6-1, L6-2, L6-3, and L6-5," Idaho National Engineering Laboratory report EGG-LOFT-5161 (October 1980). 
74. Hannu 01likkala, "Best Estimate Prediction for LOFT Anticipated Transient Slow and Fast Rod Withdrawal Experiment L6-8B," Idaho National Engineering Laboratory report EGG-LOFT-5983 (August 1982).

75. Douglas L. Reeder, "Quick-Look Report on LOFT Nuclear Experiments L6-1, L6-2, and L6-3," Idaho National Engineering Laboratory report EGG-LOFT-5270 (October 1980).

76. H. 01likkala and S. R. Behling, "Posttest Analysis of LOFT Anticipated Transient Experiments L6-1, L6-2, L6-3, and L6-5," Idaho National Engineering Laboratory report EGG-LOFT-6159 (January 1983).

77. Douglas L. Reeder, "LOFT System and Test Description (5.5-ft Nuclear Core 1 LOCES)," EG\&G Idaho, Inc. report TREE-1208, NUREG/CR-0247 (July 1978).

78. "TRAC-PD2--An Advanced Best-Estimate Computer Program for Pressurized Water Reactor Loss-of-Coolant Accident Analysis," Los Alamos National Laboratory report LA-8709-MS, NUREG/CR-2054 (April 1981). 


\section{DISTRIBUTION}

Nuclear Regulatory Commission, R4, Laurel, Maryland 298

Technical Information Center, Oak Ridge, Tennessee

Los Álamos National Laboratory, Los Alamos, New Mexico

Special Distribution to TRAC User's Group 


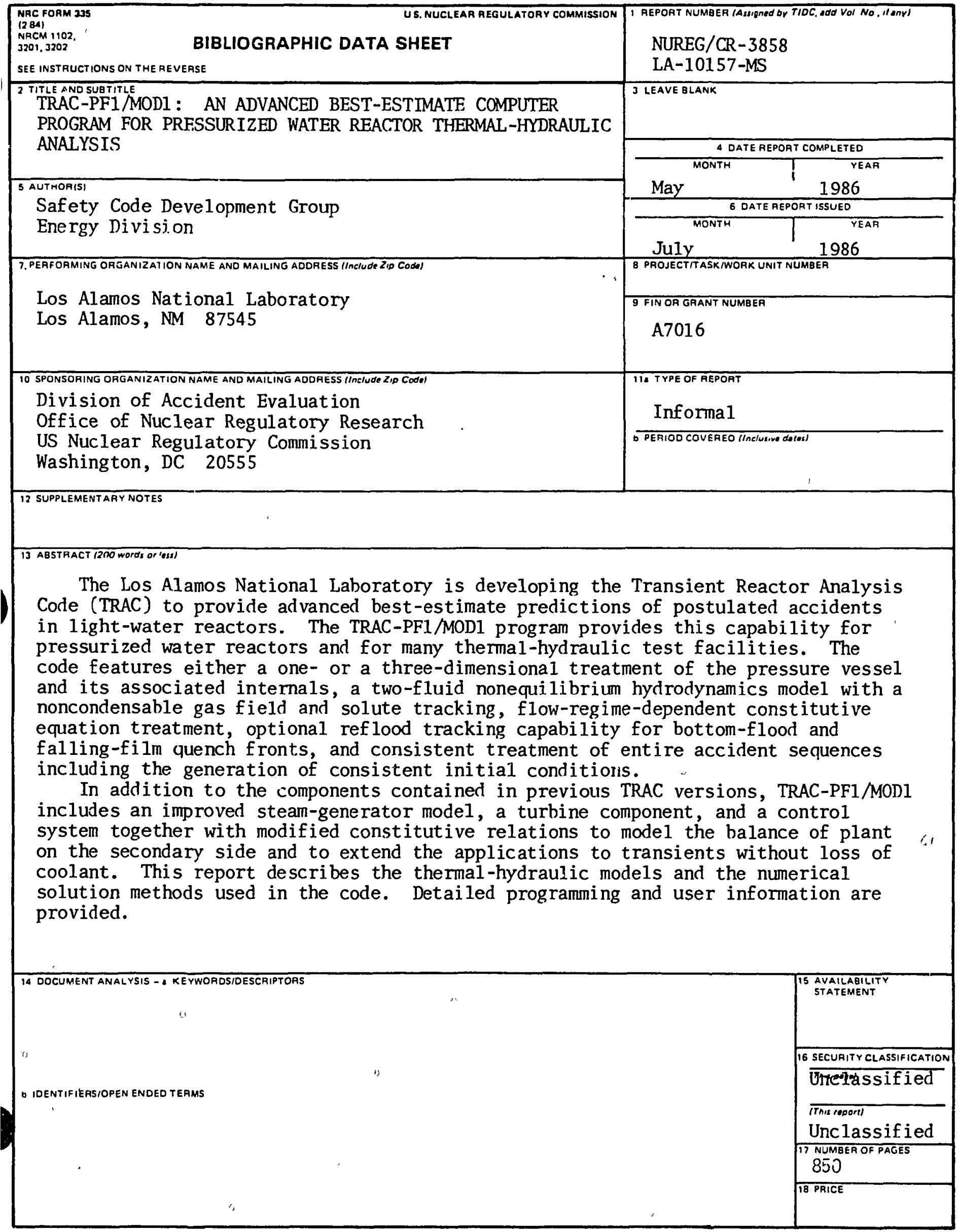

